\title{
Value Distribution of Meromorphic Functions
}

\author{
Anatoly A. Goldberg \\ Iossif V. Ostrovskii \\ Department of Mathematics and Computer Science, Bar-Ilan Uni- \\ VERSITY, RAMAT GAN, 52900 ISRAEL \\ Department of Mathematics, Bilkent University, 06800 Bilkent \\ ANKARA, Turkey \\ E-mail address: iossif@fen.bilkent.edu.tr
}


1991 Mathematics Subject Classification. Primary 30D30, 30D35;

Secondary 30D20, 30D15

Key words and phrases. Meromorphic functions, Entire functions, Nevanlinna theory, Value distribution theory, Deficient values, Asymptotic values, Riemann surfaces.

ABSTRACT. This ia a translation of the monograph originally published in 1970. It contains a comprehensive exposition of the Nevanlinna theory of meromorphic functions of one complex variable, with the emphasis on the detailed study of deficiencies, distribution of values with respect to arguments and asymptotic properties of meromorphic functions. A self-contained exposition of the inverse problem for meromorphic functions of finite order with finitely many deficiencies is given in full detail. Many of the results included in the book belong to the authors, and were previously available only in journal publications.

The exposition is completely self-contained: the only prerequisite is an undergraduate course in one complex variable.

There are two appendices: the first contains Govorov's proof of the Paley conjecture, and the second a survey of the results obtained after 1970, with extensive bibliography. 
Dedicated to our teachers: Lev Israilevich Volkovyskii and Boris Yakovlevich Levin 


\section{Contents}

Preface to the second edition vii

Preface to the first edition $\quad$ ix

Chapter 1. Characteristics of the Behavior of a Meromorphic Function and the First Fundamental Theorem 1

1. Auxiliary results

2. The Nevanlinna, Poisson-Jensen, and Shimizu-Ahlfors formulae 5

3. The Carleman and Levin formulae 10

4. The Nevanlinna characteristics. The first fundamental theorem of the value distribution theory 13

5. The angular Nevanlinna characteristics, Tsuji chracteristics and related analogs of the first fundamental theorem 23

6. Some relations between the characteristics 28

7. Connection between the growth of the modulus of a meromorphic function and the growth of its characteristic $T(r, f) \quad 37$

Chapter 2. Meromorphic Functions of Finite Order 43

1. The growth scale for meromorphic functions 43

2. Proximate order 49

3. Infinite products 54

4. Hadamard and Lindelöf theorems $\quad 57$

5. Some examples 66

$\begin{array}{lll}\text { Chapter 3. The second fundamental theorem } & 87\end{array}$

1. Lemma on the logarithmic derivative $\quad 87$

2. The Nevanlinna second fundamental theorem 95

3. Analogues of the second fundamental theorem for angular Nevanlinna characteristics and for Tsuji characteristics 104

Chapter 4. Deficient values 113

1. Exceptional values 113

2. The set of Valiron exceptional values 116

3. Deficiency relation 121

4. The structure of the set of deficient values 122

5. Entire functions having infinitely many deficient values 132

6. Stability of deficiencies 149

Chapter 5. Asymptotic properties of meromorphic functions and deficiencies 171

$\begin{array}{ll}\text { 1. Asymptotic values } & 171\end{array}$

$\begin{array}{ll}2 . & \text { Non-asymptotic deficient values } \\ & 180\end{array}$ 
3. Meromorphic functions of order less than 1/2 201

4. Bounds for the sum of two deficiencies and related problems 218

5. The growth of a meromorphic function with exceptional values 238

6. Meromorphic functions representable by series of simple fractions $\quad 252$

Chapter 6. Value distribution with respect to the arguments 261

1. Meromorphic functions with separated poles and zeros 261

2. Meromorphic functions with poles and zeros located close to a system $\begin{array}{lr}\text { of rays } & 279\end{array}$

3. Proofs of main Theorems 2.3 and $2.7 \quad 298$

4. Meromorphic functions with poles and zeros located in small angles 310

5. Entire functions with derivatives only vanishing close to the real axis 322

Chapter 7. Applications of Riemann surfaces to value distribution 337

1. Geometric meaning of deficient and index values 337

2. Quasiconformal mappings 341

3. The Teichmüller theorem 351

4. Riemann surfaces of the class $F_{q}$ and line complexes 358

5. Statement of the inverse problem of the value distribution theory.

Riemann surfaces with finitely many logarithmic ends $\quad 375$

6. Almost periodic ends 383

7. Riemann surfaces with finitely many almost periodic ends 399

8. The inverse problem of the value distribution theory 408

Appendix. On the magnitude of type of an entire function $\quad 427$

$\begin{array}{ll}\text { Appendix. Notes } & 437\end{array}$

$\begin{array}{ll}\text { Chapter } 1 & 437\end{array}$

$\begin{array}{ll}\text { Chapter } 2 & 437\end{array}$

$\begin{array}{ll}\text { Chapter } 3 & 439\end{array}$

$\begin{array}{lr}\text { Chapter } 4 & 439\end{array}$

Chapter $5 \quad 440$

Chapter $6 \quad 444$

$\begin{array}{ll}\text { Chapter } 7 & 447\end{array}$

$\begin{array}{lll}\text { Appendix. Bibliography } & 451\end{array}$

$\begin{array}{lll}\text { Appendix. Index } & 459\end{array}$

$\begin{array}{lll}\text { Appendix. Index } & 461\end{array}$

$\begin{array}{lll}\text { Appendix. } & \text { Index } & 463\end{array}$

Appendix A. A survey of some results after 1970 (by A. Eremenko and J. K. Langley) 465

$\begin{array}{lll}\text { Appendix. } & \text { Bibliography } & 479\end{array}$ 


\section{Preface to the second edition}

In this edition we corrected some errors and misprints and added references and footnotes related to recent achievements in the topics considered in the original edition. A survey of the results obtained since 1970 is in a separate appendix at the end of the book. This appendix has its own list of references. In the footnotes to the main text these references are marked with the letter A (like for example [A12]). 
Dedicated to our teachers: Lev Israilevich Volkovyskii and Boris Yakovlevich Levin 


\section{Preface to the first edition}

The origins of the value distribution theory of meromorphic functions go back to the classical theorems of Sokhotskii-Casorati (1868), Weierstrass (1876), Picard (1879). In the last decade of the XIX-th and the first two decades of the XX-th centuries, these theorems underwent further development through investigations on the zero distribution of entire functions carried out mainly by the French school (Hadamard, Borel, Valiron and others). The analytical machinery intrinsically connected with meromorphic functions was built in the 20-ies of the XX-th century by the Finnish mathematician Rolf Nevanlinna. After his work, the value distribution theory acquired, in some way, a complete form. The main classical results of the theory of entire functions have been included in Nevanlinna's theory in a natural way.

The results of Nevanlinna's theory, which, at the present time, can be regarded as classical, are considered in the first part of the present book, consisting of chapters I, II, III, and sec. 1-3 of IV. Aiming to help beginning mathematicians, we consider the subject here in detail. We foresee reproaches of more qualified readers in this respect.

While the first part of this monograph treats mostly results obtained before the 50-ies, the remainder is dedicated to modern research.

In spite of a certain completeness of the value distribution theory, the study of even some of the most classical problems has not been brought to an end. On the contrary, the study is conducted more and more extensively. A lot of important problems remain open, and new ones arise during the process of further investigations.

Achievements are so essential and diverse that they admit the free coexistence of several monographs on the theory of value distribution. In any case, the reader who is acquainted with Hayman's book [Hay64], will not find any substantial intersection with our book except for classical theorems which had been known as early as the 30-ies of XX century.

The value distribution theory of meromorphic functions occupies one of the central places in Complex Analysis. Numerous works are devoted to studying its connections with other areas of mathematics (topology, differential geometry, measure theory, potential theory and others); extending its inferences to wider classes of functions (meromorphic functions in arbitrary plane regions and Riemann surfaces, algebroid functions, functions of several variables, meromorphic curves), and also its applications, mainly to the analytic theory of differential equations.

In this book, the related topics are left aside, and the main attention is concentrated on the problems internal to the value distribution theory, which include the following problems:

(i) To what extent the main inferences of Nevanlinna's theory have final character 
and cannot be improved further;

(ii) What properties of Picard's exceptional values are preserved for a wider class of exceptional values considered in the value distribution theory;

(iii) Which are the connections between Nevanlinna's characteristics and other quantities characterizing asymptotic properties of entire and meromorphic functions;

(iv) Study of asymptotic properties and value distribution of meromorphic functions belonging to some special classes which are on one hand sufficiently narrow to give new information not implied by general theorems, and on the other hand sufficiently wide for being of interest for the general theory;

(v) Study of the value distribution with respect to arguments (not only with respect to moduli as in classical Nevanlinna theory).

We pay great attention to examples of functions with "exotic" properties. Without them the reader will get a restricted image of the theory under consideration. Examples of functions having unusual properties play in the theory a role as important as counterexamples do in Real Analysis.

Notation and Conventions. Functions meromorphic in the complex plane will be called meromorphic functions, unless otherwise explicitly stated. Uniform (absolute, or usual) convergence of a series or of an infinite product is understood as the convergence of the corresponding type of its remainder. Thus, behavior of a finite number of terms does not affect the convergence or its type.

If we consider a continuous function with a removable discontinuity, the discontinuity is assumed to be removed. For example $f(0)=1$ for $f(\lambda)=\pi \lambda \cot \pi \lambda$.

By $[\alpha]$ we denote the integer part of the number $\alpha$. Square brackets are used to separate expressions in the cases when it cannot cause any confusion. We also use square brackets for references. We denote the number $(|\alpha|+\alpha) / 2$ by $\alpha^{+}$.

The closure of the angle $\{\alpha<\arg z<\beta\}$ in the complex plane is denoted by $\{\alpha \leq \arg z \leq \beta\}$. Thus $0 \in\{\alpha \leq \arg z \leq \beta\}$.

Writing $\sum_{k} a_{k}$ or $\prod_{k} a_{k}$ we do not exclude the case when the sequence $\left\{a_{k}\right\}$ is finite or empty. In the latter case we assume $\sum_{k} a_{k}=0$ and $\prod_{k} a_{k}=0$.

Each chapter has its own numeration of formulas, the number (4.20) refers to formula number 20 in section 4 of the same chapter. Analogous numeration is used for theorems. When we refer to a formula or a theorem from another chapter, we refer to the chapter number also.

All of the facts related to the history of the problems under consideration along with references on the subject are placed in the Notes at the end of the book. References in the text are given only in the case where we refer to results lying beyond the scope of standard graduate courses in Real and Complex Analysis.

Note that results of sec. 3 and 5 of Chapter 1 and of sec. 3 of Chapter 3 are used in Chapter 6 only, and can be skipped in the first reading.

Preliminary versions of sec. 7,8 of Ch.I; Ch.II; sec. 1,2 of Ch.III; sec.1-4,6 of Ch.IV; sec 1,2 of Ch.V; Ch.VII; Appendix I have been written by A. Goldberg; sec. 1-5 of Ch.I, sec.3 of Ch.III, sec.5 of Ch.IV, sec.3-6 of Ch.V; Ch.VI have been written by I. Ostrovskii.

We express our deep gratitude to Nikolaj Govorov, Larisa Kudina, Victor Petrenko, David Potyagajlo, Elena Sergienko, and Stanislav Tushkanov for their various help in our work. Our special thanks to our editor Naum Landkof. 
Anatoly A. Goldberg and Iossif V. Ostrovskii 



\section{CHAPTER 1}

\section{Characteristics of the Behavior of a Meromorphic Function and the First Fundamental Theorem}

The basis of the value distribution theory of meromorphic functions consists of several formulae connecting the behavior of a meromorphic function with the distribution of its zeros and poles. At the beginning of this chapter we derive these formulae. Later, we use them to introduce the main characteristics of the behavior of a meromorphic function, and study connections between them. One of the most important connections is the celebrated theorem which R. Nevanlinna called "the first fundamental theorem of the value distribution theory".

\section{Auxiliary results}

Let $D$ be a bounded domain in the complex plane, whose boundary $\Gamma$ consists of finitely many piecewise-analytic curves; by $\frac{\partial}{\partial n}$ we shall denote the operator of differentiation in the direction of the inwardly directed normal vector to $\Gamma$, by $\Delta$ we denote the operator $\frac{\partial^{2}}{\partial x^{2}}+\frac{\partial^{2}}{\partial y^{2}}$.

We shall use the following formula:

$$
\iint_{D}(u \Delta v-v \Delta u) d \sigma=-\int_{\Gamma}\left(u \frac{\partial v}{\partial n}-v \frac{\partial u}{\partial n}\right) d s,
$$

where $u=u(x, y)$ and $v=v(x, y)$ are twice continuously differentiable in some domain containing the closure $\bar{D}$ of the domain $D, \sigma$ is the Lebesgue measure in the plane and $s$ is the one-dimensional Lebesgue measure (length) on $\Gamma$. This formula is usually called the second Green formula. It can be obtained from the well-known Green formula

$$
\iint_{D}\left(\frac{\partial Q}{\partial x}-\frac{\partial P}{\partial y}\right) d \sigma=\int_{\Gamma} P d x+Q d y
$$

in the following way. Let

We get the formula

$$
P=-u \frac{\partial v}{\partial y}, Q=u \frac{\partial v}{\partial x}
$$

$$
\iint_{D}\left(u \Delta v+\frac{\partial u}{\partial x} \frac{\partial v}{\partial x}+\frac{\partial u}{\partial y} \frac{\partial v}{\partial y}\right) d \sigma=\int_{\Gamma}-u \frac{\partial v}{\partial y} d x+u \frac{\partial v}{\partial x} d y
$$

which can be rewritten as

$$
\iint_{D}\left(u \Delta v+\frac{\partial u}{\partial x} \frac{\partial v}{\partial x}+\frac{\partial u}{\partial y} \frac{\partial v}{\partial y}\right) d \sigma=-\int_{\Gamma} u \frac{\partial v}{\partial n} d s
$$

Subtracting from (1.2) the formula which is obtained from (1.2) if we interchange $u$ and $v$, we get (1.1). 
We also need the notion of a Green function. The Green function of a domain $D$ is a function $G(\zeta, z)$, defined for $\zeta \in \bar{D}, z \in \bar{D}, \zeta \neq z$, and satisfying the following conditions:

(i) For each fixed $z \in D$

$$
G(\zeta, z)=\ln \frac{1}{|\zeta-z|}+h_{z}(\zeta)
$$

where $h_{z}(\zeta)$ is a function, harmonic in $D$ and continuous in $\bar{D}$.

(ii) If $\zeta \in \Gamma, z \in \bar{D}, \zeta \in \bar{D}$, and $z \in \Gamma$, then

$$
G(\zeta, z)=0 .
$$

The Green function exists and is unique. The uniqueness can be proved in the following way. Assuming that $G_{1}(\zeta, z)$ and $G_{2}(\zeta, z)$ are two Green functions of $D$, we choose $z \in D$ and consider the function $v(\zeta)=G_{1}(\zeta, z)-G_{2}(\zeta, z)$. The condition (i) implies that this function is harmonic in $D$ and continuous in $\bar{D}$, and the condition (ii) implies that it is equal to zero on $\Gamma$. By the maximum and the minimum principles for harmonic functions we get $v(\zeta)=0$, and, hence, $G_{1}(\zeta, z) \equiv G_{2}(\zeta, z)$.

We would like to observe that the condition (i) implies that there exists a disc $K_{z}$ centered at $z$ and such that $G(\zeta, z)>0$ for $\zeta \in K_{z}$. Using the minimum principle for $D \backslash K_{z}$, we conclude that $G(\zeta, z)>0$ for all $\zeta \in D, z \in D$.

We will not use the existence of the Green functions for multiply connected domains, for this reason we do not discuss this matter.

For a simply connected domain the existence of the Green function follows from classical results on conformal mappings. In fact, by the Riemann theorem there exists a conformal mapping $w=f_{z}(\zeta)$ which maps the region $D$ onto the disc $\{|w|<1\}$ in such a way that $f_{z}(z)=0$. By the theorem on the boundary behavior of conformal mappings the function $f_{z}$ is continuous on $\bar{D}$, and $\left|f_{z}(\zeta)\right|=1$ for $\zeta \in \Gamma$. Let

$$
G(\zeta, z)=\ln \frac{1}{\left|f_{z}(\zeta)\right|},
$$

it is easy to check that this function satisfies (i) and (ii).

Example 1. Let $D=\{|\zeta|<R\}$. Then

$$
f_{z}(\zeta)=\frac{R(\zeta-z)}{R^{2}-\zeta \bar{z}}, G(\zeta, z)=\ln \left|\frac{R^{2}-\zeta \bar{z}}{R(\zeta-z)}\right| .
$$

Example 2. Let $D=\{|\zeta|<R, \operatorname{Im} \zeta>0\}$. Then

$$
f_{z}(\zeta)=\frac{R(\zeta-z)}{R^{2}-\zeta \bar{z}} \frac{R^{2}-\zeta z}{R(\zeta-\bar{z})}, G(\zeta, z)=\ln \left|\frac{R^{2}-\zeta \bar{z}}{R(\zeta-z)} \frac{R(\zeta-\bar{z})}{R^{2}-\zeta z}\right|
$$

Suppose that $D$ is simply connected. Let $\gamma_{1}, \gamma_{2}, \ldots, \gamma_{p}$ be analytic curves forming $\Gamma$, let $A_{l}, l=1,2, \ldots, p$ be the common end point of $\gamma_{l}$ and $\gamma_{l+1}$ (we use the convention: $\left.\gamma_{p+1} \equiv \gamma_{1}\right)$, let $\pi \alpha_{l}\left(0<\alpha_{l} \leq 2\right), l=1,2, \ldots, p$, be the angles between $\gamma_{l}$ and $\gamma_{l+1}$. By the Riemann-Schwarz symmetry principle (see Privalov [Pri56], 
Chapter XII, $\S 2.4)$ the function $f_{z}$ has an analytic extension to a certain domain containing $\bar{D} \backslash\left\{A_{1}, \ldots, A_{p}\right\}$.

We shall assume (without mentioning this) that in the intersection of a sufficiently small neighborhood $U_{l}$ of $A_{l}$ with $D$ the function $f_{z}$ has a representation

$$
f_{z}(\zeta)=\left(\zeta-A_{l}\right)^{1 / \alpha_{l}} \varphi_{l}(\zeta)+w_{l},
$$

where $\varphi_{l}(\zeta)$ is an analytic function in $U_{l}, \varphi_{l}\left(A_{l}\right) \neq 0,\left|w_{l}\right|=1, l=1,2, \ldots, p$. We omit the discussion of the conditions on $D$ under which such a representation holds. For our purposes it is enough to observe that the domain $D=\{|\zeta|<R\}$ satisfies this condition since it is bounded by an analytic curve and there are no points $A_{l}$. The domain $D=\{|\zeta|<R, \operatorname{Im} \zeta>0\}$ also belongs to this class. In fact, in this case the points $A_{l}, l=1,2$, are the points $\zeta= \pm R, \alpha_{l}=\frac{1}{2}, l=1,2$. The equation (1.5) implies that $f_{z}(\zeta)$ is analytic at $\zeta= \pm R$ and $\frac{d}{d \zeta} f_{z}(\zeta)$ has simple zeros at $\zeta= \pm R$, hence the representation (1.6) holds.

Further we deal with the derivative $\frac{\partial G}{\partial n}$ with respect to $\zeta$ as a function of $z$. This derivative exists for all $\zeta \neq A_{l}(l=1, \ldots, p)$. Since $G(\zeta, z)>0$ for $\zeta \in D, z \in D$ and $G(\zeta, z)=0$ for $\zeta \in \Gamma, z \in D$, then $\frac{\partial G}{\partial n} \geq 0$. Along the curve $\Gamma$ the following equation is satisfied:

$$
\frac{f_{z}^{\prime}(\zeta)}{f_{z}(\zeta)} d \zeta=i \frac{\partial G}{\partial n} d s\left(\zeta \neq A_{l}, l=1, \ldots, p\right) .
$$

In fact, computing the differential of $d \ln f_{z}(\zeta)$ along the curve $\Gamma$ and using the Cauchy-Riemann conditions, we get

$$
\begin{aligned}
d \ln f_{z}(\zeta) & =\frac{\partial}{\partial s} \ln f_{z}(\zeta) d s=\left(\frac{\partial}{\partial s} \ln \left|f_{z}(\zeta)\right|+i \frac{\partial}{\partial s} \arg f_{z}(\zeta)\right) d s \\
& =i \frac{\partial}{\partial s} \arg f_{z}(\zeta) d s=-i \frac{\partial}{\partial n} \ln \left|f_{z}(\zeta)\right| d s=i \frac{\partial G}{\partial n} d s
\end{aligned}
$$

The equation (1.7) sometimes can be used to find $\frac{\partial G}{\partial n}$.

EXAmPle 1. Let $D=\{\zeta:|\zeta|<R\}$, then

$$
\frac{\partial G}{\partial n} d s=-i\left[\ln \frac{R(\zeta-z)}{R^{2}-\zeta \bar{z}}\right]^{\prime} d \zeta=\frac{R^{2}-|z|^{2}}{|\zeta-z|^{2}} d \theta, \zeta=R e^{i \theta} .
$$

The function $\frac{R^{2}-|z|^{2}}{|\zeta-z|^{2}}$ is called the Poisson kernel. Observe that

$$
\frac{R^{2}-|z|^{2}}{|\zeta-z|^{2}}=\frac{R^{2}-r^{2}}{R^{2}+r^{2}-2 R r \cos (\varphi-\theta)}=\operatorname{Re} \frac{\zeta+z}{\zeta-z}, \zeta=R e^{i \theta}, z=r e^{i \varphi} .
$$

EXAmple 2. Let $D=\{\zeta:|\zeta|<R, \operatorname{Im} \zeta>0\}$. Then

$$
\frac{\partial G}{\partial n} d s=-i\left[\ln \left(\frac{R(\zeta-z)}{R^{2}-\zeta \bar{z}} \frac{R^{2}-\zeta z}{R(\zeta-\bar{z})}\right)\right]^{\prime} d \zeta .
$$

On the semi-circle $\left\{\zeta=R e^{i \theta}, 0<\theta<\pi\right\}$ we have

$$
\frac{\partial G}{\partial n} d s=\left\{\frac{R^{2}-|z|^{2}}{|\zeta-z|^{2}}-\frac{R^{2}-|z|^{2}}{|\zeta-\bar{z}|^{2}}\right\} d \theta
$$


on the interval $\{\zeta=t,-R<t<R\}$ we have

$$
\frac{\partial G}{\partial n} d s=2\left\{\frac{r \sin \varphi}{|z-t|^{2}}-\frac{R^{2} r \sin \varphi}{\left|R^{2}-z t\right|^{2}}\right\} d t, z=r e^{i \varphi} .
$$

Since the function $f_{z}(\zeta)$ is analytic on $\Gamma$, except at the points $A_{l}, l=1, \ldots, p$, then (1.7) implies that with the exception of these points the function $\frac{\partial G}{\partial n}$ is infinitely differentiable on $\Gamma$. By (1.6) the singularities of $\frac{\partial G}{\partial n}$ at the points $A_{l}$ are such that the product $\left|\zeta-A_{l}\right|^{\frac{1}{2}} \frac{\partial G}{\partial n}\left(\leq\left|\zeta-A_{l}\right|^{1-\frac{1}{\alpha_{l}}} \frac{\partial G}{\partial n}\right)$ is bounded. This observation implies that all integrals containing $\frac{\partial G}{\partial n}$ which we will consider later are convergent.

THEOREM 1.1. Let $D$ be a simply connected domain with a piecewise analytic boundary $\Gamma$, and let $u(z)$ be a twice continuously differentiable ${ }^{1}$ function in some domain containing $\bar{D}$, excluding a finite set $\left\{c_{1}, c_{2}, \ldots, c_{q}\right\} \subset \bar{D}$. In a neighborhood of these points $u(z)$ has the form

$$
u(z)=d_{k} \ln \left|z-c_{k}\right|+u_{k}(z),
$$

where $d_{k}$ are constants and $u_{k}(z)$ are twice continuously differentiable functions in a neighborhood of points $c_{k}(k=1, \ldots, q)$. Then

$$
u(z)+\frac{1}{2 \pi} \iint_{D} G(\zeta, z) \Delta u(\zeta) d \sigma=\frac{1}{2 \pi} \int_{\Gamma} u(\zeta) \frac{\partial G}{\partial n} d s-\sum_{c_{k} \in D} d_{k} G\left(c_{k}, z\right)
$$

for $z \in D, z \neq c_{1}, \ldots, c_{q}$.

Proof. Let us exclude from $D$ discs of radius $\varepsilon$ centered at $c_{1} \ldots, c_{q}, z$, and $A_{1}, \ldots, A_{p}$. We assume that $\varepsilon$ is so small that the discs do not intersect, and that if the center of such a disc is inside $D$, then the whole disc is inside $D$. We denote the domain so obtained by $D_{\varepsilon}$ and the part of $\Gamma$ which is not in any of the discs by $\Gamma_{\varepsilon}$. By $C(\varepsilon, a)$ we denote the intersection of $\{z:|z-a|=\varepsilon\}$ and $D$.

Letting $u=u(\zeta)$ and $v=G(\zeta, z)$, we write the second Green formula for $D_{\varepsilon}$. Since $\Delta v=0$, we get ${ }^{2}$

$$
\begin{aligned}
& \iint_{D_{\varepsilon}} v \Delta u d \sigma \\
& \quad=\left(\int_{\Gamma_{\varepsilon}}+\int_{C(\varepsilon, z)}+\sum_{k=1}^{q} \int_{C\left(\varepsilon, c_{k}\right)}+\sum_{l=1}^{p} \int_{C\left(\varepsilon, A_{l}\right)}\right)\left(u \frac{\partial v}{\partial n}-v \frac{\partial u}{\partial n}\right) d s .
\end{aligned}
$$

Let us find the limits of each of the integrals as $\varepsilon \rightarrow 0$. Since $v=0$ for $\zeta \in \Gamma$, then

$$
\lim _{\varepsilon \rightarrow 0} \int_{\Gamma_{\varepsilon}}\left(u \frac{\partial v}{\partial n}-v \frac{\partial u}{\partial n}\right) d s=\int_{\Gamma} u(\zeta) \frac{\partial G}{\partial n} d s .
$$

Furthermore, by the mean value theorem, for each $a \in \bar{D}$ we have

$$
\int_{C(\varepsilon, a)}\left(u \frac{\partial v}{\partial n}-v \frac{\partial u}{\partial n}\right) d s=\left.(\text { the length of } C(\varepsilon, a))\left(u \frac{\partial v}{\partial n}-v \frac{\partial u}{\partial n}\right)\right|_{\zeta *},
$$

\footnotetext{
$1_{\text {with respect to the variables } x \text { and } y, z=x+i y}$

${ }^{2}$ If some points are repeated twice among $\left\{c_{1}, \ldots, c_{q}\right\}$ and $\left\{A_{1}, \ldots, A_{p}\right\}$, we write the corresponding integrals only once.
} 
where $\zeta^{*}$ is a point on $C(\varepsilon, a)$. Since the length of $C(\varepsilon, z)$ is $2 \pi \varepsilon$ and on $C(\varepsilon, z)$ the following asymptotic estimates hold as $\varepsilon \rightarrow 0$

$$
v=\ln \frac{1}{\varepsilon}+O(1), \frac{\partial v}{\partial n}=\frac{\partial v}{\partial \varepsilon}=-\frac{1}{\varepsilon}+O(1), u=u(z)+o(1), \frac{\partial u}{\partial n}=O(1),
$$

then

$$
\lim _{\varepsilon \rightarrow 0} \int_{C(\varepsilon, z)}\left(u \frac{\partial v}{\partial n}-v \frac{\partial u}{\partial n}\right) d s=-2 \pi u(z) .
$$

If $c_{k} \in D$, then on $C\left(\varepsilon, c_{k}\right)$ the following asymptotic estimates hold as $\varepsilon \rightarrow 0$

$$
\begin{gathered}
u=d_{k} \ln \varepsilon+O(1), \frac{\partial u}{\partial n}=\frac{\partial u}{\partial \varepsilon}=\frac{d_{k}}{\varepsilon}+O(1), \\
v=G\left(c_{k}, z\right)+o(1), \frac{\partial v}{\partial n}=O(1) .
\end{gathered}
$$

Therefore for $c_{k} \in D$ we have

$$
\lim _{\varepsilon \rightarrow 0} \int_{C\left(\varepsilon, c_{k}\right)}\left(u \frac{\partial v}{\partial n}-v \frac{\partial u}{\partial n}\right) d s=-2 \pi d_{k} G\left(c_{k}, z\right) .
$$

If $a$ is either one of the points $c_{k}$ belonging to $\Gamma$, or one of the points $A_{l}, l=$ $1, \ldots, p$, then, in any case, the length of $C(\varepsilon, a)$ is $O(\varepsilon)$, and the following asymptotic estimates are valid on $C(\varepsilon, a)$

$$
u=O(|\ln \varepsilon|), \frac{\partial u}{\partial n}=O\left(\frac{1}{\varepsilon}\right), v=o(1), \frac{\partial v}{\partial n}=O\left(\frac{1}{\sqrt{\varepsilon}}\right)
$$

(the last estimate follows from (1.6) and (1.3)). Therefore we have

$$
\lim _{\varepsilon \rightarrow 0} \int_{C(\varepsilon, a)}\left(u \frac{\partial v}{\partial n}-v \frac{\partial u}{\partial n}\right) d s=0 .
$$

Taking the limit as $\varepsilon \rightarrow 0$ in (1.14), we get the statement of the theorem.

Observe that the integral in the left-hand side of (1.13) is, by definition, assumed to be equal to

$$
\lim _{\varepsilon \rightarrow 0} \iint_{D_{\varepsilon}} G(\zeta, z) \Delta u(\zeta) d \sigma
$$

REMARK. The statement of Theorem 1.1 remains true even if the function $u(z)$, in addition to singularities $c_{k}$ on the boundary $\Gamma$ of the region $D$ whose behavior is determined by (1.12), has a finite set of singularities $\mu_{k}, k=1, \ldots, q$, such that on the $\operatorname{arcs} C\left(\varepsilon, \mu_{k}\right)$ the following asymptotic estimates hold (as $\varepsilon \rightarrow 0$ )

$$
u=o\left(\frac{1}{\sqrt{\varepsilon}}\right), \frac{\partial u}{\partial n}=O\left(\frac{1}{\varepsilon}\right) .
$$

\section{The Nevanlinna, Poisson-Jensen, and Shimizu-Ahlfors formulae}

We are going to use several formulae connecting the behavior of a meromorphic function with the distribution of its zeros and poles. Most of the formulae we need are special cases of (1.13). 
THEOREM 2.1. Let $D$ be a simply connected region with a piecewise analytic boundary $\Gamma$ and $f(z) \not \equiv 0$ be a meromorphic function in $\bar{D}$. Then

$$
\ln |f(z)|=\frac{1}{2 \pi} \int_{\Gamma} \ln |f(\zeta)| \frac{\partial G}{\partial n} d s-\sum_{a_{m} \in D} G\left(a_{m}, z\right)+\sum_{b_{n} \in D} G\left(b_{n}, z\right),
$$

where $a_{m}$ 's are zeros of $f(z)$, and $b_{n}$ 's are its poles. ${ }^{3}$

Proof. We apply Theorem 1.1 with $u(z)=\ln |f(z)|$. If $z$ is neither a pole nor a zero of $f(z)$, then $\Delta u(z)=0$. Therefore the integral in the left-hand side of (1.13) is equal to zero. If the point $c_{k}$ is a zero (pole) of $f(z)$ of order $\chi_{k}$, then the equality (1.12) with $d_{k}=\chi_{k}\left(d_{k}=-\chi_{k}\right)$ is valid in the neighborhood of $c_{k}$. Therefore the right-hand side of (1.13) is equal to the right-hand side of (2.1).

Remark. The remark after Theorem 1.1 implies that the formula (2.1) remains valid if $f(z)$ is meromorphic everywhere in $\bar{D}$ except possibly finitely many singular points $\mu_{k} \in \Gamma, k=1, \ldots, q$, such that in the intersection of a small enough neighborhood of $\mu_{k}$ with $\bar{D}$ the function $f(z)$ admits a representation

$$
f(z)=g_{k}\left(\left(z-\mu_{k}\right)^{\alpha_{k}}\right),
$$

where $g_{k}(\zeta)$ are functions meromorphic in the neighborhood of $\zeta=0$, and $\alpha_{k}>0$ are some constants $(k=1, \ldots, q)$.

One of the most important special cases of the formula (2.1) is the case when $D$ is a disc $\{|z|<R\}$.

TheOREM 2.2. Let $f(z) \not \equiv 0$ be a meromorphic function in $\{|z| \leq R\}$. Then the following formula, called the Poisson-Jensen formula, holds:

$$
\begin{aligned}
\ln |f(z)|= & \frac{1}{2 \pi} \int_{0}^{2 \pi} \ln \left|f\left(R e^{i \theta}\right)\right| \operatorname{Re} \frac{R e^{i \theta}+z}{R e^{i \theta}-z} d \theta \\
& -\sum_{\left|a_{m}\right|<R} \ln \left|\frac{R^{2}-\bar{a}_{m} z}{R\left(z-a_{m}\right)}\right|+\sum_{\left|b_{n}\right|<R} \ln \left|\frac{R^{2}-b_{n} z}{R\left(z-b_{n}\right)}\right|,
\end{aligned}
$$

where $a_{m}$ 's are zeros of $f(z)$, and $b_{n}$ 's are its poles.

The proof immediately follows from the formulae (1.4), (1.8), and (1.9).

Applying (2.1) to the half-disc $D=\{|z|<R, \operatorname{Im} z>0\}$, and using the identities (1.5), (1.6), and (1.11), we get the following result.

\footnotetext{
${ }^{3}$ Summands related to multiple zeros and poles are repeated in the right hand side of (2.1) an appropriate number of times.
} 
THEOREM 2.3. Let $f(z) \not \equiv 0$ be a meromorphic function in the half-disc $\{|z| \leq$ $R, \operatorname{Im} z \geq 0\}$. Then

$$
\begin{aligned}
\ln |f(z)|= & \frac{1}{\pi} \int_{-R}^{R} \ln |f(t)|\left\{\frac{r \sin \varphi}{|z-t|^{2}}-\frac{R^{2} r \sin \varphi}{\left|R^{2}-z t\right|^{2}}\right\} d t \\
& +\frac{1}{2 \pi} \int_{0}^{\pi} \ln \left|f\left(R e^{i \theta}\right)\right|\left\{\frac{R^{2}-r^{2}}{\left|R e^{i \theta}-z\right|^{2}}-\frac{R^{2}-r^{2}}{\left|R e^{-i \theta}-z\right|^{2}}\right\} d \theta \\
& -\sum_{\left|a_{m}\right|<R, \operatorname{Im} a_{m}>0} \ln \left|\frac{R^{2}-\bar{a}_{m} z}{R\left(z-a_{m}\right)} \cdot \frac{R\left(z-\bar{a}_{m}\right)}{R^{2}-a_{m} z}\right| \\
& +\sum_{\left|b_{n}\right|<R, \operatorname{Im} b_{n}>0} \ln \left|\frac{R^{2}-\bar{b}_{n} z}{R\left(z-b_{n}\right)} \cdot \frac{R\left(z-\bar{b}_{n}\right)}{R^{2}-b_{n} z}\right|
\end{aligned}
$$

where $a_{m}$ 's are zeros of the function $f(z)$, and $b_{n}$ 's are its poles.

REMARK. The formulae (2.2) and (2.3) are valid for functions $f(z)$ satisfying the conditions of the remark after Theorem 2.1 in the disc $\{|z| \leq R\}$ and the half-disc $\{|z| \leq R, \operatorname{Im} z \geq 0\}$, respectively.

THEOREM 2.4. Let $f(z)$ be a non-identically zero function meromorphic in the disc $\{|z| \leq R\}$. The following formulae hold (the last of them under the assumption $f(0) \neq 0, \infty)$ :

$$
\begin{aligned}
& \ln f(z)= \frac{1}{2 \pi} \int_{0}^{2 \pi} \ln \left|f\left(R e^{i \theta}\right)\right| \frac{R e^{i \theta}+z}{R e^{i \theta}-z} d \theta \\
& \quad-\sum_{\left|a_{m}\right|<R} \ln \frac{R^{2}-\bar{a}_{m} z}{R\left(z-a_{m}\right)}+\sum_{\left|b_{n}\right|<R} \ln \frac{R^{2}-\bar{b}_{n} z}{R\left(z-b_{n}\right)}+i C ; \\
& \frac{d^{p}}{d z^{p}} \ln f(z)= \frac{1}{2 \pi} \int_{0}^{2 \pi} \ln \left|f\left(R e^{i \theta}\right)\right| \frac{p ! 2 R e^{i \theta}}{\left(R e^{i \theta}-z\right)^{p+1}} d \theta \\
&+(p-1) ! \sum_{\left|a_{m}\right|<R}\left(\frac{\bar{a}_{m}^{p}}{\left(R^{2}-\bar{a}_{m} z\right)^{p}}-\frac{(-1)^{p}}{\left(z-a_{m}\right)^{p}}\right) \\
& \quad(p-1) ! \sum_{\left|b_{n}\right|<R}\left(\frac{b_{n}^{p}}{\left(R^{2}-b_{n} z\right)^{p}}-\frac{(-1)^{p}}{\left(z-b_{n}\right)^{p}}\right) \\
& \frac{d^{p}}{d z^{p}} \ln f(z) \mid=\frac{2 p !}{R^{p}} \frac{1}{2 \pi} \int_{o}^{2 \pi} \ln \left|f\left(R e^{i \theta}\right)\right| e^{-i p \theta} d \theta \\
&+(p-1) ! \sum_{\left|a_{m}\right|<R}\left(\frac{\bar{a}_{m}^{p}}{R^{2 p}}-\frac{1}{a_{m}^{p}}\right)-(p-1) ! \sum_{\left|b_{n}\right|<R}\left(\frac{b_{n}^{p}}{R^{2 p}}-\frac{1}{b_{n}^{p}}\right)
\end{aligned}
$$

where $C$ is a real constant, $p=1,2,3, \ldots ; a_{m}$ 's are zeros of the function $f(z)$, and $b_{n}$ 's are its poles.

Proof. The functions in both sides of (2.4) are analytic as functions of $z$. By Theorem 2.2 the real parts of these functions coincide. By Cauchy-Riemann equations it follows that the functions are equal up to a purely imaginary additive constant. The validity of (2.4) follows. The formula (2.5) follows from (2.4) by differentiation. Finally, the formula (2.6) follows from (2.5) if we let $z=0$. 
Observe that in the case when $f(z)$ does not have poles and zeros in $\{|z| \leq R\}$, the formula (2.4) coincides with the classical Schwartz formula written for $\ln f(z)$. We refer to (2.1) and (2.3)-(2.6) as the Nevanlinna formulae.

EXERCISE. Obtain analogues of (2.4) and (2.5) for functions meromorphic in the half-disc $\{|z| \leq R, \operatorname{Im} z \geq 0\}$.

THEOREM 2.5. Let $f(z) \not \equiv 0$ be a function meromorphic in the disc $\{|z| \leq R\}$ and let

$$
f(z)=c_{\lambda} z^{\lambda}+c_{\lambda+1} z^{\lambda+1}+\ldots, c_{\lambda} \neq 0
$$

be its Laurent expansion in the neighborhood of $z=0$. Then the following formula (called the Jensen formula) holds:

$$
\begin{aligned}
\ln \left|c_{\lambda}\right|= & \frac{1}{2 \pi} \int_{0}^{2 \pi} \ln \left|f\left(R e^{i \theta}\right)\right| d \theta-\sum_{o<\left|a_{m}\right|<R} \ln \frac{R}{\left|a_{m}\right|} \\
& +\sum_{0<\left|b_{n}\right|<R} \ln \frac{R}{\left|b_{n}\right|}-\lambda \ln R,
\end{aligned}
$$

where $a_{m}$ 's are zeros of $f(z)$, and $b_{n}$ 's are its poles.

Proof. Let $z \rightarrow 0$ in the Poisson-Jensen formula. For sums in the right-hand side we get

$$
\begin{aligned}
-\sum_{\left|a_{m}\right|<R}+\sum_{\left|b_{n}\right|<R} & =-\sum_{0<\left|a_{m}\right|<R}+\sum_{0<\left|b_{n}\right|<R}+\lambda \ln \frac{|z|}{R} \\
& =-\sum_{0<\left|a_{m}\right|<R} \ln \frac{R}{\left|a_{m}\right|}+\sum_{0<\left|b_{n}\right|<R} \ln \frac{R}{\left|b_{n}\right|}+o(1)+\lambda \ln \frac{|z|}{R} .
\end{aligned}
$$

Since in the left-hand side we get

$$
\ln |f(z)=\lambda \ln | z|+\ln | c_{\lambda} \mid+o(1)
$$

the formula (2.8) follows.

THEOREM 2.6. Let $f(z) \not \equiv 0$ be a function meromorphic in the disc $\{|z| \leq R\}$. If $f(0) \neq \infty$, then the following formula (called the Shimizu-Ahlfors formula) holds:

$$
\begin{aligned}
& \frac{1}{\pi} \iint_{|z| \leq R}\left(\ln \frac{R}{|z|}\right) \frac{\left|f^{\prime}(z)\right|^{2}}{\left(1+|f(z)|^{2}\right)^{2}} d \sigma(z) \\
& \quad=\frac{1}{2 \pi} \int_{0}^{2 \pi} \ln \sqrt{1+\left|f\left(R e^{i \theta}\right)\right|^{2}} d \theta-\ln \sqrt{1+|f(0)|^{2}}+\sum_{\left|b_{n}\right|<R} \ln \frac{R}{\left|b_{n}\right|}
\end{aligned}
$$

where $b_{n}$ 's are poles of $f(z)$. In the case $f(0)=\infty$ the right-hand side should be replaced by

$$
\frac{1}{2 \pi} \int_{0}^{2 \pi} \ln \sqrt{1+\left|f\left(R e^{i \theta}\right)\right|^{2}} d \theta-\ln \left|c_{\lambda}\right|-\lambda \ln R+\sum_{0<\left|b_{n}\right|<R} \ln \frac{R}{\left|b_{n}\right|},
$$

where $c_{\lambda}$ is the same as in (2.7). 
Proof. We use Theorem 1.1 with $u(z)=\ln \sqrt{1+|f(z)|^{2}}, D=\{|z| \leq R\}$. To compute $\Delta u$ we let $A(z)=\operatorname{Re} f(z), B(z)=\operatorname{Im} f(z)$. Then

$$
\begin{gathered}
u=\frac{1}{2} \ln \left(1+A^{2}+B^{2}\right), \frac{\partial u}{\partial x}=\frac{A \frac{\partial A}{\partial x}+B \frac{\partial B}{\partial x}}{1+A^{2}+B^{2}}, \\
\frac{\partial^{2} u}{\partial x^{2}}=\frac{1}{\left(1+A^{2}+B^{2}\right)^{2}}\left\{\left[\left(\frac{\partial A}{\partial x}\right)^{2}+\left(\frac{\partial B}{\partial x}\right)^{2}+A \frac{\partial^{2} A}{\partial x^{2}}+B \frac{\partial^{2} B}{\partial x^{2}}\right]\right. \\
\left.\times\left(1+A^{2}+B^{2}\right)-2\left(A \frac{\partial A}{\partial x}+B \frac{\partial B}{\partial x}\right)^{2}\right\}, \\
\frac{\partial^{2} u}{\partial y^{2}}=\frac{1}{\left(1+A^{2}+B^{2}\right)^{2}}\left\{\left[\left(\frac{\partial A}{\partial y}\right)^{2}+\left(\frac{\partial B}{\partial y}\right)^{2}+A \frac{\partial^{2} A}{\partial y^{2}}+B \frac{\partial^{2} B}{\partial y^{2}}\right]\right. \\
\left.\times\left(1+A^{2}+B^{2}\right)-2\left(A \frac{\partial A}{\partial y}+B \frac{\partial B}{\partial y}\right)^{2}\right\} .
\end{gathered}
$$

Since

$$
\begin{gathered}
\Delta A=\Delta B=0 \\
\left(\frac{\partial A}{\partial x}\right)^{2}+\left(\frac{\partial B}{\partial x}\right)^{2}=\left(\frac{\partial A}{\partial y}\right)^{2}+\left(\frac{\partial B}{\partial y}\right)^{2}=\left|f^{\prime}(z)\right|^{2}, \\
\frac{\partial A}{\partial x} \frac{\partial B}{\partial x}+\frac{\partial A}{\partial y} \frac{\partial B}{\partial y}=0
\end{gathered}
$$

we get

$$
\Delta u=\frac{2\left|f^{\prime}(z)\right|^{2}}{\left(1+|f(z)|^{2}\right)^{2}}
$$

Observe that in a neighborhood of a pole $b_{k}$ of order $\chi_{k}$ of the function $f(z)$, the relation (1.12) is valid with $c_{k}=b_{k}$, and $d_{k}=-\chi_{k}$. Taking into account (1.4) and (1.8), we get

$$
\begin{aligned}
& \ln \sum \sqrt{1+|f(z)|^{2}}+\frac{1}{\pi} \iint_{|\zeta| \leq R} \ln \left|\frac{R^{2}-\zeta z}{R(z-\zeta)}\right| \frac{\left|f^{\prime}(\zeta)\right|^{2}}{\left(1+|f(\zeta)|^{2}\right)^{2}} d \sigma(\zeta) \\
& \quad=\frac{1}{2 \pi} \int_{0}^{2 \pi} \frac{R^{2}-|z|^{2}}{\left|R e^{i \theta}-z\right|^{2}} \ln \sqrt{1+\left|f\left(R e^{i \theta}\right)\right|^{2}} d \theta+\sum_{\left|b_{n}\right|<R} \ln \left|\frac{R^{2}-\bar{b}_{n} z}{R\left(z-b_{n}\right)}\right| .
\end{aligned}
$$

If $f(0) \neq \infty$, then, letting $z=0$, we get (2.9). If $f(0)=\infty$, we take into account that

$$
\begin{gathered}
\ln \sqrt{1+|f(z)|^{2}}=\lambda \ln |z|+\ln \left|c_{\lambda}\right|+o(1), \\
\sum_{\left|b_{n}\right|<R} \ln \left|\frac{R^{2}-b_{n} z}{R\left(z-b_{n}\right)}\right|=\lambda \ln \frac{|z|}{R}+\sum_{0<\left|b_{n}\right|<R} \ln \frac{R}{\left|b_{n}\right|}+o(1),
\end{gathered}
$$

when $z \rightarrow 0$. It remains only to let $z \rightarrow 0$ in (2.11). 


\section{The Carleman and Levin formulae}

THEOREM 3.1. Let $f(z)$ be a non-identically-zero function in the semi-annulus $\left\{0<R_{0} \leq|z| \leq R, \operatorname{Im} z \geq 0\right\}$. Then the following formula (called the Carleman formula) holds

$$
\begin{aligned}
\sum_{m} & \left(\frac{1}{r_{m}}-\frac{r_{m}}{R^{2}}\right) \sin \varphi_{m}-\sum_{n}\left(\frac{1}{\rho_{n}}-\frac{\rho_{n}}{R^{2}}\right) \sin \psi_{n} \\
= & \frac{1}{2 \pi} \int_{R_{0}}^{R}\left(\frac{1}{t^{2}}-\frac{1}{R^{2}}\right) \ln |f(t) f(-t)| d t+\frac{1}{\pi R} \int_{0}^{\pi} \ln \left|f\left(R e^{i \theta}\right)\right| \sin \theta d \theta \\
& +Q\left(R, R_{0} ; f\right),
\end{aligned}
$$

where $r_{m} e^{i \varphi_{m}}$ 's are the zeros, and $\rho_{n} e^{i \psi_{n}}$ 's are the poles of the function $f(z)$ in $\left\{R_{0}<|z|<R, \operatorname{Im} z>0\right\}$, and $Q\left(R, R_{0} ; f\right)=O(1)$ as $R \rightarrow \infty$.

We start by proving the following analog of Theorem 1.1.

Theorem 3.2. Let $D=\left\{R_{0}<|z|<R, \operatorname{Im} z>0\right\}$, and let the function $u(z)$ satisfy the conditions of Theorem 1.1. Then

$$
\begin{aligned}
-\frac{1}{2 \pi} & \iint_{D}\left[\operatorname{Im}\left(\frac{1}{\zeta}+\frac{\zeta}{R^{2}}\right)\right] \Delta u(\zeta) d \sigma(\zeta) \\
= & \frac{1}{2 \pi} \int_{R_{0}}^{R}\left(\frac{1}{t^{2}}-\frac{1}{R^{2}}\right)[u(t)+u(-t)] d t+\frac{1}{\pi R} \int_{0}^{\pi} u\left(R e^{i \theta}\right) \sin \theta d \theta \\
& +\sum_{c_{k} \in D} d_{k} \operatorname{Im}\left(\frac{1}{c_{k}}+\frac{c_{k}}{R^{2}}\right)+Q^{(1)}\left(R, R_{0} ; u\right),
\end{aligned}
$$

where $Q^{(1)}\left(R, R_{0} ; u\right)=O(1)$.

Proof. First we consider the case when none of the points $c_{k}$ belongs to $\left\{|z|=R_{0}, \operatorname{Im} z \geq 0\right\}$. We exclude from $D$ discs of sufficiently small radius $\varepsilon$ centered at $c_{1}, \ldots, c_{q}$. We use the second Green formula for the obtained region with

$$
u=u(\zeta), v=-\operatorname{Im}\left(\frac{1}{\zeta}+\frac{\zeta}{R^{2}}\right)
$$

It is easy to see that the function $v(\zeta)$ satisfies the conditions:

a) $\Delta v=0$;

b) For $\zeta$ in the semi-circle $\{|z|=R, \operatorname{Im} z \geq 0\}$ the following conditions are satisfied

$$
v=0, \frac{\partial v}{\partial n}=\frac{2}{R^{2}} \sin \theta, \zeta=R e^{i \theta}
$$

c) For $\zeta$ in the line segments $\left\{z=t, R_{0}<t<R\right\}$ and $\left\{z=t,-R<t<-R_{0}\right\}$ the following equalities are satisfied

$$
v=0, \frac{\partial v}{\partial n}=\frac{1}{t^{2}}-\frac{1}{R^{2}}
$$

Let $\varepsilon \rightarrow 0$. Computing integrals over the boundaries of the excluded discs using an argument similar to the one used in the proof of Theorem 1.1, we get the formula 
(3.2) with

$$
\begin{aligned}
Q^{(1)}( & \left.R, R_{0} ; u\right)=\frac{1}{2 \pi} \int_{|\zeta|=R_{0}} 0 \leq \arg \zeta \leq \pi \\
= & -\frac{1}{2 \pi} \int_{0}^{\pi}\left[u \frac{\partial v}{\partial n}-v \frac{\partial u}{\partial n}\right) d s \\
\quad \times R_{0} d \theta=O(1) \text { as } R \rightarrow \infty & \left.\left(\frac{1}{R_{0}^{2}}+\frac{1}{R^{2}}\right) \sin \theta+\left(\frac{1}{R_{0}}-\frac{R_{0}}{R^{2}}\right) \sin \theta \frac{\partial}{\partial R_{0}} u\left(R_{0} e^{i \theta}\right)\right]
\end{aligned}
$$

We shall get rid of the condition that none of the points $c_{k}$ belongs to $\{|z|=$ $\left.R_{0}, \operatorname{Im} z \geq 0\right\}$ in the following way. We choose $R_{0}^{\prime}>R_{0}$ in such a way that the semi-circle $\left\{|z|=R_{0}^{\prime}, \operatorname{Im} z \geq 0\right\}$ does not contain points $c_{k}$. We write the formula (3.2) for the region $D^{\prime}=\left\{R_{0}<|z|<R_{0}^{\prime}, \operatorname{Im} z>0\right\}$. Then we set

$$
\begin{aligned}
& Q^{(1)}\left(R, R_{0} ; u\right)=Q^{(1)}\left(R, R_{0}^{\prime} ; u\right)-\frac{1}{2 \pi} \int_{R_{0}}^{R_{0}^{\prime}}\left(\frac{1}{t^{2}}-\frac{1}{R^{2}}\right)[u(t)+u(-t)] d t \\
& \quad-\sum_{c_{k} \in D \backslash D^{\prime}} d_{k} \operatorname{Im}\left(\frac{1}{c_{k}}+\frac{c_{k}}{R^{2}}\right)+\frac{1}{2 \pi} \iint_{D \backslash D^{\prime}}\left[-\operatorname{Im}\left(\frac{1}{\zeta}+\frac{\zeta}{R^{2}}\right)\right] \Delta u(\zeta) d \sigma(\zeta) .
\end{aligned}
$$

It is easy to see that $Q^{(1)}\left(R, R_{0} ; u\right)=O(1)$ as $R \rightarrow \infty$, and that the equation (3.2) is satisfied.

Theorem 3.1 can be derived from Theorem 3.2 if we let $u(\zeta)=\ln |f(\zeta)|$ (cf. proof of Theorem 2.1); we take into account, also, the equalities

$$
-\operatorname{Im}\left(\frac{1}{\zeta}+\frac{\zeta}{R^{2}}\right)=\left(\frac{1}{r}-\frac{r}{R^{2}}\right) \sin \theta, \zeta=r e^{i \theta}
$$

Letting $u(z)=\ln \sqrt{1+|f(z)|^{2}}$ in (3.2), and using (2.10), we get an analog of the Shimizu-Ahlfors formula.

THEOREM 3.3. Let $f(z) \not \equiv 0$ be a function meromorphic in the semi-annulus $\left\{0<R_{0} \leq|z| \leq R, \operatorname{Im} z \geq 0\right\}$. Then

$$
\begin{aligned}
\frac{1}{\pi} \int_{R_{0}}^{R} \int_{0}^{\pi}( & \left.\frac{1}{r}-\frac{r}{R^{2}}\right) \sin \theta \frac{\left|f^{\prime}\left(r e^{i \theta}\right)\right|^{2}}{\left(1+\left|f\left(r e^{i \theta}\right)\right|^{2}\right)^{2}} r d r d \theta \\
= & \frac{1}{2 \pi} \int_{R_{0}}^{R}\left(\frac{1}{t^{2}}-\frac{1}{R^{2}}\right) \ln \sqrt{\left(1+|f(t)|^{2}\right)\left(1+|f(-t)|^{2}\right)} d t \\
& +\frac{1}{\pi R} \int_{0}^{\pi} \ln \sqrt{1+\left|f\left(R e^{i \theta}\right)\right|^{2}} \sin \theta d \theta \\
& +\sum_{n}\left(\frac{1}{\rho_{n}}-\frac{\rho_{n}}{R^{2}}\right) \sin \psi_{n}+Q^{(2)}\left(R, R_{0} ; f\right),
\end{aligned}
$$

where $\rho_{n} e^{i \psi_{n}}$ 's are the poles of $f(z)$, and $Q^{(2)}\left(R, R_{0} ; f\right)=O(1)$ as $R \rightarrow \infty$.

Let us turn to the Levin formula. 
TheOREM 3.4. Let $f(z)$ be a non-identically-zero function, meromorphic in the domain $\left\{\left|z-i \frac{R}{2}\right| \leq \frac{R}{2}\right\} \cup\left\{|z| \leq R_{0}\right\}, 0<R_{0}<R$. Then

$$
\begin{aligned}
& \sum_{m}\left(\frac{\sin \varphi_{m}}{r_{m}}-\frac{1}{R}\right)-\sum_{n}\left(\frac{\sin \psi_{n}}{\rho_{n}}-\frac{1}{R}\right) \\
& \quad=\frac{1}{2 \pi} \int_{\arcsin \left(R_{0} R^{-1}\right)}^{\pi-\arcsin \left(R_{0} R^{-1}\right)} \ln \left|f\left(R \sin \theta e^{i \theta}\right)\right| \frac{d \theta}{R \sin ^{2} \theta}+\tilde{Q}\left(R, R_{0} ; f\right),
\end{aligned}
$$

where $r_{m} e^{i \theta}$ 's are zeros and $\rho_{n} e^{i \psi_{n}}$ 's are poles of the function $f(z)$ belonging to the $\operatorname{domain}\left\{\left|z-i \frac{R}{2}\right|<\frac{R}{2},|z|>R_{0}\right\}$; and $\tilde{Q}\left(R, R_{0} ; f\right)=O(1)$ as $R \rightarrow \infty$.

We shall derive this theorem from a suitable analog of Theorem 1.1.

TheOrem 3.5. Let $D=\left\{\left|z-i \frac{R}{2}\right|<\frac{R}{2},|z|>R_{0}\right\}$, and let $u(z)$ satisfy the conditions of Theorem 1.1 in the domain $D \cup\left\{|z|<R_{1}\right\}, R_{0}<R_{1}<R$. Then

$$
\begin{aligned}
-\frac{1}{2 \pi} \iint_{D} \operatorname{Im} & \left(\frac{1}{\zeta}+\frac{i}{R}\right) \Delta u(\zeta) d \sigma(\zeta)=\frac{1}{2 \pi} \int_{\arcsin \left(R_{0} R^{-1}\right)}^{\pi-\arcsin \left(R_{0} R^{-1}\right)} u\left(R \sin \theta e^{i \theta}\right) \\
& \times \frac{d \theta}{R \sin ^{2} \theta}+\sum_{c_{k} \in D} d_{k} \operatorname{Im}\left(\frac{1}{c_{k}}+\frac{i}{R}\right)+\tilde{Q}^{(1)}\left(R, R_{0} ; u\right),
\end{aligned}
$$

where $\tilde{Q}^{(1)}\left(R, R_{0} ; u\right)=O(1)$ as $R \rightarrow \infty$.

Proof. The argument is similar to the one used in the proof of Theorems 1.1 and 3.2. First we assume that none of the points $c_{k}$ belongs to the semi-circle $\left\{|z|=R_{0}, \operatorname{Im} z>0\right\}$. We delete from the domain $D$ discs of radius $\varepsilon$ centered at $c_{1}, \ldots, c_{q}$, and use the second Green formula for the obtained region with

$$
u=u(\zeta), v=-\operatorname{Im}\left(\frac{1}{\zeta}+\frac{i}{R}\right) .
$$

Letting $\varepsilon \rightarrow 0$ and observing that for $z$ from the set $\left\{\left|z-i \frac{R}{2}\right|=\frac{R}{2},|z| \geq R_{0}\right\}=$ $\left\{z=R \sin \theta e^{i \theta}, \arcsin \left(R_{0} R^{-1}\right) \leq \theta \leq \pi-\arcsin \left(R_{0} R^{-1}\right)\right\}$, we have

$$
v=0, \frac{\partial v}{\partial n}=\frac{1}{R^{2} \sin ^{2} \theta}
$$

we get formula (3.4), furthermore

$$
\begin{aligned}
\tilde{Q}^{(1)}\left(R, R_{o} ; u\right)= & \frac{1}{2 \pi} \int_{\arcsin \left(R_{0} R^{-1}\right)}^{\pi-\arcsin \left(R_{0} R^{-1}\right)}\left\{u\left(R_{0} e^{i \theta}\right)\left(-\frac{-\sin \theta}{R^{2}}\right)\right. \\
& \left.-\left(\frac{\sin \theta}{R_{0}}-\frac{1}{R}\right) \frac{\partial}{\partial R_{0}} u\left(R_{0} e^{i \theta}\right)\right\} R_{0} d \theta=O(1) \text { as } R \rightarrow \infty .
\end{aligned}
$$

If the semi-circle $\left\{|z|=R_{0}, \operatorname{Im} z>0\right\}$ contains some of the points $c_{k}$, then we first use the formula (3.4) for the domain $D^{\prime}=\left\{\left|z-i \frac{R}{2}\right|<\frac{R}{2},|z|>R_{0}^{\prime}\right\}$, where $R_{0}^{\prime}, R_{0}<R_{0}^{\prime}<R$, is chosen in such a way that $\left\{|z|=R_{0}^{\prime}, \operatorname{Im} z>0\right\}$ does not contain points $c_{k}$. We get the result for the region $D$ in a way similar to the one used at the end of the proof of Theorem 3.2. 
We get Theorem 3.4 from Theorem 3.5 by letting $u(\zeta)=\ln |f(\zeta)|$. If we let $u(\zeta)=\ln \sqrt{1+|f(\zeta)|^{2}}$, we get the following analog of the Shimizu-Ahlfors formula.

THEOREM 3.6. Let $f(z)$ be a non-identically-zero function meromorphic in the domain $\left\{\left|z-i \frac{R}{2}\right| \leq \frac{R}{2}\right\} \cup\left\{|z| \leq R_{0}\right\}, 0<R_{0}<R$. Then

$$
\begin{aligned}
\frac{1}{\pi} \iint_{\left|z-i \frac{R}{2}\right| \leq \frac{R}{2},|z| \geq R_{0}} & \left(\frac{\sin \theta}{r}-\frac{1}{R}\right) \frac{\left|f^{\prime}\left(r e^{i \theta}\right)\right|^{2}}{\left(1+\left|f\left(r e^{i \theta}\right)\right|^{2}\right)^{2}} r d r d \theta \\
= & \frac{1}{2 \pi} \int_{\arcsin \left(R_{0} R^{-1}\right)}^{\pi-\arcsin \left(R_{0} R^{-1}\right)} \ln \sqrt{1+\left|f\left(R \sin \theta e^{i \theta}\right)\right|^{2}} \frac{d \theta}{R \sin ^{2} \theta} \\
& +\sum_{n}\left(\frac{\sin \psi_{n}}{\rho_{n}}-\frac{1}{R}\right)+\tilde{Q}^{(2)}\left(R, R_{0} ; f\right),
\end{aligned}
$$

where $\rho_{n} e^{i \psi_{n}}$ 's are poles of $f(z)$ belonging to the domain $\left\{\left|z-i \frac{R}{2}\right|<\frac{R}{2},|z|>R_{0}\right\}$, and $\tilde{Q}^{(2)}\left(R, R_{0} ; f\right)=O(1)$ as $R \rightarrow \infty$.

\section{The Nevanlinna characteristics. The first fundamental theorem of the value distribution theory}

Let $f(z)$ be a meromorphic function. We shall introduce several real functions defined on $[0, \infty)$ which characterize the behavior of $f(z)$. The functions to be introduced will be called the Nevanlinna characteristics of $f(z)$.

The number of poles of $f(z)$ in the disc $\{|z| \leq r\}$ will be denoted by $n(r, f)$; we assume that a pole of order $m$ contributes $m$ to the value of $n(r, f)$. It is clear that $n(r, f)$ is integer-valued, non-decreasing, and right semi-continuous on $[0, \infty)$. A point $r_{0} \in(0, \infty)$ is a point of discontinuity for $n(r, f)$ if and only if the circle $\left\{|z|=r_{0}\right\}$ contains poles of $f(z)$, the value of the jump at $r_{0}$ is equal to the number of such poles. The set of points of discontinuity of $n(r, f)$ cannot have limit points in $[0, \infty)$, therefore on each interval $[a, b] \subset[0, \infty)$ the function $n(r, f)$ is piecewise constant.

Let

$$
N(r, f)=\int_{0}^{r} \frac{n(t, f)-n(0, f)}{t} d t+n(0, f) \ln r .
$$

This characteristic also describes the location of poles of $f(z)$. The function $N(r, f)$ is a continuous non-decreasing function on $(0, \infty)$. Furthermore, as a function of $\ln r$, this function is convex, that is, $N\left(e^{u}, f\right)$ is a convex function of $u$ for $-\infty<u<\infty$. In fact, since

$$
N\left(e^{u}, f\right)=\int_{-\infty}^{u}\left[n\left(e^{v}, f\right)-n(0, f)\right] d v+n(0, f) u,
$$

then $N\left(e^{u}, f\right)$ is an integral of a non-decreasing function. It is worth mentioning that the characteristics $n(r, f)$ and $N(r, f)$ take into account the absolute values of poles of $f(z)$ only.

Characteristics $N(r, f)$ is useful because it is related, in a natural way, to the Jensen formula (2.8). The Jensen formula can be written as

$$
N\left(r, \frac{1}{f}\right)-N(r, f)=\frac{1}{2 \pi} \int_{0}^{2 \pi} \ln \left|f\left(r e^{i \theta}\right)\right| d \theta-\ln \left|c_{\lambda}\right| .
$$


This statement follows from the equalities

$$
\begin{gathered}
\sum_{0<\left|b_{n}\right|<r} \ln \frac{r}{\left|b_{n}\right|}=N(r, f)-n(0, f) \ln r \\
\sum_{0<\left|a_{m}\right|<r} \ln \frac{r}{\left|a_{m}\right|}=N\left(r, \frac{1}{f}\right)-n\left(0, \frac{1}{f}\right) \ln r \\
-\lambda=n(0, f)-n\left(0, \frac{1}{f}\right),
\end{gathered}
$$

where $a_{m}$ 's are zeros of $f(z), b_{n}$ 's are its poles, and the number $\lambda$ is determined from (2.7). The validity of the third equality is obvious, the other two can be obtained by using properties of the Stieltjes integral. In fact,

$$
\begin{aligned}
\sum_{0<\left|b_{n}\right|<r} \ln \frac{r}{\left|b_{n}\right|} & =\int_{+0}^{r} \ln \frac{r}{t} d n(t, f)=\int_{0}^{r} \ln \frac{r}{t} d[n(t, f)-n(0, f)] \\
& =\left.\ln \frac{r}{t}[n(t, f)-n(0, f)]\right|_{+0} ^{r}+\int_{0}^{r} \frac{n(t, f)-n(0, f)}{t} d t \\
& =\int_{0}^{r} \frac{n(t, f)-n(0, f)}{t} d t
\end{aligned}
$$

In order to introduce other characteristics we need the function $\ln ^{+} x$, defined on the semi-axis $\{x \geq 0\}$ by the equality

$$
\ln ^{+} x=\max (\ln x, 0) .
$$

The following relations are obvious:

$$
\ln x=\ln ^{+} x-\ln ^{+} \frac{1}{x},|\ln x|=\ln ^{+} x+\ln ^{+} \frac{1}{x}, \ln ^{+} x=\ln x^{\wedge},
$$

where $x \geq 0, x^{\wedge}=\max (x, 1)$. We need also the following inequalities:

$$
\begin{gathered}
\ln ^{+}\left|\prod_{\nu=1}^{n} x_{\nu}\right| \leq \sum_{\nu=1}^{n} \ln ^{+}\left|x_{\nu}\right|, \\
\ln ^{+}\left|\sum_{\nu=1}^{n} x_{\nu}\right| \leq \sum_{\nu=1}^{n} \ln ^{+}\left|x_{\nu}\right|+\ln n \\
\left|\ln ^{+}\right| x_{1}\left|-\ln ^{+}\right| x_{2}|| \leq|\ln | \frac{x_{1}}{x_{2}}|| \\
\left|\ln ^{+}\right| x_{1}\left|-\ln ^{+}\right| x_{2}|| \leq \ln ^{+}\left|x_{1}-x_{2}\right|+\ln 2
\end{gathered}
$$

where $x_{1}, x_{2}, \ldots, x_{n}$ are arbitrary complex numbers. Inequalities (4.3) and (4.4) can be obtained in the following way:

$$
\begin{aligned}
\ln ^{+}\left|\prod_{\nu=1}^{n} x_{\nu}\right| & =\ln ^{+}\left(\prod_{\nu=1}^{n}\left|x_{\nu}\right|\right) \leq \ln ^{+}\left(\prod_{\nu=1}^{n}\left|x_{\nu}\right|^{\wedge}\right) \\
& =\ln \left(\prod_{\nu=1}^{n}\left|x_{\nu}\right|^{\wedge}\right)=\sum_{\nu=1}^{n} \ln \left|x_{\nu}\right|^{\wedge}=\sum_{\nu=1}^{n} \ln ^{+}\left|x_{\nu}\right|
\end{aligned}
$$




$$
\begin{aligned}
\ln ^{+}\left|\sum_{\nu=1}^{n} x_{\nu}\right| & \leq \ln ^{+}\left(\sum_{\nu=1}^{n}\left|x_{\nu}\right|\right) \leq \ln ^{+}\left(\sum_{\nu=1}^{n}\left|x_{\nu}\right|^{\wedge}\right) \\
& =\ln \left(\sum_{\nu=1}^{n}\left|x_{\nu}\right|^{\wedge}\right) \leq \ln \left(n \max _{1 \leq \nu \leq n}\left|x_{\nu}\right|^{\wedge}\right) \\
& =\ln \left(\max _{1 \leq \nu \leq n}\left|x_{\nu}\right|^{\wedge}\right)+\ln n \leq \sum_{\nu=1}^{n} \ln \left|x_{\nu}\right|^{\wedge}+\ln n=\sum_{\nu=1}^{n} \ln +\left|x_{\nu}\right|+\ln n .
\end{aligned}
$$

Inequalities (4.5) and (4.6) follow from (4.3) and (4.4), respectively. In fact, the inequality (4.3) implies

$$
\ln ^{+}\left|x_{1}\right|=\ln ^{+}\left|\frac{x_{1}}{x_{2}} x_{2}\right| \leq \ln ^{+}\left|\frac{x_{1}}{x_{2}}\right|+\ln ^{+}\left|x_{2}\right|,
$$

therefore, using the second of the relations (4.2), we get

$$
\ln ^{+}\left|x_{1}\right|-\ln ^{+}\left|x_{2}\right| \leq|\ln | \frac{x_{1}}{x_{2}}|| .
$$

Similarly, by (4.4)

$$
\ln ^{+}\left|x_{1}\right| \leq \ln ^{+}\left(\left|x_{1}-x_{2}\right|+\left|x_{2}\right|\right) \leq \ln ^{+}\left|x_{2}\right|+\ln ^{+}\left|x_{1}-x_{2}\right|+\ln 2,
$$

hence

$$
\ln ^{+}\left|x_{1}\right|-\ln ^{+}\left|x_{2}\right| \leq \ln ^{+}\left|x_{1}-x_{2}\right|+\ln 2 .
$$

Since the inequalities $\left(4.5^{\prime}\right)$ and $\left(4.6^{\prime}\right)$ are symmetric with respect to the variables $x_{1}$ and $x_{2}$, we can interchange their positions, and we get the inequalities (4.5) and (4.6).

Let

$$
m(r, f)=\frac{1}{2 \pi} \int_{0}^{2 \pi} \ln ^{+}\left|f\left(r e^{i \varphi}\right)\right| d \varphi .
$$

This function characterizes the growth of the function $f(z)$. Later we shall see that $m(r, f)$ is a continuous function of $r$. We would like to mention only that the behavior of this function can be less regular than the behavior of the function $N(r, f)$, which, as we have already mentioned, is a non-decreasing convex function of $\ln r$.

EXAMPLE. Let

$$
f(z)=\frac{3}{\pi^{2}} \sum_{n=1}^{\infty} \frac{1}{n^{2}(z-n)}
$$

Then

$$
|f(z)| \leq \frac{3}{\pi^{2}} \sum_{n=1}^{\infty} \frac{2}{n^{2}}=1
$$

outside the union of the discs $\left\{|z-n|<\frac{1}{2}\right\}$, therefore $m\left(n+\frac{1}{2}, f\right)=0, n=$ $1,2 \ldots$ At the same time, it is clear that $m(n, f)>0, n=1,2, \ldots$.

Let $a \neq \infty$ be a complex number. The function $m\left(r, \frac{1}{f-a}\right)$ characterizes the proximity of $f(z)$ to $a$ on the circle $\{|z|=r\}$. It is clear that the functions 
$n\left(r, \frac{1}{f-a}\right)$ and $N\left(r, \frac{1}{f-a}\right)$ characterize the moduli of the $a$-points of the function $f(z)$, that is, the moduli of roots of the equation

$$
f(z)=a .
$$

In the cases when the function $f(z)$ we deal with is clear from context, we write $m(r, a), n(r, a)$, and $N(r, a)$ instead of $m\left(r, \frac{1}{f-a}\right), n\left(r, \frac{1}{f-a}\right)$, and $N\left(r, \frac{1}{f-a}\right)$. Also, we shall write $m(r, \infty), n(r, \infty)$, and $N(r, \infty)$ instead of $m(r, f), n(r, f)$, and $N(r, f)$. We shall use also the notation $m(r, a, f), n(r, a, f), N(r, a, f)$. We introduce the characteristic

$$
T(r, f)=m(r, f)+N(r, f) .
$$

It turns out that for each non-constant function $f(z)$ the sum $m(r, a)+N(r, a)$ is "almost independent" of $a$. Roughly speaking, this means that if $f(z)$ takes the value $a$ more often than the value $b$, then it "approaches" to $a$ more slowly than it "approaches" to $b$. The precise statement of this result is given in the following theorem, which Nevanlinna named "the first fundamental theorem of the value distribution theory".

THEOREM 4.1. Let $f(z)$ be a non-constant meromorphic function. Then

$$
m(r, a)+N(r, a)=T(r, f)+\varepsilon(r, a)
$$

for each complex number $a \neq \infty$, where $\varepsilon(r, a)=O(1)$ as $r \rightarrow \infty$.

Proof. We use the Jensen formula in the form (4.1) for $f(z)-a$ (we can do this because $f(z)$ is non-constant). Since the poles of this function coincide with the poles of $f(z)$, we get

$$
N\left(r, \frac{1}{f-a}\right)-N(r, f)=\frac{1}{2 \pi} \int_{0}^{2 \pi} \ln \left|f\left(r e^{i \varphi}\right)-a\right| d \varphi-\ln \left|c_{\lambda}(a)\right|,
$$

where $c_{\lambda}(a)$ is the first non-zero coefficient in the Laurent series of $f(z)-a$ in the neighborhood of $z=0$.

Since by the first of the equalities in (4.2) we have

$$
\frac{1}{2 \pi} \int_{0}^{2 \pi} \ln \left|f\left(r e^{i \varphi}\right)-a\right| d \varphi=m(r, f-a)-m\left(r, \frac{1}{f-a}\right)
$$

then $\left(4.1^{\prime}\right)$ can be rewritten in the form

$$
N\left(r, \frac{1}{f-a}\right)+m\left(r, \frac{1}{f-a}\right)=N(r, f)+m(r, f-a)-\ln \left|c_{\lambda}(a)\right| .
$$

The inequality (4.6) implies that

$$
\left|\ln ^{+}\right| f-a\left|-\ln ^{+}\right| f|| \leq \ln ^{+}|a|+\ln 2,
$$

therefore $|m(r, f-a)-m(r, f)| \leq \ln ^{+}|a|+\ln 2$. Letting

$$
\varepsilon(r, a)=m(r, f-a)-m(r, f)-\ln \left|c_{\lambda}(a)\right|,
$$

we get (4.7). To finish the proof, it remains to observe that

$$
|\varepsilon(r, a)| \leq \ln ^{+}|a|+\ln 2+|\ln | c_{\lambda}(a)||=O(1) \text { as } r \rightarrow \infty .
$$


Let us show that for a non-constant meromorphic function $f(z)$ the characteristic $T(r, f)$ tends to $\infty$ as $r \rightarrow \infty$, and hence the term $\varepsilon(r, a)$ in (4.7) can be regarded as unessential. Let $a=f(0)$, then $n(0, a)>0$, and we have

$$
N(r, a)=\int_{0}^{r} \frac{n(t, a)-n(0, a)}{t} d t+n(0, a) \ln r \geq n(0, a) \ln r \rightarrow \infty .
$$

Since $m(r, a)$ is non-negative, then (4.7) implies

$$
T(r, f) \geq N(r, a)+O(1) \rightarrow \infty \text { as } r \rightarrow \infty .
$$

Our next purpose is to find integral representations of the characteristics $T(r, f)$. We write $f(z)$ as a quotient

$$
f(z)=\frac{f_{1}(z)}{f_{2}(z)}
$$

where $f_{1}(z)$ and $f_{2}(z)=z^{\lambda}+c_{\lambda+1} z^{\lambda+1}+\ldots(\lambda \geq 0)$ are entire functions without common zeros. Let

$$
u(z)=\max \left(\left|f_{1}(z)\right|,\left|f_{2}(z)\right|\right) .
$$

Then, despite the non-uniqueness of the choice of $f_{1}(z)$ and $f_{2}(z)$, we have

$$
T(r, f)=\frac{1}{2 \pi} \int_{0}^{2 \pi} \ln u\left(r e^{i \varphi}\right) d \varphi .
$$

In fact, it is easy to see that

$$
\ln ^{+}|f(z)|=\ln u(z)-\ln \left|f_{2}(z)\right|,
$$

on the other hand, by the Jensen formula (4.1) we have

$$
N\left(r, \frac{1}{f_{2}}\right)=\frac{1}{2 \pi} \int_{0}^{2 \pi} \ln \left|f_{2}\left(r e^{i \varphi}\right)\right| d \varphi .
$$

Since $N(r, f)=N\left(r, \frac{1}{f_{2}}\right)$, then

$$
\begin{aligned}
T(r, f)= & m(r, f)+N(r, f)=\frac{1}{2 \pi} \int_{0}^{2 \pi}\left[\ln u\left(r e^{i \varphi}\right)-\ln \left|f_{2}\left(r e^{i \varphi}\right)\right|\right] d \varphi \\
& +\frac{1}{2 \pi} \int_{0}^{2 \pi} \ln \left|f_{2}\left(r e^{i \varphi}\right)\right| d \varphi=\frac{1}{2 \pi} \int_{0}^{2 \pi} \ln u\left(r e^{i \varphi}\right) d \varphi .
\end{aligned}
$$

Shimizu and Ahlfors proved a more refined theorem than Theorem 4.1. Also, their theorem admits an interesting geometric interpretation.

Denote the Riemann sphere by $S_{0}$, and the map inverse to the stereographic projection of $S_{0}$ onto the extended complex $w$-plane by $\omega=p(w)$. Let $w$ and $a$ be two different points in the extended complex plane. Denote by $[w, a]$ the length of the line segment joining the points $p(w)$ and $p(a)$ on the sphere $S_{0}$. Since the diameter of $S_{0}$ is equal to 1 , then $[w, a] \leq 1$ for each pair of points. It is easy to check that

$$
\begin{gathered}
{[w, a]=\frac{|w-a|}{\sqrt{1+|w|^{2}} \sqrt{1+|a|^{2}}}, w, a \neq \infty} \\
{[w, \infty]=\frac{1}{\sqrt{1+|w|^{2}}} .}
\end{gathered}
$$

The ratio between the length elements on $S_{0}$ and on $w$-plane is equal to

$$
\frac{|d p(w)|}{|d w|}=\frac{1}{1+|w|^{2}},
$$


and the ratio between the area elements $d \omega(w)$ on $S_{0}$ and $d \sigma(w)$ on $w$-plane is equal to

$$
\frac{d \omega(w)}{d \sigma(w)}=\left(\frac{|d p(w)|}{|d w|}\right)^{2}=\frac{1}{\left(1+|w|^{2}\right)^{2}}
$$

We introduce the spherical derivative of a meromorphic function $f(z)$ by

$$
\stackrel{\circ}{f}(z)=\frac{\left|f^{\prime}(z)\right|}{1+|f(z)|^{2}}=\frac{|d p(f(z))|}{|d f(z)|} \frac{|d f(z)|}{|d z|}=\frac{|d p(f(z))|}{|d z|} .
$$

It is easy to see that the function $f^{\circ}(z)$ is continuous at each finite point of the $z$-plane.

Suppose that the function $w=f(z)$ maps the disc $\{|z| \leq r\}$ onto the Riemann surface $F_{r}$. The average number of sheets of the surface $F_{r}$ can be determined as the quotient of the area of $F_{r}$ in the spherical metric and the area of the sphere $S_{0}$, that is $\pi$. If we denote the average number of sheets by $\stackrel{\circ}{A}(r, f)$, we get

$$
\stackrel{\circ}{A}(r, f)=\frac{1}{\pi} \operatorname{area}\left(F_{r}\right)=\frac{1}{\pi} \iint_{F_{r}} d \omega(w)=\frac{1}{\pi} \iint_{|w| \leq \infty} n(r, w) d \omega(w) .
$$

On the other hand

$$
\stackrel{\circ}{A}(r, f)=\frac{1}{\pi} \iint_{F_{r}} d \omega(w)=\frac{1}{\pi} \iint_{|z| \leq r}\left(\frac{d \omega(w)}{d \sigma(z)}\right) d \sigma(z)=\frac{1}{\pi} \iint_{|z| \leq r}(\stackrel{\circ}{f}(z))^{2} d \sigma(z) \text {. }
$$

Let $f(z)$ be a non-constant meromorphic function. If $f(0) \neq a$, we let

$$
\stackrel{\circ}{m}(r, a)=\frac{1}{2 \pi} \int_{0}^{2 \pi} \ln \frac{1}{\left[f\left(r e^{i \varphi}\right), a\right]} d \varphi-\ln \frac{1}{[f(0), a]},
$$

if $f(0)=a$, we let

$$
\begin{gathered}
\stackrel{\circ}{m}(r, a)=\frac{1}{2 \pi} \int_{0}^{2 \pi} \ln \frac{1}{\left[f\left(r e^{i \varphi}\right), a\right]} d \varphi-\ln \frac{\sqrt{1+|a|^{2}}}{\left|c_{\lambda}(a)\right|}, a \neq \infty, \\
\stackrel{\circ}{m}(r, \infty)=\frac{1}{2 \pi} \int_{0}^{2 \pi} \ln \frac{1}{\left[f\left(r e^{i \varphi}\right), \infty\right]} d \varphi-\ln \left|c_{\lambda}(0)\right|,
\end{gathered}
$$

where $c_{\lambda}(a)$ is the first non-zero coefficient in the Laurent series of $f(z)-a$ in the neighborhood of $z=0$. Observe that the integral from the definition of $\stackrel{\circ}{m}(r, a)$ is different from the one in the definition of $m(r, a)$ : now the distance between $f\left(r e^{i \varphi}\right)$ and $a$ is not the plane distance, but the length of a chord of the Riemann sphere.

The function

$$
\stackrel{\circ}{T}(r, f)=\int_{0}^{r} \frac{\stackrel{\circ}{A}(t, f)}{t} d t
$$

is called the Shimizu-Ahlfors characteristic of $f(z)$. By the continuity of $\stackrel{\circ}{f}(z)$, the function $\stackrel{\circ}{A}(r, f)$ is a continuous function of $r$, and $\stackrel{\circ}{A}(r, f)=O\left(r^{2}\right)$ as $r \rightarrow 0$, therefore the function $\stackrel{\circ}{T}(r, f)$ is finite and continuous for $0 \leq r<\infty$. Since

$$
\frac{d \stackrel{\circ}{A}(r, f)}{d r}=\frac{r}{\pi} \int_{0}^{2 \pi}\left(\stackrel{\circ}{f}\left(r e^{i \varphi}\right)\right)^{2} d \varphi>0 \text { for } r>0
$$

then the function $\stackrel{\circ}{A}(r, f)$ is strictly increasing on $[0, \infty)$, and the function $\stackrel{\circ}{T}(r, f)$ is twice continuously differentiable and strictly increasing on $[0, \infty)$, and convex as 
a function of $\ln r$. Also, the second derivative of $\stackrel{\circ}{T}(r, f)$ with respect to $\ln r$ is continuous for $r \geq 0$ and positive for $r>0$.

TheOREM 4.2. Let $f(z)$ be a non-constant meromorphic function. Then

$$
\stackrel{\circ}{m}(r, a)+N(r, a)=\stackrel{\circ}{T}(r, f)
$$

for each complex number a from the extended plane.

Proof. We need the equality

$$
\stackrel{\circ}{T}(r, f)=\frac{1}{\pi} \iint_{|z| \leq r}\left(\ln \frac{r}{|z|}\right)(\stackrel{\circ}{f}(z))^{2} d \sigma(z),
$$

which can be obtained in the following way:

$$
\begin{aligned}
\frac{1}{\pi} \iint_{|z| \leq r} & \left(\ln \frac{r}{|z|}\right)(\stackrel{\circ}{f}(z))^{2} d \sigma(z)=\int_{0}^{r} \ln \frac{r}{t}\left\{\frac{1}{\pi} \int_{0}^{2 \pi}\left(\stackrel{\circ}{f}\left(t e^{i \varphi}\right)\right)^{2} t d \varphi\right\} d t \\
= & \int_{0}^{r} \ln \frac{r}{t} d \stackrel{\circ}{A}(t, f)=\left.\ln \frac{r}{t} \stackrel{\circ}{A}(t, f)\right|_{0} ^{r}+\int_{0}^{r} \frac{\stackrel{\circ}{A}(t, f)}{t} d t=\stackrel{\circ}{T}(r, f) .
\end{aligned}
$$

Using the introduced notation, we can rewrite (2.9) from Theorem 2.6 in the form

$$
\stackrel{\circ}{T}(r, f)=\stackrel{\circ}{m}(r, \infty)+N(r, \infty) .
$$

Thus (4.9) has been proved for $a=\infty$; observe that a similar equality for the Nevanlinna characteristic $T(r, f)$ is valid by definition.

Now assume $a \neq \infty$. Let

$$
F(z, a)=\frac{1+\bar{a} f(z)}{f(z)-a}
$$

Let us show that (a) $\stackrel{\circ}{T}(r, F(z, a))=\stackrel{\circ}{T}(r, f)$, (b) the value $\stackrel{\circ}{m}(r, \infty)$ for the function $F(z, a)$ coincides with the value $\stackrel{\circ}{m}(r, a)$ for the function $f(z)$. Therefore the result will follow if we write the equality $\left(4.9^{\prime}\right)$ for $F(z, a)$.

Using the identities

$$
F^{\prime}(z, a)=-\frac{1+|a|^{2}}{(f(z)-a)^{2}} f^{\prime}(z)
$$

and

$$
|f-a|^{2}+|1+\bar{a} f|^{2}=\left(1+|a|^{2}\right)\left(1+|f|^{2}\right)
$$

we get

$$
\stackrel{\circ}{F}(z, a)=\frac{\left|F^{\prime}(z, a)\right|}{1+|F(z, a)|^{2}}=\frac{\left(1+|a|^{2}\right)\left|f^{\prime}(z)\right|}{|f(z)-a|^{2}+|1+\bar{a} f(z)|^{2}}=\stackrel{\circ}{f}(z) .
$$

As a consequence we get $\stackrel{\circ}{A}(r, F(z, a))=\stackrel{\circ}{A}(r, f)$, and, hence, the statement (a).

The equation (4.11) implies

$$
[F, \infty]=\frac{1}{\sqrt{1+|F|^{2}}}=\frac{|f-a|}{\sqrt{|f-a|^{2}+|1+\bar{a} f|^{2}}}=\frac{|f-a|}{\sqrt{1+|a|^{2}} \sqrt{1+|f|^{2}}}=[f, a] .
$$


If $f(0) \neq a$, this equality immediately implies (b). If $f(0)=a$, we should take into account also the fact that the Laurent series for $F(z, a)$ in the neighborhood of $z=0$ is of the form

$$
F(z, a)=\frac{1+|a|^{2}}{c_{\lambda}(a)} z^{-\lambda}+\ldots
$$

REMARK. The basic idea of the argument used to derive (4.9) from its particular case $a=\infty$ is the following. The Möbius transformation

$$
w_{1}=\frac{1+\bar{a} w}{w-a}
$$

can be geometrically interpreted as the rotation of the Riemann sphere $S_{0}$ under which the image $p(a)$ of the point $a$ is mapped onto the North pole $p(\infty)$ of $S_{0}$. If we use this idea, the argument becomes intuitively obvious.

Let us prove that Theorem 4.1 is a corollary of Theorem 4.2. To do this it is enough to show that

$$
|m(r, a)-\stackrel{\circ}{m}(r, a)|<d(a),
$$

where $d(a), 0<d(a)<\infty$, depends on $a$ only.

For $a \neq \infty$ we have

$$
\begin{gathered}
\frac{1}{[f, a]}=\frac{\sqrt{1+|f|^{2}} \sqrt{1+|a|^{2}}}{|f-a|} \geq\left(\frac{1}{|f-a|}\right)^{\wedge} ; \\
\frac{1}{|f, a|} \leq \frac{\sqrt{1+(|f-a|+|a|)^{2}} \sqrt{1+|a|^{2}}}{|f-a|} \\
\leq \frac{1+|f-a|+|a|}{|f-a|} \sqrt{1+|a|^{2}} \leq\left(\frac{1+|a|}{|f-a|}+1\right)(1+|a|) \\
\leq\left[\left(\frac{1}{|f-a|}\right)^{\wedge}(1+|a|)+\left(\frac{1}{|f-a|}\right)^{\wedge}\right](1+|a|) \leq\left(\frac{1}{|f-a|}\right)^{\wedge}(2+|a|)^{2} .
\end{gathered}
$$

Hence

$$
\ln ^{+} \frac{1}{|f-a|} \leq \ln \frac{1}{[f, a]} \leq \ln ^{+} \frac{1}{|f-a|}+2 \ln (2+|a|),
$$

and we get (4.12). It is obvious that

$$
|f|^{\wedge} \leq \frac{1}{[f, \infty]} \leq|f|^{\wedge} \sqrt{2}
$$

therefore

$$
\ln ^{+}|f| \leq \ln \frac{1}{[f, \infty]} \leq \ln ^{+}|f|+\frac{1}{2} \ln 2,
$$

and (4.12) remains true for $a=\infty$.

Observe that (4.12) implies

$$
|T(r, f)-\stackrel{\circ}{T}(r, f)| \leq d_{1}(f)
$$

where $d_{1}(f)=\frac{1}{2} \ln \left(2+2|f(0)|^{2}\right)$ if $f(0) \neq \infty$, and $d_{1}(f)=\frac{1}{2} \ln 2+|\ln | c_{\lambda}(0)||$ if $f(0)=\infty$, that is, the characteristics $T(r, f)$ and $\stackrel{\circ}{T}(r, f)$ have a bounded difference as $r \rightarrow \infty$. 
Exercise. Prove the following formula, similar to (4.8):

$$
\stackrel{\circ}{T}(r, f)=\frac{1}{2 \pi} \int_{0}^{2 \pi} \ln v\left(r e^{i \varphi}\right) d \varphi-\ln v(0),
$$

where

$$
v(z)=\sqrt{\left|f_{1}(z)\right|^{2}+\left|f_{2}(z)\right|^{2}}
$$

and $f_{1}(z), f_{2}(z)$ have the same meaning as in the definition of the function $u(z)$ used in (4.8). (Hint. Use the equality $\ln v(z)=\ln \sqrt{1+|f(z)|^{2}}-\ln \left|f_{2}(z)\right|$, the Jensen formula for $f_{2}(z)$, and $\left(4.9^{\prime}\right)$.)

As we have already mentioned, the characteristic $\stackrel{\circ}{T}(r, f)$ is a non-decreasing convex function of $\ln r$. It turns out that the same statement is valid for $T(r, f)$. This fact is not a trivial one because the behavior of the term $m(r, f)$ in the sum $m(r, f)+N(r, f)$ can be quite irregular (see the example on p. 14). We shall use the following theorem of $\mathrm{H}$. Cartan.

TheOREM 4.3. Let $f(z)$ be a non-constant meromorphic function. Then

$$
T(r, f)=\frac{1}{2 \pi} \int_{0}^{2 \pi} N\left(r, e^{i \theta}\right) d \theta+d(f)
$$

where the constant $d(f)$ does not depend on $r$.

Proof. Observe that in passing we shall prove measurability of $N\left(r, e^{i \theta}\right)$ as a function of $\theta$.

Since $N(r, a)$ is non-decreasing convex in $\ln r$, then (4.13) immediately implies that $T(r, f)$ is also non-decreasing convex in $\ln r$.

We need the formula:

$$
\frac{1}{2 \pi} \int_{0}^{2 \pi} \ln \left|w-e^{i \theta}\right| d \theta=\ln ^{+}|w|
$$

and the inequality

$$
\frac{1}{2 \pi} \int_{0}^{2 \pi}|\ln | w-e^{i \theta}|| d \theta \leq \ln ^{+}|w|+2 \ln 2
$$

The formula (4.14) can be proved in the following way. We use (2.8) for $f(z)=$ $w-z$ and $R=1$. If $|w| \geq 1$, then this function does not have zeros and poles in the disc $\{|z|<1\}$, and we get

$$
\ln |w|=\frac{1}{2 \pi} \int_{0}^{2 \pi} \ln \left|w-e^{i \theta}\right| d \theta .
$$

If $0<|w|<1$, then $f(z)$ has, in $\{|z|<1\}$, a zero at $w$, and (2.8) implies

$$
\ln |w|=\frac{1}{2 \pi} \int_{0}^{2 \pi} \ln \left|w-e^{i \theta}\right| d \theta-\ln \frac{1}{|w|} .
$$

Thus (4.14) has been proved for $w \neq 0$. For $w=0$ the formula is obvious.

Further, using (4.2), we get

$$
\begin{aligned}
& \frac{1}{2 \pi} \int_{0}^{2 \pi}|\ln | w-e^{i \theta}|| d \theta \\
& \quad=-\frac{1}{2 \pi} \int_{0}^{2 \pi} \ln \left|w-e^{i \theta}\right| d \theta+2 \cdot \frac{1}{2 \pi} \int_{0}^{2 \pi} \ln ^{+}\left|w-e^{i \theta}\right| d \theta
\end{aligned}
$$


Observing that $\ln ^{+}\left|w-e^{i \theta}\right| \leq \ln ^{+}|w|+\ln 2$, and using (4.14), we get (4.15).

We turn to the proof of Theorem 4.3. First we assume that $f(0) \neq \infty$. We apply the Jensen formula (4.1) to $f(z)-e^{i \theta}$. If $f(0) \neq e^{i \theta}$, we get

$$
N\left(r, e^{i \theta}\right)-N(r, \infty)=\frac{1}{2 \pi} \int_{0}^{2 \pi} \ln \left|f\left(r e^{i \varphi}\right)-e^{i \theta}\right| d \varphi-\ln \left|f(0)-e^{i \theta}\right| .
$$

The right-hand side of this equality is measurable in $\theta$. Since the equality holds for all $\theta \in[0,2 \pi)$, except possibly one (for which $e^{i \theta}=f(0)$ ), then $N\left(r, e^{i \theta}\right.$ ) is also measurable in $\theta$ on $[0,2 \pi)$. We integrate the equality $(4.16)$ over $[0,2 \pi]$ and divide the result by $2 \pi$. We get

$$
\begin{aligned}
& \frac{1}{2 \pi} \int_{0}^{2 \pi} N\left(r, e^{i \theta}\right) d \theta-N(r, \infty) \\
& =\frac{1}{2 \pi} \int_{0}^{2 \pi}\left\{\frac{1}{2 \pi} \int_{0}^{2 \pi} \ln \left|f\left(r e^{i \varphi}\right)-e^{i \theta}\right| d \varphi\right\} d \theta-\frac{1}{2 \pi} \int_{0}^{2 \pi} \ln \left|f(0)-e^{i \theta}\right| d \theta,
\end{aligned}
$$

provided that the first integral in the right-hand side is well-defined. We shall show this and also justify the change the order of integration in this integral. By the Fubini theorem, it suffices to establish that

$$
I=\frac{1}{2 \pi} \int_{0}^{2 \pi}\left\{\frac{1}{2 \pi} \int_{0}^{2 \pi}|\ln | f\left(r e^{i \varphi}\right)-e^{i \theta}|| d \theta\right\} d \varphi<\infty .
$$

This follows from (4.15):

$$
I \leq \frac{1}{2 \pi} \int_{0}^{2 \pi}\left\{\ln ^{+}\left|f\left(r e^{i \varphi}\right)\right|+2 \ln 2\right\} d \varphi=m(r, f)+2 \ln 2<\infty .
$$

Changing the order of integration in (4.17) and using (4.14), we get

$$
\frac{1}{2 \pi} \int_{0}^{2 \pi} N\left(r, e^{i \theta}\right) d \theta-N(r, \infty)=\frac{1}{2 \pi} \int_{0}^{2 \pi} \ln ^{+}\left|f\left(r e^{i \varphi}\right)\right| d \varphi-\ln ^{+}|f(0)| .
$$

The obtained equality is equivalent to (4.13) with $d(f)=\ln ^{+}|f(0)|$.

If $f(0)=\infty$, then the right-hand side of (4.16) will contain $\ln \left|c_{\lambda}\right|$ instead of $\ln \left|f(0)-e^{i \theta}\right|$, where $c_{\lambda}$ is defined in (2.7). Therefore, using the same argument as above, we get (4.13) with $d(f)=\ln \left|c_{\lambda}\right|$.

The integral in the right-hand side of (4.13) can be regarded as an integral average of $N(r, a)$ over the circle $|a|=1$ or over the equator of the sphere $S_{0}$. One can get analogous formulas for averages of $N(r, a)$ over certain other sufficiently large subsets of the sphere $S_{0}$ (see R. Nevanlinna [NevR74, Chapter VI, §4]). We shall give here only one identity for $\stackrel{\circ}{T}(r, f)$ which will be used in Chapter IV.

THEOREM 4.4. Let $f(z)$ be a non-constant meromorphic function. Then

$$
\stackrel{\circ}{T}(r, f)=\frac{1}{\pi} \iint_{|a| \leq \infty} N(r, a) d \omega(a) .
$$

PRoof. Identity (4.18) could be obtained by a change of the order of integration:

$$
\begin{aligned}
\stackrel{\circ}{T}(r, f) & =\int_{0}^{r} \frac{\stackrel{\circ}{A}(t, f)}{t} d t=\frac{1}{\pi} \int_{0}^{r} \frac{d t}{t} \iint_{|a| \leq \infty} n(t, a) d \omega(a) \\
& =\frac{1}{\pi} \iint_{|a| \leq \infty}^{r} d \omega(a) \int_{0}^{r} \frac{n(t, a)}{t} d t=\frac{1}{\pi} \iint_{|a| \leq \infty} N(r, a) d \omega(a) .
\end{aligned}
$$


However, in order to justify the change, we need to show that $n(t, a)$ is measurable in the two variables $t$ and $a$ on $\{0 \leq t \leq r,|a| \leq \infty\}$. This would require a lengthy argument. We pass over the difficulty in the following way. We use the equality

$$
\iint_{|a| \leq \infty} \ln \frac{1}{[w, a]} d \omega(a)=\frac{\pi}{2}
$$

which is valid for an arbitrary complex number $w,|w| \leq \infty$. In fact, making the change of variables

$$
a^{\prime}=\frac{1+a \bar{w}}{a-w},
$$

which is equivalent to a rotation of the sphere $S_{0}$, under which the point $p(w)$ is mapped onto $p(\infty)$, we get

$$
\begin{aligned}
\iint_{|a| \leq \infty} & \ln \frac{1}{[w, a]} d \omega(a)=\iint_{\left|a^{\prime}\right| \leq \infty} \ln \frac{1}{\left[\infty, a^{\prime}\right]} d \omega\left(a^{\prime}\right) \\
& =\iint_{\left|a^{\prime}\right| \leq \infty} \frac{\ln \sqrt{1+\left|a^{\prime}\right|^{2}}}{\left(1+\left|a^{\prime}\right|^{2}\right)^{2}} d \sigma\left(a^{\prime}\right)=2 \pi \int_{0}^{\infty} \frac{\ln \sqrt{1+r^{2}}}{\left(1+r^{2}\right)^{2}} r d r=\frac{\pi}{2} .
\end{aligned}
$$

The equation (4.9) implies

$$
N(r, a)=\stackrel{\circ}{T}(r, f)-\frac{1}{2 \pi} \int_{0}^{2 \pi} \ln \frac{1}{\left[f\left(r e^{i \varphi}\right), a\right]} d \varphi+\ln \frac{1}{[f(0), a]}
$$

provided that $a \neq f(0)$. We integrate this equation with respect to $d \omega(a)$ over $|a| \leq \infty$. The measurability of $N(r, a)$ in $a$ follows from the measurability of the right hand side of (4.20). Using (4.19) and changing the order of integration, we get

$$
\begin{aligned}
& \frac{1}{\pi} \iint_{|a| \leq \infty} N(r, a) d \omega(a) \\
& =\stackrel{\circ}{T}(r, f)-\frac{1}{2 \pi} \int_{0}^{2 \pi} d \varphi \frac{1}{\pi} \iint_{|a| \leq \infty} \ln \frac{1}{\left[f\left(r e^{i \varphi}\right), a\right]} d \omega(a) \\
& \quad+\frac{1}{\pi} \iint_{|a| \leq \infty} \ln \frac{1}{[f(0), a]} d \omega(a)=\stackrel{\circ}{T}(r, f)-\frac{1}{2 \pi} \int_{0}^{2 \pi} \frac{1}{2} d \varphi+\frac{1}{2}=\stackrel{\circ}{T}(r, f),
\end{aligned}
$$

that is, (4.18). The legitimacy of the change of the order of integration follows immediately from Fubini's theorem, since the integrand is non-negative and measurable in $\varphi$ and $a$.

\section{The angular Nevanlinna characteristics, Tsuji chracteristics and related analogs of the first fundamental theorem}

The characteristics introduced in the previous section are fundamental for the study of functions meromorphic in the whole complex plane. These characteristics do not take into account, at least explicitly, the arguments of $a$-points. For this reason in the studies devoted to the distribution of arguments of $a$-points an essential role is played by different characteristics which we shall introduce now.

Let $f(z)$ be meromorphic in the closed half-plane $\{\operatorname{Im} z \geq 0\}$. Let $\rho_{n} e^{i \psi_{n}}$ 's be its poles; we count each of the poles according to its multiplicity. Let

$$
c(r, f)=\sum_{1<\rho_{n} \leq r} \sin \psi_{n} .
$$


The value of the characteristic $c(r, f)$ heavily depends on the arguments of poles of $f(z)$. Roughly speaking, the contribution of the pole $\rho_{n} e^{i \psi_{n}}$ to $c(r, f)$ is smaller the closer it closer to the real line $\{\operatorname{Im} z=0\}$ (in the sense that $\psi_{n}$ is close to either 0 or $\pi)$. The poles lying on the line $\{\operatorname{Im} z=0\}$ do not contribute to $c(r, f)$ at all. It is clear that $c(r, f)$ is non-decreasing, right-continuous in $r$ on $[1, \infty)$ and piecewise constant on each line segment $[a, b] \subset[1, \infty)$.

Let

$$
C(r, f)=2 \int_{1}^{r} c(t, f)\left(\frac{1}{t^{2}}+\frac{1}{r^{2}}\right) d t
$$

This characteristic is naturally connected with the Carleman formula (3.1), which can be written in the form

$$
\begin{aligned}
C\left(r, \frac{1}{f}\right)-C(r, f)= & \frac{1}{\pi} \int_{1}^{r}\left(\frac{1}{t^{2}}-\frac{1}{r^{2}}\right) \ln |f(t) f(-t)| d t \\
& +\frac{2}{\pi r} \int_{0}^{\pi} \ln \left|f\left(r e^{i \theta}\right)\right| \sin \theta d \theta+Q(r, 1 ; f) .
\end{aligned}
$$

This statement follows from the equation

$$
C(r, f)=2 \sum_{1<\rho_{n}<r}\left(\frac{1}{\rho_{n}}-\frac{\rho_{n}}{r^{2}}\right) \sin \psi_{n},
$$

which can be verified as follows:

$$
\begin{aligned}
2 \sum_{1 \leq \rho_{n} \leq r} & \left(\frac{1}{\rho_{n}}-\frac{\rho_{n}}{r^{2}}\right) \sin \psi_{n}=2 \int_{1}^{r}\left(\frac{1}{t}-\frac{t}{r^{2}}\right) d c(t, f) \\
= & \left.2\left(\frac{1}{t}-\frac{t}{r^{2}}\right) c(t, f)\right|_{1} ^{r}+2 \int_{1}^{r} c(t, f)\left(\frac{1}{t^{2}}+\frac{1}{r^{2}}\right) d t=C(r, f) .
\end{aligned}
$$

Since each summand in $\left(5.1^{\prime}\right)$ is non-negative and non-decreasing, then $C(r, f)$ is a non-decreasing in $r$.

We introduce, also, characteristics:

$$
\begin{gathered}
A(r, f)=\frac{1}{\pi} \int_{1}^{r}\left(\frac{1}{t^{2}}-\frac{1}{r^{2}}\right)\left[\ln ^{+}|f(t)|+\ln ^{+}|f(-t)|\right] d t, \\
B(r, f)=\frac{2}{\pi r} \int_{0}^{\pi} \ln ^{+} \mid f\left(r e^{i \varphi)} \mid \sin \varphi d \varphi, \quad 1 \leq r \leq \infty .\right.
\end{gathered}
$$

These functions characterize the growth of $f(z)$ as $|z| \rightarrow \infty$; the function $A$ characterizes the growth along the real $\operatorname{line}\{\operatorname{Im} z=0\}$, and the function $B$ characterizes the growth of $f$ in the interior of the half-plane $\{\operatorname{Im} z>0\}$.

When there is no danger of confusion, the functions

$$
A\left(r, \frac{1}{f-a}\right), B\left(r, \frac{1}{f-a}\right), C\left(r, \frac{1}{f-a}\right), a \neq \infty
$$

will be denoted by

$$
A(r, a), B(r, a), C(r, a),
$$

respectively. These functions characterize the proximity of $f(z)$ to $a$ and the distribution of $a$-points. We also denote

$$
A(r, \infty)=A(r, f), B(r, \infty)=B(r, f), C(r, \infty)=C(r, f) .
$$


Finally, we introduce

$$
S(r, f)=A(r, f)+B(r, f)+C(r, f) .
$$

The characteristics $A, B, C, c$, and $S$ will be called the Nevanlinna characteristics for a half-plane.

The following result is an analog of the first fundamental theorem.

THEOREM 5.1. Let $f(z)$ be non-constant function meromorphic in the upper half-plane $\{\operatorname{Im} z \geq 0\}$. Then

$$
A(r, a)+B(r, a)+C(r, a)=S(r, f)+\varepsilon_{1}(r, a)
$$

for each complex $a \neq \infty$, where $\varepsilon_{1}(r, a)=O(1)$ as $r \rightarrow \infty$.

Proof. Following the analogy with Theorem 4.1 we apply the Carleman formula in the form (5.1) to $f(z)-a$. Taking into consideration the first of the equations in (4.2), we get

$$
\begin{aligned}
& A\left(r, \frac{1}{f-a}\right)+B\left(r, \frac{1}{f-a}\right)+C\left(r, \frac{1}{f-a}\right) \\
& =A(r, f-a)+B(r, f-a)+C(r, f)+Q(r, 1 ; f-a) .
\end{aligned}
$$

Now we use (4.6) and get

$$
\begin{aligned}
|A(r, f-a)-A(r, f)| \leq & 2\left(\ln ^{+}|a|+\ln 2\right) \frac{1}{\pi} \int_{1}^{r}\left(\frac{1}{t^{2}}-\frac{1}{r^{2}}\right) d t \\
\leq & \frac{2}{\pi}\left(\ln ^{+}|a|+\ln 2\right), \\
|B(r, f-a)-B(r, f)| & \leq\left(\ln ^{+}|a|+\ln 2\right) \frac{2}{\pi r} \int_{0}^{\pi} \sin \varphi d \varphi \\
& \leq \frac{4}{\pi}\left(\ln ^{+}|a|+\ln 2\right) .
\end{aligned}
$$

Hence we get (5.2) with

$$
\left|\varepsilon_{1}(r, a)\right| \leq \frac{6}{\pi}\left(\ln ^{+}|a|+\ln 2\right)+|Q(r, 1 ; f-a)|=O(1)
$$

as $r \rightarrow \infty$.

Later we will need a more general result, dealing with functions meromorphic in an angle $\{\alpha \leq \arg z \leq \beta\}, 0<\beta-\alpha \leq 2 \pi$. Letting $k=\pi(\beta-\alpha)^{-1}, 1 \leq r<\infty$, we introduce functions, which we shall call angular Nevanlinna characteristics:

$$
\begin{gathered}
A_{\alpha \beta}(r, f)=\frac{k}{\pi} \int_{1}^{r}\left(\frac{1}{t^{k}}-\frac{t^{k}}{r^{2 k}}\right)\left[\ln ^{+}\left|f\left(t e^{i \alpha}\right)\right|+\ln ^{+}\left|f\left(t e^{i \beta}\right)\right|\right] \frac{d t}{t} \\
B_{\alpha \beta}(r, f)=\frac{2 k}{\pi r^{k}} \int_{\alpha}^{\beta} \ln ^{+}\left|f\left(r e^{i \varphi}\right)\right| \sin k(\varphi-\alpha) d \varphi \\
C_{\alpha \beta}(r, f)=2 k \int_{1}^{r} c_{\alpha \beta}(t, f)\left(\frac{1}{t^{k}}+\frac{t^{k}}{r^{2 k}}\right) \frac{d t}{t}
\end{gathered}
$$

where

$$
c_{\alpha \beta}(r, f)=\sum_{1<\rho_{n} \leq r, \alpha \leq \psi_{n} \leq \beta} \sin k\left(\psi_{n}-\alpha\right)
$$


and $\rho_{n} e^{i \psi_{n}}$ 's are poles of $f(z)$ counted according to their multiplicity;

$$
S_{\alpha \beta}(r, f)=A_{\alpha \beta}(r, f)+B_{\alpha \beta}(r, f)+C_{\alpha \beta}(r, f) .
$$

Note, that the characteristics $A(r, f), B(r, f), C(r, f)$, and $S(r, f)$ will be obtained from the angular characteristics if $\alpha=0, \beta=\pi$. For functions $A_{\alpha \beta}(r, a)$, $B_{\alpha \beta}(r, a)$, and $C_{\alpha \beta}(r, a)$ we shall use the same convention as for their half-plane analogs.

THEOREM 5.2. Let $f(z)$ be a non-constant, function meromorphic in the angle $\{\alpha \leq \arg z \leq \beta\}, 0<\beta-\alpha \leq 2 \pi$. Then

$$
A_{\alpha \beta}(r, a)+B_{\alpha \beta}(r, a)+C_{\alpha \beta}(r, a)=S_{\alpha \beta}(r, f)+\varepsilon_{2}(r, a)
$$

for each complex $a \neq \infty$, where $\varepsilon_{2}(r, a)=O(1)$ as $r \rightarrow \infty$.

Proof. This theorem is a corollary of Theorem 5.1. In fact, considering in the half-plane $\{\operatorname{Im} z \geq 0\}$ the branch of $w=z^{\frac{1}{k}}$ positive for $z>0$, we introduce the function $f_{1}(z)=f\left(z^{\frac{1}{k}} e^{i \alpha}\right)$. This function is meromorphic in the half-plane $\{\operatorname{Im} z \geq 0\}$, except at the origin. Straightforward verification shows that

$$
A\left(r, f_{1}\right)=A_{\alpha \beta}\left(r^{\frac{1}{k}}, f\right), B\left(r, f_{1}\right)=B_{\alpha \beta}\left(r^{\frac{1}{k}}, f\right), C\left(r, f_{1}\right)=C_{\alpha \beta}\left(r^{\frac{1}{k}}, f\right),
$$

hence

$$
S\left(r, f_{1}\right)=S_{\alpha \beta}\left(r^{\frac{1}{k}}, f\right) .
$$

Applying Theorem 5.1 to $f_{1}(z)$, we get the desired result.

Now we turn to the characteristics generated by the Levin formula (3.3). These characteristics will be called the Tsuji characteristics.

Let $f(z)$ be a non-constant function, meromorphic in the half-plane $\{\operatorname{Im} z \geq 0\}$. Denote by $\mathfrak{n}(r, f)$ the number of poles of $f(z)$ contained in the set

$$
\left\{\left|z-i \frac{r}{2}\right| \leq \frac{r}{2},|z|>1\right\}
$$

(each pole is counted according to its multiplicity). The characteristic $\mathfrak{n}(r, f)$ takes into account the arguments of the poles. The characteristic $\mathfrak{n}(r, f)$ can only decrease if we fix the moduli of the poles and change their arguments in such a way that they will become closer to the real $\operatorname{line}\{\operatorname{Im} z=0\}$.

Let

$$
\mathfrak{N}(r, f)=\int_{1}^{r} \frac{\mathfrak{n}(t, f)}{t^{2}} d t, 1<r<\infty
$$

With this notation the Levin formula (3.3) can be written as

$$
\mathfrak{N}\left(r, \frac{1}{f}\right)-\mathfrak{N}(r, f)=\frac{1}{2 \pi} \int_{\kappa(r)}^{\pi-\kappa(r)} \ln \left|f\left(r \sin \varphi e^{i \varphi}\right)\right| \frac{d \varphi}{r \sin ^{2} \varphi}+\tilde{Q}(r, 1 ; f),
$$

where

$$
\kappa(r)=\arcsin \left(\frac{1}{r}\right) .
$$

This statement follows from the equality

$$
\sum_{1<\rho_{n} \leq r \sin \psi_{n}}\left(\frac{\sin \psi_{n}}{\rho_{n}}-\frac{1}{r}\right)=\mathfrak{N}(r, f),
$$


where $\rho_{n} e^{i \psi_{n}}$ 's are poles of the function $f(z)$. Indeed, observe that the function $\mathfrak{n}(t, f)$ has jumps at the points $t=\rho_{n} / \sin \psi_{n}$ only, and the values of the jumps are equal to the numbers of poles of $f(z)$ lying on the circular arc

$$
\left\{\left|z-i \frac{t}{2}\right|=\frac{t}{2},|z|>1\right\} \text {. }
$$

Hence

$$
\begin{aligned}
\sum_{1<\rho_{n} \leq \sin \psi_{n}} & \left(\frac{\sin \psi_{n}}{\rho_{n}}-\frac{1}{r}\right)=\int_{1}^{r}\left(\frac{1}{t}-\frac{1}{r}\right) d \mathfrak{n}(t, f) \\
= & \left.\left(\frac{1}{t}-\frac{1}{r}\right) \mathfrak{n}(t, f)\right|_{1} ^{r}+\int_{1}^{r} \frac{\mathfrak{n}(t, f)}{t^{2}} d t=\mathfrak{N}(r, f) .
\end{aligned}
$$

We introduce also

$$
\mathfrak{m}(r, f)=\frac{1}{2 \pi} \int_{\kappa(r)}^{\pi-\kappa(r)} \ln ^{+}\left|f\left(r \sin \varphi e^{i \varphi}\right)\right| \frac{d \varphi}{r \sin ^{2} \varphi}
$$

and

$$
\mathfrak{T}(r, f)=\mathfrak{m}(r, f)+\mathfrak{N}(r, f)
$$

With the customary convention for the meaning of the symbols $\mathfrak{m}(r, a), \mathfrak{n}(r, a)$, and $\mathfrak{N}(r, a)$, we get the following analog of the first fundamental theorem.

THEOREM 5.3. Let $f(z)$ be a non-constant function, meromorphic in the halfplane $\{\operatorname{Im} z \geq 0\}$. Then

$$
\mathfrak{m}(r, a)+\mathfrak{N}(r, a)=\mathfrak{T}(r, f)+\varepsilon_{3}(r, a), \quad 1<r<\infty
$$

for each complex number $a \neq \infty$, where $\varepsilon_{3}(r, a)=O(1)$ as $r \rightarrow \infty$.

Proof. This is proved by an argument similar to those in the proofs of Theorems 5.1 and 5.2. We apply the Levin formula in the form (5.3) to $f(z)-a$. Combining this result with the observation that (4.6) implies

$$
\begin{aligned}
|\mathfrak{m}(r, f-a)-\mathfrak{m}(r, f)| & \leq\left(\ln ^{+}|a|+\ln 2\right) \frac{1}{2 \pi} \int_{\kappa(r)}^{\pi-\kappa(r)} \frac{d \varphi}{r \sin ^{2} \varphi} \\
& =\left(\ln ^{+}|a|+\ln 2\right) \frac{1}{2 \pi r} 2 \cot \kappa(r) \\
& =\left(\ln ^{+}|a|+\ln 2\right) \frac{\sqrt{r^{2}-1}}{\pi r} \leq \frac{1}{\pi}\left(\ln ^{+}|a|+\ln 2\right),
\end{aligned}
$$

we get the desired result.

Using Theorems 3.3 and 3.6 it is possible to prove analogs of the ShimizuAhlfors theorem for angular Nevanlinna characteristics and for Tsuji characteristics. Since these analogs will not be used in this book, we are not going to do this. Among the results of this type we prove the following fact only.

THEOREM 5.4. There exist continuous non-decreasing functions $\stackrel{\circ}{S}_{\alpha \beta}(r, f)$ and $\stackrel{\circ}{\mathfrak{T}}(r, f)$, such that

$$
\begin{gathered}
S_{\alpha \beta}(r, f)=\stackrel{\circ}{S}_{\alpha \beta}(r, f)+O(1), \\
\mathfrak{T}(r, f)=\stackrel{\circ}{\mathfrak{T}}(r, f)+O(1) .
\end{gathered}
$$


Proof. Let

$$
\begin{gathered}
\stackrel{\circ}{S}(r, f)=\frac{1}{\pi} \int_{1}^{r} \int_{1}^{\pi}\left(\frac{1}{t}-\frac{t}{r^{2}}\right)\left(\stackrel{\circ}{f}\left(t e^{i \theta}\right)\right)^{2} \sin \theta t d t d \theta, \\
\stackrel{\circ}{\mathfrak{T}}(r, f)=\frac{1}{\pi} \iint_{\left|z-\frac{i r}{2}\right| \leq \frac{r}{2},|z| \geq 1}\left(\frac{\sin \theta}{t}-\frac{1}{r}\right)\left(\stackrel{\circ}{f}\left(t e^{i \theta}\right)\right)^{2} t d t d \theta .
\end{gathered}
$$

Since the integrands in both integrals are non-negative and are non-decreasing as functions of $r$, then $\stackrel{\circ}{S}(r, f)$ and $\stackrel{\circ}{\mathfrak{T}}(r, f)$ are non-decreasing functions of $r$. Using the inequality

$$
\left|\ln ^{+}\right| f\left|-\ln \sqrt{1+|f|^{2}}\right| \leq \frac{1}{2} \ln 2,
$$

and Theorems 3.3 and 3.6, we get the estimates

$$
|S(r, f)-\stackrel{\circ}{S}(r, f)| \leq \frac{2}{\pi} \cdot \frac{1}{2} \ln 2+\frac{4}{\pi} \cdot \frac{1}{2} \ln 2+|Q(r, 1 ; f)|+\left|Q^{(2)}(r, 1 ; f)\right|,
$$

and

$$
|\mathfrak{T}(r, f)-\stackrel{\circ}{\mathfrak{T}}(r, f)| \leq \frac{1}{\pi} \cdot \frac{1}{2} \ln 2+|\tilde{Q}(r, 1 ; f)|+\left|\tilde{Q}^{(2)}(r, 1 ; f)\right| .
$$

These estimates imply the statement of the theorem for $S=S_{0 \pi}$ and $\mathfrak{T}$. Generalization for $S_{\alpha \beta}(r, f)$ can be achieved in the same way as in Theorem 5.2.

In many situations the characteristics $\stackrel{\circ}{S}_{\alpha \beta}(r, f)$ and $\stackrel{\circ}{\mathfrak{T}}(r, f)$ are more convenient than $S_{\alpha \beta}(r, f)$ and $\mathfrak{T}(r, f)$.

\section{Some relations between the characteristics}

The relations which we derive in this section will be used very often in this book, usually without reference.

Let $f_{1}(z), \ldots, f_{q}(z)$ be meromorphic functions. The inequalities (4.3) and (4.4) immediately imply

$$
m\left(r, \prod_{j=1}^{q} f_{j}\right) \leq \sum_{j=1}^{q} m\left(r, f_{j}\right)
$$

and

$$
m\left(r, \sum_{j=1}^{q} f_{j}\right) \leq \sum_{j=1}^{q} m\left(r, f_{j}\right)+\ln q .
$$

If $f_{j}(z)$ has a pole of order $\lambda_{j} \geq 0$ at $z_{0}$, then the order of the pole of $\prod_{j=1}^{q} f_{j}(z)$ at $z_{0}$ is at most $\sum_{j=1}^{q} \lambda_{j}$, and the order of the pole of $\sum_{j=1}^{q} f_{j}(z)$ at $z_{0}$ is at most $\max _{1 \leq j \leq q} \lambda_{j} \leq \sum_{j=1}^{q} \lambda_{j}$. Hence, if we denote the number of poles of $f_{j}(z)$ in a set $D \subset \mathbf{C}$ by $n_{j}(D)$, and the number of poles of $\prod_{j=1}^{q} f_{j}$ (or $\sum_{j=1}^{q} f_{j}$ ) in $D$ by $n(D)$, then

$$
n(D) \leq \sum_{j=1}^{q} n_{j}(D)
$$

In particular, for $D=\{0<|z| \leq t\}$ we get

$$
n\left(t, \prod_{j=1}^{q} f_{j}\right)-n\left(0, \prod_{j=1}^{q} f_{j}\right) \leq \sum_{j=1}^{q}\left[n\left(t, f_{j}\right)-n\left(0, f_{j}\right)\right]
$$




$$
n\left(t, \sum_{j=1}^{q} f_{j}\right)-n\left(0, \sum_{j=1}^{q} f_{j}\right) \leq \sum_{j=1}^{q}\left[n\left(t, f_{j}\right)-n\left(0, f_{j}\right)\right],
$$

and for $D=\{z=0\}$ we get

$$
n\left(0, \prod_{j=1}^{q} f_{j}\right) \leq \sum_{j=1}^{q} n\left(0, f_{j}\right), n\left(0, \sum_{j=1}^{q} f_{j}\right) \leq \sum_{j=1}^{q} n\left(0, f_{j}\right) .
$$

If $r \geq 1$, these inequalities imply

$$
N\left(r, \prod_{j=1}^{q} f_{j}\right) \leq \sum_{j=1}^{q} N\left(r, f_{j}\right)
$$

and

$$
N\left(r, \sum_{j=1}^{q} f_{j}\right) \leq \sum_{j=1}^{q} N\left(r, f_{j}\right)
$$

Adding (6.1) to (6.3), and (6.2) to (6.4), we get for $r \geq 1$ :

$$
\begin{gathered}
T\left(r, \prod_{j=1}^{q} f_{j}\right) \leq \sum_{j=1}^{q} T\left(r, f_{j}\right), \\
T\left(r, \sum_{j=1}^{q} f_{j}\right) \leq \sum_{j=1}^{q} T\left(r, f_{j}\right)+\ln q .
\end{gathered}
$$

Theorem 4.1 can be considered as the following property of the characteristic $T(r, f)$ :

$$
T\left(r, \frac{1}{f-a}\right)=T(r, f)+O(1) .
$$

Let us prove the following more general fact

$$
T(r, L(f))=T(r, f)+O(1),
$$

where

$$
L(w)=\frac{\alpha w+\beta}{\gamma w+\delta}, \quad \alpha \delta-\beta \gamma \neq 0,
$$

is a Möbius transformation. Properties of Möbius transformations imply that it suffices to consider the following cases: 1) $L(w)=a w, 2) L(w)=w-a, 3) L(w)=$ $\frac{1}{w}$, where $a$ is a complex number, $a \neq 0, \infty$. By (4.5) and (4.6) we have

$$
\begin{gathered}
|m(r, a f)-m(r, f)| \leq|\ln | a||, \\
|m(r, f-a)-m(r, f)| \leq \ln ^{+}|a|+\ln 2 .
\end{gathered}
$$

Since it is clear that $N(r, a f)=N(r, f), N(r, f-a)=N(r, f)$, then we get (6.8) in the cases 1) and 2). In the case 3) the relation (6.8) coincides with (6.7) for $a=0$.

Observe that if $|f(0)|=1$, then (4.1) immediately implies

$$
T\left(r, \frac{1}{f}\right)=T(r, f) \text {. }
$$


In a similar manner, for functions meromorphic in an angle $\{\alpha \leq \arg z \leq \beta\}$, $0<\beta-\alpha \leq 2 \pi$, we get $(r \geq 1)$

$$
\begin{gathered}
D_{\alpha \beta}\left(r, \prod_{j=1}^{q} f_{j}\right) \leq \sum_{j=1}^{q} D_{\alpha \beta}\left(r, f_{j}\right), \\
D_{\alpha \beta}\left(r, \sum_{j=1}^{q} f_{j}\right) \leq \sum_{j=1}^{q} D_{\alpha \beta}\left(r, f_{j}\right)+\ln q,
\end{gathered}
$$

where $D_{\alpha \beta}(r, f)$ is any of the characteristics $A_{\alpha \beta}(r, f), B_{\alpha \beta}(r, f)$, and $C_{\alpha \beta}(r, f)$;

$$
S_{\alpha \beta}(r, L(f))=S_{\alpha \beta}(r, f)+O(1) .
$$

For functions analytic in the half-plane

$$
\{\operatorname{Im} z \geq 0\}
$$

we get

$$
\begin{gathered}
D\left(r, \prod_{j=1}^{q} f_{j}\right) \leq \sum_{j=1}^{q} D\left(r, f_{j}\right), \\
D\left(r, \sum_{j=1}^{q} f_{j}\right) \leq \sum_{j=1}^{q} D\left(r, f_{j}\right)+O(1),
\end{gathered}
$$

where $D(r, f)$ is $\mathfrak{m}(r, f)$ or $\mathfrak{N}(r, f)$; and also

$$
\mathfrak{T}(r, L(f))=\mathfrak{T}(r, f)+O(1)
$$

From (6.5), (6.6), and (6.7) we get immediately

$$
T\left(r, R\left(f_{1}, \ldots, f_{q}\right)\right)=O\left(\sum_{j=1}^{q} T\left(r, f_{j}\right)\right),
$$

where $R\left(w_{1}, \ldots, w_{q}\right)$ is a rational function in $w_{1}, \ldots, w_{q}$. The equality $(6.15)$ can be made more precise in several important cases. Let

$$
F(z)=f_{0}(z)+f_{1}(z) f(z)+\ldots+f_{q}(z)[f(z)]^{q},
$$

where $f_{0}(z), \ldots, f_{q}(z), f(z)$ are meromorphic functions. Then

$$
T(r, F) \leq q T(r, f)+\sum_{j=0}^{q} T\left(r, f_{j}\right)+O(1) .
$$

This statement can be proved using induction in $q$. For $q=1$ the inequality (6.16) follows immediately from (6.5) and (6.6). Assume that (6.16) has been proved for $q-1$. Then

$$
T\left(r, F_{1}\right) \leq(q-1) T(r, f)+\sum_{j=1}^{q} T\left(r, f_{j}\right)+O(1),
$$


where $F_{1}(z)=f_{1}(z)+f_{2}(z) f(z)+\ldots+f_{q}(z)[f(z)]^{q-1}$. Since $F(z)=f_{0}(z)+$ $f(z) F_{1}(z)$, then

$$
T(r, F) \leq T\left(r, f_{0}\right)+T(r, f)+T\left(r, F_{1}\right)+O(1) \leq q T(r, f)+\sum_{j=0}^{q} T\left(r, f_{j}\right)+O(1) .
$$

The following inequalities can be proved using the same argument as were used to prove (6.16)

$$
\begin{gathered}
m(r, F) \leq q m(r, f)+\sum_{j=0}^{q} m\left(r, f_{j}\right)+O(1), \\
N(r, F) \leq q N(r, f)+\sum_{j=1}^{q} N\left(r, f_{j}\right) .
\end{gathered}
$$

(The inequality (6.18) can be also easily proved directly).

Definition. A meromorphic function $f(z)$ is called a rational function of degree $d$ if it can be written as a quotient of two polynomials, $P(z)$ and $Q(z)$, which do not have common zeros, and the maximum of degrees of $P(z)$ and $Q(z)$ is equal to $d$.

THEOREM 6.1. If $R(u)$ is a rational function of degree $d$ and $f(z)$ is a meromorphic function, then

$$
T(r, R(f))=d T(r, f)+O(1) .
$$

We start by proving the following result.

THEOREM 6.2. If $P(u)$ is a polynomial of degree $p$ and $f(z)$ is a meromorphic function, then

$$
\begin{gathered}
T(r, P(f(z)))=p T(r, f(z))+O(1), \\
m(r, P(f(z)))=p m(r, f(z))+O(1), \\
N(r, P(f(z)))=p N(r, f(z)) .
\end{gathered}
$$

The equation (6.22) is obvious, because if $f(z)$ has a pole of order $\lambda \geq 0$ at $z_{0}$, then $P(f(z))$ has a pole of order $\lambda p$ at $z_{0}$.

To prove (6.21) we need the following lemma, which will be used many times later.

Lemma 6.1. Let $K$ be a positive number and let $E$ be the set of those $\varphi$ satisfying $0 \leq \varphi<2 \pi$ for which $\left|f\left(r e^{i \varphi}\right)\right|>K$. Then

$$
\frac{1}{2 \pi} \int_{E} \ln \left|f\left(r e^{i \varphi}\right)\right| d \varphi=m(r, f)+O(1)
$$

This lemma immediately follows from the equality

$$
\frac{1}{2 \pi} \int_{E} \ln \left|f\left(r e^{i \varphi}\right)\right| d \varphi=m\left(r, \frac{f}{K}\right)+\frac{\operatorname{mes} E}{2 \pi} \ln K=m(r, f)+O(1) .
$$

To prove (6.21) we observe that (6.17) implies

$$
m(r, P(f)) \leq p m(r, f)+O(1) .
$$


Let $P(u)=a_{p} u^{p}+a_{p-1} u^{p-1}+\ldots+a_{0}, a_{p} \neq 0$. Let $K>1$ be so large that

$$
\left|a_{p}+\frac{a_{p-1}}{u}+\ldots+\frac{a_{0}}{u^{p}}\right| \geq \frac{1}{2}\left|a_{p}\right|
$$

for $|u|>K$. Then for $|f(z)|>K$ we have $|P(f(z))| \geq|f(z)|^{p}\left|\frac{a_{p}}{2}\right|$. Let us denote by $E$ the set of those arcs of $\{|z|=r\}$ for which $|f(z)|>K$. Using the lemma we get

$$
\begin{aligned}
& m(r, P(f)) \geq \frac{1}{2 \pi} \int_{E} \ln \left|P\left(f\left(r e^{i \varphi}\right)\right)\right| d \varphi \geq \frac{1}{2 \pi} \int_{E} \ln \left\{\left|f\left(r e^{i \varphi}\right)\right|^{p}\left|\frac{a_{p}}{2}\right|\right\} d \varphi \\
& =\frac{p}{2 \pi} \int_{E} \ln \left|f\left(r e^{i \varphi}\right)\right| d \varphi+O(1)=p m(r, f)+O(1) .
\end{aligned}
$$

The assertion (6.21) follows from (6.23) and (6.24). The assertion (6.20) follows from (6.21) and (6.22).

Proof of Theorem 6.1. Let $R(u)=P(u) / Q(u)$. Without loss of generality we may assume that the degree of $Q(u)$ is equal to $d$. If it is not the case we would use

$$
T\left(r, \frac{P(f)}{Q(f)}\right)=T\left(r, \frac{Q(f)}{P(f)}\right)+O(1) .
$$

Also, we may assume that the degree of $P(u)$ is strictly less than $d$, because, if the degree of $P(u)$ is $d$, we may use the equation

$$
T\left(r, \frac{P(f)}{Q(f)}\right)=T\left(r, \frac{P(f)}{Q(f)}-R(\infty)\right)+O(1)=T\left(r, \frac{P_{1}(f)}{Q(f)}\right)+O(1)
$$

where $P_{1}(u)=P(u)-Q(u) R(\infty)$ is a polynomial whose degree is strictly less than $d$. So we assume that the degree of $Q(u)$ is $d$ and the degree of $P(u)$ is strictly less than $d$. We cover the zeros of $Q(u)$ by a collection of open discs $\Gamma$ in such a way that none of the zeros of $P(u)$ is contained in the closure of $\Gamma$. Denote by $\Omega$ the complement of $\Gamma$ in the extended complex plane. Since $R(u) \rightarrow 0$ and $1 / Q(u) \rightarrow 0$ as $u \rightarrow \infty$, then the functions $R(u)$ and $1 / Q(u)$ are bounded on $\Omega$. There exist real numbers $0<M_{1}, M_{2}<\infty$ such that $M_{1}<|P(u)|<M_{2}$ for each $u \in \Gamma$. Hence for $u \in \Gamma$ we have

$$
\frac{M_{1}}{|Q(u)|} \leq|R(u)| \leq \frac{M_{2}}{|Q(u)|}
$$

Let $E_{1}$ and $E_{2}$ be the subsets of $\{|z|=r\}$ where $f(z) \in \Gamma$ and $f(z) \in \Omega$ respectively. Then

$$
\begin{aligned}
m(r, R(f)) & =\frac{1}{2 \pi} \int_{z \in E_{1}} \ln ^{+}|R(f(z))| d \varphi+\frac{1}{2 \pi} \int_{z \in E_{2}} \ln ^{+}|R(f(z))| d \varphi \\
& =\frac{1}{2 \pi} \int_{z \in E_{1}} \ln ^{+}|R(f(z))| d \varphi+O(1)
\end{aligned}
$$

Further

$$
\begin{aligned}
\frac{1}{2 \pi} \int_{z \in E_{1}} & \ln ^{+}|R(f(z))| d \varphi \leq \frac{1}{2 \pi} \int_{z \in E_{1}} \ln ^{+} \frac{M_{2}}{|Q(f(z))|} d \varphi \\
& \leq \frac{1}{2 \pi} \int_{0}^{2 \pi} \ln ^{+} \frac{M_{2}}{|Q(f(z))|} d \varphi=m\left(r, \frac{M_{2}}{Q(f)}\right)=m\left(r, \frac{1}{Q(f)}\right)+O(1)
\end{aligned}
$$




$$
\begin{aligned}
\frac{1}{2 \pi} \int_{z \in E_{1}} \ln ^{+}|R(f(z))| d \varphi & \geq \frac{1}{2 \pi} \int_{z \in E_{1}} \ln ^{+} \frac{M_{1}}{|Q(f(z))|} d \varphi \\
& =m\left(r, \frac{M_{1}}{Q(f)}\right)-\frac{1}{2 \pi} \int_{z \in E_{2}} \ln ^{+} \frac{M_{1}}{|Q(f(z))|} d \varphi=m\left(r, \frac{1}{Q(f)}\right)+O(1)
\end{aligned}
$$

Combining these estimates we get

$$
m(r, R(f))=m\left(r, \frac{1}{Q(f)}\right)+O(1) .
$$

On the other hand poles of the functions $R(f(z))$ and $1 / Q(f(z))$ coincide. In fact, if the function $f(z)$ has a pole at $z_{0}$, then the order of the pole of $Q(f(z))$ at $z_{0}$ is greater than the order of the pole of $P(f(z))$ at $z_{0}$, and $R\left(f\left(z_{0}\right)\right)=0$. Hence the function $R(f(z))$ can have poles only at zeros of the function $Q(f(z))$. The function $Q(f(z))$ has zeros only at those points at which $f(z)$ is equal to one of the zeros of $Q(u)$. Since zeros of $P(u)$ are different from zeros of $Q(u)$, then $|P(f(z))|+|Q(f(z))|>0$. Therefore the functions $R(f(z))$ and $1 / Q(f(z))$ have the same poles. Hence $N(r, R(f))=N(r, 1 / Q(f))$. Adding this equality to (6.25) we get

$$
T(r, R(f))=T(r, 1 / Q(f))+O(1)
$$

By (6.20) we have

$$
T(r, 1 / Q(f))=T(r, Q(f))+O(1)=d T(r, f)+O(1) .
$$

Theorem 6.1 has been proved.

The relations (6.14) and (6.20) are particular cases of Theorem 6.1. We would like to mention also the following

Corollary. If $R(z)$ is a rational function of degree $d$, then

$$
T(r, R(z))=d \ln r+O(1) .
$$

In fact, $T(r, z)=\ln ^{+} r$, and (6.26) follows from (6.19).

EXERCISES. (i) Let $p$ be a natural number. Prove the relation $T\left(r,[f(z)]^{p}\right)=$ $p T(r, f(z))$.

(ii) Prove that $T(r, \cosh z)=2 T\left(r, e^{z}\right)+O(1)=\frac{2}{\pi} r+O(1)$.

A theorem, analogous to Theorem 6.1, is valid for characteristics $S_{\alpha \beta}$ and $\mathfrak{T}$.

TheOREm 6.3. Let $R(u)$ be a rational function of degree $d$. If $f(z)$ is meromorphic in an angle $\{\alpha \leq \arg z \leq \beta\}, 0<\beta-\alpha \leq 2 \pi$, then

$$
S_{\alpha \beta}(r, R(f))=d S_{\alpha \beta}(r, f)+O(1) .
$$

If $f(z)$ is meromorphic in the half-plane $\{\operatorname{Im} z \geq 0\}$, then

$$
\mathfrak{T}(r, R(f))=d \mathfrak{T}(r, f)+O(1) .
$$

This theorem can be proved by minor modifications of the argument used in the proof of Theorem 6.1. We leave the proof to the reader as exercise.

Definition. A meromorphic function is called transcendental if it is not rational. 
THEOREM 6.4. A meromorphic function $f(z)$ is transcendental if and only if

$$
\liminf _{r \rightarrow \infty} \frac{T(r, f)}{\ln r}=\infty .
$$

Sufficiency of the condition (6.27) follows from (6.26). Let us prove the necessity. Assume that (6.27) does not take place, that is,

$$
\liminf _{r \rightarrow \infty} \frac{T(r, f)}{\ln r}=K<\infty .
$$

Then $f(z)$ has at most $p=[K]$ poles. In fact, let us number the poles according to their moduli, the pole with the smallest modulus comes first. If there is a pole number $(p+1)$, denote its modulus by $r_{0}$. We have

$$
\begin{aligned}
N(r, f) & \geq \int_{r_{0}}^{r} \frac{n(t, f)-n(0, f)}{t} d t+n(0, f) \ln r \\
& \geq \int_{r_{0}}^{r} \frac{p+1-n(0, f)}{t} d t+n(0, f) \ln r \\
& =[p+1-n(0, f)] \ln \frac{r}{r_{0}}+n(0, f) \ln r=(p+1) \ln r+O(1) .
\end{aligned}
$$

Hence

$$
\liminf _{r \rightarrow \infty} \frac{T(r, f)}{\ln r} \geq \liminf _{r \rightarrow \infty} \frac{N(r, f)}{\ln r} \geq p+1>K,
$$

we get a contradiction with (6.28). In a similar way we prove that $f(z)$ has at most $p$ zeros. Therefore there exists a rational function $R(z)$, such that

$$
g(z)=f(z) R(z)
$$

has neither zeros nor poles. Since

$$
T(r, g) \leq T(r, f)+T(r, R)=T(r, f)+O(\ln r),
$$

then

$$
\liminf _{r \rightarrow \infty} \frac{T(r, g)}{\ln r} \leq K_{1}<\infty .
$$

We need to show that $g(z) \equiv$ const. This statement can be derived from the following lemma.

LEMMA 6.2. Let $g(z)$ be an entire function without zeros satisfying

$$
\liminf _{r \rightarrow \infty} \frac{T(r, g)}{r^{\lambda}}=0
$$

for some $\lambda>0$. Then

$$
g(z)=e^{P(z)}
$$

where $P(z)$ is a polynomial whose degree is less than $\lambda$.

Proof. Observe that the function

$$
P(z)=\ln g(z)=\int_{0}^{z} \frac{g^{\prime}(\zeta)}{g(\zeta)} d \zeta+\ln g(0)
$$

is an entire function. Let

$$
P(z)=\sum_{p=0}^{\infty} c_{p} z^{p}
$$


By (2.6) for all $r>0$ and $p=1,2, \ldots$ we have

$$
\left.\frac{d^{p}}{d z^{p}} \ln g(z)\right|_{z=0}=\frac{2 p !}{r^{p}} \frac{1}{2 \pi} \int_{0}^{2 \pi} \ln \left|g\left(r e^{i \theta}\right)\right| e^{-i p \theta} d \theta
$$

hence

$$
\begin{aligned}
\left|c_{p}\right| & =\frac{1}{p !}\left|\frac{d^{p}}{d z^{p}} \ln g(z)\right|_{z=0}\left|\leq \frac{2}{r^{p}} \frac{1}{2 \pi} \int_{0}^{2 \pi}\right| \ln \mid g\left(r e^{i \theta}\right) \| d \theta \\
& =\frac{2}{r^{p}}\left[m(r, g)+m\left(r, \frac{1}{g}\right)\right] .
\end{aligned}
$$

Since $m(r, g) \leq T(r, g)$, and (by Theorem 4.1) $m\left(r, \frac{1}{g}\right) \leq T(r, g)+O(1)$, then

$$
\left|c_{p}\right| \leq \frac{2}{r^{p}}[2 T(r, g)+O(1)] .
$$

Letting $r \rightarrow \infty$, we see that $c_{p}=0$ for $p \geq \lambda$.

We are mostly interested in transcendental meromorphic functions. For this reason, in our estimates for $T(r, f)$, terms which are $O(\ln r)$ can be disregarded.

Theorem 6.5. Let $f(z)$ be a meromorphic function, $R_{j}(z), 0 \leq j \leq p$, be rational functions, $R_{p}(z) \not \equiv 0$; and

$$
F(z)=R_{p}(z)[f(z)]^{p}+R_{p-1}(z)[f(z)]^{p-1}+\ldots+R_{0}(z) .
$$

Then

$$
\begin{aligned}
& T(r, F)=p T(r, f)+O(\ln r), \\
& m(r, F)=p m(r, f)+O(\ln r), \\
& N(r, F)=p N(r, f)+O(\ln r) .
\end{aligned}
$$

Proof. For rational $f(z)$ the result is trivial. For this reason we assume that $f(z)$ is transcendental. Let $M$ be a finite set containing the poles of all functions $R_{j}(z), 0 \leq j \leq p$, and the zeros of $R_{p}(z)$. If the function $f(z)$ has a pole of order $\lambda(\geq 0)$ at some $z_{0} \notin M$, then $F(z)$ has a pole of order $\lambda p$ at $z_{0}$. Hence $n(r, F)=p n(r, f)+O(1)$, and we get the equality (6.31). From (6.17) we get $m(r, F) \leq p m(r, f)+O(\ln r)$. Let us show that the inverse inequality is also true. Let $R_{j}(z)=\beta_{j} z^{\alpha_{j}}(1+o(1))$ as $z \rightarrow \infty$, where $\beta_{j} \neq 0$, and $\alpha_{j}$ is an integer. Denote by $\lambda$ the largest of the differences $\alpha_{j}-\alpha_{p}, 0 \leq j \leq p-1$. Let $\chi=\max \left(1, \lambda+1, \alpha_{p}\right)$. Denote by $E$ the set of those $z$ for which

$$
|f(z)| \geq|z|^{\chi}, \Omega=\{|z|<\infty\} \backslash E .
$$

It is clear that for $z \in E,|z| \geq r_{0}$ we have

$$
\begin{gathered}
\left|1+\frac{R_{p-1}(z)}{R_{0}(z)} \frac{1}{f(z)}+\cdots+\frac{R_{0}(z)}{R_{p}(z)} \frac{1}{[f(z)]^{p}}\right| \\
=\left|1+\frac{\beta_{p-1}}{\beta_{p}} \frac{z^{\alpha_{p-1}-\alpha_{p}}(1+o(1))}{f(z)}+\cdots+\frac{\beta_{0}}{\beta_{p}} \frac{z^{\alpha_{0}-\alpha_{p}}(1+o(1))}{[f(z)]^{p}}\right| \\
\geq 1-\left|\frac{\beta_{p-1}}{\beta_{p}}\right||z|^{\lambda-\chi}(1+o(1))-\cdots-\left|\frac{\beta_{0}}{\beta_{p}}\right||z|^{\lambda-p \chi}(1+o(1))>\frac{1}{2}, \\
|F(z)| \geq \frac{\left|\beta_{p}\right|}{2}(1+o(1))|z|^{\alpha_{p}}|f(z)|^{p} .
\end{gathered}
$$


Let $E(r)=E \cap\{|z|=r\}, \Omega(r)=\Omega \cap\{|z|=r\}$. Then $\left(z=r e^{i \varphi}\right)$

$$
\begin{aligned}
m(r, F) & \geq \frac{1}{2 \pi} \int_{E(r)} \ln ^{+}|F(z)| d \varphi \geq O(\ln r)+\frac{1}{2 \pi} \int_{E(r)} \ln ^{+}\{|f(z)|\}^{p} d \varphi \\
& =O(\ln r)+p m(r, f)-\frac{1}{2 \pi} \int_{\Omega(r)} \ln ^{+}\left\{|f(z)|^{p}\right\} d \varphi \\
& \geq O(\ln r)+p m(r, f)-p \chi \ln r=p m(r, f)+O(\ln r) .
\end{aligned}
$$

We have proved (6.30). The equality (6.29) follows from (6.30) and (6.31).

COROLlary. Let $f(z)$ be a meromorphic function whose series in the neighborhood of $z=0$ is $f(z)=c_{\lambda} z^{\lambda}+c_{\lambda+1} z^{\lambda+1}+\ldots, c_{\lambda} \neq 0$. Let $f_{1}(z)=f(z) / c_{\lambda} z^{\lambda}$. Then

$$
\begin{gathered}
T\left(r, f_{1}\right)=T(r, f)+O(\ln r), m\left(r, f_{1}\right)=m(r, f)+O(\ln r), \\
N\left(r, f_{1}\right)=N(r, f)+O(\ln r), m\left(r, \frac{1}{f_{1}}\right)=m\left(r, \frac{1}{f}\right)+O(\ln r), \\
N\left(r, \frac{1}{f_{1}}\right)=N\left(r, \frac{1}{f}\right)+O(\ln r) .
\end{gathered}
$$

This corollary allows us, in many cases, to assume that transcendental function $f(z)$ under consideration is normalized by the condition $f(0)=1$.

EXERCISE. Let $f(z)$ be a meromorphic function, and $R_{j}(z)$ be rational functions, $1 \leq j \leq 4$, satisfying the condition: $R_{1}(z) R_{4}(z)-R_{2}(z) R_{3}(z) \not \equiv 0$. Set

$$
F(z)=\frac{R_{1}(z) f(z)+R_{2}(z)}{R_{3}(z) f(z)+R_{4}(z)}
$$

Show that $T(r, F)=T(r, f)+O(\ln r)$.

Proofs of the following two theorems are left to the reader as exercises.

THEOREM 6.6. Let $f(z)$ be a meromorphic function, $p$ be a natural number, $f_{1}(z)=f\left(z^{p}\right)$. Then

$$
\begin{gathered}
m\left(r, f_{1}\right)=m\left(r^{p}, f\right), N\left(r, f_{1}\right)=N\left(r^{p}, f\right) \\
T\left(r, f_{1}\right)=T\left(r^{p}, f\right) .
\end{gathered}
$$

Corollary. Let $p$ be a natural number, $f(z)$ be a meromorphic function, such that $f_{1}(z)=f\left(z^{1 / p}\right)$ is single-valued. Then

$$
\begin{gathered}
m\left(r, f_{1}\right)=m\left(r^{1 / p}, f\right), N\left(r, f_{1}\right)=N\left(r^{1 / p}, f\right), \\
T\left(r, f_{1}\right)=T\left(r^{1 / p}, f\right) .
\end{gathered}
$$

THEOREM 6.7. Let $f(z)$ be a meromorphic function, $f_{1}(z)=f(A z)$, where $A \neq 0$. Then

$$
\begin{gathered}
m\left(r, f_{1}\right)=m(|A| r, f), N\left(r, f_{1}\right)=N(|A| r, f)-n(0, f) \ln |A|, \\
T\left(r, f_{1}\right)=T(|A| r, f)-n(0, f) \ln |A| .
\end{gathered}
$$




\section{Connection between the growth of the modulus of a meromorphic function and the growth of its characteristic $T(r, f)$}

Let $f(z)$ be a meromorphic function. Let

$$
M(r, f)=\max _{|z|=r}|f(z)| .
$$

We shall study relations between $M(r, f)$ and $T(r, f)$. First we consider the case when $f$ is entire.

THEOREM 7.1. Let $f(z)$ be an entire function, and let $0<r<R$. Then

$$
T(r, f) \leq \ln ^{+} M(r, f) \leq \frac{R+r}{R-r} T(R, f) .
$$

Proof. Since $\ln ^{+}|f(z)| \leq \ln ^{+} M(r, f)$ for $|z|=r$, then

$$
T(r, f)=m(r, f) \leq \ln ^{+} M(r, f) .
$$

Next we suppose that $\left|f\left(z_{0}\right)\right|=M(r, f),\left|z_{0}\right|=r$. We use the Poisson-Jensen formula (2.2) for a disc $\{|z| \leq R\}$. We get

$$
\begin{aligned}
\ln M(r, f) & =\ln \left|f\left(z_{0}\right)\right| \\
& =\frac{1}{2 \pi} \int_{0}^{2 \pi} \ln \left|f\left(R e^{i \theta}\right)\right| \operatorname{Re} \frac{R e^{i \theta}+z_{0}}{R e^{i \theta}-z_{0}} d \theta-\sum_{\left|a_{\mu}\right|<R} \ln \left|\frac{R^{2}-\bar{a}_{\mu} z_{0}}{R\left(r_{0}-a_{\mu}\right)}\right| .
\end{aligned}
$$

Since the summands in the right-hand side of (7.2) represent the values of the Green function for a disc, they are positive. Also

$$
0<\operatorname{Re} \frac{R e^{i \theta}+z_{0}}{R e^{i \theta}-z_{0}}=\frac{R^{2}-\left|z_{0}\right|^{2}}{\left|R e^{i \theta}-z_{0}\right|^{2}} \leq \frac{R+r}{R-r} .
$$

Therefore

$$
\begin{aligned}
& \frac{1}{2 \pi} \int_{0}^{2 \pi} \ln \left|f\left(R e^{i \theta}\right)\right| \operatorname{Re} \frac{R e^{i \theta}+z_{0}}{R e^{i \theta}-z_{0}} d \theta \\
\leq & \frac{1}{2 \pi} \int_{0}^{2 \pi} \ln ^{+}\left|f\left(R e^{i \theta}\right)\right| \operatorname{Re} \frac{R e^{i \theta}+z_{0}}{R e^{i \theta}-z_{0}} d \theta \\
\leq & \frac{R+r}{R-r} \frac{1}{2 \pi} \int_{0}^{2 \pi} \ln ^{+}\left|f\left(R e^{i \theta}\right)\right| d \theta=\frac{R+r}{R-r} m(R, f)
\end{aligned}
$$

and

$$
\ln M(r, f) \leq \frac{R+r}{R-r} m(R, f)=\frac{R+r}{R-r} T(R, f) .
$$

Since the right-hand side of this inequality is positive, the right side of the inequality of (7.1) follows.

Now we consider the connection between $T(r, f)$ and $\ln M(r, f)$ for meromorphic functions. In distinction from an entire $f$, for a non-constant meromorphic $f$, it is possible that $\liminf _{r \rightarrow \infty} M(r, f)<\infty$ : in the example on page ??? we have

$$
M(n+1 / 2, f) \leq 1, n=0,1,2, \ldots
$$

Hence, in general, the left inequality in (7.1) is not valid for meromorphic functions. If $f(z)$ has a pole on the circle $\{|z|=r\}$, then $M(r, f)=+\infty$, Hence, in general, an estimate of $M(r, f)$ from above in terms of $T(R, f), R>r$, is impossible. Nevertheless for meromorphic functions there is a significantly weaker analogue of the right inequality in (7.1). We mean the following theorem due to R. Nevanlinna. 
TheOREM 7.2. Let $f(z)$ be a meromorphic function, $k>1$ be a real number. Then

$$
\frac{1}{r} \int_{0}^{r} \ln ^{+} M(t, f) d t \leq C(k) T(k r, f),
$$

where the constant $C(k)>1$ depends on $k$ only.

First we prove two lemmas, which will be used not only in the proof of Theorem 7.2 .

Lemma 7.1. Let $R^{\prime}>R>1$. Then

$$
n(R, f) \leq \frac{N\left(R^{\prime}, f\right)}{\ln \frac{R^{\prime}}{R}} \leq \frac{R^{\prime}}{R^{\prime}-R} N\left(R^{\prime}, f\right) .
$$

Proof. Indeed,

$$
\begin{aligned}
& N\left(R^{\prime}, f\right) \geq \int_{R}^{R^{\prime}} \frac{n(t, f)-n(0, f)}{t} d t+n(0, f) \ln R^{\prime} \\
\geq & {[n(R, f)-n(0, f)] \ln \frac{R^{\prime}}{R}+n(0, f) \ln R^{\prime} } \\
= & n(R, f) \ln \frac{R^{\prime}}{R}+n(0, f) \ln R \geq n(R, f) \ln \frac{R^{\prime}}{R}=n(R, f) \int_{R}^{R^{\prime}} \frac{d t}{t} \geq n(R, f) \frac{R^{\prime}-R}{R^{\prime}} .
\end{aligned}
$$

Lemma 7.2. Let $\varphi(x)$ be an even integrable real-valued function on $(-a, a)$, non-increasing on $(0, a)$. (The case $\varphi(0)=+\infty$ is allowed). Let $E \subset(-a, a)$ be a measurable subset, mes $E=2 b$. Then

$$
\int_{E} \varphi(x) d x \leq \int_{-b}^{b} \varphi(x) d x
$$

Proof. Let $E_{1}=E \cap(-b, b), E_{2}=E \backslash E_{1}, E_{3}=(-b, b) \backslash E_{1}$. It is clear that $\operatorname{mes} E_{2}=\operatorname{mes} E_{3}=2 b-\operatorname{mes} E_{1}$. For $x \in(-b, b)$ we have $\varphi(x) \geq \varphi(b)$, and for $x \notin(-b, b)$ we have $\varphi(x) \leq \varphi(b)$. Hence

Adding the integral

$$
\int_{E_{2}} \varphi(x) d x \leq \varphi(b) \operatorname{mes} E_{2}=\varphi(b) \operatorname{mes} E_{3} \leq \int_{E_{3}} \varphi(x) d x .
$$

$$
\int_{E_{1}} \varphi(x) d x
$$

to both sides of the inequality, we get the desired inequality.

Proof. (Theorem 7.2) Denote zeros of $f(z)$ by $a_{\mu}$ 's, and poles of $f(z)$ by $b_{\nu}$ 's. Let $0 \leq t \leq r, R=\sqrt{k} r$. Using the Poisson-Jensen formula for the disc $\{|z| \leq R\}$, we get

$$
\begin{aligned}
& \ln \left|f\left(t e^{i \varphi}\right)\right|=\frac{1}{2 \pi} \int_{0}^{2 \pi} \ln \left|f\left(R e^{i \theta}\right)\right| \operatorname{Re}\left(\frac{R e^{i \theta}+t e^{i \varphi}}{R e^{i \theta}-t e^{i \varphi}}\right) d \theta \\
& \quad+\sum_{\left|b_{\nu}\right|<R} \ln \left|\frac{R^{2}-\bar{b}_{\nu} t e^{i \varphi}}{R\left(t e^{i \varphi}-b_{\nu}\right)}\right|-\sum_{\left|a_{\mu}\right|<R} \ln \left|\frac{R^{2}-\bar{a}_{\mu} t e^{i \varphi}}{R\left(t e^{i \varphi}-a_{\mu}\right)}\right| \\
& \leq \frac{1}{2 \pi} \int_{0}^{2 \pi} \ln \left|f\left(R e^{i \theta}\right)\right| \operatorname{Re}\left(\frac{R e^{i \theta}+t e^{i \varphi}}{R e^{i \theta}-t e^{i \varphi}}\right) d \theta+\sum_{\left|b_{\nu}\right|<R} \ln \left|\frac{R^{2}-\bar{b}_{\nu} t e^{i \varphi}}{R\left(t e^{i \varphi}-b_{\nu}\right)}\right| .
\end{aligned}
$$


We estimate the integral in the right hand side using (7.4), and estimate the sum using

$$
\left|\frac{R^{2}-b_{\nu} t e^{i \varphi}}{R\left(t e^{i \varphi}-b_{\nu}\right)}\right| \leq \frac{R^{2}+\left|b_{\nu}\right| t}{R|t-| b_{\nu}||} \leq \frac{2 R}{|t-| b_{\nu}||} .
$$

Then we get in succession the following inequalities

$$
\begin{aligned}
\ln \left|f\left(t e^{i \varphi}\right)\right| & \leq \frac{R+t}{R-t} m(R, f)+\sum_{\left|b_{\nu}\right|<R} \ln \frac{2 R}{|t-| b_{\nu}||} ; \\
\ln ^{+} M(t, f) & \leq \frac{R+t}{R-t} m(R, f)+\sum_{\left|b_{\nu}\right| \leq R} \ln \frac{2 R}{|t-| b_{\nu}||} \\
& \leq \frac{R+r}{R-r} m(R, f)+\sum_{\left|b_{\nu}\right| \leq R} \ln \frac{2 R}{|t-| b_{\nu}||} ; \\
\frac{1}{r} \int_{0}^{r} \ln ^{+} M(t, f) d t & \leq \frac{R+r}{R-r} m(R, f)+\sum_{\left|b_{\nu}\right| \leq R} \frac{1}{r} \int_{0}^{r} \ln \frac{2 R}{|t-| b_{\nu}||} d t .
\end{aligned}
$$

Using Lemma 7.2 and straightforward transformations, we get

$$
\begin{aligned}
\int_{0}^{r} \ln \frac{2 R}{|t-| b_{\nu}||} d t & =\int_{-\left|b_{\nu}\right|}^{r-\left|b_{\nu}\right|} \ln \frac{2 R}{|\tau|} d \tau \leq \int_{-r / 2}^{r / 2} \ln \frac{2 R}{|\tau|} d \tau \\
& =2 \int_{0}^{r / 2} \ln \frac{2 R}{\tau} d \tau=r \ln \frac{4 e R}{r}=r \ln (4 e \sqrt{k}) .
\end{aligned}
$$

Hence (7.7) implies

$$
\frac{1}{r} \int_{0}^{r} \ln ^{+} M(t, f) d t \leq \frac{\sqrt{k}+1}{\sqrt{k}-1} m(\sqrt{k} r, f)+n(R, f) \ln (4 e \sqrt{k}) .
$$

Taking $R^{\prime}=\sqrt{k} R$, we estimate $n(R, f)$ from above using Lemma 7.1; also, we take into account the inequalities

$$
m(\sqrt{k} r, f) \leq T(\sqrt{k} r, f) \leq T(k r, f), N\left(R^{\prime}, f\right) \leq T\left(R^{\prime}, f\right) \leq T(k r, f) .
$$

Hence (7.8) implies (7.5) with

$$
C(k)=\frac{\sqrt{k}+1}{\sqrt{k}-1}+\frac{\ln (4 e \sqrt{k})}{\ln \sqrt{k}} .
$$

COROLlary. Under the conditions of Theorem 7.2 the inequality holds

$$
\frac{1}{r} \int_{0}^{r} \ln ^{+} M\left(t, \frac{1}{f-a}\right) d t \leq 2 C(k) T(k r, f)
$$

for $r>r_{0}, r_{0}=r_{0}(k, a)$,

To prove this corollary, it suffices to apply Theorem 7.2 to the function $1 /(f(z)-$ $a$ ), and to use the first fundamental theorem (Theorem 4.1).

We shall prove two more theorems connecting the behavior of the modulus of a meromorphic function $f$ with the behavior of $T(r, f)$. 
THEOREM 7.3. Let $f(z)$ be a meromorphic function, $k, \delta, r$ be real numbers satisfying $k>1,0<\delta \leq 2 \pi, r>1$. There exists a constant $C_{1}(k, \delta)$ such that each measurable subset $E_{r} \subset[-\pi, \pi]$ with mes $E_{r}=\delta$ satisfies

$$
\int_{E_{r}} \ln ^{+}\left|f\left(r e^{i \varphi}\right)\right| d \varphi \leq C_{1}(k, \delta) T(k r, f)
$$

where $C_{1}(k, \delta)=\frac{6 k}{k-1} \delta \ln \frac{2 \pi e}{\delta} \rightarrow 0$ as $\delta \rightarrow 0$.

Proof. First we assume that $\delta<\pi$. We write the inequality (7.6) with $t=r$ for the disc $\{|z| \leq R\}, R=\sqrt{k} r$ :

$$
\begin{aligned}
& \ln \left|f\left(r e^{i \varphi}\right)\right| \leq \frac{1}{2 \pi} \int_{0}^{2 \pi} \ln \left|f\left(R e^{i \theta}\right)\right| \operatorname{Re}\left(\frac{R e^{i \theta}+r e^{i \varphi}}{R e^{i \theta}-r e^{i \varphi}}\right) d \theta \\
&+\sum_{\left|b_{\nu}\right|<R} \ln \left|\frac{R^{2}-\bar{b}_{\nu} r e^{i \varphi}}{R\left(r e^{i \varphi}-b_{\nu}\right)}\right| \leq \frac{R+r}{R-r} m(R, f)+\sum_{\left|b_{\nu}\right|<R} \ln \frac{2 R}{\left|r e^{i \varphi}-b_{\nu}\right|} \\
& \leq \frac{R+r}{R-r} T(R, f)+n(0, f) \ln \frac{2 R}{r}+\sum_{0<\left|b_{\nu}\right|<R} \ln \frac{2 R}{\left|r e^{i\left(\varphi-\beta_{\nu}\right)}-\right| b_{\nu}||}, \\
& \beta_{\nu}=\arg b_{\nu} .
\end{aligned}
$$

The right-hand side of the inequality is non-negative, therefore we may replace $\ln \left|f\left(r e^{i \varphi}\right)\right|$ by $\ln ^{+}\left|f\left(r e^{i \varphi}\right)\right|$ in the left-hand side. Let us integrate the obtained inequality over the set $E_{r}$. We get

$$
\begin{aligned}
\int_{E_{r}} \ln ^{+}\left|f\left(r e^{i \varphi}\right)\right| d \varphi \leq & \delta \frac{R+r}{R-r} T(R, f)+\delta n(0, f) \ln \frac{2 R}{r} \\
& +\sum_{0<\left|b_{\nu}\right|<R} \int_{E_{r}} \ln \frac{2 R}{\left|r e^{i\left(\varphi-\beta_{\nu}\right)}-\right| b_{\nu}||} d \varphi .
\end{aligned}
$$

We apply Lemma 7.2 and get

$$
\begin{aligned}
& \int_{E_{r}} \ln \frac{2 R}{\left|r e^{i\left(\varphi-\beta_{\nu}\right)}-\right| b_{\nu}||} d \varphi \leq \int_{-\delta / 2}^{\delta / 2} \ln \frac{2 R}{\left|r e^{i \varphi}-\right| b_{\nu}||} d \varphi \\
\leq & \int_{-\delta / 2}^{\delta / 2} \ln \frac{2 R}{r|\sin \varphi|} d \varphi \leq 2 \int_{0}^{\delta / 2} \ln \frac{\sqrt{k \pi}}{\varphi} d \varphi=\delta \ln \frac{2 e \sqrt{k \pi}}{\delta} .
\end{aligned}
$$

Hence

$$
\begin{aligned}
\int_{E_{r}} \ln ^{+}\left|f\left(r e^{i \varphi}\right)\right| d \varphi \leq & \delta \frac{\sqrt{k}+1}{\sqrt{k}-1} T(\sqrt{k} r, f)+\delta n(0, f) \ln 2 \sqrt{k} \\
& +\{n(\sqrt{k} r, f)-n(0, f)\} \delta \ln \frac{2 \sqrt{k} e \pi}{\delta} \\
\leq & \delta \frac{\sqrt{k}+1}{\sqrt{k}-1} T(k r, f)+n(\sqrt{k} r, f) \delta \ln \frac{2 \sqrt{k} e \pi}{\delta} .
\end{aligned}
$$

We estimate $n(\sqrt{k} r, f)$ using Lemma 7.1 with $R^{\prime}=k r$. We get

$$
n(\sqrt{k} r, f) \leq \frac{N(k r, f)}{\ln \sqrt{k}} \leq \frac{T(k r, f)}{\ln \sqrt{k}} .
$$

Using (7.10) and (7.11) we get

$$
\int_{E_{r}} \ln ^{+}\left|f\left(r e^{i \varphi}\right)\right| d \varphi \leq C(k, \delta) T(k r, f),
$$


where

$$
\begin{aligned}
C(k, \delta) & =\delta\left(\frac{\sqrt{k}+1}{\sqrt{k}-1}+\frac{\ln \frac{2 \sqrt{k} e \pi}{\delta}}{\ln \sqrt{k}}\right)=\delta\left(\frac{2 \sqrt{k}}{\sqrt{k}-1}+\frac{2}{\ln k} \ln \left(\frac{2 e \pi}{\delta}\right)\right) \\
& <\left(\frac{4 k}{k-1}+\frac{2}{\ln k}\right) \delta \ln \frac{2 e \pi}{\delta}<\frac{6 k}{k-1} \delta \ln \frac{2 e \pi}{\delta}=C_{1}(k, \delta),
\end{aligned}
$$

and (7.9) is proved.

For $\pi \leq \delta \leq 2 \pi$ we use the trivial estimate

$$
\int_{E_{r}} \ln ^{+}\left|f\left(r e^{i \varphi}\right)\right| d \varphi \leq 2 \pi m(r, f) \leq 2 \pi T(r, f) .
$$

Since $T(r, f) \leq T(k r, f)$ and $2 \pi \leq 2 \delta \leq C_{1}(k, \delta)$, the inequality (7.9) has been proved in this case also.

REMARK. It is clear that we can replace $C_{1}(k, \delta)$ by $C(k, \delta)<C_{1}(k, \delta)$ in the inequality (7.9), but $C_{1}(k, \delta)$ is simpler than $C(k, \delta)$.

TheOREM 7.4. Let $E$ be a measurable subset of $[-\pi, \pi], \Phi(\varphi)$ be an integrable non-negative function on $E, R(r)$ be a positive function on $\left\{r \geq r_{0}>0\right\}$, satisfying $R(r) \rightarrow \infty$ as $r \rightarrow \infty$, and such that $R(k r)=O\{R(r)\}$. for some real $k>1$. Suppose that $f(z)$ is a meromorphic function, such that $T(r, f)=O\{R(r)\}$, and for each $\delta>0$ there exists a subset $E_{\delta} \subset E$, mes $E_{\delta}=\delta$, such that one of the following three conditions

$$
\begin{aligned}
& \text { (a) } \ln ^{+}\left|f\left(r e^{i \varphi}\right)\right| \leq \Phi(\varphi) R(r)+o(R(r)), \\
& \text { (b) } \ln ^{+}\left|f\left(r e^{i \varphi}\right)\right| \geq \Phi(\varphi) R(r)+o(R(r)), \\
& \text { (c) } \ln ^{+}\left|f\left(r e^{i \varphi}\right)\right|=\Phi(\varphi) R(r)+o(R(r))
\end{aligned}
$$

is satisfied uniformly in $\varphi$ for $\varphi \in E \backslash E_{\delta}$. Then

$$
\begin{aligned}
\left(\mathrm{a}^{\prime}\right) \limsup _{r \rightarrow \infty} \frac{1}{R(r)} \int_{E} \ln ^{+}\left|f\left(r e^{i \varphi}\right)\right| d \varphi & \leq \int_{E} \Phi(\varphi) d \varphi, \\
\left(\mathrm{b}^{\prime}\right) \liminf _{r \rightarrow \infty} \frac{1}{R(r)} \int_{E} \ln ^{+}\left|f\left(r e^{i \varphi}\right)\right| d \varphi & \geq \int_{E} \Phi(\varphi) d \varphi, \\
\left(\mathrm{c}^{\prime}\right) \lim _{r \rightarrow \infty} \frac{1}{R(r)} \int_{E} \ln ^{+}\left|f\left(r e^{i \varphi}\right)\right| d \varphi & =\int_{E} \Phi(\varphi) d \varphi,
\end{aligned}
$$

respectively.

Proof. Let us prove $\left(\mathrm{a}^{\prime}\right)$. Using (7.9) we get

$$
\begin{aligned}
\int_{E} \ln ^{+}\left|f\left(r e^{i \varphi}\right)\right| d \varphi & =\int_{E \backslash E_{\delta}} \ln ^{+}\left|f\left(r e^{i \varphi}\right)\right| d \varphi+\int_{E_{\delta}} \ln ^{+}\left|f\left(r e^{i \varphi}\right)\right| d \varphi \\
& \leq R(r) \int_{E \backslash E_{\delta}} \Phi(\varphi) d \varphi+o(R(r))+C_{1}(k, \delta) T(k r, f) \\
& \leq R(r) \int_{E} \Phi(\varphi) d \varphi+o(R(r))+C_{1}(k, \delta) O\{R(r)\} .
\end{aligned}
$$

Hence

$$
\limsup _{r \rightarrow \infty} \frac{1}{R(r)} \int_{E} \ln ^{+}\left|f\left(r e^{i \varphi}\right)\right| d \varphi \leq \int_{E} \Phi(\varphi) d \varphi+C_{1}(k, \delta) A,
$$


where $A$ is a constant. Letting $\delta \rightarrow 0$, we get $\left(\mathrm{a}^{\prime}\right)$. The proof of $\left(\mathrm{b}^{\prime}\right)$ is trivial after we observe that

$$
\lim _{\delta \rightarrow 0} \int_{E_{\delta}} \Phi(\varphi) d \varphi=0 .
$$

The equation $\left(\mathrm{c}^{\prime}\right)$ follows from $\left(\mathrm{a}^{\prime}\right)$ and $\left(\mathrm{b}^{\prime}\right)$.

REMARK 1. If we replace the function $\Phi(\varphi)$ in Theorem 7.4 by a bounded function $\Phi(\varphi, r)$ depending on a parameter $r$, and allow the set $E_{\delta}$ to be dependent on $r$ also, then, under the condition (c), we have

$$
\int_{E} \ln ^{+}\left|f\left(r e^{i \varphi}\right)\right| d \varphi=R(r) \int_{E} \Phi(\varphi, r) d \varphi+o(R(r)) .
$$

The proof follows the same lines as the proof of Theorem 7.4. In the case when the conditions (a) and (b) are satisfied, the equality in (7.15) will be replaced by the corresponding inequality.

REMARK 2. In the conditions (a), (b), and (c) of Theorem 7.4 the interval $\left[r_{0}, \infty\right)$ can be replaced by a set unbounded from above. Then $\left(\mathrm{a}^{\prime}\right),\left(\mathrm{b}^{\prime}\right)$, and $\left(\mathrm{c}^{\prime}\right)$ will take place for the same set of values of $r$. 


\section{CHAPTER 2}

\section{Meromorphic Functions of Finite Order}

\section{The growth scale for meromorphic functions}

We classify meromorphic functions according to the rate of growth of $T(r, f)$ as $r \rightarrow \infty$.

By Theorem 4.3 from Chapter 1 , the characteristic $T(r, f)$ is a non-decreasing function of $r$. Now we introduce the basic notions related to the growth scale of non-decreasing functions.

Let $\alpha(r)$ be a function defined for $r>0$, which is non-negative and nondecreasing for sufficiently large $r$ (if $\alpha(r)$ satisfies this condition, we write $\alpha(r) \in \Lambda$ ). The number

$$
\rho=\rho[\alpha]=\limsup _{r \rightarrow \infty} \frac{\ln ^{+} \alpha(r)}{\ln r}
$$

is called the order of $\alpha(r)$. If we replace the upper limit by the lower limit in this definition, we get the definition of the lower order, which will be denoted by $\lambda=\lambda[\alpha]$. The function $\alpha(r)$ is said to have finite (infinite) order if $\rho<\infty(\rho=\infty)$. Suppose that $\alpha(r)$ has finite order $\rho$. The number

$$
\sigma=\sigma[\alpha]=\limsup _{r \rightarrow \infty} \frac{\alpha(r)}{r^{\rho}}
$$

is called the magnitude of type of the function $\alpha(r)$. If $\sigma=0$, we say that $\alpha(r)$ has minimal type; if $0<\sigma<\infty$, we say that $\alpha(r)$ has normal (or mean) type; if $\sigma=\infty$, we say that $\alpha(r)$ has maximal type.

Let $\rho$ be order of $\alpha(r), \rho<\infty$, and $\mu$ an arbitrary number satisfying $\mu>\rho$. Then, for sufficiently large $r$, we have $\alpha(r)<r^{(\rho+\mu) / 2}$, and the integral

$$
\int_{1}^{\infty} \frac{\alpha(r)}{r^{\mu+1}} d r
$$

converges. On the other hand, if the integral (1.1) converges $(\mu>0)$, then the order of $\alpha(r)$ is not greater than $\mu$, furthermore, if the order of $\alpha(r)$ is equal to $\mu$, then $\alpha(r)$ is of minimal type. In fact, for $r>r_{0}(\varepsilon)$ we have

$$
\varepsilon>\int_{r}^{\infty} \frac{\alpha(t)}{t^{\mu+1}} d t \geq \alpha(r) \int_{r}^{\infty} \frac{d t}{t^{\mu+1}}=\frac{\alpha(r)}{\mu r^{\mu}}
$$

from which we get

$$
\alpha(r)<\varepsilon \mu r^{\mu} .
$$

Thus, the order of $\alpha(r)$ is equal to the infimum of those $\mu$ for which the integral (1.1) converges (if the set is empty, then $\rho[\alpha]=\infty$ ). 
Let $\alpha(r)$ be a function of finite order $\rho$. We say that $\alpha(r)$ belongs to the convergence class or to the divergence class depending on whether the integral

$$
\int_{1}^{\infty} \frac{\alpha(r)}{r^{\rho+1}} d r
$$

converges or diverges.

The reasoning presented above implies that $\alpha(r)$ has minimal type if it belongs to the convergence class. Functions of normal and maximal type belong to the divergence class.

EXAMPLE. Let $\alpha(r)=r^{\beta}(\ln r)^{\gamma}$ for $r>2,0<\beta<\infty,-\infty<\gamma<\infty$. Then $\alpha(r)$ is of order $\beta$; $\alpha(r)$ is of maximal, normal, or minimal type depending on whether $\gamma>0, \gamma=0$, or $\gamma<0$. The function $\alpha(r)$ belongs to the divergence class if $\gamma \geq-1$, and to the convergence class if $\gamma<-1$.

Definition. We say that functions $\alpha_{1}(r), \alpha_{2}(r) \in \Lambda$ are of the same growth category if they have the same order, and, if the order is finite, have the same type and either both belong to the convergence class, or both belong to the divergence class. We say that $\alpha_{2}(r)$ is of higher growth category than $\alpha_{1}(r)$ if one of the following conditions is satisfied.

1) $\rho\left[\alpha_{2}\right]>\rho\left[\alpha_{1}\right]$.

2) $\rho\left[\alpha_{1}\right]=\rho\left[\alpha_{2}\right]<\infty, \alpha_{1}(r)$ is of minimal type, and $\alpha_{2}(r)$ is of normal or maximal type.

3) $\rho\left[\alpha_{1}\right]=\rho\left[\alpha_{2}\right]<\infty, \alpha_{1}(r)$ is of normal type, and $\alpha_{2}(r)$ is of maximal type.

4) $\rho\left[\alpha_{1}\right]=\rho\left[\alpha_{2}\right]<\infty, \alpha_{1}(r)$ and $\alpha_{2}(r)$ are of minimal type, $\alpha_{1}(r)$ belongs to the convergence class, and $\alpha_{2}(r)$ belongs to the divergence class.

It is easy to see that

$$
\liminf _{r \rightarrow \infty} \frac{\alpha_{1}(r)}{\alpha_{2}(r)}=0
$$

if $\alpha_{2}(r)$ is of higher growth category than $\alpha_{1}(r)$, but the condition $\alpha_{1}(r)=o\left(\alpha_{2}(r)\right)$ does not have to be satisfied. Indeed, consider $\alpha_{2}(r)=r^{2+\sin \left(\ln ^{+} \ln ^{+} r\right)}$ and $\alpha_{1}(r)=$ $r^{2}$. It is clear that $\alpha_{1}, \alpha_{2} \in \Lambda, \rho\left[\alpha_{2}\right]=3$, and $\rho\left[\alpha_{1}\right]=2$. Therefore $\alpha_{2}(r)$ is of higher growth category than $\alpha_{1}(r)$. However, $\alpha_{2}\left(r_{n}\right)=o\left(\alpha_{1}\left(r_{n}\right)\right)$ for $r_{n}=\exp \exp \left(\frac{3}{2} \pi+2 \pi n\right)$.

On the other hand, it can happen that $\alpha_{1}(r)$ and $\alpha_{2}(r)$ are of the same growth category, but $\alpha_{1}(r)=o\left(\alpha_{2}(r)\right)$. It happens, for example, for $\alpha_{1}(r)=r \ln ^{+} r$, $\alpha_{2}(r)=r\left(\ln ^{+} r\right)^{2}$.

It is easy to verify that the functions $\alpha(r) \in \Lambda, \alpha_{1}(r)=A \alpha(r)+B(A>0)^{1}$, and $\alpha_{2}(r)=\alpha(a r+b)$, where $a>0$, are of the same growth category (here and later we define functions $\alpha_{j}(r)$ for sufficiently large $r$, the values of $\alpha_{j}(r)$ on finite intervals do not affect the growth category). The magnitudes of the types of these functions are related in the following way: $\sigma\left[\alpha_{1}\right]=A \sigma[\alpha], \sigma\left[\alpha_{2}\right]=a^{\rho} \sigma[\alpha]$. If $\alpha_{3}(r)=\alpha\left(r^{\beta}\right), \beta>0$, then $\rho\left[\alpha_{3}\right]=\beta \rho[\alpha]$ and $\sigma\left[\alpha_{3}\right]=\sigma[\alpha]$. We leave verification of these statements to interested readers.

Theorem 1.1. Let $\alpha(r) \in \Lambda, \alpha(r) \rightarrow \infty(r \rightarrow \infty)$,

$$
\alpha_{1}(r)=C+\int_{r_{0}}^{r} \frac{\alpha(t)}{t} d t, r_{0}>0 \text {. }
$$

\footnotetext{
${ }^{1}$ If $\alpha(r)=O(1)$ and $B<0$, we assume also that $\alpha_{1}(r) \in \Lambda$.
} 
Then the functions $\alpha(r)$ and $\alpha_{1}(r)$ are of the same growth category.

Proof. We have

$$
\alpha_{1}(e r)-C \geq \int_{r}^{e r} \frac{\alpha(t)}{t} d t \geq \alpha(r) \int_{r}^{e r} \frac{d t}{t}=\alpha(r) .
$$

Hence the growth category of $\alpha(r)$ cannot be higher than the growth category of $\alpha_{1}(r)$. On the other hand,

$$
\begin{gathered}
\alpha_{1}(r) \leq C+\alpha(r) \int_{r_{0}}^{r} d \ln t=C+\alpha(r) \ln \frac{r}{r_{0}} \\
\frac{\ln ^{+} \alpha_{1}(r)}{\ln r} \leq \frac{\ln ^{+} \alpha(r)}{\ln r}+\frac{\ln ^{+} \ln \frac{r}{r_{0}}}{\ln r}+O\left(\frac{1}{\ln r}\right)=\frac{\ln ^{+} \alpha(r)}{\ln r}+o(1),
\end{gathered}
$$

hence $\rho\left[\alpha_{1}\right] \leq \rho[\alpha]$. Since the inverse inequality has been already verified, then $\rho\left[\alpha_{1}\right]=\rho[\alpha]=\rho$. If $\rho=0$, then both functions $\alpha(r)$ and $\alpha_{1}(r)$ are of maximal type and thus are of the same growth category. Now let $0<\rho<\infty$. If $\sigma[\alpha]<\infty$, then for $r>r_{1}(\varepsilon)$ we have $\alpha(r)<(\sigma[\alpha]+\varepsilon) r^{\rho}$ and

$$
\alpha_{1}(r) \leq O(1)+\int_{r_{1}}^{r} \frac{(\sigma[\alpha]+\varepsilon) t^{\rho}}{t} d t \leq O(1)+\frac{\sigma[\alpha]+\varepsilon}{\rho} r^{\rho} .
$$

Therefore $\sigma\left[\alpha_{1}\right] \leq \rho^{-1} \sigma[\alpha]$. This inequality is trivial if $\sigma[\alpha]=\infty$. The inequality (1.2) implies that $\sigma[\alpha] \leq e^{\rho} \sigma\left[\alpha_{1}\right]$. Combining these inequalities, we get that $\alpha_{1}(r)$ and $\alpha(r)$ are of the same type. If $\alpha_{1}(r)$ belongs to the convergence class, then $\alpha(r)$ also belongs to the convergence class (see (1.2)). If $\alpha(r)$ belongs to the convergence class, then $\alpha(r)$ and thus, also, $\alpha_{1}(r)$ have minimal type. On the other hand

$$
\int_{1}^{r} \frac{\alpha(t)}{t^{\rho+1}} d t=\int_{1}^{r} \frac{d \alpha_{1}(t)}{t^{\rho}}=\frac{\alpha_{1}(r)}{r^{\rho}}-\alpha_{1}(1)+\rho \int_{1}^{r} \frac{\alpha_{1}(t)}{t^{\rho+1}} d t .
$$

Letting $r \rightarrow \infty$, we get

$$
\rho \int_{1}^{\infty} \frac{\alpha_{1}(t)}{t^{\rho+1}} d t=\alpha_{1}(1)+\int_{1}^{\infty} \frac{\alpha(t)}{t^{\rho+1}} d t<\infty .
$$

Hence $\alpha_{1}(r)$ also belongs to the convergence class.

Corollary. Let $f(z)$ be a meromorphic function. Then

1) $T(r, f), \stackrel{\circ}{T}(r, f)$, and $\stackrel{\circ}{A}(r, f)$ have the same growth category, except in the case when $\stackrel{\circ}{A}(r, f)=O(1)$ as $r \rightarrow \infty$ and $\stackrel{\circ}{A}(r, f)$ is not identically 0 .

2) $N(r, a)$ and $n(r, a)$ have the same growth category, except in the case when $n(r, a)=O(1)$ and $n(r, a)$ is not identically 0 . For each a the category of growth of $N(r, a)$ cannot exceed the category of growth of $T(r, f)$.

In the case when $\stackrel{\circ}{A}(r, f)=O(1)$ and $\stackrel{\circ}{A}(r, f)$ is not identically 0, it is clear that $\stackrel{\circ}{T}(r, f) \sim$ const $\cdot \ln r$. In the case when $n(r, a)=O(1)$ and $n(r, a)$ is not identically 0 , we have $N(r, a) \sim$ const $\cdot \ln r$. Thus, in these cases the categories of $\stackrel{\circ}{A}(r, f)$ and $n(r, a)$ are lower than the categories of $\stackrel{\circ}{T}(r, f)$ and $N(r, a)$, respectively.

To prove the corollary, it is enough to use the definitions of $\stackrel{\circ}{T}(r, f)$ and $N(r, a)$. We write the definition of $N(r, a)$ in the following way. Let $r_{1}$ be the least absolute value of a non-zero $a$-point of $f(z), r>r_{1}$. Then 


$$
\begin{aligned}
N(r, a) & =\int_{r_{1}}^{r} \frac{n(t, a)-n(0, a)}{t} d t+n(0, a) \ln r \\
& =\int_{r_{1}}^{r} \frac{n(t, a)}{t} d t+n(0, a) \ln r_{1} .
\end{aligned}
$$

The first fundamental theorem implies that $N(r, a) \leq T(r, f)+O(1)$, hence the growth category of $N(r, a)$ does not exceed the growth category of $T(r, f)$.

Proof of the following theorem is left to interested readers.

TheOREM 1.2. Let $\alpha_{1}(r) \in \Lambda, \alpha_{2}(r) \in \Lambda$, such that $\alpha_{2}(r)$ is not identically 0 and the growth category of $\alpha_{1}(r)$ does not exceed the growth category of $\alpha_{2}(r)$. Then the growth category of $\alpha(r)=\alpha_{1}(r)+\alpha_{2}(r)+O(1)$ coincides with the growth category of $\alpha_{2}(r)$.

Definition. The order, lower order, type, class and the growth category of a meromorphic function $f$ are defined as that of the characteristics $T(r, f)$.

THEOREM 1.3. If $f(z)$ is an entire function, then it has the same growth category as $\ln M(r, f)$.

Proof. Letting $R=k r, k>1$, in the formula (7.1) from Chapter 1, we get $T(r, f) \leq \ln ^{+} M(r, f) \leq \frac{k+1}{k-1} T(k r, f)$. Since $T(r, f)$ and $\frac{k+1}{k-1} T(k r, f)$ have the same growth category, we get the desired result.

REMARK. If $0<\rho[f]=\rho[T(r, f)]=\rho<\infty$, then the equation (7.1) from Chapter 1 implies

$$
\sigma[f] \leq \sigma\left[\ln M(r, f) \leq \frac{k+1}{k-1} k^{\rho} \sigma[f] .\right.
$$

When $k>1$, the minimum of $\frac{k+1}{k-1} k^{\rho}$ is attained at $k=k(\rho)=\frac{1}{\rho}\left(1+\sqrt{1+\rho^{2}}\right)$. The estimate

$$
\sigma\left[\ln M(r, f) \leq \frac{k(\rho)+1}{k(\rho)-1}[k(\rho)]^{\rho} \sigma[f]\right.
$$

is not precise for the class of entire functions. The following estimate holds

$$
\sigma[\ln M(r, f)] \leq \begin{cases}\pi \rho \csc \pi \rho \sigma[f], & \text { if } 0 \leq \rho \leq \frac{1}{2} \\ \pi \rho \sigma[f], & \text { if } \frac{1}{2} \leq \rho<\infty\end{cases}
$$

Proof of this estimate will be given in the Appendix, where we also show that this estimate is precise.

Relations obtained in Section 6 of Chapter 1 imply several results on growth of meromorphic functions.

THEOREM 1.4. Let $f(z)$ be a meromorphic function, $f_{1}(z)=R_{1}[f(z)], f_{2}(z)=$ $R_{2}[z, f(z)]$, where $R_{1}(u)$ is a rational function of degree $d, R_{2}(u, v)$ is a rational function of $u$ and a polynomial of degree $p$ of $v$. Then $f(z), f_{1}(z)$, and $f_{2}(z)$ are of the same growth category, and the magnitudes of their types are related by the equations $\sigma\left[f_{1}\right]=d \sigma[f]$ and $\sigma\left[f_{2}\right]=p \sigma[f]$. 
THEOREM 1.5. Let $f(z)$ be a meromorphic function, $f_{1}(z)=f\left(z^{n}\right)$, where $n$ is a positive integer. Then $\rho\left[f_{1}\right]=n \rho[f], \sigma\left[f_{1}\right]=\sigma[f]$, and the functions $f(z)$ and $f_{1}(z)$ are in the same class.

THEOREM 1.6. Let $f(z)$ be a meromorphic function, $f_{1}(z)=f(a z+b), a \neq 0$. Then $f(z)$ and $f_{1}(z)$ (as well as $N(r, f)$ and $N\left(r, f_{1}\right)$ ) are of the same growth category, furthermore $\sigma\left[f_{1}\right]=|a|^{\rho} \sigma[f]$ and $\rho=\rho[f]=\rho\left[f_{1}\right]$.

Proof. It suffices to prove Theorem 1.6 in the cases when either $b=0$ or $a=1$. In the former case the theorem follows from Theorem 6.7 from Chapter 1 . In the latter case, the obvious (for $r>|b|$ ) inequalities

$$
\begin{gathered}
\stackrel{\circ}{A}(r-|b|, f(z)) \leq \stackrel{\circ}{A}(r, f(z+b)) \leq \stackrel{\circ}{A}(r+|b|, f(z)), \\
n(r-|b|, f(z)) \leq n(r, f(z+b)) \leq n(r+|b|, f(z)),
\end{gathered}
$$

and the corollary of Theorem 1.1 imply that $f(z)$ and $f(z+b)$ (as well as $N(r, f(z))$ and $N(r, f(z+b)))$ have the same growth category.

Let $0<\rho<\infty$. Let us show that $\sigma[f(z)]=\sigma[f(z+b)]$. It is clear that $\stackrel{\circ}{A}(r, f(z))$ and $\stackrel{\circ}{A}(r, f(z+b))$ tend to $\infty$ as $r \rightarrow \infty$ (otherwise we would have $\stackrel{\circ}{T}(r, f(z))=O(\ln r)$ and $\stackrel{\circ}{T}(r, f(z+b))=O(\ln r))$. Let $r>2|b|$. Then

$$
\begin{aligned}
\stackrel{\circ}{T}(r, f(z+b)) & =\int_{0}^{r} \frac{\stackrel{\circ}{A}(t, f(z+b))}{t} d t \\
& \geq \int_{2|b|}^{r} \frac{\stackrel{\circ}{A}(t, f(z+b))}{t} d t \geq \int_{2|b|}^{r} \frac{\stackrel{\circ}{A}(t-|b|, f(z))}{t} d t \\
& =\int_{|b|}^{r-|b|} \frac{\stackrel{\circ}{A}(t, f(z))}{t+|b|} d t=(1+o(1)) \int_{0}^{r-|b|} \frac{\stackrel{\circ}{A}(t, f(z))}{t} d t \\
& =(1+o(1)) \stackrel{\circ}{T}(r-|b|, f)
\end{aligned}
$$

(we used the l'Hôpital's rule). We get

$$
\stackrel{\circ}{T}(r, f(z))=\stackrel{\circ}{T}(r, f[(z+b)-b]) \geq(1+o(1)) \stackrel{\circ}{T}(r-|b|, f(z+b)) .
$$

Hence

$$
\begin{aligned}
\stackrel{\circ}{T}(r, f(z+b)) & \geq(1+o(1)) \stackrel{\circ}{T}(r-|b|, f(z)) \\
& \geq(1+o(1)) \stackrel{\circ}{T}(r-2|b|, f(z+b)) .
\end{aligned}
$$

Therefore $\sigma[f(z+b)]=\sigma[f(z)]$.

REMARK. The following inequality (for an arbitrary a) can be proved in the same way as (1.4)

$$
\begin{aligned}
N(r, a, f(z+b)) & \geq(1+o(1)) N(r-|b|, a, f(z)) \\
& \geq(1+o(1)) N(r-2|b|, a, f(z+b)) .
\end{aligned}
$$

TheOREM 1.7. Let $f_{1}(z)$ and $f_{2}(z)$ be meromorphic functions, such that the growth category of $f_{1}(z)$ does not exceed the growth category of $f_{2}(z)$. Then the growth categories of $f_{1}(z) \pm f_{2}(z), f_{1}(z) f_{2}(z), f_{1}(z) / f_{2}(z)$, and $f_{2}(z) / f_{1}(z)$ do not exceed the growth category of $f_{2}(z)$. Furthermore, they coincide with the growth 
category of $f_{2}(z)$ if $f_{1}(z)$ is not identically 0 and the growth category of $f_{1}(z)$ is lower than the growth category of $f_{2}(z)$.

Proof. Since

$$
\begin{gathered}
T\left(r, f_{1} \pm f_{2}\right) \leq T\left(r, f_{1}\right)+T\left(r, f_{2}\right)+O(1), \\
T\left(r, f_{1} f_{2}\right) \leq T\left(r, f_{1}\right)+T\left(r, f_{2}\right)+O(1), \\
T\left(r, \frac{f_{1}}{f_{2}}\right) \leq T\left(r, f_{1}\right)+T\left(r, f_{2}\right)+O(1),
\end{gathered}
$$

Theorem 1.2 implies the first statement. We prove the second statement for $f_{1}(z) f_{2}(z)$ only (proofs for $f_{1} \pm f_{2}, f_{1} / f_{2}$, and $f_{2} / f_{1}$ are similar). Assume that the growth category of $f_{1}(z) f_{2}(z)$ is lower than the growth category of $f_{2}(z)$. Since $f_{2}(z)=$ $\left\{f_{1}(z) f_{2}(z)\right\} / f_{1}(z)$, by the first part of the theorem, the growth category of $f_{2}(z)$ does not exceed the maximum of the growth categories of $f_{1}(z) f_{2}(z)$ and $f_{1}(z)$, which are lower than the growth category of $f_{2}(z)$. We get a contradiction.

Let $\left\{r_{\nu}\right\}$ be a non-decreasing sequence of positive numbers tending to $\infty$. Denote by $\nu(r)$ the number of members of this sequence satisfying $0<r_{\nu} \leq r$. Let

$$
\nu_{1}(r)=\int_{0}^{r} \frac{\nu(t)}{t} d t
$$

It is clear that $\nu(r) \in \Lambda, \nu_{1}(r) \in \Lambda$, and by Theorem 1.1, these functions have the same growth category.

TheOREM 1.8. Let $\nu(r)$ be of order $\rho, 0<\rho<\infty$. Then the series

$$
\sum_{\nu=1}^{\infty}\left(\frac{1}{r_{\nu}}\right)^{\mu}
$$

converges if $\mu>\rho$, diverges if $\mu<\rho$. For $\mu=\rho$ the series converges or diverges depending on whether $\nu(r)$ belongs to the convergence class or to the divergence class.

Proof. Obviously (see above) it suffices to show that the series (1.6) and the integral

$$
\int_{r_{1}}^{\infty} \frac{\nu(r)}{r^{1+\mu}} d r
$$

are either both convergent of both divergent. For $0<r_{0}<r_{1}$ we have

$$
\sum_{r_{\nu} \leq r}\left(\frac{1}{r_{\nu}}\right)^{\mu}=\int_{r_{0}}^{r} \frac{d \nu(t)}{t^{\mu}}=\frac{\nu(r)}{r^{\mu}}+\mu \int_{r_{0}}^{r} \frac{\nu(t)}{t^{\mu+1}} d t .
$$

If the integral (1.7) diverges, then the inequality

$$
\sum_{r_{\nu} \leq r}\left(\frac{1}{r_{\nu}}\right)^{\mu} \geq \mu \int_{r_{0}}^{r} \frac{\nu(t)}{t^{\mu+1}} d t
$$

implies that the series (1.6) also diverges. If the integral (1.7) converges, then, as we saw, $\nu(r)=o\left(r^{\mu}\right)$, therefore, letting $r \rightarrow \infty$ in (1.8), we get

$$
\sum_{\nu=1}^{\infty}\left(\frac{1}{r_{\nu}}\right)^{\mu}=\mu \int_{r_{0}}^{\infty} \frac{\nu(t)}{t^{\mu+1}} d t
$$


Corollary. Let $f(z)$ be a meromorphic function, $z_{\nu}(a)$ be its non-zero apoints. Let $\rho=\rho[f]$ and $\rho_{1}=\rho[n(r, a)]$. Then the series

$$
\sum_{\nu} \frac{1}{\left|z_{\nu}(a)\right|^{\mu}}
$$

converges if $\mu>\rho_{1}$, and hence if $\mu>\rho$. The series (1.9) converges if $\mu=\rho_{1}$ and $n(r, a)$ belongs to the convergence class. If $\mu<\rho_{1}$, the series (1.9) diverges.

Definition. Let $f(z)$ be a transcendental meromorphic function. A complex number $a$ is called a Picard exceptional value of the function $f(z)$ if the number of its $a$-points in $\{|z|<\infty\}$ is finite, that is, if $N(r, a)=O(\ln r)$. A complex number $a$ is called a Borel exceptional value if the growth category of $n(r, a)$ is lower than the growth category of $f(z)$.

In the cases when the order of $f(z)$ is non-zero, or when $n(r, a) \rightarrow \infty$ as $r \rightarrow \infty$, we may replace $n(r, a)$ by $N(r, a)$ in the definition of a Borel exceptional value.

It is clear that a Picard exceptional value is a Borel exceptional value. The converse is not true.

Example $f(z)=e^{z^{2}} \sin z$. The function $e^{z^{2}}$ has order 2 , the function $\sin z$ has order 1 . Hence $f(z)$ has order 2 . On the other hand, $n(r, 0)=1+2[r / \pi]$. Hence, the order of $n(r, 0)$ is 1 . Thus 0 is a Borel exceptional value, but is not a Picard exceptional value.

\section{Proximate order}

A function $\rho(r)$ defined on $[0, \infty)$ is called a proximate order if it satisfies the conditions

1) $\rho(r) \geq 0$

2) $\lim _{r \rightarrow \infty} \rho(r)=\rho, 0 \leq \rho<\infty$;

3) $\rho(r)$ is continuously differentiable on $[0, \infty)$;

4) $\lim _{r \rightarrow \infty} r \rho^{\prime}(r) \ln r=0$.

Proximate orders $\rho_{1}(r)$ and $\rho_{2}(r)$ are called equivalent if $\rho_{1}(r) \rho_{2}(r)=o\left(\ln ^{-1} r\right)$. Let $\alpha(r) \in \Lambda$ be of finite order $\rho[\alpha]$. A proximate order $\rho(r)$ is called a proximate order of the function $\alpha(r)$ if $0<\sigma^{*}[\alpha]<\infty$, where

$$
\sigma^{*}[\alpha]=\limsup _{r \rightarrow \infty} \frac{\alpha(r)}{r^{\rho(r)}} .
$$

The number $\sigma^{*}[\alpha]$ is called the magnitude of type of $\alpha(r)$ with respect to the proximate order $\rho(r)$. It is clear that if $\rho(r)$ is a proximate order of $\alpha(r)$, then $\rho(r) \rightarrow \rho=\rho[\alpha]$ as $r \rightarrow \infty$. The magnitude of type $\sigma^{*}[\alpha]$ does not change if the proximate order $\rho_{1}(r)$ is replaced by a proximate order $\rho_{2}(r)$, since $r^{\rho_{1}(r)} \sim r^{\rho_{2}(r)}$. Using a proximate order $\rho(r)$ and the magnitude of type $\sigma^{*}[\alpha]$ we can introduce the notions of type, convergence class, and growth category of $\alpha(r)$ with respect to $\rho(r)$. It can be verified that all theorems of the previous section (except Theorem 1.1 in the case $\rho=0$ ) remain true if we understand the category of $\alpha(r)$ as the category with respect to a proximate order $\rho(r)$. We are not going to use this.

If a function $\alpha(r) \in \Lambda$ has finite lower order $\lambda[\alpha]$, then its lower proximate order is defined as a proximate order $\lambda(r)$ such that $0<\sigma_{*}[\alpha]<\infty$, where

$$
\sigma_{*}[\alpha]=\liminf _{r \rightarrow \infty} \frac{\alpha(r)}{r^{\lambda}(r)} .
$$


It is easy to see, that if $\lambda(r)$ is a lower proximate order of $\alpha(r)$, then $\lambda(r) \rightarrow$ $\lambda=\lambda[\alpha]$.

Applications of proximate orders are based on the following theorem.

THEOREM 2.1. Each positive continuous function $\alpha(r) \in \Lambda$ has a proximate order. Each positive continuous function $\alpha(r) \in \Lambda$ of finite lower order has a lower proximate order.

Proof. As is easy to see it suffices to find $\rho(r)$ and $\lambda(r)$ for which the condition 3 ) in the definition of a proximate order is replaced by the following weaker condition: $\rho(r)$ and $\lambda(r)$ are continuous on $[0, \infty)$, and continuously differentiable everywhere on $[0, \infty)$ except for a set of isolated points at which they have onesided derivatives. In fact, in sufficiently small neighborhoods of the points where the derivatives do not exist, the functions $\rho(r)$ and $\lambda(r)$ can be corrected by at most $o\left\{(\ln r)^{-1}\right\}$ in such a way that the resulting functions are continuously differentiable everywhere, and still satisfy the conditions 1$), 2$ ), and 4 ).

First we prove the existence of a proximate order $\rho(r)$. Suppose that $\alpha(r)$ is defined on $[0, \infty)$ and $\alpha(r) \rightarrow \infty$ as $r \rightarrow \infty$. (If $\alpha(r)=O(1)$, we let $\rho(r) \equiv 0$.)

Let $\rho$ be the order of $\alpha(r)$. Denote by $d(r)$ the function

$$
d(r)=\frac{\ln ^{+} \alpha(r)}{\ln r}
$$

Then

$$
\limsup _{r \rightarrow \infty} d(r)=\rho .
$$

There are two possibilities:

A) there exists a sequence $r_{k} \rightarrow \infty, 1 \leq k \rightarrow \infty$, such that $d\left(r_{k}\right)>\rho$;

B) $d(r) \leq \rho$ for all $r \geq r_{0}>e$.

Assume that A is satisfied. Let $\varphi(r)=\max _{x \geq r} d(x)$. It is clear that $\varphi(r)$ is well-defined and is a non-increasing, positive function, and $\varphi(r) \rightarrow \rho$ as $r \rightarrow \infty$. It is clear, also, that the set $M$ of those $r$ for which $\varphi(r)=d(r)$ is unbounded.

Let $r_{1}>e^{e^{e}}$ and $r_{1} \in M$. Set $\rho(r)=\varphi\left(r_{1}\right)$ for $0 \leq r \leq r_{1}$. Denote by $t_{1}$ the least integer, exceeding $1+r_{1}$ and such that $\varphi\left(r_{1}\right)>\varphi\left(t_{1}\right)$. Set $\rho(r)=\varphi\left(r_{1}\right)$ for $r_{1}<r \leq t_{1}$. Let $u_{1}$ be the $x$-coordinate of the first point of intersection of the curves $y=\rho\left(r_{1}\right)-\ln _{3} x+\ln _{3} t_{1}$ and $y=\varphi(x)$ for $x>t_{1}{ }^{2}$. Set $\rho(r)=$ $\rho\left(r_{1}\right)-\ln _{3} r+\ln _{3} t_{1}$ for $t_{1} \leq r \leq u_{1}$. It is clear that on this interval, $\rho(r) \geq \varphi(r)$, and the equality takes place at $r=u_{1}$ only. Let $r_{2}=\min \left(M \cap\left\{r \geq u_{1}\right\}\right)$. If $r_{2}>u_{1}$, set $\rho(r)=\varphi(r)$ for $u_{1} \leq r \leq r_{2}$. Since $\varphi(r)$ is constant when $u_{1} \leq r \leq r_{2}$, then $\rho(r)$ is also constant on this interval. We repeat the same construction with $r_{1}$ replaced by $r_{2}$. Continuing in such a manner we define $\rho(r)$ on $[0, \infty)$, since $r_{n}-r_{n-1} \geq u_{n-1}-r_{n-1} \geq t_{n-1}-r_{n-1} \geq 1$ and $r_{n} \rightarrow \infty$. The function $\rho(r)$ is continuous and has a continuous derivative everywhere except points $t_{n}$ and $u_{n}$, $n \geq 1$, where it has one-sided derivatives. Since

$$
\rho^{\prime}(r)=0 \text { or } \rho^{\prime}(r)=-\frac{1}{r \ln r \ln _{2} r},
$$

\footnotetext{
${ }^{2}$ We use the notation $\ln _{j} x$ for the $j$-th iteration of the $\operatorname{logarithm}(j \geq 0): \ln _{0} x=x$, $\ln _{j} x=\ln \left(\ln _{j-1} x\right)$. We use the notation $\exp _{j} x$ for the $j$-th iteration of the exponential function: $\exp _{0} x=x, \exp _{j} x=\exp \left(\exp _{j-1} x\right)$.
} 
the condition 4) is satisfied. Our construction is such that $\rho(r) \geq \varphi(r) \geq d(r)$ if $r \geq r_{1}, \rho\left(r_{n}\right)=\varphi\left(r_{n}\right)=d\left(r_{n}\right), n \geq 1$, and $\rho(r)$ is a non-increasing function. Hence

$$
\lim _{r \rightarrow \infty} \rho(r)=\lim _{r \rightarrow \infty} \varphi(r)=\rho,
$$

and the condition 2) is satisfied. Furthermore,

$$
\alpha(r) \leq r^{d(r)} \leq r^{\rho(r)} \text { and } \alpha\left(r_{n}\right)=r_{n}^{d\left(r_{n}\right)}=r_{n}^{\rho\left(r_{n}\right)}
$$

if $r \geq r_{1}$. Therefore

$$
\sigma^{*}[\alpha]=\limsup _{r \rightarrow \infty} \frac{\alpha(r)}{r^{\rho(r)}}=1 .
$$

Now we consider the case B. If $d\left(r_{n}\right)=\rho$ for a sequence $\left\{r_{n}\right\}$ satisfying $r_{n} \rightarrow \infty$, then we may let $\rho(r) \equiv \rho$. Now let $0<d(r)<\rho$ for all $r \geq r_{0}>\exp _{3} 1$. Set $\psi(r)=$ $\max _{r_{0} \leq x \leq r} d(x)$. It is clear that $\psi(r)$ is a continuous non-decreasing function, and the set $\bar{L}$ of those $r \geq r_{0}$ for which $d(r)=\psi(r)$ is unbounded. Let $r_{1}>r_{0}$ and let $s_{1}$ be the largest $x$-coordinate of the points of intersection of the curves $y=\rho+\ln _{3} x-\ln _{3} r_{1}$ and $y=\psi(x)$. It is clear that if $r_{1}$ is sufficiently large, then then there exists a point of intersection such that $r_{0}<s_{1}<r_{1}$ and $L \cap\left[r_{0}, s_{1}\right] \neq \emptyset$. Let $t_{1}=\max \left\{L \cap\left[r_{0}, s_{1}\right]\right\}$. Set $\rho(r) \equiv \psi\left(t_{1}\right)=d\left(t_{1}\right)$ for $0 \leq r \leq s_{1}$. Choose $r_{2}>r_{1}+1$ to be so large that the largest $x$-coordinate of points of intersection of the curves $y=\rho+\ln _{3} x-\ln _{3} r_{2}$ and $y=\psi(x)$, denoted by $s_{2}$, is greater than $r_{1}$, and $L \cap\left[r_{1}, s_{2}\right] \neq \emptyset$. Let $t_{2}=\max \left\{L \cap\left[r_{1}, s_{2}\right]\right\}$ Denote by $u_{1}, s_{1} \leq u_{1} \leq r_{1}$ the point at which $\rho+\ln _{3} x-\ln _{3} r_{1}=\psi\left(t_{2}\right)$. Set $\rho(r)=\rho+\ln _{3} r-\ln _{3} r_{1}$ for $s_{1} \leq r \leq u_{1}$ and $\rho(r) \equiv \psi\left(t_{2}\right)=d\left(t_{2}\right)$ for $u_{1} \leq r \leq s_{2}$. We continue in an obvious way. Since $r_{n}>r_{n-1}+1$, the function $\rho(r)$ is defined for all $r \geq 0$. It is clear from the construction that $\rho(r)$ is continuous for $r \geq 0$, and the inequality $\rho \geq \rho(r) \geq \psi(r) \geq d(r)$ is satisfied, furthermore $\rho\left(t_{n}\right)=\psi\left(t_{n}\right)=d\left(t_{n}\right), t_{n} \rightarrow \infty$. Therefore

$$
\sigma^{*}[\alpha]=\limsup _{r \rightarrow \infty} \frac{\alpha(r)}{r^{\rho(r)}}=1 .
$$

Since $\psi(r) \rightarrow \rho$ as $r \rightarrow \infty$, we have $\rho(r) \rightarrow \rho$. Finally, the derivative $\rho^{\prime}(r)$ is defined everywhere except the points $s_{n}, u_{n}$, it is equal either to 0 , or to $\left(r \ln r \ln _{2} r\right)^{-1}$, thus the condition 4) is satisfied.

The desired function is constructed in all cases.

To construct a lower proximate order $\lambda(r)$ we introduce a function $d_{1}(r)$ by

$$
d_{1}(r)=\frac{\ln r}{\ln r+\ln ^{+} \alpha(r)} .
$$

Then

$$
\limsup _{r \rightarrow \infty} d_{1}(r)=\frac{1}{1+\lambda}
$$

and, using the same argument as above, we can construct a proximate order $\rho_{1}(r) \rightarrow$ $(1+\lambda)^{-1}$, such that $0<\rho_{1}(r)<1$ and $d_{1}(r) \leq \rho_{1}(r)$ for all $r, r_{0} \leq r<\infty$, and, furthermore, $d_{1}\left(r_{n}\right)=\rho_{1}\left(r_{n}\right)$ for a sequence $\left\{r_{n}\right\}$ satisfying $\lim _{n \rightarrow \infty} r_{n}=\infty$.

Set $\lambda(r)=\frac{1}{\rho_{1}(r)}-1$. It is easy to see that $\lambda(r)$ is a proximate order, $\lambda(r) \rightarrow$ $\lambda=\lambda[\alpha]$, and, moreover, $\alpha(r) \geq r^{\lambda(r)}$ for all $r$ satisfying $r_{0} \leq r<\infty$, and $\alpha\left(r_{n}\right)=r_{n}^{\lambda\left(r_{n}\right)}, n=1,2, \ldots$ Hence $\lambda(r)$ is a lower proximate order of $\alpha(r)$. 
Observe that the proximate order $\rho(r)$ constructed in the proof is a monotone function, $\alpha(r) \leq r^{\rho(r)}$ for $r \geq r_{0}$, and there exists a sequence $\left\{r_{k}\right\}, r_{k} \rightarrow \infty$, such that $\alpha(r)=r^{\rho(r)}$ for $r=r_{k}$.

A proximate order $\rho(r)$ is convenient because the function $r^{\rho(r)}$ has many properties of the power function $r^{\rho}$.

Definition. A positive continuous function $L(r)$, defined for $r \geq r_{0}$, is called slowly varying if

$$
\lim _{r \rightarrow \infty} \frac{L(k r)}{L(r)}=1
$$

uniformly on each interval $0<a \leq k \leq b<\infty$.

THEOREM 2.2. If $\rho(r)$ is a proximate order, then $L(r)=r^{\rho(r)-\rho}$ is a slowly varying function.

Proof. Assume, without loss of generality, that $0<a<1$ and $b=1 / a$. Then

$$
\ln \frac{L(k r)}{L(r)}=[\rho(k r)-\rho] \ln k+[\rho(k r)-\rho(r)] \ln r .
$$

For each $\varepsilon>0$ there exists $r_{0}$, such that for $a r>r_{0}>1$ the conditions

$$
|\rho(r)-\rho|<\varepsilon
$$

and

$$
\left|\rho^{\prime}(r)\right| r \ln r<\varepsilon
$$

hold. By Lagrange's mean value theorem, applied to $\rho\left(e^{t}\right), t=\ln r$ (for $r>r_{0}$ ), there exists $k^{*}=k^{*}(r), a \leq k^{*} \leq b$, such that

$$
|\rho(k r)-\rho(r)|=k^{*} r\left|\rho^{\prime}\left(k^{*} r\right)\right||\ln k|<\varepsilon \frac{\ln b}{\ln \left(k^{*} r\right)}<\frac{\varepsilon \ln b}{\ln (a r)} .
$$

Then

$$
\left|\ln \frac{L(k r)}{L(r)}\right| \leq \varepsilon \ln b+\varepsilon \ln b \frac{\ln r}{\ln (a r)} \leq \varepsilon \ln b\left(1+\frac{\ln r_{0}}{\ln \left(a r_{0}\right)}\right) .
$$

Hence $\ln (L(k r) / L(r))$ converges uniformly to 0 , and $L(k r) L(r))$ converges uniformly to 1 .

Corollary. If $\rho(r)$ is a proximate order and $0<a<b<\infty$, then for each $\varepsilon>0$ there exists $r_{0}$, such that for all $r>r_{0}$ and $a \leq k \leq b$ the inequality

$$
(1-\varepsilon) k^{\rho} r^{\rho(r)}<(k r)^{\rho(k r)}<(1+\varepsilon) k^{\rho} r^{\rho(r)}
$$

holds.

In fact, if $L(r)$ is the function from Theorem 2.2, then

$$
\frac{L(k r)}{L(r)}=\frac{(k r)^{\rho(k r)}}{k^{\rho} r^{\rho(r)}} .
$$

THEOREM 2.3. If $\rho(r)$ is a proximate order and $\rho>0$, then the function $V(r)=r^{\rho(r)}$ is increasing for sufficiently large $r$.

In fact,

$$
V^{\prime}(r)=r^{\rho(r)-1}\left\{\rho(r)+\rho^{\prime}(r) r \ln r\right\} .
$$

The function in braces approaches $\rho>0$ as $r \rightarrow \infty$, hence, for sufficiently large $r$, we have $V^{\prime}(r)>0$. 
Theorem 2.4. Let $\rho(r)$ be a proximate order, $\rho(r) \rightarrow \rho$ as $r \rightarrow \infty$. Then

$$
\int_{1}^{r} t^{\rho(t)-q} d t \sim \frac{r^{\rho(r)+1-q}}{\rho+1-q}
$$

if $q<\rho+1$; and

$$
\int_{r}^{\infty} t^{\rho(t)-q} d t \sim \frac{r^{\rho(r)+1-q}}{q-\rho-1}
$$

if $q>\rho+1$.

The statements can be easily verified using the l'Hôpital's rule.

EXERCISE 1 . Let $0 \leq p<\rho<p+1$, and let $\rho(r)$ be a proximate order satisfying $\rho(r) \rightarrow \rho$. Prove

$$
\lim _{r \rightarrow \infty} \int_{c / r}^{\infty} \frac{(r \tau)^{\rho(r \tau)}}{r^{\rho(r)}} \frac{d \tau}{\tau^{p+1}(1+\tau)}=\int_{0}^{\infty} \frac{\tau^{\rho-p-1}}{1+\tau} d \tau=\frac{\pi}{\sin \pi(\rho-p)}
$$

that is, it is possible to pass to the limit under the integral sign.

Hint. Split the interval $[c / r, \infty)$ into three subintervals: $[c / r, \varepsilon],\left[\varepsilon, \varepsilon^{-1}\right]$, $\left[\varepsilon^{-1}, \infty\right)$, where $\varepsilon>0$ is a small positive number. The first integral is estimated as:

$$
\int_{c / r}^{\varepsilon} \frac{(r \tau)^{\rho(r \tau)}}{r^{\rho(r)}} \frac{d \tau}{\tau^{\rho+1}(1+\tau)} \leq r^{p-\rho(r)} \int_{c}^{\varepsilon r} t^{\rho(t)-p-1} d t \rightarrow_{r \rightarrow \infty} \frac{\varepsilon^{\rho-p}}{\rho-p} .
$$

The last relation can be obtained by combining Theorem 2.4 and Theorem 2.2 (instead of using Theorem 2.4 one can use the l'Hôpital's rule). The third integral can be estimated in a similar way. To estimate the second integral one can use the corollary of Theorem 2.2 .

EXERCise 2. Let $\rho(r)$ be a proximate order satisfying $\rho(r) \rightarrow \rho$. Then for each fixed $k>0$ we have

$$
\lim _{R \rightarrow \infty} \int_{1}^{k R} r^{\rho(r)-\rho(R)-1} d r=\infty .
$$

HINT. Let $L(r)=r^{\rho(r)-\rho}$, fix $0<\varepsilon<k$. We get

$$
\begin{aligned}
& \int_{1}^{k R} r^{\rho(r)-\rho(R)-1} d r=\int_{1}^{k R} \frac{L(r)}{L(R)}\left(\frac{R}{r}\right)^{\rho(R)-\rho} \frac{d r}{r} \\
& \quad \geq \int_{\varepsilon R}^{k R} \frac{L(r)}{L(R)}\left(\frac{R}{r}\right)^{\rho(R)-\rho} \frac{d r}{r} \geq\left(\min _{\varepsilon R \leq r \leq k R} \frac{L(r)}{L(R)}\right) \varepsilon^{|\rho(R)-\rho|} \ln \frac{k}{\varepsilon} .
\end{aligned}
$$

Applying Theorem 2.2 we get

$$
\liminf _{R \rightarrow \infty} \int_{1}^{k R} r^{\rho(r)-\rho(R)-1} d r \geq \ln \frac{k}{\varepsilon} .
$$

It remains to use the possibility of choosing $\varepsilon$ arbitrarily small. 


\section{Infinite products}

We assume knowledge of basic properties of infinite products of analytic functions (see, for example [Pri56, Chapter IX]). It will be convenient for us to deviate from the standard definitions, and to consider an infinite product $\prod_{k=1}^{\infty} f_{k}(z)$ as being convergent at $z_{0}$, if for some $N$ there exists a finite non-zero limit

$$
\lim _{n \rightarrow \infty} \prod_{k=N}^{n} f_{k}\left(z_{0}\right) \text {. }
$$

We consider $\prod_{k=1}^{n} f_{k}(z)$ as being uniformly convergent on a set $M$, if for some $N$ the products $\prod_{k=N}^{n} f_{k}(z)$ converge uniformly on $z \in M$ to some function $R_{N}(z) \neq$ $0, \infty$ as $n \rightarrow \infty$.

In a similar way we modify the standard definition of the absolute convergence of an infinite product.

This modification of the standard definitions is convenient for us, because, with this modification, the fact that some terms of the product are equal to 0 or $\infty$ at some points does not affect the convergence of the product and the type of this convergence.

The following theorem is presented in many texts.

THEOREM 3.1. Let $f_{k}(z), k=1,2, \ldots$, be analytic in a region $G$, such that $\left|f_{k}(z)\right| \leq a_{k}, k \geq k_{0} \geq 1$ for $z \in G$. If the series $\sum_{k} a_{k}$ is convergent, then the product $\prod_{k=1}^{\infty}\left[1+f_{k}(z)\right]$ converges uniformly and absolutely on $G$ to an analytic function.

Theorem 3.1 implies the following result.

THEOREM 3.2. Let $a_{k}, k=1,2, \ldots$, be a sequence of complex numbers satisfying $0<\left|a_{1}\right| \leq\left|a_{2}\right| \leq \ldots$, such that

$$
\sum_{k=1}^{\infty} \frac{1}{\left|a_{k}\right|^{q}}<\infty
$$

where $q$ is a positive integer. Suppose that an entire function $g(z)$ has a power series representation of the form

$$
g(z)=1+c_{q} z^{q}+c_{q+1} z^{q+1}+\ldots
$$

Then the product

$$
f(z)=\prod_{k=1}^{\infty} g\left(\frac{z}{a_{k}}\right)
$$

converges absolutely and uniformly on each bounded disc to an entire function $f(z)$.

Proof. By (3.2), $|g(z)-1| \leq A|z|^{q}$ for $|z|<r_{0}$, where $A$ is some constant. Consider a disc $\{|z| \leq R\}$ of an arbitrarily large radius $R$. The inequality $R<\left|a_{k}\right| r_{0}$ holds for $k \geq k_{0}=k_{0}(R)$. We have

$$
\prod_{k=k_{0}}^{\infty} g\left(\frac{z}{a_{k}}\right)=\prod_{k=k_{0}}^{\infty}\left\{1+\left[g\left(\frac{z}{a_{k}}\right)-1\right]\right\} .
$$

Since for $k \geq k_{0}$ and $|z| \geq R$ we have

$$
\left|g\left(\frac{z}{a_{k}}\right)-1\right| \leq A\left|\frac{z}{a_{k}}\right|^{q} \leq A R^{q} \frac{1}{\left|a_{k}\right|^{q}},
$$


by (3.1) and Theorem 3.1, the product (3.3) converges absolutely and uniformly in $\{|z|<R\}$ to an analytic function $f(z)$.

Example 1. Denote by $E(z, p)$ the entire function given by

$$
E(z, p)= \begin{cases}1-z & \text { if } p=0 \\ (1-z) e^{z+\frac{z^{2}}{2}+\cdots+\frac{z^{p}}{p}} & \text { if } p \geq 1\end{cases}
$$

The function $E(z, p)$ is called a Weierstrass primary factor. It is clear that $E(z, p)-1$ has a zero of order $(p+1)$ at $z=0$. If $p \geq 1$, we have

$$
E^{\prime}(z, p)=-z^{p} \exp \left(z+\frac{z^{2}}{2}+\cdots+\frac{z^{p}}{p}\right) .
$$

Thus, if the sequence $\left\{a_{k}\right\}$ is such that the series (3.1) converges for $q=p+1$, then, by Theorem 3.2, the product

$$
f(z)=\prod_{k=1}^{\infty} E\left(\frac{z}{a_{k}}, p\right)
$$

converges absolutely and uniformly on each bounded disc, and hence is an entire function, called the Weierstrass canonical product of genus $p$. It is clear that $f(z)$ has zeroes at points $a_{k}$, and only there.

EXAMPLE 2. Let $g(z)$ be an arbitrary entire function satisfying $g(0)=1$. Suppose that in a neighborhood of $z=0$ the function $1 / \mathrm{g}$ admits a representation of the form $1 / g(z)=1+c_{1} z+c_{2} z^{2}+\ldots$ Let $P_{n-1}(z)=1+c_{1} z+\cdots+c_{n-1} z^{n-1}$, $R_{n}(z)=\sum_{j=n}^{\infty} c_{j} z^{j}$. Then the entire function

$$
P_{n-1}(z) g(z)=\left(\frac{1}{g(z)}-R_{n}(z)\right) g(z)=1-R_{n}(z) g(z)=1-c_{n} z^{n}+\ldots
$$

satisfied the conditions of Theorem 3.2 with $q=n$.

EXAMPLE 3. If a sequence $\left\{a_{k}\right\}$ is such that the series (3.1) is convergent with $q=2$, then the product

$$
\prod_{k=1}^{\infty} \cos \frac{z}{a_{k}}
$$

converges absolutely and uniformly in each bounded disc, and represents an entire function.

Suppose that $g(z)$ and $f(z)$ satisfy the conditions of Theorem 3.2. Our next purpose is to estimate from above $\ln M(r, f)$ provided an estimate from above of $\ln M(r, g)$ is given.

THEOREM 3.3. Suppose that the conditions of Theorem 3.2 are satisfied and $\ln M(r, g)=O\left(r^{\kappa}\right)$ as $r \rightarrow \infty$, where $0<\kappa<q$. Denote by $\nu(r)$ the number of points $a_{k}$ in the disc $\{|z| \leq r\}$. Then there exists a constant $C$, depending on $g(z)$, but not on the sequence $\left\{a_{k}\right\}$, such that

$$
\ln M(r, f)<C \int_{0}^{\infty} \frac{\nu(r \tau)}{\tau^{1+\kappa}} \frac{d \tau}{1+\tau^{q-\kappa}}, 0<r<\infty .
$$


Proof. By $C$ with different indices we denote constants which depend on $g(z)$ only. Since for $r \rightarrow 0$ we have $\ln M(r, g)=O\left(r^{q}\right)$, for all $r, 0<r<\infty$, the following condition holds

$$
\ln M(r, g(z)) \leq C_{1} \Psi(r), \text { where } \Psi(r)=\frac{r^{q}}{1+r^{q-\kappa}} .
$$

By (3.3), we get

$$
\begin{aligned}
\ln M(r, f) & \leq \sum_{k=1}^{\infty} \ln M\left(r, g\left(\frac{z}{a_{k}}\right)\right) \leq C_{1} \sum_{k=1}^{\infty} \Psi\left(\frac{r}{\left|a_{k}\right|}\right) \\
& =C_{1} \int_{0}^{\infty} \Psi\left(\frac{r}{t}\right) d \nu(t)=C_{1} \Phi(r) .
\end{aligned}
$$

Convergence of the series (3.1) implies that $\nu(r)=o\left(r^{q}\right)$ as $r \rightarrow \infty$. In addition, $\nu(r)=0$ when $0 \leq r<\left|a_{1}\right|$. Taking this into account and integrating by parts we get

$$
\Phi(r)=\int_{0}^{\infty} \nu(t) \Psi^{\prime}\left(\frac{r}{t}\right) \frac{r d t}{t^{2}}=\int_{0}^{\infty} \nu(r \tau) \Psi^{\prime}\left(\frac{1}{\tau}\right) \frac{d \tau}{\tau^{2}} .
$$

It is easy to check that

$$
\frac{\kappa \tau^{1-\kappa}}{1+\tau^{q-\kappa}}<\Psi^{\prime}\left(\frac{1}{\tau}\right)=\tau^{1-\kappa} \frac{q \tau^{q-\kappa}+\kappa}{\left(1+\tau^{q-\kappa}\right)^{2}}<\frac{q \tau^{1-\kappa}}{1+\tau^{q-\kappa}} .
$$

This immediately implies the conclusion of the theorem with $C=C_{1} q$.

We will need also the following inequality

$$
\Phi(r) \geq C_{1} \kappa \int_{0}^{\infty} \frac{\nu(r \tau)}{\tau^{1+\kappa}} \frac{d \tau}{1+\tau^{q-\kappa}}, 0<r<\infty .
$$

Corollary. If $f(z)$ is a Weierstrass canonical product of genus $p(3.4)$, then

$$
\ln M(r, f)<C(p) \int_{0}^{\infty} \frac{n(r \tau, 0)}{\tau^{1+p}} \frac{d \tau}{1+\tau}=C(p) r^{p+1} \int_{0}^{\infty} \frac{n(t, 0) d t}{t^{p+1}(r+t)} .
$$

In fact, if $p \geq 1$, we can apply Theorem 3.3 with $g(z)=E(z, p), \kappa=p, q=p+1$. If $p=0$, we use the same argument as above with $\Psi(r)=\ln (1+r)$. We get

$$
\ln M(r, f) \leq C_{1} \int_{0}^{\infty} n(r \tau, 0) \Psi^{\prime}\left(\frac{1}{\tau}\right) \frac{d \tau}{\tau^{2}} .
$$

But in this case $\Psi^{\prime}\left(\frac{1}{\tau}\right)=\tau /(1+\tau)$, and we immediately get the desired inequality. Observe that, as is easy to see, if $p=0$ we can set $C(0)=1$. It can be shown that $C(p)$ can be chosen as $4(p+1)(2+\ln p)$ for $p \geq 1$ (see Hayman [Hay64]).

REMARK 1. If $f(z)$ is a Weierstrass canonical product of genus $p$, then

$$
\ln M(r, f) \leq C(p)\left\{r^{p} \int_{0}^{r} \frac{n(t, 0)}{t^{p+1}} d t+r^{p+1} \int_{r}^{\infty} \frac{n(t, 0)}{t^{p+2}} d t\right\} .
$$

In fact, for each $R, 0<R<\infty$, we have

$$
\begin{aligned}
& r^{p+1} \int_{0}^{\infty} \frac{n(t, 0)}{t^{p+1}(r+t)} d t \leq r^{p+1} \int_{0}^{R} \frac{n(t, 0)}{t^{p+1}(t+r)} d t \\
& \quad+r^{p+1} \int_{R}^{\infty} \frac{n(t, 0)}{t^{p+1}(t+r)} d t \leq r^{p} \int_{0}^{R} \frac{n(t, 0)}{t^{p+1}} d t+r^{p+1} \int_{R}^{\infty} \frac{n(t, 0)}{t^{p+2}} d t .
\end{aligned}
$$


If $R=r$, from (3.5) and (3.7) we get (3.6).

REMARK 2. If $f(z)$ is a Weierstrass canonical product of genus $p$, then

$$
\ln M(r, f)=o\left(r^{p+1}\right) .
$$

This relation can be derived from (3.5) since

$$
\int_{0}^{\infty} \frac{n(t, 0) d t}{t^{p+1}(r+t)}=o(1) \text { as } r \rightarrow \infty .
$$

The legitimacy of passing to limit under the integral sign can be easily justified, since the integrand tends to 0 monotonically as $r \rightarrow \infty$.

We establish the following convention: the value of $p$ in the Weierstrass canonical products of genus $p$ which we consider is, unless the contrary is explicitly stated, the least non-negative integer such that the series (3.1) converges for $q=p+1$. This non-negative integer will be called the genus of the sequence $\left\{a_{k}\right\}$.

TheOREM 3.4 (Borel). The order of the Weierstrass canonical product $f(z)$ is equal to the order of $n(r, 0)$.

Proof. Let $\rho_{1}$ be the order of $n(r, 0)$. This number is equal to the order of $N(r, 0)$ (see the corollary of Theorem 1.1), hence $\rho_{1} \leq \rho$, where $\rho$ is the order of the canonical product. Since $p$ is the least integer for which the series $\sum_{k}\left|a_{k}\right|^{-p-1}$ is convergent, then $p \leq \rho_{1} \leq p+1$. By Remark 2 we get $\rho \leq p+1$, therefore, if $\rho_{1}=p+1$, then $\rho_{1} \geq \rho$. Now assume $\rho_{1}<p+1$. Then for each $\rho^{\prime}, \rho_{1}<\rho^{\prime}<p+1$, we have $n(r, 0)<C_{2} r^{\rho^{\prime}}$ for all $r>0$, where $C_{2}$ is some constant. From (3.5) we get

$$
\ln M(r, f)<C(p) C_{2} r^{\rho^{\prime}} \int_{0}^{\infty} \frac{\tau^{\rho^{\prime}-p-1}}{1+\tau} d \tau=C_{3} r^{\rho^{\prime}} .
$$

Hence $\rho \leq \rho^{\prime}$ and $\rho \leq \rho_{1}$. Therefore $\rho \leq \rho_{1}$ is satisfied in all cases, and together with $\rho_{1} \leq \rho$ this proves the theorem.

\section{Hadamard and Lindelöf theorems}

Canonical products can be use to find convenient representations of meromorphic functions of finite order.

THEOREM 4.1 (Hadamard). Let $f(z)$ be a meromorphic function of finite order $\rho$, and let $\left\{a_{\nu}\right\}$ and $\left\{b_{\mu}\right\}$ be the sequences of zeros and poles of the function $f(z)$, which are different from $z=0$. Let $p_{1}$ be the genus of $\left\{a_{\nu}\right\}$ and $p_{2}$ be the genus of $\left\{b_{\mu}\right\}$. Suppose that in the neighborhood of $z=0$ the function $f(z)$ has a representation $f(z)=c_{\lambda} z^{\lambda}+c_{\lambda+1} z^{\lambda+1}+\ldots$ with $c_{\lambda} \neq 0$. Then

$$
f(z)=z^{\lambda} e^{P(z)} \frac{\prod_{a_{\nu}} E\left(\frac{z}{a_{\nu}}, p_{1}\right)}{\prod_{b_{\mu}} E\left(\frac{z}{b_{\mu}}, p_{2}\right)},
$$

where $P(z)$ is a polynomial, whose degree $q$ does not exceed $[\rho]$. 
Proof. Consider an auxiliary function

$$
F(z)=f(z) z^{-\lambda} \frac{\prod_{b_{\mu}} E\left(\frac{z}{b_{\mu}}, p_{2}\right)}{\prod_{a_{\nu}} E\left(\frac{z}{a_{\nu}}, p_{1}\right)} .
$$

It is clear that $F(z)$ is an entire function without zeros. By Theorems 1.7 and 3.4, we have

$$
\rho_{1}=\rho[F] \leq \max (\rho[f], \rho[n(r, 0, f)], \rho[n(r, \infty, f)])=\rho .
$$

Using Lemma 6.2 from Chapter 1 , we get $F(z)=\exp P(z)$, where $P(z)$ is a polynomial whose degree does not exceed $\left[\rho_{1}\right] \leq[\rho]$.

Definition. Let $f(z)$ be a meromorphic function of finite order $\rho$. Then it has a representation of the form (4.1). The number $p=\max \left(q, p_{1}, p_{2}\right)$ is called the genus of the function $f(z)$.

ExERCISE. Prove the inequality $p \leq \rho \leq p+1$.

REMARK. Suppose that the conditions of Theorem 4.1 are satisfied. Denote the sequence of zeros and poles of the function $f(z)$ by $\left\{c_{k}\right\}$. Then the representation (4.1) remains valid if we replace $p_{1}$ and $p_{2}$ by $p^{\prime}$ in the Weierstrass canonical products. The same representation remains valid if we replace $p_{1}$ and $p_{2}$ by the genus $p$ of the function $f(z)$. In fact, suppose, for example, that $p_{1}<p, p_{2}<p$. Then

$$
f(z)=z^{\lambda} e^{P(z)+P_{1}(z)+P_{2}(z)} \frac{\prod_{a_{\nu}} E\left(\frac{z}{a_{\nu}}, p\right)}{\prod_{b_{\mu}} E\left(\frac{z}{b_{\mu}}, p\right)},
$$

where

$$
\begin{gathered}
P_{1}(z)=-\frac{z^{p_{1}+1}}{p_{1}+1} \sum_{\nu} \frac{1}{a_{\nu}^{p_{1}+1}}-\cdots-\frac{z^{p}}{p} \sum_{\nu} \frac{1}{a_{\nu}^{p}}, \\
P_{2}(z)=\frac{z^{p_{2}+1}}{p_{2}+1} \sum_{\mu} \frac{1}{b_{\mu}^{p_{2}+1}}+\cdots+\frac{z^{p}}{p} \sum_{\mu} \frac{1}{b_{\mu}^{p}} .
\end{gathered}
$$

The series forming the coefficients of polynomials $P_{1}(z)$ and $P_{2}(z)$ are absolutely convergent by the definitions of the numbers $p_{1}$ and $p_{2}$. Since the degree of the polynomial $P(z)+P_{1}(z)+P_{2}(z)$ does not exceed $p$, and, hence, does not exceed $[\rho]$, then the statement is proved in this case. All other cases can be considered in a similar manner.

Denote by $N(r ; a, b), a \neq b$, the sum $N(r, a)+N(r, b)$, also let $n(r ; a, b)=$ $n(r, a)+n(r, b)$. We are going to establish connections between the orders and types of the functions $f(z)$ and $N(r ; a, b)$. It is clear that without loss of generality we may assume that $a=0, b=\infty$, and $f(0)=1$.

The easiest case is the case when the order $\rho$ of the function $f(z)$ is non-integer.

THEOREM 4.2. If the order $\rho$ of a meromorphic function $f(z)$ is non-integer, then the order of $N(r ; 0, \infty)$ is equal to $\rho$. The types of $T(r, f)$ and $N(r ; 0, \infty)$ with respect to an arbitrary proximate order $\rho(r), \rho(r) \rightarrow \rho$, coincide. 
Proof. We represent $f(z)$ in the form (4.1). It is clear that $\rho[N(r ; 0, \infty)] \leq$ $\rho[f]=\rho$. Suppose that $\rho_{1}=\rho[N(r, 0)] \geq \rho[N(r, \infty)]$. Then $\rho[N(r ; 0, \infty)]=\rho_{1}$.

By Theorem 3.4 the order of the function

$$
\pi(z)=\frac{\prod_{a_{\nu}} E\left(\frac{z}{a_{\nu}}, p_{1}\right)}{\prod_{b_{\mu}} E\left(\frac{z}{b_{\mu}}, p_{2}\right)}
$$

is equal to $\rho_{1}$, since $\rho_{1}=\rho[N(r, 0)] \leq \rho[\pi(z)] \leq \max \left(\rho[N(r, 0)], \rho[N(r, \infty)]=\rho_{1}\right.$. Since $\rho\left[e^{-P(z)}\right] \leq p<\rho$ ( $\rho$ is non-integer! $)$, then

$$
\rho_{1}=\rho[\pi(z)]=\max \left(\rho\left[f(z), \rho\left[e^{-P(z)}\right]\right)=\rho .\right.
$$

Suppose that

$$
\limsup _{r \rightarrow \infty} \frac{N(r ; 0, \infty)}{r^{\rho(r)}}=\Delta_{N}, \limsup _{r \rightarrow \infty} \frac{T(r, f)}{r^{\rho(r)}}=\Delta_{f} .
$$

By $N(r ; 0, \infty) \leq 2 T(r, f)+O(1)$, we get $\Delta_{N} \leq 2 \Delta_{f}$. Represent $f(z)$ in the form (4.1) with $p_{1}=p_{2}=p$ (see the remark to Theorem 4.1). Using the formulae (6.5) and (6.7) from Chapter 1 and the corollary of Theorem 3.3, we get

$$
\begin{aligned}
& T(r, f) \leq O\left(r^{p}\right)+\ln M\left(r, \prod_{a \nu} E\left(\frac{z}{a_{\nu}}, p\right)\right)+\ln M\left(r, \prod_{b_{\mu}} E\left(\frac{z}{b_{\mu}}, p\right)\right) \\
\leq & O\left(r^{p}\right)+C(p) \int_{0}^{\infty} \frac{n(r \tau, 0)}{\tau^{1+p}} \frac{d \tau}{1+\tau}+C(p) \int_{0}^{\infty} \frac{n(r \tau, \infty)}{\tau^{1+p}} \frac{d \tau}{1+\tau} \\
= & O\left(r^{p}\right)+C(p) \int_{0}^{\infty} \frac{n(r \tau ; 0, \infty)}{\tau^{1+p}} \frac{d \tau}{1+\tau}=O\left(r^{p}\right)+C(p) \int_{0}^{\infty} \frac{d N(r \tau ; 0, \infty)}{\tau^{p}(1+\tau)} \\
= & O\left(r^{p}\right)+\left.C(p) \frac{N(r \tau ; 0, \infty)}{\tau^{p(1+\tau)}}\right|_{\tau=0} ^{\tau=\infty}+C(p) \int_{0}^{\infty} N(r \tau ; 0, \infty) \frac{(p+1) \tau+p}{\tau^{p+1}(1+\tau)^{2}} d \tau \\
\leq & O\left(r^{p}\right)+C(p)(p+1) \int_{0}^{\infty} \frac{N(r \tau ; 0, \infty)}{\tau^{p+1}} \frac{d \tau}{1+\tau} .
\end{aligned}
$$

We have used the facts that $\rho<p+1$ and $N(r \tau ; 0, \infty)=o\left(\tau^{p+1}\right)$. Assume that $\Delta_{N}<\infty$. Then the condition $N(r ; 0, \infty) \leq\left(\Delta_{N}+\varepsilon\right) r^{\rho(r)}$ is satisfied for $r>c(\varepsilon), \varepsilon>0$. Then (4.3) implies

$$
\frac{T(r, f)}{r^{\rho(r)}} \leq o(1)+C(p)(p+1)\left(\Delta_{N}+\varepsilon\right) \int_{c / r}^{\infty} \frac{(r \tau)^{\rho(r \tau)}}{r^{\rho(r)}} \frac{d \tau}{\tau^{p+1}(1+\tau)} .
$$

Taking in this inequality limits as $r \rightarrow \infty$, and using Exercise 1 from Section 2 of this chapter, we get

$$
\Delta_{f} \leq C(p)(p+1)\left(\Delta_{N}+\varepsilon\right) \frac{\pi}{\sin \pi(\rho-p)} .
$$

Since $\varepsilon$ can be arbitrarily small, we get

$$
\Delta_{f} \leq C(p)(p+1) \frac{\pi}{\sin \pi(\rho-p)} \Delta_{N} .
$$

This inequality is obvious if $\Delta_{N}=\infty$. Together with the inequality $\Delta_{f} \geq 2^{-1} \Delta_{N}$ it concludes the proof. 
COROLlaRY. If a meromorphic function $f(z)$ has non-integer order $\rho,[\rho]=p$, then

$$
\limsup _{r \rightarrow \infty} \frac{N(r ; 0, \infty)}{T(r, f)} \geq \frac{\sin \pi(\rho-p)}{C(p)(p+1) \pi}>0 .
$$

Let $\rho(r)$ be a proximate order of $T(r, f)$. Then, by (4.4) we get

$$
\limsup _{r \rightarrow \infty} \frac{N(r ; 0, \infty)}{T(r, f)} \geq \limsup _{r \rightarrow \infty} \frac{N(r ; 0, \infty)}{\Delta_{f} r^{\rho(r)}}=\frac{\Delta_{N}}{\Delta_{f}} \geq \frac{\sin \pi(\rho-p)}{C(p)(p+1) \pi} .
$$

We consider now the case when a meromorphic function $f(z)$ has integer order $\rho \geq 1$. Then the genus of $f(z)$ is equal to $\rho-1$ or $\rho$. Using (4.1), the function $f(z)$ can be represented in the form

$$
f(z)=e^{P(z)} \frac{\prod_{a_{\nu}} E\left(\frac{z}{a_{\nu}}, \rho\right)}{\prod_{b_{\mu}} E\left(\frac{z}{b_{\mu}}, \rho\right)},
$$

where

$$
P(z)=c_{\rho} z^{\rho}+c_{\rho-1} z^{\rho-1}+\cdots+c_{1} z .
$$

First we prove the following theorem.

THEOREM 4.3. Let $f(z)$ be a meromorphic function of integer order $\rho \geq 1$, and $\rho(r)$ a proximate order, $\rho(r) \rightarrow \rho$ as $r \rightarrow \infty$. Suppose that

$$
\Delta_{n}=\limsup _{r \rightarrow \infty} \frac{n(r ; 0, \infty)}{r^{\rho(r)}}<\infty \text {. }
$$

Then $(\varepsilon>0, r \geq r(\varepsilon))$

$$
T(r, f)=\frac{r^{\rho}}{\pi}|K(r)|+\left(\Delta_{n}+\varepsilon\right) \omega_{\varepsilon}(r) r^{\rho(r)},
$$

where the value of $\left|\omega_{\varepsilon}(r)\right|$ is bounded by a constant $M_{\rho}$, which depends on $\rho$ only, and

$$
K(r)=c_{\rho}+\frac{1}{\rho} \sum_{\left|a_{\nu}\right| \leq r} a_{\nu}^{-\rho}-\frac{1}{\rho} \sum_{\left|b_{\mu}\right| \leq r} b_{\mu}^{-\rho} .
$$

Proof. Using $\left(4.1^{\prime}\right)$, we represent the function $f(z)$ as a product, $f(z)=$ $f_{1}(z) f_{2}(z)$, where

$$
\begin{gathered}
f_{1}(z)=\exp \left\{z^{\rho} K(r)\right\}, \\
f_{2}(z)=e^{c_{\rho-1} z^{\rho-1}+\cdots+c_{1} z} \cdot \frac{\prod_{\left|a_{\nu}\right| \leq r} E\left(\frac{z}{a_{\nu}}, \rho-1\right) \prod_{\left|a_{\nu}\right|>r} E\left(\frac{z}{a_{\nu}}, \rho\right)}{\prod_{\left|b_{\mu}\right| \leq r} E\left(\frac{z}{b_{\mu}}, \rho-1\right) \prod_{\left|b_{\mu}\right|>r} E\left(\frac{z}{b_{\mu}}, \rho\right)}
\end{gathered}
$$

It is easy to compute that

$$
T\left(r, f_{1}(z)\right)=\frac{r^{\rho}}{\pi}|K(r)|
$$

Since

$$
\left|T(r, f)-T\left(r, f_{1}\right)\right| \leq T\left(r, f_{2}\right)+O(1),
$$


it remains to estimate $T\left(r, f_{2}\right)$. Using the inequality (3.6) and the equality $\left(6.8^{\prime}\right)$ from Chapter 1, we get

$$
\begin{aligned}
& T\left(r, f_{2}\right) \leq T\left(r, e^{c_{\rho-1} z^{\rho-1}+\cdots+c_{1} z}\right)+T\left(r, \prod_{\left|a_{\nu}\right| \leq r} E\left(\frac{z}{a_{\nu}}, \rho-1\right)\right) \\
& +T\left(r, \prod_{\left|a_{\nu}\right|>r} E\left(\frac{z}{a_{\nu}}, \rho\right)\right)+T\left(r, \prod_{\left|b_{\mu}\right| \leq r} E\left(\frac{z}{b_{\mu}}, \rho-1\right)\right) \\
& +T\left(r, \prod_{\left|b_{\mu}\right|>r} E\left(\frac{z}{b_{\mu}}, \rho\right)\right) \\
& \leq O\left(r^{\rho-1}\right)+\ln M\left(r, \prod_{\left|a_{\nu}\right| \leq r} E\left(\frac{z}{a_{\nu}}, \rho-1\right)\right)+\ln M\left(r, \prod_{\left|a_{\nu}\right|>r} E\left(\frac{z}{a_{\nu}}, \rho\right)\right) \\
& +\ln M\left(r, \prod_{\left|b_{\mu}\right| \leq r} E\left(\frac{z}{b_{\mu}}, \rho-1\right)\right)+\ln M\left(r, \prod_{\left|b_{\mu}\right|>r} E\left(\frac{z}{b_{\mu}}, \rho\right)\right) \\
& \leq O\left(r^{\rho-1}\right)+C(\rho-1)\left\{r^{\rho-1} \int_{r_{0}}^{r} \frac{n(t ; 0, \infty)}{t^{\rho}} d t+r^{\rho} n(r ; 0, \infty) \int_{r}^{\infty} \frac{d t}{t^{\rho+1}}\right\} \\
& +C(\rho) r^{\rho+1} \int_{r}^{\infty} \frac{n(t ; 0, \infty)}{t^{\rho+2}} d t=O\left(r^{\rho-1}\right) \\
& +C(\rho-1)\left\{r^{\rho-1} \int_{r_{0}}^{r} \frac{n(t ; 0, \infty)}{t^{\rho}} d t+\frac{1}{\rho} n(r ; 0, \infty)\right\} \\
& +C(\rho) r^{\rho+1} \int_{r}^{\infty} \frac{n(t ; 0, \infty)}{t^{\rho+2}} d t, r_{0}>0 .
\end{aligned}
$$

We may assume that the inequality $n(r ; 0, \infty) \leq\left(\Delta_{n}+\varepsilon\right) r^{\rho(r)}$ is satisfied for all $r \geq r_{0}$. Taking into account Theorem 2.4, we get

$$
\begin{aligned}
T\left(r, f_{2}\right) \leq & O\left(r^{\rho-1}\right)+\left(\Delta_{n}+\varepsilon\right)\left\{C(\rho-1) r^{\rho-1} \int_{r_{0}}^{r} t^{\rho(t)-\rho} d t\right. \\
& \left.+\frac{C(\rho-1)}{\rho} r^{\rho(r)}+C(\rho) r^{\rho+1} \int_{r}^{\infty} t^{\rho(t)-\rho-2} d t\right\} \\
= & O\left(r^{\rho-1}\right)+\left(\Delta_{n}+\varepsilon\right)(1+o(1)) r^{\rho(r)}\left\{C(\rho-1)\left(1+\frac{1}{\rho}\right)+C(\rho)\right\} \\
= & \left(\Delta_{n}+\varepsilon\right)(1+o(1)) r^{\rho(r)}\left\{C(\rho-1)\left(1+\frac{1}{\rho}\right)+C(\rho)\right\},
\end{aligned}
$$

the proof is complete.

TheOREM 4.4 (Lindelöf). Suppose that the conditions of Theorem 4.3 are satisfied. Let

$$
L(r)=r^{\rho(r)-\rho}, \Delta_{K}=\limsup _{r \rightarrow \infty} \frac{|K(r)|}{L(r)}, \Omega=\max \left(\Delta_{K}, \Delta_{n}\right) .
$$

Then the function $f(z)$ has minimal, normal, or maximal type with respect to $\rho(r)$ depending on whether $\Omega=0,0<\Omega<\infty$, or $\Omega=\infty$, respectively. 
Proof. We denote by $A_{j}$ various positive constants depending on $\rho$ only. The relation (4.5) implies that, if $\Delta_{n}<\infty$, then $\Delta_{f} \leq A_{1}\left(\Delta_{K}+\Delta_{n}+\varepsilon\right)$, hence $\Delta_{f} \leq A_{2} \Omega$. If $\Delta_{n}=\infty$, the inequality is trivial. The relation (4.5) implies, also, that $\Delta_{K} \leq A_{3}\left(\Delta_{f}+\Delta_{n}+\varepsilon\right)$ if $\Delta_{n}<\infty$, hence $\Delta_{K} \leq A_{3}\left(\Delta_{f}+\Delta_{n}\right)$. It is clear that this inequality is valid also if $\Delta_{n}=\infty$. Lemma 7.1 from Chapter 1 implies that $n(r ; 0, \infty) \leq N(e r ; 0, \infty) \leq 2 T(e r, f)+O(1)$. Therefore $\Delta_{n} \leq 2 e^{\rho} \Delta_{f}$. Hence $\Delta_{K} \leq A_{3}\left(1+2 e^{\rho}\right) \Delta_{f}$. We get $\Omega \leq A_{4} \Delta_{f}$. Using the inequality $A_{4}^{-1} \Omega \leq \Delta_{f} \leq A_{2} \Omega$ we complete the proof.

REMARK. It is easy to show (cf. the proof of Theorem 1.1) that $e^{-\rho} \Delta_{n} \leq$ $\Delta_{N} \leq \rho^{-1} \Delta_{n}$. Therefore Theorem 4.4 remains true if we replace $\Omega$ in its statement by $\Omega^{\prime}=\max \left(\Delta_{K}, \Delta_{N}\right)$.

Alternatively, Theorem 4.4 can be proved without using Theorem 4.3, but using formula (2.6) from Chapter 1 instead. The idea of this proof is due to N.I. Akhiezer [Akh27] and L.A. Rubel [Rub63]. For simplicity, we restrict our attention to the case $\rho(r) \equiv \rho, L(r) \equiv 1$, leaving the general case to interested readers.

Without loss of generality we may assume that $f(0)=1$. Suppose that in a neighborhood of $z=0$ we have

$$
\ln f(z)=\sum_{k=1}^{\infty} \gamma_{k} z^{k}
$$

It is easy to derive from $\left(4.1^{\prime}\right)$ that

$$
\gamma_{k}= \begin{cases}c_{k}, & k=1, \ldots, \rho \\ -\frac{1}{k} \sum_{\nu} a_{\nu}^{-k}+\frac{1}{k} \sum_{\mu} b_{\mu}^{-k}, & k=\rho+1, \rho+2, \ldots\end{cases}
$$

Denote by $F_{k}(r)$ the Fourier coefficient of the function $\ln \left|f\left(r e^{i \varphi}\right)\right|, 0 \leq \varphi \leq 2 \pi$ :

$$
F_{k}(r)=\frac{1}{2 \pi} \int_{0}^{2 \pi} \ln \left|f\left(r e^{i \varphi}\right)\right| e^{-i k \varphi} d \varphi, k=0, \pm 1, \pm 2, \ldots
$$

It is clear that

$$
\left|F_{k}(r)\right| \leq \frac{1}{2 \pi} \int_{0}^{2 \pi}|\ln | f\left(r e^{i \varphi}\right) \| d \varphi=m(r, f)+m\left(r, \frac{1}{f}\right) \leq 2 T(r, f) .
$$

The formula (2.6) from Chapter 1 can be rewritten as $(k=1,2, \ldots)$ :

$$
\gamma_{k}=\frac{2}{r^{k}} F_{k}(r)+\frac{1}{k} \sum_{\left|a_{\nu}\right| \leq r}\left(\frac{\bar{a}_{\nu}^{k}}{r^{2 k}}-\frac{1}{a_{\nu}^{k}}\right)-\frac{1}{k} \sum_{\left|b_{\mu}\right| \leq r}\left(\frac{\bar{b}_{\mu}^{k}}{r^{2 k}}-\frac{1}{b_{\mu}^{k}}\right)
$$

If $k=\rho$ and $\gamma_{k}=c_{\rho}$, the equality (4.8) can be rewritten as:

$$
K(r)=\frac{2}{r^{\rho}} F_{\rho}(r)+\frac{1}{\rho} \sum_{\left|a_{\nu}\right| \leq r} \frac{\bar{a}_{\nu}^{\rho}}{r^{2 \rho}}-\frac{1}{\rho} \sum_{\left|b_{\mu}\right| \leq r} \frac{\bar{b}_{\mu}^{\rho}}{r^{2 \rho}} .
$$

Using (4.7) we get

$$
\begin{aligned}
|K(r)| & \leq \frac{4 T(r, f)}{r^{\rho}}+\frac{1}{\rho r^{\rho}}\left\{\sum_{\left|a_{\nu}\right| \leq r}\left(\frac{\left|\bar{a}_{\nu}\right|}{r}\right)^{\rho}+\sum_{\left|b_{\mu}\right| \leq r}\left(\frac{\left|\bar{b}_{\mu}\right|}{r}\right)^{\rho}\right\} \\
& \leq \frac{4 T(r, f)}{r^{\rho}}+\frac{n(r ; 0, \infty)}{\rho r^{\rho}} .
\end{aligned}
$$


Hence

$$
\Delta_{K} \leq 4 \Delta_{f}+\rho^{-1} \Delta_{n}
$$

Using this inequality we get, as in our first proof of Theorem 4.4, $\Omega \leq A_{5} \Delta_{f}$.

Assume now that $\Omega<\infty$. If $r>r_{0}(\varepsilon)$, then $|K(r)|<\Delta_{K}+\varepsilon$ and $n(r ; 0, \infty)<$ $\left(\Delta_{n}+\varepsilon\right) r^{\rho}, \varepsilon>0$. We estimate the Fourier coefficients $F_{k}(r)$. If $k>\rho$, from (4.8) we get

$$
-\frac{1}{k} \sum_{\left|a_{\nu}\right|>r} a_{\nu}^{-k}+\frac{1}{k} \sum_{\left|b_{\mu}\right|>r} b_{\mu}^{-k}=\frac{2}{r^{k}} F_{k}(r)+\frac{1}{k} \sum_{\left|a_{\nu}\right| \leq r} \frac{\bar{a}_{\nu}^{k}}{r^{2 k}}-\frac{1}{k} \sum_{\left|b_{\mu}\right| \leq r} \frac{\bar{b}_{\mu}^{k}}{r^{2 k}},
$$

hence

$$
\begin{aligned}
\left|F_{k}(r)\right| & \leq \frac{1}{2 k} n(r ; 0, \infty)+\frac{r^{k}}{2 k} \int_{r}^{\infty} \frac{d n(t ; 0, \infty)}{t^{k}} \\
& =\frac{r^{k}}{2} \int_{r}^{\infty} \frac{n(t ; 0, \infty)}{t^{k+1}} d t \leq \frac{\Delta_{n}+\varepsilon}{2(k-\rho)} r^{\rho}, k>\rho .
\end{aligned}
$$

If $1 \leq k \leq \rho-1$, then using (4.8) we get

$$
\begin{aligned}
\left|F_{k}(r)\right| & \leq \frac{\left|c_{k}\right|}{2} r^{k}+\frac{1}{2 k} n(r ; 0, \infty)+\frac{r^{k}}{2 k}\left\{\sum_{\left|a_{\nu}\right| \leq r}\left|a_{\nu}\right|^{-k}+\sum_{\left|b_{\mu}\right| \leq r}\left|b_{\mu}\right|^{-k}\right\} \\
& \leq \frac{\left|c_{k}\right|}{2} r^{k}+\frac{n(r ; 0, \infty)}{2 k}+\frac{r^{k}}{2 k} \int_{0}^{r} \frac{d n(t ; 0, \infty)}{t^{k}} d t \\
& =\frac{\left|c_{k}\right|}{2} r^{k}+\frac{n(r ; 0, \infty)}{k}+\frac{r^{k}}{2} \int_{0}^{r} \frac{n(t ; 0, \infty)}{t^{k+1}} d t \\
& \leq O\left(r^{\rho-1}\right)+\left(\Delta_{n}+\varepsilon\right)\left(\frac{1}{k}+\frac{1}{2(\rho-k)}\right) r^{\rho}
\end{aligned}
$$

From (4.9) we get

$$
\left|F_{\rho}(r)\right| \leq \frac{|K(r)|}{2} r^{\rho}+\frac{n(r ; 0, \infty)}{2 \rho} \leq \frac{1}{2}\left(\Delta_{K}+\varepsilon+\frac{\Delta_{n}+\varepsilon}{\rho}\right) r^{\rho} .
$$

Finally, by Jensen's formula,

$$
F_{0}(r)=N(r, 0)-N(r, \infty)
$$

and

$$
\left|F_{0}(r)\right| \leq N(r ; 0, \infty) \leq \frac{\Delta_{n}+\varepsilon}{\rho} r^{\rho}+O(1)
$$

By the Parseval's identity and the identity $F_{-k}=\overline{F_{k}(r)}$, we get

$$
\frac{1}{2 \pi} \int_{0}^{2 \pi} \ln ^{2}\left|f\left(r e^{i \varphi}\right)\right| d \varphi=\sum_{k=-\infty}^{\infty}\left|F_{k}(r)\right|^{2}=\left|F_{0}(r)\right|^{2}+2 \sum_{k=1}^{\infty}\left|F_{k}(r)\right|^{2} .
$$


Using the inequalities (4.10), (4.11), (4.12), (4.13), and the elementary inequality $(|a|+|b|)^{2} \leq 2\left(|a|^{2}+|b|^{2}\right)$, we get

$$
\begin{aligned}
& \frac{1}{2 \pi} \int_{0}^{2 \pi} \ln ^{2}\left|f\left(r e^{i \varphi}\right)\right| d \varphi \leq O\left(r^{2 \rho-2}\right)+2\left\{\left(\frac{\Delta_{n}+\varepsilon}{\rho}\right)^{2}\right. \\
& \quad+\left(\Delta_{n}+\varepsilon\right)^{2} \sum_{k=1}^{\rho-1}\left(\frac{1}{k}+\frac{1}{2(\rho-k)}\right)^{2}+\frac{\left(\Delta_{K}+\varepsilon\right)^{2}}{2}+\frac{\left(\Delta_{n}+\varepsilon\right)^{2}}{2 \rho^{2}} \\
& \left.\quad+\frac{\left(\Delta_{n}+\varepsilon\right)^{2}}{4} \sum_{k=\rho+1}^{\infty} \frac{1}{(k-\rho)^{2}}\right\} r^{2 \rho}=O\left(r^{2 \rho-2}\right)+\left\{\left(\Delta_{K}+\varepsilon\right)^{2}+A_{6}\left(\Delta_{n}+\varepsilon\right)^{2}\right\} r^{2 \rho}
\end{aligned}
$$

where

$$
A_{6}=\frac{3}{\rho^{2}}+2 \sum_{k=1}^{\rho-1}\left(\frac{1}{k}+\frac{1}{2(\rho-k)}\right)^{2}+\frac{1}{2} \sum_{k=\rho+1}^{\infty} \frac{1}{(k-\rho)^{2}}<\infty .
$$

By the Cauchy-Buniakowsky inequality we get

$$
\begin{aligned}
m(r, f)+m\left(r, \frac{1}{f}\right) & =\frac{1}{2 \pi} \int_{0}^{2 \pi}|\ln | f\left(r e^{i \varphi}\right) \| d \varphi \leq\left(\frac{1}{2 \pi} \int_{0}^{2 \pi} \ln ^{2}\left|f\left(r e^{i \varphi}\right)\right| d \varphi\right)^{1 / 2} \\
& \leq r^{\rho}\left\{\left(\Delta_{K}+\varepsilon\right)^{2}+A_{6}\left(\Delta_{n}+\varepsilon\right)^{2}+O\left(r^{-2}\right)\right\}^{1 / 2} \\
2 T(r, f) & =m(r, f)+m\left(r, \frac{1}{f}\right)+N(r ; 0, \infty) \\
\leq & r^{\rho}\left\{\left(\Delta_{K}+\varepsilon\right)^{2}+A_{6}\left(\Delta_{n}+\varepsilon\right)^{2}+O\left(r^{-2}\right)\right\}^{1 / 2}+\frac{\Delta_{n}+\varepsilon}{\rho} r^{\rho}+O(1) .
\end{aligned}
$$

Hence

$$
\begin{gathered}
2 \Delta_{f} \leq\left\{\left(\Delta_{K}+\varepsilon\right)^{2}+A_{6}\left(\Delta_{n}+\varepsilon\right)^{2}\right\}^{1 / 2}+\frac{\Delta_{n}+\varepsilon}{\rho} \\
\Delta_{f} \leq \frac{1}{2}\left\{\Delta_{K}^{2}+A_{6} \Delta_{n}^{2}\right\}^{1 / 2} \frac{\Delta_{n}}{\rho}+A_{7} \Omega .
\end{gathered}
$$

Therefore, we have proved the inequality $A_{5} \Omega \leq \Delta_{f} \leq A_{7} \Omega$, thus completing the proof of Theorem 4.4.

ExERCISE. Prove Theorem 4.2 using the formula (4.8), the estimates (4.7), (4.10), (4.11), (4.13), and the equality (4.14).

Now we consider the case of order $\rho=0$.

THEOREM 4.5. Let $f(z)$ be a transcendental meromorphic function of order $\rho=$ 0 . Then the types of $T(r, f)$ and $N(r ; 0, \infty)$ with respect to an arbitrary proximate order $\rho(r), \rho(r) \rightarrow 0$, coincide.

Proof. Let

$$
f(z)=\frac{\prod_{a_{\nu}}\left(1-\frac{z}{a_{\nu}}\right)}{\prod_{b_{\mu}}\left(1-\frac{z}{b_{\mu}}\right)}=\frac{\pi_{1}(z)}{\pi_{2}(z)}
$$


From the inequality (3.6) we get

$$
\begin{aligned}
T(r, f) \leq & T\left(r, \pi_{1}\right)+T\left(r, \pi_{2}\right) \leq \ln M\left(r, \pi_{1}\right)+\ln M\left(r, \pi_{2}\right) \\
\leq & \int_{0}^{r} \frac{n(t ; 0, \infty)}{t} d t+r \int_{r}^{\infty} \frac{n(t ; 0, \infty) d t}{t^{2}} \\
= & N(r ; 0, \infty)+r \int_{r}^{\infty} \frac{d N(t ; 0, \infty)}{t}=N(r ; 0, \infty)-N(r ; 0, \infty) \\
& +r \int_{r}^{\infty} \frac{N(t ; 0, \infty)}{t^{2}} d t=r \int_{r}^{\infty} \frac{N(t ; 0, \infty)}{t^{2}} d t .
\end{aligned}
$$

Suppose that $\ln r=o\left(r^{\rho(r)}\right)$. Then, using Theorem 2.4 it is easy to get $\Delta_{f} \leq$ $\Delta_{N}$. On the other hand, it is clear that $\Delta_{N} \leq 2 \Delta_{f}$. These two inequalities imply the statement of the theorem in this case.

If the condition $\ln r=o\left(r^{\rho(r)}\right)$ is not satisfied, then $T(r, f)$ has maximal type. If $N(r ; 0, \infty)$ has normal or minimal type, then for an infinite set of values of $r$ the relation $N(r ; 0, \infty)=O(\ln r)$ holds. In such a case the function $f(z)$ has to have finitely many zeros and poles, and hence by (4.15) is a rational function, so we get a contradiction.

REMARK 1. Under the condition of Theorem 4.5, if $f(z)$ is an entire function, then $\Delta_{N} \leq \Delta_{f}$, and hence $\Delta_{f}=\Delta_{N}$.

REMARK 2 . In the case when the proximate order $\rho(r)$ approaches 0 , the types of the functions $n(r ; 0, \infty)$ and $N(r ; 0, \infty)$ can differ. For example, if $n(r ; 0, \infty) \sim$ $K r^{\rho(r)}$, then $N(r ; 0, \infty) \sim K \int_{r_{0}}^{r} t^{\rho(t)-1} d t$ and, as is easy to check,

$$
r^{\rho(r)}=o\left(\int_{r_{0}}^{r} t^{\rho(t)-1} d t\right) .
$$

Denote by $\widehat{N}(r ; a, b)$ the function $\max (N(r, a), N(r, b))$. Suppose that the conditions of Theorem 4.5 are satisfied. We estimate $T(r, f)$ from above, using instead of the inequality

$$
T\left(r, \frac{\pi_{1}}{\pi_{2}}\right) \leq \ln M\left(r, \pi_{1}\right)+\ln M\left(r, \pi_{2}\right)+O(1)
$$

a more precise one, which can be obtained using (4.8) from Chapter 1:

$$
T\left(r, \frac{\pi_{1}}{\pi_{2}}\right) \leq \max \left\{\ln M\left(r, \pi_{1}\right), \ln M\left(r, \pi_{2}\right)\right\} .
$$

Then, in the same way as we proved (4.16), we get

$$
\begin{aligned}
& T(r, f) \leq \max \left\{\ln M\left(r, \pi_{1}\right), \ln M\left(r, \pi_{2}\right)\right\} \\
& \leq \max \left\{r \int_{r}^{\infty} \frac{N(t, 0)}{t^{2}} d t, r \int_{r}^{\infty} \frac{N(t, \infty)}{t^{2}} d t\right\} \leq r \int_{r}^{\infty} \frac{\widehat{N}(t ; 0, \infty)}{t^{2}} d t .
\end{aligned}
$$

This inequality implies $\Delta_{f} \leq \Delta_{\widehat{N}}$. On the other hand, $\widehat{N}(r ; 0, \infty) \leq T(r, f)+O(1)$ and $\Delta_{\widehat{N}} \leq \Delta_{f}$. Hence $\Delta_{f}=\Delta_{\widehat{N}}$, that is

$$
\limsup _{r \rightarrow \infty} \frac{T(r, f)}{r^{\rho(r)}}=\limsup _{r \rightarrow \infty} \frac{\widehat{N}(r ; 0, \infty)}{r^{\rho(r)}} .
$$


It is clear that 0 and $\infty$ in the last equality can be replaced by arbitrary $a$ and $b$. Let $\rho(r)$ be a proximate order of $T(r, f)$. Then

$$
1 \geq \limsup _{r \rightarrow \infty} \frac{\widehat{N}(r ; a, b)}{T(r, f)} \geq \limsup _{r \rightarrow \infty} \frac{\widehat{N}(r ; a, b)}{\Delta_{f} r^{\rho(r)}}=\frac{\Delta_{\widehat{N}}}{\Delta_{f}}=1,
$$

that is,

$$
\limsup _{r \rightarrow \infty} \frac{\widehat{N}(r ; a, b)}{T(r, f)}=1 .
$$

In the case when $f(z)$ is a rational function, the validity of (4.18) can be easily verified, using the equality (6.26) from Chapter 1 . This implies the following theorem.

THEOREM 4.6 (Valiron). Let $f(z)$ be a meromorphic function of order $\rho=0$. If the inequality

$$
\limsup _{r \rightarrow \infty} \frac{N(r, a)}{T(r, f)}<1
$$

holds for some $a$, then

$$
\limsup _{r \rightarrow \infty} \frac{N(r, b)}{T(r, f)}=1
$$

for all $b \neq a$.

\section{Some examples}

We consider some entire transcendental functions and find some relations for them. We will use the relations on many occasions ${ }^{3}$.

$1^{\circ}$ Let $p$ be a non-negative integer, $p<\eta_{1} \leq \eta_{2}<p+1$. Let $l(r)$ be a function, continuously differentiable on $[0, \infty)$, and such that

1) $l^{\prime}(r) r \ln r \rightarrow 0$ as $r \rightarrow \infty$,

2) $\liminf _{r \rightarrow \infty} l(r)=\eta_{1}, \lim \sup _{r \rightarrow \infty} l(r)=\eta_{2}$.

If $\eta_{1}=\eta_{2}$, the function $l(r)$ is a proximate order. The function $l(r)$ preserves many properties of a proximate order, sometimes with minor modifications. We mention those among these properties which will be used later. 1) The function $r^{l(r)}$ is increasing for sufficiently large $r$ (this can be proved in the same way as Theorem 2.3). 2) If $0<a \leq k \leq b<\infty$, then

$$
l(k r)-l(r)=o\left(\frac{1}{\ln r}\right) \text { and } \frac{(k r)^{l(k r)}}{(k r)^{l(r)}} \rightarrow 1,(r \rightarrow \infty)
$$

uniformly in $k$. The proof is based on the same tricks as our proof of Theorem 2.2, we leave it to interested readers. 3) The following inequalities hold:

$$
\begin{gathered}
\limsup _{r \rightarrow \infty} \frac{\int_{1}^{r} t^{l(t)-p-1} d t}{r^{l(r)-p}} \leq \frac{1}{\eta_{1}-p}, \\
\limsup _{r \rightarrow \infty} \frac{\int_{r}^{\infty} t^{l(t)-p-2} d t}{r^{l(r)-p-1}} \leq \frac{1}{p+1-\eta_{2}} .
\end{gathered}
$$

\footnotetext{
${ }^{3}$ This section could be skipped in the first reading and be consulted when we refer to it in the later sections.
} 
Let us prove, for example, the latter inequality. Using a well-known result from Analysis, we get

$$
\begin{aligned}
\limsup _{r \rightarrow \infty} & \frac{\int_{r}^{\infty} t^{l(t)-p-2} d t}{r^{l(r)-p-1}} \leq \limsup _{r \rightarrow \infty} \frac{\frac{d}{d r} \int_{r}^{\infty} t^{l(t)-p-2} d t}{\frac{d}{d r} r^{l(r)-p-1}} \\
& =\limsup _{r \rightarrow \infty} \frac{1}{p+1-l(r)-l^{\prime}(r) r \ln r}=\frac{1}{p+1-\eta_{2}} .
\end{aligned}
$$

Now we consider the Weierstrass canonical products of genus $p$ with positive zeros $1 \leq a_{1} \leq a_{2} \leq \ldots$, such that $n(r, 0) \sim \Delta r^{l(r)}(0<\Delta<\infty)$ :

$$
f(z)=\prod_{k=1}^{\infty} E\left(\frac{z}{a_{k}}, p\right)
$$

Let us find an asymptotic formula for $\ln f(z)$ in the angle $\delta<\arg z<2 \pi-\delta$ for that branch of $\ln f(z)$ for which $\ln f(0)=0$. We represent $\ln f(z)$ as:

$$
\begin{gathered}
\ln f(z)=\sum_{k=1}^{\infty} \ln E\left(\frac{z}{a_{k}}, p\right)=\int_{0}^{\infty} \ln E\left(\frac{z}{t}, p\right) d n(t, 0) \\
=\left.n(t, 0) \ln E\left(\frac{z}{t}, p\right)\right|_{t=0} ^{\infty}-\int_{0}^{\infty} n(t, 0) \frac{d}{d t} \ln E\left(\frac{z}{t}, p\right) d t \\
=-z^{p+1} \int_{0}^{\infty} n(t, 0) \frac{d t}{t^{p+1}(t-z)} .
\end{gathered}
$$

Consider an arbitrarily small number $\varepsilon, 0<\varepsilon<1$. If $r>r_{0}(\varepsilon)$, then $\mid n(r, 0)-$ $\Delta r^{l(r)} \mid<\varepsilon \Delta r^{l(r)}$. Let $z=r e^{i \varphi}$. Observe that for $\delta<\varphi<2 \pi-\delta$ we have $|t-z| \geq$ $(t+r) \sin \frac{\delta}{2}$ (this statement can be easily proved by minimizing $\left|x-e^{i \varphi}\right| /(x+1)$ over $0 \leq x<\infty)$.

There exists a constant $K \geq \Delta>0$, such that for $0<r<\infty$ the inequality $n(r, 0)<K r^{l(r)}$ holds. Let $\delta_{1}>0$ be such that $1>K_{1}=\eta_{1}-p-\delta_{1}>0$ and $K_{2}=p+1-\eta_{2}-\delta_{1}>0$. Select numbers $a$ and $b, 0<a<1<b<\infty$, such that $2 K K_{1}^{-1} b^{-K_{1}}<\varepsilon, 2 K K_{2}^{-1} b^{-K_{2}}<\varepsilon$ and find $r_{1}(\varepsilon)>a^{-1} r_{0}(\varepsilon)$, such that for $r \geq r_{1}(\varepsilon)$ the following inequalities hold:

1) $(1-\varepsilon)(k r)^{l(r)} \leq(k r)^{l(k r)} \leq(1+\varepsilon)(k r)^{l(r)}$ for $a \leq k \leq b$;

2) $\int_{1}^{a r} t^{l(t)-p-1} d t \leq K_{1}^{-1}(a r)^{l(a r)-p}$

3) $\int_{b r}^{\infty} t^{l(t)-p-2} d t \leq K_{2}^{-1}(b r)^{l(b r)-p-1}$

4) $\eta_{1}-\delta_{1} \leq l(r) \leq \eta_{2}+\delta_{1}$. 
Now we estimate for $r \geq r_{1}(\varepsilon)$ the difference

$$
\begin{aligned}
& \left|z^{p+1} \int_{0}^{\infty} \frac{n(t, 0) d t}{t^{p+1}(t-z)}-z^{p+1} \Delta \int_{0}^{\infty} \frac{t^{l(r)} d t}{t^{p+1}(t-z)}\right| \\
& \leq r^{p+1} \mid \int_{0}^{a r} \frac{n(t, 0) d t}{t^{p+1}(t-z)}+\int_{b r}^{\infty} \frac{n(t, 0) d t}{t^{p+1}(t-z)}+\int_{a r}^{b r} \frac{n(t, 0)-\Delta t^{l(t)}}{t^{p+1}(t-z)} d t \\
& +\Delta \int_{a r}^{b r} \frac{t^{l(t)}-t^{l(r)}}{t^{p+1}(t-z)} d t-\Delta \int_{0}^{a r} \frac{t^{l(r)} d t}{t^{p+1}(t-z)}-\Delta \int_{b r}^{\infty} \frac{t^{l(r)} d t}{t^{p+1}(t-z)} \\
& \leq \frac{r^{p+1}}{\sin \frac{\delta}{2}}\left\{\frac{1}{r} \int_{1}^{a r} \frac{n(t, 0)}{t^{p+1}} d t+\int_{b r}^{\infty} \frac{n(t, 0)}{t^{p+2}} d t+\int_{a r}^{b r} \frac{\left|n(t, 0)-\Delta t^{l(t)}\right|}{t^{p+1}(t+r)} d t\right. \\
& \left.+\Delta \int_{a r}^{b r} \frac{\left|t^{l(t)}-t^{l(r)}\right|}{t^{p+1}(t+r)} d t+\frac{\Delta}{r} \int_{0}^{a r} \frac{t^{l(r)} d t}{t^{p+1}}+\Delta \int_{b r}^{\infty} \frac{t^{l(r)}}{t^{p+2}} d t\right\} \\
& \leq \frac{r^{p+1}}{\sin \frac{\delta}{2}}\left\{\frac{K}{r} \int_{1}^{a r} t^{l(t)-p-1} d t+K \int_{b r}^{\infty} t^{l(t)-p-2} d t+\varepsilon \Delta \int_{a r}^{b r} \frac{t^{l(t)} d t}{t^{p+1}(t+r)}\right. \\
& +\frac{\Delta}{r^{p+1}} \int_{a}^{b} \frac{\left|(\tau r)^{l(\tau r)}-(\tau r)^{l(r)}\right|}{\tau^{p+1}(\tau+1)} d \tau+\frac{\Delta}{l(r)-p}(a r)^{l(r)-p} \frac{1}{r} \\
& \left.+\frac{\Delta}{p+1-l(r)}(b r)^{l(r)-p-1}\right\} \\
& \leq \frac{r^{p+1}}{\sin \frac{\delta}{2}}\left\{\frac{K}{r} K_{1}^{-1}(a r)^{l(a r)-p}+K K_{2}^{-1}(b r)^{l(b r)-p-1}\right. \\
& +\varepsilon \Delta r^{-p-1} \int_{a}^{b} \frac{(\tau r)^{l(\tau r)} d \tau}{\tau^{p+1}(1+\tau)}+\varepsilon \Delta r^{-p-1} \int_{a}^{b} \frac{(\tau r)^{l(r)} d \tau}{\tau^{p+1}(1+\tau)} \\
& \left.+\Delta K_{1}^{-1} r^{-p-1} a^{K_{1}} r^{l(r)}+\Delta K_{2}^{-1} r^{-p-1} b^{-K_{2}} r^{l(r)}\right\} \\
& \leq \csc \frac{\delta}{2}\left\{K K_{1}^{-1} a^{-p}(1+\varepsilon)(a r)^{l(r)}+K K_{2}^{-1} b^{-p-1}(1+\varepsilon)(b r)^{l(r)}\right. \\
& \left.+\varepsilon \Delta(2+\varepsilon) \int_{a}^{b} \frac{(\tau r)^{l(r)} d \tau}{\tau^{p+1}(1+\tau)}+\varepsilon r^{l(r)}+\varepsilon r^{l(r)}\right\} \\
& \leq r^{l(r)} \csc \frac{\delta}{2}\left\{K K_{1}^{-1} a^{K_{1}}(1+\varepsilon)+K K_{2}^{-1} b^{-K_{2}}(1+\varepsilon)\right. \\
& \left.+\varepsilon \Delta(2+\varepsilon) \int_{0}^{\infty} \frac{\tau^{l(r)-p-1} d \tau}{1+\tau}+2 \varepsilon\right\} \\
& \leq \varepsilon r^{l(r)} \csc \frac{\delta}{2}\left\{2(1+\varepsilon)+\Delta(2+\varepsilon) \frac{\pi}{\sin \pi(l(r)-p)}+2 \varepsilon\right\} \\
& \leq \varepsilon r^{l(r)} \csc \frac{\delta}{2}\left\{6+3 \Delta \pi \max \left[\csc \pi K_{1}, \csc \pi K_{2}\right]\right\}=\varepsilon A r^{l(r)} \csc \frac{\delta}{2} .
\end{aligned}
$$

The integral

$$
I(z)=\int_{0}^{\infty} \frac{t^{l(r)} d t}{t^{p+1}(t-z)}
$$


can be easily evaluated using the Residue Calculus:

$$
I(z)=-\frac{\pi e^{-i \pi(l(r)-p)}}{\sin \pi(l(r)-p)} \cdot \operatorname{res}_{t=z} \frac{t^{l(r)-p-1}}{t-z}=-\frac{\pi e^{-i \pi(l(r)-p)}}{\sin \pi(l(r)-p)} z^{l(r)-p-1} .
$$

Thus we get for $\left\{\delta<\arg z<2 \pi<2 \pi-\delta,|z|>r_{1}(\varepsilon)\right\}$ the inequality

$$
\left|\ln f(z)-\frac{\pi \Delta}{\sin \pi(l(r)-p)} e^{-i \pi(l(r)-p)} z^{l(r)}\right| \leq \varepsilon A r^{l(r)} \csc \frac{\delta}{2},
$$

that is, for $\delta<\varphi<2 \pi-\delta$ we get uniformly the following:

$$
\begin{aligned}
\ln f\left(r e^{i \varphi}\right) & =\frac{\pi \Delta}{\sin \pi l(r)} e^{i l(r)(\varphi-\pi)} r^{l(r)}+o\left(r^{l(r)}\right), \\
\ln \left|f\left(r e^{i \varphi}\right)\right| & =\pi \Delta \frac{\cos l(r)(\varphi-\pi)}{\sin \pi l(r)} r^{l(r)}+o\left(r^{l(r)}\right), \\
\arg f\left(r e^{i \varphi}\right) & =\pi \Delta \frac{\sin l(r)(\varphi-\pi)}{\sin \pi l(r)} r^{l(r)}+o\left(r^{l(r)}\right) .
\end{aligned}
$$

In the case when $l(r)=\rho(r) \rightarrow \rho, p<\rho<p+1$, that is, $l(r)$ is a proximate order, these formulas can be rewritten as

$$
\begin{array}{r}
\ln f\left(r e^{i \varphi}\right)=\frac{\pi \Delta}{\sin \pi \rho} e^{i \rho(\varphi-\pi)} r^{\rho(r)}+o\left(r^{\rho(r)}\right) \\
\ln \left|f\left(r e^{i \varphi}\right)\right|=\pi \Delta \frac{\cos \rho(\varphi-\pi)}{\sin \pi \rho} r^{\rho(r)}+o\left(r^{\rho(r)}\right) \\
\arg f\left(r e^{i \varphi}\right)=\pi \Delta \frac{\sin \rho(\varphi-\pi)}{\sin \pi \rho} r^{\rho(r)}+o\left(r^{\rho(r)}\right)
\end{array}
$$

Now we estimate $\ln M(r, f)$. We cannot use for this purpose the estimate $\left(5.2_{2}\right)$ because $\left(5.2_{2}\right)$ is known for $\delta<\varphi<2 \pi-\delta$ only. For this reason we use a more rough estimate. Inequality (3.5) and (5.1') imply

$$
\ln M(r, f)<C(p)(-1)^{p} \ln f(-r)
$$

Using $\left(5.2_{2}\right)$ we get

$$
\frac{\pi \Delta}{|\sin \pi l(r)|} r^{l(r)}+o\left(r^{l(r)}\right)<\ln M(r, f)<C(p) \frac{\pi \Delta}{|\sin \pi l(r)|} r^{l(r)}+o\left(r^{l(r)}\right)
$$

It is clear that for $p=0$ we have

$$
\ln M(r, f)=\ln f(-r)=\frac{\pi \Delta}{\sin \pi l(r)} r^{l(r)}+o\left(r^{l(r)}\right)
$$

Let us compute the Nevanlinna characteristic for the function $f(z)$, defined by (5.1). Note that if $\psi(r) \rightarrow \infty$ as $r \rightarrow \infty$, then $\{\psi(r)+o(\psi(r))\}^{+}=\{\psi(r)\}^{+}+$ $o(\psi(r))$. If, in addition, an estimate of $o(\psi(r))$ in the left-hand side is uniform with respect to some parameter, then the same is true for $o(\psi(r))$ in the right-hand side. The function $r^{l(r)}=R(r)$ satisfies the conditions of Theorem 7.4 (Chapter 1) for an arbitrary $k>1$. In fact, $R(k r) \leq(1+o(1)) k^{l(r)} R(r) \leq(1+o(1)) k^{\eta_{2}} R(r)$ and $T(r, f) \leq \ln M(r, f)=O(R(r))$. Using the formula (7.15) from Chapter 1 and 
$\left(5.2_{2}\right)$, we get

$$
\begin{aligned}
T(r, f) & =m(r, f)=\frac{1}{2 \pi} \int_{0}^{2 \pi} \ln ^{+}\left|f\left(r e^{i \varphi}\right)\right| d \varphi \\
& =r^{l(r)} \frac{\Delta}{2} \int_{0}^{2 \pi}\left\{\frac{\cos l(r)(\varphi-\pi)}{\sin \pi l(r)}\right\}^{+} d \varphi+o\left(r^{l(r)}\right) .
\end{aligned}
$$

An elementary computation shows that for $p<\lambda<p+1$ we have

$$
I_{1}(\lambda)=\frac{1}{2} \int_{0}^{2 \pi}\left\{\frac{\cos \lambda(\varphi-\pi}{\sin \pi \lambda}\right\}^{+} d \varphi= \begin{cases}\frac{p+|\sin \pi \lambda|}{\lambda|\sin \pi \lambda|}, & \text { if } p<\lambda<p+\frac{1}{2} \\ \frac{p+1}{\lambda|\sin \pi \lambda|}, & \text { if } p+\frac{1}{2} \leq \lambda<p+1\end{cases}
$$

Hence

$$
T(r, f)=\Delta I_{1}(l(r)) r^{l(r)}+o\left(r^{l(r)}\right) .
$$

Taking into account the condition 2) for $l(r)$ we get that for $r \geq r_{0}$ the value of $I_{1}(l(r))$ is bounded below and bounded above by positive constants. Therefore the function $f(z)$ has order $\eta_{2}$. The lower order of $f(z)$ is equal to $\eta_{1}$. If $l(r)=\rho(r) \rightarrow \rho$ as $r \rightarrow \infty$, then the relation (5.4) can be written as

$$
T(r, f)=\Delta I_{1}(\rho) r^{\rho(r)}+o\left(r^{\rho(r)}\right) .
$$

It is clear that $n(r, f) \equiv 0$ and $N(r, f) \equiv 0$. We find $m\left(r, \frac{1}{f}\right)$ using a similar argument:

$$
\begin{aligned}
m\left(r, \frac{1}{f}\right) & =r^{l(r)} \frac{\Delta}{2} \int_{0}^{2 \pi}\left\{-\frac{\cos l(r)(\varphi-\pi)}{\sin \pi l(r)}\right\}^{+} d \varphi+o\left(r^{l(r)}\right) \\
& =\Delta I_{2}(l(r)) r^{l(r)}+o\left(r^{l(r)}\right)
\end{aligned}
$$

where

$$
\begin{aligned}
I_{2}(\lambda) & =\frac{1}{2} \int_{0}^{2 \pi}\left\{-\frac{\cos \lambda(\varphi-\pi)}{\sin \pi \lambda}\right\}^{+} d \varphi \\
& = \begin{cases}\frac{p}{\lambda|\sin \pi \lambda|}, & \text { if } p<\lambda<p+\frac{1}{2}, \\
\frac{p+1-|\sin \pi \lambda|}{\lambda|\sin \pi \lambda|}, & \text { if } p+\frac{1}{2} \leq \lambda<p+1 .\end{cases}
\end{aligned}
$$

Let us compute $N\left(r, \frac{1}{f}\right)$. Choose an arbitrary $\varepsilon>0$. Let $r_{0}(\varepsilon)$ be such that $\left|n(r, 0)-\Delta r^{l(r)}\right|<\varepsilon r^{l(r)}$ for $r>r_{0}(\varepsilon)$. Select $a, 0<a<1$ to be so small that $a^{\eta_{1}}<\eta_{1} \varepsilon$. For $r>r_{1}(\varepsilon, a) \geq a^{-1} r_{0}(\varepsilon)$ and $a \leq k \leq 1$ the inequality $\mid(k r)^{l(r)}-$ 
$(k r)^{l(r)} \mid<\varepsilon(k r)^{l(r)}$ holds. We have the following estimate

$$
\begin{aligned}
& \left|N\left(r, \frac{1}{f}\right)-\Delta \int_{a r}^{r} t^{l(r)-1} d t\right| \leq \int_{1}^{a r} \frac{n\left(t, \frac{1}{f}\right)}{t} d t \\
& +\left|\int_{a r}^{r} \frac{n\left(t, \frac{1}{f}\right)-\Delta t^{l(t)}}{t} d t\right|+\left|\Delta \int_{a r}^{r} \frac{t^{l(t)}-t^{l(r)}}{t} d t\right| \\
\leq & K \int_{1}^{a r} t^{l(t)-1} d t+\varepsilon \int_{a r}^{r} t^{l(t)-1} d t+\Delta \int_{a}^{r} \frac{\left|(\tau r)^{l(\tau r)}-(\tau r)^{l(r)}\right|}{\tau} d \tau \\
\leq & K \int_{1}^{a r} t^{l(t)-1} d t+\varepsilon(1+\varepsilon) \int_{a}^{1} \frac{(\tau r)^{l(r)}}{\tau} d \tau+\Delta \varepsilon \int_{a}^{1} \frac{(\tau r)^{l(r)}}{\tau} d \tau \\
= & K \int_{1}^{a r} t^{l(t)-1} d t+\varepsilon(1+\Delta+\varepsilon) r^{l(r)} \int_{a}^{1} \tau^{l(r)-1} d \tau \\
\leq & K \int_{1}^{a r} t^{l(t)-1} d t+\varepsilon \frac{1+\Delta+\varepsilon}{l(r)} r^{l(r)} .
\end{aligned}
$$

On the other hand,

$$
\begin{aligned}
\limsup _{r \rightarrow \infty} \frac{\int_{1}^{a r} t^{l(t)-1} d t}{r^{l(r)}} & \leq \limsup _{r \rightarrow \infty} \frac{\frac{d}{d r} \int_{1}^{a r} t^{l(t)-1} d t}{\frac{d}{d r} r^{l(r)}} \\
& =\limsup _{r \rightarrow \infty} a^{l(r)} \frac{(a r)^{l(a r)}}{(a r)^{l(r)}\left(l(r)+l^{\prime}(r) r \ln r\right)}=\frac{a^{\eta_{1}}}{\eta_{1}}<\varepsilon .
\end{aligned}
$$

Since

$$
\int_{a r}^{r} t^{l(r)-1} d t=\frac{r^{l(r)}}{l(r)}\left(1-a^{l(r)}\right)
$$

for $r \geq r_{2} \geq r_{1}(\varepsilon, a)$ we have

$$
\left|N\left(r, \frac{1}{f}\right)-\frac{\Delta r^{l(r)}}{l(r)}\right| \leq \varepsilon r^{l(r)}\left(K+\Delta+\frac{1+\Delta+\varepsilon}{\eta_{1}}\right) .
$$

Thus

$$
N\left(r, \frac{1}{f}\right)=\frac{\Delta}{l(r)} r^{l(r)}+o\left(r^{l(r)}\right) .
$$

Let $a \neq 0, \infty$, and let $E_{\delta}(r)$ be the set $\bigcup_{k}\{|l(r)(\varphi-\pi)-\pi(k+1 / 2)|<\delta\} \cup[0, \delta] \cup$ $[2 \pi-\delta, 2 \pi]$, where the union is taken over those integer $k$ for which $-l(r)-1 / 2 \leq$ $k \leq l(r)-1 / 2$ (if $p=0$, it can happen that there are no such $k$ ). It is easy to see that mes $E_{\delta}(r) \leq A \delta$, where $A$ is a constant which does not depend on $r$. From $\left(5.2_{2}\right)$ we get that for $r \geq r_{0}$ and $\varphi \in[0,2 \pi] \backslash E_{\delta}(r)$ we have

$$
\ln ^{+} \frac{1}{\left|f\left(r e^{i \varphi}\right)-a\right|}=O(1) .
$$

Hence by Theorem 7.4 from Chapter 1 and the Remark 1 following it, we get

$$
m(r, a, f)=o\left(r^{l(r)}\right) .
$$


The First Fundamental Theorem together with (5.7) and (5.4) implies

$$
N(r, a, f)=T(r, f)+o\left(r^{l(r)}\right)=\Delta I_{1}(l(r)) r^{l(r)}+o\left(r^{l(r)}\right)
$$

We already proved an estimate for $\ln M(r, f)$, but it is sharp for $p=0$ only. Now we shall get a sharp estimate in the case when $l(r)=\rho(r) \rightarrow \rho$ as $r \rightarrow \infty$, that is, when $l(r)$ is a proximate order. We shall complement $\left(5.3_{2}\right)$ by the following asymptotic inequality:

$$
\ln \left|f\left(r e^{i \varphi}\right)\right| \leq \pi \Delta \frac{\cos \rho(\varphi-\pi)}{\sin \pi \rho} r^{\rho(r)}+o\left(r^{\rho(r)}\right)
$$

as $r \rightarrow \infty$, uniformly with respect to $\varphi, 0 \leq \varphi \leq 2 \pi$.

Denote by $D_{\delta}$ the domain $\{|z|>1,|\arg z|<\delta\}$, where $0<\delta<\frac{\pi}{2} \min \left(1, \frac{1}{\rho}\right)$. Choose an arbitrarily small $\omega>0$ and denote by $\mu$ the number

$$
\mu=\frac{\cos \rho(\delta-\pi)}{\cos \rho \delta}+\omega \frac{\sin \pi \rho}{\pi \Delta \cos \rho \delta} .
$$

Consider in the domain $D_{\delta}$ the function

$$
\psi_{\delta}(z)=f(z)[f(-z)]^{-\mu} .
$$

For the ray $\arg z=\delta$ we have the following estimate

$$
\begin{aligned}
& \ln \left|\psi_{\delta}\left(r e^{i \delta}\right)\right|=\ln \left|f\left(r e^{i \delta}\right)\right|-\mu \ln \left|f\left(r e^{i(\delta+\pi)}\right)\right| \\
& \quad=\pi \Delta \frac{\cos \rho(\delta-\pi)}{\sin \pi \rho} r^{\rho(r)}-\mu \pi \Delta \frac{\cos \rho \delta}{\sin \pi \rho} r^{\rho(r)}+o\left(r^{\rho(r)}\right)=-\omega r^{\rho(r)}+o\left(r^{\rho(r)}\right) .
\end{aligned}
$$

An analogous estimate takes place for the ray $\arg z=-\delta$. Therefore the function $\psi_{\delta}(z)$ is bounded on the boundary $D_{\delta}$ with $\infty$ excluded:

$$
\left|\psi_{\delta}(z)\right| \leq M, z \in \partial D_{\delta} \backslash\{\infty\} .
$$

Consider $\rho^{\prime}$ satisfying $\rho<\rho^{\prime}<\frac{\pi}{2 \delta}$, and an arbitrary number $\xi>0$. The function $\psi_{\delta}(z) \exp \left\{-\xi z^{\rho^{\prime}}\right\}$ is continuous in the closure of $D_{\delta}$ and in the closure of $D_{\delta}$ in the extended complex plane, if we define its value at $\infty$ as 0 . In fact, for $|\varphi| \leq \delta$ we have

$$
\begin{aligned}
& \ln \left|\psi_{\delta}\left(r e^{i \varphi}\right) e^{-\xi r^{\rho^{\prime}}} e^{i \varphi \rho^{\prime}}\right| \leq \ln M(r, f)-\mu \ln \left|f\left(r e^{i(|\varphi|+\pi)}\right)\right|-\xi r^{\rho^{\prime}} \cos \varphi \rho^{\prime} \\
& \quad \leq O\left(r^{\rho(r)}\right)-\mu \pi \Delta \frac{\cos \rho|\varphi|}{\sin \pi \rho} r^{\rho(r)}+o\left(r^{\rho(r)}\right)-\xi r^{\rho^{\prime}} \cos \delta \rho^{\prime} \\
& \quad=O\left(r^{\rho(r)}\right)-\xi r^{\rho} \cos \delta \rho^{\prime} \rightarrow-\infty \text { as } r \rightarrow \infty .
\end{aligned}
$$

Hence we may use for $\psi_{\delta}(z) \exp \left(-\xi z^{\rho^{\prime}}\right)$ the principle of maximum modulus. On the other hand, for $z \in D_{\delta}$ we have $\left|\exp \left(-\xi z^{\rho^{\prime}}\right)\right|<1$. Hence

$$
\left|\varphi_{\delta}(z) \exp \left(-\xi z^{\rho^{\prime}}\right)\right| \leq M
$$

for $z \in D_{\delta}$. Passing in this inequality to the limit as $\xi \rightarrow 0$, we get that for $z \in D_{\delta}$ the inequality $\left|\psi_{\delta}(z)\right| \leq M$ holds. For $|\varphi| \leq \delta$ we have

$$
\begin{aligned}
\ln \left|f\left(r e^{i \varphi}\right)\right| & =\mu \ln \left|f\left(r e^{i(|\varphi|+\pi)}\right)\right|+\ln \left|\psi_{\delta}\left(r e^{i \varphi}\right)\right| \\
& \leq \mu \pi \Delta \frac{\cos \rho|\varphi|}{\sin \pi \rho} r^{\rho(r)}+o\left(r^{\rho(r)}\right)+\ln M \leq \frac{\mu \pi \Delta}{\sin \pi \rho} r^{\rho(r)}+o\left(r^{\rho(r)}\right) .
\end{aligned}
$$


Let $\varepsilon>0$ be an arbitrarily small positive number. Choose $\delta>0$ and $\omega>0$ in such a way that for $0 \leq \varphi \leq \delta$ and $2 \pi-\delta \leq \varphi \leq 2 \pi$ the inequality

$$
\varepsilon+\Delta \pi \frac{\cos \rho(\varphi-\pi)}{\sin \pi \rho}>\Delta \pi \frac{\cos \rho \pi}{\sin \pi \rho}>\frac{\mu \pi \Delta}{\sin \pi \rho}-\varepsilon
$$

holds.

Then by (5.10) we get that for $0 \leq \varphi \leq \delta$ and $2 \pi-\delta \leq \varphi \leq 2 \pi$ the inequality

$$
\ln \left|f\left(r e^{i \varphi}\right)\right| \leq \Delta \pi \frac{\cos \rho(\varphi-\pi)}{\sin \pi \rho} r^{\rho(r)}+2 \varepsilon r^{\rho(r)}+o\left(r^{\rho(r)}\right)
$$

holds uniformly with respect to $\varphi$.

By $\left(5.3_{2}\right)$ this inequality holds also for $\delta \leq \varphi \leq 2 \pi-\delta$. Thus, we have proved the inequality (5.9).

The relation $\left(5.3_{2}\right)$ implies that

$$
\ln \left|f\left(r e^{i \varphi_{0}}\right)\right|=\frac{\pi \Delta}{\sin \pi \rho} r^{\rho(r)}+o\left(r^{\rho(r)}\right),
$$

where $\varphi_{0}=\pi$ for even $p$ and $\varphi_{0}=\pi+\frac{\pi}{\rho}$ for odd $p$. Together with (5.9) it implies

$$
\ln M(r, f)=\frac{\pi \Delta}{\sin \pi \rho} r^{\rho(r)}+o\left(r^{\rho(r)}\right) .
$$

The function $f(z)$ given by (5.1) will be used in several constructions. First, let us mention the following theorem.

THEOREM 5.1. Let $\rho(r)$ be a proximate order, $\rho(r) \rightarrow \rho$ as $r \rightarrow \infty, L(r)=$ $r^{\rho(r)-\rho}$. There exists a function $F(z)$ analytic in $\{|\arg z|<\pi\}$ taking positive values on the positive semi-axis, and such that for every $\delta$ satisfying $0<\delta<\pi$ the condition

$$
F\left(r e^{i \varphi}\right)=(1+o(1)) L(r)<r \rightarrow \infty
$$

holds uniformly with respect to $\varphi$ in $\{|\varphi|<\pi-\delta\}$.

Indeed, let $f(z)$ be a canonical product with positive zeros, such that $n(r, 0) \sim$ $\pi^{-1} r^{1 / 2+\rho(r)-\rho}$. The desired function $F(z)$ can be chosen in $\{|\arg z|<\pi\}$ as

$$
F(z)=\frac{\ln f(-z)}{\sqrt{z}},
$$

where the branch of $\sqrt{z}$ is chosen in such a way that $\sqrt{r}>0$ for $r>0$. Since $f(-r)>1$, then $F(r)>0$. Taking into account the inequality $\left(5.3_{1}\right)$ we get $(|\varphi|<\pi-\delta)$

$$
F\left(r e^{i \varphi}\right)=\frac{e^{i \frac{\varphi}{2}} r^{\frac{1}{2}+\rho(r)-\rho}+o\left(r^{\frac{1}{2}+\rho(r)-\rho}\right)}{e^{i \frac{\varphi}{2}} r^{\frac{1}{2}}}=L(r)+o(L(r)) .
$$

Let $f(z)$ be an entire function of order $\rho, 0<\rho<\infty$. The inequality $T(r, f) \leq$ $\ln ^{+} M(r, f) \leq 3 T(2 r, f)$ (see (7.1) in Chapter 1) implies that under the condition

$$
0<\liminf _{r \rightarrow \infty} \frac{\ln M(r, f)}{r^{\rho}} \leq \limsup _{r \rightarrow \infty} \frac{\ln M(r, f)}{r^{\rho}}<\infty
$$

we have

$$
0<\liminf _{r \rightarrow \infty} \frac{T(r, f)}{r^{\rho}} \leq \limsup _{r \rightarrow \infty} \frac{T(r, f)}{r^{\rho}}<\infty
$$


and vice versa. It is natural to ask whether the existence of the limit

$$
\lim _{r \rightarrow \infty} \frac{\ln M(r, f)}{r^{\rho}} \neq 0, \infty
$$

implies the existence of the limit

$$
\lim _{r \rightarrow \infty} \frac{T(r, f)}{r^{\rho}} \neq 0, \infty
$$

and vice versa. It turns out that in both cases the answer to this question is negative. Let us give the corresponding examples.

EXAMPLE 1. We give an example showing that the existence of the limit in (5.12) does not imply the existence of the limit in (5.13). First we let $0<\rho<1 / 2$. We construct two proximate orders, $\rho_{1}(r)$ and $\rho_{2}(r)$, satisfying for $r>r_{0}$ the inequality

$$
\rho-\frac{\ln \ln r}{\ln r} \leq \rho_{j}(r) \leq \rho, j=1,2 .
$$

Observe that $r^{\rho-\frac{\ln \ln r}{\ln r}}=\frac{r^{\rho}}{\ln r}$. Choose $\rho_{j}(r)$ in such a way that

$$
\max \left(\rho_{1}(r), \rho_{2}(r)\right) \equiv \rho \text {; }
$$

the inequality $\rho_{1}\left(r_{n}\right)=\rho-\frac{\ln \ln r_{n}}{\ln r_{n}}$ is satisfied for some sequence $r_{n} \rightarrow \infty$, and $\rho_{1}\left(r_{n}^{\prime}\right)=\rho_{2}\left(r_{n}^{\prime}\right)=\rho$ for some sequence $r_{n}^{\prime} \rightarrow \infty$. Let $V_{j}(z)$ be canonical products of genus 0 with positive zeros satisfying $n\left(r, 0, V_{j}\right) \sim r^{\rho_{j}(r)}$. Consider the function

$$
f(z)=V_{1}(z)+V_{2}(-z) .
$$

It is clear that $|f(\bar{z})|=|f(z)|$, therefore it suffices to get suitable estimates for $|f(z)|$ in the upper half-plane $\{\operatorname{Im} z \geq 0\}$. We shall use the relations $\left(5.3_{2}\right)$ and (5.11). Since

$$
M(r, f) \leq 2 \max _{j=1,2} M\left(r, V_{j}\right),
$$

we have

$$
\ln M(r, f) \leq \frac{\pi}{\sin \pi \rho} r^{\rho}+o\left(r^{\rho}\right) .
$$

On the other hand,

$$
V_{j}(r) \leq \exp \left(\pi \cot \rho \pi r^{\rho_{j}(r)}+o\left(r^{\rho_{j}(r)}\right)\right)
$$

and

$$
V_{j}(-r)=\exp \left(\frac{\pi}{\sin \rho \pi} r^{\rho_{j}(r)}+o\left(r^{\rho_{j}(r)}\right)\right) .
$$

Denote by $s(r)$ the function which is equal to 1 at those values of $r$ for which $\rho_{1}(r)=\rho$, and is equal to -1 when $\rho_{1}(r)<\rho$ (at such points $\rho_{2}(r)=\rho$ ). It is easy to see that

$$
|f(-s(r) r)|=\exp \left\{\frac{\pi}{\sin \pi \rho} r^{\rho}+o\left(r^{\rho}\right)\right\},
$$

therefore

$$
\ln M(r, f)=\frac{\pi}{\sin \pi \rho} r^{\rho}+o\left(r^{\rho}\right),
$$

and (5.12) is satisfied. For $r=r_{n}$ we have

$$
T\left(r_{n}, V_{1}\right) \leq \ln M\left(r_{n}, V_{1}\right)=O\left(r_{n}^{\rho_{1}\left(r_{n}\right)}\right)=O\left(\frac{r_{n}^{\rho}}{\ln r_{n}}\right) .
$$


Taking into account $\left(5.4^{\prime}\right)$, we get

$$
\begin{aligned}
T\left(r_{n}, f\right) & =T\left(r_{n}, V_{2}\right)+O\left(\frac{r_{n}^{\rho}}{\ln r_{n}}\right) \\
& =I_{1}(\rho) r_{n}^{\rho_{2}\left(r_{n}\right)}+o\left(r_{n}^{\rho_{2}\left(r_{n}\right)}\right)+O\left(\frac{r_{n}^{\rho}}{\ln r_{n}}\right) \\
& =I_{1}(\rho) r_{n}^{\rho}+o\left(r_{n}^{\rho}\right)=\frac{1}{\rho} r_{n}^{\rho}+o\left(r_{n}^{\rho}\right) .
\end{aligned}
$$

For $r=r_{n}^{\prime}$ and $\delta \leq \varphi \leq \pi / 2-\delta$ or $\pi / 2+\delta \leq \varphi \leq \pi-\delta, \delta>0$, by $\left(5.3_{2}\right)$ we have

$$
\ln \left|f\left(r_{n}^{\prime} e^{i \varphi}\right)\right|=\pi \frac{\max \{\cos \rho(\pi-\varphi), \cos \rho \varphi\}}{\sin \pi \rho} r_{n^{\prime}}^{\rho}+o\left(r_{n^{\prime}}^{\rho}\right) .
$$

Using Theorem 7.4 from Chapter 1 and Remark 2 following it, we get

$$
\begin{aligned}
T\left(r_{n}^{\prime}, f\right) & =m\left(r_{n}^{\prime}, f\right)=\frac{{r_{n}^{\prime}}_{n}^{\rho}}{\sin \pi \rho} \int_{0}^{\pi} \max \{\cos \rho(\pi-\varphi), \cos \rho \varphi\} d \varphi+o\left({r^{\prime}}_{n}^{\rho}\right) \\
& =\frac{2 r_{n}^{\prime \rho}}{\sin \pi \rho} \int_{0}^{\pi / 2} \cos \rho \varphi d \varphi+o\left(r_{n}^{\prime \rho}\right)=\frac{2 \sin \frac{\pi \rho}{2}}{\rho \sin \pi \rho} r_{n}^{\prime \rho}+o\left(r_{n}^{\prime \rho}\right) \\
& =\frac{1}{\rho} \sec \frac{\pi \rho}{2} r_{n}^{\prime \rho}+o\left({r_{n}^{\prime}}_{n}^{\rho}\right) .
\end{aligned}
$$

Hence

$$
\liminf _{r \rightarrow \infty} \frac{T(r, f)}{r^{\rho}} \leq \frac{1}{\rho}<\frac{1}{\rho} \sec \frac{\pi \rho}{2} \leq \limsup _{r \rightarrow \infty} \frac{T(r, f)}{r^{\rho}},
$$

that is, the limit in (5.13) does not exist. If instead of $f(z)$ we consider the functions $f\left(z^{p}\right), p=2,3,4, \ldots$, by Theorem 6.6 from Chapter 1 and the obvious inequality $\ln M\left(r, f\left(z^{p}\right)\right)=\ln M\left(r^{p}, f(z)\right)$, we get analogous examples for each $\rho, 0<\rho<\frac{p}{2}$, that is, for each $\rho, 0<\rho<\infty$.

EXAMPLE 2. Now we show that the existence of the limit in (5.13) does not imply the existence of the limit in (5.12). The idea of this example is similar to the idea of Example 1, but the construction is somewhat more technical. Let $0<\rho<1 / 2$. Consider the equation

$$
\begin{aligned}
F(x, y)= & y \cot \rho \pi\left\{\frac{y}{x \cos \rho \pi}-1\right\} \\
& +\sqrt{x^{2}\left[1+\cot ^{2} \rho \pi\left\{\frac{y}{x \cos \rho \pi}-1\right\}^{2}\right]-y^{2}} \\
& -2 \sin \frac{\rho \pi}{2} \sqrt{1+\cot ^{2} \rho \pi\left\{\frac{y}{x \cos \rho \pi}-1\right\}^{2}}=0,
\end{aligned}
$$

where the radical denotes the arithmetical value of the square root, $x$ and $y$ are real variables. It is easy to check that $F(1,1)=0, F_{x}^{\prime}(1,1)=\tan \frac{\rho \pi}{2}, F_{y}^{\prime}(1,1)=\tan \frac{\rho \pi}{2}$. Well-known theorems on implicit functions imply that the equation (5.14) defines in a sufficiently small neighborhood of $(1,1)$ an univalent continuously differentiable decreasing function $y=y(x), y(1)=1,\left|y^{\prime}(x)-(-1)\right|<1 / 2$.

Let $L_{1}(r)=1+\eta \sin \ln \ln (e+r), r \geq 0$, where $\eta>0$ is chosen to be so small that the interval $[1-\eta, 1+\eta]$ is contained in the interval where the function $y(x)$ 
with the properties described above is defined. Let $L_{2}(r)=y\left(L_{1}(r)\right)$. We may assume that $\eta$ is so small that for $1-\eta \leq x \leq+\eta$ the inequality

$$
\cos \pi \rho<y(x) / x<\sec \pi \rho
$$

is satisfied. Hence, for all $r>0$ the inequality

$$
\cos \pi \rho<L_{2}(r) / L_{1}(r)<\sec \pi \rho
$$

holds. Denote by $V_{j}(r)$ the canonical product of genus 0 with positive zeros, for which $n\left(r, 0, V_{j}\right) \sim r^{\rho} L_{j}(r), j=1,2$. It is easy to verify that the functions $\rho_{j}(r)$ defined by the equations $r^{\rho_{j}(r)}=r^{\rho} L_{j}(r)$ are proximate orders, and $\rho_{j}(r) \rightarrow \rho$ as $r \rightarrow \infty$. As in Example 1 we let

$$
f(z)=V_{1}(z)+V_{2}(-z) .
$$

Using the same argument as in Example 1, we get

$$
\begin{aligned}
\ln M(r, f) & =\max _{j=1,2} \ln M\left(r, V_{j}\right)+o\left(r^{\rho}\right) \\
& =\frac{\pi}{\sin \pi \rho} r^{\rho} \max \left\{L_{1}(r), L_{2}(r)\right\}+o\left(r^{\rho}\right) .
\end{aligned}
$$

Hence

$$
\begin{aligned}
\limsup _{r \rightarrow \infty} \frac{\ln M(r, f)}{\sin \pi \rho}= & \frac{\pi}{\sin \pi \rho} \limsup _{r \rightarrow \infty} \max \left\{L_{1}(r), L_{2}(r)\right\} \\
& =\frac{\pi}{\sin \pi \rho} \max \{(1+\eta), y(1-\eta)\} \geq(1+\eta) \frac{\pi}{\sin \pi \rho}, \\
\liminf _{r \rightarrow \infty} \frac{\ln M(r, f)}{r^{\rho}} & =\frac{\pi}{\sin \pi \rho} \liminf _{r \rightarrow \infty} \max \left\{L_{1}(r), L_{2}(r)\right\} \\
& =\frac{\pi}{\sin \pi \rho} \max \{(1-\eta), y(1+\eta)\}<\frac{\pi}{\sin \pi \rho} .
\end{aligned}
$$

Thus the limit in (5.12) does not exist. Denote by $\varphi(r), 0<\varphi(r)<\pi$, the (unique) solution of the equation

$$
L_{1}(r) \cos \rho(\pi-\varphi)=L_{2}(r) \cos \rho \varphi .
$$

The existence of the solution follows from (5.15). From $\left(5.3_{2}\right)$ it is easy to get that for each positive $\delta$ we have

$$
\ln \left|f\left(r e^{i \varphi}\right)\right|= \begin{cases}\pi r^{\rho} L_{2}(r) \frac{\cos \rho \varphi}{\sin \rho \pi}+o\left(r^{\rho}\right), & \delta \leq \varphi \leq \varphi(r)-\delta, \\ \pi r^{\rho} L_{1}(r) \frac{\cos \rho(\pi-\varphi)}{\sin \rho \pi}+o\left(r^{\rho}\right), & \varphi(r)+\delta \leq \varphi \leq \pi-\delta .\end{cases}
$$

Taking into account Theorem 7.4 from Chapter 1 and Remark 1 following it, we get

$$
\begin{aligned}
& T(r, f)=\frac{r^{\rho}}{\sin \pi \rho}\left\{L_{2}(r) \int_{0}^{\varphi(r)} \cos \rho \varphi+L_{1}(r) \int_{\varphi(r)}^{\pi} \cos \rho(\pi-\varphi) d \varphi\right\} \\
& +o\left(r^{\rho}\right)=\frac{r^{\rho}}{\rho \sin \pi \rho}\left\{L_{2}(r) \sin \rho \varphi(r)+L_{1}(r) \sin \rho(\pi-\varphi(r))\right\}+o\left(r^{\rho}\right) .
\end{aligned}
$$

Using (5.16) we get

$$
\tan \rho \varphi(r)=\cot \rho \pi\left(\frac{L_{2}(r)}{L_{1}(r) \cos \rho \pi}-1\right)
$$




$$
\begin{aligned}
\sin \rho \varphi(r) & =\frac{\cot \rho \pi\left(\frac{L_{2}(r)}{L_{1}(r) \cos \rho \pi}-1\right)}{\left\{1+\cot ^{2} \rho \pi\left(\frac{L_{2}(r)}{L_{1}(r) \cos \rho \pi}-1\right)^{2}\right\}^{\frac{1}{2}}}, \\
\sin \rho[\pi-\varphi(r)]= & \left\{1-\left[\frac{L_{2}(r)}{L_{1}(r)} \cos \rho \varphi(r)\right]^{2}\right\}^{\frac{1}{2}} \\
= & \frac{\left\{1+\cot ^{2} \rho \pi\left\{\frac{L_{2}(r)}{L_{1}(r) \cos \rho \pi}-1\right\}^{2}-\frac{L_{2}^{2}(r)}{L_{1}^{2}(r)}\right\}^{\frac{1}{2}}}{\left\{1+\cot ^{2} \rho \pi\left\{\frac{L_{2}(r)}{L_{1}(r) \cos \rho \pi}-1\right\}^{2}\right\}^{\frac{1}{2}}} .
\end{aligned}
$$

Taking into account (5.14) and the definition of the function $L_{2}(r)=y\left(L_{1}(r)\right)$, we get

$$
L_{2}(r) \sin \rho \varphi(r)+L_{1}(r) \sin \rho(\pi-\varphi(r)) \equiv 2 \sin \frac{\rho \pi}{2} .
$$

Now we can rewrite (5.17) as

$$
T(r, f)=\frac{r^{\rho}}{\rho \cos \frac{\rho \pi}{2}}+o\left(r^{\rho}\right),
$$

that is, the limit in (5.13) is defined. Considering the functions $f\left(z^{p}\right)$, as in Example 1 , we drop remove the restriction $\rho<1 / 2$.

It is worth mentioning that it is possible to construct examples of the type of Example 1 using another idea. We restrict our attention to the case $\rho=1$, because in this case the construction is simpler.

EXAMPLE $1^{\prime}$. We define an integer sequence $\left\{r_{k}\right\}$ using the following recurrent relation: $r_{1}=1, r_{k+1}=3 r_{k}^{2}$. Let $p_{k}=r_{k}^{2} ; \eta_{2 k}=1, k=0,1,2, \ldots$ Let $\eta_{2 k+1}=1$ if $r_{2 n-1} \leq 2 k+1<r_{2 n}$ and $\eta_{2 k+1}=0$ if $r_{2 n} \leq 2 k+1<r_{2 n+1}$. Let $\eta_{2 k}^{\prime}=0$ and $\eta_{2 k+1}^{\prime}=\eta_{2 k+1}$. Note that for integer $s \geq r_{k+1}$ we have

$$
\frac{p_{k}^{s}}{s !}<\frac{r_{k}^{2 s} e^{s}}{s^{s}} \leq\left(\frac{r_{k}^{2} e}{r_{k+1}}\right)^{s}=\left(\frac{e}{3}\right)^{s} \text {. }
$$

The function

$$
f(z)=\sum_{s=0}^{\infty} \eta_{s} \frac{z^{s}}{s !}
$$

has the desired properties. It is clear that

$$
\cosh r \leq M(r, f)=f(r) \leq e^{r} .
$$

Hence

$$
\lim _{r \rightarrow \infty} \frac{\ln M(r, f)}{r}=1 .
$$


If $|z|=p_{2 n-1}$ then (see (5.18)) the following inequality holds

$$
\begin{aligned}
\left|e^{z}-f(z)\right| & =\left|\sum_{s=0}^{\infty}\left(1-\eta_{s}\right) \frac{z^{s}}{s !}\right| \leq \sum_{s=0}^{r_{2 n-1}} \frac{|z|^{s}}{s !}+\sum_{s=r_{2 n}}^{\infty} \frac{|z|^{s}}{s !} \\
& \leq p_{2 n-1}^{r_{2 n-1}} \sum_{s=0}^{\infty} \frac{1}{s !}+\sum_{s=r_{2 n}}^{\infty}\left(\frac{e}{3}\right)^{s} \leq e p_{2 n-1}^{\sqrt{p_{2 n-1}}}+o(1) .
\end{aligned}
$$

Hence, for $r=p_{2 n-1}$ we have

$$
f\left(r e^{i \varphi}\right)=\exp \left(r e^{i \varphi}\right)+O(\exp (\sqrt{r} \ln r))
$$

and

$$
T(r, f)=T\left(r, e^{z}\right)+O(\sqrt{r} \ln r)=\frac{r}{\pi}+O(\sqrt{r} \ln r) .
$$

Thus

$$
\liminf _{r \rightarrow \infty} \frac{T(r, f)}{r} \leq \frac{1}{\pi}
$$

In a similar manner, for $|z|=p_{2 n}$ we get

$$
\begin{aligned}
|f(z)-\cosh z| & =\left|\sum_{s=0}^{\infty} \eta_{s}^{\prime} \frac{z^{s}}{s !}\right| \\
& \leq \sum_{s=0}^{r_{2 n}} \frac{|z|^{s}}{s !}+\sum_{s=r_{2 n+1}}^{\infty} \frac{|z|^{s}}{s !} \leq p_{2 n}^{r_{2 n}} e+o(1)=e p_{2 n}^{\sqrt{p_{2 n}}}+o(1) .
\end{aligned}
$$

Therefore for $r=p_{2 n}$ we have

$$
\begin{gathered}
f\left(r e^{i \varphi}\right)=\cosh \left(r e^{i \varphi}\right)+O(\exp (\sqrt{r} \ln r)), \\
T(r, f)=T(r, \cosh z)+O(\sqrt{r} \ln r)=\frac{2 r}{\pi}+O(\sqrt{r} \ln r) .
\end{gathered}
$$

(Here we have used the result of Exercise 2 to Theorem 6.1 from Chapter 1.) It follows that

$$
\limsup _{r \rightarrow \infty} \frac{T(r, f)}{r} \geq \frac{2}{\pi}
$$

Combining (5.19) and (5.20) we conclude that the limit of $T(r, f) / r$ as $r \rightarrow \infty$ does not exist.

$2^{\circ}$. Now we consider entire functions of integer order $\rho>0$ with positive zeros, such that $n(r, 0) \sim \Delta r^{\rho(r)}$, where $0<\Delta<\infty, \rho(r)$ is a proximate order, $\rho(r) \rightarrow \rho$ as $r \rightarrow \infty$. We shall use the notation from the proof of Theorem 4.3. In this case $f(z)=f_{1}(z) f_{2}(z)$, where

$$
\begin{gathered}
f_{1}(z)=\exp \left\{z^{\rho}\left(c_{\rho}+\frac{1}{\rho} \sum_{0<a_{\nu} \leq r} a_{\nu}^{-\rho}\right)\right\} \\
f_{2}(z)=e^{c_{\rho-1} z^{\rho-1}+\cdots+c_{1} z} \prod_{0<a_{\nu} \leq r} E\left(\frac{z}{a_{\nu}}, \rho-1\right) \prod_{a_{\nu}>r} E\left(\frac{z}{a_{\nu}}, \rho\right) \\
=e^{c_{\rho-1} z^{\rho-1}+\cdots+c_{1} z} f_{3}(z) .
\end{gathered}
$$


We consider the function $\ln f(z)$ in the angle $\{0<\delta \leq \arg z \leq 2 \pi-\delta\}$, $\ln f(0)=\ln f_{2}(0)=\ln f_{1}(0)=\ln f_{3}(0)=0$. We have

$$
\left|\ln f(z)-\ln f_{1}(z)\right|=\left|\ln f_{2}(z)\right| \leq O\left(|z|^{\rho-1}\right)+\left|\ln f_{3}(z)\right| .
$$

On the other hand, the function $\ln f_{3}(z)$ can be represented in the form (cf. computation in part $1^{\circ}$ of this section):

$$
\begin{aligned}
\ln f_{3}(z)= & -z^{\rho} \int_{0}^{r} n(t, 0) \frac{d t}{t^{\rho}(t-z)}-z^{\rho} n(r, 0) \int_{r}^{\infty} \frac{d t}{t^{\rho}(t-z)} \\
& -z^{\rho+1} \int_{r}^{\infty}\{n(t, 0)-n(r, 0)\} \frac{d t}{t^{\rho+1}(t-z)}=-z^{\rho} \int_{0}^{r} n(t, 0) \frac{d t}{t^{\rho}(t-z)} \\
& -\frac{z^{\rho}}{\rho r^{\rho}} n(r, 0)-z^{\rho+1} \int_{r}^{\infty} n(t, 0) \frac{d t}{t^{\rho+1}(t-z)} .
\end{aligned}
$$

For $\delta<\varphi<2 \pi-\delta$ we have $\left|t-r e^{i \varphi}\right| \geq \sin \frac{\delta}{2}(t+r)$, hence

$$
\left|\ln f_{3}\left(r e^{i \varphi}\right)\right| \leq \frac{1}{\sin \frac{\delta}{2}}\left\{r^{\rho} \int_{0}^{r} \frac{n(t, 0)}{t^{\rho}(t+r)} d t+\frac{1}{\rho} n(r, 0)+r^{\rho+1} \int_{r}^{\infty} \frac{n(t, 0)}{t^{\rho+1}(t+r)} d t\right\} .
$$

Estimating the sum in the braces we, as in the proof of Theorem 4.3, get

$$
\left|\ln f_{3}\left(r e^{i \varphi}\right)\right|=O\left(r^{\rho(r)}\right)
$$

uniformly with respect to $\varphi, \delta<\varphi<2 \pi-\delta$. Hence

$$
\begin{aligned}
& \ln f(z)=z^{\rho}\left(c_{\rho}+\frac{1}{\rho} \sum_{0<a_{\nu} \leq r} a_{\nu}^{-\rho}\right)+O\left(r^{\rho(r)}\right), \\
& \ln |f(z)|=\operatorname{Re}\left\{z^{\rho}\left(c_{\rho}+\frac{1}{\rho} \sum_{0<a_{\nu} \leq r} a_{\nu}^{-\rho}\right)\right\}+O\left(r^{\rho(r)}\right), \\
& \arg f(z)=\operatorname{Im}\left\{z^{\rho}\left(c_{\rho}+\frac{1}{\rho} \sum_{0<a_{\nu} \leq r} a_{\nu}^{-\rho}\right)\right\}+O\left(r^{\rho(r)}\right) .
\end{aligned}
$$

Note, that in order to prove these relations, we used the relation $n(r, 0)=O\left(r^{\rho(r)}\right)$ only. Consider first the case when the integral

$$
\int_{1}^{\infty} t^{\rho(t)-\rho-1} d t
$$

is divergent. In this case we introduce the notation $(r \geq 2)$

$$
L_{1}(r)=\int_{1}^{r} t^{\rho(t)-\rho-1} d t, r^{\rho_{1}(r)}=r^{\rho} L_{1}(r)
$$

It is easy to check that $\rho_{1}(r)$ is a proximate order,

$$
\rho_{1}(r) \rightarrow \rho, r^{\rho}=o\left(r^{\rho_{1}(r)}\right), r^{\rho(r)}=o\left(r^{\rho_{1}(r)}\right) \text { as } r \rightarrow \infty .
$$

Using $\left(5.22_{1}\right)$ we get

$$
\ln f(z)=z^{\rho} \frac{1}{\rho} \sum_{0<a_{\nu} \leq r} a_{\nu}^{-\rho}+o\left(r^{\rho_{1}(r)}\right) .
$$


By the l'Hôpital rule, we get

$$
\begin{aligned}
\frac{1}{\rho} \sum_{0<a_{\nu} \leq r} a_{\nu}^{-\rho} & =\frac{1}{\rho} \int_{0}^{r} \frac{d n(t, 0)}{t^{\rho}}=\frac{n(r, 0)}{\rho r^{\rho}}+\int_{0}^{r} \frac{n(t, 0)}{t^{\rho+1}} d t \\
& =O\left(r^{\rho(r)-\rho}\right)+\Delta(1+o(1)) \int_{1}^{r} t^{\rho(t)-\rho-1} d t=\Delta(1+o(1)) L_{1}(r) .
\end{aligned}
$$

Hence

$$
\begin{gathered}
\ln f\left(r e^{i \varphi}\right)=\Delta e^{i \rho \varphi} r^{\rho_{1}(r)}+o\left(r^{\rho_{1}(r)}\right), \\
\ln \left|f\left(r e^{i \varphi}\right)\right|=\Delta r^{\rho_{1}(r)} \cos \rho \varphi+o\left(r^{\rho_{1}(r)}\right),
\end{gathered}
$$

and these estimates are uniform with respect to $\delta<\varphi<2 \pi-\delta$. Using Theorem 4.3 we get

$$
T(r, f)=m(r, f)=\frac{\Delta}{\pi} r^{\rho_{1}(r)}+o\left(r^{\rho_{1}(r)}\right) .
$$

By Theorem 7.4 from Chapter 1 we have

$$
\begin{gathered}
N(r, f)=0, m\left(r, \frac{1}{f}\right)=\frac{\Delta}{\pi} r^{\rho_{1}(r)}+o\left(r^{\rho_{1}(r)}\right), \\
N\left(r, \frac{1}{f}\right) \sim \frac{\Delta}{\rho} r^{\rho(r)}=o\left(r^{\rho_{1}(r)}\right),
\end{gathered}
$$

and for $a \neq 0, \infty$, we have

$$
m\left(r, \frac{1}{f-a}\right)=o\left(r^{\rho_{1}(r)}\right) .
$$

Hence, by the First Fundamental Theorem,

$$
N\left(r, \frac{1}{f-a}\right)=\frac{\Delta}{\pi} r^{\rho_{1}(r)}+o\left(r^{\rho_{1}(r)}\right) .
$$

It is worth mentioning that using the method from part $1^{\circ}$, it can be shown that if in $\left(5.24_{2}\right)$ we replace the equality sign by $\leq$, the obtained inequality will be valid everywhere in the complex plane.

EXERCISE. Using the infinite product representation of the entire function $1 / \Gamma(z)$, where $\Gamma(z)$ is the Euler gamma-function, namely

$$
\frac{1}{\Gamma(z)}=z e^{\gamma z} \sum_{n=1}^{\infty} E\left(-\frac{z}{n}, 1\right)
$$

$(\gamma=0,577 \ldots$ is the Euler-Mascheroni constant), find an asymptotic formula for $\Gamma(z)$ in the region $\{|\arg z|<\pi-\delta\}, \delta>0$ :

$$
\ln \Gamma(z)=z \ln z+o(r \ln r) .
$$

Now we suppose that the integral in (5.23) converges. Let

$$
\begin{gathered}
\rho_{2}(r)=\rho+(\ln r)^{-1} \ln \left\{\int_{r}^{\infty} t^{\rho(t)-\rho-1} d t\right\}, r>1, \\
r^{\rho_{2}(r)}=r^{\rho} \int_{r}^{\infty} t^{\rho(t)-\rho-1} d t=r^{\rho} L_{2}(r) .
\end{gathered}
$$


As before, we check that $r^{\rho(r)}=o\left(r^{\rho_{2}(r)}\right), r^{\rho_{2}(r)}=o\left(r^{\rho}\right)$. In the case under consideration the function $n(r, 0)$ belongs to the convergence class, and the function $f(z)$ can be represented as

$$
f(z)=e^{c_{\rho}^{\prime} z^{\rho}+\ldots} \prod_{\nu} E\left(\frac{z}{a_{\nu}}, \rho-1\right)=f_{4}(z) f_{2}(z),
$$

where $f_{2}(z)$ is defined as before, and

$$
f_{4}(z)=\exp \left\{z^{\rho}\left(c_{\rho}^{\prime}-\frac{1}{\rho} \sum_{\left|a_{\nu}\right|>r} a_{\nu}^{-\rho}\right)\right\} .
$$

The series $\sum_{\nu} a_{\nu}^{-\rho}$ converges since $n(r, 0)$ belongs to the convergence class.

The relation (5.21) implies that uniformly in $\varphi=\arg z$ within the angle $0<$ $\delta<\arg z<2 \pi-\delta$, the relation

$$
\left|\ln f\left(r e^{i \varphi}\right)-\ln f_{4}\left(r e^{i \varphi}\right)\right| \leq O\left(r^{\rho(r)}\right)+O\left(r^{\rho-1}\right)=o\left(r^{\rho_{2}(r)}\right) .
$$

holds. In the same way as before we get

$$
\begin{aligned}
-\frac{1}{\rho} \sum_{a_{\nu}>r} a_{\nu}^{-\rho} & =-\frac{1}{\rho} \int_{r}^{\infty} \frac{d n(t, 0)}{t^{\rho}}=\frac{1}{\rho} \frac{n(r, 0)}{r^{\rho}}-\int_{r}^{\infty} \frac{n(t, 0)}{t^{1+\rho}} d t \\
& =O\left(r^{\rho(r)-\rho}\right)-\Delta(1+o(1)) \int_{r}^{\infty} t^{\rho(t)-\rho-1} d t=-\Delta(1+o(1)) L_{2}(r) .
\end{aligned}
$$

By (5.25) we get

$$
\ln f\left(r e^{i \varphi}\right)=e^{i \rho \varphi} c_{\rho}^{\prime} r^{\rho}-\Delta e^{i \rho \varphi} r^{\rho_{2}(r)}+o\left(r^{\rho_{2}(r)}\right) .
$$

If $c_{\rho}^{\prime}=0$, then

$$
\begin{gathered}
\ln f\left(r e^{i \varphi}\right)=-\Delta e^{i \rho \varphi} r^{\rho_{2}(r)}+o\left(r^{\rho_{2}(r)}\right), \\
\ln \left|f\left(r e^{i \varphi}\right)\right|=-\Delta \cos \rho \varphi r^{\rho_{2}(r)}+o\left(r^{\rho_{2}(r)}\right) .
\end{gathered}
$$

If $c_{\rho}^{\prime} \neq 0$, then

$$
\begin{array}{r}
\ln f\left(r e^{i \varphi}\right)=e^{i \rho \varphi} c_{\rho}^{\prime} r^{\rho}+o\left(r^{\rho}\right), \\
\ln \left|f\left(r e^{i \varphi}\right)\right|=\left|c_{\rho}^{\prime}\right| \cos \left(\rho \varphi+\arg c_{\rho}^{\prime}\right) r^{\rho}+o\left(r^{\rho}\right) .
\end{array}
$$

As in the previous example, it can be shown that if we replace in $\left(5.26_{2}\right)$ and $\left(5.27_{2}\right)$ the equality sign by $\leq$, then the obtained inequality will be valid everywhere in the complex plane.

For Nevanlinna characteristics we get the same formulas as in the case when the integral (5.23) diverges, but with $\rho_{1}(r)$ replaced by $\rho_{2}(r)$ in the case when $c_{\rho}^{\prime}=0$, and with $\rho_{1}(r)$ replaced by $\rho$ and $\Delta$ replaced by $\left|c_{\rho}^{\prime}\right|$ in the case when $c_{\rho}^{\prime} \neq 0$. To apply (4.5) we take into account

$$
c_{\rho}=c_{\rho}^{\prime}-\frac{1}{\rho} \sum_{\nu} a_{\nu}^{-\rho} .
$$

Finally, we consider the case $\rho=0$. Let $\rho(r)$ be a proximate order satisfying $\rho(r) \rightarrow 0$ as $r \rightarrow \infty, r^{\rho(r)}$ is monotone when $r \geq r_{0}$ and goes to $\infty$ as $r \rightarrow \infty$. Consider a canonical product of genus 0 with positive zeros, such that $n(r, 0) \sim$ 
$\Delta r^{\rho(r)}, 0<\Delta<\infty$. Without loss of generality we may assume that all zeros are greater than 1 . It is easy to check that

$$
\begin{gathered}
N(r, 0)=\int_{1}^{r} \frac{n(t, 0)}{t} d t=\Delta(1+o(1)) \int_{1}^{r} t^{\rho(t)-1} d t=\Delta(1+o(1)) L_{1}(r), \\
L_{1}\left(\frac{r}{2}\right) \sim L_{1}(r), r^{\rho(r)}=o\left(L_{1}(r)\right) \text { as } r \rightarrow \infty .
\end{gathered}
$$

Let $\delta<\varphi<2 \pi-\delta$. We apply $\left(5.1^{\prime}\right)$ to $f(z)$. We get

$$
\begin{aligned}
\ln f\left(r e^{i \varphi}\right)-N(r, 0) & =-z \int_{1}^{\infty} \frac{n(t, 0) d t}{t(t-z)}-\int_{1}^{r} \frac{n(t, 0)}{t} d t \\
& =-\int_{1}^{r} \frac{n(t, 0)}{t-z} d t-z \int_{r}^{\infty} \frac{n(t, 0)}{t(t-z)} d t, \\
\left|\ln f\left(r e^{i \varphi}\right)-N(r, 0)\right| \leq & \csc \frac{\delta}{2}\left\{\int_{1}^{r} \frac{n(t, 0)}{t+r} d t+r \int_{r}^{\infty} \frac{n(t, 0)}{t(t+r)} d t\right\} \\
\leq & \csc \frac{\delta}{2}\left\{n(r, 0)+r \int_{r}^{\infty} \frac{n(t, 0)}{t^{2}} d t\right\} \\
\leq & \Delta(1+o(1)) \csc \frac{\delta}{2}\left\{r^{\rho(r)}+r \int_{r}^{\infty} t^{\rho(t)-2} d t\right\} \\
& =2 \Delta(1+o(1)) \csc \frac{\delta}{2} r^{\rho(r)}=o(N(r, 0)) .
\end{aligned}
$$

Therefore uniformly with respect to $\varphi$ satisfying $\delta<\varphi<2 \pi-\delta$ the relations

$$
\begin{gathered}
\ln f\left(r e^{i \varphi}\right)=N(r, 0)+o(N(r, 0)), \\
\ln \left|f\left(r e^{i \varphi}\right)\right|=N(r, 0)+o(N(r, 0)), \\
\arg f\left(r e^{i \varphi}\right)=o(N(r, 0)) .
\end{gathered}
$$

hold. It is clear that $\ln M(r, f)=\ln f(-r)=N(r, 0)+o(N(r, 0))$. Since

$$
N(r, 0) \leq T(r, f) \leq \ln M(r, f),
$$

we get

$$
T(r, f)=N(r, 0)+o(N(r, 0)), m\left(r, \frac{1}{f}\right)=o(N(r, 0)) .
$$

It is easy to check that relations (5.30) take place even without the requirement of positivity of zeros. In fact, let

$$
f(z)=\prod_{\nu}\left(1-\frac{z}{a_{\nu}}\right), \check{f}(z)=\prod_{\nu}\left(1-\frac{z}{\left|a_{\nu}\right|}\right) .
$$

It is clear that $M(r, f) \leq M(r, \check{f})=\check{f}(-r)$ and $N(r, 0, f)=N(r, 0, \check{f})$. From (5.29) and (5.30) we get

$$
\begin{aligned}
N(r, 0, f) & \leq T(r, f) \leq \ln M(r, \check{f})=N(r, 0, \check{f})+o(N(r, 0, \check{f})) \\
& =N(r, 0, f)+o(N(r, 0, f)),
\end{aligned}
$$

that is, (5.30) remains true. Using the condition of positivity of zeros of $f(z)$, using $\left(5.28_{2}\right)$, the First Fundamental Theorem, and Theorem 7.4 from Chapter 1, we get

$$
m(r, a, f)=o(N(r, 0)), N(r, a)=(1+o(1)) N(r, 0), a \neq 0, \infty .
$$


$3^{\circ}$. Finally we consider the entire Mittag-Leffler function

$$
E_{\rho}(z)=\sum_{k=0}^{\infty} \frac{z^{k}}{\Gamma\left(1+\frac{k}{\rho}\right)}, 0<\rho<\infty .
$$

Using Stirling's formula, it is easy to verify that this power series has an infinite radius of convergence. The function $E_{\rho}(z)$ is a generalization of the exponential function $e^{z}$, which corresponds to the case $\rho=1$. For $\rho=1 / 2$ we have $E_{1 / 2}(z)=$ $\cosh \sqrt{z}$. We are going to find some asymptotic relations for $E_{\rho}(z)$. To do so we need the Hankel integral representation for $\Gamma$-function. Let $s=\sigma+i \tau$ be an arbitrary complex number. Denote by $L(\theta, a), \frac{\pi}{2}<\theta \leq \pi, 0<a<\infty$, the boundary of the region $\{|\arg z|<\theta,|z|>a\}$, parameterized in the negative direction.

Consider the integral

$$
F(s)=\frac{1}{2 \pi i} \int_{L(\theta, a)} e^{t} t^{-s} d t
$$

where by $t^{-s}$ we mean the principal value of the power function. It is easy to check that the integral (5.32) converges absolutely and uniformly with respect to $s$, for $|s| \leq R$, where $R$ is an arbitrarily large number. Using the Morera theorem for the function $F(s)$ and changing the order of integration, we conclude that $F(s)$ is an entire function. The integral (5.32) does not depend on $a$, since the integral of $e^{t} t^{-s}$ over the boundary of the region $\left\{|\arg z|<\theta, a_{1}<|z|<a_{2}\right\}$ is equal to zero by the Cauchy theorem. The integral (5.32) does not depend on $\theta, \frac{\pi}{2}<\theta \leq \pi$, either. In fact, let $\frac{\pi}{2}<\theta_{1}<\theta_{2} \leq \pi$. The difference

$$
\int_{L\left(\theta_{1}, a\right)} e^{t} t^{-s} d t-\int_{L\left(\theta_{2}, a\right)} e^{t} t^{-s} d t
$$

does not depend on $a$, and is equal to the integral of $e^{t} t^{-s}$ taken over the boundary of the set $\left\{\theta_{1}<|\arg z|<\theta_{2},|z|>a\right\}$. On the other hand, on the $\operatorname{arcs}\left\{\theta_{1}<|\arg z|<\right.$ $\left.\theta_{2},|z|=a\right\}$ the integrand satisfies the estimate $\left|e^{t} t^{-s}\right| \leq e^{a \cos \theta_{1}} a^{-\sigma} e^{\theta_{2} \tau}=o\left(a^{-1}\right)$ as $a \rightarrow+\infty$. Therefore the difference (5.33) approaches zero as $a \rightarrow+\infty$. Since the difference (5.33) does not depend on $a$, it should be equal to 0 .

Thus, in (5.32) we do not need to indicate the dependence of $F(s)$ on $\theta$ and $a$.

Now we let $0<s=\sigma<1$. We have

$$
\begin{aligned}
F(\sigma)= & \frac{1}{2 \pi i} \int_{L(\pi, a)} e^{t} t^{-\sigma} d t=\frac{e^{\pi i \sigma}}{2 \pi i} \int_{-\infty}^{-a} e^{x}|x|^{-\sigma} d x \\
& +\frac{1}{2 \pi} \int_{-\pi}^{\pi} e^{a e^{i \varphi}} a^{1-\sigma} e^{i(1-\sigma) \varphi} d \varphi+\frac{e^{-\pi i \sigma}}{2 \pi i} \int_{-a}^{-\infty} e^{x}|x|^{-\sigma} d x \\
= & \frac{\sin \pi \sigma}{\pi} \int_{a}^{\infty} e^{-x} x^{-\sigma} d x+a^{1-\sigma} \frac{1}{2 \pi} \int_{-\pi}^{\pi} e^{a e^{i \varphi}+i(1-\sigma) \varphi} d \varphi
\end{aligned}
$$

Letting $a \rightarrow 0$ and using the formula for the complement of the $\Gamma$-function, we get

$$
F(\sigma)=\frac{\sin \pi \sigma}{\pi} \int_{0}^{\infty} e^{-x} x^{-\sigma} d x=\frac{\sin \pi \sigma}{\pi} \Gamma(1-\sigma)=\frac{1}{\Gamma(\sigma)} .
$$


Since the entire functions $F(s)$ and $1 / \Gamma(s)$ coincide on the interval $0<\sigma=s<1$, they are identically equal. We get from (5.32) the Hankel formula

$$
\frac{1}{\Gamma(s)}=\frac{1}{2 \pi i} \int_{L(\theta, a)} e^{t} t^{-s} d t, \frac{\pi}{2}<\theta \leq \pi, 0<a<\infty .
$$

We introduce notation $\alpha=1 / \rho$. Let $|z|<1, a>1$, and $\frac{\pi}{2}<\theta \leq \pi$. Using (5.31) and (5.34) we get

$$
\begin{aligned}
E_{\rho}(z) & =\sum_{k=0}^{\infty} \frac{z^{k}}{2 \pi i} \int_{L(\theta, a)} e^{t} t^{-\alpha k-1} d t \\
& =\frac{1}{2 \pi i} \int_{L(\theta, a)} \frac{e^{t}}{t}\left\{\sum_{k=0}^{\infty}\left(\frac{z}{t^{\alpha}}\right)^{k}\right\} d t=\frac{1}{2 \pi i} \int_{L(\theta, a)} \frac{e^{t} t^{\alpha-1}}{t^{\alpha}-z} d t .
\end{aligned}
$$

The integral in the right-hand side of (5.35) defines for $|z|<a^{\alpha}$ an analytic function of $z$, which coincides with $E_{\rho}(z)$. Since $a$ can be chosen to be arbitrarily large, we get the following integral representation for $E_{\rho}(z)$ :

$$
E_{\rho}(z)=\frac{1}{2 \pi i} \int_{L(\theta, a)} \frac{e^{t} t^{\alpha-1}}{t^{\alpha}-z} d t, \frac{\pi}{2}<\theta \leq \pi, a>|z|^{\rho} .
$$

First we suppose that $1 / 2<\rho<\infty$. Assume that $|z|>1$. Let $z=r e^{i \varphi}$. Suppose that $|\varphi| \leq \frac{\pi}{2 \rho}+\delta, 0<\delta<\pi-\frac{\pi}{2 \rho}$. Then

$$
\begin{aligned}
E_{\rho}\left(r e^{i \varphi}\right) & =\frac{1}{2 \pi i} \int_{L\left(\pi,(2 r)^{\rho}\right)} \frac{e^{t} t^{\alpha-1}}{t^{\alpha}-z} d t \\
& =\frac{1}{2 \pi i} \int_{L(\pi, 1)} \frac{e^{t} t^{\alpha-1}}{t^{\alpha}-z} d t+\operatorname{res}_{t=z^{\rho}} \frac{e^{t} t^{\alpha-1}}{t^{\alpha}-z} .
\end{aligned}
$$

In writing this representation we take into account the fact that the function

$$
e^{t} t^{\alpha-1}\left(t^{\alpha}-z\right)^{-1}
$$

has in the region $\left\{|\arg t|<\pi, 1<|t|<(2 r)^{\rho}\right\}$ only one pole at $t=r^{\rho} e^{i \rho \varphi}$. Since for $|\varphi| \leq \frac{\pi}{2 \rho}+\sigma, t^{\alpha}=|t|^{\alpha} e^{ \pm i \pi \alpha}, r \geq 1,|t| \geq 1$, the absolute value $\left|\frac{t^{\alpha}}{z}-1\right|$ is bounded below by a positive constant $m$, then

$$
\left|\frac{1}{2 \pi i} \int_{L(\pi, 1)} \frac{e^{t} t^{\alpha-1}}{t^{\alpha}-z} d t\right| \leq \frac{1}{|z|} \frac{1}{2 \pi m} \int_{1}^{\infty} e^{-x} x^{\alpha-1} d x<\frac{1}{|z|} \frac{\Gamma(\alpha)}{2 \pi m} .
$$

Therefore we get, using (5.37):

$$
E_{\rho}(z)=O\left(\frac{1}{|z|}\right)+\rho e^{z^{\rho}},|\arg z| \leq \frac{\pi}{2 \rho}+\delta<\frac{\pi}{\rho} .
$$

If $\frac{\pi}{2 \rho}+\delta \leq|\varphi| \leq \pi$, we choose $\theta$ satisfying $\frac{\pi}{2}<\theta<\frac{\pi}{2}+\rho \delta$. Then

$$
E_{\rho}\left(r e^{i \varphi}\right)=\frac{1}{2 \pi i} \int_{L\left(\theta,(2 r)^{\rho}\right)} \frac{e^{t} t^{\alpha-1} d t}{t^{\alpha}-z}=\frac{1}{2 \pi i} \int_{L(\theta, 1)} \frac{e^{t} t^{\alpha-1}}{t^{\alpha}-z} d t,
$$

since under the conditions listed above we have $|\rho \varphi|>\theta, \rho \varphi-2 k \pi \rho \leq \rho \pi-2 k \pi \rho<$ $-\theta$ and $\rho \varphi+2 k \pi \geq-\pi \rho+2 k \pi>\theta$, for $k=1,2,3, \ldots$; and the integrand does not have poles in the region $\{|\arg t| \leq \theta,|t|>1\}$. In the same way as before we 
show that the integral in the right-hand side of (5.39) is $O\left(|z|^{-1}\right)$. Thus we get the following uniform estimate in the complex plane:

$$
E_{\rho}(z)= \begin{cases}\rho e^{z^{\rho}}+O\left(\frac{1}{|z|}\right), & |\arg z| \leq \frac{\pi}{2 \rho}, \\ O\left(\frac{1}{|z|}\right), & \pi \geq|\arg z| \geq \frac{\pi}{2 \rho} .\end{cases}
$$

In some cases, for $\pi \geq|\arg z| \geq \frac{\pi}{2 \rho}+\delta, \delta>0$, a more precise estimate for $E_{\rho}(z)$ than $O\left(\frac{1}{|z|}\right)$ is needed. To get it, we transform the integral (5.39) in the following way:

$$
\begin{aligned}
\frac{1}{2 \pi i} \int_{L(\theta, 1)} \frac{e^{t} t^{\alpha-1}}{t^{\alpha}-z} d t & =-\frac{1}{z} \frac{1}{2 \pi i} \int_{L(\theta, 1)} e^{t} t^{\alpha-1} d t+\frac{1}{z} \frac{1}{2 \pi i} \int_{L(\theta, 1)} \frac{e^{t} t^{2 \alpha-1}}{t^{\alpha}-z} d t \\
& =-\frac{1}{z} \frac{1}{\Gamma(1-\alpha)}+O\left(\frac{1}{|z|^{2}}\right) .
\end{aligned}
$$

We took into account (5.34). Hence

$$
E_{\rho}(z)=-\frac{1}{z} \frac{1}{\Gamma(1-\alpha)}+O\left(\frac{1}{|z|^{2}}\right) \text { for } \pi \geq|\arg z| \geq \frac{\pi}{2 \rho}+\delta .
$$

When $\rho>1 / 2$ and $\rho \neq 1$, then $1 / \Gamma(1-\alpha) \neq 0$. If $\rho=1$, the formula $\left(5.40^{\prime}\right)$ gives a more rough estimate, because $E_{1}(z)=e^{z}$. If $\rho=1 / 2$, then, as we noted above, $E_{\rho}(z)=\cosh \sqrt{z}$, and we easily get

$$
E_{1 / 2}(z)=\frac{1}{2} e^{z^{1 / 2}}+\omega(z),|\omega(z)| \leq \frac{1}{2} .
$$

If $0<\rho<1 / 2$, the investigation of the function is somewhat more complicated. Let $\left|\varphi_{0}\right| \leq \pi$. We find $\theta=\theta\left(\varphi_{0}\right), \pi \geq \theta>\frac{\pi}{2}$, and a small number $\varepsilon\left(\varphi_{0}\right)>0$, such that for $\left|\varphi-\varphi_{0}\right| \leq \varepsilon\left(\varphi_{0}\right)$ and an arbitrary integer $k$, the numbers $\rho \varphi+2 k \pi \rho$ are not equal to $\theta$ or $-\theta$. Then, as before, we get

$$
E_{\rho}(z)=\frac{1}{2 \pi i} \int_{L(\theta, 1)} \frac{e^{t} t^{\alpha-1} d t}{t^{\alpha}-z}+\sum \operatorname{res} \frac{e^{t} t^{\alpha-1}}{t^{\alpha}-z},
$$

where the sum of residues is taken over all poles of the function $e^{t} t^{\alpha-1}\left(t^{\alpha}-z\right)^{-1}$ which are in the region $\{|\arg t|<\theta,|t|>1\}$, that is, at the points $t=r^{\rho} e^{i \rho(\varphi+2 k \pi)}$, where the integers $k$ are such that $-\theta<\rho \varphi+2 k \pi \rho<\theta$. The integral in (5.42) can be estimated using the same method as before. Therefore

$$
E_{\rho}(z)=O\left(\frac{1}{|z|}\right)+\sum_{|\varphi+2 k \pi|<\frac{\theta}{\rho}} \rho e^{z^{\rho} e^{i 2 k \pi \rho}},|\arg z| \leq \pi .
$$

This estimate is uniform with respect to $\left|\varphi-\varphi_{0}\right| \leq \varepsilon\left(\varphi_{0}\right)$. Using the HeineBorel lemma, we cover the interval $[-\pi, \pi]$ by finitely many intervals $\left\{\left|\varphi-\varphi_{\mu}\right| \leq\right.$ $\left.\varepsilon\left(\varphi_{\mu}\right)\right\}, \mu=1,2, \ldots, \mu_{0}$, and conclude that the estimate (5.43) is uniform in the complex plane. It is necessary to take into account that $\theta$ in (5.43) will take, depending on $\varphi=\arg z, \mu_{0}$ different values. If we restrict our attention to the main term of the decomposition, we get from (5.43) that

$$
E_{\rho}(z)=(1+o(1)) \rho e^{z^{\rho}},
$$

uniformly with respect to $\arg z$ for $|\arg z| \leq \pi-\delta, \delta>0$.

Since the coefficients in (5.31) are positive, then $(\rho>0)$ :

$$
M\left(r, E_{\rho}(z)\right)=E_{\rho}(r)=(1+o(1)) \rho e^{r^{\rho}}, T\left(r, E_{\rho}\right) \leq r^{\rho}+O(1) .
$$


Applying Theorem 7.4 from Chapter 1, we get

$$
T\left(r, E_{\rho}\right)=\left\{\begin{array}{l}
\frac{1}{\pi \rho} r^{\rho}+o\left(r^{\rho}\right), \quad \frac{1}{2} \leq \rho<\infty, \\
\frac{\sin \pi \rho}{\pi \rho} r^{\rho}+o\left(r^{\rho}\right), 0<\rho \leq \frac{1}{2} .
\end{array}\right.
$$

Taking into account $\left(5.40^{\prime}\right)$, we conclude

$$
\begin{gathered}
m\left(r, \frac{1}{E_{\rho}}\right)=\left\{\begin{array}{l}
o\left(r^{\rho}\right), \rho \neq 1, \\
\frac{r}{\pi}, \rho=1,
\end{array}\right. \\
m\left(r, \frac{1}{E_{\rho}-a}\right)=o\left(r^{\rho}\right), 0<\rho<\infty, a \neq 0, \infty
\end{gathered}
$$

By the First Fundamental Theorem we get

$$
\begin{gathered}
N\left(r, 0, E_{\rho}\right)= \begin{cases}T\left(r, E_{\rho}\right)+o\left(r^{\rho}\right), & \rho \neq 1, \\
0, & \rho=1,\end{cases} \\
N\left(r, a, E_{\rho}\right)=T\left(r, E_{\rho}\right)+o\left(r^{\rho}\right), a \neq 0, \infty ; 0<\rho<\infty .
\end{gathered}
$$




\section{CHAPTER 3}

\section{The second fundamental theorem}

\section{Lemma on the logarithmic derivative}

The second fundamental theorem is the deepest and the most important result of the value distribution theory. As we shall see later, the classical Picard and Borel theorems are its corollaries. To prove the second fundamental theorem we need some auxiliary results, which we are going to present now.

THEOREM 1.1. Let $f(z)$ be a meromorphic function satisfying $f(0)=1$. For all $r$ and $R$ satisfying $1<r<R<\infty$ and all $\alpha, 0<\alpha<1$, the inequality

$$
\begin{aligned}
m\left(r, \frac{f^{\prime}}{f}\right) \leq & \frac{1}{\alpha} \ln ^{+} T(R, f)+\max \left(2, \frac{1}{\alpha}\right)\left\{\ln ^{+} \frac{1}{R-r}+\ln R\right\} \\
& +\frac{1}{\alpha} \ln \frac{64}{1-\alpha}
\end{aligned}
$$

holds.

We need the following lemma.

LEMma 1.1. Let $f(x)$ and $g(x)$ be non-negative measurable functions on $[a, b]$, and let

$$
A=\int_{a}^{b} g(x) d x>0 .
$$

Then the inequality

$$
\frac{1}{A} \int_{a}^{b}\left\{\ln ^{+} f(x)\right\} g(x) d x \leq \ln ^{+}\left\{\frac{1}{A} \int_{a}^{b} f(x) g(x) d x\right\}+\ln 2
$$

holds.

Proof. Note that in the case $g(x) \equiv 1$ the inequality (1.2) becomes

$$
\frac{1}{b-a} \int_{a}^{b} \ln ^{+} f(x) d x \leq \ln ^{+}\left\{\frac{1}{b-a} \int_{a}^{b} f(x) d x\right\}+\ln 2 .
$$

To prove the lemma set

$$
m=\frac{1}{A} \int_{a}^{b}[f(x)]^{\wedge} g(x) d x, \varphi(x)=[f(x)]^{\wedge}-m .
$$

Note that $m \geq 1$. It is clear that

$$
\int_{a}^{b} \varphi(x) g(x) d x=0 .
$$


We have

$$
\begin{aligned}
& \frac{1}{A} \int_{a}^{b}\left\{\ln ^{+} f(x)\right\} g(x) d x=\frac{1}{A} \int_{a}^{b}\left\{\ln [f(x)]^{\wedge}\right\} g(x) d x \\
& \quad=\ln m+\frac{1}{A} \int_{a}^{b} \ln \left(1+\frac{\varphi(x)}{m}\right) g(x) d x \leq \ln m+\frac{1}{A} \int_{a}^{b} \frac{\varphi(x)}{m} g(x) d x=\ln ^{+} m
\end{aligned}
$$

Thus

$$
\begin{aligned}
\frac{1}{A} \int_{a}^{b} & \left\{\ln [f(x)]^{\wedge}\right\} g(x) d x \leq \ln ^{+}\left\{\frac{1}{A} \int_{a}^{b}[f(x)]^{\wedge} g(x) d x\right\} \\
& \leq \ln ^{+}\left\{\frac{1}{A} \int_{a}^{b}[f(x)+1] g(x) d x\right\}=\ln ^{+}\left\{\frac{1}{A} \int_{a}^{b} f(x) g(x) d x+1\right\} \\
& \leq \ln ^{+}\left\{\frac{1}{A} \int_{a}^{b} f(x) g(x) d x\right\}+\ln 2 .
\end{aligned}
$$

Proof. (Theorem 1.1) Note that the condition $f(0)=1$ implies (see $\left(6.8^{\prime}\right)$ from Chapter 1) that $T\left(r, \frac{1}{f}\right)=T(r, f)$, therefore we have

$$
m\left(r, \frac{1}{f}\right)+m(r, f) \leq 2 T(r, f), N(r ; 0, \infty) \leq 2 T(r, f) .
$$

Using the formula (2.5) from Chapter 1 for the disc $\{|z|<s\}, s>0$, and letting $p=1$ in this formula, we get

$$
\begin{aligned}
\frac{f^{\prime}(z)}{f(z)}= & \frac{1}{2 \pi} \int_{0}^{2 \pi} \ln \left|f\left(s e^{i \theta}\right)\right| \frac{2 s e^{i \theta}}{\left(s e^{i \theta}-z\right)^{2}} d \theta \\
& +\sum_{\left|a_{m}\right|<s} \frac{s^{2}-\left|a_{m}\right|^{2}}{\left(s^{2}-\bar{a}_{m} z\right)\left(z-a_{m}\right)}-\sum_{\left|b_{n}\right|<s} \frac{s^{2}-\left|b_{n}\right|^{2}}{\left(s^{2}-\bar{b}_{n} z\right)\left(z-b_{n}\right)},|z|<s .
\end{aligned}
$$

Denote by $\left\{c_{q}\right\}$ the set-theoretic sum of the sequences $\left\{a_{m}\right\}$ and $\left\{b_{n}\right\}$. Observe that

$$
\frac{1}{2 \pi} \int_{0}^{2 \pi}|\ln | f\left(s e^{i \theta}\right)|| d \theta=m\left(s, \frac{1}{f}\right)+m(s, f) \leq 2 T(s, f),
$$

and for $\left|c_{q}\right|<s$

$$
\left|\frac{s^{2}-\left|c_{q}\right|^{2}}{\left(s^{2}-\bar{c}_{q} z\right)\left(z-c_{q}\right)}\right| \leq \frac{s^{2}-\left|c_{q}\right|^{2}}{\left(s^{2}-\left|c_{q}\right| s\right)\left|z-c_{q}\right|}=\frac{s+\left|c_{q}\right|}{s} \cdot \frac{1}{\left|z-c_{q}\right|} \leq \frac{2}{\left|z-c_{q}\right|},
$$

we have

$$
\left|\frac{f^{\prime}(z)}{f(z)}\right| \leq \frac{4 s T(s, f)}{(s-|z|)^{2}}+2 \sum_{\left|c_{q}\right|<s} \frac{1}{\left|z-c_{q}\right|},|z|<s .
$$

Using the well-known inequality

$$
\left(\sum_{k} d_{k}\right)^{\alpha} \leq \sum_{k} d_{k}^{\alpha}, d_{k} \geq 0,0<\alpha<1,
$$


we get

$$
\begin{aligned}
\int_{0}^{2 \pi}\left|\frac{f^{\prime}\left(r e^{i \varphi}\right)}{f\left(r e^{i \varphi}\right)}\right|^{\alpha} d \varphi & \leq \int_{0}^{2 \pi}\left\{\frac{4 s T(s, f)}{(s-r)^{2}}+2 \sum_{\left|c_{q}\right|<s} \frac{1}{\left|r e^{i \varphi}-c_{q}\right|}\right\}^{\alpha} d \varphi \\
& \leq \int_{0}^{2 \pi}\left\{\frac{4^{\alpha} s^{\alpha} T^{\alpha}(s, f)}{(s-r)^{2 \alpha}}+2^{\alpha} \sum_{\left|c_{q}\right|<s} \frac{1}{\left|r e^{i \varphi}-c_{q}\right|^{\alpha}}\right\} d \varphi \\
& =2 \pi \frac{4^{\alpha} s^{\alpha} T^{\alpha}(s, f)}{(s-r)^{2 \alpha}}+2^{\alpha} \sum_{\left|c_{q}\right|<s} \int_{0}^{2 \pi} \frac{d \varphi}{\left|r e^{i \varphi}-c_{q}\right|^{\alpha}} .
\end{aligned}
$$

Since

$$
\begin{aligned}
\int_{0}^{2 \pi} \frac{d \varphi}{\left|r e^{i \varphi}-c_{q}\right|^{\alpha}} & =\int_{0}^{2 \pi} \frac{d \varphi}{\left.\left|r e^{i \varphi}-\right| c_{q}\right|^{\alpha}} \leq \int_{0}^{2 \pi} \frac{d \varphi}{|r \sin \varphi|^{\alpha}} \\
& =\frac{4}{r^{\alpha}} \int_{0}^{\pi / 2} \frac{d \varphi}{\sin ^{\alpha} \varphi} \leq \frac{4}{r^{\alpha}}\left(\frac{\pi}{2}\right)^{\alpha} \int_{0}^{\pi / 2} \frac{d \varphi}{\varphi^{\alpha}}=\frac{2 \pi}{r^{\alpha}(1-\alpha)},
\end{aligned}
$$

we get the inequality

$$
\int_{0}^{2 \pi}\left|\frac{f^{\prime}\left(r e^{i \varphi}\right)}{f\left(r e^{i \varphi}\right)}\right|^{\alpha} d \varphi \leq 2 \pi \frac{4^{\alpha} s^{\alpha} T^{\alpha}(s, f)}{(s-r)^{2 \alpha}}+2 \pi \frac{2^{\alpha} n(s ; 0, \infty)}{(1-\alpha) r^{\alpha}} .
$$

Now let $s=\frac{1}{2}(R+r)$. Observe that then

$$
r<s<R, s-r=R-s=\frac{1}{2}(R-r),
$$

and since the function $T(s, f)$ is non-decreasing, we get

$$
T(s, f) \leq T(R, f)
$$

By Lemma 7.1 from Chapter 1 we get

$$
n(s ; 0, \infty) \leq \frac{R}{R-s} N(R ; 0, \infty)=\frac{2 R}{R-r} N(R ; 0, \infty) \leq \frac{4 R}{R-r} T(R, f) .
$$

Thus we get $(R>r \geq 1)$

$$
\begin{aligned}
\frac{1}{2 \pi} \int_{0}^{2 \pi} & \left|\frac{f^{\prime}\left(r e^{i \varphi}\right)}{f\left(r e^{i \varphi}\right)}\right|^{\alpha} d \varphi \leq \frac{2^{4 \alpha} R^{\alpha} T^{\alpha}(R, f)}{(R-r)^{2 \alpha}}+\frac{2^{2+2 \alpha} R T(R, f)}{(1-\alpha)(R-r) r^{\alpha}} \\
& \leq \frac{16}{1-\alpha} T^{\wedge}(R, f)\left\{\frac{1}{R^{\alpha}}\left(1-\frac{r}{R}\right)^{-2 \alpha}+\frac{1}{r^{\alpha}}\left(1-\frac{r}{R}\right)^{-1}\right\} \\
& \leq \frac{32}{1-\alpha} T^{\wedge}(R, f) r^{-\alpha}\left(1-\frac{r}{R}\right)^{-(2 \alpha)^{\wedge}} .
\end{aligned}
$$

By Lemma 1.1 we have

$$
\begin{aligned}
m\left(r, \frac{f^{\prime}}{f}\right) & =\frac{1}{\alpha} \frac{1}{2 \pi} \int_{0}^{2 \pi} \ln ^{+}\left|\frac{f^{\prime}\left(r e^{i \varphi}\right)}{f\left(r e^{i \varphi}\right)}\right|^{\alpha} d \varphi \\
& \leq \frac{1}{\alpha}\left\{\ln ^{+}\left[\frac{1}{2 \pi} \int_{0}^{2 \pi}\left|\frac{f^{\prime}\left(r e^{i \varphi}\right)}{f\left(r e^{i \varphi}\right)}\right|^{\alpha} d \varphi\right]+\ln 2\right\},
\end{aligned}
$$

therefore

$$
m\left(r, \frac{f^{\prime}}{f}\right) \leq \frac{1}{\alpha} \ln ^{+}\left\{\frac{32}{1-\alpha} T^{\wedge}(R, f) r^{-\alpha}\left(1-\frac{r}{R}\right)^{-(2 \alpha)^{\wedge}}\right\}+\frac{1}{\alpha} \ln 2 .
$$


Using the inequality (4.3) from Chapter 1, we easily get the conclusion of the theorem.

Later we shall need an estimate for $m\left(r, \frac{f^{\prime}}{f}\right)$ in terms $T(r, f)$ rather than in terms of $T(R, f), R>r$. It is clear that we cannot get such an estimate taking the limit as $R \rightarrow r$ in (1.1). We shall use for this matter the following theorem of Borel-Nevanlinna.

Theorem 1.2 (Borel-Nevanlinna). Let $u(r)$ be a continuous, non-decreasing function on $\left[r_{0}, \infty\right)$, tending to $+\infty$ as $r \rightarrow \infty$. Let $\varphi(u)$ be a continuous positive non-increasing function on $\left[u_{0}, \infty\right), u_{0}=u\left(r_{0}\right)$, having zero limit as $u \rightarrow \infty$ and satisfying

$$
\int_{u_{0}}^{\infty} \varphi(u) d u<\infty .
$$

Then for all $r \geq r_{0}$ except, possibly, a set of finite measure the inequality

$$
u\{r+\varphi(u(r))\}<u(r)+1
$$

is satisfied.

Proof. Denote by $E$ the closed subset of $\left[r_{0}, \infty\right)$ on which the inequality (1.7) is not satisfied, that is, the inequality

$$
u\{r+\varphi(u(r))\} \geq u(r)+1
$$

holds. Denote $E \cap[r, \infty)$ by $E(r)$. We assume that for all $r \geq r_{0}$ the sets $E(r)$ are non-empty, otherwise the theorem is trivial. Let $r_{1}=\min r, r \in E ; r_{1} \geq r_{0}$. Let $r_{1}^{\prime}$ be the least value of $r$ satisfying $u(r)=u\left(r_{1}\right)+1$; it is clear that $r_{1}^{\prime}>r_{1}$. On the other hand $u\left\{r_{1}+\varphi\left(u\left(r_{1}\right)\right)\right\} \geq u\left(r_{1}\right)+1=u\left(r_{1}^{\prime}\right)$. Since the function $u(r)$ is nondecreasing, we have $r_{1}+\varphi\left(u\left(r_{1}\right)\right) \geq r_{1}^{\prime}, r_{1}^{\prime}-r_{1} \leq \varphi\left(u\left(r_{1}\right)\right)$. Suppose that we have already found the values $r_{1}, \ldots, r_{n}, r_{1}^{\prime}, \ldots, r_{n}^{\prime}$. Set $r_{n+1}=\min r, r \in E\left(r_{n}^{\prime}\right)$, and let $r_{n+1}^{\prime}$ be the least number $r$ satisfying $u(r)=u\left(r_{n+1}\right)+1$ and $r_{n+1}^{\prime}>r_{n+1}$. We have $u\left\{r_{n+1}+\varphi\left(u\left(r_{n+1}\right)\right)\right\} \geq u\left(r_{n+1}\right)+1=u\left(r_{n+1}^{\prime}\right)$ and $r_{n+1}^{\prime}-r_{n+1} \leq \varphi\left(u\left(r_{n+1}\right)\right)$. Since $u\left(r_{n+1}\right) \geq u\left(r_{n}^{\prime}\right)=u\left(r_{n}\right)+1$, we get $u\left(r_{n+1}\right)-u\left(r_{n}\right) \geq 1$ and $u\left(r_{n+1}\right) \geq$ $u\left(r_{1}\right)+n \geq u_{0}+n$. Therefore $u\left(r_{n}\right) \rightarrow \infty$ and $r_{n} \rightarrow \infty$ as $n \rightarrow \infty$. The definition of the sequence $r_{n}$ implies that (1.7) holds on $\left(r_{n-1}^{\prime}, r_{n}\right), n=1,2, \ldots, r_{0}^{\prime}=r_{0}$. Hence the set $E$ is covered by the sets $\left[r_{n}, r_{n}^{\prime}\right], n=1,2, \ldots$ On the other hand

$$
\begin{aligned}
\sum_{n=1}^{\infty}\left(r_{n}^{\prime}-r_{n}\right) & \leq \sum_{n=1}^{\infty} \varphi\left(u\left(r_{n}\right)\right) \leq \sum_{n=1}^{\infty} \varphi\left(u_{0}+n-1\right) \\
& \leq \varphi\left(u_{0}\right)+\int_{u_{0}}^{\infty} \varphi(u) d u<\infty
\end{aligned}
$$

Observe that we also have proved that the set $E$ can be covered by pairwise disjoint segments with finite total lengths and end points going to infinity.

R. Nevanlinna showed that the condition (1.6) cannot be weakened. In fact, let $u_{0}$ be a real number, $\varphi(u)$ be a function satisfying the conditions of Theorem 1.2 , but

$$
\int_{u_{0}}^{\infty} \varphi(u) d u=\infty
$$


Set

$$
r(u)=\int_{u_{0}}^{u} \varphi(t) d t, u \geq u_{0} .
$$

Then the function $u(r)$ inverse to $r(u)$ satisfies all conditions of Theorem 1.2. The condition (1.9) implies that $u(r)$ is defined everywhere on $[0, \infty)$. Let us show that $(1.8)$ is satisfied everywhere on $[0, \infty)$. Indeed, by the mean value theorem we get

$$
u\{r+\varphi(u(r))\}-u(r)=u_{r}^{\prime}\{r+\theta \varphi(u(r))\} \varphi(u(r)),
$$

where $0<\theta=\theta(r)<1$. On the other hand

$$
\begin{aligned}
u_{r}^{\prime}\{r+\theta \varphi(u(r))\} & =\frac{1}{r_{u}^{\prime}\{u(r+\theta \varphi(u(r)))\}} \\
& =\frac{1}{\varphi\{u(r+\theta \varphi(u(r)))\}} \geq \frac{1}{\varphi(u(r))},
\end{aligned}
$$

hence (1.8) is satisfied.

EXERCISE 1. Let $u(r)$ be a function satisfying the conditions of Theorem 1.2, $\varepsilon>0$. Then for all $r \geq r_{0}$ except, possibly, a set of finite measure, the inequality

$$
u\left(r+\frac{1}{\ln u(r)}\right)<\{u(r)\}^{1+\varepsilon}
$$

holds.

HINT. Use the argument of Theorem 1.2 with $u_{1}(r)=\sqrt{\ln u(r)}$ instead of $u(r)$ and with $\varphi(u)=u^{-2}$.

EXERCise 2. A set $E \subset[1, \infty)$ is called a set of finite logarithmic measure if the integral $\int_{E} r^{-1} d r$ converges. For all $r \geq r_{0}$ except, possibly, a set of finite logarithmic measure, the inequality

$$
u\left(r+\frac{r}{\ln u(r)}\right)<\{u(r)\}^{1+\varepsilon}
$$

holds.

HINT. Use the result of Exercise 1 for $u\left(e^{r}\right)$, and observe that

$$
\exp \left(r+\frac{1}{\ln u\left(e^{r}\right)}\right) \geq e^{r}\left(1+\frac{1}{\ln u\left(e^{r}\right)}\right) .
$$

Let $f(z)$ be a meromorphic function. Denote by $Q(r, f)$ an arbitrary function defined on $\{0 \leq r<\infty\}$ and such that:

1) If the function $f(z)$ has finite order, then $Q(r, f)=O(\ln r)$ as $r \rightarrow \infty$.

2) If the function $f(z)$ has infinite order, then $Q(r, f)=O(\ln T(r, f)+\ln r)$ as $r$ goes to $\infty$, excluding, possibly, some set of segments in $[0, \infty)$ with finite total length.

It is clear that $Q(r, f) \pm Q(r, f)=Q(r, f), A Q(r, f)=Q(r, f)$; if $T\left(r, f_{1}\right)=$ $o(T(r, f))$, then

$$
Q\left(r, f f_{1}\right)=Q(r, f), Q\left(r, f_{1}+f\right)=Q(r, f)
$$


THEOREM 1.3 (Lemma on the logarithmic derivative). Each meromorphic function $f(z)$ satisfies

$$
m\left(r, \frac{f^{\prime}}{f}\right)=Q(r, f)
$$

Proof. Observe that it is enough to prove the theorem for functions $f(z)$ satisfying $f(0)=1$. In fact, in the general case $f(z)=A z^{p} \varphi(z)$, where $A$ is a constant, $p$ is an integer, and $\varphi(0)=1$. If we assume that for $\varphi(z)$ the theorem has already been proved, we get

$$
\begin{aligned}
m\left(r, \frac{f^{\prime}}{f}\right) & =m\left(r, \frac{p}{z}+\frac{\varphi^{\prime}}{\varphi}\right)=m\left(r, \frac{\varphi^{\prime}}{\varphi}\right)+O(1) \\
& =Q(r, \varphi)+O(1)=Q(r, f) .
\end{aligned}
$$

So let $f(0)=1$. Assume that $T(r, f)>1$ for $r \geq r_{0}>1$. Letting $R>r$, $\alpha=1 / 2$ in the inequality (1.1), we get for $r \geq r_{0}$

$$
m\left(r, \frac{f^{\prime}}{f}\right) \leq 2 \ln T(R, f)+2 \ln ^{+} \frac{1}{R-r}+2 \ln R+2 \ln 128 .
$$

If the function $f(z)$ has finite order, then $\ln T(r, f)=O(\ln r)$ and letting $R=2 r$ in (1.11), we get

$$
m\left(r, \frac{f^{\prime}}{f}\right)=O(\ln 2 r)=O(\ln r) .
$$

Now suppose that $f(z)$ has infinite order. Let $R$ in (1.11) be equal to $R=r+$ $\{T(r, f)\}^{-2} \leq 2 r$. By Theorem 1.2 everywhere except, possibly, a set of finite measure the inequality

$$
T(R, f)=T\left(r+\{T(r, f)\}^{-2}, f\right)<T(r, f)+1
$$

holds. Here we use (1.7) with $u(r)=T(r, f)$ and $\varphi(u)=u^{-2}$. Now it follows from (1.11) that outside the exceptional set of values of $r$ the inequality

$$
m\left(r, \frac{f^{\prime}}{f}\right) \leq 6 \ln T(r, f)+2 \ln r+O(1)
$$

holds, that is (1.10) is satisfied.

The question arises, whether Theorem 1.3 is valid in the general case for functions of infinite order, if in the definition of $Q(r, f)$ we drop the mention of the exceptional set, that is, whether the following estimate holds:

$$
m\left(r, \frac{f^{\prime}}{f}\right)=O(\ln T(r, f)+\ln r), r \rightarrow \infty .
$$

Observe that the estimate

$$
m\left(r, \frac{f^{\prime}}{f}\right)=O(\ln T(k r, f)+\ln r), r \rightarrow \infty
$$

holds for each $k>1$; to get this estimate it is enough to let $R=k r$ in (1.11).

Following Hayman [Hay65] we show, however, that the estimate (1.12) is not true in general. If the estimate (1.12) were valid, then for each transcendental entire 
function $f(z)$ we would have

$$
\begin{aligned}
T\left(r, f^{\prime}\right) & =m\left(r, f^{\prime}\right) \leq m(r, f)+m\left(r, \frac{f^{\prime}}{f}\right) \\
& =T(r, f)+O(\ln T(r, f)+\ln r)=(1+o(1)) T(r, f) .
\end{aligned}
$$

It turns out that not only this estimate is not always true (as R. Nevanlinna supposed), but for each positive arbitrarily rapidly increasing function $\Psi(T), \Psi(T) \rightarrow$ $\infty$ as $T \rightarrow \infty$, there exists an entire function $f(z)$, such that

$$
\limsup _{r \rightarrow \infty} \frac{T\left(r, f^{\prime}\right)}{\Psi(T(r, f))}=\infty
$$

Let $r_{n}=2^{n-1}, n=1,2, \ldots$, and let $\lambda_{1}, \lambda_{2}, \ldots$ be a strictly monotone indefinitely increasing sequence of positive integers satisfying $\lambda_{n}>n, n=o\left(\ln \lambda_{n}\right)$, $n \lambda_{n} 2^{n \lambda_{n}}=o\left(\lambda_{n+1}\right), \Psi\left(n \lambda_{n-1}\right)=o\left(\ln \lambda_{n}\right)$ as $n \rightarrow \infty$. Set

$$
f(z)=\sum_{n=1}^{\infty}\left(\frac{z}{r_{n}}\right)^{\lambda_{n}} .
$$

Then

$$
f^{\prime}(z)=\sum_{n=1}^{\infty} \frac{\lambda_{n}}{r_{n}}\left(\frac{z}{r_{n}}\right)^{\lambda_{n}-1} .
$$

For $|z|=r_{\nu}, \nu \geq 2$ we have

$$
\begin{gathered}
|f(z)| \leq \sum_{n=1}^{\infty}\left(\frac{r_{\nu}}{r_{n}}\right)^{\lambda_{n}} \leq \sum_{n=1}^{\nu-1} r_{\nu}^{\lambda_{n}}+1+\sum_{n=\nu+1}^{\infty} 2^{-\lambda_{n}} \leq(\nu-1) r_{\nu}^{\lambda_{\nu-1}}+2, \\
T\left(r_{\nu}, f\right) \leq \lambda_{\nu-1} \ln r_{\nu}+\ln (\nu-1)+O(1) \sim \nu \lambda_{\nu-1} \ln 2 .
\end{gathered}
$$

For $x \geq(\ln 2)^{-1}$, the function $x 2^{-x}$ is decreasing, therefore $\lambda_{n} 2^{-\lambda_{n}}<n 2^{-n}$ for $n \geq 2$. If $|z|=r_{\nu}, \nu \geq 2$, then

$$
\begin{aligned}
\left|f^{\prime}(z)\right| & \geq \frac{\lambda_{\nu}}{r_{\nu}}-\sum_{n=1}^{\nu-1} \frac{\lambda_{n}}{r_{n}}\left(\frac{r_{\nu}}{r_{n}}\right)^{\lambda_{n}-1}-\sum_{n=\nu+1}^{\infty} \frac{\lambda_{n}}{r_{n}}\left(\frac{r_{\nu}}{r_{n}}\right)^{\lambda_{n}-1} \\
& \geq \frac{\lambda_{\nu}}{r_{\nu}}-(\nu-1) \lambda_{\nu-1} r_{\nu}^{\lambda_{\nu-1}-1}-\frac{1}{r_{\nu}} \sum_{n=\nu+1}^{\infty} \lambda_{n}\left(\frac{r_{\nu}}{r_{n}}\right)^{\lambda_{n}} \\
& \geq \frac{1}{r_{\nu}}\left\{\lambda_{\nu}-(\nu-1) \lambda_{\nu-1} r_{\nu}^{\lambda_{\nu-1}}-\sum_{n=\nu+1}^{\infty} \lambda_{n} 2^{-\lambda_{n}}\right\} \\
& \geq \frac{1}{r_{\nu}}\left\{\lambda_{\nu}-(\nu-1) \lambda_{\nu-1} 2^{(\nu-1) \lambda_{\nu-1}}-2\right\}=(1+o(1)) \frac{\lambda_{\nu}}{r_{\nu}} .
\end{aligned}
$$

Hence

$$
T\left(r_{\nu}, f^{\prime}\right)=m\left(r_{\nu}, f^{\prime}\right) \geq \ln \lambda_{\nu}-\ln r_{\nu}+o(1)=(1+o(1)) \ln \lambda_{\nu} .
$$

Using (1.15) and (1.16) we get for $\nu \geq \nu_{0}$

$$
\frac{T\left(r_{\nu}, f^{\prime}\right)}{\Psi\left(T\left(r_{\nu}, f\right)\right)} \geq \frac{(1+o(1)) \ln \lambda_{\nu}}{\Psi\left(\nu \lambda_{\nu-1}\right)} \rightarrow \infty \text { as } \nu \rightarrow \infty,
$$

that is, (1.14) holds. 
If $T=o(\Psi(T))$ as $T \rightarrow \infty$, then

$$
\frac{m\left(r_{\nu}, f^{\prime} / f\right)}{\Psi\left(T\left(r_{\nu}, f\right)\right)} \geq \frac{T\left(r_{\nu}, f^{\prime}\right)}{\Psi\left(T\left(r_{\nu}, f\right)\right)}-\frac{T\left(r_{\nu}, f\right)}{\Psi\left(T\left(r_{\nu}, f\right)\right)} \rightarrow \infty \text { as } \nu \rightarrow \infty
$$

Thus, not only the estimate $m\left(r, f^{\prime} / f\right)=o(T(r, f))$ is not always true for functions of infinite order (this is weaker than (1.12)), but no estimate of the form $m\left(r, f^{\prime} / f\right)=O(\Psi(T(r, f)))$ is true unless we exclude some values of $r$.

For functions of finite order the estimate $m\left(r, f^{\prime} / f\right)=O(\ln r)$ can be made more precise. We show that if $f(z)$ has order $\rho$, then

$$
\limsup _{r \rightarrow \infty}(\ln r)^{-1} m\left(r, \frac{f^{\prime}}{f}\right) \leq(\rho-1)^{+} .
$$

From the argument in the beginning of the proof of Theorem 1.3 we see that without loss of generality we may assume that $f(0)=1$.

Let $R=2 r$ in (1.5). We have

$$
m\left(r, \frac{f^{\prime}}{f}\right) \leq \frac{1}{\alpha} \ln ^{+}\left\{\frac{32}{1-\alpha} T^{\wedge}(2 r, f) r^{-\alpha} 2^{2}\right\}+\frac{1}{\alpha} \ln 2 .
$$

Since for each $\varepsilon>0$ the relation $T(2 r, f)=O\left(r^{\rho+\varepsilon}\right)$ holds, we get

$$
m\left(r, \frac{f^{\prime}}{f}\right) \leq \frac{1}{\alpha} \ln ^{+}\left\{O\left(r^{\rho+\varepsilon-\alpha}\right)\right\}+O(1) \leq \frac{1}{\alpha}(\rho+\varepsilon-\alpha)^{+} \ln r+O(1),
$$

therefore

$$
\limsup _{r \rightarrow \infty}(\ln r)^{-1} m\left(r, \frac{f^{\prime}}{f}\right) \leq \frac{1}{\alpha}(\rho+\varepsilon-\alpha)^{+} .
$$

Letting $\varepsilon \rightarrow 0$ and $\alpha \rightarrow 1$ in this inequality, we get (1.17).

Observe that the function $f(z)=e^{z^{p}}$ ( $p$ is a positive integer) satisfies $\rho=p$, $m\left(r, f^{\prime} / f\right)=(\rho-1) \ln r+\ln \rho, r \geq 1$, and hence the sides of (1.17) are equal.

EXERCISE 1. Prove that for functions of order $\rho<1$ the relation

$$
m\left(r, \frac{f^{\prime}}{f}\right)=o(1), r \rightarrow \infty
$$

holds.

(We have $m\left(r, \frac{f^{\prime}}{f}\right) \leq \frac{1}{\alpha} \frac{1}{2 \pi} \int_{0}^{2 \pi}\left|\frac{f^{\prime}\left(r e^{i \varphi}\right)}{f\left(r e^{i \varphi}\right)}\right|^{\alpha} d \varphi$; we use the estimate (1.4) with $R=2 r$ for the integral; we choose the number $\alpha$ in such a way that $\rho<\alpha<1$.)

EXERCISE 2. Prove that for functions of finite lower order $\lambda$ the inequality

$$
\liminf _{r \rightarrow \infty}(\ln r)^{-1} m\left(r, \frac{f^{\prime}}{f}\right) \leq(\lambda-1)^{+}
$$

holds.

(Let $r=r_{n}, R=2 r_{n}$ in (1.5), where the sequence $\left\{r_{n}\right\}$ is such that $T\left(2 r_{n}, f\right)=$ $O\left(r_{n}^{\lambda+\varepsilon}\right)$.)

EXERCISE 3. Prove

$$
\int_{1}^{s} m\left(r, \frac{f^{\prime}}{f}\right) d r=O\left\{\int_{s / 2}^{s}[\ln T(r, f)+\ln r] d r\right\}, s \rightarrow \infty
$$

(Let $R=(s+r) / 2$ in (1.11), then integrate with respect to $r$ from 1 to $s$. ) 
EXERCISE 4. Prove that everywhere outside a set of finite logarithmic measure the relation

holds.

$$
m\left(r, \frac{f^{\prime}}{f}\right)=O(\ln T(r, f))
$$

(Use the inequality (1.5) and the Exercise 2 after Theorem 1.2.)

EXERCISE 5. Show that for non-integer $\rho$ and for $f(z)=\prod_{k=1}^{\infty} E\left(\frac{z}{k^{1 / \rho}},[\rho]\right)$ the two sides of (1.17) are equal.

First we need to prove that for every $\delta>0$ uniformly in $\varphi$ satisfying $\delta \leq \varphi \leq$ $2 \pi-\delta$ the relation

$$
\frac{f^{\prime}\left(r e^{i \varphi}\right)}{f\left(r e^{i \varphi}\right)}=\frac{\pi \rho}{\sin \pi \rho} e^{-i \rho \pi} e^{i(\rho-1) \varphi_{r} \rho-1}+o\left(r^{\rho-1}\right)
$$

holds. This can be done using the asymptotic formula (5.2 $)$ from Chapter 2 . In fact, letting $z=r e^{i \varphi}, z^{\rho}=r^{\rho} e^{i \rho \varphi}$, and using the mentioned formula we get that the function

$$
g(z)=\ln f(z)-\frac{\pi}{\sin \pi \rho} e^{-i \rho \pi} z^{\rho},
$$

analytic for $0<\varphi<2 \pi$, admits an estimate

$$
g(z)=o\left(r^{\rho}\right)
$$

for $\frac{\delta}{2} \leq \varphi \leq 2 \pi-\frac{\delta}{2}$, uniform with respect to $\varphi$.

Assuming that $z$ is in the angle $\{\delta \leq \varphi \leq 2 \pi-\delta\}$, and observing that then the circle $\left\{|\zeta-z|=|z| \sin \frac{\delta}{2}\right\}$ is contained in $\left\{\frac{\delta}{2} \leq \varphi \leq 2 \pi-\frac{\delta}{2}\right\}$, by the Cauchy formula

we get

$$
g^{\prime}(z)=\frac{1}{2 \pi i} \int_{|\zeta-z|=|z| \sin \frac{\delta}{2}} \frac{g(\zeta)}{(\zeta-z)^{2}} d \zeta
$$

uniformly for $\delta \leq \varphi \leq 2 \pi-\delta$.

$$
g^{\prime}(z)=o\left(r^{\rho-1}\right)
$$

\section{The Nevanlinna second fundamental theorem}

We shall need some auxiliary functions. Let $f(z)$ be a meromorphic function. Denote by $n_{1}(r, a, f)=n_{1}(r, a)$ the number of $a$-points of the function $f(z)$ in the disc $\{|z| \leq r\}$, an $a$-point of order $m$ is counted $m-1$ times. If the orders of $a$-points are disregarded, and each $a$-point is counted once, we get the function $\bar{n}(r, a)$. It is clear that $n(r, a)=n_{1}(r, a)+\bar{n}(r, a)$. Using the functions $n_{1}(r, a)$ and $\bar{n}(r, a)$ we define functions $N_{1}(r, a)$ and $\bar{N}(r, a)$, in the same way as we defined $N(r, a)$ using $n(r, a)$.

For example,

$$
N_{1}(r, a)=\int_{0}^{r} \frac{n_{1}(t, a)-n_{1}(0, a)}{t} d t+n_{1}(0, a) \ln r .
$$

It is clear that the function $n_{1}(r, a)$ does not count simple $a$-points at all. Since for $a \neq \infty$ to each $a$-point of order $m$ of $f(z)$ there corresponds a zero of order $m-1$ of $f^{\prime}(z)$, we have

$$
\sum_{a \neq \infty} n_{1}(r, a, f)=n\left(r, 0, f^{\prime}\right)
$$


where the sum in the left-hand side has at most a finite number of non-zero terms. To each pole of order $m$ of the function $f(z)$ there corresponds a pole of order $m+1$ of the function $f^{\prime}(z)$. Therefore $n\left(r, \infty, f^{\prime}\right)=n(r, \infty, f)+\bar{n}(r, \infty, f)$ and $n_{1}(r, \infty, f)=2 n(r, \infty, f)-n\left(r, \infty, f^{\prime}\right)$. We set $n_{1}(r, f)=n_{1}(r, \infty, f), N_{1}(r, f)=$ $N_{1}(r, \infty, f)$, etc. From the equalities above we get

$$
n_{1}(r)=\sum_{a} n_{1}(r, a, f)=n\left(r, \frac{1}{f^{\prime}}\right)+2 n(r, f)-n\left(r, f^{\prime}\right),
$$

and hence

$$
N_{1}(r)=\sum_{a} N_{1}(r, a, f)=N\left(r, \frac{1}{f^{\prime}}\right)+2 N(r, f)-N\left(r, f^{\prime}\right) .
$$

One drawback of the introduced notation $n_{1}(r)$ and $N_{1}(r)$ is that it does not reflect the function $f(z)$ which is used in the definition, but it will not cause any inconvenience.

We observe one more equality which will be needed on many occasions:

$$
N\left(r, \frac{f^{\prime}}{f}\right)=\bar{N}(r, f)+\bar{N}\left(r, \frac{1}{f}\right) .
$$

This equality immediately follows from the easily verifiable fact that $f^{\prime} / f$ has simple poles at zeros and poles of $f(z)$, and does not have any other poles.

Now we are ready to state the second fundamental theorem, which is due to R. Nevanlinna.

THEOREM 2.1. Let $f(z)$ be a non-identically-constant meromorphic function, let $a_{1}, \ldots, a_{q}$ be distinct complex numbers, one of which can be equal to $\infty$. Then

$$
\sum_{\nu=1}^{q} m\left(r, a_{\nu}\right) \leq 2 T(r, f)-N_{1}(r)+Q(r, f) .
$$

Proof. It is clear that it suffices to consider the case when all numbers $a_{\nu}$, $\nu=1, \ldots, q$, are finite, and to prove the inequality

$$
m(r, \infty)+\sum_{\nu=1}^{q} m\left(r, a_{\nu}\right)+N_{1}(r) \leq 2 T(r, f)+Q(r, f) .
$$

Consider the rational function

$$
h(w)=\sum_{\nu=1}^{q} \frac{1}{w-a_{\nu}}
$$

it has degree $q$. Let $H(z)=h(f(z))$. By Theorem 6.1 from Chapter 1 we get

$$
T(r, H)=q T(r, f)+O(1)
$$

It is easy to see that

$$
N(r, H)=\sum_{\nu=1}^{q} N\left(r, a_{\nu}, f\right) .
$$

Taking into account the first fundamental theorem, we get

$$
\begin{aligned}
m(r, H) & =T(r, H)-N(r, H) \\
& =q T(r, f)-\sum_{\nu=1}^{q} N\left(r, a_{\nu}, f\right)+O(1)=\sum_{\nu=1}^{q} m\left(r, a_{\nu}, f\right)+O(1) .
\end{aligned}
$$


Observe that by the lemma on the logarithmic derivative we have

$$
\begin{aligned}
m(r, H) & \leq m\left(r, \frac{1}{f^{\prime}}\right)+m\left(r, f^{\prime} H\right) \\
& \leq m\left(r, \frac{1}{f^{\prime}}\right)+\sum_{\nu=1}^{q} m\left(r, \frac{f^{\prime}}{f-a_{\nu}}\right)+\ln q=m\left(r, \frac{1}{f^{\prime}}\right)+Q(r, f) .
\end{aligned}
$$

From (2.3) and (2.4) we get

$$
\sum_{\nu=1}^{q} m\left(r, a_{\nu}\right) \leq m\left(r, \frac{1}{f^{\prime}}\right)+Q(r, f)
$$

Thus

$$
\begin{aligned}
m(r, \infty) & +\sum_{\nu=1}^{q} m\left(r, a_{\nu}\right)+N_{1}(r) \\
& \leq m(r, f)+m\left(r, \frac{1}{f^{\prime}}\right)+N\left(r, \frac{1}{f^{\prime}}\right)+2 N(r, f)-N\left(r, f^{\prime}\right)+Q(r, f) \\
& =T(r, f)+T\left(r, \frac{1}{f^{\prime}}\right)+N(r, f)-N\left(r, f^{\prime}\right)+Q(r, f) \\
& =T(r, f)+\left\{T\left(r, f^{\prime}\right)-N\left(r, f^{\prime}\right)\right\}+N(r, f)+Q(r, f) \\
& =T(r, f)+N(r, f)+m\left(r, f^{\prime}\right)+Q(r, f) .
\end{aligned}
$$

Since

$$
m\left(r, f^{\prime}\right) \leq m(r, f)+m\left(r, \frac{f^{\prime}}{f}\right)=m(r, f)+Q(r, f),
$$

the inequality (2.6) implies (2.2).

REMARK 1 . It is easy to check that the quantity $2 T(r, f)-N_{1}(r)$ from the right-hand side of (2.1) can be replaced by

$$
-\frac{1}{2 \pi} \int_{0}^{2 \pi} \ln f\left(r e^{i \varphi}\right) d \varphi
$$

where $\stackrel{\circ}{f}(z)$ is the spherical derivative of $f(z)$ (see section 4 of chapter 1 ).

In fact,

$$
\begin{aligned}
-\frac{1}{2 \pi} & \int_{0}^{2 \pi} \ln \frac{\left|f^{\prime}\left(r e^{i \varphi}\right)\right|}{1+\left|f\left(r e^{i \varphi}\right)\right|^{2}} d \varphi+N_{1}(r) \\
= & -\frac{1}{2 \pi} \int_{0}^{2 \pi} \ln \mid f^{\prime}\left(r e^{i \varphi}\right) d \varphi+2 \frac{1}{2 \pi} \int_{0}^{2 \pi} \ln \sqrt{1+\left|f\left(r e^{i \varphi}\right)\right|^{2}} d \varphi+N_{1}(r) \\
= & -m\left(r, f^{\prime}\right)+m\left(r, \frac{1}{f^{\prime}}\right)+2 \stackrel{\circ}{m}(r, f)+N\left(r, \frac{1}{f^{\prime}}\right)+2 N(r, f) \\
& \quad-N\left(r, f^{\prime}\right)=2 T(r, f)+O(1)-T\left(r, f^{\prime}\right)+T\left(r, \frac{1}{f^{\prime}}\right)=2 T(r, f)+O(1) .
\end{aligned}
$$

REMARK 2. The result of the Exercise 1 on p. ?? implies that for functions of order less than 1 , we may assume $Q(r, f)=O(1)$ in $(2.1)$. 
REMARK 3. The inequality (2.5) implies the following lemma which will play an important role in Chapter 6.

LEMMA 2.1. Let $a_{1}, \ldots, a_{p}$ be distinct complex numbers, different from 0 and $\infty$. The inequality

$$
\sum_{\nu=1}^{p} m\left(r, a_{\nu}\right) \leq m\left(r, \frac{f}{f^{\prime}}\right)+Q(r, f)
$$

holds. In particular, for each $a \neq 0, \infty$ the inequality

$$
m(r, a) \leq m\left(r, \frac{f}{f^{\prime}}\right)+Q(r, f)
$$

holds. that

In fact, writing $(2.5)$ for the numbers $0, a_{1}, \ldots, a_{p}(q=p+1)$, and observing

$$
m\left(r, \frac{1}{f^{\prime}}\right) \leq m\left(r, \frac{f}{f^{\prime}}\right)+m\left(r, \frac{1}{f}\right)=m\left(r, \frac{f}{f^{\prime}}\right)+m(r, 0),
$$

we get the desired inequality.

Let us write down some inequalities, immediately following from (2.1). Letting $m\left(r, a_{\nu}\right)=T(r, f)-N\left(r, a_{\nu}\right)+O(1)$ in $(2.1)$, we get

$$
(q-2) T(r, f) \leq \sum_{\nu=1}^{q} N\left(r, a_{\nu}\right)-N_{1}(r)+Q(r, f) .
$$

Replacing $N_{1}(r)$ by the quantity $\sum_{\nu=1}^{q} N_{1}\left(r, a_{\nu}\right) \leq N_{1}(r)$, we get from (2.1) and (2.8), respectively, the following inequalities:

$$
\begin{gathered}
\sum_{\nu=1}^{q}\left\{m\left(r, a_{\nu}\right)+N_{1}\left(r, a_{\nu}\right)\right\} \leq 2 T(r, f)+Q(r, f) \\
(q-2) T(r, f) \leq \sum_{\nu=1}^{q} \bar{N}\left(r, a_{\nu}\right)+Q(r, f) .
\end{gathered}
$$

Theorem 2.1 immediately implies the following theorem, which goes back to Borel.

THEOREM 2.2. A transcendental meromorphic function $f(z)$ cannot have more than two Borel exceptional values.

Proof. Assume that $f(z)$ has three Borel exceptional values $a_{1}, a_{2}, a_{3}$. From (2.10) with $q=3$ we get

$$
T(r, f) \leq \sum_{\nu=1}^{3} N\left(r, a_{\nu}\right)+Q(r, f) .
$$

If the function $f(z)$ has infinite order, then $N\left(r, a_{\nu}\right), \nu=1,2,3$, have finite order, hence, there is a constant $K<\infty$, such that for $r \geq r_{0}$ the inequality $\sum_{\nu=1}^{3} N\left(r, a_{\nu}\right) \leq r^{K}$ holds. Then outside some set $E$ of finite measure $L$ the inequalities

$$
T(r, f) \leq r^{K}+O(\ln T(r, f)+\ln r) \leq r^{K}+\frac{1}{2} T(r, f)
$$


and

$$
T(r, f) \leq 2 r^{K}
$$

hold. If $r \in E$, then there exists $r^{\prime}, r \leq r^{\prime} \leq r+L+1$, such that $r^{\prime} \notin E$. Then

$$
T(r, f) \leq T\left(r^{\prime}, f\right) \leq 2{r^{\prime}}^{K} \leq 2(r+L+1)^{K} \leq 3 r^{K},
$$

if $r$ is sufficiently large. Hence $T(r, f)$ has finite order, and we get a contradiction.

If $f(z)$ is of finite order, then $Q(r, f)=o(T(r, f))$, and (2.11) can be rewritten as

$$
(1+o(1)) T(r, f) \leq \sum_{\nu=1}^{3} N\left(r, a_{\nu}\right) .
$$

This implies immediately that the growth category of at least one of the functions $N\left(r, a_{\nu}\right), \nu=1,2,3$, is not lower than the growth category of $T(r, f)$. If $f(z)$ has positive order, we already have a contradiction. If $f(z)$ has zero order, we need to consider also the case when $n\left(r, a_{\nu}\right)=O(1)$, that is, $N\left(r, a_{\nu}\right)=O(\ln r), \nu=1,2,3$. From $(2.12)$ we get $T(r, f)=O(\ln r)$, that is, $f(z)$ is a rational function.

Corollary. (PICARD) A transcendental meromorphic function $f(z)$ cannot have more than two Picard exceptional values.

EXERCise. Prove the corollary using (2.11) directly, without using Theorem 2.2 .

THEOREM 2.3. If $f(z)$ is a meromorphic function, then $(l=1,2,3, \ldots)$

$$
\begin{gathered}
T\left(r, f^{(l)}\right) \leq(l+1) T(r, f)+Q(r, f), \\
Q\left(r, f^{(l)}\right)=Q(r, f) .
\end{gathered}
$$

Proof. Combining the inequality $(2.7)$ with $N\left(r, f^{\prime}\right)=N(r, f)+\bar{N}(r, f) \leq$ $2 N(r, f)$, we get $T\left(r, f^{\prime}\right) \leq 2 T(r, f)+Q(r, f)$. From here it is easy to derive that $Q\left(r, f^{\prime}\right)=Q(r, f)$, and prove (2.14) for an arbitrary $l \geq 1$ using induction. Then by the lemma on the logarithmic derivative,

$$
\begin{aligned}
m\left(r, f^{(l)}\right) & \leq m\left(r, \frac{f^{(l)}}{f^{(l-1)}}\right)+\cdots+m\left(r, \frac{f^{\prime}}{f}\right)+m(r, f) \\
& =Q\left(r, f^{(l-1)}\right)+\cdots+Q(r, f)+m(r, f)=m(r, f)+Q(r, f) .
\end{aligned}
$$

On the other hand,

$$
N\left(r, f^{(l)}\right)=N(r, f)+l \bar{N}(r, f) \leq(l+1) N(r, f) .
$$

The inequality (2.13) follows from (2.15) and (2.16).

COROLlaRY. If $f(z)$ is a meromorphic function, then

$$
m\left(r, \frac{f^{(l)}}{f}\right)=Q(r, f) .
$$

The second fundamental theorem can be generalized in many directions, if we consider inequalities involving not only the characteristics of the meromorphic function itself, but also characteristics of its derivatives. We consider here only the simplest generalizations of the inequality (2.11), which we will need later. 
THEOREM 2.4 (Milloux). Let $f(z)$ be a meromorphic function such that $f^{(k)}(z)$ is not identically constant for $k \geq 1$. Let $a$ and $b$ be (finite) complex numbers, $b \neq 0$. Then the inequality

$$
\begin{aligned}
T(r, f) \leq & \bar{N}(r, f)+N\left(r, \frac{1}{f-a}\right) \\
& +\bar{N}\left(r, \frac{1}{f^{(k)}-b}\right)-N\left(r, \frac{f^{(k)}-b}{f^{(k+1)}}\right)+Q(r, f)
\end{aligned}
$$

holds.

Proof. It is clear that it is enough to prove (2.17) for $a=0, b=1$, that is, to prove

$$
\begin{gathered}
T(r, f) \leq \bar{N}(r, f)+N\left(r, \frac{1}{f}\right) \\
+\bar{N}\left(r, \frac{1}{f^{(k)}-1}\right)-N\left(r, \frac{f^{(k)}-1}{f^{(k+1)}}\right)+Q(r, f),
\end{gathered}
$$

since (2.17) is obtained from $\left(2.17^{\prime}\right)$ by passing to the function $F(z)=(f(z)-a) / b$.

By the first fundamental theorem we have

$$
T(r, f)=m\left(r, \frac{1}{f}\right)+N\left(r, \frac{1}{f}\right)+O(1) .
$$

On the other hand, by the corollary to Theorem 2.3 we have

$$
\begin{aligned}
& m\left(r, \frac{1}{f}\right)=m\left(r, \frac{f^{(k)}}{f}-\frac{f^{(k)}-1}{f^{(k+1)}} \frac{f^{(k+1)}}{f}\right) \leq m\left(r, \frac{f^{(k)}}{f}\right) \\
& \quad+m\left(r, \frac{f^{(k)}-1}{f^{(k+1)}}\right)+m\left(r, \frac{f^{(k+1)}}{f}\right)+\ln 2=m\left(r, \frac{f^{(k)}-1}{f^{(k+1)}}\right)+Q(r, f) .
\end{aligned}
$$

By the first fundamental theorem

$$
m\left(r, \frac{f^{(k)}-1}{f^{(k+1)}}\right)=m\left(r, \frac{f^{(k+1)}}{f^{(k)}-1}\right)+N\left(r, \frac{f^{(k+1)}}{f^{(k)}-1}\right)-N\left(r, \frac{f^{(k)}-1}{f^{(k+1)}}\right)+O(1) .
$$

Since

we have

$$
\frac{f^{(k+1)}}{f^{(k)}-1}=\frac{\left(f^{(k)}-1\right)^{\prime}}{f^{(k)}-1}
$$

$$
m\left(r, \frac{f^{(k+1)}}{f^{(k)}-1}\right)=Q\left(r, f^{(k)}-1\right)=Q(r, f)
$$

and

$$
\begin{aligned}
N\left(r, \frac{f^{(k+1)}}{f^{(k)}-1}\right) & =\bar{N}\left(r, \frac{1}{f^{(k)}-1}\right)+\bar{N}\left(r, f^{(k)}-1\right) \\
& =\bar{N}\left(r, \frac{1}{f^{(k)}-1}\right)+\bar{N}(r, f) .
\end{aligned}
$$

Combining these estimates we get $\left(2.17^{\prime}\right)$.

It is not difficult to prove an inequality similar to (2.17) in which the characteristics $T(r, f)$ would be estimated from above through three functions $N$, but without taking into account the number of poles. 
TheOREM 2.5 (Hiong King-Lai). Let $f(z)$ be a meromorphic function such that $f^{(k)}(z)$ is not identically constant for some $k \geq 1$. Let $a, b, c$ be (finite) complex numbers, $b \neq 0, c \neq 0$. Then the inequality

$$
T(r, f) \leq N\left(r, \frac{1}{f-a}\right)+N\left(r, \frac{1}{f^{(k)}-b}\right)+N\left(r, \frac{1}{f^{(k)}-c}\right)+Q(r, f)
$$

holds.

Proof. By the first fundamental theorem

$$
T(r, f)=m(r, f-a)+N(r, f-a)+O(1)
$$

and

$$
\begin{aligned}
& m(r, f-a) \leq m\left(r, f^{(k)}\right)+m\left(r, \frac{f-a}{f^{(k)}}\right) \\
& \quad=m\left(r, f^{(k)}\right)+m\left(r, \frac{f^{(k)}}{f-a}\right)+N\left(r, \frac{f^{(k)}}{f-a}\right)-N\left(r, \frac{f-a}{f^{(k)}}\right)+O(1) .
\end{aligned}
$$

Later we shall use the equality

$$
\begin{aligned}
N\left(r, \frac{f^{(k)}}{f-a}\right)- & N\left(r, \frac{f-a}{f^{(k)}}\right)=N\left(r, f^{(k)}\right)-N\left(r, \frac{1}{f^{(k)}}\right) \\
& +N\left(r, \frac{1}{f-a}\right)-N(r, f-a),
\end{aligned}
$$

which follows easily from the Jensen formula (4.1) from Chapter 1. Since

$$
m\left(r, \frac{f^{(k)}}{f-a}\right)=Q(r, f)
$$

using (2.19) and (2.20) we get

$$
T(r, f) \leq T\left(r, f^{(k)}\right)-N\left(r, \frac{1}{f^{(k)}}\right)+N\left(r, \frac{1}{f-a}\right)+Q(r, f) .
$$

Using for $f^{(k)}(z)$ the inequality (2.8) with $q=3, a_{1}=0, a_{2}=b, a_{3}=c$, we get

$$
\begin{aligned}
T\left(r, f^{(k)}\right) \leq & N\left(r, \frac{1}{f^{(k)}}\right)+N\left(r, \frac{1}{f^{(k)}-b}\right)+N\left(r, \frac{1}{f^{(k)}-c}\right) \\
& -N_{1}\left(r, f^{(k)}\right)-N\left(r, \frac{1}{f^{(k+1)}}\right)+Q\left(r, f^{(k)}\right) .
\end{aligned}
$$

By (2.14), (2.21), (2.22) and the obvious equality $N_{1}\left(r, f^{(k)}\right)=N(r, f)+(k-$ 1) $\bar{N}(r, f)$, we get the inequality

$$
\begin{aligned}
T(r, f) \leq & N\left(r, \frac{1}{f-a}\right)+N\left(r, \frac{1}{f^{(k)}-b}\right)+N\left(r, \frac{1}{f^{(k)}-c}\right) \\
& -\left\{N(r, f)+(k-1) \bar{N}(r, f)+N\left(r, \frac{1}{f^{(k+1)}}\right)\right\}+Q(r, f) .
\end{aligned}
$$

Leaving out the expression in braces, we get (2.18).

We show that in $(2.18)$ we can delete $N\left(r, 1 /\left(f^{(k)}-c\right)\right)$ if we multiply the summands $N(r, 1 /(f-a))$ and $N\left(r, 1 /\left(f^{(k)}-b\right)\right)$ by 3 and 4 , respectively. More precisely, we prove the following theorem. 
TheOREM 2.6 (Hayman). Under the conditions of Theorem 2.4 the inequality

$$
T(r, f) \leq\left(2+\frac{1}{k}\right) N\left(r, \frac{1}{f-a}\right)+\left(2+\frac{2}{k}\right) \bar{N}\left(r, \frac{1}{f^{(k)}-b}\right)+Q(r, f)
$$

holds.

Proof. As in the proof of Theorem 2.4, it suffices to consider the case $a=0$, $b=1$. We introduce the following notation:

$$
\varphi(z)=f^{(k)}(z)-1, g(z)=\left(\frac{\varphi^{\prime}}{\varphi}\right)^{k+1} \frac{1}{\varphi} .
$$

If the function $\varphi(z)$ has a zero of order $\mu$ at $z_{0}$, then $g(z)$ has a pole of order $\mu+k+1$ at $z_{0}$. If the function $f(z)$ has a pole of order $m \geq 1$ at $z_{0}$, then $\varphi(z)$ has a pole of order $m+k$ at $z_{0},\left(\varphi^{\prime} / \varphi\right)^{k+1}$ has a pole of order $k+1$, and $g(z)$ has a zero of order $m-1$, if $m \geq 2$, and $g\left(z_{0}\right) \neq 0, \infty$, if $m=1$. The function $g(z)$ has zeros also at zeros of $\varphi^{\prime} / \varphi$, and $g(z) \neq 0, \infty$ at all points except those listed above. Let $N_{s}(r, f)$ take into account simple poles of the function $f(z)$ in the disc $\{|z| \leq r\}$ only. The argument above implies

Hence

$$
\begin{gathered}
\bar{N}(r, g)=\bar{N}\left(r, \frac{1}{\varphi}\right), \\
\bar{N}\left(r, \frac{1}{g}\right)=\bar{N}(r, f)-N_{s}(r, f)+\bar{N}\left(r, \frac{\varphi}{\varphi^{\prime}}\right) .
\end{gathered}
$$

$$
\begin{aligned}
N\left(r, \frac{g^{\prime}}{g}\right)= & \bar{N}(r, g)+\bar{N}\left(r, \frac{1}{g}\right)=\bar{N}\left(r, \frac{1}{\varphi}\right)+\bar{N}(r, f) \\
& -N_{s}(r, f)+\bar{N}\left(r, \frac{\varphi}{\varphi^{\prime}}\right) .
\end{aligned}
$$

Suppose that $f(z)$ has pole of the first order at $z_{0}$. Then in the neighborhood of $z_{0}$ the function $\varphi(z)$ and its derivatives can be represented as

$$
\begin{gathered}
\varphi(z)=\frac{A}{\left(z-z_{0}\right)^{k+1}}+h(z), \varphi^{\prime}(z)=\frac{-(k+1) A}{\left(z-z_{0}\right)^{k+2}}+h^{\prime}(z), \\
\varphi^{\prime \prime}(z)=\frac{(k+1)(k+2) A}{\left(z-z_{0}\right)^{k+3}}+h^{\prime \prime}(z),
\end{gathered}
$$

where $A \neq 0$, and $h(z)$ is a function holomorphic in the neighborhood of the point $z_{0}$. We have

$$
\frac{g^{\prime}(z)}{g(z)}=\frac{(k+1) \varphi \varphi^{\prime \prime}-(k+2) \varphi^{\prime 2}}{\varphi \varphi^{\prime}}=(k+1) \frac{\varphi^{\prime \prime}}{\varphi^{\prime}}-(k+2) \frac{\varphi^{\prime}}{\varphi} .
$$

It is easy to see that

$$
(k+1) \varphi \varphi^{\prime \prime}-(k+2) \varphi^{\prime 2}=\frac{(k+1)^{2}(k+2) A h\left(z_{0}\right)}{\left(z-z_{0}\right)^{k+3}}+P(z),
$$

where $P(z)$ has a pole of order at most $k+2$ at $z_{0}$, hence, $g^{\prime} / g$ has a zero of order at least $k$ at $z_{0}$. Thus ${ }^{1}$

$$
k N_{s}(r, f) \leq N\left(r, \frac{g}{g^{\prime}}\right)
$$

\footnotetext{
${ }^{1}$ If $g^{\prime} \equiv 0$ then $f$ is rational with $f(\infty)=$ infty and (2.23) holds trivially in this case.
} 
Using the first fundamental theorem, the lemma on the logarithmic derivative, and (2.14), we get from (2.24) and (2.25):

$$
\begin{aligned}
k N_{s}(r, f) \leq & N\left(r, \frac{g^{\prime}}{g}\right)+m\left(r, \frac{g^{\prime}}{g}\right)-m\left(r, \frac{g}{g^{\prime}}\right)+O(1) \\
\leq & N\left(r, \frac{g^{\prime}}{g}\right)+Q(r, f)=\bar{N}\left(r, \frac{1}{\varphi}\right)+\bar{N}(r, f) \\
& -N_{s}(r, f)+\bar{N}\left(r, \frac{\varphi}{\varphi^{\prime}}\right)+Q(r, f) .
\end{aligned}
$$

Since in $N(r, f)$ multiple poles are counted at least twice, then, using $\left(2.17^{\prime}\right)$, we get

$$
\begin{aligned}
N_{s}(r, f) & +2\left\{\bar{N}(r, f)-N_{s}(r, f)\right\} \leq N(r, f) \\
& \leq T(r, f) \leq \bar{N}(r, f)+N\left(r, \frac{1}{f}\right)+\bar{N}\left(r, \frac{1}{\varphi}\right)-N\left(r, \frac{\varphi}{\varphi^{\prime}}\right)+Q(r, f)
\end{aligned}
$$

and

$$
\bar{N}(r, f) \leq N_{s}(r, f)+N\left(r, \frac{1}{f}\right)+\bar{N}\left(r, \frac{1}{\varphi}\right)-N\left(r, \frac{\varphi}{\varphi^{\prime}}\right)+Q(r, f) .
$$

Multiplying this inequality by $k+1$ and adding it with the inequality (2.26) we get

$$
\begin{aligned}
k \bar{N}(r, f) \leq & (k+1) N\left(r, \frac{1}{f}\right)+(k+2) \bar{N}\left(r, \frac{1}{\varphi}\right) \\
& -k N\left(r, \frac{\varphi}{\varphi^{\prime}}\right)-N_{1}\left(r, \frac{\varphi}{\varphi^{\prime}}\right)+Q(r, f) .
\end{aligned}
$$

Substituting this inequality into $\left(2.17^{\prime}\right)$ and leaving out the negative summands, we get

$$
T(r, f) \leq\left(2+\frac{1}{k}\right) N\left(r, \frac{1}{f}\right)+\left(2+\frac{2}{k}\right) \bar{N}\left(r, \frac{1}{\varphi}\right)+Q(r, f),
$$

that is, the inequality (2.23) with $a=0, b=1$.

Using Theorems 2.4, 2.5, and 2.6 it is possible to prove analogues of Theorem 2.2 and the corollary of Theorem 2.2. For example, using Theorem 2.6 we get that if a meromorphic function $f(z)$ has a Borel (Picard) exceptional value $a \neq \infty$, then $f^{(k)}(z), k \geq 1$, cannot have a Borel (Picard) exceptional value $b \neq 0, \infty$.

In fact, if $f(z)$ has infinite order, then $N(r, a, f)$ and $N\left(r, b, f^{(k)}\right)$ have finite order, and there is a constant $K>0$, such that

$$
N(r, a, f)+N\left(r, b, f^{(k)}\right)<r^{K}
$$

for $r \geq r_{0}$. Arguing in the same way as in the proof of Theorem 2.2, but using (2.23) instead of (2.11), we conclude that $T(r, f)<3 r^{K}$ for sufficiently large $r$. Hence $f(z)$ has finite order. For transcendental functions of finite order (2.23) implies

$$
(1+o(1)) T(r, f) \leq\left(2+\frac{1}{k}\right) N(r, a, f)+\left(2+\frac{2}{k}\right) N\left(r, b, f^{(k)}\right) .
$$

The category of $N(r, a, f)$ is lower than the category of $T(r, f)$, and the category of $N\left(r, b, f^{(k)}\right)$ is lower than the category of $T\left(r, f^{(k)}\right)$, by the definition of a Borel exceptional value. On the other hand, by Theorem 2.3 the category of $T\left(r, f^{(k)}\right)$ 
does not exceed the category of $T(r, f)$. Therefore the right-hand side of $(2.27)$ has lower category than the left-hand side, and we get a contradiction.

Theorem 2.6 implies the following result.

Theorem 2.7 (Hayman). If $g(z)$ is an entire function and $g(z) g^{\prime \prime}(z)$ does not have zeros, then $g(z)=e^{A z+B}$, where $A$ and $B$ are constants, $A \neq 0$.

Proof. Let

$$
f(z)=g(z) / g^{\prime}(z)
$$

It is clear that $N(r, 0, f) \equiv 0$ and that $1 / f(z)$ is an entire function. Since

$$
f^{\prime}(z)=1-\frac{g(z) g^{\prime \prime}(z)}{\left[g^{\prime}(z)\right]^{2}}
$$

we have also $N\left(r, 1, f^{\prime}\right) \equiv 0$. Applying (2.23) with $k=1, a=0, b=1$, we get

$$
T(r, f)=Q(r, f),
$$

therefore

$$
T(r, f)=O(\ln r),
$$

and, hence, the function $f(z)$ is rational, and $1 / f(z)$ is a polynomial. It follows that $g(z)=\exp P(z)$, where $P(z)$ is a polynomial. Since $g^{\prime \prime}(z)=\left(P^{\prime 2}+P^{\prime \prime}\right) e^{P}$, then $g^{\prime \prime}(z)$ has no zeros if and only if ${P^{\prime}}^{2}+P^{\prime \prime}=$ const $\neq 0$, that is, if $P(z)=A z+B$, $A \neq 0$.

REMARK. The statement of Theorem 2.7 remains true, if we replace the condition $g(z) g^{\prime \prime}(z) \neq 0$ by the condition $g(z) g^{(n)}(z) \neq 0$ for some $n \geq 2$ (Tumura, Clunie). Proof of this result is rather deep, and we do not include it (it can be found in Hayman's book [Hay64, §3.5]).

EDITOR'S REMARK. Langley [A103] generalized Theorem 2.7 to meromorphic functions: the only meromorphic functions with the property $f(z) f^{\prime \prime}(z) \neq 0$ are the exponential function and $f(z)=c(z-a)^{-n}$. The same result with $f f^{(k)}, k \geq 3$ was earlier proved by Frank [A52].

\section{Analogues of the second fundamental theorem for angular Nevanlinna characteristics and for Tsuji characteristics}

First we prove the corresponding analogues of the lemma on the logarithmic derivative.

It is natural to expect that for the function $f(z)$ meromorphic in the angle $\alpha \leq \arg z \leq \beta, 0<\beta-\alpha \leq 2 \pi$, the relation

$$
A_{\alpha \beta}\left(r, \frac{f^{\prime}}{f}\right)+B_{\alpha \beta}\left(r, \frac{f^{\prime}}{f}\right)=o\left(S_{\alpha \beta}(r, f)\right)
$$

holds as $r \rightarrow \infty$, outside an exceptional set. However, this statement conjectured by R. Nevanlinna [NevR25b] in 1925 has not been proved or disproved yet. ${ }^{2}$ We shall estimate the sum $A_{\alpha \beta}\left(r, f^{\prime} / f\right)+B_{\alpha \beta}\left(r, f^{\prime} / f\right)$ for functions $f(z)$ meromorphic everywhere in the complex plane only, and we give it in terms of $T(r, f)$, and not $S_{\alpha \beta}(r, f)$.

\footnotetext{
${ }^{2} \mathrm{~A}$ counterexample was constructed by Goldberg [A63].
} 
Let $f(z)$ be a meromorphic function, $\{\alpha \leq \arg z \leq \beta\}$ be an angle, $k=\pi /(\beta-$ $\alpha)$. Denote by $Q_{\alpha \beta}(r, f)$ an arbitrary function defined for $\{1 \leq r<\infty\}$ and such that

1) If the function $f(z)$ satisfies the condition

$$
\int_{1}^{\infty} r^{-k-1} \ln ^{+} T(r, f) d r<\infty
$$

then $Q_{\alpha \beta}(r, f)=O(1)$,

2) If the condition (3.1) is not satisfied, then $Q_{\alpha \beta}(r, f)=O(\ln T(r, f)+\ln r)$ as $r \rightarrow \infty$, possibly omitting a set of finite measure.

THEOREM 3.1. Each meromorphic function $f(z)$ satisfies

$$
A_{\alpha \beta}\left(r, f^{\prime} / f\right)+B_{\alpha \beta}\left(r, f^{\prime} / f\right)=Q_{\alpha \beta}(r, f) .
$$

Proof. It is easy to see that

$$
B_{\alpha \beta}\left(r, \frac{f^{\prime}}{f}\right)=\frac{2 k}{\pi r^{k}} \int_{\alpha}^{\beta} \ln ^{+}\left|\frac{f^{\prime}\left(r e^{i \varphi}\right)}{f\left(r e^{i \varphi}\right)}\right| \sin k(\varphi-\alpha) d \varphi \leq \frac{4 k}{r^{k}} m\left(r, \frac{f^{\prime}}{f}\right) .
$$

If the function $f(z)$ satisfies the condition (3.1), then $\ln ^{+} T(r, f)=o\left(r^{k}\right)$ because

$$
\frac{\ln ^{+} T(r, f)}{2^{k+1} r^{k}} \leq \int_{r}^{2 r} \frac{\ln ^{+} T(t, f)}{t^{k+1}} d t=o(1) .
$$

Using the estimate (1.13) for $m\left(r, f^{\prime} / f\right)$, we get

$$
B_{\alpha \beta}\left(r, \frac{f^{\prime}}{f}\right) \leq \frac{4 k}{r^{k}}\left(o\left[(2 r)^{k}\right]+O(\ln r)\right)=o(1)=Q_{\alpha \beta}(r, f) .
$$

If the condition (3.1) is not satisfied, then the estimate $B_{\alpha \beta}\left(r, f^{\prime} / f\right)=Q_{\alpha \beta}(r, f)$ follows from (3.2) and the lemma on the logarithmic derivative.

Thus, everything is reduced to a proof of the relation

$$
A_{\alpha \beta}\left(r, \frac{f^{\prime}}{f}\right)=Q_{\alpha \beta}(r, f) .
$$

We prove the following lemma which plays the same role as Theorem 1.1 in the proof of the lemma on the logarithmic derivative.

Lemma 3.1. Let $f(z)$ be a meromorphic function satisfying $f(0)=1$. Then the inequality

$$
A_{\alpha \beta}\left(r, \frac{f^{\prime}}{f}\right) \leq K\left\{\left(\frac{R}{r}\right)^{k} \int_{1}^{R} \frac{\ln ^{+} T(t, f)}{t^{k+1}} d t+\ln ^{+} \frac{r}{R-r}+\ln \frac{R}{r}+1\right\}
$$

holds, where $1 \leq r<R<\infty$, and $K$ is a constant which does not depend on $r$ and $R$.

Proof. Our argument is based on the inequality $\left(1.3^{\prime}\right)$ (we use the same notation as in the proof of Theorem 1.1). Let $z=t e^{i \varphi}$ and $s=\sqrt{\lambda} t$, where $\lambda=R r^{-1}$ in the inequality $\left(1.3^{\prime}\right)$. We get

$$
\left|\frac{f^{\prime}\left(t e^{i \varphi}\right)}{f\left(t e^{i \varphi}\right)}\right| \leq U_{1}(t)+U_{2}(t)
$$

where

$$
U_{1}(t)=\frac{4 \sqrt{\lambda}}{t(\sqrt{\lambda}-1)^{2}} T(\sqrt{\lambda} t, f) \leq \frac{16 \lambda^{3 / 2}}{(\lambda-1)^{2}} T(\lambda t, f), t \geq 1,
$$




$$
U_{2}(t)=\frac{\sqrt{\lambda}}{\sqrt{\lambda}-1} \sum_{\left|c_{q}\right|<\sqrt{\lambda} t} \frac{1}{|t-| c_{q}||} \leq \frac{2 \lambda}{\lambda-1} \sum_{\left|c_{q}\right|<\sqrt{\lambda} t} \frac{1}{|t-| c_{q}||} .
$$

Therefore

$$
\begin{aligned}
A_{\alpha \beta}\left(r, \frac{f^{\prime}}{f}\right) & =\frac{k}{\pi} \int_{1}^{r}\left(\frac{1}{t^{k}}-\frac{t^{k}}{r^{2 k}}\right)\left[\ln ^{+}\left|\frac{f^{\prime}\left(t e^{i \alpha}\right)}{f\left(t e^{i \alpha}\right)}\right|+\ln ^{+}\left|\frac{f^{\prime}\left(t e^{i \beta}\right)}{f\left(t e^{i \beta}\right)}\right|\right] \frac{d t}{t} \\
& \leq \frac{k}{\pi} \int_{1}^{r}\left(\frac{1}{t^{k}}-\frac{t^{k}}{r^{2 k}}\right) 2 \ln ^{+}\left[U_{1}(t)+U_{2}(t)\right] \frac{d t}{t} \\
& \leq \frac{2 k}{\pi} \int_{1}^{r}\left(\frac{1}{t^{k}}-\frac{t^{k}}{r^{2 k}}\right)\left[\ln ^{+} U_{1}(t)+\ln ^{+} U_{2}(t)+\ln 2\right] \frac{d t}{t} \\
& \leq \frac{2 k}{\pi}\left\{\int_{1}^{r} t^{-k-1} \ln ^{+} U_{1}(t) d t+\int_{1}^{r}\left(\frac{1}{t^{k}}-\frac{t^{k}}{r^{2 k}}\right) \ln ^{+} U_{2}(t) \frac{d t}{t}+\frac{\ln 2}{k}\right\} \\
& =\frac{2 k}{\pi}\left\{I_{1}+I_{2}+\frac{\ln 2}{k}\right\} .
\end{aligned}
$$

Now we estimate the integrals $I_{1}$ and $I_{2}$. By $K$ we denote positive constants which do not depend on $t, r, \lambda$, and hence, on $R=\lambda r$.

We have

$$
\begin{aligned}
I_{1} & \leq \int_{1}^{r} \ln ^{+}\left\{\frac{16 \lambda^{3 / 2}}{(\lambda-1)^{2}} T(\lambda t, f)\right\} \frac{d t}{t^{k+1}} \\
& \leq \int_{1}^{r}\left\{\ln 16+\frac{3}{2} \ln \lambda+2 \ln ^{+} \frac{1}{\lambda-1}+\ln ^{+} T(\lambda t, f)\right\} \frac{d t}{t^{k+1}} \\
& \leq \int_{1}^{r} \frac{\ln ^{+} T(\lambda t, f)}{t^{k+1}} d t+K\left(\ln ^{+} \frac{1}{\lambda-1}+\ln \lambda+1\right) .
\end{aligned}
$$

In a similar way we get

$$
\begin{aligned}
I_{2} \leq & \int_{1}^{r} \ln ^{+}\left\{\frac{2 \lambda}{\lambda-1} \sum_{\left|c_{q}\right|<\sqrt{\lambda} t} \frac{1}{|t-| c_{q}||}\right\}\left(\frac{1}{t^{k}}-\frac{t^{k}}{r^{2 k}}\right) \frac{d t}{t} \\
\leq & \int_{1}^{r} \ln ^{+}\left\{\sum_{\left|c_{q}\right|<\sqrt{\lambda} t} \frac{1}{|t-| c_{q}||}\right\}\left(\frac{1}{t^{k}}-\frac{t^{k}}{r^{2 k}}\right) \frac{d t}{t} \\
& +K\left(\ln ^{+} \frac{1}{\lambda-1}+\ln \lambda+1\right)=\tilde{I}_{2}+K\left(\ln ^{+} \frac{1}{\lambda-1}+\ln \lambda+1\right) .
\end{aligned}
$$


Consider the integral $\tilde{I}_{2}$. Integrating by parts we get

$$
\begin{aligned}
\tilde{I}_{2} & =\int_{1}^{r}\left\{\int_{1}^{t} \ln ^{+}\left[\sum_{\left|c_{q}\right|<\sqrt{\lambda} \tau} \frac{1}{|\tau-| c_{q}||}\right] d \tau\right\}\left\{\frac{k+1}{t^{k+2}}+\frac{k-1}{r^{2 k}} t^{k-2}\right\} d t \\
& \leq 2(k+1) \int_{1}^{r}\left\{\int_{1}^{t} \ln ^{+}\left[\sum_{\left|c_{q}\right|<\sqrt{\lambda} \tau} \frac{1}{|\tau-| c_{q}||}\right] d \tau\right\} \frac{d t}{t^{k+2}} \\
& =4(k+1) \int_{1}^{r}\left\{\int_{1}^{t} \ln ^{+}\left[\sum_{\left|c_{q}\right|<\sqrt{\lambda} \tau} \frac{1}{|\tau-| c_{q}||}\right]^{1 / 2} d \tau\right\} \frac{d t}{t^{k+2}} \\
& \leq 4(k+1) \int_{1}^{r}\left\{\frac{1}{t-1} \int_{1}^{t} \ln ^{+}\left[\sum_{\left|c_{q}\right|<\sqrt{\lambda} \tau} \frac{1}{|\tau-| c_{q}||^{1 / 2}}\right] d \tau\right\} \frac{d t}{t^{k+1}} .
\end{aligned}
$$

To estimate the expression in braces, we use the inequality $\left(1.2^{\prime}\right)$. We get $^{3}$

$$
\begin{aligned}
\frac{1}{t-1} & \int_{1}^{t} \ln ^{+}\left[\sum_{\left|c_{q}\right|<\sqrt{\lambda} \tau} \frac{1}{\left.|\tau-| c_{q}\right|^{1 / 2}}\right] d \tau \\
& \leq \ln ^{+}\left\{\frac{1}{t-1} \int_{0}^{t}\left[\sum_{\left|c_{q}\right|<\sqrt{\lambda} t} \frac{1}{\left.|\tau-| c_{q}\right|^{1 / 2}}\right] d \tau\right\}+\ln 2 \\
& \leq \ln ^{+}\left\{\frac{1}{t-1} \sum_{\left|c_{q}\right|<\sqrt{\lambda} t} \int_{0}^{t} \frac{d \tau}{\left.|\tau-| c_{q}\right|^{1 / 2}}\right\}+\ln 2 \\
& \leq \ln ^{+}\left\{\frac{1}{t-1} n(\sqrt{\lambda} t ; 0, \infty) \int_{-t}^{t} \frac{d \tau}{|\tau|^{1 / 2}}\right\}+\ln 2 \\
& =\ln ^{+}\left\{\frac{1}{t-1} 4 \sqrt{t} n(\sqrt{\lambda} t ; 0, \infty)\right\}+\ln 2 .
\end{aligned}
$$

Since by Lemma 7.1 from Chapter 1,

$$
\begin{gathered}
n(\sqrt{\lambda} t ; 0, \infty) \leq \frac{\lambda t}{\lambda t-\sqrt{\lambda} t} N(\lambda t ; 0, \infty) \leq \\
\frac{\sqrt{\lambda}}{\sqrt{\lambda}-1} 2 T(\lambda t, f) \leq \frac{4 \lambda}{\lambda-1} T(\lambda t, f),
\end{gathered}
$$

thus we get the following estimate for $I_{2}$

$$
I_{2} \leq K\left\{\int_{1}^{r} t^{-k-1} \ln ^{+} T(\lambda t, f) d t+\ln ^{+} \frac{1}{\lambda-1}+\ln \lambda+1\right\} .
$$

\footnotetext{
${ }^{3}$ Below we use Lemma 7.2 from Chapter 1
} 
Therefore

$$
\begin{aligned}
A_{\alpha \beta}\left(r, \frac{f^{\prime}}{f}\right) & \leq K\left\{\int_{1}^{r} t^{-k-1} \ln ^{+} T(\lambda t, f) d t+\ln ^{+} \frac{1}{\lambda-1}+\ln \lambda+1\right\} \\
& \leq K\left\{\lambda^{k} \int_{1}^{\lambda r} t^{-k-1} \ln ^{+} T(t, f) d t+\ln ^{+} \frac{1}{\lambda-1}+\ln \lambda+1\right\} .
\end{aligned}
$$

Recalling that $\lambda=R r^{-1}$, we get the estimate (3.4).

Now we prove (3.3). As in the proof of Theorem 1.3 we observe that we may assume without loss of generality that $f(0)=1$. If the function $f(z)$ satisfies the condition (3.1), then, letting $R=2 r$ in (3.4), we get $A_{\alpha \beta}\left(r, f^{\prime} / f\right)=O(1)$. Now suppose that the condition (3.1) is not satisfied. The inequality (3.4) implies that

$$
A_{\alpha \beta}\left(r, \frac{f^{\prime}}{f}\right) \leq K\left\{\left(\frac{R}{r}\right)^{k} \ln ^{+} T(R, f)+\ln ^{+} \frac{R}{R-r}+\ln \frac{R}{r}+1\right\} .
$$

We set

$$
R=r+\{T(r, f)\}^{-2}
$$

and observe that then the condition $\left(1.7^{\prime}\right)$ is satisfied everywhere except a set of finite measure. We get that, outside a set of finite measure, $A_{\alpha \beta}\left(r, f^{\prime} / f\right)=$ $O(\ln T(r, f)+\ln r)$. This proves (3.3) and concludes the proof of the theorem.

It turns out to be possible to prove a complete analogue of the lemma on the logarithmic derivative for Tsuji characteristics.

Let $f(z)$ be a function, meromorphic in the half-plane $\{\operatorname{Im} z \geq 0\}$. Denote by $\mathfrak{Q}(r, f)$ an arbitrary function defined for $\{1 \leq r<\infty\}$ and such that

1) If $\stackrel{\mathfrak{T}}{\mathfrak{T}}(r, f)$ is of finite order, then $\mathfrak{Q}(r, f)=O(\ln r)$,

2) If $\stackrel{\circ}{\mathfrak{T}}(r, f)$ is of infinite order, then

$$
\mathfrak{Q}(r, f)=O(\ln \stackrel{\circ}{\mathfrak{T}}(r, f)+\ln r),
$$

as $r \rightarrow \infty$, except, possibly, a set of finite measure.

THEOREM 3.2. For each function $f(z)$ meromorphic in the half-plane $\{\operatorname{Im} z \geq$ $0\}$, the relation

$$
\mathfrak{m}\left(r, \frac{f^{\prime}}{f}\right)=\mathfrak{Q}(r, f)
$$

holds.

Proof. First we obtain the following estimate, similar to the estimate (1.1):

$$
\mathfrak{m}\left(r, \frac{f^{\prime}}{f}\right) \leq K\left(\ln ^{+} \stackrel{\circ}{\mathfrak{T}}(R, f)+\ln ^{+} \frac{1}{R-r}+\ln R+1\right),
$$

where $R$ and $r$ are arbitrary numbers satisfying the condition $2 \leq r<R<\infty$, and $K$ is a constant which does not depend on $r$ and $R$. 
We write the formula (1.3) for the disc $\left\{|z| \leq \frac{1}{2} s\right\}$ and the function $f\left(z+\frac{1}{2} i s\right)$, and after that set $z+\frac{1}{2} i s=\zeta$. We get

$$
\begin{aligned}
& \frac{f^{\prime}(\zeta)}{f(\zeta)}=\frac{1}{2 \pi} \int_{0}^{2 \pi} \ln \left|f\left(\frac{s}{2} e^{i \theta}+\frac{i s}{2}\right)\right| \frac{s e^{i \theta}}{\left(\frac{s}{2} e^{i \theta}-\zeta+\frac{i s}{2}\right)^{2}} d \theta \\
& +\sum_{\left|a_{m}-\frac{i s}{2}\right|<\frac{s}{2}} \frac{\frac{s^{2}}{4}-\left|a_{m}-\frac{i s}{2}\right|^{2}}{\left(\frac{s^{2}}{4}-\left[a_{m}-\frac{i s}{2}\right]\left[\zeta-\frac{i s}{2}\right]\right)\left(\zeta-a_{m}\right)} \\
& -\sum_{\left|b_{n}-\frac{i s}{2}\right|<\frac{s}{2}} \frac{\frac{s^{2}}{4}-\left|b_{n}-\frac{i s}{2}\right|^{2}}{\left(\frac{s^{2}}{4}-\overline{\left[b_{n}-\frac{i s}{2}\right]}\left[\zeta-\frac{i s}{2}\right]\right)\left(\zeta-b_{n}\right)},\left|\zeta-i \frac{s}{2}\right|<\frac{s}{2} .
\end{aligned}
$$

Thereafter we use the notation $\zeta=r \sin \vartheta e^{i \vartheta}, \kappa(r) \leq \vartheta \leq \pi-\kappa(r), \kappa(r)=$ $\arcsin \frac{1}{r}, s=\frac{1}{2}(R+r)$, and denote by $K$ positive constants which do not depend on $R$ and $r$. Observe that

$$
\left|\zeta-\frac{i s}{2}\right| \leq\left|r \sin \kappa(r) e^{i \kappa(r)}-\frac{i s}{2}\right|=\sqrt{1-\frac{s}{r}+\frac{s^{2}}{4}},
$$

implies

$$
\frac{s}{2}\left|\zeta-\frac{i s}{2}\right| \geq \frac{s}{2}-\sqrt{1-\frac{s}{r}+\frac{s^{2}}{4}} \geq \frac{s-r}{s t} .
$$

Formula (3.6) implies the inequality

$$
\left|\frac{f^{\prime}(\zeta)}{f(\zeta)}\right| \leq \Phi_{1}(\zeta)+\Phi_{2}(\zeta)
$$

where

$$
\begin{gathered}
\Phi_{1}(\zeta)=\frac{s^{3} r^{2}}{(s-r)^{2}} \frac{1}{2 \pi} \int_{0}^{2 \pi}|\ln | f\left(\frac{s}{2} e^{i \theta}+\frac{i s}{2}\right) \| d \theta, \\
\Phi_{2}(\zeta)=\frac{s^{2} r}{s-r} \sum_{\left|c_{q}-\frac{i s}{2}\right|<\frac{s}{2}} \frac{1}{\left|\zeta-c_{q}\right|},
\end{gathered}
$$

and $\left\{c_{q}\right\}=\left\{a_{m}\right\} \cup\left\{b_{n}\right\}$. By (3.7) we

$$
\begin{aligned}
\mathfrak{m}\left(r, \frac{f^{\prime}}{f}\right) \leq & \frac{1}{2 \pi} \int_{\kappa(r)}^{\pi-\kappa(r)} \ln ^{+}\left[\Phi_{1}(\zeta)+\Phi_{2}(\zeta)\right] \frac{d \vartheta}{r \sin ^{2} \vartheta} \\
\leq & \frac{1}{2 \pi} \int_{\kappa(r)}^{\pi-\kappa(r)} \ln ^{+} \Phi_{1}(\zeta) \frac{d \vartheta}{r \sin ^{2} \vartheta}+\frac{1}{2 \pi} \int_{\kappa(r)}^{\pi-\kappa(r)} \ln ^{+} \Phi_{2}(\zeta) \frac{d \vartheta}{r \sin ^{2} \vartheta} \\
& +\frac{\ln 2}{2 \pi} \int_{\kappa(r)}^{\pi-\kappa(r)} \frac{d \vartheta}{r \sin ^{2} \vartheta}=I_{1}+I_{2}+\frac{\ln 2}{2 \pi} \frac{2}{r} \cot \kappa(r) .
\end{aligned}
$$

Let us estimate the integrals $I_{1}$ and $I_{2}$. To estimate $I_{1}$ observe, that taking $\left[-\frac{\pi}{2}, \frac{3 \pi}{2}\right]$ as an interval of integration instead of $[0,2 \pi]$, and making the change of 
variable $\theta=2 \tau-\frac{\pi}{2}$, we get

$$
\begin{aligned}
\Phi_{1}(\zeta)= & \frac{s^{3} r^{2}}{(s-r)^{2}} \frac{1}{\pi} \int_{0}^{\pi}|\ln | f\left(s \sin \tau e^{i \tau}\right)|| d \tau \\
= & \frac{s^{3} r^{2}}{(s-r)^{2}}\left\{\frac{1}{\pi} \int_{\kappa(s)}^{\pi-\kappa(s)} s \sin ^{2} \tau|\ln | f\left(s \sin \tau e^{i \tau}\right)|| \frac{d \tau}{s \sin ^{2} \tau}\right. \\
& \left.+\frac{1}{\pi}\left[\int_{0}^{\kappa(s)}+\int_{\pi-\kappa(s)}^{\pi}\right]|\ln | f\left(s \sin \tau e^{i \tau}\right)|| d \tau\right\} \\
\leq & \frac{s^{3} r^{2}}{(s-r)^{2}}\{2 s(\mathfrak{m}(s, 0)+\mathfrak{m}(s, \infty))+K\} .
\end{aligned}
$$

Since by Theorem 5.3 from Chapter 1 the inequality $\mathfrak{m}(s, a) \leq \mathfrak{T}(s, f)+O(1)$ holds, and, by Theorem 5.4 from Chapter $1, \mathfrak{T}(s, f)=\stackrel{\mathfrak{T}}{\mathfrak{T}}(s, f)+O(1)$, we have

$$
\Phi_{1}(\zeta) \leq K \frac{R^{6}}{(R-r)^{2}}[\stackrel{\circ}{\mathfrak{T}}(R, f)+1]
$$

Therefore

$$
I_{1} \leq K\left\{\ln ^{+} \stackrel{\circ}{\mathfrak{T}}(R, f)+\ln ^{+} \frac{1}{R-r}+\ln R+1\right\} \frac{2}{\pi r} \cot \kappa(r)
$$

and since $\frac{2}{\pi r} \cot \kappa(r) \leq 1$, we get

$$
I_{1} \leq K\left\{\ln ^{+} \stackrel{\circ}{\mathfrak{T}}(R, f)+\ln ^{+} \frac{1}{R-r}+\ln R+1\right\}
$$

We turn to an estimate of $I_{2}$. We have

$$
\begin{aligned}
I_{2} \leq & \frac{1}{2 \pi} \int_{\kappa(r)}^{\pi-\kappa(r)} \ln ^{+}\left(\sum_{\left|c_{q}-\frac{i s}{2}\right|<\frac{s}{2}} \frac{1}{\left|\zeta-c_{q}\right|}\right) \frac{d \vartheta}{r \sin ^{2} \vartheta} \\
& +K\left(\ln ^{+} \frac{1}{R-r}+\ln R\right)=\tilde{I}_{2}+K\left(\ln ^{+} \frac{1}{R-r}+\ln R\right) .
\end{aligned}
$$

Also we have

$$
\begin{aligned}
\tilde{I}_{2} & =\frac{2}{\pi} \int_{\kappa(r)}^{\pi-\kappa(r)} \ln ^{+}\left(\sum_{\left|c_{q}-i \frac{s}{2}\right|<\frac{s}{2}} \frac{1}{\left|\zeta-c_{q}\right|}\right)^{1 / 4} \frac{d \vartheta}{r \sin ^{2} \vartheta} \\
& \leq \frac{2}{\pi} \int_{\kappa(r)}^{\pi-\kappa(r)} \ln ^{+}\left(\sum_{\left|c_{q}-\frac{i s}{2}\right|<\frac{s}{2}} \frac{1}{\left|\zeta-c_{q}\right|^{1 / 4}}\right) \frac{d \vartheta}{r \sin ^{2} \vartheta}
\end{aligned}
$$


Applying Lemma 1.1 we get

$$
\begin{aligned}
\tilde{I}_{2} \leq & \frac{2}{\pi} \frac{2}{r} \cot \kappa(r) \\
& \times\left\{\ln ^{+}\left[\frac{r}{2 \cot \kappa(r)} \int_{\kappa(r)}^{\pi-\kappa(r)}\left(\sum_{\left|c_{q}-\frac{i s}{2}\right|<\frac{s}{2}} \frac{1}{\left|\zeta-c_{q}\right|^{1 / 4}}\right) \frac{d \vartheta}{r \sin ^{2} \vartheta}\right]+\ln 2\right\} \\
\leq & K\left\{\ln ^{+}\left[\sum_{\left|c_{q}-\frac{i s}{2}\right|<\frac{s}{2}} \int_{\kappa(r)}^{\pi-\kappa(r)} \frac{1}{\left|\zeta-c_{q}\right|^{1 / 4}} \frac{d \vartheta}{r \sin ^{2} \vartheta}\right]+1\right\} \\
\leq & K\left\{\ln ^{+}\left[\sum_{\left|\sum_{q}-\frac{i s}{2}\right|<\frac{s}{2}} \int_{\kappa(r)}^{\pi-\kappa(r)} \frac{d \vartheta}{\left|\zeta-c_{q}\right|^{1 / 4}}\right]+1\right\} .
\end{aligned}
$$

Denoting $\beta_{q}=\arg c_{q}$ we have

$$
\begin{aligned}
\int_{\kappa(r)}^{\pi-\kappa(r)} \frac{d \vartheta}{\left|\zeta-c_{q}\right|^{1 / 4}} & =\int_{\kappa(r)}^{\pi-\kappa(r)} \frac{d \vartheta}{\left|r \sin \vartheta e^{i\left(\vartheta-\beta_{q}\right)}-\right| c_{q}||^{1 / 4}} \\
& \leq \int_{0}^{\pi} \frac{d \vartheta}{\left|r \sin \vartheta \sin \left(\vartheta-\beta_{q}\right)\right|^{1 / 4}} \\
& \leq\left(\int_{0}^{\pi} \frac{d \vartheta}{(\sin \vartheta)^{1 / 2}} \int_{0}^{\pi} \frac{d \vartheta}{\left|\sin \left(\vartheta-\beta_{q}\right)\right|^{1 / 2}}\right)^{1 / 2}=\int_{0}^{\pi} \frac{d \vartheta}{(\sin \vartheta)^{1 / 2}}
\end{aligned}
$$

Therefore $^{4}$

$$
\tilde{I}_{2} \leq K\left\{\ln r+\ln ^{+} \mathfrak{n}(s ; 0, \infty)+1\right\}
$$

Since

$$
\begin{aligned}
\mathfrak{n}(s ; 0, \infty) & \leq \frac{R^{2}}{R-s} \int_{s}^{R} \frac{\mathfrak{n}(t ; 0, \infty)}{t^{2}} d t \leq \frac{R^{2}}{R-s} \mathfrak{N}(R ; 0, \infty) \\
& \leq \frac{R^{2}}{R-s}(2 \stackrel{\circ}{\mathfrak{T}}(R, f)+O(1)) \leq \frac{K R^{2}}{R-r}(\stackrel{\mathfrak{T}}{ }(R, f)+O(1)),
\end{aligned}
$$

we get

$$
\tilde{I}_{2} \leq K\left\{\ln ^{+} \stackrel{\circ}{\mathfrak{T}}(R, f)+\ln ^{+} \frac{1}{R-r}+\ln R+1\right\} .
$$

Using (3.9), (3.10), and (3.11) we get (3.5).

To prove Theorem 3.2 it is enough to let $R=r+\{\stackrel{\circ}{\mathfrak{T}}(r, f)\}^{-2}$ in (3.5).

We turn to analogues of the second fundamental theorem. We say that the characteristics $C_{\alpha \beta}(r, a)$ and $\mathfrak{N}(r, a)$ correspond to the characteristic $N(r, a)$, the characteristics $A_{\alpha \beta}(r, a)+B_{\alpha \beta}(r, a)$ and $\mathfrak{m}(r, a)$ correspond to the characteristic $m(r, a)$, and the characteristics $S_{\alpha \beta}(r, f)$ and $\stackrel{\circ}{\mathfrak{T}}(r, f)$ correspond to the characteristic $T(r, f)$. The characteristics corresponding to $\bar{N}(r, a)$ are the characteristics

\footnotetext{
${ }^{4}$ We set $\mathfrak{n}(r ; a, b)=\mathfrak{n}(r, a)+\mathfrak{n}(r, b) ; \mathfrak{N}(r ; a, b)=\mathfrak{N}(r, a)+\mathfrak{N}(r, b)$.
} 
$\bar{C}_{\alpha \beta}(r, a)$ and $\overline{\mathfrak{N}}(r, a)$ defined in the following way:

$$
\begin{gathered}
\bar{C}_{\alpha \beta}(r, a)=2 \sum_{\substack{1<r_{m}<r \\
\alpha<\varphi_{m}<\beta}}\left(\frac{1}{r_{m}^{k}}-\frac{r_{m}^{k}}{r^{2 k}}\right) \sin k\left(\varphi_{m}-\alpha\right), \\
\overline{\mathfrak{N}}(r, a)=\sum_{1<r_{m}<r \sin \varphi_{m}}\left(\frac{\sin \varphi_{m}}{r_{m}}-\frac{1}{r}\right),
\end{gathered}
$$

where $r_{m} e^{i \varphi_{m}}$ are the $a$-points of the function $f(z)$ counted without multiplicity. We also define in a natural way the characteristics $C_{1 \alpha \beta}(r)$ and $\mathfrak{N}_{1}(r)$ corresponding to the characteristic $N_{1}(r)$, and the characteristics $C_{1 \beta}(r, a)$ and $\mathfrak{N}_{1}(r, a)$ corresponding to the characteristic $N_{1}(r, a)$.

Analyzing the proofs of Theorems 2.1, 2.4, 2.5, and 2.6, we see that they use only those properties of the Nevanlinna characteristic which were described in Theorems 4.1 and 6.1 from Chapter 1, relations (6.1), (6.2), (6.3), (6.4), (4.1) from Chapter 1, and in Theorem 1.3 from Chapter 3. On the other hand, similar theorems and relations are valid also for angular Nevanlinna characteristics (Theorems 5.1 and 6.3 from Chapter 1, relations (6.9), (6.10), (6.12), (6.13) from Chapter 1, Theorem 3.1 from Chapter 3), and for Tsuji characteristics (Theorem 5.3 and 6.3 from Chapter 1, relations (6.9), (6.10), (6.12), (6.13) from Chapter 1, Theorem 3.2 from Chapter 3).

Therefore we have the following theorem.

Theorem 3.3. Theorems 2.1, 2.3, 2.4, 2.5, 2.6 and Lemma 2.1 remain true, if the Nevanlinna characteristics are replaced by the corresponding angular Nevanlinna characteristics, and $Q(r, f)$ is replaced by $Q_{\alpha \beta}(r, f)$.

Theorems 2.1, 2.3, 2.4, 2.5, 2.6 and Lemma 2.1 remain true, if the Nevanlinna characteristics are replaced by the corresponding Tsuji characteristics, and $Q(r, f)$ is replaced by $\mathfrak{Q}(r, f)$. 


\section{CHAPTER 4}

\section{Deficient values}

\section{Exceptional values}

By the first fundamental theorem the relation $m(r, a)+N(r, a)=T(r, f)+O(1)$ holds for each $a$, so the dependence of the sum $m(r, a)+N(r, a)$ on $a$ is inessential. On the other hand, the contribution to this sum of each of the summands can depend heavily on $a$, although, as we shall see later, for "majority" of values of $a$ the summand $N(r, a)$ is dominant. We introduce the following notation:

$$
\begin{gathered}
\delta(a, f)=\delta(a)=\liminf _{r \rightarrow \infty} \frac{m(r, a)}{T(r, f)}=1-\limsup _{r \rightarrow \infty} \frac{N(r, a)}{T(r, f)}, \\
\Delta(a, f)=\Delta(a)=\limsup _{r \rightarrow \infty} \frac{m(r, a)}{T(r, f)}=1-\liminf _{r \rightarrow \infty} \frac{N(r, a)}{T(r, f)}
\end{gathered}
$$

The quantity $\delta(a)$ is called the Nevanlinna deficiency (or simply deficiency) of the function $f(z)$ at the point $a$, the quantity $\Delta(a)$ is called the Valiron deficiency. It is clear that

$$
0 \leq \delta(a) \leq \Delta(a) \leq 1
$$

If $\Delta(a)>0$, then $a$ is called a Valiron exceptional value, if $\delta(a)>0$, then $a$ is called a Nevanlinna exceptional value, or deficient value. Denote by $E_{P}(f), E_{B}(f)$, $E_{N}(f)$, and $E_{V}(f)$ the sets of Picard, Borel, Nevanlinna, and Valiron exceptional values, respectively.

Let $f(z)$ be a transcendental meromorphic function. We already observed in Section 1 of Chapter 2 that $E_{P}(f) \subset E_{B}(f)$. The inequality (1.1) immediately implies that $E_{N}(f) \subset E_{V}(f)$. Since for $a \in E_{P}(f)$ we have $N(r, a)=O(\ln r)$, it is easy to see that $a \in E_{P}(f)$ implies $\delta(a)=\Delta(a)=1$.

Thus

$$
E_{P}(f) \subset E_{N}(f) \subset E_{V}(f) .
$$

Let us show that $E_{B}(f) \subset E_{V}(f)$. In fact, if $a \notin E_{V}(f)$, then $\Delta(a)=0$ and $N(r, a) \sim T(r, f)$. If $n(r, a) \rightarrow \infty$ as $r \rightarrow \infty$, then, by Theorem 1.1 from Chapter 2 the growth category of $n(r, a)$ coincides with the growth category of $N(r, a)$, and thus $a \notin E_{B}(f)$. On the other hand, if $n(r, a)=O(1)$, then $a \in E_{P}(f)$ and, hence, $a$ belongs both to $E_{B}(f)$ and $E_{V}(f)$. Thus we obtain also

$$
E_{P}(f) \subset E_{B}(f) \subset E_{V}(f) .
$$

The question arises: is it possible to find an inclusion between the sets $E_{B}(f)$ and $E_{N}(f)$, and thus to write (1.2) and (1.3) as one inclusion? Examples given below (see Remark 3 at the end of Section 4) and Examples 3 and 4 in Section 2 of Chapter 5 show that there is no general set-theoretical dependence between the sets $E_{B}(f)$ 
and $E_{N}(f)$, namely, for some $a$ and $f$ we can have 1) $a \in E_{B}(f) \cap E_{N}(f)$; 2) $a \in E_{B}(f) \backslash E_{N}(f)$; 3) $a \in E_{N}(f) \backslash E_{B}(f)$.

The problem of the structure of the sets $E_{P}(f)$ and $E_{B}(f)$ is relatively simple. In Theorem 2.2 from Chapter 3 and its Corollary it was shown that for transcendental meromorphic functions $f(z)$ the sets $E_{P}(f)$ and $E_{B}(f)$ consist of at most two points. For meromorphic functions of non-integer order $\rho$ and for meromorphic functions of zero order this statement can be made more precise.

THEOREM 1.1. Transcendent meromorphic function of non-integer or zero order cannot have more than one Borel and hence Picard exceptional value.

Proof. Assume the contrary, that is, the function $f(z)$ has two Borel exceptional values. Without loss of generality we may assume that 0 and $\infty$ are the Borel exceptional values, otherwise we can get into such a situation by using a linear-fractional transformation. First we consider the case $\rho=0$. Then, if $n(r, 0)$ and $n(r, \infty)$ have lower category than $T(r, f)$, we have $n(r, 0)=O(1)$ and $n(r, \infty)=O(1)$. By the Hadamard Theorem (Theorem 4.1 from Chapter 2), the function $f(z)$ is rational, contrary to the assumption. Now we consider the case when $\rho$ (the order of $f(z))$ is non-integer. If both $N(r, 0)$ and $N(r, \infty)$ have lower category than $T(r, f)$, then the same property is shared by $N(r ; 0, \infty)$. By Theorem 4.2 (Chapter 2) it follows that the orders and types of the functions $T(r, f)$ and $N(r ; 0, \infty)$ coincide.

Let us prove that if $N(r ; 0, \infty)$ has convergence class, then $T(r, f)$ also has convergence class. We assume, without loss of generality, that $f(0) \neq 0, \infty$. Let

$$
\int_{0}^{\infty} \frac{N(r ; 0, \infty)}{r^{\rho+1}} d r=M<\infty .
$$

Using the inequality (4.3) from Chapter 2, we get

$$
\begin{aligned}
\int_{1}^{\infty} \frac{T(r, f)}{r^{\rho+1}} d r \leq & O(1) \int_{1}^{\infty} r^{p-\rho-1} d r \\
& +C(p)(p+1) \int_{1}^{\infty} \frac{d r}{r^{\rho+1}} \int_{0}^{\infty} \frac{N(r \tau ; 0, \infty)}{\tau^{p+1}} \frac{d \tau}{1+\tau} \\
= & O(1)+C(p)(p+1) \int_{0}^{\infty} \frac{d \tau}{\tau^{p+1}(1+\tau)} \int_{1}^{\infty} \frac{N(r \tau ; 0, \infty)}{r^{\rho+1}} d r \\
\leq & O(1)+C(p)(p+1) \int_{0}^{\infty} \frac{\tau^{\rho-p-1} d \tau}{1+\tau} \int_{0}^{\infty} \frac{N(t ; 0, \infty)}{t^{\rho+1}} d t \\
= & O(1)+C(p)(p+1) M \frac{\pi}{\sin \pi(\rho-p)}<\infty
\end{aligned}
$$

Legitimacy of the change of order of integration follows from the standard tests. We get a contradiction with the assumption that the category of $N(r ; 0, \infty)$ is lower than the category of $T(r, f)$.

Now we give examples showing that the statements about $E_{P}(f)$ and $E_{B}(f)$ cannot be made more precise. Detailed analysis of these examples is left as an exercise for interested readers.

A. Order $\rho=0$. Each transcendental meromorphic function of zero order with infinitely many zeros and poles and such that $n(r, \infty)=o(n(r, 0))$, does not have Picard and Borel exceptional values. Each transcendental entire function of zero order has $\infty$ as its only Picard and Borel exceptional value. 
B. Order $\rho=n=1,2, \ldots$ The function $\exp \left(z^{n}\right)$ has two Picard exceptional values: 0 and $\infty$. For $f(z)=\sin z^{n}$ the sets $E_{P}(f)=E_{B}(f)$ consist of one point $\infty$. The function $\left(\sin z^{n}\right) / \sinh z^{n}$ does not have Borel exceptional values.

C. The order $\rho$ is non-integer. Let

$$
f(z)=\prod_{n=1}^{\infty} E\left(\frac{z}{n^{1 / \rho}}, p\right), p=[\rho] .
$$

$\infty$ is the only Borel and Picard exceptional value of $f(z)$. The function $f(z) / f(-z)$ does not have Borel (and Picard) exceptional values.

D. The order $\rho=\infty$. The function $\exp \left(e^{z}\right)$ has two Picard exceptional values: 0 and $\infty$; the function $\sin e^{z}$ has $\infty$ as its only Borel and Picard exceptional value; the function $\left(\sin e^{z}\right) / \sinh e^{z}$ does not have Borel exceptional values.

In all of the examples Picard exceptional values were 0 and $\infty$. Using linearfractional transformations they can be replaced by arbitrary numbers $a$ and $b$.

The problem of the characterization of the structure of $E_{V}(f)$ and $E_{N}(f)$ is much more complicated. We shall study this problem in the forthcoming sections.

Note that by Theorems 6.6 and 6.7 from Chapter 1 the sets of exceptional values in all senses under consideration (Picard, Borel, Nevanlinna, and Valiron) of the functions $f(z), f\left(z^{n}\right)$, and $f(A z), n=1,2, \ldots, A \neq 0$, coincide, they also have the same Valiron and Nevanlinna deficiencies. This observation will be repeatedly used later on.

EXERCISE 1. Let $f(z)$ be a canonical product of genus $p \geq 0$ with positive zeros, such that $n(r, 0) \sim A r^{\rho(r)}$, where $0<A<\infty, \rho(r)$ is a proximate order, $\rho(r) \rightarrow \rho$. Using results of Section 5 from Chapter 2 show that

(1) If $\rho=0$, then $\Delta(a)=0$ for all $a \neq \infty$;

(2) If $\rho>0$ and $\rho=p$ or $\rho=p+1$, then $\delta(\infty)=\delta(0)=1$ and $\Delta(a)=0$ for all $a \neq 0, \infty$

(3) If $p<\rho<p+1$, then $\Delta(a)=0$ for all $a \neq 0, \infty$, and

$$
\delta(0, f)=\Delta(0, f)=\omega(\rho)= \begin{cases}\frac{p}{p+|\sin \rho \pi|}, & p<\rho \leq p+\frac{1}{2} \\ \frac{p+1-|\sin \rho \pi|}{p+1}, & p+\frac{1}{2} \leq \rho<p+1\end{cases}
$$

EXERCISE 2. Let $f(z)$ be a canonical product of genus $p \geq 0$ with positive zeros, such that $n(r, 0) \sim A r^{l(r)}$, where $0<A<\infty, l(r)$ is a function differentiable on $\left[r_{0}, \infty\right), r l^{\prime}(r) \ln r \rightarrow 0$ as $r \rightarrow \infty$,

$$
\begin{gathered}
\limsup _{r \rightarrow \infty} l(r)=l_{2}<p+1, \\
\liminf _{r \rightarrow \infty} l(r)=l_{1}>p .
\end{gathered}
$$

Prove that $\Delta(a, f)=0$ for all $a \neq 0, \infty$, and

$$
\begin{aligned}
\delta(0, f) & =\min _{l_{1} \leq t \leq l_{2}} \omega(t), \\
\Delta(0, f) & =\max _{l_{1} \leq t \leq l_{2}} \omega(t),
\end{aligned}
$$

where $\omega(t)$ is defined by (1.4). 
EXERCise 3. Prove that for the Mittag-Leffler function $E_{\rho}(z)$, where $0<\rho<$ $\infty, \rho \neq 1$, the deficiency $\Delta(a)=0$ for all $a \neq \infty$.

ExERCise 4. Let $f(z)$ be a meromorphic function of normal type of order $\rho$, $0<\rho<\infty$. Prove that if $\delta(a)=1$, then $a$ is a Borel exceptional value.

\section{The set of Valiron exceptional values}

We start with the following Valiron theorem.

THEOREM 2.1. For each meromorphic non-identically-constant function $f(z)$ the two-dimensional Lebesgue measure of $E_{V}(f)$ is zero.

Proof. This theorem explains why the values $a$ for which $\Delta(a)>0$ are called exceptional. More precise estimates of "smallness" of the set $E_{V}(f)$ are known, they are due to Valiron and Ahlfors. We are not going to present them, because their statements and proofs require additional background in Potential Theory. ${ }^{1}$

We shall use the notation from Section 4 of Chapter 1. The measure of a measurable set $D$ in an $a$-plane will be denoted by $|D|$, and the two-dimensional measure of the corresponding set $\tilde{D}=p(D) \subset S_{0}$ will be denoted by $|D|_{s}$.

We shall use the following equality:

$$
\iint_{|a| \leq \infty} \ln \frac{1}{[\omega, a]} d \omega(a)=\frac{\pi}{2}
$$

which was proved in Section 4 of Chapter 1 (see the equality (4.19)).

The equality (4.20) from Chapter 1 implies the equality

$$
N(r, a) \leq \stackrel{\circ}{T}(r, f)+\ln \frac{1}{[f(0), a]} .
$$

Let $D$ be a measurable subset of an extended $a$-plane. Integrating the inequality (2.2) over $D$ and using (2.1), we get

$$
\begin{aligned}
& \iint_{D} N(r, a) d \omega(a) \leq \stackrel{\circ}{T}(r, f)|D|_{s}+\iint_{D} \ln \frac{1}{[f(0), a]} d \omega(a) \\
& \quad \leq \stackrel{\circ}{T}(r, f)|D|_{s}+\iint_{|a| \leq \infty} \ln \frac{1}{[f(0), a]} d \omega(a)=\stackrel{\circ}{T}(r, f)|D|_{s}+\frac{\pi}{2} .
\end{aligned}
$$

Let $0<\eta<1 / 2$. Denote by $B(r)$ the set of those values of $a$ for which the inequality

$$
N(r, a) \leq \stackrel{\circ}{T}(r, f)-\stackrel{\circ}{T}^{\frac{1}{2}+\eta}(r, f)
$$

holds.

Since $N(r, a)$ is measurable as a function of $a$, the set $B(r)$ is measurable. Let us show that the inequality

$$
|B(r)|_{s}<\frac{\pi}{\{\stackrel{\circ}{T}(r, f)\}^{\frac{1}{2}+\eta}}
$$

holds. Assume the contrary, that is, assume that

$$
|B(r)|_{s} \geq \frac{\pi}{\{\stackrel{\circ}{T}(r, f)\}^{\frac{1}{2}+\eta}} .
$$

\footnotetext{
${ }^{1}$ Nevanlinna proved that $E_{V}(f)$ always has zero logarithmic capacity.
} 
Denote by $B_{1}(r)$ some subset of $B(r)$ satisfying

$$
\left|B_{1}(r)\right|_{s}=\frac{\pi}{\{\stackrel{\circ}{T}(r, f)\}^{\frac{1}{2}+\eta}},
$$

and let $C(r)=\{|a| \leq \infty\} \backslash B_{1}(r)$. Then, combining (4.18) from Chapter 1 with (2.3), (2.4), and (2.7), we get

$$
\begin{aligned}
\stackrel{\circ}{T}(r, f) & =\frac{1}{\pi} \iint_{B_{1}(r)} N(r, a) d \omega(a)+\frac{1}{\pi} \iint_{C(r)} N(r, a) d \omega(a) \\
& \leq \frac{1}{\pi}\left\{\stackrel{\circ}{T}(r, f)-\stackrel{\circ}{T}^{\frac{1}{2}+\eta}(r, f)\right\}\left|B_{1}(r)\right|_{s}+\frac{1}{\pi} \stackrel{\circ}{T}(r, f)|C(r)|_{s}+\frac{1}{2} \\
& =\stackrel{\circ}{T}(r, f) \frac{\left|S_{0}\right|_{s}}{\pi}-\circ^{\frac{1}{2}+\eta}(r, f) \frac{\left|B_{1}(r)\right|_{s}}{\pi}+\frac{1}{2}=\stackrel{\circ}{T}(r, f)-\frac{1}{2},
\end{aligned}
$$

a contradiction.

Note that for $x>1$ the function $x-x^{\frac{1}{2}+\eta}$ is strictly increasing. Choose $r_{1}$ in such a way that $\stackrel{\circ}{T}\left(r_{1}, f\right)>1$, and define a sequence $\left\{r_{n}\right\}$ using the following recurrence: $r_{n}$ is the (uniquely determined) solution of the equation

$$
\stackrel{\circ}{T}(r, f)-\stackrel{\circ}{T}^{\frac{1}{2}+\eta}(r, f)=\stackrel{\circ}{T}\left(r_{n-1}, f\right), n=2,3, \ldots
$$

Since

$$
\stackrel{\circ}{T}\left(r_{n}, f\right)-\stackrel{\circ}{T}\left(r_{n-1}, f\right)=\stackrel{\circ}{T}^{\frac{1}{2}+\eta}\left(r_{n}, f\right)>1
$$

we have $\stackrel{\circ}{T}\left(r_{n}, f\right) \rightarrow \infty$ as $n \rightarrow \infty$. It is clear that the sequence $\left\{r_{n}\right\}$ is increasing and $\lim _{n \rightarrow \infty} r_{n}=\infty$. If $a \notin B\left(r_{n}\right)$, then the inequality

$$
N(r, a) \geq \stackrel{\circ}{T}(r, f)-2 \stackrel{\circ}{T}^{\frac{1}{2}+\eta}(r, f)
$$

holds for $r_{n} \leq r \leq r_{n+1}$. In fact,

$$
\begin{aligned}
N(r, a) \geq & N\left(r_{n}, a\right)>\stackrel{\circ}{T}\left(r_{n}, f\right)-\stackrel{\circ}{T}^{\frac{1}{2}+\eta}\left(r_{n}, f\right) \\
\geq & \stackrel{\circ}{T}\left(r_{n}, f\right)-\stackrel{\circ}{T}^{\frac{1}{2}+\eta}\left(r_{n}, f\right)+\left\{\stackrel{\circ}{T}(r, f)-\stackrel{\circ}{T}^{\frac{1}{2}+\eta}(r, f)\right\} \\
& -\left\{\stackrel{\circ}{T}\left(r_{n+1}, f\right)-\stackrel{\circ}{T}^{\frac{1}{2}+\eta}\left(r_{n+1}, f\right)\right\} \\
= & \stackrel{\circ}{T}(r, f)-\stackrel{\circ}{T}^{\frac{1}{2}+\eta}(r, f)-\stackrel{\circ}{T}^{\frac{1}{2}+\eta}\left(r_{n}, f\right) \geq \stackrel{\circ}{T}(r, f)-2 \stackrel{\circ}{T}^{\frac{1}{2}+\eta}(r, f) .
\end{aligned}
$$

Hence, if $a \notin \bigcup_{k=n}^{\infty} B\left(r_{k}\right)$, then the inequality (2.9) holds for all $r \geq r_{n}$, thus $\Delta(a)=0$. Therefore

$$
E_{V}(f) \subset \bigcup_{k=n}^{\infty} B\left(r_{k}\right)
$$


for each $n$. On the other hand, by (2.5) and (2.8), we get

$$
\begin{aligned}
& \left|\bigcup_{k=n}^{\infty} B\left(r_{k}\right)\right|_{s} \leq \sum_{k=n}^{\infty}\left|B\left(r_{k}\right)\right|_{s} \leq \pi \sum_{k=n}^{\infty} \stackrel{\circ}{T}^{-\frac{1}{2}-\eta}\left(r_{k}, f\right) \\
& \quad=\pi \sum_{k=n}^{\infty} \frac{\stackrel{\circ}{T}\left(r_{k}, f\right)-\stackrel{\circ}{T}\left(r_{k-1}, f\right)}{\left\{\stackrel{\circ}{T}\left(r_{k}, f\right)\right\}^{1+2 \eta}}=\pi \sum_{k=n}^{\infty} \int_{r_{k-1}}^{r_{k}} \frac{d \stackrel{\circ}{T}(r, f)}{\left\{\stackrel{\circ}{T}\left(r_{k}, f\right)\right\}^{1+2 \eta}} \\
& \quad \leq \pi \sum_{k=n}^{\infty} \int_{r_{k-1}}^{r_{k}} \frac{d \stackrel{\circ}{T}(r, f)}{\{\stackrel{\circ}{T}(r, f)\}^{1+2 \eta}}=\pi \int_{r_{n-1}}^{\infty} \frac{d \stackrel{\circ}{T}(r, f)}{\{\stackrel{\circ}{T}(r, f)\}^{1+2 \eta}}=\frac{\pi}{2 \eta} \frac{1}{\left\{\stackrel{\circ}{T}\left(r_{n-1}, f\right)\right\}^{2 \eta}} .
\end{aligned}
$$

Hence

$$
\lim _{n \rightarrow \infty}\left|\bigcup_{k=n}^{\infty} B\left(r_{k}\right)\right|_{s}=0
$$

and

$$
\left|E_{V}(f)\right|_{s}=0
$$

Denote by $E_{V}^{n}(f)$ the intersection $E_{V}(f) \cap\{|a|<n\}, n=1,2, \ldots$. Since

$$
0=\left|E_{V}^{n}(f)\right|_{s}=\iint_{E_{V}^{n}(f)} \frac{d \sigma(a)}{\left(1+|a|^{2}\right)^{2}} \geq \frac{1}{\left(1+n^{2}\right)^{2}} \iint_{E_{V}^{n}(f)} d \sigma(a)=\frac{\left|E_{V}^{n}(f)\right|}{\left(1+n^{2}\right)^{2}}
$$

we get $\left|E_{V}^{n}(f)\right|=0$. The set $E_{V}(f)$ can differ from $\bigcup_{n=1}^{\infty} E_{V}^{n}(f)$ by one point $a=\infty$ only and has zero two-dimensional measure.

EXERCISE. Using Cartan's identity (4.13) from Chapter 1, prove that the intersection of $E_{V}(f)$ with the unit circle has one-dimensional measure zero. Use this result to show that the intersection of $E_{V}(f)$ with an arbitrary circle or line has one-dimensional measure zero.

ExAmple. The set $E_{V}(f)$ can have the cardinality of a continuum. ${ }^{2}$

Denote $N_{p}=2^{3^{p}}, p=0,1,2, \ldots, q(p)=\left[\log _{2} p\right]+1, p \geq 1\left(\log _{2} p\right.$ denotes the logarithm with base 2). We select $\{\theta(n)\}, n=1,2, \ldots$, to be an integer-valued sequence, so rapidly increasing that $\theta(n+1)>\theta(n)+1$ and

$$
2^{-\theta(q(p))}<\frac{N_{p} !}{\left(2 N_{p}\right) !}
$$

It is clear that for $n \leq N_{p}$ the inequality

$$
2^{-\theta(q(p))}<\frac{n !}{(2 n) !}
$$

holds.

Denote by $M$ the set of those real numbers $x \in\left(\frac{1}{2}, 1\right)$ which can be represented in the form

$$
x=\frac{1}{2}+\frac{1}{2}\left\{\frac{\alpha_{1}}{2^{\theta(1)}}+\frac{\alpha_{2}}{2^{\theta(2)}}+\cdots+\frac{\alpha_{n}}{2^{\theta(n)}}+\ldots\right\},
$$

where $\alpha_{1}, \alpha_{2}, \ldots, \alpha_{n}, \ldots$ is an arbitrary sequence of 0 and 1 with infinitely many 1. The cardinality of the set of such sequences coincides with the cardinality of the

\footnotetext{
${ }^{2}$ Hayman [A72] constructed an example of an entire function such that $E_{V}(f)$ contains an arbitrary given $F_{\sigma}$ set of zero capacity.
} 
interval $(0,1)$ of real numbers. Since the representation of a real number $x$ in the form (2.12) is unique, the set $M$ has cardinality of the continuum.

If $p=\gamma_{1}+\gamma_{2} 2+\cdots+\gamma_{q} 2^{q-1}, q=q(p), \gamma_{j}=0,1$, we let

$$
a_{p}=\frac{1}{2}+\frac{1}{2}\left\{\frac{\gamma_{1}}{2^{\theta(1)}}+\frac{\gamma_{2}}{2^{\theta(2)}}+\cdots+\frac{\gamma_{q}}{2^{\theta(q)}}\right\} .
$$

Now we let $b_{0}=b_{1}=b_{2}=3 / 4$,

$$
b_{n}=a_{p} \text { for } N_{p-1}<n \leq N_{p}, p=1,2, \ldots
$$

It is clear that $1 / 2<b_{j}<1, j \geq 0$. Denote by $\varphi(z)$ the entire function

$$
\varphi(z)=\sum_{j=0}^{\infty} \frac{b_{j}}{j !} z^{j} .
$$

It is clear that $M(r, \varphi)=\varphi(r), \frac{1}{2} e^{r}<\varphi(r)<e^{r}$. Consider the entire function $\psi(z)=2 e^{z}-\varphi(z)$. It is easy to see that $M(r, \psi)=\psi(r)$ and $e^{r}<\psi(r)<2 e^{r}$. Using the inequality (7.1) from Section 7 of Chapter 1, we get

$$
T(r, \psi) \geq \frac{1}{3} \ln \psi\left(\frac{r}{2}\right)>\frac{1}{6} r .
$$

Let $a_{0}$ be a complex number satisfying $\Delta\left(a_{0}, \psi\right)=0$, that is, $N\left(r, a_{0}, \psi\right)=$ $(1+o(1)) T(r, \psi)>\frac{1}{6} r(1+o(1))$. The existence of such a number $a_{0}$ follows from Theorem 2.1. The function

$$
f(z)=\left(\varphi(z)+a_{0}\right) e^{-z}
$$

has the desired properties.

First of all, we have

$$
T(r, f) \leq \ln M(r, f) \leq \ln M\left(r, e^{-z}\right)+\ln M(r, \varphi)+O(1) \leq 2 r+O(1) .
$$

Let us estimate $N(r, 2, f)$ from below. Solutions of the equation $f(z)=2$ coincide with solutions of the equation $2 e^{z}-\varphi(z)=a_{0}$, therefore $N(r, 2, f) \equiv$ $N\left(r, a_{0}, \psi\right)>\frac{1}{6}(1+o(1))$. Hence we get

$$
T(r, f) \geq N(r, 2, f)+O(1) \geq \frac{1}{6} r(1+o(1)) .
$$

Now we show that each $x \in M$ satisfies $\Delta(x, f)=1$. Choose $x \in M$,

$$
x=\frac{1}{2}+\frac{1}{2}\left\{\frac{\gamma_{1}}{2^{\theta(1)}}+\frac{\gamma_{2}}{2^{\theta(2)}}+\cdots+\frac{\gamma_{n}}{2^{\theta(n)}}+\ldots\right\}, \gamma_{j}=0,1 .
$$

Let $\left\{\gamma_{n_{k}}\right\}_{k=1}^{\infty}$ be a subsequence of the sequence $\left\{\gamma_{n}\right\}$ consisting of those members which are equal to 1 . We introduce a correspondence which assigns to $x$ the sequence

$$
p_{k}=\gamma_{1}+\gamma_{2} 2+\cdots+\gamma_{n_{k}} 2^{n_{k}-1}, k=1,2, \ldots
$$

of positive integers. It is clear that $n_{k}=q\left(p_{k}\right)$ and that $p_{k} \rightarrow \infty$ as $k \rightarrow \infty$. Let $r_{k}=\frac{1}{3} N_{p_{k}}$. We estimate $N\left(r_{k}, x, f\right)$ from above. Since the solutions of the equation

$$
\left(\varphi(z)+a_{0}\right) e^{-z}=x
$$

coincide with the solutions of

$$
x e^{z}-\varphi(z)-a_{0}=0,
$$


we have $N\left(r_{k}, x, f\right) \equiv N\left(r_{k}, 0, \Phi(z)\right)$, where

$$
\Phi(z)=x e^{z}-\varphi(z)-a_{0}=-a_{0}+\sum_{j=0}^{\infty} \frac{x-b_{j}}{j !} z^{j} .
$$

Since $x \in\left(\frac{1}{2}, 1\right)$ and $b_{j} \in\left(\frac{1}{2}, 1\right)$, then $\left|x-b_{j}\right|<\frac{1}{2}$ for all $j \geq 0$. By (2.13), (2.16), and (2.11), for $N_{p_{k-1}}<j \leq N_{p_{k}}$ we have

$$
\begin{aligned}
x-b_{j} & =x-a_{p_{k}}=\frac{1}{2} \sum_{n=n_{k}+1}^{\infty} \frac{\gamma_{n}}{2^{\theta(n)}} \leq \frac{1}{2} \sum_{n=n_{k}+1}^{\infty} 2^{-\theta(n)} \\
& <\frac{1}{2} \sum_{n=\theta\left(n_{k}\right)}^{\infty} 2^{-n}=2^{-\theta\left(n_{k}\right)}=2^{-\theta\left(q\left(p_{k}\right)\right)}<\frac{j !}{(2 j) !} .
\end{aligned}
$$

Then for $|z|=r_{k}$ the inequality

$$
\begin{aligned}
|\Phi(z)| & \leq\left|a_{0}\right|+\sum_{j=0}^{\infty} \frac{\left|x-b_{j}\right|}{j !} r_{k}^{j} \\
& \leq\left|a_{0}\right|+\frac{1}{2} \sum_{j=0}^{N_{p_{k}-1}} \frac{r_{k}^{j}}{j !}+\sum_{j=N_{p_{k}-1}+1}^{N_{p_{k}}} \frac{j !}{(2 j) !} \frac{1}{j !} r_{k}^{j}+\frac{1}{2} \sum_{j=N_{p_{k}}+1}^{\infty} \frac{r_{k}^{j}}{j !}
\end{aligned}
$$

holds. Now we estimate each of the sums from the right-hand side of (2.17). We get

$$
\begin{aligned}
\sum_{j=0}^{N_{p_{k}}-1} \frac{r_{k}^{j}}{j !} & \leq r_{k}^{N p_{k}-1} \sum_{j=0}^{\infty} \frac{1}{j !}=e \exp \left\{N_{p_{k}-1} \ln r_{k}\right\} \\
& =e \exp \left\{\left(3 r_{k}\right)^{1 / 3} \ln r_{k}\right\}=o\left(e^{\sqrt{r_{k}}}\right),
\end{aligned}
$$

since $N_{p-1}=N_{p}^{1 / 3}$. We also have

$$
\begin{gathered}
\sum_{j=N_{p_{k}}+1}^{\infty} \frac{r_{k}^{j}}{j !} \leq \sum_{j=N_{p_{k}}+1}^{\infty}\left(\frac{e r_{k}}{j}\right)^{j}<\sum_{j=N_{p_{k}}+1}^{\infty}\left(\frac{e r_{k}}{N_{p_{k}}}\right)^{j}<\sum_{j=1}^{\infty}\left(\frac{e}{3}\right)^{j}=\frac{e}{3-e}, \\
\sum_{j=N_{p_{k}-1}+1}^{N_{p_{k}}} \frac{r_{k}^{j}}{(2 j) !}<\sum_{j=0}^{\infty} \frac{r_{k}^{j}}{(2 j) !}=\cosh \sqrt{r_{k}}=\left\{\frac{1}{2}+o(1)\right\} e^{\sqrt{r_{k}}} .
\end{gathered}
$$

Combining these estimates, we get

Now,

$$
\begin{gathered}
M\left(r_{k}, \Phi\right) \leq\left(\frac{1}{2}+o(1)\right) e^{\sqrt{r_{k}}}, \\
T\left(r_{k}, \Phi\right) \leq \ln M\left(r_{k}, \Phi\right)=\sqrt{r_{k}}+O(1) .
\end{gathered}
$$

$$
N\left(r_{k}, x, f\right)=N\left(r_{k}, 0, \Phi\right) \leq T\left(r_{k}, \Phi\right)+O(1) \leq \sqrt{r_{k}}+O(1) .
$$

From (2.15) and (2.18) we get

$$
\liminf _{r \rightarrow \infty} \frac{N(r, x, f)}{T(r, f)} \leq \liminf _{k \rightarrow \infty} \frac{N\left(r_{k}, x, f\right)}{T\left(r_{k}, f\right)} \leq \lim _{k \rightarrow \infty} \frac{\sqrt{r_{k}}+O(1)}{\frac{1}{6} r_{k}}=0,
$$

that is, $\Delta(x, f)=1$. 
Thus the function $f(z)$ has all desired properties.

\section{Deficiency relation}

In Section 2 of Chapter 3 (see (2.9)) we proved the inequality

$$
\sum_{\nu=1}^{q}\left\{m\left(r, a_{\nu}\right)+N_{1}\left(r, a_{\nu}\right)\right\} \leq 2 T(r, f)+Q(r, f),
$$

where $a_{1}, \ldots, a_{q}$ are arbitrary pairwise distinct complex numbers. Denote by $\varepsilon(a)$ the quantity

$$
\varepsilon(a)=\liminf _{r \rightarrow \infty} \frac{N_{1}(r, a)}{T(r, f)},
$$

and call it the index of ramification (or simply the index) of the function $f(z)$ at the point $a$. If $\varepsilon(a)>0$, then $a$ is called an index value. We choose a sequence $r_{k} \rightarrow \infty$ such that $Q\left(r_{k}, f\right)=o\left(T\left(r_{k}, f\right)\right)$. Such a sequence exists by the definition of $Q(r, f)$ (for rational functions $f(z)$ we use Remark 2 on page ???). Let $r=r_{k}$ in (3.1), divide the obtained inequality by $T\left(r_{k}, f\right)$, and pass to the lower limit. We get

from where we get

$$
\sum_{\nu=1}^{q}\left\{\liminf _{k \rightarrow \infty} \frac{m\left(r_{k}, a_{\nu}\right)}{T\left(r_{k}, f\right)}+\liminf _{k \rightarrow \infty} \frac{N_{1}\left(r_{k}, a_{\nu}\right)}{T\left(r_{k}, f\right)}\right\} \leq 2,
$$

$$
\sum_{\nu=1}^{q}\left\{\delta\left(a_{\nu}\right)+\varepsilon\left(a_{\nu}\right)\right\} \leq 2 .
$$

Since $m(r, a)+N_{1}(r, a) \leq m(r, a)+N(r, a)=T(r, f)+O(1)$, we get

$$
0 \leq \delta(a)+\varepsilon(a) \leq 1 .
$$

We show that the set of those points $a$ for which $\delta(a)+\varepsilon(a)>0$ is at most countable. In fact, let $E$ be the set of those numbers $a$ for which $\delta(a)+\varepsilon(a)>0$, and $E_{k}$ be the set of those numbers $a$ for which $\delta(a)+\varepsilon(a)>\frac{1}{k}$. It is clear that $E=\bigcup_{k=1}^{\infty} E_{k}$, therefore it is enough to show that the sets $E_{k}$ are finite. To do this, suppose that for some $k$ the set $E_{k}$ contains more than $2 k$ points $a_{\nu}, \nu=1, \ldots, n_{k}$, $n_{k}>2 k$. Choosing $q=n_{k}$ in (3.2) we get a contradiction.

If the set of points $a$ for which $\delta(a)+\varepsilon(a)>0$ is finite, then we may let $\left\{a_{\nu}\right\}$ in (3.2) be the set of all these points. If this set can be listed as a sequence $\left\{a_{1}, a_{2}, a_{3}, \ldots\right\}$, then, taking limit in (3.2) as $q \rightarrow \infty$, we get

$$
\sum_{\nu=1}^{\infty}\left\{\delta\left(a_{\nu}\right)+\varepsilon\left(a_{\nu}\right)\right\} \leq 2 .
$$

We combine these cases (of a finite and of a countable set of values $a$ ) writing

$$
\sum_{a}\{\delta(a)+\varepsilon(a)\} \leq 2 .
$$

Despite the fact that the sum is taken over all complex numbers $a$, the summands in this sum are positive for a finite or countable set of values of $a$ only, and the sum is to be understood as a sum of finitely many numbers or a sum of a series with positive terms. The inequality (3.4) is called the deficiency relation, it is one of the main results of the Nevanlinna theory. Since for Picard exceptional values $\delta(a)=1$, Picard theorem follows from (3.4): the set $E_{P}(f)$ contains at most 2 points. Before 
(see Theorem 2.2 from Chapter 3) this theorem was derived as a corollary of the Borel theorem. Note, that we have also proved the following result, which we state as a separate theorem.

THEOREM 3.1. The set $E_{N}(f)$ is at most countable.

EXERCise. Prove that for $f(z)=\sin z$ we have $\Delta(a)=0$ for all $a \neq \infty$, $\delta(\infty)=1, \varepsilon(1)=\varepsilon(-1)=1 / 2$.

\section{The structure of the set of deficient values}

For meromorphic functions of zero order the statement of Theorem 3.1 can be made more precise. In fact, Valiron's theorem (Theorem 4.6, Chapter 2) can be restated in the following way: a meromorphic function of zero order cannot have more than one deficient value.

For meromorphic functions of order $\rho, 0<\rho \leq \infty$, Theorem 3.1 cannot be improved (if we do not impose any restrictions onto meromorphic function in addition to prescribing the magnitude of order).

Theorem 4.1. Let $0<\rho \leq \infty$ and let $M$ be an arbitrary at most countable subset of the extended complex plane ( $M$ can be empty). Then there exists a meromorphic function of order $\rho$ whose set of deficient values coincides with $M$.

Proof. First we prove that for each $\rho, 0 \leq \rho \leq \infty$, there exists a meromorphic function of order $\rho$ which does not have deficient values. Let $\left\{p_{n}\right\}$ be an arbitrary sequence of non-negative integers.

Consider the function

$$
f(z)=\frac{1}{z}+\sum_{n=1}^{\infty} \frac{1}{2^{n}}\left(\frac{1}{z-2^{n}}\right)^{p_{n}+2}=\frac{1}{z}+\psi(z) .
$$

It is clear that the series in (4.1) converges absolutely and uniformly on bounded sets in the complex plane. We show that for an arbitrary choice of the sequence $\left\{p_{n}\right\}$ the set $E_{N}(f)$ is empty. Let $r_{\nu}=2^{\nu-2} 3=2^{\nu} \frac{3}{4}$. For $|z|=r_{\nu}, \nu \geq 3$, we have

$$
\begin{aligned}
|\psi(z)| & \leq \sum_{n=1}^{\infty} \frac{1}{2^{n}}\left(\frac{1}{\left|z-2^{n}\right|}\right)^{p_{n}+2} \leq \sum_{n=1}^{\infty} \frac{1}{2^{n}}\left(\frac{1}{\left|r_{\nu}-2^{n}\right|}\right)^{p_{n}+2} \\
& \leq \sum_{n=1}^{\infty} \frac{1}{2^{n}} \frac{1}{\left|r_{\nu}-2^{n}\right|^{2}}=\sum_{n=1}^{\nu-1} \frac{1}{r_{\nu}^{2} 2^{n}} \frac{1}{\left(1-\frac{2^{n}}{r_{\nu}}\right)^{2}}+\sum_{n=\nu}^{\infty} \frac{1}{2^{3 n}} \frac{1}{\left(1-r_{\nu} 2^{-n}\right)^{2}} \\
& <\frac{1}{r_{\nu}^{2}} \sum_{n=1}^{\nu-1} \frac{1}{2^{n}} \frac{1}{\left(1-\frac{2^{\nu-1}}{r_{\nu}}\right)^{2}}+\sum_{n=\nu}^{\infty} \frac{1}{2^{3 n}} \frac{1}{\left(1-r_{\nu} 2^{-\nu}\right)^{2}} \\
& =\frac{9}{r_{\nu}^{2}} \sum_{n=1}^{\nu-1} \frac{1}{2^{n}}+16 \sum_{n=\nu}^{\infty} \frac{1}{2^{3 n}}<\frac{9}{r_{\nu}^{2}}+\frac{128}{7} \frac{1}{2^{3 \nu}}=\frac{9}{r_{\nu}^{2}}+\frac{54}{7} \frac{1}{r_{\nu}^{3}} .
\end{aligned}
$$

The equality (4.1) implies that

$$
|f(z)|=\frac{1}{r_{\nu}}+O\left(\frac{1}{r_{\nu}^{2}}\right)
$$


holds for $|z|=r_{\nu}$. Hence for $0<|a| \leq \infty$ we have $m\left(r_{\nu}, a, f\right)=O(1), r_{\nu} \rightarrow \infty$, and $m\left(r_{\nu}, 0, f\right)=\ln r_{\nu}+o(1)$. Therefore $\delta(a)=0$ for each $a$ and $E_{N}(f)=\emptyset$.

Now we show that choosing a suitable sequence $\left\{p_{n}\right\}$ we get a function $f(z)$ with prescribed order $\rho$. Note that if $|z|=r$, where $\left|r-2^{n}\right| \geq 1, n=1,2, \ldots$, $r \geq 3$, then

$$
|f(z)| \leq \frac{1}{r}+\sum_{n=1}^{\infty} \frac{1}{2^{n}}\left(\frac{1}{\left|z-2^{n}\right|}\right)^{p_{n}+2} \leq \frac{1}{r}+\sum_{n=1}^{\infty} \frac{1}{2^{n}} \leq \frac{4}{3}
$$

and

$$
m(r, f)=O(1) .
$$

Now, for $2^{n-1} \leq r<2^{n}$ we have

$$
n(r, f)=2 n-1+p_{1}+p_{2}+\cdots+p_{n-1} .
$$

If all $p_{n} \equiv 0$, then $n(r, f) \sim \frac{2}{\ln 2} \ln r, N(r, f) \sim \frac{\ln ^{2} r}{\ln 2}$. Denote by $I$ the set $[3, \infty)$ with the intervals $\left|r-2^{n}\right|<1, n=2,3, \ldots$ removed. By (4.2) we have $T(r, f) \sim \frac{\ln ^{2} r}{\ln 2}$ on $I$, and by monotonicity of $T(r, f)$ this relation remains true for all $r$. For $p_{n} \equiv 0$ the function $f(z)$ has zero order.

Now let $0<\rho<\infty$. Set $p_{n}=\left[2^{n \rho}\right]$. Then (4.3) implies $\left(2^{n-1} \leq r<2^{n}\right)$ :

$$
\begin{gathered}
n(r, f) \sim \frac{2^{n \rho}}{2^{\rho}-1}, \\
(1+o(1)) \frac{r^{\rho}}{2^{\rho}-1} \leq n(r, f) \leq(1+o(1)) \frac{2^{\rho} r^{\rho}}{2^{\rho}-1}, \\
(1+o(1)) \frac{r^{\rho}}{\rho\left(2^{\rho}-1\right)} \leq N(r, f) \leq(1+o(1)) \frac{2^{\rho} r^{\rho}}{\rho\left(2^{\rho}-1\right)} .
\end{gathered}
$$

Using (4.2) as before, we show that

$$
\ln T(r, f) \sim \rho \ln r,
$$

that is, the function $f(z)$ has order $\rho$.

If we set $p_{n}=2^{(n+1)^{2}}$, we get $\left(2^{n-1} \leq r<2^{n}\right)$

$$
\begin{gathered}
n(r, f) \geq p_{n-1}=2^{n^{2}} \geq 2^{\left(\frac{\ln r}{\ln 2}\right)^{2}} \\
\ln T(r, f) \geq \ln N(r, f) \geq \ln n\left(\frac{r}{e}, f\right) \geq \frac{(\ln r-1)^{2}}{\ln 2},
\end{gathered}
$$

that is, the function $f(z)$ has order $\rho=\infty$.

It is easy to give an example of a meromorphic function $f(z)$ of order zero, such that $E_{N}(f)$ consists of one point $a$. If $a=\infty$, we let $f(z)$ be an arbitrary entire function $g(z)$ of zero order; if $a \neq \infty$, we set $f(z)=a+1 / g(z)$.

Now let $0<\rho \leq \infty, M$ be an arbitrary nonempty at most countable set. We show that there exists a meromorphic function $f(z)$ of order $\rho$, for which $E_{N}(f)=$ $M$. It is clear that without loss of generality we may assume that $\infty \notin M$.

We start with the case $\rho=1$. Let $\theta_{j}, j=0, \pm 1, \pm 2, \pm 3, \ldots$, be a sequence of numbers from the interval $(-\pi, \pi), \pi>\theta_{j+1}-\theta_{j}>0, \theta_{j} \rightarrow \pi$ as $j \rightarrow+\infty, \theta_{j} \rightarrow-\pi$ as $j \rightarrow-\infty$. Let $a_{j}, j=0, \pm 1, \pm 2, \ldots$, be a sequence of complex numbers whose set of terms coincides with $M$. 
Let $\left\{c_{j}\right\}$ be a sequence of positive numbers such that

$$
\sum_{j=-\infty}^{\infty} c_{j}\left|a_{j}\right|=S_{1}<\infty, \sum_{j=-\infty}^{\infty} c_{j}=S_{2}<\infty .
$$

Set

$$
\begin{aligned}
f_{1}(z) & =\sum_{j=-\infty}^{\infty} c_{j} a_{j} \exp \left(z e^{-i \theta_{j}}\right), \\
f_{2}(z) & =\sum_{j=-\infty}^{\infty} c_{j} \exp \left(z e^{-i \theta_{j}}\right) .
\end{aligned}
$$

It is clear that these series converge absolutely and uniformly on bounded sets, and represent entire functions. Let

$$
f(z)=\frac{f_{1}(z)}{f_{2}(z)}
$$

We show that the meromorphic function $f(z)$ has the desired properties.

For $|z|=r$ we have $\left|\exp \left(z e^{-i \theta_{j}}\right)\right| \leq e^{r}$, hence

$$
\begin{gathered}
\left|f_{1}(z)\right| \leq \sum_{j=-\infty}^{\infty} c_{j}\left|a_{j}\right| e^{r}=S_{1} e^{r}, \\
\left|f_{2}(z)\right| \leq \sum_{j=-\infty}^{\infty} c_{j} e^{r}=S_{2} e^{r} .
\end{gathered}
$$

Therefore

$$
\begin{aligned}
T(r, f) & \leq T\left(r, f_{1}\right)+T\left(r, f_{2}\right)+O(1) \\
& \leq \ln M\left(r, f_{1}\right)+\ln M\left(r, f_{2}\right)+O(1) \leq 2 r+O(1) .
\end{aligned}
$$

We fix an integer $\nu$. Let $\theta_{j}^{\prime} \equiv \theta_{j}(\bmod 2 \pi), \theta_{\nu}-\pi<\theta_{j}^{\prime} \leq \theta_{\nu}+\pi$. It is clear that $\theta_{\nu \pm 1}^{\prime}=\theta_{\nu \pm 1}$. Let $\eta=\eta(\nu)>0$. We consider the function $f(z)$ in the angle

$$
\frac{\theta_{\nu}+\theta_{\nu-1}}{2}+\eta<\varphi=\arg z<\frac{\theta_{\nu+1}+\theta_{\nu}}{2}-\eta, 0<\eta<\frac{\theta_{\nu+1}-\theta_{\nu-1}}{4} .
$$

We have

$$
\begin{aligned}
f(z)-a_{\nu} & =\frac{f_{1}(z)-a_{\nu} f_{2}(z)}{f_{2}(z)}=\frac{\sum_{j \neq \nu} c_{j}\left(a_{j}-a_{\nu}\right) \exp \left(z e^{-i \theta_{j}^{\prime}}\right)}{\sum_{j} c_{j} \exp \left(z e^{-i \theta_{j}^{\prime}}\right)} \\
& =\frac{\sum_{j \neq \nu} c_{j}\left(a_{j}-a_{\nu}\right) \exp \left\{z\left(e^{-i \theta_{j}^{\prime}}-e^{-i \theta_{\nu}}\right)\right\}}{c_{\nu}+\sum_{j \neq \nu} c_{j} \exp \left\{z\left(e^{-i \theta_{j}^{\prime}}-e^{-i \theta_{\nu}}\right)\right\}} .
\end{aligned}
$$

Observe that

$$
\begin{aligned}
\left|\exp \left\{z\left(e^{-i \theta_{j}^{\prime}}-e^{-i \theta_{\nu}}\right)\right\}\right| & =\exp \left\{r\left(\cos \left(\varphi-\theta_{j}^{\prime}\right)-\cos \left(\varphi-\theta_{\nu}\right)\right)\right\} \\
& =\exp \left\{2 r \sin \left(\varphi-\frac{\theta_{j}^{\prime}+\theta_{\nu}}{2}\right) \sin \frac{\theta_{j}^{\prime}-\theta_{\nu}}{2}\right\} .
\end{aligned}
$$

Let $\theta_{\nu+1} \leq \theta_{j}^{\prime} \leq \theta_{\nu}+\pi$. Then

$$
\eta \leq \frac{\theta_{j}^{\prime}-\theta_{\nu+1}}{2}+\eta<\frac{\theta_{j}^{\prime}+\theta_{\nu}}{2}-\varphi<\frac{\theta_{j}^{\prime}-\theta_{\nu-1}}{2}-\eta<\pi-\eta
$$


and

$$
\sin \left(\varphi-\frac{\theta_{j}^{\prime}+\theta_{\nu}}{2}\right) \sin \frac{\theta_{j}^{\prime}-\theta_{\nu}}{2} \leq-\sin \eta \sin \frac{\theta_{\nu+1}-\theta_{\nu}}{2}=-A_{\nu}^{\prime} \sin \eta .
$$

If $\theta_{\nu}-\pi<\theta_{j}^{\prime} \leq \theta_{\nu-1}$, then

$$
\eta \leq \frac{\theta_{\nu-1}-\theta_{j}^{\prime}}{2}+\eta<\varphi-\frac{\theta_{j}^{\prime}+\theta_{\nu}}{2}<\frac{\theta_{\nu+1}-\theta_{j}^{\prime}}{2}-\eta<\pi-\eta
$$

and

$$
\sin \left(\varphi-\frac{\theta_{j}^{\prime}+\theta_{\nu}}{2}\right) \sin \frac{\theta_{j}^{\prime}-\theta_{\nu}}{2} \leq-\sin \eta \sin \frac{\theta_{\nu}-\theta_{\nu-1}}{2}=-A_{\nu}^{\prime \prime} \sin \eta .
$$

If we denote $A_{\nu}=\min \left(A_{\nu}^{\prime}, A_{\nu}^{\prime \prime}\right)$, then by (4.9) we get the inequality

$$
\left|\exp \left\{z\left(e^{-i \theta_{j}^{\prime}}-e^{-i \theta_{\nu}}\right)\right\}\right| \leq \exp \left\{-2 r A_{\nu} \sin \eta\right\},
$$

valid for all $j \neq \nu$. Now,

$$
\begin{gathered}
\left|\sum_{j \neq \nu} c_{j} \exp \left\{z\left(e^{-i \theta_{j}^{\prime}}-e^{-i \theta_{\nu}}\right)\right\}\right| \leq S_{2} \exp \left\{-2 r A_{\nu} \sin \eta\right\}, \\
\left|\sum_{j \neq \nu} c_{j}\left(a_{j}-a_{\nu}\right) \exp \left\{z\left(e^{-i \theta_{j}^{\prime}}-e^{-i \theta_{\nu}}\right)\right\}\right| \leq\left(S_{1}+\left|a_{\nu}\right| S_{2}\right) \exp \left\{-2 r A_{\nu} \sin \eta\right\} .
\end{gathered}
$$

If $r \geq r_{\nu}(\eta)$, the right-hand side of (4.10) is less than $c_{\nu} / 2$, and by (4.8) and (4.11) we get

$$
\begin{gathered}
\left|f(z)-a_{\nu}\right| \leq \frac{S_{1}+\left|a_{\nu}\right| S_{2}}{c_{\nu}-\frac{c_{\nu}}{2}} \exp \left\{-2 r A_{\nu} \sin \eta\right\} \\
\ln ^{+} \frac{1}{\left|f(z)-a_{\nu}\right|} \geq 2 r A_{\nu} \sin \eta+O(1)
\end{gathered}
$$

uniformly in the angle (4.7). Now

$$
\begin{aligned}
m\left(r, a_{\nu}\right) & \geq \frac{1}{2 \pi} \int_{\frac{\theta_{\nu}+\theta_{\nu-1}}{2}+\eta}^{\frac{\theta_{\nu+1}+\theta_{\nu}}{2}-\eta} \ln ^{+} \frac{1}{\left|f\left(r e^{i \varphi}\right)-a_{\nu}\right|} d \varphi \\
& \geq \frac{1}{\pi}\left(\frac{\theta_{\nu+1}-\theta_{\nu-1}}{2}-2 \eta\right) r A_{\nu} \sin \eta+O(1)=B_{\nu}(\eta) r+O(1),
\end{aligned}
$$

where $B_{\nu}(\eta)>0$. From (4.6) and (4.13) we get

$$
\delta\left(a_{\nu}\right)=\liminf _{r \rightarrow \infty} \frac{m\left(r, a_{\nu}\right)}{T(r, f)} \geq \frac{B_{\nu}(\eta)}{2}>0 .
$$

Thus we have shown that $M \subset E_{N}(f)$. The inequalities (4.6) and (4.13) imply also

$$
B_{0}\left(\eta_{0}\right) r+O(1) \leq T(r, f) \leq 2 r+O(1)
$$

where

Hence $f(z)$ is of order 1 .

$$
0<\eta_{0}<\frac{\theta_{1}-\theta_{-1}}{4} .
$$

Let $\delta$ be an arbitrary positive number. The segment $[-\pi+\delta, \pi-\delta]$ contains finitely many points of the form $\left(\theta_{j}+\theta_{j+1}\right) / 2$. We cover these points by intervals 
of length $2 \eta$ centered at them, where $\eta>0$ is so small that the total length of the intervals is less than $\delta$. Denote by $I$ the union of the intervals. If $\varphi \in[-\pi+\delta, \pi-$ $\delta] \backslash I$, then by (4.12) the functions $\left|f\left(r e^{i \varphi}\right)\right|$ and $\left|f\left(r e^{i \varphi}\right)-a\right|^{-1}, a \notin M, a \neq \infty$ converge uniformly to some bounded functions as $r \rightarrow \infty$.

By Theorem 7.4 from Chapter 1 we conclude that

$$
m(r, a)=o(T(r, f)) \text { and } \delta(a)=0
$$

for $a \notin M$. Thus we have proved that $M=E_{N}(f)$.

Now we show how to change the construction above in order to get similar example in the case $0<\rho<1 / 2$. As before, we choose the sequences $\theta_{j}, a_{j}, c_{j}$. Now the role of $e^{z}$ will be played by the function

$$
V(z)=\prod_{n=1}^{\infty}\left(1+\frac{z}{n^{1 / \rho}}\right)
$$

Results of Section 5 from Chapter 2 imply that

$$
\ln \left|V\left(r e^{i \varphi}\right)\right|=\frac{\pi \cos \rho \varphi}{\sin \pi \rho} r^{\rho}+o\left(r^{\rho}\right), r \rightarrow \infty
$$

uniformly in $\varphi \in[-\pi+\delta, \pi-\delta]$, furthermore, if we replace $=$ by $\leq$ in (4.14), the obtained inequality is satisfied uniformly in $\varphi \in[-\pi, \pi]$. In addition,

$$
\ln M(r, V)=\frac{\pi}{\sin \pi \rho} r^{\rho}+o\left(r^{\rho}\right)
$$

Now we set

$$
\begin{aligned}
f_{1}(z) & =\sum_{j=-\infty}^{\infty} c_{j} a_{j} V\left(z e^{-i \theta_{j}}\right), \\
f_{2}(z) & =\sum_{j=-\infty}^{\infty} c_{j} V\left(z e^{-i \theta_{j}}\right),
\end{aligned}
$$

and, as before, $f(z)=f_{1}(z) / f_{2}(z)$. Instead of (4.6) we get

$$
T(r, f) \leq \ln M\left(r, f_{1}\right)+\ln M\left(r, f_{2}\right)+O(1) \leq \frac{2 \pi}{\sin \pi \rho} r^{\rho}+o\left(r^{\rho}\right) .
$$

Fixing $\theta_{\nu}$ and defining $\theta_{j}^{\prime}$ as before, we get that the equality

$$
f(z)-a_{\nu}=\frac{\sum_{j \neq \nu} c_{j}\left(a_{j}-a_{\nu}\right) \frac{V\left(z e^{-i \theta_{j}^{\prime}}\right)}{V\left(z e^{-i \theta_{\nu}}\right)}}{c_{\nu}+\sum_{j \neq \nu} c_{j} \frac{V\left(z e^{-i \theta_{j}^{\prime}}\right)}{V\left(z e^{-i \theta_{\nu}}\right)}} .
$$

holds in the angle (4.7). Denote by $c_{\rho}(\varphi)$ the periodic with period $2 \pi$ extension of the function $\cos \rho \varphi$ from $[-\pi, \pi]$ to the whole real line. Then the role of the equality (4.9) will be played by the inequality

$$
\ln \left|\frac{V\left(r e^{i\left(\varphi-\theta_{j}^{\prime}\right)}\right)}{V\left(r e^{i\left(\varphi-\theta_{\nu}\right)}\right)}\right| \leq r^{\rho} \frac{\pi}{\sin \pi \rho}\left\{c_{\rho}\left(\varphi-\theta_{j}^{\prime}\right)-c_{\rho}\left(\varphi-\theta_{\nu}\right)\right\}+o\left(r^{\rho}\right) .
$$

To derive (4.18) we use (4.14) with the equality $\operatorname{sign}$ for $\ln \left|V\left(r e^{i\left(\varphi-\theta_{\nu}\right)}\right)\right|$ and with the inequality sign for $\ln \left|V\left(r e^{i\left(\varphi-\theta_{j}^{\prime}\right)}\right)\right|$. In order to continue the proof on the same 
lines as before we need to show that there exists a positive constant $K_{\nu}(\eta)$ such that for $\varphi$ satisfying the inequality (4.7) and for all $j \neq \nu$ the inequality

$$
c_{\rho}\left(\varphi-\theta_{j}^{\prime}\right)-c_{\rho}\left(\varphi-\theta_{\nu}\right)<-K_{\nu}(\eta) .
$$

holds.

The proof of (4.19) is based on simple trigonometric transformations, although often requires tedious computations. We leave the detailed proof of (4.19) to interested readers, we provide only a short sketch of one of the possible proofs.

a) Let $0<\theta \leq \pi$, then $c_{\rho}(\psi)>c_{\rho}(\psi-\theta)$ for $\theta / 2>\psi>\theta / 2-\pi$.

b) Let $-\pi \leq \theta<0$, then $c_{\rho}(\psi)>c_{\rho}(\psi-\theta)$ for $\theta / 2<\psi<\theta / 2+\pi$.

c) Let $0<\theta^{\prime \prime} \leq \theta \leq \pi,-\pi \leq \psi \leq \theta^{\prime \prime} / 2$. Then

$$
\left.\max \left\{c_{\rho}(\psi-\pi), c_{\rho}\left(\psi-\theta^{\prime \prime}\right)\right\} c_{\rho}\right\} \geq c_{\rho}(\psi-\theta) .
$$

d) Let $-\pi \leq \theta \leq \theta^{\prime}<0, \theta^{\prime} / 2 \leq \psi \leq \pi$. Then

$$
\max \left\{c_{\rho}(\psi-\pi), c_{\rho}\left(\psi-\theta^{\prime}\right)\right\} \geq c_{\rho}(\psi-\theta) .
$$

e) If $-\pi \leq \theta^{\prime}<0<\theta^{\prime \prime} \leq \pi, \theta^{\prime} / 2<\psi<\theta^{\prime \prime} / 2, \theta \in[-\pi, \pi] \backslash\left[\theta^{\prime}, \theta^{\prime \prime}\right]$, then

$$
c_{\rho}(\psi)>\max \left\{c_{\rho}\left(\psi-\theta^{\prime}\right), c_{\rho}\left(\psi-\theta^{\prime \prime}\right)\right\} \geq c_{\rho}(\psi-\theta) .
$$

f) If $-\pi \leq \theta^{\prime}<\theta^{\prime} / 2+\eta<0<\theta^{\prime \prime} / 2-\eta<\theta^{\prime \prime} \leq \pi$ and $\theta^{\prime} / 2+\eta \leq \psi \leq \theta^{\prime \prime} / 2-\eta$, then, by the Weierstrass theorem,

$$
c_{\rho}(\psi)-\max \left\{c_{\rho}\left(\psi-\theta^{\prime}\right), c_{\rho}\left(\psi-\theta^{\prime \prime}\right)\right\} \geq A_{\rho}\left(\eta, \theta^{\prime}, \theta^{\prime \prime}\right)>0
$$

and

$$
c_{\rho}(\psi)-c_{\rho}(\psi-\theta) \geq A_{\rho}\left(\eta, \theta^{\prime}, \theta^{\prime \prime}\right) .
$$

for $\theta \in[-\pi, \pi] \backslash\left[\theta^{\prime}, \theta^{\prime \prime}\right]$.

Now it is easy to see that in (4.19) we can set

$$
K_{\nu}(\eta)=A_{\rho}\left(\eta, \theta_{\nu-1}-\theta_{\nu}, \theta_{\nu+1}-\theta_{\nu}\right) .
$$

In order to see this, it is enough to let $\psi=\varphi-\theta_{\nu}, \theta^{\prime}=\theta_{\nu-1}-\theta_{\nu}, \theta^{\prime \prime}=\theta_{\nu+1}-\theta_{\nu}$ in (4.19).

Now, after proving the inequality (4.19), by (4.17), (4.18), and (4.19) we get

$$
\left|f(z)-a_{\nu}\right| \leq \frac{S_{1}+\left|a_{\nu}\right| S_{2}}{c_{\nu}-\frac{c_{\nu}}{2}} \exp \left\{-r^{\rho} \frac{\pi K_{\nu}(\eta)}{\sin \pi \rho}+o\left(r^{\rho}\right)\right\}
$$

for sufficiently large $r$ and for $\varphi$ satisfying (4.7).

Using the inequalities (4.16) and (4.20) as we used the inequalities (4.6) and (4.12) before, we get that $E_{N}(f)=M$ and that $f(z)$ has order $\rho, 0<\rho<1 / 2$.

Considering the functions $f\left(z^{n}\right), n=1,2, \ldots$, we get examples of meromorphic functions with $E_{N}(f)=M$ and an arbitrary order $\rho, 0<\rho<n / 2$. Since $n$ can be chosen to be arbitrarily large, then the order $\rho$ can be an arbitrary positive finite number. Of course, there is no need to consider the case $\rho=1$ separately. We started with the case $\rho=1$ because in this case the function $f(z)$ is particularly simple, and it will be convenient to use it in our construction of an example for $\rho=\infty$

Let $f_{1}(z)$ and $f_{2}(z)$ be given by (4.4) and (4.5), respectively. Set

$$
F_{1}(z)=f_{1}\left(e^{z}\right), F_{2}(z)=f_{2}\left(e^{z}\right), F(z)=F_{1}(z) / F_{2}(z) .
$$

Let

$$
\psi(y)=\sup _{-\infty<j<\infty} \cos \left(y-\theta_{j}\right),-\infty<y<\infty .
$$


It is easy to see that $\psi(y)=\cos \left(y-\theta_{j}\right)$ for $\left(\theta_{j-1}+\theta_{j}\right) / 2 \leq y \leq\left(\theta_{j}+\theta_{j+1}\right) / 2$, $\psi( \pm \pi)=1$. The function $\psi(y)$ is continuous for $-\infty<y<\infty$, periodic with period $2 \pi$, and satisfying the inequality $0<\psi(y) \leq 1$. We easily get

$$
\begin{aligned}
\left|F_{2}(x+i y)\right| & \leq \sum_{j=-\infty}^{\infty} c_{j} \exp \left\{e^{x} \cos \left(y-\theta_{j}\right)\right\} \\
& \leq \sum_{j=-\infty}^{\infty} c_{j} \exp \left\{e^{x} \psi(y)\right\}=S_{2} \exp \left\{e^{x} \psi(y)\right\}
\end{aligned}
$$

and

$$
\left|F_{1}(x+i y)\right| \leq S_{1} \exp \left\{e^{x} \psi(y)\right\} .
$$

Later on we shall need the following

LEMMA 4.1 (Fuchs, Hayman). Let $\varphi(t)$ be a bounded, non-negative function, integrable on each segment $0 \leq t \leq r$, such that there exists a finite limit

$$
\lim _{r \rightarrow \infty} \frac{1}{r} \int_{0}^{r} \varphi(t) d t=l .
$$

Then

$$
I(r)=\frac{1}{2 \pi} \int_{0}^{\pi / 2} e^{r \cos \theta} \varphi(r \sin \theta) d \theta=\frac{\{l+o(1)\} e^{r}}{2 \sqrt{2 \pi r}}, r \rightarrow \infty .
$$

Proof. First we observe that

$$
\frac{1}{2 \pi} \int_{0}^{\pi / 2} e^{r \cos \theta} d \theta=(1+o(1)) \frac{e^{r}}{2 \sqrt{2 \pi r}}, r \rightarrow \infty .
$$

This follows from the equalities

$$
\begin{gathered}
\frac{1}{2 \pi} \int_{0}^{\pi / 2} e^{r \cos \theta} d \theta=\frac{1}{4 \pi} \int_{-\pi / 2}^{\pi / 2} e^{r \cos \theta} d \theta=\frac{1}{4 \pi} \int_{-\pi}^{\pi} e^{r \cos \theta} d \theta+O(1), \\
\frac{1}{2 \pi} \int_{-\pi}^{\pi} e^{r \cos \theta} d \theta=I_{0}(r)=(1+o(1)) \frac{e^{r}}{\sqrt{2 \pi r}} .
\end{gathered}
$$

where $I_{0}(r)=J_{0}(i r)$ is a cylindric function of imaginary parameter.

For convenience of readers who are not acquainted with the formula (4.24), we include an independent proof of formula (4.23).

We start with

$$
\int_{0}^{\pi / 2} e^{r \cos \theta} d \theta=e^{r} \int_{0}^{\pi / 2} e^{r(\cos \theta-1)} d \theta=e^{r} \int_{0}^{\pi / 2} e^{-2 r \sin ^{2} \frac{\theta}{2}} d \theta .
$$

On the other hand,

$$
\begin{aligned}
\int_{0}^{\pi / 2} e^{-2 r \sin ^{2} \frac{\theta}{2}} d \theta & =\int_{0}^{r^{-1 / 4}} e^{-2 r \sin ^{2} \frac{\theta}{2}} d \theta+\int_{r^{-1 / 4}}^{\pi / 2} e^{-2 r \sin ^{2} \frac{\theta}{2}} d \theta \\
& =\int_{0}^{r^{-1 / 4}} e^{-2 r \sin ^{2} \frac{\theta}{2}} d \theta+O\left(e^{-\frac{1}{3} \sqrt{r}}\right), r \rightarrow \infty .
\end{aligned}
$$

But for $0 \leq \theta \leq r^{-1 / 4}$ we have

$$
h(r)\left(\frac{\theta}{2}\right)^{2} \leq \sin ^{2} \frac{\theta}{2} \leq\left(\frac{\theta}{2}\right)^{2},
$$


where $h(r) \rightarrow 1$ as $r \rightarrow \infty$. Therefore

$$
\begin{aligned}
& \int_{0}^{r^{-1 / 4}} e^{-2 r \sin ^{2} \frac{\theta}{2}} d \theta \leq \int_{0}^{r^{-1 / 4}} e^{-\frac{r}{2} h(r) \theta^{2}} d \theta<\int_{0}^{\infty} e^{-\frac{r}{2} h(r) \theta^{2}} d \theta \\
& =\sqrt{\frac{\pi}{2}} \frac{1}{\sqrt{r h(r)}}=(1+o(1)) \sqrt{\frac{\pi}{2 r}}, \\
& \int_{0}^{r^{-1 / 4}} e^{-2 r \sin ^{2} \frac{\theta}{2}} d \theta \geq \int_{0}^{r^{-1 / 4}} e^{-\frac{r}{2} \theta^{2}} d \theta=\int_{0}^{\infty} e^{-\frac{r}{2} \theta^{2}} d \theta-\int_{r^{-1 / 4}}^{\infty} e^{-\frac{r}{2} \theta^{2}} d \theta \\
& \geq \sqrt{\frac{\pi}{2 r}}-\int_{r^{-1 / 4}}^{1} e^{-\frac{r}{2} \theta^{2}} d \theta-\int_{1}^{\infty} e^{-\frac{r}{2} \theta} d \theta \\
& >\sqrt{\frac{\pi}{2 r}}-e^{-\frac{\sqrt{r}}{2}}-\frac{2}{r} e^{-\frac{r}{2}}=(1+o(1)) \sqrt{\frac{\pi}{2 r}} .
\end{aligned}
$$

Hence

$$
\int_{0}^{r^{-1 / 4}} e^{-2 r \sin ^{2} \frac{\theta}{2}} d \theta=(1+o(1)) \sqrt{\frac{\pi}{2 r}}
$$

Using (4.26) and (4.25), we get (4.23).

To complete the proof of the lemma, it is enough to show that

$$
I(r)-\frac{l}{2 \pi} \int_{0}^{\pi / 2} e^{r \cos \theta} d \theta=o\left(\frac{e^{r}}{\sqrt{r}}\right), r \rightarrow \infty .
$$

Since $\varphi(r \sin \theta)=O(1)$

$$
\begin{gathered}
\int_{0}^{r^{-2 / 3}} e^{r \cos \theta} d \theta=O\left(e^{r} r^{-\frac{2}{3}}\right)=o\left(\frac{e^{r}}{\sqrt{r}}\right), \\
\int_{\pi / 3}^{\pi / 2} e^{r \cos \theta} d \theta=O\left(e^{\frac{r}{2}}\right)=o\left(\frac{e^{r}}{\sqrt{r}}\right),
\end{gathered}
$$

in order to prove (4.27), it is enough to check that

$$
\begin{aligned}
\int_{r^{-2 / 3}}^{\pi / 3} e^{r \cos \theta} \varphi(r \sin \theta) d \theta & -l \int_{r^{-2 / 3}}^{\pi / 3} e^{r \cos \theta} d \theta \\
& =\int_{r^{-2 / 3}}^{\pi / 3} e^{r \cos \theta}\{\varphi(r \sin \theta)-l\} d \theta=o\left(\frac{e^{r}}{\sqrt{r}}\right) .
\end{aligned}
$$

Introduce

$$
\chi(\theta)=\frac{e^{r \cos \theta}}{r \cos \theta}, \Phi(\theta)=\int_{0}^{r \sin \theta} \varphi(t) d t-l r \sin \theta
$$

It is easy to see that for $r^{-2 / 3} \leq \theta \leq r^{-1 / 4}$ and for sufficiently large $r$ we have $\chi^{\prime}(\theta)<0$, and also that $\Phi(\theta)=o(r \sin \theta)$ by the condition of the lemma and the 
fact that $r \sin \theta \geq(1+o(1)) r^{1 / 3}$ uniformly in $\theta$ as $r \rightarrow \infty$. Now,

$$
\begin{aligned}
\int_{r^{-2 / 3}}^{\pi / 3} e^{r \cos \theta}\{\varphi(r \sin \theta) & -l\} d \theta=\int_{r^{-2 / 3}}^{\pi / 3} \chi(\theta) d \Phi(\theta) \\
& =\left.\chi(\theta) \Phi(\theta)\right|_{r^{-2 / 3}} ^{\pi / 3}-\int_{r^{-2 / 3}}^{\pi / 3} \chi^{\prime}(\theta) \Phi(\theta) d \theta \\
& =\left.o(\chi(\theta) r \sin \theta)\right|_{r^{-2 / 3}} ^{\pi / 3}-o(1) \int_{r^{-2 / 3}}^{\pi / 3} \chi^{\prime}(\theta) r \sin \theta d \theta \\
& =\left.o(\chi(\theta) r \sin \theta)\right|_{r^{-2 / 3}} ^{\pi / 3}+o(1) \int_{r^{-2 / 3}}^{\pi / 3} \chi(\theta) r \cos \theta d \theta \\
& =o\left(e^{\frac{r}{2}}\right)+o\left(e^{r} r^{-\frac{2}{3}}\right)+o(1) \int_{r^{-2 / 3}}^{\pi / 3} e^{r \cos \theta} d \theta=o\left(\frac{e^{r}}{\sqrt{r}}\right) .
\end{aligned}
$$

Here we used (4.23). Hence the relation (4.28) and thus also the lemma have been proved.

We continue our construction of the example. By (4.21) and (4.22) we have

$$
\begin{aligned}
T(r, F) & \leq T\left(r, F_{1}\right)+T\left(r, F_{2}\right)+O(1)=m\left(r, F_{1}\right)+m\left(r, F_{2}\right)+O(1) \\
& \leq 2 \cdot \frac{1}{2 \pi} \int_{-\pi}^{\pi} e^{r \cos \theta} \psi(r \sin \theta) d \theta+O(1) \\
& =\frac{1}{\pi} \int_{-\pi / 2}^{\pi / 2} e^{r \cos \theta} \psi(r \sin \theta) d \theta+O(1) \\
& =\frac{1}{\pi} \int_{0}^{\pi / 2} e^{r \cos \theta}\{\psi(r \sin \theta)+\psi(-r \sin \theta)\} d \theta+O(1) .
\end{aligned}
$$

Now we apply Lemma 4.1 with $\varphi(t)=\psi(t)+\psi(-t)$. Since the function $\psi(y)$ is periodic with period $2 \pi$, we have

$$
\lim _{r \rightarrow \infty} \frac{1}{r} \int_{0}^{r} \varphi(t) d t=\lim _{r \rightarrow \infty} \frac{1}{r} \int_{-r}^{r} \psi(t) d t=\frac{1}{\pi} \int_{-\pi}^{\pi} \psi(t) d t=A, 0<A<\infty .
$$

The inequality (4.29) and Lemma 4.1 imply that

$$
T(r, F) \leq(1+o(1)) \frac{A e^{r}}{\sqrt{2 \pi r}}, r \rightarrow \infty .
$$

Now we fix $\nu$. Define the function $\psi_{\nu}(y)$ by the equation $\psi_{\nu}(y)=2 A_{\nu} \sin \eta$ for

$$
\frac{\theta_{\nu}+\theta_{\nu-1}}{2}+\eta<y<\frac{\theta_{\nu+1}+\theta_{\nu}}{2}-\eta
$$

where $\eta$ and $A_{\nu}$ are defined in the same way as in the construction of the example for $\rho=1$. If

$$
-\pi \leq y \leq \frac{\theta_{\nu}+\theta_{\nu-1}}{2}+\eta \text { and } \frac{\theta_{\nu+1}+\theta_{\nu}}{2}-\eta \leq y \leq \pi,
$$

we set $\psi_{\nu}(y)=0$. We extend the function $\psi_{\nu}(y)$ as periodic with the period $2 \pi$ from the segment $[-\pi, \pi]$ to the whole real line $\{-\infty<y<\infty\}$. By $\left(4.12_{1}\right)$ we have

$$
\ln ^{+} \frac{1}{\left|F(x+i y)-a_{\nu}\right|}=\ln ^{+} \frac{1}{\left|f\left(e^{x+i y}\right)-a_{\nu}\right|} \geq e^{x} \psi_{\nu}(y)+O(1)
$$


Hence

$$
m\left(r, a_{\nu}, F\right) \geq \frac{1}{2 \pi} \int_{-\pi}^{\pi} e^{r \cos \theta} \psi_{\nu}(r \sin \theta) d \theta+O(1) .
$$

As in the argument above we show that

$$
\begin{aligned}
& \frac{1}{2 \pi} \int_{-\pi}^{\pi} e^{r \cos \theta} \psi_{\nu}(r \sin \theta) d \theta \\
& \quad=\frac{1}{\pi} \int_{0}^{\pi / 2} e^{r \cos \theta}\left\{\psi_{\nu}(r \sin \theta)+\psi_{\nu}(-r \sin \theta)\right\} d \theta+O(1) \\
& \quad=(1+o(1)) \frac{B_{\nu} e^{r}}{\sqrt{2 \pi r}}, r \rightarrow \infty,
\end{aligned}
$$

where

$$
B_{\nu}=\lim _{r \rightarrow \infty} \frac{1}{r} \int_{-r}^{r} \psi_{\nu}(t) d t=\frac{1}{\pi} \int_{-\pi}^{\pi} \psi_{\nu}(t) d t=\frac{1}{\pi}\left(\frac{\theta_{\nu+1}-\theta_{\nu}}{2}-2 \eta\right) 2 A_{\nu} \sin \eta .
$$

The relations (4.31) and (4.32) imply that

$$
m\left(r, a_{\nu}, F\right) \geq(1+o(1)) \frac{B_{\nu} e^{r}}{\sqrt{2 \pi r}}
$$

which, together with (4.30), implies

$$
\delta\left(a_{\nu}, F\right) \geq \frac{B_{\nu}}{A}>0
$$

that is, $M \subset E_{N}(F)$. The proof of the fact that $a \notin M$ implies $a \notin E_{N}(F)$ requires much more complicated estimates and tedious computations. We do not include it.

REMARK 1 . In the case when the set $M$ is finite, the construction of a meromorphic function $f(z)$ for which $E_{N}(f)=M$ becomes much simpler. Constructing the functions $f_{1}(z)$ and $f_{2}(z)$ it is enough to consider finite sums.

REMARK 2. Investigation of the constructed examples would be much simpler and more complete if we use some deep theorems from the theory of entire functions (B. Ya. Levin [Lev80, Chapters II and VI]). Readers acquainted with the material of the mentioned chapters are invited to prove as an exercise, that $a \notin M$ implies $a \notin E_{N}(F)$, where $F(z)$ is a function of infinite order, constructed at the end of the proof.

REMARK 3. It is easy to see that there exists a meromorphic function of an arbitrary order having a deficient value which is not a Borel exceptional value. If $\rho>0$, it is enough to consider a meromorphic function with three deficient values. At least one of them is not a Borel exceptional value by the Borel theorem. If $\rho=0$ the example can be constructed in the following way. Let $g_{1}(z)$ and $g_{2}(z)$ be two Weierstrass canonical products of genus zero with positive zeros such that

$$
n\left(r, 0, g_{1}\right) \sim \ln r, n\left(r, 0, g_{2}\right) \sim \ln ^{2} r .
$$

Then $\infty$ is a deficient value with $\delta(\infty)=1$ for the function $f(z)=g_{2}(z) / g_{1}(-z)$, but $\infty \notin E_{B}(f)$. We leave verification of this statement to interested readers. (Hint: use the asymptotic formulas from Section 5 of Chapter 2.) 


\section{Entire functions having infinitely many deficient values}

Since $\delta(\infty, f)=1$ for each entire function $f(z)$, speaking of deficient values of entire functions we will mean finite deficient values. The problem of the description of the structure of this set for entire functions of finite order was elusive until recently. Although the complete solution is not known yet, ${ }^{3}$ the following result is available.

TheOREM 5.1 (N. U. Arakelyan). Let $M=\left\{a_{k}\right\}_{k=1}^{\infty}$ be an arbitrary countable set, $\rho$ be an arbitrary number satisfying the condition $1 / 2<\rho<\infty$. Then there exists an entire function of order $\rho$ whose set of deficient values contains the set $M$.

The restriction $\rho>1 / 2$ in this theorem is necessary. We shall show in Chapter 5 , page ???, that entire functions of order $\rho \leq 1 / 2$ cannot have deficient values. We note that in proving Theorem 5.1 N. U. Arakelyan disproved R. Nevanlinna's conjecture ([NevR74, Ch. 10, sect. 222]) that an entire function of order $\rho>1 / 2$ cannot have more than $[2 \rho]$ deficient values.

The idea of the proof of Theorem 5.1 can be described in the following way. Let $M=\left\{a_{k}\right\}_{k=1}^{\infty}$, and let $\left\{\Omega_{k}\right\}_{k=1}^{\infty}$ be a sequence of pairwise disjoint sets, such that for $r \geq r(k)$ the circle $\{|z|=r\}$ intersects the set $\Omega_{k}$ along an arc of length $\geq s_{k} r$, where $s_{k}>0, k=1,2, \ldots$ Define on the set $\bigcup_{k=1}^{\infty} \Omega_{k}$ a piece-wise constant function $\varphi(z)$ by the equalities

$$
\varphi(z)=a_{k}, z \in \Omega_{k} .
$$

By the theorems from approximation theory, which we prove below, it turns out that for a special choice of the sets $\Omega_{k}$ it is possible to construct an entire function $G(z)$ of normal type and of order $\rho$, such that

$$
|\varphi(z)-G(z)|<e^{-q_{k}|z|^{\rho}}, z \in \Omega_{k},|z| \geq r(k),
$$

where $q_{k}>0(k=1,2, \ldots)$. Since the set of values $\theta$ for which $\left|G\left(r e^{i \theta}\right)-a_{k}\right|<$ $e^{-q_{k} r^{\rho}}$ for $r \geq r(k)$ has measure $\geq s_{k}$, we have $m\left(r, a_{k}, G\right) \geq \frac{1}{2 \pi} s_{k} q_{k} r^{\rho}(r \geq r(k))$, and hence $\delta\left(a_{k}, G\right)>0, k=1,2, \ldots$.

Now we present the necessary results from the approximation theory.

TheOREM 5.2 (M. V. Keldysh). Let $L$ be a rectifiable curve joining points a and $b$. For each $\varepsilon$ and $d, 0<\varepsilon<1,0<d<1$, there exists a polynomial $P(z)$ such that

a) For all $z$ which are not in the d-neighborhood of $L$ the inequality

$$
\left|\frac{1}{z-a}-P\left(\frac{1}{z-b}\right)\right|<\varepsilon
$$

holds;

b) For all $z$ satisfying $\{|z-b| \geq d\}$, the inequality

$$
\left|P\left(\frac{1}{z-b}\right)\right|<\exp \left\{C\left(1+\ln \frac{1}{\varepsilon d}\right) e^{\frac{C \cdot \operatorname{length}(L)}{d}}\right\}
$$

holds, where $C>1$ is an absolute constant.

\footnotetext{
${ }^{3}$ Now this question is completely solved by Eremenko [A43]: for every at most countable set $E$ in the plane and every $\rho>1 / 2$, there exists an entire function $f$ of order $\rho$ for which $E_{N}(f)=E$.
} 
Proof. Let $l$ be the length of the curve $L, m=\left[\frac{4 l}{d}\right]+1$. We choose on the curve $L$ points $z_{0}=a, z_{1}, z_{2}, \ldots, z_{m-1}, z_{m}=b$ in such a way, that $\max _{0 \leq k \leq m-1} \mid z_{k+1}-$ $z_{k} \mid \leq \frac{1}{4} d$. Now we shall perform a construction using which to each point $z_{k}, k=$ $0,1, \ldots, m$ we assign a polynomial $P_{k}(z)$. The polynomial $P(z)$ whose existence is claimed in the theorem will be equal to $P_{m}(z)$.

Polynomials $P_{k}(z)$ are defined inductively. Let $P_{0}(z) \equiv z$. Suppose that we have already constructed the polynomial $P_{k}(z)$ of degree $n_{k}>k$. We construct the polynomial $P_{k+1}(z)$ in the following way.

The function $P_{k}\left(\frac{1}{z-z_{k}}\right)$ is holomorphic in the region $\left\{\left|z-z_{k+1}\right|>\left|z_{k}-z_{k+1}\right|\right\}$ and is bounded as $z \rightarrow \infty$. Therefore in the described region it is represented by a Laurent series

$$
P_{k}\left(\frac{1}{z-z_{k}}\right)=\sum_{q=0}^{\infty} \frac{A_{q}^{(k)}}{\left(z-z_{k+1}\right)^{q}} .
$$

The polynomial $P_{k+1}(z)$ will be of the form $\sum_{q=0}^{n_{k+1}} A_{q}^{(k)} z^{q}$, where the number $n_{k+1}>k+1$ is suitably chosen.

At the moment we use the notation $n_{k+1}$ for an arbitrary positive integer. Let us estimate the quantity

$$
U\left(n_{k+1}\right)=\max _{\left|z-z_{k+1}\right| \geq d}\left|P_{k}\left(\frac{1}{z-z_{k}}\right)-\sum_{q=0}^{n_{k+1}} \frac{A_{q}^{(k)}}{\left(z-z_{k+1}\right)^{q}}\right| .
$$

For $\left|z-z_{k+1}\right|>r>\left|z_{k}-z_{k+1}\right|$ we have

$$
\begin{aligned}
P_{k} & \left(\frac{1}{z-z_{k}}\right)-\sum_{q=0}^{n_{k+1}} \frac{A_{q}^{(k)}}{\left(z-z_{k+1}\right)^{q}}=\sum_{q=n_{k+1}+1}^{\infty} \frac{A_{q}^{(k)}}{\left(z-z_{k+1}\right)^{q}} \\
& =\sum_{q=n_{k+1}+1}^{\infty} \frac{1}{\left(z-z_{k+1}\right)^{q}} \frac{1}{2 \pi i} \int_{\left|t-z_{k+1}\right|=r} P_{k}\left(\frac{1}{t-z_{k}}\right)\left(t-z_{k+1}\right)^{q-1} d t \\
& =\frac{1}{2 \pi i} \int_{\left|t-z_{k+1}\right|=r} P_{k}\left(\frac{1}{t-z_{k}}\right)\left\{\sum_{q=n_{k+1}+1}^{\infty} \frac{\left(t-z_{k+1}\right)^{q-1}}{\left(z-z_{k+1}\right)^{q}}\right\} d t \\
& =\frac{1}{2 \pi i} \int_{\left|t-z_{k+1}\right|=r} P_{k}\left(\frac{1}{t-z_{k}}\right)\left(\frac{t-z_{k+1}}{z-z_{k+1}}\right)^{n_{k+1}} \frac{d t}{z-t} .
\end{aligned}
$$

Set

$$
M_{k}=\max _{\left|z-z_{k}\right| \geq d}\left|P_{k}\left(\frac{1}{z-z_{k}}\right)\right|
$$

Note that the function $\left(t-z_{k}\right)^{n_{k}} P_{k}\left(\frac{1}{t-z_{k}}\right)$ is holomorphic in the disc $\left\{\left|t-z_{k}\right|<d\right\}$, and on the circle its modulus does not exceed $d^{n_{k}} M_{k}$. By the maximum modulus principle, its modulus does not exceed $d^{n_{k}} M_{k}$ inside the circle also. Hence we have the estimate

$$
\left|P_{k}\left(\frac{1}{t-z_{k}}\right)\right| \leq \frac{d^{n_{k}} M_{k}}{\left|t-z_{k}\right|^{n_{k}}},\left|t-z_{k}\right|<d .
$$


For $\left|t-z_{k+1}\right|=\frac{1}{2} d$ we have $\left|t-z_{k}\right| \geq\left|t-z_{k+1}\right|-\left|z_{k+1}-z_{k}\right| \geq \frac{1}{2} d-\frac{1}{4} d=\frac{1}{4} d$, therefore

$$
\left|P_{k}\left(\frac{1}{t-z_{k}}\right)\right| \leq 4^{n_{k}} M_{k},\left|t-z_{k+1}\right|=\frac{1}{2} d .
$$

Letting $r=\frac{1}{2} d,\left|z-z_{k+1}\right| \geq d$ in (5.1) and estimating the integral, we get the inequality

$$
U\left(n_{k+1}\right) \leq 4^{n_{k}} M_{k}\left(\frac{\frac{1}{2} d}{d}\right)^{n_{k+1}}=M_{k} 2^{2 n_{k}-n_{k+1}} .
$$

If we set

$$
n_{k+1}=2 n_{k}+k+2+\left[\frac{\ln ^{+}\left(M_{k} / \varepsilon\right)}{\ln 2}\right]
$$

then, as is easy to see, we have

$$
U\left(n_{n+1}\right)<\frac{\varepsilon}{2^{k+1}}
$$

Defining $n_{k+1}$ by the formula (5.3), we get the desired polynomial $P_{k+1}(z)$.

Now we check that the polynomial $P(z)=P_{m}(z)$ satisfies the conditions a) and b).

Outside the $d$-neighborhood of the curve $L$ we have

$$
\begin{aligned}
& \left|\frac{1}{z-a}-P\left(\frac{1}{z-b}\right)\right|=\left|P_{0}\left(\frac{1}{z-z_{0}}\right)-P_{m}\left(\frac{1}{z-z_{m}}\right)\right| \\
& \leq \sum_{k=0}^{m-1}\left|P_{k}\left(\frac{1}{z-z_{k}}\right)-P_{k+1}\left(\frac{1}{z-z_{k+1}}\right)\right| \leq \sum_{k=0}^{m-1} U\left(n_{k+1}\right)<\sum_{k=0}^{m-1} \frac{\varepsilon}{2^{k+1}}<\varepsilon .
\end{aligned}
$$

Hence the condition a) is satisfied.

To show that the condition b) is satisfied, we have to estimate $M_{m}$. To do this we need to consider the behavior of the quantities $M_{k}$ and $n_{k}$.

Since the region $\left\{\left|z-z_{k+1}\right|>d\right\}$ is contained in the region $\left\{\left|z-z_{k}\right| \geq \frac{3}{4} d\right\}$, we have

$$
\begin{aligned}
M_{k+1} & =\max _{\left|z-z_{k+1}\right| \geq d}\left|P_{k+1}\left(\frac{1}{z-z_{k+1}}\right)\right| \\
& \leq \max _{\left|z-z_{k+1}\right| \geq d}\left|P_{k+1}\left(\frac{1}{z-z_{k+1}}\right)-P_{k}\left(\frac{1}{z-z_{k}}\right)\right|+\max _{\left|z-z_{k}\right| \geq \frac{3}{4} d}\left|P_{k}\left(\frac{1}{z-z_{k}}\right)\right| .
\end{aligned}
$$

Using the inequalities (5.4) and (5.2), we get the relation

$$
M_{k+1}<\frac{\varepsilon}{2^{k+1}}+\left(\frac{4}{3}\right)^{n_{k}} M_{k} .
$$

Letting $\tilde{M}_{k}=\max \left(M_{k}, \varepsilon\right)$ we have

$$
\tilde{M}_{k+1}<2^{n_{k}} \tilde{M}_{k}
$$

Observe that by (5.3) the inequality $n_{k}<\frac{1}{2} n_{k+1}$ holds, therefore we get from (5.5) that

$$
\tilde{M}_{k+1}<\tilde{M}_{0} 2^{n_{k}+n_{k-1}+\cdots+n_{0}}<\tilde{M}_{0} 2^{n_{k}\left(1+\frac{1}{2}+\frac{1}{2^{2}}+\cdots+\frac{1}{2^{k}}\right)}<\tilde{M}_{0} 2^{2 n_{k}}
$$


Further, by (5.3) we have

$$
n_{k+1}<2\left(n_{k}+k+1+\ln \frac{\tilde{M}_{0} 2^{2 n_{k}}}{\varepsilon}\right) .
$$

Taking into account that $n_{k}>k, n_{0}=1, n_{k-1}<\frac{1}{2} n_{k}$, we have

$$
\begin{gathered}
n_{k+1}<2\left(4 n_{k}+\ln \frac{\tilde{M}_{0}}{\varepsilon}\right), \\
n_{k+1}+\ln \frac{\tilde{M}_{0}}{\varepsilon}<8\left(n_{k}+\ln \frac{\tilde{M}_{0}}{\varepsilon}\right),
\end{gathered}
$$

from where we get

$$
\begin{gathered}
n_{k+1}+\ln \frac{\tilde{M}_{0}}{\varepsilon}<8^{k+1}\left(1+\ln \frac{\tilde{M}_{0}}{\varepsilon}\right), \\
n_{k+1}<2^{3(k+1)}\left(1+\ln ^{+} \frac{M_{0}}{\varepsilon}\right) .
\end{gathered}
$$

Since $M_{0}=\frac{1}{d}$ and hence, $\ln ^{+} \frac{M_{0}}{\varepsilon}=\ln \frac{1}{\varepsilon d}$, and $m<\frac{4 l}{d}+1$, we have

$$
n_{m-1}<2^{\frac{12 l}{d}}\left(1+\ln \frac{1}{\varepsilon d}\right)<e^{\frac{9 l}{d}}\left(1+\ln \frac{1}{\varepsilon d}\right) .
$$

This inequality together with (5.6) imply

$$
\begin{aligned}
M_{m} & <\tilde{M}_{0} 2^{2 n_{m-1}}<\exp \left\{2 n_{m-1}+\ln \frac{1}{\varepsilon d}\right\} \\
& <\exp \left\{2 e^{\frac{9 l}{d}}\left(1+\ln \frac{1}{\varepsilon d}\right)+\ln \frac{1}{\varepsilon d}\right\}<\exp \left\{3 e^{\frac{9 l}{d}}\left(1+\ln \frac{1}{\varepsilon d}\right)\right\} .
\end{aligned}
$$

The proof of the theorem is complete.

We shall need the following lemma, which is a corollary of Theorem 5.2.

Lemma 5.1. Let $\gamma$ be a closed rectifiable curve, $l$ be a rectifiable curve joining a point of the curve $\gamma$ with a point $b$ which does not belong to $\gamma$. Let $0<\varepsilon<1$, $0<d<1$ be given. Then there exists a function $Q(\zeta, z)$, rational in $z$ and having its only pole at $b$, which is defined for $\{\zeta \in \gamma\} \times\{|z|<\infty\}$, piece-wise constant ${ }^{4}$ in $\zeta$ and is such that the following conditions are satisfied:

$\left.\mathrm{a}^{\prime}\right)$ For all $z$ outside the $d$-neighborhood of the curve $\gamma \cup l$ and all $\zeta \in \gamma$ the inequality

holds;

$$
\left|Q(\zeta, z)-\frac{1}{\zeta-z}\right|<\varepsilon
$$

$\left.\mathrm{b}^{\prime}\right)$ For all $z$ from $\{|z-b| \geq d\}$ and all $\zeta \in \gamma$ the inequality

$$
|Q(\zeta, z)|<\exp \left\{C\left(1+\ln \frac{1}{\varepsilon d}\right) e^{\frac{C}{d}\{\operatorname{length}(\gamma)+\text { length }(l)\}}\right\}
$$

holds, where $C>1$ is an absolute constant.

${ }^{4}$ Piece-wise constant means that the curve $\gamma$ can be cut into finitely many pieces $\gamma_{j}$ in such a way that the restriction of $Q(\zeta, z)$ to the set $\left\{\zeta \in \gamma_{j}\right\} \times\{|z|<\infty\}$ does not depend on $\zeta$. 
Proof. Let $\zeta$ be an arbitrary point on the curve $\gamma$. Letting $a=\zeta$ and choosing as $L$ the curve joining $a$ and $b$, and consisting of the curve $l$ and a part of the curve $\gamma$, we apply Theorem 5.2. We denote the obtained function $P\left(\frac{1}{z-b}\right)$ by $R(\zeta, z)$. It is clear that the conditions $\left.\mathrm{a}^{\prime}\right)$ and $\mathrm{b}^{\prime}$ ) will be satisfied for the function $R(\zeta, z)$, but possibly, it will not be piece-wise constant in $\zeta$.

Denote by $U$ the complement to the $d$-neighborhood of the curve $\gamma \cup l$. Since $U$ is a closed set and $R(\zeta, z) \rightarrow 0$ as $z \rightarrow \infty$, the condition $\left.\mathrm{a}^{\prime}\right)$ for $R(\zeta, z)$ implies that

$$
\max _{z \in U}\left|R(\zeta, z)-\frac{1}{\zeta-z}\right|<\varepsilon .
$$

Choose $\varepsilon_{1}(\zeta), 0<\varepsilon_{1}(\zeta)<\varepsilon$, in such a way that the last inequality remains true if we replace $\varepsilon$ by $\varepsilon_{1}(\zeta)$. Then for $\zeta^{\prime} \in \gamma,\left|\zeta-\zeta^{\prime}\right|<\varepsilon_{2}(\zeta)=d^{2}\left(\varepsilon-\varepsilon_{1}(\zeta)\right)$ the inequality

$$
\begin{aligned}
& \max _{z \in U}\left|R(\zeta, z)-\frac{1}{\zeta^{\prime}-z}\right| \\
\leq & \max _{z \in U}\left(\left|R(\zeta, z)-\frac{1}{\zeta-z}\right|+\left|\frac{1}{\zeta-z}-\frac{1}{\zeta^{\prime}-z}\right|\right) \leq \varepsilon_{1}(\zeta)+\frac{\left|\zeta-\zeta^{\prime}\right|}{d^{2}}<\varepsilon
\end{aligned}
$$

holds. By the Heine-Borel lemma there is a finite subset $\left\{a_{j}\right\}$ of $\gamma$ such that for each $\zeta \in \gamma$ there is $a_{j} \in\left\{a_{j}\right\}$ satisfying the condition

$$
\left|a_{j}-\zeta\right|<\varepsilon_{2}\left(a_{j}\right) \text {. }
$$

Denoting by $a_{j(\zeta)}$ one of the points satisfying this condition we can easily check that the function $Q(\zeta, z)=R\left(a_{j(\zeta)}, z\right)$ satisfies the conditions of the lemma.

THEOREM 5.3 (M.V. Keldysh). Let the function $f(z)$ be analytic inside the angle $\{|\arg z|<\beta\}, 0<\beta<\pi$, and satisfy inside this angle the condition

$$
\ln |f(z)|<K\left(1+|z|^{\rho}\right)
$$

where $K>0, \rho>1 / 2$, are constants. For each $\alpha, 0<\alpha<\min \left(\beta, \pi-\frac{\pi}{2 \rho}\right)$, there exists an entire function $G(z)$ whose growth does not exceed the normal type of order $\rho$, such that

$$
G(z)=f(z)+g(z),|\arg z| \leq \alpha,
$$

where $g(z)$ is bounded for $|\arg z| \leq \alpha$.

Proof. Let $\alpha<\gamma<\min \left(\beta, \pi-\frac{\pi}{2 \rho}\right), \kappa=\frac{\pi}{2 \pi-2 \gamma}$. Note that $\kappa<\rho$. We construct an auxiliary function $G(z, \zeta, A)$, which is entire in variable $z$, jointly continuous with respect to the variables $z, \zeta, A$ on the set $\{|z|<\infty\} \times\{|\arg \zeta| \geq$ $\beta\} \times\{A>0\}$, and such that the following conditions are satisfied: $C_{2}<1$

a) $\left|\frac{1}{\zeta-z}-G(z, \zeta, A)\right|<e^{-A C_{1}|\zeta|^{\kappa}}$ for $z \in\{|\arg z| \leq \alpha\} \cup\left\{1<|z|<C_{2}|\zeta|\right\}$,

b) $|G(z, \zeta, A)|<e^{A C_{3}|z|^{\kappa}}$ for $1<|\zeta|<C_{2}^{-1}|z|+1,|z|>1$, where $C_{1}, C_{2}, C_{3}, \ldots$ here and below denote positive constants depending on $\alpha, \beta$, and $\gamma$ only.

Denote by $L(R, \theta)$ the boundary of the region $D(R, \theta)=\{|\pi-\arg z|<\theta,|z|>$ $R\}$ parameterized in the negative direction. We consider the branch of the function $(-z)^{\kappa}$ in $D(R, \theta)$ taking positive values on the ray $\{\arg z=\pi\}$. Observe that the values of the function $(-z)^{\kappa}$ on the rays $\{\arg z=\pi \pm(\pi-\gamma)\}$ are pure imaginary. 
If $z$ is outside the closed region $\bar{D}(R, \pi-\gamma)$ and $\zeta \in\{|\arg \zeta-\pi| \leq \pi-\beta\}$, then by the Cauchy theorem,

$$
\begin{aligned}
\frac{1}{2 \pi i} \int_{L\left(R^{\prime}, \pi-\gamma\right)} \frac{e^{A(-t)^{\kappa}} d t}{(t-\zeta)(t-z)} & -\frac{1}{2 \pi i} \int_{L(R, \pi-\gamma)} \frac{e^{A(-t)^{\kappa}} d t}{(t-\zeta)(t-z)} \\
& = \begin{cases}0 & \text { if }|\zeta|<R<R^{\prime} \\
\frac{e^{A(-\zeta)^{\kappa}}}{\zeta-z} & \text { if } R<|\zeta|<R^{\prime}\end{cases}
\end{aligned}
$$

For $R>|\zeta|$ and $z \notin \bar{D}(R, \pi-\gamma)$ set

$$
G(z, \zeta, A)=\frac{e^{-A(-\zeta)^{\kappa}}}{2 \pi i} \int_{L(R, \pi-\gamma)} \frac{e^{A(-t)^{\kappa}} d t}{(t-\zeta)(t-z)}
$$

It is clear that the integral in (5.9) is an analytic function of $z$ outside the region $D(R, \pi-\gamma)$. By (5.8) the right-hand side of (5.9) does not change if we replace the integration over $L(R<\pi-\gamma)$ by the integration over $L\left(R^{\prime}, \pi-\gamma\right)$ with $R^{\prime}>R$. We infer that the function $G(z, \zeta, A)$ has an analytic continuation into the whole plane $\{|z|<\infty\}$ and thus determines an entire function. We keep the notation $G(z, \zeta, A)$ for this entire function and show that it satisfies the conditions a) and $b)$.

First we verify that a) is satisfied. If $z$ is not in the region $D\left(\frac{1}{2} R, \pi-\alpha\right)$ ( $D(R, \pi-\gamma))$, and $\zeta \in D(R, \pi-\beta)$ then, by (5.8), we have

$$
G(z, \zeta, A)=\frac{1}{\zeta-z}+\frac{e^{-A(-\zeta)^{\kappa}}}{2 \pi i} \int_{L(R, \pi-\gamma)} \frac{e^{A(-t)^{\kappa}} d t}{(t-\zeta)(t-z)}
$$

Since for $|\arg \zeta-\pi| \leq \pi-\beta$ the condition $\operatorname{Re}(-\zeta)^{\kappa} \geq \cos \{\kappa(\pi-\beta)\}|\zeta|^{\kappa}>0$ holds, whereas for $|\arg t-\pi|=\pi-\gamma$ we have $\operatorname{Re}(-t)^{\kappa}=0$, we get the estimate

$$
\begin{aligned}
& \left|G(z, \zeta, A)-\frac{1}{\zeta-z}\right| \leq \frac{e^{-A C_{5}|\zeta|^{\kappa}}}{2 \pi} \\
& \quad \times\left\{\frac{e^{A R^{\kappa}}}{|\zeta|-R} \int_{\substack{|t|=R \\
|\pi-\arg t| \leq \pi-\gamma}} \frac{|d t|}{|t-z|}+\int_{\substack{R<|t|<\infty \\
|\pi-\arg t|=\pi-\gamma}} \frac{|d t|}{|t-\zeta||t-z|}\right\} \\
& =\frac{e^{-A C_{5}|\zeta|^{\kappa}}}{2 \pi}\left\{\frac{e^{A R^{\kappa}}}{|\zeta|-R} I_{1}+I_{2}\right\}, 0<C_{5}<1 .
\end{aligned}
$$

Since the distance between the arc $\{|t|=R,|\arg t-\pi| \leq \pi-\gamma\}$ and the exterior of the region $D\left(\frac{1}{2} R, \pi-\alpha\right)$ is not less than $C_{6} R$, we have $I_{1} \leq C_{7}$. Let us estimate $I_{2}$. Observe that for $|\arg t-\pi|=\pi-\gamma$ and $|\arg \zeta-\pi| \leq \pi-\beta$, the inequality $|t-\zeta| \geq|t| \sin (\beta-\gamma)$ holds. Therefore for $|z| \leq \frac{1}{2} R$ we have

$$
I_{2} \leq 2 \int_{R}^{\infty} \frac{d u}{u \sin (\beta-\gamma)\left(u-\frac{1}{2} R\right)} \leq \frac{4}{R \sin (\beta-\gamma)}
$$

If $|\pi-\arg z| \geq \pi-\alpha$, taking into account $|t-z| \geq|t| \sin (\gamma-\alpha)$, we have

$$
I_{2} \leq 2 \int_{R}^{\infty} \frac{d u}{(u \sin (\beta-\gamma))(u \sin (\gamma-\alpha))} \leq \frac{2}{R \sin (\beta-\gamma) \sin (\gamma-\alpha)}
$$

Hence, if $z$ is outside the region $D\left(\frac{1}{2} R, \pi-\alpha\right)$, then $I_{2} \leq C_{8} R^{-1}$. 
Now we set $R=C_{9}|\zeta|$ in $(5.10)$, where $C_{9}=\left(\frac{1}{2} C_{5}\right)^{1 / \kappa}<1$, and assume that $|z|>1$. Then $R>2|z|>2$, and we get the estimate

$$
\begin{aligned}
\left|G(z, \zeta, A)-\frac{1}{\zeta-z}\right| & \leq \frac{e^{-A C_{5}|\zeta|^{\kappa}}}{2 \pi}\left\{\frac{e^{\frac{1}{2} A C_{5}|\zeta|^{\kappa}}}{\left(C_{9}^{-1}-1\right) R} C_{7}+C_{8} R^{-1}\right\} \\
& \leq C_{10} e^{-\frac{1}{2} A C_{5}|\zeta|^{\kappa}}, 1<|z|<\frac{1}{2} C_{9}|\zeta|,
\end{aligned}
$$

QED.

To verify that the condition b) is satisfied, we set $R=2 C_{2}^{-1}|z|+2$ in (5.9).

We get $\left(1<|\zeta|<C_{2}^{-1}|z|+1=\frac{1}{2} R\right)$

$$
\begin{aligned}
|G(z, \zeta, A)| & \\
& \leq \frac{e^{-A C_{5}|\zeta|^{\kappa}}}{2 \pi}\left\{\frac{e^{C_{2}^{-\kappa} A 4^{\kappa}|z|^{\kappa}}}{R-|\zeta|} 4(\pi-\gamma)+\int_{\substack{R<|t|<\infty \\
|\pi-\arg t|=\pi-\gamma}} \frac{|d t|}{(|t|-|\zeta|)(|t|-|z|)}\right\} \\
& \leq \frac{1}{2 \pi}\left\{e^{A C_{11}|z|^{\kappa}} 4 \pi+2 \int_{R}^{\infty} \frac{d u}{(u-|z|)^{2}}\right\} \\
& \leq \frac{1}{2 \pi}\left\{e^{A C_{11}|z|^{\kappa}} 4 \pi+2\right\} \leq 3 e^{A C_{11}|z|^{\kappa}} .
\end{aligned}
$$

Thus, we have constructed the auxiliary function. Set

$$
G(z, \zeta)=G\left(z, \zeta, 2 K C_{1}^{-1}|\zeta|^{\rho-\kappa}\right),
$$

where $K$ is the same constant as in (5.7). The function $G(z, \zeta)$ is entire in the variable $z$, jointly continuous on the set $\{|z|<\infty\} \times\{|\arg \zeta| \geq \beta\}$, and satisfies the conditions

c)

$$
\left|\frac{1}{\zeta-z}-G(z, \zeta)\right|<e^{-2 K|\zeta|^{\rho}}
$$

for $|\arg z| \leq \alpha$ and for $1<|z|<C_{2}|\zeta|,\left(C_{2}<1\right)$;

$$
|G(z, \zeta)|<e^{K C_{12}|z|^{\rho}}
$$

for $1<|\zeta|<|z|$ (to derive this condition from b) we take into account that $\kappa<\rho$ ).

Now we turn to the construction of the desired entire function $G(z)$. Set

$$
G_{n}(z)=\frac{1}{2 \pi i} \int_{\substack{C_{2}^{-1}<|\zeta|<n \\ \arg \zeta= \pm \beta}} f(\zeta) G(z, \zeta) d \zeta+\frac{1}{2 \pi i} \int_{\substack{|\zeta|=n \\|\arg \zeta| \leq \beta}} f(\zeta) \frac{d \zeta}{\zeta-z}
$$

(the paths here and below are traversed in the following way: the ray $\{\arg \zeta=\beta\}$ is traversed from $\infty$ to 0 , the ray $\{\arg \zeta=-\beta\}$ is traversed from 0 to $\infty$, the circle $\{|\zeta|=$ const $\}$ is traversed counterclockwise).

The function $G_{n}(z)$ is analytic if $|z|<n$. It is clear that for $m>n>C_{2}^{-1}$ we have

$$
\begin{aligned}
& G_{m}(z)-G_{n}(z) \\
& =\frac{1}{2 \pi i} \int_{\substack{n<|\zeta|<m \\
\arg \zeta= \pm \beta}} f(\zeta) G(z, \zeta) d \zeta+\frac{1}{2 \pi i}\left\{\int_{\substack{|\zeta|=m \\
|\arg \zeta| \leq \beta}}-\int_{\substack{|\zeta|=n \\
|\arg \zeta| \leq \beta}}\right\} f(\zeta) \frac{d \zeta}{\zeta-z}
\end{aligned}
$$


By the Cauchy theorem the equality

$$
0=\frac{1}{2 \pi i}\left\{\int_{\substack{n<|\zeta|<m \\ \arg \zeta= \pm \beta}}+\int_{\substack{|\zeta|=m \\|\arg \zeta| \leq \beta}}-\int_{\substack{|\zeta|=n \\|\arg \zeta| \leq \beta}}\right\} f(\zeta) \frac{d \zeta}{\zeta-z} .
$$

holds for $|z|<n$. Subtracting the equality (5.15) from (5.14), we get

$$
G_{m}(z)-G_{n}(z)=\frac{1}{2 \pi i} \int_{\substack{n<|\zeta|<m \\ \arg \zeta= \pm \beta}} f(\zeta)\left\{G(z, \zeta)-\frac{1}{\zeta-z}\right\} d \zeta,|z|<n .
$$

From here, using (5.11), we have for $1<|z|<C_{2} n$

$$
\left|G_{m}(z)-G_{n}(z)\right| \leq \frac{1}{2 \pi} \int_{\substack{n<|\zeta|<m \\ \arg \zeta= \pm \beta}} \exp \left\{K\left(1+|\zeta|^{\rho}\right)-2 K|\zeta|^{\rho}\right\}|d \zeta| .
$$

Since the right-hand side goes to 0 as $n, m \rightarrow \infty$, the sequence $\left\{G_{n}(z)\right\}$ converges to some entire function $G(z)$ uniformly in each disc of finite radius. Let us show that this function has the desired properties.

By the Cauchy theorem for $z \in\left\{C_{2}^{-1}<|z|<n,|\arg z|<\beta\right\}$ we have

$$
f(z)=\frac{1}{2 \pi i}\left\{-\int_{\substack{|\zeta|=C_{2}^{-1} \\|\arg \zeta| \leq \beta}}+\int_{\substack{C_{2}^{-1}<|\zeta|<n \\ \arg \zeta= \pm \beta}}+\int_{\substack{|\zeta|=n \\|\arg \zeta|=\beta}}\right\} f(\zeta) \frac{d \zeta}{\zeta-z} .
$$

Subtracting (5.17) from (5.13) we get

$$
\begin{aligned}
G_{n}(z)-f(z)= & \frac{1}{2 \pi i} \int_{C_{2}^{-1}<|\zeta|<n}^{\arg \zeta= \pm \beta}\left\{f(\zeta)\left\{G(z, \zeta)-\frac{1}{\zeta-z}\right\} d \zeta\right. \\
& -\frac{1}{2 \pi i} \int_{\substack{|\zeta|=C_{2}^{-1} \\
|\arg \zeta| \leq \beta}} f(\zeta) \frac{d \zeta}{\zeta-z} .
\end{aligned}
$$

For $|\arg z| \leq \alpha, 2 C_{2}^{-1}<|z|<n$, by (5.11), we get the estimate

$$
\left|G_{n}(z)-f(z)\right| \leq \frac{1}{2 \pi} \int_{\substack{C_{2}^{-1}<|\zeta|<n \\ \arg \zeta= \pm \beta}} \exp \left\{K\left(1+|\zeta|^{\rho}\right)-2 K|\zeta|^{\rho}\right\}|d \zeta|+e^{K C_{13}}
$$

from where, letting $n \rightarrow \infty$, we infer the relation $\left(|\arg z| \leq \alpha,|z|>C_{2}^{-1}\right)$

$$
|G(z)-f(z)| \leq \frac{1}{2 \pi} \int_{\substack{C_{2}^{-1}<|\zeta|<\infty \\ \arg \zeta= \pm \beta}} \exp \left\{K\left(1+|\zeta|^{\rho}\right)-2 K|\zeta|^{\rho}\right\}|d \zeta|+e^{K C_{13}} .
$$

Thus we have proved that the difference $G(z)-f(z)$ is bounded as $|\arg z| \leq \alpha$.

Now we estimate the growth of the function $G(z)$. Letting $m \rightarrow \infty$ in (5.16) we get $\left(1<|z|<C_{2} n\right)$

$$
\left|G(z)-G_{n}(z)\right| \leq \frac{1}{2 \pi} \int_{\substack{n<|\zeta|<\infty \\ \arg \zeta= \pm \beta}} \exp \left\{K\left(1+|\zeta|^{\rho}\right)-2 K|\zeta|^{\rho}\right\}|d \zeta| .
$$

Hence, for sufficiently large $n$ and for $1<|z|<C_{2} n$, the inequality

$$
\left|G(z)-G_{n}(z)\right|<1
$$

holds, therefore

$$
\left|G(z)-G_{\left[C_{2}^{-1}|z|\right]+1}(z)\right|<1
$$


for sufficiently large $|z|$. Furthermore, by (5.7), (5.12), and (5.13) for $n=\left[C_{2}^{-1}|z|\right]+$ 1 the inequality

$$
\begin{aligned}
\left|G_{n}(z)\right|< & \frac{1}{2 \pi} \int_{\substack{C_{2}^{-1}<|\zeta|<n \\
\arg \zeta= \pm \beta}} \exp \left\{K\left(1+|\zeta|^{\rho}\right)+K C_{12}|z|^{\rho}\right\}|d \zeta| \\
& +\frac{1}{2 \pi} \exp \left\{K\left(1+n^{\rho}\right)\right\} 2 \beta n \leq e^{K C_{15} n^{\rho}} \leq e^{K C_{16}|z|^{\rho}}
\end{aligned}
$$

holds.

By (5.18) and (5.19) for sufficiently large $|z|$ we have $|G(z)|<\exp \left\{K C_{17}|z|^{\rho}\right\}$. The proof of the theorem is complete.

We shall need the following lemma, which can be derived from Theorem 5.3.

Lemma 5.2. Let $f(z)$ be a function satisfying the conditions of Theorem 5.3 for $\frac{1}{2}<\rho \leq 1$. Then, for each $\alpha, 0<\alpha<\min \left(\beta, \frac{1}{2}\left(\pi-\frac{\pi}{2 \rho}\right)\right)$, there exists an entire function $G(z)$ whose growth does not exceed the normal type of order $\rho$, such that

$$
G(z)=f(z)+g(z)
$$

for $|\arg z| \leq \alpha$, where $g(z)$ is a function satisfying the estimate

$$
|g(z)|<e^{-|z|^{\rho}}
$$

in the angle $\{|\arg z| \leq \alpha\}$ for sufficiently large $|z|$.

Proof. For each $\delta, 0<\delta<\frac{1}{2}\left(\pi-\frac{\pi}{2 \rho}\right)$, we can find an entire function $\omega_{\rho}(z ; \delta)$ not exceeding the normal type of order $\rho$ satisfying the estimate

$$
e^{-K(\delta)|z|^{\rho}} \leq\left|\omega_{\rho}(z ; \delta)\right| \leq e^{-2|z|^{\rho}}, 2<K(\delta)<\infty .
$$

in the angle $\left\{|\arg z| \leq \frac{1}{2}\left(\pi-\frac{\pi}{2 \rho}\right)-\delta\right\}$ for sufficiently large $|z|$. If $1 / 2<\rho<1$, this can be done in the following way. Let

$$
\omega(z)=\prod_{k=1}^{\infty}\left(1-\frac{z}{k^{1 / \rho}}\right) .
$$

The asymptotic formula $\left(5.3_{2}\right)$ from Chapter 2 implies that for $0<\delta \leq \arg z \leq$ $\pi-\frac{\pi}{2 \rho}-\delta$ and for sufficiently large $|z|$ the estimate

$$
e^{-K_{1}(\delta)|z|^{\rho}} \leq|\omega(z)| \leq e^{-K_{2}(\delta)|z|^{\rho}}
$$

holds, where $0<K_{2}(\delta)<K_{1}(\delta)<\infty$. Therefore we can let

$$
\omega_{\rho}(z ; \delta)=\omega\left(\lambda_{\delta} z e^{\frac{i}{2}\left(\pi-\frac{\pi}{2 \rho}\right)}\right), \lambda_{\delta}=\left(\frac{2}{K_{2}(\delta)}\right)^{1 / \rho} .
$$

If $\rho=1$, set

$$
\omega_{1}(z ; \delta)=e^{-4 z} .
$$

Choose $\delta$ in such a way that $\{|\arg z| \leq \alpha\} \subset\left\{|\arg z|<\frac{1}{2}\left(\pi-\frac{\pi}{2 \rho}\right)-\delta\right\} \subset$ $\{|\arg z| \leq \beta\}$. Consider the function

$$
f_{1}(z)=f(z) / \omega_{\rho}(z ; \delta) .
$$


It is clear that this function is analytic in the angle $\left\{|\arg z| \leq \frac{1}{2}\left(\pi-\frac{\pi}{2 \rho}\right)-\delta\right\}$ and can be estimated there as

$$
\left|f_{1}(z)\right| \leq e^{K\left(1+|z|^{\rho}\right)}
$$

$(0<K<\infty$ does not depend on $z)$. We apply Theorem 5.3 to $f_{1}(z)$ (the role of the angle $\{|\arg z|<\beta\}$ is played by the angle $\left.\left\{|\arg z|<\frac{1}{2}\left(\pi-\frac{\pi}{2 \rho}\right)-\delta\right\}\right)$. We get an entire function $G_{1}(z)$ not exceeding the normal type of order $\rho$, such that

$$
G_{1}(z)=f_{1}(z)+g_{1}(z)
$$

where $g_{1}(z)$ is bounded for $|\arg z|<\alpha$. Letting $G(z)=G_{1}(z) \omega_{\rho}(z ; \delta)$, we see that we get an entire function with the desired properties.

Now we turn to proof of Theorem 5.1.

Proof. Observe that it suffices to consider the case $1 / 2<\rho \leq 1$. In fact, if $\rho>1$, we choose a positive integer $N$ such that $N<\rho \leq 2 N$. We construct an entire function $G(z)$ of order $\frac{\rho}{2 N}$ whose set of deficient values contains $\left\{a_{k}\right\}_{k=1}^{\infty}$. The function $G\left(z^{2 N}\right)$ has order $\rho$ and, by the remark from Section 1 (p.???) its set of deficient values also contains $\left\{a_{k}\right\}_{k=1}^{\infty}$.

Some notation which we are going to use:

$\alpha$ : a number satisfying $0<\alpha<\frac{1}{2}\left(\pi-\frac{\pi}{2 \rho}\right)$.

$\left\{\theta_{k}\right\}_{k=1}^{\infty}$ : a sequence of positive numbers satisfying the condition $\theta_{k} \uparrow \alpha$ as $k \uparrow+\infty$.

$\theta_{-k}=-\theta_{k}, k=1,2, \ldots, \theta_{0}=0$.

$\eta_{k}=\frac{1}{2} \min \left(\left|\theta_{k+1}-\theta_{k}\right|,\left|\theta_{k}-\theta_{k-1}\right|\right)$.

$\varepsilon_{k}=\exp \left(-2^{10} C \eta_{k}^{-1}\right)$, where $C$ is the constant from Lemma 5.1.

$\delta_{k n}=\exp \left(-\varepsilon_{k} 2^{n \rho}\right)$.

$\Delta_{k}(\kappa)=\left\{\left|\arg z-\theta_{k}\right| \leq \kappa \eta_{k}\right\}, 0<\kappa<1$.

$\sigma_{n}(\lambda)=\left\{(1-\lambda) 2^{n} \leq|z| \leq(1+\lambda) 2^{n+1}\right\}, 0<\lambda<1$.

$E_{k n}^{i}=\Delta_{k}\left(\frac{i+1}{4}\right) \cap \sigma_{n}\left(2^{i-4}\right), i=0,1,2$ (for $k>0$ see Figure 1 ).

$\gamma_{k n}^{i}$ is the boundary of the set $E_{k n}^{i}$.

$l_{k n}^{i}=\left\{|z|=\left(1+2^{i-4}\right) 2^{n+1},(-1)^{n} \theta_{k} \leq(-1)^{n} \arg z \leq \pi\right\}$.

Let $\left\{n_{k}\right\}_{k=1}^{\infty}$ be a sequence consisting of even positive integers, $n_{k} \uparrow \infty$ as $k \uparrow \infty$; define the sequence $\left\{n_{-k}\right\}_{k=1}^{\infty}$ by the equality $n_{-k}=n_{k}+1, k=1,2, \ldots$ Set (see Figure 2)

$$
E_{i}=\bigcup_{|k|=1}^{\infty} \bigcup_{m=0}^{\infty} E_{k, n_{k}+2 m}^{i}
$$

Denote by $\Gamma_{i}$ the boundary of the set $E_{i}$. It is easy to see that

$$
\Gamma_{i}=\bigcup_{|k|=1}^{\infty} \bigcup_{m=0}^{\infty} \gamma_{k, n_{k}+2 m}^{i}
$$


We choose the sequence $\left\{n_{k}\right\}_{k=1}^{\infty}$ to be so rapidly increasing that the conditions (5.21)-(5.24) hold.

$$
\begin{gathered}
\prod_{|k|=1}^{\infty} \prod_{n=n_{k}}^{\infty} \frac{1+\delta_{k n}}{1-\delta_{k n}}<\frac{16}{15}, \\
\varepsilon_{k}^{-1}<2^{n_{k} \rho}, \\
\max \left(2^{n+8},\left|a_{|k|}\right|\right)<\exp \left(\varepsilon_{k} 2^{n \rho-1}\right) \text { for } n \geq n_{k}, \\
\int_{\Gamma_{1}}\left|\zeta^{-2}\right||d \zeta|<1 .
\end{gathered}
$$

Note that $E_{k n}^{0} \subset E_{k n}^{1} \subset E_{k n}^{2}$, and hence $E_{0} \subset E_{1} \subset E_{2}$. Later on we shall use the following convention: we consider the sets $E_{k n}^{i}, \gamma_{k n}^{i}, l_{k n}^{i}$ only for those pairs $(k, n)$ of indices which participate in forming the sets $E_{i}$ (that is, $k \neq 0$, $\left.n=n_{k}+2 m, m=0,1,2, \ldots\right)$.

Our proof is organized in the following way. We would like to approximate the function $\varphi(z)$ defined by the equalities

$$
\varphi(z)=a_{|k|}, \quad z \in E_{k n}^{1},
$$

by an entire function $G(z)$ having normal type of order $\rho$ in such a way that the inequality

$$
|\varphi(z)-G(z)| \leq \exp \left\{-\frac{1}{2} \varepsilon_{k}|z|^{\rho}\right\}, z \in E_{k n}^{0},
$$

holds. To do this, first we construct (Lemma 5.3) a function $\omega(z)$, analytic in the half-plane $\{\operatorname{Re} z>-1\}$ and such that

$$
\begin{gathered}
\left|e^{-\varepsilon_{k} z^{\rho}}\right| \leq|\omega(z)| \leq 2\left|e^{-\varepsilon_{k} z^{\rho}}\right| \text { for } z \in E_{k n}^{1}, \\
|\omega(z)| \leq \exp \left\{(|z|+1)^{\rho}\right\} \text { for } \operatorname{Re} z>-1
\end{gathered}
$$

Next, with the help of the function $\omega(z)$ we construct (Lemma 5.4) a function $F(z)$, analytic in the half-plane $\{\operatorname{Re} z>0\}$, and such that

$$
\begin{gathered}
|\varphi(z)-F(z)|<\frac{1}{2}\left|\exp \left\{-\frac{1}{2} \varepsilon_{k} z^{\rho}\right\}\right|, z \in E_{k n}^{0}, \\
\ln |F(z)| \leq O\left((|z|+1)^{\rho}\right), \operatorname{Re} z>0 .
\end{gathered}
$$

Finally, approximating, by Lemma 5.2, the function $F(z)$ in the angle $\{|\arg z| \leq \alpha\}$ by an entire function $G(z)$, we get the desired function.

In our proofs of Lemmas 5.3 and 5.4 we use an auxiliary function $Q_{i}(\zeta, z)$, defined on the set $\left\{\zeta \in \Gamma_{i}\right\} \times\{|z|<\infty\}, i=0,1,2$. We construct this function first. It is easy to check that the curves $\gamma_{k n}^{i}$ and $l_{k n}^{i}(i=1,2)$ do not intersect the set $E_{i-1}$, and their distance from this set is not less than $d_{k n}=\eta_{k} 2^{n-4}$. Denote by $D_{k n}^{i}$ the $d_{k n}$-neighborhood of the curve $\gamma_{k n}^{i} \cup l_{k n}^{i}$ (see Figure 3). We apply Lemma 5.1 with $\gamma=\gamma_{k n}^{i}, l=l_{k n}^{i}, b=-\left(1+2^{i-4}\right) 2^{n+1}, \varepsilon=4^{-n-2} \exp \left\{-5 \varepsilon_{k} 2^{n \rho}\right\}, d=d_{k n}$. We get a function $Q_{k n}^{i}(\zeta, z)$, defined on the set $\left\{\zeta \in \gamma_{k n}^{i}\right\} \times\{|z|<\infty\}$, holomorphic 
with respect to $z$ in the half-plane $\{\operatorname{Re} z>-1\}$, piecewise-constant with respect to $\zeta$ and satisfying the conditions ${ }^{5}$ :

$$
\begin{gathered}
\left|Q_{k n}^{i}(\zeta, z)-\frac{1}{\zeta-z}\right|<4^{-n-2} \exp \left(-5 \varepsilon_{k} 2^{n \rho}\right), \zeta \in \gamma_{k n}^{i}, z \in C D_{k n}^{i}, \\
\left|Q_{k n}^{i}(\zeta, z)\right|<\exp \left(\varepsilon_{k}^{1 / 2} 2^{n \rho-1}\right), \zeta \in \gamma_{k n}^{i}, \operatorname{Re} z>-1 .
\end{gathered}
$$

We derive the inequality (5.26) from the statement $b^{\prime}$ ) of Lemma 5.1. We have

$$
\frac{1}{\varepsilon d}=\frac{1}{4^{-n-2} \exp \left(-5 \varepsilon_{k} 2^{n \rho}\right) \eta_{k} 2^{n-4}}=\frac{1}{\eta_{k}} 2^{n+8} \exp \left(5 \varepsilon_{k} 2^{n \rho}\right),
$$

but by (5.22), for $n \geq n_{k}$ we have

$$
\frac{1}{\eta_{k}}=\frac{1}{2^{10} C} \ln \frac{1}{\varepsilon_{k}} \leq \frac{1}{\varepsilon_{k}} \leq 2^{n_{k} \rho} \leq 2^{n}<2^{n+8},
$$

and by (5.23) the inequality

$$
2^{n+8}<\exp \left(\varepsilon_{k} 2^{n \rho-1}\right)<\exp \left(\varepsilon_{k} 2^{n \rho}\right)
$$

holds. Therefore

$$
\frac{1}{\varepsilon d}<\exp \left(7 \varepsilon_{k} 2^{n \rho}\right), \ln \frac{1}{\varepsilon d}<7 \varepsilon_{k} 2^{n \rho},
$$

and, taking into account (5.22) again, we get

$$
1+\ln \frac{1}{\varepsilon d}<1+7 \varepsilon_{k} 2^{n \rho}<8 \varepsilon_{k} 2^{n \rho}=\varepsilon_{k} 2^{n \rho+3} .
$$

Further, it is easy to check that length $\left(\gamma_{k n}^{i}\right)<2^{n+3}$, length $\left(l_{k n}^{i}\right)<2^{n+3}$, therefore

$$
\frac{1}{d}(\text { length }(\gamma)+\operatorname{length}(l)) \leq \frac{2^{n+4}}{\eta_{k} 2^{n-4}}=\frac{2^{8}}{\eta_{k}} .
$$

Thus

$$
\begin{aligned}
& \exp \left\{C\left(1+\ln \frac{1}{\varepsilon d}\right) e^{\frac{C}{d}(\text { length }(\gamma)+\operatorname{length}(l))}\right\} \\
& <\exp \left\{C \varepsilon_{k} 2^{n \rho+3} e^{\frac{2^{8} C}{\eta_{k}}}\right\}=\exp \left\{C \varepsilon_{k}^{3 / 4} 2^{n \rho+3}\right\} .
\end{aligned}
$$

Observing that ${ }^{6} C \varepsilon_{k}^{1 / 4}=C \exp \left\{-\frac{2^{8} C}{\eta_{k}}\right\} \leq \frac{\eta_{k}}{2^{8}} e^{-1}<\frac{\pi}{2^{10} e}<2^{-9}$, we get the inequality (5.26).

The function $Q_{k n}^{i}(\zeta, z)$ is defined on the set $\left\{\zeta \in \gamma_{k n}^{i}\right\} \times\{|z|<\infty\}$. Since for fixed $i$ the sets $\gamma_{k n}^{i}$ are pairwise disjoint and their union is equal to $\Gamma_{i}$, we can define on the set $\left\{\zeta \in \Gamma_{i}\right\} \times\{|z|<\infty\}$ a function $Q_{i}(\zeta, z)$ by letting

$$
Q_{i}(\zeta, z)=Q_{k n}^{i}(\zeta, z) \text { for } \zeta \in \gamma_{k n}^{i},|z|<\infty .
$$

As is easy to see, the inequalities (5.25) and (5.26) will remain valid if we replace $Q_{k n}^{i}(\zeta, z)$ by $Q_{i}(\zeta, z)$.

We define a function $\psi(z)$ on the set $E_{2}$ by

$$
\psi(z)=e^{-\varepsilon_{k} z^{\rho}}, z \in E_{k n}^{2} .
$$

\footnotetext{
${ }^{5}$ In this section by $C D$ we denote the complement of the set $D$ in the complex plane.

${ }^{6}$ It is easy to see that the function $e^{x} / x$ takes its minimum value on $(0, \infty)$ at $x=1$, hence $e^{-x} \leq(e x)^{-1}$ for $x>0$.
} 
Observe that for $z \in E_{k n}^{i}$ the inequality

$$
\exp \left\{-\frac{5}{2} \varepsilon_{k} 2^{n \rho}\right\} \leq|\psi(z)| \leq \exp \left\{-\varepsilon_{k} 2^{n \rho-1}\right\}
$$

holds.

Lemma 5.3. There exists a function $\omega(z)$ analytic in the half-plane $\{\operatorname{Re} z>$ $-1\}$, such that

$$
\begin{gathered}
1<|\omega(z) / \psi(z)|<2, \quad z \in E_{1}, \\
|\omega(z)|<\exp \left\{(|z|+1)^{\rho}\right\}, \operatorname{Re} z>-1 .
\end{gathered}
$$

Proof. Set

$$
\omega_{k n}(z)=1+\frac{1}{2 \pi i} \int_{\gamma_{k n}^{2}}\{\psi(\zeta)-1\} Q_{2}(\zeta, z) d \zeta
$$

Since $Q_{i}(\zeta, z)$ is a piecewise-constant function of $\zeta$, and it is analytic with respect to $z$ in the half-plane $\{\operatorname{Re} z>-1\}$, the integral in (5.30) makes sense, and the function $\omega_{k n}(z)$ is analytic in the half-plane $\{\operatorname{Re} z>-1\}$. Let us show that the following inequalities hold:

$$
\begin{gathered}
\left|\omega_{k n}(z)\right|<\exp \left(\varepsilon_{k}^{1 / 2} 2^{n \rho+1}\right), \operatorname{Re} z>-1, \\
3 / 4<\left|\omega_{k n}(z) / \psi(z)\right|<5 / 4, z \in E_{k n}^{1}, \\
\left|\omega_{k n}(z)-1\right|<\delta_{k n}, \quad z \in C D_{k n}^{2} \backslash E_{k n}^{2} .
\end{gathered}
$$

Since length $\left(\gamma_{k n}^{2}\right)<2^{n+3}$ and $|\psi(\zeta)|<1$, using (5.26) we get

$$
\left|\omega_{k n}(z)\right| \leq 1+\frac{1}{2 \pi} \cdot 2 \cdot 2^{n+3} \exp \left(\varepsilon_{k}^{1 / 2} 2^{n \rho}\right)<2^{n+3} \exp \left(\varepsilon_{k}^{1 / 2} 2^{n \rho}\right) .
$$

On the other hand, by $(5.23)$, we have $2^{n+3}<\exp \left(\varepsilon_{k} 2^{n \rho-1}\right)<\exp \left(\varepsilon_{k}^{1 / 2} 2^{n \rho}\right)$, and the inequality (5.31) is proved.

Further we set

$$
\psi_{k n}(z)=1+\frac{1}{2 \pi i} \int_{\gamma_{k n}^{2}}\{\psi(\zeta)-1\} \frac{d \zeta}{\zeta-z} .
$$

By the Cauchy formula,

$$
\psi_{k n}(z)=\left\{\begin{array}{l}
\psi(z), z \in E_{k n}^{1} \\
1, z \in C E_{k n}^{2}
\end{array}\right.
$$

Since

$$
\omega_{k n}(z)-\psi_{k n}(z)=\frac{1}{2 \pi i} \int_{\gamma_{k n}^{2}}\{\psi(\zeta)-1\}\left\{Q_{2}(\zeta, z)-\frac{1}{\zeta-z}\right\} d \zeta,
$$

by application of (5.25), we get that for $z \in C D_{k n}^{2}$ the inequality

$$
\begin{aligned}
\left|\omega_{k n}(z)-\psi_{k n}(z)\right| & \leq \frac{1}{2 \pi} \cdot 2 \cdot 2^{n+3} \cdot 4^{-n-2} \exp \left(-5 \varepsilon_{k} 2^{n \rho}\right) \\
& <\frac{1}{4} \exp \left(-5 \varepsilon_{k} 2^{n \rho}\right)
\end{aligned}
$$

holds, from where, by (5.34) and (5.27), we get

$$
\left|\omega_{k n}(z)-\psi(z)\right|<\frac{1}{4}|\psi(z)|, z \in E_{k n}^{1} \cap C D_{k n}^{2}=E_{k n}^{1},
$$


thus (5.32) is proved. The relation (5.33) follows immediately from (5.35) and (5.34).

We define the desired function $\omega(z)$ as

$$
\omega(z)=\frac{3}{2} \prod_{|k|=1}^{\infty} \prod_{m=0}^{\infty} \omega_{k, n_{k}+2 m}(z)
$$

The infinite product converges absolutely and uniformly in each bounded region $\Omega$ from $\{\operatorname{Re} z>-1\}$, and, hence, the function $\omega(z)$ is analytic in this half-plane. In fact, for sufficiently large $n$ we have $\Omega \subset C D_{k n}^{2} \backslash E_{k n}^{2}$, hence, all $\omega_{k, n_{k}+2 m}(z)$ except possibly finitely many satisfy the inequality (5.33). Since (5.21) implies the convergence of the series

$$
\sum_{|k|=1}^{\infty} \sum_{m=0}^{\infty} \delta_{k, n_{k}+2 m}
$$

we can apply Theorem 3.1 from Chapter 2 .

Now let $z \in E_{1}$. Since the sets $E_{k n}^{1}$ are pairwise disjoint, $z$ belongs to only one of them; for the corresponding multiplier $\omega_{k n}(z)$ the inequality (5.32) holds. The other sets $E_{k n}^{1}$ not only do not contain $z$, but even $z \in C D_{k n}^{2} \backslash E_{k n}^{2}$ holds, and the corresponding multipliers satisfy (5.33). Taking into account (5.21) we have (prime near a product sign means that one of the terms is omitted)

$$
\begin{aligned}
|\omega(z)| & \leq \frac{3}{2} \cdot \frac{5}{4}|\psi(z)| \prod_{|k|=1}^{\infty} \prod_{m=0}^{\infty}\left(1+\delta_{k, n_{k}+2 m}\right) \\
& \leq \frac{15}{8}|\psi(z)| \prod_{|k|=1}^{\infty} \prod_{n=n_{k}}^{\infty} \frac{1+\delta_{k n}}{1-\delta_{k n}} \leq 2|\psi(z)|, \\
|\omega(z)| & \geq \frac{3}{2} \cdot \frac{3}{4}|\psi(z)| \prod_{|k|=1}^{\infty} \prod_{m=0}^{\infty}\left(1-\delta_{k, n k+2 m}\right) \\
& \geq \frac{9}{8}|\psi(z)| \prod_{|k|=1}^{\infty} \prod_{n=n_{k}}^{\infty} \frac{1-\delta_{k n}}{1+\delta_{k n}} \geq|\psi(z)| .
\end{aligned}
$$

Let us prove the inequality (5.29). For each $z \in\{\operatorname{Re} z>-1\} \cap\{|z| \geq 2\}$ we choose a positive integer $N$ such that $2^{N} \leq|z|<2^{N+1}$. Then $z \in C D_{k n}^{2} \backslash E_{k n}^{2}$ for all sets of indices $k$ and $n$, except possibly those for which $n=N-1, N, N+1$.

We divide the set of all pairs $(k, n)$ of indices used in the construction of the set $E_{2}$ into two subsets: the set $V_{1}$ of those pairs for which $z \in C D_{k n}^{2} \backslash E_{k n}^{2}$, and the set $V_{2}$ of the remaining pairs. It is clear that by (5.33) and (5.21) we have

$$
\prod_{(k, n) \in V_{1}}\left|\omega_{k n}(z)\right| \leq \prod_{(k, n) \in V_{1}}\left(1+\delta_{k n}\right) \leq \prod_{|k|=1}^{\infty} \prod_{n=n_{k}}^{\infty} \frac{1+\delta_{k n}}{1-\delta_{k n}} \leq \frac{16}{15}
$$


Now, if $V_{2}$ is non-empty, by (5.31) we get

$$
\begin{aligned}
\prod_{(k, n) \in V_{2}}\left|\omega_{k n}(z)\right| & \leq \prod_{(k, n) \in V_{2}} \exp \left(\varepsilon_{k}^{1 / 2} 2^{n \rho}\right) \leq \prod_{|k|=1}^{\infty} \prod_{n=N-1}^{N+1} \exp \left(\varepsilon_{k}^{1 / 2} 2^{n \rho}\right) \\
& \leq \prod_{|k|=1}^{\infty} \exp \left(3 \varepsilon_{k}^{1 / 2} 2^{(N+1) \rho}\right)=\exp \left(3 \cdot 2^{(N+1) \rho} \sum_{|k|=1}^{\infty} \varepsilon_{k}^{1 / 2}\right) .
\end{aligned}
$$

Since

$$
\sum_{|k|=1}^{\infty} \varepsilon_{k}^{1 / 2}=\sum_{|k|=1}^{\infty} \exp \left(-\frac{2^{9} C}{\eta_{k}}\right) \leq \sum_{|k|=1}^{\infty} \frac{\eta_{k}}{2^{9} C}<\frac{\pi}{2^{10}}<\frac{1}{2^{8}},
$$

we get the estimate

$$
\begin{aligned}
|\omega(z)| & \leq \frac{3}{2} \cdot \frac{16}{15} \exp \left(2^{N \rho-5}\right)<\exp \left(2^{N \rho-5}+1\right) \\
& <\exp \left\{\left(2^{N}+1\right)^{\rho}\right\}<\exp \left\{(|z|+1)^{\rho}\right\} .
\end{aligned}
$$

The lemma is proved.

Define a function $\varphi(z)$ on $E_{1}$ by the equality

$$
\varphi(z)=a_{|k|}, z \in E_{k n}^{1} \text {. }
$$

Lemma 5.4. There exists a function $F(z)$ analytic in the half-plane $\{\operatorname{Re} z>0\}$, such that

$$
\begin{gathered}
|\varphi(z)-F(z)|<\frac{1}{2}|\psi(z)|, \quad z \in E_{0}, \\
|F(z)| \leq \exp \left\{D_{1}(|z|+1)^{\rho}\right\}, \quad \operatorname{Re} z>0,
\end{gathered}
$$

where $D_{1}>0$ is a constant.

Proof. By (5.27) the inequality (5.25) implies

$$
\left|Q_{1}(\zeta, z)-\frac{1}{\zeta-z}\right|<\left|\frac{\psi(\zeta)}{\zeta}\right|^{2}, \zeta \in \gamma_{k n}^{1}, z \in C D_{k n}^{1} .
$$

Observe that this inequality is valid, in particular, for $\zeta \in \Gamma_{1}, z \in E_{0}$, and also for $\zeta \in \Gamma_{1},|z| \leq \frac{1}{4}|\zeta|$. Since for $\zeta \in \gamma_{k n}^{1}$ the inequality $|\zeta|^{\rho} \geq 2^{n \rho-1} \geq \varepsilon_{k}^{1 / 2} 2^{n \rho-1}$ holds, the inequality (5.26) implies

$$
\left|Q_{1}(\zeta, z)\right| \leq \exp \left(|\zeta|^{\rho}\right), \quad \zeta \in \Gamma_{1}, z \in\{\operatorname{Re} z>-1\} .
$$

Set

$$
\Gamma_{1 n}=\bigcup_{n_{k}+2 m \leq n} \gamma_{k, n_{k}+2 m}^{1}, n \geq n_{1} .
$$

Observe that $\Gamma_{1 n}$ is contained in the $\operatorname{disc}\left\{|z| \leq \frac{9}{4} 2^{n}\right\}, \Gamma_{1}=\bigcup_{n=n_{1}}^{\infty} \Gamma_{1 n}$, and $\Gamma_{1} \backslash \Gamma_{1 n}$ is contained in the complement of the disc $\left\{|z|<\frac{7}{8} 2^{n}\right\}$.

Consider a sequence of functions analytic in the half-plane $\{\operatorname{Re} z>-1\}$ :

$$
H_{n}(z)=\frac{1}{2 \pi i} \int_{\Gamma_{1 n}} \frac{\varphi(\zeta)}{\omega(\zeta)} Q_{1}(\zeta, z) d \zeta
$$


Let us show that this sequence converges uniformly in each bounded region contained in $\{\operatorname{Re} z>0\}$, and that the function $H(z)=\lim _{n \rightarrow \infty} H_{n}(z)$ satisfies the conditions

$$
\begin{gathered}
\left|H(z)-\frac{\varphi(z)}{\omega(z)}\right|<\frac{1}{4}, z \in E_{0} ; \\
|H(z)| \leq \exp \left\{D_{2}(|z|+1)^{\rho}\right\}, \operatorname{Re} z>0,
\end{gathered}
$$

where $D_{2}>0$ is a constant.

Let $N$ be such that $|z| \leq 2^{N}$ and $m>n>N+2$. Since, by the Cauchy theorem,

$$
0=\frac{1}{2 \pi i} \int_{\Gamma_{1 m} \backslash \Gamma_{1 n}} \frac{\varphi(\zeta)}{\omega(\zeta)} \frac{d \zeta}{\zeta-z}
$$

we have

$$
H_{m}(z)-H_{n}(z)=\frac{1}{2 \pi i} \int_{\Gamma_{1 m} \backslash \Gamma_{1 n}} \frac{\varphi(\zeta)}{\omega(\zeta)}\left\{Q_{1}(\zeta, z)-\frac{1}{\zeta-z}\right\} d \zeta .
$$

Using (5.28), (5.23), and (5.27) in succession, we get

$$
\left|\frac{\varphi(\zeta)}{\omega(\zeta)}\right| \leq \frac{\left|a_{|k|}\right|}{|\psi(\zeta)|} \leq \frac{\exp \left(\varepsilon_{k} 2^{n \rho-1}\right)}{|\psi(\zeta)|} \leq \frac{1}{|\psi(\zeta)|^{2}}, \zeta \in \gamma_{k n}^{1}
$$

therefore using (5.38) we get

$$
\left|H_{m}(z)-H_{n}(z)\right| \leq \frac{1}{2 \pi} \int_{\Gamma_{1 m} \backslash \Gamma_{1 n}}\left|\zeta^{-2} d \zeta\right| \leq \frac{1}{2 \pi} \int_{\Gamma_{1} \backslash \Gamma_{1 n}}\left|\zeta^{-2} d \zeta\right|
$$

By (5.24), this implies that the sequence $\left\{H_{n}(z)\right\}$ converges uniformly in each bounded region contained in $\{\operatorname{Re} z>0\}$.

Now, by the Cauchy formula we have

$$
\frac{\varphi(z)}{\omega(z)}=\frac{1}{2 \pi i} \int_{\Gamma_{1 n}} \frac{\varphi(\zeta)}{\omega(\zeta)} \frac{d \zeta}{\zeta-z}, z \in E_{0},|z| \leq 2^{n}
$$

therefore

$$
\begin{gathered}
H_{n}(z)-\frac{\varphi(z)}{\omega(z)}=\frac{1}{2 \pi i} \int_{\Gamma_{1 n}} \frac{\varphi(\zeta)}{\omega(\zeta)}\left\{Q_{1}(\zeta, z)-\frac{1}{\zeta-z}\right\} d \zeta, \\
z \in E_{0},|z| \leq 2^{n} .
\end{gathered}
$$

Using (5.42), (5.38), and (5.24), we get

$$
\left|H_{n}(z)-\frac{\varphi(z)}{\omega(z)}\right| \leq \frac{1}{2 \pi} \int_{\Gamma_{1 n}}\left|\zeta^{-2} d \zeta\right|<\frac{1}{4}, z \in E_{0},|z| \leq 2^{n},
$$

whence the inequality (5.40) follows.

Let us prove (5.41). Letting $m \rightarrow+\infty$ in (5.43), we see that

$$
\left|H(z)-H_{n}(z)\right|<\frac{1}{4},|z| \leq 2^{N}, n>N+2,
$$

and, in particular,

$$
\left|H(z)-H_{N+3}(z)\right|<\frac{1}{4},|z| \leq 2^{N} .
$$


Suppose that $N$ is chosen in such a way that $2^{N-1} \leq|z|<2^{N}$. Using (5.42) and (5.39) we have

$$
\begin{aligned}
\left|H_{N+3}(z)\right| & =\left|\frac{1}{2 \pi i} \int_{\Gamma_{1, N+3}} \frac{\varphi(\zeta)}{\omega(\zeta)} Q_{1}(\zeta, z) d \zeta\right| \\
& \leq \frac{1}{2 \pi} \int_{\Gamma_{1, N+3}}\left|\frac{1}{\psi(\zeta)}\right|^{2} \exp \left(|\zeta|^{\rho}\right)|d \zeta| \\
& \leq \frac{1}{2 \pi} \max _{\zeta \in \Gamma_{1, N+3}}\left\{\left|\frac{\zeta}{\psi(\zeta)}\right|^{2} \exp \left(|\zeta|^{\rho}\right)\right\} \int_{\Gamma_{1, N+3}}\left|\zeta^{-2} d \zeta\right| \\
& \leq \frac{1}{2 \pi} \max _{\zeta \in \Gamma_{1, N+3}}\left\{|\zeta|^{2} \exp \left(3|\zeta|^{\rho}\right)\right\} .
\end{aligned}
$$

Since for $\zeta \in \Gamma_{1, N+3}, 2^{N-1} \leq|z|<2^{N}$ the inequality

$$
|\zeta|<\frac{9}{4} 2^{N+3}<36 \cdot 2^{N-1} \leq 36|z|
$$

holds, we have

$$
\left|H_{N+3}(z)\right| \leq \exp \left\{D_{3}(|z|+1)^{\rho}\right\},
$$

where $D_{3}>0$ is an absolute constant. Using this and (5.44) we get (5.41).

The function $F(z)=H(z) \omega(z)$ satisfies the conditions (5.36) and (5.37). In fact, (5.40) implies

$$
|F(z)-\varphi(z)|<\frac{1}{4}|\omega(z)|, \quad z \in E_{0},
$$

from where, using (5.28), we get (5.36). The estimate (5.37) follows from (5.41) and (5.29). The lemma is proved.

Theorem 5.1 follows from Lemmas 5.2 and 5.4. Let us show this. Let $F(z)$ be the function from Lemma 5.4. By Lemma 5.2 there exists an entire function $G(z)$ not exceeding normal type of order $\rho$, such that

$$
G(z)=F(z)+g(z),
$$

where $g(z)$ admits the estimate (5.20) in the angle $\{|\arg z| \leq \alpha\}$ for sufficiently large $|z|$. Let us show that each of the numbers $a_{k} \in\left\{a_{k}\right\}_{k=1}^{\infty}$ is a deficient value for the function $G(z)$.

It is clear that for sufficiently large $|z|$ the inequality $|g(z)|<\frac{1}{2}|\psi(z)|(|\arg z|<$ $\alpha$ ) holds, therefore (5.45) and (5.36) imply

$$
|G(z)-\varphi(z)| \leq|\psi(z)|, z \in E_{0},|z|>r_{0} .
$$

Fix a positive integer $k$. If $r>2^{n_{k}}$, the circle $\{|z|=r\}$ intersects the part of $E_{0}$ for which $\varphi(z)=a_{k}$ in one or two arcs of length $\frac{1}{2} \eta_{k} r$. For each of these arcs, by (5.46) the inequality $\left(|z|>\max \left(2^{n_{k}}, r_{0}\right)\right)$

$$
\left|G(z)-a_{k}\right| \leq \exp \left(-\varepsilon_{k} r^{\rho} \cos \rho \varphi\right) \leq \exp \left(-\frac{1}{2} \varepsilon_{k} r^{\rho}\right), z=r e^{i \varphi}
$$

holds. Therefore

$$
\begin{aligned}
m\left(r, a_{k}, G\right) & =\frac{1}{2 \pi} \int_{0}^{2 \pi} \ln ^{+} \frac{1}{\left|G\left(r e^{i \varphi}\right)_{-} a_{k}\right|} d \varphi \\
& >\frac{1}{2 \pi} \frac{\eta_{k}}{2} \frac{1}{2} \varepsilon_{k} r^{\rho}>\frac{1}{8 \pi} \eta_{k} \varepsilon_{k} r^{\rho}, r>\max \left(2^{n_{k}}, r_{0}\right) .
\end{aligned}
$$


Since $T(r, G) \leq \ln ^{+} M(r, G)=O\left(r^{\rho}\right)$, we have $\delta\left(a_{k}, G\right)>0$. The theorem is proved.

\section{Stability of deficiencies}

Let $f(z)$ be a meromorphic function in the complex plane. All functions of the form $f(a z+b), a \neq 0$ map the complex plane onto the same Riemann surface $F$. Studying the asymptotic behavior of meromorphic functions given by the Riemann surfaces onto which they map their domains, it makes sense to study only those properties which are the same for all functions of the form $f(a z+b)$. From quantities characterizing the asymptotic behavior of $f(z)$ it is natural to require that they do not change when we pass to $f(a z+b)$.

In Theorem 6.7 from Chapter 1 we described the changes which occur to the main quantities of the Nevanlinna theory when we pass from $f(z)$ to $f(a z), a \neq 0$. We saw (Theorem 1.6 from Chapter 2) that $f(z)$ and $f(a z)$ have the same growth category and, as it was noted at the end of Section 1, the sets $E_{P}, E_{B}, E_{N}, E_{V}$ of the functions $f(z)$ and $f(a z)$ are the same, and the Nevanlinna and Valiron deficiencies are the same. Therefore it suffices to compare the functions $f(z)$ and $f(z+h)$.

We write $f(z+h)=f_{h}(z)$ and mark all quantities related to $f_{h}(z)$ with a subscript $h$; the absence of this subscript means that the quantity is related to $f(z)$. In Section 1 of Chapter 2 (see the proof of Theorem 1.6) we obtained the following inequalities

$$
\begin{gathered}
n(r-2|h|, a) \leq n_{h}(r-|h|, a) \leq n(r, a)(r>2|h|), \\
(1+o(1)) N(r-2|h|, a) \leq N_{h}(r-|h|, a) \leq(1+o(1)) N(r, a), \\
(1+o(1)) T(r-2|h|, f) \leq T_{h}(r-|h|, f) \leq(1+o(1)) T(r, f) .
\end{gathered}
$$

These inequalities imply that $n(r, a)$ and $n_{h}(r, a), N(r, a)$ and $N_{h}(r, a), T(r, f)$ and $T_{h}(r, f)$ have, respectively, the same growth categories (Theorem 1.6 from Chapter 2). Therefore the sets of Borel exceptional values for $f(z)$ and $f(z+h)$ are the same. For Picard exceptional values this is obvious. This makes the example constructed by Dugué even more unexpected. Dugué constructed an example of functions $f(z)$ and $f(z+h)$ with different deficiencies.

EXAmple 1. (Dugué) Let $\psi(z)=e^{e^{z}}-1$. The entire function $\psi(z)$ has simple zeros at points $z_{p q}=\ln (2 \pi p)+i\left(\frac{\pi}{2}+\pi q\right), p=1,2,3, \ldots, q=0, \pm 1, \pm 2, \pm 3, \ldots$. All these zeros are in the half-plane $\{\operatorname{Re} z>0\}$. Let us show that

$$
T(r, \psi)=(1+o(1)) \frac{e^{r}}{\sqrt{2 \pi^{3} r}}, r \rightarrow \infty
$$


We have

$$
\begin{aligned}
T(r, \psi) & =T\left(r, e^{e^{z}}\right)+O(1)=m\left(r, e^{e^{z}}\right)+O(1) \\
& =\frac{1}{2 \pi} \int_{0}^{2 \pi}\left\{\operatorname{Re} e^{r e^{i \varphi}}\right\}^{+} d \varphi+O(1) \\
& =\frac{1}{2 \pi} \int_{0}^{2 \pi} e^{r \cos \varphi}\{\cos (r \sin \varphi)\}^{+} d \varphi+O(1) \\
& =\frac{1}{\pi} \int_{0}^{\pi / 2} e^{r \cos \varphi}\{\cos (r \sin \varphi)\}^{+} d \varphi+O(1) .
\end{aligned}
$$

Applying Lemma 4.1 with $\varphi(t)=\{\cos t\}^{+}$, and taking into account that

$$
\lim _{r \rightarrow \infty} \frac{1}{r} \int_{0}^{r}\{\cos t\}^{+} d t=\frac{1}{\pi}
$$

we get (6.4).

Applying the second main Nevanlinna inequality (2.8) from Chapter 3 to $\psi(z)$ we get the inequality

$$
(1+o(1)) T(r, \psi)<N(r, 0, \psi)+N(r,-1, \psi)+N(r, \infty, \psi)=N(r, 0, \psi),
$$

which is valid outside some set of intervals of finite total length. Since the function $N(r, 0, \psi)$ is increasing, the inequality is valid everywhere on the real line. In fact, if $r$ is in an exceptional interval, then the left end of the interval is $r^{\prime}=r+o(1)$, and

$$
\begin{aligned}
N(r, 0, \psi) & \geq N\left(r^{\prime}, 0, \psi\right) \geq(1+o(1)) T\left(r^{\prime}, \psi\right) \\
& =(1+o(1)) \frac{e^{r^{\prime}}}{\sqrt{2 \pi^{3} r^{\prime}}}=(1+o(1)) \frac{e^{r}}{\sqrt{2 \pi^{3} r}}=(1+o(1)) T(r, \psi) .
\end{aligned}
$$

In addition, we have $N(r, 0, \psi) \leq T(r, \psi)+O(1)$, therefore

$$
N(r, 0, \psi)=(1+o(1)) T(r, \psi) \text {. }
$$

Now we consider the function

$$
f(z)=\psi(z) / \psi(-z) .
$$

The function $f(z)$ has simple zeros at points $z_{p q}$ and simple poles at points $-z_{p q}$, therefore $N(r, 0, f)=N(r, \infty, f)=N(r, 0, \psi)$.

Observe that for $x>0$ the inequality

$$
|\psi(-z)| \leq 1+\left|e^{e^{-z}}\right|=1+e^{e^{-x} \cos y} \leq 1+e, z=x+i y,
$$

holds, and that $|\psi(z)| \leq 1+e$ for $x \leq 0$. Therefore

$$
\begin{aligned}
m(r, f) & \geq \frac{1}{2 \pi} \int_{-\pi / 2}^{\pi / 2} \ln ^{+}\left|\frac{\psi\left(r e^{i \varphi}\right)}{\psi\left(-r e^{i \varphi}\right)}\right| d \varphi \\
& \geq \frac{1}{2 \pi} \int_{-\pi / 2}^{\pi / 2} \ln ^{+} \frac{\left|\psi\left(r e^{i \varphi}\right)\right|}{1+e} d \varphi=\frac{1}{2 \pi} \int_{-\pi / 2}^{\pi / 2} \ln ^{+}\left|\psi\left(r e^{i \varphi}\right)\right| d \varphi+O(1) \\
& =m(r, \psi)+O(1)=T(r, \psi)+O(1) .
\end{aligned}
$$

Now, by (6.5) and (6.6), we get

$$
T(r, f) \leq T(r, \psi(z))+T(r, \psi(-z))+O(1)=2 T(r, \psi)+O(1)
$$

and

$$
T(r, f)=m(r, f)+N(r, f) \geq(1+o(1)) 2 T(r, \psi) .
$$


Hence

$$
\begin{gathered}
T(r, f)=(2+o(1)) T(r, \psi), \\
\Delta(0, f)=\delta(0, f)=1-\lim _{r \rightarrow \infty} \frac{N(r, 0, f)}{T(r, f)}=1-\lim _{r \rightarrow \infty} \frac{N(r, 0, \psi)}{2 T(r, \psi)}=\frac{1}{2}, \\
\Delta(\infty, f)=\delta(\infty, f)=1-\lim _{r \rightarrow \infty} \frac{N(r, \infty, \psi)}{2 T(r, \psi)}=\frac{1}{2} .
\end{gathered}
$$

Let $h$ be a non-zero real number. Consider the function $f_{h}(z)=f(z+h)$. It can be investigated in the same way as above. First of all,

$$
\begin{aligned}
T\left(r, \psi_{h}(z)\right) & =T\left(r, e^{e^{z+h}}\right)+O(1)=\frac{1}{2 \pi} \int_{-\pi}^{\pi}\left\{\operatorname{Re} e^{h+r e^{i \varphi}}\right\}^{+} d \varphi+O(1) \\
& =e^{h} \frac{1}{2 \pi} \int_{-\pi}^{\pi}\left\{\operatorname{Re} e^{r e^{i \varphi}}\right\}^{+} d \varphi+O(1)=e^{h} T\left(r, e^{e^{z}}\right)+O(1) .
\end{aligned}
$$

In the same way as we got (6.5) we get

$$
N\left(r, 0, \psi_{h}(z)\right)=(1+o(1)) T\left(r, \psi_{h}(z)\right), r \rightarrow \infty .
$$

Thus

$$
\begin{gathered}
N\left(r, 0, f_{h}\right)=N\left(r, 0, \psi_{h}(z)\right)=(1+o(1)) e^{h} T\left(r, e^{e^{z}}\right), \\
\begin{aligned}
N\left(r, \infty, f_{h}\right)=N(r, 0, \psi(-z-h)) & =N\left(r, 0, \psi_{-h}(-z)\right) \\
& =N\left(r, 0, \psi_{-h}(z)\right)=(1+o(1)) e^{-h} T\left(r, e^{e^{z}}\right) .
\end{aligned}
\end{gathered}
$$

Also,

$$
T\left(r, f_{h}\right) \leq T\left(r, \psi_{h}(z)\right)+T\left(r, \psi_{-h}(-z)\right)+O(1)=\left(e^{h}+e^{-h}\right) T\left(r, e^{e^{z}}\right)+O(1) .
$$

Since for $\operatorname{Re} z>0$ the inequality $|\psi(-z-h)| \leq 1+e^{e^{-h}}$ holds, and for $\operatorname{Re} z \leq 0$ we have $|\psi(z+h)| \leq 1+e^{e^{h}}$, in the same way as we proved (6.6) we get $m\left(r, f_{h}\right) \geq$ $T\left(r, \psi_{h}\right)+O(1)$ and $T\left(r, f_{h}\right)=m\left(r, f_{h}\right)+N\left(r, f_{h}\right) \geq(1+o(1))\left(e^{h}+e^{-h}\right) T\left(r, e^{e^{z}}\right)$. Thus

$$
T\left(r, f_{h}\right)=(1+o(1))\left(e^{h}+e^{-h}\right) T\left(r, e^{e^{z}}\right), r \rightarrow \infty .
$$

Now we easily get

$$
\begin{gathered}
\Delta\left(0, f_{h}\right)=\delta\left(0, f_{h}\right)=\frac{e^{-h}}{e^{h}+e^{-h}}, \\
\Delta\left(\infty, f_{h}\right)=\delta\left(\infty, f_{h}\right)=\frac{e^{h}}{e^{h}+e^{-h}} .
\end{gathered}
$$

If $h=0$ we get the same formulas as before.

Thus, we see that the deficiencies of the function $f(z+h)$ at 0 and $\infty$ are different from the deficiencies of the function $f(z)$. This could be shown in a more elementary way, following Dugué, but for us it is important to note that in the presented example the Nevanlinna and Valiron deficiencies coincide for 0 and for $\infty$.

In Dugué's example the function $f(z)$ is of infinite order. Besides, although the deficiencies $\delta_{h}(0)$ and $\delta_{h}(\infty)$ change as $h$ varies, they remain positive. We shall give an example of a meromorphic function $f(z)$ having the maximal type of order one, for which $\delta(\infty)=0$ but $\delta_{-2}(\infty)=1$. We start by investigating some auxiliary functions. 
Consider the entire function

$$
g(z)=g\left(z ; r_{k}, n_{k}\right)=\prod_{k=1}^{\infty}\left\{1-\left(\frac{z}{r_{k}}\right)^{n_{k}}\right\}
$$

where $n_{k}$ are arbitrary positive integers, $r_{k}$ are arbitrary positive numbers, satisfying the conditions $r_{k+1} \geq 2 r_{k}, r_{1}>1$. Let us show that $m(r, 0, g) \leq 4$ for each such function. In fact, $g(0)=1$ implies $T(r, g)=m(r, 0, g)+N(r, 0, g)$. On the other hand,

$$
\begin{aligned}
T(r, g) \leq \ln M(r, g) & \leq \sum_{k=1}^{\infty} \ln \left\{1+\left(\frac{r}{r_{k}}\right)^{n_{k}}\right\} \\
& =\left(\sum_{r_{k}>r}+\sum_{r_{k} \leq r}\right) \ln \left\{1+\left(\frac{r}{r_{k}}\right)^{n_{k}}\right\} \\
& \leq \sum_{r_{k}>r}\left(\frac{r}{r_{k}}\right)^{n_{k}}+\sum_{r_{k} \leq r} n_{k} \ln \frac{r}{r_{k}}+\sum_{r_{k} \leq r}\left(\frac{r_{k}}{r}\right)^{n_{k}} \\
& \leq \sum_{k=0}^{\infty} 2^{-k}+N(r, 0, g)+\sum_{k=0}^{\infty} 2^{-k}=N(r, 0, g)+4 .
\end{aligned}
$$

Hence, $m(r, 0, g) \leq 4$.

Consider, now, the meromorphic function

$$
\begin{gathered}
\Phi(z)=\Phi\left(z ; a_{j}, b_{j}, m_{j}, \sigma_{j}, \varepsilon\right)=\prod_{j=1}^{\infty} \varphi_{j}(z), \\
\varphi_{j}(z)=\left(\frac{z-b_{j}}{z-a_{j}}\right)^{m_{j}},
\end{gathered}
$$

where $0<\varepsilon<\frac{1}{2} ;\left\{a_{j}\right\}$ is an arbitrary sequence of distinct complex numbers approaching $\infty ;\left|a_{j}\right|>1 ; m_{j}$ are arbitrary positive integers; $\sigma_{j}$ is a sequence of positive numbers, such that the discs $\left\{\left|z-a_{j}\right|<\sigma_{j}\right\}$ are pairwise disjoint, and

$$
\sigma_{j}<1, \sum_{j=1}^{\infty} m_{j} \sigma_{j}<\infty
$$

holds; complex numbers $b_{j}$ are so close to $a_{j}$, that for $\left|z-a_{j}\right| \geq \sigma_{j}$ the inequalities $\left|\varphi_{j}(z)-1\right|<\varepsilon 2^{-j-1}$ and $\left|1 / \varphi_{j}(z)-1\right|<\varepsilon 2^{-j-1}$ hold. It is clear that under these conditions the product (6.8) is absolutely and uniformly convergent in each disc in the complex plane, moreover, for $z \notin \bigcup_{j}\left\{\left|z-a_{j}\right|<\sigma_{j}\right\}$ the inequality

$$
\begin{aligned}
|\Phi(z)-1| & =\left|\prod_{j=1}^{\infty}\left\{1+\left(\varphi_{j}(z)-1\right)\right\}-1\right| \\
& \leq \max \left[\left\{\prod_{j=1}^{\infty}\left(1+\frac{\varepsilon}{2^{j+1}}\right)-1\right\},\left\{1-\prod_{j=1}^{\infty}\left(1-\frac{\varepsilon}{2^{j+1}}\right)\right\}\right] \\
& \leq \max \left[e^{\frac{\varepsilon}{2}}-1,1-e^{-\frac{8}{7} \frac{\varepsilon}{2}}\right]<\varepsilon
\end{aligned}
$$


holds. We have used the inequality $\ln (1-x) \geq-\frac{x}{1-x} \geq-\frac{8}{7} x$ valid for $0<x<\frac{1}{8}$.

The inequality (6.10) implies, in particular, that $|\Phi(z)| \leq 2$ for $z \notin \bigcup_{j}\left\{\left|z-a_{j}\right|<\sigma_{j}\right\}$.

By the maximum modulus principle

$$
|\Phi(z)| \leq 2\left(\frac{\sigma_{j}}{\left|z-a_{j}\right|}\right)^{m_{j}} .
$$

for each $z$ in the disc $\left\{\left|z-a_{j}\right|<\sigma_{j}\right\}$. Therefore $\left(a_{j}=\left|a_{j}\right| e^{i \alpha_{j}}\right)$,

$$
\begin{aligned}
m(r, \Phi) & \leq O(1)+\sum_{j=1}^{\infty} \frac{1}{2 \pi} \int_{\left|z-a_{j}\right|<\sigma_{j}} \ln \left\{2\left(\frac{\sigma_{j}}{\left|z-a_{j}\right|}\right)^{m_{j}}\right\} d \varphi \\
& \leq O(1)+\sum_{j=1}^{\infty} \frac{m_{j}}{2 \pi} \int_{-\pi}^{\infty} \ln ^{+} \frac{\sigma_{j}}{\left|r e^{i \varphi}-\right| a_{j}\left|e^{i \alpha_{j}}\right|} d \varphi .
\end{aligned}
$$

On the other hand,

$$
\begin{aligned}
& \int_{-\pi}^{\pi} \ln ^{+} \frac{\sigma_{j}}{\left|r e^{i \varphi}-\right| a_{j} \mid e^{i \alpha_{j} \mid}} d \varphi=\int_{\alpha_{j}-\frac{\pi}{2}}^{\alpha_{j}+\frac{\pi}{2}} \ln ^{+} \frac{\sigma_{j}}{|r-| a_{j} \mid e^{i\left(\alpha_{j}-\varphi\right) \mid}} d \varphi \\
& \quad \leq \int_{\alpha_{j}-\frac{\pi}{2}}^{\alpha_{j}+\frac{\pi}{2}} \ln ^{+} \frac{\sigma_{j}}{\left|a_{j}\right|\left|\sin \left(\alpha_{j}-\varphi\right)\right|} d \varphi \leq \int_{-\pi / 2}^{\pi / 2} \ln ^{+} \frac{\sigma_{j}}{|\sin \theta|} d \theta \\
& \quad \leq 2 \int_{0}^{\pi / 2} \ln ^{+} \frac{2 \sigma_{j}}{\pi \theta} d \theta=2 \int_{0}^{2 \sigma_{j} / \pi} \ln \frac{2 \sigma_{j}}{\pi \theta} d \theta=\frac{4 \sigma_{j}}{\pi} .
\end{aligned}
$$

Therefore, by (6.9),

$$
m(r, \Phi) \leq O(1)+\frac{2}{\pi^{2}} \sum_{j=1}^{\infty} m_{j} \sigma_{j}=O(1) .
$$

In the same way we show

$$
m(r, 1 / \Phi)=O(1)
$$

The relations (6.11) and (6.12) imply

$$
N(r, \infty, \Phi)=N(r, 0, \Phi)+O(1) .
$$

We shall assume that zeros of $g(z)$ and poles of $\Phi(z)$ are distinct. Then, by (6.11), (6.12), (6.13), we easily get for $f(z)=g(z) \Phi(z)$ that

$$
\begin{aligned}
& m(r, 0, f) \leq m(r, 0, g)+m(r, 0, \Phi)=O(1), \\
& N(r, \infty, f)=N(r, \infty, \Phi)=\sum_{j=1}^{\infty} m_{j} \ln ^{+} \frac{r}{\left|a_{j}\right|}, \\
T(r, f)= & N(r, 0, f)+m(r, 0, f)+O(1) \\
= & N(r, 0, g)+N(r, 0, \Phi)+O(1)=N(r, 0, g)+N(r, \infty, \Phi)+O(1) \\
= & \sum_{k=1}^{\infty} n_{k} \ln ^{+} \frac{r}{r_{k}}+\sum_{j=1}^{\infty} m_{j} \ln ^{+} \frac{r}{\left|a_{j}\right|}+O(1) .
\end{aligned}
$$

Since there is much freedom in the choice of $n_{k}, r_{k}, m_{j}, a_{j}$ from the definition of $f(z)$, this class of functions is suitable for construction of various examples. 
ExAmPLE 2. Choose the sequence $\left\{r_{k}\right\}$ to be so rapidly increasing, that

$$
\sum_{k=1}^{\kappa-1} r_{k}\left(\ln r_{k}\right)^{4}<\ln r_{\kappa}
$$

Let $a_{k}=r_{k}-1, n_{k}=\left[r_{k}\left(\ln r_{k}\right)^{4}\right], m_{k}=\left[r_{k}\left(\ln r_{k}\right)^{3}\right]$. Then, by (6.16),

$$
\begin{aligned}
T\left(r_{\kappa}, f\right) & =\sum_{k=1}^{\kappa-1} r_{k}\left(\ln r_{k}\right)^{4} \ln \frac{r_{\kappa}}{r_{k}}+\sum_{k=1}^{\kappa} r_{k}\left(\ln r_{k}\right)^{3} \ln \frac{r_{\kappa}}{r_{k}-1}+O\left(\ln r_{\kappa}\right) \\
& =O\left(\ln ^{2} r_{\kappa}\right)+r_{\kappa}\left(\ln r_{\kappa}\right)^{3} \ln \frac{r_{\kappa}}{r_{\kappa}-1}=(1+o(1)) \ln ^{3} r_{\kappa} .
\end{aligned}
$$

By (6.15),

$$
N\left(r_{\kappa}, \infty, f\right)=\sum_{k=1}^{\kappa} r_{k}\left(\ln r_{k}\right)^{3} \ln \frac{r_{\kappa}}{r_{k}-1}+O\left(\ln r_{\kappa}\right)=(1+o(1)) \ln ^{3} r_{\kappa} .
$$

Hence, $N\left(r_{\kappa}, \infty, f\right) \sim T\left(r_{\kappa}, f\right)$ and $\delta(\infty, f)=0$.

Now we consider the function $f_{-2}(z)=f(z-2)$. Let $r_{\kappa}+1 \leq r<r_{\kappa+1}+1$. Then

$$
N\left(r, \infty, f_{-2}\right)=\sum_{k=1}^{\kappa}\left[r_{k}\left(\ln r_{k}\right)^{3}\right] \ln \frac{r}{r_{k}+1} \leq \sum_{k=1}^{\kappa} r_{k}\left(\ln r_{k}\right)^{3} \ln \frac{r}{r_{k}-1} .
$$

Observe that for sufficiently large $r_{k}, k \geq \kappa_{0}$, the measure (in radians) of the arc $\left\{|z|=r_{k}\right\}$ contained in the disc $\left\{|z-2|<r_{k}-1\right\}$ exceeds $\pi / 2$ (the measure approaches $2 \pi / 3$ as $k \rightarrow \infty)$. Estimating $N\left(r, 0, g_{-2}\right)$ from below we shall count only those zeros of $g(z)$ which are in the angle $\{|\arg z-\pi|<\pi / 4\}$. Then

$$
\begin{aligned}
& T\left(r, f_{-2}\right) \geq N\left(r, 0, f_{-2}\right)+O(1) \geq N\left(r, 0, g_{-2}\right)+O(1) \\
& \geq \sum_{k=\kappa_{0}}^{\kappa} \frac{1}{4} r_{k}\left(\ln r_{k}\right)^{4} \ln \frac{r}{r_{k}-1}+O(\ln r)
\end{aligned}
$$

Fix some $\kappa_{1}>\kappa_{0}$. Then from (6.17) and $\left(6.17^{\prime}\right)$ we get, for $r_{\kappa}+1 \leq r<r_{\kappa+1}+1$,

$$
\begin{aligned}
N\left(r, \infty, f_{-2}\right) & \leq \sum_{k=\kappa_{1}}^{\kappa} r_{k}\left(\ln r_{k}\right)^{3} \ln \frac{r}{r_{k}-1}+O(\ln r), \\
T\left(r, f_{-2}\right) & \geq \frac{1}{4} \sum_{k=\kappa_{1}}^{\kappa} r_{k}\left(\ln r_{k}\right)^{4} \ln \frac{r}{r_{k}-1}+O(\ln r) \\
& \geq \frac{1}{4} \ln r_{\kappa_{1}} \sum_{k=\kappa_{1}}^{\kappa} r_{k}\left(\ln r_{k}\right)^{3} \ln \frac{r}{r_{k}-1}+O(\ln r) \\
& \geq \frac{1}{4}\left(\ln r_{\kappa_{1}}\right) N\left(r, \infty, f_{-2}\right)+O(\ln r) .
\end{aligned}
$$

Hence

$$
\limsup _{r \rightarrow \infty} \frac{N\left(r, \infty, f_{-2}\right)}{T\left(r, f_{-2}\right)} \leq \frac{4}{\ln r_{\kappa_{1}}} .
$$

Letting $\kappa_{1} \rightarrow \infty$ in this inequality, we get

$$
\limsup _{r \rightarrow \infty} \frac{N\left(r, \infty, f_{-2}\right)}{T\left(r, f_{-2}\right)}=0
$$


that is, $\delta\left(\infty, f_{-2}\right)=1$.

It is easy to show that the functions $n(r, 0, g)$ and $n(r, \infty, \Phi)$ have the maximal type of order one, and, by (6.16), the function $f(z)$ also has the maximal type of order one.

EXAMPLE 3. If we let $n_{k}=\left[r_{k}\left(\ln r_{k}\right)^{3}\right], m_{k}=\left[r_{k}\left(\ln r_{k}\right)^{4}\right]$ in the previous example, leaving all other parameters unchanged, we get an example with varying Valiron deficiency: $\Delta(\infty, f(z))=0, \Delta(\infty, f(z-2))=1$. We leave details of this example to interested readers.

Observe that in Example 2 we have $\Delta(\infty, f)=1>\delta(\infty, f)=0$, but if, before passing to the limit, we exclude from consideration values of $r$ from the segments $\left[r_{k}-1, r_{k}+1\right]$, we would have $\delta(\infty, f)=1$. The situation is similar for $f(z-2)$ in Example 3. This is a fundamental difference between Examples 2 and 3, and Dugué's Example 1. Examples 2 and 3 show how we should "correct" the definition of deficiency to get its shift invariance for meromorphic functions of finite order.

Let $E$ be a measurable set, $E \subset(1, \infty), \chi(r, E)$ be the indicator function of this set, $C E=(1, \infty) \backslash E$. We write $E \in L$ if

$$
\limsup _{r \rightarrow \infty} \frac{1}{\ln r} \int_{1}^{r} \chi(t, E) d t<\infty .
$$

It is easy to show that for $E \in L$

$$
\int_{1}^{\infty} \chi(t, E) t^{-\sigma} d t<\infty
$$

for each $\sigma>0$. In fact, if (6.18) is satisfied, then $\int_{1}^{r} \chi(t, E) d t=O(\ln r)$, and therefore

$$
\begin{aligned}
\int_{1}^{r} \chi(t, E) t^{-\sigma} d t & =r^{-\sigma} \int_{1}^{r} \chi(t, E) d t+\sigma \int_{1}^{r} \frac{d t}{t^{1+\sigma}} \int_{1}^{t} \chi(\tau, E) d \tau \\
& =r^{-\sigma} O(\ln r)+\sigma \int_{1}^{r} \frac{O(\ln t) d t}{t^{1+\sigma}}=O(1) .
\end{aligned}
$$

In particular $(\sigma=1)$ all sets of the class $L$ have finite logarithmic measure (see the definition in Exercise 2 from Chapter 3, Section 1). Observe also that, if $E^{\prime} \in L$ and $E^{\prime \prime} \in L$, then $E^{\prime} \cup E^{\prime \prime} \in L, E^{\prime} \cap E^{\prime \prime} \in L$.

Now we introduce the following definitions (all limits are taken as $r \rightarrow \infty$ ):

$$
\begin{gathered}
\delta^{*}(a)=\sup _{C E \in L} \liminf _{r \in E} \frac{m(r, a)}{T(r, f)}=1-\inf _{C E \in L} \limsup _{r \in E} \frac{N(r, a)}{T(r, f)} \\
\Delta^{*}(a)=\inf _{C E \in L} \limsup _{r \in E} \frac{m(r, a)}{T(r, f)}=1-\sup _{C E \in L} \liminf _{r \in E} \frac{N(r, a)}{T(r, f)} .
\end{gathered}
$$

It is easy to check that in Example 2 the following relations hold: $\delta(\infty)=0$, $\Delta(\infty)=1, \delta_{-2}(\infty)=\Delta_{-2}(\infty)=1, \delta^{*}(\infty)=\Delta^{*}(\infty)=1, \delta_{-2}^{*}(\infty)=\Delta_{-2}^{*}(\infty)=1$.

THEOREM 6.1. For meromorphic functions of finite order the equalities

$$
\delta_{h}^{*}(a)=\delta^{*}(a), \Delta_{h}^{*}(a)=\Delta^{*}(a)
$$

are satisfied for each $h$. 
Proof. We give proof for $\delta^{*}(a)$, the proof for $\Delta^{*}(a)$ is similar. In this section we shall use the spherical characteristic $T(r, f)$, without reflecting this in our notation. Denote by $M_{\varepsilon}$ the set of intervals on which $\frac{d}{d r} \ln T(r)<\varepsilon, \varepsilon>0$. Let us show that $C M_{\varepsilon} \in L$. In fact, let $\Delta_{r}=C M_{\varepsilon} \cap(1, r)$. Then

$$
\ln T(r)-\ln T(1) \geq \int_{\Delta_{r}} d \ln T(r) \geq \varepsilon \int_{\Delta_{r}} d r=\varepsilon \int_{1}^{r} \chi\left(t, C M_{\varepsilon}\right) d t .
$$

Since the function $f(z)$ has finite order $\rho$, we have

$$
\limsup _{r \rightarrow \infty} \frac{1}{\ln r} \int_{1}^{r} \chi\left(t, C M_{\varepsilon}\right) d t \leq \frac{\rho}{\varepsilon}<\infty,
$$

that is, $C M_{\varepsilon} \in L$. By (6.2) and (6.3) we get

$$
\frac{N_{h}(r-|h|, a)}{T_{h}(r-|h|)} \leq(1+o(1)) \frac{N(r, a)}{T(r-2|h|)} .
$$

Since $T(r)$ is a convex function of $\ln r$, for $r>4|h|, 0<\theta=\theta(r)<1$, we have

$$
\begin{aligned}
T(r)= & T(r-2|h|)+\frac{d T(r-\theta 2|h|)}{d \ln r} \ln \frac{r}{r-2|h|} \leq T(r-2|h|) \\
& +\frac{d T(r)}{d \ln r} \frac{2|h|}{r-2|h|}=T(r-2|h|)+T^{\prime}(r) \frac{2|h| r}{r-2|h|} .
\end{aligned}
$$

If $r \in M_{\varepsilon}, \varepsilon<(8|h|)^{-1}$, then (6.21) implies

$$
\begin{aligned}
& \frac{T(r)}{T(r-2|h|)} \leq 1+\frac{2|h| r}{r-2|h|} \frac{T^{\prime}(r)}{T(r)} \frac{T(r)}{T(r-2|h|)} \\
& \quad \leq 1+4|h| \varepsilon \frac{T(r)}{T(r-2|h|)}, \frac{T(r)}{T(r-2|h|)} \leq \frac{1}{1-4|h| \varepsilon}<1+8|h| \varepsilon .
\end{aligned}
$$

By (6.20) and (6.22) we get, for $r \in M_{\varepsilon}$,

$$
\frac{N_{h}(r-|h|, a)}{T_{h}(r-|h|)} \leq(1+o(1))(1+8|h| \varepsilon) \frac{N(r, a)}{T(r)} .
$$

Let $E_{\varepsilon}$ be an arbitrary set satisfying $E_{\varepsilon} \subset M_{\varepsilon}$ and $C E_{\varepsilon} \in L$. Then

$$
\begin{gathered}
\limsup _{r \in E_{\varepsilon}} \frac{N_{h}(r-|h|, a)}{T_{h}(r-|h|)} \leq(1+8|h| \varepsilon) \limsup _{r \in E_{\varepsilon}} \frac{N(r, a)}{T(r)}, \\
\quad \inf _{\varepsilon, E_{\varepsilon}} \limsup _{r \in E_{\varepsilon}} \frac{N_{h}(r-|h|, a)}{T_{h}(r-|h|)} \leq \inf _{\varepsilon, E_{\varepsilon}} \limsup _{r \in E_{\varepsilon}} \frac{N(r, a)}{T(r)}
\end{gathered}
$$

(the infimum is taken over all $E_{\varepsilon}$ for all $0<\varepsilon<(8|h|)^{-1}$ ).

But

$$
\inf _{\varepsilon, E_{\varepsilon}} \limsup _{r \in E_{\varepsilon}} \frac{N}{T}=\inf _{C E \in L} \limsup _{r \in E} \frac{N}{T}
$$

In fact, on one hand

$$
\inf _{C E \in L} \limsup _{r \in E} \frac{N}{T} \leq \inf _{\substack{E \subset M_{\varepsilon} \\ C E \in L}} \limsup _{r \in E} \frac{N}{T}=\inf _{\varepsilon, E_{\varepsilon}} \limsup _{r \in E_{\varepsilon}} \frac{N}{T} .
$$

On the other hand, if $C E \in L$, then $C\left(E \cap M_{\varepsilon}\right)=C E \cup C M_{\varepsilon} \in L$, therefore

$$
\inf _{C E \in L} \limsup _{r \in E} \frac{N}{T} \geq \inf _{C E \in L} \limsup _{r \in E \cap M_{\varepsilon}} \frac{N}{T}=\inf _{\varepsilon, E_{\varepsilon}} \limsup _{r \in E_{\varepsilon}} \frac{N}{T} .
$$


By (6.23) and (6.24) we get

$$
\inf _{C E \in L} \limsup _{r \in E} \frac{N_{h}(r-|h|, a)}{T_{h}(r-|h|)} \leq \inf _{C E \in L} \limsup _{r \in E} \frac{N(r, a)}{T(r)},
$$

that is, $\delta^{*}(a, f(z+h)) \geq \delta^{*}(a, f(z))$.

Applying this relation to $f(z+h)$ and $f((z+h)-h)$, we have

$$
\delta^{*}(a, f(z)) \geq \delta^{*}(a, f(z+h))
$$

and

$$
\delta^{*}(a, f(z))=\delta^{*}(a, f(z+h)) .
$$

REMARK. We could define the deficiency in such a way that it would be shift invariant for functions (not necessarily of finite order) satisfying

$$
\lim _{r \rightarrow \infty} r^{-1} \ln T(r, f)=0 .
$$

It suffices to define the deficiency in the same way as $\delta^{*}(a)$, but with $L$ consisting of sets $E$ with zero relative measure, that is, satisfying

$$
\lim _{r \rightarrow \infty} \frac{1}{r} \int_{1}^{r} \chi(t, E) d t=0
$$

The Dugue example shows that if we drop the restriction (6.25), the shift invariance of deficiency cannot be achieved no matter what sets (even the whole interval $(1, \infty)$ without a sequence $r_{n} \rightarrow \infty$ ) we would exclude from consideration.

We preferred to define $\delta^{*}(a)$ and $\Delta^{*}(a)$ as above, since in the value distribution theory there are many results which are valid for $r$ outside intervals of finite logarithmic measure, whereas consideration of larger exceptional sets would require significant extensions of the Nevanlinna theory.

THEOREM 6.2. The deficiencies of meromorphic functions satisfy the relation

$$
\sum_{a} \delta^{*}(a) \leq 2 .
$$

Proof. Consider arbitrary distinct points $a_{j}, j=1,2, \ldots, q$. By the definition of $\delta^{*}(a)$ for each $\varepsilon>0$ there exists a set $E_{j}$ such that $C E_{j}$ has finite logarithmic measure and $m\left(r, a_{j}\right) / T(r, f) \geq \delta^{*}\left(a_{j}\right)-\varepsilon$ for $r \in E_{j}, j=1, \ldots, q$. The set $E=\bigcap_{j=1}^{q} E_{j}$ is non-empty because the set $C E$ has finite logarithmic measure. By Theorem 2.1 from Chapter 3 the inequalities

$$
\begin{gathered}
\sum_{j=1}^{q} \frac{m\left(r, a_{j}\right)}{T(r, f)} \leq 2+\frac{O(\ln r)+O(\ln T(r, f))}{T(r, f)} \\
\sum_{j=1}^{q} \delta^{*}\left(a_{j}\right) \leq 2+\varepsilon q+\frac{O(\ln r)+O(\ln T(r, f))}{T(r, f)}
\end{gathered}
$$

hold on $E$, since the intervals on which the inequalities are not satisfied have finite measure, and without loss of generality we may assume that they are contained in $C E$. 
Letting $r \rightarrow \infty$ and taking into account that $\varepsilon$ can be chosen arbitrarily, we get the inequality

$$
\sum_{j=1}^{q} \delta^{*}\left(a_{j}\right) \leq 2,
$$

hence

$$
\sum_{a} \delta^{*}(a) \leq 2
$$

THEOREM 6.3. The following relation holds:

$$
\delta(a) \leq \delta^{*}(a) \leq \Delta^{*}(a) \leq \Delta(a) .
$$

Proof. Only the middle inequality has to be verified, the other two follow from the definitions of $\delta^{*}(a)$ and $\Delta^{*}(a)$. Consider a set $E_{1}$ such that $C E_{1} \in L$ and

$$
\delta^{*}(a) \geq \liminf _{r \in E_{1}} \frac{m(r, a)}{T(r, f)}>\delta^{*}(a)-\varepsilon,
$$

and $E_{2}$ such that $C E_{2} \in L$ and

$$
\Delta^{*}(a) \leq \limsup _{r \in E_{2}} \frac{m(r, a)}{T(r, f)}<\Delta^{*}(a)+\varepsilon .
$$

Then

$$
\begin{aligned}
\delta^{*}(a)-\varepsilon<\liminf _{r \in E_{1}} \frac{m(r, a)}{T(r, f)} & \leq \liminf _{r \in E_{1} \cap E_{2}} \frac{m(r, a)}{T(r, f)} \\
& \leq \limsup _{r \in E_{1} \cap E_{2}} \frac{m(r, a)}{T(r, f)} \leq \limsup _{r \in E_{2}} \frac{m(r, a)}{T(r, f)}<\Delta^{*}(a)+\varepsilon .
\end{aligned}
$$

Since $\varepsilon$ can be chosen to be arbitrarily small, we get $\delta^{*}(a) \leq \Delta^{*}(a)$.

The inequality (6.26) implies that $\delta^{*}(a)$ is a more precise characteristic of the distribution of exceptional $a$-points than the Nevanlinna deficiency $\delta(a)$. The following result is also a corollary of (6.26).

Theorem 6.4. If $\delta(a, f)=\Delta(a, f)$, then $\delta(a, f)=\delta^{*}(a, f)=\Delta^{*}(a, f)$.

Together with Theorem 6.1 this result implies that for functions of finite order the equality $\delta(a, f)=\Delta(a, f)$ implies $\delta_{h}^{*}(a, f)=\Delta_{h}^{*}(a, f)=\delta(a, f)$. For functions of infinite order this implication does not hold, as is shown in Example 1.

Observe also, that it can happen that $\delta^{*}(a)>\delta_{h}(a)$ for each $h$ satisfying $0 \leq|h|<\infty$. To show this it is enough to modify Example 2 in the following way. In addition to $a_{k}=r_{k}-1$ we introduce

$$
a_{k}^{\prime}=-r_{k}+1, a_{k}^{\prime \prime}=i\left(r_{k}-1\right), a_{k}^{\prime \prime \prime}=-i\left(r_{k}-1\right), m_{k}^{\prime}=m_{k}^{\prime \prime}=m_{k}^{\prime \prime \prime}=m_{k} .
$$

The modified function $\Phi(z)$ is

$$
\Phi(z)=\prod_{k=1}^{\infty}\left\{\frac{\left(z-b_{k}\right)\left(z-b_{k}^{\prime}\right)\left(z-b_{k}^{\prime \prime}\right)\left(z-b_{k}^{\prime \prime \prime}\right)}{\left(z-a_{k}\right)\left(z-a_{k}^{\prime}\right)\left(z-a_{k}^{\prime \prime}\right)\left(z-a_{k}^{\prime \prime \prime}\right)}\right\}^{m_{k}},
$$

where $b_{k}, b_{k}^{\prime}, b_{k}^{\prime \prime}, b_{k}^{\prime \prime \prime}$ are sufficiently close to $a_{k}, a_{k}^{\prime}, a_{k}^{\prime \prime}, a_{k}^{\prime \prime \prime}$, the rest of the construction remains unchanged. Repeating the argument from the example with minor modifications, we get that $\delta_{h}(\infty)=0$ for each $h$ whereas $\delta^{*}(\infty)=1$.

Our next result distinguishes a rather wide class of functions for which $\delta^{*}(a)=$ $\delta(a)$. 
THEOREM 6.5. If the difference between the order and the lower order for a meromorphic function $f(z)$ satisfies $\rho-\lambda<1$, then

$$
\delta^{*}(a)=\delta(a), \Delta^{*}(a)=\Delta(a)
$$

for all a. The equality (6.27) takes place also for all meromorphic functions whose growth does not exceed the normal type of order one.

Corollary. If the conditions of Theorem 6.5 are satisfied, then $\delta_{h}(a)=\delta(a)$ for each $h$, that is, the deficiencies are shift invariant.

Proof. To prove $\delta^{*}(a)=\delta(a)$ and $\Delta^{*}(a)=\Delta(a)$, it suffices to show that for each $0<K<\infty$ and each $R, r<R<r+K \ln r$ the inequality

$$
(1+o(1)) \frac{N(r, a)}{T(r, f)} \leq \frac{N(R, a)}{T(R, f)} \leq(1+o(1)) \frac{N(r+K \ln r, a)}{T(r+K \ln r, f)}
$$

holds, since this inequality shows that exclusion from consideration of sets of class $L$ does not affect the value of $\lim \sup \frac{N}{T}$ and $\lim \inf \frac{N}{T}$. On the other hand,

$$
\frac{T(r, f)}{T(r+K \ln r, f)} \frac{N(r, a)}{T(r, f)} \leq \frac{N(R, a)}{T(R, f)} \leq \frac{N(r+K \ln r, a)}{T(r+K \ln r, f)} \frac{T(r+K \ln r, f)}{T(r, f)} .
$$

Therefore in order to prove (6.28) it is enough to show that

$$
\lim _{r \rightarrow \infty} \frac{T(r+K \ln r)}{T(r)}=1 .
$$

We have

$$
\frac{T(r+K \ln r)}{T(r)}=1+\frac{T^{\prime}(r+\theta(r) K \ln r)}{T(r)} K \ln r,
$$

where $0 \leq \theta(r) \leq 1$. Assume that (6.29) does not hold. Then there exists a sequence $r_{n} \rightarrow \infty$ such that

$$
\frac{T^{\prime}\left(r_{n}^{\prime}\right)}{T\left(r_{n}\right)}>\frac{\eta}{\ln r_{n}}, \eta>0, r_{n}^{\prime}=r_{n}+\theta\left(r_{n}\right) K \ln r_{n} .
$$

Since

$$
\frac{d}{d r} r T^{\prime}(r)=\stackrel{\circ}{A}(r)>0,
$$

the function $r T^{\prime}(r)$ is an increasing function and therefore

$$
T^{\prime}(r) \geq \frac{r_{n}^{\prime} T^{\prime}\left(r_{n}^{\prime}\right)}{r}, r \geq r_{n}^{\prime},
$$

whence

$$
\begin{gathered}
\int_{r_{n}^{\prime}}^{e r_{n}^{\prime}} T^{\prime}(r) d r \geq \int_{r_{n}^{\prime}}^{e r_{n}^{\prime}} \frac{r_{n}^{\prime} T^{\prime}\left(r_{n}^{\prime}\right)}{r} d r, \\
T\left(e r_{n}^{\prime}\right) \geq r_{n}^{\prime} T^{\prime}\left(r_{n}^{\prime}\right)+T\left(r_{n}^{\prime}\right)>\frac{r_{n}^{\prime} \eta}{\ln r_{n}} T\left(r_{n}\right) .
\end{gathered}
$$

For all $n \geq n_{0}(\varepsilon)$ we have $r_{n} \leq r_{n}^{\prime}<2 r_{n}$ and $T\left(r_{n}\right)>r_{n}^{\lambda-\varepsilon}, \varepsilon>0$.

From (6.30) we get

$$
T\left(6 r_{n}\right)>\frac{r_{n}^{1+\lambda-\varepsilon}}{\ln r_{n}} .
$$


Hence, $\rho \geq 1+\lambda-\varepsilon$ and, letting $\varepsilon \rightarrow 0$, we get $\rho \geq 1+\lambda$. Hence, if (6.27) does not hold, then $\rho-\lambda \geq 1$. Since for each transcendental meromorphic function $\lim _{r \rightarrow \infty} \frac{T(r)}{\ln r}=\infty$, the inequality (6.30) implies

$$
\lim _{n \rightarrow \infty} \frac{T\left(6 r_{n}\right)}{6 r_{n}}=\infty
$$

that is, if (6.27) does not hold, then $f(z)$ has growth of at least maximal type of order one.

Examples 2, 3, and other examples based on the same ideas (we leave the constructions of such examples to interested readers) show that the assumptions of Theorem 6.5 cannot be significantly weakened. Nevertheless some additions to it can be made. For example, (6.30) immediately implies

THEOREM 6.6. Let $f(z)$ be a meromorphic function satisfying $\rho=1+\lambda$. If one of the following conditions is satisfied:

(a) $\lim _{r \rightarrow \infty} T(r) / r^{\lambda} \ln r=\infty$ and $\lim \sup _{r \rightarrow \infty} T(r) / r^{\rho}<\infty$,

(b) $\liminf \inf _{r \rightarrow \infty} T(r) / r^{\lambda}>0$ and $T(r)=o\left(r^{\rho}(\ln r)^{-1}\right)$, then (6.27) holds.

Shift invariance of deficiencies takes place under weaker assumptions.

THEOREM 6.7. Let $f(z)$ be a meromorphic function satisfying $\rho=1+\lambda$. If one of the following conditions is satisfied:

(a) $\lim _{r \rightarrow \infty} T(r) / r^{\lambda}=\infty$ and $\limsup _{r \rightarrow \infty} T(r) / r^{\rho}<\infty$,

(b) $\liminf \operatorname{in}_{r \rightarrow \infty} T(r) / r^{\lambda}>0$ and $T(r)=o\left(r^{\rho}\right)$, then

$$
\delta_{h}(a)=\delta(a), \Delta_{h}(a)=\Delta(a) .
$$

for all $h$.

The proof is similar to the proof of Theorem 6.5. For (6.31) to be satisfied it is enough that for each $K, 0<K<\infty$ the equality

$$
\lim _{r \rightarrow \infty} \frac{T(r+K)}{T(r)}=1
$$

holds. It is clear that it is enough to show that

$$
\limsup _{r \rightarrow \infty} \frac{T(r+1)}{T(r)}=1 .
$$

But $T(r+1)=T(r)+T^{\prime}(r+\theta(r)), 0 \leq \theta(r) \leq 1$. If (6.33) does not take place, then there exists a sequence $r_{n} \rightarrow \infty$ such that

$$
\frac{T^{\prime}\left(r_{n}^{\prime}\right)}{T\left(r_{n}\right)}>\eta, \eta>0
$$

where $r_{n}^{\prime}=r_{n}+\theta\left(r_{n}\right)$. As before we get, that $T\left(e r_{n}^{\prime}\right) \geq r_{n}^{\prime} T^{\prime}\left(r_{n}^{\prime}\right)+T\left(r_{n}^{\prime}\right)$, therefore

$$
T\left(e r_{n}^{\prime}\right)>\eta r_{n}^{\prime} T\left(r_{n}\right) \text {. }
$$

Theorem 6.7 follows immediately from the inequality (6.34).

Note that the conditions of Theorem 6.7 may be satisfied even when $\delta^{*}(a)>$ $\delta(a)$ or $\Delta^{*}(a)<\Delta(a)$. We only give an example for which the condition (a) of Theorem 6.7 is satisfied and $\delta^{*}(\infty)>\delta(\infty)$. 
Example 4 . Let $\lambda>0, \rho=\lambda+1$. Consider the equation

$$
b^{\rho} \ln \frac{r}{b}=r^{\lambda}(\ln r)^{1 / 8}, b>2^{2 \lambda+8} .
$$

Taking into account the fact that the left-hand side of the equation is a linear function of $\ln r$ and the right-hand side is convex with respect to $\ln r$, it is easy to see that the equation (6.35) has two solutions in $[b, \infty)$, and these solutions, denotes by $R_{1}(b)$ and $R_{2}(b), R_{1}(b)<R_{2}(b)$, satisfy $b<R_{1}(b)<2 b, \ln R_{2}(b) \sim \frac{\rho}{\lambda} \ln b$ as $b \rightarrow \infty$. If $R_{1}(b)<r<R_{2}(b)$, the left-hand side of (6.35) is greater than the right-hand side.

We take the function $g\left(z ; r_{k}, n_{k}\right)$ (see (6.7)), where the parameters $r_{k}$ and $n_{k}$ are chosen in the following way. Let $r_{1}>1$ be so large that $R_{2}(b) \geq 2 b$ for each $b \geq r_{1}$. Set $r_{k+1}=R_{2}\left(r_{k}\right), k=1,2, \ldots$, and $n_{k}=\left[r_{k}^{\rho}\right]$. Consider the meromorphic function $\Phi\left(z ; a_{j}, b_{j}, m_{j}, \sigma_{j}, \varepsilon\right)($ see $(6.8))$, where $a_{k}=r_{k}-\sqrt{\ln r_{k}}, m_{k}=\left[r_{k}^{\rho}\left(\ln r_{k}\right)^{-\frac{1}{4}}\right]$. Let $f(z)=g(z) \Phi(z)$. By (6.16) we have $T(r, f)=N(r, 0, g)+N(r, \infty, \Phi)+O(1)$. It is easy to see that $n(r, 0, g) \leq\left(1-2^{-\rho}\right)^{-1} r^{\rho}$ and hence $N(r, 0, g)=O\left(r^{\rho}\right)$ and $N(r, \infty, \Phi)=o\left(r^{\rho}\right)$. Hence $T(r, f)=O\left(r^{\rho}\right)$. If $r \in\left[r_{k}, r_{k+1}\right)$, taking into account (6.35) we get

$$
\begin{aligned}
T(r, f) \geq N(r, 0, g)+O(1) & \geq r_{k-1}^{\rho} \ln \frac{r}{r_{k-1}}+r_{k}^{\rho} \ln \frac{r}{r_{k}}+O(\ln r) \\
& \geq r_{k-1}^{\rho} \ln \frac{r_{k}}{r_{k-1}}+r_{k}^{\rho} \ln \frac{r}{r_{k}}+O(\ln r) \\
& =r_{k}^{\lambda}\left(\ln r_{k}\right)^{1 / 8}+r_{k}^{\rho} \ln \frac{r}{r_{k}}+O(\ln r) .
\end{aligned}
$$

For $r_{k} \leq r<2 r_{k}$ the inequality

$$
T(r, f) \geq r_{k}^{\lambda}\left(\ln r_{k}\right)^{1 / 8}+O(\ln r)
$$

holds, and for $2 r_{k} \leq r<r_{k+1}$ the inequality

$$
T(r, f) \geq r_{k}^{\rho} \ln \frac{r}{r_{k}}+O(\ln r) \geq r^{\lambda}(\ln r)^{1 / 8}+O(\ln r)
$$

holds. Hence

$$
\liminf _{r \rightarrow \infty} \frac{T(r, f)}{r^{\lambda}(\ln r)^{1 / 8}}>0,
$$

and the function $f(z)$ satisfies the condition (a) of Theorem 6.7. Using (6.15) we get

$$
\begin{gathered}
N\left(r_{k}, \infty, f\right)=N\left(r_{k}, \infty, \Phi\right) \sim \sum_{j=1}^{k} r_{j}^{\rho}\left(\ln r_{j}\right)^{-\frac{1}{4}} \ln \frac{r_{k}}{r_{j}-\sqrt{\ln r_{j}}} \\
\geq r_{k}^{\rho}\left(\ln r_{k}\right)^{-\frac{1}{4}} \ln \frac{r_{k}}{r_{k}-\sqrt{\ln r_{k}}} \sim r_{k}^{\lambda}\left(\ln r_{k}\right)^{\frac{1}{4}} \\
N\left(r_{k}, 0, g\right) \leq \sum_{j=1}^{k} r_{j}^{\rho} \ln \frac{r_{k}}{r_{j}}=\sum_{j=1}^{k-1} r_{j}^{\rho} \ln \frac{r_{k}}{r_{j}} \\
=O\left(r_{k-1}^{\rho} \ln \frac{r_{k}}{r_{k-1}}\right)=O\left(r_{k}^{\lambda}\left(\ln r_{k}\right)^{1 / 8}\right), \\
T\left(r_{k}, f\right)=N\left(r_{k}, 0, g\right)+N\left(r_{k}, \infty, \Phi\right)+O(1) \sim N\left(r_{k}, \infty, \Phi\right)=N\left(r_{k}, \infty, f\right) .
\end{gathered}
$$


Hence $\delta(\infty, f)=0$ and, by Theorem $6.7, \delta_{h}(\infty, f)=0$ for all $h$.

Now let $r_{k}+\sqrt{\ln r_{k}} \leq r \leq r_{k+1}-\sqrt{\ln r_{k+1}}$. Then

$$
\begin{gathered}
N(r, \infty, f) \leq \sum_{j=1}^{k} r_{j}^{\rho}\left(\ln r_{j}\right)^{-\frac{1}{4}} \ln \frac{r}{r_{j}-\sqrt{\ln r_{j}}}=O\left(r_{k}^{\rho}\left(\ln r_{k}\right)^{-\frac{1}{4}} \ln \frac{r}{r_{k}-\sqrt{\ln r_{k}}}\right), \\
N(r, 0, g) \geq r_{k}^{\rho} \ln \frac{r}{r_{k}} .
\end{gathered}
$$

Since for $r_{k}+\sqrt{\ln r_{k}} \leq r \leq r_{k+1}-\sqrt{\ln r_{k+1}}$ the inequality

$$
\frac{\ln \frac{r}{r_{k}-\sqrt{\ln r_{k}}}}{\ln \frac{r}{r_{k}}} \leq \frac{\ln \frac{r_{k}+\sqrt{\ln r_{k}}}{r_{k}-\sqrt{\ln r_{k}}}}{\ln \frac{r_{k}+\sqrt{\ln r_{k}}}{r_{k}}} \rightarrow 2, k \rightarrow \infty,
$$

holds, we have $N(r, \infty, f)=o(N(r, 0, g))$ as $r \rightarrow \infty$, if we exclude from $[0, \infty)$ the set $E=\bigcup_{k=1}^{\infty}\left\{\left|r-r_{k}\right|<\sqrt{\ln r_{k}}\right\}$. It is easy to see that $E \in L$. Since for $r \in C E$ the relation $N(r, \infty, f)=o(N(r, 0, g))=o(T(r, f))$ holds, we get $\delta^{*}(\infty, f)=1$, that is, $\delta^{*}(\infty, f)>\delta(\infty, f)$.

REMARK 1. In many situations the easiest way to verify that $\delta^{*}(a)=\delta(a)$ is to check that the condition (6.33) is satisfied.

REMARK 2. It is clear that for fixed $a$ the statements of Theorems 6.5, 6.6, and 6.7 remain true if the conditions of the theorems are imposed onto $N(r, a)$ instead of $T(r, f)$, for example, if in Theorem 6.5 we require

$$
\rho[N(r, a)]-\lambda[N(r, a)]<1 .
$$

In fact, $N(r, a)$ is also an increasing function convex with respect to the logarithm, and our proof is based on these properties of $T(r, f)$ only.

Passage from $f(z)$ to $f(z+h)$ can be considered as a passage from exhaustion of the plane by circles $\{|z|=r\}$ to exhaustion of the plane by circles $\{|z+h|=r\}$. We can generalize this problem and consider the exhaustion of the complex $z$-plane by a family $\gamma_{r}$ of curves which are images of the family of circles $\{|z|=r\}$ under a homeomorphic mapping of the complex plane onto itself. We assume, for simplicity, that for $r \leq r_{0}$ we have $\gamma_{r}=\{|z|=r\}$. Let $D_{r}$ be the closed region bounded by the curve $\gamma_{r}$. Denote by $n_{\gamma}(r, a)$ the number of $a$-points of the function $f(z)$ in $D_{r}$, by $\pi \stackrel{\circ}{A}_{\gamma}(r, f)$ the (measured in the spherical metric) area of the piece of the Riemann surface onto which the function $w=f(z)$ maps $D_{r}$. After this the quantities $N_{\gamma}(r, a), T_{\gamma}(r, f), \delta_{\gamma}(a, f), \Delta_{\gamma}(a, f), \delta_{\gamma}^{*}(a, f)$, and $\Delta_{\gamma}^{*}(a, f)$ are introduced in the usual manner. Let $r_{1}(r)=\min _{z \in \gamma_{r}}|z|, r_{2}(r)=\max _{z \in \gamma_{r}}|z|$.

THEOREM 6.8. If

$$
A r-B<r_{1}(r) \leq r_{2}(r)<A r+B, 0<A, B<\infty,
$$

then $\rho\left[T_{\gamma}(r)\right]=\rho, \lambda\left[T_{\gamma}(r)\right]=\lambda$ and

(1) If $\rho<\infty$, then $\delta_{\gamma}^{*}(a)=\delta^{*}(a), \Delta_{\gamma}^{*}(a)=\Delta^{*}(a)$,

(2) If $\rho-\lambda<1$, then $\delta_{\gamma}^{*}(a)=\delta_{\gamma}(a), \Delta_{\gamma}^{*}(a)=\Delta_{\gamma}(a)$. 
We may without loss of generality assume that $A=1$. Analysis of proofs of Theorems 6.1 and 6.5, and also of Theorem 1.6 from Chapter 2 shows that we did not use the fact that the exhaustion is done by concentric circles, but only the fact that $\{|z+h|=r\}$ lies between $\{|z|=r+|h|\}$ and $\{|z|=r-|h|\}$. Therefore the same argument works in the present situation.

THEOREM 6.9. Let

$$
\lim _{r \rightarrow \infty} \frac{r_{2}(r)}{r_{1}(r)}=1
$$

If there exist constants $K_{1}$ and $K_{2}$ such that $(0<\rho<\infty)$

$$
0<K_{1} \leq r^{-\rho} T(r, f) \leq K_{2}<\infty, r \geq r_{0},
$$

then $\delta_{\gamma}(a)=\delta(a)$ and $\Delta_{\gamma}(a)=\Delta(a)$ for all $a$.

Proof. It is easy to see (compare the proof of Theorem 6.5), that in order to establish $\delta_{\gamma}(a)=\delta(a)$ and $\Delta_{\gamma}(a)=\Delta(a)$ it suffices to show that for each function $\varepsilon(r)>0$ satisfying $\lim _{r \rightarrow \infty} \varepsilon(r)=0$ the equality

$$
\lim _{r \rightarrow \infty} \frac{T(r(1+\varepsilon(r)))}{T(r)}=1
$$

holds.

We have

$$
\frac{T(r(1+\varepsilon(r)))}{T(r)}=1+\frac{T^{\prime}(r(1+\theta(r) \varepsilon(r)))}{T(r)} r \varepsilon(r),
$$

where $0 \leq \theta(r) \leq 1$.

If (6.38) is not satisfied, then there exist a function $\varepsilon_{1}(r)>0, \varepsilon_{1}(r) \rightarrow 0$ as $r \rightarrow \infty$, and a sequence $r_{n} \uparrow \infty, r_{n} \geq r_{0}$, such that

$$
\frac{T^{\prime}\left(r_{n}^{\prime}\right)}{T\left(r_{n}\right)}>\frac{\eta}{r_{n} \varepsilon_{1}\left(r_{n}\right)}, \eta>0 ; r_{n}^{\prime}=r_{n}\left(1+\theta\left(r_{n}\right) \varepsilon_{1}\left(r_{n}\right)\right)<2 r_{n} .
$$

Therefore, arguing in the same way as in the proof of (6.30), we get

$$
T\left(e r_{n}^{\prime}\right)>r_{n}^{\prime} T^{\prime}\left(r_{n}^{\prime}\right)+T\left(r_{n}^{\prime}\right)>\frac{r_{n}^{\prime} \eta}{r_{n} \varepsilon\left(r_{n}\right)} T\left(r_{n}\right) \geq \frac{\eta}{\varepsilon\left(r_{n}\right)} T\left(r_{n}\right)>\frac{\eta}{\varepsilon\left(r_{n}\right)} K_{1} r_{n}^{\rho}
$$

Since $r_{n}^{\prime}<2 r_{n}$, we get $T\left(e r_{n}^{\prime}\right)<K_{2}(2 e)^{\rho} r_{n}^{\rho}$, a contradiction with (6.39).

REMARK. If the condition (6.36) is satisfied and, furthermore, $r_{1}(r) \sim K r$, $0<K<\infty$, then we may use $T_{\gamma}(r, f)$ instead of $T(r, f)$ in the definition of the order and the type of a function $f(z)$, and also replace $T(r, f)$ by $T_{\gamma}(r, f)$ in the condition (6.37) of Theorem 6.9. In fact, the inequality $\stackrel{\circ}{A}\left(r_{1}(r)\right) \leq \stackrel{\circ}{A_{\gamma}}(r) \leq \stackrel{\circ}{A}\left(r_{2}(r)\right)$ implies that for $r>r_{0}(\varepsilon)$ the inequality

$$
\stackrel{\circ}{A}((K-\varepsilon) r) \leq \stackrel{\circ}{A}_{\gamma}(r) \leq \stackrel{\circ}{A}((K+\varepsilon) r), K-\varepsilon>0,
$$

holds, from where we get

$$
\int_{r_{0}(K-\varepsilon)}^{r(K-\varepsilon)} \frac{\stackrel{\circ}{A}(t)}{t} d t \leq \int_{r_{0}}^{r} \frac{\stackrel{\circ}{A}(t)}{t} d t \leq \int_{r_{0}(K+\varepsilon)}^{r(K+\varepsilon)} \frac{\stackrel{\circ}{A}(t)}{t} d t,
$$

$$
T((K-\varepsilon) r)+O(1) \leq T_{\gamma}(r) \leq T((K+\varepsilon) r)+O(1) .
$$


The inequality (6.40) immediately implies that $T(r)$ and $T_{\gamma}(r)$ have the same order and the same type, and also, that the following inequality is a necessary and sufficient condition for (6.37)

$$
0<K_{1}^{\prime} \leq r^{-\rho} T_{\gamma}(r) \leq K_{2}^{\prime}<\infty, r \geq r_{0}^{\prime} .
$$

The proof of the theorem is completed.

The condition (6.37) of Theorem 6.9, generally speaking, cannot be weakened. We give only one example of this type, showing that if $K_{2}$ is a function of $r$, $K_{2}=K_{2}(r)$, arbitrarily slowly approaching $\infty$ as $r \rightarrow \infty$, then it can happen that $\delta_{\gamma}(a) \neq \delta(a)$. An analogous example with $K_{1}=K_{1}(r) \rightarrow 0$ can be constructed in a similar way.

EXAMPLE 5. Without loss of generality we may assume that $K_{2}(r)>1$ is an increasing slowly varying function. Take the function

$$
w=F(\zeta)=g\left(\zeta ; r_{k}, n_{k}\right) \Phi\left(\zeta ; a_{k}, b_{k}, m_{k}, \sigma_{k}, \varepsilon\right)
$$

(see (6.7), (6.8)) with $\varepsilon<1 / 2, r_{k}=2^{k}, a_{k}=r_{k}-2$,

$$
n_{k}=m_{k}=\left\{\begin{array}{cc}
{\left[2^{\rho k}\right],} & k \neq k_{i}, \quad i=1,2, \ldots, \\
{\left[2^{k \rho}\left(2^{k}\right) K_{2}\right],} & k=k_{i},
\end{array}\right\}
$$

where the choice of $k_{i}(\geq 4)$ will be specified later, now we require only that $K_{2}\left(r_{k_{i}}\right)>64$.

Independently of the choice of the sequence $\left\{k_{i}\right\}$ the inequalities

$$
\begin{aligned}
& n(r, 0, g) \geq\left(2^{\rho}-1\right)^{-1} r^{\rho}, \\
& n(r, \infty, \Phi) \geq\left(2^{\rho}-1\right)^{-1} r^{\rho}
\end{aligned}
$$

hold. Therefore, taking into account (6.16) we get $T(r, f) \geq\left\{\rho\left(2^{\rho}-1\right)\right\}^{-1} r^{\rho}+O(1)$. On the other hand

$$
\begin{gathered}
n(r, 0, g)=O\left(r^{\rho} K_{2}(r)\right), \\
n(r, \infty, \Phi)=O\left(r^{\rho} K_{2}(r)\right), \\
T(r, f)=O\left(r^{\rho} K_{2}(r)\right) .
\end{gathered}
$$

It is clear now that the sequence $\left\{k_{i}\right\}$ can be chosen to be so rapidly increasing that the inequality

$$
N\left(r_{k_{i}}, 0, g\right) \leq C r_{k_{i}}^{\rho}
$$

holds, where $C$ is a constant greater than $2^{\rho}\left\{\rho\left(2^{\rho}-1\right)\right\}^{-1}$, because, if $n_{k}=\left[r_{k}^{\rho}\right]$ for all $k$, then we would have

$$
\limsup _{r \rightarrow \infty} \frac{n(r, 0, g)}{r^{\rho}} \leq \frac{2^{\rho}}{2^{\rho}-1} .
$$

It is easy to check that $N(r, 0, g) \sim N(r, \infty, \Phi)$, and to get, using the formulas (6.14), (6.15), (6.16), that $\delta(\infty, F(\zeta))=\Delta(\infty, F(\zeta))=1 / 2, \delta(0, F(\zeta))=$ $\Delta(0, F(\zeta))=0$.

Consider the strips

$$
S_{i}=\left\{r_{k_{i}}-1>\operatorname{Re} \zeta>r_{k_{i}}\left(1-K_{2}^{-\frac{1}{2}}\left(r_{k_{i}}\right)\right)-1,|\operatorname{Im} \zeta|<\eta_{i}\right\},
$$

where the sequence $\left\{\eta_{i}\right\}$ is chosen to be so rapidly converging to 0 , that the measure in the spherical metric area of the regions on the Riemann surface corresponding 
to $\bigcup_{i=1}^{\infty} S_{i}$ under the mapping $w=F(\zeta)$ is finite. Now we consider a homeomorphic mapping $z=z(\zeta)$ of the complex plane onto itself satisfying the following conditions: $z(\zeta)=\zeta$ outside $\bigcup_{i=1}^{\infty} S_{i}$, and on $S_{i}$ the mapping $z(\zeta)$ leaves fixed the boundary of $S_{i}$ and maps the point $r_{k_{i}}-2$ onto the point $r_{k_{i}}\left(1-K_{2}^{-\frac{1}{2}}\left(r_{k_{i}}\right)\right)$. We denote the inverse of the mapping $z(\zeta)$ by $\zeta(z)$. Let $\gamma_{r}$ be the image of $\{|z|=r\}$ under the mapping $\zeta(z)$. It is easy to compute that

$$
\begin{aligned}
& N_{\gamma}\left(r_{k_{i}}, \infty, F\right) \sim r_{k_{i}}^{\rho} K_{2}\left(r_{k_{i}}\right) \ln \left(1-K_{2}^{-\frac{1}{2}}\left(r_{k_{i}}\right)\right)^{-1} \sim r_{k_{i}}^{\rho} \sqrt{K_{2}\left(r_{k_{i}}\right)}, \\
& T_{\gamma}\left(r_{k_{i}}, F\right) \sim N\left(r_{k_{i}}, 0, F\right) \\
& \sim O\left(r_{k_{i}}^{\rho}\right)+r_{k_{i}}^{\rho} K_{2}\left(r_{k_{i}}\right) \ln \left(1-K_{2}^{-\frac{1}{2}}\left(r_{k_{i}}\right)\right)^{-1} \sim r_{k_{i}}^{\rho} \sqrt{K_{2}\left(r_{k_{i}}\right)} .
\end{aligned}
$$

Hence $\delta_{\gamma}(\infty, F)=\delta_{\gamma}(0, F)=0$ and $\delta_{\gamma}(\infty, F)<\delta(\infty, F)$.

Note that it is still unknown whether the deficiencies can be non-shift-invariant for entire functions of finite order. For entire functions of infinite order such an example was constructed by Hayman [Hay53]. We reproduce this example.

EXAMPle 6. Consider the entire function

$$
f(z)=\prod_{n=1}^{\infty}\left\{1+\left(\frac{z}{n}\right)^{3 n}\right\}^{2^{n}} .
$$

Since for $|z| \leq r$ we have

$$
\sum_{n=1}^{\infty} 2^{n}\left(\frac{|z|}{n}\right)^{3 n} \leq \sum_{n=1}^{\infty}\left(\frac{2 r^{3}}{n^{3}}\right)^{n}<\infty,
$$

the infinite product in (6.43) is absolutely and uniformly convergent in each disc $\{|z| \leq r\}$, and hence represents an entire function.

Since for $n \rightarrow \infty$ we have

$$
\left(\frac{n+\frac{1}{2}}{n+1}\right)^{3(n+1)} \rightarrow e^{-\frac{3}{2}},\left(\frac{n}{n+\frac{1}{2}}\right)^{3 n} \rightarrow e^{-\frac{3}{2}},
$$

and

$$
\ln \left(1-e^{-\frac{3}{2}}\right)>-0.3, e^{-\frac{3}{2}}<0.3
$$

there exists $N_{0}>1$ such that for $n \geq N_{0}$ the inequalities

$$
\begin{gathered}
\left|\ln \left\{1-\left(\frac{n}{n+\frac{1}{2}}\right)^{3 n}\right\}\right|<0.3 ; \\
\left(\frac{n+\frac{1}{2}}{n+1}\right)^{3(n+1)}<0.3 ; \\
\left(\frac{1}{n+\frac{1}{2}}\right)^{3}<\left(\frac{n}{n+\frac{1}{2}}\right)^{3 n} ; \\
\ln \left(\frac{n+\frac{1}{2}}{k}\right)>\frac{8}{9} \frac{n+\frac{1}{2}-k}{k}, k=n-2, n-1, n
\end{gathered}
$$


hold. Now we estimate $|f(z)|$ on the circle $\left\{|z|=n+\frac{1}{2}\right\}, n \geq N_{0}$. We have

$$
\begin{aligned}
\ln |f(z)|=\sum_{k=1}^{\infty} 2^{k} \ln \left|1+\left(\frac{z}{k}\right)^{3 k}\right| \\
=\sum_{k=1}^{n} 2^{k} 3 k \ln \frac{|z|}{k}+\sum_{k=1}^{n} 2^{k} \ln \left|1+\left(\frac{k}{z}\right)^{3 k}\right|+\sum_{k=n+1}^{\infty} 2^{k} \ln \left|1+\left(\frac{z}{k}\right)^{3 k}\right| \\
=\sum_{k=1}^{n} 2^{k} 3 k \ln \frac{n+\frac{1}{2}}{k}+\Phi_{1}(z)+\Phi_{2}(z) \\
=N\left(n+\frac{1}{2}, 0, f\right)+\Phi_{1}(z)+\Phi_{2}(z) .
\end{aligned}
$$

Taking into account the equality $\sum_{k=0}^{\infty} k x^{k}=x(1-x)^{-2},|x|<1$, we get

$$
\begin{aligned}
N\left(n+\frac{1}{2}, 0, f\right) & =\sum_{k=1}^{n} 2^{k} 3 k \ln \left(1+\frac{n+\frac{1}{2}-k}{k}\right) \\
& <\sum_{k=1}^{n} 2^{k} 3 k \frac{n+\frac{1}{2}-k}{k}=3 \cdot 2^{n} \sum_{\nu=0}^{n-1}\left(\nu+\frac{1}{2}\right) 2^{-\nu} \\
& <3 \cdot 2^{n} \sum_{\nu=0}^{\infty}\left(\nu+\frac{1}{2}\right) 2^{-\nu}=9 \cdot 2^{n} .
\end{aligned}
$$

On the other hand, taking into account (6.47) we get

$$
\begin{aligned}
& N\left(n+\frac{1}{2}, 0, f\right)>\sum_{k=n-2}^{n} 2^{k} 3 k \ln \frac{n+\frac{1}{2}}{k} \\
& \quad>\frac{8}{3} \sum_{k=n-2}^{n} 2^{k}\left(n+\frac{1}{2}-k\right)=\frac{8}{3}\left(\frac{1}{2}+\frac{1}{2} \cdot \frac{3}{2}+\frac{1}{4} \cdot \frac{5}{2}\right) 2^{n}=5 \cdot 2^{n} .
\end{aligned}
$$

An elementary investigation of the function $\left(\frac{x}{n+\frac{1}{2}}\right)^{3 x}$ shows that it decreases on the interval $\left[1,\left(n+\frac{1}{2}\right) e^{-1}\right]$ and increases on the interval $\left[\left(n+\frac{1}{2}\right) e^{-1}, \infty\right)$. Taking into account the inequality (6.46) we see that this function attains its maximum on the interval $[1, n]$ at the point $x=n$, and its minimal value on the interval $[n+1, \infty)$ at the point $x=n+1$. Therefore

$$
\begin{gathered}
\left(\frac{k}{n+\frac{1}{2}}\right)^{3 k} \leq\left(\frac{n}{n+\frac{1}{2}}\right)^{3 n}, 1 \leq k \leq n, \\
\left(\frac{n+\frac{1}{2}}{k}\right)^{3 k} \leq\left(\frac{n+\frac{1}{2}}{n+1}\right)^{3(n+1)}<0.3, k \geq n+1,
\end{gathered}
$$

and (6.44) implies the following inequality

$$
\left|\ln \left\{1-\left(\frac{k}{n+\frac{1}{2}}\right)^{3 k}\right\}\right|<0.3,1 \leq k \leq n \text {. }
$$


Now we estimate $\left|\Phi_{1}(z)\right|$ for $|z|=n+\frac{1}{2}$. Using (6.52) and the fact that for $0<$ $r<1,0 \leq \varphi<2 \pi$ the inequality

$$
|\ln | 1+r e^{i \varphi}|| \leq|\ln (1-r)| \leq \frac{r}{1-r}
$$

holds, we get

$$
\begin{aligned}
\left|\Phi_{1}(z)\right| & \leq \sum_{k=1}^{n} 2^{k}|\ln | 1+\left(\frac{k}{z}\right)^{3 k}|| \\
& \leq \sum_{k=1}^{n} 2^{k}\left|\ln \left\{1-\left(\frac{k}{n+\frac{1}{2}}\right)^{3 k}\right\}\right|<0.3 \sum_{k=1}^{n} 2^{k}<2^{n} \cdot 0.6 .
\end{aligned}
$$

Now we turn to an estimate of $\left|\Phi_{2}(z)\right|$ for $|z|=n+1 / 2$. Using (6.51) and (6.53) we get

$$
\begin{aligned}
\left|\Phi_{2}(z)\right| & \leq \sum_{k=n+1}^{\infty} 2^{k}|\ln | 1+\left(\frac{z}{k}\right)^{3 k}|| \\
& \leq \sum_{k=n+1}^{\infty} 2^{k} \frac{\left(\frac{n+\frac{1}{2}}{k}\right)^{3 k}}{1-\left(\frac{n+\frac{1}{2}}{k}\right)^{3 k}}<\frac{1}{0.7} \sum_{k=n+1}^{\infty} 2^{k}\left(\frac{n+\frac{1}{2}}{k}\right)^{3 k}=\frac{1}{0.7} \sum_{k=n+1}^{\infty} u_{k} .
\end{aligned}
$$

For $k \geq n+1$ we have

$$
\begin{aligned}
\frac{u_{k+1}}{u_{k}} & =2^{k+1}\left(\frac{n+\frac{1}{2}}{k+1}\right)^{3(k+1)} \frac{1}{2^{k}}\left(\frac{k}{n+\frac{1}{2}}\right)^{3 k} \\
& =2\left(\frac{n+\frac{1}{2}}{k+1}\right)^{3}\left\{\left(1+\frac{1}{k}\right)^{k}\right\}^{-3}<2\left\{\left(1+\frac{1}{k}\right)^{k}\right\}^{-3}<\frac{1}{4},
\end{aligned}
$$

hence

Therefore, by (6.45),

$$
u_{k} \leq \frac{1}{4^{k-n-1}} u_{n+1}
$$

$$
\begin{aligned}
& \left|\Phi_{2}(z)\right| \leq \frac{1}{0.7} \sum_{k=n+1}^{\infty} \frac{1}{4^{k-n-1}} u_{n+1}=\frac{1}{0.7} \cdot \frac{4}{3} u_{n+1} \\
& =\frac{1}{0.7} \cdot \frac{4}{3} 2^{n+1}\left(\frac{n+\frac{1}{2}}{n+1}\right)^{3(n+1)}<\frac{1}{0.7} \cdot \frac{4}{3} 2^{n+1} \cdot 0.3=\frac{8}{7} \cdot 2^{n}<1.2 \cdot 2^{n} .
\end{aligned}
$$

The relations (6.48), (6.49), (6.50), (6.54), and (6.55) imply that on the circle $\left\{|z|=n+\frac{1}{2}\right\}, n \geq N_{0}$, the inequality

$$
3 \cdot 2^{n}<3.2 \cdot 2^{n}<\ln |f(z)|<10.8 \cdot 2^{n}<11 \cdot 2^{n}
$$

holds. The inequality (6.56) implies that the inequality $|f(z)|>1$ holds on the circles $\left\{|z|=n+\frac{1}{2}\right\}$, hence $m\left(n+\frac{1}{2}, 0, f\right)=0$ and $\delta(0, f)=0$.

Now we prove that $\delta_{1}(0, f)>0$. Let $0<\eta<\frac{1}{4} e^{-22}, z_{0}=n e^{i \theta}$ be a zero of the function $f(z)$ of order $2^{n}, n \geq N_{0}$. Then the inequality

$$
\ln |f(z)|<2^{n-1} \ln \eta
$$


holds in the disc $\left\{\left|z-z_{0}\right|<\eta\right\}$.

In fact, since $\left\{\left|z-z_{0}\right| \leq \frac{1}{2}\right\} \subset\left\{|z| \leq n+\frac{1}{2}\right\}$, using (6.56) we conclude that the inequality $\ln |f(z)| \leq 11 \cdot 2^{n}$ holds in the disc $\left\{\left|z-z_{0}\right| \leq \frac{1}{2}\right\}$. On the circle $\left\{\left|z-z_{0}\right|=\frac{1}{2}\right\}$ we have

$$
\left|\frac{f(z)}{\left(z-z_{0}\right)^{2^{n}}}\right| \leq \frac{e^{11 \cdot 2^{n}}}{2^{-2^{n}}}=e^{11 \cdot 2^{n}} 2^{2^{n}},
$$

therefore, applying the maximum modulus principle for the disc $\left\{\left|z-z_{0}\right|<\eta\right\}$, we get

$$
|f(z)| \leq e^{11 \cdot 2^{n}} 2^{2^{n}}\left|z-z_{0}\right|^{2^{n}}<\left(e^{11} 2 \eta\right)^{2^{n}} \leq\left(\frac{1}{\sqrt{\eta}} \eta\right)^{2^{n}}=\eta^{2^{n-1}}
$$

which is equivalent to (6.57).

Now let $r$ be an arbitrary number exceeding $N_{0}+1$, and let $p=p_{r}$ be an integer satisfying $r-1<p<r+1$. The circles $\{|z|=p\}$ and $\{|z-1|=r\}$ intersect, therefore there exists $\varphi_{0}$ such that $\left|p e^{i \varphi_{0}}-1\right|=r$. We show that for $\left|\varphi-\varphi_{0}\right| \leq \frac{1}{4} \eta$ the inequality

$$
|| p e^{i \varphi}-1|-r|<\frac{1}{2} \eta
$$

holds. In fact, by the Lagrange theorem, we get

$$
|| p e^{i \varphi}-1|-r|=\left|\frac{d}{d \varphi}\right| p e^{i \varphi}-1||_{\varphi=\varphi^{\prime}}\left|\varphi-\varphi_{0}\right|,
$$

where $\varphi^{\prime}$ is a number between $\varphi$ and $\varphi_{0}$. But

$$
\left|\frac{d}{d \varphi}\right| p e^{i \varphi}-1||=\left|\frac{d}{d \varphi} \sqrt{p^{2}-2 p \sin \varphi+1}\right|=\left|\frac{-p \cos \varphi}{\left|p e^{i \varphi}-1\right|}\right| \leq \frac{p}{p-1}<2,
$$

therefore

$$
|| p e^{i \varphi}-1|-r|<2\left|\varphi-\varphi_{0}\right| \leq \frac{1}{2} \eta .
$$

Since the circle $\{|z|=p\}$ contains $3 p$ zeros of the function $f(z)$ (of order $2^{p}$ each), and these zeros divide the circle into arcs of $2 \pi / 3 p$ radians each, then the arc $\left\{|z|=p,\left|\arg z-\varphi_{0}\right| \leq \frac{1}{4} \eta\right\}$ contains at least

$$
\nu=\left[\frac{\eta}{2} \frac{3 p}{2 \pi}-1\right]=\left[\frac{3 \eta}{4 \pi} p-1\right], p \geq \frac{8 \pi}{3 \eta},
$$

zeros $z_{j}=p e^{i \varphi_{j}}, \varphi_{1}<\varphi_{2}<\cdots<\varphi_{\nu},\left|\varphi_{j}-\varphi_{0}\right| \leq \frac{1}{4} \eta$. Since each zero $z_{j}$ satisfies (6.58), we can find a point $z_{j}^{\prime}=1+r e^{i \theta_{j}}$ such that $\left|z_{j}^{\prime}-z_{j}\right|<\frac{1}{2} \eta$. Denote the set of $\operatorname{arcs} C_{r}^{j}=\left\{z-1=r e^{i \theta},\left|\theta-\theta_{j}\right|<\eta /(2 r)\right\}, 1 \leq j \leq \nu$, by $C_{r}$. The inequality $\left|z-z_{j}^{\prime}\right|=\left|(z-1)-\left(z_{j}^{\prime}-1\right)\right|=\left|r e^{i \theta}-r e^{i \theta_{j}}\right|=r\left|e^{i \theta}-e^{i \theta_{j}}\right|<r\left|\theta-\theta_{j}\right|<\frac{1}{2} \eta$ holds on $C_{r}^{j}$, therefore $\left|z-z_{j}\right| \leq\left|z-z_{j}^{\prime}\right|+\left|z_{j}^{\prime}-z_{j}\right|<\eta$. The $\operatorname{arcs} C_{r}^{j}$ are pairwise 
disjoint. In fact, if there is a point $z$ in the intersection of $C_{r}^{j}$ and $C_{r}^{j+1}$, then $\left|z_{j}-z_{j+1}\right|<\left|z_{j}-z\right|+\left|z-z_{j+1}\right|<2 \eta$. On the other hand,

$$
\begin{aligned}
\left|z_{j}-z_{j+1}\right| & =\left|p e^{i \varphi_{j}}-p e^{i \varphi_{j+1}}\right|=p\left|1-e^{i\left(\varphi_{j+1}-\varphi_{j}\right)}\right| \\
& =p\left|1-e^{2 \pi i / 3 p}\right| \geq p \sin \frac{2 \pi}{3 p} \geq p \frac{2}{\pi} \cdot \frac{2 \pi}{3 p}=\frac{4}{3},
\end{aligned}
$$

and we get a contradiction. Thus the total measure of $C_{r}=\bigcup_{j=1}^{\nu} C_{r}^{j}$ in radians is

$$
\frac{\eta}{r} \nu=\frac{\eta}{r}\left[\frac{3 \eta}{4 \pi} p-1\right] \text {. }
$$

Since $p / r \rightarrow 1$ as $r \rightarrow \infty$, the measure approaches $3 \eta^{2} /(4 \pi)$ as $r \rightarrow \infty$, and is greater than $\eta^{2} /(2 \pi)$ when $r>r_{0}(\eta)=r_{0}$.

Now we consider the function $f(z+1)$ on the circle $\{|z|=r\}, r>r_{0}$. Let $n=[r+3 / 2]$. Then for $|z|=r$ the inequality $|z+1| \leq n+1 / 2$ holds. By (6.56), for $\{|z|=r\}$ we have

$$
\ln |f(z+1)|<11 \cdot 2^{n}
$$

and

$$
T(r, f(z+1)) \leq \ln ^{+} M(r, f(z+1))<11 \cdot 2^{n} .
$$

On the other hand, the circle $\{|z+1|=n-1\}$ intersects the circle $\{|z|=r\}$. It is clear that $r-1<n-1<r+1$, and we can use the estimates presented above. For each $\operatorname{arc} C_{r}^{j}$ there exists a zero $z_{j}=(n-1) e^{i \varphi_{j}}$ of the function $f(z)$ such that $\left|z-z_{j}\right|<\eta$ if $z \in C_{r}^{j}$. Therefore by (6.57), for $z \in C_{r}$, the inequality

$$
\ln \left|f\left(1+r e^{i \theta}\right)\right|=\ln |f(z)|<2^{n-2} \ln \eta
$$

holds. Since the measure of the set $K_{r}$ of those $\theta$ for which (6.60) holds exceeds $\eta^{2} /(2 \pi)$ for $r>r_{0}$, we have

$$
\begin{aligned}
m(r, 0, f(z+1)) & \geq \frac{1}{2 \pi} \int_{K_{r}} \ln ^{+} \frac{1}{\left|f\left(1+r e^{i \theta}\right)\right|} d \theta \\
& \geq \frac{1}{2 \pi} \int_{K_{r}} 2^{n-2} \ln \frac{1}{\eta} d \theta>\frac{1}{2 \pi} 2^{n-2}\left(\ln \frac{1}{\eta}\right) \frac{\eta^{2}}{2 \pi}=2^{n} \frac{\eta^{2}}{16 \pi^{2}} \ln \frac{1}{\eta} .
\end{aligned}
$$

Taking into account (6.59) we get

$$
\begin{aligned}
\delta_{1}(0, f) & =\delta(0, f(z+1)) \\
& =\liminf _{r \rightarrow \infty} \frac{m(r, 0, f(z+1))}{T(r, f(z+1))} \geq \frac{2^{n} \frac{\eta^{2}}{16 \pi^{2}} \ln \frac{1}{\eta}}{2^{n} 11}=\frac{\eta^{2}}{176 \pi^{2}} \ln \frac{1}{\eta}>0 .
\end{aligned}
$$

Thus, zero is a deficient value for $f(z+1)$, but is not a deficient value for $f(z)$.

Observe also, that for $f_{1}(z)=f(z+1)$ we have

$$
\lim _{r \rightarrow \infty} \frac{\ln \mu\left(r, f_{1}\right)}{\ln M\left(r, f_{1}\right)}=-\infty, \text { where } \mu\left(r, f_{1}\right)=\min _{|z|=r}\left|f_{1}(z)\right| .
$$

In fact, (6.60) implies that $\ln \mu\left(r, f_{1}\right) \leq 2^{n-2} \ln \eta$, and (6.59) implies that $\ln M\left(r, f_{1}\right)<11 \cdot 2^{n}$. Therefore for $r>r_{0}(\eta)$ the inequality

$$
\frac{\ln \mu\left(r, f_{1}\right)}{\ln M\left(r, f_{1}\right)}<\frac{-2^{n-2} \ln \frac{1}{\eta}}{11 \cdot 2^{n}}=-\frac{1}{44} \ln \frac{1}{\eta}
$$

holds. Since $\eta$ can be chosen arbitrarily small, we get (6.61). 
In conclusion we mention that it is possible to do the work similar to that done in this section for the index $\varepsilon(a)$ instead of the defect $\delta(a)$ considered here (see (6.19)). That is, we can introduce the values $\varepsilon^{*}(a), \varepsilon_{\gamma}(a), \varepsilon_{\gamma}^{*}(a)$, and prove for them analogues of all theorems of this section except Theorem 6.3. The analogue of Theorem 6.4 is stated in the following way: if the limit

$$
\lim _{r \rightarrow \infty} \frac{N_{1}(r, a)}{T(r, f)}=\varepsilon(a)
$$

exists, then $\varepsilon(a)=\varepsilon^{*}(a)$. 


\section{CHAPTER 5}

\section{Asymptotic properties of meromorphic functions and deficiencies}

\section{Asymptotic values}

Let $f(z)$ be a meromorphic function, $C$ a continuous curve in $\{|z|<\infty\}$ given by an equation $z=z(t), 0 \leq t<\infty,|z(t)|<\infty$, and $z(t) \rightarrow \infty$ as $t \rightarrow \infty$. If there exists a finite or infinite $a$ such that

$$
\lim _{\substack{z \rightarrow \infty \\ z \in C}} f(z)=\lim _{t \rightarrow \infty} f(z(t))=a,
$$

then $a$ is called an asymptotic value of the function $f(z)$, and $C$ is called an asymptotic curve. The pair $\{a, C\}$ is also called an asymptotic spot (sometimes such pair is called an asymptotic value). Two asymptotic spots $\left\{a_{1}, C_{1}\right\}$ and $\left\{a_{2}, C_{2}\right\}$ are considered as equal if:

(1) $a_{1}=a_{2}=a$.

(2) There exist a sequence of continuous curves $\gamma_{k}$ such that one end of $\gamma_{k}$ belongs to $C_{1}$, the other end belongs to $C_{2}$, and

$$
\lim _{k \rightarrow \infty} \min _{z \in \gamma_{k}}|z|=\infty, \lim _{\substack{z \in \infty \\ z \in \cup_{k} \gamma_{k}}} f(z)=a .
$$

It is clear that asymptotic spots $\left\{a_{1}, C_{1}\right\}$ and $\left\{a_{2}, C_{2}\right\}$ are equal if the curves $C_{1}$ and $C_{2}$ have points of intersection in each neighborhood of infinity.

Significant amount of research is devoted to relations between exceptional and asymptotic values of a meromorphic functions, however, many problems are still open. We present some of the known results.

First of all, it is clear that an asymptotic value does not have to be exceptional in any sense. Indeed the value $w=0$ is an asymptotic value for $f(z)=\sin z / z$ (it corresponds to two different asymptotic spots with asymptotic curves $C_{1}: z(t)=$ $t$ and $C_{2}: z(t)=-t$ ) without being a Valiron exceptional value, and hence without being an exceptional value in any other sense. The following theorem due to $\mathrm{F}$. Iversen is a result in the opposite direction.

TheOREM 1.1. Each Picard exceptional value is an asymptotic value.

To prove this theorem we need the following lemma.

Lemma 1.1. Let $G$ be an unbounded region, let $\Gamma$ be the part of the boundary of $G$ in the complex plane (that is, $\infty$ is not considered as a point in $\Gamma$ ). Let $f(z)$ be analytic in $G$ and continuous in $G \cup \Gamma$. If $|f(z)|$ is bounded in $G$ and $|f(z)| \leq M$ for $z \in \Gamma$, then $|f(z)| \leq M$ in $G$.

Proof. If $f(z)$ is constant, the result is obvious. So suppose that $f(z)$ is nonconstant and $|f(z)| \leq K$ for $z \in G$. We may assume that $K>M$ because 
otherwise there is nothing to prove. We suppose that $z_{0} \in G$ is such that $\left|f\left(z_{0}\right)\right|>$ $M$ and prove that in such a case for an arbitrary $z^{\prime} \in G, z^{\prime} \neq z_{0}$ we have $\left|f\left(z^{\prime}\right)\right| \leq$ $\left|f\left(z_{0}\right)\right|$. It leads in contradiction with the maximum modulus principle. Let $\varepsilon$ be an arbitrarily small positive number. Consider a disc $D=\left\{\left|z-z_{0}\right| \leq \eta\right\}$ with so small radius that it is contained in $G$, does not contain $z^{\prime}$, and the inequality $|f(z)| \leq\left|f\left(z_{0}\right)\right|+\varepsilon$ holds for $z$ in $\gamma=\left\{\left|z-z_{0}\right|=\eta\right\}$. Let $1>\delta>0$. We consider the function $\varphi_{\delta}(z)=f(z)\left(\eta /\left(z-z_{0}\right)\right)^{\delta}$ in the region $G \backslash D=G_{\eta}$. This function is multivalent, but it has a univalent modulus. It is analytic in $G_{\eta}$, continuous in the closure of $G_{\eta}$, including $z=\infty$, and $\varphi_{\delta}(\infty)=0$. We have $\left|\eta /\left(z-z_{0}\right)\right|=1$ for $z \in \gamma$ and $\left|\eta /\left(z-z_{0}\right)\right|<1$ for $z \in \Gamma$. Therefore $\left|\varphi_{\delta}(z)\right| \leq\left|f\left(z_{0}\right)\right|+\varepsilon$ for $z \in \gamma$ and $\left|\varphi_{\delta}(z)\right| \leq M<\left|f\left(z_{0}\right)\right|+\varepsilon$ for $z \in \Gamma$. Applying the maximum modulus principle to $\varphi_{\delta}(z)$ we get $\left|\varphi_{\delta}\left(z^{\prime}\right)\right| \leq\left|f\left(z_{0}\right)\right|+\varepsilon$. Letting $\varepsilon \rightarrow 0$ and $\delta \rightarrow 0$ in this inequality, we get $\left|f\left(z^{\prime}\right)\right| \leq\left|f\left(z_{0}\right)\right|$.

Proof. Now we turn to the proof of Theorem 1.1. Without loss of generality we assume that $a=\infty$ is a Picard exceptional value. Otherwise we would consider the function $F(z)=1 /\{f(z)-a\}$. Thus the transcendental function $f(z)$ has at most finitely many poles. First we assume that there are no poles, that is, the function is entire. We need to show the existence of a continuous curve $C$ approaching infinity, such that $f(z)$ approaches infinity along this curve as $z \rightarrow \infty$.

Denote by $G_{n}, n=1,2, \ldots$, the open set of those $z$ for which $|f(z)|>n$. The equality $|f(z)|=n$ holds for each point of the boundary of $G_{n}$ in the complex plane. Each connected component of $G_{n}$ is an unbounded region, since otherwise, by the maximum modulus principle, we would have $|f(z)| \leq n$ for each $z \in G_{n}$. By Lemma 1.1 each connected component of $G_{n}$ contains at least one point of $G_{n+1}$. Therefore we can find a sequence $\left\{G_{n}^{\prime}\right\}_{n=1}^{\infty}$ of connected components of sets $G_{n}$ such that $G_{1}^{\prime} \supset G_{2}^{\prime} \supset G_{3}^{\prime} \supset \ldots$. Since the function $f(z)$ is bounded in each disc $\{|z| \leq R\}$, then, starting with some $n=n(R)$ the regions $G_{n}^{\prime}$ do not intersect this set. Consider in each region $G_{n}^{\prime}$ a point $z_{n}$ and join it by a Jordan $\operatorname{arc} C_{n} \subset G_{n}^{\prime}$ with $z_{n+1}$. Then $C=C_{1} \cup C_{2} \cup \ldots$ is the desired curve. In fact, if $z$ successively traverses $C_{1}, C_{2}, \ldots$, then $z \rightarrow \infty$ since $\bigcup_{j=n(R)}^{\infty} C_{j}$ does not intersect the disc $\{|z| \leq R\}$. Besides, $\bigcup_{j=n}^{\infty} C_{j} \subset G_{n}^{\prime}$ and hence $|f(z)|>n$ for $z \in \bigcup_{j=n}^{\infty} C_{j}$, that is, $f(z) \rightarrow \infty$ as $z \rightarrow \infty, z \in C$.

If $f(z)$ has poles at finitely many points $\zeta_{1}, \ldots, \zeta_{p}$, we denote by $H_{j}(z)$ the principal part of the Laurent expansion of $f(z)$ in the neighborhood of $\zeta_{j}$. The function $H_{j}(z)$ is rational, has zero at $\infty$ and the only pole at $\zeta_{j}$. The function

$$
g(z)=f(z)-\sum_{j=1}^{p} H_{j}(z)
$$

is an entire transcendental function, hence, as we have proved above, there exists an asymptotic curve $C$ such that $g(z) \rightarrow \infty$ along $C$. The function $\sum_{j=1}^{p} H_{j}(z)$ approaches zero along $C$, hence $f(z)$ approaches $\infty$.

REMARK. The argument used in the proof of Theorem 1.1 can be used to prove the following statement. Let $G$ be an unbounded region, $\Gamma$ be the part of its boundary in the complex plane. Let $f(z)$ be analytic in $G$ and continuous in $G \cup \Gamma$. If the function $f(z)$ is bounded on $\Gamma$ but is unbounded on $G$, then there exists an asymptotic curve in $G$ approaching $\infty$ and such that $f(z)$ approaches $\infty$ along $C$. 
ExERCISE. Prove that for each transcendental entire function $f(z)$ and each positive integer $n$ there exists an asymptotic curve $C_{n}$ such that $f(z) z^{-n} \rightarrow \infty$ as $z \rightarrow \infty, z \in C_{n}$.

Hint. Apply Theorem 1.1 to $\varphi(z)=z^{-n} f(z)$.

Later we shall see that, in general, Borel or Nevanlinna exceptional values, and hence, Valiron exceptional values are not necessarily asymptotic values.

Now we prove the following important theorem due to Ahlfors.

THEOREM 1.2. If an entire function $f(z)$ has $p \geq 2$ different asymptotic spots $\left\{\infty, C_{j}\right\}, 1 \leq j \leq p$, then

$$
\liminf _{r \rightarrow \infty} r^{-\frac{p}{2}} T(r, f)>0
$$

The statement remains valid for $p=1$ if we assume, in addition, that

$$
\mu(r, f)=\min _{|z|=r}|f(z)|=O(1), r \rightarrow \infty .
$$

Proof. We may assume, without loss of generality, that the curves $C_{j}$ do not intersect each other. Consider the open set $G_{n}$ of those points for which $|f(z)|>n$. Denote by $G_{n}^{(j)}$ the connected component of the set $G_{j}$ containing $C_{j} \cap\{|z|>R\}$ for sufficiently large $R$. It is clear that the region $G_{n}^{(j)}$ is uniquely determined by this condition. The regions $G_{n}^{(j)}, 1 \leq j \leq p$, are pairwise disjoint for sufficiently large $n$. In fact, if we assume that the regions $G_{n}^{\left(j_{1}\right)}$ and $G_{n}^{\left(j_{2}\right)}$ intersect for all $n \geq 1$, they would coincide: $G_{n}^{\left(j_{1}\right)}=G_{n}^{\left(j_{2}\right)}$, and joining the curves $C_{j_{1}}$ and $C_{j_{2}}$ by continuous curves $\gamma_{k}$ lying in $G_{k}^{\left(j_{1}\right)}=G_{k}^{\left(j_{2}\right)}$, we can easily show that the asymptotic spots $\left\{\infty, C_{j_{1}}\right\}$ and $\left\{\infty, C_{j_{2}}\right\}$ coincide, a contradiction. Increasing $n$, if necessary, we get into the situation in which none of the regions $G_{n}^{(j)}$ completely contains any of the circles $\{|z|=r\}$. If $p=1$ the possibility to choose such $n$ is guaranteed by the condition (1.2): it suffices to choose $n>\sup _{0<r<\infty} \mu(r, f)$. For $p \geq 2$ such $n$ can be chosen because the complement of $G_{n}^{(j)}$ contains at least one unbounded region. Thus, we have found $p$ unbounded pairwise disjoint regions $G_{n_{0}}^{(j)}, 1 \leq j \leq p$, in the complex plane having the following properties:

(a) The region $G_{n_{0}}^{(j)}$ does not completely contain any of the circles $\{|z|=r\}$.

(b) The function $f(z)$ is unbounded in $G_{n_{0}}^{(j)}$ and satisfies the inequality $|f(z)|>$ $n_{0}$ in $G_{n_{0}}^{(j)}$.

(c) $|f(z)|=n_{0}$ on the part of the boundary of $G_{n_{0}}^{(j)}$ in the complex plane.

We may assume, without loss of generality, that $n_{0}=1$, we would consider the function $f(z) / n_{0}$ otherwise. In what follows we assume that $n_{0}=1$ and drop the lower index $n_{0}$ for $G_{n_{0}}^{(j)}$.

We need the following Wirtinger inequality. Let $y(x)$ be a continuously differentiable function on $[a, b], y(a)=y(b)=0$. Then

$$
\int_{a}^{b}\left[y^{\prime}(x)\right]^{2} d x \geq\left(\frac{\pi}{b-a}\right)^{2} \int_{a}^{b}[y(x)]^{2} d x .
$$

We may assume, without loss of generality, that $a=0, b=\pi$, since the general case can be reduced to this case using the substitution $x=a+\frac{b-a}{\pi} t$. So, we need 
to prove

$$
\int_{0}^{\pi}\left[y^{\prime}(x)\right]^{2} d x \geq \int_{0}^{\pi}[y(x)]^{2} d x .
$$

Since $y(0)=y(\pi)=0$, we have $y(x)=O(x)$ as $x \rightarrow 0$ and $y(x)=O(\pi-x)$ as $x \rightarrow \pi$. Therefore the function $y^{2}(x) \cot x$ is continuous on the segment $[0, \pi]$ and vanishes at the ends of the segment, and the function $y(x) \cot x$ is continuous on $[0, \pi]$.

From here we get

$$
\begin{aligned}
0=\int_{0}^{\pi} d\left(y^{2} \cot x\right) & =\int_{0}^{\pi}\left\{2 y y^{\prime} \cot x-y^{2}\left(1+\cot ^{2} x\right)\right\} d x \\
& =\int_{0}^{\pi}\left\{\left(y^{\prime}\right)^{2}-y^{2}-\left(y^{\prime}-y \cot x\right)^{2}\right\} d x \leq \int_{0}^{\pi}\left(\left(y^{\prime}\right)^{2}-y^{2}\right) d x
\end{aligned}
$$

that is, the inequality $\left(1.3^{\prime}\right)$.

Let $r_{0}$ be a so large number that the circle $\left\{|z|=r_{0}\right\}$ intersects all of the regions $G^{(j)}, 1 \leq j \leq p$. We fix a region $G^{(j)}$. Denote by $r_{0}^{(j)}$ the minimal value of $|z|$ for $z \in \bar{G}^{(j)}$, and by $S^{(j)}(r)$ the intersection $G^{(j)} \cap\{|z|=r\}$ for $r>r_{0}^{(j)}$. The set $S^{(j)}(r)$ consists of finitely many arcs since the part of the boundary of $G^{(j)}$ contained in an arbitrary disc $\{|z| \leq r\}$ consists of finitely many analytic curves. The last statement can be easily verified using the fact that $|f(z)|=1$ on the boundary of $G^{(j)}$. Denote by $r l_{j}(r)$ the length of the longest arc among the arcs forming $S^{(j)}(r)$. It is clear that

$$
\sum_{j=1}^{p} l_{j}(r) \leq 2 \pi
$$

Consider the function $\ln \left|f\left(r e^{i \varphi}\right)\right|=\ln \left|f\left(e^{t+i \varphi}\right)\right|=u(t, \varphi), t=\ln r$ in the region $G^{(j)}$. It is clear that $u(\ln r, \varphi)>0$ in the region $G^{(j)}$, and that $u(t, \varphi)=$ $\operatorname{Re} \ln f\left(e^{t+i \varphi}\right)$ is a harmonic function. Therefore

$$
\frac{\partial^{2} u}{\partial t^{2}}+\frac{\partial^{2} u}{\partial \varphi^{2}}=0
$$

We introduce the notation

$$
\begin{gathered}
m_{j}(r)=\int_{S^{(j)}(r)}\left(\ln \left|f\left(r e^{i \varphi}\right)\right|\right)^{2} d \varphi \\
\mu_{j}(t)=m_{j}\left(e^{t}\right)=\int_{S^{(j)}(r)} u^{2}(t, \varphi) d \varphi .
\end{gathered}
$$

Taking into account the fact that $u(t, \varphi)=0$ on the ends of the intervals of integration, we get

$$
\begin{gathered}
\mu_{j}^{\prime}(t)=2 \int_{S^{(j)}(r)} u \frac{\partial u}{\partial t} d \varphi \\
\mu_{j}^{\prime \prime}(t)=2 \int_{S^{(j)}(r)}\left\{u \frac{\partial^{2} u}{\partial t^{2}}+\left(\frac{\partial u}{\partial t}\right)^{2}\right\} d \varphi .
\end{gathered}
$$

Integrating by parts and using (1.5), we get

$$
\int_{S^{(j)}(r)} u \frac{\partial^{2} u}{\partial t^{2}} d \varphi=-\int_{S^{(j)}(r)} u \frac{\partial^{2} u}{\partial \varphi^{2}} d \varphi=\int_{S^{(j)}(r)}\left(\frac{\partial u}{\partial \varphi}\right)^{2} d \varphi .
$$


Substituting into the equality (1.6) we have

$$
\mu_{j}^{\prime \prime}(t)=2 \int_{S^{(j)}(r)}\left\{\left(\frac{\partial u}{\partial t}\right)^{2}+\left(\frac{\partial u}{\partial \varphi}\right)^{2}\right\} d \varphi .
$$

Obviously, for $t>\ln r_{0}^{(j)}$ we have $\mu_{j}^{\prime \prime}(t) \geq 0$, hence $\mu_{j}^{\prime}(t)$ is nondecreasing. Since $\mu_{j}(t)>0$ for $t>\ln r_{0}^{(j)}$, and $\mu_{j}(t) \rightarrow 0$ as $t \rightarrow \ln r_{0}^{(j)}$, we have, as is easy to see, $\mu_{j}^{\prime}(t)>0$ for $t>\ln r_{0}^{(j)}$. When $r e^{i \varphi} \in S^{(j)}(r)$, the variable $\varphi$ ranges over the finite system of intervals $\left(\varphi_{1}, \varphi_{1}^{\prime}\right),\left(\varphi_{2}, \varphi_{2}^{\prime}\right), \ldots,\left(\varphi_{s}, \varphi_{s}^{\prime}\right)$, where $s, \varphi_{1}, \varphi_{1}^{\prime}, \ldots, \varphi_{s}, \varphi_{s}^{\prime}$ depend on $j$ and $r$. We use for each of the intervals $\left(\varphi_{\sigma}, \varphi_{\sigma}^{\prime}\right)$ the Wirtinger inequality (1.3) and get

$$
\int_{\varphi_{\sigma}}^{\varphi_{\sigma}^{\prime}}\left(\frac{\partial u}{\partial \varphi}\right)^{2} d \varphi \geq\left(\frac{\pi}{\varphi_{\sigma}^{\prime}-\varphi_{\sigma}}\right)^{2} \int_{\varphi_{\sigma}}^{\varphi_{\sigma}^{\prime}} u^{2} d \varphi \geq\left(\frac{\pi}{l_{j}(r)}\right)^{2} \int_{\varphi_{\sigma}}^{\varphi_{\sigma}^{\prime}} u^{2} d \varphi .
$$

Adding these inequalities for all $\sigma$ from 1 to $s$, we get

$$
\int_{S^{(j)}(r)}\left(\frac{\partial u}{\partial \varphi}\right)^{2} d \varphi \geq\left(\frac{\pi}{l_{j}(r)}\right)^{2} \int_{S^{(j)}(r)} u^{2} d \varphi=\left(\frac{\pi}{l_{j}(r)}\right)^{2} \mu_{j}(t) .
$$

Using the Cauchy-Buniakowsky inequality we get

$$
\begin{aligned}
\left(\mu_{j}^{\prime}(t)\right)^{2}=4\left\{\int_{S^{(j)}(r)} u \frac{\partial u}{\partial t} d \varphi\right\}^{2} & \leq 4 \int_{S^{(j)}(r)} u^{2} d \varphi \int_{S^{(j)}(r)}\left(\frac{\partial u}{\partial t}\right)^{2} d \varphi \\
& =4 \mu_{j}(t) \int_{S^{(j)}(r)}\left(\frac{\partial u}{\partial t}\right)^{2} d \varphi .
\end{aligned}
$$

From (1.7), (1.8), and (1.9) we derive

$$
\mu_{j}^{\prime \prime}(t) \geq \frac{1}{2} \frac{\left\{\mu_{j}^{\prime}(t)\right\}^{2}}{\mu_{j}(t)}+2\left(\frac{\pi}{l_{j}(r)}\right)^{2} \mu_{j}(t)
$$

from where, taking into account

$$
\left(\frac{\mu_{j}^{\prime}}{\mu_{j}}\right)^{\prime}=\frac{\mu_{j}^{\prime \prime}}{\mu_{j}}-\left(\frac{\mu_{j}^{\prime}}{\mu_{j}}\right)^{2}
$$

we get

This implies

$$
2 \omega_{j}^{\prime \prime}(t)+{\omega_{j}^{\prime}}^{2}(t) \geq\left(\frac{2 \pi}{l_{j}(r)}\right)^{2}, \omega_{j}(t)=\ln \mu_{j}(t) .
$$

$$
\left\{\omega_{j}^{\prime}(t)+\frac{\omega_{j}^{\prime \prime}(t)}{\omega_{j}^{\prime}(t)}\right\}^{2} \geq{\omega_{j}^{\prime}}^{2}(t)+2 \omega_{j}^{\prime \prime}(t) \geq\left(\frac{2 \pi}{l_{j}(r)}\right)^{2} .
$$

Since $\omega_{j}^{\prime}(t)>0$ for $t>\ln r_{0}^{(j)}$, the last inequality implies that

$$
\omega_{j}^{\prime}(t)+\frac{\omega_{j}^{\prime \prime}(t)}{\omega_{j}^{\prime}(t)} \geq \frac{2 \pi}{l_{j}(r)} .
$$

It is easy to check that the left-hand side of the of (1.10) is equal to

$$
\frac{d}{d t}\left\{\ln \left(\frac{d}{d t} e^{\omega_{j}(t)}\right)\right\}=\frac{d}{d \ln r}\left\{\ln \left(\frac{d m_{j}(r)}{d \ln r}\right)\right\},
$$


therefore $\left(r \geq r_{0}\right)$

$$
\frac{d}{d \ln r}\left\{\ln \left(\frac{d m_{j}(r)}{d \ln r}\right)\right\} \geq \frac{2 \pi}{l_{j}(r)} .
$$

Integrating this inequality we get

$$
\frac{d m_{j}(r)}{d \ln r} \geq A_{j} \exp \left\{\int_{r_{0}}^{r} \frac{2 \pi}{t l_{j}(t)} d t\right\},
$$

where

$$
A_{j}=\left.\frac{d m_{j}(r)}{d \ln r}\right|_{r=r_{0}}>0,
$$

and

$$
m_{j}(r) \geq m_{j}\left(r_{0}\right)+A_{j} \int_{r_{0}}^{r} \frac{d \tau}{\tau} \exp \left\{\int_{r_{0}}^{\tau} \frac{2 \pi}{l_{j}(t) t} d t\right\} .
$$

From (1.11) we get $\left(A=\min _{1 \leq j \leq p} A_{j}\right)$

$$
\begin{aligned}
m_{j}(e r) & \geq A \int_{r}^{e r} \frac{d \tau}{\tau} \exp \left\{\int_{r_{0}}^{\tau} \frac{2 \pi}{l_{j}(t) t} d t\right\} \\
& \geq A \exp \left\{\int_{r_{0}}^{r} \frac{2 \pi}{l_{j}(t) t} d t\right\} \int_{r}^{e r} \frac{d \tau}{\tau}=A \exp \left\{\int_{r_{0}}^{r} \frac{2 \pi}{l_{j}(t) t} d t\right\},
\end{aligned}
$$

from where

$$
\ln m_{j}(e r) \geq 2 \pi \int_{r_{0}}^{r} \frac{d t}{l_{j}(t) t}+\ln A .
$$

Since $m_{j}(e r) \leq 2 \pi \ln ^{2} M(e r, f)$, we get

$$
\ln \ln M(e r, f) \geq \pi \int_{r_{0}}^{r} \frac{d t}{l_{j}(t) t}+\frac{1}{2} \ln \frac{A}{2 \pi} .
$$

Adding these inequalities we get

$$
\ln \ln M(e r, f) \geq \frac{\pi}{p} \int_{r_{0}}^{r} \frac{d t}{t} \sum_{j=1}^{p} \frac{1}{l_{j}(t)}+\frac{1}{2} \ln \frac{A}{2 \pi} .
$$

Using the the Cauchy-Buniakowsky inequality and (1.4) we obtain

$$
p^{2}=\left(\sum_{j=1}^{p} \sqrt{l_{j}(t)} \frac{1}{\sqrt{l_{j}(t)}}\right)^{2} \leq \sum_{j=1}^{p} l_{j}(t) \sum_{j=1}^{p} \frac{1}{l_{j}(t)} \leq 2 \pi \sum_{j=1}^{p} \frac{1}{l_{j}(t)} .
$$

Therefore $\left(1.12^{\prime}\right)$ implies

$$
\ln \ln M(e r, f) \geq \frac{p}{2} \int_{r_{0}}^{r} \frac{d t}{t}+\frac{1}{2} \ln \frac{A}{2 \pi}=\ln \left(K r^{p / 2}\right),
$$

where $K=r_{0}^{-p / 2} \sqrt{A /(2 \pi)}$. If $r / 2>r_{0}$, we get

$$
\ln M\left(\frac{r}{2}, f\right) \geq K_{1} r^{p / 2}, K_{1}=K(2 e)^{-p / 2} .
$$

Hence

$$
T(r, f) \geq \frac{1}{3} \ln M\left(\frac{r}{2}, f\right) \geq \frac{1}{3} K_{1} r^{p / 2},
$$

that is, (1.1) is satisfied. 
Our next example shows that the estimate given by Theorem 1.2 is sharp.

Let $f(z)=\cos \left(z^{p / 2}\right), p=1,2, \ldots$ It is clear that $f(z)$ is an entire function. Using Theorem 6.1 from Chapter 1 or the following asymptotic equality directly $\left(z=r e^{i \varphi},|\varphi|<\pi\right)$

$$
\left|\cos \left(z^{p / 2}\right)\right|=\frac{1}{2} \exp \left(r^{p / 2}\left|\cos \frac{p \varphi}{2}\right|\right)+O(1),
$$

we get

$$
T(r, f) \sim \frac{2}{\pi} r^{p / 2}
$$

If $p$ is an even number, $p=2 m$, the function $f(z)$ has $p$ asymptotic spots $\left\{\infty, C_{k}\right\}$, where $C_{k}$ is the ray $\left\{\arg z=\varphi_{k}=\frac{\pi}{2 m}+k \frac{\pi}{m}\right\}, k=-m,-m+1, \ldots, 0, \ldots, m-$ 1. In fact, if $z=r e^{i \varphi_{k}}$, then $\cos \left(z^{p / 2}\right)=\cos z^{m}=\cos \left\{r^{m} i(-1)^{k}\right\}=\cosh r^{m}$. Asymptotic spots $\left\{\infty, C_{k}\right\}$ are different since the inequality $\left|\cos z^{m}\right| \leq 1$ holds on the rays $\left\{\arg z=\varphi_{k}^{\prime}=k \frac{\pi}{m}\right\}, k=-m,-m+1, \ldots, m$. If $p$ is odd, $p=2 m-$ 1 , then the function $f(z)$ has $p$ asymptotic spots $\left\{\infty, C_{k}\right\}$, where $C_{k}$ is the ray $\left\{\arg z=\theta_{k}=\frac{\pi}{2 m-1}+k \frac{2 \pi}{2 m-1}\right\}, k=-m+1, \ldots, m-1$. In fact, for $z=r e^{i \theta_{k}}$, the equality $\cos \left(z^{p / 2}\right)=\cos \left\{r^{p / 2} i(-1)^{k}\right\}=\cosh r^{p / 2}$ holds.

Since $\left|\cos \left(z^{p / 2}\right)\right| \leq 1$ on the rays $\left\{\arg z=\theta_{k}^{\prime}=k \frac{2 \pi}{2 m-1}\right\}, k=-m+1,-m+$ $2, \ldots, m-1$, then the asymptotic spots $\left\{\infty, C_{k}\right\}$ are distinct in this case, too.

The following classical theorem is an immediate corollary of Theorem 1.2.

TheOREm 1.3 (Wiman, Heins). If the lower order $\lambda$ of an entire function $f(z)$ is less than $1 / 2$, then

$$
\limsup _{r \rightarrow \infty} \mu(r, f)=\infty
$$

The conclusion remains true if

$$
\liminf _{r \rightarrow \infty} r^{-1 / 2} T(r, f)=0 .
$$

The relation (1.13) means that there exists a sequence of circles $\left\{|z|=r_{k}\right\}$, $r_{k} \rightarrow \infty$ on which $|f(z)|$ uniformly with respect to $\arg z$ approaches $\infty$.

To prove Theorem 1.3 note, that by Theorem 1.1 the function $f(z)$ has at least one asymptotic spot at $\infty$. If the condition (1.13) were not satisfied, then the relation (1.2) would take place, and, by (1.1), we would have

$$
\liminf _{r \rightarrow \infty} r^{-1 / 2} T(r, f)>0,
$$

a contradiction.

COROLlaRY 1. If the lower order $\lambda$ of an entire function $f(z)$ is less than $1 / 2$, then $\delta(a, f)=0$ for all $a \neq \infty$.

In fact, suppose that $\mu\left(r_{k}, f\right) \rightarrow \infty$ as $r_{k} \rightarrow \infty$. Then $\left|f\left(r_{k} e^{i \varphi}\right)-a\right| \geq \mu\left(r_{k}, f\right)-$ $|a| \rightarrow \infty$ and $m\left(r_{k}, a, f\right)=0$ for all $k \geq k_{0}$.

In Section 3 we show that for $\lambda=1 / 2$ an entire function $f(z)$ also cannot have finite deficient values. If $\lambda>1 / 2$, then, as we have seen in Section 5 of Chapter 4 , the set $E_{N}(f)$ can contain an arbitrary preassigned countable set. 
Corollary 2. Deficiencies of an entire function of order $\rho<3 / 2$ are shift invariant.

In fact, if $\lambda<1 / 2$, then, by Corollary 1 , there are no finite deficient values, and if $\lambda \geq 1 / 2, \rho<3 / 2$, then $\rho-\lambda<1$, and we can apply the corollary of Theorem 6.5 from Chapter 4 .

Corollary 3. Let $f(z)$ be an entire function of finite lower order $\lambda$. Then

$$
\limsup _{r \rightarrow \infty} \frac{\ln \mu(r, f)}{\ln M(r, f)} \geq-[2 \lambda] .
$$

If $\lambda<1 / 2$, the inequality (1.15) follows from (1.13). Therefore we may assume that $\lambda \geq 1 / 2$ and, hence, $n=[2 \lambda]+1 \geq 2$. We form the function

$$
F(z)=f(z) f(\omega z) \ldots f\left(\omega^{n-1} z\right), \omega=e^{2 \pi i / n} .
$$

It is clear that $F(z)=\Phi\left(z^{n}\right)$, where $\Phi(z)$ is an entire function. Since $M(r, \Phi)=$ $M\left(r^{n}, F\right), M(r, F) \leq\{M(r, f)\}^{n}$, it is easy to see that the lower order of the function $F(z)$ does not exceed $\lambda$, and the lower order of the function $\Phi(z)$ does not exceed $\lambda / n<1 / 2$. The equality (1.13) implies that there exists a sequence $r_{j} \rightarrow \infty$ such that $\mu\left(r_{j}, \Phi\right)>1$. Then $\mu\left(R_{j}, F\right)>1$, where $R_{j}=r_{j}^{n}$. But

$$
1<\mu\left(R_{j}, F\right) \leq \mu\left(R_{j}, f\right)\left\{M\left(R_{j}, f\right)\right\}^{n-1},
$$

from where $\ln \mu\left(R_{j}, f\right)>-(n-1) \ln M\left(R_{j}, f\right)=-[2 \lambda] \ln M\left(R_{j}, f\right)$, and we get (1.15).

Note also, that as is shown by Example 6 from Section 6 of Chapter 4 (see (6.61)), without the assumption $\lambda<\infty$ we cannot claim that the upper limit in (1.15) is finite.

The estimate (1.15) is not sharp for $\lambda \neq 1 / 2$. A sharp estimate in the case $\lambda \leq 1$ is obtained in Theorem 3.4 and Corollary 2 from it. No sharp estimate is known $^{1}$ for $\lambda>1$.

Theorem 1.3 can be generalized in the following way.

THEOREM 1.3'. Let $f(z)$ be an entire function of finite lower order $\lambda$, and let $l$ be a number satisfying the conditions

$$
0<l \leq 2 \pi, l<\frac{\pi}{\lambda}
$$

Then there exists a sequence of arcs $\left\{|z|=r_{k}, \theta_{k} \leq \arg z \leq \theta_{k}+l\right\}, r_{k} \rightarrow \infty$, $0 \leq \theta_{k}<2 \pi$ on which $|f(z)|$ approaches $\infty$ uniformly with respect to $\arg z$.

PRoof. If $\limsup _{r \rightarrow \infty} \mu(r, f)=\infty$, we can let $l=2 \pi$, therefore it suffices to consider the case when $\mu(r, f)=O(1)$. In such a case we are under conditions of Theorem 1.2. Using the same notation as in the proof of Theorem 1.2, we can write the inequality (1.12). If the inequality $\limsup _{r \rightarrow \infty} l_{j}(r)<\frac{\pi}{\lambda}$ were satisfied, then, taking $\gamma$ satisfying $\lim \sup _{r \rightarrow \infty} l_{j}(r)<\gamma<\frac{\pi}{\lambda}$, by (1.12) we would get

$$
\ln \ln M(e r, f) \geq \frac{\pi}{\gamma} \ln r+O(1)
$$

\footnotetext{
${ }^{1}$ Sharp estimate is still unknown. See the editor's Appendix in the end of the book for a survey of known results.
} 
Since $\frac{\pi}{\gamma}>\lambda$, we would get a contradiction with the assumption that the lower order of $f(z)$ is equal to $\lambda$. Thus $\lim \sup _{r \rightarrow \infty} l_{j}(r) \geq \frac{\pi}{\lambda}$.

We see from here that if the number $l$ satisfies the conditions $\left(1.14^{\prime}\right)$, then there exists a sequence $r_{k}^{(1)} \rightarrow \infty$ such that $l_{j}\left(r_{k}^{(1)}\right)>l, k=1,2, \ldots$ By the definition of $l_{j}(r)$, this indicates that there exists a sequence of $\operatorname{arcs} S_{k}^{(1)}=\left\{|z|=r_{k}^{(1)}, \theta_{k}^{(1)} \leq\right.$ $\left.\arg z \leq \theta_{k}^{(1)}+l\right\}$, such that $|f(z)| \geq 1$ for $z \in S_{k}^{(1)}, k=1,2, \ldots$ Considering instead of $f(z)$ the function $f(z) / n$, where $n$ is an arbitrary positive integer, we get a sequence of $\operatorname{arcs} S_{k}^{(n)}=\left\{|z|=r_{k}^{(n)}, \theta_{k}^{(n)} \leq \arg z \leq \theta_{k}^{(n)}+l\right\}, 0 \leq \theta_{k}^{(n)}<2 \pi$, $\lim _{k \rightarrow \infty} r_{k}^{(n)}=\infty$, such that $|f(z)| \geq n$ for $z \in S_{k}^{(n)}, k=1,2, \ldots$ The sequence $S_{n}^{(n)}, n=1,2, \ldots$, has the desired property.

Theorem 1.4 (Denjoy, Carleman, Ahlfors). If an entire function $f(z)$ has $p$ asymptotic spots $\left\{a_{j}, C_{j}\right\}, 1 \leq j \leq p, a_{j} \neq \infty$, then it satisfies (1.1).

Proof. Let $r_{0}$ be so large that the circle $\left\{|z|=r_{0}\right\}$ intersects each curve $C_{j}$, $1 \leq j \leq p$, and the curves are pairwise disjoint for $|z| \geq r_{0}$. We may assume without loss of generality that the initial points $z_{j}$ of curves $C_{j}$ lie on the circle $\left\{|z|=r_{0}\right\}$, and the rest of the curve is situated in $\left\{|z|>r_{0}\right\}$ (if necessary, we delete the part of $C_{j}$ between the initial point and the last point of intersection with $\left\{|z|=r_{0}\right\}$ ). We may assume that the curves $C_{j}$ are numbered in such a way that indexes of the points $z_{1}, \ldots, z_{p}$ are growing as we follow circle $\left\{|z|=r_{0}\right\}$ in the positive direction. The curves $C_{1}, \ldots, C_{p}$ separate the region $\left\{r_{0}<|z|<\infty\right\}$ into simply connected regions $D_{1}, \ldots, D_{p}$; the region $D_{j}$ lies between the curves $C_{j}$ and $C_{j+1}, C_{p+1}=C_{1}$. Obviously the function $f(z)$ is bounded on the boundary of $D_{j}$ in the complex plane. Let us show that $f(z)$ is unbounded in the region $D_{j}$.

LEMma 1.2 (Lindelöf). Let $\Phi(z)$ be analytic and bounded in $\{\operatorname{Re} z>0\}$, and continuous in $\{\operatorname{Re} z \geq 0\}$. Let $\Phi(i y) \rightarrow a_{1}$ as $y \rightarrow+\infty$ and $\Phi(i y) \rightarrow a_{2}$ as $y \rightarrow-\infty$. Then $a_{1}=a_{2}=a$, and the function $\Phi(z)$ approaches $a$ as $z \rightarrow \infty, \operatorname{Re} z \geq 0$.

Proof. First we suppose that $a_{1}=a_{2}=a$. For each $\varepsilon>0$ there exists $r_{1}=r_{1}(\varepsilon)$ such that $|\Phi(i y)-a|<\varepsilon$ for $|y| \geq r_{1}(\varepsilon)$. Let the number $q>r_{1}$ be so large that

$$
\left|\frac{z}{z+q}\{\Phi(z)-a\}\right| \leq \frac{r_{1}}{q-r_{1}} \max _{z \in \gamma}|\Phi(z)-a|<\varepsilon
$$

on the $\operatorname{arc} \gamma=\left\{|z|=r_{1}, \operatorname{Re} z \geq 0\right\}$. Since

$$
\left|\frac{z}{z+q}\right|<1 \text { for } \operatorname{Re} z \geq 0,
$$

on the boundary of the region $G=\left\{|z|>r_{1}\right\} \cap\{\operatorname{Re} z>0\}$ in the complex plane we have

$$
\left|\frac{z}{z+q}\{\Phi(z)-a\}\right| \leq \varepsilon,
$$

and the left-hand side of (1.16) is bounded in the region $G$. Lemma 1.1 implies that the inequality (1.16) holds everywhere in $G$. Now let $|z|>q, \operatorname{Re} z \geq 0$. The inequality (1.16) implies

$$
|\Phi(z)-a| \leq \varepsilon\left|\frac{z+q}{z}\right| \leq \varepsilon\left(1+\frac{q}{|z|}\right)<2 \varepsilon,
$$


that is, $\Phi(z)$ approaches $a$ as $z \rightarrow \infty, \operatorname{Re} z \geq 0$.

Now we assume that $a_{1} \neq a_{2}$. Then the function

$$
F(z)=\left\{\Phi(z)-\frac{a_{1}+a_{2}}{2}\right\}^{2}
$$

satisfies the condition of the lemma and $F(i y) \rightarrow\left(\frac{a_{1}-a_{2}}{2}\right)^{2}$ as $y \rightarrow+\infty$ and $y \rightarrow-\infty$. As was proved above the function $F(z)$ approaches $\left(\frac{a_{1}-a_{2}}{2}\right)^{2}$ as $z \rightarrow \infty, \operatorname{Re} z \geq 0$.

From (1.17) we see that there is a single-valued of $\sqrt{F(z)}$ in $\{\operatorname{Re} z \geq 0\}$ such that

$$
\Phi(z)-\frac{a_{1}+a_{2}}{2}=\sqrt{F(z)} .
$$

But $\sqrt{F(i y)}$ approaches either $\left(a_{1}-a_{2}\right) / 2$ or $\left(a_{2}-a_{1}\right) / 2$ as $|y| \rightarrow \infty$, that is, $\Phi(i y)$ approaches $a_{1}$ or $a_{2}$ as $|y| \rightarrow \infty$, it contradicts the assumption that $\Phi(i y)$ has different limits as $y \rightarrow+\infty$ and $y \rightarrow-\infty$.

We continue the proof of the theorem. Assume that the function $f(z)$ is bounded in the region $D_{j}$. Let function $z=\omega(\zeta)$ be a conformal univalent mapping of the half-plane $\{\operatorname{Re} \zeta>0\}$ onto $D_{j}, \omega(\infty)=\infty$. Then the function $\Phi(\zeta)=f(\omega(\zeta))$ satisfies the conditions of Lemma 1.2 with the roles of $a_{1}$ and $a_{2}$ played by $a_{j+1}$ and $a_{j}$, respectively. The lemma implies that $a_{j+1}=a_{j}=a$ and $\Phi(\zeta)$ approaches $a$ as $\zeta \rightarrow \infty, \operatorname{Re} \zeta \geq 0$ and, hence, as $\zeta \rightarrow \infty, \operatorname{Re} \zeta \geq 0,|\zeta|=k, k=1,2, \ldots$ Denote by $\gamma_{k}$ the image of the semi-circle $\{\operatorname{Re} \zeta \geq 0,|\zeta|=k\}$ under the mapping $z=\omega(\zeta)$. It is clear that the distance between $\gamma_{k}$ and $z=0$ approaches $\infty$ as $k \rightarrow \infty$. Then $f(z) \rightarrow a$ as $z \rightarrow \infty, z \in \bigcup_{k} \gamma_{k}$. According to the definition the asymptotic spots $\left\{a_{j}, C_{j}\right\}$ and $\left\{a_{j+1}, C_{j+1}\right\}$ coincide, we get a contradiction. Thus the function $f(z)$ is unbounded in the region $D_{j}, 1 \leq j \leq p$. By the remark to Theorem 1.1 there exists an asymptotic curve $C_{j}^{\prime}$ in $D_{j}$ such that $f(z)$ approaches $\infty$ along it. Obviously $\left\{\infty, C_{j}^{\prime}\right\}, 1 \leq j \leq p$, are different asymptotic spots, because the function $f(z)$ is bounded on $C_{j}$. Hence we are under the conditions of Theorem 1.2 , therefore $(1.1)$ is satisfied.

COROLlary. An entire function of finite lower order $\lambda$ cannot have more than $[2 \lambda]$ different finite asymptotic values and even $[2 \lambda]$ different asymptotic spots with finite asymptotic values (the second statement is stronger).

ExERCISE. Use the example

$$
f(z)=z^{-p / 2} \sin \left(z^{p / 2}\right), p=1,2, \ldots,
$$

to show that the estimate from the corollary is attained.

\section{Non-asymptotic deficient values}

For a long time the following conjecture of R. Nevanlinna was considered as plausible: an entire function of a finite order $\rho$ can have at most $[2 \rho]$ finite deficient values. The point is that it seemed likely that each deficient value of an entire function is asymptotic. Theorem 5.1 from Chapter 4 not only disproves this conjecture, but also shows that an entire function of an arbitrary order $\rho, 1 / 2<\rho<\infty$, can have an infinite set of deficient nonasymptotic values, because the number of 
asymptotic values does not exceed $[2 \rho]$. The restriction $\rho>1 / 2$ is natural for entire functions, since for $\rho \leq 1 / 2$ an entire function, as will be shown in Section 3 (for $\rho<1 / 2$ this was shown in Corollary 1 from Theorem 1.3), does not have finite deficient values, and a Picard exceptional value at $\infty$ is necessarily an asymptotic value. Now we show that for meromorphic functions a deficient value can be nonasymptotic even for $0<\rho \leq 1 / 2$. It is still unknown ${ }^{2}$ whether this is true for $\rho=0$.

Since the construction of the example is rather simple we will not restrict ourselves to the case $0<\rho \leq 1 / 2$, but will consider the general case $0<\rho<\infty$.

Example 1. Let $a=4^{\rho}, 0<\rho<\infty$. Consider the function

$$
\begin{aligned}
f(z) & =\sum_{k=1}^{\infty}\left(\frac{3 \cdot 4^{k}}{z-5 \cdot 4^{k}}\right)^{\left[a^{k}\right]}+\sum_{k=1}^{\infty}\left(\frac{6 \cdot 4^{k}}{z+10 \cdot 4^{k}}\right)^{\left[a^{k}\right]} \\
& =\sum_{k=1}^{\infty} H_{k}(z)+\sum_{k=1}^{\infty} H_{-k}(z) .
\end{aligned}
$$

It is easy to check, using the convergence of the series $\sum_{k=1}^{\infty}\left(\frac{3}{5}\right)^{\left[a^{k}\right]}$, that both series from (2.1) are absolutely and uniformly convergent in each disc of finite radius, and therefore, the function $f(z)$ determined by (2.1) is meromorphic.

We introduce the following notation $(k=1,2, \ldots)$ :

$$
\begin{aligned}
& C_{+k}=\left\{\left|z-5 \cdot 4^{k}\right|<3 \cdot 4^{k}\right\}, \\
& \tilde{C}_{+k}=\left\{\left|z-5 \cdot 4^{k}\right|<4 \cdot 4^{k}\right\}, \\
& C_{-k}=\left\{\left|z+10 \cdot 4^{k}\right|<6 \cdot 4^{k}\right\}, \\
& \tilde{C}_{-k}=\left\{\left|z+10 \cdot 4^{k}\right|<8 \cdot 4^{k}\right\}
\end{aligned}
$$

It is easy to check that the $\operatorname{discs} C_{j}, j= \pm 1, \pm 2, \ldots$, are pairwise disjoint and that none of the point in the plane is covered by more than two discs $\tilde{C}_{j}$. Note that for $z \notin \tilde{C}_{k}(k \geq 1)$ the inequality

$$
\left|\frac{3 \cdot 4^{k}}{z-5 \cdot 4^{k}}\right| \leq \frac{3 \cdot 4^{k}}{4 \cdot 4^{k}}=\frac{3}{4}
$$

holds, and for $z \notin \tilde{C}_{k}(k \leq-1)$ the inequality

$$
\left|\frac{6 \cdot 4^{|k|}}{z+10 \cdot 4^{|k|}}\right| \leq \frac{6 \cdot 4^{|k|}}{8 \cdot 4^{|k|}}=\frac{3}{4}
$$

holds. Therefore, if $z \notin \bigcup_{j \in A} \tilde{C}_{j}$, where $A$ is an arbitrary subset of the set $B$ of nonzero integers, then

$$
\left|\sum_{j \in A} H_{j}(z)\right| \leq \sum_{j \in A}\left(\frac{3}{4}\right)^{\left[a^{|j|}\right]} \leq 2 \sum_{j=1}^{\infty}\left(\frac{3}{4}\right)^{a^{j}}=M<\infty .
$$

It is easy to see that outside $\bigcup_{j \in B} C_{j}$ the function $f(z)$ is bounded. In fact, if $z \notin \bigcup_{j \in B} \tilde{C}_{j}$, then, by $(2.2)$, we have $|f(z)| \leq M$. If $z$ is covered by $\operatorname{discs} \tilde{C}_{j}$, then

\footnotetext{
${ }^{2}$ Ter-Israelian [A150] constructed a meromorphic function of order 0 with a deficient nonasymptotic value.
} 
it is covered by at most two such discs, so it is either covered by $\tilde{C}_{j_{0}}$ only, or by $\tilde{C}_{j_{0}}$ and $\tilde{C}_{j_{0}+1}$. Consider, for example, the second case. Let $z \notin \tilde{C}_{j}, j \neq 0, j_{0}, j_{0}+1$; $z \in \tilde{C}_{j}, j=j_{0}, j_{0}+1$. By $(2.2)$ we have

$$
|f(z)| \leq\left|H_{j_{0}}(z)\right|+\left|H_{j_{0}+1}(z)\right|+\left|\sum_{j \neq 0, j_{0}, j_{0}+1} H_{j}(z)\right| \leq\left|H_{j_{0}}(z)\right|+\left|H_{j_{0}+1}(z)\right|+M .
$$

But if $z \notin C_{j}$, then $\left|H_{j}(z)\right| \leq 1$. Therefore $|f(z)| \leq 2+M$. If $z$ belongs to only one of the sets $\tilde{C}_{j_{0}}$, using a similar argument we get $|f(z)| \leq 1+M$. In any case we have

$$
|f(z)| \leq 2+M=M_{1}, z \notin \bigcup_{j \in B} C_{j} .
$$

Since the discs $C_{j}$ are pairwise disjoint, it is obvious that for each continuous curve $\gamma$ tending to $\infty$ we have

$$
\liminf _{\substack{z \rightarrow \infty \\ z \in \gamma}}|f(z)| \leq M_{1}
$$

and hence, $\infty$ cannot be an asymptotic value for $f(z)$.

If $z \in C_{j}$ then, using (2.2) and using the same argument as in the proof of (2.3), we get

$$
\left|H_{j}(z)\right|-M_{1} \leq|f(z)| \leq\left|H_{j}(z)\right|+M_{1}, z \in C_{j} .
$$

By (2.3) and (2.4) everywhere in the complex plane the relations

$$
\ln ^{+}|f(z)|=\sum_{j \in B} \ln ^{+}\left|H_{j}(z)\right|+\Omega(z), \quad|\Omega(z)| \leq \ln \left(2 M_{1}\right)
$$

hold, hence

$$
m(r, f)=\sum_{j \in B} m\left(r, H_{j}\right)+O(1)
$$

Let $k \geq 1$. Then

$$
\begin{array}{r}
m\left(r, H_{k}\right)=\left[a^{k}\right] \frac{1}{2 \pi} \int_{0}^{2 \pi} \ln ^{+} \frac{3 \cdot 4^{k}}{\left|r e^{i \varphi}-5 \cdot 4^{k}\right|} d \varphi \\
=\left[a^{k}\right] \frac{1}{2 \pi} \int_{0}^{2 \pi} \ln ^{+} \frac{3}{\left|4^{-k} r e^{i \varphi}-5\right|} d \varphi=\left[a^{k}\right] I_{1}\left(\frac{r}{4^{k}}\right),
\end{array}
$$

where the function

$$
I_{1}(t)=\frac{1}{2 \pi} \int_{0}^{2 \pi} \ln ^{+} \frac{3}{\left|t e^{i \varphi}-5\right|} d \varphi
$$

is continuous for $t \geq 0$, positive for $2<t<8$, and is equal to zero outside this interval. Similarly

$$
m\left(r, H_{-k}\right)=\left[a^{k}\right] I_{2}\left(\frac{r}{4^{k}}\right)
$$

where the function

$$
I_{2}(t)=\frac{1}{2 \pi} \int_{0}^{2 \pi} \ln ^{+} \frac{6}{\left|t e^{i \varphi}+10\right|} d \varphi
$$

is continuous for $t \geq 0$, positive for $4<t<16$, and is equal to zero outside this interval. 
Let $2 \cdot 4^{k} \leq r \leq 8 \cdot 4^{k}, k=2,3, \ldots$ Then

$$
\begin{aligned}
m(r, f)+O(1) & =\sum_{j=1}^{\infty}\left[a^{j}\right] I_{1}\left(\frac{r}{4^{j}}\right)+\sum_{j=1}^{\infty}\left[a^{j}\right] I_{2}\left(\frac{r}{4^{j}}\right) \\
& =\left[a^{k}\right] I_{1}\left(\frac{r}{4^{k}}\right)+\left[a^{k}\right] I_{2}\left(\frac{r}{4^{k}}\right)+\left[a^{k-1}\right] I_{2}\left(\frac{r}{4^{k-1}}\right) \\
& =O(1)+a^{k}\left\{I_{1}\left(\frac{r}{4^{k}}\right)+I_{2}\left(\frac{r}{4^{k}}\right)+\frac{1}{a} I_{2}\left(\frac{r}{4^{k-1}}\right)\right\} .
\end{aligned}
$$

Let $r 4^{-k}=t$. The function

$$
I(t)=I_{1}(t)+I_{2}(t)+\frac{1}{a} I_{2}(4 t)
$$

is continuous and positive on the closed interval $[2,8]$ because $I_{j}(t) \geq 0, j=1,2$, $I_{1}(t)>0$ on the interval $(2,8), I_{2}(t)>0$ on the interval $(4,16)$, and $I_{2}(4 t)>0$ is positive on the interval $(1,4)$. Therefore

$$
0<\mu_{1}=\min _{[2,8]} I(t) \leq \max _{[2,8]} I(t)=\mu_{2}<\infty .
$$

Relation (2.8) implies that for $2 \cdot 4^{k} \leq r \leq 8 \cdot 4^{k}$ the inequality

$$
\mu_{1} a^{k}+O(1) \leq m(r, f) \leq \mu_{2} a^{k}+O(1)
$$

holds. But $a=4^{\rho}$, therefore

$$
\left(\frac{r}{8}\right)^{\rho}=a^{k}=\left(4^{k}\right)^{\rho} \leq\left(\frac{r}{2}\right)^{\rho}
$$

and

$$
8^{-\rho} \mu_{1} r^{\rho}+O(1) \leq m(r, f) \leq 2^{-\rho} \mu_{2} r^{\rho}+O(1) .
$$

It is easy to see that

$$
n(r, f)=\sum_{k=1}^{p(r)}\left[a^{k}\right]+\sum_{k=1}^{q(r)}\left[a^{k}\right], p(r)=\left[\frac{\ln \frac{r}{5}}{\ln 4}\right], q(r)=\left[\frac{\ln \frac{r}{10}}{\ln 4}\right] .
$$

Therefore

$$
\begin{array}{r}
n(r, f) \leq \sum_{k=1}^{p(r)} a^{k}+\sum_{k=1}^{q(r)} a^{k}=\frac{a^{p(r)+1}-a}{a-1}+\frac{a^{q(r)+1}-a}{a-1} \\
\leq \frac{a}{a-1}\left\{a^{p(r)}+a^{q(r)}\right\} \leq \frac{2 a}{a-1} a^{p(r)} \leq \frac{2 a}{a-1} 4^{\rho \frac{\ln \frac{r}{5}}{\ln 4}}=\frac{2 a}{a-1}\left(\frac{r}{5}\right)^{\rho}=K_{1} r^{\rho} .
\end{array}
$$

Hence $(r>20)$

$$
N(r, f) \leq \frac{K_{1}}{\rho} r^{\rho}=K_{2} r^{\rho} .
$$

The formulas (2.9) and (2.10) imply that

$$
T(r, f) \leq\left(2^{-\rho} \mu_{2}+K_{2}\right) r^{\rho}+O(1)=K r^{\rho}+O(1) .
$$

From (2.9) and (2.11) we get

$$
\delta(\infty, f)=\liminf _{r \rightarrow \infty} \frac{m(r, f)}{T(r, f)} \geq \frac{8^{-\rho} \mu_{1}}{K}>0,
$$

that is, $\infty$ is a deficient value. Formulas (2.9) and (2.11) imply that the order of $f(z)$ is equal to $\rho$. Thus the function $f(z)$ has the desired properties. 
We see that in Iversen's Theorem 1.1 the condition $a \in E_{P}(f)$ cannot be replaced by a weaker condition $a \in E_{N}(f)$. Now we show that even the condition $\delta(a, f)=1$, and also the condition $a \in E_{B}(f)$, does not imply that $a$ is an asymptotic value. Here is an example.

ExAmPLE 2. Let $1<\rho<\infty$. Let $l_{j}(r), j=1,2$, be two continuously differentiable functions on $[0, \infty)$ satisfying the conditions:

A. $\alpha \leq l_{j}(r) \leq \rho$, where $\alpha$ is a number satisfying

$$
\frac{\rho}{2 \rho-1}<\alpha<1
$$

B. $\left|l_{j}^{\prime}(r)\right|=O\left\{\left(r \ln r \ln _{2} r \ln _{3} r\right)^{-1}\right\}$ as $r \rightarrow \infty$, where $\ln _{j} r=\ln \ln _{j-1} r$, $\ln _{1} r=\ln r$.

C. There exist sequences of $\left[a_{n}^{(j)}, b_{n}^{(j)}\right]$ of closed intervals $n=1,2, \ldots ; j=1,2$, such that $a_{n}^{(j)} \rightarrow \infty, b_{n}^{(j)}-a_{n}^{(j)} \geq n^{2}$, and $l_{j}(r) \equiv \alpha$ for $a_{n}^{(j)} \leq r \leq b_{n}^{(j)}$.

D. $l(r)=\max \left\{l_{1}(r), l_{2}(r)\right\} \equiv \rho$ for $0 \leq r<\infty$.

It is easy to see that such functions $l_{j}(r)$ can be constructed.

In what follows $z=r e^{i \varphi},|\varphi| \leq \pi$. We consider the finctions analytic in the plane with a cut along the negative ray

$$
\lambda_{j}(z)=z \int_{0}^{\infty} \frac{l_{j}(t) d t}{(t+z)^{2}}, j=1,2 .
$$

We show that in each angle $\{|\varphi| \leq \pi-\delta\}, \delta>0$, the relation

$$
\lambda_{j}(z)=l_{j}(r)+o\left(\frac{1}{\ln r}\right)
$$

holds uniformly with respect to $\varphi$. As we already observed in Section 5 of Chapter 2 , for $|\varphi| \leq \pi-\delta, 0 \leq t<\infty$, the inequality

$$
|t+z| \geq(t+r) \sin \frac{\delta}{2}
$$

holds. Since

$$
z \int_{0}^{\infty} \frac{d t}{(t+z)^{2}}=1 \text { for }|\varphi|<\pi
$$

we have

$$
\begin{aligned}
\left|\lambda_{j}(z)-l_{j}(r)\right| & =\left|z \int_{0}^{\infty} \frac{l_{j}(t)-l_{j}(r)}{(t+z)^{2}} d t\right| \leq \frac{r}{\sin ^{2} \frac{\delta}{2}} \int_{0}^{\infty} \frac{\left|l_{j}(t)-l_{j}(r)\right|}{(t+r)^{2}} d t \\
& =\frac{r}{\sin ^{2} \frac{\delta}{2}}\left(\int_{0}^{\frac{r}{(\ln r)^{2}}}+\int_{\frac{r}{(\ln r)^{2}}}^{r(\ln r)^{2}}+\int_{r(\ln r)^{2}}^{\infty} \frac{\left|l_{j}(t)-l_{j}(r)\right|}{(t+r)^{2}} d t .\right.
\end{aligned}
$$

In the first and the third integrals we use the estimate $\left|l_{j}(t)-l_{j}(r)\right| \leq \rho$. If $r(\ln r)^{-2} \leq t \leq r(\ln r)^{2}$, then $|\ln (t / r)| \leq 2 \ln _{2} r$. Using the mean value theorem, we get

$$
l_{j}(t)-l_{j}(r)=\left.\frac{d l_{j}(t)}{d \ln t}\right|_{t=\xi} \ln \frac{t}{r}, r(\ln r)^{-2} \leq \xi \leq r(\ln r)^{2},
$$

from where, using the condition B we obtain

$$
\left|l_{j}(t)-l_{j}(r)\right|=O\left\{\left(\ln \xi \ln _{2} \xi \ln _{3} \xi\right)^{-1}\right\} 2 \ln _{2} r=O\left\{\left(\ln r \ln _{3} r\right)^{-1}\right\}, r \rightarrow \infty .
$$


Substituting these estimates into (2.13) we get

$$
\begin{aligned}
\mid \lambda_{j}(z)- & l_{j}(r) \mid \\
\leq & \frac{r}{\sin ^{2} \frac{\delta}{2}}\left(\rho \int_{0}^{r(\ln r)^{-2}} \frac{d t}{(t+r)^{2}}+O\left\{\left(\ln r \ln _{3} r\right)^{-1}\right\} \int_{r(\ln r)^{-2}}^{r(\ln r)^{2}} \frac{d t}{(t+r)^{2}}\right. \\
& \left.+\rho \int_{r(\ln r)^{2}}^{\infty} \frac{d t}{(t+r)^{2}}\right) \leq \frac{r}{\sin ^{2} \frac{\delta}{2}}\left(\frac{\rho}{r^{2}} \int_{0}^{r(\ln r)^{-2}} d t\right. \\
& \left.+O\left\{\left(\ln r \ln _{3} r\right)^{-1}\right\} \int_{r(\ln r)^{-2}}^{r(\ln r)^{2}} \frac{d t}{(t+r)^{2}}+\rho \int_{r(\ln r)^{2}}^{\infty} \frac{d t}{t^{2}}\right) \\
= & O\left\{(\ln r)^{-2}\right\}+O\left\{\left(\ln r \ln _{3} r\right)^{-1}\right\}=o\left\{(\ln r)^{-1}\right\},
\end{aligned}
$$

that is, (2.12) has been proved. Note that (2.12) remains true, if the condition A is replaced by $l_{j}(r)=o(\ln r)$. The condition (2.12) implies that the relation

$$
z^{\lambda_{j}(z)}=(1+o(1)) z^{l_{j}(r)}, r \rightarrow \infty .
$$

in the angle $\{|\varphi| \leq \pi-\delta\}$.

Let number $\theta$ be such that

$$
1<\theta<\frac{2 \rho \alpha}{\rho+\alpha}<2
$$

Denote by $\Gamma_{j}(t)$ the boundary of the region $D_{j}(t)=\left\{r>t,|\varphi|<\pi \theta / 2 l_{j}(r)\right\}, t \geq 1$, oriented in such a way that the region $D_{j}(t)$ is on the right-hand side of $\Gamma_{j}(t)$. By (2.14) it is clear that for $z \in \Gamma_{j}(1)$ we have

$$
\operatorname{Re} z^{\lambda_{j}(z)}=r^{l_{j}(r)}\left(\cos \frac{\pi}{2} \theta+o(1)\right) .
$$

In the complement to $\bar{D}(1)$ we define an analytic function $F_{j}(z)$ by a Cauchy-type integral

$$
F_{j}(z)=\frac{1}{2 \pi i} \int_{\Gamma_{j}(1)} \frac{\exp \left(\zeta^{\lambda_{j}(\zeta)}\right)}{\zeta-z} d \zeta, z \notin \bar{D}_{j}(1)
$$

and the consider an analytic continuation of $F_{j}(z)$ to the complex $z$-plane, setting

$$
F_{j}(z)=\frac{1}{2 \pi i} \int_{\Gamma_{j}(t)} \frac{\exp \left(\zeta^{\lambda_{j}(\zeta)}\right)}{\zeta-z} d \zeta, t>|z| .
$$

Thus, $F_{j}(z)$ is an entire function.

Let $\theta^{\prime}$ and $\theta^{\prime \prime}$ be arbitrary numbers satisfying $1<\theta^{\prime}<\theta<\theta^{\prime \prime}<2$. We denote the functions which we get if in the definition of $\Gamma_{j}(t)$ and $D_{j}(t)$ we replace $\theta$ by $\theta^{\prime}$ (or $\theta^{\prime \prime}$ ) by $\Gamma_{j}^{\prime}(t)$ and $D_{j}^{\prime}(t)$ (or by $\Gamma_{j}^{\prime \prime}(t)$ and $D_{j}^{\prime \prime}(t)$, respectively). Using (2.14) it is easy to check that the inequality

$$
\operatorname{Re} z^{\lambda_{j}(z)} \leq r^{l_{j}(r)}\left(\cos \frac{\pi}{2} \theta^{\prime}+o(1)\right)
$$

holds on $\operatorname{arcs}\left\{|z|=r, \pi \theta^{\prime} / 2 l_{j}(r)<|\varphi|<\pi \theta^{\prime \prime} / 2 l_{j}(r)\right\}$, therefore, applying the integral Cauchy theorem (compare with Example 3 from Section 5, Chapter 2), we get:

1) If $z \notin D_{j}(1)$, then

$$
F_{j}(z)=\frac{1}{2 \pi i} \int_{\Gamma_{j}^{\prime}(1)} \frac{\exp \left(\zeta^{\lambda_{j}(\zeta)}\right)}{\zeta-z} d \zeta
$$


2) If $z \in D_{j}(1)$, then

$$
F_{j}(z)=\frac{1}{2 \pi i} \int_{\Gamma_{j}^{\prime \prime}(t)} \frac{\exp \left(\zeta^{\lambda_{j}(\zeta)}\right)}{\zeta-z} d \zeta, t>|z| .
$$

If $|z|>R$, where $R>1$ is sufficiently large, then $z \notin D_{j}(1)$ implies that the distance from $z$ to $\Gamma_{j}^{\prime}(1)$ is greater than 1 , and $z \in D_{j}(1)$ implies that the distance for $z$ to $\Gamma_{j}^{\prime \prime}(1)$ is greater than 1 . Assume that $|z|>R$. If $z \notin D_{j}(1)$, then

$$
\left|F_{j}(z)\right| \leq \frac{1}{2 \pi} \int_{\Gamma_{j}^{\prime}(1)} \exp \left(\operatorname{Re} \zeta^{\lambda_{j}(\zeta)}\right)|d \zeta|<\infty .
$$

If $z \in D_{j}(1)$, then

$$
F_{j}(z)=\frac{1}{2 \pi i} \int_{\Gamma_{j}^{\prime \prime}(1)} \frac{\exp \left(\zeta^{\lambda_{j}(\zeta)}\right)}{\zeta-z} d \zeta+\frac{1}{2 \pi i} \int_{\Gamma_{j}^{\prime \prime}(t)-\Gamma_{j}^{\prime \prime}(1)} \frac{\exp \left(\zeta^{\lambda_{j}(\zeta)}\right)}{\zeta-z} d \zeta,
$$

where $\Gamma_{j}^{\prime \prime}(t)-\Gamma_{j}^{\prime \prime}(1)$ is the boundary of the region $\left\{1<r<t,|\varphi|<\pi \theta^{\prime \prime} / 2 l_{j}(r)\right\}$. Since

$$
\left|\frac{1}{2 \pi i} \int_{\Gamma_{j}^{\prime \prime}(1)} \frac{\exp \left(\zeta^{\lambda_{j}(\zeta)}\right)}{\zeta-z} d \zeta\right| \leq \frac{1}{2 \pi} \int_{\Gamma_{j}^{\prime \prime}(1)} \exp \left(\operatorname{Re} \zeta^{\lambda_{j}(\zeta)}\right)|d \zeta|<\infty
$$

and

$$
\frac{1}{2 \pi i} \int_{\Gamma_{j}^{\prime \prime}(t)-\Gamma_{j}^{\prime \prime}(1)} \frac{\exp \left(\zeta^{\lambda_{j}(\zeta)}\right)}{\zeta-z} d \zeta=\exp \left(z^{\lambda_{j}(z)}\right)
$$

we have

$$
F_{j}(z)=\exp \left(z^{\lambda_{j}(z)}\right)+O(1) .
$$

As a result we get for the entire function $F_{j}(z)$ the following relations:

$$
F_{j}(z)= \begin{cases}O(1), & z \notin D_{j}(1) \\ \exp \left(z^{\lambda_{j}(z)}\right)+O(1), & z \in D_{j}(1) .\end{cases}
$$

Denote by $\Delta_{1}$ the region $D_{1}(1)$, and by $\Delta_{2}$ the region symmetric to $D_{2}(1)$ about the imaginary axis. By the condition D,

$$
\frac{1}{l_{1}(r)}+\frac{1}{l_{2}(r)} \leq \frac{1}{\rho}+\frac{1}{\alpha}<\frac{2}{\theta}, r \geq 0
$$

therefore

$$
\pi-\frac{\pi \theta}{2 l_{2}(r)}>\frac{\pi \theta}{2 l_{1}(r)},
$$

and the closed regions $\bar{\Delta}_{1}$ and $\bar{\Delta}_{2}$ do not intersect. Denote by $\Delta_{3}$ the complement of $\Delta_{1} \cup \Delta_{2}$ in the complex plane. Then the function $\Psi(z)=F_{1}(z)+F_{2}(-z)$ satisfies the condition:

$$
\Psi(z)= \begin{cases}\exp \left\{z^{\lambda_{1}(z)}\right\}+O(1), & z \in \Delta_{1}, \\ \exp \left\{(-z)^{\lambda_{2}(-z)}\right\}+O(1), & z \in \Delta_{2}, \\ O(1), & z \in \Delta_{3}\end{cases}
$$


Let $\alpha<\beta<1$,

$$
V(z)=\prod_{n=1}^{\infty}\left(1-\frac{z}{n^{1 / \beta}}\right), \Phi(z)=V(z) V(-z) .
$$

Since all zeros of $V(z)$ are positive and $n(r, 0, V)=\left[r^{\beta}\right]$, we can use for $V(z)$ the asymptotic formulas $\left(5.3_{2}\right)$ from Chapter 2 . Let $\eta$ be an arbitrarily small number satisfying $0<\eta<\pi \theta / 2 \rho$. Then

$$
\ln \left|V\left(r e^{i \varphi}\right)\right|=r^{\beta} \frac{\pi}{\sin \pi \beta} \cos \beta(\varphi-\pi)+o\left(r^{\beta}\right)
$$

uniformly in $\varphi$ satisfying $\eta \leq \varphi \leq 2 \pi-\eta$. If we replace the equality sign in (2.16) by $\leq$, we get a relation valid for all $\varphi, 0 \leq \varphi<2 \pi$. We need also the following lemma. Then

Lemma 2.1. Let $\mu(r, V)=\min _{|z|=r}|V(z)|, r_{n}=\left(n+\frac{1}{2}\right)^{1 / \beta}, n=1,2,3, \ldots$.

$$
\ln \mu\left(r_{n}, V\right)=\ln \left|V\left(r_{n}\right)\right|=r_{n}^{\beta} \pi \cot \pi \beta+o\left(r_{n}^{\beta}\right) .
$$

Joining (2.16) and (2.17), it is easy to see that (2.16) holds for all $\varphi, 0 \leq \varphi<2 \pi$ as $r=r_{n} \rightarrow \infty$. We do not want to interrupt our presentation of Example 2, for this reason we shall prove Lemma 2.1 later.

Since

$$
\Phi(z)=\prod_{n=1}^{\infty}\left\{1-\left(\frac{z}{n^{1 / \beta}}\right)^{2}\right\},
$$

then obviously, $\mu(r, \Phi)=|\Phi(r)|=|V(r)||V(-r)|=\mu(r, V) M(r, V)$. Therefore

$$
\begin{array}{r}
\ln \mu\left(r_{n}, \Phi\right)=\ln \mu\left(r_{n}, V\right)+\ln M\left(r_{n}, V\right) \\
=r_{n}^{\beta}\left\{\pi \cot \pi \beta+\frac{\pi}{\sin \pi \beta}\right\}+o\left(r_{n}^{\beta}\right)=r_{n}^{\beta} \pi \cot \frac{\pi \beta}{2}+o\left(r_{n}^{\beta}\right) .
\end{array}
$$

Hence $\mu\left(r_{n}, \Phi\right) \rightarrow \infty$ as $n \rightarrow \infty$. The relation (2.16) implies that for $\eta \leq|\varphi| \leq \pi-\eta$

$$
\begin{array}{r}
\ln \left|\Phi\left(r e^{i \varphi}\right)\right|=(1+o(1)) A(\beta) \cos \beta\left(|\varphi|-\frac{\pi}{2}\right) r^{\beta}, \\
A(\beta)=\pi \csc \frac{\pi \beta}{2}
\end{array}
$$

holds uniformly in $\varphi$, furthermore, for $r=r_{n} \rightarrow \infty$ the relation (2.19) holds uniformly in $\varphi,|\varphi| \leq \pi$.

We show that the function $f(z)=\Psi(z) / \Phi(z)$ has the desired properties. The relation (2.19) implies that for $\eta \leq|\varphi| \leq \pi-\eta$ the function $\left|\Phi\left(r e^{i \varphi}\right)\right|$ uniformly approaches $\infty$ as $r \rightarrow \infty$. By (2.15) the function $f(z)$ approaches 0 uniformly as $z \rightarrow \infty, z \in \Delta_{3}$. For $r=r_{n}$ and $|\varphi| \leq \pi$ we get from (2.15) and (2.19) that

$$
\begin{array}{r}
\left|f\left(r e^{i \varphi}\right)\right|=\exp \left\{( 1 + o ( 1 ) ) \left[r^{l_{j}(r)} \cos \left(l_{j}(r) \varphi\right)\right.\right. \\
\left.\left.-A(\beta) \cos \beta\left(|\varphi|-\frac{\pi}{2}\right) r^{\beta}\right]\right\}+o(1) \text { for } r e^{i \varphi} \in \Delta_{j}, j=1,2 .
\end{array}
$$

Taking into account the condition $\mathrm{D}$, we get

$$
(1+o(1)) \frac{1}{\pi \rho} r_{n}^{\rho} \leq m\left(r_{n}, f\right) \leq(1+o(1)) \frac{2}{\pi \rho} r_{n}^{\rho} .
$$


It is easy to see that $n(r, f)=n(r, 0, \Phi)+O(1) \sim 2 r^{\beta}$,

$$
N(r, f) \sim \frac{2}{\beta} r^{\beta} .
$$

From (2.21) and (2.22) we get

$$
(1+o(1)) \frac{1}{\pi \rho} r_{n}^{\rho} \leq T\left(r_{n}, f\right) \leq(1+o(1)) \frac{2}{\pi \rho} r_{n}^{\rho} .
$$

Since the function $T(r, f)$ is increasing and $r_{n+1} / r_{n} \rightarrow 1$, for all $r>0$ we have

$$
(1+o(1)) \frac{1}{\pi \rho} r^{\rho} \leq T(r, f) \leq(1+o(1)) \frac{2}{\pi \rho} r^{\rho} .
$$

The formulas (2.22) and (2.23) imply that $\delta(\infty, f)=1$ and that $\infty$ is a Borel exceptional value.

Now we show that $\infty$ is not an asymptotic value. Since $\beta>1 / 2$, we have $r_{n+1}-r_{n}=O(n)=o\left(n^{2}\right)$. Taking into account the condition $\mathrm{C}$ we see that for all sufficiently large $k$ the segment $\left[a_{k}^{(j)}, b_{k}^{(j)}\right]$ contains a point $r_{n_{k}}^{(j)}$. On the arcs $\left\{|z|=r_{n_{k}}^{(j)}, z \in \Delta_{j}\right\}, j=1,2$, by the condition $\mathrm{C}$ and the relation (2.20) we have $\left(r=r_{n_{k}}^{(j)}\right)$

$$
\begin{aligned}
\left|f\left(r e^{i \varphi}\right)\right| & =\exp \left\{\left(1+o(1)\left[O\left(r^{\alpha}\right)-A(\beta) \cos \beta\left(|\varphi|-\frac{\pi}{2}\right) r^{\beta}\right]\right\}\right. \\
& +o(1) \leq \exp \left\{-(1+o(1)) \pi \cot \frac{\pi \beta}{2} r^{\beta}\right\}+o(1)=o(1) .
\end{aligned}
$$

Since, as we already observed, $|f(z)|=o(1)$ when $z \rightarrow \infty, z \in \Delta_{3}$, it is clear that $\infty$ cannot be an asymptotic value.

A slight modification of the example is necessary in the case $\rho=\infty$. Let $1 / 2<\alpha<\beta<1,1<\theta<2 \alpha$. We choose the functions $l_{j}(r)$ in such a way that they satisfy the conditions $\mathrm{B}$ and $\mathrm{C}$, and also the following conditions:

$\mathrm{A}^{\prime} . \alpha \leq l_{j}(r)<\infty$.

$\mathrm{D}^{\prime} . l(r) \rightarrow \infty, l(r)=o(\ln r)$ as $r \rightarrow \infty$.

$\mathrm{E}^{\prime}$. If $l_{1}(r)<2 \theta \alpha /(2 \alpha-\theta)$, then $l_{2}(r)>\theta \alpha$, and if $l_{2}(r)<2 \theta \alpha /(2 \alpha-\theta)$, then $l_{1}(r)>\theta \alpha$.

With these modifications the previous argument can be used. We leave verification of this as an exercise for interested readers. Later in this section we shall construct an example of an entire function of infinite order for which $\delta(0, f)=1$, but 0 is not an asymptotic value. However, in this example $0 \notin E_{B}(f)$.

Now we prove Lemma 2.1.

Proof. It is clear that $\mu(r, V)=|V(r)|$. Since (2.16) with $=$ replaced by $\leq$ holds also for $\varphi=0$, it remains to show that

$$
\ln \left|V\left(r_{n}\right)\right| \geq r_{n}^{\beta} \pi \cot \pi \beta+o\left(r_{n}^{\beta}\right) .
$$

We have

$$
\begin{gathered}
\ln \left|V\left(r_{n}\right)\right|=\sum_{k=1}^{n} \ln \left(\frac{r_{n}}{k^{1 / \beta}}-1\right)+\sum_{k=n+1}^{\infty} \ln \left(1-\frac{r_{n}}{k^{1 / \beta}}\right) \\
=\sum_{k=1}^{n} \ln \frac{r_{n}}{k^{1 / \beta}}+\sum_{k=1}^{n} \ln \left[1-\left(\frac{k}{n+\frac{1}{2}}\right)^{1 / \beta}\right]+\sum_{k=n+1}^{\infty} \ln \left[1-\left(\frac{n+\frac{1}{2}}{k}\right)^{1 / \beta}\right] .
\end{gathered}
$$


Since $n(r, 0, V)=(1+o(1)) r^{\beta}$, we get

$$
\sum_{k=1}^{n} \ln \frac{r_{n}}{k^{1 / \beta}}=N\left(r_{n}, 0, V\right)=(1+o(1)) \frac{r_{n}^{\beta}}{\beta} .
$$

It is easy to check that the functions $\ln \left(1-x^{\beta}\right)$ and $\ln \left(1-x^{-\beta}\right)$ are concave on intervals $(0,1)$ and $(1, \infty)$, respectively. Therefore for $|t| \leq 1 / 2$ we have

$$
\ln \left[1-\left(\frac{k}{n+\frac{1}{2}}\right)^{1 / \beta}\right] \geq \frac{1}{2}\left\{\ln \left[1-\left(\frac{k+t}{n+\frac{1}{2}}\right)^{1 / \beta}\right]+\ln \left[1-\left(\frac{k-t}{n+\frac{1}{2}}\right)^{1 / \beta}\right]\right\}
$$

if $k \leq n$, and

$$
\ln \left[1-\left(\frac{n+\frac{1}{2}}{k}\right)^{1 / \beta}\right] \geq \frac{1}{2}\left\{\ln \left[1-\left(\frac{n+\frac{1}{2}}{k+t}\right)^{1 / \beta}\right]+\ln \left[1-\left(\frac{n+\frac{1}{2}}{k-t}\right)^{1 / \beta}\right]\right\}
$$

if $k \geq n+1$. Integrating the first of these inequalities with respect to $t$ from 0 to $1 / 2$, we get:

$$
\begin{gathered}
\ln \left[1-\left(\frac{k}{n+\frac{1}{2}}\right)^{1 / \beta}\right] \geq \int_{0}^{1 / 2}\left\{\ln \left[1-\left(\frac{k+t}{n+\frac{1}{2}}\right)^{1 / \beta}\right]+\ln \left[1-\left(\frac{k-t}{n+\frac{1}{2}}\right)^{1 / \beta}\right]\right\} d t \\
\quad=\int_{-1 / 2}^{1 / 2} \ln \left[1-\left(\frac{k+t}{n+\frac{1}{2}}\right)^{1 / \beta}\right] d t=\int_{k-1 / 2}^{k+1 / 2} \ln \left[1-\left(\frac{x}{n+\frac{1}{2}}\right)^{1 / \beta}\right] d x, k \leq n,
\end{gathered}
$$

similarly,

$$
\ln \left[1-\left(\frac{n+\frac{1}{2}}{k}\right)^{1 / \beta}\right] \geq \int_{k-1 / 2}^{k+1 / 2} \ln \left[1-\left(\frac{n+\frac{1}{2}}{x}\right)^{1 / \beta}\right] d x, k \geq n+1 .
$$

Hence

$$
\begin{aligned}
\sum_{k=1}^{n} & \ln \left[1-\left(\frac{k}{n+\frac{1}{2}}\right)^{1 / \beta}\right]+\sum_{k=n+1}^{\infty} \ln \left[1-\left(\frac{n+\frac{1}{2}}{k}\right)^{1 / \beta}\right] \\
& \geq \int_{1 / 2}^{n+1 / 2} \ln \left[1-\left(\frac{x}{n+\frac{1}{2}}\right)^{1 / \beta}\right] d x+\int_{n+1 / 2}^{\infty} \ln \left[1-\left(\frac{n+\frac{1}{2}}{x}\right)^{1 / \beta}\right] d x \\
& \geq \int_{0}^{n+1 / 2} \ln \left[1-\left(\frac{x}{n+\frac{1}{2}}\right)^{1 / \beta}\right] d x+\int_{n+1 / 2}^{\infty} \ln \left[1-\left(\frac{n+\frac{1}{2}}{x}\right)^{1 / \beta}\right] d x \\
& =\beta r_{n}^{\beta}\left\{\int_{0}^{1} u^{\beta-1} \ln (1-u) d u+\int_{1}^{\infty} u^{\beta-1} \ln \frac{u-1}{u} d u\right\}=\beta r_{n}^{\beta} I(\beta) .
\end{aligned}
$$

Here we made the change of variable $x=\left(n+\frac{1}{2}\right) u^{\beta}=r_{n}^{\beta} u^{\beta}$. Now we compute $I(\beta), 0<\beta<1$. Note that the function

$$
I(z)=\int_{0}^{1} u^{z-1} \ln (1-u) d u+\int_{1}^{\infty} u^{z-1} \ln \frac{u-1}{u} d u
$$

is analytic in the strip $\{|\operatorname{Re} z|<1\}$. Let us compute $I(z)$ for $z=-\sigma, 0<\sigma<1$. We can rewrite

$$
I(-\sigma)=\int_{0}^{\infty} u^{-\sigma-1} \ln |1-u| d u-\int_{1}^{\infty} u^{-\sigma-1} \ln u d u .
$$


The first of these integrals can be easily computed using the residue calculus:

$$
\begin{aligned}
& \int_{0}^{\infty} u^{-\sigma-1} \ln |1-u| d u \\
&=\lim _{\varepsilon \rightarrow+0}\left\{\int_{1+\varepsilon}^{\infty} u^{-\sigma-1} \ln (u-1) d u+\int_{0}^{1-\varepsilon} u^{-\sigma-1} \ln (1-u) d u\right\} \\
&=\lim _{\varepsilon \rightarrow+0}\left\{-\left.\frac{1}{\sigma} u^{-\sigma} \ln (u-1)\right|_{1+\varepsilon} ^{\infty}+\frac{1}{\sigma} \int_{1+\varepsilon}^{\infty} \frac{u^{-\sigma}}{u-1} d u\right. \\
& \\
&\left.-\left.\frac{1}{\sigma} u^{-\sigma} \ln (1-u)\right|_{0} ^{1-\varepsilon}+\frac{1}{\sigma} \int_{0}^{1-\varepsilon} \frac{u^{-\sigma}}{u-1} d u\right\} \\
&= \lim _{\varepsilon \rightarrow+0} \frac{1}{\sigma} \ln \varepsilon\left\{(1+\varepsilon)^{-\sigma}-(1-\varepsilon)^{-\sigma}\right\}+\frac{1}{\sigma} \mathrm{v} \cdot \mathrm{p} \cdot \int_{0}^{\infty} \frac{u^{-\sigma}}{u-1} d u \\
&= \frac{\pi}{\sigma} \cot \pi \sigma
\end{aligned}
$$

the second integral can be easily computed using integration by parts:

$$
\int_{1}^{\infty} u^{-\sigma-1} \ln u d u=\frac{1}{\sigma^{2}} .
$$

Thus

$$
I(-\sigma)=\frac{\pi}{\sigma} \cot \pi \sigma-\frac{1}{\sigma^{2}}
$$

The function

$$
\frac{\pi}{z} \cot \pi z-\frac{1}{z^{2}}
$$

is analytic in the strip $\{|\operatorname{Re} z|<1\}$ and coincides with $I(z)$ on the interval $\{\operatorname{Im} z=$ $0,-1<\operatorname{Re} z<0\}$. Hence

$$
I(z)=\frac{\pi}{z} \cot \pi z-\frac{1}{z^{2}}
$$

everywhere in the strip $\{|\operatorname{Re} z|<1\}$, in particular,

$$
I(\beta)=\frac{\pi}{\beta} \cot \pi \beta-\frac{1}{\beta^{2}} .
$$

Joining (2.25), (2.26), (2.27), and (2.33), we get (2.24).

REMARK. If we use the representation

$$
\pi \cot \pi z=\frac{1}{z}+\sum_{n=1}^{\infty} \frac{2 z}{z^{2}-n^{2}},
$$

we can get the equality (2.32) in an easier way. Replacing $u$ by $u^{-1}$ in the second integral in (2.28), we get

$$
\begin{aligned}
I(z) & =\int_{0}^{1} u^{z-1} \ln (1-u) d u+\int_{0}^{1} u^{-z-1} \ln (1-u) d u \\
& =-\int_{0}^{1}\left\{\sum_{n=1}^{\infty} \frac{u^{n+z-1}+u^{n-z-1}}{n}\right\} d u=2 \sum_{n=1}^{\infty} \frac{1}{z^{2}-n^{2}}=\frac{\pi}{z} \cot \pi z-\frac{1}{z^{2}} .
\end{aligned}
$$

It is worth mentioning that we will use the equalities (2.29) and (2.30) in Section 3 (Lemma 3.3). 
Now we construct an example showing that a meromorphic function of order $\rho, 0<\rho<\infty$, can have a Borel exceptional value which is not asymptotic. In the same example a Borel exceptional value is not deficient.

ExAmPle 3. Let $0<\rho<\infty, 0<\alpha<\beta<\min (\rho, 1 / 2), n$ is an odd integer exceeding $2 \rho$. Let $V(z)$ be a function from Lemma 2.1. Take a continuously differentiable for $r \geq 0$ function $l(r)$ such that $l^{\prime}(r) r \ln r \rightarrow 0$ as $r \rightarrow \infty$, $\alpha / n \leq l(r) \leq \rho / n<1 / 2$, where $l\left(r_{k}^{n}\right)=\rho / n$ for some sequence $r_{k} \rightarrow \infty$ and for some increasing sequence $\left\{n_{j}\right\}$ of positive integers we have

$$
l\left(\left(n_{j}+\frac{1}{2}\right)^{n / \beta}\right)=\frac{\alpha}{n} .
$$

It is easy to see that such function $l(r)$ exists. Let $\Phi(z)$ be a canonical product of genus zero with positive zeros $a_{j}$ such that $n(r, 0, \Phi) \sim r^{l(r)}$ as $r \rightarrow \infty$.

Using the relations $\left(5.2_{2}^{\prime}\right)$ and (5.4) from Chapter 2 we get

$$
\begin{gathered}
\ln M(r, \Phi) \sim \pi \csc (\pi l(r)) r^{l(r)}, \\
T(r, \Phi) \sim \frac{1}{l(r)} r^{l(r)} .
\end{gathered}
$$

Let $\Psi(z)=\Phi\left(z^{n}\right), l_{1}(r)=l\left(r^{n}\right)$. By Theorem 6.6 from Chapter 1 we have

$$
\begin{gathered}
\ln M(r, \Psi) \sim \pi \csc \left(\pi l_{1}(r)\right) r^{n l_{1}(r)}, \\
T(r, \Psi) \sim \frac{1}{l_{1}(r)} r^{n l_{1}(r)} .
\end{gathered}
$$

Since $n$ is odd, then $\Psi(z)$ does not have negative integers. The function $f(z)=$ $\Psi(z) / V(-z)$ has the desired properties. It is obvious that $N(r, \infty, f)=N(r, 0, V) \sim$ $\frac{1}{\beta} r^{\beta}$. The function $\Psi(z)$ has order $\rho$ and the function $V(-z)$ has order $\beta<\rho$, therefore the order of the function $f(z)$ is equal to $\rho$ and $\infty \in E_{B}(f)$. We show that $\infty$ is not an asymptotic value. Let $q_{j}=\left(n_{j}+\frac{1}{2}\right)^{1 / \beta}$. By $(2.34)$ we have $n l_{1}\left(q_{j}\right)=\alpha$.

Taking into account (2.17) and (2.35) we get

$$
\begin{aligned}
\ln M\left(q_{j}, f\right) & \leq \ln M\left(q_{j}, \Psi\right)-\ln \mu\left(q_{j}, V\right) \\
& =q_{j}^{\alpha} \pi \csc \frac{\pi \alpha}{n}+o\left(q_{j}^{\alpha}\right)-q_{j}^{\beta} \pi \cot \pi \beta+o\left(q_{j}^{\beta}\right) \\
& =-q_{j}^{\beta} \pi \cot \pi \beta+o\left(q_{j}^{\beta}\right) \rightarrow-\infty .
\end{aligned}
$$

Hence $M\left(q_{j}, f\right)=o(1)$ and $\infty$ cannot be an asymptotic value.

By (2.36) we have

$$
\begin{gathered}
T\left(q_{j}, f\right) \geq T\left(q_{j}, V\right)-T\left(q_{j}, \Psi\right)+O(1) \\
=\frac{1}{\beta} q_{j}^{\beta}+o\left(q_{j}^{\beta}\right)-\frac{1}{l_{1}\left(q_{j}\right)} q_{j}^{\alpha}+o\left(q_{j}^{\alpha}\right)=\frac{1}{\beta} q_{j}^{\beta}+o\left(q_{j}^{\beta}\right), \\
N\left(q_{j}, \infty, f\right)=\frac{1}{\beta} q_{j}^{\beta}+o\left(q_{j}^{\beta}\right)
\end{gathered}
$$

hence $\delta(\infty, f)=0$. 
REMARK 1. Using the same idea it is possible to construct similar example with $\rho=\infty$. It will be necessary to replace the function $\Psi(z)$ by the entire function which was constructed in Section 6 of Chapter 4 (see (6.7) in Chapter 4).

REMARK 2. In Example 2 the value $\infty$ was not only nonasymptotic Borel exceptional value, but also the order of $N(r, \infty, f)$ was less than the lower order of $T(r, f)$. We do not $\mathrm{know}^{3}$ whether it is possible to construct such example with $\rho \leq 1$. In Example 3 we can take $\rho \leq 1$, but in this example the order of $N(r, \infty, f)$ exceeds the order of $T(r, f)$.

We saw that if for a meromorphic function $f(z)$ the order of $N(r, a, f)$ is less than the order of $T(r, f)$, the value $a$ does not have to be asymptotic. However the following is true:

THEOREM 2.1. Let $f(z)$ be a meromorphic function of order $\rho<\infty$, and orders of $N(r, a)$ and $N(r, b), a \neq b$, be less than $\rho$. Then $a$ and $b$ are asymptotic values.

Proof. It is clear that without loss of generality we may assume that $a=0$, $b=\infty$. The values 0 and $\infty$ are Borel exceptional values, hence, as it was shown in Section 1 of Chapter 4, the order $\rho$ is a positive integer. Let $\pi_{1}(z)$ and $\pi_{2}(z)$ be canonical products built using zeros and poles of $f(z)$, respectively. The order of $\pi_{j}(z)$ is equal to $\rho_{j}<\rho$, and genus is equal to $p_{j} \leq \rho-1$, here $\rho_{1}$ is the order of $N(r, 0)$, and $\rho_{2}$ is the order of $N(r, \infty)$.

The function $f(z)$ can be represented in the form (see Theorem 4.1 from Chapter 2):

$$
f(z)=z^{m} e^{\alpha z^{\rho}} e^{Q(z)} \frac{\pi_{1}(z)}{\pi_{2}(z)},
$$

where $m$ is an integer, $\alpha \neq 0, Q(z)$ is a polynomial of degree at most $\rho-1$. Let

$$
1>\varepsilon>0 ; \rho_{j}+\varepsilon<\rho, j=1,2 ; h=\max \left(\rho-1+\varepsilon, \rho_{1}+\varepsilon, \rho_{2}+\varepsilon\right)<\rho .
$$

Since for $r \leq r_{0}$ the inequalities $\ln M\left(r, e^{-Q}\right) \leq r^{h}, \ln M\left(r, \pi_{j}\right) \leq r^{h}$ hold, then

$$
\begin{aligned}
\ln |f(z)| & \geq \operatorname{Re}\left\{\alpha z^{\rho}\right\}+\ln \left|\pi_{1}(z)\right|+m \ln |z|-\ln M\left(r, e^{-Q}\right)-\ln M\left(r, \pi_{2}\right) \\
& =\operatorname{Re}\left\{\alpha z^{\rho}\right\}+\ln \left|\pi_{1}(z)\right|+O\left(|z|^{h}\right) .
\end{aligned}
$$

Let $a_{j} \neq 0$ be a sequence of zeros of the function $f(z)$. Then for $r \geq r_{1}$ we have $n(r, 0, f) \leq r^{\rho_{1}+\frac{\varepsilon}{2}}$ and

$$
\sum_{j}\left|a_{j}\right|^{-h}<\infty
$$

Let $|z|=r>1, R=2 r$. Denote by $g_{R}(z)$ the function

$$
g_{R}(z)=\pi_{1}(z) \prod_{\left|a_{j}\right| \leq R} \frac{R^{2}-\bar{a}_{j} z}{R\left(z-a_{j}\right)}=\pi_{1}(z) \omega_{R}(z) .
$$

Since $\left|\omega_{R}(z)\right|=1$ for $|z|=R$, the equality

$$
T\left(R, g_{R}\right)=m\left(R, g_{R}\right)=m\left(R, \pi_{1}\right)=T\left(R, \pi_{1}\right)
$$

holds. We have

$$
\ln \left|\pi_{1}(z)\right| \geq \ln \mu\left(|z|, g_{R}\right)-\ln \left|\omega_{R}(z)\right|
$$

\footnotetext{
${ }^{3}$ This seems to be still unknown.
} 
But

$$
\begin{aligned}
\ln \mu\left(r, g_{R}\right) & =-\ln M\left(r, \frac{1}{g_{R}}\right) \geq-\frac{R+r}{R-r} T\left(R, \frac{1}{g_{R}}\right) \\
& =-3 T\left(R, \frac{1}{g_{R}}\right)=-3\left\{T\left(R, g_{R}\right)-\ln \left|g_{R}(0)\right|\right\} \\
& =-3\left\{T\left(R, \pi_{1}\right)-\ln \left|\omega_{R}(0)\right|\right\} \geq-3 T\left(R, \pi_{1}\right) \\
& \geq-3 R^{h}=-3 \cdot 2^{h} r^{h} .
\end{aligned}
$$

We exclude from the complex plane the set $M=\bigcup_{j}\left\{\left|z-a_{j}\right| \leq\left|a_{j}\right|^{-h}\right\}$. Outside this set for $|z|=r \geq r_{1}$ the inequality

$$
\begin{aligned}
\ln \left|\omega_{R}(z)\right| & =\sum_{\left|a_{j}\right| \leq R} \ln \left|\frac{R^{2}-\bar{a}_{j} z}{R\left(z-a_{j}\right)}\right| \leq \sum_{\left|a_{j}\right| \leq R} \ln \frac{2 R}{\left|z-a_{j}\right|} \\
& \leq \sum_{\left|a_{j}\right| \leq R} \ln \left(2 R\left|a_{j}\right|^{h}\right) \leq n(R, 0, f) \ln \left(2 R^{h+1}\right) \\
& \leq R^{\rho_{1}+\frac{\varepsilon}{2}} \ln \left(2 R^{h+1}\right)<R^{h}=2^{h} r^{h}
\end{aligned}
$$

holds.

Joining the inequalities $(2.38),(2.40),(2.41),(2.42)$ we get that outside the set $M$ the inequality

$$
\ln |f(z)| \geq \operatorname{Re}\left\{\alpha z^{\rho}\right\}+O\left(|z|^{h}\right)
$$

holds. Let $0<\eta<\frac{\pi}{2}$. In the angle $W=\left\{|\arg \alpha+\rho \arg z|<\frac{\pi}{2}-\eta\right\}$ the inequality $\operatorname{Re}\left\{\alpha z^{\rho}\right\} \geq|z|^{\rho}|\alpha| \sin \eta$ holds. On the other hand, the sum of radiuses of the discs forming $M$ is finite. Therefore the open set $W \backslash M$ has one unbounded connected component, in which we can draw a continuous curve $C$ approaching $\infty$. On the curve $C$

$$
\left.\ln |f(z) \geq| z\right|^{\rho}|\alpha| \sin \eta+O\left(|z|^{h}\right)=(1+o(1))|z|^{\rho}|\alpha| \sin \eta \rightarrow \infty
$$

as $z \rightarrow \infty$, that is, the curve $C$ is an asymptotic curve with $\infty$ as an asymptotic value. Applying this statement to $1 / f(z)$ we get that 0 is also an asymptotic value.

It is still unknown whether Theorem 2.1 remains true ${ }^{4}$ without the assumption $\rho<\infty$.

The assumption that the orders of $N(r, a)$ and $N(r, b)$ are less than the order of $T(r, f)$ in Theorem 2.1 cannot be replaced by the assumption that $a$ and $b$ are Borel exceptional values. It is shown in our next example.

EXAMPLE 4. We construct an entire function $f(z)$ of the first order such that $0 \in E_{B}(f)$, but 0 is not an asymptotic value. Let $f(z)$ be a following canonical product of genus 1 ,

$$
f(z)=\prod_{m=1}^{\infty}\left(1-\frac{z}{a_{m}}\right) e^{\frac{z}{a_{m}}}
$$

\footnotetext{
${ }^{4}$ No. Hayman [A76] constructed an entire function $f$ of infinite lower order, such that $N(r, 0, f)$ has order $1 / 2$, but zero is not an asymptotic value.
} 
such that its roots are real,

$$
n(r, 0)=O(r)
$$

and there exists a sequence $4<r_{1}<r_{2}<r_{3}<\ldots$, such that the following conditions are satisfied:

(a)

$$
r_{3 k+3}>2 e^{r_{3 k+2}}, k=0,1, \ldots
$$

(b) The function $f(z)$ does not have zeros in the annuluses $Q_{k}=\left\{2 r_{3 k+2} \leq\right.$ $\left.|z| \leq r_{3 k+3}\right\}, k=0,1, \ldots$

$$
\sum_{\left|a_{m}\right| \leq r_{3 k+1}} \frac{1}{a_{m}}>k+2, k=0,1, \ldots
$$

$$
\sum_{\left|a_{m}\right|<r} \frac{1}{a_{m}}=0, r_{3 k+2}<r<r_{3 k+3}, k=0,1, \ldots
$$

Let us assume for a moment that sequences $\left\{a_{m}\right\}$ and $\left\{r_{k}\right\}$ having the desired properties exist and show that $f(z)$ is the desired function.

The order of $n(r, 0)$ cannot be less than one because otherwise the series $\sum_{m} \frac{1}{\left|a_{m}\right|}$ would converge in contradiction with (2.46). Since $f(z)$ is a canonical product of genus one, its order is also equal to one. To determine the type of $T(r, f)$ we use the Lindelöf theorem (Theorem 4.4 from Chapter 2). For $r=r_{3 k+1}$ we have, using (2.46),

$$
K(r)=\sum_{\left|a_{m}\right| \leq r} \frac{1}{a_{m}}>k \rightarrow \infty,
$$

therefore $T(r, f)$ has maximal type and, by $(2.44), 0 \in E_{B}(f)$.

Let us show that zero is not an asymptotic value. For this it is enough to establish that the function $f(z)$ uniformly with respect to $\arg z$ approaches $\infty$ on the sequence $\left\{|z|=R_{k}=\exp \left(\frac{1}{2} r_{3 k+2}\right)\right\}$ of circles. It is clear that $2 r_{3 k+2}<R_{k}<$ $\frac{1}{2} r_{3 k+3}$. Since $f(z)$ does not have zeros in the annuluses $Q_{k}$, by $(2.47)$, we can write

$$
f(z)=g_{k}(z) h_{k}(z), k=0,1,2, \ldots,
$$

where

$$
g_{k}(z)=\prod_{\left|a_{m}\right|<2 r_{3 k+2}}\left(1-\frac{z}{a_{m}}\right), h_{k}(z)=\prod_{\left|a_{m}\right|>r_{3 k+3}} E\left(\frac{z}{a_{m}}, 1\right) .
$$


Since for $|u|<1 / 2$ the inequality $|\ln E(u, 1)| \leq \frac{|u|^{2}}{2}\left(1+|u|+|u|^{2}+\ldots\right) \leq|u|^{2}$ holds, then, taking into account (2.44), we have

$$
\begin{aligned}
\left|\ln h_{k}(z)\right| & \leq \sum_{\left|a_{m}\right|>r_{3 k+3}}\left|\ln E\left(\frac{z}{a_{m}}, 1\right)\right| \leq \sum_{\left|a_{m}\right|>r_{3 k+3}}\left|\frac{z}{a_{m}}\right|^{2} \\
& =R_{k}^{2} \int_{r_{3 k+3}}^{\infty} \frac{d n(t, 0)}{t^{2}} \leq 2 R_{k}^{2} \int_{r_{3 k+3}}^{\infty} \frac{n(t, 0)}{t^{3}} d t \\
& =O\left(R_{k}^{2} \int_{r_{3 k+3}}^{\infty} t^{-2} d t\right)=O\left(R_{k}^{2} r_{3 k+3}^{-1}\right)=O(1)
\end{aligned}
$$

for $|z|=R_{k}$ as $k \rightarrow \infty$. On the other hand, for $|z|=R_{k}$ we have

$$
\ln \left|g_{k}(z)\right| \geq \sum_{\left|a_{m}\right|<2 r_{3 k+2}} \ln \left(\frac{R_{k}}{\left|a_{m}\right|}-1\right) \geq \ln \left(\frac{R_{k}}{r_{3 k+2}}-1\right) \rightarrow \infty .
$$

Therefore $\ln |f(z)| \geq \ln \left|g_{k}(z)\right|-|\ln | h_{k}(z)||=\ln \left|g_{k}(z)\right|+O(1)$ on the circles $\{|z|=$ $\left.R_{k}\right\}$, and the function $f(z)$ uniformly approaches $\infty$. From here it follows, among other things, that $\delta(0, f)=0$.

Now we describe the construction of sequences $\left\{a_{m}\right\}$ and $\left\{r_{k}\right\}$. This construction is based on the divergence of the series $\sum_{n=1}^{\infty} \frac{1}{n}$.

Consider an interval $\left(0, r_{1}\right)$, where $r_{1}>4$ is not an integer. Assume that the interval is so long that

$$
\sum_{n \in\left(0, r_{1}\right)} \frac{1}{n}>2
$$

Now we consider an interval $\left(r_{1}, r_{2}\right), r_{1}<r_{2}$, where $r_{2}$ is not an integer and is such that

$$
\sum_{n \in\left(0, r_{1}\right)} \frac{1}{n}-\sum_{n \in\left(r_{1}, r_{2}\right)} \frac{1}{n} \geq 0, \sum_{n \in\left(0, r_{1}\right)} \frac{1}{n}-\sum_{n \in\left(r_{1}, r_{2}+1\right)} \frac{1}{n}<0 .
$$

We include into the sequence $\left\{a_{m}\right\}$ all integers from $\left(0, r_{1}\right)$ and all integers from $\left(-r_{2},-r_{1}\right)$.

Further, we include into $\left\{a_{m}\right\}$ a number $b_{1}$ from the interval $\left(-\left[r_{2}\right]-1,-\left[r_{2}\right]\right)$, chosen in such a way that

$$
\sum_{n \in\left(0, r_{1}\right)} \frac{1}{n}-\sum_{n \in\left(r_{1}, r_{2}\right)} \frac{1}{n}+\frac{1}{b_{1}}=0 .
$$

The inequality (2.50) implies that such choice is possible.

Let $r_{3}$ be an arbitrary non-integer satisfying $r_{3}>2 e^{r_{2}}$.

Now we choose an interval $\left(r_{3}, r_{4}\right), r_{3}<r_{4}$, where $r_{4}$ is non-integer, in such a way that

$$
\sum_{n \in\left(r_{3}, r_{4}\right)} \frac{1}{n}>3
$$

and interval $\left(r_{4}, r_{5}\right), r_{4}<r_{5}, r_{5}$ is non-integer, in such a way that

$$
\sum_{n \in\left(r_{3}, r_{4}\right)} \frac{1}{n}-\sum_{n \in\left(r_{4}, r_{5}\right)} \frac{1}{n} \geq 0, \sum_{n \in\left(r_{3}, r_{4}\right)} \frac{1}{n}-\sum_{n \in\left(r_{4}, r_{5}+1\right)} \frac{1}{n}<0 .
$$


Include into $\left\{a_{m}\right\}$ all integers from $\left(r_{3}, r_{4}\right)$ and all integers from $\left(-r_{5},-r_{4}\right)$, and also a number $b_{2}$ satisfying $-\left[r_{5}\right]-1<b_{2}<-\left[r_{5}\right]$ and such that

$$
\sum_{n \in\left(r_{3}, r_{4}\right)} \frac{1}{n}-\sum_{n \in\left(r_{4}, r_{5}\right)} \frac{1}{n}+\frac{1}{b_{2}}=0
$$

Further, we choose a non-integer $r_{6}>2 e^{r_{5}}$ and continue in an obvious way. It is clear that the sequences $\left\{a_{m}\right\}$ and $\left\{r_{k}\right\}$ chosen using this procedure satisfy have the desired properties.

REMARK 1. The constructed function $f(z)$ satisfies $m\left(R_{k}, 0, f\right) \rightarrow 0$, hence $\delta(0, f)=0$. Thus $0 \in E_{B}(f) \backslash E_{N}(f)$.

REMARK 2. The lower order of the constructed function $f(z)$ is equal to zero. In fact, by (2.48), (2.49), and (2.44), we get

$$
\begin{aligned}
\ln M\left(R_{k}, f\right) & =\ln M\left(R_{k}, g_{k}\right)+O(1) \\
& \leq \sum_{\left|a_{m}\right|<2 r_{3 k+2}} \ln \left(1+\frac{R_{k}}{\left|a_{m}\right|}\right)+O(1) \leq n\left(2 r_{3 k+2}, 0\right) \ln \left(1+R_{k}\right)+O(1) \\
& =O\left(r_{3 k+2} \ln R_{k}\right)=O\left(\ln ^{2} R_{k}\right) .
\end{aligned}
$$

The order $\rho$ of the function $f(z)$ in our example is equal to 1 . If we take $F(z)=f\left(z^{n}\right), n=2,3, \ldots$, we get an analogous example with $\rho=n$. It is not known whether such example exist for functions of infinite order. ${ }^{5}$

We see that in Theorem 2.1 the condition that the orders of $N(r, a)$ and $N(r, b)$ are lower than the order of $T(r, f)$ cannot be replaced by the condition that the categories of $N(r, a)$ and $N(r, b)$ are lower than the category of $T(r, f)$. However, Edrei and Fuchs [EF59a] proved the following theorem: Let $f(z)$ be a meromorphic function of a finite order and $\delta(a)=\delta(b)=1, a \neq b$ (that is, $N(r, a)=o(T(r, f))$ and $N(r, b)=o(T(r, f)))$. Then $a$ and $b$ are asymptotic values. ${ }^{6}$ We cannot present here a proof of this theorem, we only give an example showing that this theorem is not valid for functions of infinite order.

EXAMPLE 5. We construct an entire function $f(z)$ of infinite order such that $\delta(0, f)=1$, but 0 is not an asymptotic value (since now we are dealing with functions of infinite order, this example is stronger than Example 2).

First of all, we need a function $E(z)$ defined in the following way. Let $a$ be a real number, $A(a)$ be a half-strip $\{x>a,|y|<\pi\}, z=x+i y, L(a)$ be the boundary of $A(a)$ oriented in such a way that $A(a)$ is on the right. We define the function $E(z)$ in the complement of $\overline{A(0)}$ by the Cauchy-type integral:

$$
E(z)=\frac{1}{2 \pi i} \int_{L(0)} \frac{e^{e^{t}}}{t-z} d t .
$$

Obviously, the integral

$$
\frac{1}{2 \pi i} \int_{L(a)} \frac{e^{e^{t}}}{t-z} d t
$$

\footnotetext{
${ }^{5}$ Now it exists: Hayman [A76].

${ }^{6}$ For meromorphic functions of finite lower order with sum of deficiencies two all deficient values are asymptotic. See the Appendix at the end of the book.
} 
for $a>0$ is an analytic continuation of $E(z)$ onto the complement of $\overline{A(a)}$. Hence the function (2.51) has an analytic continuation to the whole complex plane, that is, is an entire function.

Since by the integral Cauchy theorem the integral of the function $e^{e^{t}}$ over the boundary of the rectangle $\left\{a_{1}<x<a_{2},|y|<\pi\right\}$ is equal to zero, we have

$$
\frac{1}{2 \pi i} \int_{L\left(a_{1}\right)} e^{e^{t}} d t=\frac{1}{2 \pi i} \int_{L\left(a_{2}\right)} e^{e^{t}} d t
$$

and the integral

$$
I=\frac{1}{2 \pi i} \int_{L(a)} e^{e^{t}} d t
$$

does not depend on $a$. Since the function $e^{e^{t}}$ is periodic with period $2 \pi i$, the integrals over the rays $\{y= \pm \pi, x \geq a\}$ cancel each other, and we get

$$
I=\frac{1}{2 \pi} \int_{-\pi}^{\pi} e^{e^{a+i y}} d y
$$

Letting $a \rightarrow-\infty$ in the right-hand side of this equality, we get $I=1$. Let $z \in \overline{A(0)}$. Then

$$
\begin{aligned}
E(z)+\frac{1}{z} & =\frac{1}{2 \pi i} \int_{L(2)} \frac{e^{e^{t}}}{t-z} d t+\frac{1}{2 \pi i} \int_{L(2)} \frac{e^{e^{t}}}{z} d t=\frac{1}{2 \pi i} \int_{L(2)} \frac{t}{z(t-z)} e^{e^{t}} d t \\
& =\frac{1}{z^{2}} \frac{1}{2 \pi i} \int_{L(2)}\left(-t+\frac{t^{2}}{t-z}\right) e^{e^{t}} d t=\frac{1}{z^{2}} \Omega(z) .
\end{aligned}
$$

If the distance from $z$ to $L(2)$ is not less than $\pi / 3$, then we have $\left|-t+t^{2}(t-z)^{-1}\right| \leq$ $|t|+|t|^{2} \leq 2|t|^{2}$. Since on the rays $\{x>1, y= \pm \pi\}$ the equality $\left|\exp \left(e^{x+i y}\right)\right|=$ $\exp \left(-e^{x}\right)$ holds, the function $\Omega(z)$ is bounded by a constant which does no depend on $z$. If the distance from $z$ to $L(2)$ is less than $\pi / 3$, we replace the part of $L(2)$ which is inside the disc $\{|t-z|<\pi / 3\}$ by an arc of the circle $\{|t-z|=\pi / 3\}$ lying in $A(2)$. By the integral Cauchy theorem the value of the last integral in (2.52) is not affected by this change of the path of integration. As before we have $\left|-t+t^{2}(t-z)^{-1}\right| \leq 2|t|^{2}$, and for points $z$ on the arc $\{|t-z|=\pi / 3\}$ we have the inequality $\left|\exp \left(e^{t}\right)\right| \leq \exp \left(-\frac{1}{2} e^{\operatorname{Re} t}\right)$. Thus in this case the function $\Omega(z)$ is also bounded. If $z \in L(0)$ we can use similar reasoning. We get that

$$
E(z)=-\frac{1}{z}+O\left(\frac{1}{|z|^{2}}\right), z \notin A(0) .
$$

Now let $z \in A(0)$. Than, taking $a>\operatorname{Re} z>0$, we have

$$
\begin{aligned}
E(z)= & \frac{1}{2 \pi i} \int_{L(a)} \frac{e^{e^{t}}}{t-z} d t=\frac{1}{2 \pi i} \int_{L(-2)} \frac{e^{e^{t}}}{t-z} d t \\
& +\frac{1}{2 \pi i} \int_{L(a)-L(-2)} \frac{e^{e^{t}}}{t-z} d t=\frac{1}{2 \pi i} \int_{L(-2)} \frac{e^{e^{t}}}{t-z} d t+e^{e^{z}}
\end{aligned}
$$

As before, we get that

$$
E(z)-e^{e^{z}}+\frac{1}{z}=\frac{1}{z^{2}} \frac{1}{2 \pi i} \int_{L(-2)}\left(-t+\frac{t^{2}}{t-z}\right) e^{e^{t}} d t .
$$


The boundedness of the last integral is proved as before; only in this case, when the distance from $z$ to $L(-2)$ is less than $\pi / 3$ we replace the part of $L(-2)$ by an arc of the circle $\{|t-z|=\pi / 3\}$ lying outside $A(-2)$. Thus

$$
E(z)=e^{e^{z}}-\frac{1}{z}+O\left(\frac{1}{|z|^{2}}\right), z \in A(0) .
$$

We shall write $A$ instead of $A(0)$. The relations (2.53) and (2.54) imply that

$$
E(z)= \begin{cases}e^{e^{z}}+\frac{\Psi_{1}(z)}{z}, & z \in A, \\ \frac{\Psi_{2}(z)}{z}, & z \notin A,\end{cases}
$$

where $\left|\Psi_{j}(z)\right|<2$ for $|z|>r_{0} \geq 1, j=1,2$.

Let $K>8$ be a real number such that the open set $G \subset A$ defined as the set of all solutions of the inequality $\left|e^{e^{z}}+K\right|<7$ lies in the half-plane $\left\{x>4 r_{0}\right\}$ and the diameters of all connected components of $G$ are less than $\pi$. Obviously, sufficiently large numbers $K$ have this property. Let

$$
\begin{gathered}
q_{n}=\sqrt{\left(2 \pi 2^{n}\right)^{2}-1}, \theta_{n}=\arcsin \left(2 \pi 2^{n}\right)^{-1}, \\
\Phi(z)=K+\sum_{n=1}^{\infty} E\left\{e^{i \theta_{n}}\left(z-i q_{n}\right)\right\} .
\end{gathered}
$$

The uniform convergence of the series (2.56) in each finite disc can be easily checked, hence $\Phi(z)$ is an entire function.

Denote by $A^{0}$ the half-strip $\{x>0,|y|<\pi / 2\}$, and by $A_{n}, A_{n}^{0}, G_{n}$ the images of $A, A^{0}, G$, respectively, under the mapping $\lambda_{n}(z)=e^{-i \theta_{n}} z+i q_{n}$. It is easy to see that half-strips $A_{n}$ are pairwise disjoint, $G_{n} \subset A_{n}$ and $\bigcup_{n=1}^{\infty} G_{n} \subset H=\left\{x>2 r_{0}\right\}$. Denote by $C_{n}$ the disc $\left\{\left|z-i q_{n}\right|<r_{0}\right\}$. Let us show that

$$
\Omega(z)=\sum_{n=1}^{\infty}\left|z-i q_{n}\right|^{-1}<3
$$

for $z \notin C=\bigcup_{n=1}^{\infty} C_{n}$. In fact, if $q_{m} \leq y \leq q_{m+1}, m=1,2, \ldots$, then

$$
\begin{aligned}
\Omega(z) & \leq 2+\sum_{n=1}^{m-1}\left|z-i q_{n}\right|^{-1}+\sum_{n=m+2}^{\infty}\left|z-i q_{n}\right|^{-1} \\
& \leq 2+\sum_{n=1}^{m-1}\left|y-q_{n}\right|^{-1}+\sum_{n=m+2}^{\infty}\left|y-q_{n}\right|^{-1} \\
& \leq 2+\sum_{n=1}^{m-1}\left(q_{m}-q_{n}\right)^{-1}+\sum_{n=m+2}^{\infty}\left(q_{n}-q_{m+1}\right)^{-1} \\
& \leq 2+\sum_{n=1}^{m-1} \frac{3}{2 \pi 2^{m}}+\sum_{n=m+2}^{\infty} \frac{3}{2 \pi 2^{n}} \\
& \leq 2+\frac{3}{2 \pi} \frac{m-1}{2^{m}}+\frac{3}{8 \pi} \leq 2+\frac{3}{8 \pi}+\frac{3}{8 \pi}<3 .
\end{aligned}
$$


If $y \leq q_{1}$, the same estimate works. The relations (2.55), (2.56), (2.57) imply that the relation

$$
\Phi(z)= \begin{cases}Q(z), & z \notin\left\{\bigcup_{n=1}^{\infty} A_{n}\right\} \cup C \\ \exp \exp \left\{e^{i \theta_{n}}\left(z-i q_{n}\right)\right\}+Q(z), & z \in A_{n} \backslash C\end{cases}
$$

holds, where $|Q(z)-K|<6$. Denote by $D$ the set of those $z \notin C$ for which $|\Phi(z)|<1$. It is easy to see that $D \subset \bigcup_{n=1}^{\infty} G_{n}$. In fact, $|Q(z)| \geq K-6 \geq 2$, therefore $D \subset \bigcup_{n=1}^{\infty} A_{n}$. Let $z \in D \cap A_{n}$. Then

$$
\left|\exp \exp \left\{e^{i \theta_{n}}\left(z-i q_{n}\right)\right\}+K\right| \leq|\Phi(z)|+|K-Q(z)|<1+6=7,
$$

that is, $z \in G_{n}$. Hence $D \subset H$ and the diameters of all connected components of $D$ are less than $\pi$.

Consider the function $g(z)=\Phi(z) \exp \exp z$. Let us show that zero is not an asymptotic value for this function. In the half-plane $H_{1}=\left\{x \leq 2 r_{0}\right\}$ the inequality $|\exp \exp z| \geq \exp \left(-e^{2 r_{0}}\right)=\mu, 0<\mu<1$, holds, and for $z \notin C$ we have $|\Phi(z)| \geq 1$ since $H_{1}$ does not intersect $D$. Hence, in $H_{1} \backslash C$ we have $|g(z)| \geq \mu$. The sets $B_{k}=\left\{x \geq 2 r_{0},|y-2 \pi k| \leq \pi / 2\right\} \backslash D, k=0, \pm 1, \pm 2, \ldots$, are unbounded continua, since the diameters of all connected components of $D$ are less than $\pi$. On the other hand, on $B_{k}$ we have $|\Phi(z)| \geq 1$ and $|\exp \exp z|=\exp \left\{e^{x} \cos y\right\} \geq 1,|g(z)| \geq 1$. On the rays $S_{n}=\left\{\arg \left(z-i q_{n}\right)=-\theta_{n}, x \geq 2 r_{0}\right\}, n \geq 1$, the inequalities

$$
\begin{aligned}
|\Phi(z)|= & |\exp \exp | z-i q_{n}|+Q(z)| \\
\geq & \exp \exp \left|z-i q_{n}\right|+\operatorname{Re} Q(z)>\exp \exp \left|z-i q_{n}\right| \\
= & \exp \exp \left\{x \sec \theta_{n}\right\}, \\
& |g(z)|=\exp \left\{e^{x \sec \theta_{n}}+e^{x} \cos y\right\} \geq 1
\end{aligned}
$$

hold. Obviously, the open set $H \backslash(B \cup S)$, where $B=\bigcup_{k=-\infty}^{\infty} B_{k}, S=\bigcup_{n=1}^{\infty} S_{n}$, does not have unbounded connected components.

As the final result we get that the open set of those $z$ for which $|g(z)|<\mu$ does not have unbounded connected components. Hence zero is not an asymptotic value for $g(z)$.

Let us estimate $T(r, \Phi(z))$. It is convenient for us to estimate $T(r, \Phi(z-1))$ first. Observe that by the maximum modulus principle $|\Phi(z)|=O(1)$ for $z \in C$. Let $z=r e^{i \theta}$. Then, by (2.58), we get

$$
\begin{aligned}
T(r, \Phi(z-1)) & =\frac{1}{2 \pi} \int_{|z|=r} \ln ^{+}|\Phi(z-1)| d \theta \\
& \leq O(1)+\sum_{n} \frac{1}{2 \pi} \int_{\substack{|z|=r \\
z-1 \in A_{n}^{0}}} \ln ^{+}|\Phi(z-1)| d \theta \\
& =O(1)+\sum_{n} \frac{1}{2 \pi} \int_{\substack{|z|=r \\
z-1 \in A_{n}^{0}}} \operatorname{Re} \exp \left\{e^{i \theta_{n}}\left(z-1-i q_{n}\right)\right\} d \theta .
\end{aligned}
$$

But

$$
-1-i q_{n}=-2 \pi 2^{n} e^{i\left(\frac{\pi}{2}-\theta_{n}\right)}=-i 2 \pi 2^{n} e^{-i \theta_{n}},
$$


therefore $\exp \left\{e^{i \theta_{n}}\left(z-1-i q_{n}\right)\right\}=\exp \left\{r e^{i\left(\theta+\theta_{n}\right)}\right\}$. Suppose that the intersection of the set of those $z$, for which $z-1 \in A_{n}^{0}$ with the circle $\{|z|=r\}$ is non-empty, that is, $2 \pi 2^{n}-\frac{\pi}{2} \leq r$. Then

$$
\begin{aligned}
\int_{\substack{|z|=r \\
z-1 \in A_{n}^{0}}} \operatorname{Re} \exp \left\{r e^{i\left(\theta+\theta_{n}\right)}\right\} d \theta & =\int_{\alpha_{n}-\theta_{n}}^{\beta_{n}-\theta_{n}} e^{r \cos \left(\theta+\theta_{n}\right)} \cos \left\{r \sin \left(\theta+\theta_{n}\right)\right\} d \theta \\
& =\int_{\alpha_{n}}^{\beta_{n}} e^{r \cos \theta} \cos \{r \sin \theta\} d \theta
\end{aligned}
$$

where

$$
\begin{gathered}
\alpha_{n}=\arcsin \frac{2 \pi 2^{n}-\pi / 2}{r}, \\
\beta_{n}=\arcsin \min \left(1, \frac{2 \pi 2^{n}+\pi / 2}{r}\right) .
\end{gathered}
$$

Substituting (2.60) into (2.59) we get

$$
T(r, \Phi(z-1)) \leq O(1)+\sum_{n=1}^{\nu(r)} \frac{1}{2 \pi} \int_{\alpha_{n}}^{\beta_{n}} e^{r \cos \theta} \cos \{r \sin \theta\} d \theta,
$$

where $\nu(r)=\left[(\ln 2)^{-1} \ln \frac{r+\pi / 2}{2 \pi}\right]$. We define a continuous function $\varphi(y)$ on $[0, \infty)$ in the following way. We let $\varphi(y)=\cos y$ for $\left|y-2 \pi 2^{n}\right| \leq \frac{\pi}{2}, n=1,2, \ldots$, and let $\varphi(y)=0$ for all other $y \geq 0$. Then the inequality (2.61) can be rewritten in the following way:

$$
T(r, \Phi(z-1)) \leq O(1)+\frac{1}{2 \pi} \int_{0}^{\pi / 2} e^{r \cos \theta} \varphi(r \sin \theta) d \theta .
$$

It is clear that

$$
\frac{1}{r} \int_{0}^{r} \varphi(y) d y \rightarrow 0 \text { as } r \rightarrow \infty
$$

Applying to the integral in the right-hand side of (2.62) Lemma 4.1 from Chapter 4 , we get $T(r, \Phi(z-1))=o\left(e^{r} / \sqrt{r}\right)$. Since $T(r, \Phi(z)) \leq(1+o(1)) T(r+1, \Phi(z-1))$ (see (1.4) in Chapter 2), we have

$$
T(r, \Phi(z))=o\left(e^{r} / \sqrt{r}\right) .
$$

On the other hand, as it was shown in the construction of Example 1 in Section 6 Chapter 4, we have

$$
T\left(r, e^{e^{z}}\right)=(1+o(1)) \frac{e^{r}}{\sqrt{2 \pi^{3} r}}, r \rightarrow \infty .
$$

Since $T\left(r, e^{e^{z}}\right)-T\left(r, \Phi^{-1}\right) \leq T(r, g) \leq T\left(r, e^{e^{z}}\right)+T(r, \Phi)$, the relations (2.63) and (2.64) imply that

$$
T(r, g)=(1+o(1)) \frac{e^{r}}{\sqrt{2 \pi^{3} r}}, r \rightarrow \infty .
$$

But $N(r, 0, g)=N(r, 0, \Phi) \leq T(r, \Phi)+O(1)=o(T(r, g))$. Hence $\delta(0, g)=1$ and the function $g(z)$ has the desired properties. 


\section{Meromorphic functions of order less than $1 / 2$}

By the Wiman-Heins Theorem 1.3 for an entire function of order $\lambda<1 / 2$ there exists a sequence of circles $\left\{|z|=r_{k}\right\}, r_{k} \rightarrow \infty$, on which $f(z)$ approaches $\infty$ uniformly in $\arg z$. The following theorem is a generalization of this result for meromorphic functions.

THEOREM 3.1. Let $f(z)$ be a meromorphic function of lower order $\lambda<1 / 2$. If

$$
\delta(\infty, f)>1-\cos \pi \lambda,
$$

then there exists a sequence of circles $\left\{|z|=r_{k}\right\}, r_{k} \rightarrow \infty$, on which $f(z)$ approaches $\infty$ uniformly in $\arg z$.

Observe, that if we replace the condition (3.1) by the condition $\delta(a, f)>1-$ $\cos \pi \lambda, a \neq \infty$, we can claim that there exists a sequence of circles $\left\{|z|=r_{k}\right\}$, $r_{k} \rightarrow \infty$, on which $f(z)$ approaches $a$ uniformly in $\arg z$. In fact, for the function $f_{1}(z)=\frac{1}{f(z)-a}$ we have $\lambda\left[f_{1}\right]=\lambda[f]=\lambda, \delta\left(\infty, f_{1}\right)=\delta(a, f)$, so it suffices to apply Theorem 3.1 to $f_{1}(z)$. By virtue of this remark, Theorem 3.1 immediately implies the following corollary, containing the corollary of Theorem 1.3 and Valiron's Theorem 4.6 from Chapter 2.

Corollary. Let $f(z)$ be a meromorphic function of lower order $\lambda<1 / 2$. If $\delta(a, f)>1-\cos \pi \lambda$, then $a$ is the only deficient value of the function $f(z)$. In particular, a meromorphic function of lower order $\lambda=0$ cannot have more than one deficient value.

In fact, since the function $f(z)$ approaches $a$ on the sequence of circles $\{|z|=$ $\left.r_{k}\right\}, r_{k} \rightarrow \infty$, uniformly in $\arg z$, for $b \neq a$ we have $m\left(r_{k}, b\right)=O(1)$ and hence $\delta(b, f)=0$.

Theorem 3.1 is a corollary of a more subtle theorem giving a quantitative estimate of the degree of convergence of $f(z)$ to $\infty$.

THEOREM 3.2. Let $f(z)$ be a meromorphic function of lower order $\lambda<1 / 2$. Then the inequality

$$
\limsup _{r \rightarrow \infty} \frac{\ln ^{+} \mu(r, f)}{T(r, f)} \geq \frac{\pi \lambda}{\sin \pi \lambda}(\delta(\infty, f)-1+\cos \pi \lambda)
$$

holds.

The proof of Theorem 3.2 relies on several lemmas. The first of them will be also used in Section 4, for this reason we prove it in a more general formulation than is needed for the proof of Theorem 3.2.

LEMma 3.1. Let $f(z), f(0)=1$, be a meromorphic function with zeros $a_{\mu}$ and poles $b_{\nu}$, and $q \geq 0$ be an integer. The following representation holds for each $R>0$ :

$$
f(z)=\alpha_{R, q}(z) \omega_{R, q}(z)
$$

where

$$
\alpha_{R, q}(z)=\prod_{\left|a_{\mu}\right|<R} E\left(\frac{z}{a_{\mu}}, q\right)\left\{\prod_{\left|b_{\nu}\right|<R} E\left(\frac{z}{b_{\nu}}, q\right)\right\}^{-1}
$$


and $\ln \omega_{R, q}(z)$ (the branch of $\ln$ is chosen in such a way that $\ln \omega_{R, q}(0)=0$ ) satisfies the estimate

$$
\begin{gathered}
\left|\ln \omega_{R, q}(z)\right| \leq K_{1}\left(\frac{r}{R}\right)^{q+1} T(2 R, f)+K_{2} q\left(r^{q}+1\right), \\
|z|=r \leq \frac{1}{2} R,
\end{gathered}
$$

where $K_{1}$ and $K_{2}, 0<K_{1}, K_{2}<\infty$ are constants which do not depend on $r$ and $R$.

Proof. By formula (2.5) from Chapter 1 we have

$$
\begin{aligned}
\{\ln f(\zeta)\}^{(q+1)}= & (-1)^{q} q ! \sum_{\left|a_{\mu}\right|<R} \frac{1}{\left(\zeta-a_{\mu}\right)^{q+1}}-(-1)^{q} q ! \sum_{\left|b_{\nu}\right|<R} \frac{1}{\left(\zeta-b_{\nu}\right)^{q+1}} \\
& +I_{R, q}(\zeta)+J_{R, q}(\zeta)
\end{aligned}
$$

where

$$
\begin{gathered}
I_{R, q}(\zeta)=\frac{(q+1) !}{2 \pi} \int_{0}^{2 \pi} \ln \left|f\left(\operatorname{Re}^{i \theta}\right)\right| \frac{2 \operatorname{Re}^{i \theta}}{\left(\operatorname{Re}^{i \theta}-\zeta\right)^{q+2}} d \theta \\
J_{R, q}(\zeta)=q ! \sum_{\left|a_{\mu}\right|<R}\left(\frac{a_{\mu}}{R^{2}-\bar{a}_{\mu} \zeta}\right)^{q+1}-q ! \sum_{\left|b_{\nu}\right|<R}\left(\frac{b_{\nu}}{R^{2}-b_{\nu} \zeta}\right)^{q+1} .
\end{gathered}
$$

Straightforward calculation shows that

$$
\left\{\ln \alpha_{R, q}(\zeta)\right\}^{(q+1)}=(-1)^{q} q ! \sum_{\left|a_{\mu}\right|<R} \frac{1}{\left(\zeta-a_{\mu}\right)^{q+1}}-(-1)^{q} q ! \sum_{\left|b_{\nu}\right|<R} \frac{1}{\left(\zeta-b_{\nu}\right)^{q+1}} .
$$

Subtracting this equality from (3.6) we get

$$
\left\{\ln \omega_{R, q}(\zeta)\right\}^{(q+1)}=I_{R, q}(\zeta)+J_{R, q}(\zeta) .
$$

Let us estimate $I_{R, q}(\zeta)$ and $J_{R, q}(\zeta)$ for $|\zeta| \leq \frac{1}{2} R$. We have

$$
\begin{aligned}
\left|I_{R, q}(\zeta)\right| & \leq \frac{(q+1) ! 2 R}{(R-|\zeta|)^{q+2}} \frac{1}{2 \pi} \int_{0}^{2 \pi}|\ln | f\left(\operatorname{Re}^{i \theta}\right)|| d \theta \\
& \leq \frac{(q+1) ! 2^{q+3}}{R^{q+1}}\left(m(R, f)+m\left(R, \frac{1}{f}\right)\right) \leq \frac{(q+1) ! 2^{q+4}}{R^{q+1}} T(R, f) \\
\left|J_{R, q}(\zeta)\right| & \leq q !\left(\frac{R}{R^{2}-R|\zeta|}\right)^{q+1}\left(n(R, f)+n\left(R, \frac{1}{f}\right)\right) \\
& \leq \frac{q ! 2^{q+1}}{R^{q+1}} n(R ; 0, \infty) \leq \frac{q ! 2^{q+2}}{R^{q+1}} N(2 R ; 0, \infty) \leq \frac{q ! 2^{q+3}}{R^{q+1}} T(2 R, f)
\end{aligned}
$$

(passing from $n(R ; 0, \infty)$ to $N(2 R ; 0, \infty)$ we used Lemma 7.1 from Chapter 1 ).

Thus

$$
\left|\left\{\ln \omega_{R, q}(\zeta)\right\}^{(q+1)} \leq \frac{(q+1) ! 2^{q+5}}{R^{q+1}} T(2 R, f),\right| \zeta \mid \leq \frac{1}{2} R
$$

Later on we shall need the following relation $(|z|<R)$ 


$$
\ln \omega_{R, q}(z)=\frac{1}{q !} \int_{0}^{z}(z-\zeta)^{q}\left\{\ln \omega_{R, q}(\zeta)\right\}^{(q+1)} d \zeta+\sum_{k=0}^{q} \frac{z^{k}}{k !}\left\{\ln \omega_{R, q}(\zeta)\right\}_{\zeta=0}^{(k)}
$$

(the integral is taken over a line segment joining 0 and $z$ ). This relation follows from the observation that the derivatives of order $q+1$ of the different sides are the same, and the derivatives of orders $k=0,1, \ldots, q$ coincide at $z=0$.

Using (3.7) and (3.8) we get the estimate $(z=|z| \leq R / 2)$

$$
\left|\ln \omega_{R, q}(z)\right| \leq(q+1) 2^{q+5}\left(\frac{r}{R}\right)^{q+1} T(2 R, f)+\left|\sum_{k=0}^{q} \frac{z^{k}}{k !}\left\{\ln \omega_{R, q}(\zeta)\right\}_{\zeta=0}^{(k)}\right| .
$$

The values of $\left\{\ln \omega_{R, q}(\zeta)\right\}_{\zeta=0}^{(k)}, k=0,1, \ldots, q$, do not depend on $R \operatorname{since} \ln \omega_{R, q}(\zeta)=$ $\ln f(\zeta)-\ln \alpha_{R, q}(\zeta)$ and $\left\{\ln \alpha_{R, q}(\zeta)\right\}_{\zeta=0}^{(k)}=0, k=0,1, \ldots, q$. Besides, $\ln \omega_{R, q}(0)=0$, therefore the estimate

$$
\left|\sum_{k=0}^{q} \frac{z^{k}}{k !}\left\{\ln \omega_{R, q}(\zeta)\right\}_{\zeta=0}^{(k)}\right| \leq K_{2} q\left(r^{q}+1\right)
$$

holds, where $0<K_{2}<\infty$ does not depend on $R$ and $r$. The proof of the lemma is completed.

Lemma 3.2. Let $f(z), f(0)=1$, be a meromorphic function with zeros $a_{\mu}$ and poles $b_{\nu}$. Set

$$
H_{R}(r)=\sum_{\left|a_{\mu}\right|<R} \ln \left(1+\frac{r}{\left|a_{\mu}\right|}\right)+\sum_{\left|b_{\nu}\right|<R} \ln \left(1+\frac{r}{\left|b_{\nu}\right|}\right) .
$$

The estimate

$$
H_{R}(r) \leq 4 T(2 R, f), 0 \leq r \leq R,
$$

holds.

Proof. By the inequality $\ln (1+x) \leq \ln ^{+} x+\ln 2,0 \leq x<\infty$, we have

$$
\begin{aligned}
H_{R}(r) & \leq \sum_{\left|a_{\mu}\right|<R}\left(\ln ^{+} \frac{r}{\left|a_{\mu}\right|}+\ln 2\right)+\sum_{\left|b_{\nu}\right|<R}\left(\ln ^{+} \frac{r}{\left|b_{\nu}\right|}+\ln 2\right) \\
& \leq N(r, 0)+n(R, 0) \ln 2+N(r, \infty)+n(R, \infty) \ln 2 \\
& =N(r ; 0, \infty)+n(R ; 0, \infty) \ln 2 .
\end{aligned}
$$

Using Lemma 7.1 from Chapter 1 we get

$$
H_{R}(r) \leq N(r ; 0, \infty)+N(2 R ; 0, \infty) \leq 2 T(r, f)+2 T(2 R, f) \leq 4 T(2 R, f),
$$

Lemma 3.3. For each $a$ and $b, 0<a<b$, and each $\sigma, 0<\sigma<1$, the inequalities

$$
\begin{array}{r}
\int_{a}^{b}\left\{\ln |1-x|-\pi \sigma \cot \pi \sigma \ln ^{+} x\right\} x^{-1-\sigma} d x \\
\geq C_{1} a^{-\sigma} \ln (1+a)-C_{2} b^{-\sigma} \ln (1+b)
\end{array}
$$




$$
\begin{gathered}
\int_{a}^{b}\left\{\pi \sigma \csc \pi \sigma \ln ^{+} x-\ln (1+x)\right\} x^{-1-\sigma} d x \\
\geq C_{3} a^{-\sigma} \ln (1+a)-C_{4} b^{-\sigma} \ln (1+b)
\end{gathered}
$$

hold, where $C_{k}, 0<C_{k}<\infty, k=1,2,3,4$, are constants which do not depend on $a$ and $b$.

Proof. We shall use the equalities
(a) $\int_{0}^{\infty} \frac{\ln ^{+} x}{x^{1+\sigma}} d x=\frac{1}{\sigma^{2}}$;
(b) $\int_{0}^{\infty} \frac{\ln (1+x)}{x^{1+\sigma}} d x=\frac{\pi}{\sigma \sin \pi \sigma}$;

$$
\text { (c) } \int_{0}^{\infty} \frac{\ln |1-x|}{x^{1+\sigma}} d x=\frac{\pi}{\sigma \tan \pi \sigma} .
$$

To get (b) we need to integrate by parts and to use the Residue Calculus:

$$
\int_{0}^{\infty} \frac{\ln (1+x)}{x^{1+\sigma}} d x=\frac{1}{\sigma} \int_{0}^{\infty} \frac{d x}{x^{\sigma}(1+x)}=\frac{\pi}{\sigma \sin \pi \sigma} .
$$

The equalities (a) and (c) were proved in Section 2 (see (2.29) and (2.30)).

Consider the function

$$
\varphi(a)=\int_{a}^{\infty}\left\{\ln |1-x|-\pi \sigma \cot \pi \sigma \ln ^{+} x\right\} x^{-1-\sigma} d x, 0 \leq a<\infty .
$$

By the equalities (a) and (c) we have $\varphi(0)=0$; it is clear that $\lim _{a \rightarrow \infty} \varphi(a)=0$. Since the derivative

$$
\varphi^{\prime}(a)=-\left\{\ln |1-a|-\pi \sigma \cot \pi \sigma \ln ^{+} a\right\} a^{-1-\sigma},
$$

as is easy to check, changes sign only once on the half-axis $\{0<a<\infty\}$, from plus to minus, we have $\varphi(a)>0$ for $0<a<\infty$.

Set

$$
\psi(a)=\frac{\varphi(a)}{a^{-\sigma} \ln (1+a)} .
$$

This function is continuous and positive for $0<a<\infty$. Using the l'Hôpital rule we find its limits as $a \rightarrow+0$ and $a \rightarrow+\infty$ and see that both are finite and positive. Therefore there exist constants $C_{1}$ and $C_{2}, 0<C_{1}<C_{2}<\infty$, such that

$$
C_{1} \leq \psi(a) \leq C_{2}, \quad 0<a<\infty .
$$

The last inequality can be rewritten in the form

$$
C_{1} a^{-\sigma} \ln (1+a) \leq \varphi(a) \leq C_{2} a^{-\sigma} \ln (1+a), 0<a<\infty,
$$

from where we get

$$
\varphi(a)-\varphi(b) \geq C_{1} a^{-\sigma} \ln (1+a)-C_{2} b^{-\sigma} \ln (1+b), 0<a<b<\infty,
$$

the last inequality is equivalent to (3.11).

To prove the inequality (3.12) we use similar argument for

$$
\varphi(a)=\int_{a}^{\infty}\left\{\pi \sigma \csc \pi \sigma \ln ^{+} x-\ln (1+x)\right\} x^{-1-\sigma} d x .
$$


LEMma 3.4. Let $f(z), f(0)=1$, be a meromorphic function with lower order $\lambda<1$. Then for each $\sigma, \lambda<\sigma<1$, the inequality

$$
\limsup _{r \rightarrow \infty}\left\{\ln \mu(r, f)+\frac{\pi \sigma}{\sin \pi \sigma}[N(r, \infty)-\cos \pi \sigma N(r, 0)]\right\} \geq 0
$$

holds.

Proof. We may assume without loss of generality that $f(z)$ is not identically constant. Let $a_{\mu}$ be zeros of the function $f(z), b_{\nu}$ be its poles. Using the change of variable in the integrals from the inequalities (3.11) and (3.12) we get the inequalities

$$
\begin{aligned}
\int_{\xi}^{\eta} & \left\{\ln \left|1-\frac{r}{\left|a_{\mu}\right|}\right|-\pi \sigma \cot \pi \sigma \ln ^{+} \frac{r}{\left|a_{\mu}\right|}\right\} \frac{d r}{r^{1+\sigma}} \\
& \geq C_{1} \xi^{-\sigma} \ln \left(1+\frac{\xi}{\left|a_{\mu}\right|}\right)-C_{2} \eta^{-\sigma} \ln \left(1+\frac{\eta}{\left|a_{\mu}\right|}\right) ; \\
\int_{\xi}^{\eta} & \left\{\frac{\pi \sigma}{\sin \pi \sigma} \ln ^{+} \frac{r}{\left|b_{\nu}\right|}-\ln \left(1+\frac{r}{\left|b_{\nu}\right|}\right)\right\} \frac{d r}{r^{1+\sigma}} \\
& \geq C_{3} \xi^{-\sigma} \ln \left(1+\frac{\xi}{\left|b_{\nu}\right|}\right)-C_{4} \eta^{-\sigma} \ln \left(1+\frac{\eta}{\left|b_{\nu}\right|}\right),
\end{aligned}
$$

where $0<\sigma<1$, and $\xi$ and $\eta$ are arbitrary numbers satisfying the condition $o<\xi<\eta<\infty$. We add inequalities of the form (3.15) over all $a_{\mu}$ satisfying $\left|a_{\mu}\right|<R$, and add all inequalities of the form (3.16) over all $b_{\nu}$ satisfying $\left|b_{\nu}\right|<R$. We get two inequalities. Adding them together, we get

$$
\begin{gathered}
\int_{\xi}^{\eta}\left\{\ln \left|\check{\alpha}_{R, 0}(r)\right|-\frac{\pi \sigma}{\tan \pi \sigma} N(r, 0)+\frac{\pi \sigma}{\sin \pi \sigma} N(r, \infty)\right\} \frac{d r}{r^{1+\sigma}} \\
\geq C_{5} \xi^{-\sigma} H_{R}(\xi)-C_{6} \eta^{-\sigma} H_{R}(\eta), 0<\xi<\eta<R,
\end{gathered}
$$

where $C_{5}=C_{1}+C_{3}, C_{6}=C_{2}+C_{4}, H_{R}(r)$ is determined by (3.9), and

$$
\check{\alpha}_{R, 0}(z)=\prod_{\left|a_{\mu}\right|<R}\left(1-\frac{z}{\left|a_{\mu}\right|}\right)\left\{\prod_{\left|b_{\nu}\right|<R}\left(1+\frac{z}{\left|b_{\nu}\right|}\right)\right\}^{-1} .
$$

By Lemma 3.1 with $q=0$, we have a representation

$$
f(z)=\alpha_{R, 0}(z) \omega_{R, 0}(z),
$$

where

$$
\alpha_{R, 0}(z)=\prod_{\left|a_{\mu}\right|<R}\left(1-\frac{z}{a_{\mu}}\right)\left\{\prod_{\left|b_{\nu}\right|<R}\left(1-\frac{z}{b_{\nu}}\right)\right\}^{-1},
$$

and $\ln \omega_{R, 0}(z)$ admits the estimate

$$
\left|\ln \omega_{R, 0}(z)\right| \leq K_{1} \frac{r}{R} T(2 R, f),|z|=r \leq \frac{1}{2} R
$$

( $K_{1}$ does not depend on $r$ and $R$ ). Obviously,

$$
\left.\mu\left(r, \alpha_{R, 0}\right) \geq \mid \check{\alpha}_{R, 0}\right)(r) \mid,
$$

therefore

$$
\mu(r, f) \geq \mu\left(r, \alpha_{R, 0}\right) \mu\left(r, \omega_{R, 0}\right) \geq\left|\check{\alpha}_{R, 0}(r)\right| \mu\left(r, \omega_{R, 0}\right),
$$


from where, using the estimate (3.5) we get

$\ln \left|\check{\alpha}_{R, 0}(r)\right| \leq \ln \mu(r, f)+\left|\ln \mu\left(r, \omega_{R, 0)}\right)\right| \leq \ln \mu(r, f)+K_{1} \frac{r}{R} T(2 R, f), 0 \leq r \leq \frac{1}{2} R$,

whence

$$
\int_{\xi}^{\eta} \ln \left|\check{\alpha}_{R, 0}(r)\right| \frac{d r}{r^{1+\sigma}} \leq \int_{\xi}^{\eta} \ln \mu(r, f) \frac{d r}{r^{1+\sigma}}+\frac{K_{1}}{1-\sigma} \frac{\eta^{1-\sigma}}{R} T(2 R, f),
$$

$0<\sigma<1,0<\xi<\eta \leq \frac{1}{2} R$. The next inequality follows from (3.17) and (3.20):

$$
\begin{aligned}
\int_{\xi}^{\eta}\{ & \left.\ln \mu(r, f)-\frac{\pi \sigma}{\tan \pi \sigma} N(r, 0)+\frac{\pi \sigma}{\sin \pi \sigma} N(r, \infty)\right\} \frac{d r}{r^{1+\sigma}} \\
& \geq C_{5} \xi^{-\sigma} H_{R}(\xi)-C_{6} \eta^{-\sigma} H_{R}(\eta)-\frac{K_{1}}{1-\sigma} \frac{\eta^{1-\sigma}}{R} T(2 R, f) .
\end{aligned}
$$

Let $\eta=\frac{1}{2} R$ in this inequality. Since, by Lemma 3.2 , the inequality $H_{R}(\eta) \leq$ $4 T(2 R, f)$ holds, we get the inequality

$$
\begin{gathered}
\int_{\xi}^{\frac{1}{2} R}\left\{\ln \mu(r, f)-\frac{\pi \sigma}{\tan \pi \sigma} N(r, 0)+\frac{\pi \sigma}{\sin \pi \sigma} N(r, \infty)\right\} \frac{d r}{r^{1+\sigma}} \\
\geq C_{5} \xi^{-\sigma} H_{R}(\xi)-C_{7} R^{-\sigma} T(2 R, f), 0<\xi<\frac{1}{2} R,
\end{gathered}
$$

where the constant $C_{7}, 0<C_{7}<\infty$, does not depend on $\xi$ and $R$.

Let $\xi_{0}>0$ be an arbitrarily large number. Choose $\xi>\xi_{0}$, such that $H_{R}(\xi)>0$ for $R>2 \xi$. Such choice is possible because otherwise the function $f(z)$ would be an entire function without zeros, and Lemma 6.2 from Chapter 1 would imply $f(z) \equiv f(0)=1$.

Assume that $\sigma$ in the inequality (3.21) satisfies the condition $\lambda<\sigma<1$. Then $\liminf _{R \rightarrow \infty} R^{-\sigma} T(2 R, f)=0$, therefore the number $R>2 \xi$ can be chosen in such a way that the right-hand side of (3.21) is positive. From here it follows that for some $r>\xi_{0}$ the integrand in the left-hand side of (3.21) is also positive. Since $\xi_{0}$ is arbitrarily large, we get the statement of the lemma.

Proof. Now we proof Theorem 3.2. We may assume that the function is transcendent, because for rational functions the theorem is trivial.

By Theorem 2.1 from Chapter 4 we can find a number $a \neq \infty$, such that $N(r, a, f) \sim T(r, f)$. Let

$$
f_{1}(z)=\frac{f(z)-a}{c_{k} z^{k}}
$$

where $c_{k}$ is the first non-zero coefficient of the Laurent series of the function $f(z)-a$ at $z=0$. Observe that $f_{1}(0)=1$,

$$
\begin{aligned}
& N\left(r, \infty, f_{1}\right)=N(r, \infty, f)+O(\ln r), \\
& N\left(r, 0, f_{1}\right)=N(r, a, f)+O(\ln r) \sim T(r, f), \\
& \ln ^{+} \mu\left(r, f_{1}\right) \leq \ln ^{+} \mu(r, f)+O(\ln r) .
\end{aligned}
$$

By Lemma 3.4, for each $\sigma, \lambda<\sigma<1$, and each $\varepsilon>0$ there exists a sequence $r_{n} \rightarrow \infty$, such that

$$
\ln ^{+} \mu\left(r_{n}, f_{1}\right) \geq \frac{\pi \sigma}{\sin \pi \sigma}\left\{\cos \pi \sigma N\left(r_{n}, 0, f_{1}\right\}-N\left(r_{n}, \infty, f_{1}\right)\right\}-\varepsilon
$$


Dividing both parts of this relation by $T\left(r_{n}, f\right)$ and using the relations (3.22) we get

$$
\limsup _{r \rightarrow \infty} \frac{\ln ^{+} \mu(r, f)}{T(r, f)} \geq \frac{\pi \sigma}{\sin \pi \sigma}\{\cos \pi \sigma-1+\delta(\infty, f)\} .
$$

Letting $\sigma$ tend to $\lambda$, we get the statement of the theorem. Note that we have not used the condition $\lambda<1 / 2$, however, for $1 / 2 \leq \lambda<1$ the right-hand side of (3.2) cannot be positive and the theorem becomes trivial.

The following corollary of Lemma 3.4, complementing Corollary 1 of Theorem 1.3 , is of interest.

COROLlary. An entire function $f(z)$ of lower order $\lambda \leq 1 / 2$ cannot have finite deficient values.

Obviously, it suffices to prove that $\delta(0, f)=0$. We may assume without loss of generality that $f(0)=1$. By Lemma 3.4, for each $\varepsilon>0$ and each $\sigma, 1 / 2<\sigma<1$, there exists a sequence $r_{k} \rightarrow \infty$, such that

$$
\ln \mu\left(r_{k}, f\right) \geq \pi \sigma \cot \pi \sigma N\left(r_{k}, 0\right)-\varepsilon
$$

whence

$$
\ln \frac{1}{\mu\left(r_{k}, f\right)} \leq-\pi \sigma \cot \pi \sigma N\left(r_{k}, 0\right)+\varepsilon .
$$

Since the right-hand side of this inequality is positive $(1 / 2<\sigma<1)$, we can replace $\ln$ by $\ln ^{+}$in the left-hand side. Taking this into account, we get

$$
\begin{aligned}
\delta(0, f) & =\liminf _{r \rightarrow \infty} \frac{m(r, 0)}{T(r, f)} \leq \liminf _{r \rightarrow \infty} \frac{\ln ^{+} \frac{1}{\mu(r, f)}}{T(r, f)} \\
& \leq \liminf _{k \rightarrow \infty} \frac{\ln ^{+} \frac{1}{\mu\left(r_{k}, f\right)}}{T\left(r_{k}, f\right)} \leq-\pi \sigma \cot \pi \sigma \limsup _{k \rightarrow \infty} \frac{N\left(r_{k}, 0\right)}{T\left(r_{k}, f\right)} \\
& \leq \pi \sigma \cot \pi \sigma(1-\delta(0, f)),
\end{aligned}
$$

whence

$$
\delta(0, f) \leq \frac{-\pi \sigma \cot \pi \sigma}{1-\pi \sigma \cot \pi \sigma}
$$

Letting $\sigma$ tend to $1 / 2$, we get $\delta(0, f)=0$.

The method used in the proof of Theorem 3.2 can be used to get many different relations connecting the asymptotic behavior of a meromorphic function with the distribution of its zeros and poles. We restrict our attention by the following result.

THEOREM 3.3. Let $f(z)$ be a meromorphic function of lower order $\lambda<1 / 2$ such that

$$
\lim _{r \rightarrow \infty} \frac{\ln M(r, f)}{\ln r}=+\infty .
$$

If $0<\lambda<1 / 2$, the inequality

$$
\limsup _{r \rightarrow \infty} \frac{\ln \mu(r, f)}{\ln M(r, f)}+\pi \lambda \sin \pi \lambda \limsup _{r \rightarrow \infty} \frac{N(r, f)}{\ln M(r, f)} \geq \cos \pi \lambda
$$

holds. This inequality remains true for $\lambda=0$ provided

$$
\limsup _{r \rightarrow \infty} \frac{N(r, f)}{\ln M(r, f)}<\infty .
$$


To prove Theorem 3.3 we need the following lemma, analogous to Lemma 3.3.

Lemma 3.5. For all $a$ and $b, 0<a<b<\infty$, and $\sigma, 0<\sigma<\frac{1}{2}$, the inequalities

$$
\begin{gathered}
\int_{a}^{b}\{\ln |1-x|-\cos \pi \sigma \ln (1+x)\} x^{-1-\sigma} d x \\
\geq C_{8} a^{-\sigma} \ln (1+a)-C_{9} b^{-\sigma} \ln (1+b), \\
\int_{a}^{b}\left\{\cos \pi \sigma \ln |1-x|-\ln (1+x)+\pi \sigma \sin \pi \sigma \ln ^{+} x\right\} x^{-1-\sigma} d x \\
\geq C_{10} a^{-\sigma} \ln (1+a)-C_{11} b^{-\sigma} \ln (1+b)
\end{gathered}
$$

hold, where $C_{k}, 0<C_{k}<\infty, k=8,9,10,11$, are constants which do not depend on $a$ and $b$.

This lemma is a corollary of Lemma 3.3. In fact, if we multiply (3.12) by $\cos \pi \sigma$ and add the result to (3.11), we get (3.24). If we multiply (3.11) by $\cos \pi \sigma$ and add the result to (3.12), we get (3.25) (we take into account that $0<\sigma<1 / 2$ and hence $\cos \pi \sigma>0)$.

Next we prove a lemma analogous to Lemma 3.4.

LEMMA 3.6. Let $f(z), f(0)=1$, be a meromorphic function of lower order $\lambda<1 / 2$. Then for each $\sigma, \lambda<\sigma<1 / 2$, the inequality

$$
\limsup _{r \rightarrow \infty}\{\ln \mu(r, f)+\pi \sigma \sin \pi \sigma N(r, \infty)-\cos \pi \sigma \ln M(r, f)\} \geq 0
$$

holds.

Proof. Using the change of variable in (3.24) and (3.25) we get the inequalities

$$
\begin{gathered}
\int_{\xi}^{\eta}\left\{\ln \left|1-\frac{r}{\left|a_{\mu}\right|}\right|-\cos \pi \sigma \ln \left(1+\frac{r}{\left|a_{\mu}\right|}\right)\right\} r^{-1-\sigma} d r \\
\geq C_{8} \xi^{-\sigma} \ln \left(1+\frac{\xi}{\left|a_{\mu}\right|}\right)-C_{9} \eta^{-\sigma} \ln \left(1+\frac{\eta}{\left|a_{\mu}\right|}\right) \\
\int_{\xi}^{\eta}\left\{\cos \pi \sigma \ln \left|1-\frac{r}{\left|b_{\nu}\right|}\right|-\ln \left(1+\frac{r}{\left|b_{\nu}\right|}\right)+\pi \sigma \sin \pi \sigma \ln ^{+} \frac{r}{\left|b_{\nu}\right|}\right\} \frac{d r}{r^{1+\sigma}} \\
\geq C_{10} \xi^{-\sigma} \ln \left(1+\frac{\xi}{\left|b_{\nu}\right|}\right)-C_{11} \eta^{-\sigma} \ln \left(1+\frac{\eta}{\left|b_{\nu}\right|}\right),
\end{gathered}
$$

where $\xi$ and $\eta$ are arbitrary numbers satisfying the condition $0<\xi<\eta<\infty, a_{\mu}$ are zeros of the function $f(z), b_{\nu}$ are its poles. Using these inequalities and the argument similar to the used in Lemma 3.4, we get

$$
\begin{aligned}
& \int_{\xi}^{\eta}\left\{\ln \left|\check{\alpha}_{R, 0}(r)\right|-\cos \pi \sigma \ln \left|\check{\alpha}_{R, 0}(-r)\right|\right. \\
& \quad+\pi \sigma \sin \pi \sigma N(r, \infty)\} \frac{d r}{r^{1+\sigma}} \geq C_{12} \xi^{-\sigma} H_{R}(\xi)-C_{13} \eta^{-\sigma} H_{R}(\eta),
\end{aligned}
$$

where $0<\xi<\eta<R, C_{12}=C_{8}+C_{11}, C_{13}=C_{9}+C_{11}$, and $\check{\alpha}_{R, 0}(z)$ is defined by the formula (3.18).

As in the proof of Lemma 3.4 we represent the function $f(z)$ in the form (3.19). Obviously

$$
M\left(r, \alpha_{R, 0}\right) \leq\left|\check{\alpha}_{R, 0}(-r)\right|
$$


therefore

$$
M(r, f) \leq M\left(r, \alpha_{R, 0}\right) M\left(r, \omega_{R, 0}\right) \leq\left|\check{\alpha}_{R, 0}(-r)\right| M\left(r, \omega_{R, 0}\right),
$$

from where, using the estimate (3.5), we get

$$
\begin{aligned}
& -\ln \left|\check{\alpha}_{R, 0}(-r)\right| \leq-\ln M(r, f)+\ln M\left(r, \omega_{R, 0}\right) \\
& \leq-\ln M(r, f)+K_{1} \frac{r}{R} T(2 R, f), 0 \leq r \leq \frac{1}{2} R,
\end{aligned}
$$

whence

$$
\begin{gathered}
\int_{\xi}^{\eta}\left(-\ln \left|\check{\alpha}_{R, 0}(-r)\right|\right) \frac{d r}{r^{1+\sigma}} \leq \int_{\xi}^{\eta}(-\ln M(r, f)) \frac{d r}{r^{1+\sigma}}+\frac{K_{1}}{1-\sigma} \frac{\eta^{1-\sigma}}{R} T(2 R, f) \\
0<\sigma<\frac{1}{2}, 0<\xi<\eta \leq \frac{1}{2} R
\end{gathered}
$$

Using $\left(3.17^{\prime}\right),(3.20)$ and (3.28) we get the inequality

$$
\begin{aligned}
& \int_{\xi}^{\eta}\{\ln \mu(r, f)-\cos \pi \sigma \ln M(r, f)+\pi \sigma \sin \pi \sigma N(r, \infty)\} \frac{d r}{r^{1+\sigma}} \\
& \leq C_{12} \xi^{-\sigma} H_{R}(\xi)-C_{13} \eta^{-\sigma} H_{R}(\eta)-\frac{2 K_{1}}{1-\sigma} \frac{\eta^{1-\sigma}}{R} T(2 R, f) .
\end{aligned}
$$

Repeating the argument used in the proof of Lemma 3.3 to derive (3.14) from (3.21), we get the relation $(3.26)$.

Proof of Theorem 3.3. We apply Lemma 3.6 to the function $f_{1}(z)=\frac{f(z)}{c_{k} z^{k}}$, where $c_{k}$ is the first non-zero Laurent coefficient of $f(z)$ at $z=0$. We get that for each $\sigma, \lambda<\sigma<1 / 2$, and each $\varepsilon>0$ there exists a sequence $r_{n} \rightarrow \infty$ such that

$$
\ln \mu\left(r_{n}, f_{1}\right)+\pi \sigma \sin \pi \sigma N\left(r_{n}, \infty, f_{1}\right) \geq \cos \pi \sigma \ln M\left(r_{n}, f_{1}\right)-\varepsilon .
$$

Taking into account

$$
N\left(r, \infty, f_{1}\right)=N(r, \infty, f)+O(\ln r),
$$

$$
\ln M\left(r, f_{1}\right)=\ln M(r, f)+O(\ln r), \ln \mu\left(r, f_{1}\right)=\ln \mu(r, f)+O(\ln r),
$$

and dividing both parts of (3.29) by $\ln M\left(r_{n}, f\right)$, we get

$$
\limsup _{r \rightarrow \infty} \frac{\ln \mu(r, f)}{\ln M(r, f)}+\pi \sigma \sin \pi \sigma \limsup _{r \rightarrow \infty} \frac{N(r, \infty, f)}{\ln M(r, f)} \geq \cos \pi \sigma .
$$

Letting $\sigma$ tend to $\lambda$, we get the relation (3.23).

Exercise. Prove that

$$
\limsup _{r \rightarrow \infty} \frac{T(r, f)}{\ln M(r, f)} \geq \frac{\sin \pi \lambda}{\pi \lambda}
$$

for each entire function $f(z)$ of lower order $0 \leq \lambda<1$, with the help of the method used in the proofs of Theorems 3.2 and 3.3.

HinT. It suffices to prove that

$$
\limsup _{r \rightarrow \infty} \frac{N(r, 0)}{\ln M(r, f)} \geq \frac{\sin \pi \lambda}{\pi \lambda}
$$


We may assume without loss of generality that $f(0)=1$. Let $a_{\mu}$ be zeros of the function $f(z)$. Using the inequality (3.12) we get

$$
\begin{aligned}
\int_{\xi}^{\eta}\left\{\frac{\pi \sigma}{\sin \pi \sigma} \ln ^{+} \frac{r}{\left|a_{\mu}\right|}-\ln \left(1+\frac{r}{\left|a_{\mu}\right|}\right)\right\} \frac{d r}{r^{1+\sigma}} \\
\quad \geq C_{3} \xi^{-\sigma} \ln \left(1+\frac{\xi}{\left|a_{\mu}\right|}\right)-C_{4} \eta^{-\sigma} \ln \left(1+\frac{\eta}{\left|a_{\mu}\right|}\right), 0<\sigma<1,0<\xi<\eta<\infty .
\end{aligned}
$$

Adding over all $a_{\mu},\left|a_{\mu}\right|<R$, we have

$$
\begin{aligned}
\int_{\xi}^{\eta}\{ & \left.\frac{\pi \sigma}{\sin \pi \sigma} N(r, 0)-\ln \left|\check{\alpha}_{R, 0}(-r)\right|\right\} \frac{d r}{r^{1+\sigma}} \\
& \geq C_{3} \xi^{-\sigma} H_{R}(\xi)-C_{4} \eta^{-\sigma} H_{R}(\eta), 0<\sigma<1,0<\xi<\eta<R,
\end{aligned}
$$

where $\check{\alpha}_{R, 0}(z)$ are defined by the relation (3.18) (poles $b_{\nu}$ in this case are absent). Next, using the representation (3.19) and the estimate (3.28) we arrive at the inequality $\left(0<\sigma<1,0<\xi<\eta \leq \frac{1}{2} R\right)$

$$
\begin{aligned}
\int_{\xi}^{\eta}\{ & \left.\frac{\pi \sigma}{\sin \pi \sigma} N(r, 0)-\ln M(r, f)\right\} \frac{d r}{r^{1+\sigma}} \\
& \geq C_{3} \xi^{-\sigma} H_{R}(\xi)-C_{4} \eta^{-\sigma} H_{R}(\eta)-\frac{K_{1}}{1-\sigma} \frac{\eta^{1-\sigma}}{R} T(2 R, f),
\end{aligned}
$$

from where we get (3.31) using the same argument as at the end of the proof of Lemma 3.3.

The relation between $\ln \mu(r, f)$ and $\ln M(r, f)$ is of interest for entire functions.

TheOREm 3.4. Let $f(z)$ be an entire function of lower order $\lambda<1$. Then

$$
\limsup _{r \rightarrow \infty} \frac{\ln \mu(r, f)}{\ln M(r, f)} \geq \cos \pi \lambda .
$$

Proof. In the case $0 \leq \lambda<1 / 2$ this theorem is an immediate corollary of Theorem 3.3. Consider the case $1 / 2 \leq \lambda<1$.

Observe that the inequality (3.24) takes place also for $0<\sigma<1$. To show this, it is enough to set

$$
\varphi(a)=\int_{a}^{\infty}\{\ln |1-x|-\cos \pi \sigma \ln (1+x)\} \frac{d x}{x^{1+\sigma}}
$$

and, using (3.13) (b) and (c), to argue in the same way as in the proof of (3.11) in Lemma 3.3. Therefore (3.24) holds for $0<\sigma<1$ also. From here, adding over $a_{\mu}$, $\left|a_{\mu}\right|<R$, we get

$$
\begin{aligned}
& \int_{\xi}^{\eta}\left\{\ln \left|\check{\alpha}_{R, 0}(r)\right|-\cos \pi \sigma \ln \left|\check{\alpha}_{R, 0}(-r)\right|\right\} \frac{d r}{r^{1+\sigma}} \\
& \quad \geq C_{8} \xi^{-\sigma} H_{R}(\xi)-C_{9} \eta^{-\sigma} H_{R}(\eta), \lambda<\sigma<1,0<\xi<\eta \leq \frac{1}{2} R,
\end{aligned}
$$

where $\check{\alpha}_{R, 0}(z)$ is defined by the inequality (3.18) (poles $b_{\mu}$ in this case are absent). Next we use the representation of $f(z)$ in the form (3.19).

Since $\cos \pi \sigma<0$, we cannot, using (3.20) and (3.28), get from (3.33) and inequality with the left-hand side equal to

$$
\int_{\xi}^{\eta}\{\ln \mu(r, f)-\cos \pi \sigma \ln M(r, f)\} \frac{d r}{r^{1+\sigma}} .
$$


To prove the theorem we argue in the following way.

By (3.5) we have $\left(0<\xi<\eta \leq \frac{1}{2} R\right)$

$$
\int_{\xi}^{\eta} \frac{\ln \mu\left(r, \omega_{R, 0}\right)}{r^{1+\sigma}} d r \geq-\frac{K_{1}}{1-\sigma} \frac{\eta^{-\sigma}}{R} T(2 R, f) .
$$

Multiplying this inequality by $1-\cos \pi \sigma$, and adding the resulting inequality to $(3.33)$, we get

$$
\begin{gathered}
\int_{\xi}^{\eta}\left\{\ln \left|\check{\alpha}_{R, 0}(r) \mu\left(r, \omega_{R, 0}\right)\right|-\cos \pi \sigma \ln \left|\check{\alpha}_{R, 0}(-r) \mu\left(r, \omega_{R, 0}\right)\right|\right\} \frac{d r}{r^{1+\sigma}} \\
\geq C_{8} \xi^{-\sigma} H_{R}(\xi)-C_{9} \eta^{-\sigma} H_{R}(\eta)-\frac{2 K_{1}}{1-\sigma} \frac{\eta^{-\sigma}}{R} T(2 R, f), \\
0<\sigma<1,0<\xi<\eta \leq \frac{1}{2} R .
\end{gathered}
$$

From here, arguing in the same way as at the end of the proof of Lemma 3.4, we conclude that for each fixed $\sigma, \lambda<\sigma<1$, there exist sequences $\left\{R_{k}\right\}_{k=1}^{\infty}$ and $\left\{r_{k}\right\}_{k=1}^{\infty}, \lim _{k \rightarrow \infty} R_{k}=\lim _{k \rightarrow \infty} r_{k}=\infty, r_{k} \leq \frac{1}{2} R_{k}$, such that for $R=R_{k}, r=r_{k}$, the inequality

$$
\ln \left|\check{\alpha}_{R, 0}(r) \mu\left(r, \omega_{R, 0}\right)\right|-\cos \pi \sigma \ln \left|\check{\alpha}_{R, 0}(-r) \mu\left(r, \omega_{R, 0}\right)\right| \geq 0
$$

holds. Adding $\ln \left|\check{\alpha}_{R, 0}(-r) \mu\left(r, \omega_{R, 0}\right)\right|$ to both sides and exponentiating, we get

$$
\left|\check{\alpha}_{R, 0}(r) \check{\alpha}_{R, 0}(-r)\right| \mu^{2}\left(r, \omega_{R, 0}\right) \geq\left|\check{\alpha}_{R, 0}(-r) \mu\left(r, \omega_{R, 0}\right)\right|^{1+\cos \pi \sigma} .
$$

If $z_{0}$ is a point on the circle $\{|z|=r\}$ for which $\left|\alpha_{R, 0}(z)\right|=\mu\left(r, \alpha_{R, 0}\right)$, then we have

$$
\begin{aligned}
M\left(r, \alpha_{R, 0}\right) \mu\left(r, \alpha_{R, 0}\right) & \geq\left|\alpha_{R, 0}(-z) \alpha_{R, 0}\left(z_{0}\right)\right| \\
& =\prod_{\left|a_{\mu}\right|<R}\left|1-\frac{z_{0}^{2}}{a_{\mu}^{2}}\right| \geq \prod_{\left|a_{\mu}\right|<R}\left|1-\frac{r^{2}}{\left|a_{\mu}\right|^{2}}\right|=\left|\check{\alpha}_{R, 0}(r) \check{\alpha}_{R, 0}(-r)\right| .
\end{aligned}
$$

Therefore, by (3.34) and (3.27) we get

$$
M\left(r, \alpha_{R, 0}\right) \mu\left(r, \alpha_{R, 0}\right) \mu^{2}\left(r, \omega_{R, 0}\right) \geq\left\{M\left(r, \alpha_{R, 0}\right) \mu\left(r, \omega_{R, 0}\right)\right\}^{1+\cos \pi \sigma,}
$$

whence

$$
\mu\left(r, \alpha_{R, 0}\right) \mu\left(r, \omega_{R, 0}\right) \geq\left\{M\left(r, \alpha_{R, 0}\right) \mu\left(r, \omega_{R, 0}\right)\right\}^{\cos \pi \sigma} .
$$

Observing that

$$
M(r, f) \geq M\left(r, \alpha_{R, 0}\right) \mu\left(r, \omega_{R, 0}\right),
$$

and using $\left(3.19^{\prime}\right)$, we get

$$
\mu(r, f) \geq\{M(r, f)\}^{\cos \pi \sigma}, r=r_{k},
$$

and thus

$$
\limsup _{r \rightarrow \infty} \frac{\ln \mu(r, f)}{\ln M(r, f)} \geq \cos \pi \sigma .
$$

Letting $\sigma$ tend to $\lambda$ we conclude the proof of the theorem. 
Theorems 3.2 and 3.4 imply the following result

Corollary 1. Each entire function $f(z)$ of lower order 0 satisfies:

$$
\limsup _{r \rightarrow \infty} \frac{\ln \mu(r, f)}{T(r, f)}=\limsup _{r \rightarrow \infty} \frac{\ln \mu(r, f)}{\ln M(r, f)}=\limsup _{r \rightarrow \infty} \frac{T(r, f)}{\ln M(r, f)}=1 .
$$

In fact, Theorems 3.2 and 3.4 imply the inequalities

$$
\limsup _{r \rightarrow \infty} \frac{\ln \mu(r, f)}{T(r, f)} \geq 1, \limsup _{r \rightarrow \infty} \frac{\ln \mu(r, f)}{\ln M(r, f)} \geq 1 .
$$

Since $\ln \mu(r, f) \leq m(r, f)=T(r, f) \leq \ln ^{+} M(r, f)$, then the inequalities in the opposite direction are trivial. If the inequality

$$
\limsup _{r \rightarrow \infty} \frac{T(r, f)}{\ln M(r, f)}<1
$$

were true ${ }^{7}$, we would have

$$
\limsup _{r \rightarrow \infty} \frac{\ln \mu(r, f)}{T(r, f)}>\limsup _{r \rightarrow \infty} \frac{\ln \mu(r, f)}{\ln M(r, f)} .
$$

Theorem 3.4 implies also

Corollary 2. Each entire function $f(z)$ of lower order $\lambda \leq 1$ satisfies

$$
\limsup _{r \rightarrow \infty} \frac{\ln \mu(r, f)}{\ln M(r, f)} \geq-1 \text {. }
$$

In fact, let $F(z)=f(z) f(-z)$. Then $\lambda[F(\sqrt{z})]=\lambda_{1} \leq 1 / 2$. The inequality (3.32) implies, that

$$
\limsup _{r \rightarrow \infty} \frac{\ln \mu(r, F(z))}{\ln M(r, F(z))}=\limsup _{r \rightarrow \infty} \frac{\ln \mu(r, F(\sqrt{z}))}{\ln M(r, F(\sqrt{z}))} \geq \cos \pi \lambda_{1} \geq 0 .
$$

Hence for each $\varepsilon>0$ there exists a sequence $r_{k} \rightarrow \infty$ such that

$$
\ln \mu\left(r_{k}, F(z)\right) \geq-\varepsilon \ln M\left(r_{k}, F(z)\right) .
$$

But it is clear that $\mu(r, F(z)) \leq \mu(r, f(z)) M(r, f(z)), M(r, F(z)) \leq\{M(r, f(z))\}^{2}$. Therefore

$$
\ln \mu\left(r_{k}, f\right) \geq-(1+2 \varepsilon) \ln M\left(r_{k}, f\right),
$$

from where the statement of Corollary 2 follows. The exactness of the obtained estimate is shown by the example $f(z)=e^{z}$.

Now we consider examples showing the exactness of Theorems $3.1-3.4$.

To verify the exactness of Theorems 3.2 and 3.3 it suffices to show that for each $\lambda, 0 \leq \lambda<1 / 2$, there exists a meromorphic function $f(z)$ for which (3.2) and (3.23) are equalities. We can ask the following more general question. Let the numbers $0 \leq \lambda<1 / 2, \lambda \leq \rho \leq \infty, 1-\cos \pi \lambda \leq \delta_{0} \leq 1$ be given. Does there exist a meromorphic function $f(z)$ of order $\rho$, lower order $\lambda$, with $\delta(\infty, f)=\delta_{0}$, and such that (3.2) and (3.23) are equalities for $f(z)$ ? An answer to this question in the full generality is unknown. Now we shall get the affirmative answer under the additional conditions $\lambda>0$ and $\rho<1$.

\footnotetext{
${ }^{7}$ Note that for entire functions of lower order 0 the inequality $\limsup _{r \rightarrow \infty} T(r, f) / \ln M(r, f) \geq 1$ follows also from (3.30).
} 
EXAMPLE 1. Let $0<\lambda \leq \rho<1, f(z, u)$ be a canonical product of genus 0 with positive zeros, such that $n(r, 0) \sim u r^{l(r)}$, where $0<u \leq 1, l(r)$ is a differentiable on $\left[r_{0}, \infty\right)$ function, $\lim _{r \rightarrow \infty} r l^{\prime}(r)=0$,

$$
\limsup _{r \rightarrow \infty} l(r)=\rho, \liminf _{r \rightarrow \infty} l(r)=\lambda .
$$

By the asymptotic formula $\left(5.2_{2}\right)$ from Chapter 2 , for each $\eta>0$ the relation

$$
\ln \left|f\left(r e^{i \varphi}, u\right)\right|=\pi u \frac{\cos l(r)(\varphi-\pi)}{\sin \pi l(r)} r^{l(r)}+o\left(r^{l(r)}\right)
$$

holds uniformly in $\varphi, \eta \leq \varphi \leq 2 \pi-\eta$. Defining $f(z, 0) \equiv 1$, consider the function

$$
f_{u v}(z)=\frac{f(z, u)}{f(-z, v)}, u \geq v \geq 0, u>0 .
$$

By (3.36), for each $\eta>0$ the relation

$$
\ln \left|f_{u v}\left(r e^{i \varphi}\right)\right|=\frac{\pi}{\sin \pi l(r)}\{u \cos l(r)(\varphi-\pi)-v \cos l(r) \varphi\} r^{l(r)}+o\left(r^{l(r)}\right)
$$

holds uniformly in $\varphi, \eta \leq \varphi \leq \pi-\eta$. Since $f_{u v}(z)=\overline{f_{u v}(\bar{z})}$, we have

$$
m\left(r, f_{u v}\right)=\frac{1}{\pi} \int_{0}^{\pi} \ln ^{+}\left|f_{u v}\left(r e^{i \varphi}\right)\right| d \varphi
$$

and using Theorem 7.4 from Chapter 1, we get

$$
m\left(r, f_{u v}\right)=\frac{1}{l(r)} I(u, v, l(r)) r^{l(r)}+o\left(r^{l(r)}\right),
$$

where

$$
I(u, v, \gamma)=\frac{\gamma}{\sin \pi \gamma} \int_{0}^{\pi}\{u \cos \gamma(\varphi-\pi)-v \cos \gamma \varphi\}^{+} d \varphi, 0<\gamma<1 .
$$

By formula (5.6) from Chapter 2 we have

$$
N\left(r, f_{u v}\right)=N(r, 0, f(z, v))=\frac{v}{l(r)} r^{l(r)}+o\left(r^{l(r)}\right),
$$

and hence

$$
\begin{gathered}
T\left(r, f_{u v}\right)=\frac{1}{l(r)}\{I(u, v, l(r))+v\} r^{l(r)}+o\left({ }^{l(r)}\right), \\
\delta\left(\infty, f_{u v}\right)=\liminf _{r \rightarrow \infty} \frac{I(u, v, l(r))}{I(u, v, l(r))+v} .
\end{gathered}
$$

By virtue of (3.35) and (3.39) the function $f_{u v}(z)$ has order $\rho$ and lower order $\lambda$.

Straightforward computation gives:

$I(u, v, \gamma)=u-v$, if $u \cos \gamma \pi \geq v$,

$$
I(u, v, \gamma)=\left\{u^{2}+\left(\frac{v-u \cos \gamma \pi}{\sin \gamma \pi}\right)^{2}\right\}^{1 / 2}-v, \text { if } u \geq v \geq u \cos \gamma \pi .
$$

It is not difficult to determine that $I(u, v, \gamma)$ is a non-decreasing function of $\gamma$. Therefore

$$
\delta\left(\infty, f_{u v}\right)=\frac{I(u, v, \lambda)}{I(u, v, \lambda)+v}
$$


Imposing the conditions $0<\lambda<1 / 2$ and $u \cos \lambda \pi \geq v$ on the parameters $\lambda, u$, and $v$, we get

$$
\delta\left(\infty, f_{u v}\right)=\frac{u-v}{u} .
$$

It is easy to see that selecting $v, 0 \leq v \leq u \cos \lambda \pi$, in a suitable way, we can give $\delta\left(\infty, f_{u v}\right)$ an arbitrary preassigned value $\delta_{0}, 1-\cos \lambda \pi \leq \delta_{0} \leq 1$.

Now we estimate from above

$$
\limsup _{r \rightarrow \infty} \frac{\ln \mu\left(r, f_{u v}\right)}{T\left(r, f_{u v}\right)} .
$$

Obviously,

$$
\ln \mu\left(r, f_{u v}\right)=\ln \left|f_{u v}(r)\right|=\ln |f(r, u)|-\ln |f(-r, v)| .
$$

The formula (3.36) does not allow to find an asymptotic formula for $\ln |f(r, u)|$. However, since $\left|f\left(r e^{i \eta}, u\right)\right| \geq|f(r, u)|$ for each $\eta>0$, we have the following estimate from above:

$$
\begin{aligned}
\ln \mu\left(r, f_{u v}\right) & \leq \ln \left|f\left(r e^{i \eta}, u\right)\right|-\ln |f(-r, v)| \\
& =\frac{\pi}{\sin \pi l(r)}\{u \cos l(r)(\pi-\eta)-v\} r^{l(r)}+o\left(r^{l(r)}\right) .
\end{aligned}
$$

Using (3.39) we get

$$
\limsup _{r \rightarrow \infty} \frac{\ln \mu\left(r, f_{u v}\right)}{T\left(r, f_{u v}\right)} \leq \limsup _{r \rightarrow \infty} \frac{\vartheta(\mu, v, l(r), \eta)}{I(u, v, l(r))+v}
$$

where

$$
\vartheta(u, v, \gamma, \eta)=\frac{\pi \gamma}{\sin \pi \gamma}(u \cos \gamma(\pi-\eta)-v), 0<\gamma<1 .
$$

It is easy to see that

$$
\frac{1}{\pi} \frac{\partial}{\partial \gamma} \vartheta(u, v, \gamma, 0)=u \frac{\sin 2 \pi \gamma-2 \pi \gamma}{2 \sin ^{2} \pi \gamma}+v \frac{\pi \gamma \cos \pi \gamma-\sin \pi \gamma}{\sin ^{2} \pi \gamma} .
$$

Since for $0<\gamma<1$ the inequalities $2 \pi \gamma>\sin 2 \pi \gamma$ and $\sin \pi \gamma>\pi \gamma \cos \pi \gamma$ hold, then

$$
\frac{\partial}{\partial \gamma} \vartheta(u, v, \gamma, 0)<0,0<\gamma<1
$$

therefore the number $\eta>0$ can be chosen to be so small that the conditions

$$
\frac{\partial}{\partial \gamma} \vartheta(u, v, \gamma, \eta)<0, \frac{\lambda}{2} \leq \gamma<1
$$

are satisfied. Then the function $\vartheta(u, v, \gamma, \eta)$ is non-increasing for $\frac{\lambda}{2}<\gamma<1$. Taking into account that $I(u, v, \gamma)$ is a non-decreasing function of $\gamma$, we get

$$
\limsup _{r \rightarrow \infty} \frac{\ln \mu\left(r, f_{u v}\right)}{T\left(r, f_{u v}\right)} \leq \frac{\vartheta(u, v, \lambda, \eta)}{I(u, v, \lambda)+v}
$$

Since this inequality holds for each sufficiently small $\eta>0$, we have

$$
\limsup _{r \rightarrow \infty} \frac{\ln \mu\left(r, f_{u v}\right)}{T\left(r, f_{u v}\right)} \leq \frac{\vartheta(u, v, \lambda, 0)}{I(u, v, \lambda)+v}=\frac{\pi \lambda}{\sin \pi \lambda} \frac{u \cos \pi \lambda-v}{u} .
$$

Together with $\left(3.40^{\prime}\right)$ this gives

$$
\limsup _{r \rightarrow \infty} \frac{\ln \mu\left(r, f_{u v}\right)}{T\left(r, f_{u v}\right)} \leq \frac{\pi \lambda}{\sin \pi \lambda}\left\{\cos \pi \lambda-1+\delta\left(\infty, f_{u v}\right)\right\}
$$


moreover, since the right-hand side of this inequality is non-negative, we may replace $\ln \mu\left(r, f_{u v}\right)$ by $\ln ^{+} \mu\left(r, f_{u v}\right)$ in the left-hand side.

Comparing with (3.2) we see that for the function $f_{u v}(z)$ it becomes an equality.

Let us show that for the function $f_{u v}(z)$ the inequality (3.23) also becomes an equality.

Since for each $\eta>0$ the inequality $\left|f\left(r e^{i \eta}, v\right)\right| \geq|f(r, v)|$ holds, we have

$$
\begin{aligned}
\ln M\left(r, f_{u v}\right) & =\ln |f(-r, u)|-\ln |f(r, v)| \geq \ln |f(-r, u)|-\ln \left|f\left(r e^{i \eta}, v\right)\right| \\
& =\frac{\pi}{\sin \pi l(r)}\{u-v \cos l(r)(\pi-\eta)\} r^{l(r)}+o\left(r^{l(r)}\right),
\end{aligned}
$$

from where, by (3.41) and (3.38) we get

$$
\begin{aligned}
& \limsup _{r \rightarrow \infty} \frac{\ln \mu\left(r, f_{u v}\right)}{\ln M\left(r, f_{u v}\right)} \leq \limsup _{r \rightarrow \infty} \vartheta_{1}(u, v, l(r), \eta), \\
& \limsup _{r \rightarrow \infty} \frac{N\left(r, f_{u v}\right)}{\ln M\left(r, f_{u v}\right)} \leq \limsup _{r \rightarrow \infty} \vartheta_{2}(u, v, l(r), \eta),
\end{aligned}
$$

where

$$
\begin{gathered}
\vartheta_{1}(u, v, \gamma, \eta)=\frac{u \cos \gamma(\pi-\eta)-v}{u-v \cos \gamma(\pi-\eta)}, \\
\vartheta_{2}(u, v, \gamma, \eta)=\frac{\sin \pi \gamma}{\pi \gamma} \frac{v}{u-v \cos \gamma(\pi-\eta)} .
\end{gathered}
$$

Since $\vartheta_{j}(u, v, \gamma, \eta), j=1,2$, are non-increasing functions of $\gamma$ for sufficiently small $\eta$, we may replace $l(r)$ by $\lambda$ in the right-hand sides of (3.42) and (3.43). Letting, after that, $\eta$ tend to zero, we get

$$
\begin{gathered}
\limsup _{r \rightarrow \infty} \frac{\ln \mu\left(r, f_{u v}\right)}{\ln M\left(r, f_{u v}\right)} \leq \frac{u \cos \lambda \pi-v}{u-v \cos \lambda \pi}, \\
\limsup _{r \rightarrow \infty} \frac{N\left(r, f_{u v}\right)}{\ln M\left(r, f_{u v}\right)} \leq \frac{\sin \pi \lambda}{\pi \lambda} \frac{v}{u-v \cos \lambda \pi} .
\end{gathered}
$$

Thus

$$
\begin{aligned}
\limsup _{r \rightarrow \infty} \frac{\ln \mu\left(r, f_{u v}\right)}{\ln M\left(r, f_{u v}\right)} & +\pi \lambda \sin \pi \lambda \limsup _{r \rightarrow \infty} \frac{N\left(r, f_{u v}\right)}{\ln M\left(r, f_{u v}\right)} \\
& \leq \frac{u \cos \lambda \pi-v}{u-v \cos \lambda \pi}+\sin ^{2} \pi \lambda \frac{v}{u-v \cos \lambda \pi}=\cos \lambda \pi .
\end{aligned}
$$

Hence, if $0<\lambda<1 / 2$, then the sides of (3.23) are equal for function $f_{u v}(z)$.

Observe, that in our proof of the inequality (3.44) we did not use the conditions $\lambda<1 / 2, u \cos \pi \lambda \geq v$, hence, the inequality (3.44) holds for $0<\lambda<1$ and $u \geq v$. For $v=0$ we get

$$
\limsup _{r \rightarrow \infty} \frac{\ln \mu\left(r, f_{u 0}\right)}{\ln M\left(r, f_{u 0}\right)} \leq \cos \lambda \pi .
$$

But the function $f_{u 0}(z) \equiv f(z, u)$ is entire and, thus, for each $\lambda, 0<\lambda<1$ and each $\rho, \lambda \leq \rho<1$, we have an example of an entire function of order $\rho$, lower order $\lambda$, for which (3.32) is an equality. 
ExERCISE. Prove that the function $f_{u 0}(z)$ satisfies:

$$
\limsup _{r \rightarrow \infty} \frac{T\left(r, f_{u 0}\right)}{\ln M\left(r, f_{u 0}\right)}= \begin{cases}\frac{\sin \pi \lambda}{\pi \lambda}, & 0<\lambda<\frac{1}{2}, \\ \frac{1}{\pi \lambda}, & \frac{1}{2} \leq \lambda<1 .\end{cases}
$$

Thus, for each $\lambda$ and $\rho, 0<\lambda \leq 1 / 2, \lambda \leq \rho<1$, there exists an entire function of order $\rho$ and lower order $\lambda$, for which (3.30) is an equality. ${ }^{8}$

EXAMPLE 2. We show that for each $\delta_{0}, 0 \leq \delta_{0} \leq 1$, there exists a meromorphic function $f(z)$ with $\lambda=\rho=0$ such that $\delta(\infty, f)=\delta_{0}$ and

$$
\begin{gathered}
\limsup _{r \rightarrow \infty} \frac{\ln \mu(r, f)}{T(r, f)}=\delta(\infty, f), \\
\limsup _{r \rightarrow \infty} \frac{\ln \mu(r, f)}{\ln M(r, f)}=1, \limsup _{r \rightarrow \infty} \frac{N(r, f)}{\ln M(r, f)}<\infty,
\end{gathered}
$$

that is, there is an equality in (3.2) and (3.23).

Let $\rho(r) \rightarrow 0$ be a proximate order, such that $r^{\rho(r) \uparrow} \infty$ as $r \uparrow \infty$. Denote by $g(z, u)$ the canonical product of genus 0 with positive zeros, such that $n(r, 0) \sim$ $u r^{\rho(r)}$. By the asymptotic formula $\left(5.28_{2}\right)$ from Chapter 2 , for each $\eta>0$ the relation

$$
\ln \left|g\left(r e^{i \varphi}, u\right)\right|=(1+o(1)) N(r, 0, g(z, u))=(u+o(1)) \int_{1}^{r} t^{\rho(t)-1} d t
$$

holds uniformly in $\varphi, \eta \leq \varphi \leq 2 \pi-\eta$. Defining $g(z, 0) \equiv 1$, consider the function

$$
g_{u v}(z)=\frac{g(z, u)}{g(-z, v)}, 0 \leq v \leq u<\infty, u>0 .
$$

The relation (3.47) implies that for each $\eta>0$ the relation

$$
\ln \left|g_{u v}\left(r e^{i \varphi}\right)\right|=(u-v+o(1)) \int_{1}^{r} t^{\rho(t)-1} d t
$$

holds uniformly in $\varphi, \eta \leq \varphi \leq 2 \pi-\eta$. Whence, with the help of Theorem 7.4 from Chapter 1, we get

$$
m\left(r, g_{u v}\right)=\frac{1}{\pi} \int_{0}^{\pi} \ln ^{+}\left|g_{u v}\left(r e^{i \varphi}\right)\right| d \varphi=(u-v+o(1)) \int_{1}^{r} t^{\rho(t)-1} d t .
$$

Since

$$
N\left(r, g_{u v}\right)=N(r, 0, g(z, v))=(v+o(1)) \int_{1}^{r} t^{\rho(t)-1} d t
$$

we have

$$
T\left(r, g_{u v}\right)=m\left(r, g_{u v}\right)+N\left(r, g_{u v}\right)=(u+o(1)) \int_{1}^{r} t^{\rho(t)-1} d t
$$

${ }^{8}$ There exists an entire function $g(z)$ of order $\rho$ and lower order $\lambda, 0 \leq \lambda \leq \rho \leq \infty$, for which

$$
\limsup _{r \rightarrow \infty} \frac{T(r, g)}{\ln M(r, g)}= \begin{cases}\frac{\sin \pi \lambda}{\pi \lambda}, & 0 \leq \lambda \leq \frac{1}{2}, \\ \frac{1}{\pi \lambda}, & \frac{1}{2} \leq \lambda \leq \infty,\end{cases}
$$

(see M.N. Sheremeta [She69]). 
and, hence,

$$
\delta\left(\infty, g_{u v}\right)=\liminf _{r \rightarrow \infty} \frac{m\left(r, g_{u v}\right)}{T\left(r, g_{u v}\right)}=\frac{u-v}{u} .
$$

Obviously, fixing $u>0$ and choosing $v, 0 \leq v \leq u$, we can give $\delta\left(\infty, g_{u v}\right)$ an arbitrary preassigned value $\delta_{0}, 0 \leq \delta_{0} \leq 1$. Since $\ln \mu(r, f) \leq m(r, f)$, we have

$$
\limsup _{r \rightarrow \infty} \frac{\ln \mu\left(r, g_{u v}\right)}{T\left(r, g_{u v}\right)} \leq \limsup _{r \rightarrow \infty} \frac{m\left(r, g_{u v}\right)}{T\left(r, g_{u v}\right)}=\frac{u-v}{u}=\delta\left(\infty, g_{u v}\right) .
$$

Comparing with (3.2) for $\lambda=0$, we see, that (3.45) holds for $f(z)=g_{u v}(z)$.

Since $\ln ^{+} M(r, f) \geq m(r, f)$, for $v<u$ we have

$$
\limsup _{r \rightarrow \infty} \frac{N\left(r, g_{u v}\right)}{\ln ^{+} M\left(r, g_{u v}\right)} \leq \limsup _{r \rightarrow \infty} \frac{N\left(r, g_{u v}\right)}{m\left(r, g_{u v}\right)}=\frac{v}{u-v}<\infty,
$$

that is, the second relation from (3.46) holds. The first of the relations from (3.46) follows from the trivial inequality $\mu(r, f) \leq M(r, f)$ and the inequality (3.23) for $\lambda=0$.

EXAMPLE 3. We show that Theorem 3.1 ceases to be true if we replace $<$ by $\leq$ in (3.1) (it is clear that this does not follow from the previous examples).

For this, for arbitrary $\lambda$ and $\rho$ satisfying $0<\lambda \leq \rho<1 / 2$, we construct a meromorphic function $f(z)$ of order $\rho$ and lower order $\lambda$, such that $|f(-r)| \leq 2$, $0 \leq r<\infty$ and $\delta(\infty, f)=1-\cos \pi \lambda$.

Let $g(z, u)$ be a function defined in the same way as $f(z, u)$ in Example 1, with the only difference that the condition (3.35) is replaced by the condition

$$
\liminf _{r \rightarrow \infty} l(r)=2 \lambda, \limsup _{r \rightarrow \infty} l(r)=2 \rho .
$$

Let

$$
g_{1}(z)=\frac{g(z, 1)}{g(-z, 1)} .
$$

As in Example 1, we get that for each $\eta>0$ the relation

$$
\begin{aligned}
\ln \left|g_{1}\left(r e^{i \varphi}\right)\right| & =\frac{\pi}{\sin \pi l(r)}\{\cos l(r)(\varphi-\pi)-\cos l(r) \varphi\} r^{l(r)}+o\left(r^{l(r)}\right) \\
& =\frac{\pi}{\cos \frac{\pi l(r)}{2}}\left\{\sin l(r)\left(\varphi-\frac{\pi}{2}\right)\right\} r^{l(r)}+o\left(r^{l(r)}\right)
\end{aligned}
$$

holds uniformly in $\varphi, \eta \leq \varphi \leq \pi-\eta$.

Consider the function

$$
g_{2}(z)=g_{1}(z)+g_{1}(-z) \text {. }
$$

From (3.48) we see that

$$
g_{1}\left(r e^{i \varphi}\right) \rightarrow 0, g_{1}\left(-r e^{i \varphi}\right) \rightarrow \infty \text { as } r \rightarrow+\infty
$$

uniformly in $\varphi$ for $\eta \leq \varphi \leq \frac{\pi}{2}-\eta$, and, hence

$$
g_{2}\left(r e^{i \varphi}\right)=(1+o(1)) g_{1}\left(-r e^{i \varphi}\right),
$$

and

$$
g_{1}\left(r e^{i \varphi}\right) \rightarrow \infty, g_{1}\left(-r e^{i \varphi}\right) \rightarrow 0 \text { as } r \rightarrow+\infty
$$


uniformly in $\varphi$ satisfying $\frac{\pi}{2}+\eta \leq \varphi \leq \pi-\eta$, and hence

$$
g_{2}\left(r e^{i \varphi}\right)=(1+o(1)) g_{1}\left(r e^{i \varphi}\right) .
$$

Therefore the relation

$$
\ln \left|g_{2}\left(r e^{i \varphi}\right)\right|=\frac{\pi}{\cos \frac{\pi l(r)}{2}}\left\{\sin \left(l(r)\left|\varphi-\frac{\pi}{2}\right|\right)\right\} r^{l(r)}+o\left(r^{l(r)}\right)
$$

holds uniformly in $\varphi$ satisfying $\eta \leq \varphi \leq \frac{\pi}{2}-\eta$ or $\frac{\pi}{2}+\eta \leq \varphi \leq \pi-\eta$. Applying Theorem 7.4 from Chapter 1 we have

$$
\begin{aligned}
m\left(r, g_{2}\right) & =\frac{1}{\pi} \int_{0}^{\pi} \ln ^{+}\left|g_{2}\left(r e^{i \varphi}\right)\right| d \varphi \\
& =r^{l(r)} \sec \frac{\pi l(r)}{2} \int_{0}^{\pi} \sin \left(l(r)\left|\varphi-\frac{\pi}{2}\right|\right) d \varphi+o\left(r^{l(r)}\right) \\
& =r^{l(r)} \frac{2}{l(r)} \sec \frac{\pi l(r)}{2}\left(1-\cos \frac{\pi l(r)}{2}\right)+o\left(r^{l(r)}\right) .
\end{aligned}
$$

Since

$$
N\left(r, \infty, g_{2}\right)=2 N\left(r, \infty, g_{1}\right)=\frac{2}{l(r)} r^{l(r)}+o\left(r^{l(r)}\right),
$$

we get

$$
T\left(r, g_{2}\right)=\frac{2}{l(r)} \sec \frac{\pi l(r)}{2} r^{l(r)}+o\left(r^{l(r)}\right) .
$$

Since the function $g_{2}(z)$ is even, the function $f(z)=g_{2}(\sqrt{z})$ is univalent and, hence, meromorphic. By the corollary of Theorem 6.6 from Chapter 1 we have

$$
\begin{aligned}
T(r, f) & =T\left(\sqrt{r}, g_{2}\right) \\
& =\frac{2}{l(\sqrt{r})} \sec \frac{\pi l(\sqrt{r})}{2} r^{\frac{1}{2} l(\sqrt{r})}+o\left(r^{\frac{1}{2} l(\sqrt{r})}\right), \\
\delta(\infty, f) & =\liminf _{r \rightarrow \infty} \frac{m(r, f)}{T(r, f)}=\liminf _{r \rightarrow \infty} \frac{m\left(\sqrt{r}, g_{2}\right)}{T\left(\sqrt{r}, g_{2}\right)} \\
& =\liminf _{r \rightarrow \infty}\left(1-\cos \frac{\pi l(\sqrt{r})}{2}\right)=1-\cos \pi \lambda,
\end{aligned}
$$

and also, it is clear from (3.49) that $f(z)$ has order $\rho$ and lower order $\lambda$.

Since $g(z, 1)$ is a canonical product with positive zeros, we have $g(z, 1)=\overline{g(\bar{z}, 1)}$. Therefore $|g(i y, 1)|=|g(-i y, 1)|,-\infty<y<\infty$, and hence $\left|g_{1}(i y)\right|=1$ and $\left|g_{2}(i y)\right| \leq 2,-\infty<y<\infty$. Thus $|f(-r)| \leq 2,0 \leq r<\infty$, Q.E.D.

\section{Bounds for the sum of two deficiencies and related problems}

In Section 1 of Chapter 4 we saw that a meromorphic function of non-integer order cannot have more than one Borel exceptional value. Examples given in Section 4 of Chapter 4 show that this result does not immediately carry over to deficient values. However, it turns out that a meromorphic function $f(z)$ of a non-integer order $\rho$ cannot have two deficient values, $a$ and $b$, with the sum $\delta(a, f)+\delta(b, f)$ close to 2 . 
More precisely, for each non-integer $\rho>0$ we can find a positive number $d(\rho)$, such that for each meromorphic function of order $\rho$ and for each $a \neq b$, the inequality

$$
\delta(a, f)+\delta(b, f) \leq 2-d(\rho)
$$

holds.

This result can be easily obtained using the corollary of Theorem 4.2 from Chapter 2 (p. ???). First we observe, that it is enough to prove (4.1) for $a=0$, $b=\infty$. In fact, if $a \neq \infty, b \neq \infty$, for the function $f_{1}(z)=\frac{f(z)-a}{f(z)-b}$ we have $\rho\left[f_{1}\right]=\rho[f]=\rho, \delta\left(0, f_{1}\right)=\delta(a, f), \delta\left(\infty, f_{1}\right)=\delta(b, f)$, and the inequality $\delta\left(0, f_{1}\right)+$ $\delta\left(\infty, f_{1}\right) \leq 2-d(\rho)$ implies (4.1). If $a \neq \infty, b=\infty$, we let $f_{1}(z)=f(z)-a$. Further, since

$$
\delta(0, f)+\delta(\infty, f) \leq 2-\limsup _{r \rightarrow \infty} \frac{N(r ; 0, \infty)}{T(r, f)},
$$

and, by the corollary of Theorem 4.2 from Chapter 2

$$
\limsup _{r \rightarrow \infty} \frac{N(r ; 0, \infty)}{T(r, f)} \geq \frac{\sin \pi(\rho-[\rho])}{C_{1}([\rho])},
$$

where $0<C_{1}([\rho])<\infty$ does not depend on $f(z)$, we get (4.1) with $d(\rho)=$ $|\sin \pi \rho| / C_{1}([\rho])$.

The problem about the best possible value of $d(\rho)$ arises. By (4.2) this problem is connected with the problem of the best estimate from below in terms of $\rho$ of the value

$$
\kappa(f)=\limsup _{r \rightarrow \infty} \frac{N(r ; 0, \infty)}{T(r, f)}
$$

for meromorphic functions $f(z)$ of order $\rho$. More general is a problem of the best estimate from below of $\kappa(f)$ for meromorphic functions $f(z)$ of order $\rho$ and lower order $\lambda$.

At present none of these problems is completely solved. ${ }^{9}$ The existing results are summarized in the following theorem.

THEOREM 4.1. Let

$$
\nu(x)= \begin{cases}1, & 0 \leq x \leq 1 / 2, \\ \sin \pi x, & 1 / 2 \leq x \leq 1 \\ |\sin \pi x|\{2,2 x+0,5|\sin \pi x|\}^{-1}, & 1 \leq x<\infty \\ 0, & x=\infty\end{cases}
$$

For each meromorphic function $f(z)$ of order $\rho \leq \infty$ and lower order $\lambda<\infty$, the estimates

$$
\begin{gathered}
\kappa(f) \geq \max _{\lambda \leq x \leq \rho} \nu(x), \\
\delta(a, f)+\delta(b, f) \leq 2-\max _{\lambda \leq x \leq \rho} \nu(x), a \neq b
\end{gathered}
$$

hold.

In particular, for each meromorphic function $f(z)$ of order $\rho$ the estimates

$$
\kappa(f) \geq \nu(\rho)
$$

\footnotetext{
${ }^{9}$ If $\lambda<\infty$ and the sum of deficiencies is 2 , then $\rho=\lambda=n / 2$ where $n$ is an integer [A44].
} 


$$
\delta(a, f)+\delta(b, f) \leq 2-\nu(\rho), a \neq b
$$

hold.

To characterize the accuracy of the estimates $(4.4)$ and $\left(4.4^{\prime}\right)$, define a function $\nu_{1}(x), x \geq 0$, as:

$$
\nu_{1}(x)= \begin{cases}|\sin \pi x|\{[x]+|\sin \pi x|\}^{-1}, & {[x] \leq x \leq[x]+\frac{1}{2},} \\ |\sin \pi x|\{[x]+1\}^{-1}, & {[x]+\frac{1}{2} \leq x \leq[x]+1 .}\end{cases}
$$

Obviously

$$
\begin{gathered}
\nu_{1}(x)=\nu(x), 0 \leq x \leq 1, \\
0,3 \nu_{1}(x) \leq \nu(x) \leq \nu_{1}(x), x \geq 1
\end{gathered}
$$

(where the equality in the last inequality takes place for integer $x$ only).

Exercise 1 from Section 1 of Chapter 4 contains an example of a function of order $\rho$, such that

$$
1-\delta(0, f)=\kappa(f)=\nu_{1}(\rho), \delta(\infty, f)=1 .
$$

Thus, the estimates (4.4) and $\left(4.4^{\prime}\right)$ are the best possible for $0<\rho \leq 1$, and, in certain sense, are close to the best possible for $\rho>1$.

As for the accuracy of the estimates (4.3) and $\left(4.3^{\prime}\right)$, note that Exercise 2 from Section 1 of Chapter 4, for each integer $p \geq 0$ and for numbers $\lambda$ and $\rho$ satisfying $p<\lambda \leq \rho<p+1$, contains an example of a function of order $\rho$ and lower order $\lambda$, such that

$$
1-\delta(0, f)=\kappa(f)=\max _{\lambda \leq x \leq \rho} \nu_{1}(x), \delta(\infty, f)=1 .
$$

Thus the estimates (4.3) and $\left(4.3^{\prime}\right)$ are the best possible for $0<\lambda \leq \rho<1$ and, in certain sense, are close to the best possible if $[\rho]<\lambda \leq \rho,[\rho] \geq 1$.

We can also add that the estimates $(4.3)$ and $\left(4.3^{\prime}\right)$ are the best possible for $0 \leq \lambda \leq 1 / 2$. In fact, in this case $\max _{\lambda \leq x \leq \rho} \nu(x)=1$ for each $\rho \geq \lambda$. On the other hand, for each entire function $f(z)$ of order $\rho$ and lower order $\lambda$, the relations

$$
1-\delta(0, f)=\kappa(f)=\limsup _{r \rightarrow \infty} \frac{N(r, 0)}{T(r, f)} \leq 1, \delta(\infty, f)=1
$$

hold, and hence there is an equality in (4.3) and (4.3').

Note that Theorem 4.1 immediately implies the following statement, which was already proved as a corollary of Lemma 3.4: an entire function of lower order $\lambda \leq 1 / 2$ cannot have finite deficient values. In fact, since $\delta(\infty, f)=1$ and $\max _{\lambda \leq x \leq \rho} \nu(x)=1$, letting $b=\infty$ in $\left(4.3^{\prime}\right)$, we get $\delta(a, f)=0$ for each $a \neq \infty$.

Theorem 4.1 is a corollary of the following two theorems.

TheOREM 4.2. Let

$$
\nu_{2}(x)=|\sin \pi x|\{2,2 x+0,5|\sin \pi x|\}^{-1}, x \geq 0 .
$$

For each meromorphic function $f(z)$ of order $\rho \leq \infty$ and lower order $\infty>\lambda>$ 0 the estimate

$$
\kappa(f) \geq \max _{\lambda \leq x \leq \rho} \nu_{2}(x)
$$

holds. 

estimate

TheOREM 4.3. For each meromorphic function of lower order $0 \leq \lambda<1$ the holds.

In order to derive Theorem 4.1 from these theorems, it suffices to observe, that for $\lambda \geq 1$ the estimate (4.3) coincides with (4.5), and for $\lambda<1$

$$
\max _{\lambda \leq x \leq \rho} \nu(x)=\max \left\{\nu(\lambda), \max _{\lambda \leq x \leq \rho} \nu_{2}(x)\right\}
$$

and the estimate (4.3) follows from (4.5) and (4.6). The estimate $\left(4.3^{\prime}\right)$ is a easy corollary of (4.3) and (4.2).

Now we turn to lemmas used in the proof of Theorem 4.2.

Lemma 4.1. Let $f(z), f(0)=1$, be a meromorphic function, and $q \geq 0$ be an integer. If $0 \leq r \leq \frac{1}{2} R$, the inequality

$$
\begin{aligned}
2 T(r, f)-N(r ; 0, \infty) \leq & r^{q} \int_{0}^{R} n(t ; 0, \infty) \Phi\left(\frac{t}{r}\right) t^{-q-1} d t \\
& +C_{1}\left(\frac{r}{R}\right)^{q+1} T(2 R, f)+C_{2} q\left(r^{q}+1\right),
\end{aligned}
$$

holds, where $0<C_{1}, C_{2}<\infty$ are constants, which do not depend on $r$ and $R$, and

$$
\Phi(t)=\frac{1}{2 \pi} \int_{0}^{2 \pi} \frac{d \theta}{\left|t e^{i \theta}-1\right|} .
$$

Proof. We have

$$
\begin{aligned}
2 T(r, f)-N(r ; 0, \infty) & =m(r, f)+m\left(r, \frac{1}{f}\right) \\
& =\frac{1}{2 \pi} \int_{0}^{2 \pi}|\ln | f\left(r e^{i \theta}\right)|| d \theta .
\end{aligned}
$$

Applying Lemma 3.1 we represent the function $f(z)$ in the form (3.3). We get

$$
\begin{aligned}
\frac{1}{2 \pi} \int_{0}^{2 \pi} & |\ln | f\left(r e^{i \theta}\right)|| d \theta \\
& \leq \frac{1}{2 \pi} \int_{0}^{2 \pi}|\ln | \alpha_{R, q}\left(r e^{i \theta}\right)|| d \theta+\frac{1}{2 \pi} \int_{0}^{2 \pi}|\ln | \omega_{R, q}\left(r e^{i \theta}\right)|| d \theta
\end{aligned}
$$

from where, by (3.4) and (3.5) we get the inequality

$$
\begin{aligned}
\frac{1}{2 \pi} \int_{0}^{2 \pi}|\ln | f\left(r e^{i \theta}\right)|| d \theta \leq \sum_{\left|a_{\mu}\right|<R} I\left(a_{\mu}\right)+\sum_{\left|b_{\nu}\right|<R} I\left(b_{\nu}\right) \\
\quad+K_{1}\left(\frac{r}{R}\right)^{q+1} T(2 R, f)+K_{2} q\left(r^{q}+1\right), 0 \leq r \leq \frac{1}{2} R,
\end{aligned}
$$

where

$$
I(a)=\frac{1}{2 \pi} \int_{0}^{2 \pi}|\ln | E\left(\frac{r e^{i \theta}}{a}, q\right)|| d \theta, 0<|a|<\infty .
$$


Let us estimate $I(a)$. Since

$$
\frac{\partial}{\partial t} \ln \left|E\left(t e^{i \theta}, q\right)\right|=R e \frac{\partial}{\partial t} \ln E\left(t e^{i \theta}, q\right)=R e \frac{t^{q} e^{i(q+1) \theta}}{t e^{i \theta}-1},
$$

for $0<\theta<2 \pi, 0<t<\infty$, we have

$$
|\ln | E\left(r e^{i \theta}, q\right)|| \leq \int_{0}^{r}\left|\frac{\partial}{\partial t} \ln \right| E\left(t e^{i \theta}, q\right)|| d t \leq \int_{0}^{r} \frac{t^{q} d t}{\left|t e^{i \theta}-1\right|}
$$

and hence, we get

$$
\begin{aligned}
I(a)=I(|a|) & =\frac{1}{2 \pi} \int_{0}^{2 \pi}|\ln | E\left(\frac{r}{|a|} e^{i \theta}, q\right)|| d \theta \\
& \leq \int_{0}^{r /|a|}\left\{\frac{1}{2 \pi} \int_{0}^{2 \pi} \frac{d \theta}{\left|t e^{i \theta}-1\right|}\right\} t^{q} d t=\int_{0}^{r /|a|} t^{q} \Phi(t) d t .
\end{aligned}
$$

It is easy to see that $\Phi(t)=\frac{1}{t} \Phi\left(\frac{1}{t}\right)$, therefore we have the estimate

$$
I(a) \leq \int_{0}^{r /|a|} t^{q-1} \Phi\left(\frac{1}{t}\right) d t=r^{q} \int_{|a|}^{\infty} u^{-q-1} \Phi\left(\frac{u}{r}\right) d u .
$$

Using this estimate we get

$$
\begin{aligned}
\sum_{\left|a_{\mu}\right|<R} I\left(a_{\mu}\right)+ & \sum_{\left|b_{\nu}\right|<R} I\left(b_{\nu}\right) \\
\leq & r^{q} \sum_{\left|a_{\mu}\right|<R} \int_{\left|a_{\mu}\right|}^{\infty} u^{-q-1} \Phi\left(\frac{u}{r}\right) d u+r^{q} \sum_{\left|b_{\nu}\right|<R} \int_{\left|b_{\nu}\right|}^{\infty} u^{-q-1} \Phi\left(\frac{u}{r}\right) d u \\
\leq & r^{q} \int_{0}^{R} d n(s ; 0, \infty) \int_{s}^{\infty} u^{-q-1} \Phi\left(\frac{u}{r}\right) d u \\
= & r^{q}\left\{n(R ; 0, \infty) \int_{R}^{\infty} u^{-q-1} \Phi\left(\frac{u}{r}\right) d u\right. \\
& \left.+\int_{0}^{R} n(s ; 0, \infty) s^{-q-1} \Phi\left(\frac{s}{r}\right) d s\right\} .
\end{aligned}
$$

For $0 \leq r \leq \frac{1}{2} R, u \geq R$, the inequality

$$
\Phi\left(\frac{u}{r}\right)=\frac{1}{2 \pi} \int_{0}^{2 \pi} \frac{d \theta}{\left|\frac{u}{r} e^{i \theta}-1\right|} \leq \frac{r}{u-r} \leq 2 \frac{r}{u}
$$

holds, and therefore

$$
\int_{R}^{\infty} u^{-q-1} \Phi\left(\frac{u}{r}\right) d u \leq 2 r \int_{R}^{\infty} u^{-q-2} d u=\frac{2}{q+1} \frac{r}{R^{q+1}} .
$$

Taking into the account the inequality $n(R ; 0, \infty) \leq 4 T(2 R, f)$ (Lemma 7.1 from Chapter 1), we get the estimate

$$
\begin{aligned}
& \sum_{\left|a_{\mu}\right|<R} I\left(a_{\mu}\right)+\sum_{\left|b_{\nu}\right|<R} I\left(b_{\nu}\right) \\
& \quad \leq r^{q}\left\{\frac{8}{q+1} \frac{r}{R^{q+1}} T(2 R, f)+\int_{0}^{R} n(s ; 0, \infty) \Phi\left(\frac{s}{r}\right) s^{-q-1} d s\right\} .
\end{aligned}
$$

The conclusion of the lemma follows from (4.7), (4.9), and (4.10). 
LEMMA 4.2. Let

$$
J(\beta)=\int_{0}^{\infty} t^{-\beta-1} \Phi\left(\frac{1}{t}\right) d t, 0<\beta<1 .
$$

The estimate

$$
J(\beta) \leq 4.4 \csc \pi \beta
$$

holds.

Proof. We have

$$
J(\beta)=\frac{1}{\pi} \int_{0}^{\pi} d \theta \int_{0}^{\infty} \frac{t^{-\beta} d t}{\sqrt{t^{2}-2 t \cos \theta+1}} .
$$

Making the change of variable

$$
t=\cos \theta+\sin \theta \tan \varphi=\frac{\cos (\theta-\varphi)}{\cos \varphi}
$$

we get

$$
J(\beta)=\frac{1}{\pi} \int_{0}^{\pi} d \theta \int_{\theta-\frac{\pi}{2}}^{\pi / 2}\{\cos (\theta-\varphi)\}^{-\beta}\{\cos \varphi\}^{\beta-1} d \varphi .
$$

Changing the order of integration, we have

$$
J(\beta)=\frac{1}{\pi} \int_{-\pi / 2}^{\pi / 2}\{\cos \varphi\}^{\beta-1} d \varphi \int_{0}^{\varphi+\frac{\pi}{2}}\{\cos (\theta-\varphi)\}^{-\beta} d \theta .
$$

Since the second integral in the right-hand side of the equality

$$
\int_{0}^{\varphi+\frac{\pi}{2}}\{\cos (\theta-\varphi)\}^{-\beta} d \theta=\int_{0}^{\pi / 2}\{\cos \omega\}^{-\beta} d \omega+\int_{-\varphi}^{0}\{\cos \omega\}^{-\beta} d \omega
$$

is an odd function of $\varphi$, we get

$$
J(\beta)=\frac{1}{\pi} \int_{-\pi / 2}^{\pi / 2}\{\cos \varphi\}^{\beta-1} d \varphi \int_{0}^{\pi / 2}\{\cos \omega\}^{-\beta} d \omega .
$$

Using the well-known formula

$$
\int_{0}^{\pi / 2}\{\cos \alpha\}^{x} d \alpha=\frac{\sqrt{\pi} \Gamma\left(\frac{x+1}{2}\right)}{\Gamma\left(\frac{x}{2}+1\right)},-1<x<\infty
$$

we get

$$
J(\beta)=\frac{\Gamma\left(\frac{\beta}{2}\right) \Gamma\left(\frac{1-\beta}{2}\right)}{2 \Gamma\left(\frac{\beta+1}{2}\right) \Gamma\left(1-\frac{\beta}{2}\right)},
$$

from where, by virtue of $\Gamma(x) \Gamma(1-x)=\pi \csc \pi x$, we obtain

$$
J(\beta)=\frac{\pi^{2}}{\sin \pi \beta \Gamma^{2}\left(\frac{1+\beta}{2}\right) \Gamma^{2}\left(1-\frac{\beta}{2}\right)} .
$$

Letting $t=\frac{1}{4}-\frac{\beta}{2}$, we see that $-\frac{1}{4}<t<\frac{1}{4}$, and

$$
\Gamma\left(\frac{1+\beta}{2}\right) \Gamma\left(1-\frac{\beta}{2}\right)=\Gamma\left(\frac{3}{4}+t\right) \Gamma\left(\frac{3}{4}-t\right) .
$$


Let us find the minimum of the function $g(t)=\Gamma\left(\frac{3}{4}+t\right) \Gamma\left(\frac{3}{4}-t\right)$ on the interval $\left(-\frac{1}{4}, \frac{1}{4}\right)$. Using the well-known formula (see Whittaker, Watson [WW96, 12.16])

$$
\frac{\Gamma^{\prime}(z)}{\Gamma(z)}=-\gamma-\frac{1}{z}-\sum_{n=1}^{\infty}\left\{\frac{1}{z+n}-\frac{1}{n}\right\}
$$

( $\gamma$ is the Euler-Mascheroni constant), we get

$$
\frac{g^{\prime}(t)}{g(t)}=\frac{\Gamma^{\prime}\left(\frac{3}{4}+t\right)}{\Gamma\left(\frac{3}{4}+t\right)}-\frac{\Gamma^{\prime}\left(\frac{3}{4}-t\right)}{\Gamma\left(\frac{3}{4}-t\right)}=\sum_{n=0}^{\infty} \frac{2 t}{\left(n+\frac{3}{4}\right)^{2}-t^{2}} .
$$

Hence we see that

$$
\operatorname{sign} g^{\prime}(t)=\operatorname{sign} t
$$

on the interval $\left(-\frac{1}{4}, \frac{1}{4}\right)$. It indicates that

$$
\min _{-\frac{1}{4}<t<\frac{1}{4}} g(t)=g(0)=\Gamma^{2}\left(\frac{3}{4}\right) \text {. }
$$

Thus,

$$
J(\beta) \leq \frac{\pi^{2}}{\sin \pi \beta \Gamma^{4}\left(\frac{3}{4}\right)}<\frac{4.4}{\sin \pi \beta} .
$$

Q.E.D.

Lemma 4.3. Let $f(z), f(0)=1$, be a meromorphic function, $q \geq 0$ be an integer, $\kappa^{\prime}>\kappa(f), \varepsilon>0$. It is possible to find $R_{0} \geq 1$ such that for $R \geq 2 R_{0}$, $q+\varepsilon \leq x \leq q+1-\varepsilon$, the inequality

$$
\left(\nu_{2}(x)-\kappa^{\prime}\right) \int_{R_{0}}^{\frac{1}{2} R} T(r, f) r^{-x-1} d r \leq C_{3} R^{-x} T(2 R, f)+C_{4}
$$

holds, where $0<C_{3}, C_{4}<\infty$ are constants which do not depend on $R$ and $x$.

Proof. Let $R_{0} \geq 1$ be such that $N(r ; 0, \infty) \leq \kappa^{\prime} T(r, f)$ holds for $r \geq R_{0}$. Lemma 4.1 implies the inequality

$$
\begin{aligned}
\left(2-\kappa^{\prime}\right) T(r, f) \leq & r^{q} \int_{0}^{R} n(t ; 0, \infty) \Phi\left(\frac{t}{r}\right) t^{-q-1} d t \\
& +C_{1}\left(\frac{r}{R}\right)^{q+1} T(2 R, f)+2 C_{2} q r^{q}, R_{0} \leq r \leq \frac{1}{2} R .
\end{aligned}
$$

We multiply both parts of this inequality by $r^{-x-1}$ and integrate with respect to $r$ from $R_{0}$ to $\frac{1}{2} R$. We have

$$
\begin{aligned}
\left(2-\kappa^{\prime}\right) \int_{R_{0}}^{\frac{1}{2} R} T(r, f) r^{-x-1} d r & \\
\leq & \int_{R_{0}}^{\frac{1}{2} R} r^{q-x-1} d r \int_{0}^{R} n(t ; 0, \infty) \Phi\left(\frac{t}{r}\right) t^{-q-1} d t \\
& +\frac{C_{1}}{q+1-x} R^{-x} T(2 R, f)+\frac{2 C_{2} q}{x-q} .
\end{aligned}
$$


Denote the iterated integral in the right-hand side of (4.12) by $I$. Changing the order of integration, we get

$$
\begin{aligned}
I= & \int_{0}^{R} n(t ; 0, \infty) t^{-q-1} d t \int_{R_{0}}^{\frac{1}{2} R} r^{q-x-1} \Phi\left(\frac{t}{r}\right) d r \\
= & \int_{0}^{R} n(t ; 0, \infty) t^{-x-1} d t \int_{R_{0} / t}^{R /(2 t)} u^{q-x-1} \Phi\left(\frac{1}{u}\right) d u \\
\leq & \int_{0}^{R} n(t ; 0, \infty) t^{-x-1} d t \int_{0}^{\infty} u^{q-x-1} \Phi\left(\frac{1}{u}\right) d u \\
= & J(x-q) \int_{0}^{R} n(t ; 0, \infty) t^{-x-1} d t \\
= & J(x-q)\left\{N(R ; 0, \infty) R^{-x}+x \int_{0}^{R} N(t ; 0, \infty) t^{-x-1} d t\right\} \\
= & J(x-q)\left\{N(R ; 0, \infty) R^{-x}+x \int_{\frac{1}{2} R}^{R} N(t ; 0, \infty) t^{-x-1} d t\right. \\
& \left.+x \int_{R_{0}}^{\frac{1}{2} R} N(t ; 0, \infty) t^{-x-1} d t+x \int_{0}^{R_{0}} N(t ; 0, \infty) t^{-x-1} d t\right\}
\end{aligned}
$$

Since

$$
\begin{gathered}
N(R ; 0, \infty) \leq 2 T(R, f) \leq 2 T(2 R, f), \\
x \int_{\frac{1}{2} R}^{R} N(t ; 0, \infty) t^{-x-1} d t \leq N(R ; 0, \infty)\left(\frac{1}{2} R\right)^{-x} \leq 2^{q+2} R^{-x} T(2 R, f), \\
x \int_{R_{0}}^{\frac{1}{2} R} N(t ; 0, \infty) t^{-x-1} d t \leq x \kappa^{\prime} \int_{R_{0}}^{\frac{1}{2} R} T(t, f) t^{-x-1} d t \\
x \int_{0}^{R_{0}} N(t ; 0, \infty) t^{-x-1} d t \leq(q+1)\left\{\int_{0}^{1} N(t ; 0, \infty) t^{-q-2} d t\right. \\
\left.+\int_{1}^{R_{0}} N(t ; 0, \infty) t^{-q-1} d t\right\},
\end{gathered}
$$

and, by Lemma 4.2,

$$
J(x-q) \leq 4.4|\csc \pi x|,
$$

we get the estimate

$$
I \leq \frac{4.4 x \kappa^{\prime}}{|\sin \pi x|} \int_{R_{0}}^{\frac{1}{2} R} T(r, f) r^{-x-1} d r+C_{5} R^{-x} T(2 R, f)+C_{6},
$$

where $0<C_{5}, C_{6}<\infty$ do not depend on $R$ and $x, q+\varepsilon<x<q+1-\varepsilon$. Using this estimate, from (4.12) we get

$$
\left(2-\kappa^{\prime}-\frac{4.4 x \kappa^{\prime}}{|\sin \pi x|}\right) \int_{R_{0}}^{\frac{1}{2} R} T(r, f) r^{-x-1} d r \leq C_{7} R^{-x} T(2 R, f)+C_{8},
$$


where $0<C_{7}, C_{8}<\infty$ do not depend on $R$ and $x, q+\varepsilon<x<q+1-\varepsilon$. Since $2-\kappa^{\prime}-\frac{4.4 x \kappa^{\prime}}{|\sin \pi x|}=\left(\nu_{2}(x)-x^{\prime}\right)\left(1+\frac{4.4 x}{|\sin \pi x|}\right)$, multiplying both parts of the relation (4.13) by $\left(1+\frac{4.4 x}{|\sin \pi x|}\right)^{-1}$, we get the inequality (4.11).

Proof. Now we prove Theorem 4.2. We consider separately the following two cases: (a) $0<\lambda<\rho \leq \infty$, (b) $1 \leq[\rho]<\rho=\lambda$. We do not need to consider the case $1 \leq[\rho]=\rho=\lambda$, since in this case the right-hand side of the inequality (4.5) vanishes, and the conclusion of Theorem 4.2 is trivial.

The case (a). By the continuity of the function $\nu_{2}(x)$ it suffices to prove that for each non-integer $x, \lambda<x<\rho$, the inequality

$$
\kappa(f) \geq \nu_{2}(x)
$$

holds. Letting $q=[x], \varepsilon=\min (x-q, q+1-x)$, we apply Lemma 4.3. Note that the quantities $C_{3}$ and $C_{4}$ in the inequality (4.11) could depend on $q$ and $\varepsilon$, therefore now they can depend on $x$, but this does not interfere with our argument.

Since $x<\rho$, we get

$$
\lim _{R \rightarrow \infty} \int_{R_{0}}^{\frac{1}{2} R} T(r, f) r^{-x-1} d r=\infty .
$$

Since $x>\lambda$, there exists a sequence $R_{k} \rightarrow \infty$, such that

$$
\lim _{k \rightarrow \infty} R_{k}^{-x} T\left(2 R_{k}, f\right)=0 .
$$

Therefore, letting $R$ tend to $\infty$ along the sequence $R_{k}$ in (4.11), we get

$$
\nu_{2}(x)-\kappa^{\prime} \leq 0 \text {. }
$$

Since this inequality holds for each $\kappa^{\prime}>\kappa(f)$, it holds also for $\kappa^{\prime}=\kappa(f)$, that is (4.14) holds.

Case (b). We prove that

$$
\kappa(f) \geq \nu_{2}(\lambda)
$$

Let $\lambda(r)$ be the lower proximate order of the function $T(r, f), q=[\lambda], \varepsilon=\frac{1}{2} \min (\lambda-$ $q, q+1-\lambda)$. We choose $R_{0}^{\prime} \geq R_{0}$ so large, that for $r \geq R_{0}^{\prime}$ the inequalities $q+\varepsilon<\lambda(r)<q+1-\varepsilon$ and $T(r, f) \geq \frac{1}{2} r^{\lambda(r)}$ hold. Letting $x=\lambda(R)$ in (4.11), we get the inequality

$$
\left(\nu_{2}(\lambda(R))-\kappa^{\prime}\right) \int_{R_{0}}^{\frac{1}{2} R} T(r, f) r^{-\lambda(R)-1} d r \leq C_{3} R^{-\lambda(R)} T(2 R, f)+C_{4} .
$$

By the result of Exercise 2 from Section 2 of Chapter 2 we have

$$
\begin{array}{rl}
\lim _{R \rightarrow \infty} \int_{R_{0}}^{\frac{1}{2} R} & T(r, f) r^{-\lambda(R)-1} d r \\
& \quad \geq \lim _{R \rightarrow \infty}\left\{\int_{R_{0}}^{R_{0}^{\prime}} T(r, f) r^{-\lambda(R)-1} d r+\frac{1}{2} \int_{R_{0}^{\prime}}^{\frac{1}{2} R} r^{\lambda(r)-\lambda(R)-1} d r\right\}=\infty .
\end{array}
$$

The definition of a lower proximate order implies that there exists a sequence $R_{k} \rightarrow \infty$, such that

$$
\lim _{k \rightarrow \infty} R_{k}^{-\lambda\left(R_{k}\right)} T\left(2 R_{k}, f\right)<\infty
$$


Letting $R$ tend to $\infty$ along the sequence $R_{k}$, we get the inequality $\nu_{2}(\lambda)-\kappa^{\prime} \leq 0$, which, in turn, implies (4.5). The proof of Theorem 4.2 is completed.

Now we turn to lemmas used in the proof of Theorem 4.3.

LEMMA 4.4. Let

$$
\alpha(z)=\prod_{\mu}\left(1-\frac{z}{a_{\mu}}\right)\left\{\prod_{\nu}\left(1-\frac{z}{b_{\nu}}\right)\right\}^{-1}
$$

be a meromorphic function of genus zero. Let

$$
\check{\alpha}(z)=\prod_{\mu}\left(1-\frac{z}{\left|a_{\mu}\right|}\right)\left\{\prod_{\nu}\left(1+\frac{z}{\left|b_{\nu}\right|}\right)\right\}^{-1} .
$$

Then

$$
m(r, \check{\alpha}) \geq m(r, \alpha), T(r, \check{\alpha}) \geq T(r, \alpha) .
$$

Proof. Let $a_{\mu}=\left|a_{\mu}\right| e^{i \alpha_{\mu}}, b_{\nu}=\left|b_{\nu}\right| e^{i \beta_{\nu}}$. We fix $r>0$ and denote by $D$ the set of those values of $\theta,-\pi \leq \theta<\pi$, for which $\left|\alpha\left(r e^{i \theta}\right)\right| \geq 1$. Let $D\left(a_{\mu}\right)$ be the set of those values of $\theta,-\pi \leq \theta<\pi$, for which $\theta+\alpha_{\mu}+\pi \in D(\bmod 2 \pi)$, and $D\left(b_{\nu}\right)$ be the set of those values of $\theta,-\pi \leq \theta<\pi$, for which $\theta+\beta_{\nu} \in D(\bmod 2 \pi)$. It is clear that $d=\operatorname{mes} D=\operatorname{mes} D\left(a_{\mu}\right)=\operatorname{mes} D\left(b_{\nu}\right)$. We have

$$
\begin{aligned}
m(r, \alpha) & =\frac{1}{2 \pi} \int_{-\pi}^{\pi} \ln ^{+}\left|\alpha\left(r e^{i \theta}\right)\right| d \theta=\frac{1}{2 \pi} \int_{D} \ln \left|\alpha\left(r e^{i \theta}\right)\right| d \theta \\
& =\sum_{\mu} \frac{1}{2 \pi} \int_{D} \ln \left|1-\frac{r e^{i \theta}}{a_{\mu}}\right| d \theta+\sum_{\nu} \frac{1}{2 \pi} \int_{D}-\ln \left|1-\frac{r e^{i \theta}}{b_{\nu}}\right| d \theta \\
& =\sum_{\mu} \frac{1}{2 \pi} \int_{D\left(a_{\mu}\right)} \ln \left|1+\frac{r e^{i \theta}}{\left|a_{\mu}\right|}\right| d \theta+\sum_{\nu} \frac{1}{2 \pi} \int_{D\left(b_{\nu}\right)}-\ln \left|1-\frac{r e^{i \theta}}{\left|b_{\nu}\right|}\right| d \theta .
\end{aligned}
$$

Since the functions $\ln \left|1+\frac{r e^{i \theta}}{\left|a_{\mu}\right|}\right|$ and $-\ln \left|1-\frac{r e^{i \theta}}{\left|b_{\nu}\right|}\right|$ are decreasing on $[0, \pi]$ and even, by Lemma 7.2 from Chapter 1, we have

$$
\begin{gathered}
\int_{D\left(a_{\mu}\right)} \ln \left|1+\frac{r e^{i \theta}}{\left|a_{\mu}\right|}\right| d \theta \leq \int_{-\frac{1}{2} \operatorname{mes} D\left(a_{\mu}\right)}^{\frac{1}{2} \operatorname{mes} D\left(a_{\mu}\right)} \ln \left|1+\frac{r e^{i \theta}}{\left|a_{\mu}\right|}\right| d \theta=\int_{-\frac{1}{2} d}^{\frac{1}{2} d} \ln \left|1+\frac{r e^{i \theta}}{\left|a_{\mu}\right|}\right| d \theta, \\
\int_{D\left(b_{\nu}\right)}-\ln \left|1-\frac{r e^{i \theta}}{\left|b_{\nu}\right|}\right| d \theta \leq \int_{-\frac{1}{2} \operatorname{mes} D\left(b_{\nu}\right)}^{\frac{1}{2} \operatorname{mes} D\left(b_{\nu}\right)}-\ln \left|1-\frac{r e^{i \theta}}{\left|b_{\nu}\right|}\right| d \theta=\int_{-\frac{1}{2} d}^{\frac{1}{2} d}-\ln \left|1-\frac{r e^{i \theta}}{\left|b_{\nu}\right|}\right| d \theta .
\end{gathered}
$$

We get

$$
\begin{aligned}
m(r, \alpha) & \leq \sum_{\mu} \frac{1}{2 \pi} \int_{-\frac{1}{2} d}^{\frac{1}{2} d} \ln \left|1+\frac{r e^{i \theta}}{\left|a_{\mu}\right|}\right| d \theta+\sum_{\nu} \frac{1}{2 \pi} \int_{-\frac{1}{2} d}^{\frac{1}{2} d}-\ln \left|1-\frac{r e^{i \theta}}{\left|b_{\nu}\right|}\right| d \theta \\
& =\frac{1}{2 \pi} \int_{-\frac{1}{2} d}^{\frac{1}{2} d} \ln \left|\check{\alpha}\left(-r e^{i \theta}\right)\right| d \theta \leq \frac{1}{2 \pi} \int_{-\pi}^{\pi} \ln ^{+}\left|\check{\alpha}\left(-r e^{i \theta}\right)\right| d \theta=m(r, \check{\alpha}) .
\end{aligned}
$$

Since $N(r, \alpha)=N(r, \check{\alpha})$, we conclude

$$
T(r, \alpha)=m(r, \alpha)+N(r, \alpha) \leq m(r, \check{\alpha})+N(r, \check{\alpha})=T(r, \check{\alpha})
$$

Q.E.D. 
Lemma 4.5. Let $f(z), f(0)=1$, be a meromorphic function of of genus zero with positive zeros and negative poles. Denote by $A$ the (possibly, empty) set of those values of $r, 0<r<\infty$, for which at least one of the following equalities is true

$$
T(r, f)=N(r, f), T(r, f)=N\left(r, \frac{1}{f}\right) .
$$

For each $r \notin A$ there exists $\beta=\beta(r), 0<\beta(r)<\pi$, such that the equality

$$
T(r, f)=\int_{0}^{\infty} N\left(t, \frac{1}{f}\right) K(t, r, \beta) d t+\int_{0}^{\infty} N(t, f) K(t, r, \pi-\beta) d t
$$

takes place, where

$$
K(t, r, \varphi)=\frac{1}{\pi} \frac{r \sin \varphi}{t^{2}+r^{2}-2 r t \cos \varphi}=\frac{1}{\pi} \operatorname{Im} \frac{1}{t-r e^{i \varphi}}, 0<\varphi<\pi .
$$

Proof. We choose a single-valued of $\ln f(z)$ satisfying $\ln f(0)=0$ in the region $\{\operatorname{Im} z>0\} \cup\{|z|<q\}$, where $q$ is so small that $f(z) \neq 0, \infty$ for $|z|<q$. We have the representation

$$
\ln f(z)=\int_{0}^{\infty} \ln \left(1-\frac{z}{t}\right) d n\left(t, \frac{1}{f}\right)-\int_{0}^{\infty} \ln \left(1+\frac{z}{t}\right) d n(t, f), \operatorname{Im} z>0,
$$

from where, integrating by parts twice, we get

$$
\ln f(z)=-z \int_{0}^{\infty} N\left(t, \frac{1}{f}\right) \frac{d t}{(t-z)^{2}}-z \int_{0}^{\infty} N(t, f) \frac{d t}{(t+z)^{2}}, \operatorname{Im} z>0 .
$$

Fix $r \notin A$, then $m(r, f)>0$ and $m\left(r, \frac{1}{f}\right)>0$. Since the function $\ln \left|f\left(r e^{i \theta}\right)\right|$ is an increasing function of $\theta$ on $[0, \pi]$, we get from here that $\ln |f(r)|<0$ and $\ln |f(-r)|>0$. Denote by $\beta=\beta(r), 0<\beta(r)<\pi$ the number determined by the condition $\ln \left|f\left(r e^{i \beta}\right)\right|=0$. Observing that $\ln \left|f\left(r e^{i \theta}\right)\right|$ is an even function of $\theta$, we get for $r \notin A$

$$
m(r, f)=\frac{1}{\pi} \int_{0}^{\pi} \ln ^{+}\left|f\left(r e^{i \theta}\right)\right| d \theta=\frac{1}{\pi} \int_{\beta}^{\pi} \ln \left|f\left(r e^{i \theta}\right)\right| d \theta=\lim _{\delta \rightarrow \pi} \frac{1}{\pi} \operatorname{Im} \int_{r e^{i \beta}}^{r e^{i \delta}} \ln f(z) \frac{d z}{z} .
$$

By virtue of the representation (4.17) we get

$$
\begin{aligned}
m(r, f)= & -\frac{1}{\pi} \lim _{\delta \rightarrow \pi}\left\{\operatorname{Im} \int_{0}^{\infty} N\left(t, \frac{1}{f}\right) d t \int_{r e^{i \beta}}^{r e^{i \delta}} \frac{d z}{(z-t)^{2}}\right. \\
& \left.+\operatorname{Im} \int_{0}^{\infty} N(t, f) d t \int_{r e^{i \beta}}^{r e^{i \delta}} \frac{d z}{(z+t)^{2}}\right\} \\
= & \frac{1}{\pi} \lim _{\delta \rightarrow \pi}\left\{\int_{0}^{\infty} N\left(t, \frac{1}{f}\right) \operatorname{Im}\left[\frac{1}{r e^{i \delta}-t}-\frac{1}{r e^{i \beta}-t}\right] d t\right. \\
& \left.+\int_{0}^{\infty} N(t, f) \operatorname{Im}\left[\frac{1}{r e^{i \delta}+t}-\frac{1}{r e^{i \beta}+t}\right] d t\right\} \\
= & \int_{0}^{\infty} N\left(t, \frac{1}{f}\right) K(t, r, \beta) d t+\int_{0}^{\infty} N(t, f) K(t, r, \pi-\beta) d t \\
& -\lim _{\delta \rightarrow \pi} \int_{0}^{\infty} N\left(t, \frac{1}{f}\right) K(t, r, \delta) d t-\lim _{\delta \rightarrow \pi} \int_{0}^{\infty} N(t, f) K(t, r, \pi-\delta) d t .
\end{aligned}
$$


From the estimate

$$
K(t, r, \delta) \leq \frac{1}{\pi} \frac{r \sin \delta}{r^{2}+t^{2}}, \frac{\pi}{2} \leq \delta<\pi
$$

and the convergence of the integral

$$
\int_{0}^{\infty} N\left(t, \frac{1}{f}\right) t^{-2} d t
$$

it follows easily, that

$$
\lim _{\delta \rightarrow \pi} \int_{0}^{\infty} N\left(t, \frac{1}{f}\right) K(t, r, \delta) d t=0 .
$$

We show that

$$
\lim _{\delta \rightarrow \pi} \int_{0}^{\infty} N(t, f) K(t, r, \pi-\delta) d t=N(r, f) .
$$

Choose an arbitrary $\varepsilon>0$. By the continuity of the function $N(t, f)$ we can choose $\eta>0$ such that for $|t-r|<\eta$ the inequality $|N(t, f)-N(r, f)|<\varepsilon$ holds. We have

$$
K(t, r, \pi-\delta) \leq \frac{1}{\pi} \frac{r \sin \delta}{(r-t)^{2}}
$$

Since $\int_{-\infty}^{\infty} K(t, r, \varphi) d t=1,0<\varphi<\pi$, it follows that

$$
\begin{aligned}
\int_{0}^{\infty} N(t, f) K(t, r, \pi-\delta) d t-N(r, f) & \\
= & \int_{0}^{\infty} N(t, f) K(t, r, \pi-\delta) d t-\int_{-\infty}^{\infty} N(r, f) K(t, r, \pi-\delta) d t \\
= & \int_{|t-r|<\eta}\{N(t, f)-N(r, f)\} K(t, r, \pi-\delta) d t \\
& +\int_{\substack{|t-r| \geq \eta \\
t>0}} N(t, f) K(t, r, \pi-\delta) d t-\int_{|t-r| \geq \eta} N(r, f) K(t, r, \pi-\delta) d t \\
= & I_{1}+I_{2}-I_{3} .
\end{aligned}
$$

Noticing that $K(t, r, \varphi)>0,0<\varphi<\pi$, we get

$$
\left|I_{1}\right| \leq \varepsilon \int_{|t-r|<\eta} K(t, r, \pi-\delta) d t<\varepsilon \int_{-\infty}^{\infty} K(t, r, \pi-\delta) d t=\varepsilon .
$$

Next, by virtue of the estimate (4.19) we have

$$
\begin{aligned}
& \left|I_{2}\right| \leq \frac{1}{\pi} \sin \delta \int_{\substack{|t-r| \geq \eta \\
t>0}} N(t, f) \frac{r}{(r-t)^{2}} d t, \\
& \left|I_{3}\right| \leq \frac{1}{\pi} \sin \delta N(r, f) \int_{|t-r| \geq \eta} \frac{r}{(r-t)^{2}} d t .
\end{aligned}
$$

Therefore for all $\delta$ sufficiently close to $\pi$, the inequality $\left|I_{2}\right|+\left|I_{3}\right|<\varepsilon$ holds, hence, $\left|I_{1}\right|+\left|I_{2}\right|+\left|I_{3}\right|<2 \varepsilon$. Thus, we have proved the equality (4.18). So we have $(r \notin A)$

$$
m(r, f)=\int_{0}^{\infty} N\left(t, \frac{1}{f}\right) K(t, r, \beta) d t+\int_{0}^{\infty} N(t, f) K(t, r, \pi-\beta) d t-N(r, f)
$$

an equality, equivalent to (4.16). 
LEMMA 4.6. Let $V(r)>0$ be a continuous non-decreasing function of $r$.

(A) If for some $\sigma, 0<\sigma<\infty$,

$$
(\alpha) \liminf _{r \rightarrow \infty} r^{-\sigma} V(r)=0, \quad(\beta) \limsup _{r \rightarrow \infty} r^{-\sigma} V(r)=\infty,
$$

then for each $\varepsilon, 0<\varepsilon<1$, there are two sequences, $r_{k} \rightarrow \infty$ and $R_{k} \rightarrow \infty$, such that

$$
\text { (a) } V(t) \leq\left(\frac{t}{r_{k}}\right)^{\sigma} V\left(r_{k}\right), 1 \leq t \leq R_{k} ; \quad \text { (b) } r_{k} R_{k}^{-1}<\varepsilon \text {. }
$$

(B) If

$$
\limsup _{r \rightarrow \infty} \frac{\ln V(r)}{\ln r}=\rho<\infty,
$$

then for each $\varepsilon, 0<\varepsilon<1$, there exists a sequence $r_{k} \rightarrow \infty$, such that

$$
\begin{gathered}
V(t) \leq\left(\frac{t}{r_{k}}\right)^{\rho-\varepsilon} V\left(r_{k}\right), 1 \leq t \leq r_{k}, \\
V(t) \leq\left(\frac{t}{r_{k}}\right)^{\rho+\varepsilon} V\left(r_{k}\right), r_{k} \leq t<\infty .
\end{gathered}
$$

Proof. We prove the statement (B) first. Since lim $\sup _{r \rightarrow \infty} r^{-\rho+\varepsilon} V(r)=\infty$, the set of those values of $r$ for which

$$
r^{-\rho+\varepsilon} V(r)=\max _{1 \leq t \leq r}\left\{t^{-\rho+\varepsilon} V(t)\right\},
$$

is not bounded from above. We select in this set a sequence $s_{k} \uparrow \propto$. Since $\lim _{t \rightarrow \infty} t^{-\rho-\varepsilon} V(t)=0$, for each $s_{k}$ we can find $r_{k} \geq s_{k}$ such that

$$
r_{k}^{-\rho-\varepsilon} V\left(r_{k}\right)=\max _{s_{k} \leq t \leq<\infty} t^{-\rho-\varepsilon} V(t) .
$$

The sequence $\left\{r_{k}\right\}$ has the desired properties. In fact, the validity of (4.22) follows from (4.23) and the inequality $r_{k} \geq s_{k}$.

Next, for $1 \leq t \leq s_{k}$ we have

$$
\frac{V(t)}{t^{\rho-\varepsilon}} \leq \frac{V\left(s_{k}\right)}{s_{k}^{\rho-\varepsilon}}=\frac{V\left(s_{k}\right)}{s_{k}^{\rho+\varepsilon}} s_{k}^{2 \varepsilon} \leq \frac{V\left(r_{k}\right)}{r_{k}^{\rho+\varepsilon}} s_{k}^{2 \varepsilon} \leq \frac{V\left(r_{k}\right)}{r_{k}^{\rho+\varepsilon}} r_{k}^{2 \varepsilon}=\frac{V\left(r_{k}\right)}{r_{k}^{\rho-\varepsilon}}
$$

and for $s_{k} \leq t \leq r_{k}$ we have

$$
\frac{V(t)}{t^{\rho-\varepsilon}}=\frac{V(t)}{t^{\rho+\varepsilon}} t^{2 \varepsilon} \leq \frac{V\left(r_{k}\right)}{r_{k}^{\rho+\varepsilon}} t^{2 \varepsilon} \leq \frac{V\left(r_{k}\right)}{r_{k}^{\rho+\varepsilon}} r_{k}^{2 \varepsilon}=\frac{V\left(r_{k}\right)}{r_{k}^{\rho-\varepsilon}} .
$$

Therefore the inequality (4.21) is also valid.

Now we prove the statement (A). By $(\alpha)$ there exists a sequence $R_{k} \uparrow \infty$ such that $\lim _{k \rightarrow \infty} R_{k}^{-\sigma} V\left(R_{k}\right)=0$. Since $V(r)$ is non-decreasing, we have

$$
\max _{\varepsilon R_{k} \leq r \leq R_{k}} r^{-\sigma} V(r) \leq \varepsilon^{-\sigma} R_{k}^{-\sigma} V\left(R_{k}\right) \rightarrow 0 \text { when } k \rightarrow \infty .
$$

Denote by $F$ the closed set consisting of those $r \geq 1$ for which

$$
r^{-\sigma} V(r)=\max _{1 \leq t \leq r} t^{-\sigma} V(t) .
$$

By $(\beta)$ the set $F$ is not bounded from above and $\lim _{\substack{r \rightarrow \infty \\ r \in F}} r^{-\sigma} V(r)=\infty$. Therefore $\left(4.23^{\prime}\right)$ implies that for $k \geq k_{0}$ none of the segments $\left[\varepsilon R_{k}, R_{k}\right]$ intersects the set $F$. 
Denote by $r_{k}$ the largest number in the set $F \cap\left[1, \varepsilon R_{k}\right], k \geq k_{0}$. The sequences $\left\{r_{k}\right\}_{k_{0}}^{\infty}$ and $\left\{R_{k}\right\}_{k_{0}}^{\infty}$ have the desired properties. In fact, since $r_{k} \in F \cap\left[1, \varepsilon R_{k}\right]$, the inequality (b) from (4.20) holds. The validity of the part (a) of (4.20) for $1 \leq t \leq r_{k}$ follows from $r_{k} \in F$. Since the half-open interval $\left(r_{k}, R_{k}\right]$ does not intersect the set $F$, the part (a) of (4.20) holds, also, for $r_{k}<t \leq R_{k}$.

The proof of the lemma is completed.

Proof. Now we prove Theorem 4.3. We may assume without loss of generality that $f(0)=1$. We consider separately two cases: (a) $\lambda<\rho$, (b) $\lambda=\rho$ (by the assumptions of the theorem we have $0 \leq \lambda<1$ in both cases).

CASE (a). Using Lemma 3.1 we represent the function $f(z)$ in the form $f(z)=$ $\alpha_{R, 0}(z) \omega_{R, 0}(z)$. By virtue of the estimate (3.5) and Lemma 4.4 we have $\left(r \leq \frac{1}{2} R\right)$

$$
T(r, f) \leq T\left(r, \alpha_{R, 0}\right)+T\left(r, \omega_{R, 0}\right) \leq T\left(r, \check{\alpha}_{R, 0}\right)+K_{1} \frac{r}{R} T(2 R, f),
$$

where $K_{1}$ is a constant which does not depend on $r$ and $R$.

Let $\sigma$ be an arbitrary number satisfying the condition $\lambda<\sigma<\min (\rho, 1)$. Then the function $V(r)=T(r, f)$ satisfies the conditions $(\alpha)$ and $(\beta)$ of Lemma 4.6(A), and therefore, for each $\varepsilon, 0<\varepsilon<1 / 4$, we can find sequences $\left\{r_{k}\right\}$ and $\left\{R_{k}\right\}$ satisfying (4.20),(a),(b). In what follows we assume

$$
r=r_{k}, 2 R=R_{k} .
$$

Note that then

$$
T(t, f) \leq\left(\frac{t}{r}\right)^{\sigma} T(r, f), 1 \leq t \leq 2 R \text { and } \frac{r}{R}<2 \varepsilon,
$$

and the inequality (4.24) implies

$$
T(r, f) \leq T\left(r, \check{\alpha}_{R, 0}\right)+2 K_{1} \varepsilon^{1-\sigma} T(r, f) .
$$

To estimate $T\left(r, \check{\alpha}_{R, 0}\right)$ we use Lemma 4.5.

Suppose that there is an infinite sequence of pairs $(r, R)$ satisfying the conditions (4.25), and such that

$$
T\left(r, \check{\alpha}_{R, 0}\right)=N\left(r, \check{\alpha}_{R, 0}\right) .
$$

Since for $r \leq R$ we have

$$
N\left(r, \check{\alpha}_{R, 0}\right)=N(r, f),
$$

by $(4.27)$ we get

$$
T(r, f) \leq N(r, f)+2 K_{1} \varepsilon^{1-\sigma} T(r, f),
$$

whence

$$
\limsup _{r \rightarrow \infty} \frac{N(r, f)}{T(r, f)} \geq 1-2 K_{1} \varepsilon^{1-\sigma},
$$

hence, a fortiori,

$$
\kappa(f) \geq 1-2 K_{1} \varepsilon^{1-\sigma} .
$$

Letting $\varepsilon$ tend to 0 , we get $\kappa(f) \geq 1$, and the inequality (4.6) is proved in this case. Similarly, the inequality (4.6) holds also in the case when there is an infinite sequence of pairs $(r, R)$, satisfying the conditions $(4.25)$, such that

$$
T\left(r, \check{\alpha}_{R, 0}\right)=N\left(r, 1 / \check{\alpha}_{R, 0}\right) .
$$


If there is neither an infinite sequence of pairs $(r, R)$ satisfying (4.25) and (4.28), nor an infinite sequence of pairs satisfying (4.25) and (4.29), then by Lemma 4.5 for all pairs $(r, R)$ satisfying (4.25) for $k \geq k_{0}$ there is $\beta=\beta(r), 0<\beta<\pi$, such that the equality

$$
\begin{aligned}
T\left(r, \check{\alpha}_{R, 0}\right)= & \int_{0}^{\infty} N\left(t, 1 / \check{\alpha}_{R, 0}\right) K(t, r, \beta) d t \\
& +\int_{0}^{\infty} N\left(t, \check{\alpha}_{R, 0}\right) K(t, r, \pi-\beta) d t=I_{1}+I_{2}
\end{aligned}
$$

holds. Since for $t \leq R$ the equality $N\left(t, \check{\alpha}_{R, 0}\right)=N(t, f)$ holds, and for $t>R$ we have

$$
\begin{aligned}
N\left(t, \check{\alpha}_{R, 0}\right) & =N(R, f)+n(R, f) \ln \frac{t}{R} \\
& \leq T(R, f)+2 T(2 R, f) \ln \frac{t}{R} \leq 2 T(2 R, f) \ln \frac{e t}{R},
\end{aligned}
$$

we conclude

$$
I_{2} \leq \int_{0}^{R} N(t, f) K(t, r, \pi-\beta) d t+2 T(2 R, f) \int_{R}^{\infty} \ln \left(\frac{e t}{R}\right) K(t, r, \pi-\beta) d t
$$

and, observing that for $t \geq R(\geq 2 r)$ the inequality

$$
K(t, r, \beta) \leq \frac{1}{\pi} \frac{r \sin \beta}{(r-t)^{2}} \leq 2 \frac{r}{t^{2}}
$$

holds, and

$$
\int_{R}^{\infty} t^{-2} \ln \left(\frac{e t}{R}\right) d t=\frac{2}{R}
$$

we get

$$
I_{2} \leq \int_{0}^{R} N(t, f) K(t, r, \pi-\beta) d t+8 \frac{r}{R} T(2 R, f) .
$$

Similarly we get

$$
I_{1} \leq \int_{0}^{R} N\left(t, \frac{1}{f}\right) K(t, r, \beta) d t+8 \frac{r}{R} T(2 R, f) .
$$

The combination of (4.27), (4.30), (4.31), (4.32), and (4.26) implies the inequality

$$
\begin{aligned}
T(r, f) \leq & \int_{0}^{R} N\left(t, \frac{1}{f}\right) K(t, r, \beta) d t \\
& +\int_{0}^{R} N(t, f) K(t, r, \pi-\beta) d t+\left(2 K_{1}+32\right) \varepsilon^{1-\sigma} T(r, f) .
\end{aligned}
$$

Let $\gamma=\min (\beta, \pi-\beta)$. Then, obviously, $0<\gamma \leq \frac{\pi}{2}$ and

$$
K(t, r, \gamma)=\max (K(t, r, \beta), K(t, r, \pi-\beta)) .
$$

Therefore (4.33) implies

$$
T(r, f) \leq \int_{0}^{R} N(t ; 0, \infty) K(t, r, \gamma) d t+\left(2 K_{1}+32\right) \varepsilon^{1-\sigma} T(r, f) .
$$


Let $\kappa^{\prime}>\kappa(f)$. Then there is $R_{0} \geq 1$, such that for $t \geq R_{0}$ the inequality $N(t ; 0, \infty) \leq \kappa^{\prime} T(t, f)$ holds. Taking into account (4.26), we derive from (4.35) the inequality

$$
\begin{aligned}
T(r, f) \leq & \kappa^{\prime} T(r, f) \int_{R_{0}}^{R}\left(\frac{t}{r}\right)^{\sigma} K(t, r, \gamma) d t \\
& +\frac{N\left(R_{0} ; 0, \infty\right) r R_{0}}{\pi\left(r-R_{0}\right)^{2}}+\left(2 K_{1}+32\right) \varepsilon^{1-\sigma} T(r, f) .
\end{aligned}
$$

Next we observe that

$$
\int_{R_{0}}^{R}\left(\frac{t}{r}\right)^{\sigma} K(t, r, \gamma) d t \leq \int_{0}^{\infty}\left(\frac{t}{r}\right)^{\sigma} K(t, r, \gamma) d t=\int_{0}^{\infty} u^{\sigma} K(u, 1, \gamma) d u
$$

and that using the Residue Calculus, it is easy get

$$
\int_{0}^{\infty} u^{\sigma} K(u, 1, \varphi) d u=\frac{\sin (\pi-\varphi) \sigma}{\sin \pi \sigma}, 0<\varphi<\pi,-1<\sigma<1 .
$$

Therefore (4.36) implies the inequality

$$
1 \leq \kappa^{\prime} \frac{\sin (\pi-\gamma) \sigma}{\sin \pi \sigma}+\left(2 K_{1}+32\right) \varepsilon^{1-\sigma}+\frac{N\left(R_{0} ; 0, \infty\right) r R_{0}}{\pi\left(r-R_{0}\right)^{2} T(r, f)}
$$

Letting $r$ tend to $\infty$ in this inequality, and then letting $\varepsilon$ tend to 0 , we get

$$
1 \leq \kappa^{\prime} \max _{0 \leq \gamma \leq \frac{\pi}{2}} \frac{\sin (\pi-\gamma) \sigma}{\sin \pi \sigma}
$$

But

$$
\max _{0 \leq \gamma \leq \frac{\pi}{2}} \frac{\sin (\pi-\gamma) \sigma}{\sin \pi \sigma}=\left\{\begin{array}{ll}
1, & 0<\sigma \leq \frac{1}{2} \\
\frac{1}{\sin \pi \sigma}, & \frac{1}{2}<\sigma<1
\end{array}\right\}=\frac{1}{\nu(\sigma)},
$$

therefore (4.38) can be rewritten in the form

$$
\kappa^{\prime} \geq \nu(\sigma) \text {. }
$$

Here $\sigma$ is an arbitrary number satisfying the inequality $\lambda<\sigma<\min (\rho, 1)$, and $\kappa^{\prime}$ is an arbitrary number satisfying the inequality $\kappa^{\prime}>\kappa(f)$. Therefore the validity of (4.40) implies the validity of (4.6).

CASE (b). We prove that for each function $f(z), f(0)=1$, of order $\rho<1$ the inequality $\kappa(f) \geq \nu(\rho)$ holds (we shall not use the condition $\lambda=\rho$ ). First we assume in addition that all zeros of the function $f(z)$ are positive, and all of its poles are negative. Then, since $f(z)$ has genus zero, we can apply Lemma 4.5.

If there is an infinite sequence of values of $r$ for which at least one of the equalities

$$
T(r, f)=N(r, f), T(r, f)=N\left(r, \frac{1}{f}\right)
$$

is valid, then $\kappa(f) \geq 1$, and hence (4.6) holds. Therefore, by Lemma 4.5, we may assume that for all sufficiently large $r$ there is $\beta=\beta(r), 0<\beta(r)<\pi$, such that the inequality (4.16) holds. Letting, as in the case (a), $\gamma=\min (\beta, \pi-\beta)$, and using (4.34) we get

$$
T(t, f) \leq \int_{0}^{\infty} N(t ; 0, \infty) K(t, r, \gamma) d t
$$


Let $\varepsilon, 0<\varepsilon<\min \left(\frac{1}{4}, 1-\rho\right)$ be an arbitrary prescribed number. Let $V(r)=$ $T(r, f)$ in Lemma 4.6(B) and denote by $\left\{r_{k}\right\}$ the sequence whose existence is established in the lemma. In what follows we assume that $r$ takes values belonging to the sequence $\left\{r_{k}\right\}$ only. Note that by Lemma 4.6(B) we have

$$
\begin{gathered}
T(t, f) \leq\left(\frac{t}{r}\right)^{\rho-\varepsilon} T(r, f), 1 \leq t \leq r, \\
T(t, f) \leq\left(\frac{t}{r}\right)^{\rho+\varepsilon} T(r, f), r \leq t<\infty .
\end{gathered}
$$

Let $\kappa^{\prime}>\kappa(f)$ and let $R_{0} \geq 1$ be such that for $t \geq R_{0}$ the inequality $N(t ; 0, \infty) \leq$ $\kappa^{\prime} T(t, f)$ holds. Taking into account $(4.42),\left(4.42^{\prime}\right)$, we get from (4.41) the inequality

$$
\begin{aligned}
T(r, f) \leq & \kappa^{\prime} T(r, f)\left\{\int_{R_{0}}^{r}\left(\frac{t}{r}\right)^{\rho-\varepsilon} K(t, r, \gamma) d t\right. \\
& \left.+\int_{r}^{\infty}\left(\frac{t}{r}\right)^{\rho+\varepsilon} K(t, r, \gamma) d t\right\}+\frac{N\left(R_{0} ; 0, \infty\right) r R_{0}}{\pi\left(r-R_{0}\right)^{2}} .
\end{aligned}
$$

We estimate the expression in braces. We have

$$
\begin{aligned}
& \int_{R_{0}}^{r}\left(\frac{t}{r}\right)^{\rho-\varepsilon} K(t, r, \gamma) d t+\int_{r}^{\infty}\left(\frac{t}{r}\right)^{\rho+\varepsilon} K(t, r, \gamma) d t \\
& \quad=\int_{R_{0} / r}^{1} u^{\rho-\varepsilon} K(u, 1, \gamma) d u+\int_{1}^{\infty} u^{\rho+\varepsilon} K(u, 1, \gamma) d u \\
& \quad \leq \int_{0}^{\infty} u^{\rho+\varepsilon} K(u, 1, \gamma) d u+\int_{0}^{1}\left(u^{\rho-\varepsilon}-u^{\rho+\varepsilon}\right) K(u, 1, \gamma) d u .
\end{aligned}
$$

By virtue of $\left(4.37^{\prime}\right)$ we have

$$
\int_{0}^{\infty} u^{\rho+\varepsilon} K(u, 1, \gamma) d u=\frac{\sin (\pi-\gamma)(\rho+\varepsilon)}{\sin \pi(\rho+\varepsilon)} .
$$

Next, since $1-u^{2 \varepsilon}<8 \varepsilon u^{-\frac{1}{4}}, u^{\rho-\varepsilon}<u^{-\frac{1}{4}}$ for $0<u<1$, we get

$$
\begin{aligned}
& \int_{0}^{1}\left(u^{\rho-\varepsilon}-u^{\rho+\varepsilon}\right) K(u, 1, \gamma) d u \leq 8 \varepsilon \int_{0}^{1} u^{-\frac{1}{2}} K(u, 1, \gamma) d u \\
&<8 \varepsilon \int_{0}^{\infty} u^{-\frac{1}{2}} K(u, 1, \gamma) d u=8 \varepsilon \sin \frac{\pi-\gamma}{2} \leq 8 \varepsilon
\end{aligned}
$$

Therefore (4.43) implies

$$
1 \leq \kappa^{\prime}\left\{\frac{\sin (\pi-\gamma)(\rho+\varepsilon)}{\sin \pi(\rho+\varepsilon)}+8 \varepsilon\right\}+\frac{N\left(R_{0} ; 0, \infty\right) r R_{0}}{\pi\left(r-R_{0}\right)^{2} T(r, f)} .
$$

Letting $r$ tend to $\infty$, we get

$$
1 \leq \kappa^{\prime}\left\{\max _{0 \leq \gamma \leq \frac{\pi}{2}} \frac{\sin (\pi-\gamma)(\rho+\varepsilon)}{\sin \pi(\rho+\varepsilon)}+8 \varepsilon\right\},
$$

whence, by (4.39), it follows that

$$
1 \leq \kappa^{\prime}\left\{\frac{1}{\nu(\rho+\varepsilon)}+8 \varepsilon\right\} .
$$


Letting $\varepsilon$ tend to 0 , and then letting $\kappa^{\prime}$ tend to $\kappa(f)$, we get $\kappa(f) \geq \nu(\rho)$, Q.E.D.

Now we get rid of the assumption that all zeros of the function $f(z)$ are positive and all of its poles are negative. Let $f(z), f(0)=1$, be a meromorphic function of order $\rho<1$. We form the function $\check{f}(z)$. Obviously,

$$
N(r, f)=N(r, \check{f}), N\left(r, \frac{1}{f}\right)=N\left(r, \frac{1}{\check{f}}\right) .
$$

Since the functions $f(z)$ and $\check{f}(z)$ are quotients of canonical products, using Theorem 3.4 from Chapter 2 we get

$$
\begin{aligned}
& \rho[f]=\max \left\{\rho[N(r, f)], \rho\left[N\left(r, \frac{1}{f}\right)\right]\right\}, \\
& \rho[\check{f}]=\max \left\{\rho\left[N(r, \check{f}], \rho\left[N\left(r, \frac{1}{\check{f}}\right)\right]\right\},\right.
\end{aligned}
$$

and hence $\rho[f]=\rho[\check{f}]=\rho$. All zeros of the function $\check{f}(z)$ are positive and all of its poles are negative, therefore, since the result has been already proven in this case, $\kappa(\check{f}) \geq \nu(\rho)$. But $\kappa(f) \geq \kappa(\check{f})$, it follows from (4.44) and the inequality $T(r, f) \leq T(r, \check{f})$, which is a consequence of Lemma 4.4. The proof of Theorem 4.3 is completed.

The method used in the proof of Theorem 4.3 can be used to prove the following result about deficiencies.

THEOREM 4.4. Let $f(z)$ be a meromorphic function of lower order $\lambda<1, a \neq b$ be two numbers from the extended plane. Let

$$
u=1-\delta(a, f), v=1-\delta(b, f) .
$$

The inequality

$$
u^{2}+v^{2}-2 u v \cos \pi \lambda \geq \sin ^{2} \pi \lambda
$$

holds, where $v=1$ if $u<\cos \pi \lambda$, and $u=1$ if $v<\cos \pi \lambda$.

Observe that the equation $u^{2}+v^{2}-2 u v \cos \pi \lambda=\sin ^{2} \pi \lambda$ represents an ellipse in the $(u, v)$-plane, with $\{u=v\}$ and $\{u=-v\}$ being its axes of symmetry, inscribed into the square $\{-1 \leq u \leq 1,-1 \leq v \leq 1\}$. This ellipse is tangent to the lines $\{u=1\}$ and $\{v=1\}$ at the points $(1, \cos \pi \lambda)$ and $(\cos \pi \lambda, 1)$, respectively. For $1 / 2<\lambda<1$ both points of tangency are outside the square $Q=\{0 \leq u \leq 1,0 \leq$ $v \leq 1\}$, and the statement of Theorem 4.4 means that the point $(u, v)$ lies in the square $Q$, but not in the interior of the ellipse. For $0<\lambda \leq 1 / 2$ the statement of Theorem 4.4 means that the point $(u, v)$ either lies on one of the lines $\{u=1\}$ and $\{v=1\}$, or in the piece of the square $Q$ near the vertex $(1,1)$, which is cut off the square by the arc of the ellipse joining the points $(1, \cos \pi \lambda)$ and $(\cos \pi \lambda, 1)$ (Figure 4, (a) shows the case $0 \leq \lambda<1 / 2$, Figure 4 , (b) shows the case $1 / 2 \leq \lambda<1$ In both cases the set of possible values of $(u, v)$ is shaded.)

For $\lambda=0$ the ellipse degenerates into a line segment joining the points $(-1,-1)$ and $(1,1)$, and the statement of Theorem 4.4 means that the point $(u, v)$ lies on one of the lines $\{u=1\}$ and $\{v=1\}$. This last result was obtained earlier (see the corollary of Theorem 3.1). 
Proof. Proving Theorem 4.4 we may assume without loss of generality that $a=0, b=\infty, f(0)=1$. As in the proof of Theorem 4.3 we consider two cases: (a) $\lambda<\rho$, (b) $\lambda=\rho$.

CASE (a) Arguing in the same way as in the proof of Theorem 4.3 we arrive at the inequality (4.27). If there is a sequence of pairs $(r, R)$ satisfying the conditions (4.25) and (4.28), then $\left(4.28^{\prime}\right)$ implies $\delta(\infty, f) \leq 2 K_{1} \varepsilon^{1-\sigma}$, and, since $\varepsilon$ can be chosen arbitrarily small, $\delta(\infty, f)=0, v=1-\delta(\infty, f)=1$. Similarly, if there is a sequence of pairs $(r, R)$ satisfying the conditions $(4.25)$ and $(4.29)$, then $\delta(0, f)=0$, $u=1$. It is clear that points of the form $(u, 1)$ and $(1, v)$ satisfy the conditions of Theorem 4.4 .

If there is neither a sequence of pairs $(r, R)$ satisfying the conditions (4.25) and (4.28), nor a sequence of pairs satisfying the conditions (4.25) and (4.29), then, arguing in the same way as in the proof of Theorem 4.3, we arrive at the inequality (4.33).

Let $X$ and $Y$ be an arbitrary pair of numbers satisfying the conditions $X>u$, $Y>v$. Then there is $R_{0} \geq 1$ such that for $t \geq R_{0}$ the inequalities $N\left(t, \frac{1}{f}\right) \leq$ $X T(t, f), N(t, f) \leq Y T(t, f)$ hold. Taking into account (4.26) and (4.33) we get

$$
\begin{aligned}
T(r, f) \leq & X T(r, f) \int_{R_{0}}^{R}\left(\frac{t}{r}\right)^{\sigma} K(t, r, \beta) d t \\
& +Y T(r, f) \int_{R_{0}}^{R}\left(\frac{t}{r}\right)^{\sigma} K(t, r, \pi-\beta) d t+\frac{N\left(R_{0} ; 0, \infty\right) r R_{0}}{\pi\left(r-R_{0}\right)^{2}} \\
& +\left(2 K_{1}+32\right) \varepsilon^{1-\sigma} T(r, f),
\end{aligned}
$$

where $r$ and $R$ satisfy the condition (4.25).

Hence, using (4.37) and $\left(4.37^{\prime}\right)$, we have

$$
1 \leq X \frac{\sin (\pi-\beta) \sigma}{\sin \pi \sigma}+Y \frac{\sin \beta \sigma}{\sin \pi \sigma}+\left(2 K_{1}+32\right) \varepsilon^{1-\sigma}+\frac{N\left(R_{0} ; 0, \infty\right) r R_{0}}{\pi\left(r-R_{0}\right)^{2} T(r, f)}
$$

Since

$$
\begin{aligned}
X \sin (\pi & -\beta) \sigma+Y \sin \beta \sigma \\
& =(Y-X \cos \pi \sigma) \sin \beta \sigma+(X \sin \pi \sigma) \cos \beta \sigma \\
& \leq\left\{(Y-X \cos \pi \sigma)^{2}+(X \sin \pi \sigma)^{2}\right\}^{1 / 2}=\left\{X^{2}+Y^{2}-2 X Y \cos \pi \sigma\right\}^{1 / 2},
\end{aligned}
$$

the inequality (4.46) implies

$$
1 \leq \frac{1}{\sin \pi \sigma}\left\{X^{2}+Y^{2}-2 X Y \cos \pi \sigma\right\}^{1 / 2}+\left(2 K_{1}+32\right) \varepsilon^{1-\sigma}+\frac{N\left(R_{0} ; 0, \infty\right) r R_{0}}{\pi\left(r-R_{0}\right)^{2} T(r, f)} .
$$

Letting $r$ tend to $\infty$ in this inequality, and then $\varepsilon$ tend to 0 and $\sigma$ to $\lambda$, we get

$$
\sin ^{2} \pi \lambda \leq X^{2}+Y^{2}-2 X Y \cos \pi \lambda .
$$

Since $X$ and $Y$ are arbitrary numbers satisfying the inequalities $X>u$ and $Y>v$, the inequality (4.45) is proved.

The fact that $v=1$ for $u<\cos \pi \lambda$ and that $u=1$ for $v<\cos \pi \lambda$ follows immediately from the corollary of Theorem 3.1

The case (b) can be treated in the same way as the case (b) in Theorem 4.3, we leave the details to the reader. 
It turns out that Theorem 4.4 cannot be made more precise, that is, for each pair $u, v$ of numbers, $0 \leq u, v \leq 1$, satisfying the conditions described in the statement of the theorem, there exists a meromorphic function $f(z)$ of lower order $\lambda$ such that $\delta(a, f)=1-u, \delta(b, f)=1-v$. We do not prove this result in the general case, referring to the paper [EF60]. We give only the following example.

ExAmPLE. Let numbers $\rho$ and $\lambda, 0<\lambda \leq \rho<1$, and $u$ and $v, 0 \leq u, v \leq 1$, $u^{2}+v^{2}-2 u v \cos \pi \lambda=\sin ^{2} \pi \lambda$ be given. If $\lambda<1 / 2$, we assume in addition that $u, v \geq \cos \pi \lambda$. We shall find a meromorphic function $f(z)$ of order $\rho$ and lower order $\lambda$, such that

$$
\delta(0, f)=1-u, \delta(\infty, f)=1-v
$$

In the case $u \geq v$, the function $f_{u v}(z)$ considered in Example 1 from Section 3 has these properties. In fact, by (3.40) we have

$$
1-\delta\left(\infty, f_{u v}\right)=\frac{v}{I(u, v, \lambda)+v}
$$

But for $u$ and $v$ satisfying the conditions described above the equality

$$
I(u, v, \lambda)=\frac{1}{\sin \pi \lambda}\left\{u^{2}+v^{2}-2 u v \cos \pi \lambda\right\}^{1 / 2}-v=1-v
$$

holds, therefore $\delta\left(\infty, f_{u v}\right)=1-v$. Next, since

$$
N\left(r, \frac{1}{f_{u v}}\right)=N(r, 0, f(z, u))=\frac{u}{l(r)} r^{l(r)}+o\left(r^{l(r)}\right),
$$

using (3.38) and (4.48) we get

$$
1-\delta\left(0, f_{u v}\right)=\limsup _{r \rightarrow \infty} \frac{N\left(r, \frac{1}{f_{u v}}\right)}{T\left(r, f_{u v}\right)}=\limsup _{r \rightarrow \infty} \frac{u}{I(u, v, l(r))+v}=\frac{u}{I(u, v, \lambda)+v}=u
$$

and, hence $\delta\left(0, f_{u v}\right)=1-u$.

If $v \leq u$, then $1 / f_{u v}(z)$ is a desired function.

If instead of the just considered restriction on $u$ and $v$, we require $u=1$, $0 \leq v<\cos \pi \lambda$, then the function $f_{u v}(z)$ also satisfies (4.47). It follows from the relation $\left(3.40^{\prime}\right)$ and the equality $\delta\left(0, f_{u v}\right)=0$, which is true by the corollary of Theorem 3.1. If $0 \leq u<\cos \pi \lambda, v=1$, then $1 / f_{u v}(z)$ is a desired function.

Theorem 4.4 also implies the following result containing both the corollary of Theorem 3.1 and the corollary of Lemma 3.4.

THEOREM 4.5. Let $f(z)$ be a meromorphic function of lower order $\lambda \leq 1 / 2$. If $\delta(a, f) \geq 1-\cos \pi \lambda, 0<\lambda \leq 1 / 2$, or $\delta(a, f)>0, \lambda=0$, then a is the only deficient value of the function $f(z)$.

By the corollary of Theorem 3.1 it is enough to consider the case $\delta(a, f)=$ $1-\cos \pi \lambda, 0<\lambda \leq 1 / 2$. Let $b \neq a$ and $u=1-\delta(a, f), v=1-\delta(b, f)$. Then $u=\cos \pi \lambda, 0 \leq v \leq 1$, and the inequality (4.45) implies $v \geq 1$. Therefore $v=1$, $\delta(b, f)=0$, Q.E.D. 


\section{The growth of a meromorphic function with exceptional values}

The presence of one, even Picard, exceptional value does not impose any restrictions onto the order and lower order of a meromorphic function. Let us show that for arbitrary $\lambda$ and $\rho$ satisfying $0 \leq \lambda \leq \rho \leq \infty$ there is an entire function of order $\rho$ and lower order $\lambda$.

Example 1. (The case $0<\lambda \leq \rho<1$.) Take a differentiable on $[1, \infty)$ function $l(r)$, such that

$$
\liminf _{r \rightarrow \infty} l(r)=\lambda, \limsup _{r \rightarrow \infty} l(r)=\rho .
$$

Let $f(z)$ be a canonical product of genus 0 with positive zeros, such that $n(r, 0) \sim r^{l(r)}$. By the formula (5.4) from Chapter 2 the function $f(z)$ has order $\rho$ and lower order $\lambda$.

ExAmPle 2. (The case $0<\lambda \leq \rho<\infty$.) Let $n \geq 2 \rho$ be a positive integer, $\lambda_{1}=\lambda / n, \rho_{1}=\rho / n$. Then $0<\lambda_{1} \leq \rho_{1} \leq 1 / 2$. Let $f(z)$ be an entire function of order $\rho_{1}$ and lower order $\lambda_{1}$. The function $f\left(z^{n}\right)$ has order $\rho$ and lower order $\lambda$.

Example 3. (The case $\lambda=0,0<\rho<\infty$.) In Section 6 of Chapter 4 we proved (p. ???) that the entire function

$$
g(z)=\prod_{k=1}^{\infty}\left\{1-\left(\frac{z}{r_{k}}\right)^{n_{k}}\right\}
$$

where $n_{k}$ are arbitrary positive integers, and $r_{k}$ are arbitrary positive integers satisfying the condition $r_{k+1} \geq 2 r_{k}, r_{1}>1$, satisfy the relation

$$
T(r, g)=N(r, 0)+O(1) .
$$

Let $r_{1}=e, r_{k+1}=e^{r_{k}}, n_{k}=\left[r_{k}^{\rho}\right]$. Then

$$
n(r, 0)=\sum_{r_{k} \leq r}\left[r_{k}^{\rho}\right]=O\left(r^{\rho}\right), n\left(r_{n}, 0\right) \sim r_{n}^{\rho}
$$

Since $\rho[N(r, 0)]=\rho[n(r, 0)]$, the function $g(z)$ has order $\rho$. Next,

$$
\begin{aligned}
N\left(r_{n}, 0\right) & =\int_{e}^{r_{n}} \frac{n(t, 0)}{t} d t \leq n\left(r_{n-1}, 0\right) \ln \frac{r_{n}}{e} \\
& =O\left(r_{n-1}^{\rho}\right) \ln \frac{r_{n}}{e}=O\left(\left(\ln r_{n}\right)^{\rho+1}\right) .
\end{aligned}
$$

Hence the lower order of the function $g(z)$ is equal to 0 .

Example 4. (The case $\lambda=0, \rho=\infty$.) Consider somewhat more general case. Let $\varphi(r)$ and $\psi(r)$ be two arbitrary non-decreasing functions, such that $\psi(r) \geq$ $\varphi(r) \geq 1$ and

$$
\lim _{r \rightarrow \infty} \frac{\varphi(r)}{\ln r}=\infty
$$

We construct an entire function $g(z)$ for which

$$
\liminf _{r \rightarrow \infty} \frac{T(r, g)}{\varphi(r)} \leq 1, \limsup _{r \rightarrow \infty} \frac{T(r, g)}{\psi(r)} \geq 1 .
$$


Select a sequence $\left\{r_{k}\right\}$ of positive numbers, such that $r_{1}>1, r_{k+1} \geq 2 r_{k}$,

$$
\sum_{k=1}^{n-1}\left[\psi\left(r_{k} e\right)\right] \ln \frac{r_{n}}{r_{k}} \leq \varphi\left(r_{n}\right) .
$$

This is possible since $\ln r=o(\varphi(r))$. Letting $n_{k}=\left[\psi\left(r_{k} e\right)\right]$, consider the function (5.1). The relation (5.3) is equivalent to

$$
N\left(r_{n}, 0\right) \leq \varphi\left(r_{n}\right) .
$$

Besides, it is clear that

$$
N\left(e r_{n}, 0\right) \geq n\left(r_{n}, 0\right) \geq\left[\psi\left(r_{n} e\right)\right] .
$$

Therefore the function (5.1) satisfies the conditions (5.2).

EXAMPLE 5. (The case $0<\lambda<\infty, \rho=\infty$.) Let $g(z)$ be an entire function of order $\infty$ and lower order $0, h_{\lambda}(z)$ be a canonical product with positive zeros, such that $n\left(r, 0, h_{\lambda}\right) \sim r^{\lambda}$. Then $\ln T\left(r, h_{\lambda}\right) \sim \lambda \ln r$, therefore the lower order of the function $f(z)=g(z) h_{\lambda}(z)$ is equal to $\lambda$, but the order of $f(z)$ is infinite.

In the cases $\lambda=\rho=0, \lambda=\rho=\infty$ the corresponding examples are trivial.

If a function $f(z)$ has two Picard exceptional values, then necessarily $\rho[f]=$ $\lambda[f]$, and it is either a positive integer or $\infty$. This result follows from the next theorem.

THEOREM 5.1. If a function $f(z)$ is such that for some $a$ and $b, a \neq b$, the inequality $\rho[N(r ; a, b)]<\rho[f]$ holds, then $\rho[f]=\lambda[f]$, and it is either a positive integer or $\infty$.

Proof. We may assume without loss of generality that $a=0, b=\infty, f(0) \neq$ $0, \infty$. Let $\pi_{1}(z)$ be a canonical product, whose zeros and their multiplicities coincide with such of the function $f(z)$, and $\pi_{2}(z)$ be a canonical product, whose zeros and their multiplicities coincide with poles and their multiplicities of $f(z)$. Then we have a representation

$$
f(z)=g(z) \frac{\pi_{1}(z)}{\pi_{2}(z)}
$$

where $g(z)$ is an entire function without zeros. Since

$$
\begin{aligned}
& T(r, f) \leq T(r, g)+T\left(r, \frac{\pi_{1}}{\pi_{2}}\right), \\
& T(r, g) \leq T(r, f)+T\left(r, \frac{\pi_{1}}{\pi_{2}}\right),
\end{aligned}
$$

and

$$
\rho\left[\frac{\pi_{1}}{\pi_{2}}\right]=\rho[N(r ; 0, \infty)],
$$

we have the inequalities

$$
\begin{aligned}
& \rho[f] \leq \max \{\rho[g], \rho[N(r ; 0, \infty]\}=\rho[g], \\
& \lambda[g] \leq \max \{\lambda[f], \rho[N(r ; 0, \infty)]\} .
\end{aligned}
$$


But $\rho[g]=\lambda[g]$, and is either an integer or $\infty$. In fact, if $\lambda[g]<\infty$, then by Lemma 6.2 from Chapter 1 we have $g(z)=e^{P(z)}$, where $P(z)$ is a polynomial, hence $\lambda[g]=\rho[g]$ and is equal to the degree of the polynomial $P(z)$.

Therefore (5.4) implies that $\rho[f] \leq \max \{\lambda[f], \rho[N(r ; 0, \infty)]\}=\lambda[f]$, whence $\rho[f]=\lambda[f]$, and is either an integer or $\infty$. It remains to observe that $\rho[f]>0$, because $\rho[f]>\rho[N(r ; 0, \infty)]$.

However, presence of two Borel exceptional values does not imply, in general, the equality $\rho[f]=\lambda[f]$, although, in this case, by Theorem 1.1 from Chapter 4 , the number $\rho[f]$ should be either a positive integer ${ }^{10}$ or $\infty$.

EXAMPLE 6 . We show that for each positive integer $\rho$ and each $\lambda, 0 \leq \lambda \leq \rho$, there exists an entire function $g_{\lambda \rho}(z)$ of order $\rho$ and lower order $\lambda$, for which zero is a Borel exceptional value.

As $g_{01}(z)$ we take the function $f(z)$ from Example 4 of Section 2 (its lower order is equal to zero by Remark 2 to that example). In the case $0<\lambda<1$ we let

$$
g_{\lambda 1}(z)=g_{01}(z) \omega_{\lambda}(z)
$$

where

$$
\omega_{\lambda}(z)=\prod_{n=1}^{\infty}\left(1-\frac{z}{n^{1 / \lambda}}\right) .
$$

Since $g_{01}(z)=f(z)$ is a function of maximal type of order 1 , and the order of $\omega_{\lambda}(z)$ is equal to $\lambda<1$, then $g_{\lambda 1}(z)$ is also of maximal type of order 1. Taking into account that $n(r, 0, f)=O(r)$, we get $n\left(r, 0, g_{\lambda 1}\right)=O(r)$, and hence $0 \in E_{B}\left(g_{\lambda 1}\right)$. Since $\ln T\left(r, \omega_{\lambda}\right) \sim \lambda \ln r$, and the lower order of the function $g_{01}(z)=f(z)$ is equal to zero, the lower order of the function $g_{\lambda 1}(z)$ is equal to $\lambda$.

Let also $g_{11}(z)=e^{z}$. Thus, the function $g_{\lambda \rho}(z)$ is constructed for $\rho=1$, $0 \leq \lambda \leq 1$. Let now $\rho$ be an arbitrary positive integer, $0 \leq \lambda \leq \rho$. Then, letting

$$
g_{\lambda \rho}(z)=g_{\mu 1}\left(z^{\rho}\right), \text { where } \mu=\lambda / \rho,
$$

we get, as is easy to see, a desired function.

If the function $f(z)$ has two deficient values, then by the Corollary of Theorem 3.1 necessarily $\lambda[f]>0$. Let us show that for arbitrary $\lambda$ and $\rho, 0<\lambda \leq \rho<\infty$, there exists a meromorphic function $f(z)$ such that $\lambda[f]=\lambda, \rho[f]=\rho, \delta(0, f)=$ $\delta(\infty, f)>0$.

ExAmple 7. First let $0<\lambda \leq \rho \leq 1 / 2$. Letting $u=v=\cos \frac{\pi \lambda}{2}$ in Example 1 from Section 4 , we get a function $f_{\lambda \rho}(z)$ of order $\rho$ and lower order $\lambda$ with $\delta\left(0, f_{\lambda \rho}\right)=\delta\left(\infty, f_{\lambda \rho}\right)=1-\cos \frac{\pi \lambda}{2}$. If $\lambda$ and $\rho$ are arbitrary numbers satisfying $0<\lambda \leq \rho<\infty$, a desired function can be obtained by selecting a positive integer $n \geq 2 \rho$ and letting

$$
f_{\lambda \rho}(z)=f_{\lambda_{1} \rho_{1}}\left(z^{n}\right), \text { where } \lambda_{1}=\lambda / n, \rho_{1}=\rho / n .
$$

It turns out that existence of two deficient values $a$ and $b, a \neq b$ with deficiencies $\delta(a, f)$ and $\delta(b, f)$ close to one, implies proximity of $\rho[f]$ and $\lambda[f]$ to some positive integer.

\footnotetext{
${ }^{10}$ The possibility $\rho[f]=0$ is excluded, since in this case $n(r ; a, b)=O(1)$ implies that $f(z)$ is a rational function.
} 
5. THE GROWTH OF A MEROMORPHIC FUNCTION WITH EXCEPTIONAL VALUES 241

We may assume without loss of generality that $a=0, b=\infty$. By (4.2) the inequality

$$
\{1-\delta(0, f)\}+\{1-\delta(\infty, f)\} \geq \kappa(f)
$$

holds. We show that smallness of $\kappa(f)$ implies the proximity of $\rho[f]$ and $\lambda[f]$ to some positive integer.

THEOREM 5.2. Let $f(z)$ be a meromorphic function of order $\rho \leq \infty$ and lower order $\lambda<\infty$, and $p$ be an integer, defined by the condition

$$
p-\frac{1}{2} \leq \lambda<p+\frac{1}{2} \text {. }
$$

If

$$
\kappa(f) \leq \frac{\varepsilon}{1.1(p+1)}, \text { where } 0 \leq \varepsilon<\frac{1}{2}
$$

then $p \geq 1$ and

$$
p-\varepsilon \leq \lambda \leq \rho \leq p+\varepsilon .
$$

Proof. By Theorem 4.1 the inequality

$$
\kappa(f) \geq \max _{\lambda \leq x \leq \rho} \nu(x)
$$

holds, therefore we have

$$
\max _{\lambda \leq x \leq \rho} \nu(x) \leq \frac{\varepsilon}{1.1(p+1)} .
$$

Since $p \geq 0$, the right-hand side of this inequality is less than one. But $\nu(x)=1$ for $0 \leq x \leq 1 / 2$, therefore (5.6) implies that $\lambda>1 / 2$ and $p \geq 1$.

Next, since

$$
\nu(x) \geq \frac{|\sin \pi x|}{2.2 x+0.5|\sin \pi x|},
$$

by (5.6) we get that the inequality

$$
\frac{|\sin \pi x|}{2.2 x+0.5|\sin \pi x|} \leq \frac{\varepsilon}{1.1(p+1)}
$$

should hold for all $x, \lambda \leq x \leq \rho$. But it does not hold for $x=p+1 / 2$, therefore $\rho<p+1 / 2$. Since for $|x-p|<1 / 2$ the inequality $|\sin \pi x| \geq 2|x-p|$ holds, we come to the conclusion that for all $x, \lambda \leq x \leq \rho$ the inequality

$$
\frac{2|x-p|}{2.2 x+|x-p|} \leq \frac{\varepsilon}{1.1(p+1)}
$$

should hold, a fortiori the inequality

$$
\frac{2|x-p|}{2.2\left(p+\frac{1}{2}\right)+\frac{1}{2}} \leq \frac{\varepsilon}{1.1(p+1)}
$$

should hold. Therefore for all $x, \lambda \leq x \leq \rho$, the inequality $|x-p| \leq \varepsilon$ holds. From here we get (5.5). 
Theorem 5.2 and the corollary of Theorem 6.5 from Chapter 4 immediately imply

Corollary. Let $f(z)$ be a meromorphic function of finite lower order $\lambda$. If

$$
\kappa(f)<\frac{1}{2.2\left(\lambda+\frac{3}{2}\right)}
$$

then the deficiencies of the function $f(z)$ are shift-invariant, that is deficiencies of $f(z+h)$ are independent of $h$.

We mention also the following theorem which can be proved with the help of Theorem 5.2.

THEOREM 5.3. Let $f(z)$ be a meromorphic function of order $\rho<\infty$ and lower order $\lambda$, and $p$ is an integer defined by the condition

$$
p-\frac{1}{2} \leq \lambda<p+\frac{1}{2}
$$

If

$$
\sum_{a \neq \infty} \delta(a, f) \geq 1-\gamma, \delta(\infty, f) \geq 1-\gamma
$$

where

$$
\gamma=\frac{\varepsilon}{5(p+1)}, 0 \leq \varepsilon<\frac{1}{2}
$$

then $p \geq 1$ and

$$
p-\varepsilon \leq \lambda \leq \rho \leq p+\varepsilon .
$$

COROLlary 1. Under the conditions of Theorem 5.3 all deficiencies are shiftinvariant.

COROLlary 2. If $f(z)$ is an entire function of finite order and

$$
\sum_{a \neq \infty} \delta(a, f)=1
$$

then $\rho[f]=\lambda[f]$ is a positive integer and all deficiencies are shift-invariant.

To prove Theorem 5.3 we need the following lemma.

LEMMA 5.1. Let $f(z)$ be a meromorphic function of finite order and let the conditions (5.7), where $0 \leq \gamma<1$, be satisfied. Then

$$
\liminf _{r \rightarrow \infty} \frac{T\left(r, f^{\prime}\right)}{T(r, f)} \geq 1-\gamma, \limsup _{r \rightarrow \infty} \frac{T\left(r, f^{\prime}\right)}{T(r, f)} \leq 1+\gamma
$$

and

$$
\kappa\left(f^{\prime}\right) \leq \frac{4 \gamma}{1-\gamma^{2}}
$$


Proof. We have

$$
\begin{aligned}
T\left(r, f^{\prime}\right)= & m\left(r, f^{\prime}\right)+N\left(r, f^{\prime}\right) \leq m(r, f)+m\left(r, \frac{f^{\prime}}{f}\right) \\
& +2 N(r, f)=T(r, f)+N(r, f)+m\left(r, \frac{f^{\prime}}{f}\right) \\
\leq & T(r, f)+(1-\delta(\infty, f)+o(1)) T(r, f)+O(\ln r)
\end{aligned}
$$

(we used Theorem 1.3 from Chapter 3). The function $f(z)$ is transcendental since a rational function cannot have more that one deficient value. Therefore $\ln r=$ $o(T(r, f))$, and we get

$$
\limsup _{r \rightarrow \infty} \frac{T\left(r, f^{\prime}\right)}{T(r, f)} \leq 2-\delta(\infty, f) \leq 1+\gamma
$$

that is, the right-hand side inequality in (5.9) is proved.

To prove the left-hand side inequality in (5.9) we use the inequality (2.5) from Chapter 3. Adding $N\left(r, \frac{1}{f^{\prime}}\right)$ to both sides of the latter, we get

$$
T\left(r, f^{\prime}\right) \geq N\left(r, \frac{1}{f^{\prime}}\right)+\sum_{\nu=1}^{q} m\left(r, a_{\nu}, f\right)+O(\ln r),
$$

where $\left\{a_{1}, \ldots, a_{q}\right\}$ is an arbitrary finite collection of (finite) complex numbers. We shall take for $a_{1}, \ldots, a_{q}$ finite deficient values of the function $f(z)$. From (5.11) we get

$$
\liminf _{r \rightarrow \infty} \frac{T\left(r, f^{\prime}\right)}{T(r, f)} \geq \liminf _{r \rightarrow \infty} \frac{N\left(r, \frac{1}{f^{\prime}}\right)}{T(r, f)}+\sum_{\nu=1}^{q} \liminf _{r \rightarrow \infty} \frac{m\left(r, a_{\nu}, f\right)}{T(r, f)} \geq \sum_{\nu=1}^{q} \delta\left(a_{\nu}, f\right) .
$$

Since $q$ is an arbitrary positive integer, we get from here the left-hand side inequality from (5.9).

Now we prove the inequality (5.10). We have

$$
\begin{aligned}
\limsup _{r \rightarrow \infty} & \frac{N\left(r, f^{\prime}\right)}{T\left(r, f^{\prime}\right)} \leq \limsup _{r \rightarrow \infty} \frac{2 N(r, f)}{T\left(r, f^{\prime}\right)} \\
& \leq \limsup _{r \rightarrow \infty} \frac{2 N(r, f)}{T(r, f)} \limsup _{r \rightarrow \infty} \frac{T(r, f)}{T\left(r, f^{\prime}\right)} \leq 2(1-\delta(\infty, f)) \frac{1}{1-\gamma} \leq \frac{2}{1-\gamma} .
\end{aligned}
$$

Next, the inequality (5.11) implies

$$
\begin{aligned}
\limsup _{r \rightarrow \infty} \frac{N\left(r, \frac{1}{f^{\prime}}\right)}{T\left(r, f^{\prime}\right)} & \leq 1-\liminf _{r \rightarrow \infty} \frac{1}{T\left(r, f^{\prime}\right)} \sum_{\nu=1}^{q} m\left(r, a_{\nu}, f\right) \\
& \leq 1-\left\{\liminf _{r \rightarrow \infty} \frac{T(r, f)}{T\left(r, f^{\prime}\right)}\right\} \liminf _{r \rightarrow \infty} \sum_{\nu=1}^{q} \frac{m\left(r, a_{\nu}, f\right)}{T(r, f)} \\
& \leq 1-\left\{\liminf _{r \rightarrow \infty} \frac{T(r, f)}{T\left(r, f^{\prime}\right)}\right\} \sum_{\nu=1}^{q} \delta\left(a_{\nu}, f\right) .
\end{aligned}
$$


Since $q$ can be chosen arbitrarily, we get

$$
\begin{aligned}
\limsup _{r \rightarrow \infty} \frac{N\left(r, \frac{1}{f^{\prime}}\right)}{T\left(r, f^{\prime}\right)} & \leq 1-\left\{\liminf _{r \rightarrow \infty} \frac{T(r, f)}{T\left(r, f^{\prime}\right)}\right\} \sum_{a \neq \infty} \delta(a, f) \\
& \leq 1-\frac{1}{1+\gamma}(1-\gamma)=\frac{2 \gamma}{1+\gamma} .
\end{aligned}
$$

Thus

$$
\kappa\left(f^{\prime}\right)=\limsup _{r \rightarrow \infty} \frac{N\left(r, \frac{1}{f^{\prime}}\right)+N\left(r, f^{\prime}\right)}{T\left(r, f^{\prime}\right)} \leq \frac{2 \gamma}{1+\gamma}+\frac{2 \gamma}{1-\gamma}=\frac{4 \gamma}{1-\gamma^{2}} .
$$

Proof. Now let $f(z)$ be a function satisfying the conditions of Theorem 5.3. Then by (5.9) we get $\ln T\left(r, f^{\prime}\right) \sim \ln T(r, f)$ and hence, $\lambda\left[f^{\prime}\right]=\lambda[f]=\lambda, \rho\left[f^{\prime}\right]=$ $\rho[f]=\rho$. By (5.10) and (5.8) we have $\kappa\left(f^{\prime}\right) \leq \frac{\varepsilon}{1.1(p+1)}$, therefore Theorem 5.2 is applicable to $f^{\prime}(z)$, and we conclude that $p \geq 1, p-\varepsilon \leq \lambda \leq \rho \leq p+\varepsilon$.

REMARK 1. It is not known whether the condition $\rho<\infty$ in Theorem 5.3 is essential. $^{11}$

Remark 2. Performing a linear-fractional transformation on the function $f(z)$, we see that the condition (5.7) in Theorem 5.3 can be replaced by the condition

$$
\sum_{a \neq b} \delta(a, f) \geq 1-\gamma, \delta(b, f) \geq 1-\gamma
$$

where $b$ is an arbitrary number from the extended complex plane.

In connection with Theorem 5.3 the question arises: whether the proximity of the sum of all deficiencies $\sum_{a} \delta(a, f)$ to 2 implies the proximity of $\rho[f]$ and $\lambda[f]$ to a positive integer. It turns out that this question has a negative answer.

EXAMPLE 8. For each integer $q \geq 3$ there exists a meromorphic function $f(z)$ such that ${ }^{12}$

$$
\sum_{a} \delta(a, f)=2, \rho[f]=\frac{q}{2}
$$

Let

$$
I_{n}(z)=\sum_{k=0}^{\infty} \frac{\left(\frac{1}{2} z\right)^{n+2 k}}{k ! \Gamma(n+k+1)},|\arg z|<\pi,
$$

be the Bessel function with purely imaginary argument. We assume that the parameter $n$ is real and $|n|<1 / 2$. Then for each $\varepsilon, 0<\varepsilon<\pi / 2$, the following asymptotic formulas hold uniformly with respect to $\arg z$ in the region $\left\{|\arg z|<\frac{\pi}{2}-\varepsilon\right\}$ (see [WW96, 17.7]).

$$
I_{n}(z) \sim \frac{e^{z}}{(2 \pi z)^{1 / 2}}, z \rightarrow \infty
$$

\footnotetext{
${ }^{11}$ Toda was able to remove this condition.

${ }^{12}$ Other examples of functions with such properties will be given in Section 5 of Chapter VII.
} 


$$
I_{-n}(z)-I_{n}(z) \sim 2 \sin n \pi \frac{e^{-z}}{2 \pi z)^{1 / 2}}, z \rightarrow \infty .
$$

For given integer $q \geq 3$ let

$$
w_{1}(z)=z^{1 / 2} I_{1 / q}\left(2 z^{q / 2}\right), w_{2}(z)=z^{1 / 2} I_{-1 / q}\left(2 z^{q / 2}\right) .
$$

Since

$$
w_{1}(z)=z \sum_{k=0}^{\infty} \frac{z^{k q}}{k ! \Gamma\left(\frac{1}{q}+k+1\right)}, w_{2}(z)=\sum_{k=0}^{\infty} \frac{z^{k q}}{k ! \Gamma\left(-\frac{1}{q}+k+1\right)},
$$

the functions $w_{1}(z)$ and $w_{2}(z)$ are entire. By (5.12) and (5.13) for each $\varepsilon, 0<\varepsilon<$ $\pi / q$ the asymptotic formulas

$$
\begin{gathered}
w_{1}(z) \sim z^{1 / 2} \frac{e^{2 z^{q / 2}}}{\left(4 \pi z^{q / 2}\right)^{1 / 2}}, w_{2}(z) \sim z^{1 / 2} \frac{e^{2 z^{q / 2}}}{\left(4 \pi z^{q / 2}\right)^{1 / 2}}, \\
w_{2}(z)-w_{1}(z) \sim z^{1 / 2} 2 \sin \frac{\pi}{q} \frac{e^{-2 z^{q / 2}}}{\left(4 \pi z^{q / 2}\right)^{1 / 2}}, z \rightarrow \infty
\end{gathered}
$$

hold uniformly in $\arg z$ in the region $\left\{|\arg z|<\frac{\pi}{q}-\varepsilon\right\}$. We need the relations

$$
\begin{gathered}
T\left(r, w_{l}\right)=\frac{4 r^{q / 2}}{\pi}+o\left(r^{q / 2}\right), l=1,2, \\
m\left(r, \frac{w_{1}}{w_{2}}\right)=o\left(r^{q / 2}\right), \\
m\left(r, \frac{w_{2}}{w_{1}-w_{2}}\right) \geq \frac{8 r^{q / 2}}{q \pi}+o\left(r^{q / 2}\right) .
\end{gathered}
$$

To prove these relations observe that $(5.15)$ and $\left(5.15^{\prime}\right)$ imply that for each $\varepsilon$, $0<\varepsilon<\frac{\pi}{q}$, uniformly in $\varphi,|\varphi|<\frac{\pi}{q}-\varepsilon$, the relations

$$
\begin{array}{r}
\ln \left|w_{l}\left(r e^{i \varphi}\right)\right|=2 r^{q / 2} \cos \frac{q}{2} \varphi+O(\ln r), l=1,2, \\
\ln \left|\frac{w_{2}\left(r e^{i \varphi}\right)}{w_{1}\left(r e^{i \varphi}\right)-w_{2}\left(r e^{i \varphi}\right)}\right|=4 r^{q / 2} \cos \frac{q}{2} \varphi+O(\ln r)
\end{array}
$$

hold. From (5.14) we see that $M\left(r, w_{l}\right)=w_{l}(r), l=1,2,0 \leq r<\infty$, and hence $T\left(r, w_{l}\right) \leq \ln ^{+} M\left(r, w_{l}\right)=2 r^{q / 2}+O(\ln r)$. By (5.14) we have also

$$
w_{1}(\omega z)=\omega w_{1}(z), w_{2}(\omega z)=w_{2}(z),
$$

where $\omega=\exp \{2 \pi i / q\}$, therefore

$$
\begin{gathered}
T\left(r, w_{l}\right)=m\left(r, w_{l}\right)=\frac{q}{2 \pi} \int_{-\pi / q}^{\pi / q} \ln ^{+}\left|w_{l}\left(r e^{i \varphi}\right)\right| d \varphi, \\
m\left(r, \frac{w_{1}}{w_{2}}\right)=\frac{q}{2 \pi} \int_{-\pi / q}^{\pi / q} \ln ^{+}\left|\frac{w_{1}\left(r e^{i \varphi}\right)}{w_{2}\left(r e^{i \varphi}\right)}\right| d \varphi .
\end{gathered}
$$


Using (5.17) and Theorem 7.4 from Chapter 1 we get

$$
\begin{gathered}
T\left(r, w_{l}\right)=2 r^{q / 2} \frac{q}{2 \pi} \int_{-\pi / q}^{\pi / q} \cos \frac{q}{2} \varphi d \varphi+o\left(r^{q / 2}\right)=\frac{4 r^{q / 2}}{\pi}+o\left(r^{q / 2}\right), \\
m\left(r, \frac{w_{1}}{w_{2}}\right)=o\left(r^{q / 2}\right), \\
m\left(r, \frac{w_{2}}{w_{1}-w_{2}}\right) \geq \frac{1}{2 \pi} \int_{-\pi / q}^{\pi / q} \ln ^{+}\left|\frac{w_{2}\left(r e^{i \varphi}\right)}{w_{1}\left(r e^{i \varphi}\right)-w_{2}\left(r e^{i \varphi}\right)}\right| d \varphi \\
=4 r^{q / 2} \frac{1}{2 \pi} \int_{-\pi / q}^{\pi / q} \cos \frac{q}{2} \varphi d \varphi+o\left(r^{q / 2}\right)=\frac{8 r^{q / 2}}{q \pi}+o\left(r^{q / 2}\right) .
\end{gathered}
$$

Consider the meromorphic function

$$
f(z)=\frac{w_{1}(z)}{w_{2}(z)} .
$$

We show that $\rho[f]=q / 2, \delta\left(\omega^{k}, f\right)=2 / q(k=0,1, \ldots, q-1)$. This indicates that $f(z)$ is a desired example.

We have

$$
\begin{aligned}
T(r, f) & =N(r, f)+m(r, f) \leq N\left(r, \frac{1}{w_{2}}\right)+m\left(r, \frac{w_{1}}{w_{2}}\right) \\
& \leq T\left(r, w_{2}\right)+o\left(r^{q / 2}\right)=\frac{4 r^{q / 2}}{\pi}+o\left(r^{r / 2}\right) .
\end{aligned}
$$

Next,

$$
m\left(r, \frac{1}{f-1}\right)=m\left(r, \frac{w_{2}}{w_{1}-w_{2}}\right) \geq \frac{8 r^{q / 2}}{q \pi}+o\left(r^{q / 2}\right) .
$$

The inequalities (5.19) and (5.20) imply that $\rho[f]=q / 2, \delta(1, f) \geq 2 / q$.

By (5.18) we have $f\left(\omega^{-k} z\right)=\omega^{-k} f(z), k=0,1, \ldots, q-1$. As it was observed at the end of Section 1 of Chapter 4 (p. ???) deficiencies of the functions $f(z)$ and $f(a z), a \neq 0$ coincide. Therefore $\delta(1, f(z))=\delta\left(1, f\left(\omega^{-k} z\right)\right)=\delta\left(1, \omega^{-k} f(z)\right)$. But, obviously, $\delta(1, a f(z))=\delta\left(a^{-1}, f(z)\right), a \neq 0$, and hence

$$
\delta\left(\omega^{k}, f\right)=\delta(1, f) \geq 2 / q, k=0,1, \ldots, q-1 .
$$

Since $\sum_{k=0}^{q-1} \delta\left(\omega^{k}, f\right)=q \delta(1, f) \leq 2$, there is an equality in (5.21), Q.E.D.

Another question arising in connection with Theorem 5.3 is the the following. What can be said about the connection between $\rho[f]$ and $\lambda[f]$ in the case when $f(z)$ has at least two deficient values with one of the deficiencies close to one? The answer to this question is unknown. It is possible that such relation does not exist at all and all values of $\rho[f]$ and $\lambda[f]$ satisfying the condition $1 / 2 \leq \lambda[f] \leq \rho[f] \leq \infty$ are admissible. The last sentence is supported by following example.

ExAmple 9. There exists an entire function $f(z)$ of order $\rho$ and lower order $\lambda, 1 / 2<\lambda \leq \rho<\infty$, such that $\delta(0, f)>0$. 
Note that the restriction $\lambda>1 / 2$ cannot be dropped (see Corollary 1 from Theorem 1.3 and Theorem 4.5). It is an open problem whether the condition $\rho<\infty$ can be dropped. ${ }^{13}$

Let $\alpha$ be a given number satisfying $1 / 2<\alpha<\min (1, \lambda)$, and $n$ be a positive integer satisfying $n>\rho$. Suppose that there exists an entire function $g(z)$ having the following properties:

(a) The function $g(z)$ can be represented by a power series of the form

$$
g(z)=1+c_{n} z^{n}+c_{n+1} z^{n+1}+\ldots
$$

with real coefficients and $c_{n}<0$;

(b) $|g(x)|<1$ for $0<x<\infty$;

(c) There exists $\eta, 0<\eta<\frac{\pi}{2}|\cot \pi \alpha|$, such that for $r>R_{0}(\eta), 0 \leq \varphi \leq 2 \pi$, the inequality

$$
\ln \mid g\left(r e^{i \varphi}\right)<r^{\alpha}\left\{\eta+\frac{\pi \cos \alpha(\varphi-\pi)}{\sin \pi \alpha}\right\}
$$

holds.

We postpone the proof of existence of such function $g(z)$ because we do not want to interrupt the principal argument.

Let $r_{k}$ be a sequence satisfying $1<r_{1} \leq r_{2} \leq r_{3} \leq \ldots, r_{k} \rightarrow \infty$ as $k \rightarrow \infty$, and such that

$$
\sum_{k=1}^{\infty} r_{k}^{-n}<\infty
$$

By Theorem 3.2 from Chapter 2 the absolutely convergent infinite product

$$
f(z)=\prod_{k=1}^{\infty} g\left(\frac{z}{r_{k}}\right)
$$

represents an entire function. Denote by $\nu(r)$ the number of points $r_{k}$ on the segment $[0, r]$. Since (5.23) implies that $\ln M(r, g)=O\left(r^{\alpha}\right)$, by Theorem 3.3 from Chapter 2 we get

$$
\ln M(r, f)<C \int_{0}^{\infty} \frac{\nu(r \tau)}{\tau^{1+\alpha}} \frac{d \tau}{1+\tau^{n-\alpha}}, 0<r<\infty,
$$

where $C$ is a positive constant depending on $g(z)$ only.

The representation (5.22) implies that

$$
\ln \left|g\left(r e^{i \varphi}\right)\right|=c_{n} r^{n} \cos n \varphi+O\left(r^{n+1}\right)
$$

as $r \rightarrow \infty$, whence there exist $R_{1}, 0<R_{1}<R_{0}$, and $\varphi_{1}, 0<\varphi_{1}<\frac{\pi}{2 n}$, such that for all $r \in\left[0, R_{1}\right]$ and $|\varphi| \leq \varphi_{1}$ the inequality

$$
\ln \left|g\left(r e^{i \varphi}\right)\right| \leq-C_{1} r^{n}
$$

holds (we use the notation $C_{j}$ for positive constants depending on $g(z)$ only). The inequality (5.23) implies the existence of $R_{2}>R_{0}$ and $\varphi_{2}, 0<\varphi_{2}<\pi\left(1-\frac{1}{2 \alpha}\right)$

\footnotetext{
${ }^{13}$ There exist similar examples with $\rho=\infty$, L. Kotman, An entire function with irregular growth and more than one deficient value. Complex analysis Joensuu, Lect. Notes. Math., 747, Springer, Berlin, 1979.
} 
such that for all $r>R_{2}$ and $|\varphi| \leq \varphi_{2}$ the inequality

$$
\ln \left|g\left(r e^{i \varphi}\right)\right| \leq-C_{2} r^{\alpha}
$$

holds. If $x \in\left[R_{1}, R_{2}\right]$, by the condition (b) we have $\ln |g(x)|<0$. Since the function $\left|g\left(r e^{i \varphi}\right)\right|$ is uniformly continuous for $r \in\left[R_{1}, R_{2}\right], \varphi \in[-\pi, \pi]$, there exists $\varphi_{3}, 0<\varphi_{3}<\pi$, such that for all $r \in\left[R_{1}, R_{2}\right]$ and $|\varphi| \leq \varphi_{3}$ the estimate

$$
\ln \left|g\left(r e^{i \varphi}\right)\right| \leq-C_{3}
$$

holds. Combining (5.26), (5.27), and (5.28) we get that for all $r, 0 \leq r<\infty$, and $\varphi,|\varphi| \leq \varphi_{0}=\min \left(\varphi_{1}, \varphi_{2}, \varphi_{3}\right)$, the inequality

$$
\ln \left|g\left(r e^{i \varphi}\right)\right| \leq-C_{4} \Psi(r), \Psi(r)=\frac{r^{n}}{1+r^{n-\alpha}}
$$

holds. Hence, for $|\varphi| \leq \varphi_{0}$, we have

$$
\ln \left|f\left(r e^{i \varphi}\right)\right| \leq-C_{4} \sum_{k=1}^{\infty} \Psi\left(\frac{r}{r_{k}}\right)=-C_{4} \Phi(r)
$$

and

$$
m(r, 0, f) \geq \frac{1}{2 \pi} \int_{-\varphi_{0}}^{\varphi_{0}} \ln \frac{1}{\left|f\left(r e^{i \varphi}\right)\right|} d \varphi \geq \frac{\varphi_{0}}{\pi} C_{4} \Phi(r) .
$$

Taking into account the following estimate of $\Phi(r)$ from below:

$$
\Phi(r) \geq C_{5} \int_{0}^{\infty} \frac{\nu(r \tau)}{\tau^{1+\alpha}} \frac{d \tau}{1+\tau^{n-\alpha}}, 0<r<\infty
$$

(the estimate was obtained in the proof of Theorem 3.3 from Chapter 2), we get

$$
m(r, 0, f) \geq C_{6} \int_{0}^{\infty} \frac{\nu(r \tau)}{\tau^{1+\alpha}} \frac{d \tau}{1+\tau^{n-\alpha}}, 0<r<\infty .
$$

Comparing (5.25) and (5.29) we find that

$$
\delta(0, f) \geq \liminf _{r \rightarrow \infty} \frac{m(r, 0, f)}{\ln M(r, f)} \geq \frac{C_{6}}{C}>0 .
$$

Thus, for each sequence $\left\{r_{k}\right\}$ satisfying the condition (5.24) we have $0 \in E_{N}(f)$.

Now we show that the sequence $\left\{r_{k}\right\}$ can be chosen in such a way that the function $f(z)$ has prescribed order $\rho$ and lower order $\lambda$. Observe that (5.25) and (5.29) imply

$$
C Q(r) \geq T(r, f) \geq C_{6} Q(r), Q(r)=\int_{0}^{\infty} \frac{\nu(r \tau)}{\tau^{1+\alpha}} \frac{d \tau}{1+\tau^{n-\alpha}},
$$

therefore the order and the lower order of the functions $T(r, f)$ and $Q(r)$ coincide.

If $\rho=\lambda$, we choose the sequence $\left\{r_{k}\right\}$ in such a way that $\nu(r)=\left[r^{\rho}\right]$ for $r \geq 2^{1 / \rho}=r_{0}$ and $\nu(r)=0$ for $0 \leq r<r_{0}$. Then for all $r \geq r_{0}$ we have $\frac{1}{2} r^{\rho} \leq \nu(r) \leq r^{\rho}$ and

$$
\frac{1}{2} r^{\rho} I(\rho)-\frac{1}{2} r^{\rho} \int_{0}^{r_{0} / r} \frac{\tau^{\rho-\alpha-1}}{1+\tau^{n-\alpha}} d \tau \leq Q(r) \leq r^{\rho} I(\rho),
$$

where

$$
I(\rho)=\int_{0}^{\infty} \frac{\tau^{\rho-\alpha-1}}{1+\tau^{n-\alpha}}<\infty
$$


and, hence, the order and lower order of the function $f(z)$ are equal to $\rho$. Though, in the case $\rho=\lambda$ there exist easier ways to construct an example (see Exercise 1 from Section 1, Chapter 4).

Now let $\lambda<\rho$. We choose a number $q_{1}>1$, such that $r^{\rho} \geq \frac{1}{2} r^{\rho}+1 \geq r^{\lambda} \geq$ $\frac{1}{2} r^{\lambda}+1$ for $r \geq q_{1}$. We define sequences $q_{k}, p_{k}, p_{k}^{\prime}, s_{k}, t_{k}, k=1,2, \ldots$, by the following recursions: $p_{k}=\exp \exp q_{k}, p_{k}^{\prime}=p_{k}^{\rho / \lambda}, q_{k+1}=\exp \exp p_{k}^{\prime}, s_{k}=\exp q_{k}$, $t_{k}=\exp p_{k}^{\prime}, k=1,2, \ldots$. It is clear that these sequences tend to $\infty$. We select a sequence $r_{k}$ in such a way that

$$
\nu(r)= \begin{cases}0 & \text { for } 0 \leq r<q_{1}, r^{\rho}>r^{\rho} \\ & \text { for } q_{k} \leq r \leq p_{k}, r^{\lambda}>r^{\lambda} \\ & \text { for } p_{k}^{\prime} \leq r<q_{k+1}, p_{k}^{\rho}>p_{k}^{\rho} \\ =\left[p_{k}^{\prime \lambda}\right] & \text { for } p_{k} \leq r \leq p_{k}^{\prime} .\end{cases}
$$

Then for all $r \geq q_{1}$ we have $\frac{1}{2} r^{\lambda} \leq \nu(r) \leq r^{\rho}$. Since $\rho<n$, the condition (5.24) is satisfied. It is easy to see that

$$
\frac{1}{2} I(\lambda) r^{\lambda}+o\left(r^{\lambda}\right)=\frac{1}{2} I(\lambda) r^{\lambda}-\frac{1}{2} r^{\lambda} \int_{0}^{q_{1} / r} \frac{\tau^{\lambda-\alpha-1}}{\tau^{n-\alpha}+1} d \tau \leq Q(r) \leq I(\rho) r^{\rho} .
$$

Hence

$$
\lambda \leq \liminf _{r \rightarrow \infty} \frac{\ln Q(r)}{\ln r} \leq \limsup _{r \rightarrow \infty} \frac{\ln Q(r)}{\ln r} \leq \rho .
$$

On the other hand

$$
\begin{aligned}
Q\left(s_{k}\right) & =\int_{0}^{\infty} \frac{\nu\left(\tau s_{k}\right) d \tau}{\tau^{1+\alpha}\left(\tau^{n-\alpha}+1\right)} \geq \int_{q_{k} / s_{k}}^{p_{k} / s_{k}} \frac{\nu\left(\tau s_{k}\right) d \tau}{\tau^{1+\alpha}\left(\tau^{n-\alpha}+1\right)} \\
& \geq \frac{1}{2} s_{k}^{\rho} \int_{q_{k} / \exp q_{k}}^{p_{k} / \ln p_{k}} \frac{\tau^{\rho-\alpha-1} d \tau}{\tau^{n-\alpha}+1}=\frac{1}{2} s_{k}^{\rho}\{I(\rho)+o(1)\} .
\end{aligned}
$$

Therefore

$$
\limsup _{r \rightarrow \infty} \frac{\ln Q(r)}{\ln r} \geq \limsup _{k \rightarrow \infty} \frac{\ln Q\left(s_{k}\right)}{\ln s_{k}} \geq \rho,
$$

together with (5.30) this shows that the order of the function $Q(r)$ is equal to $\rho$. Next,

$$
Q\left(t_{k}\right)=\left\{\int_{p_{k}^{\prime} / t_{k}}^{q_{k+1} / t_{k}}+\int_{q_{k+1} / t_{k}}^{\infty}+\int_{0}^{p_{k}^{\prime} / t_{k}}\right\} \frac{\nu\left(\tau t_{k}\right) d \tau}{\tau^{1+\alpha}\left(\tau^{n-\alpha}+1\right)} .
$$

We estimate from above each of the three integrals:

$$
\begin{gathered}
\int_{p_{k}^{\prime} / t_{k}}^{q_{k+1} / t_{k}} \frac{\nu\left(\tau t_{k}\right) d \tau}{\tau^{1+\alpha}\left(\tau^{n-\alpha}+1\right)} \leq t_{k}^{\lambda} \int_{p_{k}^{\prime} / t_{k}}^{q_{k+1} / t_{k}} \frac{\tau^{\lambda-\alpha-1} d \tau}{\tau^{n-\alpha}+1} \leq t_{k}^{\lambda} I(\lambda), \\
\int_{q_{k+1} / t_{k}}^{\infty} \frac{\nu\left(\tau t_{k}\right) d \tau}{\tau^{1+\alpha}\left(\tau^{n-\alpha}+1\right)} \leq t_{k}^{\rho} \int_{q_{k+1} / t_{k}}^{\infty} \frac{\tau^{\rho-\alpha-1} d \tau}{\tau^{n-\alpha}+1} \leq t_{k}^{\rho} \int_{q_{k+1} / t_{k}}^{\infty} \tau^{\rho-n-1} d \tau \\
=\frac{t_{k}^{\rho}}{n-\rho} \frac{t_{k}^{n-\rho}}{q_{k+1}^{n-\rho}}=\frac{1}{n-\rho} \frac{t_{k}^{n}}{\left(\exp t_{k}\right)^{n-\rho}}=o(1),
\end{gathered}
$$




$$
\begin{aligned}
\int_{0}^{p_{k}^{\prime} / t_{k}} & \frac{\nu\left(\tau t_{k}\right) d \tau}{\tau^{1+\alpha}\left(\tau^{n-\alpha}+1\right)} \leq t_{k}^{\rho} \int_{0}^{p_{k}^{\prime} / t_{k}} \frac{\tau^{\rho-\alpha-1} d \tau}{\tau^{n-\alpha}+1} \\
& \leq t_{k}^{\rho} \int_{0}^{\ln t_{k} / t_{k}} \tau^{\rho-\alpha-1} d \tau=\frac{t_{k}^{\rho}}{\rho-\alpha}\left(\frac{\ln t_{k}}{t_{k}}\right)^{\rho-\alpha}=\frac{t_{k}^{\alpha}}{\rho-\alpha}\left(\ln t_{k}\right)^{\rho-\alpha}=o\left(t_{k}^{\lambda}\right) .
\end{aligned}
$$

Joining these estimates, we get

$$
Q\left(t_{k}\right) \leq t_{k}^{\lambda} I(\lambda)+o\left(t_{k}^{\lambda}\right),
$$

therefore

$$
\liminf _{r \rightarrow \infty} \frac{\ln Q(r)}{\ln r} \leq \liminf _{k \rightarrow \infty} \frac{\ln Q\left(t_{k}\right)}{\ln t_{k}} \leq \lambda .
$$

Together with (5.30) this shows that the lower order of $Q(r)$ is equal to $\lambda$. As was mentioned above this proves that the order and the lower order of the function $f(z)$ are equal to $\rho$ and $\lambda$, respectively.

It remains to show that there exists a function $g(z)$ having the properties (a), (b), and (c). Take

$$
V(z)=\prod_{k=1}^{\infty}\left(1-\frac{z}{k^{1 / \alpha}}\right) .
$$

The formula $\left(5.3_{2}\right)$ from Chapter 2 implies that the entire function

$$
g(z)=R(z) V(z)
$$

where $R(z)$ is an arbitrary rational function, all poles of which are zeros of $V(z)$, satisfies the condition (c). We show that the function $R(z)$ can be selected in such a way that the function (5.31) satisfies also the conditions (a) and (b).

Let $p$ be a positive integer, $p>2 n$; the choice of $p$ will be specified later. Let

$$
V_{p}(z)=\prod_{k=p+1}^{\infty}\left(1-\frac{z}{k^{1 / \alpha}}\right), v_{p}(z)=\prod_{k=1}^{p}\left(1-\frac{z}{k^{1 / \alpha}}\right) .
$$

We have a power series representation

$$
\frac{1}{V_{p}(z)}=\sum_{k=0}^{\infty} a_{k} z^{k}, a_{k}=a_{k}^{(p)}, a_{0}=1,
$$

in the circle $\left\{|z|<(p+1)^{1 / \alpha}\right\}$. Since

$$
\frac{1}{V_{p}(z)}=\prod_{k=p+1}^{\infty} \sum_{j=0}^{\infty}\left(\frac{z}{k^{1 / \alpha}}\right)^{j}
$$

obviously $a_{k}>0, k=0,1,2, \ldots$ The function $V_{p}(z)$ tends to 1 as $p \rightarrow \infty$ uniformly over the circle $\{|z|=1\}$. Therefore $\left|V_{p}(z)\right| \geq 1 / 2$ for $\{|z|=1\}$ if $p \geq p_{0}$, and

$$
0<a_{k}=\left|\frac{1}{2 \pi i} \int_{|z|=1} \frac{d z}{z^{k+1} V_{p}(z)}\right| \leq 2, k=0,1,2, \ldots
$$

Take $R(z)=\sum_{k=0}^{n-1} a_{k} z^{k} / v_{p}(z)$ in (5.31), then

$$
g(z)=\frac{\sum_{k=0}^{n-1} a_{k} z^{k}}{v_{p}(z)} V(z)=V_{p}(z) \sum_{k=0}^{n-1} a_{k} z^{k}=1+c_{n} z^{n}+c_{n+1} z^{n+1}+\ldots
$$


(see Example 2 from Section 3 of Chapter 2). Since the function $V_{p}(z)$ takes real values on the real axis, then, as is easy to see, all coefficients $c_{k}, k \geq n$, are real numbers. Since

$$
V_{p}(z) \sum_{k=0}^{n-1} a_{k} z^{k}=1-V_{p}(z)\left(\frac{1}{V_{p}(z)}-\sum_{k=0}^{n-1} a_{k} z^{k}\right)=1-V_{p}(z) \sum_{k=n}^{\infty} a_{k} z^{k}
$$

for $|z|<(p+1)^{1 / \alpha}$, it is clear that $c_{n}=-a_{n}<0$. Thus the function $g(z)$ satisfies the condition (a).

We show that $|V(x)|<1$ for $0<x<\infty$. For $x>0, x \neq k^{1 / \alpha}, k=1,2, \ldots$, the inequality

$$
0<\frac{1-\frac{x}{k^{1 / \alpha}}}{1-\frac{x^{2 \alpha}}{k^{2}}}=\frac{1-\frac{x}{k^{1 / \alpha}}}{1-\left(\frac{x}{k^{1 / \alpha}}\right)^{2 \alpha}}<1
$$

holds, therefore

$$
|V(x)|=\prod_{k=1}^{\infty} \frac{1-\frac{x}{k^{1 / \alpha}}}{1-\frac{x^{2 \alpha}}{k^{2}}}\left|1-\frac{x^{2 \alpha}}{k^{2}}\right| \leq \prod_{k=1}^{\infty}\left|1-\frac{x^{2 \alpha}}{k^{2}}\right|=\frac{\left|\sin \pi x^{\alpha}\right|}{\pi x^{\alpha}}<1 .
$$

For $0<x<(p+1)^{1 / \alpha}$ we have

$$
|g(x)|=g(x)=V_{p}(x) \sum_{k=0}^{n-1} a_{k} x^{k}<V_{p}(x) \sum_{k=0}^{\infty} a_{k} x^{k}=1 .
$$

For $x \geq(p+1)^{1 / \alpha}$ the inequality

$$
|g(x)|=|V(x)| \frac{\sum_{k=0}^{n-1} a_{k} x^{k}}{\left|v_{p}(x)\right|}<\frac{\sum_{k=0}^{n-1} a_{k} x^{k}}{\left|v_{p}(x)\right|}
$$

holds.

Letting $x^{\alpha}=\xi, \xi \geq p+1$, and using the inequality $a^{1 / \alpha}-1 \geq \frac{1}{\alpha}(a-1), a \geq 1$, we get

$$
\begin{aligned}
\left|v_{p}(x)\right| & =\prod_{k=1}^{p}\left(\frac{x}{k^{1 / \alpha}}-1\right)=\prod_{k=1}^{p}\left\{\left(\frac{\xi}{k}\right)^{1 / \alpha}-1\right\} \\
& \geq \frac{1}{\alpha^{p}} \prod_{k=1}^{p}\left(\frac{\xi}{k}-1\right)=\frac{1}{\alpha^{p} p !} \prod_{k=1}^{2 n}(\xi-k) \prod_{k=2 n+1}^{p}(\xi-k) \\
& \geq \frac{1}{a^{p} p !}(\xi-2 n)^{2 n}(p-2 n) ! \geq \frac{1}{\alpha^{p} p^{2 n}}(\xi-2 n)^{2 n} .
\end{aligned}
$$

Taking into account (5.33), (5.35), and $1 / 2<\alpha<1$, for $p \geq p_{0}$ we get

$$
|g(x)|<\frac{2 n \xi^{n / \alpha} \alpha^{p} p^{2 n}}{(\xi-2 n)^{2 n}}<2 n\left(\frac{\xi}{\xi-2 n}\right)^{2 n} p^{2 n} \alpha^{p} \leq 2 n\left(\frac{p+1}{p+1-2 n}\right)^{2 n} p^{2 n} \alpha^{p} .
$$

Since for $p \rightarrow \infty$ the right-hand side of the last inequality tends to 0 , the number $p$ can be chosen so large that it is less than 1. Taking into account (5.34) we find that $|g(x)|<1$ for $0<x<\infty$, that is the condition (b) is satisfied. We have proved the existence of a desired function $g(z)$.

REMARK. If in Example 9 we required the inequality $1<\lambda \leq \rho<\infty$ only, using the same idea it would be possible to construct a much easier example. Let 
$g(z)=e^{-z} \sum_{k=0}^{n-1} z^{k} / k !$ and $\alpha=1$. We can repeat the previous argument (the inequality (5.23) will be replaced by

$$
\ln \left|g\left(r e^{i \varphi}\right)\right|<r(\eta-\cos \varphi)
$$

for $r>R_{0}(\eta)$ ), but the investigation of properties of the function $g(z)$ is significantly simplified.

\section{Meromorphic functions representable by series of simple fractions}

In this section we consider meromorphic functions of the form

$$
f(z)=\sum_{k=1}^{\infty} \frac{A_{k}}{z-h_{k}}, h_{k} \rightarrow \infty,
$$

where the series is assumed to be absolutely convergent, as is

$$
\sum_{\left|h_{k}\right|>1}\left|A_{k} / h_{k}\right|<\infty
$$

Obviously, rational functions admitting representations of the form

$$
R(z)=\sum_{k=1}^{n} \frac{A_{k}}{z-h_{k}}(1 \leq n<\infty)
$$

satisfy the following conditions:

(a) $R(z) \rightarrow 0$ as $z \rightarrow \infty$.

(b) For each $a \neq 0$ the number of $a$-points of $R(z)$ is equal to $n$, and the number of zeros is equal to $n-1$, if $\sum_{k=1}^{n} A_{k} \neq 0$.

The following two theorems, showing that transcendental meromorphic functions of the form (6.1) also have properties, which are in certain sense close to (a) and (b), are the main results of this section.

TheOREM 6.1. Let $f(z)$ be a meromorphic function of the form (6.1). Then

$$
m(r, f)=o(1), r \rightarrow \infty,
$$

besides, for each $0<p<1$ the relation

$$
\int_{0}^{2 \pi}\left|f\left(r e^{i \varphi}\right)\right|^{p} d \varphi=o(1), r \rightarrow \infty
$$

holds.

THEOREM 6.2. Let $f(z)$ be a meromorphic function of the form (6.1), for which $\lambda[f]<\infty$. Then $\delta(a, f)=0$ for all $a \neq 0$, and the order of $N(r, a)$ is equal to the order of $T(r, f)$. This statement remains true also for $a=0$, if we assume, in addition, that

$$
\sum_{k=1}^{\infty}\left|A_{k}\right|<\infty
$$

and

$$
\sum_{k=1}^{\infty} A_{k} \neq 0 .
$$

We shall assume, without loss of generality, that $h_{k} \neq 0, k=1,2, \ldots$.

To prove Theorem 6.1 we need the following lemma. 
Lemma 6.1. If a function $F(z)$ is analytic in the disc $\{|z|<R\}$, and is such that the functions $\operatorname{Re} F(z)$ and $\operatorname{Im} F(z)$ have constant sign, then the inequality

$$
\int_{0}^{2 \pi} u^{p}(\varphi) d \varphi \leq \frac{2 \pi}{\cos \frac{\pi p}{2}}|F(0)|^{p}
$$

holds for each $0<p<1$, where

$$
u(\varphi)=\liminf _{\substack{z \rightarrow \operatorname{Ri}^{i \varphi} \\|z|<R}}|F(z)| .
$$

Proof. We may assume without loss of generality that $\operatorname{Re} f(z)>0$ for $|z|<R$. The function $F(z)$ does not have zeros in the disc $\{|z|<R\}$, and we can fix a branch of $\arg F(z)$ in such a way, that $|\arg F(z)|<\pi / 2$ for $|z|<R$. The function

$$
F^{p}(z)=|F(z)|^{p} \exp \{i p \arg F(z)\}
$$

is analytic in the disc $\{|z|<R\}$ and hence, $\operatorname{Re}\{F(z)\}^{p}$ is harmonic there. Since

$$
\operatorname{Re}\{F(z)\}^{p}=|F(z)|^{p} \cos (p \arg F(z)) \geq|F(z)|^{p} \cos \frac{p \pi}{2},
$$

we have

$$
\int_{0}^{2 \pi}\left|F\left(r e^{i \varphi}\right)\right|^{p} d \varphi \leq \frac{1}{\cos \frac{p \pi}{2}} \int_{0}^{2 \pi} \operatorname{Re}\left\{F\left(r e^{i \varphi}\right)\right\}^{p} d \varphi=\frac{2 \pi}{\cos \frac{p \pi}{2}} \operatorname{Re}\{F(0)\}^{p}
$$

thus

$$
\int_{0}^{2 \pi}\left|F\left(r e^{i \varphi}\right)\right|^{p} \leq \frac{2 \pi}{\cos \frac{p \pi}{2}}|F(0)|^{p}, r<R .
$$

Taking limits as $r \rightarrow R$ and using the Fatou lemma, we complete the proof of the lemma.

Proof. We prove Theorem 6.1. The relation (6.3) follows from (6.4) and the inequality $\ln ^{+} a \leq \frac{1}{p} a^{p}, a>0$, therefore it suffices to prove (6.4).

Let $\theta_{k}=\arg h_{k}, A_{k}=\alpha_{k}+i \beta_{k}$. Fixing $R>0$, we write the function $f(z)$ in the form

$$
\begin{aligned}
f(z)= & \sum_{\left|h_{k}\right|>R} \frac{{ }^{i \theta_{k}} \operatorname{Re}\left(A_{k} e^{-i \theta_{k}}\right)}{z-h_{k}}+\sum_{\left|h_{k}\right|>R}{ }^{\prime \prime}+i \sum_{\left|h_{k}\right|>R} \frac{{ }^{\prime} e^{i \theta_{k}} \operatorname{Im}\left(A_{k} e^{-i \theta_{k}}\right)}{z-h_{k}} \\
& +i \sum_{\left|h_{k}\right|>R}{ }^{\prime \prime}+\sum_{\left|h_{k}\right| \leq R}{ }^{\prime} \frac{\alpha_{k}}{z-h_{k}}+\sum_{\left|h_{k}\right| \leq R}{ }^{\prime \prime}+i \sum_{\left|h_{k}\right| \leq R}{ }^{\prime} \frac{\beta_{k}}{z-h_{k}}+i \sum_{\left|h_{k}\right| \leq R}{ }^{\prime \prime} \\
= & F_{1}(z)+\cdots+F_{8}(z),
\end{aligned}
$$

where the sums $\sum^{\prime}$ contain those terms for which $\operatorname{Re}\left(A_{k} e^{-i \theta_{k}}\right), \operatorname{Im}\left(A_{k} e^{-i \theta_{k}}\right), \alpha_{k}$, $\beta_{k}$, respectively, are positive, and the sums $\sum^{\prime \prime}$ contain all other terms.

Since for $\left|h_{k}\right|>R,|z|<R$, the inequality

$$
\operatorname{Re} \frac{e^{i \theta_{k}}}{z-h_{k}}<0
$$


holds, the functions $F_{j}(z), j=1,2,3,4$, satisfy the conditions of Lemma 6.1 , hence $(j=1,2,3,4)$

$$
\int_{0}^{2 \pi}\left|F_{j}\left(\operatorname{Re}^{i \varphi}\right)\right|^{p} d \varphi \leq \frac{2 \pi}{\cos \frac{p \pi}{2}}\left|F_{j}(0)\right|^{p} \leq \frac{2 \pi}{\cos \frac{p \pi}{2}}\left(\sum_{\left|h_{k}\right|>R}\left|\frac{A_{k}}{h_{k}}\right|\right)^{p} .
$$

Since for $|z|=R$ the equality

$$
\left|\sum_{\left|h_{k}\right| \leq R}{ }^{\prime} \frac{\alpha_{k}}{z-h_{k}}\right|=\left|\sum_{\left|h_{k}\right| \leq R}{ }^{\prime} \frac{R \alpha_{k}}{R^{2}-h_{k} z}\right|
$$

holds, and for $\left|h_{k}\right| \leq R,|z|<R$, the inequality

$$
\operatorname{Re} \frac{R}{R^{2}-h_{k} z}>0
$$

holds, considering the function

$$
F_{5}^{(1)}(z)=\sum_{\left|h_{k}\right| \leq R}^{\prime} \frac{R \alpha_{k}}{R^{2}-h_{k} z}
$$

we see that it satisfies the conditions of Lemma 6.1, besides, we have $\left|F_{5}^{(1)}\left(\operatorname{Re}^{i \varphi}\right)\right|=$ $\left|F_{5}\left(\operatorname{Re}^{i \varphi}\right)\right|, 0 \leq \varphi<2 \pi$. Hence

$$
\int_{0}^{2 \pi}\left|F_{5}\left(\operatorname{Re}^{i \varphi}\right)\right|^{p} d \varphi \leq \frac{2 \pi}{\cos \frac{p \pi}{2}}\left|F_{5}^{(1)}(0)\right|^{p} \leq \frac{2 \pi}{\cos \frac{p \pi}{2}}\left(\frac{1}{R} \sum_{\left|h_{k}\right| \leq R}\left|A_{k}\right|\right)^{p} .
$$

In a similar way we get

$$
\int_{0}^{2 \pi}\left|F_{j}\left(\operatorname{Re}^{i \varphi}\right)\right|^{p} d \varphi \leq \frac{2 \pi}{\cos \frac{p \pi}{2}}\left(\frac{1}{R} \sum_{\left|h_{k}\right| \leq R}\left|A_{k}\right|\right)^{p}, j=6,7,8 .
$$

Using the obtained estimates, we get the following conclusion:

$$
\begin{aligned}
\int_{0}^{2 \pi} & \left|f\left(\operatorname{Re}^{i \varphi}\right)\right|^{p} d \varphi \leq \sum_{j=1}^{8} \int_{0}^{2 \pi}\left|F_{j}\left(\operatorname{Re}^{i \varphi}\right)\right|^{p} d \varphi \\
& \leq \frac{8 \pi}{\cos \frac{p \pi}{2}}\left\{\left(\sum_{\left|h_{k}\right|>R}\left|\frac{A_{k}}{h_{k}}\right|\right)^{p}+\left(\frac{1}{R} \sum_{\left|h_{k}\right| \leq R}\left|A_{k}\right|\right)^{p}\right\} \\
& \leq \frac{8 \pi}{\cos \frac{p \pi}{2}}\left\{\left(\sum_{\left|h_{k}\right|>R}\left|\frac{A_{k}}{h_{k}}\right|\right)^{p}+\left(\frac{1}{\sqrt{R}} \sum_{\left|h_{k}\right|<\sqrt{R}}\left|\frac{A_{k}}{h_{k}}\right|+\sum_{\sqrt{R} \leq\left|h_{k}\right| \leq R}\left|\frac{A_{k}}{h_{k}}\right|\right)^{p}\right\} \\
& =o(1), R \rightarrow \infty .
\end{aligned}
$$

Thus the relation (6.4) has been proved.

Theorem 6.1 immediately implies

Corollary. Functions of the form (6.1) satisfy the relations

$$
T(r, f)=N(r, f)+o(1), \delta(\infty, f)=0 .
$$


Let $\varphi(r)$ be an arbitrary function such that $\frac{d \varphi(r)}{d \ln r}$ monotonically tends to $+\infty$. If we choose poles of the function (6.1) in such a way that

$$
n(r, f)=\left[\frac{d \varphi(r)}{d \ln r}\right]=\frac{d \varphi(r)}{d \ln r}+O(1),
$$

then

$$
T(r, f)=N(r, f)+o(1)=\varphi(r)+O(\ln r),
$$

that is, the characteristic $T(r, f)$ has the prescribed asymptotic behavior.

With the help of a modification of the method used in the proof of Theorem 6.1 we can get an information on the behavior of a function of the form (6.1) along the rays $\{\arg z=$ const $\}$.

THEOREM 6.3. Let $f(z)$ be a meromorphic function of the form (6.1). Then, for arbitrary $\alpha$ and $p, 0 \leq \alpha<2 \pi, 0<p<1$, the relations

$$
\begin{gathered}
\int_{1}^{\infty} \frac{\ln ^{+}\left|f\left(t e^{i \alpha}\right)\right|}{t^{1+p}} d t<\infty \\
\int_{1}^{\infty} \frac{\left|f\left(t e^{i \alpha}\right)\right|^{p}}{t^{2}} d t<\infty
\end{gathered}
$$

hold.

We need the following lemma, similar to Lemma 6.1.

Lemma 6.2. If the function $g(z)$ is analytic in the half-plane $\{\operatorname{Im} z>0\}$ and is such that the functions $\operatorname{Re} g(z)$ and $\operatorname{Im} g(z)$ have a constant sign, then for each $p, 0<p<1$, the inequality

$$
\int_{-\infty}^{\infty} \frac{v^{p}(t)}{1+t^{2}} d t \leq \frac{\pi}{\cos \frac{p \pi}{2}}|g(i)|^{p}
$$

holds, where

$$
v(t)=\liminf _{\substack{z \rightarrow t \\ \operatorname{Im} z>0}}|g(z)|
$$

Proof. The function $F(z)=g\left(\frac{1}{i} \frac{z-1}{z+1}\right)$ satisfies the conditions of Lemma 6.1 $(R=1)$. Observing that for this function we have $u(\varphi)=v\left(\frac{1}{i} \frac{e^{i \varphi}-1}{e^{i \varphi}+1}\right)=v\left(\tan \frac{\varphi}{2}\right)$ and $F(0)=g(i)$, we get the inequality

$$
\int_{-\pi}^{\pi} v^{p}\left(\tan \frac{\varphi}{2}\right) d \varphi \leq \frac{2 \pi}{\cos \frac{p \pi}{2}}|g(i)|^{p} .
$$

Making the change of variables $t=\tan \frac{\varphi}{2}$, we get the desired inequality.

Proof. Obviously, it is enough to prove Theorem 6.3 for $\alpha=0$. Observe that the relation (6.7) is a corollary of (6.8). In fact, using the inequality $\ln ^{+} a<\frac{1}{p} a^{p}$, 
$p>0$, we get

$$
\begin{aligned}
\int_{1}^{\infty} \frac{\ln ^{+}|f(t)|}{t^{1+p}} d t & \leq \int_{1}^{\infty} \frac{\ln ^{+}\left|f(t) t^{\frac{p-1}{p}}\right|+\frac{1-p}{p} \ln t}{t^{1+p}} d t \\
& \leq \frac{1}{p} \int_{1}^{\infty} \frac{|f(t)|^{p}}{t^{2}} d t+\frac{1-p}{p} \int_{1}^{\infty} \frac{\ln t}{t^{1+p}} d t
\end{aligned}
$$

We let $A_{k}=\alpha_{k}+i \beta_{k}$ and we represent the function $f(z)$ in the form

$$
\begin{aligned}
f(z)= & \sum_{\operatorname{Im} h_{k} \leq 0} \frac{\alpha_{k}}{z-h_{k}}+\sum_{\operatorname{Im} h_{k} \leq 0}^{\prime \prime}+i \sum_{\operatorname{Im} h_{k} \leq 0} \frac{\beta_{k}}{z-h_{k}}+i \sum_{\operatorname{Im} h_{k} \leq 0}^{\prime \prime} \\
& +\sum_{\operatorname{Im} h_{k}>0} \frac{\alpha_{k}}{z-h_{k}}+\sum_{\operatorname{Im} h_{k}>0}^{\prime \prime}+i \sum_{\operatorname{Im} h_{k}>0}^{\prime} \frac{\beta_{k}}{z-h_{k}}+i \sum_{\operatorname{Im} h_{k}>0}^{\prime \prime} \\
= & g_{1}(z)+\cdots+g_{8}(z),
\end{aligned}
$$

where the sums $\sum^{\prime}$ contain those terms for which $\alpha_{k}$ and $\beta_{k}$ are positive, and the sums $\sum^{\prime \prime}$ contain all other terms.

Since for $\operatorname{Im} h_{k} \leq 0, \operatorname{Im} z>0$ the inequality

$$
\operatorname{Im} \frac{1}{z-h_{k}}<0
$$

holds, Lemma 6.2 can be applied to functions $g_{j}(z), j=1,2,3,4$, hence

$$
\int_{-\infty}^{\infty} \frac{\left|g_{j}(t)\right|^{p}}{1+t^{2}} d t \leq \frac{\pi}{\cos \frac{\pi p}{2}}\left|g_{j}(i)\right|^{p} \leq \frac{\pi}{\cos \frac{\pi p}{2}}\left(\sum_{\operatorname{Im} h_{k} \leq 0}\left|\frac{A_{k}}{h_{k}}\right|\right)^{p}, j=1,2,3,4
$$

Observe, that Lemma 6.2 can be applied also to functions $g_{j}(-z), j=5,6,7,8$, hence

$$
\begin{array}{r}
\int_{-\infty}^{\infty} \frac{\left|g_{j}(t)\right|^{p}}{1+t^{2}} d t \leq \frac{\pi}{\cos \frac{\pi p}{2}}\left|g_{j}(-i)\right|^{p} \leq \frac{\pi}{\cos \frac{\pi p}{2}}\left(\sum_{\operatorname{Im} h_{k}>0}\left|\frac{A_{k}}{h_{k}}\right|\right)^{p} \\
j=5,6,7,8
\end{array}
$$

Thus

$$
\begin{aligned}
\int_{-\infty}^{\infty} \frac{|f(t)|^{p}}{1+t^{2}} d t & \leq \sum_{j=1}^{8} \int_{-\infty}^{\infty} \frac{\left|g_{j}(t)\right|^{p}}{1+t^{2}} d t \\
& \leq \frac{4 \pi}{\cos \frac{\pi p}{2}}\left\{\left(\sum_{\operatorname{Im} h_{k} \leq 0}\left|\frac{A_{k}}{h_{k}}\right|\right)^{p}+\left(\sum_{\operatorname{Im} h_{k}>0}\left|\frac{A_{k}}{h_{k}}\right|\right)^{p}\right\}<\infty
\end{aligned}
$$

hence $\int_{1}^{\infty}|f(t)|^{p} t^{-2} d t<\infty$, Q.E.D.

Now we turn to the proof of Theorem 6.2. If $a=\infty$, the statement of Theorem 6.2 is contained in the corollary of Theorem 6.1. The statement of Theorem 6.2 for $a \neq 0, \infty$ follows from its statement for $a=0$. In fact, for $a \neq 0, \infty$ we have

$$
\frac{f(z)-a}{z}=-\frac{a}{z}+\sum_{k=1}^{\infty} \frac{A_{k}}{z\left(z-h_{k}\right)}=\frac{-a-\sum_{k=1}^{\infty} A_{k} / h_{k}}{z}+\sum_{k=1}^{\infty} \frac{A_{k} / h_{k}}{z-h_{k}} .
$$


Letting

$$
f_{1}(z)=\frac{f(z)-a}{z}, h_{0}=0, B_{0}=-a-\sum_{k=1}^{\infty} A_{k} / h_{k}, B_{k}=A_{k} / h_{k}(k \geq 1),
$$

we have the representation

$$
f_{1}(z)=\sum_{k=0}^{\infty} \frac{B_{k}}{z-h_{k}}
$$

and, obviously,

$$
\begin{gathered}
T\left(r, f_{1}\right)=T(r, f)+O(\ln r), N\left(r, 0, f_{1}\right)=N(r, a, f)+O(\ln r), \\
\delta\left(0, f_{1}\right)=\delta(a, f), \sum_{k=0}^{\infty}\left|B_{k}\right|<\infty, \sum_{k=0}^{\infty} B_{k}=-a \neq 0 .
\end{gathered}
$$

Now we prove the statement of Theorem 6.2 in the case $a=0$.

By virtue of (6.5) and (6.6) we may assume without loss of generality that $\sum_{k=1}^{\infty} A_{k}=1$. Let

$$
\psi(z)=z f(z)-1=\sum_{k=1}^{\infty} A_{k}\left(\frac{z}{z-h_{k}}-1\right)=\sum_{k=1}^{\infty} \frac{A_{k} h_{k}}{z-h_{k}} .
$$

The function $\psi(z)$ satisfies the conditions of Theorem 6.1, therefore for each $p$, $0<p<1$, the relation

$$
\int_{0}^{2 \pi}\left|\psi\left(r e^{i \varphi}\right)\right|^{p} d \varphi=o(1), r \rightarrow \infty
$$

holds. Denote by $E_{r}$ the set of those values of $\varphi \in[0,2 \pi)$ for which $\left|\psi\left(r e^{i \varphi}\right)\right| \geq 1 / 2$, and let $\varepsilon_{r}=$ mes $E_{r}$. Observe that

$$
\varepsilon_{r} \leq 2^{p} \int_{E_{r}}\left|\psi\left(r e^{i \varphi}\right)\right|^{p} d \varphi \leq 2^{p} \int_{0}^{2 \pi}\left|\psi\left(r e^{i \varphi}\right)\right|^{p} d \varphi=o(1), r \rightarrow \infty .
$$

For $\varphi \in C E_{r}=[0,2 \pi) \backslash E_{r}$ the inequality $\left|f\left(r e^{i \varphi}\right)\right| \geq \frac{1}{2 r}$ holds, hence, we have

$$
m\left(r, \frac{1}{f}\right) \leq \frac{1}{2 \pi} \int_{E_{r}} \ln ^{+} \frac{1}{\left|f\left(r e^{i \varphi}\right)\right|} d \varphi+\ln (2 r) .
$$

By Theorem 7.3 from Chapter 1 (p. ???) for each $k>1$ the estimate

$$
\frac{1}{2 \pi} \int_{E_{r}} \ln ^{+} \frac{1}{\left|f\left(r e^{i \varphi}\right)\right|} d \varphi \leq\left(\frac{6 r}{k-1} \varepsilon_{r} \ln \frac{2 \pi e}{\varepsilon_{r}}\right) T\left(k r, \frac{1}{f}\right)
$$

holds. Since $\ln (2 r)=o(T(r, f))$ (we assume that the function $f(z)$ is transcendental), and $T\left(r, \frac{1}{f}\right)=T(r, f)+O(1)$, we obtain the relation

$$
m\left(r, \frac{1}{f}\right) \leq \theta(k, r) T(k r, f)
$$

where $\theta(k, r)=o(1)$ as $r \rightarrow \infty$ and $k$ is fixed.

Suppose that $\delta(0, f)>0$. Then for all sufficiently large $r$ the inequality

$$
T(r, f) \leq \frac{2}{\delta(0, f)} m\left(r, \frac{1}{f}\right) \leq \frac{2 \theta(k, r)}{\delta(0, f)} T(k r, f)
$$


holds. Hence for each $\beta>1$ we can find $r_{0}=r_{0}(k, \beta)$ such that for $r \geq r_{0}$ the inequality

$$
T(k r, f) \geq \beta T(r, f)
$$

holds. From here we get

$$
T\left(k^{n} r_{0}, f\right) \geq \beta^{n} T\left(r_{0}, f\right), n=1,2,3, \ldots
$$

Letting

$$
n=\left[\frac{\ln r-\ln r_{0}}{\ln k}\right], r \geq r_{0}
$$

we get

$$
r \geq k^{n} r_{0}, \beta^{n} \geq \beta^{-\frac{\ln r_{0}}{\ln k}-1} r^{\frac{\ln \beta}{\ln k}}
$$

and hence the inequality

$$
T(r, f) \geq r^{\frac{\ln \beta}{\ln k}} \beta^{-\frac{\ln r_{0}}{\ln k}-1} T\left(r_{0}, f\right)
$$

holds.

Thus $\lambda[f] \geq \frac{\ln \beta}{\ln k}$. Since $k$ can be chosen to be arbitrarily close to 1 , we get $\lambda[f]=\infty$ contrary to the condition of the theorem.

The statement that the orders of $N(r, 0)$ and $T(r, f)$ coincide requires a separate proof because (see Example 3 in Section 2) a Borel exceptional value can have zero deficiency.

Adding $N\left(r, \frac{1}{f}\right)$ to both sides of $(6.9)$ we get the relation

$$
T(r, f) \leq \vartheta(k, r) T(k r, f)+N\left(r, \frac{1}{f}\right),
$$

where $\vartheta(k, r)=o(1)$ as $r \rightarrow \infty$ and $k$ is fixed.

Suppose that the order of $N\left(r, \frac{1}{f}\right)$ is less than the order of $T(r, f)$ and choose a number $\mu$ situated between these orders. Then $N\left(r, \frac{1}{f}\right)<r^{\mu}$ for $r>r_{1}$. Fixing $k>1$ we choose $r_{2}>r_{1}$ in such a way that $T\left(r_{2}, f\right) \geq 2 r_{2}^{\mu}$ and $4 k^{\mu} \vartheta(k, r)<1$ for $r \geq r_{2}$. Then by (6.10) we have

$$
T\left(k r_{2}, f\right) \geq \frac{1}{\vartheta\left(k, r_{2}\right)}\left\{T\left(r_{2}, f\right)-N\left(r_{2}, \frac{1}{f}\right)\right\} \geq \frac{1}{2 \vartheta\left(k, r_{2}\right)} T\left(r_{2}, f\right) \geq 2\left(k r_{2}\right)^{\mu} .
$$

Using (6.10) repeatedly we get

$$
T\left(k^{n} r_{2}, f\right) \geq \frac{1}{2^{n} \vartheta\left(k, r_{2}\right) \vartheta\left(k, k r_{2}\right) \ldots \vartheta\left(k, r^{n-1} r_{2}\right)} T\left(r_{2}, f\right) \geq\left(2 k^{\mu}\right)^{n} T\left(r_{2}, f\right) .
$$

From here, selecting $n=\left[\frac{\ln r-\ln r_{2}}{\ln k}\right], r \geq r_{2}$, we get the inequality

$$
T(r, f)>\left(2 k^{\mu}\right)^{\frac{\ln r-\ln r_{2}}{\ln k}-1} T\left(r_{2}, f\right), r \geq r_{2},
$$

and conclude that $\lambda[f] \geq \frac{\mu \ln k+\ln 2}{\ln k}$, and, because $k$ can be chosen to be arbitrarily close to 1 , we again get a contradiction. 
REMARK. The statement of Theorem 6.2 is no longer true, if we omit the condition (6.6).

In fact, assume that $\delta(0, f)=0$ for each function $f(z)$ of the form (6.1), satisfying the condition (6.5). We deduce from this assumption the following corollary: meromorphic functions of finite order cannot have deficient values different from 0 and $\infty$. It is clearly a nonsense.

Let $g(z)$ be a meromorphic function of finite order. Denoting the genus of this function by $p$, we can, by the remark to Theorem 4.1 from Chapter 2 (p. ???) represent the function $g(z)$ in the form

$$
g(z)=z^{s} e^{Q(z)} \frac{\prod_{m} E\left(\frac{z}{a_{m}}, p\right)}{\prod_{n} E\left(\frac{z}{b_{n}}, p\right)},
$$

where $s$ is an integer, $Q(z)$ is a polynomial of degree at most $p, a_{m}$ are zeros of the function $g(z), b_{n}$ are its poles. It follows from here that

$$
\frac{g^{\prime}(z)}{g(z)}=\frac{s}{z}+Q^{\prime}(z)+z^{p} \sum_{m} \frac{1}{a_{m}^{p}\left(z-a_{m}\right)}-z^{p} \sum_{n} \frac{1}{b_{n}^{p}\left(z-b_{n}\right)} .
$$

Let $h(z)=\left(z-c_{1}\right) \ldots\left(z-c_{p}\right)$, where $c_{1}, \ldots, c_{p}$ are pairwise distinct and are different from $a_{m}, b_{n}$, and 0 . It is easy to see that the function

$$
f(z)=\frac{g^{\prime}(z)}{h(z) g(z)}
$$

is representable in the form (6.1), moreover, the condition (6.5) holds.

By virtue of the assumption made above, the condition $\delta(0, f)=0$ should hold, hence, there exists a sequence $\left\{r_{n}\right\}_{n=1}^{\infty}, r_{n} \uparrow \infty$, such that

$$
m\left(r_{n}, 0, f\right)=o\left(T\left(r_{n}, f\right)\right) .
$$

Since

$$
\begin{gathered}
m(r, 0, f)=m\left(r, \frac{h g}{g^{\prime}}\right) \geq m\left(r, \frac{g}{g^{\prime}}\right)+O(\ln r), \\
T(r, f)=T\left(r, \frac{g^{\prime}}{h g}\right) \leq T\left(r, \frac{g^{\prime}}{g}\right)+O(\ln r), \\
T\left(r, \frac{g^{\prime}}{g}\right)=m\left(r, \frac{g^{\prime}}{g}\right)+N\left(r, \frac{g^{\prime}}{g}\right) \\
=O(\ln r)+\bar{N}(r, g)+\bar{N}\left(r, \frac{1}{g}\right) \leq 2 T(r, g)+O(\ln r),
\end{gathered}
$$

we have

$$
m\left(r_{n}, \frac{g}{g^{\prime}}\right)=o\left(T\left(r_{n}, g\right)\right) .
$$

Using Lemma 2.1 from Chapter 3 (p. ???), we get that the condition

$$
m\left(r_{n}, a, g\right)=o\left(T\left(r_{n}, g\right)\right)
$$

holds for each $a \neq 0, \infty$, hence $\delta(a, g)=0$. 



\section{CHAPTER 6}

\section{Value distribution with respect to the arguments}

As was already mentioned in Section 4 of Chapter 1, the function $N(r, a)$ characterizes the moduli of $a$-points only. Therefore Chapters 4 and 5 do not contain any information on the role of the arguments of $a$-points.

In the present chapter we show that the restrictions on the arguments of $a$ points can have an important impact onto the asymptotic properties of a meromorphic function. In particular, restrictions of such type can imply: (a) growth estimates; (b) regularity of growth; (c) presence of deficient values.

Note, that among theorems presented in previous chapters it is easy to point out theorems in which (a) or (b) follows from restrictions imposed onto the moduli of $a$-points. For example, by Theorem 2.2 from Chapter 3 , if the order of $N(r, a)$ does not exceed $\rho$ for 3 different values of $a$, then the order of $f(z)$ does not exceed $\rho$. By Theorem 5.1 from Chapter 5 , if the order of $N(r, a)$ is less than the order of $f(z)$ for two different values of $a$, then $f(z)$ has regular growth in the sense that $\rho[f]=\lambda[f]$. Finally, we note that from the very definition of the quantity $\delta(a, f)$,

$$
\delta(a, f)=1-\limsup _{r \rightarrow \infty} \frac{N(r, a)}{T(r, f)},
$$

we see that the condition $\delta(a, f)>0$ can be considered as a condition imposed onto $N(r, a)$, that is, onto moduli of $a$-points.

Results related to (a) are considered in Sections 2, 4, and 5; results related to (b) are considered in Sections 1 and 2; results related to (c) are considered in Section 1. Section 3 contains proofs of results on asymptotic properties of functions meromorphic in an angle, which are used in Section 2.

The methods of Sections 2-5 make substantial use of the apparatus of angular Nevanlinna characteristics, Tsuji characteristics play essential role in Section 5.

\section{Meromorphic functions with separated poles and zeros}

Let $f(z)$ be a meromorphic function with zeros $a_{m}$ and poles $b_{n}, p$ be a positive integer, and $\eta$ be a number satisfying the condition $0 \leq \eta<\frac{\pi}{2 p}$. Let

$$
\begin{gathered}
D_{1}^{p}(\eta)=\bigcup_{j=0}^{p-1}\left\{\left|\arg z-\pi \frac{2 j}{p}\right| \leq \eta\right\}, \\
D_{2}^{p}(\eta)=\bigcup_{j=0}^{p-1}\left\{\left|\arg z-\pi \frac{2 j+1}{p}\right| \leq \eta\right\} .
\end{gathered}
$$


DEFINITION. If the condition

$$
\sum_{a_{m} \notin D_{1}^{p}(\eta)} \frac{1}{\left|a_{m}\right|^{p}}+\sum_{b_{n} \notin D_{2}^{p}(\eta)} \frac{1}{\left|b_{n}\right|^{p}}<\infty
$$

is satisfied, we say that zeros and poles of $f(z)$ are $(p, \eta)$-separated.

In particular, zeros and poles of a function $f(z)$ are $(p, \eta)$-separated, if all of its zeros are in $D_{1}^{p}(\eta)$, and all of its poles are in $D_{2}^{p}(\eta)$.

In what follows we assume, without loss of generality, that $f(0)=1$.

THEOREM 1.1. If poles and zeros of $f(z)$ are $(p, \eta)$-separated, then the limit $\lim _{r \rightarrow \infty} r^{-p} T(r, f)$, finite or infinite, exists.

This theorem shows that the growth of $f(z)$ cannot be arbitrarily irregular. In particular, it immediately implies that if $\lambda[f]<p$, then $\rho[f] \leq p$.

To prove Theorem 1.1 we need the following lemma, which will be used repeatedly in this section.

LEMMA 1.1. If zeros and poles of a function $f(z)$ are $(p, \eta)$-separated, then the inequality

$$
\begin{aligned}
\frac{1}{2 \pi} & \int_{0}^{2 \pi} \ln \left|f\left(r e^{i \varphi}\right)\right| \cos p \varphi d \varphi \\
& \geq \cos p \eta \int_{0}^{r} n(t ; 0, \infty) \cosh \left(p \ln \frac{r}{t}\right) \frac{d t}{t}+O\left(r^{p}\right) \\
& =\cos p \eta\left\{N(r ; 0, \infty)+p \int_{0}^{r} N(t ; 0, \infty) \sinh \left(p \ln \frac{r}{t}\right) \frac{d t}{t}\right\}+O\left(r^{p}\right)
\end{aligned}
$$

holds.

Proof. We start from the formula (2.6) from Chapter 1. Separating the real part, we get the relation

$$
\begin{aligned}
\frac{1}{2 \pi} \int_{0}^{2 \pi} \ln \left|f\left(r e^{i \varphi}\right)\right| \cos p \varphi d \varphi=\frac{1}{2 p} \sum_{\left|a_{m}\right|<r}\left(\frac{r^{p}}{\left|a_{m}\right|^{p}}-\frac{\left|a_{m}\right|^{p}}{r^{p}}\right) \cos p \alpha_{m} \\
-\frac{1}{2 p} \sum_{\left|b_{n}\right|<r}\left(\frac{r^{p}}{\left|b_{n}\right|^{p}}-\frac{\left|b_{n}\right|^{p}}{r^{p}}\right) \cos p \beta_{n}+\left.r^{p} \operatorname{Re} \frac{d^{p}}{d z^{p}} \ln f(z)\right|_{z=0},
\end{aligned}
$$

where $\alpha_{m}=\arg a_{m}, \beta_{n}=\arg b_{n}$. This relation can be written in the form

$$
\begin{aligned}
\frac{1}{2 \pi} \int_{0}^{2 \pi} \ln \left|f\left(r e^{i \varphi}\right)\right| \cos p \varphi d \varphi=\frac{1}{p} \sum_{\left|a_{m}\right|<r} \sinh \left(p \ln \frac{r}{\left|a_{m}\right|}\right) \cos p \alpha_{m} \\
-\frac{1}{p} \sum_{\left|b_{n}\right|<r} \sinh \left(p \ln \frac{r}{\left|b_{n}\right|}\right) \cos p \beta_{n}+\left.r^{p} \operatorname{Re} \frac{d^{p}}{d z^{p}} \ln f(z)\right|_{z=0} .
\end{aligned}
$$


Since for $a_{m} \in D_{1}^{p}(\eta)$ the inequality $\cos p \alpha_{m} \geq \cos p \eta>0$ holds, we have

$$
\begin{aligned}
& \sum_{\left|a_{m}\right|<r} \sinh \left(p \ln \frac{r}{\left|a_{m}\right|}\right) \cos p \alpha_{m}=\sum_{\substack{\left|a_{m}\right|<r \\
a_{m} \in D_{1}^{p}(\eta)}} \sinh \left(p \ln \frac{r}{\left|a_{m}\right|}\right) \cos p \alpha_{m} \\
&+\sum_{\substack{\left|a_{m}\right|<r \\
a_{m} \notin D_{1}^{p}(\eta)}} \sinh \left(p \ln \frac{r}{\left|a_{m}\right|}\right) \cos p \alpha_{m} \geq \cos p \eta \sum_{\substack{\left|a_{m}\right|<r \\
a_{m} \in D_{1}^{p}(\eta)}} \sinh \left(p \ln \frac{r}{\left|a_{m}\right|}\right) \\
&-\sum_{\substack{\left|a_{m}\right|<r \\
a_{m} \notin D_{1}^{p}(\eta)}} \sinh \left(p \ln \frac{r}{\left|a_{m}\right|}\right) \\
&=\cos p \eta \sum_{\left|a_{m}\right|<r} \sinh \left(p \ln \frac{r}{\left|a_{m}\right|}\right)-(1+\cos p \eta) \sum_{\substack{\left|a_{m}\right|<r \\
a_{m} \notin D_{1}^{p}(\eta)}} \sinh \left(p \ln \frac{r}{\left|a_{m}\right|}\right) .
\end{aligned}
$$

By the inequality $\sinh x \leq \frac{1}{2} e^{x}$ and the condition (1.1) we get

$$
\sum_{\substack{\left|a_{m}\right|<r \\ a_{m} \notin D_{1}^{p}(\eta)}} \sinh \left(p \ln \frac{r}{\left|a_{m}\right|}\right) \leq \frac{1}{2} \sum_{\substack{\left|a_{m}\right|<r \\ a_{m} \notin D_{1}^{p}(\eta)}}\left(\frac{r}{\left|a_{m}\right|}\right)^{p} \leq \frac{1}{2} r^{p} \sum_{a_{m} \notin D_{1}^{p}(\eta)} \frac{1}{\left|a_{m}\right|^{p}}=O\left(r^{p}\right),
$$

therefore

$$
\begin{aligned}
\sum_{\left|a_{m}\right|<r} & \sinh \left(p \ln \frac{r}{\left|a_{m}\right|}\right) \cos p \alpha_{m} \geq \cos p \eta \sum_{\left|a_{m}\right|<r} \sinh \left(p \ln \frac{r}{\left|a_{m}\right|}\right)+O\left(r^{p}\right) \\
& =\cos p \eta \int_{0}^{r} \sinh \left(p \ln \frac{r}{t}\right) d n(t, 0)+O\left(r^{p}\right) \\
& =p \cos p \eta \int_{0}^{r} n(t, 0) \cosh \left(p \ln \frac{r}{t}\right) \frac{d t}{t}+O\left(r^{p}\right) \\
& =p \cos p \eta\left\{N(r, 0)+p \int_{0}^{r} N(t, 0) \sinh \left(p \ln \frac{r}{t}\right) \frac{d t}{t}\right\}+O\left(r^{p}\right) .
\end{aligned}
$$

Using similar argument we conclude that

$$
\begin{aligned}
-\sum_{\left|b_{n}\right|<r} & \sinh \left(p \ln \frac{r}{\left|b_{n}\right|}\right) \cos p \beta_{n} \\
& \geq p \cos p \eta \int_{0}^{r} n(t, \infty) \cosh \left(p \ln \frac{r}{t}\right) \frac{d t}{t}+O\left(r^{p}\right) \\
& =p \cos p \eta\left\{N(r, \infty)+p \int_{0}^{r} N(t, \infty) \sinh \left(p \ln \frac{r}{t}\right) \frac{d t}{t}\right\}+O\left(r^{p}\right) .
\end{aligned}
$$

Relations (1.2), (1.3), and (1.4) imply the conclusion of the lemma.

Proof of TheOrem 1.1. Obviously it is sufficient to consider the case when $\liminf _{r \rightarrow \infty} r^{-p} T(r, f)<\infty$.

Since

$$
\frac{1}{2 \pi} \int_{0}^{2 \pi} \ln \left|f\left(r e^{i \varphi}\right)\right| \cos p \varphi d \varphi \leq m(r, f)+m\left(r, \frac{1}{f}\right) \leq 2 T(r, f)
$$


by Lemma 1.1 we get

$$
2 T(r, f) \geq \cos p \eta \int_{0}^{r} n(t ; 0, \infty) \cosh \left(p \ln \frac{r}{t}\right) \frac{d t}{t}+O\left(r^{p}\right) .
$$

Since $\cosh x \geq \frac{1}{2} e^{x}$, it follows that

$$
2 T(r, f) \geq \frac{1}{2} \cos p \eta \cdot r^{p} \int_{0}^{r} n(t ; 0, \infty) t^{-p-1} d t+O\left(r^{p}\right)
$$

Dividing both sides of this inequality by $r^{p}$ and letting $r$ tend to $\infty$ along a sequence $r_{k} \uparrow \infty$ such that $T\left(r_{k}, f\right)=O\left(r_{k}^{p}\right)$, we come to the conclusion that the integral

converges.

$$
\int_{0}^{\infty} n(t ; 0, \infty) t^{-p-1} d t
$$

Convergence of this integral implies, by Theorem 1.8 from Chapter 2, the convergence of the series

$$
\sum_{m} \frac{1}{\left|a_{m}\right|^{p}} \text { and } \sum_{n} \frac{1}{\left|b_{n}\right|^{p}},
$$

therefore (see Theorem 3.2 from Chapter 2 and Example 1 on p. ???) the canonical products

$$
\pi_{1}(z)=\prod_{m} E\left(\frac{z}{a_{m}}, p-1\right), \pi_{2}(z)=\prod_{n} E\left(\frac{z}{b_{n}}, p-1\right)
$$

converge. The function

$$
F(z)=f(z) \frac{\pi_{2}(z)}{\pi_{1}(z)}
$$

is an entire function without zeros. Since

$$
T(r, F) \leq T(r, f)+T\left(r, \pi_{1}\right)+T\left(r, \pi_{2}\right)
$$

and, by Remark 2 to Theorem 3.3 from Chapter 2, $T\left(r, \pi_{j}\right)=o\left(r^{p}\right), j=1,2$, we have $\liminf _{r \rightarrow \infty} r^{-p} T(r, F)<\infty$. By Lemma 6.2 from Chapter 1 we get a representation $F(z)=e^{P(z)}$, where $P(z)=c_{p} z^{p}+\cdots+c_{0}$ is a polynomial of degree at most $p$. Thus

$$
f(z)=e^{P(z)} \frac{\pi_{1}(z)}{\pi_{2}(z)}
$$

from where

$$
T(r, f)=T\left(r, e^{P(z)}\right)+o\left(r^{p}\right)=\frac{\left|c_{p}\right|}{\pi} r^{p}+o\left(r^{p}\right),
$$

and hence the limit $\lim _{r \rightarrow \infty} r^{-p} T(r, f)$ exists. The theorem has been proved.

Let us mention the following fact, established in our proof of Theorem 1.1.

REMARK. If zeros and poles of a function $f(z)$ are $(p, \eta)$-separated and

$$
\liminf _{r \rightarrow \infty} r^{-p} T(r, f)<\infty,
$$

then

$$
\int_{0}^{\infty} \frac{n(t ; 0, \infty)}{t^{p+1}} d t<\infty, \sum_{m} \frac{1}{\left|a_{m}\right|^{p}}+\sum_{n} \frac{1}{\left|b_{n}\right|^{p}}<\infty .
$$


Now we turn to corollaries of Theorem 1.1.

COROLlaRY 1. Let $f(z)$ be an entire function of a finite lower order $\lambda$ with positive zeros. Then the growth of $f(z)$ does not exceed the minimal type of order $[\lambda]+1$. If $\lambda$ is a positive integer, then there exists a finite or infinite limit $\lim _{r \rightarrow \infty} r^{-\lambda} T(r, f)$. In any case, the numbers $\rho[f]$ and $\lambda[f]$ belong to the same segment $[k-1, k]$, where $k$ is a positive integer.

In fact, zeros and poles (the latter are absent) of $f(z)$ are $(p, 0)$-separated for each positive integer $p$. For $p=[\lambda]+1$ we have $\liminf _{r \rightarrow \infty} r^{-p} T(r, f)=0$. By Theorem 1.1 we then also have $\lim _{r \rightarrow \infty} r^{-p} T(r, f)=0$, that is, the growth of $f(z)$ does not exceed the minimal type of order $p=[\lambda]+1$. If $\lambda$ is a positive integer, we can apply Theorem 1.1 with $p=\lambda$.

The statement of Corollary 1 is sharp in the sense that for each $\rho$ and $\lambda(\lambda \leq \rho)$ belonging to the same segment $[k-1, k]$, where $k$ is a positive integer, there exist entire functions with positive zeros of order $\rho$ and lower order $\lambda$. For $k-1<\lambda \leq$ $\rho<k$ the canonical products considered in $\mathrm{n}$. $1^{\circ}$ of Section 5 , Chapter 2, p. ??? (we let $\eta_{1}=\lambda, \eta_{2}=\rho$ in their construction) are such functions. Now we construct an example of an entire function with positive zeros and $\rho=k, \lambda=k-1, k=1,2, \ldots$.

EXAMPLE 1. Let $a_{1}=e, a_{n+1}=\exp \exp a_{n}(n=1,2, \ldots), q_{n}=a_{n}^{k}\left(\ln a_{n}\right)^{-2}$. Since the series

$$
\sum_{n=1}^{\infty} q_{n} a_{n}^{-k}
$$

converges, by Theorem 3.2 from Chapter 2, the canonical product

$$
f_{k}(z)=\prod_{n=1}^{\infty}\left\{E\left(\frac{z}{a_{n}}, k-1\right)\right\}^{q_{n}}
$$

converges. Since

$$
\sum_{n=1}^{m} \frac{a_{n}^{k}}{\left(\ln a_{n}\right)^{2}}=\frac{a_{m}^{k}}{\left(\ln a_{m}\right)^{2}}(1+o(1)), m \rightarrow \infty,
$$

for $r=a_{m}$ we have

$$
n\left(r, 0, f_{k}\right)=\frac{r^{k}}{(\ln r)^{2}}(1+o(1)), r \rightarrow \infty
$$

and

$$
n\left(r, 0, f_{k}\right) \leq \frac{r^{k}}{(\ln r)^{2}}(1+o(1)), r \rightarrow \infty,
$$

for all $r>0$. Thus, the order of $n\left(r, 0, f_{k}\right)$ is equal to $k$. By Theorem 3.4 from Chapter 2 the order of $f_{k}(z)$ is also equal to $k$, moreover, by Remark 2 to Theorem 3.3 from Chapter 2,

$$
\ln M\left(r, f_{k}\right)=o\left(r^{k}\right)
$$


Next, by the estimate (3.6) from Chapter 2 (p. ???) we have

$$
\ln M\left(r, f_{k}\right) \leq C(k-1)\left\{r^{k-1} \int_{0}^{r} \frac{n\left(t, 0, f_{k}\right)}{t^{k}} d t+r^{k} \int_{r}^{\infty} \frac{n\left(t, 0, f_{k}\right)}{t^{k+1}} d t\right\} .
$$

For $r=e^{a_{m}}=\ln a_{m+1}$ we have

$$
\begin{gathered}
\int_{0}^{r} \frac{n\left(t, 0, f_{k}\right)}{t^{k}} d t \leq n\left(a_{m}, 0, f_{k}\right) \int_{1}^{r} \frac{d t}{t^{k}} \leq \frac{n\left(a_{m}, 0, f_{k}\right)}{k-1} \\
=\frac{1}{k-1} \frac{a_{m}^{k}}{\left(\ln a_{m}\right)^{2}}(1+o(1))=O\left(\ln ^{k} r\right), \text { if } k>1, \\
\int_{0}^{r} \frac{n\left(t, 0, f_{1}\right)}{t} d t \leq n\left(a_{m}, 0, f_{1}\right) \int_{1}^{r} \frac{d t}{t}=n\left(a_{m}, 0, f_{1}\right) \ln r \\
=\frac{a_{m}}{\left(\ln a_{m}\right)^{2}}(1+o(1)) \ln r=O\left(\ln ^{2} r\right), \\
\int_{r}^{\infty} \frac{n\left(t, 0, f_{k}\right)}{t^{k+1}} d t=n\left(a_{m}, 0, f_{k}\right) \int_{r}^{a_{m+1}} \frac{d t}{t^{k+1}}+\int_{a_{m+1}}^{\infty} \frac{n\left(t, 0, f_{k}\right)}{t^{k+1}} d t \\
\leq \frac{n\left(a_{m}, 0, f_{k}\right)}{k r^{k}}+2 \int_{a_{m+1}}^{\infty} \frac{d t}{t(\ln t)^{2}} \\
=\frac{1}{k r^{k}} \frac{a_{m}^{k}}{\left(\ln a_{m}\right)^{2}}(1+o(1))+\frac{2}{\ln a_{m+1}}=O\left(\frac{\ln ^{k} r}{r^{k}}\right)+O\left(\frac{1}{r}\right) .
\end{gathered}
$$

Thus, for $r=e^{a_{m}}=\ln a_{m+1}$ we have

$$
\ln M\left(r, f_{k}\right)=O\left(r^{k-1} \ln ^{k} r\right), k>1 ; \ln M\left(r, f_{1}\right)=O\left(\ln ^{2} r\right),
$$

hence, the lower order of the function $f_{k}(z)$ does not exceed $k-1$. Since the order of $f_{k}(z)$ is equal to $k$, then, by Theorem 1.1, the lower order of $f_{k}(z)$ cannot be less than $k-1$.

We leave a construction of an example of an entire function with positive zeros and $k-1=\lambda \leq \rho<k$ or $k-1<\lambda \leq \rho=k(k=1,2, \ldots)$ as an exercise.

Corollary 1 and Theorems 6.5 and 6.7 from Chapter 4 immediately imply the following result.

Corollary 2. Deficiencies of entire functions of finite lower order with positive zeros are shift-invariant.

Using the observation that poles and zeros of a function $f(z)$ with positive zeros and negative poles are $(p, 0)$-separated for each odd $p$, we get from Theorem 1.1 the following corollary.

COROLlaRY 3. Let $f(z)$ be a meromorphic function of finite lower order $\lambda$, with positive zeros and negative poles. Then the growth of the function $f(z)$ does not exceed the minimal type of order $2\left[\frac{\lambda+1}{2}\right]+1$. If $\lambda \geq 1$ is an odd number, then the limit $\lim _{r \rightarrow \infty} r^{-\lambda} T(r, f)$ exists. In any case, the numbers $\rho[f]$ and $\lambda[f]$ belong to the same segment of the form $[2 k-3,2 k-1]$, where $k$ is a positive integer.

The following corollary of Theorem 1.1 is also of interest. 
COROLlaRY 4. Let $f(z)$ be an entire function of finite lower order $\lambda$, zeros $a_{m}$ of which satisfy the condition

$$
\sum_{m}\left|\operatorname{Im} \frac{1}{a_{m}}\right|<\infty
$$

Then the growth of $f(z)$ does not exceed the minimal type of order $2\left[\frac{\lambda}{2}\right]+2$. If $\lambda \geq 2$ is an even number, then the limit $\lim _{r \rightarrow \infty} r^{-\lambda} T(r, f)$ exists. In any case, the numbers $\rho[f]$ and $\lambda[f]$ belong to the same segment of the form $[2 k-2,2 k]$, where $k$ is a positive integer.

To prove this corollary it suffices to observe that, by the condition (1.8), zeros and poles of the function $f(z)$ are $\left(p, \frac{\pi}{4 p}\right)$-separated for each even $p \geq 2$.

Now we show that functions with sufficiently large growth, having separated zeros and poles, have "few" zeros and poles in the sense that the number

$$
\kappa(f)=\limsup _{r \rightarrow \infty} \frac{N(r ; 0, \infty)}{T(r, f)}
$$

is strictly less than 2 .

THEOREM 1.2. Let zeros and poles of a function $f(z)$ be $(p, \eta)$-separated.

(a) If $0<\limsup _{r \rightarrow \infty} r^{-p} T(r, f)<\infty$, then $\kappa(f)=0$.

(b) If $\limsup _{r \rightarrow \infty} r^{-p} T(r, f)=\infty$, then

$$
\kappa(f) \leq \frac{2}{1+\cos p \eta}
$$

Proof. In the case (a), by Theorem 1.1 we have $\lim _{r \rightarrow \infty} r^{-p} T(r, f)>0$, that is, $r^{p}=O(T(r, f))$. By the remark to Theorem 1.1 on p. ???, the relation $n(r ; 0, \infty)=$ $o\left(r^{p}\right)$ holds, hence $N(r ; 0, \infty)=o\left(r^{p}\right)$. Therefore $\kappa(f)=0$, Q.E.D.

In the case (b), by Theorem 1.1, we have $\lim _{r \rightarrow \infty} r^{-p} T(r, f)=\infty$, that is $r^{p}=$ $o(T(r, f))$. Therefore, by Lemma 1.1 and relation (1.5), it follows that

$$
m(r, f)+m\left(r, \frac{1}{f}\right) \geq \cos p \eta N(r ; 0, \infty)+o(T(r, f)) .
$$

Adding $N(r ; 0, \infty)$ to both sides, we get

$$
2 T(r, f) \geq(1+\cos p \eta) N(r ; 0, \infty)+o(T(r, f)) .
$$

Dividing both sides by $T(r, f)$ and letting $r$ tend to $\infty$, we get the inequality (1.9).

Let us show that the parameter $\kappa(f)$ can be equal to 2 for a function with $(p, 0)$-separated zeros and poles, and such that $\lim _{r \rightarrow \infty} r^{-p} T(r, f)=0$.

EXAMPle 2. For $p=1$ the function

$$
f(z)=f_{1}(z) / f_{1}(-z),
$$


where $f_{k}(z)$ is a function from Example 1, is a desired example. In fact, poles and zeros of the function $f(z)$ are $(1,0)$-separated. Since

$$
T(r, f) \leq 2 T\left(r, f_{1}\right) \leq 2 \ln ^{+} M\left(r, f_{1}\right)
$$

the relation (1.6) implies that $\lim _{r \rightarrow \infty} r^{-1} T(r, f)=0$. Now we show that $\kappa(f)=2$. From (1.7) and (1.10) we get that

$$
T(r, f)=O\left(\ln ^{2} r\right)
$$

for $r=e^{a_{m}}$ and thus the function $f(z)$ has lower order zero. By the corollary of Theorem 3.1 from Chapter 5 (p. ???), the function $f(z)$ cannot have more than one deficient value. Since, obviously, $N(r, f)=N\left(r, \frac{1}{f}\right)$, we have $\delta(0, f)=\delta(\infty, f)$ and hence $\delta(0, f)=\delta(\infty, f)=0$. From here we get

$$
\kappa(f)=\limsup _{r \rightarrow \infty} \frac{N(r, f)+N\left(r, \frac{1}{f}\right)}{T(r, f)}=2 \limsup _{r \rightarrow \infty} \frac{N(r, f)}{T(r, f)}=2(1-\delta(\infty, f))=2 .
$$

To get an example for $p>1$ we consider $f\left(z^{p}\right)$.

As we already mentioned in Chapter 5 , the inequality $\kappa(f) \leq 2-\delta(0, f)-$ $\delta(\infty, f)$ holds. The question arises: is it possible to strengthen Theorem 1.2 supplementing it with the statement that $2-\delta(0, f)-\delta(\infty, f)<2$, that is, $\delta(0, f)+\delta(\infty, f)>0$. In the case (a) such strengthening is possible: the relation $\kappa(f)=0$ and the obvious inequality

$$
\min \{\delta(0, f), \delta(\infty, f)\} \geq 1-\kappa(f)
$$

imply that $\delta(0, f)=\delta(\infty, f)=1$. We show that in the case (b) for each value of $\eta>0$ it is possible that $\delta(0, f)=\delta(\infty, f)=0$.

EXAMPLE 3. Let a positive integer $p$ and a number $\eta, 0<\eta<\frac{\pi}{2 p}$ be given. Let $\rho$ be a non-integer satisfying $\rho \geq \frac{\pi}{2 \eta}$. We will construct a meromorphic function $f(z)$ of order $\rho$ zeros of which are lying on the rays $\{\arg z=0\}$ and $\left\{\arg z=\frac{\pi}{\rho}\right\}$, and poles are lying on the rays $\left\{\arg z=\frac{\pi}{p}\right\}$ and $\left\{\arg z=\frac{\pi}{p}+\frac{\pi}{\rho}\right\}$, and such that $\delta(0, f)=\delta(\infty, f)=0$. Since $\frac{\pi}{\rho} \leq 2 \eta$, zeros and poles of the function $f(z)$ are $(p, \eta)$-separated, and since $\rho \geq \frac{\pi}{2 \eta}>\rho$, we have $\limsup _{r \rightarrow \infty} r^{-p} T(r, f)=\infty$. Thus, the desired example will be constructed.

Let $r_{1}=1, r_{m+1}=\exp \exp r_{m}(m=1,2, \ldots)$. Denote by $g_{1}(z)\left(g_{2}(z)\right)$ the canonical product of genus $q=[\rho]$ with zeros at the points $k^{1 / \rho}, k=1,2, \ldots$, getting into the set $\bigcup_{j=1}^{\infty}\left[r_{2 j-1}, r_{2 j}\right)\left(\bigcup_{j=1}^{\infty}\left[r_{2 j}, r_{2 j+1}\right)\right)$. Let us show that the function

$$
f(z)=\frac{g_{1}(z) g_{1}\left(z e^{-i \alpha}\right)}{g_{2}\left(z e^{-i \beta}\right) g_{2}\left(z e^{-i \gamma}\right)}, \alpha=\frac{\pi}{\rho}, \beta=\frac{\pi}{p}, \gamma=\frac{\pi}{\rho}+\frac{\pi}{p}
$$

has all the desired properties.

It is clear that its poles and zeros belong to the indicated rays and that the order of $f(z)$ does not exceed $\rho$. 
Since

$$
n\left(r, 0, g_{1}\right)+n\left(r, 0, g_{2}\right)=\left[r^{\rho}\right]
$$

we have

$$
n(r ; 0, \infty)=n(r, f)+n\left(r, \frac{1}{f}\right)=2\left[r^{\rho}\right]
$$

and

$$
N(r ; 0, \infty) \sim \frac{2}{\rho} r^{\rho}
$$

Therefore

$$
T(r, f) \geq\left(\frac{1}{\rho}+o(1)\right) r^{\rho}
$$

and the order of the function $f(z)$ is equal to $\rho$.

Let us prove that $\delta(0, f)=\delta(\infty, f)=0$.

Using the estimate

$$
\ln M\left(r, g_{1}\right) \leq C(q)\left\{r^{q} \int_{0}^{r} \frac{n\left(t, 0, g_{1}\right)}{t^{q+1}} d t+r^{q+1} \int_{r}^{\infty} \frac{n\left(t, 0, g_{1}\right)}{t^{q+2}} d t\right\},
$$

which is valid by Remark 1 to Theorem 3.3 from Chapter 2, and taking into account the fact, that for $r=e^{r_{2 j}}=\ln r_{2 j+1}$ the inequalities

$$
\begin{gathered}
\int_{0}^{r} \frac{n\left(t, 0, g_{1}\right)}{t^{q+1}} d t \leq \frac{n\left(r_{2 j}, 0, g_{1}\right)}{q}=O\left(r_{2 j}^{\rho}\right)=O\left(\ln ^{\rho} r\right) \\
\int_{r}^{\infty} \frac{n\left(t, 0, g_{1}\right)}{t^{q+2}} d t \leq \frac{n\left(r_{2 j}, 0, g_{1}\right)}{(q+1) r^{q+1}}+\int_{r_{2 j+1}}^{\infty} \frac{n\left(t, 0, g_{1}\right)}{t^{q+2}} d t \\
=O\left(\frac{\ln ^{\rho} r}{r^{q+1}}\right)+\int_{r_{2 j+1}}^{\infty} \frac{O\left(t^{\rho}\right)}{t^{q+2}} d t=O\left(\frac{\ln ^{\rho} r}{r^{q+1}}\right)+O\left(r_{2 j+1}^{\rho-q-1)}\right) \\
=O\left(\frac{\ln ^{\rho} r}{r^{q+1}}\right)+O\left(e^{r(\rho-q-1)}\right)=O\left(\frac{\ln ^{\rho} r}{r^{q+1}}\right)
\end{gathered}
$$

hold, we get

$$
\ln M\left(r, g_{1}\right)=O\left(r^{q} \ln ^{\rho} r\right)+O\left(\ln ^{\rho} r\right)=o\left(r^{\rho}\right), r=e^{r_{2 j}} .
$$

In a similar manner, we get

$$
\ln M\left(r, g_{2}\right)=o\left(r^{\rho}\right), r=e^{r_{2 j+1}} .
$$

The function

$$
g(z)=g_{1}(z) g_{2}(z)
$$

is a canonical product with zeros at points $k^{1 / \rho}, k=1,2, \ldots$, and by the asymptotic formula $\left(5.3_{2}\right)$ from Chapter 2, for each $\delta>0$ the relation

$$
\ln \left|g\left(r e^{i \varphi}\right)\right|=\frac{\pi}{\sin \pi \rho} \cos \rho(\varphi-\pi) r^{\rho}+o\left(r^{\rho}\right)
$$

holds uniformly in $\varphi, \delta \leq \varphi \leq 2 \pi-\delta$. Therefore the function

$$
G(z)=g(z) g\left(z e^{-i \alpha}\right), \alpha=\frac{\pi}{\rho},
$$


for each $\delta, 0<\delta<\frac{\pi}{2 \rho}$, uniformly in $\varphi$, satisfies the asymptotic equalities:

$$
\begin{gathered}
\ln \left|G\left(r e^{i \varphi}\right)\right|=2 \pi \sin \rho \varphi r^{\rho}+o\left(r^{\rho}\right), \delta \leq \varphi \leq \alpha-\delta, \\
\ln \left|G\left(r e^{i \varphi}\right)\right|=o\left(r^{\rho}\right), \alpha+\delta \leq \varphi \leq 2 \pi-\delta .
\end{gathered}
$$

Applying Theorem 7.4 from Chapter 1, we get

$$
m\left(r, \frac{1}{G}\right)=\left\{\frac{1}{2 \pi} \int_{0}^{\alpha}(-2 \pi \sin \rho \varphi)^{+} d \varphi\right\} r^{\rho}+o\left(r^{\rho}\right)=o\left(r^{\rho}\right) .
$$

The function $f(z)$ can be represented in the form

$$
f(z)=\frac{G(z)}{g_{2}\left(z e^{-i \beta}\right) g_{2}\left(z e^{-i \gamma}\right) g_{2}(z) g_{2}\left(z e^{-i \alpha}\right)},
$$

from where we get

$$
m\left(r, \frac{1}{f}\right) \leq m\left(r, \frac{1}{G}\right)+4 m\left(r, g_{2}\right) \leq m\left(r, \frac{1}{G}\right)+4 \ln ^{+} M\left(r, g_{2}\right) .
$$

Taking into account (1.15) and (1.14) we get

$$
m\left(r, \frac{1}{f}\right)=o\left(r^{\rho}\right), r=e^{r_{2 j+1}}
$$

applying (1.12), we conclude that $\delta(0, f)=0$.

Similarly, representing the function $f(z)$ in the form

$$
f(z)=\frac{g_{1}(z) g_{1}\left(z e^{-i \alpha}\right) g_{1}\left(z e^{-i \beta}\right) g_{1}\left(z e^{-i \gamma}\right)}{G(z)},
$$

and using (1.15), (1.13), and (1.12) we conclude that $\delta(\infty, f)=0$.

The question: is it possible that $\delta(0, f)=\delta(\infty, f)=0$ holds under the conditions of Theorem 1.2 (b) with $\eta=0$, in full generality is still unanswered. Under some additional conditions the answer to this question turns out to be negative.

The following two theorems are fairly easy to prove.

THEOREM 1.3. Suppose that the conditions of Theorem 1.2 (b) with $\eta=0$ are satisfied, and suppose, in addition, that the limits

$$
q_{1}=\liminf _{r \rightarrow \infty} \frac{N(r, f)}{N\left(r, \frac{1}{f}\right)} \quad \text { and } \quad q_{2}=\limsup _{r \rightarrow \infty} \frac{N(r, f)}{N\left(r, \frac{1}{f}\right)}
$$

are finite and positive. Then

$$
\delta(0, f) \geq \frac{q_{1}}{1+q_{1}}>0, \delta(\infty, f) \geq \frac{1}{1+q_{2}}>0 .
$$

THEOREM 1.4. Suppose that the conditions of Theorem 1.2 (b) with $\eta=0$ are satisfied, and suppose that, in addition, there exist numbers $\mu>1$ and $1>\nu>0$, such that

$$
\frac{N(r ; 0, \infty)}{N(\mu r ; 0, \infty)} \geq \nu, r \geq r_{0}
$$

Then

$$
\min \{\delta(0, f), \delta(\infty, f)\} \geq \frac{\nu(\cosh (p \ln \mu)-1)}{2+\nu(\cosh (p \ln \mu)-1)}>0
$$


Proof of Theorem 1.3. We have

$$
\begin{aligned}
& \kappa(f)=\limsup _{r \rightarrow \infty} \frac{N(r ; 0, \infty)}{T(r, f)} \geq \limsup _{r \rightarrow \infty} \frac{N\left(r, \frac{1}{f}\right)}{T(r, f)} \liminf _{r \rightarrow \infty}\left(1+\frac{N(r, f)}{N\left(r, \frac{1}{f}\right)}\right) \\
&=(1-\delta(0, f))\left(1+q_{1}\right), \\
& \kappa(f) \geq \limsup _{r \rightarrow \infty} \frac{N(r, f)}{T(r, f)} \liminf _{r \rightarrow \infty}\left(1+\frac{N\left(r, \frac{1}{f}\right)}{N(r, f)}\right)=(1-\delta(\infty, f))\left(1+\frac{1}{q_{2}}\right) .
\end{aligned}
$$

Since the inequality (1.9) with $\eta=0$ gives us $\kappa(f) \leq 1$, we get the inequalities (1.16).

Proof of Theorem 1.4. First we establish that for functions satisfying the conditions of Theorem 1.2 (b) with $\eta \geq 0$ and the condition (1.17) the following, somewhat more sharp than (1.9) estimate holds:

$$
\kappa(f) \leq \frac{2}{1+\cos p \eta\{1-\nu+\nu \cosh (p \ln \mu)\}} .
$$

Using Lemma 1.1 and the inequality (1.5), we get

$$
\begin{aligned}
2 T(r, f) \geq & (1+\cos p \eta) N(r ; 0, \infty) \\
& +p \cos p \eta \int_{0}^{r} N(t ; 0, \infty) \sinh \left(p \ln \frac{r}{t}\right) \frac{d t}{t}+o(T(r, f)) .
\end{aligned}
$$

By the condition (1.17) we have

$$
\begin{array}{rl}
p \int_{0}^{r} & N(t ; 0, \infty) \sinh \left(p \ln \frac{r}{t}\right) \frac{d t}{t} \geq p \int_{r / \mu}^{r} N(t ; 0, \infty) \sinh \left(p \ln \frac{r}{t}\right) \frac{d t}{t} \\
& \geq N\left(\frac{r}{\mu} ; 0, \infty\right) p \int_{r / \mu}^{r} \sinh \left(p \ln \frac{r}{t}\right) \frac{d t}{t}=N\left(\frac{r}{\mu} ; 0, \infty\right)\{\cosh (p \ln \mu)-1\} \\
& \geq N(r ; 0, \infty) \nu\{\cosh (p \ln \mu)-1\}
\end{array}
$$

We come to the relation

$$
2 T(r, f) \geq\{1+\cos p \eta(1-\nu+\nu \cosh (p \ln \mu))\} N(r ; 0, \infty)+o(T(r, f)),
$$

whence (1.19) follows.

¿From (1.19) with $\eta=0$ we get

$$
\kappa(f) \leq \frac{2}{2+\nu(\cosh (p \ln \mu)-1)}<1
$$

and, using (1.11), get (1.18).

The proof of the following theorem is much more difficult.

THEOREM 1.5. If the conditions of Theorem $1.2(\mathrm{~b})$ are satisfied and, in addition, there exists a number $\xi<\infty$, such that

$$
\begin{gathered}
\sum_{m} \frac{1}{\left|a_{m}\right|^{\xi}}+\sum_{n} \frac{1}{\left|b_{n}\right|^{\xi}}<\infty, \\
\eta \leq \frac{\pi}{60 \xi}
\end{gathered}
$$


then

$$
\min \{\delta(0, f), \delta(\infty, f)\} \geq A>0
$$

where $A$ is an absolute constant.

Let us mention some of the corollaries of Theorem 1.5.

COROLlary 1. If the conditions of Theorem 1.2 (b) with $\eta=0$ are satisfied and, in addition, there exists a number $\xi<\infty$, such that (1.20) is satisfied, then (1.21) holds.

Observe that (1.20) is always true for $\xi>\rho[f]$. Therefore Corollary 1 immediately implies

COROLlary 2. If the conditions of Theorem 1.2 (b) with $\eta=0$ are satisfied and, in addition, the function $f(z)$ has finite order, then (1.21) holds.

Let us mention, also, the following statements.

COROllary 3. Let $f(z)$ be a function with positive zeros and negative poles. If $f(z)$ has finite lower order and $\limsup _{r \rightarrow \infty} r^{-1} T(r, f)=\infty$, then $(1.21)$ holds.

COROllary 4. Let $f(z)$ be an entire function with real zeros. If $f(z)$ has a finite lower order and $\limsup _{r \rightarrow \infty} r^{-2} T(r, f)=\infty$, then $(1.21)$ holds.

Corollary 3 follows from Corollary 2, since, by Corollary 3 of Theorem 1.1 the function $f(z)$ has finite order. Corollary 4 can be derived from Corollary 2 using Corollary 4 of Theorem 1.1 .

Now we turn to auxiliary results needed to prove Theorem 1.5.

LEMMA 1.2. Let $\left\{c_{k}\right\}_{k=1}^{\infty}$ be a sequence of points in the complex plane, lying in the angle $\left\{|\arg z| \leq \frac{\pi}{6}-\varepsilon\right\}$, where $0<\varepsilon \leq \frac{\pi}{6}$, and satisfying the condition

$$
\sum_{k=1}^{\infty} \frac{1}{\left|c_{k}\right|^{3}}<\infty
$$

Let $^{1}$

$$
g(z)=\prod_{k=1}^{\infty} E\left(\frac{z}{c_{k}}, 2\right)
$$

Then

$$
\int_{\frac{\pi}{3}-\frac{\varepsilon}{2}}^{\frac{\pi}{3}+\frac{\varepsilon}{2}} \ln ^{+}\left|\frac{g\left(r e^{i \varphi}\right)}{g\left(-r e^{i \varphi}\right)}\right| d \varphi \geq \varepsilon \sin \frac{\varepsilon}{2} N(r, 0, g) .
$$

Proof. It is easy to see that for $\operatorname{Im} \zeta>0$ the equality

$$
\ln \frac{E(\zeta, 2)}{E(-\zeta, 2)}=\ln \left(\frac{1-\zeta}{1+\zeta}\right)+2 \zeta=2 \int_{0}^{\zeta} \frac{t^{2} d t}{t^{2}-1}
$$

\footnotetext{
${ }^{1}$ By Theorem 3.2 from Chapter 2, the product converges absolutely and uniformly in each finite disc.
} 
holds, where the branches on the logarithm in both cases are chosen in such a way that they have zero value at $\zeta=0$, and the integral is taken over the segment joining the points 0 and $\zeta$. Separating the real part, we get

$$
\ln \left|\frac{E\left(\rho e^{i \theta}, 2\right)}{E\left(-\rho e^{i \theta}, 2\right)}\right|=2 \operatorname{Re}\left\{\int_{0}^{\rho} \frac{\tau^{2} e^{2 i \theta}}{\tau^{2} e^{2 i \theta}-1} e^{i \theta} d \tau\right\}=2 \int_{0}^{\rho} \frac{\tau^{4} \cos \theta-\tau^{2} \cos 3 \theta}{\tau^{4}-2 \tau^{2} \cos 2 \theta+1} d \tau
$$

whence

$$
\ln \left|\frac{E\left(\frac{r e^{i \varphi}}{c_{k}}, 2\right)}{E\left(-\frac{r e^{i \varphi}}{c_{k}}, 2\right)}\right|=2 \int_{0}^{r /\left|c_{k}\right|} \frac{\tau^{4} \cos \left(\varphi-\varphi_{k}\right)-\tau^{2} \cos 3\left(\varphi-\varphi_{k}\right)}{\tau^{4}-2 \tau^{2} \cos 2\left(\varphi-\varphi_{k}\right)+1} d \tau,
$$

where $\varphi_{k}=\arg c_{k}$.

Since $\left|\varphi_{k}\right| \leq \frac{\pi}{6}-\varepsilon$, for $\left|\varphi-\frac{\pi}{3}\right| \leq \frac{\varepsilon}{2}$ we have

$$
\frac{\pi}{6}+\frac{\varepsilon}{2} \leq \varphi-\varphi_{k} \leq \frac{\pi}{2}-\frac{\varepsilon}{2}
$$

hence

$$
\begin{gathered}
\cos \left(\varphi-\varphi_{k}\right) \geq \cos \left(\frac{\pi}{2}-\frac{\varepsilon}{2}\right)=\sin \frac{\varepsilon}{2} \\
\cos 3\left(\varphi-\varphi_{k}\right) \leq \cos 3\left(\frac{\pi}{6}+\frac{\varepsilon}{2}\right)=-\sin \frac{3 \varepsilon}{2} .
\end{gathered}
$$

Therefore (1.22) implies that for $\left|\varphi-\frac{\pi}{3}\right| \leq \frac{\varepsilon}{2}$ the inequality

$$
\ln \left|\frac{E\left(\frac{r e^{i \varphi}}{c_{k}}, 2\right)}{E\left(-\frac{r e^{i \varphi}}{c_{k}}, 2\right)}\right| \geq 2 \int_{0}^{r /\left|c_{k}\right|} \frac{\tau^{4} \sin \frac{\varepsilon}{2}+\tau^{2} \sin \frac{3 \varepsilon}{2}}{\tau^{4}+2 \tau^{2}+1} d \tau \geq 2 \sin \frac{\varepsilon}{2} \int_{0}^{r /\left|c_{k}\right|} \frac{\tau^{2} d \tau}{\tau^{2}+1}
$$

holds. Adding these inequalities for different values of $k$, we get $\left(\left|\varphi-\frac{\pi}{3}\right| \leq \frac{\varepsilon}{2}\right)$

$$
\begin{aligned}
\ln \left|\frac{g\left(r e^{i \varphi}\right)}{g\left(-r e^{i \varphi}\right)}\right| & \geq 2 \sin \frac{\varepsilon}{2} \sum_{k=1}^{\infty} \int_{0}^{r /\left|c_{k}\right|} \frac{\tau^{2} d \tau}{\tau^{2}+1} \\
& =2 \sin \frac{\varepsilon}{2} \int_{0}^{\infty} d n(t, 0, g) \int_{0}^{r / t} \frac{\tau^{2} d \tau}{\tau^{2}+1}=2 r^{3} \sin \frac{\varepsilon}{2} \int_{0}^{\infty} \frac{n(t, 0, g)}{t^{2}\left(r^{2}+t^{2}\right)} d t \\
& \geq 2 r^{3} \sin \frac{\varepsilon}{2} \int_{0}^{r} \frac{n(t, 0, g)}{t^{2}\left(r^{2}+t^{2}\right)} d t \geq \sin \frac{\varepsilon}{2} \int_{0}^{r} \frac{n(t, 0, g)}{t} d t=\sin \frac{\varepsilon}{2} N(r, 0, g) .
\end{aligned}
$$

Integrating with respect $\varphi$ from $\frac{\pi}{3}-\frac{\varepsilon}{2}$ to $\frac{\pi}{3}+\frac{\varepsilon}{2}$, we get the desired inequality.

LEMMA 1.3. Let $G(z), G(0)=1$, be a meromorphic function with all zeros in the angle $\left\{|\arg z| \leq \frac{\pi}{60}\right\}$ and all poles in the angle $\left\{|\pi-\arg z| \leq \frac{\pi}{60}\right\}$, and let

$$
\lim _{r \rightarrow \infty} r^{-1} T(r, G)=\infty
$$

Suppose, in addition, that $G(z)$ is of the form

$$
G(z)=e^{S(z)} G_{1}(z)
$$

where $G_{1}(z)$ is a meromorphic function of genus at most 2 , and $S(z)$ is an entire function. 
Then

$$
\min \{\delta(0, G), \delta(\infty, G)\} \geq A>0,
$$

where $A$ is an absolute constant.

Proof. First we consider the case when the genus of the function $G(z)$ itself does not exceed 2. Let

$$
g(z)=\prod_{m} E\left(\frac{z}{a_{m}}, 2\right) \prod_{n} E\left(-\frac{z}{b_{n}}, 2\right) .
$$

Then

$$
\frac{G(z)}{G(-z)}=e^{C z} \frac{g(z)}{g(-z)}
$$

where $C$ is some constant. Applying Lemma 1.2 with $\varepsilon=\frac{9}{10} \cdot \frac{\pi}{6}$, we get

$$
\begin{aligned}
\int_{\frac{\pi}{3}-\frac{\varepsilon}{2}}^{\frac{\pi}{3}+\frac{\varepsilon}{2}} & \ln ^{+}\left|\frac{G\left(r e^{i \varphi}\right)}{G\left(-r e^{i \varphi}\right)}\right| d \varphi \geq \int_{\frac{\pi}{3}-\frac{\varepsilon}{2}}^{\frac{\pi}{3}+\frac{\varepsilon}{2}} \ln ^{+}\left|\frac{g\left(r e^{i \varphi}\right)}{g\left(-r e^{i \varphi}\right)}\right| d \varphi-|C| \varepsilon r \\
& \geq \varepsilon \sin \frac{\varepsilon}{2} N(r, 0, g)+O(r) \\
& =\left(\frac{3 \pi}{20} \sin \frac{3 \pi}{40}\right)\{N(r, 0, G)+N(r, \infty, G)\}+O(r) .
\end{aligned}
$$

Zeros and poles of the function $G(z) / G(-z)$, obviously, are $\left(1, \frac{\pi}{60}\right)$-separated.

By Lemma 1.1 we have the inequality

$$
\begin{aligned}
\frac{1}{2 \pi} \int_{0}^{2 \pi} \ln \left|\frac{G\left(r e^{i \varphi}\right)}{G\left(-r e^{i \varphi}\right)}\right| \cos \varphi d \varphi & \geq \cos \frac{\pi}{60}\left\{N\left(r, \frac{G(z)}{G(-z)}\right)+N\left(r, \frac{G(-z)}{G(z)}\right)\right\} \\
+O(r) & =2 \cos \frac{\pi}{60}\{N(r, 0, G)+N(r, \infty, G)\}+O(r) .
\end{aligned}
$$

By $2 \pi$-periodicity of the function $G\left(r e^{i \varphi}\right)$ in $\varphi$, we have the equality

$$
\frac{1}{2 \pi} \int_{0}^{2 \pi} \ln \left|\frac{G\left(-r e^{i \varphi}\right)}{G\left(r e^{i \varphi}\right)}\right| d \varphi=0
$$

Adding it to the preceding inequality we get

$$
\frac{1}{2 \pi} \int_{0}^{2 \pi} \ln \left|\frac{G\left(-r e^{i \varphi}\right)}{G\left(r e^{i \varphi}\right)}\right|(1-\cos \varphi) d \varphi \geq 2 \cos \frac{\pi}{60}\{N(r, 0, G)+N(r, \infty, G)\}+O(r) .
$$

Since

$$
\begin{aligned}
& 2 m\left(r, \frac{G(-z)}{G(z)}\right) \geq \frac{1}{2 \pi} \int_{0}^{2 \pi} \ln ^{+}\left|\frac{G\left(-r e^{i \varphi}\right)}{G\left(r e^{i \varphi}\right)}\right|(1-\cos \varphi) d \varphi \\
& =\frac{1}{2 \pi} \int_{0}^{2 \pi} \ln ^{+}\left|\frac{G\left(r e^{i \varphi}\right)}{G\left(-r e^{i \varphi}\right)}\right|(1-\cos \varphi) d \varphi+\frac{1}{2 \pi} \int_{0}^{2 \pi} \ln \left|\frac{G\left(-r e^{i \varphi}\right)}{G\left(r e^{i \varphi}\right)}\right|(1-\cos \varphi) d \varphi
\end{aligned}
$$


it follows from here, that $\left(\varepsilon=\frac{9}{10} \cdot \frac{\pi}{6}\right)$

$$
\begin{aligned}
2 m\left(r, \frac{G(-z)}{G(z)}\right) \geq & \frac{1}{2 \pi} \int_{0}^{2 \pi} \ln ^{+}\left|\frac{G\left(r e^{i \varphi}\right)}{G\left(-r e^{i \varphi}\right)}\right|(1-\cos \varphi) d \varphi \\
& +2 \cos \frac{\pi}{60}\{N(r, 0, G)+N(r, \infty, G)\}+O(r) \\
\geq & \frac{1}{2 \pi}\left(1-\cos \frac{\pi}{4}\right) \int_{\frac{\pi}{3}-\frac{\varepsilon}{2}}^{\frac{\pi}{3}+\frac{\varepsilon}{2}} \ln ^{+}\left|\frac{G\left(r e^{i \varphi}\right)}{G\left(-r e^{i \varphi}\right)}\right| d \varphi \\
& +2 \cos \frac{\pi}{60}\{N(r, 0, G)+N(r, \infty, G)\}+O(r) .
\end{aligned}
$$

Using (1.26) we get

$$
\begin{aligned}
2 m & \left(r, \frac{G(-z)}{G(z)}\right) \\
& \geq\left\{\frac{3}{40}\left(1-\cos \frac{\pi}{4}\right) \sin \frac{3 \pi}{40}+2 \cos \frac{\pi}{60}\right\}\{N(r, 0, G)+N(r, \infty, G)\}+O(r) .
\end{aligned}
$$

Since

$$
\begin{aligned}
m\left(r, \frac{G(-z)}{G(z)}\right) & \leq m(r, G)+m\left(r, \frac{1}{G}\right) \\
& =2 T(r, G)-\{N(r, 0, G)+N(r, \infty, G)\}=2 T(r, G)-N(r ; 0, \infty),
\end{aligned}
$$

we have

$$
T(r, G) \geq \frac{1}{2}\left\{1+\frac{3}{80}\left(1-\cos \frac{\pi}{4}\right) \sin \frac{3 \pi}{40}+\cos \frac{\pi}{60}\right\} N(r ; 0, \infty)+O(r) .
$$

Straightforward computation shows that the number in braces exceeds 2.0004. Denoting this number by $2+2 B$, where $B>0.0002$, we get

$$
T(r, G) \geq(1+B) N(r ; 0, \infty)+O(r),
$$

by (1.23), this inequality implies

$$
\kappa(G) \leq 1 /(1+B) .
$$

Using the inequality (1.11) and letting $A=B /(1+B)$, we get (1.25).

In the case when the genus of $G(z)$ exceeds 2 , the function $S(z)$ in the representation (1.24) is either a polynomial of degree at least 3 , or an entire transcendent function. In both cases, by Lemma 6.2, we have

$$
r^{3}=O\left(T\left(r, e^{S(z)}\right)\right) .
$$

Since the genus of $G_{1}(z)$ does not exceed 2, we have

$$
T\left(r, G_{1}\right)=o\left(r^{3}\right),
$$

and hence

$$
\begin{gathered}
N(r, 0, G)=N\left(r, 0, G_{1}\right)=o\left(r^{3}\right), N(r, \infty, G)=N\left(r, \infty, G_{1}\right)=o\left(r^{3}\right), \\
r^{3}=O\left(T\left(r, e^{S(z)}\right)+T\left(r, G_{1}\right)\right)=O(T(r, G)) .
\end{gathered}
$$

Therefore $\delta(0, G)=1, \delta(\infty, G)=1$. The proof of the lemma is completed. 
Now we turn to the proof of Theorem 1.5.

Without loss of generality we may assume that all zeros of the function $f(z)$ are in the region $D_{1}^{p}(\eta)$ and all of its poles are in the region $D_{2}^{p}(\eta)$. In fact, by (1.1) the products

$$
\pi_{1}(z)=\prod_{a_{m} \notin D_{1}^{p}(\eta)} E\left(\frac{z}{a_{m}}, p-1\right), \pi_{2}(z)=\prod_{b_{n} \notin D_{2}^{p}(\eta)} E\left(\frac{z}{b_{n}}, p-1\right)
$$

are absolutely convergent. Let

$$
f_{1}(z)=f(z) \frac{\pi_{2}(z)}{\pi_{1}(z)}
$$

Then all zeros of the function $f_{1}(z)$ are in $D_{1}^{p}(\eta)$, and all of its poles are in $D_{2}^{p}(\eta)$, the function $f_{1}(z)$ also satisfies (1.20), and since $T\left(r, \pi_{j}\right)=o\left(r^{p}\right), j=1,2$, we have

$$
\begin{gathered}
T\left(r, f_{1}\right)=T(r, f)+o\left(r^{p}\right), \\
N\left(r, \infty, f_{1}\right)=N(r, \infty, f)+o\left(r^{p}\right), N\left(r, 0, f_{1}\right)=N(r, 0, f)+o\left(r^{p}\right),
\end{gathered}
$$

and because (see the proof of Theorem $1.2(\mathrm{~b})) r^{p}=o(T(r, f)$ ), we get

$$
\lim _{r \rightarrow \infty} r^{-p} T\left(r, f_{1}\right)=\infty, \delta\left(0, f_{1}\right)=\delta(0, f), \delta\left(\infty, f_{1}\right)=\delta(\infty, f) .
$$

We may assume also, that

$$
\sum_{m} \frac{1}{\left|a_{m}\right|^{p}}+\sum_{n} \frac{1}{\left|b_{n}\right|^{p}}=\infty
$$

since otherwise we have $N(r, \infty, f)=o\left(r^{p}\right), N(r, 0, f)=o\left(r^{p}\right)$, and hence $\delta(0, f)=$ $\delta(\infty, f)=1$.

Thus, we assume that all zeros of the function $f(z)$ are in the region $D_{1}^{p}(\eta)$, and all of its poles are in the region $D_{2}^{p}(\eta)$, and the condition (1.27) is satisfied. Note that then $\xi>p$, where $\xi$ is the number appearing in the statement of the theorem; and denote by $q$ a positive integer satisfying

$$
\sum_{m} \frac{1}{\left|a_{m}\right|^{q}}+\sum_{n} \frac{1}{\left|b_{n}\right|^{q}}=\infty ; \sum_{m} \frac{1}{\left|a_{m}\right|^{q+1}}+\sum_{n} \frac{1}{\left|b_{n}\right|^{q+1}}<\infty .
$$

It is clear that the inequality

$$
p \leq q \leq[\xi]
$$

holds.

By (1.28) the function $f(z)$ can be represented in the form

$$
f(z)=e^{H(z)} \frac{\prod_{m} E\left(\frac{z}{a_{m}}, q\right)}{\prod_{n} E\left(\frac{z}{b_{n}}, q\right)},
$$

where $H(z)$ is an entire function. We choose an odd number $s$ in such a way, that

$$
p s \leq q<p(s+2),
$$

and let

$$
l=p s, \omega=\exp \left(\frac{2 \pi i}{l}\right)
$$


Consider the function

$$
F(z)=\prod_{k=0}^{l-1} f\left(\omega^{k} z\right)
$$

Using the equality $(\nu=0,1,2, \ldots)$

$$
\sum_{k=0}^{l-1} \omega^{k \nu}= \begin{cases}l, & \text { if } \nu \text { is divisible by } l, \\ 0, & \text { if } \nu \text { is not divisible by } l,\end{cases}
$$

we easily get

$$
\prod_{k=0}^{l-1} E\left(\omega^{k} z, q\right)=E\left(z^{l},\left[\frac{q}{l}\right]\right), \sum_{k=0}^{l-1} H\left(\omega^{k} z\right)=S\left(z^{l}\right)
$$

where $S(z)$ is an entire function. Therefore we have

$$
F(z)=e^{S\left(z^{l}\right)} \frac{\prod_{m} E\left(\frac{z^{l}}{a_{m}^{l}},\left[\frac{q}{l}\right]\right)}{\prod_{n} E\left(\frac{z^{l}}{b_{n}^{l}},\left[\frac{q}{l}\right]\right)} .
$$

Let us show that the meromorphic function

$$
G(z)=F\left(z^{1 / l}\right)=e^{S(z)} \frac{\prod_{m} E\left(\frac{z}{a_{m}^{l}},\left[\frac{q}{l}\right]\right)}{\prod_{n} E\left(\frac{z}{b_{n}^{l}},\left[\frac{q}{l}\right]\right)}
$$

satisfies the conditions of Lemma 1.3 and the inequalities

$$
\delta(0, G) \leq \delta(0, f), \delta(\infty, G) \leq \delta(\infty, f)
$$

hold.

Since (1.29) implies $q<l+2 p \leq 3 l$, we have $\left[\frac{q}{l}\right] \leq 2$. The conditions

$$
\left|\arg a_{m}-\pi \frac{2 j}{p}\right| \leq \eta,\left|\arg b_{n}-\pi \frac{2 j+1}{p}\right| \leq \eta
$$

( $j \geq 0$ is an integer) imply that

$$
\left|\arg \left(a_{m}^{l}\right)-s \pi 2 j\right| \leq l \eta,\left|\arg \left(b_{n}^{l}\right)-s \pi(2 j+1)\right| \leq l \eta,
$$

where $s$ is defined by (1.29). Taking into account that $s$ is odd, and $l \eta \leq q \eta \leq[\xi] \eta \leq$ $\frac{\pi}{60}$, we observe that all zeros of the function $G(z)$ are in the angle $\left\{|\arg z| \leq \frac{\pi}{60}\right\}$, and all of its poles are in the angle $\left\{|\pi-\arg z| \leq \frac{\pi}{60}\right\}$.

Now we show that zeros and poles of different factors in (1.30) cannot cancel each other, this fact will be used later on. Zeros of all function $f\left(\omega^{k} z\right), k=$ $0,1, \ldots, l-1$, belong to the set

$$
\bigcup_{k=0}^{l-1} \bigcup_{j=0}^{p}\left\{\left|\arg z-k \frac{2 \pi}{l}-\pi \frac{2 j}{p}\right| \leq \eta, 0<|z|<\infty\right\},
$$


and their poles belong to the set

$$
\bigcup_{k=0}^{l-1} \bigcup_{j=0}^{p}\left\{\left|\arg z-k \frac{2 \pi}{l}-\pi \frac{2 j+1}{p}\right| \leq \eta, 0<|z|<\infty\right\} .
$$

Let us show that these sets have an empty intersection.

Assume the contrary, let $z$ be a point belonging to both sets. Then there exist integers $k_{1}, j_{1}, k_{2}, j_{2}$, such that

$$
\left|\arg z-\pi \frac{2 k_{1}}{l}-\pi \frac{2 j_{1}}{p}\right| \leq \eta,\left|\arg z-\pi \frac{2 k_{2}}{l}-\pi \frac{2 j_{2}+1}{p}\right| \leq \eta
$$

Hence

$$
\left|\pi \frac{2 k_{1}}{l}+\pi \frac{2 j_{1}}{p}-\pi \frac{2 k_{2}}{l}-\pi \frac{2 j_{2}+1}{p}\right| \leq 2 \eta,
$$

and since $l=p s$, we have

$$
\frac{\pi}{p}\left|\frac{2\left(k_{1}-k_{2}\right)}{s}-2\left(j_{2}-j_{1}\right)-1\right| \leq 2 \eta .
$$

Because the distance from a fraction with an even numerator and the odd denominator $s$ to an arbitrary odd number is not less than $1 / s$, we get

$$
\frac{\pi}{p s} \leq 2 \eta
$$

and since $p s \leq q<\xi, \eta \leq \frac{\pi}{60 \xi}$, we get a contradiction.

It is clear that all points $a_{m}^{l}$ are zeros of the function $G(z)$, and all points $b_{n}^{l}$ are its poles. If we assumed that $\liminf _{r \rightarrow \infty} r^{-1} T(r, G)<\infty$, we would get

$$
\sum_{m} \frac{1}{\left|a_{m}^{l}\right|}+\sum_{n} \frac{1}{\left|b_{n}^{l}\right|}<\infty
$$

But this contradicts (1.28) since $l \leq q$. Therefore (1.23) holds, and hence the function $G(z)$ satisfies the conditions of Lemma 1.3.

To prove (1.31) observe that (1.30) implies

$$
T(r, G)=T\left(r^{1 / l}, F\right) \leq l T\left(r^{1 / l}, f\right) .
$$

Next, since, as we have already shown, zeros and poles of different factors in (1.30) cannot cancel each other, the equalities

$$
\begin{array}{r}
N(r, \infty, G)=N\left(r^{1 / l}, \infty, F\right)=l N\left(r^{1 / l}, \infty, f\right) \\
N(r, 0, G)=N\left(r^{1 / l}, 0, F\right)=l N\left(r^{1 / l}, 0, f\right)
\end{array}
$$

hold. The inequalities (1.31) follow immediately from (1.32) and (1.33).

Applying Lemma 1.3 to the function $G(z)$ and using (1.31), we get the conclusion of Theorem 1.5. 


\section{Meromorphic functions with poles and zeros located close to a system of rays}

In this section we shall use systematically angular Nevanlinna characteristics and the results of Sections 5 and 6 from Chapter 1, and Section 3 from Chapter 3.

Denote by $D=D\left(\alpha_{1}, \ldots, \alpha_{n}\right)$ the system of rays

$$
\bigcup_{j=1}^{n}\left\{\arg z=\alpha_{j}\right\}, \alpha_{1}<\alpha_{2}<\cdots<\alpha_{n}<\alpha_{n+1}=\alpha_{1}+2 \pi .
$$

Let

$$
\omega_{j}(D)=\frac{\pi}{\alpha_{j+1}-\alpha_{j}}(j=1, \ldots, n), \omega(D)=\max _{1 \leq j \leq \eta} \omega_{j}(D) .
$$

Let $f(z)$ be a meromorphic function, and $a$ be a number from the extended complex plane. We introduce the function ${ }^{2}$

$$
U_{D}(r, a, f)=\max _{1 \leq t \leq r} \sum_{j=1}^{n} t^{\omega_{j}(D)-\omega(D)} \bar{C}_{\alpha_{j} \alpha_{j+1}}(t, a, f) .
$$

The growth of the function $U_{D}(r, a, f)$ as $r \rightarrow \infty$ is one of the natural ways to measure how distant are the $a$-points of the function $f(z)$ from the system $D\left(\alpha_{1}, \ldots, \alpha_{n}\right)$ of rays. Note that the relations $U_{D}(r, a, f) \equiv 0$ means that all $a$-points of the function $f(z)$ lying outside the disc $\{|z| \leq 1\}$ belong to the system $D\left(\alpha_{1}, \ldots, \alpha_{n}\right)$ of rays. The relation $U_{D}(r, a, f)=O(1)$ holds if the number of $a$-points not lying on the rays of the system $D\left(\alpha_{1}, \ldots, \alpha_{n}\right)$ is finite, but it can also hold in cases when the number of such points is infinite.

EXAmple 1 . The condition $U_{D}(r, a, f)=O(1)$ for the system $D(0, \pi)$ is equivalent to the condition

$$
\sum_{r_{m}>1} \frac{\left|\sin \varphi_{m}\right|}{r_{m}}<\infty
$$

where $r_{m} e^{i \varphi_{m}}$ are $a$-points of the function $f(z)$, listed without taking into account their multiplicities. In fact, we have

$$
\begin{aligned}
& \bar{C}_{0 \pi}(r, a, f)=2 \sum_{\substack{1<r_{m}<r \\
0<\varphi_{m}<\pi}}\left(\frac{1}{r_{m}}-\frac{r_{m}}{r^{2}}\right) \sin \varphi_{m} \leq 2 \sum_{\substack{1<r_{m}<r \\
0<\varphi_{m}<\pi}} \frac{\sin \varphi_{m}}{r_{m}} \\
& \leq 2 \sum_{\substack{r_{m}>1 \\
0<\varphi_{m}<\pi}} \frac{\sin \varphi_{m}}{r_{m}} ; \\
& \bar{C}_{0 \pi}(r, a, f) \geq 2 \sum_{\substack{1<r_{m}<\frac{r}{2} \\
0<\varphi_{m}<\pi}}\left(\frac{1}{r_{m}}-\frac{r_{m}}{r^{2}}\right) \sin \varphi_{m} \\
&=2 \sum_{\substack{1<r_{m}<\frac{r}{2} \\
0<\varphi_{m}<\pi}}\left(1-\frac{r_{m}^{2}}{r^{2}}\right) \frac{\sin \varphi_{m}}{r_{m}} \geq 2 \cdot \frac{3}{4} \sum_{\substack{1<r_{m}<\frac{r}{2} \\
0<\varphi_{m}<\pi}} \frac{\sin \varphi_{m}}{r_{m}} .
\end{aligned}
$$

\footnotetext{
${ }^{2}$ The parameters $\bar{C}_{\alpha \beta}(r, a, f)$ were defined on p.???
} 
Estimating in a similar way the parameter $\bar{C}_{\pi, 2 \pi}(r, a, f)$ and observing that for the system $D(0, \pi)$ we have $\omega_{1}(D)=\omega_{2}(D)=\omega(D)=1$ and

$$
U_{D}(r, a, f)=\max _{1 \leq t \leq r}\left\{\bar{C}_{0 \pi}(t, a, f)+\bar{C}_{\pi, 2 \pi}(t, a, f)\right\}=\bar{C}_{0 \pi}(r, a, f)+\bar{C}_{\pi, 2 \pi}(r, a, f),
$$

we verify the claim stated above. It is clear that (2.1) holds, for example, when $r_{m}=m, \varphi_{m}=1 / m$.

Observe that letting $z_{m}=r_{m} e^{i \varphi_{m}}$, we can rewrite the condition $(2.1)$ in the form

$$
\sum_{\left|z_{m}\right|>1}\left|\operatorname{Im} \frac{1}{z_{m}}\right|<\infty
$$

For $a \neq b$ we let

$$
U_{D}(r, a, b ; f)=U_{D}(r, a, f)+U_{D}(r, b, f),
$$

in the cases when $f$ is clear from context, we write $U_{D}(r, a, b)$ and $U_{D}(r, a)$ instead of $U_{D}(r, a, b ; f)$ and $U_{D}(r, a, f)$, respectively.

The quantity

$$
\mu(E)=\limsup _{r \rightarrow \infty} r^{-1} \operatorname{mes}(E \cap(0, r)),
$$

defined for a measurable subset $E \subset[0, \infty)$, will be called the upper relative measure of $E$.

It is easy to see that the following statements are true.

(a) $0 \leq \mu(E) \leq 1$ for each $E$.

(b) If $E \subset \bigcup_{k=1}^{m} E_{k}, m<\infty$, then $\mu(E) \leq \sum_{k=1}^{m} \mu\left(E_{k}\right)$.

(c) If the logarithmic measure of $E$ is finite (that is, $\int_{E \cap[1, \infty)} r^{-1} d r<\infty$ ), then $^{3} \mu(E)=0$.

Denote by $L^{\prime}$ the class of all measurable subsets $E \subset[0, \infty)$ satisfying $\mu(E)<1$. We shall repeatedly use the property of the class $L^{\prime}$ described in the following lemma.

LEMma 2.1. Let $u(r)$ and $v(r)$ be two non-decreasing functions such that $u(r) \leq$ $v(r)$ for all $r \geq 0$, excluding, possibly, a set $E \in L^{\prime}$. Then there exists a constant $K, 1 \leq K<\infty$, such that for all sufficiently large $r$ the inequality $u(r) \leq v(K r)$ holds.

Proof. Suppose that there exists a sequence $r_{n} \uparrow \infty$, such that

$$
u\left(r_{n}\right)>v\left(\frac{2}{1-\mu(E)} r_{n}\right)
$$

\footnotetext{
${ }^{3}$ In fact,

$$
\operatorname{mes}(E \cap(0, r))=\int_{E \cap(0, \sqrt{r})} d t+\int_{E \cap(\sqrt{r}, r)} d t \leq \sqrt{r}+r \int_{E \cap(\sqrt{r}, r)} t^{-1} d t=o(r) .
$$
}


Consider the set $E_{1}=\bigcup_{n=1}^{\infty}\left(r_{n}, r_{n 1}\right)$, where $r_{n 1}=\frac{2}{1-\mu(E)} r_{n}$. It is clear that

$$
\begin{aligned}
\mu\left(E_{1}\right) & \geq \limsup _{n \rightarrow \infty} r_{n 1}^{-1} \operatorname{mes}\left(E_{1} \cap\left(0, r_{n 1}\right)\right) \\
& \geq \limsup _{n \rightarrow \infty} r_{n 1}^{-1}\left(r_{n 1}-r_{n}\right)=\frac{1+\mu(E)}{2}>\mu(E) .
\end{aligned}
$$

Therefore there exists a point $r \in E_{1} \backslash E$. Let $r \in\left(r_{k}, r_{k 1}\right)$, then we have $u\left(r_{k}\right) \leq u(r) \leq v(r) \leq v\left(r_{k 1}\right)=v\left(\frac{2}{1-\mu(E)} r_{k}\right)$, we get a contradiction.

We introduce the parameter

$$
\delta^{\prime}(a, f)=\sup _{C E \in L^{\prime}} \liminf _{\substack{r \rightarrow \infty \\ r \in E}} \frac{m(r, a)}{T(r, f)}=1-\inf _{C E \in L^{\prime}} \limsup _{\substack{r \rightarrow \infty \\ r \in E}} \frac{N(r, a)}{T(r, f)} .
$$

Comparing with the parameter $\delta(a, f)$ and the parameter $\delta^{*}(a, f)$ introduced in Section 6 of Chapter 4 (p. ???), we see that

$$
\delta(a, f) \leq \delta^{*}(a, f) \leq \delta^{\prime}(a, f),
$$

thus, for each deficient value $a$ the inequality $\delta^{\prime}(a, f)>0$ holds.

The following theorem is the main result of this section.

TheOREM 2.1. Let $D=D\left(\alpha_{1}, \ldots, \alpha_{n}\right)$ be a system of rays, $f(z)$ be a meromorphic function satisfying $\delta^{\prime}(a, f)>0$ for some $a \neq 0, \infty$. Then the growth category of $f(z)$ does not exceed the growth category of

$$
r^{\omega(D)}\left\{U_{D}(r, 0, \infty)+1\right\} .
$$

Let us agree for the remainder of this section that $K$ will be used for different finite, positive, independent of $r$ constants.

The condition $\delta^{\prime}(a, f)>0$ implies that the inequality

$$
T(r, f) \leq \frac{2}{\delta^{\prime}(a, f)} m(r, a), r \notin B_{1}
$$

holds, where $B_{1} \subset[0, \infty)$ is a set satisfying $\mu\left(B_{1}\right)<1$. By Lemma 2.1 from Chapter 3 (p. ???) we have

$$
m(r, a) \leq m\left(r, \frac{f}{f^{\prime}}\right)+Q(r, f) .
$$

We assume that $f(z)$ is transcendental, since otherwise Theorem 2.1 is trivial. Then

$$
Q(r, f)=O(\ln r)+O(\ln T(r, f)) \leq \frac{1}{2} T(r, f)
$$

outside some set $B_{2} \subset[0, \infty)$ of finite measure, and (2.2) and (2.3) imply that for $r \notin B_{3}=B_{1} \cup B_{2}$ the inequality

$$
T(r, f) \leq K m\left(r, \frac{f}{f^{\prime}}\right)
$$

holds. Observe that $\mu\left(B_{3}\right) \leq \mu\left(B_{1}\right)+\mu\left(B_{2}\right)=\mu\left(B_{1}\right)<1$.

Next, we need to estimate $m\left(r, \frac{f}{f^{\prime}}\right)$ from above in terms of $U_{D}(r, 0, \infty)$. Since such estimates are of independent interest, we state them as a separate theorem. 
TheOREm 2.2. Let $D=D\left(\alpha_{1}, \ldots, \alpha_{n}\right)$ be a system of rays, $f(z)$ be a meromorphic function, $\varepsilon>0$ and $d>1$ be preassigned numbers.

(A) For all $r \geq 0$ except, possibly, a subset $E \subset[0, \infty)$ satisfying $\mu(E)<\varepsilon$, the inequality

$$
m\left(r, \frac{f}{f^{\prime}}\right) \leq K r^{d \omega(D)}\left\{U_{D}(r, 0, \infty)+\ln r+1\right\}^{d}\left\{\ln ^{+} T(r, f)+1\right\}^{d}
$$

holds.

(B) If the function $f(z)$ satisfies the condition

$$
\int_{1}^{\infty} r^{-q(D)-1} \ln ^{+} T(r, f) d r<\infty, q(D)=\min _{1 \leq j \leq n} \omega_{j}(D),
$$

then for all $r \geq 0$ except, possibly, a subset $E \subset[0, \infty)$ satisfying $\mu(E)<\varepsilon$, the inequality

$$
m\left(r, \frac{f}{f^{\prime}}\right) \leq K r^{\omega(D)}\left\{U_{D}(d r, 0, \infty)+1\right\}
$$

holds.

To prove Theorem 2.2 we need estimates from above for

$$
m_{\alpha \beta}(r, f)=\frac{1}{2 \pi} \int_{\alpha}^{\beta} \ln ^{+}\left|f\left(r e^{i \varphi}\right)\right| d \varphi
$$

where $f(z)$ is an arbitrary meromorphic function and $0<\beta-\alpha \leq 2 \pi$, in terms of the Nevanlinna characteristic $S_{\alpha \beta}(r, f)$. Such estimates are given by the following theorem. Its proof is rather tedious, and will be postponed till Section 3 of the present chapter.

THEOREM 2.3. Let $f(z)$ be a function meromorphic in the angle $\{\alpha \leq \arg z \leq$ $\beta\}, 0<\beta-\alpha \leq 2 \pi ; \varepsilon>0$ and $d>1$ be preassigned numbers; $\omega=\pi /(\beta-\alpha)$. For all $r \geq 0$ except, possibly, a subset $E \subset[0, \infty)$ satisfying $\mu(E)<\varepsilon$, the inequalities

$$
\begin{aligned}
& m_{\alpha \beta}(r, f) \leq K r^{\omega}\left\{S_{\alpha \beta}(r, f)+1\right\}^{d}, \\
& m_{\alpha \beta}(r, f) \leq K r^{\omega}\left\{S_{\alpha \beta}(d r, f)+1\right\}
\end{aligned}
$$

hold.

Now we prove the statement (A) of Theorem 2.2.

By Theorem 2.3 for all $r \geq 0$ except, possibly, a subset $E_{j} \subset[0, \infty)$ satisfying $\mu\left(E_{j}\right)<\frac{\varepsilon}{n}$, the inequality

$$
m_{\alpha_{j} \alpha_{j+1}}\left(r, \frac{f}{f^{\prime}}\right) \leq K r^{\omega_{j}(D)}\left\{S_{\alpha_{j} \alpha_{j+1}}\left(r, \frac{f}{f^{\prime}}\right)+1\right\}^{d}
$$

holds $(j=1, \ldots, n)$. Applying Theorem 5.2 from Chapter 1 (p. ???) and Theorem 3.1 from Chapter 3 (p. ???), we get

$$
\begin{aligned}
& S_{\alpha_{j} \alpha_{j+1}}\left(r, \frac{f}{f^{\prime}}\right)=A_{\alpha_{j} \alpha_{j+1}}\left(r, \frac{f^{\prime}}{f}\right)+B_{\alpha_{j} \alpha_{j+1}}\left(r, \frac{f^{\prime}}{f}\right) \\
& \quad+C_{\alpha_{j} \alpha_{j+1}}\left(r, \frac{f^{\prime}}{f}\right)+O(1)=C_{\alpha_{j} \alpha_{j+1}}\left(r, \frac{f^{\prime}}{f}\right)+Q_{\alpha_{j} \alpha_{j+1}}(r, f) .
\end{aligned}
$$

Recalling that, by the definition of $Q_{\alpha_{j} \alpha_{j+1}}(r, f)$ (see p. ???),

$$
Q_{\alpha_{j} \alpha_{j+1}}(r, f)=O(\ln r)+O(\ln T(r, f))
$$


outside some set $E_{j}^{(1)} \subset[0, \infty)$ of finite measure, and using the obvious equality

$$
C_{\alpha_{j} \alpha_{j+1}}\left(r, \frac{f^{\prime}}{f}\right)=\bar{C}_{\alpha_{j} \alpha_{j+1}}(r, f)+\bar{C}_{\alpha_{j} \alpha_{j+1}}\left(r, \frac{1}{f}\right)
$$

we get that for $r \notin E=\bigcup_{j=1}^{n}\left(E_{j} \cup E_{j}^{(1)}\right)$ the inequality

$$
\begin{aligned}
m\left(r, \frac{f}{f^{\prime}}\right)=\sum_{j=1}^{n} m_{\alpha_{j} \alpha_{j+1}}\left(r, \frac{f}{f^{\prime}}\right) \\
\leq K \sum_{j=1}^{n} r^{\omega_{j}(D)}\left\{\bar{C}_{\alpha_{j} \alpha_{j+1}}(r, f)+\bar{C}_{\alpha_{j} \alpha_{j+1}}\left(r, \frac{1}{f}\right)+\ln r+\ln ^{+} T(r, f)\right\}^{d} \\
\leq K r^{d \omega(D)} \sum_{j=1}^{n}\left\{r ^ { \omega _ { j } ( D ) - \omega ( D ) } \left(\bar{C}_{\alpha_{j} \alpha_{j+1}}(r, f)\right.\right. \\
\left.\left.\quad+\bar{C}_{\alpha_{j} \alpha_{j+1}}\left(r, \frac{1}{f}\right)+\ln r+\ln ^{+} T(r, f)\right)\right\}^{d}
\end{aligned}
$$

holds. Using the inequality $\sum_{j} a_{j}^{d} \leq\left(\sum_{j} a_{j}\right)^{d}, a_{j} \geq 0, d>1$, we get from here, that for $r \notin E$ the inequality

$$
\begin{aligned}
m\left(r, \frac{f}{f^{\prime}}\right) \leq & K r^{d \omega(D)}\left\{\sum _ { j = 1 } ^ { n } r ^ { \omega _ { j } ( D ) - \omega ( D ) } \left(\bar{C}_{\alpha_{j} \alpha_{j+1}}(r, f)\right.\right. \\
& \left.\left.+\bar{C}_{\alpha_{j} \alpha_{j+1}}\left(r, \frac{1}{f}\right)+\ln r+\ln ^{+} T(r, f)\right)\right\}^{d} \\
\leq & K r^{d \omega(D)}\left\{U_{D}(r, 0, \infty)+\ln r+\ln ^{+} T(r, f)\right\}^{d} \\
\leq & K r^{d \omega(D)}\left\{U_{D}(r, 0, \infty)+\ln r+1\right\}^{d}\left\{\ln ^{+} T(r, f)+1\right\}^{d}
\end{aligned}
$$

holds. Since

$$
\mu(E) \leq \sum_{j=1}^{n} \mu\left(E_{j} \cup E_{j}^{(1)}\right) \leq \sum_{j=1}^{n}\left\{\mu\left(E_{j}\right)+\mu\left(E_{j}^{(1)}\right)\right\}<\varepsilon,
$$

the statement $(\mathrm{A})$ of Theorem 2.2 has been proved.

Next, by Theorem 2.3, for all $r \geq 0$ except, possibly, a subset $E_{j}^{\prime} \subset[0, \infty)$ satisfying $\mu\left(E_{j}^{\prime}\right)<\frac{\varepsilon}{n}$, the inequality

$$
m_{\alpha_{j} \alpha_{j+1}}\left(r, \frac{f}{f^{\prime}}\right) \leq K r^{\omega_{j}(D)}\left\{S_{\alpha_{j} \alpha_{j+1}}\left(d r, \frac{f}{f^{\prime}}\right)+1\right\}
$$

holds $(j=1, \ldots, n)$. Using $(2.8),(2.9)$ and observing, that by the condition (2.6) and the definition of $Q_{\alpha_{j} \alpha_{j+1}}(r, f)$ (see p. ???) the relation $Q_{\alpha_{j} \alpha_{j+1}}(r, f)=O(1)$ 
holds, we conclude that for $r \notin E^{\prime}=\bigcup_{j=1}^{n} E_{j}^{\prime}$ the inequality

$$
\begin{aligned}
m\left(r, \frac{f}{f^{\prime}}\right) & =\sum_{j=1}^{n} m_{\alpha_{j} \alpha_{j+1}}\left(r, \frac{f}{f^{\prime}}\right) \\
& \leq K \sum_{j=1}^{n} r^{\omega_{j}(D)}\left\{\bar{C}_{\alpha_{j} \alpha_{j+1}}(d r, f)+\bar{C}_{\alpha_{j} \alpha_{j+1}}\left(d r, \frac{1}{f}\right)+K\right\} \\
& \leq K r^{\omega(D)}\left\{U_{D}(d r, 0, \infty)+1\right\}
\end{aligned}
$$

holds. Since $\mu\left(E^{\prime}\right) \leq \sum_{j=1}^{n} \mu\left(E_{j}^{\prime}\right)<\varepsilon$, we have proved the statement (B) of Theorem 2.2.

Now we turn to the proof of Theorem 2.1.

We may assume that the order of the function $U_{D}(r, 0, \infty)$ is finite, otherwise Theorem 2.1 is trivial.

First we show that this assumption implies that the order of the function $f(z)$ is also finite.

Let $B_{3}$ be a set outside which the inequality (2.4) holds. We choose $\varepsilon>0$ to be so small that $\mu\left(B_{3}\right)+\varepsilon<1$. To estimate $m\left(r, \frac{f}{f^{\prime}}\right)$ we use the statement (A) of Theorem 2.2. Let $E$ be a set, such that $\mu(E)<\varepsilon$ and for $r \notin E$ the inequality (2.5) holds. The inequalities (2.4) and (2.5) imply that for $r \notin B_{4}=B_{3} \cup E$ the inequality

$$
T(r, f) \leq K r^{d \omega(D)}\left\{U_{D}(r, 0, \infty)+\ln r+1\right\}^{d}\left\{\ln ^{+} T(r, f)+1\right\}^{d}
$$

holds. For each $\varepsilon_{1}, 0<\varepsilon_{1}<1$, we can find $r_{1}>0$, such that

$$
\left\{\ln ^{+} T(r, f)+1\right\}^{d} \leq T^{\varepsilon_{1}}(r, f), r \geq r_{1} .
$$

Therefore (2.10) implies that for $r \notin B_{5}=B_{4} \cup\left(0, r_{1}\right)$ we have

$$
T(r, f) \leq K r^{\frac{d \omega(D)}{1-\varepsilon_{1}}}\left\{U_{D}(r, 0, \infty)+1\right\}^{\frac{d}{1-\varepsilon_{1}}} .
$$

We denote the function from the right-hand side of this inequality by $v(r)$. It is a non-decreasing function, and for all $r \notin B_{5}$ the inequality $T(r, f) \leq v(r)$ holds. Since

$$
\mu\left(B_{5}\right) \leq \mu\left(B_{3}\right)+\mu(E)+\mu\left(\left(0, r_{1}\right)\right)<\mu\left(B_{3}\right)+\varepsilon<1,
$$

by Lemma 2.1, for all sufficiently large $r$ the inequality $T(r, f) \leq v(K r)$ holds. Since $U_{D}(r, 0, \infty)$, and hence $v(r)$ have finite order, then $f(z)$ also has finite order.

To prove Theorem 2.1 it suffices to get the inequality

$$
T(r, f) \leq K r^{\omega(D)}\left\{U_{D}(K r, 0, \infty)+1\right\}, r \geq K .
$$

This inequality can be obtained using (2.4) and the statement (B) of Theorem 2.2 (the condition (2.6) is satisfied since, as we have proved, the order of $f(z)$ is finite). In fact, choose so small $\varepsilon>0$, that $\mu\left(B_{3}\right)+\varepsilon<1$, and denote by $E$ a set satisfying $\mu(E)<\varepsilon$ and such that for $r \notin E$ the inequality (2.7) holds. The inequalities (2.4) and (2.7) imply that for $r \notin B_{4}=B_{3} \cup E$ the inequality

$$
T(r, f) \leq K r^{\omega(D)}\left\{U_{D}(d r, 0, \infty)+1\right\}
$$

holds. Since $\mu\left(B_{4}\right) \leq \mu\left(B_{3}\right)+\varepsilon<1$, applying Lemma 2.1 we get (2.13). Our proof of Theorem 2.1 is complete. 
The statement of Theorem 2.1 is no longer true if we drop the condition that for some $a \neq 0, \infty$ the inequality $\delta^{\prime}(a, f)>0$ holds. In fact, for each entire function $f(z)$ without zeros the condition $\delta(0, f)=\delta(\infty, f)=1$ holds, and for each system $D$ of rays we have $U_{D}(r, 0, \infty) \equiv 0$. However the growth of such entire function $f(z)$ can be arbitrarily rapid.

We consider also the following example.

EXAMPLE 2. Let $\left\{h_{k}\right\}_{k=1}^{\infty}$ and $\left\{A_{k}\right\}_{k=1}^{\infty}$ be sequences of positive numbers such that $\lim _{k \rightarrow \infty} h_{k}=\infty, \sum_{k=1}^{\infty} A_{k}<\infty$. Consider the meromorphic function

$$
f(z)=\sum_{k=1}^{\infty} \frac{A_{k}}{z-h_{k}}
$$

By the corollary of Theorem 6.1 from Chapter 5 (p. ???) we have

$$
T(r, f)=N(r, f)+o(1) \text {. }
$$

Since the growth of $N(r, f)$ depends on the choice of the sequence $h_{k}$ only, then, choosing an appropriate sequence we can assure that the function $f(z)$ has a preassigned growth category. Next, since for $\operatorname{Im} z \neq 0$ we have

$$
\operatorname{Im} f(z)=-\operatorname{Im} z \cdot \sum_{k=1}^{\infty} \frac{A_{k}}{\left|z-h_{k}\right|^{2}} \neq 0
$$

and for real negative $z$ the function $f(z)$ is negative, then all real non-negative values the function $f(z)$ takes on the ray $\{\arg z=0\}$ only. This ray can be considered as the system $D=D(0)$, for which $\omega(D)=1 / 2$.

Thus, there exist functions of an arbitrary preassigned growth, such that for infinitely many values of $b$ the equality $U_{D(0)}(r, b) \equiv 0$ holds. It shows that, if we do not suppose that $\delta^{\prime}(a, f)>0$ for some $a \neq 0, \infty$, then we cannot estimate the growth category of $f(z)$ not only by means of the growth category of the function $r^{\omega(D)}\left\{U_{D}(r, 0, \infty)+1\right\}$, but even by means of the growth categories of the functions of the form

$$
r^{\omega(D)}\left\{\sum_{\nu=1}^{q} U_{D}\left(r, b_{\nu}\right)+1\right\},
$$

for an arbitrarily large $q$.

Let us mention some corollaries of Theorem 2.1.

Corollary 1. Let $a, b$, and $c$ be three different numbers from the extended complex plane, $D=D\left(\alpha_{1}, \ldots, \alpha_{n}\right)$ be a system of rays. If a meromorphic function $f(z)$ satisfies the condition $\delta^{\prime}(a, f)>0$, then its category of growth does not exceed the category of the function $r^{\omega(D)}\left\{U_{D}(r, b, c)+1\right\}$.

To prove this corollary it suffices to apply Theorem 2.1 to the function $L(f(z))$, where $L(w)$ is a linear-fractional transformation mapping the points $b$ and $c$ onto the points 0 and $\infty$, respectively.

COROllary 2. Let $a, b$, and $c$ be three different numbers from the extended complex plane, $D=D\left(\alpha_{1}, \ldots, \alpha_{n}\right)$ be a system of rays. If a meromorphic function $f(z)$ satisfies the conditions $\delta^{\prime}(a, f)>0, U_{D}(r, b, c)=O(1)$, then the growth of the function $f(z)$ does not exceed normal type of order $\omega(D)$. 
Since for an entire function $f(z)$ the condition $\delta(\infty, f)=1$ holds, we have

Corollary 3. Let $a$ and $b, a \neq b$, be complex numbers, $D=D\left(\alpha_{1}, \ldots, \alpha_{n}\right)$ be a system of rays, $f(z)$ be an entire function. If the condition $U_{D}(r, a, b)=O(1)$ holds, then the growth of $f(z)$ does not exceed normal type of order $\omega(D)$.

This growth estimate cannot be improved.

ExAMPLE 3. Let us show that for each system $D=D\left(\alpha_{1}, \ldots, \alpha_{n}\right)$ of rays it is possible to construct an entire function $\Phi_{D}(z)$ of normal type of order $\omega(D)$ such that for each $a$ the relation $U_{D}(r, a)=O(1)$ holds.

Let $\left\{\alpha_{j_{0}} \leq \arg z \leq \alpha_{j_{0}+1}\right\}$ be an angle formed by rays of the system $D$, such that $\omega_{j_{0}}(D)=\omega(D)$. Denoting, as in Section 5 of Chapter 2, the Mittag-Leffler function by $E_{\rho}(z)$, let

$$
\Phi_{D}(z)=E_{\omega(D)}\left(z \exp \left(-i \frac{\alpha_{j_{0}}+\alpha_{j_{0}+1}}{2}\right)\right) .
$$

Let us show that $\Phi_{D}(z)$ is a desired function.

By the result proved in Section 5 of Chapter 2 (see $(5.40)$ ), the function $\Phi_{D}(z)$ is a function of normal type of order $\omega(D)$, and its modulus is bounded outside the angle $\left\{\alpha_{j_{0}}<\arg z<\alpha_{j_{0}+1}\right\}$. Therefore we have $(j=1, \ldots, n)$

$$
A_{\alpha_{j} \alpha_{j+1}}\left(r, \Phi_{D}\right)=O(1), B_{\alpha_{j} \alpha_{j+1}}\left(r, \Phi_{D}\right)=O(1)
$$

and hence $(j=1, \ldots, n)$

$$
S_{\alpha_{j} \alpha_{j+1}}\left(r, \Phi_{D}\right)=O(1) .
$$

But then, by Theorem 5.1 from Chapter 1, for each $a$ we have

$$
C_{\alpha_{j} \alpha_{j+1}}(r, a) \leq S_{\alpha_{j} \alpha_{j+1}}\left(r, \Phi_{D}\right)+O(1)=O(1),
$$

from where

$$
U_{D}(r, a)=\max _{1 \leq t \leq r} \sum_{j=1}^{n} t^{\omega_{j}(D)-\omega(D)} \bar{C}_{\alpha_{j} \alpha_{j+1}}(t, a) \leq \sum_{j=1}^{n} C_{\alpha_{j} \alpha_{j+1}}(r, a)=O(1) .
$$

Observe that the condition $U_{D}(r, a)=O(1)$ will also be satisfied for the entire function

$$
\sum_{j=1}^{n} E_{\omega_{j}(D)}\left(z \exp \left(-i \frac{\alpha_{j}+\alpha_{j+1}}{2}\right)\right) .
$$

Applying Corollary 3 to the system $D(0, \pi)$ and using the comments made at the beginning of this section (see Example 1) we get

COROLlary 4. Let $f(z)$ be an entire function, $a \neq b$ be complex numbers, $\left\{z_{m}\right\}$ be the set of all solutions of the equations $f(z)=a$ and $f(z)=b$, in which each solution is listed only once, regardless its multiplicity. If the condition

$$
\sum_{\left|z_{m}\right|>1}\left|\operatorname{Im} \frac{1}{z_{m}}\right|<\infty
$$

is satisfied, then the growth of the function $f(z)$ does not exceed normal type of order 1 .

Note that the function $f(z)=\sin z$ takes all real values $a,|a| \leq 1$, on the real line only. 
We cannot deduce the following result from Theorem 2.1.

Theorem 2.4. Let $D=D\left(\alpha_{1}, \ldots, \alpha_{n}\right)$ be a system of rays, $f(z)$ be a meromorphic function having a Borel exceptional value $a \neq 0, \infty$. Then the category of growth of the function $f(z)$ does not exceed the category of growth the function $r^{\omega(D)}\left\{U_{D}(r, 0, \infty)+1\right\}$.

Proof. By the first fundamental theorem we have

$$
T(r, f)=N(r, a)+m(r, a)+O(1) .
$$

Using (2.3) and assuming that the function $f(z)$ is transcendental, we get that for all $r \geq 0$ except, possibly, a set of finite length, the inequality

$$
T(r, f) \leq K\left\{N(r, a)+m\left(r, \frac{f}{f^{\prime}}\right)\right\}
$$

holds. To estimate $m\left(r, \frac{f}{f^{\prime}}\right)$ we use the statement (A) of Theorem 2.2. For all $r \geq 0$ except, possibly, a set $B_{1}$ such that $\mu\left(B_{1}\right)<\varepsilon<1$ we have

$$
\begin{aligned}
T(r, f) & \leq K N(r, a)+K r^{d \omega(D)}\left\{U_{D}(r, 0, \infty)+\ln r+1\right\}^{d}\left\{\ln ^{+} T(r, f)+1\right\}^{d} \\
& \leq K\left\{N(r, a)+r^{d \omega(D)}\left(U_{D}(r, 0, \infty)+\ln r+1\right)^{d}\right\}\left\{\ln ^{+} T(r, f)+1\right\}^{d} .
\end{aligned}
$$

Taking into account $(2.11)$ we get

$$
\begin{gathered}
T(r, f) \leq K\left\{N(r, a)+r^{d \omega(D)}\left(U_{D}(r, 0, \infty)+\ln r+1\right)^{d}\right\}^{\frac{1}{1-\varepsilon_{1}}} \\
\left(r \notin B_{1}, 0<\varepsilon_{1}<1\right) .
\end{gathered}
$$

Denote by $v(r)$ the function in the right-hand side. By Lemma 2.1, for sufficiently large $r$ the inequality $T(r, f) \leq v(K r)$ holds. By the definition of a Borel exceptional value, the order of $N(r, a)$ is finite. We may assume that the order of the function $U_{D}(r, 0, \infty)$ is also finite, otherwise Theorem 2.4 is trivial. Therefore the order of $v(r)$, and hence of the function $f(z)$, is finite.

To estimate $m\left(r, \frac{f}{f^{\prime}}\right)$ we use the statement (B) of Theorem 2.2. We get that for all $r \geq 0$ except, possibly some set $B_{2}$ satisfying $\mu\left(B_{2}\right)<\varepsilon<1$, the inequality

$$
T(r, f) \leq K\left\{N(r, a)+r^{\omega(D)}\left(U_{D}(d r, 0, \infty)+1\right)\right\}
$$

holds. Using Lemma 2.1 we get that for sufficiently large $r$ the estimate

$$
T(r, f) \leq K\left\{N(K r, a)+r^{\omega(D)}\left(U_{D}(K r, 0, \infty)+1\right)\right\}
$$

holds. Since the category of the characteristic $N(r, a)$ is lower than the category of the characteristic $T(r, f)$, the conclusion of the theorem follows.

The condition of positivity of $\delta^{\prime}(a, f)$ for some $a \neq 0, \infty$ in Theorem 2.1 can be replaced by a condition of more general character which can be stated in the following way: there exist a finite set $\left\{a_{1}, \ldots, a_{q}\right\}, a_{\nu} \neq 0, \infty, \nu=1, \ldots, q$ of numbers, such that the quantity

$$
\delta^{\prime}\left(a_{1}, \ldots, a_{q} ; f\right)=\sup _{C E \in L^{\prime}} \liminf _{\substack{r \rightarrow \infty \\ r \in E}} \frac{\sum_{\nu=1}^{q} m\left(r, a_{\nu}\right)}{T(r, f)}
$$


is positive. In fact, this condition implies that outside some set $B_{1}, \mu\left(B_{1}\right)<1$, the inequality

$$
T(r, f) \leq \frac{2}{\delta^{\prime}\left(a_{1}, \ldots, a_{q} ; f\right)} \sum_{\nu=1}^{q} m\left(r, a_{\nu}\right)
$$

holds. By Lemma 2.1 from Chapter 3 we have

$$
\sum_{\nu=1}^{q} m\left(r, a_{\nu}\right) \leq m\left(r, \frac{f}{f^{\prime}}\right)+Q(r, f) .
$$

Therefore we again get the inequality (2.4) outside some set of relative upper measure $<1$, from here, as it was already shown, the conclusion of Theorem 2.1 follows.

Theorem 2.1 admits also the following generalization.

TheOREM 2.5. Let $D=D\left(\alpha_{1}, \ldots, \alpha_{n}\right)$ be a system of rays, $f(z)$ be a meromorphic function, such that for some $a \neq 0, \infty$ and some integer $l \geq 0$ the inequality $\delta^{\prime}\left(a, f^{(l)}\right)>0$ holds. Then the category of the function $f(z)$ does not exceed the category of the function

$$
r^{\omega(D)}\left\{U_{D}(r, 0, \infty ; f)+1\right\} .
$$

Proof. Now the role of the inequality (2.4) will be played by the inequality

$$
T(r, f) \leq K \sum_{\nu=0}^{l} m\left(r, \frac{f^{(\nu)}}{f^{(\nu+1)}}\right) .
$$

Let us show that this inequality takes place for all $r \geq 0$ except, possibly, some set $B_{1} \subset[0, \infty)$ such that $\mu\left(B_{1}\right)<1$.

The condition $\delta^{\prime}\left(a, f^{(l)}\right)>0$ together with Lemma 2.1 from Chapter 3, applied to $f^{(l)}(z)$, imply that for $r \notin B_{2}$, where $B_{2} \subset[0, \infty)$ is some set satisfying $\mu\left(B_{2}\right)<1$, the inequality

$$
T\left(r, f^{(l)}\right) \leq \frac{2}{\delta^{\prime}\left(a, f^{(l)}\right)} m\left(r, a, f^{(l)}\right) \leq \frac{2}{\delta^{\prime}\left(a, f^{(l)}\right)} m\left(r, \frac{f^{(l)}}{f^{(l+1)}}\right)+Q\left(r, f^{(l)}\right)
$$

holds. Since

$$
\begin{aligned}
T(r, f) & =N(r, f)+m(r, f) \leq N\left(r, f^{(l)}\right)+m\left(r, \frac{f}{f^{\prime}} \cdot \frac{f^{\prime}}{f^{\prime \prime}} \cdots \frac{f^{(l-1)}}{f^{(l)}} f^{(l)}\right) \\
& \leq N\left(r, f^{(l)}\right)+\sum_{\nu=0}^{l-1} m\left(r, \frac{f^{(\nu)}}{f^{(\nu+1)}}\right)+m\left(r, f^{(l)}\right) \\
& =T\left(r, f^{(l)}\right)+\sum_{\nu=0}^{l-1} m\left(r, \frac{f^{(\nu)}}{f^{(\nu+1)}}\right),
\end{aligned}
$$

we get

$$
T(r, f) \leq \frac{2}{\delta^{\prime}\left(a, f^{(l)}\right)} \sum_{\nu=0}^{l} m\left(r, \frac{f^{(\nu)}}{f^{(\nu+1)}}\right)+Q\left(r, f^{(l)}\right) .
$$

By Theorem 2.3 from Chapter 3, for all $r \geq 0$ except, possibly, a set of finite measure, the inequality

$$
Q\left(r, f^{(l)}\right)=Q(r, f) \leq \frac{1}{2} T(r, f)
$$


holds (we suppose that the function $f(z)$ is transcendental). ¿From here it follows that (2.15) holds for all $r \geq 0$ except, possibly, some set $B_{1} \subset[0, \infty)$ satisfying $\mu\left(B_{1}\right)<1$.

Next, we need to estimate $m\left(r, \frac{f^{(\nu)}}{f^{(\nu+1)}}\right), \nu=0, \ldots, l$, from above in terms of $U_{D}(r, 0, \infty ; f)$. For this we need, besides Theorem 2.2 , the following inequality:

$$
U_{D}\left(r, 0, \infty ; f^{(\nu)}\right) \leq K U_{D}(r, 0, \infty ; f)+\sum_{j=1}^{n} Q_{\alpha_{j} \alpha_{j+1}}(r, f) .
$$

To prove this inequality we use the induction. For $\nu=0$ the inequality is trivial. Suppose that it is true for some $\nu \geq 0$. We have

$$
\begin{gathered}
\bar{C}_{\alpha_{j} \alpha_{j+1}}\left(r, f^{(\nu+1)}\right)=\bar{C}_{\alpha_{j} \alpha_{j+1}}\left(r, f^{(\nu)}\right) \\
\bar{C}_{\alpha_{j} \alpha_{j+1}}\left(r, \frac{1}{f^{(\nu+1)}}\right) \leq \bar{C}_{\alpha_{j} \alpha_{j+1}}\left(r, \frac{1}{f^{(\nu)}}\right)+\bar{C}_{\alpha_{j} \alpha_{j+1}}\left(r, \frac{f^{(\nu)}}{f^{(\nu+1)}}\right) .
\end{gathered}
$$

By Theorem 5.2 from Chapter 1 the inequality

$$
\begin{aligned}
& \bar{C}_{\alpha_{j} \alpha_{j+1}}\left(r, \frac{f^{(\nu)}}{f^{(\nu+1)}}\right) \leq S_{\alpha_{j} \alpha_{j+1}}\left(r, \frac{f^{(\nu+1)}}{f^{(\nu)}}\right)+O(1) \\
& \quad=A_{\alpha_{j} \alpha_{j+1}}\left(r, \frac{f^{(\nu+1)}}{f^{(\nu)}}\right)+B_{\alpha_{j} \alpha_{j+1}}\left(r, \frac{f^{(\nu+1)}}{f^{(\nu)}}\right)+C_{\alpha_{j} \alpha_{j+1}}\left(r, \frac{f^{(\nu+1)}}{f^{(\nu)}}\right)+O(1)
\end{aligned}
$$

holds. Using Theorem 3.1 from Chapter 3 and the fact that the relation (2.14) from Chapter 3 (p. ???) remains true if we replace $Q(r, f)$ by $Q_{\alpha_{j} \alpha_{j+1}}(r, f)$ (this was established in Theorem 3.3 from Chapter 3), we get

$$
A_{\alpha_{j} \alpha_{j+1}}\left(r, \frac{f^{(\nu+1)}}{f^{(\nu)}}\right)+B_{\alpha_{j} \alpha_{j+1}}\left(r, \frac{f^{(\nu+1)}}{f^{(\nu)}}\right)=Q_{\alpha_{j} \alpha_{j+1}}\left(r, f^{(\nu)}\right)=Q_{\alpha_{j} \alpha_{j+1}}(r, f),
$$

hence

$$
\begin{aligned}
\bar{C}_{\alpha_{j} \alpha_{j+1}}\left(r, \frac{f^{(\nu)}}{f^{(\nu+1)}}\right) & \leq Q_{\alpha_{j} \alpha_{j+1}}(r, f)+C_{\alpha_{j} \alpha_{j+1}}\left(r, \frac{f^{(\nu+1)}}{f^{(\nu)}}\right) \\
& =\bar{C}_{\alpha_{j} \alpha_{j+1}}\left(r, f^{(\nu)}\right)+\bar{C}_{\alpha_{j} \alpha_{j+1}}\left(r, \frac{1}{f^{(\nu)}}\right)+Q_{\alpha_{j} \alpha_{j+1}}(r, f) .
\end{aligned}
$$

¿From here and from (2.17) we get the inequality

$$
\begin{aligned}
& \bar{C}_{\alpha_{j} \alpha_{j+1}}\left(r, f^{(\nu+1)}\right)+\bar{C}_{\alpha_{j} \alpha_{j+1}}\left(r, \frac{1}{f^{(\nu+1)}}\right) \\
& \quad \leq 2\left\{\bar{C}_{\alpha_{j} \alpha_{j+1}}\left(r, f^{(\nu)}\right)+\bar{C}_{\alpha_{j} \alpha_{j+1}}\left(r, \frac{1}{f^{(\nu)}}\right)\right\}+Q_{\alpha_{j} \alpha_{j+1}}(r, f),
\end{aligned}
$$

and we conclude that

$$
U_{D}\left(r, 0, \infty ; f^{(\nu+1)}\right) \leq 2 U_{D}\left(r, 0, \infty ; f^{(\nu)}\right)+\sum_{j=1}^{n} Q_{\alpha_{j} \alpha_{j+1}}(r, f) .
$$

Using the induction hypothesis, we get (2.16). 
Let $\varepsilon>0$ be so small that $\mu\left(B_{1}\right)+l \varepsilon<1$, where $B_{1}$ is a set, outside which the inequality (2.15) holds. By the statement (A) of Theorem 2.2, for all $r \geq 0$ except, possibly, a subset $E_{\nu} \subset[0, \infty), \mu\left(E_{\nu}\right)<\varepsilon$, the following inequality holds $(d>1)$.

$$
m\left(r, \frac{f^{(\nu)}}{f^{(\nu+1)}}\right) \leq K r^{d \omega(D)}\left\{U_{D}\left(r, 0, \infty ; f^{(\nu)}\right)+\ln r+1\right\}^{d}\left\{\ln ^{+} T\left(r, f^{(\nu)}\right)+1\right\}^{d} .
$$

Using the inequality (2.16) and Theorem 2.3 from Chapter 3 we get that for all $r \geq 0$ except, possibly, a subset $E_{\nu}^{(1)} \subset[0, \infty)$ satisfying $\mu\left(E_{\nu}^{(1)}\right)<\varepsilon$, the inequality

$$
m\left(r, \frac{f^{(\nu)}}{f^{(\nu+1)}}\right) \leq K r^{d \omega(D)}\left\{U_{D}(r, 0, \infty ; f)+\ln r+1\right\}^{d}\left\{\ln ^{+} T(r, f)+1\right\}^{d}
$$

holds. From here, using (2.15) and (2.11) we conclude that the inequality (2.12) holds outside a set whose upper relative measure does not exceed $\mu\left(B_{1}\right)+l \varepsilon<1$, and, as in the proof of Theorem 2.1, we derive the conclusion that the function $f(z)$ has finite order.

Next, using the statement (B) of Theorem 2.2 and the inequality (2.16) we get that for all $r \geq 0$ except, possibly a set $E_{\nu}^{(2)}$ satisfying $\mu\left(E_{\nu}^{(2)}\right)<\varepsilon$, the inequality

$$
m\left(r, \frac{f^{(\nu)}}{f^{(\nu+1)}}\right) \leq K r^{\omega(D)}\left\{U_{D}\left(d r, 0, \infty ; f^{(\nu)}\right)+1\right\} \leq K r^{\omega(D)}\left\{U_{D}(d r, 0, \infty ; f)+1\right\}
$$

holds. From here and from (2.15) we conclude that outside a set whose upper relative measure does not exceed $\mu\left(B_{1}\right)+l \varepsilon<1$, the inequality (2.14) holds. Q.E.D.

Corollary. Let $D=D\left(\alpha_{1}, \ldots, \alpha_{n}\right)$ be a system of rays, $f(z)$ be a meromorphic function, such that for some $a \neq 0, \infty$ and some integer $l \geq 0$ the inequality $\delta^{\prime}\left(a, f^{(l)}\right)>0$ holds. If $U_{D}(r, 0, \infty ; f)=O(1)$, then the growth of $f(z)$ does not exceed normal type of order $\omega(D)$.

Under a more restrictive assumption than $U_{D}(r, 0, \infty ; f)=O(1)$, we can get an additional information about the asymptotic behavior of the function $f(z)$.

ThEOREM 2.6. Let $D=D\left(\alpha_{1}, \ldots, \alpha_{n}\right)$ be a system of rays, $f(z)$ be a meromorphic function, such that for some $a \neq 0, \infty$ and some integer $l \geq 0$ the inequality $\delta^{\prime}\left(a, f^{(l)}\right)>0$ holds. If for some $b \neq 0, \infty$ and some integer $m \geq 0$ the relation

$$
\sum_{j=1}^{n}\left\{C_{\alpha_{j} \alpha_{j+1}}(r, 0, f)+C_{\alpha_{j} \alpha_{j+1}}(r, \infty, f)+C_{\alpha_{j} \alpha_{j+1}}\left(r, b, f^{(m)}\right)\right\}=O(1)
$$

holds, then the growth of the function $f(z)$ does not exceed normal type of order $\omega(D)$, besides, as $r \rightarrow \infty$ missing, possibly, a set of finite logarithmic measure, we have, uniformly in $\varphi, \alpha_{j} \leq \varphi \leq \alpha_{j+1}, j=1, \ldots, n$, the relation

$$
\ln \left|f\left(r e^{i \varphi}\right)\right|=r^{\omega_{j}(D)} c_{j} \sin \left\{\omega_{j}(D)\left(\varphi-\alpha_{j}\right)\right\}+o\left(r^{\omega_{j}(D)}\right),
$$

where $c_{j}, j=1, \ldots, n$, are real constants.

The proof of Theorem 2.6 is based on the following theorem about the behavior of functions with the bounded characteristic $S_{\alpha \beta}(r, f)$ in the angle $\{\alpha \leq \arg z \leq \beta\}$. 
THEOREM 2.7. Let $f(z)$ be a function meromorphic in the angle $\{\alpha \leq \arg z \leq$ $\beta\}, 0<\beta-\alpha \leq 2 \pi$, and such that $S_{\alpha \beta}(r, f)=O(1)$. As $r \rightarrow \infty$ missing, possibly, some set of finite logarithmic measure, the relation

$$
\ln \left|f\left(r e^{i \varphi}\right)\right|=r^{\omega} c \sin \{\omega(\varphi-\alpha)\}+o\left(r^{\omega}\right)
$$

holds uniformly in $\varphi, \alpha \leq \varphi \leq \beta$, where $c$ is a constant and $\omega=\pi /(\beta-\alpha)$.

Theorem 2.7 will be proved in Section 3 .

We need also the following lemma.

LEMMa 2.2. Let $f(z)$ be a function meromorphic in the angle $\{\alpha \leq \arg z \leq \beta\}$. For each $b \neq 0, \infty$ and for each integer $m \geq 0$ the inequality

$$
S_{\alpha \beta}(r, f) \leq C_{\alpha \beta}(r, \infty, f)+C_{\alpha \beta}(r, 0, f)+C_{\alpha \beta}\left(r, b, f^{(m)}\right)+Q_{\alpha \beta}(r, f)
$$

holds.

This lemma follows immediately from an analogue of the relation (2.17) from Chapter 3 for Nevanlinna characteristics for half-plane. The validity of this analogue was established in Theorem 3.3 from Chapter 3.

Now we prove Theorem 2.6. Since the condition $(2.18)$ implies $U_{D}(r, 0, \infty ; f)=$ $O(1)$, then, by Theorem 2.5 , the growth of the function $f(z)$ does not exceed normal type of order $\omega(D)$. Next, we apply Lemma 2.2 to the function $f(z)$ in each of the angles $\left\{\alpha_{j} \leq \arg z \leq \alpha_{j+1}\right\}, j=1,2, \ldots, n$. Since the function $f(z)$ has the finite order, we have $Q_{\alpha_{j} \alpha_{j+1}}(r, f)=O(1), j=1, \ldots, n$, therefore using the condition (2.18) and Lemma 2.2 we get $S_{\alpha_{j} \alpha_{j+1}}(r, f)=O(1), j=1, \ldots, n$. Applying Theorem 2.7 in each of the angles $\left\{\alpha_{j} \leq \arg z \leq \alpha_{j+1}\right\}$, we get the statement of Theorem 2.6 .

REMARK 1. If a meromorphic function $f(z)$ and a system $D=D\left(\alpha_{1}, \ldots, \alpha_{n}\right)$ of rays satisfy the condition (2.18) for some $b \neq 0, \infty$ and some integer $m \geq 0$, and besides

$$
\int_{1}^{\infty} \frac{\ln ^{+} T(r, f)}{r^{q(D)+1}} d r<\infty, q(D)=\min _{1 \leq j \leq n} \omega_{j}(D),
$$

then, as $r \rightarrow \infty$ missing, possibly, a set of finite logarithmic measure, the relation

$$
\ln \left|f\left(r e^{i \varphi}\right)\right|=r^{\omega_{j}(D)} c_{j} \sin \left\{\omega_{j}(D)\left(\varphi-\alpha_{j}\right)\right\}+o\left(r^{\omega_{j}(D)}\right)
$$

holds uniformly in $\varphi, \alpha_{j} \leq \varphi \leq \alpha_{j+1}, j=1, \ldots, n$.

In fact, the condition (2.20) implies that $Q_{\alpha_{j} \alpha_{j+1}}(r, f)=O(1)$. Therefore, using the condition (2.18) and Lemma 2.2 we again get $S_{\alpha_{j} \alpha_{j+1}}(r, f)=O(1)$, after that we use Theorem 2.7.

REMARK 2. Under the conditions of Theorem 2.6, as $r \rightarrow \infty$, outside some set of finite logarithmic measure the relations

$$
\ln \left|f\left(r e^{i \alpha_{j}}\right)\right|=o\left(r^{q_{j}(D)}\right), q_{j}(D)=\min \left(\omega_{j-1}(D), \omega_{j}(D)\right)
$$

hold $(j=1, \ldots, n)$. These relations can be supplemented by the following relations:

$$
\int_{1}^{\infty} \frac{|\ln | f\left(t e^{i \alpha_{j}}\right)||}{t^{q_{j}}(D)+1} d t<\infty, j=1, \ldots, n .
$$


In fact, in the proof of Theorem 2.6 we established that $S_{\alpha_{j} \alpha_{j+1}}(r, f)=O(1)$, $j=1, \ldots, n$. Therefore by Theorem 5.2 from Chapter 1 we have $A_{\alpha_{j} \alpha_{j+1}}(r, f)+$ $A_{\alpha_{j} \alpha_{j+1}}\left(r, \frac{1}{f}\right)=O(1), j=1, \ldots, n$. Since $\left(\omega_{j}=\omega_{j}(D), j=1, \ldots, n\right)$

$$
\begin{aligned}
A_{\alpha_{j} \alpha_{j+1}}(r, f) & +A_{\alpha_{j} \alpha_{j+1}}\left(r, \frac{1}{f}\right) \\
& =\frac{\omega_{j}}{\pi} \int_{1}^{r}\left(\frac{1}{t^{\omega_{j}}}-\frac{t^{\omega_{j}}}{r^{2 \omega_{j}}}\right)\left\{|\ln | f\left(t e^{i \alpha_{j}}\right)||+|\ln | f\left(t e^{i \alpha_{j+1}}\right)||\right\} \frac{d t}{t} \\
& \geq \frac{\omega_{j}}{\pi} \int_{1}^{r / 2}\left(\frac{1}{t^{\omega_{j}}}-\frac{t^{\omega_{j}}}{r^{2 \omega_{j}}}\right)\left\{|\ln | f\left(t e^{i \alpha_{j}}\right)||+|\ln | f\left(t e^{i \alpha_{j+1}}\right)||\right\} \frac{d t}{t} \\
& \geq \frac{\omega_{j}}{\pi}\left(1-\left(\frac{1}{2}\right)^{\omega_{j}}\right) \int_{1}^{r / 2} t^{-\omega_{j}}\left\{|\ln | f\left(t e^{i \alpha_{j}}\right)||+|\ln | f\left(t e^{i \alpha_{j+1}}\right)||\right\} \frac{d t}{t},
\end{aligned}
$$

we easily get $(2.21)$.

REMARK 3. The statement of Theorem 2.6 remains true if we replace $f(z)$ in the relation (2.19) by $\frac{\alpha f^{(l)}(z)+\beta}{\gamma f^{(l)}(z)+\delta}$, where $\alpha \delta-\beta \gamma \neq 0$, and $l \geq 0$ is an integer. This follows from the relations

$$
\begin{gathered}
S_{\alpha_{j} \alpha_{j+1}}\left(r, \frac{\alpha f+\beta}{\gamma f+\delta}\right)=S_{\alpha_{j} \alpha_{j+1}}(r, f)+O(1), \alpha \delta-\beta \gamma \neq 0, \\
S_{\alpha_{j} \alpha_{j+1}}\left(r, f^{(l)}\right) \leq(l+1) S_{\alpha_{j} \alpha_{j+1}}(r, f)+Q_{\alpha_{j} \alpha_{j+1}}(r, f) .
\end{gathered}
$$

The first of these relations holds by (6.11) from Chapter 1 (p. ???), and the second relation is an analogue of (2.13) from Chapter 3 (p. ???), it holds by Theorem 3.3 from Chapter 3.

The following corollary of Theorem 2.6 can be obtained using Remark 2 .

COROllary 1. Let $f(z)$ be an entire function, $a \neq b$ be complex numbers, $\left\{z_{m}\right\}$ be the set of all roots of the equations $f(z)=a$ and $f(z)=b$, each root is listed according to its multiplicity. If the condition

$$
\sum_{m}\left|\operatorname{Im} \frac{1}{z_{m}}\right|<\infty
$$

is satisfied, then the function $f(z)$ has growth of at most normal type of order 1 , besides, as $r \rightarrow \infty$ outside some set of finite logarithmic measure, the relation

$$
\ln \left|f\left(r e^{i \varphi}\right)\right|=\left\{\begin{array}{l}
r c_{1} \sin \varphi+o(r), 0 \leq \varphi \leq \pi \\
r c_{2} \sin \varphi+o(r), \pi \leq \varphi \leq 2 \pi
\end{array}\right.
$$

holds uniformly in $\varphi$, where $c_{1}$ and $c_{2}$ are constants, and

$$
\int_{-\infty}^{\infty} \frac{|\ln | f(t)||}{1+t^{2}} d t<\infty
$$

The assumptions of this corollary are close to, but are somewhat stronger (we take into account the multiplicity of $a$ - and $b$-points) the assumptions of Corollary 3 of Theorem 2.1 for the system $D(0, \pi)$. 
Observe that the statement of Corollary 1 remains true for each entire function $f(z)$ having at most normal type of order 1 , and satisfying the condition

$$
\int_{-\infty}^{\infty} \frac{\ln ^{+}|f(t)|}{1+t^{2}} d t<\infty
$$

In fact, for such function we have

$$
\begin{aligned}
S_{0 \pi}(r, f)=\frac{1}{\pi} \int_{1}^{r} & \left(\frac{1}{t^{2}}-\frac{1}{r^{2}}\right)\left\{\ln ^{+}|f(t)|+\ln ^{+}|f(-t)|\right\} d t \\
& +\frac{2}{\pi r} \int_{0}^{\pi} \ln ^{+}\left|f\left(r e^{i \varphi}\right)\right| \sin \varphi d \varphi=O(1),
\end{aligned}
$$

and, similarly, $S_{\pi, 2 \pi}(r, f)=O(1)$. Therefore the desired conclusion follows from Theorem 2.7 and Remark 2 .

Theorem 2.6 and Remark 3 immediately imply

COROLlARY 2. Under the assumptions of Theorem 2.6 for each number $d$ from the extended complex plane and each integer $p \geq 0$ the relation

$$
m\left(r, d, f^{(p)}\right)=c r^{\omega(D)}+o\left(r^{\omega(D)}\right)
$$

holds as $r \rightarrow \infty$ missing, possibly, some set of finite logarithmic measure ( $c \geq 0$ is a constant which, in general, depends on $d$ and $p)$.

Now we prove the following theorem.

TheOREM 2.8. Let $D=D\left(\alpha_{1}, \ldots, \alpha_{n}\right)$ be a system of rays, $f(z)$ be a meromorphic function, such that for some $a \neq 0, \infty$ the relation $\delta^{\prime}(a, f)>0$ holds. If for some $b \neq 0, \infty$ and some integer $m \geq 0$ the relation (2.18) holds, and, in addition

$$
\limsup _{r \rightarrow \infty} r^{-\omega(D)} T(r, f)>0,
$$

then

$$
\liminf _{r \rightarrow \infty} r^{-\omega(D)} T(r, f)>0
$$

and the function $f(z)$ has at most $s$ deficient values, where $s$ is the number of angles $\left\{\alpha_{j} \leq \arg z \leq \alpha_{j+1}\right\}$, such that $\omega_{j}(D)=\omega(D)$.

Proof. For all $r \geq 0$ except, possibly, a subset $B_{1} \subset[0, \infty)$ satisfying $\mu\left(B_{1}\right)<$ 1 , the inequality

$$
T(r, f) \leq \frac{2}{\delta^{\prime}(a, f)} m(r, a, f)
$$

holds. By Corollary 2 of Theorem 2.6 the relation

$$
m(r, a, f)=c r^{\omega(D)}+o\left(r^{\omega(D)}\right), c \geq 0,
$$

holds, as $r \rightarrow \infty$ missing, possibly, a set $B_{2}$ of finite logarithmic measure. If the constant $c$ in $(2.24)$ were 0 , then outside a set $B_{3}$ satisfying $\mu\left(B_{3}\right)<1$, the condition

$$
T(r, f)=o\left(r^{\omega(D)}\right)
$$

would hold. By Lemma 2.1 we would derive from here that the function $f(z)$ has growth of at most minimal type of order $\omega(D)$, which would contradict $(2.22)$. Thus 
we have $c>0$ in (2.24), and since $T(r, f) \geq m(r, a, f)+O(1)$, we have

$$
T(r, f) \geq c r^{\omega(D)}+o\left(r^{\omega(D)}\right), r \notin B_{2} .
$$

Take an arbitrary sequence $\left\{r_{k}\right\}_{k=1}^{\infty}, r_{k} \uparrow \infty$. If the condition $T\left(r_{k}, f\right)=$ $o\left(r_{k}^{\omega(D)}\right)$ were satisfied, since the function $T(r, f)$ is non-decreasing, we would have

$$
\max _{r_{k} / 2 \leq r \leq r_{k}} T(r, f)=o\left(r_{k}^{\omega(D)}\right) .
$$

Since the set $\bigcup_{k=1}^{\infty}\left(\frac{1}{2} r_{k}, r_{k}\right)$ has an infinite logarithmic measure, we can find in it a sequence $\left\{\rho_{\nu}\right\}_{\nu=1}^{\infty}, \rho_{\nu} \uparrow \infty$, such that $\rho_{\nu} \notin B_{2}$. By (2.25) we would get $T\left(\rho_{\nu}, f\right) \geq c \rho_{\nu}^{\omega(D)}+o\left(\rho_{\nu}^{\omega(D)}\right)$, which contradicts (2.26). Thus we have established the validity of $(2.23)$.

By Remark 3 to Theorem 2.6 for each $d \neq \infty$ the relation

$$
\ln \left|f\left(r e^{i \varphi}\right)-d\right|=r^{\omega_{j}(D)} c_{j}(d) \sin \left\{\omega_{j}(D)\left(\varphi-\alpha_{j}\right)\right\}+o\left(r^{\omega_{j}(D)}\right)
$$

holds uniformly in $\varphi, \alpha_{j} \leq \varphi \leq \alpha_{j+1}$, as $r \rightarrow \infty$ missing, possibly, some set $B_{4}$ of finite logarithmic measure. If all $c_{j}(d)$, for which $\omega_{j}(D)=\omega(D)$ are non-negative, then (2.27) implies that for $r \notin B_{4}$ the relation $m(r, d, f)=o\left(r^{\omega(D)}\right)$ holds; taking into account $(2.23)$ we get $\delta(d, f)=0$. Thus, if $\delta(d, f)>0$, then for some $j$ the conditions $c_{j}(d)<0$ and $\omega_{j}(D)=\omega(D)$ should be satisfied simultaneously. But it is obvious that if $c_{j}(d)<0$, then $c_{j}\left(d^{\prime}\right)=0$ for each $d^{\prime} \neq d$. Therefore the number of deficient values cannot exceed the number of values of $j$ for which $\omega_{j}(D)=\omega(D)$, Q.E.D.

The estimate for the number of deficient values in Theorem 2.8 cannot be improved in general.

ExAmPLE 4 . Let $D$ be a system of rays $\bigcup_{j=1}^{q}\left\{\arg z=\frac{2 j-1}{q} \pi\right\}$. For this system we have $\omega(D)=q / 2$, and the number $s$, defined in the statement of Theorem 2.8 is equal to $q$. Let $f(z)$ be the meromorphic function from Example 8 from Section 5 of Chapter 5 . The number of deficient values of this function is equal to $q$. Let us show that the function $f(z)$ satisfies the conditions of Theorem 2.8 with the mentioned above system $D$ of rays. Here we shall use the notation of Example 8 from Section 5 of Chapter 5 .

The condition (2.22) holds, since by relation (5.20) (p. ???) we have

$$
T(r, f) \geq \frac{8}{q \pi} r^{q / 2}+o\left(r^{q / 2}\right)
$$

To verify that the relation (2.18) holds, we show that for an arbitrary number $b$ from the extended complex plane the relation

$$
\sum_{j=1}^{q} C_{\alpha_{j} \alpha_{j+1}}(r, b, f)=O(1), \alpha_{j}=\frac{2 j-1}{q} \pi, j=1, \ldots, q,
$$

holds.

By the formula (see $[\mathbf{W W 9 6}, 17 \cdot 7]$ )

$$
I_{n}(z)=\frac{z^{n}}{2^{n} \Gamma\left(\frac{1}{2}\right) \Gamma\left(n+\frac{1}{2}\right)} \int_{0}^{\pi} \cosh (z \cos \varphi) \sin ^{2 n} \varphi d \varphi, n>-\frac{1}{2},
$$


we get that for $|\arg z|=\frac{\pi}{2}$ the relation

$$
I_{n}(z)=O\left(|z|^{n}\right),|z| \rightarrow \infty,
$$

holds. Therefore for $|\arg z|=\frac{\pi}{q}$ we have

$$
w_{l}(z)=O(|z|),|z| \rightarrow \infty, l=1,2 .
$$

This relation, by the equalities (5.18), p.???, holds for all rays of the system $D$. Therefore for all $j=1,2, \ldots, q$ the relation

$$
\begin{aligned}
& A_{\alpha_{j} \alpha_{j+1}}\left(r, w_{l}\right) \\
& \quad=\frac{q}{2 \pi} \int_{1}^{r}\left(\frac{1}{t^{q / 2}}-\frac{t^{q / 2}}{r^{q}}\right)\left\{\ln ^{+}\left|w_{l}\left(t e^{i \alpha_{j}}\right)\right|+\ln ^{+}\left|w_{l}\left(t e^{i \alpha_{j+1}}\right)\right|\right\} \frac{d t}{t}=O(1)
\end{aligned}
$$

holds. The relation (5.16), p. ???, implies that

$$
\begin{aligned}
B_{\alpha_{j} \alpha_{j+1}}\left(r, w_{l}\right) & =\frac{q}{\pi r^{q / 2}} \int_{\alpha_{j}}^{\alpha_{j+1}} \ln ^{+}\left|w_{l}\left(r e^{i \varphi}\right)\right| \sin \frac{q}{2}\left(\varphi-\alpha_{j}\right) d \varphi \\
& \leq \frac{2 q}{r^{q / 2}} T\left(r, w_{l}\right)=O(1) .
\end{aligned}
$$

Thus,

$$
S_{\alpha_{j} \alpha_{j+1}}\left(r, w_{l}\right)=A_{\alpha_{j} \alpha_{j+1}}\left(r, w_{l}\right)+B_{\alpha_{j} \alpha_{j+1}}\left(r, w_{l}\right)=O(1)
$$

and using the relations (6.11) and (6.12) from Chapter 1 we get

$$
\begin{aligned}
S_{\alpha_{j} \alpha_{j+1}}(r, f) & \leq S_{\alpha_{j} \alpha_{j+1}}\left(r, w_{1}\right)+S_{\alpha_{j} \alpha_{j+1}}\left(r, \frac{1}{w_{2}}\right) \\
& =S_{\alpha_{j} \alpha_{j+1}}\left(r, w_{1}\right)+S_{\alpha_{j} \alpha_{j+1}}\left(r, w_{2}\right)+O(1)=O(1) .
\end{aligned}
$$

By Theorem 5.2 from Chapter 1 , for each $b$ we have

$$
C_{\alpha_{j} \alpha_{j+1}}(r, b, f) \leq S_{\alpha_{j} \alpha_{j+1}}(r, f)+O(1)=O(1), j=1,2, \ldots, q,
$$

and the relation (2.28) has been proved.

Theorems of this section, proved above, have applications to the study of considered in Section 6 of Chapter 5 functions of the form

$$
f(z)=\sum_{k=1}^{\infty} \frac{A_{k}}{z-h_{k}}, h_{k} \rightarrow \infty
$$

(the series is assumed to be absolutely convergent). These applications are based on the following lemma.

Lemma 2.3. If a function $f(z)$ is representable in the form (2.29), then for each complex number $b$ and each $\alpha$ and $\beta$ satisfying $0<\beta-\alpha \leq 2 \pi$, the relation

$$
C_{\alpha \beta}(r, b, f) \leq C_{\alpha \beta}(r, \infty, f)+O(1)
$$

holds.

Proof. By Theorem 5.2 from Chapter 1 we have

$$
C_{\alpha \beta}(r, b, f) \leq S_{\alpha \beta}(r, f)+O(1)=A_{\alpha \beta}(r, f)+B_{\alpha \beta}(r, f)+C_{\alpha \beta}(r, f)+O(1) .
$$


Since $(w=\pi /(\beta-\alpha))$

$$
\begin{aligned}
A_{\alpha \beta}(r, f) & =\frac{\omega}{\pi} \int_{1}^{r}\left(\frac{1}{t^{\omega}}-\frac{t^{\omega}}{r^{2 \omega}}\right)\left\{\ln ^{+}\left|f\left(t e^{i \alpha}\right)\right|+\ln ^{+}\left|f\left(t e^{i \beta}\right)\right|\right\} \frac{d t}{t} \\
& \leq \frac{\omega}{\pi} \int_{1}^{\infty} \frac{\ln ^{+}\left|f\left(t e^{i \alpha}\right)\right|}{t^{1+\omega}} d t+\frac{\omega}{\pi} \int_{1}^{\infty} \frac{\ln ^{+}\left|f\left(t e^{i \beta}\right)\right|}{t^{1+\omega}} d t
\end{aligned}
$$

using Theorem 6.3 from Chapter 5 we get

$$
A_{\alpha \beta}(r, f)=O(1) \text {. }
$$

Since

$$
B_{\alpha \beta}(r, f)=\frac{2 \omega}{\pi r^{\omega}} \int_{\alpha}^{\beta} \ln ^{+}\left|f\left(r e^{i \varphi}\right)\right| \sin \omega(\varphi-\alpha) d \varphi \leq \frac{4 \omega}{r^{\omega}} m(r, f),
$$

by Theorem 6.1 from Chapter 5 ,

$$
B_{\alpha \beta}(r, f)=o(1) .
$$

Let $f(z)$ be a function of the form $(2.29), D=D\left(\alpha_{1} \ldots, \alpha_{n}\right)$ be a system of rays. Lemma 2.3 implies that for each complex number $b$ the relation

$$
U_{D}(r, b, f) \leq U_{D}(r, \infty, f)+O(1)
$$

holds. Therefore Corollary 1 of Theorem 2.1 implies the following result.

TheOREM 2.9. Let $D=D\left(\alpha_{1}, \ldots, \alpha_{n}\right)$ be a system of rays, $f(z)$ be a meromorphic function of the form (2.29) such that for some a the inequality $\delta^{\prime}(a, f)>0$ holds. Then the category of growth of the function $f(z)$ does not exceed the category of growth of the function

$$
r^{\omega(D)}\left\{U_{D}(r, \infty, f)+1\right\} .
$$

Note that, by Theorem 6.1 from Chapter 5, functions of the form (2.29) satisfy $\delta(\infty, f)=\delta^{\prime}(\infty, f)=0$. By Theorem 6.2 from Chapter 5 , for $a \neq 0, \infty$ we have $\delta(a, f)=0$, but we do not know whether $\delta^{\prime}(a, f)=0$ always holds. The cases when $\delta(0, f)>0$ are possible, we discussed this matter at the end of Section 6 from Chapter 5.

We would like to mention the following corollary of Theorem 2.9.

Corollary. Let $D=D\left(\alpha_{1}, \ldots, \alpha_{n}\right)$ be a system of rays, $f(z)$ be a meromorphic function of the form (2.29) such that for some a the inequality $\delta^{\prime}(a, f)>0$ holds. If $U_{D}(r, \infty, f)=O(1)$, then the growth of the function $f(z)$ does not exceed normal type of order $\omega(D)$.

This corollary shows that Theorem 6.2 from Chapter 5 can be complemented with the following statement. If a function $f(z)$ of the form (2.29) has growth at least maximal type of order $\omega(D)$ and $U_{D}(r, \infty, f)=O(1)$, then (regardless of whether the conditions $\sum_{k=1}^{\infty}\left|A_{k}\right|<\infty$ and $\sum_{k=1}^{\infty} A_{k} \neq 0$ are satisfied) the equality $\delta^{\prime}(0, f)=0$ holds.

By Theorem 2.4 the statements of Theorem 2.9 and its corollary remain true if the condition of positivity of $\delta^{\prime}(a, f)$ for some $a$ is replaced by the condition that $a$ is a Borel exceptional value. 
Using Theorem 2.6, Remark 2 to it, and Lemma 2.3, we get the following theorem.

THEOREM 2.10. Let $D=D\left(\alpha_{1}, \ldots, \alpha_{n}\right)$ be a system of rays, $f(z)$ be a meromorphic function of the form (2.29), such that for some a the inequality $\delta^{\prime}(a, f)>0$ holds. If

$$
\sum_{j=1}^{n} C_{\alpha_{j} \alpha_{j+1}}(r, \infty, f)=O(1)
$$

then the growth of the function $f(z)$ does not exceed normal type of order $\omega(D)$, and the integrals

$$
\int_{1}^{\infty} \frac{|\ln | f\left(t e^{i \alpha_{j}}\right)||}{t^{1+q_{j}(D)}} d t, q_{j}(D)=\min \left\{\omega_{j-1}(D), \omega_{j}(D)\right\}, j=2, \ldots, n+1,
$$

converge. Besides, as $r \rightarrow \infty$ missing, possibly, some set of finite logarithmic measure, the relation

$$
\ln \left|f\left(r e^{i \varphi}\right)\right|=r^{\omega_{j}(D)} c_{j} \sin \left\{\omega_{j}(D)\left(\varphi-\alpha_{j}\right)\right\}+o\left(r^{\omega_{j}(D)}\right)
$$

holds uniformly in $\varphi, \alpha_{j} \leq \varphi \leq \alpha_{j+1}, j=1, \ldots, n$, where $c_{j}, j=1, \ldots, n$, are some constants.

COROLlaRY 1. Let $f(z)$ be a meromorphic function of the form (2.29), such that for some a the inequality $\delta^{\prime}(a, f)>0$ holds. If the condition

$$
\sum_{k}\left|\operatorname{Im} \frac{1}{h_{k}}\right|<\infty
$$

is satisfied, then the growth of the function $f(z)$ does not exceed normal type of order 1 and, in addition, for $r \rightarrow \infty$ outside some set of finite logarithmic measure the relation

$$
\ln \left|f\left(r e^{i \varphi}\right)\right|=\left\{\begin{array}{l}
r c_{1} \sin \varphi+o(r), 0 \leq \varphi \leq \pi \\
r c_{2} \sin \varphi+o(r), \pi \leq \varphi \leq 2 \pi
\end{array}\right.
$$

holds uniformly in $\varphi$, where $c_{1}$ and $c_{2}$ are constants, and

$$
\int_{-\infty}^{\infty} \frac{|\ln | f(t)||}{1+t^{2}} d t<\infty
$$

COROLlary 2. If zeros $\left\{a_{k}\right\}$ of an entire function $f(z)$ satisfy the condition

$$
\sum_{k}\left|\operatorname{Im} \frac{1}{a_{k}}\right|<\infty
$$

and, in addition, the function $1 / f(z)$ is representable in the form (2.29), then the statement of Corollary 1 remains true for the function $f(z)$.

The growth estimate in Corollary 2 is sharp, since the function $\left(z^{2}-1\right) \sin z$ is exactly of normal type of order 1 , and $\frac{1}{\left(z^{2}-1\right) \sin z}$ is representable in the form (2.29), namely,

$$
\frac{1}{\left(z^{2}-1\right) \sin z}=\frac{1}{2 \sin 1} \cdot \frac{1}{z-1}+\frac{1}{2 \sin 1} \cdot \frac{1}{z+1}+\sum_{k=-\infty}^{\infty} \frac{(-1)^{k}}{\left\{(k \pi)^{2}-1\right\}(z-k \pi)} .
$$




\section{Proofs of main Theorems 2.3 and 2.7}

We prove Theorems 2.3 and 2.7 under the assumption that the angle $\{\alpha \leq$ $\arg z \leq \beta\}$ is a half-plane

$$
\Pi=\{\operatorname{Im} z \geq 0\}
$$

The general case can be easily reduced to this case using the transformation $w=$ $z^{1 / \omega} e^{i \alpha}, \omega=\pi /(\beta-\alpha)$, which maps the half-plane $\Pi$ onto the angle $\{\alpha \leq \arg w \leq$ $\beta$. We assume, without mentioning this explicitly, that all functions $f(z)$ appearing in the statements of theorems of this section are of the form $f(z)=g\left(z^{\omega}\right)$, where $\omega>0$, and $g(\zeta)$ is a function meromorphic in the angle $\{0 \leq \arg \zeta \leq \pi / \omega\}$.

The proof of Theorem 2.7 is based on the following representation of functions with the bounded characteristic $S(r, f)=S_{0 \pi}(r, f)$.

THEOREM 3.1. If

$$
S(r, f)=O(1)
$$

then the formula $\left(z=r e^{i \varphi}, 0<\varphi<\pi\right)$

$$
\begin{gathered}
\ln |f(z)|=\frac{r \sin \varphi}{\pi} \int_{-\infty}^{\infty} \frac{\ln |f(t)|}{r^{2}+t^{2}-2 r t \cos \varphi} d t \\
-\sum_{m} \ln \left|\frac{z-\bar{a}_{m}}{z-a_{m}}\right|+\sum_{n} \ln \left|\frac{z-\bar{b}_{n}}{z-b_{n}}\right|+\eta r \sin \varphi
\end{gathered}
$$

holds, where $a_{m}$ are zeros of the function $f(z)$, and $b_{n}$ are its poles, belonging to $\Pi, \eta$ is a constant which does not depend on $z$. The integral and the series in the right-hand side of (3.1) are absolutely convergent.

Proof. Observe that the condition $S(r, f)=O(1)$ implies the convergence of the integral

$$
\int_{-\infty}^{\infty} \frac{|\ln | f(t)||}{1+t^{2}} d t
$$

and of the series

$$
\sum_{m} \frac{\sin \varphi_{m}}{r_{m}}, \sum_{n} \frac{\sin \psi_{n}}{\rho_{n}}
$$

where $a_{m}=r_{m} e^{i \varphi_{m}}, b_{n}=\rho_{n} e^{i \psi_{n}}$. This can be shown using the argument used in the proof of Remark 2 to Theorem 2.6 and in Example 1 in Section 2.

By Theorem 2.3 from Chapter 1 (p. ???) and the remark after it the formula $\left(z=r e^{i \varphi}\right)$

$$
\begin{aligned}
& \ln |f(z)|=\frac{1}{\pi} \int_{-R}^{R} \ln |f(t)|\left\{\frac{r \sin \varphi}{|z-t|^{2}}-\frac{R^{2} r \sin \varphi}{\left|R^{2}-z t\right|^{2}}\right\} d t \\
& \quad+\frac{1}{2 \pi} \int_{0}^{\pi} \ln \left|\left(R e^{i \theta}\right)\right|\left\{\frac{R^{2}-r^{2}}{\left|R e^{i \theta}-z\right|^{2}}-\frac{R^{2}-r^{2}}{\left|R e^{-i \theta}-z\right|^{2}}\right\} d \theta \\
& \quad-\sum_{\left|a_{m}\right|<R} \ln \left|\frac{R^{2}-\bar{a}_{m} z}{R\left(z-a_{m}\right)} \cdot \frac{R\left(z-\bar{a}_{m}\right)}{R^{2}-a_{m} z}\right|+\sum_{\left|b_{n}\right|<R} \ln \left|\frac{R^{2}-\bar{b}_{n} z}{R\left(z-b_{n}\right)} \cdot \frac{R\left(z-\bar{b}_{n}\right)}{R^{2}-b_{n} z}\right| \\
& =I_{1}(R)+I_{2}(R)-I_{3}(R)+I_{4}(R)
\end{aligned}
$$

holds in the half-disc $\{|z|<R, \operatorname{Im} z>0\}$. To get the formula (3.1) we consider the limits as $R \rightarrow \infty$. 
The convergence of the integral (3.2) clearly implies the absolute convergence of the integral (3.1). Therefore the limit

$$
\lim _{R \rightarrow \infty} \frac{1}{\pi} \int_{-R}^{R} \ln |f(t)| \frac{r \sin \varphi}{|z-t|^{2}} d t=\frac{1}{\pi} \int_{-\infty}^{\infty} \ln |f(t)| \frac{r \sin \varphi}{|z-t|^{2}} d t
$$

exists.

Next we note that

$$
\begin{aligned}
& \left|\int_{-R}^{R} \ln \right| f(t)\left|\frac{R^{2} r \sin \varphi}{\left|R^{2}-z t\right|^{2}} d t\right| \leq \int_{-R}^{R}|\ln | f(t)|| \frac{R^{2} r \sin \varphi}{\left(R^{2}-r t\right)^{2}} d t \\
& \leq \frac{r}{(R-r)^{2}} \int_{-R}^{R}|\ln | f(t)|| d t \leq \frac{r}{(R-r)^{2}}\left\{(1+R) \int_{-\sqrt{R}}^{\sqrt{R}} \frac{|\ln | f(t)||}{1+t^{2}} d t\right. \\
& \left.\quad+\left(1+R^{2}\right) \int_{\sqrt{R}}^{R} \frac{|\ln | f(t)||}{1+t^{2}} d t+\left(1+R^{2}\right) \int_{-R}^{-\sqrt{R}} \frac{|\ln | f(t)||}{1+t^{2}} d t\right\} \rightarrow 0
\end{aligned}
$$

as $R \rightarrow \infty$. Thus, the limit

$$
\lim _{R \rightarrow \infty} I_{1}(R)=\frac{r \sin \varphi}{\pi} \int_{-\infty}^{\infty} \frac{\ln |f(t)|}{r^{2}+t^{2}-2 r t \cos \varphi} d t\left(z=r e^{i \varphi}\right)
$$

exists.

The convergence of the series $\sum_{m} r_{m}^{-1} \sin \varphi_{m}$ implies the convergence of the series

$$
\sum_{m} \ln \left|\frac{z-\bar{a}_{m}}{z-a_{m}}\right|
$$

since for $\left|a_{m}\right|>2|z|$ we have

$$
\ln \left|\frac{z-\bar{a}_{m}}{z-a_{m}}\right|=\ln \left|1+\frac{z 2 i \operatorname{Im} \frac{1}{a_{m}}}{1-\frac{z}{a_{m}}}\right| \leq \frac{2|z|\left|\operatorname{Im} \frac{1}{a_{m}}\right|}{\left|1-\frac{z}{a_{m}}\right|} \leq 4|z| \frac{\sin \varphi_{m}}{r_{m}} .
$$

Therefore the following limit exists:

$$
\lim _{R \rightarrow \infty} \sum_{\left|a_{m}\right|<R} \ln \left|\frac{z-\bar{a}_{m}}{z-a_{m}}\right|=\sum_{m} \ln \left|\frac{z-\bar{a}_{m}}{z-a_{m}}\right| .
$$

Next we observe that

$$
\begin{aligned}
& \left|\sum_{\left|a_{m}\right|<R} \ln \right| \frac{R^{2}-\bar{a}_{m} z}{R^{2}-a_{m} z}||=\sum_{\left|a_{m}\right|<R} \ln \left|\frac{R^{2}-a_{m} z}{R^{2}-\bar{a}_{m} z}\right| \\
& =\sum_{\left|a_{m}\right|<R} \ln \left|1-\frac{z 2 i \operatorname{Im} a_{m}}{R^{2}-\bar{a}_{m} z}\right| \leq \sum_{\left|a_{m}\right|<R} \frac{2|z|\left|\operatorname{Im} a_{m}\right|}{\left|R^{2}-\bar{a}_{m} z\right|} \\
& \leq \frac{2 r}{R(R-r)} \sum_{\left|a_{m}\right|<R} r_{m} \sin \varphi_{m} \\
& \leq \frac{2 r}{R(R-r)}\left\{R \sum_{\left|a_{m}\right|<\sqrt{R}} \frac{\sin \varphi_{m}}{r_{m}}+R^{2} \sum_{\sqrt{R} \leq\left|a_{m}\right| \leq R} \frac{\sin \varphi_{m}}{r_{m}}\right\} \rightarrow 0
\end{aligned}
$$


as $R \rightarrow \infty$. Thus, the following limit exists:

$$
\lim _{R \rightarrow \infty} I_{3}(R)=\sum_{m} \ln \left|\frac{z-\bar{a}_{m}}{z-a_{m}}\right| .
$$

In a similar way we show the existence of the limit

$$
\lim _{R \rightarrow \infty} I_{4}(R)=\sum_{n} \ln \left|\frac{z-\bar{b}_{n}}{z-b_{n}}\right| .
$$

Since the left-hand side of the equality $\ln |f(z)|=I_{1}(R)+I_{2}(R)-I_{3}(R)+I_{4}(R)$ does not depend on $R$, the $\operatorname{limit} \lim _{R \rightarrow \infty} I_{2}(R)$ also exists. We need to show that

$$
\lim _{R \rightarrow \infty} I_{2}(R)=\eta r \sin \varphi
$$

where $\eta$ does not depend on $r$ and $\varphi$. It suffices to show that

$$
\lim _{R \rightarrow \infty} J(R)=0
$$

where

$$
J(R)=I_{2}(R)-r \sin \varphi \frac{2}{\pi R} \int_{0}^{\pi} \ln \left|f\left(\operatorname{Re}^{i \theta}\right)\right| \sin \theta d \theta
$$

We have

$$
\begin{aligned}
J(R)= & \frac{1}{2 \pi} \int_{0}^{\pi} \ln \left|f\left(R e^{i \theta}\right)\right| \frac{\left(R^{2}-r^{2}\right) 4 R r \sin \varphi \sin \theta d \theta}{\left(R^{2}+r^{2}-2 R r \cos (\varphi-\theta)\right)\left(R^{2}+r^{2}-2 R r \cos (\varphi+\theta)\right)} \\
& -r \sin \varphi \frac{2}{\pi R} \int_{0}^{\pi} \ln \left|f\left(R e^{i \theta}\right)\right| \sin \theta d \theta \\
= & r \sin \varphi \frac{2}{\pi R} \int_{0}^{\pi} \ln \left|f\left(R e^{i \theta}\right)\right| \sin \theta \cdot K(R, r, \varphi, \theta) d \theta,
\end{aligned}
$$

where $K(R, r, \varphi, \theta)=O\left(R^{-1}\right)$ uniformly in $\theta, 0 \leq \theta \leq \pi$ as $R \rightarrow \infty$, and $r$ and $\varphi$ are fixed. Therefore

$$
\begin{aligned}
|J(R)| & \leq r \frac{2}{\pi R} \int_{0}^{\pi}|\ln | f\left(R e^{i \theta}\right)|| \sin \theta d \theta \cdot O\left(R^{-1}\right) \\
& =r\{B(R, f)+B(R, 1 / f)\} \cdot O\left(R^{-1}\right) \\
& \leq r\{2 S(R, f)+O(1)\} \cdot O\left(R^{-1}\right) \rightarrow 0 \text { as } R \rightarrow \infty
\end{aligned}
$$

Q.E.D.

REMARK. In passing we proved, that

$$
\eta=\lim _{R \rightarrow \infty}\{B(R, f)-B(R, 1 / f)\}
$$

Let $\left(z=r e^{i \varphi}, 0<\varphi<\pi\right)$

$$
\begin{aligned}
u(z)= & \frac{r \sin \varphi}{\pi} \int_{-\infty}^{\infty} \frac{|\ln | f(t)||}{r^{2}+t^{2}-2 r t \cos \varphi} d t \\
& +\sum_{m} \ln \left|\frac{z-\bar{a}_{m}}{z-a_{m}}\right|+\sum_{n} \ln \left|\frac{z-\bar{b}_{n}}{z-b_{n}}\right| .
\end{aligned}
$$

Theorem 3.1 implies that

$$
|\ln | f\left(r e^{i \varphi}\right)|-\eta r \sin \varphi| \leq u\left(r e^{i \varphi}\right) .
$$

Whence to prove Theorem 2.7 it suffices to prove the following lemma. 
LEMMA 3.1. For the function $u(z)$ given by (3.5) the relation

$$
u\left(r e^{i \varphi}\right)=o(r)
$$

holds uniformly in $\varphi, 0<\varphi<\pi$, as $r \rightarrow \infty$ missing some set $A \subset[1, \infty)$ of finite logarithmic measure.

A proof of the lemma could be based on estimates of the integral and the series from the right-hand side of (3.5). Doing so we would repeat twice essentially the same argument. This can be avoided if we write the right-hand side of (3.5) as a Lebesgue-Stieltjes integral over the half-plane $\Pi$.

We proceed in the following way. We define on Borel subsets $E \subset \Pi$ a LebesgueStieltjes measure $\nu(E)$ by the formula

$$
\begin{aligned}
\nu(E)= & \frac{1}{\pi} \int_{E \cap(-\infty, \infty)} \frac{|\ln | f(t)||}{1+t^{2}} d t \\
& +\sum_{a_{m} \in E} \frac{2 r_{m} \sin \varphi_{m}}{1+r_{m}^{2}}+\sum_{b_{n} \in E} \frac{2 \rho_{n} \sin \psi_{n}}{1+\rho_{n}^{2}} .
\end{aligned}
$$

This measure takes finite values only, since by the convergence of the integral (3.2) and the series (3.3) we have

$$
\nu(\Pi)=\frac{1}{\pi} \int_{-\infty}^{\infty} \frac{|\ln | f(t)||}{1+t^{2}} d t+\sum_{m} \frac{2 r_{m} \sin \varphi_{m}}{1+r_{m}^{2}}+\sum_{n} \frac{2 \rho_{n} \sin \psi_{n}}{1+\rho_{n}^{2}}<\infty .
$$

Let $\left(\zeta=\xi+i \eta, \eta \geq 0, z=r e^{i \varphi}\right)$

$$
K(z, \zeta)= \begin{cases}\frac{1+|\zeta|^{2}}{2 \eta} \ln \left|\frac{z-\bar{\zeta}}{z-\zeta}\right|, & \eta>0 \\ \frac{\left(1+\xi^{2}\right) r \sin \varphi}{r^{2}+\xi^{2}-2 r \xi \cos \varphi}, & \eta=0\end{cases}
$$

Then we have

$$
u(z)=\iint_{\Pi} K(z, \zeta) d \nu(\zeta) .
$$

Having in mind also the proof of Theorem 2.3, we obtain the following very general theorem about estimates of integrals of the form (3.8).

ThEOREM 3.2. Let $\nu(E)$ be a Lebesgue-Stieltjes measure defined on Borel subsets $E \subset \Pi$ and such that $\nu(\Pi)<\infty$. Define a function $u(z ; \nu), z \in \Pi$, by the equality

$$
u(z ; \nu)=\iint_{\Pi} K(z, \zeta) d \nu(\zeta)
$$

where $K(z, \zeta)$ is given by (3.7)

For each $q>0$ the inequality

$$
\begin{aligned}
u\left(r e^{i \varphi} ; \nu\right) \leq & \frac{D}{q} \nu(\Pi) r \\
& +\frac{4}{r} \iint_{|\zeta|<\frac{1}{2} r}\left(1+|\zeta|^{2}\right) d \nu(\zeta)+18 r \iint_{|\zeta|>\frac{3}{2} r} d \nu(\zeta), \quad 0<\varphi<\pi,
\end{aligned}
$$


where $D$ is an absolute constant, holds for all $r \in[1, \infty)$ except, possibly, some subset $A_{q} \subset[1, \infty)$ satisfying

$$
\int_{A_{q}} r^{-1} d r \leq q .
$$

Before turning to the proof of Theorem 3.2 we derive from it a corollary containing Lemma 3.1.

COROllary. Let $u(z ; \nu)$ be a function defined in the statement of Theorem 3.2. Then the relation

$$
u\left(r e^{i \varphi} ; \nu\right)=o(r)
$$

holds uniformly in $\varphi, 0<\varphi<\pi$, as $r \rightarrow \infty$ missing some subset $A \subset[1, \infty)$ having finite logarithmic measure.

Let $q$ be a positive integer, $A_{q}$ be the corresponding set from the statement of Theorem 3.2. Choose a so large number $R_{q}$, that $R_{q}>q$ and

$$
\int_{A_{q} \cap\left(R_{q}, \infty\right)} r^{-1} d r<\frac{1}{2^{q}} .
$$

Let

$$
A=\bigcup_{q=1}^{\infty}\left\{A_{q} \cap\left(R_{q}, \infty\right)\right\}
$$

Obviously,

$$
\int_{A} r^{-1} d r \leq \sum_{q=1}^{\infty} 2^{-q}=1 .
$$

Let $r$ be sufficiently large, $r \notin A, q(r)$ be the largest integer $q$ satisfying $R_{q}<r$. Then the inequality (3.9) holds with $q=q(r)$. Obviously $q(r) \rightarrow \infty$ as $r \rightarrow \infty$, therefore

$$
\frac{D}{q(r)} \nu(\Pi) r=o(r) .
$$

Since

$$
\begin{aligned}
& \frac{4}{r} \iint_{|\zeta|<\frac{1}{2} r}\left(1+|\zeta|^{2}\right) d \nu(\zeta) \\
&=\frac{4}{r}\left\{\iint_{|\zeta|<\sqrt{r}}\left(1+|\zeta|^{2}\right) d \nu(\zeta)+\iint_{\sqrt{r} \leq|\zeta|<\frac{1}{2} r}\left(1+|\zeta|^{2}\right) d \nu(\zeta)\right\} \\
& \leq \frac{4}{r}\left\{(1+r) \nu(\Pi)+\left(1+\frac{1}{4} r^{2}\right) \iint_{|\zeta| \geq \sqrt{r}} d \nu(\zeta)\right\}=o(r), \\
& 18 r \iint_{|\zeta|>\frac{3}{2} r} d \nu(\zeta)=o(r),
\end{aligned}
$$

the corollary and, thus, Lemma 3.1 have been proved.

Now we prove Theorem 3.2. 
Denote by $B$ the set of those values of $r \geq 1$ for which the following is true: for each $h, 0<h \leq \frac{1}{2} r$, the inequality

$$
\nu(\{r-h \leq|z| \leq r+h\} \cap \Pi)<\frac{8 \nu(\Pi) h}{q r}
$$

holds. The set $B$ is Borel since it can be represented in the form

$$
B=\left\{r: \sup _{0 \leq h \leq \frac{1}{2} r} \frac{F(r+h)-F(r-h-0)}{h} \leq \frac{8 \nu(\Pi)}{r}\right\},
$$

where $F(r)=\nu(\{|z| \leq r\})$ is a non-decreasing function. Therefore the set $A_{q}=$ $[1, \infty) \backslash B$ is also Borel. Let us show that

$$
\int_{A_{q}} r^{-1} d r \leq \frac{3}{2} q
$$

Let $A_{q 1}$ be an arbitrary compact subset of the set $A_{q}$. The definition of the set $B$ implies that for each $x \in A_{q 1}$ there exists an interval $I_{x}=\left(x-h_{x}, x+h_{x}\right)$, $0<h_{x} \leq \frac{1}{2} x$, such that

$$
\nu\left(\left\{|z| \in \bar{I}_{x}\right\} \cap \Pi\right) \geq \frac{8 \nu(\Pi) h_{x}}{q x},
$$

where $\bar{I}_{x}=\left[x-h_{x}, x+h_{x}\right]$. By the Heine-Borel lemma there is a finite system $\left\{I_{x_{k}}\right\}$ of intervals covering $A_{q 1}$. Discarding intervals containing in unions of two other intervals, we may assume that each point of the set $A_{q 1}$ is covered by at most two intervals of the system $\left\{I_{x_{k}}\right\}$. It follows from here that each point of the set $A_{q 1}$ belongs to at most three segments $\bar{I}_{x_{k}}$. Therefore we have

$$
\sum_{k} \nu\left(\left\{|z| \in \bar{I}_{x_{k}}\right\} \bigcap \Pi\right) \leq 3 \nu(\Pi) .
$$

Hence, using (3.12), we get

$$
\begin{aligned}
\int_{A_{q 1}} r^{-1} d r & \leq \sum_{k} \int_{\bar{I}_{x_{k}}} r^{-1} d r=\sum_{k} \ln \left(1+\frac{2 h_{x_{k}}}{x_{k}-h_{x_{k}}}\right) \leq 4 \sum_{k} \frac{h_{x_{k}}}{x_{k}} \\
& \leq 4 \frac{q}{8 \nu(\Pi)} \sum_{k} \nu\left(\left\{|z| \in \bar{I}_{x_{k}}\right\} \bigcap \Pi\right) \leq \frac{3}{2} q .
\end{aligned}
$$
that

Let $A_{q 1}^{(1)} \subset A_{q 1}^{(2)} \subset \ldots$ be a sequence of compact subsets of the set $A_{q}$, such

$$
\operatorname{mes}\left(A_{q} \backslash \bigcup_{n=1}^{\infty} A_{q 1}^{(n)}\right)=0 \text {. }
$$

Then we have

$$
\int_{A_{q}} r^{-1} d r=\int_{\cup_{n=1}^{\infty} A_{q 1}^{(n)}} r^{-1} d r=\lim _{n \rightarrow \infty} \int_{A_{q 1}^{(n)}} r^{-1} d r \leq \frac{3}{2} q,
$$

the inequality (3.11) has been proved.

Now we show that (3.9) is satisfied for $r \in B$, thus completing our proof of Theorem 3.2. 
We need the following estimate of the function $K(z, \zeta)$ :

$$
K(z, \zeta) \leq \frac{\left(1+|\zeta|^{2}\right) y}{|z-\zeta|^{2}}(z=x+i y, y>0)
$$

For $\eta=0(\zeta=\xi+i \eta)$ this estimate is trivial. For $\eta>0$ we have

$$
\begin{aligned}
& K(z, \zeta)=\frac{1+|\zeta|^{2}}{4 \eta} \ln \frac{(x-\xi)^{2}+(y+\eta)^{2}}{(x-\xi)^{2}+(y-\eta)^{2}} \\
& \quad=\frac{1+|\zeta|^{2}}{4 \eta} \ln \left(1+\frac{4 y \eta}{(x-\xi)^{2}+(y-\eta)^{2}}\right) \leq \frac{\left(1+|\zeta|^{2}\right) y}{(x-\xi)^{2}+(y-\eta)^{2}}=\frac{\left(1+|\zeta|^{2}\right) y}{|z-\zeta|^{2}} .
\end{aligned}
$$

We write the equality $\left(z=r e^{i \varphi}, 0<\varphi<\pi, r>1\right)$

$$
\begin{aligned}
u(z ; \nu)= & \iint_{|\zeta|<\frac{1}{2} r} K(z, \zeta) d \nu(\zeta)+\iint_{\frac{1}{2} r \leq|\zeta| \leq \frac{3}{2} r} K(z, \zeta) d \nu(\zeta) \\
& +\iint_{|\zeta|>\frac{3}{2} r} K(z, \zeta) d \nu(\zeta)=I_{1}+I_{2}+I_{3} .
\end{aligned}
$$

Integrals $I_{1}$ and $I_{3}$ can be easily estimated, using (3.13), in the following way:

$$
\begin{aligned}
I_{1} \leq \iint_{|\zeta|<\frac{1}{2} r} \frac{\left(1+|\zeta|^{2}\right) y}{|z-\zeta|^{2}} d \nu(\zeta) & \leq \frac{4 y}{|z|^{2}} \iint_{|\zeta|<\frac{1}{2} r}\left(1+|\zeta|^{2}\right) d \nu(\zeta) \\
& \leq \frac{4}{r} \iint_{|\zeta|<\frac{1}{2} r}\left(1+|\zeta|^{2}\right) d \nu(\zeta) \\
I_{3} \leq \iint_{|\zeta|>\frac{3}{2} r} \frac{\left(1+|\zeta|^{2}\right) y}{|z-\zeta|^{2}} d \nu(\zeta) & \leq 9 y \iint_{|\zeta|>\frac{3}{2} r} \frac{1+|\zeta|^{2}}{|\zeta|^{2}} d \nu(\zeta) \\
& \leq 18 y \iint_{|\zeta|>\frac{3}{2} r} d \nu(\zeta) .
\end{aligned}
$$

Let us estimate the integral $I_{2}$. Fixing a point $z=r e^{i \varphi}, 0<\varphi<\pi, r>1$, denote by $C_{n}, n=0, \pm 1, \pm 2, \ldots$, the (possibly, empty) set of all points $\zeta \in \Pi$, satisfying the following two conditions

$$
2^{n-1} y \leq|z-\zeta|<2^{n} y, \frac{1}{2} r \leq|\zeta| \leq \frac{3}{2} r(y=\operatorname{Im} z) .
$$

Obviously the sets $C_{n}$ are pairwise disjoint and

$$
\bigcup_{n=-\infty}^{\infty} C_{n}=\left\{\frac{1}{2} r \leq|\zeta| \leq \frac{3}{2} r\right\} \backslash\{z\} .
$$

Since $r \in B$, by (3.10) we have

$$
\nu(\{z\}) \leq \nu(\{|z|=r\})=0,
$$

hence

$$
I_{2}=\sum_{n=-\infty}^{\infty} \iint_{C_{n}} K(z, \zeta) d \nu(\zeta)
$$

Note that the condition $r \in B$, by (3.10), implies

$$
\nu\left(C_{n}\right) \leq \nu(\{r-h \leq|\zeta| \leq r+h\})<\frac{8 \nu(\Pi)}{q r} 2^{n} y, h=\min \left(2^{n} y, \frac{r}{2}\right) .
$$


For $\zeta \in C_{n}$ the inequality (3.13) implies

$$
K(z, \zeta) \leq \frac{\left(1+|\zeta|^{2}\right) y}{\left(2^{n-1} y\right)^{2}} \leq \frac{1+\frac{9}{4} r^{2}}{4^{n-1} y} \leq \frac{16 r^{2}}{4^{n} y}
$$

therefore

$$
\sum_{n=0}^{\infty} \iint_{C_{n}} K(z, \zeta) d \nu(\zeta) \leq \sum_{n=0}^{\infty} \frac{16 r^{2}}{4^{n} y} \nu\left(C_{n}\right) \leq \frac{16 r^{2}}{y} \cdot \frac{8 \nu(\Pi) y}{q r} \sum_{n=0}^{\infty} \frac{1}{2^{n}} \leq \frac{256 \nu(\Pi) r}{q} .
$$

For $\zeta \in C_{n}, n<0$, the condition $\eta \geq \frac{1}{2} y>0$ holds, therefore we have

$$
\begin{aligned}
K(z, \zeta)=\frac{1+|\zeta|^{2}}{2 \eta} \ln \left|\frac{z-\bar{\zeta}}{z-\zeta}\right| & \leq \frac{1+\frac{9}{4} r^{2}}{y} \ln \left(1+\frac{2 y}{|z-\zeta|}\right) \\
& \leq \frac{1+\frac{9}{4} r^{2}}{y} \ln \left(1+\frac{2 y}{2^{n-1} y}\right) \leq \frac{4 r^{2}}{y} \ln \left(6 \cdot 2^{|n|}\right)
\end{aligned}
$$

and

$$
\begin{aligned}
\sum_{n=-\infty}^{-1} \iint_{C_{n}} K(z, \zeta) d \nu(\zeta) & \leq \sum_{n=-\infty}^{-1} \frac{4 r^{2}}{y} \ln \left(6 \cdot 2^{|n|}\right) \nu\left(C_{n}\right) \\
& \leq \frac{4 r^{2}}{y} \cdot \frac{8 \nu(\Pi) y}{q r} \sum_{n=-\infty}^{-1} 2^{-|n|} \ln \left(6 \cdot 2^{|n|}\right) \leq \frac{128 \nu(\Pi) r}{q}
\end{aligned}
$$

Thus,

$$
I_{2} \leq \frac{384 \nu(\Pi) r}{q}
$$

The formulas (3.14), (3.15), and (3.16) imply (3.9). As we have already mentioned, this completes the proof of Theorem 3.2.

We obtain Theorem 2.3 as a corollary of the following theorem.

THEOREM 3.3. Let $k(r) \geq 1$ be a continuous non-decreasing function, $U(r)=$ $r\left(1+\frac{1}{k(r)}\right)$. Then for each $\varepsilon, 0<\varepsilon<1$, and for all $r \geq 1$ except, possibly, some set $A_{\varepsilon} \subset[1, \infty)$ satisfying $\mu\left(A_{\varepsilon}\right)<\varepsilon$, the inequality

$$
\ln ^{+}\left|f\left(r e^{i \varphi}\right)\right| \leq C k^{3}(r) r\{S(U(r), f)+1\}, 0<\varphi<\pi,
$$

holds, where $C$ is a positive constant which does not depend on $r$ and $\varphi$.

To get the second inequality of Theorem 2.3 for $r \notin A_{\varepsilon}$, we choose $k(r) \equiv \frac{1}{d-1}$. Then $U(r) \equiv d r$ and, integrating (3.17) with respect to $\varphi$ from 0 to $\pi$, we get the desired inequality:

$$
m_{0 \pi}(r, f) \leq C \pi(d-1)^{-3} r\{S(d r, f)+1\} .
$$

The remaining assertions of Theorem 2.3 can be obtained in the following way. By Theorem 5.4 from Chapter 1 (p. ???) we have $S(r, f)=\stackrel{\circ}{S}(r, f)+O(1)$, where $\stackrel{\circ}{S}(r, f)$ is a non-decreasing function. Increasing, if necessary, the constant $C$, we can replace $S(r, f)$ by $\stackrel{\circ}{S}(r, f)$ in $(3.17)$, after that we let $k(r)=\ln \{\stackrel{\circ}{S}(r, f)+1\}$. 
Applying the corollary of the Borel-Nevanlinna theorem stated in Exercise 2 of Section 1 from Chapter 3 (p. ???) to the function $u(r)=\stackrel{\circ}{S}(r, f)+1$, we get

$$
\stackrel{\circ}{S}(U(r), f)+1 \leq\{\stackrel{\circ}{S}(r, f)+1\}^{\frac{1+d}{2}}
$$

for $r \notin A$, where $A$ is a set of finite logarithmic measure. For $r \notin A \cup A_{\varepsilon}$ we have

$$
\begin{aligned}
\ln ^{+}\left|f\left(r e^{i \varphi}\right)\right| & \leq C \ln ^{3}\{\stackrel{\circ}{S}(r, f)+1\} r\{\stackrel{\circ}{S}(r, f)+1\}^{\frac{1+d}{2}} \\
& \leq C_{1} r\{\stackrel{\circ}{S}(r, f)+1\}^{d} \leq C_{2} r\{S(r, f)+1\}^{d}, 0<\varphi<\pi,
\end{aligned}
$$

where $0<C_{1}<C_{2}<\infty$ are constants which do not depend on $r$ and $\varphi$. Integrating with respect to $\varphi$ from 0 to $\pi$, we get $\left(r \notin A \cup A_{3}\right)$

$$
m_{0 \pi}(r, f) \leq C_{2} \pi r\{S(r, f)+1\}^{d} .
$$

It remains to observe that $\mu\left(A \cup A_{\varepsilon}\right) \leq \mu(A)+\mu\left(A_{\varepsilon}\right)<\varepsilon$.

Now we turn to the proof of Theorem 3.3.

First we prove the inequality

$$
\begin{aligned}
\ln ^{+}\left|f\left(r e^{i \varphi}\right)\right| \leq & \frac{r \sin \varphi}{\pi} \int_{-R}^{R} \frac{\ln ^{+}|f(t)|}{r^{2}+t^{2}-2 r t \cos \varphi} d t \\
& +\sum_{\left|b_{n}\right|<R} \ln \left|\frac{z-\bar{b}_{n}}{z-b_{n}}\right|+2\left(\frac{R}{R-r}\right)^{3} r B(R, f) \\
(z & \left.=r e^{i \varphi}, 0<r<R, 0<\varphi<\pi\right) .
\end{aligned}
$$

Now we use the formula (3.4). Observe that the expression in braces from the formulas for $I_{1}(R)$ and $I_{2}(R)$ are non-negative by formulas (1.10) and (1.11) from Chapter 1. Therefore

$$
\begin{gathered}
I_{1}(R) \leq \frac{1}{\pi} \int_{-R}^{R} \ln ^{+}|f(t)|\left\{\frac{r \sin \varphi}{|z-t|^{2}}-\frac{R^{2} r \sin \varphi}{\left|R^{2}-z t\right|^{2}}\right\} d t \\
\leq \frac{r \sin \varphi}{\pi} \int_{-R}^{R} \frac{\ln ^{+}|f(t)|}{r^{2}+t^{2}-2 r t \cos \varphi} d t \\
I_{2}(R) \leq \frac{1}{2 \pi} \int_{0}^{\pi} \ln ^{+}\left|f\left(R e^{i \theta}\right)\right|\left\{\frac{R^{2}-r^{2}}{\left|R e^{i \theta}-z\right|^{2}}-\frac{R^{2}-r^{2}}{\left|R e^{-i \theta}-z\right|^{2}}\right\} d \theta \\
=\frac{1}{2 \pi} \int_{0}^{\pi} \ln ^{+}\left|f\left(R e^{i \theta}\right)\right| \frac{\left(R^{2}-r^{2}\right) 4 R r \sin \varphi \sin \theta d \theta}{\left(R^{2}+r^{2}-2 R r \cos (\varphi-\theta)\right)\left(R^{2}+r^{2}-2 R r \cos (\varphi+\theta)\right)} \\
\leq \frac{\left(R^{2}-r^{2}\right) 4 R r}{(R-r)^{4}} \cdot \frac{1}{2 \pi} \int_{0}^{\pi} \ln ^{+}\left|f\left(R e^{i \theta}\right)\right| \sin \theta d \theta \leq 2\left(\frac{R}{R-r}\right)^{3} r B(R, f) .
\end{gathered}
$$

The summands in $I_{3}(R)$ and $I_{4}(R)$ represent, by formula (1.5) from Chapter 1 , values of the Green function for the half-disc $\{|z|<R, \operatorname{Im} z>0\}$, and hence, are non-negative. Discarding $I_{3}(R)$ in (3.4) and observing that

$$
\left|\frac{R^{2}-\bar{b}_{n} z}{R\left(z-b_{n}\right)} \cdot \frac{R\left(z-\bar{b}_{n}\right)}{R^{2}-b_{n} z}\right| \leq\left|\frac{z-\bar{b}_{n}}{z-b_{n}}\right|
$$

we get the inequality (3.18). 
Let us estimate the function

$$
u\left(r e^{i \varphi}\right)=\frac{r \sin \varphi}{\pi} \int_{-R}^{R} \frac{\ln ^{+}|f(t)| d t}{r^{2}+t^{2}-2 r t \cos \varphi}+\sum_{\left|b_{n}\right|<R} \ln \left|\frac{z-\bar{b}_{n}}{z-b_{n}}\right|
$$

using Theorem 3.2. This function can be represented in the form (3.8) by letting

$$
\nu(E)=\frac{1}{\pi} \int_{(-R, R) \cap E} \frac{\ln ^{+}|f(t)|}{1+t^{2}} d t+\sum_{\substack{b_{n} \in E \\\left|b_{n}\right|<R}} \frac{2 \rho_{n} \sin \psi_{n}}{1+\rho_{n}^{2}}\left(b_{n}=\rho_{n} e^{i \psi_{n}}\right) .
$$

By Theorem 3.2 the inequality

$$
\begin{aligned}
u\left(r e^{i \varphi}\right) & \leq \frac{D}{q} \nu(\Pi) r+\frac{4}{r} \iint_{|\zeta|<\frac{1}{2} r}\left(1+|\zeta|^{2}\right) d \nu(\zeta)+18 r \iint_{|\zeta|>\frac{3}{2} r} d \nu(\zeta) \\
& \leq \frac{D}{q} \nu(\Pi) r+\frac{4}{r}\left(1+\frac{1}{4} r^{2}\right) \iint_{|\zeta|<\frac{1}{2} r} d \nu(\zeta)+18 r \iint_{|\zeta|>\frac{3}{2} r} d \nu(\zeta) \\
& \leq \frac{D}{q} \nu(\Pi) r+18 r \nu(\Pi)=\left(\frac{D}{q}+18\right) \nu(\Pi) r, 0<\varphi<\pi,
\end{aligned}
$$

holds for all $r \geq 1$ except, possibly, some subset $A_{q} \subset[1, \infty)$ satisfying

$$
\int_{A_{q}} r^{-1} d r \leq q
$$

Let us estimate

$$
\nu(\Pi)=\frac{1}{\pi} \int_{-R}^{R} \frac{\ln ^{+}|f(t)|}{1+t^{2}} d t+\sum_{\left|b_{n}\right|<R} \frac{2 \rho_{n} \sin \psi_{n}}{1+\rho_{n}^{2}} .
$$

Let $U>R$. We have

$$
\begin{aligned}
& A(U, f)=\frac{1}{\pi} \int_{1}^{U}\left(\frac{1}{t^{2}}-\frac{1}{U^{2}}\right)\left\{\ln ^{+}|f(t)|+\ln ^{+}|f(-t)|\right\} d t \\
& \geq \frac{1}{\pi} \int_{1}^{R}\left(\frac{1}{t^{2}}-\frac{1}{U^{2}}\right)\left\{\ln ^{+}|f(t)|+\ln ^{+}|f(-t)|\right\} d t \\
& \geq\left(1-\frac{R^{2}}{U^{2}}\right) \frac{1}{\pi} \int_{1}^{R} t^{-2}\left\{\ln ^{+}|f(t)|+\ln ^{+}|f(-t)|\right\} d t \\
& \geq \frac{U-R}{U \pi}\left\{\int_{-R}^{R} \frac{\ln ^{+}|f(t)|}{1+t^{2}} d t-\int_{-1}^{1} \frac{\ln ^{+}|f(t)|}{1+t^{2}} d t\right\}, \\
& C(U, f)=2\left.\sum_{1<\left|b_{n}\right|<U}\left(\frac{1}{\rho_{n}}-\frac{\rho_{n}}{U^{2}}\right) \sin \psi_{n}\right\} \\
& \sum_{1<\left|b_{n}\right|<R}\left(\frac{1}{\rho_{n}}-\frac{\rho_{n}}{U^{2}}\right) \sin \psi_{n} \geq\left(1-\frac{R^{2}}{U^{2}}\right) 2 \sum_{1<\left|b_{n}\right|<R} \frac{\sin \psi_{n}}{\rho_{n}} \\
& \geq \frac{U}{U}\left\{\sum_{\left|b_{n}\right|<R} \frac{2 \rho_{n} \sin \psi_{n}}{1+\rho_{n}^{2}} \sum_{\left|b_{n}\right| \leq 1} \frac{2 \rho_{n} \sin \psi_{n}}{1+\rho_{n}^{2}}\right\} .
\end{aligned}
$$


From here we get

$$
\begin{aligned}
\nu(\Pi) \leq & \frac{U}{U-R}\{A(U, f)+C(U, f)\} \\
& +\frac{1}{\pi} \int_{-1}^{1} \frac{\ln ^{+}|f(t)|}{1+t^{2}} d t+\sum_{\left|b_{n}\right| \leq 1} \frac{2 \rho_{n} \sin \psi_{n}}{1+\rho_{n}^{2}}
\end{aligned}
$$

In what follows the letter $K$ will be used to denote finite positive constants which do not depend on variables denoted by $r, R, \varphi, t, q, U$, and $\varepsilon$.

By (3.18) and (3.19), for $0<r<R, 0<\varphi<\pi$, the inequality

$$
\ln ^{+}\left|f\left(r e^{i \varphi}\right)\right| \leq u\left(r e^{i \varphi}\right)+2\left(\frac{R}{R-r}\right)^{3} r B(R, f)
$$

holds. ¿From here, using (3.20) and (3.22), we get that for $r \in[1, R) \backslash A_{q}, 0<\varphi<\pi$, the inequality

$$
\begin{aligned}
\ln ^{+}\left|f\left(r e^{i \varphi}\right)\right| \leq & K\left(\frac{1}{q}+1\right) \frac{U r}{U-R}\{A(U, f)+C(U, f)+1\} \\
& +2\left(\frac{R}{R-r}\right)^{3} r B(R, f),(U>R),
\end{aligned}
$$

holds. Since, by Theorem 5.4 from Chapter 1 , the function $S(r, f)$ is, up to a bounded summand, non-decreasing, we have

$$
B(R, f) \leq S(R, f) \leq S(U, f)+K .
$$

Taking into account $A(U, f)+C(U, f) \leq S(U, f)$, we get the inequality

$$
\ln ^{+}\left|f\left(r e^{i \varphi}\right)\right| \leq K r\left\{\left(\frac{1}{q}+1\right) \frac{U}{U-R}+\left(\frac{R}{R-r}\right)^{3}\right\}\{S(U, f)+1\},
$$

where $r \in[1, R) \backslash A_{q}, 0<\varphi<\pi, U>R$.

Let $(k(r)$ and $\varepsilon$ are from the statement of Theorem 3.3)

$$
\begin{gathered}
U(t)=t\left(1+\frac{1}{k(t)}\right), U_{1}(t)=\max _{0 \leq \tau \leq t} U(\tau) \\
R(t)=t\left(1+\frac{1}{2 k(t)}\right), t^{\prime}=t\left(1+\frac{1}{4 k(t)}\right), q(t)=\frac{\varepsilon}{16 k(t)},
\end{gathered}
$$

and denote by $j(t)$ the interval $\left[t, t^{\prime}\right]$. Since

$$
\frac{U(t)}{U(t)-R(t)} \leq 4 k(t), \frac{1}{q(t)}+1 \leq \frac{17 k(t)}{\varepsilon},
$$

and for $r \in j(t)$ the inequality

$$
\frac{R(t)}{R(t)-r} \leq 6 k(t)
$$

holds, the inequality (3.24) implies that for $r \in j(t) \backslash A_{q(t)}$ the inequality

$$
\ln ^{+}\left|f\left(r e^{i \varphi}\right)\right| \leq K r k^{3}(t)\{S(U(t), f)+1\}, 0<\varphi<\pi,
$$

holds. Since, obviously, $t \leq r$ for $r \in j(t) \backslash A_{q(t)}$, we have also $k(t) \leq k(r), U(t) \leq$ $U_{1}(t) \leq U_{1}(r)$, and, by Theorem 5.4 from Chapter 1 ,

$$
S(U(t), f) \leq S\left(U_{1}(r), f\right)+K .
$$


Therefore for $r \in j(t) \backslash A_{q(t)}$ the inequality

$$
\ln ^{+}\left|f\left(r e^{i \varphi}\right)\right| \leq K r k^{3}(r)\left\{S\left(U_{1}(r), f\right)+1\right\}, 0<\varphi<\pi,
$$

also holds.

We show that the inequality (3.25) holds for all $r \geq 1$ except, possibly, some subset $E_{\varepsilon} \subset[1, \infty)$ satisfying $\mu\left(E_{\varepsilon}\right) \leq \varepsilon$.

Denote by $E(t)$ the set $j(t) \cap A_{q(t)}$. Using (3.21) and the choice of $q(t)$ we get

$$
\operatorname{mes} E(t) \leq t^{\prime} \int_{E(t)} \frac{d \tau}{\tau} \leq t^{\prime} \int_{A_{q(t)}} \frac{d \tau}{\tau} \leq t^{\prime} q(t) \leq 2 t \frac{\varepsilon}{16 k(t)}=\frac{t \varepsilon}{8 k(t)},
$$

whence

$$
\operatorname{mes} E(t) \leq \frac{\varepsilon}{2} \operatorname{mes} j(t) .
$$

Consider a sequence of intervals $j\left(t_{k}\right), k=0,1, \ldots$, letting

$$
t_{0}=1, t_{k}=t_{k-1}^{\prime}, k=1,2, \ldots
$$

These intervals cover the whole semiaxis $[1, \infty)$. In fact, otherwise we would have $\lim _{k \rightarrow \infty} t_{k}=\alpha<\infty$. Choosing $\delta>0$ and $n$ in such a way, that $4 k(\alpha)<\delta^{-1}$ and

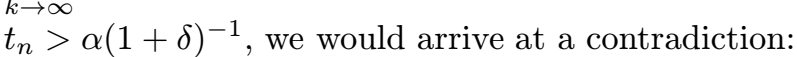

$$
t_{n+1}=t_{n}^{\prime}=t_{n}\left(1+\frac{1}{4 k\left(t_{n}\right)}\right) \geq t_{n}\left(1+\frac{1}{4 k(\alpha)}\right)>\frac{\alpha}{1+\delta}(1+\delta)=\alpha .
$$

Take

$$
E_{\varepsilon}=\bigcup_{k=0}^{\infty} E\left(t_{k}\right)
$$

Obviously, for $r \in[1, \infty) \backslash E_{\varepsilon}$ the inequality (3.25) holds. Besides, by (3.26), we have

$$
\begin{aligned}
\mu\left(E_{\varepsilon}\right) & =\limsup _{r \rightarrow \infty} r^{-1} \operatorname{mes}\left\{E_{\varepsilon} \cap(0, r)\right\} \\
& \leq \limsup _{r \rightarrow \infty} r^{-1} \sum_{t_{k}<r} \operatorname{mes} E\left(t_{k}\right) \leq \frac{\varepsilon}{2} \limsup _{r \rightarrow \infty} r^{-1} \sum_{t_{k}<r} \operatorname{mes} j\left(t_{k}\right) \\
& \leq \frac{\varepsilon}{2} \lim _{r \rightarrow \infty} r^{-1} \operatorname{mes}\left\{\left[1, r\left(1+\frac{1}{4 k(r)}\right)\right]\right\} \leq \frac{\varepsilon}{2} \cdot \frac{5}{4}<\varepsilon .
\end{aligned}
$$

The theorem will be proved is we establish that for all $r \geq 1$ except, possibly, a set of finite logarithmic measure, the equality $U_{1}(r)=U(r)$ holds. Denote by $E$ the set on which $U_{1}(r)>U(r)$. Since the functions $U_{1}(r)$ and $U(r)$ are continuous, the set $E$ is open and hence is a union of a finite or a countable set of pairwise disjoint intervals $\left(a_{\alpha}, b_{\alpha}\right)$.

Since $\lim _{r \rightarrow \infty} U(r)=\infty$, none of the intervals can be infinite.

Obviously, $U\left(a_{\alpha}\right)=U\left(b_{\alpha}\right)$, that is,

$$
a_{\alpha}\left(1+\frac{1}{k\left(a_{\alpha}\right)}\right)=b_{\alpha}\left(1+\frac{1}{k\left(b_{\alpha}\right)}\right) .
$$

This implies

$$
b_{\alpha}-a_{\alpha}=\frac{a_{\alpha}}{k\left(a_{\alpha}\right)}-\frac{b_{\alpha}}{k\left(b_{\alpha}\right)} \leq a_{\alpha}\left(\frac{1}{k\left(a_{\alpha}\right)}-\frac{1}{k\left(b_{\alpha}\right)}\right),
$$


and we get

$$
\int_{E} \frac{d t}{t}=\sum_{\alpha} \int_{a_{\alpha}}^{b_{\alpha}} \frac{d t}{t} \leq \sum_{\alpha} \frac{b_{\alpha}-a_{\alpha}}{a_{\alpha}} \leq \sum_{\alpha}\left(\frac{1}{k\left(a_{\alpha}\right)}-\frac{1}{k\left(b_{\alpha}\right)}\right) .
$$

Using Leibniz's theorem on alternating series we get the desired conclusion.

\section{Meromorphic functions with poles and zeros located in small angles}

Consider a system of rays

$$
\begin{gathered}
D^{\prime}=D\left(\alpha_{1}, \beta_{1}, \alpha_{2}, \beta_{2}, \ldots, \alpha_{n}, \beta_{n}\right) \\
\alpha_{1}<\beta_{1}<\alpha_{2}<\beta_{2}<\cdots<\alpha_{n}<\beta_{n}<\alpha_{n+1}=\alpha_{1}+2 \pi,
\end{gathered}
$$

such that

$$
\max _{1 \leq j \leq n}\left(\beta_{j}-\alpha_{j}\right)<\min _{1 \leq j \leq n}\left(\alpha_{j+1}-\beta_{j}\right) .
$$

The angles $\left\{\alpha_{j} \leq \arg z \leq \beta_{j}\right\}$ will be called small angles of the system $D^{\prime}$. Let

$$
\begin{gathered}
\omega_{j}^{\prime}\left(D^{\prime}\right)=\pi /\left(\beta_{j}-\alpha_{j}\right), \omega_{j}^{\prime \prime}\left(D^{\prime}\right)=\pi /\left(\alpha_{j+1}-\beta_{j}\right), j=1, \ldots, n, \\
\omega^{\prime}\left(D^{\prime}\right)=\min _{1 \leq j \leq n} \omega_{j}^{\prime}\left(D^{\prime}\right), \omega^{\prime \prime}\left(D^{\prime}\right)=\max _{1 \leq j \leq n} \omega_{j}^{\prime \prime}\left(D^{\prime}\right) .
\end{gathered}
$$

Note that by the condition (4.3), the inequality

$$
\omega^{\prime}\left(D^{\prime}\right)>\omega^{\prime \prime}\left(D^{\prime}\right)
$$

holds.

Definition. If the condition

$$
\sum_{j=1}^{n} r^{\omega_{j}^{\prime \prime}\left(D^{\prime}\right)-\omega^{\prime \prime}\left(D^{\prime}\right)} \bar{C}_{\beta_{j} \alpha_{j+1}}(r, a, f)=O(1), r \rightarrow \infty,
$$

is satisfied, we say that almost all $a$-points of the function $f(z)$ are in small angles of the system $D^{\prime}$.

This condition is obviously satisfied if all $a$-points of the function $f(z)$ except, possibly, finitely many are in small angles of the system $D^{\prime}$.

The following theorem is the main result of this section.

TheOREM 4.1. Let $f(z)$ be a meromorphic function of order $\rho<\infty$, almost all of whose zeros and poles are in small angles of the system $D^{\prime}$. If the inequality

$$
\delta(a, f)>1-\cos \frac{\pi \rho}{2 \omega^{\prime}\left(D^{\prime}\right)}
$$

holds for some $a \neq 0, \infty$, then the relation

$$
\omega^{\prime \prime}\left(D^{\prime}\right)<\rho<\omega^{\prime}\left(D^{\prime}\right)
$$

cannot be true. 
In particular, if the system $D^{\prime}$ consists of two rays, $D^{\prime}=D(\alpha, \beta), 0<\beta-\alpha<\pi$, then $\omega^{\prime}\left(D^{\prime}\right)=\pi /(\beta-\alpha), \omega^{\prime \prime}\left(D^{\prime}\right)=\pi /(2 \pi-\beta+\alpha)$, and Theorem 4.1 immediately implies

COROllary 1. Let $f(z)$ be a meromorphic function of order $\rho<\infty$, almost all of whose poles and zeros are in the small angle of the system $D^{\prime}=D(\alpha, \beta)$, $0<\beta-\alpha<\pi$. If the inequality

$$
\delta(a, f)>1-\cos \frac{\rho(\beta-\alpha)}{2}
$$

holds for some $a \neq 0, \infty$, then the relation

$$
\frac{\pi}{2 \pi-\beta+\alpha}<\rho<\frac{\pi}{\beta-\alpha}
$$

cannot be true.

¿From here we get

Corollary 2. Let $f(z)$ be an entire function of order $\rho$, all of $b$ - and $c$ points of which $(b \neq c ; b, c \neq \infty)$ except, possibly, finitely many, are in the angle $\{\alpha \leq \arg z \leq \beta\}, 0<\beta-\alpha<\pi$. Then either $\rho \geq \pi /(\beta-\alpha)$, or $\rho \leq \pi /(2 \pi-\beta+\alpha)$.

In fact, consider the system $D^{\prime}=D(\alpha, \beta)$ and the meromorphic function $f_{1}(z)=\frac{f(z)-b}{f(z)-c}$. Almost all zeros and poles of this function are in the small angle of the system $D^{\prime}=D(\alpha, \beta)$, and $\delta\left(1, f_{1}\right)=1$. If $\rho<\pi /(\beta-\alpha)$, then the function $f_{1}(z)$ satisfies the condition $\left(4.5^{\prime}\right)$ with $a=1$, and by Corollary 1 the inequality $\rho>\pi /(2 \pi-\beta+\alpha)$ cannot be true.

We show that for each $\rho \geq \pi /(\beta-\alpha)$ and each $\rho \leq \pi /(2 \pi-\beta+\alpha)$ it is possible to construct an entire function of order $\rho$, such that for some $b$ and $c$ $(b \neq c ; b, c \neq \infty)$, all $b$ - and $c$-points of $f(z)$, except, possibly, finitely many are in the angle $\{\alpha \leq \arg z \leq \beta\}$.

Example 1. As it was proved in n. $3^{\circ}$ from Section 5 of Chapter 2, the Mittag-Leffler function $E_{\rho}(z)$ has order $\rho$. We shall assume that $\rho>1 / 2$. By the formula (5.40) from Chapter 2 the value of

$$
M=\sup _{\frac{\pi}{2 \rho} \leq \arg z \leq 2 \pi-\frac{\pi}{2 \rho}}\left|E_{\rho}(z)\right|
$$

is finite. Therefore, if the numbers $b$ and $c$ satisfy the condition $\min (|b|,|c|)>M$, then all $b$ - and $c$-points of the function $E_{\rho}(z)$ are in the angle $\left\{-\frac{\pi}{2 \rho} \leq \arg z \leq \frac{\pi}{2 \rho}\right\}$.

Thus, for each $\rho \geq \pi /(\beta-\alpha)$ all $b$ - and $c$-points of the function $E_{\rho}\left(z e^{-i\left(\alpha+\frac{\pi}{2 \rho}\right)}\right)$ are in the angle $\left\{\alpha \leq \arg z \leq \alpha+\frac{\pi}{\rho}\right\} \subset\{\alpha \leq \arg z \leq \beta\}$

Consider now the case $\rho \leq \pi /(2 \pi-\beta+\alpha)$. The formula (5.40) from Chapter 2 implies that the number

$$
m=\inf _{\substack{\frac{\pi}{2 \rho} \leq \arg z \leq \frac{\pi}{2 \rho} \\|z| \geq R}}\left|E_{\rho}(z)\right|
$$


is positive if $R>0$ is sufficiently large. Therefore, if the numbers $b$ and $c$ satisfy the condition $\max (|b|,|c|)<m$, then all $b$ - and $c$-points of the function $E_{\rho}(z)$ except, possibly, finitely many are in the angle $\left\{\frac{\pi}{2 \rho} \leq \arg z \leq 2 \pi-\frac{\pi}{2 \rho}\right\}$. Then all $b$ - and $c$-points of the function $E_{\rho}\left(z e^{-i\left(\alpha-\frac{\pi}{2 \rho}\right)}\right)$ except, possibly, finitely many are in the angle $\left\{\alpha \leq \arg z \leq \alpha+2 \pi-\frac{\pi}{\rho}\right\} \subset\{\alpha \leq \arg z \leq \beta\}$.

Later on we shall show that Corollary 1 is no longer true if the sign $>$ in the condition $\left(4.5^{\prime}\right)$ is replaced by $\geq$. Now we observe that the condition $\left(4.5^{\prime}\right)$ cannot be dropped. In fact, Example 2 from Section 2 shows that even for a function whose zeros and poles lie on a ray, the growth can be absolutely arbitrary.

Now we point to a connection between Theorems 4.1 and 2.1.

Let all zeros and poles except, possibly, finitely many of a meromorphic function $f(z)$ of order $\rho$ lie on rays of the system $D=D\left(\alpha_{1}, \alpha_{2}, \ldots, \alpha_{n}\right)$, and $\delta(a, f)>0$ for some $a \neq 0, \infty$. Then, by Theorem 2.1, the inequality $\rho \leq \omega(D)$ holds. Under the additional assumption that $\rho<\infty$ this estimate can be derived, also, from Theorem 4.1. In fact, we choose numbers $\beta_{j}$ in such a way that the inequalities (4.2) and (4.3) hold. Then almost all zeros and poles of the function $f(z)$ are in the small angles of the system $D^{\prime}=D\left(\alpha_{1}, \beta_{1}, \ldots, \alpha_{n}, \beta_{n}\right)$. Choosing $\beta_{j}$ sufficiently close to $\alpha_{j}$, we can make $\omega^{\prime}\left(D^{\prime}\right)$ arbitrarily large, in particular, so large that (4.5) holds and $\rho<\omega^{\prime}\left(D^{\prime}\right)$. By Theorem 4.1 we get $\rho \leq \omega^{\prime \prime}\left(D^{\prime}\right)$ and, since $\omega^{\prime \prime}\left(D^{\prime}\right) \rightarrow \omega(D)$ as $\beta_{j} \rightarrow \alpha_{j}$, we conclude that $\rho \leq \omega(D)$.

Note also, that if we formally pass to the limit as $\beta_{j} \rightarrow \alpha_{j}(j=1, \ldots, n)$ in Theorem 4.1, its conditions will take the form

$$
U_{D}(r, 0, \infty)=O(1), \delta(a, f)>0,
$$

where $D=D\left(\alpha_{1}, \alpha_{2}, \ldots, \alpha_{n}\right)$, and the statement will be reduced to the inequality $\rho \leq \omega(D)$, that is, we get a special case of Theorem 2.1.

Now we turn to auxiliary results used in the proof of Theorem 4.1.

LEMMA 4.1. Let

$$
\chi(z)=\frac{\prod_{\nu=1}^{\infty}\left(1-\frac{z}{\bar{p}_{\nu}}\right)}{\prod_{\nu=1}^{\infty}\left(1-\frac{z}{p_{\nu}}\right)}
$$

be a meromorphic function of genus zero, all poles $p_{\nu}$ of which are in the half-plane $\{\operatorname{Im} z>0\}$. Suppose that the order of the function $\chi(z)$ is equal to $\eta<1$. Then for each $\sigma, \eta<\sigma<1$, the inequality

$$
\int_{b}^{\infty} \frac{m(t, \chi)}{t^{\sigma+1}} d t \leq\left(\sec \frac{\pi \sigma}{2}-1\right) \int_{b}^{\infty} \frac{N(t, \chi)}{t^{\sigma+1}} d t, 0<b<\infty,
$$

holds.

Proof. First we establish the inequality

$$
\int_{b}^{\infty} \frac{q(t)}{t^{1+\sigma}} d t \leq\left(\sec \frac{\pi \sigma}{2}-1\right) \int_{b}^{\infty} \frac{\ln ^{+} t}{t^{1+\sigma}} d t, 0<b<\infty, 0<\sigma<1,
$$


where

$$
q(t)=\frac{1}{2 \pi} \int_{-\pi / 2}^{\pi / 2} \ln \left|\frac{1+t e^{i \theta}}{1-t e^{i \theta}}\right| d \theta .
$$

Choosing arbitrarily a branch of the function $\arg \left(\frac{1+z}{1-z}\right)$ on the semicircle $C_{t}=$ $\left\{|z|=t,-\frac{\pi}{2} \leq \arg z \leq \frac{\pi}{2}\right\}$ oriented counterclockwise, by the Cauchy-Riemann equations we have $(t \neq 1)$

$$
\begin{aligned}
q^{\prime}(t) & =\frac{1}{2 \pi} \int_{-\pi / 2}^{\pi / 2} \frac{\partial}{\partial t} \ln \left|\frac{1+t e^{i \theta}}{1-t e^{i \theta}}\right| d t \\
& =\frac{1}{2 \pi t} \int_{-\pi / 2}^{\pi / 2} \frac{\partial}{\partial \theta} \arg \left(\frac{1+t e^{i \theta}}{1-t e^{i \theta}}\right) d \theta=\frac{1}{2 \pi t} \Delta_{C_{t}} \arg \left(\frac{1+t e^{i \theta}}{1-t e^{i \theta}}\right) .
\end{aligned}
$$

Therefore

$$
\begin{aligned}
& q^{\prime}(t)=\frac{2}{\pi t} \arctan t, 0<t<1 \\
& q^{\prime}(t)=\frac{2}{\pi t} \arctan t-\frac{1}{t}, t>1
\end{aligned}
$$

hence,

$$
q(t)=\frac{2}{\pi} \int_{0}^{t} \arctan s \frac{d s}{s}-\ln ^{+} t .
$$

Below we use the argument employed in the proof of Lemma 3.3 from Chapter 5 . Consider the function

$$
\varphi(b)=\int_{b}^{\infty}\left\{\left(\sec \frac{\pi \sigma}{2}-1\right) \ln ^{+} t-q(t)\right\} \frac{d t}{t^{1+\sigma}} .
$$

Since

$$
\begin{gathered}
\int_{0}^{\infty} \frac{q(t)}{t^{1+\sigma}} d t=\frac{1}{\sigma} \int_{0}^{\infty} \frac{q^{\prime}(t)}{t^{\sigma}} d t=\frac{2}{\pi \sigma} \int_{0}^{\infty} \frac{\arctan t}{t^{1+\sigma}} d t-\frac{1}{\sigma} \int_{1}^{\infty} \frac{d t}{t^{1+\sigma}} \\
=\frac{2}{\pi \sigma^{2}} \int_{0}^{\infty} \frac{d t}{t^{\sigma}\left(1+t^{2}\right)}-\frac{1}{\sigma^{2}}=\frac{1}{\sigma^{2}}\left(\sec \frac{\pi \sigma}{2}-1\right) \\
\int_{0}^{\infty} \frac{\ln ^{+} t}{t^{1+\sigma}} d t=\frac{1}{\sigma^{2}}
\end{gathered}
$$

we have $\varphi(0)=0$. Obviously $\lim _{b \rightarrow \infty} \varphi(b)=0$.

The integrand in (4.9) is negative for $0<t<1$, for $t>1$ we have

$$
\left\{\left(\sec \frac{\pi \sigma}{2}-1\right) \ln ^{+} t-q(t)\right\}^{\prime}=\frac{1}{t}\left\{\sec \frac{\pi \sigma}{2}-\frac{2}{\pi} \arctan t\right\}>0 .
$$

Therefore the function $\varphi^{\prime}(b)$ change its sign only once on the semiaxis $\{0<b<\infty\}$, from plus to minus. Since $\lim _{b \rightarrow \infty} \varphi(b)=0$, we have $\varphi(b)>0$ for all $b, 0<b<\infty$, thus, the inequality (4.8) has been proved.

Using Lemma 4.4 from Chapter 5 and the notation from its statement, we have

$$
m(r, \chi) \leq m(r, \check{\chi})
$$


But, obviously,

$$
\begin{aligned}
m(r, \check{\chi})=\frac{1}{2 \pi} \int_{0}^{2 \pi} \ln ^{+}\left|\prod_{\nu=1}^{\infty} \frac{1-\frac{r e^{i \theta}}{\left|p_{\nu}\right|} \mid d e^{i \theta}}{\left|p_{\nu}\right|}\right| d \theta \\
=\frac{1}{2 \pi} \int_{\pi / 2}^{3 \pi / 2} \ln \left|\prod_{\nu=1}^{\infty} \frac{1-\frac{r e^{i \theta}}{\left|p_{\nu}\right|} \mid}{1+\frac{r e^{i \theta}}{\left|p_{\nu}\right|} \mid}\right| d \theta \\
=\frac{1}{2 \pi} \int_{-\pi / 2}^{\pi / 2} \ln \left|\prod_{\nu=1}^{\infty} \frac{1+\frac{r e^{i \theta}}{\left|p_{\nu}\right|}}{1-\frac{r e^{i \theta}}{\left|p_{\nu}\right|}}\right| d \theta=\sum_{\nu=1}^{\infty} q\left(\frac{r}{\left|p_{\nu}\right|}\right) .
\end{aligned}
$$

By (4.8) for each $b>0$ we have

$$
\int_{b}^{\infty} \frac{q\left(\frac{r}{\left|p_{\nu}\right|}\right)}{r^{1+\sigma}} d r \leq\left(\sec \frac{\pi \sigma}{2}-1\right) \int_{b}^{\infty} \frac{\ln ^{+} \frac{r}{\left|p_{\nu}\right|}}{r^{1+\sigma}} d r .
$$

Assuming that $\eta<\sigma<1$ and summing over $\nu$, we get

$$
\int_{b}^{\infty} \frac{m(r, \check{\chi})}{r^{1+\sigma}} d r \leq\left(\sec \frac{\pi \sigma}{2}-1\right) \int_{b}^{\infty} \frac{N(r, \chi)}{r^{1+\sigma}} d r
$$

from where, by (4.10), the inequality (4.7) follows.

Lemma 4.2. Let a function $g(t) \geq 0,-\infty<t<\infty$, be such that for some $\lambda$, $0 \leq \lambda \leq 2$, the inequality

$$
\int_{-\infty}^{\infty} \frac{g(t)}{1+|t|^{\lambda}}<\infty
$$

holds. Let

$$
u(z)=\frac{y}{\pi} \int_{-\infty}^{\infty} \frac{g(t) d t}{(x-t)^{2}+y^{2}}, z=x+i y, y>0 .
$$

Then

$$
u(z) \leq K|z|^{\lambda} y^{-1},|z|>1,
$$

where $K$ is a positive constant which does not depend on $z$.

The statement of the lemma follows from the estimate

$$
\begin{aligned}
\frac{\pi u(z)}{y}= & \int_{-\infty}^{\infty} \frac{g(t)}{|z-t|^{2}} d t=\int_{|t|<2|z|} \frac{g(t)}{1+|t|^{\lambda}} \cdot \frac{1+|t|^{\lambda}}{|z-t|^{2}} d t \\
& +\int_{|t| \geq 2|z|} \frac{g(t)}{1+|t|^{\lambda}} \cdot \frac{1+|t|^{\lambda}}{t^{2}} \cdot \frac{d t}{\left|z t^{-1}-1\right|^{2}} \\
\leq & \frac{1+2^{\lambda}|z|^{\lambda}}{y^{2}} \int_{|t|<2|z|} \frac{g(t) d t}{1+|t|^{\lambda}}+\left(\frac{1}{|z|^{2}}+\frac{2^{\lambda}}{|z|^{2-\lambda}}\right) \int_{|t| \geq 2|z|} \frac{g(t) d t}{1+|t|^{\lambda}} .
\end{aligned}
$$

LEMMA 4.3. Let $f(z)$ be a meromorphic function of finite order, $a \neq 0, \infty$ be $a$ complex number, $0<\beta-\alpha \leq 2 \pi, \omega=\pi /(\beta-\alpha)$. Suppose that

$$
\bar{C}_{\alpha \beta}(r, f)+\bar{C}_{\alpha \beta}\left(r, \frac{1}{f}\right)=O\left(r^{p}\right), p>0, r \rightarrow \infty .
$$


For an arbitrary $\varepsilon$ satisfying $0<\varepsilon<\frac{1}{2}(\beta-\alpha)$ the estimate

$$
m_{\alpha+\varepsilon, \beta-\varepsilon}\left(r, \frac{1}{f-a}\right)=O\left(r^{\omega+p}\right), r \rightarrow \infty,
$$

holds, and for each $\varepsilon_{1}>0$ the integral

$$
I(\varphi)=\int_{1}^{\infty} \ln ^{+} \frac{1}{\left|f\left(r e^{i \varphi}\right)-a\right|} \frac{d r}{r^{1+p+\omega+\varepsilon_{1}}}
$$

converges for all $\varphi \in(\alpha+\varepsilon, \beta-\varepsilon)$ except, possibly, some set $E$ satisfying mes $E=0$.

Proof. It is easy to see that

$$
m_{\alpha+\varepsilon, \beta-\varepsilon}\left(r, \frac{1}{f-a}\right) \leq \frac{r^{\omega}}{4 \omega \sin \omega \varepsilon} B_{\alpha \beta}\left(r, \frac{1}{f-a}\right) .
$$

Using the analogue of Lemma 2.1 from Chapter 3 for angular Nevanlinna characteristics, valid by Theorem 3.3 from Chapter 3 , we have

$B_{\alpha \beta}\left(r, \frac{1}{f-a}\right) \leq A_{\alpha \beta}\left(r, \frac{f}{f^{\prime}}\right)+B_{\alpha \beta}\left(r, \frac{f}{f^{\prime}}\right)+Q_{\alpha \beta}(r, f) \leq S_{\alpha \beta}\left(r, \frac{f}{f^{\prime}}\right)+O(1)$.

Now we use Theorems 5.2 from Chapter 1 and 3.1 from Chapter 3, and get

$$
\begin{array}{r}
S_{\alpha \beta}\left(r, \frac{f}{f^{\prime}}\right)=A_{\alpha \beta}\left(r, \frac{f^{\prime}}{f}\right)+B_{\alpha \beta}\left(r, \frac{f^{\prime}}{f}\right)+C_{\alpha \beta}\left(r, \frac{f^{\prime}}{f}\right)+O(1) \\
=\bar{C}_{\alpha \beta}(r, f)+\bar{C}_{\alpha \beta}\left(r, \frac{1}{f}\right)+O(1) .
\end{array}
$$

Thus,

$$
m_{\alpha+\varepsilon, \beta-\varepsilon}\left(r, \frac{1}{f-a}\right) \leq \frac{r^{\omega}}{4 \omega \sin \omega \varepsilon}\left\{\bar{C}_{\alpha \beta}(r, f)+\bar{C}_{\alpha \beta}\left(r, \frac{1}{f}\right)+O(1)\right\}=O\left(r^{\omega+p}\right) .
$$

Dividing relation (4.11) by $r^{1+p+\omega+\varepsilon_{1}}$ and integrating with respect to $r$ from 1 to $\infty$, we get

$$
\begin{aligned}
\infty & >\int_{1}^{\infty} m_{\alpha+\varepsilon, \beta-\varepsilon}\left(r, \frac{1}{f-a}\right) \frac{d r}{r^{1+p+\omega+\varepsilon_{1}}} \\
& =\frac{1}{2 \pi} \int_{1}^{\infty} \frac{d r}{r^{1+p+\omega+\varepsilon_{1}}} \int_{\alpha+\varepsilon}^{\beta-\varepsilon} \ln ^{+} \frac{1}{\left|f\left(r e^{i \varphi}\right)-a\right|} d \varphi \\
& =\frac{1}{2 \pi} \int_{\alpha+\varepsilon}^{\beta-\varepsilon} d \varphi \int_{1}^{\infty} \ln ^{+} \frac{1}{\left|f\left(r e^{i \varphi}\right)-a\right|} \cdot \frac{d r}{r^{1+p+\omega+\varepsilon_{1}}}=\frac{1}{2 \pi} \int_{\alpha+\varepsilon}^{\beta-\varepsilon} I(\varphi) d \varphi .
\end{aligned}
$$

¿From here it follows that $I(\varphi)<\infty$ for all $\varphi \in(\alpha+\varepsilon, \beta-\varepsilon)$, except, possibly, some set $E$, mes $E=0$. Q.E.D.

Now we turn to a proof of Theorem 4.1. Suppose that the order $\rho$ of the function $f(z)$ satisfies the inequality $\omega^{\prime \prime}\left(D^{\prime}\right)<\rho<\omega^{\prime}\left(D^{\prime}\right)$, and let us prove that for each $a \neq 0, \infty$ the inequality

$$
\delta(a, f) \leq 1-\cos \frac{\pi \rho}{2 \omega^{\prime}\left(D^{\prime}\right)}
$$

holds. 
Denote by $D_{\varepsilon}^{\prime}$ the system $D_{\varepsilon}^{\prime}=D\left(\alpha_{1}-\varepsilon, \beta_{1}+\varepsilon, \alpha_{2}-\varepsilon, \beta_{2}+\varepsilon, \ldots, \alpha_{n}-\varepsilon, \beta_{n}+\varepsilon\right)$ of rays, where $\varepsilon>0$ is so small that

$$
\begin{gathered}
\max _{1 \leq j \leq n}\left(\beta_{j}-\alpha_{j}\right)+4 \varepsilon<\min _{1 \leq j \leq n}\left(\alpha_{j+1}-\beta_{j}\right), \\
\omega^{\prime \prime}\left(D^{\prime}\right)<\omega^{\prime \prime}\left(D_{\varepsilon}^{\prime}\right)<\omega^{\prime \prime}\left(D_{2 \varepsilon}^{\prime}\right)<\rho<\omega^{\prime}\left(D_{2 \varepsilon}^{\prime}\right)<\omega^{\prime}\left(D_{\varepsilon}^{\prime}\right)<\omega^{\prime}\left(D^{\prime}\right) .
\end{gathered}
$$

We have

$$
\begin{aligned}
m(r, a) & =\sum_{j=1}^{n} m_{\alpha_{j}-\varepsilon, \beta_{j}+\varepsilon}\left(r, \frac{1}{f-a}\right)+\sum_{j=1}^{n} m_{\beta_{j}+\varepsilon, \alpha_{j+1}-\varepsilon}\left(r, \frac{1}{f-a}\right) \\
& =\Phi_{1}(r)+\Phi_{2}(r) .
\end{aligned}
$$

The estimate for the sum $\Phi_{2}(r)$ is obtained using Lemma 4.3. Since almost all of zeros and poles of the function $f(z)$ are in small angles of the system $D^{\prime}$, then

$$
\bar{C}_{\beta_{j} \alpha_{j+1}}(r, f)+\bar{C}_{\beta_{j} \alpha_{j+1}}\left(r, \frac{1}{f}\right)=O\left(r^{\omega^{\prime \prime}\left(D^{\prime}\right)-\omega_{j}^{\prime \prime}\left(D^{\prime}\right)}\right), j=1, \ldots, n .
$$

Applying Lemma 4.3, we get the estimate

$$
m_{\beta_{j}+\varepsilon, \alpha_{j+1}-\varepsilon}\left(r, \frac{1}{f-a}\right)=O\left(r^{\omega^{\prime \prime}\left(D^{\prime}\right)}\right), j=1, \ldots, n
$$

hence,

$$
\Phi_{2}(r)=O\left(r^{\omega^{\prime \prime}\left(D^{\prime}\right)}\right)
$$

The estimate of $\Phi_{1}(r)$ is significantly more complicated.

By Lemma 4.3 there are numbers $\varphi_{j}$ and $\psi_{j}, \alpha_{j}-2 \varepsilon<\varphi_{j}<\alpha_{j}-\varepsilon, \beta_{j}+\varepsilon<$ $\psi_{j}<\beta_{j}+2 \varepsilon$, such that for $\theta=\varphi_{j}, \psi_{j}(j=1, \ldots, n)$ we have

$$
\int_{1}^{\infty} \ln ^{+} \frac{1}{\left|f\left(r e^{i \theta}\right)-a\right|} \frac{d r}{r^{1+\omega^{\prime \prime}\left(D_{\varepsilon}^{\prime}\right)}}<\infty .
$$

We map the angle $\left\{\varphi_{j} \leq \arg z \leq \psi_{j}\right\}$ onto the half-plane $\{\operatorname{Im} \zeta \geq 0\}$ using the mapping $\zeta=\left(z e^{-i \varphi_{j}}\right)^{\pi /\left(\psi_{j}-\varphi_{j}\right)}$. Under this mapping the angle $\left\{\alpha_{j}-\varepsilon \leq \arg z \leq\right.$ $\left.\beta_{j}+\varepsilon\right\}$ is mapped onto an angle of the form $\left\{\xi_{j} \leq \arg \xi \leq \eta_{j}\right\}, 0<\xi_{j}<\eta_{j}<\pi$. Let

$$
F_{j}(\zeta)=\left\{f\left(e^{i \varphi_{j}} \zeta^{\left(\psi_{j}-\varphi_{j}\right) / \pi}\right)-a\right\}^{-1}
$$

It is easy to see that

$$
m_{\alpha_{j}-\varepsilon, \beta_{j}+\varepsilon}\left(r, \frac{1}{f-a}\right)=\frac{\psi_{j}-\varphi_{j}}{\pi} m_{\xi_{j} \eta_{j}}\left(r^{\frac{\pi}{\psi_{j}-\varphi_{j}}}, F_{j}\right),
$$

therefore to estimate the sum $\Phi_{1}$ it suffices to estimate the quantities $m_{\xi_{j} \eta_{j}}\left(r, F_{j}\right)$, $j=1, \ldots, n$.

Let us show that the function $F_{j}(z)$ satisfies

$$
S\left(r, F_{j}\right)=O(1)
$$

and hence, Theorem 3.1 is applicable to it.

Note that

$$
\int_{-\infty}^{\infty} \frac{\ln ^{+}\left|F_{j}(t)\right|}{1+|t|^{1+\mu_{j}}} d t<\infty
$$


where $\mu_{j}=\left(\psi_{j}-\varphi_{j}\right) \omega^{\prime \prime}\left(D_{\varepsilon}^{\prime}\right) / \pi$. In fact, by (4.16) and (4.15), we have

$$
\begin{aligned}
\int_{-\infty}^{\infty} \frac{\ln ^{+}\left|F_{j}(t)\right|}{1+|t|^{1+\mu_{j}}} d t= & \int_{-1}^{1} \frac{\ln ^{+}\left|F_{j}(t)\right|}{1+|t|^{1+\mu_{j}}} d t+\int_{1}^{\infty} \frac{\ln ^{+}\left|F_{j}(t)\right|}{t^{1+\mu_{j}}} d t \\
& +\int_{-\infty}^{-1} \frac{\ln ^{+}\left|F_{j}(t)\right|}{|t|^{1+\mu_{j}}}=\int_{-1}^{1} \frac{\ln ^{+}\left|F_{j}(t)\right|}{1+|t|^{1+\mu_{j}}} d t \\
& +\frac{\pi}{\psi_{j}-\varphi_{j}} \int_{1}^{\infty} \frac{\ln ^{+}\left|\left\{f\left(t e^{i \varphi_{j}}\right)-a\right\}^{-1}\right|}{t^{1+\omega^{\prime \prime}\left(D_{\varepsilon}^{\prime}\right)}} d t \\
& +\frac{\pi}{\psi_{j}-\varphi_{j}} \int_{1}^{\infty} \frac{\ln ^{+}\left|\left\{f\left(t e^{i \psi_{j}}\right)-a\right\}^{-1}\right|}{t^{1+\omega^{\prime \prime}\left(D_{\varepsilon}^{\prime}\right)}} d t<\infty .
\end{aligned}
$$

Next, since

$$
\frac{\psi_{j}-\varphi_{j}}{\pi}<\left\{\omega^{\prime}\left(D_{2 \varepsilon}^{\prime}\right)\right\}^{-1}
$$

by (4.13) we have $\mu_{j}<\left\{\omega^{\prime}\left(D_{2 \varepsilon}^{\prime}\right)\right\}^{-1} \omega^{\prime \prime}\left(D_{\varepsilon}^{\prime}\right)<1$. Therefore (4.19) implies

$$
A\left(r, F_{j}\right) \leq \frac{1}{\pi} \int_{1}^{r}\left\{\ln ^{+}\left|F_{j}(t)\right|+\ln ^{+}\left|F_{j}(-t)\right|\right\} \frac{d t}{t^{2}}=O(1) .
$$

The quantity $B\left(r, F_{j}\right)$ can be estimated in the following way:

$$
\begin{aligned}
B\left(r, F_{j}\right) \leq \frac{4}{r} m_{0 \pi}\left(r, F_{j}\right) & =\frac{4 \pi}{\left(\psi_{j}-\varphi_{j}\right) r} m_{\varphi_{j} \psi_{j}}\left(r^{\frac{\psi_{j}-\varphi_{j}}{\pi}}, \frac{1}{f-a}\right) \\
& \leq \frac{4 \pi}{\left(\psi_{j}-\varphi_{j}\right) r}\left\{T\left(r^{\frac{\psi_{j}-\varphi_{j}}{\pi}}, f\right)+O(1)\right\} .
\end{aligned}
$$

By $(4.13)$ and $\left(4.19^{\prime}\right)$ we have $\frac{\psi_{j}-\varphi_{j}}{\pi} \rho<1$, and get

$$
B\left(r, F_{j}\right)=o(1) \text {. }
$$

In a similar way we get

$$
B\left(r, 1 / F_{j}\right)=o(1) .
$$

Denoting $a$-points of the function $f(z)$ by $r_{\nu} e^{i \theta_{\nu}}$, we have

$$
C\left(r, F_{j}\right)=C_{\varphi_{j} \psi_{j}}\left(r^{\frac{\psi_{j}-\varphi_{j}}{\pi}}, \frac{1}{f-a}\right) \leq 2 \sum_{\substack{r_{\nu}>1 \\ \varphi_{j} \leq \theta_{\nu} \leq \psi_{j}}} r_{\nu}^{-\frac{\pi}{\psi_{j}-\varphi_{j}}}<\infty,
$$

since $\frac{\pi}{\psi_{j}-\varphi_{j}}>\rho$.

Thus (4.18) has been proved, and, by Theorem 3.1, the function $F_{j}(\zeta)$ admits a representation

$$
\begin{aligned}
\ln \left|F_{j}(\zeta)\right|= & \frac{r \sin \varphi}{\pi} \int_{-\infty}^{\infty} \frac{\ln \left|F_{j}(t)\right| d t}{r^{2}+t^{2}-2 r t \cos \varphi} \\
& -\sum_{\kappa} \ln \left|\frac{\zeta-\bar{q}_{\kappa}}{\zeta-q_{\kappa}}\right|+\sum_{\nu}\left|\frac{\zeta-\bar{p}_{\nu}}{\zeta-p_{\nu}}\right|+\eta r \sin \varphi, \zeta=r e^{i \varphi}
\end{aligned}
$$


where $q_{\kappa}$ are zeros of the function $F_{j}(\zeta)$, and $p_{\nu}$ are its poles (lying in $\{\operatorname{Im} \zeta>0\}$ ), $\eta$ is a constant which does not depend on $\zeta$. Estimates (4.20) and (4.21) and the remark to Theorem 3.1 imply that $\eta=0$. Therefore (4.22) implies that

$$
\ln ^{+}\left|F_{j}(\zeta)\right| \leq \frac{y}{\pi} \int_{-\infty}^{\infty} \frac{\ln ^{+}\left|F_{j}(t)\right| d t}{(x-t)^{2}+y^{2}}+\sum_{\nu} \ln \left|\frac{\zeta-\bar{p}_{\nu}}{\zeta-p_{\nu}}\right|, \zeta=x+i y
$$

The relation (4.19) and Lemma 4.2 imply that the estimate

$$
\frac{y}{\pi} \int_{-\infty}^{\infty} \frac{\ln ^{+}\left|F_{j}(t)\right|}{(x-t)^{2}+y^{2}} d t=O\left(|\zeta|^{\mu_{j}}\right), \zeta=x+i y
$$

holds in the angle $\left\{\xi_{j} \leq \arg \zeta \leq \eta_{j}\right\}$. Defining a function $\chi(z)$ by the equality (4.6), we get that

$$
\ln ^{+}\left|F_{j}\left(r e^{i \varphi}\right)\right| \leq O\left(r^{\mu_{j}}\right)+\ln \left|\chi\left(r e^{i \varphi}\right)\right|, \xi_{j} \leq \arg \zeta \leq \eta_{j},
$$

from where, integrating with respect to $\varphi$ from $\xi_{j}$ to $\eta_{j}$, we conclude that

$$
m_{\xi_{j} \eta_{j}}\left(r, F_{j}\right) \leq K_{j} r^{\mu_{j}}+m_{\xi_{j} \eta_{j}}(r, \chi) \leq K_{j} r^{\mu_{j}}+m(r, \chi), r \geq 1,
$$

where $K_{j}$ is a constant which does not depend on $r$.

Let us agree to use the notation $N_{\alpha \beta}(r, f), 0<\beta-\alpha \leq 2 \pi, r \geq 0$, for the sum

$$
N_{\alpha \beta}(r, f)=\sum_{\alpha \leq \arg a_{n} \leq \beta} \ln ^{+} \frac{r}{\left|a_{n}\right|},
$$

where $a_{n}$ are poles of the function $f(z)$ counted according to their multiplicity. Obviously,

$$
N(r, \chi)=\frac{\pi}{\psi_{j}-\varphi_{j}} N_{\varphi_{j} \psi_{j}}\left(r^{\frac{\psi_{j}-\varphi_{j}}{\pi}}, \frac{1}{f-a}\right)
$$

Therefore

$$
N(r, \chi) \leq \frac{\pi}{\psi_{j}-\varphi_{j}} N\left(r^{\frac{\psi_{j}-\varphi_{j}}{\pi}}, \frac{1}{f-a}\right) \leq \frac{\pi}{\psi_{j}-\varphi_{j}} T\left(r^{\frac{\psi_{j}-\varphi_{j}}{\pi}}, f\right)+O(1) .
$$

¿From here it follows that the order of the function $N(r, \chi)$ does not exceed the number $\frac{\psi_{j}-\varphi_{j}}{\pi} \rho<\left\{\omega^{\prime}\left(D_{2 \varepsilon}^{\prime}\right)\right\}^{-1} \rho<1$. Since $N(r, \chi)=N(r, 1 / \chi)$, and the function $\chi(z)$ is a quotient of two canonical products of genus zero, then (Theorem 3.4 from Chapter 2) the order of this function also does not exceed $\frac{\psi_{j}-\varphi_{j}}{\pi} \rho$.

Using Lemma 4.1, we write the inequality (4.7) for our function $\chi(z)$. It holds for all $b>0$ and $\sigma$ satisfying the condition $\frac{\psi_{j}-\varphi_{j}}{\pi} \rho<\sigma<1$.

We divide (4.23) by $r^{1+\sigma}$ and integrate it with respect to $r$ from $b>1$ to $\infty$. Taking into account (4.7) we get

$$
\int_{b}^{\infty} \frac{m_{\xi_{j} \eta_{j}}\left(r, F_{j}\right)}{r^{1+\sigma}} d r \leq\left(\sec \frac{\pi \sigma}{2}-1\right) \int_{b}^{\infty} \frac{K_{j}^{\prime} r^{\mu_{j}}+N(r, \chi)}{r^{1+\sigma}} d r
$$

where $K_{j}^{\prime}$ is a constant which does not depend on $r$ and $b$. Using the equalities (4.17) and (4.24), and making the change of variable $r=t^{\pi /\left(\psi_{j}-\varphi_{j}\right)}$ in the integrals, 
we have that for $\frac{\psi_{j}-\varphi_{j}}{\pi} \rho<\sigma<1$ and for all $b>1$ the inequality

$$
\begin{aligned}
& \int_{b}^{\infty} \frac{m_{\alpha_{j}-\varepsilon, \beta_{j}+\varepsilon}\left(t, \frac{1}{f-a}\right)}{t^{1+\frac{\sigma \pi}{\psi_{j}-\varphi_{j}}}} d t \\
& \quad \leq\left(\sec \frac{\pi \sigma}{2}-1\right) \int_{b}^{\infty} \frac{K_{j}^{\prime \prime} t^{\omega^{\prime \prime}\left(D_{\varepsilon}^{\prime}\right)}+N_{\varphi_{j} \psi_{j}}\left(t, \frac{1}{f-a}\right)}{t^{1+\frac{\sigma \pi}{\psi_{j}-\varphi_{j}}}} d t
\end{aligned}
$$

holds, where $K_{j}^{\prime \prime}$ is a constant which does not depend on $t$ and $b$. Let $\eta=\frac{\sigma \pi}{\psi_{j}-\varphi_{j}}$ and note that as $\sigma$ ranges over the interval $\left(\rho \frac{\psi_{j}-\varphi_{j}}{\pi}, 1\right)$, the parameter $\eta$ ranges over the interval $\left(\rho, \frac{\pi}{\psi_{j}-\varphi_{j}}\right)$, which contains the interval $\left(\rho, \omega^{\prime}\left(D_{2 \varepsilon}^{\prime}\right)\right)$. Note, also, that $\sigma<\eta\left\{\omega^{\prime}\left(D_{2 \varepsilon}^{\prime}\right)\right\}^{-1}$. Then we can claim that for $\rho<\eta<\omega^{\prime}\left(D_{2 \varepsilon}^{\prime}\right)$ and $b>1$ the inequality

$$
\begin{aligned}
& \int_{b}^{\infty} \frac{m_{\alpha_{j}-\varepsilon, \beta_{j}+\varepsilon}\left(t, \frac{1}{f-a}\right)}{t^{1+\eta}} d t \\
& \quad \leq\left(\sec \frac{\pi \eta}{2 \omega^{\prime}\left(D_{2 \varepsilon}^{\prime}\right)}-1\right) \int_{b}^{\infty} \frac{K_{j}^{\prime \prime} t^{\omega^{\prime \prime}\left(D_{\varepsilon}^{\prime}\right)}+N_{\varphi_{j} \psi_{j}}\left(t, \frac{1}{f-a}\right)}{t^{1+\eta}} d t
\end{aligned}
$$

holds. Adding these inequalities over $j=1, \ldots, n$ and taking into account that $\sum_{j+1}^{n} N_{\varphi_{j} \psi_{j}}\left(t, \frac{1}{f-a}\right) \leq N\left(t, \frac{1}{f-a}\right), t \geq 1$, since the intervals $\left(\varphi_{j}, \psi_{j}\right)$ do not intersect, we get

$$
\int_{b}^{\infty} \frac{\Phi_{1}(t)}{t^{1+\eta}} d t \leq\left(\sec \frac{\pi \eta}{2 \omega^{\prime}\left(D_{2 \varepsilon}^{\prime}\right)}-1\right) \int_{b}^{\infty} \frac{K t^{\omega^{\prime \prime}\left(D_{\varepsilon}^{\prime}\right)}+N(t, a)}{t^{1+\eta}} d t
$$

where $K$ is a constant which does not depend on $b$ and $t, \rho<\eta<\omega^{\prime}\left(D_{2 \varepsilon}^{\prime}\right), b>1$.

The relation (4.14) implies that for $\eta>\rho\left(>\omega^{\prime \prime}\left(D_{\varepsilon}^{\prime}\right)>\omega^{\prime \prime}\left(D^{\prime}\right)\right)$ the inequality

$$
\int_{b}^{\infty} \frac{\Phi_{2}(t)}{t^{1+\eta}} d t \leq \int_{b}^{\infty} \frac{K^{\prime} t^{\omega^{\prime \prime}\left(D_{\varepsilon}^{\prime}\right)}}{t^{1+\eta}} d t
$$

holds, where $K^{\prime}$ is a constant which does not depend on $b$ and $t$. Adding with (4.25) we get the inequality

$$
\int_{b}^{\infty} \frac{m(t, a)}{t^{1+\eta}} d t \leq\left(\sec \frac{\pi \eta}{2 \omega^{\prime}\left(D_{2 \varepsilon}^{\prime}\right)}-1\right) \int_{b}^{\infty} \frac{K^{\prime \prime} t^{\omega^{\prime \prime}\left(D_{\varepsilon}^{\prime}\right)}+N(t, a)}{t^{1+\eta}} d t,
$$

where $K^{\prime \prime}$ does not depend on $b$ and $t, \rho<\eta<\omega^{\prime}\left(D_{2 \varepsilon}^{\prime}\right), b>1$.

Since $\rho>\omega^{\prime \prime}\left(D_{\varepsilon}^{\prime}\right)$, for each $\varepsilon_{1}>0$ there is a sequence $\left\{b_{q}\right\}, b_{q} \uparrow \infty$, such that

$$
\int_{b_{q}}^{\infty} \frac{K^{\prime \prime} t^{\omega^{\prime \prime}\left(D_{\varepsilon}\right)}}{t^{1+\eta}} d t \leq \varepsilon_{1} \int_{b_{q}}^{\infty} \frac{T(t, f)}{t^{1+\eta}} d t
$$

Therefore we have the inequality

$$
\int_{b_{q}}^{\infty} \frac{m(t, a)}{t^{1+\eta}} d t \leq\left(\sec \frac{\pi \eta}{2 \omega^{\prime}\left(D_{2 \varepsilon}^{\prime}\right)}-1\right) \int_{b_{q}}^{\infty} \frac{N(t, a)+\varepsilon_{1} T(t, f)}{t^{1+\eta}} d t
$$


This inequality implies that there exists a number $t_{q}>b_{q}$, such that

$$
m\left(t_{q}, a\right) \leq\left(\sec \frac{\pi \eta}{2 \omega^{\prime}\left(D_{2 \varepsilon}^{\prime}\right)}-1\right)\left\{N\left(t_{q}, a\right)+\varepsilon_{1} T\left(t_{q}, f\right)\right\} .
$$

Dividing both sides of (4.26) by $T\left(t_{q}, f\right)$ and taking the limit as $t_{q} \rightarrow \infty$, we get

$$
\delta(a, f) \leq\left(\sec \frac{\pi \eta}{2 \omega^{\prime}\left(D_{2 \varepsilon}^{\prime}\right)}-1\right)\left\{1-\delta(a, f)+\varepsilon_{1}\right\} .
$$

Letting here $\varepsilon$ and $\varepsilon_{1}$ tend to 0 , and $\eta$ tend to $\rho$, we get the inequality

$$
\delta(a, f) \leq\left(\sec \frac{\pi \rho}{2 \omega^{\prime}\left(D^{\prime}\right)}-1\right)\{1-\delta(a, f)\},
$$

which is equivalent to (4.12). This completes the proof of the theorem.

Now we construct an example showing that the $\operatorname{sign}>$ in the condition $\left(4.5^{\prime}\right)$ from Corollary 1 cannot be replaced by $\geq$.

EXAMPLE 2. For arbitrary preassigned $\gamma$ and $\rho, 0<\gamma<\pi, 0<\rho<\pi \gamma^{-1}$, we construct a meromorphic function $f(z)$ of order $\rho$, all zeros and poles of which are in the angle $\left\{|\arg z| \leq \frac{\gamma}{2}\right\}$, and $\delta(1, f)=1-\cos \frac{\rho \gamma}{2}$.

Let

$$
\Phi(z ; \eta)=\prod_{\nu=1}^{\infty} \frac{1+z \nu^{-\frac{1}{\eta}}}{1-z \nu^{-\frac{1}{\eta}}}, 0<\eta<1 .
$$

The function $\Phi(z ; \eta)$ is a special case of the function $f_{u v}(z)$ considered in Example 1 of Section 3 from Chapter $5(u=v=1, l(r) \equiv \eta)$. Therefore, by the formulas (3.38) and (3.39) from Chapter 5 we have

$$
\begin{gathered}
N(r, \Phi(z ; \eta))=\frac{1}{\eta} r^{\eta}+o\left(r^{\eta}\right) \\
T(r, \Phi(z ; \eta))=\frac{1}{\eta}\{I(1,1, \eta)+1\} r^{\eta}+o\left(r^{\eta}\right)=\frac{1}{\eta} \sec \frac{\pi \eta}{2} r^{\eta}+o\left(r^{\eta}\right) .
\end{gathered}
$$

Note that

$$
|\Phi(z ; \eta)| \leq 1 \text { for } \operatorname{Re} z \leq 0 \text {. }
$$

Denote by $L(\theta, a)$ the boundary of the region $D(\theta, a)=\{|\arg z|<\theta,|z|>a\}$, oriented clockwise, by $D_{1}(\theta, a)$ we denote the region on the left from $L(\theta, a)$. For $z \in D_{1}\left(\frac{\gamma}{2}, \frac{1}{2}\right)$ we let

$$
\Psi(z)=\frac{1}{2 \pi i} \int_{L\left(\frac{\gamma}{2}, \frac{1}{2}\right)} \frac{\Phi\left(\zeta^{\pi / \gamma} ; \frac{\rho \gamma}{\pi}\right)}{(\zeta+1)^{2}(\zeta-z)} d \zeta
$$

The inequality (4.29) implies that

$$
\left|\Phi\left(\zeta^{\pi / \gamma} ; \frac{\rho \gamma}{\pi}\right)\right| \leq 1 \text { for } \frac{\gamma}{2} \leq|\arg \zeta| \leq \gamma
$$

therefore the integral in (4.30) is absolutely convergent, and the function $\Psi(z)$ is analytic in $D_{1}\left(\frac{\gamma}{2}, \frac{1}{2}\right)$. 
Using the residue theorem we get the following relation for $R \geq \frac{1}{2}, R \neq \nu^{1 / \rho}$, $\nu=1,2, \ldots,\left(z \in D_{1}\left(\frac{\gamma}{2}, \frac{1}{2}\right)\right)$

$$
\begin{aligned}
& \frac{1}{2 \pi i} \int_{L\left(\frac{\gamma}{2}, \frac{1}{2}\right)} \frac{\Phi\left(\zeta^{\pi / \gamma} ; \frac{\rho \gamma}{\pi}\right)}{(\zeta+1)^{2}(\zeta-z)} d \zeta \\
& \quad=\frac{1}{2 \pi i} \int_{L\left(\frac{\gamma}{2}, R\right)} \frac{\Phi\left(\zeta^{\pi / \gamma} ; \frac{\rho \gamma}{\pi}\right)}{(\zeta+1)^{2}(\zeta-z)} d \zeta-\sum_{\nu=1}^{\left[R^{\rho}\right]} \operatorname{res}_{\zeta=\nu^{1 / \rho}} \frac{\Phi\left(\zeta^{\pi / \gamma} ; \frac{\rho \gamma}{\pi}\right)}{(\zeta+1)^{2}(\zeta-z)} .
\end{aligned}
$$

The right-hand side of this relation represents a function meromorphic in the region $D_{1}\left(\frac{\gamma}{2}, R\right)$. Since $R$ can be chosen to be arbitrarily large, the function $\Psi(z)$ can be extended to a meromorphic function in the whole complex plane. We shall keep the notation $\Psi(z)$ for this extension.

We show that for $z \in D\left(\gamma, \frac{1}{2}\right)$ the formula

$$
\Psi(z)=\frac{1}{2 \pi i} \int_{L\left(\nu, \frac{1}{2}\right)} \frac{\Phi\left(\zeta^{\pi / \gamma} ; \frac{\rho \gamma}{\pi}\right)}{(\zeta+1)^{2}(\zeta-z)} d \zeta+\frac{\Phi\left(z^{\pi / \gamma} ; \frac{\rho \gamma}{\pi}\right)}{(z+1)^{2}}
$$

holds.

For $z \in D\left(\gamma, \frac{1}{2}\right)$ the function $\Psi(z)$ can be represented by the right-hand side of the relation (4.32) with $R>|z|$. Using the Cauchy theorem and (4.31) it is easy to get that the integral from the right-hand side of (4.32) does not change if the path of integration is changed from $L\left(\frac{\gamma}{2}, R\right)$ to $L(\gamma, R)$. On the other hand, by the residue theorem we have $\left(z \in D\left(\gamma, \frac{1}{2}\right), R>|z|\right)$

$$
\begin{aligned}
\frac{1}{2 \pi i} \int_{L(\gamma, R)} \frac{\Phi\left(\zeta^{\pi / \gamma} ; \frac{\rho \gamma}{\pi}\right)}{(\zeta+1)^{2}(\zeta-z)} d \zeta= & \frac{1}{2 \pi i} \int_{L\left(\gamma, \frac{1}{2}\right)} \frac{\Phi\left(\zeta^{\pi / \gamma} ; \frac{\rho \gamma}{\pi}\right)}{(\zeta+1)^{2}(\zeta-z)} d \zeta \\
& +\sum_{\nu=1}^{\left[R^{\rho}\right]} \operatorname{res}_{\zeta=\nu^{1 / \rho}} \frac{\Phi\left(\zeta^{\pi / \gamma} ; \frac{\rho \gamma}{\pi}\right)}{(\zeta+1)^{2}(\zeta-z)}+\frac{\Phi\left(z^{\pi / \gamma} ; \frac{\rho \gamma}{\pi}\right)}{(z+1)^{2}}
\end{aligned}
$$

Thus the formula (4.33) has been proved.

Using (4.31), we get from (4.30) and (4.33) the following relations

$$
\begin{array}{r}
|\Psi(z)| \leq c /(|z|+1), \frac{\gamma}{2} \leq \arg z \leq 2 \pi-\frac{\gamma}{2}, \\
\left|\Psi(z)-\frac{\Phi\left(z^{\pi / \gamma} ; \frac{\rho \gamma}{\pi}\right)}{(z+1)^{2}}\right| \leq c /(|z|+1),|\arg z| \leq \frac{\gamma}{2},
\end{array}
$$

where $c$ is a constant which does not depend on $z$. These relations imply the following equalities:

$$
N(r, \Psi)=N\left(r^{\pi / \gamma}, \Phi\left(z ; \frac{\rho \gamma}{\pi}\right)\right)
$$




$$
\begin{aligned}
m(r, \Psi) & =m_{-\frac{\gamma}{2}, \frac{\gamma}{2}}(r, \Psi)+O(1)=m\left(r^{\pi / \gamma}, \Phi\left(z ; \frac{\rho \gamma}{\pi}\right)\right)+O(\ln r) \\
& =T\left(r^{\pi / \gamma}, \Phi\left(z ; \frac{\rho \gamma}{\pi}\right)\right)-N\left(r^{\pi / \gamma}, \Phi\left(z ; \frac{\rho \gamma}{\pi}\right)\right)+O(\ln r) .
\end{aligned}
$$

Taking into account (4.27) and (4.28) we get

$$
\begin{gathered}
N(r, \Psi)=\frac{\pi}{\rho \gamma} r^{\rho}+o\left(r^{\rho}\right), \\
T(r, \Psi)=\frac{\pi}{\rho \gamma} \sec \frac{\rho \gamma}{2} r^{\rho}+o\left(r^{\rho}\right),
\end{gathered}
$$

whence $\delta(\infty, \Psi)=1-\cos \frac{\rho \gamma}{2}$.

By (4.34) the quantity

$$
Q=\sup _{\frac{\gamma}{2} \leq \arg z \leq 2 \pi-\frac{\gamma}{2}}|\Psi(z)|
$$

is finite. Therefore for each $b,|b|>Q$, all $b$-points of the function $\Psi(z)$ are in the angle $\{|\arg z| \leq \gamma / 2\}$. Choose arbitrarily two complex numbers $b$ and $c$, $\min (|b|,|c|)>Q$, and consider the function

$$
f(z)=\frac{\Psi(z)-b}{\Psi(z)-c} .
$$

It is clear that it is a desired function.

\section{Entire functions with derivatives only vanishing close to the real axis}

As is known, zeros of a polynomial with real zeros are real. The direct analogue of this result for entire transcendental functions does not hold. For example, all zeros of the function $f(z)=\left(z^{2}-1\right) e^{z^{2} / 2}$ are real, while $f^{\prime}(z)=\left(z^{3}+z\right) e^{z^{2} / 2}$ has non-real zeros also. Sufficient conditions under which zeros of all derivatives of an entire function with real zeros are real, are given by the following classical theorem.

TheOREM 5.1 (Laguerre). If an entire function $f(z)$ is representable in the form

$$
f(z)=e^{-\gamma z^{2}} h(z),
$$

where $\gamma \geq 0$ and $h(z)$ is an entire function of genus at most 1 , real on the real line, and having only real zeros, then zeros of all derivatives of the function $f(z)$ are real.

Proof. The formula (5.1) implies that

$$
f^{\prime}(z)=e^{-\gamma z^{2}} h_{1}(z)
$$

where

$$
h_{1}(z)=h^{\prime}(z)-2 \gamma z h(z) .
$$

Theorem 5.1 will be proved, if we establish that $h_{1}(z)$ is an entire function of genus at most 1 , real on the real line and having only real zeros.

The function $h(z)$ is representable in the form

$$
h(z)=z^{m} e^{a z+b} \prod_{k}\left(1-\frac{z}{a_{k}}\right) e^{\frac{z}{a_{k}}}
$$


where $m \geq 0$ is an integer, $a, b$, and $a_{k}$ are real numbers, and

$$
\sum_{k} a_{k}^{-2}<\infty
$$

Therefore we have

$$
\frac{f^{\prime}(z)}{f(z)}=-2 \gamma z+\frac{m}{z}+a+\sum_{k}\left(\frac{1}{z-a_{k}}+\frac{1}{a_{k}}\right)
$$

whence

$$
\operatorname{Im} \frac{f^{\prime}(z)}{f(z)}=-\left\{2 \gamma+\frac{m}{|z|^{2}}+\sum_{k} \frac{1}{\left|z-a_{k}\right|^{2}}\right\} \operatorname{Im} z .
$$

We may assume that $f(z)$ is not of the form $e^{a z+b}$, because otherwise the theorem is trivial. Then the expression in braces in (5.4) is different from zero and hence

$$
\operatorname{Im} \frac{f^{\prime}(z)}{f(z)} \neq 0 \text { for } \operatorname{Im} z \neq 0 \text {. }
$$

This means that all zeros of $f^{\prime}(z)$, and hence, of $h_{1}(z)$ are real.

It remains to prove that the genus of the function $h_{1}(z)$ does not exceed 1 . Since the genus of the function $h(z)$ does not exceed 1, then, by Remark 2 to Theorem 3.3 from Chapter 2, the relation $T(r, h)=o\left(r^{2}\right)$ holds. By Theorem 2.3 from Chapter 3 we have, also, $T\left(r, h^{\prime}\right)=o\left(r^{2}\right)$, and hence, $T\left(r, h_{1}\right)=T\left(r, h^{\prime}-2 \gamma z h\right)=o\left(r^{2}\right)$. Therefore, in order to show that the genus of the function $h_{1}(z)$ does not exceed 1 , it suffices to establish the convergence of the series

$$
\sum_{k} a_{k 1}^{-2}
$$

where $a_{k 1}$ are zeros of the function $h_{1}(z)$ away from $z=0$ (multiple zeros are repeated according to their multiplicity).

The equality (5.3) implies

$$
\left(\frac{f^{\prime}(x)}{f(x)}\right)^{\prime}=-2 \gamma-\sum_{k} \frac{1}{\left(x-a_{k}\right)^{2}}
$$

For real $x$ the expression in the right-hand side is negative. Therefore in the interval between two consecutive zeros of the function $f(x)$ the function $\frac{f^{\prime}(x)}{f(x)}$ is decreasing and is varying from $\infty$ to $-\infty$. Thus, between two consecutive zeros of the function $f(x)$ there is exactly one simple root of the function $f^{\prime}(x)$. Therefore the convergence of the series (5.2) implies the convergence of the series (5.5), Q.E.D.

We say that an entire function $f(z)$ belongs to the class $A$ (notation: $f(z) \in A$ ) if its zeros $\left\{z_{n}\right\}$ satisfy the condition

$$
\sum_{\left|z_{n}\right|>1}\left|\operatorname{Im} \frac{1}{z_{n}}\right|<\infty
$$

(multiple zeros are repeated according to their multiplicity). As we mentioned in Section 2 (see Example 1), a function $f(z)$ belongs to the class $A$ if and only if

$$
C(r, 0, f(z))+C(r, 0, f(-z))=O(1) .
$$

If is clear that all functions with real zeros belong to the class $A$. 
We shall find conditions under which all derivatives of a function from the class $A$ belong to the class $A$.

THEOREM 5.2. Let $f(z)$ be an entire function belonging to the class $A$, which is representable in the form

$$
f(z)=e^{g(z)} h(z)
$$

where $g(z)$ is an entire function which does not exceed the normal type of order 1 and which satisfies the condition

$$
\int_{-\infty}^{\infty} \frac{\ln ^{+}\left|g^{\prime}(t)\right|}{1+t^{2}} d t<\infty
$$

and $h(z)$ is an entire function satisfying the condition

$$
\int_{1}^{\infty} t^{-2} \ln ^{+} T(t, h) d t<\infty .
$$

Then all derivatives of the function $f(z)$ belong to the class $A$.

Theorem 5.2 can be considered as an analogue of Laguerre's Theorem 5.1 in the case when the condition that all zeros of $f(z)$ are real is replaced by a weaker condition: the function $f(z)$ belongs to the class $A$.

To prove the theorem it suffices to establish that the function $f^{\prime}(z)$ belongs to the class $A$ and is representable in the form

$$
f^{\prime}(z)=e^{g(z)} h_{1}(z),
$$

where $h_{1}(z)$ is an entire function satisfying the condition

$$
\int_{1}^{\infty} t^{-2} \ln ^{+} T\left(t, h_{1}\right) d t<\infty .
$$

We need the following lemma.

LEMMA 5.1. If a function $f(z)$ satisfies the conditions of Theorem 5.2 , then

$$
S\left(r, \frac{f^{\prime}}{f}\right)=O(1)
$$

Proof. Since $f(z)$ belongs to the class $A$, it is clear that

$$
C\left(r, \frac{f^{\prime}}{f}\right)=\bar{C}(r, 0, f) \leq C(r, 0, f)=O(1) .
$$

Further, we have

$$
\begin{aligned}
A\left(r, \frac{f^{\prime}}{f}\right)+B\left(r, \frac{f^{\prime}}{f}\right) & =A\left(r, g^{\prime}+\frac{h^{\prime}}{h}\right)+B\left(r, g^{\prime}+\frac{h^{\prime}}{h}\right) \\
& \leq A\left(r, g^{\prime}\right)+B\left(r, g^{\prime}\right)+A\left(r, \frac{h^{\prime}}{h}\right)+B\left(r, \frac{h^{\prime}}{h}\right)+O(1) .
\end{aligned}
$$

The condition (5.7) implies that $A\left(r, g^{\prime}\right)=O(1)$. Since $T(r, g)=O(r)$, by the inequality (2.13) from Chapter 3, we have $T\left(r, g^{\prime}\right)=O(r)$ and hence

$$
B\left(r, g^{\prime}\right) \leq \frac{4}{r} m\left(r, g^{\prime}\right)=O(1) .
$$

By Theorem 1.3 from Chapter 3,

$$
A\left(r, \frac{h^{\prime}}{h}\right)+B\left(r, \frac{h^{\prime}}{h}\right)=Q_{0 \pi}(r, h) .
$$


Recalling the definition of $Q_{0 \pi}(r, h)$ (page ???), from the condition (5.8) we get

$$
A\left(r, \frac{h^{\prime}}{h}\right)+B\left(r, \frac{h^{\prime}}{h}\right)=O(1) .
$$

Thus,

$$
S\left(r, \frac{f^{\prime}}{f}\right)=A\left(r, \frac{f^{\prime}}{f}\right)+B\left(r, \frac{f^{\prime}}{f}\right)+C\left(r, \frac{f^{\prime}}{f}\right)=O(1) .
$$

Lemma has been proved.

Since

$$
C\left(r, 0, f^{\prime}\right) \leq C\left(r, \frac{f}{f^{\prime}}\right)+C(r, 0, f) \leq S\left(r, \frac{f^{\prime}}{f}\right)+C(r, 0, f)+O(1)
$$

(we used Theorem 5.1 from Chapter 1), we have $C\left(r, 0, f^{\prime}\right)=O(1)$, if the function $f(z)$ satisfies the conditions of Theorem 5.2. Considering the function $f_{1}(z)=$ $f(-z)$, in an analogous way we get $C\left(r, 0, f_{1}^{\prime}\right)=O(1)$. Thus we have proved that $f^{\prime}(z)$ belongs to the class $A$.

It is clear that the function $f^{\prime}(z)$ can be represented in the form (5.9) with $h_{1}(z)=g^{\prime}(z) h(z)+h^{\prime}(z)$. We have

$$
T\left(r, h_{1}\right) \leq T\left(r, g^{\prime}\right)+T(r, h)+T\left(r, h^{\prime}\right)+\ln 2 \leq T(r, h)+T\left(r, h^{\prime}\right)+O(r) .
$$

Since

$$
T\left(r, h^{\prime}\right)=m\left(r, h^{\prime}\right) \leq m(r, h)+m\left(r, \frac{h^{\prime}}{h}\right)=T(r, h)+m\left(r, \frac{h^{\prime}}{h}\right),
$$

using the estimate (1.13) from Chapter 3, we get

$$
\begin{gathered}
T\left(r, h_{1}\right) \leq 2 T(r, h)+O(\ln r+\ln T(2 r, h))+O(r) \leq 3 T(2 r, h)+O(r), \\
\ln ^{+} T\left(r, h_{1}\right) \leq \ln ^{+} T(2 r, h)+O(\ln r) .
\end{gathered}
$$

Therefore (5.8) implies (5.10).

Observe that the conditions of Theorem 5.2 are satisfied for each entire function $h(z)$ belonging to the class $A$, and satisfying the condition (5.8). In particular, Theorem 5.2 has the following corollary.

CoROllary. If an entire function of finite order belongs to the class $A$, then all of its derivatives belong to the class $A$.

If we weaken somewhat the conditions of Theorem 5.2 and suppose only that the function $f(z)$ belongs to the class $A$ and satisfies the condition $\ln ^{+} T(r, f)=O(r)$, the statement of Theorem 5.2 is no longer true. It suffices to consider the example $f(z)=e^{\cosh z}$. It is clear that $\ln ^{+} T(r, f)=O(r), f(z) \in A$, but $f^{\prime}(z)=\sinh z e^{\cosh z}$ does not belong to $A$.

In 1914 G. Pólya and A. Wiman posed the following problem. Whether the conditions indicated in Theorem 5.1 are not only sufficient, but also necessary for a real-valued on the real line entire function with real zeros to have derivatives with only real zeros.

Complete answer to this problem is still unknown.

An analogous problem can be posed in connection with Theorem 5.2: whether its conditions are not only sufficient, but are also necessary for an entire function of the class $A$ to have all of its derivatives in the class $A$ ? Clearly, any results on 
the latter problem can be considered as progress towards a solution of the problem of G. Pólya and A. Wiman.

It seems likely that the following conjecture is true. If an entire function $f(z)$ is such that $f(z) \in A$ and $f^{\prime \prime}(z) \in A$, then $f(z)$ is representable in the form (5.6), where $g(z)$ is an entire function which does not exceed normal type of order 1 and satisfies (5.7), and $h(z)$ satisfies the condition (5.8). We can prove the following theorem.

THEOREM 5.3. Let $f(z)$ be an entire function, such that $f(z) \in A, f^{\prime \prime}(z) \in A$. Suppose that the function $f(z)$ is representable in the form

$$
f(z)=e^{g(z)} h(z),
$$

where $g(z)$ is an entire function, and $h(z)$ satisfies the condition (5.8). Then the function $g(z)$ does not exceed normal type of order 1 and satisfies the condition (5.7).

Note that the condition $f^{\prime \prime}(z) \in A$ cannot be replaced by the condition $f^{\prime}(z) \in$ $A$. This can be seen from the example

$$
f(z)=\exp \left\{\int_{0}^{z} G(\zeta) d \zeta\right\}
$$

where $G(\zeta)$ is an arbitrary entire function without zeros.

One can observe some analogy between Theorem 5.3 and Theorem 2.7 from Chapter 3. One of the assumptions in the latter was that $f(z) f^{\prime \prime}(z)$ does not have zeros, and the condition $f(z) \in A, f^{\prime \prime}(z) \in A$ means, roughly speaking, that $f(z) f^{\prime \prime}(z)$ is "rarely" equal to zero in each of the half-planes $\{\operatorname{Im} z>0\}$ and $\{\operatorname{Im} z<0\}$.

In the proof of Theorem 2.7 from Chapter 3 we considered the function

$$
F(z)=\frac{f(z)}{f^{\prime}(z)}
$$

and studied it using the inequality (2.23) from Chapter 3. Proving Theorem 5.3 we shall also consider this function, but now we shall use analogues of the inequality (2.23) from Chapter 3 for Tsuji characteristics and for angular Nevanlinna characteristics. As opposed to the proof of Theorem 2.7 from Chapter 3, where we succeeded in establishing $T(r, F)=O(\ln r)$ and $\frac{f^{\prime}(z)}{f(z)} \equiv$ const, now we will be able to get only some estimates for $m(r, F)$ and $m(r, 1 / F)$. We need an estimate for $m(r, 1 / F)=m\left(r, f^{\prime} / f\right)$ only. It is given by the following theorem, interesting by itself.

THEOREM 5.4. If $f(z)$ is an entire function and $f(z) \in A, f^{\prime \prime}(z) \in A$, then the estimate

$$
\int_{R}^{\infty} \frac{m\left(r, \frac{f^{\prime}}{f}\right)}{r^{3}} d r=O\left(\frac{\ln R}{R}\right), R \rightarrow \infty
$$

holds.

To prove this theorem we need the following lemma, connecting the quantity $m_{0 \pi}(r, f)$ with the Tsuji characteristic $\mathfrak{m}(r, f)$. 
LEMMA 5.2. If a function $f(z)$ is meromorphic in the half-plane $\{\operatorname{Im} z>0\}$, then the inequality

$$
\int_{R}^{\infty} \frac{m_{0 \pi}(r, f)}{r^{3}} d r \leq \int_{R}^{\infty} \frac{\mathfrak{m}(r, f)}{r^{2}} d r, R \geq 1,
$$

holds.

Observe that the statement of the lemma is non-trivial only in the case when the integral in the right-hand side of (5.11) is convergent.

Consider the integral

$$
I(R)=\frac{1}{2 \pi} \iint_{\Omega_{R}} \frac{\ln ^{+}\left|f\left(r e^{i \varphi}\right)\right|}{r^{3}} d r d \varphi
$$

where $\Omega_{R}=\{r>R \sin \varphi, r>1,0<\varphi<\pi\}$. Making the change of variables $\rho=r / \sin \varphi, \theta=\varphi$, we get

$$
I(R)=\int_{R}^{\infty} \frac{d \rho}{\rho^{2}}\left\{\frac{1}{2 \pi} \int_{\arcsin \rho^{-1}}^{\pi-\arcsin \rho^{-1}} \ln ^{+}\left|f\left(\rho \sin \theta e^{i \theta}\right)\right| \frac{d \theta}{\rho \sin ^{2} \theta}\right\}=\int_{R}^{\infty} \frac{\mathfrak{m}(\rho, f)}{\rho^{2}} d \rho .
$$

Next, we consider the integral

$$
J(R)=\frac{1}{2 \pi} \iint_{K_{R}} \frac{\ln ^{+}\left|f\left(r e^{i \varphi}\right)\right|}{r^{3}} d r d \varphi
$$

where $K_{R}=\{r>R, 0<\varphi<\pi\}$. Because of $K_{R} \subset \Omega_{R}$ we have $J(R) \leq I(R)$. Since

$$
J(R)=\int_{R}^{\infty} \frac{d r}{r^{3}}\left\{\frac{1}{2 \pi} \int_{0}^{\pi} \ln ^{+}\left|f\left(r e^{i \varphi}\right)\right| d \varphi\right\}=\int_{R}^{\infty} \frac{m_{0 \pi}(r, f)}{r^{3}} d r
$$

the inequality (5.11) has been proved.

Now we prove Theorem 5.4. Let $F(z)=f(z) / f^{\prime}(z)$. Since $f(z) \in A$, all zeros $\left\{\rho_{n} e^{i \psi_{n}}\right\}$ of the function $F(z)$ lying in the half-plane $\{\operatorname{Im} z>0\}$ (all of them are simple and are zeros of $f(z))$ satisfy the condition

$$
\sum_{\rho_{n}>1} \frac{\sin \psi_{n}}{\rho_{n}}<\infty
$$

The relation

$$
\mathfrak{N}(r, 0, F)=\sum_{1<\rho_{n} \leq r \sin \psi_{n}}\left(\frac{\sin \psi_{n}}{\rho_{n}}-\frac{1}{r}\right)
$$

implies (cf. Section 5 of Chapter 1, p. ???)

$$
\mathfrak{N}(r, 0, F)=O(1) \text {. }
$$

Since

$$
F^{\prime}(z)=1-\frac{f(z) f^{\prime \prime}(z)}{\left\{f^{\prime}(z)\right\}^{2}}
$$

and $f(z) \in A, f^{\prime \prime}(z) \in A$, then the zeros $\left\{\rho_{n}^{\prime} e^{i \psi_{n}^{\prime}}\right\}$ of the function $F^{\prime}(z)-1$ lying in the half-plane $\{\operatorname{Im} z>0\}$ also satisfy the condition

$$
\sum_{\rho_{n}^{\prime}>1} \frac{\sin \psi_{n}^{\prime}}{\rho_{n}^{\prime}}<\infty
$$


hence

$$
\mathfrak{N}\left(r, 1, F^{\prime}\right)=O(1)
$$

By Theorem 3.3 from Chapter 3, the analogue of the inequality (2.23) from Chapter 3 holds for Tsuji characteristics. In particular, the inequality

$$
\mathfrak{T}(r, F) \leq 3 \mathfrak{N}(r, 0, F)+4 \mathfrak{N}\left(r, 1, F^{\prime}\right)+\mathfrak{Q}(r, F)
$$

holds. Taking into account (5.12) and (5.14), we get from here:

$$
\mathfrak{T}(r, F)=\mathfrak{Q}(r, F) .
$$

By the definition of $\mathfrak{Q}(r, F)$ it means that outside some set $E$ of finite measure the relation

$$
\mathfrak{T}(r, F)=O(\ln r)+O(\ln \mathfrak{T}(r, F)), r \rightarrow \infty,
$$

holds, hence

$$
\mathfrak{T}(r, F)=O(\ln r), r \rightarrow \infty,
$$

for $r \notin E$.

By Theorem 5.4 from Chapter 1 the function $\mathfrak{T}(r, F)$ is up to a bounded summand non-decreasing. Therefore, applying Lemma 2.1 we conclude that (5.15) holds for $r \in E$.

By Theorem 5.3 from Chapter 1, the relation (5.15) implies

$$
\mathfrak{m}\left(r, \frac{1}{F}\right)=O(\ln r) .
$$

Applying Lemma 5.2 we get the estimate

$$
\int_{R}^{\infty} \frac{m_{0 \pi}(r, 1 / F)}{r^{3}} d r \leq \int_{R}^{\infty} \frac{\mathfrak{m}(r, 1 / F)}{r^{2}} d r=O\left(\frac{\ln R}{R}\right) .
$$

Clearly, the argument above remains true for $F_{1}(z)=F(-z)$, therefore we can replace $F$ by $F_{1}$ in the relation (5.16).

Since

$$
m\left(r, \frac{f^{\prime}}{f}\right)=m_{0 \pi}\left(r, \frac{1}{F}\right)+m_{0 \pi}\left(r, \frac{1}{F_{1}}\right),
$$

Theorem 5.4 has been proved.

Now we turn to the proof of Theorem 5.3.

First we show that

$$
\int_{1}^{\infty} t^{-2} \ln ^{+} T\left(t, \frac{f^{\prime}}{f}\right) d t<\infty
$$

Obviously

$$
N\left(r, \frac{f^{\prime}}{f}\right) \leq N\left(r, \frac{1}{h}\right) \leq T(r, h)+O(1)
$$


hence

$$
\begin{aligned}
\ln ^{+} T\left(r, \frac{f^{\prime}}{f}\right) & \leq \ln ^{+} T(r, h)+\ln ^{+} m\left(r, \frac{f^{\prime}}{f}\right)+O(1) \\
& \leq \ln ^{+} T(r, h)+\ln ^{+}\left\{r^{-1} m\left(r, \frac{f^{\prime}}{f}\right)\right\}+O(\ln r) \\
& \leq \ln ^{+} T(r, h)+r^{-1} m\left(r, \frac{f^{\prime}}{f}\right)+O(\ln r) .
\end{aligned}
$$

Dividing by $r^{2}$ and integrating with respect to $r$ from 1 to $\infty$, by the condition (5.8) and Theorem 5.4, we get (5.17).

Now we establish

$$
S\left(r, \frac{f^{\prime}}{f}\right)=O(1) .
$$

We argue in the same way as in the proof of Theorem 5.4, but instead of Tsuji characteristics we use Nevanlinna characteristics for the half-plane. Let $F(z)=$ $f(z) / f^{\prime}(z)$ as before. Since $f(z) \in A, f^{\prime \prime}(z) \in A$ we get (taking into account (5.13)) the relations $C(r, 0, F)=O(1), C\left(r, 1, F^{\prime}\right)=O(1)$. By the analogue of the inequality (2.23) from Chapter 3 for Nevanlinna characteristics for half-plane we have

$$
S(r, F) \leq 3 C(r, 0, F)+4 C\left(r, 1, F^{\prime}\right)+Q_{0 \pi}(r, F)
$$

hence,

$$
S(r, F)=Q_{0 \pi}(r, F) .
$$

Since (5.17) is equivalent to the condition $\int_{1}^{\infty} t^{-2} \ln ^{+} T(t, F) d t<\infty$, the definition of $Q_{0 \pi}(r, F)$ implies that $Q_{0 \pi}(r, F)=O(1)$. Thus, we have proved $S\left(r, \frac{f^{\prime}}{f}\right)=S\left(r, \frac{1}{F}\right)=O(1)$.

$$
\frac{f^{\prime}(z)}{f(z)}=g^{\prime}(z)+\frac{h^{\prime}(z)}{h(z)}
$$

we have

$$
S\left(r, g^{\prime}\right) \leq S\left(r, \frac{f^{\prime}}{f}\right)+S\left(r, \frac{h^{\prime}}{h}\right)+O(1)
$$

Applying Lemma 5.1 to the function $h(z)$, we see that $S\left(r, \frac{h^{\prime}}{h}\right)=O(1)$. Taking into account (5.18) we get

$$
S\left(r, g^{\prime}\right)=O(1)
$$

The argument above can be applied also to the function $f_{1}(z)=f(-z)$. Therefore the relation

$$
S\left(r, g_{1}^{\prime}\right)=O(1)
$$

where $g_{1}(z)=g(-z)$, also holds.

Applying Theorem 2.7 to the functions $g(z)$ and $g_{1}(z)$, we see that the relation

$$
\ln \left|g^{\prime}\left(r e^{i \varphi}\right)\right|=O(r)
$$


holds uniformly in $\varphi, 0 \leq \varphi<2 \pi$, as $r \rightarrow \infty$, possibly omitting some set $E$ of finite logarithmic measure. Hence for $r \notin E$ we have

$$
\ln M\left(r, g^{\prime}\right)=O(r)
$$

Since $g^{\prime}(z)$ is an entire function, the function $\ln M\left(r, g^{\prime}\right)$ is monotonically increasing with $r$ and, by Lemma 2.1, the relation (5.20) holds, also, for $r \in E$. The equality

$$
g(z)=g(0)+\int_{0}^{z} g^{\prime}(\zeta) d \zeta
$$

implies

$$
M(r, g) \leq|g(0)|+r M\left(r, g^{\prime}\right)
$$

and by (5.20) we get

$$
\ln M(r, g)=O(r)
$$

whence

$$
T(r, g)=O(r)
$$

The validity of (5.7) follows from the relation $A\left(r, g^{\prime}\right)=O(1)$, which follows immediately from (5.19). The proof of the theorem is completed.

Corollary. Let $G(z)$ be an entire function, such that the function $G^{2}(z)+$ $G^{\prime}(z)$ belongs to the class $A$. Then $G(z)$ does not exceed normal type of order 1 , and

$$
\int_{-\infty}^{\infty} \frac{\ln ^{+}|G(t)|}{1+t^{2}} d t<\infty
$$

To prove the corollary we consider the function

$$
f(z)=\exp \left\{\int_{0}^{z} G(\zeta) d \zeta\right\}
$$

This function does not have zeros and

$$
f^{\prime \prime}(z)=\left\{G^{2}(z)+G^{\prime}(z)\right\} f(z) \in A .
$$

By Theorem 5.3 the growth of the function $g(z)=\int_{0}^{z} G(\zeta) d \zeta$ does not exceed the normal type of order 1 , and the relation (5.7) holds for this function. Since $g^{\prime}(z)=G(z)$ and, by Theorem 2.3 from Chapter $3, T(r, G) \leq 2 T(r, g)+O(\ln r)$, we get the desired result.

Using Theorem 5.3 we can get an affirmative solution of the G. Pólya-A. Wiman problem under the additional assumption that the considered function has finitely many zeros. We are not going to present this result here, we refer the reader to the paper B. Ja. Levin and I. V. Ostrovskiı [LO60].

The condition of representability of the function $f(z)$ in the form (5.6), where $h(z)$ satisfies (5.8), imposes a priori restrictions onto the moduli of zeros of the function $f(z)$, since

$$
N\left(r, \frac{1}{f}\right)=N\left(r, \frac{1}{h}\right) \leq T(r, h)+O(1) .
$$


In the next theorem there are no such restrictions, but we have to introduce restrictions of another character.

THEOREM 5.5. If an entire function $f(z)$ is real on the real line, has only real zeros, and $f^{\prime \prime}(z) \in A$, then

$$
\ln T(r, f)=O(r \ln r) .
$$

Note that under the conditions of Theorem 5.3 we have a stronger estimate $\ln T(r, f)=O(r)$. This estimate cannot be improved. In fact, among functions satisfying the conditions of Theorem 5.2 may obviously be such that

$$
\liminf _{r \rightarrow \infty} r^{-1} \ln T(r, f)>0
$$

(for example, $e^{e^{i z}}, e^{\cos z}$, etc.). All derivatives of these functions belong to the class A.

Let us prove necessary auxiliary results.

Lemma 5.3. Let $g(z)$ be analytic in the half-plane $\{\operatorname{Im} z>0\}$ and satisfy there the condition $\operatorname{Im} g(z)>0$. Then the estimate

$$
\frac{1}{4}|g(i)| \frac{\sin \varphi}{r} \leq\left|g\left(r e^{i \varphi}\right)\right| \leq 4|g(i)| \frac{r}{\sin \varphi}, 0<\varphi<\pi
$$

holds.

Proof. First we show that if a function $G(\zeta)$ is analytic in the disc $\{|\zeta|<1\}$ and satisfies the condition $\operatorname{Re} G(\zeta)>0$, then the estimate

$$
|G(\zeta)| \leq \frac{2|G(0)|}{1-|\zeta|}
$$

holds. In fact, by the Schwarz formula, we have

$$
G(\zeta)=\frac{1}{2 \pi} \int_{0}^{2 \pi} \frac{R e^{i \theta}+\zeta}{R e^{i \theta}-\zeta} \operatorname{Re}\left\{G\left(R e^{i \theta}\right)\right\} d \theta+i \operatorname{Im}\{G(0)\},|\zeta|<R<1 .
$$

Hence

$$
\begin{aligned}
|G(\zeta)| & \leq \frac{R+|\zeta|}{R-|\zeta|} \cdot \frac{1}{2 \pi} \int_{0}^{2 \pi} \operatorname{Re}\left\{G\left(R e^{i \theta}\right)\right\} d \theta+|\operatorname{Im}\{G(0)\}| \\
& \leq \frac{R+|\zeta|}{R-|\zeta|}|G(0)|+|G(0)|=\frac{2 R}{R-|\zeta|}|G(0)| .
\end{aligned}
$$

Letting $R$ tend to 1 , we get (5.23).

Now let $g(z)$ be a function satisfying the conditions of the lemma. Then the function

$$
G(\zeta)=\frac{1}{i} g\left(\frac{1}{i} \frac{\zeta-1}{\zeta+1}\right)
$$

is analytic for $|\zeta|<1$ and $\operatorname{Re} G(\zeta)>0$. Applying the inequality (5.23) to it, and then letting $\zeta=-\frac{z-i}{z+i}$, we get

$$
|g(z)| \leq \frac{2|z+i|}{|z+i|-|z-i|}|g(i)|=\frac{2|z+i|(|z+i|+|z-i|)}{|z+i|^{2}-|z-i|^{2}}|g(i)| .
$$

Noting, that $|z+i|^{2}-|z-i|^{2}=4 r \sin \varphi\left(z=r e^{i \varphi}\right),|z \pm i| \leq 2 r(r \geq 1)$, we get the right half of the inequality (5.22). To get the left half of the inequality it suffices to apply the right part to $g_{1}(z)=-1 / g(z)$. 
LEMMA 5.4. Let $\left\{a_{k}\right\}_{k=N}^{\infty}$ and $\left\{b_{k}\right\}_{k=N}^{\infty},-\infty \leq N<0$, be two sequences of real numbers, such that

$$
a_{N}<b_{N}<a_{N+1}<b_{N+1}<\cdots<a_{0}<b_{0}<0<a_{1}<b_{1}<\ldots, \lim _{k \rightarrow+\infty} a_{k}=+\infty,
$$

if $N>-\infty$, and

$$
\cdots<a_{0}<b_{0}<0<a_{1}<b_{1}<\ldots, \lim _{k \rightarrow-\infty} a_{k}=-\infty, \lim _{k \rightarrow+\infty} a_{k}=+\infty,
$$

if $N=-\infty$. Let

$$
\Pi(z)=\prod_{k=N}^{\infty} \frac{1-\frac{z}{b_{k}}}{1-\frac{z}{a_{k}}}
$$

Then the infinite product $\Pi(z)$ converges absolutely and uniformly on each bounded subset of a complex plane, hence $\Pi(z)$ is a meromorphic function with simple poles at the points $a_{k}$ and simple zeros at the points $b_{k}$. This function satisfies the following conditions:

(a) $\operatorname{Im} \Pi(z) \cdot \operatorname{Im} z>0$ for $\operatorname{Im} z \neq 0$.

(b) For each $\eta>0$ the relation

$$
\Pi\left(r e^{i \varphi}\right)=O(r)
$$

holds uniformly in $\varphi$ outside the angles $\{|\arg z|<\eta\},\{|\pi-\arg z|<\eta\}$.

(c) $m(r, \Pi)+m(r, 1 / \Pi)=O(\ln r)$

(d) The relation

$$
\ln \left|\Pi\left(r e^{i \varphi}\right)\right|=O(r)
$$

holds uniformly in $\varphi, 0 \leq \varphi<2 \pi$, as $r \rightarrow \infty$ missing, possibly, some set $E$ of finite logarithmic measure.

Proof. Note that by the Leibniz theorem on alternating series we have

$$
\sum_{k=N}^{\infty}\left(\frac{1}{a_{k}}-\frac{1}{b_{k}}\right)<\infty
$$

Since for $\left|a_{k}\right|>2|z|$ the relation

$$
\left|\frac{1-\frac{z}{b_{k}}}{1-\frac{z}{a_{k}}}-1\right|=|z| \frac{\left(\frac{1}{a_{k}}-\frac{1}{b_{k}}\right)}{\left|1-\frac{z}{a_{k}}\right|} \leq 2|z|\left(\frac{1}{a_{k}}-\frac{1}{b_{k}}\right)
$$

holds, the absolute and uniform convergence of the infinite product $\Pi(z)$ on each bounded set follows from Theorem 3.1 from Chapter 2 .

Let $\arg (z-\alpha)$, where $\alpha$ is a real number, $\operatorname{Im} z>0$, be the principal value of the argument, and

$$
\arg \frac{1-\frac{z}{b_{k}}}{1-\frac{z}{a_{k}}}=\arg \left(z-b_{k}\right)-\arg \left(z-a_{k}\right)
$$


The difference $\arg \left(z-b_{k}\right)-\arg \left(z-a_{k}\right)$ represents the size of the angle which the segment $\left[a_{k}, b_{k}\right]$ subtends at the point $z(\operatorname{Im} z>0)$. Therefore the number

$$
\arg \Pi(z)=\sum_{k=N}^{\infty} \arg \frac{1-\frac{z}{b_{k}}}{1-\frac{z}{a_{k}}}=\sum_{k=N}^{\infty}\left\{\arg \left(z-b_{k}\right)-\arg \left(z-a_{k}\right)\right\}
$$

satisfies the inequality

$$
0<\arg \Pi(z)<\pi, \operatorname{Im} z>0
$$

Hence

$$
\operatorname{Im} \Pi(z)>0 \text { for } \operatorname{Im} z>0 .
$$

Similarly we prove that $\operatorname{Im} \Pi(z)<0$ for $\operatorname{Im} z<0$. Thus we have proved that the function $\Pi(z)$ satisfies the condition (a).

Applying Lemma 5.3 we get the inequality

$$
\frac{1}{4}|\Pi(i)| \frac{|\sin \varphi|}{r} \leq\left|\Pi\left(r e^{i \varphi}\right)\right| \leq 4|\Pi(i)| \frac{r}{|\sin \varphi|}, 0 \leq \varphi \leq 2 \pi, r \geq 1 .
$$

From here it follows easily that the function $\Pi(z)$ satisfies the conditions (b) and (c).

To prove the last assertion of the lemma we establish that

$$
S(r, \Pi(z))+S(r, \Pi(-z))=O(1),
$$

then the desired conclusion follows by Theorem 2.7.

Let

$$
q(z)=\frac{\Pi(z)-i}{\Pi(z)+i} .
$$

Since $\operatorname{Im} \Pi(z)>0$ for $\operatorname{Im} z>0$, the function $q(z)$ is analytic in the half-plane $\{\operatorname{Im} z>0\}$ and admits there the estimate $|q(z)|<1$. Therefore $A(r, q)=B(r, q)=$ $C(r, q) \equiv 0$ and hence, $S(r, q) \equiv 0$. Using the relation (6.11) from Chapter 1, we get $S(r, \Pi(z))=S(r, q)+O(1)=O(1)$. Similarly we get $S(r, \Pi(-z)))=O(1)$. This completes the proof of the lemma.

Now we prove Theorem 5.5.

We may restrict ourselves to the case when the set of zeros of the function $f(z)$ is not bounded above. In fact, if the set of zeros of this function is finite, then $f(z)=e^{g(z)} h(z)$, where $g(z)$ is an entire function and $h(z)$ is a polynomial, and using Theorem 5.3 we get the estimate $\ln T(r, f)=O(r)$, which is more precise than (5.21). If the set of zeros is infinite, but is bounded from the right, we consider the function $f(-z)$ instead of $f(z)$.

We enumerate zeros of the function $f(z)$ into a sequence $\left\{a_{k}\right\}_{k=N}^{\infty},-\infty \leq N<$ $\infty$, in such a way that the inequalities

$$
\cdots<a_{k-2}<a_{k-1}<a_{k}<a_{k+1}<\ldots
$$

are satisfied (multiple zeros are counted only once). By the Rolle theorem, each of the intervals $\left(a_{k}, a_{k+1}\right)$ contains zeros of the function $f^{\prime}(z)$. We choose one zero of $f^{\prime}(z)$ in each of the intervals, and denote it by $b_{k}$. Considering, if necessary, the function $f(z+\alpha)$ instead of $f(z)$, where $\alpha$ is a suitably chosen real number, we may assume that the sequences $\left\{a_{k}\right\}_{k=N}^{\infty}$ and $\left\{b_{k}\right\}_{k=N}^{\infty}$ satisfy the conditions of Lemma 5.4 . 
Let $\Pi(z)$ be a function determined by the relation (5.24). Since the function $\Pi(z)$ has simple poles at the points $a_{k}$ and simple zeros at the points $b_{k}$, the function $\frac{f^{\prime}(z)}{f(z) \Pi(z)}$ is an entire function. We denote it by $\psi(z)$, then we have the equality

$$
\frac{f^{\prime}(z)}{f(z)}=\psi(z) \Pi(z) .
$$

Let us estimate the growth of the function $\psi(z)$. The equality (5.25) implies

$$
m(r, \psi) \leq m\left(r, \frac{f^{\prime}}{f}\right)+m\left(r, \frac{1}{\Pi}\right) .
$$

We divide both sides of this inequality by $r^{3}$ and integrate it with respect to $r$ from $R \geq 1$ to $\infty$. We get

$$
\int_{R}^{\infty} \frac{m(r, \psi)}{r^{3}} d r \leq \int_{R}^{\infty} \frac{m\left(r, \frac{f^{\prime}}{f}\right)}{r^{3}} d r+\int_{R}^{\infty} \frac{m\left(r, \frac{1}{\Pi}\right)}{r^{3}} d r
$$

Using Theorem 5.4 and taking into account that by Lemma 5.4 the relation

$$
m\left(r, \frac{1}{\Pi}\right)=O(\ln r)
$$

holds, we arrive at the estimate

$$
\int_{R}^{\infty} \frac{m(r, \psi)}{r^{3}} d r=O\left(\frac{\ln R}{R}\right)
$$

Since $\psi(z)$ is an entire function, we have $m(r, \psi)=T(r, \psi)$ and, by monotonicity of the characteristic $T(r, \psi)$, we get

$$
\frac{T(R, \psi)}{2 R^{2}} \leq \int_{R}^{\infty} \frac{T(r, \psi)}{r^{3}} d r=O\left(\frac{\ln R}{R}\right),
$$

whence

$$
T(R, \psi)=O(R \ln R)
$$

Letting $R=2 r$ in the inequality (7.1) from Chapter 1, we get

$$
\ln ^{+} M(r, \psi) \leq 3 T(2 r, \psi)=O(r \ln r)
$$

Since the function $\Pi(z)$ satisfies the conditions (b) and (d) of Lemma 5.4 then, taking into account the relation $(5.25)$ and the estimate $(5.26)$, we come to the conclusion that for each $\eta, 0<\eta<\frac{\pi}{2}$ and all $z=r e^{i \varphi}$ except, possibly, those for which

$$
r \in E,|\varphi|<\eta \text { or } r \in E,|\pi-\varphi|<\eta,
$$

where $E \subset(0, \infty)$ is some set of finite logarithmic measure, the inequality

$$
\left|\frac{f^{\prime}(z)}{f(z)}\right| \leq \exp \{K r \ln r\}
$$

holds, where $K>0$ is a constant.

Now we estimate the growth of the function $f(z)$. Let $z=r e^{i \varphi}, r \notin E$. We integrate both sides of the inequality (5.27) along a path $L$ which starts at 0 , goes to the point ir along the ray $\left\{\arg z=\frac{\pi}{2}\right\}$, and then continues along the arc of the 
circle $\{|z|=r\}$ in the counterclockwise direction from the point ir to the point $r e^{i \varphi}$. We get

$$
|\ln | \frac{f(z)}{f(0)}||=\left|\operatorname{Re} \int_{L} \frac{f^{\prime}(\zeta)}{f(\zeta)} d \zeta\right| \leq \int_{L}\left|\frac{f^{\prime}(\zeta)}{f(\zeta)}\right||d \zeta| \leq(2 \pi+1) r \exp \{K r \ln r\} .
$$

Hence, for $r \notin E$ the relation

$$
\ln ^{+} \ln ^{+} M(r, f)=O(r \ln r)
$$

holds. Since the function $M(r, f)$ is non-decreasing, by Lemma 2.1, the relation (5.28) holds, also, for $r \in E$. Since $T(r, f) \leq \ln ^{+} M(r, f)$, we get the conclusion of the theorem.

Theorem 5.5 shows that, working on the problem of G. Pólya and A. Wiman, it suffices to consider entire functions $f(z)$ satisfying $\ln T(r, f)=O(r \ln r)$. 



\section{CHAPTER 7}

\section{Applications of Riemann surfaces to value distribution}

In this Chapter we will use some facts about Riemann surfaces. Familiarity with the corresponding chapter of the well-known course of S. Stoillow [Sto62, Vol. II, Chapter VII] is much more than sufficient for understanding of this chapter.

\section{Geometric meaning of deficient and index values}

Let $w=f(z)$ be a function meromorphic in the complex $z$-plane. It is a one-toone mapping of the complex plane $\{|z|<\infty\}$ onto some simply connected Riemann surface $F$, that is, onto a Riemann surface which is homeomorphic to the plane ${ }^{1}$ If the function $w=f(z)$ is meromorphic in the extended complex plane, that is, if it is rational, then it maps the extended $z$-plane onto a simply connected closed Riemann surface (of genus zero), that is, onto a surface which is homeomorphic to the closed sphere. In this case the surface $F$ is finite-sheeted, and the number $n$ of sheets is equal to the degree of the rational function. If the function $f(z)$ is transcendental, then the Riemann surface $F$ is open and infinite-sheeted.

On the other hand, it is known (one of the simplest proofs can be found in the book by G. M. Goluzin [Gol69, Chapter XI, §2]), that for each open simply connected Riemann surface $F$ there exists a function $w=f(z)$ meromorphic in the $\operatorname{disc}\{|z|<R\}, 0<R \leq \infty$, which maps $\{|z|<R\}$ onto $F$. If the surface $F$ is a simply connected closed Riemann surface without one point, then the function $f(z)$ is rational, and the surface $F$ is called a surface of elliptic type. Otherwise the surface $F$ is of parabolic or hyperbolic type depending on whether $R=\infty$ or $R<\infty$. In all cases the function $w=f(z)$ will be called a mapping function of the surface $F$, and the Riemann surface $F$ will be called the surface corresponding to $w=f(z)$.

We will be mainly interested in functions which are meromorphic and transcendental in $\{|z|<\infty\}$, and map $\{|z|<\infty\}$ onto surfaces of parabolic type. For this reason, unless stated otherwise, by a Riemann surface we mean an open simply connected Riemann surface of parabolic type.

A mapping function of a given Riemann surface is determined up to a conformal univalent mapping of the complex plane onto itself, that is, if $f(z)$ is a mapping function of $F$, then all functions of the form $f(a z+b), a \neq 0$, and only such functions, are mapping functions of $F$. If we normalize mapping functions in an

\footnotetext{
${ }^{1}$ Strictly speaking, the mentioned one-to-one mapping is realized by a function $p=\tilde{f}(z)$, $p \in F$, such that $T(\tilde{f}(z))=f(z)$, where $T(p)$ is the projection of a point $p$ onto the $w$-plane. We follow the tradition not to distinguish in $\tilde{f}(z)$ and $f(z)$ both in our notation and in our terminology, the meaning will always be clear from context. We agree also to say that the point $p$ lies over $T(p)$.
} 
appropriate way ${ }^{2}$, we get a one-to-one correspondence between Riemann surfaces and mapping functions. Thus, meromorphic functions can be defined by means of the corresponding Riemann surfaces. Therefore it is not surprising that, as it was shown by L. Ahlfors ([Ahl35], see also S. Stoïlow [Sto62, Vol. II, Chapter $\mathrm{X}, \S \S 1,2])$, that the main results of the value distribution theory and their generalizations can be obtained in a purely geometric way, by studying the corresponding Riemann surfaces. We will not present the theory of L. Ahlfors here. We shall be concerned with the determination of the geometric meaning of deficient and index values only.

Universal necessary and sufficient conditions under which certain value is deficient for a function $f(z)$, which is a mapping function of a surface $F$, in terms of the structure of the surface $F$ only, cannot be given in principle. In fact, as we saw in Section 6 of Chapter 4, the function $f(z+h)$, which is also a mapping function of $F$, can have different deficient values for different $h$. Of course, if we normalize the mapping function, the mentioned reason will disappear, but it shows that the problem is very complicated. Up to now necessary and sufficient conditions for existence of deficient values are known for very restricted classes of Riemann surfaces only. The same can be said about index values.

Here we only present conditions which are necessary for $a$ to be a deficient or index value. It is convenient to state them as conditions which are sufficient for absence of deficient or index values.

THEOREM 1.1. If the number of algebraic ramification points of a Riemann surface $F$ lying over the point a is finite, then the corresponding mapping functions satisfy $\varepsilon(a)=0$.

Suppose that the sum of orders of algebraic ramification points lying over $a$ is equal to $q$. Algebraic ramification points correspond to multiple $a$-points of the mapping function $f(z)$, moreover, if an algebraic ramification point has order $m$, then the corresponding multiple $a$-point has order $m+1$. All other points of $F$ lying over $a$ correspond to simple $a$-points of the function $f(z)$. Therefore $n_{1}(r, a)=q$ for $r>r_{0}$, from where we get $N_{1}(r, a) \sim q \ln r$. Since the function $f(z)$ is transcendental, we have $\ln r=o(T(r, f))$ and $\varepsilon(a)=0$.

THEOREM 1.2. Suppose that points of a Riemann surface $F$ whose projections belong to the $\eta$-neighborhood $U(a, \eta)$ of a point a form a set of regions $F_{\nu} \subset F$.

Suppose that each point from $U(a, \eta)$ is a projection of exactly $\lambda_{\nu}$ points of $F_{\nu}$ (each algebraic ramification point of order $m$ is counted $m+1$ times), where $1 \leq \lambda_{\nu} \leq \Lambda<\infty$, that is, the Riemann surface $F_{\nu}$ has $\lambda_{\nu}$ sheets and covers $U(a, \eta)$ completely.

Then the value a is not deficient for the mapping function $f(z)$.

We may assume without loss of generality that $a=0$ and $1<\eta<\infty$. In fact, if this condition is not satisfied, we consider the meromorphic function $w=$ $f_{1}(z)=L(f(z))$, where $L(w)$ is a linear-fractional function mapping $U(a, \eta)$ onto $\left\{|w|<\eta_{1}\right\}, L(a)=0,1<\eta_{1}<\infty$. It is clear that $\delta(a, f)=\delta\left(0, f_{1}\right)$ and that the Riemann surface corresponding to the function $f_{1}(z)$ satisfies the conditions of Theorem 1.2 with $a=0, \eta=\eta_{1}$. Denote the closed set of points from $F_{\nu}$ lying

\footnotetext{
${ }^{2}$ For example, we can require that the point $z=0$ is mapped onto a preassigned simple point of the Riemann surface $F$ (that is, a point which is not an algebraic ramification point), and $f^{\prime}(0)=1$.
} 
over $\{|w| \leq 1\}$ by $F_{\nu}^{\prime}$. Obviously $F_{\nu}^{\prime}$ consists of at most $\Lambda$ closed regions. Let $D_{\nu}$ and $D_{\nu}^{\prime}$ be the pre-images of the sets $F_{\nu}$ and $F_{\nu}^{\prime}$, respectively, under the mapping $w=f(z)$. Since $F_{\nu}$ covers $\{|w|<\eta\}$ completely, then $D_{\nu}$, as well as $D_{\nu}^{\prime}$ contains as many $e^{i \theta}$-points of the function $f(z)$, as zeros, namely $\lambda_{\nu}$. Let $\Gamma_{r}$ be a closed curve on $F$, which is the image of the circle $\{|z|=r\}, r>1$, under the mapping $w=f(z)$, and

$$
L(r)=\int_{0}^{2 \pi} \stackrel{\circ}{f}\left(r e^{i \varphi}\right) r d \varphi
$$

is the length of $\Gamma_{r}$ with respect to the spherical metric. Denote by $l$ the distance on the Riemann sphere between the circles whose stereographic projections are the circles $\{|w|=1\}$ and $\{|w|=\eta\}$.

All $e^{i \theta}$-points and zeros of the function $f(z)$ are contained in $\bigcup_{\nu} D_{\nu}^{\prime} \subset \bigcup_{\nu} D_{\nu}$. If $D_{\nu}^{\prime} \subset\{|z| \leq r\}$, it contributes $\lambda_{\nu}$ to the functions $n\left(r, e^{i \theta}\right)$ and $n(r, 0)$. Therefore, if we denote by $k(r)$ the number of sets $D_{\nu}^{\prime}$ such that $D_{\nu}^{\prime}$ have nonempty intersections both with $\{|z| \leq r\}$ and with $\{|z|>r\}$, then

$$
n\left(r, e^{i \theta}\right)-n(r, 0) \leq \Lambda k(r),
$$

since the number of $e^{i \theta}$-points in $D_{\nu}^{\prime}$ does not exceed $\Lambda$. If $k(r) \geq 2$, then the circle $\{|z|=r\}$ cannot be entirely contained in one of the domains $D_{\nu}$, that is, the curve $\Gamma_{r}$ is not contained in one of the regions $F_{\nu}$. The curve $\Gamma_{r}$ intersects $k(r) \geq 2$ different sets $F_{\nu}^{\prime}$, hence, its projection intersects the ring $\{1<|w|<\eta\}$ at least $2 k(r)$ times, joining points on the circles $\{|w|=1\}$ and $\{|w|=\eta\}$. Hence $L(r) \geq 2 k(r) l$. ¿From (1.1) we get the inequality

$$
\begin{aligned}
n\left(r, e^{i \theta}\right)-n(r, 0) & \leq \Lambda \max (1, k(r)) \\
& \leq \Lambda \max \left(1, \frac{1}{2 l} L(r)\right) \leq \Lambda\left(1+\frac{1}{2 l} L(r)\right) .
\end{aligned}
$$

Since

$$
N(r, a)-N(1, a)=\int_{1}^{r} \frac{n(t, a)}{t} d t
$$

integrating the inequality (1.2) with respect to $\ln r$ we get

$$
N\left(r, e^{i \theta}\right)-N\left(1, e^{i \theta}\right)-N(r, 0)+N(1,0) \leq \Lambda\left(\ln r+\frac{1}{2 l} \int_{1}^{r} \frac{L(t)}{t} d t\right) .
$$

Integrating this inequality with respect to $\theta$ and using Cartan's formula (4.13) from Chapter 1 , we get

$$
T(r, f)-T(1, f)-N(r, 0)+N(1,0) \leq \Lambda\left(\ln r+\frac{1}{2 l} \int_{1}^{r} \frac{L(t)}{t} d t\right),
$$

from where, by Jensen's formula (4.1) from Chapter 1,

$$
m(r, 0)-m(1,0) \leq \Lambda\left(\ln r+\frac{1}{2 l} \int_{1}^{r} \frac{L(t)}{t} d t\right) .
$$


Now we estimate the integral in the right-hand side of (1.4) using the CauchyBuniakowsky inequality. We have

$$
\begin{aligned}
\int_{1}^{r} \frac{L(t)}{t} d t & =\int_{1}^{r} \int_{0}^{2 \pi} \stackrel{\circ}{f}\left(t e^{i \theta}\right) d \theta d t \\
& \leq\left\{\int_{1}^{r} \int_{0}^{2 \pi}\left\{\stackrel{\circ}{f}\left(t e^{i \theta}\right)\right\}^{2} t d t d \theta\right\}^{\frac{1}{2}}\left\{\int_{1}^{r} \int_{0}^{2 \pi} \frac{d t d \theta}{t}\right\}^{\frac{1}{2}} \\
& \leq \sqrt{2 \pi \ln r}\left\{\int_{0}^{r} \int_{0}^{2 \pi}\left\{\stackrel{\circ}{f}\left(t e^{i \theta}\right)\right\}^{2} t d t d \theta\right\}^{\frac{1}{2}}=\sqrt{2 \pi \ln r} \sqrt{\pi \stackrel{\circ}{A}(r, f)},
\end{aligned}
$$

by the definition of $\stackrel{\circ}{A}(r, f)$ from Section 4 of Chapter 1. Thus (1.4) implies

$$
m(r, 0) \leq m(1,0)+\Lambda\left(\ln r+\frac{\pi}{\sqrt{2 l}} \sqrt{\ln r} \sqrt{\stackrel{\circ}{A}(r, f)}\right) .
$$

Now we use the following lemma.

LEMMA 1.1. Let $\varphi(t)$ be a continuously differentiable, positive, non-decreasing for $t \geq t_{0}$ function. Then the inequality $\varphi^{\prime}(t) \leq\{\varphi(t)\}^{1+\varepsilon}, \varepsilon>0$ holds for all $t \geq t_{0}$ except, possibly, a set $\Delta$ of intervals, having finite total length.

In fact, the inequality $\varphi^{\prime}(t)>\{\varphi(t)\}^{1+\varepsilon}$ holds on $\Delta$, therefore

$$
\int_{\Delta} d t \leq \int_{\Delta} \frac{\varphi^{\prime}(t)}{\{\varphi(t)\}^{1+\varepsilon}} d t \leq \int_{t_{0}}^{\infty} \frac{\varphi^{\prime}(t) d t}{\{\varphi(t)\}^{1+\varepsilon}} \leq \frac{1}{\varepsilon} \frac{1}{\left\{\varphi\left(t_{0}\right)\right\}^{\varepsilon}}
$$

By the definition of the Shimizu-Ahlfors spherical characteristic $\stackrel{\circ}{T}(r, f)$ (see Section 4 of Chapter 1), we have

$$
\stackrel{\circ}{A}(r, f) \ln r=\frac{d \stackrel{\circ}{T}(r, f)}{d \ln r} \ln r=\frac{d \stackrel{\circ}{T}(r, f)}{d \ln \ln r} .
$$

Therefore, applying Lemma 1.1 to the function $\stackrel{\circ}{T}(r, f)$ with $t=\ln \ln r, \varepsilon=1 / 2$, we get that outside a set $\Delta$ of intervals, for which

$$
\int_{\Delta} d \ln \ln r<\infty
$$

the inequality $\stackrel{\circ}{A}(r, f) \ln r \leq\{\stackrel{\circ}{T}(r, f)\}^{3 / 2}$ holds. Since $\ln r=o(T(r, f))$, we get from (1.5) that outside $\Delta$, that is, on an unbounded from above set of values of $r$ the relation

$$
m(r, 0) \leq O(1)+\Lambda\left\{\ln r+\frac{\pi}{\sqrt{2 l}}(\stackrel{\circ}{T}(r, f))^{3 / 4}\right\}=o(\stackrel{\circ}{T}(r, f)), r \rightarrow \infty,
$$

holds, that is, $\delta(0, f)=0$.

It is obvious that the conditions of Theorem 1.2 are not satisfied if $a$ is an asymptotic value for $f(z)$. In fact, if the conditions of the theorem are satisfied even with the weaker condition $1 \leq \lambda_{\nu}<\infty$, then the regions in which $|f(z)-a|<\eta^{\prime}<\eta$ (or $|f(z)|>\frac{1}{\eta^{\prime}}>\frac{1}{\eta}$, if $a=\infty$ ) are all bounded, and $a$ cannot be an asymptotic value.

Theorem 1.2 does not remain true if we omit the condition $\lambda_{\nu} \leq \Lambda$. 
Consider, for example, the meromorphic function $f(z)$ from the example 1 of Section 2 from Chapter 5. The set $D$ of those $z$ for which $|f(z)|>K$, where $K>M$ and $M$ is the constant defined in the analysis of the example, consists of bounded connected components $D_{\nu}$ since $D \subset \bigcup_{j \in B} C_{j}$. Obviously, $D_{\nu}$ contains finitely many poles (in our example it is one multiple pole, since each disc $C_{j}$ contains one multiple pole). By the known theorems (see S. Stoïlow [Sto62, Vol. II, Ch. V, §2]) the image of $D_{\nu}$ under the mapping $f(z)$ onto the Riemann surface $F$ is a finite-sheeted region $F_{\nu} \subset F$ covering entirely the disc $\{|w|>K\}$. Hence all the conditions of Theorem 1.2 except $\lambda_{\nu} \leq \Lambda$, are satisfied. In fact, in our example the number $\lambda_{\nu}$ of sheets of $F_{\nu}$ is equal to the order of the pole of $f(z)$ lying in $D_{\nu}$, and the orders of poles of $f(z)$ are not bounded. On the other hand, as it was shown in the analysis of the example $1, \infty$ is a deficient value for $f(z)$.

\section{Quasiconformal mappings}

Let $w=f(z)$ be a meromorphic function which is a one-to-one mapping of the complex plane onto a Riemann surface $F$. This mapping is conformal and univalent in sufficiently small neighborhoods of all points $z$ except multiple $a$-points of the function $f(z)$, which correspond to algebraic ramification points on $F$. For brevity we call such mapping conformal, without mentioning the exceptional, at most countable, set. Thus, the problem of determination of a mapping function for a given Riemann surface $F$ can be described in other words as a problem of determination of a conformal mapping of $F$ onto $\{|z|<\infty\}$. With very few exceptions it is not known how to construct such mapping effectively. For this reason so-called quasiconformal mappings of the Riemann surface $F$ onto the complex plane are constructed. The definition of a quasiconformal mapping which we give is not the most general, but it is sufficiently general for our purposes.

We say that a function $w=\varphi(z)=u(z)+i v(z)$ is continuously differentiable in the closed region $\bar{D}$ if there exist continuous in $\bar{D}$ functions $a(z), b(z), c(z)$, and $d(z)$, such that for each point $z$ the equalities $(z=x+i y)$

$$
\left.\begin{array}{l}
u(z+\Delta z)-u(z)=a(z) \Delta x+b(z) \Delta y+o(|\Delta z|) \\
v(z+\Delta z)-v(z)=c(z) \Delta x+d(z) \Delta y+o(|z|)
\end{array}\right\}
$$

hold as $\Delta z \rightarrow 0, z+\Delta z \in \bar{D}$. The number $J=J[\varphi]=a(z) d(z)-c(z) b(z)$ is called the Jacobian of the function $\varphi(z)$ at the point $z$. It is clear that at all interior points of $\bar{D}$ the equalities

$$
a(z)=\frac{\partial u(z)}{\partial x}, b(z)=\frac{\partial u(z)}{\partial y}, c(z)=\frac{\partial v(z)}{\partial x}, d(z)=\frac{\partial v(z)}{\partial y}
$$

hold.

The same equalities hold for those points on the boundary of $\bar{D}$, where the corresponding partial derivatives exist. However, the partial derivatives of $u$ and $v$ can be undefined at some boundary points. We shall consider the equalities (2.2) as definitions of the partial derivatives on $\partial D$. The equalities (2.1) imply that the image of a smooth curve $C \subset \bar{D}$ under the mapping $w=\varphi(z)$ is a smooth curve if $J[\varphi] \neq 0$ for $z \in C$.

Let $D_{1}$ and $D_{2}$ be two closed regions bounded by finite number of piece-wise smooth closed Jordan curves. A mapping $w=\varphi(z)$ of the region $D_{1}$ onto the region 
$D_{2}$ is called quasiconformal ${ }^{3}$ if: (1) the mappings $\varphi(z)$ and if: (1) the mappings $\varphi(z)$ and $\varphi^{-1}(w)$ are one-to-one and continuous on $D_{1}$ and $D_{2}$, respectively; (2) the region $D_{1}$ can be decomposed by a finite set of piece-wise smooth curves into closed regions, in each of which the function $\varphi(z)$ is continuously differentiable and has a positive Jacobian. It is clear in such a case that the inverse mapping $\varphi^{-1}(w)$ is also quasiconformal.

Let $F$ be a simply connected Riemann surface covering the $w$-plane (the case of a plane region is not excluded). Let $p_{0}$ be a point on $F$, whose projection is $w_{0}$; we let $m=1$ if $p_{0}$ is a simple point, and $m=k+1$ if $p_{0}$ is a ramification point of order $k$. For sufficiently small $\eta=\eta\left(p_{0}\right)>0$ the function $w=w_{0}+\zeta^{m}$ maps the disc $\{|\zeta| \leq \eta\}$ onto a closed neighborhood of the point $p_{0}$. Let $q=\psi(p)$ be a one-to-one mapping of the Riemann surface $F$ onto the Riemann surface $F_{1}$ which covers the $z$-plane, $p \in F, q \in F_{1}$, and $T(q)$ be the projection of the point $q$ in the $z$-plane. The mapping $q=\psi(p)$ is called quasiconformal if the mapping $z=T\left\{\psi\left(w_{0}+\zeta^{m}\right)\right\}$ of the disc $\{|\zeta| \leq \eta\}$ is quasiconformal for each point $p_{0} \in F$. In the case when $F_{1}$ is a region in the $z$-plane we have $T\left\{\psi\left(w_{0}+\zeta^{m}\right)\right\} \equiv \psi\left(w_{0}+\zeta^{m}\right)$.

Thus, the definition of a quasiconformal mapping of a Riemann surface (including the case of open plane regions) is reduced to the definition of a quasiconformal mapping of a closed plane region.

Suppose that a Riemann surface $F$ is mapped conformally onto $\{|z|<\infty\}$ and quasiconformally onto $\{|\zeta|<\infty\}$. Then the mapping $\{|z|<\infty\} \rightarrow F \rightarrow\{|\zeta|<\infty\}$ is a quasiconformal mapping $\zeta=\zeta(z)$ of the $z$-plane onto the $\zeta$-plane. The inverse mapping $z=z(\zeta)$ is also quasiconformal. We may assume without loss of generality that $z(0)=0$. As we have already mentioned usually it is not known how to construct a conformal mapping $w=f(z)$ of the $z$-plane onto the Riemann surface $F$, but a quasiconformal mapping $w=\varphi(\zeta)$ of the $\zeta$-plane onto $F$ can sometimes be efficiently constructed.

Suppose that such a mapping has been constructed. A root $\zeta_{0}$ of the equation $\varphi(\zeta)=a(1 / \varphi(\zeta)=0$ for $a=\infty)$ will be called an $a$-point of the function $\varphi(\zeta)$, moreover, if $\zeta_{0}$ is mapped onto a simple point of $F$, then $\zeta_{0}$ is considered as an $a$ point of the first order, if $\zeta_{0}$ is mapped onto an algebraic ramification point of order $m$, then $\zeta_{0}$ is considered as an $a$-point of order $m+1$. If $\varphi(\zeta)$ is a meromorphic function, this definition is equivalent to the usual definition of an $a$-point. We denote the number of $a$-points of the function $\varphi(\zeta)$ in the disc $\{|\zeta| \leq r\}$ by $n(r, a, \varphi)$, and the spherical area of the Riemann surface $F_{r}$ which is the image of $\{|\zeta|<r\}$ under $w=\varphi(\zeta)$ by $\pi \stackrel{\circ}{A}(r, \varphi)$. The curves $\gamma_{r}$, which are images of the circles $\{|\zeta|=r\}$ under the mapping $z=z(\zeta)$, exhaust the $z$-plane. It is clear that

$$
n_{\gamma}(r, a, f) \equiv n(r, a, \varphi), \stackrel{\circ}{A_{\gamma}}(r, f) \equiv \stackrel{\circ}{A}(r, f),
$$

where the quantities $n_{\gamma}(r, a, f)$ and $\stackrel{\circ}{A}_{\gamma}(r, f)$ have the same meaning as in Section 6 of Chapter . Thus, knowledge of the quasiconformal mapping $w=\varphi(\zeta)$ allows us to study the value distribution of the meromorphic function $w=f(z)$ in the case when we exhaust the complex plane $\{|z|<\infty\}$ by the family of the curves $\gamma_{r}$, moreover, we can find $T_{\gamma}(r, f), N_{\gamma}(r, a, f), \delta_{\gamma}(a, f), \varepsilon_{\gamma}(a, f)$ etc. Since we are interested in the usual deficiencies and indices, $\delta(a, f)$ and $\varepsilon(a, f)$, rather than in the quantities $\delta_{\gamma}(a, f)$ and $\varepsilon_{\gamma}(a, f)$, we need conditions on the family $\gamma_{r}$ of curves, and on $T(r, f)$,

\footnotetext{
${ }^{3}$ This definition is different from the modern one.
} 
under which the equalities $\delta_{\gamma}(a, f)=\delta(a, f)$ and $\varepsilon_{\gamma}(a, f)=\varepsilon(a, f)$ hold. Such conditions were found in Section 6 of Chapter 4. In Theorem 6.9 from Chapter 4 the following condition was imposed on the family $\gamma_{r}$ of curves:

$$
r_{2}(r) \sim r_{1}(r), r \rightarrow \infty
$$

where $r_{2}(r)=\max _{z \in \gamma_{r}}|z|, r_{1}(r)=\min _{z \in \gamma_{r}}|z|$. Since in our case

$$
r_{2}(r)=\max _{|\zeta|=r}|z(\zeta)|, r_{1}(r)=\min _{|\zeta|=r}|z(\zeta)|,
$$

it is clear that the condition (2.3) holds if

$$
\lim _{\zeta \rightarrow \infty} \frac{|z(\zeta)|}{|\zeta|}=\lim _{z \rightarrow \infty} \frac{|z|}{|\zeta(z)|}=A, 0<A<\infty .
$$

In the next section we shall find a rather wide class of quasiconformal mappings $z(\zeta)$ for which the relation (2.4) takes place.

Now we consider some properties of quasiconformal mappings. We restrict our attention to quasiconformal mappings of plane regions (as we saw, quasiconformal mappings of Riemann surfaces can be reduced to this case).

First we observe that a composition of two quasiconformal mappings is also a quasiconformal mapping. Let $w=\psi(z)=u(z)+i v(z)$ be a function which maps quasiconformally a region $G$ onto a region $D$. Let $z_{0}$ be such point in $G$ that the function $\psi(z)$ is continuously differentiable in its sufficiently small neighborhood. Let $d z=|d z| e^{i \varphi}$, then

$$
|d w|^{2}=(d u)^{2}+(d v)^{2}=\left(E \cos ^{2} \varphi+2 F \sin \varphi \cos \varphi+G \sin ^{2} \varphi\right)|d z|^{2}
$$

where $E=\left(\frac{\partial u}{\partial x}\right)^{2}+\left(\frac{\partial v}{\partial x}\right)^{2}, F=\frac{\partial u}{\partial x} \frac{\partial u}{\partial y}+\frac{\partial v}{\partial x} \frac{\partial v}{\partial y}, G=\left(\frac{\partial u}{\partial y}\right)^{2}+\left(\frac{\partial v}{\partial y}\right)^{2}$. The modulus of the derivative in the direction $\arg \left(z-z_{0}\right)$ is equal to

$$
\left|\frac{d w}{d z}\right|_{\varphi}=\sqrt{E \cos ^{2} \varphi+2 F \sin \varphi \cos \varphi+G \sin ^{2} \varphi}=\sqrt{h(\varphi)} .
$$

It is easy to compute that

$$
\begin{aligned}
& \max _{0 \leq \varphi \leq 2 \pi} h(\varphi)=\frac{1}{2}\left\{E+G+\sqrt{(E+G)^{2}-4 J^{2}}\right\} \\
& \min _{0 \leq \varphi \leq 2 \pi} h(\varphi)=\frac{1}{2}\left\{E+G-\sqrt{(E+G)^{2}-4 J^{2}}\right\} .
\end{aligned}
$$

The quantity

$$
p\left(z_{0}, w_{0}\right)=p_{\psi}\left(z_{0}, w_{0}\right)=\left(\max _{0 \leq \varphi \leq 2 \pi}\left|\frac{d w}{d z}\right|_{\varphi}\right) /\left(\min _{0 \leq \varphi \leq 2 \pi}\left|\frac{d w}{d z}\right|_{\varphi}\right), \quad w_{0}=\psi\left(z_{0}\right),
$$

is called the characteristic of the quasiconformal mapping $w=\psi(z)$. At those points of $G$ for which there is no neighborhood in which $\psi(z)$ is a continuously differentiable function, the characteristics $p\left(z_{0}, w_{0}\right)$ is not defined. However, as is easy to see, even at these points there exists the derivative $|d w / d z|_{\varphi}$ in an arbitrary direction, continuously depending on $\varphi$. By the Heine-Borel lemma, in each closed subregion of the region $G$ the characteristic $p(z, w)$ is defined and is continuous everywhere except, possibly, a finite set of piecewise smooth curves. If the mapping 
$w=\psi(z)$ is conformal at $z_{0}$, then $|d w / d z|$ does not depend on the direction $\varphi$, and $p\left(z_{0}, w_{0}\right)=1$. Conversely, if $p\left(z_{0}, w_{0}\right)=1$, then the mapping is conformal at $z_{0}$. In fact, if $p\left(z_{0}, w_{0}\right)=1$, then $(E+G)^{2}-4 J^{2}=0$, that is,

$$
\begin{aligned}
(E & +G-2 J)(E+G+2 J) \\
& =\left\{\left(\frac{\partial u}{\partial x}-\frac{\partial v}{\partial y}\right)^{2}+\left(\frac{\partial u}{\partial y}+\frac{\partial v}{\partial x}\right)^{2}\right\}\left\{\left(\frac{\partial u}{\partial x}+\frac{\partial v}{\partial y}\right)^{2}+\left(\frac{\partial u}{\partial y}-\frac{\partial v}{\partial x}\right)^{2}\right\}=0 .
\end{aligned}
$$

The expression in the second braces cannot be equal to zero, since otherwise we would have $\frac{\partial u}{\partial x}=-\frac{\partial v}{\partial y}, \frac{\partial u}{\partial y}=\frac{\partial v}{\partial x}, J=-\left(\frac{\partial u}{\partial x}\right)^{2}-\left(\frac{\partial u}{\partial y}\right)^{2} \leq 0$, contrary to the assumption $J>0$. Hence the expression in the first braces is equal to zero, that is $w=\psi(z)$ satisfies the Cauchy-Riemann equations at the point $z_{0}$, together with the condition $J>0$ the implies that the mapping is conformal.

If $p(z, w)=1$ at all points of $G$ where the characteristic is defined, then the function $w=\psi(z)$ is analytic in $G$. In fact, the function $\psi(z)$ is continuous in $G$ and is analytic in $G$ everywhere except, possibly a set whose intersection with each closed subregion of $G$ consists of finitely many piecewise smooth curves. By the well-known result from the theory of analytic functions, the function $w=\psi(z)$ is analytic everywhere in the region $G$. Since $J[\psi]>0$, we have $\psi^{\prime}(z) \neq 0$, and the mapping $w=\psi(z)$ is conformal in $G$.

It is easy to verify that at the point $z_{0}$ where the characteristic $p\left(z_{0}, w_{0}\right)$ exists, the mapping $w=\psi(z)$ transfers the element $d z=|d z| e^{i \varphi}$ into a certain element $d w=|d w| e^{i \theta}$, where $\theta$ gets an increment $2 \pi$ if $\varphi$ gets an increment $2 \pi$. Therefore

$$
\begin{gathered}
\max _{\varphi}|d w / d z|_{\varphi}=\frac{1}{\min _{\theta}|d z / d w|_{\theta}} ; \\
\min _{\varphi}|d w / d z|_{\varphi}=\frac{1}{\max _{\theta}|d z / d w|_{\theta}} ; \\
p\left(z_{0}, w_{0}\right)=\frac{\max _{\varphi}|d w / d z|_{\varphi}}{\min _{\varphi}|d w / d z|_{\varphi}}=\frac{\max _{\theta}|d z / d w|_{\theta}}{\min _{\theta}|d z / d w|_{\theta}}=p\left(w_{0}, z_{0}\right) .
\end{gathered}
$$

In what follows, when writing relations containing the characteristics, we assume that the characteristics are defined at the corresponding points. Note, first of all, an obvious inequality: $1 \leq p(z, w)<\infty$.

Next, if a quasiconformal mapping $w=\psi(z)$ is obtained as a composition of two quasiconformal mappings $w=w(\zeta)$ and $\zeta=\zeta(z)$, then

$$
p(z, w) \leq p(z, \zeta) p(\zeta, w) .
$$

In fact,

$$
\begin{aligned}
p(z, w) & =\frac{\max |d w / d z|}{\min |d w / d z|}=\frac{\max \{|d w / d \zeta||d \zeta / d z|\}}{\min \{|d w / d \zeta||d \zeta / d z|\}} \\
& \leq \frac{\max |d w / d \zeta| \max |d \zeta / d z|}{\min |d w / d \zeta| \min |d \zeta / d z|}=p(\zeta, w) p(z, \zeta)
\end{aligned}
$$

If one of the mappings $w=w(\zeta)$ and $\zeta=\zeta(z)$ is conformal, then the equality is attained in (2.6). In fact, let, for example, $\zeta=\zeta(z)$ be a conformal mapping. Then 
$|d \zeta / d z|_{\varphi}=|d \zeta / d z|$ does not depend on $\varphi$ and

$$
p(z, w)=\frac{\max \{|d w / d \zeta||d \zeta / d z|\}}{\min \{|d w / d \zeta||d \zeta / d z|\}}=\frac{\max |d w / d \zeta|}{\min |d w / d \zeta|}=p(\zeta, w) .
$$

Similarly, if the mapping $w=w(\zeta)$ is conformal, then

$$
p(z, w)=p(z, \zeta) \text {. }
$$

It is also easy to see that if we, along with the quasiconformal mapping $w=$ $\psi(z)$ consider the "symmetric" mapping $w=\psi_{1}(z)=\overline{\psi(\bar{z})}$, we have $p_{\psi}(z, w)=$ $p_{\psi_{1}}(\bar{z}, \bar{w})$. The equalities (2.7) and (2.8) show that the characteristic of a quasiconformal mapping is a conformal invariant.

The equalities $\left(2.5^{\prime}\right)$ and $\left(2.5^{\prime \prime}\right)$ imply that

$$
\begin{aligned}
p(z, w) & =\frac{E+G}{2 J}+\sqrt{\left(\frac{E+G}{2 J}\right)^{2}-1} \\
& =\frac{\max _{\varphi}|d w / d z|_{\varphi}^{2}}{J}=\frac{J}{\min _{\varphi}|d w / d z|_{\varphi}^{2}}=K+\sqrt{K^{2}-1}
\end{aligned}
$$

where $K=(E+G) / 2 J$. From here we get the estimate

$$
p(z, w) \leq(E+G) / J=2 K .
$$

Now we introduce several examples of quasiconformal mapping which we shall refer to in what follows.

EXAMPle 1. The function $w=\psi(z)=x l_{1} / l+i y$ maps quasiconformally the rectangle $\{0 \leq x \leq l, 0 \leq y \leq 1\}$ onto the rectangle $\left\{0 \leq u \leq l_{1}, 0 \leq v \leq 1\right\}$. In this case $J=l_{1} / l, E=\left(l_{1} / l\right)^{2}, G=1$. Using the formula (2.9) we get

$$
p(z, w)=\frac{1+\left(l_{1} / l\right)^{2}+\left|1-\left(l_{1} / l\right)^{2}\right|}{2 l_{1} / l}=\max \left(\frac{l_{1}}{l}, \frac{l}{l_{1}}\right) .
$$

EXAMPLE 2. The function

$$
w=\psi(z)=x+i\left\{\left(\frac{q_{2}-q_{1}}{l} x+q_{1}\right) \frac{y}{m}+\frac{a}{l} x\right\},
$$

where $-\infty<a<\infty, 0<q_{1}, q_{2}<\infty, 0<l<\infty$, maps the rectangle $\{0 \leq x \leq$ $l, 0 \leq y \leq m\}$ quasiconformally onto the trapezoid with vertices at the points $w=0, q_{1} i, l+\left(a+q_{2}\right) i, l+a i$. For this mapping we have

$$
\begin{gathered}
\frac{\max \left(q_{1}, q_{2}\right)}{m} \geq J=\frac{\partial v}{\partial y}=\left(\frac{q_{2}-q_{1}}{l} x+q_{1}\right) \frac{1}{m} \geq \frac{\min \left(q_{1}, q_{2}\right)}{m}, \\
E+G=1+\left(\frac{q_{2}-q_{1}}{l} \cdot \frac{y}{m}+\frac{a}{l}\right)^{2}+\left(\frac{q_{2}-q_{1}}{l} x+q_{1}\right)^{2} \frac{1}{m^{2}}, \\
2 K=J+\frac{1}{J}+\frac{1}{J}\left(\frac{q_{2}-q_{1}}{l} \cdot \frac{y}{m}+\frac{a}{l}\right)^{2} .
\end{gathered}
$$

Taking into account (2.10), we get the estimate

$$
p(z, w) \leq \frac{m}{\min \left(q_{1}, q_{2}\right)}+\frac{\max \left(q_{1}, q_{2}\right)}{m}+m \frac{\max \left\{a^{2},\left(q_{2}-q_{1}+a\right)^{2}\right\}}{l^{2} \min \left(q_{1}, q_{2}\right)} .
$$


In some special cases the estimate can be simplified. If $q_{1}=q_{2}=m$, then $J \equiv 1$, $E+G=2+(a / l)^{2}, K=1+\frac{1}{2}(a / l)^{2}$,

$$
p(z, w) \equiv 1+\frac{1}{2}\left(\frac{a}{l}\right)^{2}+\frac{|a|}{l} \sqrt{\frac{1}{4}\left(\frac{a}{l}\right)^{2}+1} \leq 2+\left(\frac{a}{l}\right)^{2} .
$$

If $a=0, m=q_{1}=1, q_{2}=q$, then (2.12) implies

$$
p(z, w) \leq 1+\max \left(q, \frac{1}{q}\right)+\frac{(q-1)^{2}}{l^{2} \min (1, q)} .
$$

Suppose that a closed region $G$ is mapped quasiconformally onto a closed region $D$ by a function $w=\psi(z)$. Let $C$ be a smooth curve, $C \subset G$. If the equality $|d w / d z|_{\varphi}=$ const holds on $C$, where the derivative is taken in the direction of the tangent to $C$, we say that the mapping has constant distortion on $C$. If the curve $C$ is piecewise-smooth, and the distortion is equal to the same constant on each of the smooth pieces forming $C$, we say that the mapping has a constant distortion on $C$. It is easy to verify that in the both preceding examples the distortion is constant on each of the edges of the mapped quadrilaterals.

REMARK. We will have to use quasiconformal mappings which can be obtained from the considered above by additional entire linear mappings or by passage to "symmetric" mappings. It is clear that the estimates for the characteristic obtained above will remain true. Therefore referring to one of the examples we assume that, if needed, readers will make these additional transformations themselves. This remark will be applied, also, to the examples considered below.

ExAmPLE 3. Let $u=\omega(x)$ be a continuously differentiable on $\{0 \leq x \leq l\}$ function, such that $\omega(0)=0, \omega(l)=l, 0<1 / M \leq \omega^{\prime}(x) \leq M<\infty$. Our purpose is to construct a quasiconformal mapping of the rectangle $\{0 \leq x \leq l, 0 \leq y \leq 1\}$ onto the same rectangle $\{0 \leq u \leq l, 0 \leq v \leq 1\}$, such that the points $z=x+i$ are mapped to the points $w=\omega(x)+i$, and all points on the other three edges of the rectangle are fixed by the mapping. The mapping

$$
\left\{\begin{array}{l}
u=(\omega(x)-x) y+x, \\
v=y
\end{array}\right.
$$

is a desired mapping. For this mapping we have

$$
\begin{gathered}
J=\left(\omega^{\prime}(x)-1\right) y+1, \min \left(1, \omega^{\prime}\right) \leq J \leq \max \left(1, \omega^{\prime}\right), \\
E+G=1+\left\{\left(\omega^{\prime}(x)-1\right) y+1\right\}^{2}+(\omega(x)-x)^{2} .
\end{gathered}
$$

Then, by (2.10),

$$
\begin{aligned}
p(z, w) \leq & \frac{1}{J}+J+\frac{(\omega(x)-x)^{2}}{J} \leq 1+\max \left(\omega^{\prime}, \frac{1}{\omega^{\prime}}\right) \\
& +\frac{(\omega(x)-x)^{2}}{\min \left(1, \omega^{\prime}\right)} \leq 1+M+M l^{2} .
\end{aligned}
$$

EXAMPLE 4. Let $\omega_{j}(x)(j=1,2)$ be continuously differentiable on $\{0 \leq x \leq l\}$ functions, $\omega_{j}(0)=0, \omega_{j}(l)=l, 0<1 / M \leq \omega_{j}^{\prime}(x) \leq M<\infty, j=1,2$, and $\omega_{j}(y)$ $(j=3,4)$ be continuously differentiable on $\{0 \leq y \leq 1\}$ functions, $\omega_{j}(0)=0$, $\omega_{j}(1)=1,0<1 / M \leq \omega_{j}^{\prime}(y) \leq M<\infty, j=3,4$. Our purpose is to map the 
rectangle $R=\{0 \leq x \leq l, 0 \leq y \leq 1\}$ quasiconformally onto the same rectangle $\{0 \leq u \leq l, 0 \leq v \leq 1\}$ in such a way, that the points $z=x$ are mapped to the points $w=\omega_{1}(x)$, the points $z=x+i$ are mapped to the points $w=\omega_{2}(x)+i$, the points $z=i y$ are mapped to the points $w=i \omega_{3}(y)$, and the points $z=l+i y$ are mapped to the points $w=l+i \omega_{4}(y)$.

Thus we are given certain continuous one-to-one mapping of the boundary of the rectangle $\{0 \leq x \leq l, 0 \leq y \leq 1\}$ onto the boundary of the same rectangle $\{0 \leq u \leq l, 0 \leq v \leq 1\}$, which preserves vertices and satisfies additional smoothness conditions. We need to extend this mapping to a quasiconformal mapping of $\{0 \leq$ $x \leq l, 0 \leq y \leq 1\}$ onto $\{0 \leq u \leq l, 0 \leq v \leq 1\}$.

The desired mapping can be constructed in the following way. First we map the rectangle $R$ onto itself in such a way that points on vertical edges are fixed, and points on horizontal edges are mapped in the described way. In order to get such a mapping we apply the mappings from Example 3 to the rectangles $\{0 \leq x \leq l, 0 \leq y \leq 1 / 2\}$ and $\{0 \leq x \leq l, 1 / 2 \leq y \leq 1\}$, the points of the intervals $\{x=0,0 \leq y \leq 1\},\{x=l, 0 \leq y \leq 1\}$, and $\{y=1 / 2,0 \leq x \leq l\}$ are fixed points of these mappings. After this, in a similar way, we construct a quasiconformal mapping of $R$ onto $R$, such that points on horizontal edges are fixed, and points on vertical edges are mapped in the described way (to do this we divide $R$ into two rectangles: $\{0 \leq x \leq l / 2,0 \leq y \leq 1\}$ and $\{l / 2 \leq x \leq l, 0 \leq y \leq 1\})$. The composition of these two quasiconformal mappings is a desired mapping. Using (2.6) and (2.14) it is not difficult to get an estimate for its characteristic. We mention only that $p(z, w)$ is bounded by a constant depending on $M$ and $l$ only.

EXAMPLE 5. Let $F$ be a closed Riemann surface of genus zero, covering the $w$-plane, $D$ be a closed simply connected region on $F$, bounded by a closed Jordan curve $C$ having the following properties:

(1) $C$ does not pass through the algebraic ramification points of the surface $F$.

(2) There are four points $A_{1}, A_{2}, A_{3}, A_{4}$ on $C$, listed according to their order on $C$, such that the $\operatorname{arcs} A_{1} A_{2}, A_{2} A_{3}, A_{3} A_{4}, A_{4} A_{1}$, which partition $C$, have continuous curvature.

(3) In a sufficiently small neighborhood of the point $A_{j}$, the $\operatorname{arcs} A_{j-1} A_{j}$ and $A_{j} A_{j+1}, j=1,2,3,4, A_{5}=A_{1}, A_{0}=A_{4}$, either consist of two orthogonal line segments, or of one line segment and one circular arc, also orthogonal at $A_{j}$, moreover the region $D$ has at $A_{j}$ an angle of magnitude $\pi / 2$.

Our purpose is to construct a quasiconformal mapping $z=\psi(w)$, which maps $D$ onto some rectangle $R=\{0 \leq x \leq l, 0 \leq y \leq 1\}$ in the $(z=x+i y)$-plane, such that the points $A_{1}, A_{2}, A_{3}, A_{4}$ are mapped to the vertices of the rectangle, and the mapping has a constant distortion on each of the $\operatorname{arcs} A_{j} A_{j+1}$. To solve this problem we need some theorems from the theory of conformal mappings, all results we need can be found, for example, in the book G.M. Goluzin [Gol69].

We may assume without loss of generality that the projection of $C$ is contained in the $w$-plane. As is known (see $[\mathbf{G o l 6 9}, \mathbf{C h . I V ,}, \S 6]$ ), for a closed region $D$ there exists a one-to-one conformal mapping of $D$ onto a rectangle $R^{\prime}=\{0 \leq \xi \leq l, 0 \leq$ $\eta \leq 1\}$ in the plane $\zeta=\xi+i \eta$, where the constant $l$ depends on $D$ and on the choice of the points $A_{j}$; such that the points $A_{1}, A_{2}, A_{3}$, and $A_{4}$ are mapped to the points $\zeta=0, l, l+i$, and $i$, respectively. Denote such a mapping by $\zeta=\zeta(w)$. Since the 
curves $A_{j} A_{j+1}$ have continuous curvature, by Kellogg's theorem (see G.M. Goluzin $[$ Gol69, Ch.X, $\S 1$, Thm. 6]), it is easy to conclude that the meromorphic function $w=w(\zeta)$, inverse to $\zeta(w)$, has a continuous derivative $w^{\prime}(\zeta) \neq 0$ everywhere on the boundary of $R^{\prime}$, except the vertices of $R^{\prime}$. But, by the condition (3) and the symmetry principle, the function $w(\zeta)$ can be analytically continued to certain sufficiently small neighborhoods of the vertices, moreover, it establishes a univalent conformal mapping of these neighborhoods. Hence the derivative $w^{\prime}(\zeta)$ exists also in neighborhoods of vertices of $R^{\prime}$, where it is also continuous and nonzero. Hence everywhere on the boundary $\partial R^{\prime}$ the derivative $w^{\prime}(\zeta) \neq 0$ exists and is continuous. Hence the inequality $0<1 / M_{1} \leq\left|w^{\prime}(\zeta)\right| \leq M_{1}<\infty$ holds, where $M_{1}$ is some constant, $\zeta \in \partial R^{\prime}$.

Let $z=T(w)$ be a function establishing a continuous one-to-one correspondence between the curve $C$ and the boundary $\partial R$ of the rectangle, such that the points $A_{1}, A_{2}, A_{3}, A_{4}$ are mapped onto the vertices $z=0, l, l+i, i$, respectively, and the distortion is constant on each of the $\operatorname{arcs} A_{j} A_{j+1}$, that is,

$$
\left|\frac{d T}{d w}\right|=\left|\frac{d z}{d w}\right|= \begin{cases}l / \operatorname{length}\left(A_{1} A_{2}\right) & \text { for } w \in A_{1} A_{2}, \\ 1 / \text { length }\left(A_{2} A_{3}\right) & \text { for } w \in A_{2} A_{3} \\ l / \text { length }\left(A_{3} A_{4}\right) & \text { for } w \in A_{3} A_{4} \\ 1 / \text { length }\left(A_{1} A_{4}\right) & \text { for } w \in A_{1} A_{4}\end{cases}
$$

It is clear that $0<1 / M_{2} \leq|d z / d w| \leq M_{2}<\infty$. On the boundary $\partial R^{\prime}$ we have defined a function $z=\omega(\zeta)=T(w(\zeta))$ which maps $\partial R^{\prime}$ onto $\partial R$ in such a way, that vertices are mapped onto vertices. The function $z=\omega(\zeta)$ has a continuous derivative on each of the sides of $\partial R^{\prime}$, moreover

$$
\left.1 /\left(M_{1} M_{2}\right) \leq\left|\omega^{\prime}(\zeta)\right|=\left|T^{\prime}(w)\right| \mid w^{\prime}(\zeta)\right) \leq M_{1} M_{2}
$$

Using the result of Example 4, we can extend this mapping $z=\omega(\zeta)$ to a quasiconformal mapping $z=\Omega(\zeta)$ of the rectangle $R^{\prime}$ onto $R$, moreover, the characteristic $p(\zeta, z)$ does not exceed certain constant depending on $l$ and $M_{1} M_{2}$ only. The quasiconformal mapping $z=\Omega(\zeta(w))$ has the desired properties. In fact, by the equality (2.7), we have $p(w, z) \equiv p(\zeta(w), z)$, and the equality $z=\Omega(\zeta(w))=$ $\omega(\zeta(w))=T(w(\zeta))=T(w)$ holds on $C$, and, by (2.15), the distortion is constant on each $\operatorname{arc} A_{j} A_{j+1}$.

EXAMPLE 6. Let $w=\psi(z)$ be a quasiconformal mapping of the disc $\{|z| \leq R\}$ onto the disc $\{|w| \leq R\}$, such that the points $z=r e^{i \varphi}, 0 \leq r \leq R, 0 \leq \varphi \leq 2 \pi$, are mapped to the point $w=r e^{i \theta(\varphi)}$, where $\theta(\varphi)$ is a continuously differentiable function on $[0,2 \pi], \theta^{\prime}(\varphi)>0, \theta(0)=0, \theta(2 \pi)=2 \pi$. Let us estimate the characteristic of this mapping. In this connection it is convenient to consider an auxiliary mapping of the half-strip $\{\sigma<\ln R, 0<\tau<2 \pi\}, \zeta=\sigma+i \tau$ onto the half-strip $\left\{\sigma_{1}<\ln R, 0<\tau_{1}<2 \pi\right\}, \zeta_{1}=\sigma_{1}+i \tau_{1}$ by means of the function $\zeta_{1}=\ln \psi\left(e^{\zeta}\right)$. It is clear that $p\left(\zeta, \zeta_{1}\right)=p(z, w)$. Therefore it suffices to estimate $p\left(\zeta, \zeta_{1}\right)$. Since $\sigma_{1}=\sigma, \tau_{1}=\theta(\tau)$, we have $J=\theta^{\prime}, E+G=1+{\theta^{\prime}}^{2}$, and, using the formulas (2.9) 
we get

$$
\begin{aligned}
p(z, w) & =p\left(\zeta, \zeta_{1}\right)=\frac{1}{2}\left(\frac{1}{\theta^{\prime}}+\theta^{\prime}\right)+\sqrt{\frac{1}{4}\left(\frac{1}{\theta^{\prime}}+\theta^{\prime}\right)^{2}-1} \\
& =\frac{1}{2}\left(\frac{1}{\theta^{\prime}}+\theta^{\prime}\right)+\frac{1}{2}\left|\frac{1}{\theta^{\prime}}-\theta^{\prime}\right|=\max \left(\theta^{\prime}, \frac{1}{\theta^{\prime}}\right) .
\end{aligned}
$$

Thus

$$
p(z, w) \leq \max \left\{\max _{[0,2 \pi]} \theta^{\prime}(\varphi), \frac{1}{\min _{[0,2 \pi]} \theta^{\prime}(\varphi)}\right\} .
$$

EXAMPLE 7. Our purpose is to find a quasiconformal mapping of the disc $\{|z| \leq R\}$ onto the disc $\{|w| \leq R\}$ in such a way that the point $z=x_{0}, 0<x_{0}<R$, is mapped onto $w=0$, and the points $\{|z|=R\}$ are fixed points of the mapping.

First we carry out a linear-fractional transformation of the disc $\{|z| \leq R\}$ onto the disc $\{|\zeta| \leq R\}$, mapping the point $z=x_{0}$ onto $\zeta=0$, and $z=R$ onto $\zeta=R$ :

$$
\zeta=\zeta(z)=\frac{R^{2}\left(z-x_{0}\right)}{R^{2}-z x_{0}} .
$$

Under this mapping each point $\zeta=R e^{i \theta}$ has a unique pre-image $z=R e^{i \varphi(\theta)}$, where $\varphi(\theta)$ is an odd continuously differentiable strictly increasing function on $[-\pi, \pi]$, $\varphi(-\pi)=-\pi, \varphi(0)=0, \varphi(\pi)=\pi$. Now we construct a quasiconformal mapping which maps the disc $\{|\zeta| \leq R\}$ onto the disc $\{|w| \leq R\}$ in such a way that the point $\zeta=|\zeta| e^{i \theta},|\theta| \leq \pi, 0<|\zeta| \leq R$, is mapped onto the point $w=|\zeta| e^{i \varphi(\theta)}, w(0)=0$. It is easy to see that the mapping $w=w(z)=w[\zeta(z)]$ has the desired properties.

Let us estimate the characteristic $p(z, w)$ of the mapping. Since the mapping $\zeta(z)$ is conformal, we have $p(z, w)=p(\zeta, w)$. The equality (2.16) implies that

$$
p(z, w)=\max \left(\varphi^{\prime}, \frac{1}{\varphi^{\prime}}\right)=\max \left(\frac{d \theta}{d \varphi}, \frac{d \varphi}{d \theta}\right)
$$

if $\arg z=\varphi$. But

$$
\begin{gathered}
\frac{d \theta}{d \varphi}=\left|\frac{d \zeta}{d z}\right|_{z=R e^{i \varphi}}=\frac{R^{2}-x_{0}^{2}}{\left|R-e^{i \varphi} x_{0}\right|^{2}}, \\
\frac{R-x_{0}}{R+x_{0}} \leq \frac{d \theta}{d \varphi} \leq \frac{R+x_{0}}{R-x_{0}} .
\end{gathered}
$$

Hence

$$
p(z, w) \leq \frac{R+x_{0}}{R-x_{0}}=\frac{1+\frac{x_{0}}{R}}{1-\frac{x_{0}}{R}} .
$$

This estimate is sharp because

$$
p(z, w)=\left.\frac{d \theta}{d \varphi}\right|_{\varphi=0}=\frac{1+\frac{x_{0}}{R}}{1-\frac{x_{0}}{R}}
$$

for $z=x, 0<x<R$. 
EXAMPLE 8. Let $F_{1}$ be the Riemann surface of the function $\sqrt[n]{z}, n=2,3, \ldots$, over the disc $\{|z|<R\}, F_{2}$ be the Riemann surface of the function $\sqrt[n]{w-a}, 0<$ $|a|<R$, over the disc $\{|w|<R\}$. Our purpose is to find a quasiconformal mapping of $F_{1}$ onto $F_{2}$, such that the algebraic ramification point (of order $n-1$ ) of $F_{1}$ over the point $z=0$ is mapped onto the algebraic ramification point of $F_{2}$ over $w=a$, and the boundary points remain fixed. Such a mapping can be obtained as $w=w(z)=w\left\{z_{2}\left(z_{1}(z)\right)\right\}$, where $z_{1}(z)=z e^{-i \alpha}, \alpha=\arg a, z_{2}=z_{2}\left(z_{1}\right)$ is the inverse mapping to the mapping considered in Example 7 (the role of $x_{0}$ is played by $|a|)$, and $w\left(z_{2}\right)=z_{2} e^{i \alpha}$. Here

$$
p(z, w) \leq \frac{1+\frac{|a|}{R}}{1-\frac{|a|}{R}}
$$

ExAmple 9. Let $F$ be a closed Riemann surface of genus zero, covering the $w$-plane, $D$ be a closed simply connected region on $F$, bounded by a closed Jordan curve $C$, which does not pass through algebraic ramification points of $F$ and has a continuous curvature. There exists a quasiconformal mapping $z=\psi(w)$ with a bounded characteristic of the region $D$ onto the disc $\{|z| \leq R\}$, which has a constant distortion on $C$.

Such a quasiconformal mapping can be constructed similarly to the construction of Example 5, but instead of Example 4 we use Example 6, and some parts of the argument are simpler. Note that the estimate of the characteristic $p(w, z)$ does not depend on the radius of the disc $\{|z| \leq R\}$, since we can pass from this disc to any other disc using an additional entire linear transformation.

EXAmPLe 10. Let $W$ be a Riemann surface constructed in the following way. Take two copies, $G_{1}$ and $G_{2}$, of the disc $\{|w|<2\}$ with a cut along the segment joining the points $w= \pm 1$. We paste together the upper boundary of the cut in $G_{1}$ with the lower boundary of the cut in $G_{2}$, and vice versa. We get a two-sheeted Riemann surface $W$ with two algebraic ramification points of the first order over $w=1$ and $w=-1$. It is clear that the surface $W$ is doubly-connected and its boundary consists of two circles, $C_{1}$ and $C_{2}$, lying over $\{|w|=2\}, C_{1}=\partial G_{1}$, $C_{2}=\partial G_{2}$. Our purpose is to construct a quasiconformal mapping with bounded characteristic of the Riemann surface $W$ onto the one-sheeted annulus $\{1 / 2<$ $|z|<2\}$, such that the point $2 e^{i \theta} \in C_{1}$ is mapped onto the point $2 e^{i \theta}$, and the point $2 e^{-i \theta} \in C_{2}$ is mapped onto the point $e^{i \theta} / 2$. We are not interested in precise numerical bounds for the characteristic.

We map the Riemann surface $W$ conformally $^{4}$ onto the annulus $\{1 / R<|\zeta|<$ $R$, where $R$ is some constant, $1<R<\infty$ in such a way, that $G_{1}$ is mapped onto $\{1<|\zeta|<R\}, G_{2}$ is mapped onto $\{1 / R<|\zeta|<1\}$, and the algebraic ramification points over $w= \pm 1$ are mapped onto $\zeta= \pm 1$, respectively, and the points $\pm 2 \in C_{1}$ are mapped onto the points $\zeta= \pm R$, and the points $\pm 2 \in C_{2}$ are mapped onto $\zeta= \pm 1 / R$. To construct such a mapping it suffices to map $\{|w|<2, \operatorname{Im} w>0\}$ onto $\{1<|\zeta|<R, \operatorname{Im} \zeta>0\}$ conformally in such a way that the points $-2,-1,1,2$ are mapped onto the points $-R,-1,1, R$, respectively, and to continue the obtained

\footnotetext{
${ }^{4}$ The mapping function can be written explicitly using elliptic functions (see L.I. Volkovyskiı̌, G.L. Lunts, and I.G. Aramanovich [VLA91, Problem 1370, 7], or L.I. Volkovyskiǔ [Vol54, p. 146]).
} 
function using the symmetry principle. The symmetry principle implies that there exists a continuous, odd, increasing on $[-\pi, \pi]$ function $\varphi=\varphi(\theta)$, such that $\varphi(0)=$ $0, \varphi( \pm \pi)= \pm \pi$, the point $2 e^{i \theta} \in C_{1}$ is mapped onto the point $R e^{i \varphi(\theta)}$, and the point $2 e^{-i \theta} \in C_{2}$ is mapped onto the point $\frac{1}{R} e^{i \varphi(\theta)}$. Since the mapping is conformal in the points lying on $C_{1}$ and $C_{2}$, then $\varphi$ has a continuous derivative $\varphi^{\prime}(\theta)>0$ on $[-\pi, \pi]$. Using the function $\zeta_{1}=\ln \zeta$ we map $\{1 / R<|\zeta|<R,|\arg \zeta|<\pi\}$ onto the rectangle $R_{1}=\left\{\left|\operatorname{Re} \zeta_{1}\right|<\ln R,\left|\operatorname{Im} \zeta_{1}\right|<\pi\right\}$. Now we map $R_{1}$ quasiconformally onto the rectangle $R_{2}=\left\{\left|\operatorname{Re} \zeta_{2}\right|<\ln 2,\left|\operatorname{Im} \zeta_{2}\right|<\pi\right\}$ in such a way, that the point $\xi_{1} \pm i \pi,\left|\xi_{1}\right|<\ln R$, is mapped onto the point $\frac{\ln 2}{\ln R} \xi_{1} \pm i \pi=\xi_{2} \pm i \pi$, and the point $\pm \ln R+i \varphi(\theta)$ is mapped onto the point $\pm \ln 2+i \theta$.

Examples 1 and 4 imply that the mapping $\zeta_{2}=\psi\left(\zeta_{1}\right)$ can be constructed in such a way, that it characteristic satisfies $p\left(\zeta_{1}, \zeta_{2}\right) \leq Q$, where $Q$ is some constant. Now, in order to get the desired mapping, it remains to map $R_{2}$ onto $\{1 / 2<|z|<$ $2,|\arg z|<\pi\}$ by means of the function $z=e^{\zeta_{2}}$, and to observe that the function $z=z(\zeta)=\exp \{\psi(\ln \zeta)\}$ remains univalent and continuous on the negative radius of the annulus $\{1 / R<|\zeta|<R\}$. Our mapping of the Riemann surface $W$ onto $\{1 / 2<|z|<2\}$ is obtained as a composition of mapping out of which all mappings, except the mapping $\zeta_{2}=\psi\left(\zeta_{1}\right)$, were conformal. Hence, $p(w, z)=p\left(\zeta_{1}, \zeta_{2}\right) \leq Q$.

\section{The Teichmüller theorem}

In this section we prove the following theorem.

Theorem 3.1 (O. Teichmüller). Let $w=w(z)=u(z)+i v(z)$ be a homeomorphic mapping of the complex $z$-plane onto $\{|w|<R \leq \infty\}$, which is quasiconformal for $|z| \geq r_{0}$, and

$$
\iint_{|z| \geq r_{0}}\left\{p\left(r e^{i \varphi}, w\right)-1\right\} \frac{d r d \varphi}{r}<\infty .
$$

Then $R$ is necessarily equal to $\infty$ and the limit

$$
\lim _{z \rightarrow \infty} \frac{|w(z)|}{|z|}=A, 0<A<\infty,
$$

exists as a finite positive limit.

We introduce some notation. Let

$$
h(t)=\iint_{|z| \geq t}\left\{p\left(r e^{i \varphi}, w\right)-1\right\} \frac{d r d \varphi}{r}, t \geq r_{0} .
$$

The inequality (3.1) implies that $h(t)$ has finite values and monotonically tends to 0 as $t \rightarrow \infty$. Let $z=r e^{i \varphi}, w=\rho e^{i \theta}$, and let $d \sigma(z)$ be the area differential at $z$. We can rewrite the condition (3.1) in the following way:

$$
\iint_{|z| \geq r_{0}}\{p(z, w)-1\} d \sigma(\ln z)=\iint_{|z| \geq r_{0}}\{p(z, w)-1\} \frac{d \sigma(z)}{|z|^{2}}<\infty .
$$

The circle $\{|w|=\rho\}$ corresponds to a piecewise smooth closed Jordan curve $\Gamma_{\rho}$ in the $z$-plane. It is clear that if $\rho_{0}$ is sufficiently large, then for $\rho \geq \rho_{0}$ the curve $\Gamma_{\rho}$ lies in $\left\{r_{0}<|z|<\infty\right\}$, contains the point $z=0$ in its interior, and the increment of $\arg z$ over $\Gamma_{\rho}$ is equal to $2 \pi$. Let

$$
r_{1}(\rho)=\min _{z \in \Gamma_{\rho}}|z|, r_{2}(\rho)=\max _{z \in \Gamma_{\rho}}|z|, \omega(\rho)=\ln \frac{r_{2}(\rho)}{r_{1}(\rho)} .
$$


It is easy to see that $r_{1}(\rho)$ and $r_{2}(\rho)$ are continuous functions, which tend monotonically to $\infty$ as $\rho \rightarrow \infty$. In fact, the regions $G_{\rho}, \rho>\rho_{0}$, which are the images of the discs $\{|w|<\rho\}$ under the mapping $z=z(w)$, contain $z=0$ and exhaust $\{|z|<\infty\}$. Therefore, for each disc $\{|z| \leq r\}$, if $\rho$ is sufficiently large, the region $G_{\rho}$ contains the disc, hence $r_{2}(\rho) \geq r_{1}(\rho) \rightarrow \infty$. The continuity of the functions $r_{1}(\rho)$ and $r_{2}(\rho)$ follows immediately from the continuity of the mapping $z=z(w)$.

The proof of the theorem is rather long. Therefore we start with an outline of its plan, and divide the proof into steps. First we show that $R=\infty$ (Step I). Then (3.2) is equivalent to the relation

$$
\lim _{w \rightarrow \infty} \frac{|z(w)|}{|w|}=B, 0<B=1 / A<\infty .
$$

To prove $\left(3.2^{\prime}\right)$ it suffices to establish that

$$
\lim _{\rho \rightarrow \infty} \frac{r_{1}(\rho)}{\rho}=\lim _{\rho \rightarrow \infty} \frac{r_{2}(\rho)}{\rho}=B .
$$

We prove the existence of a finite limit $\lim _{\rho \rightarrow \infty} \ln \frac{r_{1}(\rho)}{\rho}$ using the Bolzano-Cauchy criterion. Let $\rho_{2}>\rho_{1} \geq \rho$. We need to estimate the difference

$$
Q\left(\rho_{1}, \rho_{2}\right)=\ln \frac{r_{1}\left(\rho_{2}\right)}{\rho_{2}}-\ln \frac{r_{1}\left(\rho_{1}\right)}{\rho_{1}}=\ln \frac{r_{1}\left(\rho_{2}\right)}{r_{1}\left(\rho_{1}\right)}-\ln \frac{\rho_{2}}{\rho_{1}}
$$

both from below and from above. Such estimates will be found in Step II of the proof. In order to show that $Q\left(\rho_{1}, \rho_{2}\right)$ tends to 0 as $\rho_{1} \rightarrow \infty$, we need to show that $\omega(\rho) \rightarrow 0$ as $\rho \rightarrow \infty$. The proof of this fact is the contents of Step III, the most complicated step in the proof. In the final Step IV, using the fact that $\omega(\rho) \rightarrow 0$ as $\rho \rightarrow \infty$ and the estimates for $Q\left(\rho_{1}, \rho_{2}\right)$ found in Step II, we prove the existence of the $\operatorname{limit}_{\rho \rightarrow \infty} \lim _{1}(\rho) / \rho=B$, and also the existence of the limit

$$
\lim _{\rho \rightarrow \infty} \frac{r_{2}(\rho)}{\rho}=\lim _{\rho \rightarrow \infty} e^{\omega(\rho)} \frac{r_{1}(\rho)}{\rho}=B .
$$

Now we turn to the proof.

STEP I. We show that $R=\infty$. Let $r^{\prime}$ be so large that $r^{\prime} \geq r_{0}$, and the image of the disc $\left\{|z|<r^{\prime}\right\}$ under the mapping $w=w(z)$ contains the point $w=0$. Denote by $D$ the image of the region $\left\{r^{\prime}<|z|<\infty\right\}$ under this mapping. If we assume that $R<\infty$, we get that

$$
K=\iint_{D} \frac{d \rho d \theta}{\rho}=\iint_{D} d \sigma(\ln w)=\iint_{|z|>r^{\prime}} \frac{d \sigma(\ln w)}{d \sigma(\ln z)} d \sigma(\ln z)<\infty .
$$

Since the increment of $\arg w(z)$ as $z$ runs over the circle $\{|z|=r\}, r>r^{\prime}$, is equal to $2 \pi$, we have

$$
2 \pi \leq \int_{|z|=r}|d \ln w(z)|=\int_{|z|=r}\left|\frac{d \ln w}{d \ln z}\right| d \varphi=\int_{|z|=r}\left|\frac{z}{w}\right|\left|\frac{d w}{d z}\right| d \varphi .
$$

We divide both sides of this inequality by $r$ and integrate with respect to $r$ from $r^{\prime}$ to $r^{\prime \prime}>r^{\prime}$. We get the inequality

$$
2 \pi \ln \frac{r^{\prime \prime}}{r^{\prime}} \leq \iint_{r^{\prime}<|z|<r^{\prime \prime}}\left|\frac{z}{w}\right|\left|\frac{d w}{d z}\right| d \sigma(\ln z) .
$$


From (2.9) we get that

$$
\left|\frac{z}{w}\right|\left|\frac{d w}{d z}\right| \leq\left|\frac{z}{w}\right| \max \left|\frac{d w}{d z}\right|=\left|\frac{z}{w}\right| \sqrt{p(z, w) J[w(z)]}=\sqrt{p(z, w) \frac{d \sigma(\ln w)}{d \sigma(\ln z)}} .
$$

The inequality (3.3) implies

$$
2 \pi \ln \frac{r^{\prime \prime}}{r^{\prime}} \leq \iint_{r^{\prime}<|z|<r^{\prime \prime}} \sqrt{p(z, w) \frac{d \sigma(\ln w)}{d \sigma(\ln z)}} d \sigma(\ln z) .
$$

Applying the Cauchy-Buniakowsky inequality we get

$$
\begin{aligned}
\left(2 \pi \ln \frac{r^{\prime \prime}}{r^{\prime}}\right)^{2} \leq & \iint_{r^{\prime}<|z|<r^{\prime \prime}} \frac{d \sigma(\ln w)}{d \sigma(\ln z)} d \sigma(\ln z) \iint_{r^{\prime}<|z|<r^{\prime \prime}} p(z, w) d \sigma(\ln z) \\
\leq & \iint_{r^{\prime}<|z|<r^{\prime \prime}} \frac{d \sigma(\ln w)}{d \sigma(\ln z)} d \sigma(\ln z)\left\{\iint_{|z|>r^{\prime}}\{p(z, w)-1\} d \sigma(\ln z)\right. \\
& \left.+\iint_{r^{\prime}<|z|<r^{\prime \prime}} d \sigma(\ln z)\right\}
\end{aligned}
$$

Hence

$$
\left(2 \pi \ln \frac{r^{\prime \prime}}{r^{\prime}}\right)^{2} \leq K\left\{h\left(r^{\prime}\right)+2 \pi \ln \frac{r^{\prime \prime}}{r^{\prime}}\right\}
$$

and

$$
2 \pi \ln \frac{r^{\prime \prime}}{r^{\prime}} \leq K\left\{1+\frac{h\left(r^{\prime}\right)}{2 \pi \ln \frac{r^{\prime \prime}}{r^{\prime}}}\right\} .
$$

Letting $r^{\prime \prime} \rightarrow \infty$ we get a contradiction, since the left-hand side of this inequality tends to $\infty$, but the right-hand side remains bounded. Thus $R=\infty$.

STEP II. The purpose of this step is to get estimates of the quantity $Q\left(\rho_{1}, \rho_{2}\right)$ in terms of $\omega(\rho)$ and $h(\rho)$. We fix the value of $\ln z$ at some point $A \in \Gamma_{\rho}$, and map $\Gamma_{\rho}$ onto some curve $\gamma_{\rho}$ in the $\zeta$-plane by the branch of $\ln z$ which is continuous on $\Gamma_{\rho}$ (that is, such that the function $\ln z\left(\rho e^{i \theta}\right)$ is continuous as a function of $\theta$ on some interval of length $2 \pi$ ). The point $A$ will correspond to the ends of the curve $\gamma_{\rho}: \zeta=a$ and $\zeta=b, b-a=2 \pi i$. If $\zeta=\xi+i \eta$, then

$$
\max _{\zeta \in \gamma_{\rho}} \xi=\ln r_{1}(\rho), \max _{\zeta \in \gamma_{\rho}} \xi=\ln r_{2}(\rho) .
$$

Denote by $c$ and $d$ some points on $\gamma_{\rho}$ for which

$$
\operatorname{Re} c=\ln r_{1}(\rho), \operatorname{Re} d=\ln r_{2}(\rho) .
$$

Suppose that the points $c$ and $d$ are located on the curve $\gamma_{\rho}$ in such a way that the order of the points on the curve is $a, c, d, b$ (we do not exclude the possibilities $a=c$ and $d=b$ ). We map the part of the curve $\gamma_{\rho}$ located between the points $c$ and $d$ symmetrically with respect to the line $\operatorname{Re} \zeta=\ln r_{1}(\rho)$, and shift to the left onto $2\left(\ln r_{2}(\rho)-\ln r_{1}(\rho)\right)=2 \omega(\rho)$ the part of the curve $\gamma_{\rho}$ located between the points $d$ and $b$. We get a continuous curve $\gamma_{\rho}^{\prime}$ with ends at the points $\zeta=a$ and $\zeta=b-2 \omega(\rho)=a-2 \omega(\rho)+2 \pi i$. Hence the length of the curve $\gamma_{\rho}^{\prime}$ is at least $\sqrt{4 \pi^{2}+4 \omega^{2}(\rho)}$. But the length of the curve $\gamma_{\rho}$ is the same as the length of the 
curve $\gamma_{\rho}^{\prime}$, hence it is also at least $\sqrt{4 \pi^{2}+4 \omega^{2}(\rho)}$. If the order of the points on the curve $\gamma_{\rho}$ is $a, d, c, b$, we use a similar argument. Thus

$$
2 \sqrt{\pi^{2}+\omega^{2}(\rho)} \leq \int_{\gamma_{\rho}}|d \zeta|=\int_{\Gamma_{\rho}}|d \ln z|=\int_{|w|=\rho}\left|\frac{d \ln z}{d \ln w}\right||d \ln w|=\int_{0}^{2 \pi}\left|\frac{d \ln z}{d \ln w}\right| d \theta .
$$

Applying the Cauchy-Buniakowsky inequality we get

$$
2 \pi+\frac{2 \omega^{2}(\rho)}{\pi} \leq \int_{0}^{2 \pi}\left|\frac{d \ln z}{d \ln w}\right|^{2} d \theta
$$

from where, dividing by $\rho$ and integrating with respect to $\rho$ from $\rho_{1}$ to $\rho_{2}, \rho_{0} \leq$ $\rho_{1}<\rho_{2}<\infty$, we get

$$
2 \pi \ln \frac{\rho_{2}}{\rho_{1}}+\frac{2}{\pi} \int_{\rho_{1}}^{\rho_{2}} \frac{\omega^{2}(\rho)}{\rho} d \rho \leq \int_{\rho_{1}}^{\rho_{2}} \int_{0}^{2 \pi}\left|\frac{d \ln z}{d \ln w}\right|^{2} \frac{d \rho d \theta}{\rho} .
$$

By (2.9) we get

$$
\left|\frac{d \ln z}{d \ln w}\right|^{2} \leq\left|\frac{w}{z}\right|^{2} \max \left|\frac{d z}{d w}\right|^{2}=\left|\frac{w}{z}\right|^{2} p(w, z) J[z(w)]=p(z, w) \frac{d \sigma(\ln z)}{d \sigma(\ln w)} .
$$

Thus, we have

$$
\int_{\rho_{1}}^{\rho_{2}} \int_{0}^{2 \pi} p(z, w) \frac{d \sigma(\ln z)}{d \sigma(\ln w)} \frac{d \rho d \theta}{\rho} \geq 2 \pi \ln \frac{\rho_{2}}{\rho_{1}}+\frac{2}{\pi} \int_{\rho_{1}}^{\rho_{2}} \frac{\omega^{2}(\rho)}{\rho} d \rho .
$$

But

$$
\begin{aligned}
\int_{\rho_{1}}^{\rho_{2}} \int_{0}^{2 \pi} & p(z, w) \frac{d \sigma(\ln z)}{d \sigma(\ln w)} d \sigma(\ln w) \leq \iint_{r_{1}\left(\rho_{1}\right) \leq|z| \leq r_{2}\left(\rho_{2}\right)} p(z, w) d \sigma(\ln z) \\
& =\iint_{r_{1}\left(\rho_{1}\right) \leq|z| \leq r_{2}\left(\rho_{2}\right)}\{p(z, w)-1\} d \sigma(\ln z)+\iint_{r_{1}\left(\rho_{1}\right) \leq|z| \leq r_{2}\left(\rho_{2}\right)} d \sigma(\ln z) \\
& \leq \iint_{r_{1}\left(\rho_{1}\right) \leq|z|<\infty}\{p(z, w)-1\} d \sigma(\ln z)+2 \pi \ln \frac{r_{2}\left(\rho_{2}\right)}{r_{1}\left(\rho_{1}\right)} \\
& =h\left(r_{1}\left(\rho_{1}\right)\right)+2 \pi\left(\ln \frac{r_{1}\left(\rho_{2}\right)}{r_{1}\left(\rho_{1}\right)}+\omega\left(\rho_{2}\right)\right) .
\end{aligned}
$$

Substituting this inequality into (3.5) we get

$$
\ln \frac{r_{1}\left(\rho_{2}\right)}{r_{1}\left(\rho_{1}\right)}+\omega\left(\rho_{2}\right)+\frac{1}{2 \pi} h\left(r_{1}\left(\rho_{1}\right)\right) \geq \ln \frac{\rho_{2}}{\rho_{1}}+\frac{1}{\pi^{2}} \int_{\rho_{1}}^{\rho_{2}} \frac{\omega^{2}(\rho)}{\rho} d \rho,
$$

that is,

$$
\begin{aligned}
Q\left(\rho_{1}, \rho_{2}\right) & \geq \frac{1}{\pi^{2}} \int_{\rho_{1}}^{\rho_{2}} \frac{\omega^{2}(\rho)}{\rho} d \rho-\omega\left(\rho_{2}\right)-\frac{1}{2 \pi} h\left(r_{1}\left(\rho_{1}\right)\right) \\
& \geq-\omega\left(\rho_{2}\right)-\frac{1}{2 \pi} h\left(r_{1}\left(\rho_{1}\right)\right) .
\end{aligned}
$$

Denote the function $h\left(r_{1}(\rho)\right), \rho_{0} \leq \rho<\infty$, by $h_{1}(\rho)$. It is clear that the function $h_{1}(\rho)$ monotonically tends to zero as $\rho \rightarrow \infty$.

Assume that $r_{1}\left(\rho_{2}\right)>r_{2}\left(\rho_{1}\right)$. Letting $r^{\prime \prime}=r_{1}\left(\rho_{2}\right)$ and $r^{\prime}=r_{2}\left(\rho_{1}\right)$ in (3.4) and taking into account

$$
\iint_{r_{2}\left(\rho_{1}\right)<|z|<r_{1}\left(\rho_{2}\right)} \frac{d \sigma(\ln w)}{d \sigma(\ln z)} d \sigma(\ln z) \leq \iint_{\rho_{1}<|w|<\rho_{2}} d \sigma(\ln w)=2 \pi \ln \frac{\rho_{2}}{\rho_{1}}
$$


we find that

$$
\begin{gathered}
\left(2 \pi \ln \frac{r_{1}\left(\rho_{2}\right)}{r_{2}\left(\rho_{1}\right)}\right)^{2} \leq 2 \pi \ln \frac{\rho_{2}}{\rho_{1}}\left\{h\left(r_{2}\left(\rho_{1}\right)\right)+2 \pi \ln \frac{r_{1}\left(\rho_{2}\right)}{r_{2}\left(\rho_{1}\right)}\right\} \\
\leq 2 \pi \ln \frac{\rho_{2}}{\rho_{1}}\left\{h_{1}\left(\rho_{1}\right)+2 \pi \ln \frac{r_{1}\left(\rho_{2}\right)}{r_{2}\left(\rho_{1}\right)}\right\} \\
2 \pi \ln \frac{\rho_{2}}{\rho_{1}} \geq \frac{\left(2 \pi \ln \frac{r_{1}\left(\rho_{2}\right)}{r_{2}\left(\rho_{1}\right)}\right)^{2}}{2 \pi \ln \frac{r_{1}\left(\rho_{2}\right)}{r_{2}\left(\rho_{1}\right)}+h_{1}\left(\rho_{1}\right)} \geq 2 \pi \ln \frac{r_{1}\left(\rho_{2}\right)}{r_{2}\left(\rho_{1}\right)}-h_{1}\left(\rho_{1}\right), \\
\ln \frac{\rho_{2}}{\rho_{1}} \geq \ln \frac{r_{1}\left(\rho_{2}\right)}{r_{2}\left(\rho_{1}\right)}-\frac{1}{2 \pi} h_{1}\left(\rho_{1}\right)=\ln \frac{r_{1}\left(\rho_{2}\right)}{r_{1}\left(\rho_{1}\right)}-\omega\left(\rho_{1}\right)-\frac{1}{2 \pi} h_{1}\left(\rho_{1}\right),
\end{gathered}
$$

that is,

$$
Q\left(\rho_{1}, \rho_{2}\right) \leq \omega\left(\rho_{1}\right)+\frac{1}{2 \pi} h_{1}\left(\rho_{1}\right) .
$$

It is clear that the inequality (3.9) remains true even when $r_{1}\left(\rho_{2}\right) \leq r_{2}\left(\rho_{1}\right)$. The inequalities (3.7) and (3.9) imply that for arbitrary $\rho_{1}$ and $\rho_{2}$ satisfying $\rho_{0} \leq \rho_{1} \leq$ $\rho_{2}<\infty$, the inequality

$$
\left|Q\left(\rho_{1}, \rho_{2}\right)\right| \leq \max \left\{\omega\left(\rho_{1}\right), \omega\left(\rho_{2}\right)\right\}+\frac{1}{2 \pi} h_{1}\left(\rho_{1}\right)
$$

holds. Thus we have achieved the main purpose of Step II.

SteP III. Now we show that $\omega(\rho) \rightarrow 0$ as $\rho \rightarrow \infty$. First we observe that (3.6) and (3.8) imply the inequality

$$
\omega\left(\rho_{2}\right)+\omega\left(\rho_{1}\right) \geq \frac{1}{\pi^{2}} \int_{\rho_{1}}^{\rho_{2}} \frac{\omega^{2}(\rho)}{\rho} d \rho-\frac{1}{\pi} h_{1}\left(\rho_{1}\right),
$$

for all $\rho_{1}, \rho_{2}$, satisfying $\rho_{0} \leq \rho_{1} \leq \rho_{2}<\infty$. Let $\rho \geq \max \left(\rho_{0}^{2}, 2\right)$. We introduce the following notation:

$$
\begin{aligned}
\tau_{1}(\rho) & =\min _{\sqrt{\rho} \leq x \leq \rho}\left\{2 \omega(x)-\frac{1}{\pi^{2}} \int_{x}^{\rho} \frac{\omega^{2}(t)}{t} d t\right\} \\
& =2 \omega\left(\rho_{1}\right)-\frac{1}{\pi^{2}} \int_{\rho_{1}}^{\rho} \frac{\omega^{2}(t)}{t} d t, \sqrt{\rho} \leq \rho_{1} \leq \rho, \\
\tau_{2}(\rho) & =\min _{\rho \leq x \leq \rho \sqrt{\rho}}\left\{2 \omega(x)-\frac{1}{\pi^{2}} \int_{\rho}^{x} \frac{\omega^{2}(t)}{t} d t\right\} \\
& =2 \omega\left(\rho_{2}\right)-\frac{1}{\pi^{2}} \int_{\rho}^{\rho_{2}} \frac{\omega^{2}(t)}{t} d t, \rho \leq \rho_{2} \leq \rho \sqrt{\rho} .
\end{aligned}
$$


We rewrite the inequality (3.11), where $\rho_{1}$ and $\rho_{2}$ are the numbers defined in (3.12) and (3.13), in the following way:

$$
\begin{aligned}
\omega\left(\rho_{1}\right)+\omega\left(\rho_{2}\right) \leq & \left\{2 \omega\left(\rho_{2}\right)-\frac{1}{\pi^{2}} \int_{\rho}^{\rho_{2}} \frac{\omega^{2}(t)}{t} d t\right\} \\
& +\left\{2 \omega\left(\rho_{1}\right)-\frac{1}{\pi^{2}} \int_{\rho_{1}}^{\rho} \frac{\omega^{2}(t)}{t} d t\right\}+\frac{1}{\pi} h_{1}\left(\rho_{1}\right) \\
= & \tau_{1}(\rho)+\tau_{2}(\rho)+\frac{1}{\pi} h_{1}\left(\rho_{1}\right) .
\end{aligned}
$$

Now we estimate $\tau_{1}(\rho)$ and $\tau_{2}(\rho)$. Let

$$
\alpha(x, \rho)=\operatorname{sgn}(x-\rho) \frac{1}{\pi^{2}} \int_{\rho}^{x} \frac{\omega^{2}(t)}{t} d t .
$$

Assume that $\rho \sqrt{\rho} \geq x \geq \rho$ and $\tau_{2}(\rho)>0$. Then (3.13) implies that

$$
\begin{gathered}
2 \omega(x) \geq \alpha(x, \rho)+\tau_{2}(\rho), \\
\left\{\alpha(x, \rho)+\tau_{2}(\rho)\right\}^{2} \leq 4 \omega^{2}(x)=4 \pi^{2} x \frac{d \alpha(x, \rho)}{d x},
\end{gathered}
$$

whence

$$
\begin{gathered}
\frac{1}{2} \ln \rho=\int_{\rho}^{\rho \sqrt{\rho}} \frac{d x}{x} \leq 4 \pi^{2} \int_{\rho}^{\rho \sqrt{\rho}} \frac{d \alpha(x, \rho)}{\left\{\alpha(x, \rho)+\tau_{2}(\rho)\right\}^{2}} \\
\leq 4 \pi^{2} \int_{\alpha=0}^{\infty} \frac{d \alpha}{\left\{\alpha+\tau_{2}(\rho)\right\}^{2}}=\frac{4 \pi^{2}}{\tau_{2}(\rho)}, \\
\tau_{2}(\rho) \leq \frac{8 \pi^{2}}{\ln \rho} .
\end{gathered}
$$

The inequality (3.15) remains true for $\tau_{2}(\rho) \leq 0$. In a similar way we treat the case when $\sqrt{\rho} \leq x \leq \rho$ and $\tau_{1}(\rho)>0$. In this case (3.12) implies that

$$
\begin{gathered}
2 \omega(x) \geq \tau_{1}(\rho)+\alpha(x, \rho), \\
\left\{\tau_{1}(\rho)+\alpha(x, \rho)\right\}^{2} \leq 4 \omega^{2}(x)=4 \pi^{2} x \frac{d\{-\alpha(x, \rho)\}}{d x},
\end{gathered}
$$

whence

$$
\begin{gathered}
\frac{1}{2} \ln \rho=\int_{\sqrt{\rho}}^{\rho} \frac{d x}{x} \leq 4 \pi^{2} \int_{\sqrt{\rho}}^{\rho} \frac{d\{-\alpha(x, \rho)\}}{\left\{\tau_{1}(\rho)+\alpha(x, \rho)\right\}^{2}} \\
\leq 4 \pi^{2} \int_{\beta=-\infty}^{0} \frac{d \beta}{\left\{\tau_{1}(\rho)-\beta\right\}^{2}}=\frac{4 \pi^{2}}{\tau_{1}(\rho)}, \\
\tau_{1}(\rho) \leq \frac{8 \pi^{2}}{\ln \rho} .
\end{gathered}
$$

The last inequality, obviously, holds also when $\tau_{1}(\rho) \leq 0$. Substituting $(3.15)$ and (3.16) into (3.14) we get

$$
\omega\left(\rho_{1}\right)+\omega\left(\rho_{2}\right) \leq \frac{16 \pi^{2}}{\ln \rho}+\frac{1}{\pi} h_{1}\left(\rho_{1}\right) .
$$


Let $\eta>\max \left\{\omega\left(\rho_{1}\right), \omega\left(\rho_{2}\right)\right\}$, the choice of $\eta$ will make more precise later. Denote by $E$ the set of $t, \rho_{1} \leq t \leq \rho_{2}$, for which $\omega(t)>\eta$, and by $\Delta$ the logarithmic measure of this set. The inequalities (3.11) and (3.17) imply that

$$
\begin{aligned}
& \frac{16 \pi^{2}}{\ln \rho}+\frac{1}{\pi} h_{1}\left(\rho_{1}\right) \geq \frac{1}{\pi^{2}} \int_{\rho_{1}}^{\rho_{2}} \frac{\omega^{2}(\rho)}{\rho} d \rho-\frac{1}{\pi} h_{1}\left(\rho_{1}\right) \\
& \geq \frac{\eta^{2}}{\pi^{2}} \int_{E} \frac{d \rho}{\rho}-\frac{1}{\pi} h_{1}\left(\rho_{1}\right)=\frac{\eta^{2}}{\pi^{2}} \Delta-\frac{1}{\pi} h_{1}\left(\rho_{1}\right), \\
& \frac{\eta^{2}}{\pi^{2}} \Delta \leq \frac{16 \pi^{2}}{\ln \rho}+\frac{2}{\pi} h_{1}\left(\rho_{1}\right) \leq \frac{16 \pi^{2}}{\ln \rho}+\frac{2}{\pi} h_{1}(\sqrt{\rho}), \\
& \Delta \leq \frac{16 \pi^{4}}{\eta^{2} \ln \rho}+\frac{2 \pi}{\eta^{2}} h_{1}(\sqrt{\rho}) .
\end{aligned}
$$

Assume that $\omega(\rho)>\eta$ and that $\left(\rho^{\prime}, \rho^{\prime \prime}\right)$ is the component of the set $E$ containing $\rho$. Then $\rho_{1}<\rho^{\prime}<\rho<\rho^{\prime \prime}<\rho_{2}, \omega\left(\rho^{\prime}\right)=\omega\left(\rho^{\prime \prime}\right)=\eta$. The inequality (3.18) implies that

$$
\ln \frac{\rho^{\prime \prime}}{\rho^{\prime}} \leq \Delta \leq \frac{16 \pi^{4}}{\eta^{2} \ln \rho}+\frac{2 \pi}{\eta^{2}} h_{1}(\sqrt{\rho}) .
$$

It is clear that

$$
\omega(\rho) \leq \ln \frac{r_{2}\left(\rho^{\prime \prime}\right)}{r_{1}\left(\rho^{\prime}\right)}=\ln \frac{r_{1}\left(\rho^{\prime \prime}\right)}{r_{1}\left(\rho^{\prime}\right)}+\omega\left(\rho^{\prime \prime}\right)=\ln \frac{r_{1}\left(\rho^{\prime \prime}\right)}{r_{1}\left(\rho^{\prime}\right)}+\eta .
$$

Replacing in (3.8) $\rho_{1}$ and $\rho_{2}$ by $\rho^{\prime}$ and $\rho^{\prime \prime}$, respectively, we get

$$
\ln \frac{r_{1}\left(\rho^{\prime \prime}\right)}{r_{1}\left(\rho^{\prime}\right)} \leq \omega\left(\rho^{\prime}\right)+\ln \frac{\rho^{\prime \prime}}{\rho^{\prime}}+\frac{1}{2 \pi} h_{1}\left(\rho^{\prime}\right) \leq \eta+\ln \frac{\rho^{\prime \prime}}{\rho^{\prime}}+\frac{1}{2 \pi} h_{1}(\sqrt{\rho}) .
$$

The inequalities (3.19), (3.20), and (3.21) imply that

$$
\omega(\rho) \leq 2 \eta+\frac{1}{\eta^{2}}\left\{\frac{16 \pi^{4}}{\ln \rho}+2 \pi h_{1}(\sqrt{\rho})\right\}+\frac{1}{2 \pi} h_{1}(\sqrt{\rho}) .
$$

It is clear that the inequality (3.22) holds also when $\omega(\rho) \leq \eta$. Now we choose $\eta$ in such a way that the right-hand side of the inequality (3.22) takes the least possible value. If $A>0$, then the function $2 \eta+A \eta^{-2}$, for positive $\eta$ takes the least value $3 \sqrt[3]{A}$, achieved for $\eta=\sqrt[3]{A}$. We should let

$$
\eta=\sqrt[3]{\frac{16 \pi^{4}}{\ln \rho}+2 \pi h_{1}(\sqrt{\rho})},
$$

we have right to do so if the inequality $\eta>\max \left\{\omega\left(\rho_{1}\right), \omega\left(\rho_{2}\right)\right\}$ holds. But the inequality (3.17) implies that it is sufficient that the inequality

$$
\frac{16 \pi^{2}}{\ln \rho}+\frac{1}{\pi} h_{1}(\sqrt{\rho})<\sqrt[3]{\frac{16 \pi^{4}}{\ln \rho}+2 \pi h_{1}(\sqrt{\rho})}
$$

holds, it is obviously the case when the expression under the radical sign is less than 1 , that is, when $\rho>\bar{\rho}$, where $\bar{\rho} \geq \max \left(\rho_{0}^{2}, 2\right)$ is sufficiently large. Thus for $\rho>\bar{\rho}$ we choose $\eta$ according to (3.23), substituting it into (3.22), we get

$$
\omega(\rho) \leq \sqrt[3]{\frac{16 \pi^{4}}{\ln \rho}+2 \pi h_{1}(\sqrt{\rho})}+\frac{1}{2 \pi} h_{1}(\sqrt{\rho}),
$$


whence $\omega(\rho) \rightarrow 0$ as $\rho \rightarrow \infty$.

Now we can complete the proof.

STEP IV. Let again $\rho_{1}$ and $\rho_{2}$ be arbitrary numbers satisfying $\rho_{0} \leq \rho_{1} \leq \rho_{2}<$ $\infty$. We see that the right-hand side of the inequality (3.10) tends to 0 as $\rho_{1} \rightarrow \infty$, $\rho_{2} \geq \rho_{1}$. By the Bolzano-Cauchy criterion there exists a finite limit

$$
\lim _{\rho \rightarrow \infty} \ln \frac{r_{1}(\rho)}{\rho}=B=e^{\alpha},
$$

that is, there exists the limit

$$
\lim _{\rho \rightarrow \infty} \frac{r_{1}(\rho)}{\rho}=B=e^{\alpha}, 0<B<\infty .
$$

Since

$$
\frac{r_{2}(\rho)}{\rho}=\frac{r_{1}(\rho)}{\rho} e^{\omega(\rho)} \rightarrow B, \rho \rightarrow \infty
$$

we have

$$
\lim _{w \rightarrow \infty} \frac{|z(w)|}{|w|}=B
$$

from where the equality (3.2) with $A=1 / B$ follows.

\section{Riemann surfaces of the class $F_{q}$ and line complexes}

Surfaces of the so-called class $F_{q}=F_{q}\left(a_{1}, a_{2}, \ldots, a_{q}\right)$ have a particularly simple structure among all Riemann surfaces. Let us describe this class. Let $a_{1}, \ldots, a_{q}$ be distinct $q \geq 2$ points in the extended complex $w$-plane, and let $L$ be a closed Jordan curve passing through the points $a_{1}, \ldots, a_{q}$. Without loss of generality we assume, for simplicity of the description, that $a_{j} \neq \infty, 1 \leq j \leq q$, and $L$ does not pass through $w=\infty$. We assume that the points $a_{j}$ are labelled in such a way that they are arranged on $L$ according to the positive orientation, $a_{q+1}=a_{1}, a_{q}=a_{0}$. The curve $L$ divides the extended $w$-plane into two simply connected regions: the interior $H_{i}$ and the exterior $H_{e}$, which will be called half-sheets. The part of the closed Jordan curve $L$ lying between the points $a_{j}$ and $a_{j+1}$, excluding these points, will be denoted by $L_{j}$. The regions obtained from the closed regions $\bar{H}_{i}$ and $\bar{H}_{e}$ after removal of the points $a_{j}$ will be denoted by $H_{i}^{\circ}$ and $H_{e}^{\circ}$, respectively.

Consider a Riemann surface $F_{0}$ consisting of a finite or countable set of halfsheets, which are "pasted" in such a way that for each half-sheet $H_{i}\left(H_{e}\right)$ and each arc $L_{j}, 1 \leq j \leq q$, there is a half-sheet $H_{e}\left(H_{i}\right)$ which is pasted with a given halfsheet along the arc $L_{j}$ (that is, the points of $L_{j}$ from the half-sheets $H_{i}^{\circ}$ and $H_{e}^{\circ}$ are identified).

We fix some point $a \in H_{i}$ and some point $b \in H_{e}$. Denote by $C_{j}$ a Jordan arc joining the points $a$ and $b$ in the extended complex $w$-plane and intersecting the curve $L$ only at one point lying on $L_{j}$. Besides, we require that the curves $C_{j}, 1 \leq j \leq q$ intersect each other at the ends $a$ and $b$ only. Let $\tilde{a}$ and $\tilde{b}$ be points from $F_{0}$ that project to $a$ and $b$, respectively, and let $\tilde{C}_{j}$ be curves whose projections are $C_{j}$. It is clear that the disjoint curves $\tilde{C}_{j}$ establish a one-to-one correspondence between their ends $\tilde{a}$ and $\tilde{b}$. As a result, for fixed $j$, we get a oneto-one correspondence between the points $\tilde{a}$ and and the points $\tilde{b}$. It follows that the (finite or countable) numbers of half-sheets $H_{i}$ and $H_{e}$ in $F_{0}$ are the same. For 
uniformity of notation we denote the half-sheets contained in $F_{0}$, whose projections are $H_{i}$ and $H_{e}$, by $\tilde{H}_{i}$ and $\tilde{H}_{e}$, respectively.

Consider the open set in $F_{0}$ whose projection is the region $D_{j}$ bounded by the curves $C_{j-1}$ and $C_{j}\left(1 \leq j \leq q, C_{0}=C_{q}, C_{q+1}=C_{1}\right)$ and containing the point $a_{j}$. This set is decomposed into connected components $\tilde{D}_{j}^{(k)}$ which can be finite-sheeted or infinite-sheeted. If a component $\tilde{D}_{j}^{(k)}$ is $n$-sheeted, $1 \leq n<\infty$, then the part of its boundary whose projection is $C_{j-1} \cup C_{j}$ is a closed Jordan curve consisting of $n$ $\operatorname{arcs} \tilde{C}_{j}$ and $n \operatorname{arcs} \tilde{C}_{j-1}$, which are alternating as we go around the Jordan curve. It is clear that the function $z=\left(\frac{w-a_{j}}{w-b_{j}}\right)^{1 / n}, b_{j} \notin \bar{D}_{j}$, maps $\tilde{D}_{j}^{(k)}$ bijectively onto some doubly-connected region in the $z$-plane, whose boundary consists of some closed Jordan curve and the point $z=0$. If we add to $\tilde{D}_{j}^{(k)}$ (and $F_{0}$ ) a point whose projection is $a_{j}$, then $\tilde{D}_{j}^{(k)}$ becomes a simply connected one-sheeted region if $n=1$, and it becomes a simply connected neighborhood of an algebraic ramification point of order $n-1$, whose projection coincides with $a_{j}$. We denote by $F_{0}^{\prime}$ the surface $F_{0}$ complemented in the described way by algebraic ramification points. If the component $\tilde{D}_{j}^{(k)}$ is infinite-sheeted, then the part of its boundary whose projection coincides with $C_{j-1} \cup C_{j}$ is an open Jordan curve consisting of infinitely many arcs $\tilde{C}_{j}$ and $\tilde{C}_{j-1}$, which are alternating as we go around the Jordan curve. It is easy to see that the function $z=\ln \left\{\left(w-a_{j}\right) /\left(w-b_{j}\right)\right\}, b_{j} \notin \bar{D}_{j}$, maps $\tilde{D}_{j}^{(k)}$ bijectively onto some simply connected region in the $z$-plane, which is lying to the left of some open Jordan arc located in a vertical strip and such that $\operatorname{Im} z \rightarrow+\infty$ and $\operatorname{Im} z \rightarrow-\infty$ as $z$ moves along the curve in one or the other direction. It is clear that $\tilde{D}_{j}^{(k)}$ is a neighborhood of a logarithmic ramification point of the surface $F_{0}$ (and $F_{0}^{\prime}$ ) whose projection is $a_{j}$.

If the surface $F_{0}^{\prime}$ is simply connected, we say that $F_{0}^{\prime}$ belongs to the class $F_{q}$.

It is clear that the condition $\infty \notin L$ was imposed only for the sake of simplicity of the verbal description of the construction of surfaces of the class $F_{q}$. If $\infty \in L$, then, to define $H_{i}$ and $H_{e}$, we need only to orient the curve $L$ beforehand, and writing the functions mapping $\tilde{D}_{j}^{(k)}$ take into account the possibility of $a_{j}=\infty$.

The curve $L$ will be called a base curve, and the points $a_{1}, \ldots, a_{q}$ will be called base points.

Finite-sheeted surfaces $F_{0}^{\prime}$ are obviously closed and, if they belong to the class $F_{q}$, they have genus zero. It is easy to see that, conversely, each closed Riemann surface of genus zero belongs to the class $F_{q}$. In fact, a closed Riemann surface $F$ of genus zero is topologically equivalent to the extended complex plane and hence is simply connected. We draw a closed Jordan curve $L$ through the projections $a_{1}, \ldots, a_{q}$ of the algebraic ramification points, and then make cuts of $F$ along all curves whose projections are in $L$. The surface $F$ decomposes into a finite number of simply connected regions which will play the role of half-sheets $\tilde{H}_{i}$ and $\tilde{H}_{e}$. It is easy to see that in order to reconstruct the surface $F$ from these half-sheets we have to paste the half-sheets $\tilde{H}_{i}$ and $\tilde{H}_{e}$ in the same way as it was done in the description of surfaces from $F_{q}$.

Open simply connected Riemann surfaces do not have to belong to a class $F_{q}$. Take, for example, the Riemann surface corresponding to the function $w=e^{z}-z$. 
The derivative of this entire function has zeros of order one at the points $z_{k}=2 k \pi i$, $k=0, \pm 1, \pm 2, \ldots$, hence the Riemann surface has algebraic ramification points of the first order, whose projections are the points $w_{k}=1-2 k \pi i$. Since the set of all such points $w_{k}$ is infinite, the surface cannot belong to the class $F_{q}$. It is easy to see that if an open surface $F$ of parabolic type belongs to the class $F_{q}$, then the mapping meromorphic function $w=f(z)$ can have multiple $a$-points or have $a$ as an asymptotic value only when $a$ is one of the base points. In this case asymptotic spots of $f(z)$ correspond bijectively to logarithmic branch points of $F$.

Let us show that, conversely, if a meromorphic in $\{|z|<\infty\}$ function $w=f(z)$ has multiple $a$-points or asymptotic values $a$ only for a finite set $a=a_{1}, \ldots, a_{q}$, then the Riemann surface $F$ corresponding to the function $f(z)$ belongs to the class $F_{q}\left(a_{1}, \ldots, a_{q}\right)$.

Let $\Delta$ be an arbitrary simply connected region in the extended $w$-plane which does not contain the points $a_{1}, \ldots, a_{q}$. Let $w_{0} \in \Delta$, and let $K$ be an arbitrary continuous curve lying in $\Delta$ and having $w_{0}$ as its starting point. We show that an arbitrary analytic element $z=P_{j}\left(w, w_{0}\right)$ of the function $z=f^{-1}(w)$ centered at the point $w_{0}$ admits an analytic continuation along the curve $K$. Suppose that $P_{j}\left(w, w_{0}\right)$ has an analytic continuation up to the point $w_{1} \in K$, excluding the point $w_{1}$. Let $K_{1}$ be the part of the curve $K$ between $w_{0}$ and $w_{1}, K_{1}: w=w(t), 0 \leq t \leq 1$, $w(0)=w_{0}, w(1)=w_{1}$, we denote by $K_{1}^{\prime}$ the curve $K_{1}$ without the point $w_{1}$, $K_{1}^{\prime}: w=w(t), 0 \leq t<1$. Let $Q: z=z(t), 0 \leq t<1$ be the continuous curve in $\{|z|<\infty\}$ corresponding to $K_{1}^{\prime}$ under the mapping based on the analytic continuation of $z=P_{j}\left(w, w_{0}\right)$. It is obvious that $f(z(t)) \rightarrow w_{1} \in \Delta$ as $t \rightarrow 1$.

Suppose that the function $z=z(t)$ has at least two limit points in the extended $z$-plane as $t \rightarrow 1$. Denote one of them by $z_{0}, z_{0} \neq \infty$. It is clear that $f\left(z_{0}\right)=w_{1}$. Then $f(z) \neq w_{1}$ if $z$ is in a sufficiently small neighborhood of $z_{0}$ and $z \neq z_{0}$. Let $\eta>0$ be so small that the circle $\left\{\left|z-z_{0}\right|=\eta\right\}$ is contained in this neighborhood and at least one of the limit points of $z(t)$ is outside the circle. The curve $Q$ intersects the circle $\left\{\left|z-z_{0}\right|=\eta\right\}$ infinitely many times. Hence there exists a sequence $t_{n} \rightarrow 1$ such that $z\left(t_{n}\right) \rightarrow z_{1},\left|z\left(t_{n}\right)-z_{0}\right|=\eta$. Then $f\left(z_{1}\right)=w_{1}$, we get a contradiction with the condition that $f(z) \neq w_{1}$ on the circle $\left\{\left|z-z_{0}\right|=\eta\right\}$. Hence the function $z(t)$ has a (finite or infinite) limit as $t \rightarrow 1$.

Let $z(t) \rightarrow z_{0} \neq \infty$ as $t \rightarrow 1$. Then $f\left(z_{0}\right)=w_{1}$, moreover, $z_{0}$ is not a multiple $w_{1}$-point of the function $f(z)$, since otherwise the point $z_{0}$ would be mapped onto an algebraic ramification point on $F$, whose projection is $w_{1} \neq a_{j}, 1 \leq j \leq q$. Since the function $f(z)$ is univalent in a sufficiently small neighborhood of the point $z_{0}$, continuing the element $P_{j}\left(w, w_{0}\right)$ along $K_{1}^{\prime}$ we can continue it to the point $w_{1} \in K_{1}$, getting an analytic element $P_{j}\left(w, w_{1}\right), P_{j}\left(w_{1}, w_{1}\right)=z_{0}$. We contradicts the assumption.

If $z(t) \rightarrow \infty$ as $t \rightarrow 1$, then the curve $Q$ is an asymptotic curve for the function $f(z)$, and $w_{1}$ is an asymptotic value, but again, it is impossible because $w_{1} \neq a_{j}$, $1 \leq j \leq q$.

Thus, we have shown that the analytic element $P_{j}\left(w, w_{0}\right)$ admits an analytic continuation along an arbitrary continuous curve in $\Delta$, hence, by the monodromy theorem (A.I. Markushevich [Mar77, Vol. 3, Ch.VIII,§40], S. Stoïlow [Sto62, Vol. 1 , Ch. IV $, \S 1,6]^{5}$ the function obtained as a result of such analytic continuation is defined in the region $\Delta$ and is single valued in it. In other words, the set of points

\footnotetext{
${ }^{5}$ Or L. Ahlfors, Complex Analysis, Ch. 8, 1.6.
} 
of the Riemann surface $F$ which are projected into $\Delta$ decomposes into infinitely many one-sheeted regions, forming a complete covering of the region $\Delta$.

We draw a closed Jordan curve $L$ through the points $a_{1}, \ldots, a_{q}$. The curve $L$ divides the extended $w$-plane into two simply connected regions $H_{i}$ and $H_{e}$. Onesheeted regions on $F$ forming a complete covering of $H_{i}$ and $H_{e}$ make up half-sheets $\tilde{H}_{i}$ and $\tilde{H}_{e}$. Since for fixed $j, 1 \leq j \leq q$, the region $H_{i} \cup L_{j} \cup H_{e}$ is also simply connected and the parts of the surface $F$ forming a complete covering of this region are one-sheeted, each half-sheet $\tilde{H}_{i}\left(\tilde{H}_{e}\right)$ is pasted along each the arc $\tilde{C}_{j}$ to one half-sheet $\tilde{H}_{e}\left(\tilde{H}_{i}\right)$. Hence the surface $F$ belongs to the class $F_{q}$.

The discussion above shows, in particular, that in the case when $F$ is a closed Riemann surface of genus zero or an open Riemann surface of parabolic type, then belonging of $F$ to the class $F_{q}$ does not depend on the choice of the base curve $L$, since we gave a complete characterization of mapping meromorphic functions which does not involve base curves. Similar conclusion can be derived for surfaces of hyperbolic type.

The Riemann surfaces corresponding to the functions (1) $w=e^{z}$ (base points $\left.a_{1}=0, a_{2}=\infty\right) ;(2) w=e^{e^{z}}$ (base points $\left.a_{1}=0, a_{2}=1, a_{3}=\infty\right) ;(3) w=\sin z$ (base points $a_{1}=-1, a_{2}=1, a_{3}=\infty$ ) are examples of open Riemann surfaces from the class $F_{q}$.

Riemann surfaces of the class $F_{q}$ can be conveniently presented using so-called line complexes. By a line complex $B$ of order $q, 2 \leq q<\infty$, we mean a connected set in the extended $z$-plane having the following properties:

(1) $B$ consists of a finite or countable set of Jordan arcs which are called edges, and their ends are called vertices. Two different edges can intersect at vertices only.

(2) All vertices are divided into two classes: internal and external. Each edge has, as its ends, one external vertex and one internal vertex.

(3) Edges are labelled using numbers from 1 to $q$. Each vertex is an end of $q$ edges, which are located around the edge according to their labels, the order is positive (anti-clockwise) if the vertex is interior and is negative if the vertex is exterior.

(4) If $B$ contains infinitely many vertices, then $B$ does not contain $\infty$ and $z=\infty$ is the only limit point of vertices.

Line complexes $B_{1}$ and $B_{2}$ are regarded as the same if one of them can be mapped onto the other by a homeomorphism of the extended $z$-plane, which maps edges to edges with the same label, internal vertices onto internal vertices, and external vertices onto external vertices.

The complement of $B$ in $\{|z| \leq \infty\}$, if $B$ has a finite number of vertices, and the complement of $B$ in $\{|z|<\infty\}$, if $B$ has infinitely many vertices, is an open set. Each component of this set is a region bounded by either a finite set of edges (elementary algebraic region), or by an infinite set of edges (elementary logarithmic region). By the condition (4) elementary logarithmic regions are unbounded. It is easy to see that when we go around an elementary region edges with labels $j-1$ and $j(1 \leq j \leq q$, the label 0 is equivalent to the label $q$, the label $q+1$ is equivalent to the label 1) alternate. Such elementary region is called a $j$-region. It is clear that the boundary of an algebraic elementary region consists of an even number of edges. Conversely, it is easy to see that each Jordan curve on $B$, closed or approaching 
$z=\infty$ and consisting of alternating edges with labels $j-1$ and $j$ is a boundary of a $j$-region.

Several edges with consecutive labels, having common ends lying in one internal and one external vertices form a so-called edge bundle. Clearly, two edges belonging to the same edge bundle and having labels $j-1$ and $j$ form a boundary of a digon.

Consider the class of Riemann surfaces $F_{q}\left(a_{1}, \ldots, a_{q}\right)$ and fix a base curve $L$. Now we describe a one-to-one correspondence between Riemann surfaces $F \in$ $F_{q}\left(a_{1}, \ldots, a_{q}\right)$ and line complexes $B$.

$1^{\circ}$. Let a Riemann surface $F \in F_{q}$ be given. If $F$ is open, we map it homeomorphically onto the complex $z$-plane, if $F$ is closed, we map it homeomorphically onto the extended complex $z$-plane. Then the set of points on $F$, whose projections are in $\bigcup_{j=1}^{q} C_{j}$ is mapped onto a line complex $B$, moreover, $\tilde{C}_{j}$ are mapped onto edges with label $j$, points $\tilde{a}$ are mapped onto internal vertices, and points $\tilde{b}$ are mapped onto external vertices. It is easy to check that the conditions (1)-(4) are satisfied. Hence we get a line complex. This complex is uniquely determined by $F$ and by the base curve. In fact, if we chose points $a^{\prime} \in H_{i}, b^{\prime} \in H_{e}$, and curves $C_{j}^{\prime}, 1 \leq j \leq p$, differently, then, as is easy to see that it is possible to map the extended complex $w$-plane homeomorphically onto itself ${ }^{6}$ in such a way that the points $a_{j}, 1 \leq j \leq p$, are fixed, $L$ is mapped onto $L, a^{\prime}$ onto $a, b^{\prime}$ onto $b$, and $C_{j}^{\prime}$ onto $C_{j}$. If we map all half-sheets $\tilde{H}_{i}$ and $\tilde{H}_{e}$, and all curves $\tilde{L}_{j}$ using the same homeomorphism, the Riemann surface $F$ will be mapped homeomorphically onto itself, moreover, points $\tilde{a}^{\prime}$ will be mapped on $\tilde{a}$, point $\tilde{b}^{\prime}$ will be mapped onto $\tilde{b}$, and curves $\tilde{C}_{j}^{\prime}$ will be mapped onto the curves $C_{j}$. Hence the line complex $B$, corresponding to the surface $F$, does not depend on the choice of curves $C_{j}$, and hence is uniquely determined by the surface $F$. We would like to mention that there exist examples (see V.G. Tairova [Tai62], [Tai64]) showing that with different choices of the base curve, the line complexes corresponding to the same Riemann surface $F \in F_{q}$ can be different.

$2^{\circ}$. Now suppose that we are given a line complex $B$. Since we a given a base curve $L$, the regions $H_{i}$ and $H_{e}$ are determined. We consider a one-to-one correspondence which maps each internal vertex of $B$ onto a copy of the region $H_{i}$ and each external vertex of $B$ onto a copy of the region $H_{e}$. We paste copies of $H_{i}$ and $H_{e}$ along the arc $L_{j}, 1 \leq j \leq q$, if an only if the corresponding internal and external vertices are the ends of the same edge from $B$ with label $j$. By the property (3) of line complexes we get a Riemann surface $F_{0}$ (see above). As before, we can choose points $a \in H_{i}, b \in H_{e}$, and curves $C_{j}$, define regions $\tilde{D}_{j}^{(k)}$, and complement the surface $F_{0}$ to the surface $F_{0}^{\prime}$. Now we need to show that the surface $F_{0}^{\prime}$ is simply connected.

Mark in each algebraic $j$-region of the line complex $B$ a point $a_{j}^{\prime}$; if the $j$-region is logarithmic, we place the corresponding point $a_{j}^{\prime}$ into the boundary point $z=\infty$. Each edge with label $j$ is contained in the boundary of one $(j-1)$-region and one $j$-region. We join the corresponding points $a_{j-1}^{\prime}$ and $a_{j}^{\prime}$ by a Jordan arc $l_{j}$ which

\footnotetext{
${ }^{6}$ Here and later on we use the following fact, without mentioning it explicitly. A homeomorphic mapping between boundaries of two regions bounded by closed Jordan curves can be extended to a homeomorphic mapping between the regions. In the case when the regions are unit discs, the statement is obvious. The general case can be reduced to this special case by mapping the regions conformally onto the unit disc.
} 
is contained in the union of the considered $(j-1)$-region and $j$-region, except for one point on the edge with label $j$ and, possibly, the points $a_{j-1}^{\prime}$ and $a_{j}^{\prime}$, if one or both of them coincide with $\infty$. We draw these Jordan arcs in such a way that they are pairwise disjoint, it is easy to see that it is always possible. Then the $z$-plane (the extended $z$-plane if the number of vertices is finite) is divided into regions $S$, each of which contains one vertex, and its boundary consists of $q$ Jordan curves $l_{j}$ with ends at points $a_{j}^{\prime}$ and $a_{j+1}^{\prime}$, where, as the index increases, the points $a_{1}^{\prime}, \ldots, a_{q}^{\prime}$ follow along the boundary in the positive direction if the vertex is internal, and in the negative direction if the vertex is external. We established a one-to-one correspondence between half-sheets $\tilde{H}_{i}\left(\tilde{H}_{e}\right)$ of our surface $F_{0}^{\prime}$ and internal (external) vertices. We map homeomorphically each half-sheet $\tilde{H}_{i}$ or $\tilde{H}_{e}$ onto the region $S$ containing the corresponding vertex in such a way that the mapping is continuous on the closure of these half-sheets, and in addition the boundary $\operatorname{arcs} \tilde{L}_{j}$ are mapped onto the $\operatorname{arcs} l_{j}$, and the points $\tilde{a}_{j}$ on the boundary $\tilde{H}_{i}$ or $\tilde{H}_{e}$ are mapped onto the points $a_{j}^{\prime}$. Observe that a point $a_{j}^{\prime}$, corresponding to a point $\tilde{a}_{j} \in F_{0}^{\prime}$, lies inside an algebraic $j$-region. The constructed mapping $F_{0}^{\prime}$ onto the (possibly extended) $z$-plane is not, generally speaking, single valued on $\operatorname{arcs} \tilde{L}_{j}$, since when we map half-sheets $\tilde{H}_{i}$ and $\tilde{H}_{e}$, pasted along $\tilde{L}_{j}$, we can map the same point from $\tilde{L}_{j}$ onto two different points in the $z$-plane. However, using some additional topological transformations of the closed regions $\bar{S}$ we can eliminate this multivalence. In fact, regions $S$ can be arranged into a (finite or infinite) sequence $S_{1}, S_{2}, \ldots$ such that $\bar{S}_{k}$ has at least one common $\operatorname{arc} l_{j}$ with $\bigcup_{j=1}^{k-1} \bar{S}_{j}$. This is equivalent to writing the line complex as a sequence, such that each vertex with number $k$ is joined with at least one of the vertices having smaller number by an edge. Since the line complex is connected, we can always choose such a sequence of vertices. No we subject $\bar{S}_{2}$ to an additional topological transformation, mapping $\bar{S}_{2}$ onto $\bar{S}_{2}$ in such a way that all points $a_{j}^{\prime} \in \partial S_{2}$ remain fixed, and the arcs $l_{j}$ belonging to $\partial S_{1} \cap \partial S_{2}$ are transformed in such a way that points on the corresponding $\operatorname{arcs} \tilde{L}_{j} \subset F_{0}^{\prime}$ will have the same image in the $z$-plane both under the mapping onto $\partial S_{1}$ and the mapping onto $\partial S_{2}$. Then we transform $\bar{S}_{3}$ topologically into $\bar{S}_{3}$ in such a way that all points $a_{j}^{\prime} \in \partial S_{3}$ remain fixed, and $\operatorname{arcs} l_{j}$ belonging to $\partial\left(\bar{S}_{1} \cup \bar{S}_{2}\right) \cap \partial S_{3}$ are transformed as above. Continuing this process, we get a topological transformation of the whole Riemann surface $F_{0}^{\prime}$ onto $\{|z|<\infty\}$ or $\{|z| \leq \infty\}$ depending on whether $F_{0}^{\prime}$ is a closed or open surface. Thus, the surface is simply connected and belongs to the class $F_{q}$.

It is easy to see that if we use the rule described in $1^{\circ}$ to get a line complex $B_{1}$ corresponding to the obtained Riemann surface $F_{0}^{\prime}$, the complex $B_{1}$ would coincide with the line complex $B$ with which we started our construction of the surface $F_{0}^{\prime}$.

Thus, we established a one-to-one (for a fixed base curve $L$ ) correspondence between Riemann surfaces of the class $F_{q}$ and line complexes. The line complex corresponding to a Riemann surface will be called the line complex of this surface. On our diagrams internal vertices of a complex will be marked by circles, external vertices will be marked by crosses. The label of an elementary region (the point $a_{j}$ ) will be written inside the region. We will not write labels inside digons. It is clear, that if on a line complex the labels of elementary regions are marked, it is 
not necessary to label edges, since the edge between the $j$-region and the $(j+1)$ region is the edge label $j$. If some $j$-region is a digon, then, as is easy to see, the corresponding point $\tilde{a}_{j} \in F_{0}^{\prime}$ is not a ramification point. If a $j$-region is an $2 m$-gon, $2 \leq m<\infty$, then the corresponding point $\tilde{a}_{j}$ is an algebraic ramification point of order $m-1$.

We suggest the reader to analyze the following examples of line complexes of Riemann surfaces, for which the mapping functions are indicated. In all examples the base curve is the real line, $H_{i}=\{\operatorname{Im} w<0\}, H_{e}=\{\operatorname{Im} w>0\}$.

(1) $w=z^{2}$ (Fig. 5); (2) $w=z^{3}$ (Fig. 6); (3) $w=\frac{1}{2}\left(z+\frac{1}{z}\right)$ (Fig. 7);

(4) $w=\frac{1}{2}\left(z^{3}+\frac{1}{z^{3}}\right)$ (Fig. 8); (5) $w=e^{z}$ (Fig. 9); (6) $w=e^{z^{2}}$ (Fig. 10);

(7) $w=e^{z^{3}}$ (Fig. 11); (8) $w=e^{e^{z}}$ (Fig. 12); (9) $w=\sin z$ (Fig. 13); (10) $w=\sin z^{2}$

(Fig. 14); (11) $w=\cos \sqrt{z}$ (Fig. 15).

We suggest, also, to sketch the location of regions $S$. As an example we sketch the corresponding diagrams for the functions (4), (5), and (6) (Fig. 16, 17, and 18).

Now we turn our attention to closed Riemann surfaces $F$ of genus zero. Suppose that $a_{1}, \ldots, a_{q}$ are projections of their ramification points (we do not exclude the case when there are no ramification points above some point $a_{j}$ ). Suppose that $a_{j}$ is the projection of $\nu_{j}$ algebraic ramification points of $F$ with orders $\lambda_{1}\left(a_{j}\right), \ldots, \lambda_{\nu_{j}}\left(a_{j}\right)$. Let $n$ be the number of sheets of $F$ and $\bar{n}(a)$ be the number of points of the Riemann surface whose projection is $a$, here each ramification point is counted once. It is clear that $\bar{n}(a) \leq n$ for all $a$, moreover

$$
\bar{n}(a)= \begin{cases}n & \text { for } a \neq a_{j}, j=1, \ldots, q, \\ n-\lambda_{1}\left(a_{j}\right)-\cdots-\lambda_{\nu_{j}}\left(a_{j}\right) & \text { for } a=a_{j} .\end{cases}
$$

Let $B$ be the line complex of a Riemann surface $F$. Elementary regions, edges and vertices of $B$ for on the Riemann sphere a topological polyhedron. Using the wellknown Euler formula, we get the equality $K-S+E=2$, where $K$ is the number of elementary regions, $S$ is the number of edges, $E$ is the number of vertices in the line complex $B$. The number $K$ is equal to the number of all points of the surface $F$ lying over the base points $a_{1}, \ldots, a_{q}$, that is, $\sum_{j=1}^{q} \bar{n}\left(a_{j}\right)$. The number of vertices of $E$ is equal to $2 n$, where the number of both internal and external vertices is equal to $n$. Since exactly $q$ edges leave each internal vertex, moreover, each edge has as one of its ends one and only one internal vertex, we have $S=n q$. Hence

$$
\begin{gathered}
\sum_{j=1}^{n} \bar{n}\left(a_{j}\right)-n q+2 n=2, \\
2(n-1)=\sum_{j=1}^{q}\left\{n-\bar{n}\left(a_{j}\right)\right\}=\sum_{j=1}^{q} \sum_{k=1}^{\nu_{j}} \lambda_{k}\left(a_{j}\right) .
\end{gathered}
$$

The equality (4.1) represents the well-known Riemann-Hurwitz formula.

The following question arises in a natural way. Assume that we are given complex numbers $a_{1}, \ldots, a_{q}$ and natural numbers $n, \nu_{j}, \lambda_{k}\left(a_{j}\right)$, satisfying the inequality 
(4.1) and the inequalities

$$
\Lambda\left(a_{j}\right)=\sum_{k=1}^{\nu_{j}} \lambda_{k}\left(a_{j}\right) \leq n-\nu_{j}, j=1, \ldots, q .
$$

Is it possible to construct a closed Riemann surface $F$ of genus zero, which is $n$ sheeted, has $\lambda_{k}\left(a_{j}\right)$, with $\nu_{j}$ ramification points whose projection is $a_{j}$, with orders $\lambda_{k}\left(a_{j}\right), 1 \leq k \leq \nu_{j}, 1 \leq j \leq q ?$

It turns out that it is not always possible.

EXAMPLE 1. Let $n=4, q=3, \nu_{1}=\nu_{2}=2, \nu_{3}=1, \lambda_{1}\left(a_{1}\right)=\lambda_{2}\left(a_{1}\right)=$ $\lambda_{1}\left(a_{2}\right)=\lambda_{2}\left(a_{2}\right)=1, \lambda_{1}\left(a_{3}\right)=2$. It is easy to see that these numbers satisfy the relations (4.1) and (4.2), but it is impossible to construct the corresponding Riemann surface. In fact, if there were the corresponding surface, its line complex would have eight vertices, its elementary 1- and 2-regions would be quadrangles, one 3-region $G_{1}$ would be a hexagon, and the other would be a digon $G_{2}$. The boundary of the 3-region $G_{1}$ would contain six vertices, each of which is an end of some edge lying outside $G_{1}$. There are only two vertices outside $\bar{G}_{1}$. Suppose that there are two edges leaving from two vertices on the boundary of $G_{1}$ which end at one end outside $\bar{G}_{1}$. The exterior of $G_{1}$ is divided into two Jordan polygons, a quadrangle and a hexagon. The second vertex lying outside $G_{1}$ is either inside the quadrangle or inside the hexagon. Hence one of these Jordan polygons is an elementary region, adjoining to $G_{1}$ along two or four consecutive sides of $G_{1}$, which is impossible. If both ends of some edge lying outside $G_{1}$ are on the boundary of $G_{1}$, then this edge divides the exterior of $G_{1}$ into two quadrangles and, arguing as above, we get a contradiction. Hence, from each of the vertices on the boundary of $G_{1}$ there is an edge lying outside $G_{1}$ which ends at a vertex lying outside $\bar{G}_{1}$, where different edges correspond to different vertices. Thus, there are at least six vertices outside $\bar{G}_{1}$, and we again get a contradiction.

We denote by $\mathfrak{F}=\mathfrak{F}\left(n ; a_{1}: \lambda_{1}\left(a_{1}\right), \ldots, \lambda_{\nu_{1}}\left(a_{1}\right) ; \ldots ; a_{q}: \lambda_{1}\left(a_{q}\right), \ldots, \lambda_{\nu_{q}}\left(a_{q}\right)\right)$, where $a_{1}, \ldots, a_{q}$ are distinct complex numbers, $n, \nu_{j}, \lambda_{k}\left(a_{j}\right)$ are positive integers satisfying the conditions (4.1) and (4.2), the class of closed Riemann surfaces of genus zero, which are $n$-sheeted with $\nu_{j}$ ramification points of orders $\lambda_{k}\left(a_{j}\right)$, whose projections are $a_{j}$. Sometimes it will be convenient for us to allow $\lambda_{k}\left(a_{j}\right)$ to take zero values also (we will always mention this explicitly), this will mean that we require the Riemann surfaces from the class $\mathfrak{F}$ to have over $a_{j}$ at least as many unramified sheets, as many zeros are there among the numbers $\lambda_{1}\left(a_{j}\right), \ldots, \lambda_{\nu_{j}}\left(a_{j}\right)$. If there is $a_{j}: \Lambda\left(a_{j}\right)$ instead of $a_{j}: \lambda_{1}\left(a_{j}\right), \ldots, \lambda_{\nu_{j}}\left(a_{j}\right)$ in the notation of the class $\mathfrak{F}$, it means that we require only that the sum of orders of the ramification points projected into $a_{j}$ is equal to $\Lambda\left(a_{j}\right)$. It is clear that the numbers $\Lambda\left(a_{j}\right)$ should satisfy the relations

$$
\begin{gathered}
\sum_{j=1}^{q} \Lambda\left(a_{j}\right)=2(n-1), \\
\Lambda\left(a_{j}\right) \leq n-1, j=1, \ldots, q .
\end{gathered}
$$

Obviously, for $\Lambda\left(a_{j}\right)=\lambda_{1}\left(a_{j}\right)+\cdots+\lambda_{\nu_{j}}\left(a_{j}\right)$ we have

$$
\mathfrak{F}\left(n ; \ldots ; a_{j}: \lambda_{1}\left(a_{j}\right), \ldots, \lambda_{\nu_{j}}\left(a_{j}\right) ; \ldots\right) \subset \mathfrak{F}\left(n ; \ldots ; a_{j}: \Lambda\left(a_{j}\right) ; \ldots\right) .
$$

Example 1 shows that the class $\mathfrak{F}\left(4 ; a_{1}: 1,1 ; a_{2}: 1,1 ; a_{3}: 2\right)$ is empty. 
TheOREM 4.1. The classes $\mathfrak{F}\left(n ; a_{1}: \lambda_{1}\left(a_{1}\right) ; \ldots ; a_{q}: \lambda_{1}\left(a_{q}\right)\right), \lambda_{1}\left(a_{j}\right) \geq 0$, are non-empty.

Theorem 4.1 immediately implies

Corollary. The classes $\mathfrak{F}\left(n ; a_{1}: \Lambda\left(a_{1}\right) ; \ldots ; a_{q}: \Lambda\left(a_{q}\right)\right), \Lambda\left(a_{j}\right) \geq 0$, are non-empty.

Before proving Theorem 4.1 we state two more theorems which will be needed in Section 6.

First we introduce some definitions. A Jordan $\operatorname{arc} C$ on a Riemann surface $F$ (the case of one-sheeted region $F$ is not excluded) will be called a $J$-curve, if it has the following properties: (1) different points of $C$ are projected onto different points, only the ends of $C$ can be projected onto the same point; (2) $C$ does not contain algebraic ramification points of $F$, the only possible exception is the ends of $C$; (3) there exists a Jordan $\operatorname{arc} C_{1} \subset C$ with ends in the interior points of $C$ such that $C \backslash C_{1}$ consists of two line segments; (4) $C$ has a continuous curvature. In some cases by a $J$-curve we shall mean a Jordan arc satisfying (1)-(4) without one end or without both ends. Whether or not a $J$-curve includes the ends will be clear from context.

A closed Jordan curve $C$ in the plane is called a $J^{\prime}$-curve with ends at a point $a \in C$, if it has a continuous curvature at all points except, possibly, the point $a$, and in a sufficiently small neighborhood of $a$ the curve $C$ has the form of a linear angle with a vertex at the point $a$.

It is clear that the projection of an arbitrary $J$-curve on a Riemann surface is either a $J$ - or a $J^{\prime}$-curve in the plane.

TheOREM 4.2. Let $q \geq 2$, let $a_{1}, \ldots, a_{q}$ be distinct complex numbers, and let $\gamma$ be a $J$-curve joining the points $a_{1}$ and $a_{2}$, which does not pass through the points $a_{j}$, $3 \leq j \leq q$. Then each of the classes listed below contains a Riemann surface $F$, on which one can draw a $J$-curve $\Gamma$ with ends $\mathfrak{a}_{1}$ and $\mathfrak{a}_{2} \in F$, such that $\Gamma$ is projected into $\gamma$ in a one-to-one way, and $\mathfrak{a}_{j}$ is a ramification point of order $\lambda_{1}\left(a_{j}\right) \geq 0$, lying above $a_{j}, j=1,2$. The classes of Riemann surfaces mentioned above are:

(a) $\mathfrak{F}\left(n ; a_{1}: \lambda_{1}\left(a_{1}\right), \lambda_{2}\left(a_{1}\right) ; a_{2}: \lambda_{1}\left(a_{2}\right), \lambda_{2}\left(a_{2}\right) ; a_{3}: \Lambda\left(a_{3}\right) ; a_{4}: \lambda_{1}\left(a_{4}\right) ; \ldots\right.$; $\left.a_{q}: \lambda_{1}\left(a_{q}\right)\right)$

(b) $\mathfrak{F}\left(n ; a_{1}: \lambda_{1}\left(a_{1}\right), \lambda_{2}\left(a_{1}\right) ; a_{2}: \lambda_{1}\left(a_{2}\right) ; a_{3}: \lambda_{1}\left(a_{3}\right) ; \ldots ; a_{q}: \lambda_{1}\left(a_{q}\right)\right)$, where $\lambda_{1}\left(a_{1}\right) \geq 0$

(c) $\mathfrak{F}\left(n ; a_{1}: \lambda_{1}\left(a_{1}\right) ; a_{2}: \lambda_{1}\left(a_{2}\right), \lambda\left(a_{2}\right) ; a_{3}: \lambda_{1}\left(a_{3}\right) ; \ldots ; a_{q}: \lambda_{1}\left(a_{q}\right)\right)$, where $\lambda_{1}\left(a_{2}\right) \geq 0$

(d) $\mathfrak{F}\left(n ; a_{1}: \lambda_{1}\left(a_{1}\right), \lambda_{2}\left(a_{1}\right) ; a_{2}: 0, \lambda_{2}\left(a_{2}\right) ; a_{3}: \lambda_{1}\left(a_{3}\right) ; \ldots ; a_{q}: \lambda_{1}\left(a_{q}\right)\right)$;

(e) $\mathfrak{F}\left(n ; a_{1}: 0, \lambda_{2}\left(a_{1}\right) ; a_{2}: \lambda_{1}\left(a_{2}\right), \lambda_{2}\left(a_{2}\right) ; a_{3}: \lambda_{1}\left(a_{3}\right) ; \ldots ; a_{q}: \lambda_{1}\left(a_{q}\right)\right)$;

(f) $\mathfrak{F}\left(n ; a_{1}: \lambda_{1}\left(a_{1}\right) ; a_{2}: \lambda_{1}\left(a_{2}\right) ; a_{3}: \lambda_{1}\left(a_{3}\right) ; \ldots ; a_{q}: \lambda_{1}\left(a_{q}\right)\right)$ where $\lambda_{1}\left(a_{1}\right) \geq 0, \lambda_{1}\left(a_{2}\right) \geq 0$

(g) $\mathfrak{F}\left(n ; a_{1}: \lambda_{1}\left(a_{1}\right), \lambda_{2}\left(a_{1}\right) ; a_{2}: 0 ; a_{3}: \lambda_{1}\left(a_{3}\right) \ldots ; a_{q}: \lambda_{1}\left(a_{q}\right)\right)$, where $\lambda_{1}\left(a_{1}\right) \geq 0$

(h) $\mathfrak{F}\left(n ; a_{1}: 0 ; a_{2}: \lambda_{1}\left(a_{2}\right), \lambda_{2}\left(a_{2}\right) ; a_{3}: \lambda_{1}\left(a_{3}\right) ; \ldots ; a_{q}: \lambda_{1}\left(a_{q}\right)\right)$, where $\lambda_{1}\left(a_{2}\right) \geq 0$

(i) $\mathfrak{F}\left(n ; a_{1}: 0, \lambda_{2}\left(a_{1}\right) ; a_{2}: 0, \lambda_{2}\left(a_{2}\right) ; a_{3}: \lambda_{1}\left(a_{3}\right) ; \ldots ; a_{q}: \lambda_{1}\left(a_{q}\right)\right)$, where $q \geq 4$. 
TheOREM 4.3. Let $q \geq 3$, let $a_{1}, \ldots, a_{q}$ be distinct complex numbers, and let $\gamma$ be a $J^{\prime}$-curve with ends at the point $a_{1}$, moreover, let the point $a_{2}$ be inside the curve $\gamma$, and the points $a_{3}, \ldots, a_{q}$ be outside $\gamma$. Then each of the following two classes:

(j) $\mathfrak{F}\left(n ; a_{1}: \lambda_{1}\left(a_{1}\right), \lambda_{2}\left(a_{1}\right), \lambda_{3}\left(a_{1}\right) ; a_{2}: \lambda_{1}\left(a_{2}\right) ; a_{3}: \lambda_{1}\left(a_{3}\right) ; \ldots ; a_{q}: \lambda_{1}\left(a_{q}\right)\right)$, where $\lambda_{k}\left(a_{1}\right) \geq 0, k=1,2,3 ; \lambda_{1}\left(a_{2}\right) \geq 2$

(k) $\mathfrak{F}\left(n ; a_{1}: \lambda_{1}\left(a_{1}\right), \lambda_{2}\left(a_{1}\right) ; a_{2}: \lambda_{1}\left(a_{2}\right) ; \ldots ; a_{q} ; \lambda_{1}\left(a_{q}\right)\right)$, where $\lambda_{k}\left(a_{1}\right) \geq 0, k=1,2$

contains a Riemann surface $F$, on which one can draw a J-curve $\Gamma$ with ends $\mathfrak{a}_{1}$ and $\mathfrak{a}_{2} \in F$, such that the curve $\Gamma$ without ends is projected in a one-to-one way into $\gamma \backslash\left\{a_{1}\right\}$, the point $\mathfrak{a}_{k}$ is a ramification point of order $\lambda_{k}\left(a_{1}\right)$ lying over $a_{1}, k=1,2$.

Proofs of Theorems 4.1, 4.2, and 4.3 are not difficult, but they are rather cumbersome and are using topological constructions, which are far from the main topics of this book. Readers who do not wish to analyze these proofs and are ready to believe the results, can omit the proofs without harm to understanding of the subsequent results.

Before starting the proofs we fix some notation. Let $G_{1}$ be a Jordan $m_{1}$ gon, we join it with a Jordan $m_{2}$-gon, lying outside $G_{1}$ in such a way that $\partial G_{2} \cap$ $\partial G_{1}$ is a Jordan arc, consisting of $m$ Jordan arcs which are sides of $G_{1}$ and $G_{2}$, $m \leq \min \left(m_{1}-1, m_{2}-1\right)$, following one after another starting with a vertex $\alpha$ in the negative direction of the boundary of $G_{1}$. This operation will be denoted by $U\left(G_{1}, G_{2}, \alpha, m\right)$ (Fig. 19).

Proof of Theorem 4.1. The theorem is trivial if $n=1$. We assume that $n \geq 2$ and construct the corresponding line complexes. If $q=2$, then necessarily $\lambda_{1}\left(a_{1}\right)=\lambda_{1}\left(a_{2}\right)=n-1$, and the line complex is a closed Jordan curve consisting of $2 n$ edges (see, for example, Fig. 5 and 6 ). Now let $q \geq 3$. We take the region $G_{1}$ which is a Jordan $\left(2 \lambda_{1}\left(a_{1}\right)+2\right)$-gon as an elementary 1-region. We fix on the boundary of $G_{1}$ an internal vertex $\alpha$. Let $p$ be the largest integer satisfying

$$
2+2 \sum_{j=1}^{p} \lambda_{1}\left(a_{j}\right) \leq 2 n
$$

Since, by (4.1), we have

$$
2 \sum_{j=1}^{q} \lambda_{1}\left(a_{j}\right)=4(n-1),
$$

then $1<p<q$. Suppose that there is an equality in (4.3). We join to $G_{1}$ and elementary 2-region $G_{2}$, which is a Jordan $\left(2 \lambda_{1}\left(a_{2}\right)+2\right)$-gon using the operation $U\left(G_{1}, G_{2}, \alpha, 1\right)$. Denote the region $\left(\bar{G}_{1} \cup \bar{G}_{2} \backslash \partial\left(\bar{G}_{1} \cup \bar{G}_{2}\right)\right.$, which is a Jordan $\left(2 \lambda_{1}\left(a_{1}\right)+2 \lambda_{1}\left(a_{2}\right)+2\right)$-gon, by $\Delta_{2}$. We join to $\Delta_{2}$ an elementary 3 -region $G_{3}$, which is a Jordan $\left(2 \lambda_{1}\left(a_{3}\right)+2\right)$-gon, using $U\left(\Delta_{2}, G_{3}, \alpha, 1\right)$. Suppose that we have already joined an elementary $s$-region $G_{s}, 1<s \leq p-1$. Let $\Delta_{s}=\left(\bigcup_{1}^{s} \bar{G}_{j}\right) \backslash \partial\left(\bigcup_{1}^{s} \bar{G}_{j}\right)$. We join to $\Delta_{s}$ and elementary $(s+1)$-region $G_{s+1}$ which is a Jordan $\left(2 \lambda_{1}\left(a_{s+1}\right)+2\right)$ gon, using $U\left(\Delta_{s}, G_{s+1}, \alpha, 1\right)$. The boundary of $\Delta_{p}$ contains $2 \sum_{j=1}^{p} \lambda_{1}\left(a_{j}\right)+2=2 n$ vertices. We join to $\Delta_{p}$ an elementary $(p+1)$-region $G_{p+1}$, which is a Jordan $\left(2 \lambda_{1}\left(a_{p+1}\right)+2\right)$-gon, using $U\left(\Delta_{p}, G_{p+1}, \alpha, 2 \lambda_{1}\left(a_{p+1}\right)+1\right)$. The boundary of $\Delta_{p+1}$ 
contains $2 n-2 \lambda_{1}\left(a_{p+1}\right)$ vertices. Joining to $\Delta_{s}, p \leq s \leq q-2$, an elementary $(s+1)$ region $G_{s+1}$, which is a $\left(2 \lambda_{1}\left(a_{s+1}\right)+2\right)$-gon, using $U\left(\Delta_{s}, G_{s+1}, \alpha, 2 \lambda_{1}\left(a_{s+1}\right)+1\right)$, we get a Jordan region $\Delta_{s+1}$, the boundary of which contains $2 n-2 \sum_{p+1}^{s+1} \lambda_{1}\left(a_{j}\right)$ vertices. The boundary of the region $\Delta_{q-1}$ contains

$$
2 n-2 \sum_{j=p+1}^{q-1} \lambda_{1}\left(a_{1}\right)=2(n-1)-2 \sum_{j=p+1}^{q-1} \lambda_{1}\left(a_{j}\right)+2
$$

vertices. By (4.1) and the assumption that there is an equality in (4.3), we have

$$
\begin{gathered}
2 \sum_{j=1}^{p} \lambda_{1}\left(a_{j}\right)=2(n-1)=\sum_{j=1}^{q} \lambda_{1}\left(a_{j}\right), \\
\sum_{j=1}^{p} \lambda_{1}\left(a_{j}\right)=\sum_{j=p+1}^{q} \lambda_{1}\left(a_{j}\right)=n-1 .
\end{gathered}
$$

Therefore the boundary of $\Delta_{q-1}$ contains

$$
2 \sum_{j=p+1}^{q} \lambda_{1}\left(a_{j}\right)-2 \sum_{j=p+1}^{q-1} \lambda_{1}\left(a_{j}\right)+2=2 \lambda_{1}\left(a_{q}\right)+2
$$

vertices. As an elementary $q$-region $G_{q}$ we take the complement of $\bar{\Delta}_{q-1}$ in the extended complex plane.

Thus, we have constructed all needed elementary regions different from digons. Our system of edges does not yet satisfy the condition (3) from the definition of line complexes. However, it, as is easy to see, has the property that if some internal (external) vertex lies on the boundary of more than two elementary regions, then the labels of these elementary regions increase (decrease) as we go around the vertex in the positive direction. Clearly, some edges can be replaced by bundles in such a way, that we get a line complex having all required properties, that is, representing a Riemann surface belonging to the class $\mathfrak{F}\left(n ; a_{1}: \lambda_{1}\left(a_{1}\right) ; \ldots ; a_{q}: \lambda_{1}\left(a_{q}\right)\right), \lambda_{1}\left(a_{j}\right) \geq 0$.

If there is a strict inequality in (4.3), then the construction is changed at one point only. We construct the elementary 1-, 2-, ., p-regions $G_{1}, G_{2}, \ldots, G_{p}$, and the regions $\Delta_{2}, \Delta_{3}, \ldots, \Delta_{p}$ in the same way as before, but now there are $m_{1}$ vertices on the boundary of $\Delta_{p}$, where

$$
m_{1}=2+2 \sum_{j=1}^{p} \lambda_{1}\left(a_{j}\right)<2 n .
$$

On the other hand,

$$
2 \lambda_{1}\left(a_{p+1}\right)+m_{1}=2+2 \sum_{j=1}^{p+1} \lambda_{1}\left(a_{j}\right)>2 n .
$$

The inequality (4.4) implies that

$$
\lambda_{1}\left(a_{p+1}\right)+\sum_{j=1}^{p} \lambda_{1}\left(a_{j}\right)<(n-1)+(n-1)=2(n-1),
$$

therefore, by (4.1), we have $p+1<q$. Now we join to $\Delta_{p}$ and elementary $(p+1)$ region $G_{p+1}$, which is a Jordan $\left(2 \lambda_{1}\left(a_{p+1}\right)+2\right)$-gon, using $U\left(\Delta_{p}, G_{p+1}, \alpha, m_{2}+1\right)$, where $m_{2}=2 \lambda_{1}\left(a_{p+1}\right)+m_{1}-2 n$. We can use this operation since $m_{2}+1<m_{1}$ 
and $m_{2}+1<2 \lambda\left(a_{p+1}\right)+2$. Since the number $m_{2}+1$ is odd, the end of the Jordan arc $\partial \Delta_{p} \cap \partial G_{p+1}$, different from $\alpha$, is an external vertex. It is easy to count that there are $m_{1}+\left(2 \lambda_{1}\left(a_{p+1}\right)+2\right)-\left(m_{2}+2\right)=2 n$ vertices in $\bar{\Delta}_{p+1}$, moreover, there are $2 n-m_{2}$ vertices on the boundary of $\Delta_{p+1}$.

After the construction of the region $\Delta_{p+1}$, we construct the elementary regions $G_{s}$ and the regions $\Delta_{s}, p+2 \leq s \leq q-1$ in the same way as before. The boundary of $\Delta_{q-1}$ contains

$$
\begin{aligned}
2 n-m_{2} & -2 \sum_{j=p+1}^{q-1} \lambda_{1}\left(a_{j}\right)=4 n-m_{1}-2 \lambda_{1}\left(a_{p+1}\right)-2 \sum_{j=p+2}^{q-1} \lambda_{1}\left(a_{j}\right) \\
& =2\{2(n-1)\}+4-2 \sum_{j=1}^{p} \lambda_{1}\left(a_{j}\right)-2-2 \sum_{j=p+1}^{q-1} \lambda_{1}\left(a_{j}\right) \\
& =2 \sum_{j=1}^{q} \lambda_{1}\left(a_{j}\right)+2-2 \sum_{j=1}^{q-1} \lambda_{1}\left(a_{j}\right)=2 \lambda_{1}\left(a_{q}\right)+2
\end{aligned}
$$

vertices. After that the construction of the line complex is completed in the same way as in the previous case. Fig. 20 and 21 show examples of line complexes corresponding to the cases when there is an equality and the strict inequality in $(4.3)$.

REMARK. Later on we shall use the observation that in the construction that we used in the proof of Theorem 4.1 there is an internal vertex $\alpha$ which lies simultaneously on the boundaries of the elementary $j$-regions $G_{1}, G_{2}, \ldots, G_{q}$, which are Jordan $\left(2 \lambda_{1}\left(a_{j}\right)+2\right)$-gons, that is, all ramification points of the Riemann surface are lying on the boundary of one internal half-sheet.

Proof of Theorem 4.2. We draw a base curve $L$ through the points $a_{1}, \ldots$, $a_{q}$ in such a way that the curve $\gamma$, except its ends, is inside $L$. Now, obviously, it suffices to construct a line complex with prescribed ramification and satisfying the additional condition that the elementary regions corresponding to the points $\mathfrak{a}_{1}$ and $\mathfrak{a}_{2}$ have on their boundary at least one common internal vertex.

CASE (a). Since $\tau=\lambda_{1}\left(a_{1}\right)+\lambda_{2}\left(a_{1}\right)+\lambda_{1}\left(a_{2}\right)+\lambda_{2}\left(a_{2}\right)<2(n-2)<2(n-1)$, then $n \geq 3, q \geq 3$, and $\sum_{j=3}^{q} \Lambda\left(a_{j}\right) \geq 2$. Assume first that $\tau \leq n-1$. In this case we consider surfaces of the class $\mathfrak{F}\left(n ; a_{1}: \lambda_{1}\left(a_{1}\right), \lambda_{2}\left(a_{1}\right) ; a_{2}: \lambda_{1}\left(a_{2}\right), \lambda_{2}\left(a_{2}\right) ; a_{3}: \lambda_{1}\left(a_{3}\right)\right.$; $\left.\ldots ; a_{q}: \lambda_{1}\left(a_{q}\right)\right)$, where $\lambda_{1}\left(a_{3}\right)=\Lambda\left(a_{3}\right)$. Take as an elementary 1-region $G_{1}^{\prime}$ a Jordan $\left(2 \lambda_{2}\left(a_{1}\right)+2\right)$-gon and mark some internal vertex $\alpha^{\prime}$ on its boundary. We join to $G_{1}^{\prime}$ and elementary 2-region $G_{2}^{\prime}$, which is a Jordan $\left(2 \lambda_{2}\left(a_{2}\right)+2\right)$-gon using $U\left(G_{1}^{\prime}, G_{2}^{\prime}, \alpha^{\prime}, 1\right)$. Let $\beta$ be the next after $\alpha^{\prime}$ external vertex, as we go around the boundary of $G_{2}^{\prime}$ in the negative direction. We join to $\Delta^{\prime}=\left(\bar{G}_{1}^{\prime} \cup \bar{G}_{2}^{\prime}\right) \backslash \partial\left(\bar{G}_{1}^{\prime} \cup \bar{G}_{2}^{\prime}\right)$ an elementary 1-region $G_{1}^{\prime \prime}$, which is a Jordan $\left(2 \lambda_{1}\left(a_{1}\right)+2\right)$-gon, using $U\left(\Delta^{\prime}, G_{1}^{\prime \prime}, \beta, 1\right)$. Let $\alpha$ be the next after $\beta$ internal vertex, as we go around the boundary of $G_{1}^{\prime \prime}$ in the negative direction. We join to $\Delta^{\prime \prime}=\left(\bar{G}_{1}^{\prime \prime} \cup \bar{\Delta}^{\prime}\right) \backslash \partial\left(\bar{G}_{1}^{\prime \prime} \cup \bar{\Delta}^{\prime}\right)$ an elementary 2-region $G_{2}^{\prime \prime}$, which is a Jordan $\left(2 \lambda_{1}\left(a_{2}\right)+2\right)$-gon using the operation $U\left(\Delta^{\prime \prime}, G_{2}^{\prime \prime}, \alpha, 1\right)$. Let $\Delta_{2}=\left(\bar{\Delta}^{\prime \prime} \cup \bar{G}_{2}^{\prime \prime}\right) \backslash \partial\left(\bar{\Delta}^{\prime \prime} \cup \bar{G}_{2}^{\prime \prime}\right)$. It is easy to count that the boundary of $\Delta_{2}$ contains $2+2 \lambda_{1}\left(a_{1}\right)+2 \lambda_{1}\left(a_{2}\right)+2 \lambda_{2}\left(a_{1}\right)+2 \lambda_{2}\left(a_{2}\right)=2+2 \tau \leq 2 n$ vertices, and there are no vertices inside $\Delta_{2}$. After this we join to $\Delta_{2}$ successively elementary 3-, 
..., q-regions and construct a line complex in exactly the same way as in the proof of Theorem 4.1, in addition, the internal vertex $\alpha$ is lying on the boundary of the elementary regions $G_{1}^{\prime \prime}, G_{2}^{\prime \prime}, G_{3}, \ldots, G_{q}$ (only the fact that $\alpha$ belongs to $\partial G_{1}^{\prime \prime} \cap \partial G_{2}^{\prime \prime}$ is important for us).

A line complex corresponding to the case when $\tau \leq n-1$ is shown in Fig. 22 .

Now we consider the case when $\tau \geq n$. If $\Lambda\left(a_{3}\right)>1$, we construct a surface of the class $\mathfrak{F}\left(n ; a_{1}: \lambda_{1}\left(a_{1}\right), \lambda_{2}\left(a_{1}\right) ; a_{2}: \lambda_{1}\left(a_{2}\right), \lambda_{2}\left(a_{2}\right) ; a_{3}: \lambda_{1}\left(a_{3}\right), \lambda_{2}\left(a_{3}\right) ; a_{4}\right.$ : $\left.\lambda_{1}\left(a_{4}\right) ; \ldots ; a_{q}: \lambda_{1}\left(a_{q}\right)\right)$, where $\lambda_{2}\left(a_{3}\right)=1, \lambda_{1}\left(a_{3}\right)=\Lambda\left(a_{3}\right)-1$; if $\Lambda\left(a_{3}\right)=1$ then necessarily $q \geq 4$, and wee construct a surface of the class $\mathfrak{F}\left(n ; a_{1}: \lambda_{1}\left(a_{1}\right), \lambda_{2}\left(a_{1}\right)\right.$; $\left.a_{2}: \lambda_{1}\left(a_{2}\right), \lambda_{2}\left(a_{2}\right) ; a_{3}: \lambda_{1}\left(a_{3}\right) ; \ldots ; a_{q}: \lambda_{1}\left(a_{q}\right)\right)$, where $\lambda_{1}\left(a_{3}\right)=1$. At first we assume $\tau=n$. The corresponding line complex can be constructed in the following way. Let $Q_{1}$ be the square with vertices $A=(-1,-1), B=(-1,1), C=(1,1)$, $D=(1,-1), Q_{2}$ be the square with the vertices $A^{\prime}=(-2,-2), B^{\prime}=(-2,2), C^{\prime}=$ $(2,2), D^{\prime}=(2,-2)$. The square $Q_{1}$ will play the role of an elementary 3-region. Using segments of bisectors of the coordinate angles we join the corresponding vertices of the squares $Q_{1}$ and $Q_{2}$, we divide the region $Q_{2} \backslash \bar{Q}_{1}$ into four trapezoids $T_{1}, \ldots, T_{4}$, where $T_{1}$ is the lower, $T_{2}$ is the left, $T_{3}$ is the upper, and $T_{4}$ is the right trapezoid. We place internal vertices in the points $\alpha=A^{\prime}, C^{\prime}, B, D$, and external vertices in the remaining vertices of $Q_{1}$ and $Q_{2}$. Now we place $\left(2 \lambda_{1}\left(a_{1}\right)-2\right)$ vertices in the interval $A^{\prime} D^{\prime},\left(2 \lambda_{1}\left(a_{2}\right)-2\right)$ vertices in the interval $A^{\prime} B^{\prime},\left(2 \lambda_{2}\left(a_{1}\right)-2\right)$ in the interval $B^{\prime} C^{\prime},\left(2 \lambda_{2}\left(a_{2}\right)-2\right)$ in the interval $C^{\prime} D^{\prime}$. We shall regard $T_{1}$ and $T_{2}$ as elementary 1-regions and $T_{2}$ and $T_{4}$ as elementary 2-regions. The closed square $\bar{Q}_{2}$ will contain $2\left(\lambda_{1}\left(a_{1}\right)+\lambda_{1}\left(a_{2}\right)+\lambda_{2}\left(a_{1}\right)+\lambda_{2}\left(a_{2}\right)-4\right)+8=2 \tau=2 n$ vertices, $2 n-4$ of them lie on the boundary of $Q_{2}$. If $\Lambda\left(a_{3}\right)>1$, we join to $Q_{2}$ successively elementary $3-, 4-, \ldots,(q-1)$-regions, which are Jordan $\left(2 \lambda_{1}\left(a_{j}\right)+2\right)$ gons, using the operations $U\left(\Delta_{j-1}, G_{j}, \alpha, 2 \lambda_{1}\left(a_{j}\right)+1\right), 3 \leq j \leq q-1$, where $\Delta_{2}=Q_{2}, \Delta_{j}=\left(\bar{\Delta}_{j-1} \cup \bar{G}_{j}\right) \backslash \partial\left(\bar{\Delta}_{j-1} \cup \bar{G}_{j}\right)$. It is easy to count that the boundary of $\Delta_{q-1}$ contains $2 n-4-2 \sum_{j=3}^{q-1} \lambda_{1}\left(a_{j}\right)$ vertices. But (4.1) implies that

$$
\sum_{j=3}^{q} \lambda_{1}\left(a_{j}\right)=2(n-1)-\tau-\lambda_{2}\left(a_{3}\right)=2(n-1)-n-1=n-3
$$

therefore $2 n-4=2 \sum_{j=3}^{q} \lambda_{1}\left(a_{j}\right)+2$, and the boundary of the region $\Delta_{q-1}$ contains $2 \lambda\left(a_{q}\right)+2$ vertices. We take the complement of $\bar{\Delta}_{q-1}$ in the extended complex plane as an elementary $q$-region. Replacing some of the edges by bundles we get, as before, a required line complex. If $\Lambda\left(a_{3}\right)=1$, we carry out the construction in the same way, the only difference is that we do not need to take an elementary 3-region in the complement of $Q_{2}$. Both cases are illustrated on Fig. 23 and 24 .

Now we assume that $p=\tau-n>0$. Then $\lambda_{1}\left(a_{1}\right)+\lambda_{2}\left(a_{1}\right)=\tau-\lambda_{1}\left(a_{2}\right)-$ $\lambda_{2}\left(a_{2}\right) \geq n+p-(n-2)=p+2$ and, similarly, $\lambda_{1}\left(a_{2}\right)+\lambda_{2}\left(a_{2}\right) \geq p+2$. We subtract one from the largest of the numbers $\lambda_{1}$ and $\lambda_{2}$ and from the largest of the numbers $\lambda_{3}$ and $\lambda_{4}$. We get numbers, which we denote $\lambda_{1}^{(1)}, \lambda_{2}^{(1)}, \lambda_{3}^{(1)}, \lambda_{4}^{(1)}$. We repeat the same operation with the new numbers, and get $\lambda_{1}^{(2)}, \ldots, \lambda_{4}^{(2)}$. Repeating the same operation $p$ times, we get the numbers $\lambda_{1}^{(p)}, \ldots, \lambda_{4}^{(p)}$. It is easy to see that $\lambda_{1}^{(p)}, \ldots, \lambda_{4}^{(p)} \geq 1$. Let $p_{13}$ be the number of ones which were subtracted simultaneously from $\lambda_{1}$ and $\lambda_{3}$ in the process of all $p$ operations. The numbers $p_{14}, p_{23}, p_{24}, p_{31}, p_{32}, p_{41}, p_{42}$ are defined similarly. It is easy to see that $p_{j k}=p_{k j}$, 
$\lambda_{1}^{(p)}=\lambda_{1}-p_{13}-p_{14}, \lambda_{2}^{(p)}=\lambda_{2}-p_{23}-p_{24}, \lambda_{3}^{(p)}=\lambda_{3}-p_{31}-p_{32}, \lambda_{4}^{(p)}=\lambda_{4}-p_{41}-p_{42}$, $p_{13}+p_{14}+p_{23}+p_{24}=p_{31}+p_{32}+p_{41}+p_{42}=p$.

Now we construct the squares $Q_{1}$ and $Q_{2}$ and trapezoids $T_{1}, \ldots, T_{4}$, and place vertices of the line complex in the vertices of these squares and trapezoids in the same way as we did for $\tau=n$. Now in the intervals, joining the points shown in the table below, we place the number of vertices shown in the right column of the table:

$$
\begin{array}{c|cc|c}
A^{\prime} D^{\prime} & 2 \lambda_{1}^{(p)}-2 & A^{\prime} A & 2 p_{13} \\
A^{\prime} B^{\prime} & 2 \lambda_{3}^{(p)}-2 & B^{\prime} B & 2 p_{23} \\
B^{\prime} C^{\prime} & 2 \lambda_{2}^{(p)}-2 & C^{\prime} C & 2 p_{24} \\
C^{\prime} D^{\prime} & 2 \lambda_{4}^{(p)}-2 & D^{\prime} D & 2 p_{14}
\end{array}
$$

The boundary of $T_{1}$ contains $\left(2 \lambda_{1}^{(p)}-2\right)+2 p_{13}+2 p_{14}+4=2 \lambda_{1}+2=2 \lambda_{1}\left(a_{1}\right)+2$ vertices, the boundary of $T_{2}$ contains $\left(2 \lambda_{1}\left(a_{2}\right)+2\right)$ vertices, the boundary of $T_{3}$ contains $\left(2 \lambda_{2}\left(a_{1}\right)+2\right)$ vertices, and the boundary of $T_{4}$ contains $\left(2 \lambda_{2}\left(a_{2}\right)+2\right)$ vertices. We take $Q_{1}$ as an elementary 3-region, $T_{1}$ and $T_{3}$ as elementary 1-regions, $T_{2}$ and $T_{4}$ as elementary 2-regions. The boundary of $Q_{2}$ contains $2\left(\lambda_{1}^{(p)}-1\right)+\cdots+$ $2\left(\lambda_{4}^{(p)}-1\right)+4=2\left(\lambda_{1}-1-p_{13}-p_{14}\right)+\cdots+2\left(\lambda_{4}-1-p_{41}-p_{42}\right)+4=2 \tau-4 p-4=$ $2 n-2 p-4$ vertices, and the interior of $Q_{2}$ contains $(2 p+4)$ vertices. Now we successively join to $Q_{2}$ elementary 3-, 4-, $\ldots,(q-1)$-regions, if $\Lambda\left(a_{3}\right)>1$, or 4-, $\ldots,(q-1)$-regions if $\Lambda\left(a_{3}\right)=1$, in the same way as we did this in the case $\tau=n$. Now the boundary of $\Delta_{q-1}$ contains $\sigma=2 n-2 p-4-2\left(\Lambda\left(a_{3}\right)-1\right)-2 \sum_{j=4}^{q-1} \lambda_{1}\left(a_{j}\right)$ vertices. Since $2 n-2=\tau+\Lambda\left(a_{3}\right)+\sum_{j=4}^{q} \lambda_{1}\left(a_{j}\right)$ and $\tau=n+p$, we have

$$
\tau=2 p+2+\Lambda\left(a_{3}\right)+\sum_{j=4}^{q} \lambda_{1}\left(a_{j}\right)
$$

and

$$
\sigma=2 \tau-4 p-2-2 \Lambda\left(a_{3}\right)-2 \sum_{j=4}^{q-1} \lambda_{1}\left(a_{j}\right)=2 \lambda_{1}\left(a_{q}\right)+2 .
$$

We take as an elementary $q$-region the complement of $\bar{\Delta}_{q-1}$ in the extended complex plane, and then complete the proof as in the previous case. A line complex with $\tau>n$ is shown on Fig. 25 .

CASE (b). Denote $\tau=\lambda_{1}\left(a_{1}\right)+\lambda_{2}\left(a_{1}\right)+\lambda_{1}\left(a_{2}\right)$. It is clear that $\tau \leq n-2+n-1=$ $2 n-3$, therefore $q \geq 3$. At first we assume $\tau \leq n-1$. Take as a 1-region $G_{1}^{\prime}$ a Jordan $\left(2 \lambda\left(a_{1}\right)+2\right)$-gon and mark an interior vertex $\alpha$ on its boundary. Then we join to $G_{1}^{\prime}$, as a 2 -region $G_{2}$, a Jordan $\left(2 \lambda_{1}\left(a_{2}\right)+2\right)$-gon using the operation $U\left(G_{1}^{\prime}, G_{2}, \alpha, 1\right)$. We join to the region $\Delta_{1}=\left(\bar{G}_{1}^{\prime} \cup \bar{G}_{2}\right) \backslash \partial\left(\bar{G}_{1}^{\prime} \cup \bar{G}_{2}\right)$ a 1-region $G_{1}^{\prime \prime}$, which is a Jordan $\left(2 \lambda_{2}\left(a_{1}\right)+2\right)$-gon, using the operation $U\left(\Delta_{1}, G_{1}^{\prime \prime}, \beta, 1\right)$, where $\beta$ is an exterior vertex on the boundary of $G_{2}$, next after $\alpha$ as we go around the boundary in the negative direction. The boundary of the region $\Delta_{2}=\left(\bar{\Delta}_{1} \cup \bar{G}_{1}^{\prime \prime}\right) \backslash \partial\left(\bar{\Delta}_{1} \cup \bar{G}_{1}^{\prime \prime}\right)$ contains $2 \lambda_{1}\left(a_{1}\right)+2+2 \lambda_{1}\left(a_{2}\right)+2 \lambda_{2}\left(a_{1}\right)=2 \tau+2 \leq 2 n$ vertices, After that we complete the construction of a line complex in the same way as in the proof of Theorem 4.1. If $\tau \geq n$, then $\tau=n-1+p, p \geq 1$. It is easy to see that $p=$ $\lambda_{1}\left(a_{1}\right)+\lambda_{2}\left(a_{1}\right)+\lambda_{1}\left(a_{2}\right)-(n-1) \leq \lambda_{1}\left(a_{1}\right)+\lambda_{2}\left(a_{1}\right)$. Therefore we can find numbers $p_{1}, p_{2}$, such that $0 \leq p_{1} \leq \lambda_{1}\left(a_{1}\right), 0 \leq p_{2} \leq \lambda_{2}\left(a_{1}\right), p=p_{1}+p_{2}$. Now we carry out the construction of a line complex in the same way as in the case $\tau \leq n-1$, with the 
only difference that we join $G_{2}$ to $G_{1}^{\prime}$ using the operation $U\left(G_{1}^{\prime}, G_{2}, \alpha, 2 p_{1}+1\right)$, and join $G_{1}^{\prime \prime}$ to $\Delta_{1}$ using the operation $U\left(\Delta_{1}, G_{1}^{\prime \prime}, \beta, 2 p_{2}+1\right)$. Now the closed region $\bar{\Delta}_{2}$ contains $2 \lambda_{1}\left(a_{1}\right)+2+2 \lambda_{2}\left(a_{1}\right)+2+2 \lambda_{1}\left(a_{2}\right)-\left(2 p_{1}+1\right)-\left(2 p_{2}+1\right)=2 \tau+2-2 p=2 n$ vertices, and the boundary of $\Delta_{2}$ contains $2 n-2 p_{1}-2 p_{2}=2(n-p)$ vertices. Now we again join successively $3-, \ldots,(q-1)$-regions as in the proof of Theorem 4.1, and get a region $\Delta_{q-1}$ whose boundary contains

$$
\begin{aligned}
2(n-p) & -2 \sum_{j=3}^{q-1} \lambda_{1}\left(a_{j}\right)=2 n+2 n-2-2 \tau-2 \sum_{j=3}^{q-1} \lambda_{1}\left(a_{j}\right) \\
& =2\{2(n-1)\}+2-2 \tau-2 \sum_{j=3}^{q-1} \lambda_{1}\left(a_{j}\right) \\
& =2\left(\tau+\sum_{j=3}^{q} \lambda_{1}\left(a_{j}\right)\right)+2-2 \tau-2 \sum_{j=3}^{q-1} \lambda_{1}\left(a_{j}\right)=2 \lambda_{1}\left(a_{q}\right)+2
\end{aligned}
$$

vertices. After that the construction of a line complex is completed in the usual way. Line complexes corresponding to the case (b) for $\tau \leq n-1$ and $\tau \geq n$ are shown on Fig. 26 and Fig. 27.

CASE (c). First we construct a line complex for a Riemann surface of the class $\mathfrak{F}\left(n ; a_{1}: \lambda_{1}\left(a_{2}\right), \lambda_{2}\left(a_{2}\right) ; a_{2}: \lambda_{1}\left(a_{1}\right) ; a_{3}: \lambda_{1}\left(a_{q}\right) ; a_{4}: \lambda_{1}\left(a_{q-1}\right) ; \ldots ; a_{q}: \lambda_{1}\left(a_{3}\right)\right)$ in the same way as we did it in the case (b). Then we take a mirror image of this line complex and the images of 2-regions consider as 1-regions, the images of 1 regions as 2-regions, the images of $q$-regions as 3 -regions, the images of $(q-1)$-regions as 4 -regions, ..., the images of 3 regions as $q$-regions. It is easy to verify that as a result of this transformation (we denote it by $S$ ) we get a line complex satisfying all the conditions imposed in the case (c). The line complex obtained as the result of the transformation $S$ for the line complex from Fig. 27 is shown on Fig. 28.

CASE (d). Consider the line complex which we constructed in our study of case (b), where $\lambda_{1}\left(a_{2}\right)$ is equal to the number $\lambda_{2}\left(a_{2}\right)$ prescribed in the case $(\mathrm{d})$, and $\lambda_{1}\left(a_{1}\right) \geq 1$. Let $\alpha^{\prime}$ be the exterior vertex on the boundary of the elementary 1 region $G_{1}^{\prime}, \beta^{\prime}$ be the interior vertex following after $\alpha^{\prime}$ as we go around the boundary of $G_{1}^{\prime}$ in the positive direction. Some elementary 2-region $G_{2}^{\prime}$ has the edge joining $\alpha^{\prime}$ and $\beta^{\prime}$ as its common boundary with $G_{1}^{\prime}$. If $\tau \leq n-1$, then clearly $G_{2}^{\prime} \neq G_{2}$. If $\tau \geq n$, we observe, that $\lambda_{1}\left(a_{2}\right) \leq n-2$ in our case, therefore $p=\lambda_{1}\left(a_{1}\right)+\lambda_{2}\left(a_{1}\right)+$ $\lambda_{1}\left(a_{2}\right)-(n-1) \leq \lambda_{1}\left(a_{1}\right)+\lambda_{2}\left(a_{2}\right)-1$, and we can take $0 \leq p_{1} \leq \lambda_{1}\left(a_{1}\right)-1$, $0 \leq p_{2} \leq \lambda_{2}\left(a_{1}\right)$. With this choice of $p_{1}$ we get that $G_{2}^{\prime} \neq G_{2}$ for $\tau \geq n$ also. Thus $G_{2}^{\prime}$ is a digon. The line complex satisfies all the conditions, there is an interior vertex $\alpha^{\prime}$ in $\partial G_{1} \cap \partial G_{2}^{\prime}$.

CASE (e). We get the solution by using the solution of the case (d) in the same way as we used the solution of the problem in the case (b) to solve it in the case (c).

CAse (f). The solution is contained in Theorem 4.1 and the remark after it.

CAsE (g). First we a construct a line complex disregarding the existence of the base point $a_{2}$, that is, assuming that there are $q-1$ base points: $a_{1}, a_{3}, \ldots$, $a_{q}$. Then the construction is reduced to the case (b). If we take into account the base point $a_{2}$, we need only to replace each edge separating 1 - and 3-regions by a digon. Since in our construction in the case (b) the regions $G_{1}^{\prime}$ and $G_{2}$ in the constructed complex have in their boundaries at least one common edge, in our 
case the boundary of the 1-region $G_{1}^{\prime}$, which is a Jordan $\left(2 \lambda_{1}\left(a_{1}\right)+2\right)$-gon, and the boundary of some 2-region which is a digon have one common internal vertex.

CASE (h). The argument is similar to the case (g).

CASE (i). We construct a line complex in the same way as in the proof of Theorem 4.1 for a Riemann surface of the class $\mathfrak{F}\left(n ; a_{2}: \lambda_{2}\left(a_{2}\right) ; a_{3}: \lambda_{1}\left(a_{3}\right) ; \ldots ; a_{q}\right.$ : $\left.\lambda_{1}\left(a_{q}\right) ; a_{1}: \lambda_{2}\left(a_{1}\right)\right)$, that is, the region $G_{j}$ is an elementary $(j+1)$-region, $1 \leq j \leq q-1$, and the region $G_{q}$ is an elementary 1-region. The first and the second vertices on the boundary of $G_{2}$, counting starting at $\alpha$ in the negative direction, we denote by $\beta$ and $\alpha^{\prime}$, respectively; the vertex $\beta$ is external, and the vertex $\alpha^{\prime}$ is internal. These vertices do not lie on the boundary of $G_{1}$ since it is a Jordan $\left(2 \lambda_{2}\left(a_{2}\right)+2\right)$-gon, and $2 \lambda_{2}\left(a_{2}\right)+2 \leq 2 n-2$ since $\lambda_{2}\left(a_{2}\right) \leq n-2$. The vertices $\beta$ and $\alpha^{\prime}$ lie on the common part of the boundary of a 3-region and a 4-region, therefore they also belong to the common part of the boundary of two digons, which are elementary 1- and 2-regions. Since $\alpha^{\prime}$ is an internal vertex, the constructed line complex has all required properties.

Note that in the case (i) the requirement $q \geq 4$ is essential. For $q=3$ the class $\mathfrak{F}\left(n ; a_{1}: 0, \lambda_{2}\left(a_{1}\right) ; a_{2}: 0, \lambda_{2}\left(a_{2}\right) ; a_{3}: \lambda_{1}\left(a_{3}\right)\right)$ is nonempty, as is easy to show referring to Theorem 4.1. However, it is easy to verify that for $q=3$ the digons which are elementary 1- and 2-regions have disjoint boundaries.

REMARK. In Theorem 4.2 we assume that $\lambda_{1}\left(a_{j}\right) \geq 1,3 \leq j \leq q$ in all cases. This is done for simplicity of the proof only. It is easy to see that it would suffice to require $\lambda_{1}\left(a_{j}\right) \geq 0,3 \leq j \leq q$ only, in addition, in the case (a) for one of the points $a_{j}, 3 \leq j \leq q$, we require that $\Lambda\left(a_{j_{0}}\right) \geq 1$ if $\lambda_{1}\left(a_{j}\right)=0$ for $3 \leq j<j_{0}$; and in the case (i) we require that $\lambda_{1}\left(a_{j}\right) \geq 1$ for at least two values $j, 3 \leq j \leq q$. In fact, in our construction of the line complex we can first disregard those base points for which $\Lambda\left(a_{j}\right)=0$, and then complete the constructed line complex with the corresponding digons.

Proof of Theorem 4.3. First we note that since

$$
\begin{gathered}
\lambda_{1}\left(a_{1}\right)+\lambda_{2}\left(a_{1}\right)+\lambda_{3}\left(a_{1}\right) \leq n-3, \\
\lambda_{1}\left(a_{2}\right) \leq n-1,
\end{gathered}
$$

the condition (4.1) implies that the requirement $q \geq 3$ in the statement of the theorem is necessary.

We can draw the base curve $L$ in such a way that it intersects the curve $\gamma$ in two points only, one of which is $a_{1}$. We select interior and exterior vertices on the curve $\gamma$, and the arc of $\gamma$ joining these vertices and not passing through $a_{1}$ take as the curve $C_{2}$. We select the other curves, $C_{1}, C_{3}, \ldots, C_{q}$, used in the construction of a line complex, without any additional requirements. Now it is easy to verify that it suffices to construct a line complex with the prescribed ramification and the additional restriction that on the boundary of an elementary 1-region corresponding to the point $\mathfrak{a}_{1}$ (point $\mathfrak{a}_{2}$ ) there is an internal (external) vertex with the property that these vertices are the ends of the same edge with label 2.

CASE $(\mathrm{j})$. We shall distinguish two subcases: $\left(\mathrm{j}_{1}\right)$ when $2 \Lambda\left(a_{1}\right)+2 \lambda_{1}\left(a_{2}\right)+2 \leq$ $2 n ;\left(\mathrm{j}_{2}\right)$ when $2 \Lambda\left(a_{1}\right)+2 \lambda_{1}\left(a_{2}\right)+2=2 n+2 p, p>0$.

SuBCASE $\left(\mathrm{j}_{1}\right)$. Take as an elementary 1-region a Jordan $\left(2 \lambda_{1}\left(a_{1}\right)+2\right)$-gon. Mark an internal vertex $\alpha$ on its boundary and join as an elementary 2-region $G_{2}$ a Jordan $\left(2 \lambda_{1}\left(a_{2}\right)+2\right)$-gon using the operation $U\left(G_{1}, G_{2}, \alpha, 1\right)$. We mark on the 
boundary of $G_{2}$ an external vertex $\beta$, which is the first after $\alpha$ if we go along the boundary of $G_{2}$ in the negative direction, and an internal vertex $\alpha^{\prime}$ separated from $\alpha$ by four edges. We join to the region $\Delta=\left(\bar{G}_{1} \cup \bar{G}_{2}\right) \backslash \partial\left(\bar{G}_{1} \cup \bar{G}_{2}\right)$ an elementary 1region $G_{3}$ which is a Jordan $\left(2 \lambda_{2}\left(a_{1}\right)+2\right)$-gon, using the operation $U\left(\Delta_{2}, G_{3}, \beta, 1\right)$. Then we join to $\Delta_{3}=\left(\bar{\Delta}_{2} \cup \bar{G}_{3}\right) \backslash \partial\left(\bar{\Delta}_{2} \cup \bar{G}_{3}\right)$ an elementary 1-region $G_{4}$ which is a Jordan $\left(2 \lambda_{3}\left(a_{1}\right)+2\right)$-gon using the operation $U\left(\Delta_{3}, G_{4}, \alpha^{\prime}, 1\right)$. It is easy to see that the boundary of the region $\Delta_{4}=\left(\bar{\Delta}_{3} \cup \bar{G}_{4}\right) \backslash \partial\left(\bar{\Delta}_{3} \cup \bar{G}_{4}\right)$ contains

$$
2\left(\lambda_{1}\left(a_{1}\right)+\lambda_{2}\left(a_{1}\right)+\lambda_{3}\left(a_{1}\right)+\lambda_{1}\left(a_{2}\right)\right)+2=2 \Lambda\left(a_{1}\right)+2 \lambda_{1}\left(a_{2}\right)+2<2 n
$$

vertices. After that we successively join to $\Delta_{4}$ elementary $3-, 4-, \ldots, q$-regions in the same way as in the proof of Theorem 4.1 , but now the role of the region $G_{1}$ is played by the region $\Delta_{4}$, and the number $p, 2 \leq p<q$ is defined as the largest integer satisfying $2+2 \Lambda\left(a_{1}\right)+2 \sum_{j=2}^{p} \lambda_{1}\left(a_{j}\right) \leq 2 n$ (cf. (4.3). We leave the details of the construction to interested readers. Examples of line complexes, corresponding to the cases when $p=2$ and when $p=3$ are shown on Fig. 29 and Fig. 30.

$\operatorname{SubCASE}\left(\mathrm{j}_{2}\right)$. By (4.6) we have $p \leq \Lambda\left(a_{1}\right)$. Therefore we can write $p=$ $p_{1}+p_{2}+p_{3}$, where $0 \leq p_{k} \leq \lambda_{k}\left(a_{1}\right), k=1,2,3$. Take as an elementary 1region $G_{1}$ a Jordan $\left(2 \lambda_{1}\left(a_{1}\right)+2\right)$-gon. We mark on its boundary an internal vertex $\alpha$ and join as an elementary 2-region $G_{2}$ a Jordan $\left(2 \lambda_{1}\left(a_{2}\right)+2\right)$-gon using the operation $U\left(G_{1}, G_{2}, \alpha, 2 p_{1}+1\right)$. The inequality (4.5) implies that $2 p \leq 2 \lambda_{1}\left(a_{2}\right)-4=$ $\left(2 \lambda_{1}\left(a_{2}\right)+2\right)-6$. Therefore the indicated operation of joining, as well as the next two, is admissible. We mark on the boundary of $G_{2}$ an external vertex $\beta$ which is the first after $\alpha$ as we go around the boundary of $G_{2}$ in the negative direction. We join to the region $\Delta_{2}=\left(\bar{G}_{1} \cup \bar{G}_{2}\right) \backslash \partial\left(\bar{G}_{1} \cup \bar{G}_{2}\right)$ an elementary 1-region $G_{3}$ which is a Jordan $\left(2 \lambda_{2}\left(a_{1}\right)+2\right)$-gon using the operation $U\left(\Delta_{2}, G_{3}, \beta, 2 p_{2}+1\right)$. The Jordan arc $\partial G_{3} \cap \partial \Delta_{2}$ has two ends: one is in the vertex $\beta$, the other is in an internal vertex, which we denote by $\beta^{\prime}$. We mark on the boundary of $G_{2}$ an external vertex $\alpha^{\prime}$, which is the first external vertex after $\beta^{\prime}$ if we go around the boundary of $G_{2}$ in the negative direction. We join to the region $\Delta_{3}=\left(\bar{\Delta}_{2} \cup \bar{G}_{3}\right) \backslash \partial\left(\bar{\Delta}_{2} \cup \bar{G}_{3}\right)$ an elementary 1-region $G_{4}$, which is a Jordan $\left(2 \lambda_{3}\left(a_{1}\right)+2\right)$-gon, using the operation $U\left(\Delta_{3}, G_{4}, \alpha^{\prime}, 2 p_{3}+1\right)$. It is easy to count that the region $\Delta_{4}=\left(\bar{\Delta}_{3} \cup \bar{G}_{4}\right) \backslash \partial\left(\bar{\Delta}_{3} \cup \bar{G}_{4}\right)$ contains $2\left(p_{1}+p_{2}+p_{3}\right)=2 p$ vertices, and the boundary of $\Delta_{4}$ contains $2 n-2 p$ vertices. Let

$$
\Delta_{s}=\left(\Delta_{s-1} \cup \bar{G}_{s}\right) \backslash \partial\left(\bar{\Delta}_{s-1} \cup \bar{G}_{s}\right),
$$

$5 \leq s \leq q+1, G_{s}$ be an elementary $(s-2)$-region, which is a Jordan $\left(2 \lambda_{1}\left(a_{s-2}\right)+2\right)$ gon, joined to $\Delta_{s-1}$ using the operation $U\left(\Delta_{s-1}, G_{s}, \alpha, 2 \lambda_{1}\left(a_{s-2}\right)+1\right)$. It is easy to count that the boundary of $\Delta_{q+1}$ contains

$$
\begin{aligned}
2 n-2 p-2 \sum_{j=3}^{q-1} \lambda_{1}\left(a_{j}\right) & =2 n-\left(2 \Lambda\left(a_{1}\right)+2 \lambda_{1}\left(a_{2}\right)+2-2 n\right)-2 \sum_{j=3}^{q-1} \lambda_{1}\left(a_{j}\right) \\
& =4(n-1)+2-2 \Lambda\left(a_{1}\right)-2 \sum_{j=2}^{q-1} \lambda_{1}\left(a_{j}\right)=2 \lambda_{1}\left(a_{q}\right)+2
\end{aligned}
$$

vertices. We take as an elementary $q$-region the complement of $\bar{\Delta}_{q+1}$ in the extended complex plane. We have constructed all elementary regions, and, replacing some of 
the edges by edge bundles, we get the desired line complex. An example of a line complex corresponding to the case $\left(\mathrm{j}_{2}\right)$ is shown on Fig. 31.

CASE (k). The argument is completely analogous to the argument in the case (j), the only difference is that we do not need to join to $\Delta_{3}$ the third elementary 1-region. We leave the detailed argument in this case to interested readers. An example of a line complex corresponding to the case $(\mathrm{k})$ is shown on Fig. 32. Note also, that if the relation (4.5) is satisfied with $\lambda_{1}\left(a_{2}\right) \geq 2$, then the case $(\mathrm{k})$ is reduced to the case $(\mathrm{j})$ with $\lambda_{3}\left(a_{1}\right)=0$.

REMARK. It is clear that in the condition of Theorem 4.3 we may assume that the point $a_{2}$ is outside $\gamma$, and the points $a_{3}, \ldots, a_{q}$ are inside this curve.

EXERCISE. Prove the following statement. Let $q \geq 4$ distinct complex numbers $a_{1}, \ldots, a_{q}$ and a $J^{\prime}$-curve $\gamma$ with ends at the point $a_{1}$ be given, moreover, let points $a_{2}, \ldots, a_{r}, 2<r<q$, be inside the curve $\gamma$, and the points $a_{r+1}, \ldots, a_{q}$ be outside the curve $\gamma$. Then each of the classes

(l) $\mathfrak{F}\left(n ; a_{1}: \lambda_{1}\left(a_{1}\right), \lambda_{2}\left(a_{1}\right), \lambda_{3}\left(a_{1}\right), a_{2}: \lambda_{1}\left(a_{2}\right) ; a_{3}: \lambda_{1}\left(a_{3}\right), 0 \ldots ; a_{q-1}:\right.$ $\left.\lambda_{1}\left(a_{q-1}\right), 0 ; a_{q}: \lambda_{1}\left(a_{q}\right)\right)$, where $\lambda_{k}\left(a_{1}\right) \geq 0, k=1,2,3 ; \lambda_{1}\left(a_{2}\right) \geq 2$;

(m) $\mathfrak{F}\left(n ; a_{1}: \lambda_{1}\left(a_{1}\right), \lambda_{2}\left(a_{1}\right) ; a_{2}: \lambda_{1}\left(a_{2}\right) ; a_{3}: \lambda_{1}\left(a_{3}\right), 0 ; \ldots ; a_{q-1}: \lambda_{1}\left(a_{q-1}\right)\right.$; $\left.a_{q}: \lambda_{1}\left(a_{q}\right)\right)$, where $\lambda_{k}\left(a_{1}\right) \geq 0, k=1,2$;

contains a Riemann surface $F$ on which a $J$-curve $\Gamma$ with the ends $\mathfrak{a}_{1}$ and $\mathfrak{a}_{2} \in F$, such that the curve $\Gamma$ without ends is projected in a one-to-one way onto $\gamma \backslash\left\{a_{1}\right\}$, and the point $\mathfrak{a}_{k}$ is a ramification point of order $\lambda_{k}\left(a_{1}\right)$ lying over $a_{1}, k=1,2$.

Fig. 33, containing a "typical" line complex corresponding to the case (l) can be considered as a hint showing the changes which should be made in the proof of Theorem 4.3 (cf. Fig. 31) in order to get the desired statement.

\section{Statement of the inverse problem of the value distribution theory. Riemann surfaces with finitely many logarithmic ends}

The direct problem of the value distribution theory is the problem of determination of the distribution of deficiencies and indices of a given meromorphic function. The inverse problem consists in finding a meromorphic function having the prescribed distribution of deficiencies and indices. In the general form the inverse problem of the value distribution theory for meromorphic function (later on we shall call it briefly 'the inverse problem') is stated in the following way:

Let $a_{1}, a_{2}, \ldots$, be a (finite or infinite) sequence of complex numbers. Suppose that for each member of this sequence numbers $\delta\left(a_{k}\right)$ and $\varepsilon\left(a_{k}\right)$ are given, and these numbers satisfy the conditions

$$
\begin{gathered}
0<\delta\left(a_{k}\right)+\varepsilon\left(a_{k}\right) \leq 1, k=1,2, \ldots, \\
\sum_{k}\left\{\delta\left(a_{k}\right)+\varepsilon\left(a_{k}\right)\right\} \leq 2 .
\end{gathered}
$$

The problem is to find a meromorphic function having at the points $a_{k}, k=1,2, \ldots$, deficiencies $\delta\left(a_{k}\right)$ and indices $\varepsilon\left(a_{k}\right)$, and having no other deficient or index values in the complex plane. 
The most interesting is the study of the distribution of deficiencies. For this reason the inverse problem is often considered in more narrow form, with no restriction on indices. Thus, the "restricted" inverse problem, as we shall call it, is the following

Let $\left\{a_{k}\right\}$ be a (finite or infinite) sequence, let $\delta\left(a_{k}\right)$ be numbers corresponding to the elements of the sequence and satisfying the conditions $0<\delta\left(a_{k}\right) \leq 1$ and

$$
\sum_{k} \delta\left(a_{k}\right) \leq 2 .
$$

The problem is to find a meromorphic function $f(z)$ such that $\delta\left(a_{k}, f\right)=\delta\left(a_{k}\right)$, $k=1,2, \ldots$, and $\delta(a, f)=0$ for all $a \neq a_{k}, k=1,2, \ldots$; or to prove that such functions do not exist.

Solutions of the inverse problem can be sought among meromorphic functions belonging to some class $C$, for example, to the class of entire functions (in this case we, of course, require $a_{1}=\infty$ and $\delta(\infty)=1$ ) or to the class of meromorphic functions of finite order. In such a case we say that the inverse problem is solved in the class $C$.

The complete solution of the inverse problem is still unknown. ${ }^{7}$ Fuchs and Hayman [FH62] (see also [Hay64, §4.1]) found a complete solution of the "restricted" inverse problem in the class of entire functions of infinite order. For this result we refer the reader to the mentioned book of Hayman. All other case in which the solutions of the inverse problem are known today will be presented in this chapter.

We shall consider the inverse problem in the class of meromorphic functions of finite order without mentioning this explicitly. Further, we restrict our attention to the case when the number of points $\left\{a_{k}\right\}$ is finite: $a_{1}, a_{2}, \ldots, a_{q}$. Although, as we saw in Chapter 4, the set of deficient values of a meromorphic function of a finite positive order can be an arbitrarily prescribed countable set, the deficiencies in the points of this set cannot be prescribed arbitrarily, since besides the inequality (5.3) they satisfy some additional relations (see the commentary to Chapter 7 ). The fact that the deficiencies, besides the inequality (5.3) should satisfy some additional conditions, which are almost unexplored yet, makes the solution of the inverse problem for countable sets $\left\{a_{k}\right\}$ extremely complicated. As for the set of index values, we can easily get rid of the requirement of its finiteness and introduce this requirement only to make our presentation more accessible. In fact, as we shall see, in the case when the set $\left\{a_{k}\right\}$ is finite it suffices to consider rather simple class $F_{q}$ of Riemann surfaces. However, in the corresponding place we shall provide hints which can be used to generalize the obtained results to the case when the set of index values is infinite.

So we shall solve the inverse problem assuming that the set $\left\{a_{k}\right\}=\left\{a_{1}, a_{2}, \ldots\right.$, $\left.a_{q}\right\}$ is finite. Besides, we shall assume that all numbers $a_{j}, 1 \leq j \leq q$, are finite. Obviously the latter assumption does not reduce the generality because we can pass to the general case using a linear-fractional transformation.

Now we shall prove one theorem due to R. Nevanlinna [NevR32b] (see, also [NevR74, n. 257]). Although this theorem will be considerably generalized in subsequent sections, we present it here not so much because it is historically the first example of a rather general solution of the inverse problem, but because the

\footnotetext{
${ }^{7}$ Now it is solved. About this and other problems mentioned in this page, see the survey in the end of the book.
} 
main idea of the proof (it is different from the original proof due to R. Nevanlinna) will be used in what follows, and here it is not concealed by technicalities.

Theorem 5.1. Let $q \geq 2$, let $a_{1}, \ldots, a_{q}$ be complex numbers, and $\delta\left(a_{j}\right), 0<$ $\delta\left(a_{j}\right) \leq 1$, be rational numbers satisfying $\sum_{j=1}^{q} \delta\left(a_{j}\right)=2$. Then there exists a meromorphic function $f(z)$ whose deficiency at the point $a_{j}$ is equal to $\delta\left(a_{j}\right), 1 \leq j \leq q$.

First we introduce a class of Riemann surfaces, the so-called surfaces with finitely many logarithmic ends, and study the value distribution of the meromorphic mapping functions.

Let $a_{1}, \ldots, a_{q}$ be a set of distinct complex numbers $(q \geq 2)$, for simplicity we assume that they are finite.

Take a finite sequence of numbers $b_{1}, b_{2}, \ldots, b_{p}$, all members of which are taken from the set $\left\{a_{1}, \ldots, a_{q}\right\}$, moreover, the number $a_{j}(1 \leq j \leq q)$ is repeated $p_{j}$ times in the sequence $\left\{b_{k}\right\}$, also we assume that the sequence satisfies $b_{k} \neq b_{k-1}, b_{k+1}$ $\left(b_{0}=b_{p}, b_{p+1}=b_{1}\right)$. It is clear that $\sum_{j=1}^{q} p_{j}=p$ and $p_{j} \leq p / 2$ for $1 \leq j \leq q$. Take a simply connected finite-sheeted Riemann surface $K$ with boundary, ${ }^{8}$ bounded by a closed Jordan curve $C$, consisting of $p J$-curves $\sigma_{k}=B_{k} B_{k+1}$, where the point $B_{k}$ is projected onto the point $b_{k}$. Here and later on all indices $k$ will be taken modulo $p$, that is, $k=0$ should be replaced by $k=p$, and $k=p+1$ should be replaced by $k=1$. The surface $K$ can be constructed, for example, in the following way. We draw a closed Jordan curve $c$ through the points $a_{1}, a_{2}, \ldots, a_{q}$ in the $w$-plane, having a continuous curvature and consisting of line segments in sufficiently small neighborhoods of points $a_{j}$. Let $w_{0}$ be a point inside $c$. We consider a path on the curve $c$ which passes through points $b_{1}, b_{2}, \ldots, b_{p}, b_{1}$ moving along $c$ in the positive direction only (of course, when we move along $c$ from $b_{j}$ to $b_{j+1}$ we can pass through some other points $\left.b_{k}\right)$. Then $\arg \left(w-w_{0}\right)$ will get an increment $2 \pi \nu$, where $\nu$ is a positive integer. We can take as $K$ a part of the Riemann surface of the function $\left(w-w_{0}\right)^{1 / \nu}$ (that is, a part of the Riemann surface corresponding to the function $w=w_{0}+z^{\nu}$ ), covering the interior of the curve $c$. Then the boundary of $K$ will play the role of $C$, and, obviously, the points $B_{k}$ can be placed on the curve $C$ in the desired way. If $p_{j}=1$ for all $j$, we can take as $K$ the interior of the curve $c$.

Let $\Lambda$ be a Riemann surface corresponding to the function $w=\left(b e^{z}-a\right) /\left(e^{z}-1\right)$ (that is, the Riemann surface of the function $z=\operatorname{Ln}\{(w-a) /(w-b)\}$ ), with logarithmic ramification points over $a$ and $b, a \neq b$. If we join these logarithmic ramification points by a $J$-curve $\Gamma$ on $\Lambda$ (this curve does not have ends since the surface $\Lambda$ does not cover the points $a$ and $b$, but as a point moves along $\Gamma$ in each of the two possible directions, it tends to the corresponding logarithmic ramification points), and make a cut along this curve, the surface $\Lambda$ will be decomposed into two parts $\Lambda_{1}$ and $\Lambda_{2}$, each of them is called a logarithmic end. The curve $\Gamma$ oriented in such a way that the region $\Lambda_{j}(j=1,2)$ is on the left, is called the boundary of the logarithmic end $\Lambda_{j}$. For each of the logarithmic ends we shall say that it has logarithmic ramification points over $a$ and $b$.

${ }^{8}$ S. Stoïlow [Sto62, Vol. 2, Ch.VII, $\left.\S 5\right]$ uses the term polyhedral regions for Riemann surfaces with boundary. 
Now we take $p$ logarithmic ends $\Lambda_{k}, 1 \leq k \leq p$, where the end $\Lambda_{k}$ has logarithmic ramification points over $b_{k}$ and $b_{k+1}$, and its boundary is the $J$-curve $\sigma_{k}=B_{k+1} B_{k}$. We paste to the Riemann surface $K$ with the points $B_{k}, 1 \leq k \leq p$, excluded, the logarithmic ends $\Lambda_{k}$ along the curves $\sigma_{k}$ for all $k, 1 \leq k \leq p$. We get a Riemann surface $F$ which is called a Riemann surface with $p$ logarithmic ends. The part $K$ of the Riemann surface $F$ is called its pseudokernel (clearly it is not uniquely determined). If we exclude from $K$ sufficiently small one-sheeted neighborhoods of the boundary points $B_{k}, 1 \leq k \leq p$, we get a Riemann surface with boundary $K_{0}$ which is called the kernel of the Riemann surface $F$. The Riemann surface $F$ has at most finitely many algebraic ramification points, all of which belong to $K$, and $p$ logarithmic ramification points $\tilde{B}_{1}, \ldots, \tilde{B}_{p}$, which are projected onto the points $a_{1}, \ldots, a_{q}$, moreover, there are $p_{j}$ logarithmic ramification points over $a_{j}$.

Sufficiently small $\varepsilon$-neighborhood of the logarithmic ramification point $\tilde{B}_{k}$ contains an $\varepsilon$-neighborhood of the boundary point $B_{k}$ on $K$ and intersects the logarithmic ends $\Lambda_{k}$ and $\Lambda_{k-1}$. It is clear that the Riemann surface $F$ belongs to the class $F_{q_{1}}, q_{1} \geq q$. The line complex of a Riemann surface with five logarithmic ends (the sequence $b_{k}$ is of the form $a_{1}, a_{2}, a_{1}, a_{3}, a_{2}, \nu=3$ ) is shown in Fig. 34. See, also, Fig. 10 and 11. The line complex of a surface with $p$ logarithmic ends has $p$ elementary logarithmic regions, which are separated from each other by parts of the complex which have the form of a sequence of vertices, each of which is the end of exactly two bundles, so that bundles and vertices alternate in the following way: bundle, vertex, bundle, vertex, .... These parts of the line complex are also often called logarithmic ends because they, in some sense, correspond to logarithmic ends of the Riemann surface. More precisely, if a point moves along a logarithmic end of the line complex visiting successively all vertices contained in it, then the corresponding point on the Riemann surface is moving on certain logarithmic end $\Lambda$, moving from sheet to sheet, and moving away from the boundary of $\Lambda$.

Now we construct a homeomorphic mapping of the Riemann surface $F$ onto the complex $\zeta$-plane, which is quasiconformal on $F \backslash K_{0}$. Let us agree that to all regions on $F$, which we consider, we join their boundary points belonging to $F$.

Take pairwise disjoint $2 \varepsilon$-neighborhoods $U\left(2 \varepsilon, \tilde{B}_{k}\right)$ of the logarithmic ramification points $\tilde{B}_{k}$, where $\varepsilon$ is so small that $U\left(2 \varepsilon, \tilde{B}_{k}\right) \cap K$ is a disc sector with the vertex at $B_{k}$, which does not contain algebraic ramification points and is bounded by two radii and an arc which is projected into the circle $\left\{\left|w-b_{k}\right|=2 \varepsilon\right\}$. The radian measure of the arc can exceed $2 \pi$, so the sector is not necessarily one-sheeted. We make a cut in $U\left(\varepsilon, \tilde{B}_{k}\right) \cap K$ along a radius

$$
S_{k}=\left\{\left|w-b_{k}\right| \leq \varepsilon, \arg w=\alpha_{k}\right\},
$$

going out of $B_{k}$. Then $U\left(\varepsilon, \tilde{B}_{k}\right)$ decomposes into two parts, $U^{+}\left(\varepsilon, \tilde{B}_{k}\right)$ and $U^{-}(\varepsilon$, $\left.\tilde{B}_{k}\right)$, where $U^{+}\left(\varepsilon, \tilde{B}_{k}\right)$ has a nonempty intersection with $\Lambda_{k}$ and $U^{-}\left(\varepsilon, \tilde{B}_{k}\right)$ has a nonempty intersection with $\Lambda_{k-1}$. Denote the kernel $K \backslash \bigcup_{k=1}^{p} U\left(\varepsilon, \tilde{B}_{k}\right)$ by $K_{0}$. Let

$$
\begin{gathered}
\lambda_{k}=\Lambda_{k} \backslash\left\{U\left(\varepsilon, \tilde{B}_{k}\right) \cup U\left(\varepsilon, \tilde{B}_{k+1}\right)\right\}, \\
\Lambda_{k}^{\prime}=\Lambda_{k} \cup U^{+}\left(\varepsilon, \tilde{B}_{k}\right) \cup U^{-}\left(\varepsilon, \tilde{B}_{k+1}\right)=\lambda_{k} \cup U^{+}\left(\varepsilon, \tilde{B}_{k}\right) \cup U^{-}\left(\varepsilon, \tilde{B}_{k+1}\right) .
\end{gathered}
$$


It is easy that

$$
F=K_{0} \cup\left(\bigcup_{k=1}^{q} \Lambda_{k}^{\prime}\right) .
$$

We map $\Lambda_{k}^{\prime}$ quasiconformally onto the upper half-plane

$$
H_{k}=\left\{\operatorname{Im} \zeta_{k}>0\right\}
$$

with some trapezoid removed. To construct such mapping we do the following. We map $U^{-}\left(\varepsilon, \tilde{B}_{k+1}\right)$ conformally onto

$$
H_{k}^{-}=\left\{\xi_{k}<-1, \eta_{k}>0\right\}, \zeta_{k}=\xi_{k}+i \eta_{k},
$$

using the function

$$
\zeta_{k}=\ln \frac{w-b_{k+1}}{\varepsilon}-1-i \alpha_{k+1},
$$

where the branch of the logarithm is chosen in such a way that the point $w \in S_{k+1}$ is mapped onto $\zeta_{k}=\xi_{k}=\ln \frac{\left|w-b_{k+1}\right|}{\varepsilon}-1$. Similarly, we map $U^{+}\left(\varepsilon, \tilde{B}_{k}\right)$ conformally onto

$$
H_{k}^{+}=\left\{\xi_{k}>1, \eta_{k}>0\right\}
$$

using the function

$$
\zeta_{k}=\ln \frac{\varepsilon}{w-b_{k}}+1+i \alpha_{k}
$$

where the branch of the logarithm is chosen in such a way that the point $w \in S_{k}$ is mapped onto $\zeta_{k}=\xi_{k}=\ln \frac{\varepsilon}{\left|w-b_{k}\right|}+1$.

We cut $\Lambda_{k}$ along all $J$-curves lying over $\sigma_{k}$. Then the Riemann surface $\lambda_{k}$ decomposes into one-sheeted regions $\Phi_{j}^{(k)}, j=1,2, \ldots$, each of which has the form of the extended complex $w$-plane with the discs $\left\{\left|w-b_{k}\right|<\varepsilon\right\}$ and $\left\{\mid w-b_{k+1}<\varepsilon\right\}$ removed, and a cut $\sigma_{k}^{0}$ along those part of the curve $\sigma_{k}$ which lies outside these discs. We denote the boundaries of this cut by $\sigma_{k}^{+}$and $\sigma_{k}^{-}$depending on whether the positive direction on this boundary (that is, the direction for which the region is on the left) coincides with the direction from $b_{k}$ to $b_{k+1}$ on $\sigma_{k}$, or with the direction from $b_{k+1}$ to $b_{k}$. We map $\Phi_{j}^{(k)}$ quasiconformally onto the rectangle

$$
Q=\left\{-1<\xi_{k}<1,0<\eta_{k}<2 \pi\right\}
$$

in such a way that the circle $\left\{\left|w-b_{k+1}\right|=\varepsilon\right\}$ is mapped onto the segment $\left\{\xi_{k}=\right.$ $\left.-1,0 \leq \eta_{k} \leq 2 \pi\right\}$, the circle $\left\{\left|w-b_{k}\right|=\varepsilon\right\}$ is mapped onto the segment $\left\{\xi_{k}=\right.$ $\left.1,0 \leq \eta_{k} \leq 2 \pi\right\}, \sigma_{k}^{+}$is mapped onto the segment $\left\{\eta_{k}=2 \pi,-1 \leq \xi_{k} \leq 1\right\}$, and $\sigma_{k}^{-}$is mapped onto the segment $\left\{\eta_{k}=0,-1 \leq \xi_{k} \leq 1\right\}$, moreover, the distortion is constant on each of the sides of the rectangle. The existence of such quasiconformal mapping with a bounded characteristic follows from Example 5 from Section 2. Each point from $\lambda_{k}$ which is projected into $\sigma_{k}$, lies simultaneously on the boundary $\sigma_{k}^{+}$of some $\Phi_{j}^{(k)}$, and on the boundary $\sigma_{k}^{-}$of the region $\Phi_{j+1}^{(k)}$. Under the mapping onto $Q$ this point is mapped either onto the point $(\xi, 0)$, or onto the point $(\xi, 2 \pi)$ depending on whether we mapped $\Phi_{j+1}^{(k)}$ or $\Phi_{j}^{(k)}$, but the $x$-coordinate in both cases will be the same, since the distortion $\left|d w / d \zeta_{k}\right|$ on the horizontal sides of the rectangle is constant and is equal to $\frac{1}{2} \operatorname{length}\left(\sigma_{k}^{0}\right)$. Therefore, making additional 
vertical shifts of the rectangles $Q$ corresponding to $\Phi_{j}^{(k)}, j=1,2, \ldots$, we can paste them and get a half-strip $L_{k}=\left\{-1<\xi_{k}<1, \eta_{k}>0\right\}$. In such a way we get a quasiconformal mapping $\zeta_{k}=\omega_{k}(w)$ of the surface $\lambda_{k}$ onto $L_{k}$, moreover, $\omega_{k}(w)$ $\operatorname{maps} \Phi_{j}^{(k)}$ onto

$$
Q_{j}^{(k)}=\left\{-1<\xi_{k}<1,2 \pi(j-1)<\eta_{k}<2 \pi j\right\} .
$$

The mapping $\omega_{k}(w)$ has a bounded characteristic, and a constant distortion

$$
\left|d \zeta_{k} / d w\right|=1 / \varepsilon
$$

on the part of the boundary of $\lambda_{k}$ which is projected into $\left\{\left|w-b_{k}\right|=\varepsilon\right\}$ or $\{\mid w-$ $\left.b_{k+1} \mid=\varepsilon\right\}$.

The function $\zeta_{k}=\omega_{k}(w)$ maps the part of the boundary of $\lambda_{k}$, which is projected into $\left\{\left|w-b_{k+1}\right|=\varepsilon\right\}$, into $\left\{\xi_{k}=-1, \eta_{k} \geq 0\right\}$. But the same set of points considered as a part of the boundary of $U^{-}\left(\varepsilon, \tilde{B}_{k+1}\right)$ is mapped by the function (5.4) into $\left\{\xi_{k}=-1, \eta_{k} \geq \theta_{k+1}^{-}\right\}$, where $\theta_{k+1}^{-}>0$ is the angle between $S_{k+1}$ and $\sigma_{k}$ at the point $B_{k+1}$, moreover, the distortion is constant and satisfies the relation (5.6). Similarly, the part of the boundary of $\lambda_{k}$ which is projected into $\left\{\left|w-b_{k}\right|=\varepsilon\right\}$, on one hand, is mapped by the function $\omega_{k}(w)$ into $\left\{\xi_{k}=1, \eta_{k} \geq 0\right\}$, and on the other hand, is mapped by the function (5.5) into $\left\{\xi_{k}=1, \eta_{k} \geq \theta_{k}^{+}\right\}$, where $\theta_{k}^{+}>0$ is the angle between $S_{k}$ and $\sigma_{k}$ at the point $B_{k}$.

Denote by $T_{k}$ the trapezoid with vertices at the points $(-1,0),(1,0),\left(1, \theta_{k}^{+}\right)$, $\left(-1, \theta_{k+1}^{-}\right)$. We map quasiconformally $L_{k}$ onto $L_{k} \backslash T_{k}$ using the mapping

$$
\zeta_{k}=\tilde{\omega}_{k}\left(\zeta_{k}\right)=\xi_{k}+i\left\{\eta_{k}+\frac{\theta_{k}^{+}-\theta_{k+1}^{-}}{2} \xi_{k}+\frac{\theta_{k}^{+}+\theta_{k+1}^{-}}{2}\right\} .
$$

This quasiconformal mapping (cf. Example 2 from Section 2) is affine, has a bounded characteristic, and reduced to vertical shifts on the vertical sides of $L_{k}$. Each rectangle $Q_{j}^{(k)}$ is mapped onto some parallelogram by the mapping $\tilde{\omega}_{k}\left(\zeta_{k}\right)$. The function $\zeta_{k}=\Omega_{k}(w)=\tilde{\omega}_{k}\left(\omega_{k}(w)\right)$ maps $\lambda_{k}$ onto $L_{k} \backslash T_{k}$ quasiconformally and with the bounded characteristic $p\left(w, \zeta_{k}\right) \leq M$, moreover now points of the part of the boundary of $\lambda_{k}$ which are projected into $\left\{\left|w-b_{k+1}\right|=\varepsilon\right\}$ (into $\left\{\left|w-b_{k}\right|=\varepsilon\right\}$ ) are mapped onto the same points on $\left\{\xi_{k}=-1, \eta_{k} \geq \theta_{k+1}^{-}\right\}$(on $\left.\left\{\xi_{k}=1, \eta_{k} \geq \theta_{k}^{+}\right\}\right)$ both under the mapping $\zeta_{k}=\Omega_{k}(w)$ and under the mapping (5.4) (mapping (5.5)).

We have got a quasiconformal mapping $\zeta_{k}=\Omega_{k}^{0}(w)$ of the surface $\Lambda_{k}^{\prime}$ onto $H_{k} \backslash T_{k}$, which is represented by the function (5.4) on $U^{-}\left(\varepsilon, \tilde{B}_{k+1}\right)$, by the function (5.5) on $U^{+}\left(\varepsilon, \tilde{B}_{k}\right)$, and by the function $\Omega_{k}(w)$ on $\lambda_{k}$, where the first two functions are conformal mappings, and the last function is a quasiconformal mapping with a bounded characteristic. Therefore

$$
\begin{aligned}
\iint_{H_{k} \backslash T_{k}}\left\{p\left(\zeta_{k}, w\right)-1\right\} d \sigma\left(\ln \zeta_{k}\right) \\
=\iint_{L_{k} \backslash T_{k}}\left\{p\left(\zeta_{k}, w\right)-1\right\} d \sigma\left(\ln \zeta_{k}\right) \leq M \iint_{L_{k} \backslash T_{k}} d \sigma\left(\ln \zeta_{k}\right) \\
=M \iint_{L_{k} \backslash T_{k}} \frac{d \sigma\left(\zeta_{k}\right)}{\left|\zeta_{k}\right|^{2}} \leq M^{\prime}<\infty
\end{aligned}
$$

Let $w=\psi_{k}\left(\zeta_{k}\right)$ be the inverse function of $\zeta_{k}=\Omega_{k}^{0}(w)$, mapping $H_{k} \backslash T_{k}$ onto $\Lambda_{k}^{\prime}$. Denote by $\nu_{k}(r, a)$ the number of $a$-points of the function $\psi_{k}\left(\zeta_{k}\right)$ in $\left(H_{k} \backslash T_{k}\right) \cap\left\{\left|\zeta_{k}\right| \leq\right.$ 
$r\}$. It is easy to see that $\psi_{k}\left(\zeta_{k}\right) \neq b_{k}, b_{k+1}$ and $\nu\left(r, b_{k}\right) \equiv 0, \nu\left(r, b_{k+1}\right) \equiv 0$. If $a \neq$ $b_{k}, b_{k+1}$, then the $a$-points $\psi_{k}\left(\zeta_{k}\right)$ form a sequence $\zeta_{k}^{(0)}(a)+2 \pi i m, m=0,1,2, \ldots$ Therefore $\nu_{k}(r, a) \sim r /(2 \pi)$ as $r \rightarrow \infty$ for all $a \neq b_{k}, b_{k+1}$.

Now we observe that the point $w \in S_{k}$, as we have already mentioned, is mapped onto the point $\xi_{k}=\ln \frac{\varepsilon}{\left|w-b_{k}\right|}+1$ on the boundary of $H_{k} \backslash T_{k}$, and onto the point $\xi_{k-1}=\ln \frac{\left|w-b_{k}\right|}{\varepsilon}-1=-\xi_{k}$ on the boundary of $H_{k-1} \backslash T_{k-1}$. Therefore, if we map $H_{k} \backslash T_{k}$ using the function

$$
\zeta=\zeta_{k}^{\frac{2}{p}} e^{i \frac{2 \pi}{p}(k-1)}, k=1,2, \ldots, p,
$$

onto some region $\Xi_{k}$, which is the angle $\left\{\frac{2 \pi}{p}(k-1)<\arg \zeta<\frac{2 \pi}{p} k\right\}$ with some bounded region deleted, then the points on the surface $F$ which correspond to a point $\zeta$ from $\partial \Xi_{k-1} \cap \partial \Xi_{k}=\left\{\arg \zeta=\frac{2 \pi}{p}(k-1),|\zeta| \geq 1\right\}$ under the mappings $w=\psi_{k}\left((-1)^{k-1} \zeta^{p / 2}\right)$ and $w=\psi_{k-1}\left((-1)^{k} \zeta^{p / 2}\right)$ are the same. Then the function

$$
\zeta=\zeta(w)=\left\{\Omega_{k}^{0}(w)\right\}^{\frac{2}{p}} e^{i \frac{2 \pi}{p}(k-1)}, k=1,2, \ldots, p,
$$

realizes a quasiconformal mapping of the part of the simply connected Riemann surface $F \backslash K_{0}=\bigcup_{k=1}^{p} \Lambda_{k}^{\prime}$ onto $\bigcup_{k=1}^{p} \bar{\Xi}_{k}$. We map the Riemann surface $K_{0}$ homeomorphically onto the region $\{|\zeta|<\infty\} \backslash \bigcup_{k=1}^{p} \bar{\Xi}_{k}$ in such a way that the points $w \in \partial K_{0}$ are mapped in the same way as by the function $\zeta=\zeta(w)$ defined by the formulas (5.9). Thus we get a homeomorphic mapping $\zeta=\zeta(w)$ of the Riemann surface $F$ onto $\{|\zeta|<\infty\}^{9}$. Thus we have proved that the Riemann surface $F$ is simply connected. Let $z=z(w)$ be a conformal mapping of $F$ onto $\{|z|<R\}, R \leq \infty$. We define a homeomorphic mapping $z=z(\zeta)$ of the complex $\zeta$-plane onto $\{|z|<R\}$ as a composition of two mappings $\{|\zeta|<\infty\} \rightarrow F \rightarrow\{|z|<R\}$, moreover on $\bigcup_{k=1}^{p} \bar{\Xi}_{k}$ this mapping is quasiconformal with the characteristic $p(\zeta, z)=p(\zeta, w)=p\left(\zeta_{k}, w\right)$, where $\zeta$ and $\zeta_{k}$ are connected by the formulas (5.8).

Taking into account (5.7) we get

$$
\begin{aligned}
\iint_{\bigcup_{k=1}^{p} \Xi_{k}}\{p(\zeta, w) & -1\} d \sigma(\ln \zeta)=\sum_{k=1}^{p} \iint_{\Xi_{k}}\{p(\zeta, w)-1\} d \sigma(\ln \zeta) \\
& =\sum_{k=1}^{p} \iint_{H_{k} \backslash T_{k}}\left\{p\left(\zeta_{k}, w\right)-1\right\} \frac{d \sigma(\ln \zeta)}{d \sigma\left(\ln \zeta_{k}\right)} d \sigma\left(\ln \zeta_{k}\right) \\
& =\sum_{k=1}^{p} \frac{1}{p^{2}} \iint_{H_{k} \backslash T_{k}}\left\{p\left(\zeta_{k}, w\right)-1\right\} d \sigma\left(\ln \zeta_{k}\right) \leq \frac{1}{p} M^{\prime}<\infty .
\end{aligned}
$$

Thus, the mapping $z=z(\zeta)$ satisfies the condition of Theorem 3.1 (O. Teichmüller theorem). Hence $R=\infty$ and the surface $F$ is of parabolic type. The meromorphic

\footnotetext{
${ }^{9}$ It is possible to map $K_{0}$ by a quasiconformal mapping also, then the mapping $\zeta=\zeta(w)$ would be quasiconformal everywhere on $F$.
} 
function $w=f(z)$ mapping $\{|z|<\infty\}$ onto $F$ can be normalized in such a way that the function $\zeta=\zeta(z)=\zeta[f(z)]$, mapping the complex $z$-plane onto the complex $\zeta$-plane satisfies the condition

$$
\lim _{z \rightarrow \infty}\left|\frac{\zeta(z)}{z}\right|=\lim _{\zeta \rightarrow \infty}\left|\frac{z(\zeta)}{\zeta}\right|=1
$$

Let $w=\varphi(\zeta)$ be the inverse function to $\zeta=\zeta(w), \varphi$ maps $\{|\zeta|<\infty\}$ onto the surface $F$. Outside $\bigcup_{k=1}^{p} \bar{\Xi}_{k}$ the function $\varphi(\zeta)$ has at most finitely many $a$-points for each $a$. It is not difficult to count, using (5.8), that

$$
\begin{gathered}
n(r, a, \varphi)=O(1)+\sum_{k=1}^{p} \nu_{k}\left(r^{p / 2}, a\right) \sim \\
\frac{p}{2 \pi} r^{\frac{p}{2}} \text { for } a \neq a_{1}, \ldots, a_{q}, \\
\frac{p-2 p_{j}}{2 \pi} r^{\frac{p}{2}}+O(1) \text { for } a=a_{j}, 1 \leq j \leq q,
\end{gathered}
$$

since for $a=a_{j}$ exactly $2 p_{j}$ of the functions $\nu_{k}(r, a)$ are identically zero. The summand $O(1)$ in (5.12) is essential only for those $a_{j}$, for which $2 p_{j}=p$, if there are any. Note that the pseudokernel $K$ can always be chosen in such a way that it does not cover the points $a_{j}, 1 \leq j \leq q$ (this is what happens in the construction of a pseudokernel which was given as an example). Then the summand $O(1)$ in (5.12) is absent (it appears because of the points on $K_{0}$ which are projected onto $a_{j}$ ), and if $2 p_{j}=p$, the function $f(z)$ does not take the value $a_{j}$.

The equality (5.10) implies

$$
n(r, a, f) \sim \begin{cases}\frac{p}{2 \pi} r^{\frac{p}{2}} & \text { for } a \neq a_{1}, \ldots, a_{q}, \\ \frac{p-2 p_{j}}{2 \pi} r^{\frac{p}{2}}+O(1) & \text { for } a=a_{j}, 1 \leq j \leq q\end{cases}
$$

so

$$
N(r, a, f) \sim \frac{1}{\pi} r^{\frac{p}{2}} \quad \text { for } \quad a \neq a_{1}, \ldots, a_{q}
$$

and

$$
N(r, a, f) \sim \frac{1}{\pi}\left(1-\frac{2 p_{j}}{p}\right) r^{\frac{p}{2}}+O(\ln r) \quad \text { for } \quad a=a_{j}, 1 \leq j \leq q .
$$

Take $a \neq a_{1}, \ldots, a_{q}$ such that the Valiron deficiency $\Delta(a, f)=0$. Then (5.13) implies that

$$
T(r, f) \sim \frac{1}{\pi} r^{\frac{p}{2}} .
$$

On one hand, the relation (5.14') implies that the function $f(z)$ has a normal type of order $\rho=p / 2$, on the other hand, using (5.13) and (5.14) we find that

$$
\delta(a, f)=\Delta(a, f)= \begin{cases}0 & \text { for } a \neq a_{1}, \ldots, a_{q}, \\ \frac{2 p_{j}}{p} & \text { for } a=a_{j}, 1 \leq j \leq q,\end{cases}
$$


moreover, by the corollary of Theorem 6.5 from Chapter $4,(5.15)$ remains true for each normalization of the function $f(z)$ mapping $\{|z|<\infty\}$ onto $F$, that is, for all $f(A z+B), A \neq 0$. We have

$$
\sum_{j=1}^{p} \delta\left(a_{j}, f\right)=\frac{2}{p} \sum_{j=1}^{p} p_{j}=2 .
$$

If we let $p$ be an odd number $\geq 3$, we get examples of meromorphic functions with properties similar to the properties of functions from Example 8 from Section 5 of Chapter 5.

Now it is not difficult to prove Theorem 5.1. We write the rational numbers $\delta\left(a_{j}\right) / 2$ in the form $\delta\left(a_{j}\right) / 2=p_{j} / p$, where $p_{j}$ and $p$ are positive integers. Since $\sum_{j=1}^{p} \delta\left(a_{j}\right)=2$, we have $\sum_{j=1}^{q} p_{j}=p$. Besides, it is obvious that $p_{j} \leq p / 2$, $1 \leq j \leq q$. It is clear that we can find a sequence $b_{1}, \ldots, b_{p}$ whose elements belong to the set $\left\{a_{1}, \ldots, a_{q}\right\}$, and the value $a_{j}(1 \leq j \leq q)$ occurs $p_{j}$ times in the sequence $b_{1}, \ldots, b_{p}$, and $b_{k} \neq b_{k-1}, b_{k+1}$. Constructing for such sequence $b_{1}, \ldots, b_{p}$ a surface $F$ with $p$ logarithmic ends in the way described above, we get, that for the mapping function $f(z)$ the formulas (5.1) hold, that is, the deficiency at the point $a_{j}$ is equal to $\delta\left(a_{j}\right), 1 \leq j \leq q$, and there are no other deficient values.

If $\delta\left(a_{j}\right)=1$, then the surface $F$ can be chosen in such a way that it does not cover the point $a_{j}$, that is, $f(z) \neq a_{j}$ for all $z$.

\section{Almost periodic ends}

In the construction of surfaces with logarithmic ends, which was presented in the previous section, the main role was played by the notion of a logarithmic end, a sort of a "half" of the Riemann surface of a logarithmic function. In order to solve the inverse problem for a wider class of cases than the one for which the solution is given by Theorem 5.1, we generalize the class of Riemann surfaces with finitely many logarithmic ends, to do this we first generalize the notion of a logarithmic end.

Consider a sequence $\left\{\Phi_{k}\right\}, k= \pm 1, \pm 2, \ldots$, of closed Riemann surfaces of genus zero, all ramification points of which are projected into the points $a_{1}, a_{2}, \ldots, a_{q}$ only, where the points $a_{1}, \ldots, a_{q}$ are distinct. For simplicity we shall assume that the complex numbers $a_{1}, \ldots, a_{q}$ are finite. Let $b_{1}$ and $b_{2}$ be complex numbers, such that either $b_{1}=a_{1}, b_{2}=a_{2}$, or $b_{1}=b_{2}=a_{1}$. We assume that $\Phi_{k}=\Phi_{-k}, k \geq 1$. For each Riemann surface $\Phi_{k}$ we make a cut $\Gamma_{k}$ along some $J$-curve with ends $B_{1}^{k}, B_{2}^{k} \in \Phi_{k}$, which is projected to a fixed curve $\gamma$ with ends $b_{1}$ and $b_{2}$, which does not pass through the points $a_{j}$, unless $a_{j} \neq b_{1}, b_{2}$. The case $b_{1}=b_{2}$, which corresponds to a closed Jordan curve $\gamma$ is not excluded, but, obviously, in this case the requirement of possibility making the cut $\Gamma_{k}$ imposes certain restriction on the surface $\Phi_{k}$, for example, $\Phi_{k}$ cannot be a one-sheeted surface. The Riemann surface obtained from $\Phi_{k}$ after making the cut $\Gamma_{k}$ will be called a brick $\tilde{\Phi}_{k}, \tilde{\Phi}_{k}=\tilde{\Phi}_{-k}$.

Considering $\gamma$ as a cut, denote its boundaries by $\gamma^{+}$and $\gamma^{-}$, so that in the sufficiently small neighborhood of $b_{2}$ the angle between $\gamma^{-}$and $\gamma^{+}$with the vertex at $b_{2}$ is equal to $2 \pi$. Transferring this notation to each of the cuts $\Gamma_{k}$ we get the boundaries $\Gamma_{k}^{+}$and $\Gamma_{k}^{-}$. Now we paste $\Gamma_{k}^{+}$with $\Gamma_{k+1}^{-}, k \neq-1,0$, and $\Gamma_{-1}^{+}$with $\Gamma_{1}^{-}$. We get an open simply connected Riemann surface $\Phi$ which has two logarithmic ramification points $\tilde{B}_{1}$ and $\tilde{B}_{2}$ which are projected onto $b_{1}$ and $b_{2}$, respectively (if $b_{1}=b_{2}$, then the correspondence between logarithmic ramification points and $b_{j}$, 
$j=1,2$ is established in an arbitrary, but fixed way). The cut on the surface $\phi$ along the curve $\Gamma=\Gamma_{1}^{-}=\Gamma_{-1}^{+}$divides $\Phi$ into two parts, $\Lambda_{1}$ and $\Lambda_{2}$, each of which is called an end. The boundary of the end $\Lambda_{j}$ is defined in the same way as for logarithmic ends.

We shall distinguish between surfaces $\Phi$ with $b_{1} \neq b_{2}$, and with $b_{1}=b_{2}$. Following L.I. Volkovyski1 [Vol50], we call the ends arising from $\Phi$ the ends of the first and the second kind, respectively.

If all $\Phi_{k}$ are one-sheeted, then, as is easy to see, the surface $\Phi$ is the Riemann surface of the function $\ln \frac{w-a_{2}}{w-a_{1}}$, and the ends $\Lambda_{j}$ are logarithmic. In the case when all bricks $\tilde{\Phi}_{k}$ are the same, the ends $\Lambda_{j}$ are called periodic ${ }^{10}$. Ends satisfying somewhat less restrictive assumptions are called almost periodic. Before listing the assumption, we introduce some notation.

Let $m_{k}$ be the number of sheets on the surface $\Phi_{k}$.

Denote by $\Phi^{\nu}, \nu=1,2, \ldots$, the part of the Riemann surface $\Phi$ consisting of $\bigcup_{k=1}^{\nu}$ of the points of $\Phi^{\nu}$ lying over $a$, and $n_{\nu}(a)$ is the number of the same points, but each ramification point of order $m$ is counted $m+1$ times. The number

$$
n_{\nu}=\sum_{k=1}^{\nu} m_{k}
$$

is called the number of sheets of the surface $\Phi^{\nu}$. Besides, we let $n_{0}=n_{0}(a)=0$ for all $a$. Obviously, $\bar{n}_{\nu}(a) \leq n_{\nu}(a) \leq n_{\nu}$, besides, $n_{\nu}(a)=n_{\nu}$ for all $a \neq b_{1}, b_{2}$. In the case when $b_{1}=b_{2}=a_{1}$, we denote the sum over $k, 1 \leq k \leq \nu$, of numbers of points (in the case of algebraic ramification points we take into account their orders) of the Riemann surfaces $\Phi_{k} \backslash\left\{B_{j}^{k}\right\}$, lying over the point $a_{1}$ by $n_{\nu}^{\prime}\left(b_{j}\right), j=1,2$. It is easy to see that

$$
2 n_{\nu}-n_{\nu}^{\prime}\left(b_{1}\right)-n_{\nu}^{\prime}\left(b_{2}\right)=n_{\nu}-n_{\nu}\left(a_{1}\right) .
$$

Later on we shall write $n_{\nu}\left(b_{j}\right)$ instead of $n_{\nu}^{\prime}\left(b_{j}\right)$, because it does not lead to any confusion, but will allow us not to consider the cases $b_{1}=b_{2}$ and $b_{1} \neq b_{2}$ separately in some formulas. By the Riemann-Hurwitz formula (4.1) the sum of all algebraic ramification points of the surface $\Phi_{k}$ is equal to $2 m_{k}$, if we agree to count the orders of the points $B_{j}^{k}, j=1,2$, according to the number of sheets included in the cycle, that is, we add one to the order of a ramification point (or to zero, in the case of a simple point). Then it is easy to check that

$$
\begin{aligned}
\sum_{j=1}^{2}\left(n_{\nu}-\bar{n}_{\nu}\left(a_{j}\right)\right) & +\sum_{j=3}^{q}\left(n_{\nu}\left(a_{j}\right)-\bar{n}_{\nu}\left(a_{j}\right)\right) \\
& =\sum_{j=1}^{2}\left(n_{\nu}-n_{\nu}\left(a_{j}\right)\right)+\sum_{j=1}^{q}\left(n_{\nu}\left(a_{j}\right)-\bar{n}_{\nu}\left(a_{j}\right)\right)=2 n_{\nu}
\end{aligned}
$$

\footnotetext{
${ }^{10}$ In the case of periodic ends the requirement that the curves $\Gamma_{k} \equiv \Gamma_{1}$ are $J$-curves is superfluous, since the equal bricks $\tilde{\Phi}_{k} \equiv \tilde{\Phi}_{1}$ can be pasted into a surface $\Phi$ if the cuts in $\Phi_{k}=\Phi_{1}$ are made along an arbitrary Jordan curve, the same for all $\Phi_{k}$.
} 
for $b_{1} \neq b_{2}$, and

$$
\begin{aligned}
n_{\nu}-\bar{n}_{\nu}\left(a_{1}\right) & +\sum_{j=2}^{q}\left(n_{\nu}\left(a_{j}\right)-\bar{n}_{\nu}\left(a_{j}\right)\right) \\
& =\left(n_{\nu}-n_{\nu}\left(a_{1}\right)\right)+\sum_{j=1}^{q}\left(n_{\nu}\left(a_{j}\right)-\bar{n}_{\nu}\left(a_{j}\right)\right)=2 n_{\nu}
\end{aligned}
$$

for $b_{1}=b_{2}=a_{1}$.

The quantities

$$
\begin{gathered}
\delta_{g}(a)=\delta_{g}\left(a, \Lambda_{1}\right)=1-\limsup _{\nu \rightarrow \infty} \frac{n_{\nu}(a)}{n_{\nu}}=\liminf _{\nu \rightarrow \infty} \frac{n_{\nu}-n_{\nu}(a)}{n_{\nu}}, \\
\varepsilon_{g}(a)=\varepsilon_{g}\left(a, \Lambda_{1}\right)=\liminf _{\nu \rightarrow \infty} \frac{n_{\nu}(a)-\bar{n}_{\nu}(a)}{n_{\nu}}
\end{gathered}
$$

will be called the geometric deficiencies and indices, respectively, of the end $\Lambda_{1}$, as well as of the end $\Lambda_{2}$, and of the surface $\Phi$. It is clear that for $a \neq b_{1}, b_{2}$ we have $\delta_{g}(a)=0$, and for $a \neq a_{1}, \ldots, a_{q}$, we have $\varepsilon_{g}(a)=0$. It is easy to see that $\varepsilon_{g}(a)+\delta_{g}(a) \leq 1, \varepsilon_{g}(a) \geq 0, \delta_{g}(a) \geq 0$. Besides, the equalities (6.2) and (6.3) imply that

$$
\sum_{a}\left(\delta_{g}(a)+\varepsilon_{g}(a)\right) \leq 2,
$$

moreover, the equality here takes place if and only if all geometric deficiencies and indices are obtained as exact limits, that is, for all $a$ the relations $n_{\nu}(a)=$ $n_{\nu}\left(1-\delta_{g}(a)\right)+o\left(n_{\nu}\right)$ and $n_{\nu}(a)-\bar{n}_{\nu}(a)=\varepsilon_{g}(a) n_{\nu}+o\left(n_{\nu}\right)$ hold.

For $b_{1}=b_{2}=a_{1}$ we will consider, in addition to $\delta_{g}\left(a_{1}\right)$, the quantity

$$
\delta_{g}^{\prime}\left(b_{j}\right)=\delta_{g}^{\prime}\left(b_{j}, \Lambda_{1}\right)=1-\limsup _{\nu \rightarrow \infty} \frac{n_{\nu}^{\prime}\left(b_{j}\right)}{n_{\nu}}, j=1,2 .
$$

The equality (6.1) implies that, if $n_{\nu}^{\prime}\left(b_{j}\right) / n_{\nu}=1-\delta_{g}^{\prime}\left(b_{j}\right)+o(1)$ as $\nu \rightarrow \infty$, $j=1,2$, then $\delta_{g}\left(a_{1}\right)=\delta_{g}^{\prime}\left(b_{1}\right)+\delta_{g}^{\prime}\left(b_{2}\right)$. Later on we shall write $\delta_{g}\left(b_{j}\right)$ and $\delta_{g}\left(b_{j}, \Lambda_{1}\right)$ instead of $\delta_{g}^{\prime}\left(b_{j}\right)$ and $\delta_{g}^{\prime}\left(b_{j}, \Lambda_{1}\right)$.

It is clear that the surface $\Phi$ belongs to the class $F_{q}$ and can be represented by means of a line complex. It will be convenient for us to choose base curves in a special way. If $\Phi$ has ends of the first kind, we choose the base curve in such a way that it includes the curve $\gamma$. If $\Phi$ has ends of the second kind, then $\gamma$ is a closed Jordan curve, and we choose the base curve in such a way that it intersects $\gamma$ at two points only: at $a_{1}=b_{1}=b_{2}$ and at one more point $b$. In this case as vertices we choose one point on each of the arcs on which the curve $\gamma$ is divided by the points $a_{1}$ and $b$. It is easy to see that with such choice of the base curve the boundary between the parts of the line complex of the surface $\Phi$ corresponding to the neighboring bricks passes either through one edge $\left(b_{1} \neq b_{2}\right)$, or through two vertices joined by one edge $\left(b_{1}=b_{2}\right)$. A line complex of the surface $\Phi$ with ends of the first kind is shown on Fig. 9 and 35, a line complex of the surface with ends of the second kind is shown on Fig. 13 and 36. In the case shown on Fig. 9, the surface $\Phi$ has two logarithmic ends, in the case shown on Fig. 13, the surface $\Phi$ has two periodic ends, which in this special case are called sine-ends. 
It is easy to single out those parts of the line complex of $\Phi$ which correspond to surfaces $\Phi^{\nu}$, and, as it easy to verify, the geometric deficiencies and indices of the surface $\Phi$ can be computed directly from the line complex of the surface $\Phi$.

Now we assume that $\delta_{g}\left(b_{1}\right)>0$ and $\delta_{g}\left(b_{2}\right)>0$. We cut from $\Phi_{k}$ pairwise disjoint $\varepsilon$-neighborhoods $U\left(\varepsilon, B_{j}^{k}\right), j=1,2$, of the points $B_{1}^{k}$ and $B_{2}^{k}$, where $\varepsilon>0$ is chosen to be so small that $2 \varepsilon$-neighborhoods of the points $B_{1}^{k}$ and $B_{2}^{k}$ do not contain other ramification points of $\Phi_{k}$, and the part of $\Gamma_{k}$ containing in each of the neighborhoods $U\left(2 \varepsilon, B_{j}^{k}\right)$ consists of one line segment. We find a quasiconformal mapping of the Riemann surface

$$
\Phi_{k}^{\prime}=\tilde{\Phi}_{k} \backslash\left(U\left(\varepsilon, B_{1}^{k}\right) \cup U\left(\varepsilon, B_{2}^{k}\right)\right)
$$

onto the rectangle $R_{k}=\left\{-1 / \delta_{g}\left(b_{2}\right) \leq \xi \leq 1 / \delta_{g}\left(b_{1}\right), 0 \leq \eta \leq 2 \pi m_{k}\right\}, \zeta=\xi+i \eta$, such that the part of the boundary of $\Phi_{k}^{\prime}$ lying over $\left\{\left|w-b_{2}\right|=\varepsilon\right\}$ and over $\left\{\left|w-b_{1}\right|=\varepsilon\right\}$ is mapped onto the left and the right sides of the rectangle $R_{k}$, respectively; and the part of the boundary of $\Phi_{k}^{\prime}$ lying on $\Gamma_{k}^{-}$and on $\Gamma_{k}^{+}$, is mapped onto the lower side and the upper side of $R_{k}$, respectively; moreover the distortion of the quasiconformal mapping is constant on each side of the rectangle. The existence of the required quasiconformal mapping follows from Example 5 of Section 2. These quasiconformal mappings are not uniquely determined. Denote by $p_{k}$ the infimum of the set of numbers $\sup _{w \in \Phi_{k}^{\prime}} p(w, \zeta)$ over all quasiconformal mappings $\Phi_{k}^{\prime}$ on $R_{k}$ with the described correspondence between the boundaries. It ic clear that $p_{-k}=p_{k}$. Since $\Phi_{k}^{\prime}$ depends on $\varepsilon$, then $p_{k}$ depends on $\varepsilon$. We shall assume that $\varepsilon$ is fixed ${ }^{11}$.

Denote the function realizing the quasiconformal mapping of $\Phi_{k}^{\prime}$ onto $R_{k}$ with the indicated correspondence between the boundaries by

$$
\zeta=\varphi_{k}(w),
$$

we also assume that $p\left(w, \varphi_{k}(w)\right)<2 p_{k}$ for $w \in \Phi_{k}^{\prime}$.

Definition. Ends $\Lambda_{1}$ and $\Lambda_{2}$ of the Riemann surface $\Phi$ are called almost periodic, if the following conditions are satisfied:

$1^{\circ} 1-\frac{n_{\nu}\left(b_{j}\right)}{n_{\nu}}+O\left(\frac{1}{n_{\nu}}\right)=\delta_{g}\left(b_{j}\right)>0, j=1,2 ;$

$2^{\circ} \sum_{\nu=1}^{\infty} \frac{p_{\nu} m_{\nu}}{n_{\nu}^{2}}<\infty$;

$3^{\circ}$ The following limits exists: $\lim _{\nu \rightarrow \infty} \frac{n_{\nu}(a)-\bar{n}_{\nu}(a)}{n_{\nu}}=\varepsilon_{g}(a) \geq 0$;

$4^{\circ} \lim _{\nu \rightarrow \infty} \frac{m_{\nu}}{n_{\nu}}=0$.

In the case when the surface $\Phi$ has periodic ends, $m_{k} \equiv m_{1}, n_{\nu}=\nu m_{1}=\nu n_{1}$, $n_{\nu}(a)=\nu n_{1}(a), \bar{n}_{\nu}(a)=\nu \bar{n}_{1}(a), n_{\nu}\left(b_{j}\right)=\nu n_{1}\left(b_{j}\right), j=1,2, p_{\nu} \equiv p_{1}$. It is clear that the conditions $1^{\circ}-4^{\circ}$ hold, hence periodic ends are almost periodic. It is easy to see that all geometric deficiencies and indices of a surface $\Phi$ with periodic ends are rational numbers, and if $\varepsilon_{g}(a)>0$, then $\varepsilon_{g}(a)+\delta_{g}(a)<1$.

\footnotetext{
${ }^{11}$ Since, as we shall see, the sequence $p_{k}$ is used in the definition of an almost periodic end, the almost periodicity of an end depends, generally speaking, not only on $\Lambda_{j}$, but also on the choice of $\varepsilon$. This deficiency of the definition can be eliminated, but we are not going to do this, because the present definition will no cause any inconvenience.
} 
The conditions $1^{\circ}$ and $3^{\circ}$ together with (6.2) and (6.3) imply that for a surface $\Phi$ with almost periodic ends the condition

$$
\sum_{a}\left(\delta_{g}(a)+\varepsilon_{g}(a)\right)=2
$$

holds.

In what follows we consider surfaces $\Phi$ with almost periodic ends only without mentioning this explicitly.

We construct a quasiconformal mapping of the surface $\Phi$ onto the complex $\zeta$ plane, $\zeta=\xi+i \eta$. To this end we map the $\varepsilon$-neighborhoods $U\left(\varepsilon, \tilde{B}_{1}\right)$ and $U\left(\varepsilon, \tilde{B}_{2}\right)$ of the logarithmic ramification points using the function

$$
\begin{gathered}
\zeta=\frac{1}{\delta_{g}\left(b_{2}\right)} \ln \frac{w-b_{2}}{\varepsilon e}+i \alpha_{2}, \\
\zeta=\frac{1}{\delta_{g}\left(b_{1}\right)} \ln \frac{\varepsilon e}{w-b_{1}}+i \alpha_{1}
\end{gathered}
$$

onto the regions $G_{2}=\left\{\xi<-1 / \delta_{g}\left(b_{2}\right)\right\}$ and $G_{1}=\left\{\xi>1 / \delta_{g}\left(b_{1}\right)\right\}$, respectively. We choose the real constants $\alpha_{2}$ in (6.5) and $\alpha_{1}$ in (6.6) in such a way that these mappings map the line segment $\Gamma \cap U\left(\varepsilon, \tilde{B}_{2}\right)$ onto $\left\{\eta=0, \xi<-1 / \delta_{g}\left(b_{2}\right)\right\}$, and the line segment $\Gamma \cap U\left(\varepsilon, \tilde{B}_{1}\right)$ onto $\left\{\eta=0, \xi>1 / \delta_{g}\left(b_{1}\right)\right\}$. We place the vertices $A_{1}^{k}, A_{2}^{k}, A_{3}^{k}, A_{4}^{k}$ on the boundary $\Phi_{k}^{\prime}$ in the order corresponding to the positive (counterclockwise) orientation of the boundary, in such a way that $A_{1}^{k} A_{2}^{k}=\Gamma_{k}^{-}$, $A_{3}^{k} A_{4}^{k}=\Gamma_{k}^{+}$, the curve $A_{2}^{k} A_{3}^{k}$ lies over $\left\{\left|w-b_{1}\right|=\varepsilon\right\}$, and the curve $A_{4}^{k} A_{1}^{k}$ lies over $\left\{\left|w-b_{2}\right|=\varepsilon\right\}$. Under the mapping (6.5) the point $A_{1}^{k}$ is mapped onto the point

$$
\begin{aligned}
& D_{1}^{k}=-\frac{1}{\delta_{g}\left(b_{2}\right)}+\frac{2 \pi i}{\delta_{g}\left(b_{2}\right)}\left(n_{k-1}-n_{k-1}\left(b_{2}\right)\right) \text { for } k \geq 1, \\
& D_{1}^{k}=-\frac{1}{\delta_{g}\left(b_{2}\right)}-\frac{2 \pi i}{\delta_{g}\left(b_{2}\right)}\left(n_{-k}-n_{-k}\left(b_{2}\right)\right) \text { for } k \leq-1,
\end{aligned}
$$

the point $A_{4}^{k}$ is mapped onto the point $D_{4}^{k}=D_{1}^{k+1}, D_{1}^{0}=D_{1}^{1}$. Under the mapping (6.6) the point $A_{2}^{k}$ is mapped onto the point

$$
\begin{aligned}
& D_{2}^{k}=\frac{1}{\delta_{g}\left(b_{1}\right)}+\frac{2 \pi i}{\delta_{g}\left(b_{1}\right)}\left(n_{k-1}-n_{k-1}\left(b_{1}\right)\right) \text { for } k \geq 1, \\
& D_{2}^{k}=\frac{1}{\delta_{g}\left(b_{1}\right)}-\frac{2 \pi i}{\delta_{g}\left(b_{1}\right)}\left(n_{-k}-n_{-k}\left(b_{1}\right)\right) \text { for } k \leq-1,
\end{aligned}
$$

the point $A_{3}^{k}$ is mapped onto the point $D_{3}^{k}=D_{2}^{k+1}$.

We consider a quasiconformal mapping of $\Phi_{k}^{\prime}$ onto the trapezoid $T_{k}$ with vertices at the points $D_{1}^{k}, D_{2}^{k}, D_{3}^{k}, D_{4}^{k}$ in such a way that the point $A_{j}^{k}$ is mapped onto the point $D_{j}^{k}, j=1,2,3,4$, and the distortion is constant on each of the sides of the trapezoid. To this end, we first map $\Phi_{k}^{\prime}$ onto the rectangle using the function $\zeta^{\prime}=$ $\varphi_{k}(w)$ onto the rectangle $R_{k}=\left\{-1 / \delta_{g}\left(b_{2}\right) \leq \xi^{\prime} \leq 1 / \delta_{g}\left(b_{1}\right), 0 \leq \eta^{\prime} \leq 2 \pi m_{k}\right\}$. Then we map the rectangle $R_{k}$ onto the trapezoid $T_{k}$ using a quasiconformal mapping $\zeta=\psi_{k}\left(\zeta^{\prime}\right)$, such that $\psi_{k}\left(-1 / \delta_{g}\left(b_{2}\right)\right)=D_{1}^{k}, \psi_{k}\left(1 / \delta_{g}\left(b_{1}\right)\right)=D_{2}^{k}, \psi_{k}\left(1 / \delta_{g}\left(b_{1}\right)+\right.$ $\left.i 2 \pi m_{k}\right)=D_{3}^{k}, \psi_{k}\left(-1 / \delta_{g}\left(b_{2}\right)+i 2 \pi m_{k}\right)=D_{4}^{k}$, and the distortion is constant on each of the sides of $R_{k}$. We show that the functions $\psi_{k}\left(\zeta^{\prime}\right)$ can be chosen in such a 
way that the characteristic $p\left(\zeta^{\prime}, \zeta\right)$ is bounded by a constant which does not depend on $k$. In fact, if we use the inequality (2.12), we get the estimate $(k \geq 1)$

$$
\begin{aligned}
p\left(\zeta^{\prime}, \zeta\right) \leq & \frac{2 \pi m_{k}}{\min \left(q_{1}^{k}, q_{2}^{k}\right)}+\frac{\max \left(q_{1}^{k}, q_{2}^{k}\right)}{2 \pi m_{k}} \\
& +\frac{2 \pi m_{k}}{h^{2} \min \left(q_{1}^{k}, q_{2}^{k}\right)} \max \left\{\alpha_{k}^{2},\left(q_{2}^{k}-q_{1}^{k}-q_{1}^{k}+\alpha_{k}\right)^{2}\right\}
\end{aligned}
$$

where

$$
\begin{gathered}
h=\frac{1}{\delta_{g}\left(b_{1}\right)}+\frac{1}{\delta_{g}\left(b_{2}\right)} \\
q_{j}^{k}=2 \pi \frac{\left\{n_{k}-n_{k}\left(b_{j}\right)\right\}-\left\{n_{k-1}-n_{k-1}\left(b_{j}\right)\right\}}{\delta_{g}\left(b_{j}\right)}, j=1,2 ; \\
\alpha_{k}=2 \pi\left\{\frac{n_{k-1}-n_{k-1}\left(b_{1}\right)}{\delta_{g}\left(b_{1}\right)}-\frac{n_{k-1}-n_{k-1}\left(b_{2}\right)}{\delta_{g}\left(b_{2}\right)}\right\} .
\end{gathered}
$$

If $k \leq-1$, the estimate (6.7) remains true with $\alpha_{k}=\alpha_{|k|}, q_{j}^{k}=q_{j}^{|k|}, j=1,2$. It is clear that it suffices to consider the estimate (6.7) for $k \geq 1$. The condition $1^{\circ}$ implies that $n_{\nu}-n_{\nu}\left(b_{j}\right)=n_{\nu} \delta_{g}\left(b_{j}\right)+O(1), j=1,2$. Therefore

$$
\begin{gathered}
\alpha_{k}=O(1), q_{j}^{k}=2 \pi\left(n_{k}-n_{k-1}\right)+O(1)=2 \pi m_{k}+O(1), \\
q_{2}^{k}-q_{1}^{k}+\alpha_{k}=O(1), \frac{q_{j}^{k}}{2 \pi m_{k}}=1+O\left(\frac{1}{m_{k}}\right)=O(1) .
\end{gathered}
$$

Besides, since the quantities $n_{k}-n_{k}\left(b_{j}\right)$ form a non-decreasing sequence, $q_{j}^{k} \geq$ $2 \pi / \delta_{g}\left(b_{j}\right), j=1,2, k \geq 1$. Therefore

$$
\frac{2 \pi m_{k}}{q_{j}^{k}}=\frac{1}{1+O\left(1 / m_{k}\right)}=O(1) .
$$

These estimates imply that the right-hand side of (6.7) is uniformly bounded by some constant $L$. Then the characteristic of the quasiconformal mapping $\zeta=$ $\Psi_{k}(w)=\psi_{k}\left(\varphi_{k}(w)\right)$ of the surface $\Phi_{k}^{\prime}$ onto the trapezoid $T_{k}$ is bounded by $2 L p_{k}$.

Taking into account the fact that under the mapping of $U\left(\varepsilon, \tilde{B}_{1}\right), U\left(\varepsilon, \tilde{B}_{2}\right)$, and $\Phi_{k}^{\prime}, k= \pm 1, \pm 2, \ldots$, onto $G_{1}, G_{2}$, and $T_{k}$, respectively, the distortion on the common part of the boundary of two of these regions is equal to the same constant, it is easy to see, that we have constructed a quasiconformal mapping $\zeta=\zeta(w)$ of the whole Riemann surface $\Phi$ onto $\{|\zeta|<\infty\}$, moreover, in $U\left(\varepsilon, \tilde{B}_{1}\right)$ and $U\left(\varepsilon, \tilde{B}_{2}\right)$ this mapping is conformal. Under this mapping the end $\Lambda_{1}$ is mapped into $\{\eta>0\}$, the end $\Lambda_{2}$ is mapped into $\{\eta<0\}$, and the curve $\Gamma$ is mapped into $\{\eta=0\}$.

We estimate the integral

$$
\begin{aligned}
\iint_{\zeta \bar{\in} T_{1} \cup T_{-1}} p(\zeta, w) d \sigma(\ln \zeta)=\sum_{|k|=2}^{\infty} \iint_{T_{k}} p(\zeta, w) d \sigma(\ln \zeta) \\
\leq \sum_{|k|=2}^{\infty} 2 L p_{k} \iint_{T_{k}} d \sigma(\ln \zeta)=4 L \sum_{k=2}^{\infty} p_{k} \iint_{T_{k}} d \sigma(\ln \zeta) .
\end{aligned}
$$


But for sufficiently large $k$ we have

$$
\begin{aligned}
\iint_{T_{k}} d \sigma(\ln \zeta) & =\iint_{T_{k}} \frac{d \sigma(\zeta)}{|\zeta|^{2}} \leq \frac{1}{\left(\min _{\zeta \in T_{k}}|\zeta|\right)^{2}} \iint_{T_{k}} d \sigma(\zeta) \\
& \leq \frac{h}{2\left\{\min \left(\left|D_{1}^{k}\right|,\left|D_{2}^{k}\right|\right)\right\}^{2}}\left\{\left|D_{3}^{k}-D_{2}^{k}\right|+\left|D_{4}^{k}-D_{1}^{k}\right|\right\} \\
& \leq \frac{h\left(q_{1}^{k}+q_{2}^{k}\right)}{2\left\{\min _{\mu=1,2}\left(\frac{2 \pi}{\delta_{g}\left(b_{\mu}\right)}\left[n_{k-1}-n_{k-1}\left(b_{\mu}\right)\right]\right)\right\}^{2}} \\
& =\frac{h}{2} \frac{4 \pi m_{k}+O(1)}{\left\{2 \pi n_{k-1}+O(1)\right\}^{2}}=\frac{h}{2} \frac{4 \pi m_{k}+O(1)}{\left\{2 \pi n_{k}-2 \pi m_{k}+O(1)\right\}^{2}} \\
& =\frac{h}{2} \cdot \frac{m_{k}}{n_{k}^{2}} \frac{4 \pi+O\left(1 / m_{k}\right)}{\left\{2 \pi-2 \pi \frac{m_{k}}{n_{k}}+O\left(1 / n_{k}\right)\right\}^{2}}=O\left(\frac{m_{k}}{n_{k}^{2}}\right) .
\end{aligned}
$$

We used the condition $4^{\circ}$ here.

By the condition $2^{\circ}$ the inequality (6.9) implies that the series in the right-hand side of (6.8) converges. Hence

$$
\begin{aligned}
& \iint_{|\zeta| \geq 1}\{p(\zeta, w)-1\} d \sigma(\ln \zeta)\{p(\zeta, w)-1\} d \sigma(\ln \zeta) \\
& \quad=\iint_{|\zeta| \geq 1,-1 / \delta_{g}\left(b_{2}\right) \leq \xi \leq 1 / \delta_{g}\left(b_{1}\right)} p(\zeta, w) d \sigma(\ln \zeta)<\infty . \\
& \quad \leq \iint_{|\zeta| \geq 1,-1 / \delta_{g}\left(b_{2}\right) \leq \xi \leq 1 / \delta_{g}\left(b_{1}\right)} p .
\end{aligned}
$$

Let $z=z(w)$ be a conformal mapping of the surface $\Phi$ onto $\{|z|<R\}$. Then the quasiconformal mapping $z=z(\zeta)$, obtained as a composition of the mappings $\{|\zeta|<\infty\} \rightarrow \Phi \rightarrow\{|z|<R\}$ with $p(\zeta, z)=p(\zeta, w)$, by (6.10), satisfies the conditions of Theorem 3.1. Hence $R=\infty$ and with a suitable normalization of the mapping of $\Phi$ onto $\{|z|<\infty\}$ we have

$$
\lim _{z \rightarrow \infty}\left|\frac{\zeta(z)}{z}\right|=\lim _{\zeta \rightarrow \infty}\left|\frac{\zeta}{z(\zeta)}\right|=1 .
$$

Let us study the value distribution of the meromorphic function $w=\varphi(\zeta)$, which maps the complex $z$-plane onto $\Phi$. By (6.11) it suffices to study the value distribution of the function $w=\varphi(\zeta)$ inverse to the function $\zeta=\zeta(w)$ constructed above. The function $w=\varphi(\zeta)$ establishes a quasiconformal mapping of $\{|\zeta|<\infty\}$ onto $\Phi$. It is easy to see that for each $a, a$-points of the function $\varphi(z)$ lie in some vertical strip $\left\{|\xi|<\xi_{0}\right\}, \xi_{0}=\xi_{0}(a)>0$. On the other hand, the mapping $\zeta=\zeta(w)$ maps the brick $\tilde{\Phi}_{k}$ into the region $S_{k}$ consisting of the trapezoid $T_{k}$ and two adjoining to its vertical sides horizontal half-strips. The region $S_{k}$ is contained in the horizontal strip

$$
\begin{aligned}
\min _{\mu=1,2}\left\{\frac{2 \pi}{\delta_{g}\left(b_{\mu}\right)}\left[n_{|k|-1}-n_{|k|-1}\left(b_{\mu}\right)\right]\right\} & \leq(-1)^{\operatorname{sign} k} \eta \\
& \leq \max _{\mu=1,2}\left\{\frac{2 \pi}{\delta_{g}\left(b_{\mu}\right)}\left[n_{|k|}-n_{|k|}\left(b_{\mu}\right)\right]\right\} .
\end{aligned}
$$


Using the conditions $1^{\circ}$ and $4^{\circ}$ we see that the width of this strip is equal to $2 \pi\left(n_{|k|}-n_{|k|-1}\right)+O(1)=2 \pi m_{|k|}+O(1)=o\left(n_{|k|}\right)$. Since the set

$$
\left(\bigcup_{j=1}^{k} S_{j}\right) \cup\left(\bigcup_{j=-1}^{-k} S_{j}\right), k \geq 1,
$$

contains $2 n_{k}(a) a$-points of the function $\varphi(\zeta)\left(2 \bar{n}_{k}(a) a\right.$-points, if we do not take into account the multiplicity of $a$-points), then, using the conditions $1^{\circ}, 3^{\circ}$, and $4^{\circ}$, it is easy to show that

$$
\begin{aligned}
& n(r, a, \varphi)= \begin{cases}\frac{r}{\pi}+o(r) & \text { for } a \neq b_{1}, b_{2}, \\
\frac{r}{\pi}\left(1-\delta_{g}\left(a_{j}\right)\right)+o(r) & \text { for } a=a_{j}, j=1,2\left(\text { if } b_{1} \neq b_{2}\right), \\
\frac{r}{\pi}\left(1-\delta_{g}\left(a_{1}\right)\right)+o(r) & \text { for } a=a_{1}\left(\text { if } b_{1}=b_{2}=a_{1}\right),\end{cases} \\
& n_{1}(r, a, \varphi)=\varepsilon_{g}(a) \frac{r}{\pi}+o(r) \text { for all } a .
\end{aligned}
$$

Taking into account (6.11) we see that the relations (6.12) and (6.13) remains true if we replace $n(r, a, \varphi)$ by $n(r, a, f)$ and $n_{1}(r, a, \varphi)$ by $n_{1}(r, a, f)$ in them. Now we easily find, that

$$
\begin{aligned}
N(r, a, f)= & \begin{array}{ll}
\frac{r}{\pi}+o(r) & \text { for } a \neq b_{1}, b_{2}, \\
\frac{r}{\pi}\left(1-\delta_{g}\left(a_{j}\right)\right)+o(r) & \text { for } a=a_{j}, j=1,2\left(\text { if } b_{1} \neq b_{2}\right), \\
\frac{r}{\pi}\left(1-\delta_{g}\left(a_{1}\right)\right)+o(r) & \text { for } a=a_{1}\left(\text { if } b_{1}=b_{2}=a_{1}\right),
\end{array} \\
& N_{1}(r, a, f)=\varepsilon_{g}(a) \frac{r}{\pi}+o(r) \quad \text { for all } a .
\end{aligned}
$$

Taking $a \neq b_{1}, b_{2}$ such that $\Delta(a, f)=0$, we get

$$
T(r, f)=\frac{r}{\pi}+o(r)
$$

The relation (6.16) implies that the type, and also deficiencies and indices are the same for all functions $f(A z+B), A \neq 0$, that is, for all normalizations of functions mapping the complex $z$-plane onto the surface $\Phi$. Taking into account (6.14), (6.15), and (6.16), we get the following theorem.

THEOREM 6.1. If the meromorphic functions $w=f(z)$ maps the complex $z$ plane onto the Riemann surface $\Phi$ with almost periodic ends, then the function $f(z)$ has the normal type of the first order, and its deficiencies and indices coincide with the geometric deficiencies and indices of the surface $\Phi$.

Taking into account this theorem we shall write simply $\delta\left(a, \Lambda_{1}\right)$ and $\varepsilon\left(a, \Lambda_{1}\right)$ instead of $\delta_{g}\left(a, \Lambda_{1}\right)$ and $\varepsilon_{g}\left(a, \Lambda_{1}\right)$. However we cannot omit the subscript $g$ in the notation $\delta_{g}(a)$ and $\varepsilon_{g}(a)$, because it can lead to a confusion.

ExERCISE. Suppose that the surface $\Phi$ has periodic ends and $\Phi_{k} \equiv \Phi_{1}$. Suppose that a rational function $w=R_{1}(z)$ maps the extended $z$-plane onto $\Phi_{1}$, moreover $R_{1}\left(\beta_{j}\right)=B_{j}^{1} \in \Phi_{1}, j=1,2 ; R(z)=R_{1}(L(z))$, where $L(z)$ is a linear-fractional 
transformation, mapping the points 0 and $\infty$ onto the points $\beta_{1}$ and $\beta_{2}$, respectively. Prove that the function $w=R\left(e^{z}\right)$ maps $\{|z|<\infty\}$ onto $\Phi$. Conversely, each meromorphic function of the form $w=R\left(e^{z}\right)$, where $R(z)$ is some rational function, maps the complex $z$-plane onto some surface $\Phi$ with periodic ends.

We would like to make some remarks in connection with the study if surfaces $\Phi$, undertaken above. This remarks will be used later on.

REMARK 1. When we constructed the quasiconformal mapping $\zeta=\zeta(w)$ of the Riemann surface $\Phi$ onto $\{|\zeta|<\infty\}$, the part of the Riemann surface which was mapped into the strip $\left\{-1 / \delta_{g}\left(b_{2}\right) \leq \xi \leq 1 / \delta_{g}\left(b_{1}\right)\right\}$ was $\Phi \backslash\left(U\left(\varepsilon, \tilde{B}_{1}\right) \cup U\left(\varepsilon, \tilde{B}_{2}\right)\right)$, where $\varepsilon$ is the fixed number from the definition of the quantity $p_{k}$ entering the condition $2^{\circ}$. Let us show that the quasiconformal mapping $\zeta_{1}=\zeta_{1}(w)$ with similar properties can be constructed if we take instead of $\varepsilon$ an arbitrary number $\varepsilon_{1}$ satisfying $0<\varepsilon_{1}<\varepsilon$. In fact, let us map $U\left(\varepsilon_{1}, \tilde{B}_{2}\right)$ and $U\left(\varepsilon_{1}, \tilde{B}_{1}\right)$ onto $\left\{\xi_{1}<-1 / \delta_{g}\left(b_{2}\right)\right\}$ and $\left\{\xi_{1}>1 / \delta_{g}\left(b_{1}\right)\right\}$, respectively, using the functions (6.5) and (6.6), with $\varepsilon_{1}$ instead of $\varepsilon$. We map $\Phi \backslash\left(U\left(\varepsilon_{1}, \tilde{B}_{1}\right) \cup U\left(\varepsilon_{1}, \tilde{B}_{2}\right)\right)$ onto the strip

$$
\left\{-\frac{1}{\delta_{g}\left(b_{2}\right)} \ln \frac{\varepsilon e}{\varepsilon_{1}} \leq \xi \leq \frac{1}{\delta_{g}\left(b_{1}\right)} \ln \frac{\varepsilon e}{\varepsilon_{1}}\right\}
$$

using the function $\zeta=\zeta(w)$, and then map this strip using the function

$$
\xi_{1}+i \eta_{1}=\frac{\xi}{\ln \frac{\varepsilon e}{\varepsilon_{1}}}+i \eta
$$

onto the strip $\left\{-1 / \delta_{g}\left(b_{2}\right) \leq \xi \leq 1 / \delta_{g}\left(b_{1}\right)\right\}$. The quasiconformal mapping (6.17) has characteristic $p\left(\zeta, \zeta_{1}\right) \equiv$ const, therefore it is easy to verify that the relations (6.10), (6.12), and (6.13) are valid also for the function $w=\varphi_{1}\left(\zeta_{1}\right)$ which is the inverse of $\zeta_{1}=\zeta_{1}(w)$. Therefore in what follows we shall use these formulas, and also the formulas (6.5) and (6.6), regarding $\varepsilon$ as a sufficiently small number.

REMARK 2. The constructed quasiconformal mapping $w=\varphi(\zeta)$ maps the upper half-plane $\{\eta>0\}$ onto the almost periodic end $\Lambda_{1}$. Let $\alpha$ and $\beta$ be some positive numbers. Denote by $H_{\alpha \beta}$ the region in the $\zeta_{1}$-plane, $\zeta_{1}=\xi_{1}+i \eta_{1}$, whose boundary is the polygonal line consisting of $\left\{\eta_{1}=\alpha, \xi_{1} \leq-1 / \delta_{g}\left(b_{2}\right)\right\},\left\{\eta_{1}=\right.$ $\left.\beta, \xi_{1} \geq 1 / \delta_{g}\left(b_{1}\right)\right\}$, and the line segment joining the points $\left(-1 / \delta_{g}\left(b_{2}\right)\right)+i \alpha$ and $\left(1 / \delta_{g}\left(b_{1}\right)\right)+i \beta$, where the ray $\left\{\xi_{1}=0, \eta_{1}>\max (\alpha, \beta)\right\}$ is contained in $H_{\alpha \beta}$. We map $H_{\alpha \beta}$ onto $\{\eta>0\}$ using the mapping

$$
\zeta=\xi+i \eta= \begin{cases}\xi_{1}+\left(\eta_{1}-\alpha\right) i & \text { for } \xi_{1} \leq-1 / \delta_{g}\left(b_{2}\right), \\ \xi_{1}+\left(\eta_{1}-\beta\right) i & \text { for } \xi_{1} \geq 1 / \delta_{g}\left(b_{1}\right), \\ \xi_{1}+\left(\eta_{1}-\frac{\beta-\alpha}{h} \xi_{1}-\frac{\alpha / \delta_{g}\left(b_{1}\right)+\beta / \delta_{g}\left(b_{2}\right)}{h}\right) i & \text { for } \frac{-1}{\delta_{g}\left(b_{2}\right)} \leq \xi_{1} \leq \frac{1}{\delta_{g}\left(b_{1}\right)}\end{cases}
$$

It is clear that $p\left(\zeta_{1}, \zeta\right) \equiv 1$ for $\xi_{1}>1 / \delta_{g}\left(b_{1}\right)$ and for $\xi_{1}<-1 / \delta_{g}\left(b_{2}\right)$, and $p\left(\zeta_{1}, \zeta\right) \equiv$ $C=$ const for $-1 / \delta_{g}\left(b_{2}\right)<\xi_{1}<1 / \delta_{g}\left(b_{1}\right)$. Denote by $w=\varphi_{\alpha \beta}\left(\zeta_{1}\right)=\varphi\left(\zeta\left(\zeta_{1}\right)\right)$ a function which establishes a quasiconformal mapping of the region $H_{\alpha \beta}$ onto the almost periodic end $\Lambda_{1}$. It is easy to see that the function $\zeta=\zeta(w)$, inverse to $w=\varphi_{\alpha \beta}(\zeta)$, has the form (6.5) and (6.6) in $U\left(\varepsilon, \tilde{B}_{2}\right)$ and $U\left(\varepsilon, \tilde{B}_{1}\right)$, but with 
different constants $\alpha_{1}$ and $\alpha_{2}$. Next, by (6.10) we have

$$
\begin{aligned}
\iint_{H_{\alpha \beta}}\{ & \left.p\left(\zeta_{1}, w\right)-1\right\} d \sigma\left(\ln \zeta_{1}\right) \\
& =\iint_{H_{\alpha \beta} \cap\left\{-1 / \delta_{g}\left(b_{2}\right)<\xi_{1}<1 / \delta_{g}\left(b_{1}\right)\right\}}\left\{p\left(\zeta_{1}, w\right)-1\right\} d \sigma\left(\ln \zeta_{1}\right) \\
& \leq C \iint_{\left\{-1 / \delta_{g}\left(b_{2}\right)<\xi<1 / \delta_{g}\left(b_{1}\right),|\zeta|>1\right\}} p(\zeta, w) \frac{d \sigma\left(\ln \zeta_{1}\right)}{d \sigma(\ln \zeta)} d \sigma(\ln \zeta)+C_{1} \\
& =C \iint_{\left\{-1 / \delta_{g}\left(b_{2}\right)<\xi<1 / \delta_{g}\left(b_{1}\right),|\zeta|>1\right\}} p(\zeta, w)\left|\frac{\zeta}{\zeta_{1}}\right|^{2} d \sigma(\ln \zeta)+C_{1} \\
& \leq C_{2} \iint_{\left\{-1 / \delta_{g}\left(b_{2}\right)<\xi<1 / \delta_{g}\left(b_{1}\right),|\zeta|>1\right\}} p(\zeta, w) d \sigma(\ln \zeta)+C_{1}<\infty,
\end{aligned}
$$

where $C_{1}$ and $C_{2}$ are some constants.

Denote by $n\left(r, a, \varphi_{\alpha \beta}\right)$ or $\bar{n}\left(r, a, \varphi_{\alpha \beta}\right)$ the numbers of $a$-points of the function $w=\varphi_{\alpha \beta}(\zeta)$ lying in $H_{\alpha \beta} \cap\{|\zeta| \leq r\}$, depending on whether we count or no the order of $a$-points. Let $n_{1}\left(r, a, \varphi_{\alpha \beta}\right)=n\left(r, a, \varphi_{\alpha \beta}\right)-\bar{n}\left(r, a, \varphi_{\alpha \beta}\right)$. Using (6.12) and $(6.13)$, it is easy to get

$$
\begin{aligned}
n\left(r, a, \varphi_{\alpha \beta}\right)= & \begin{cases}\frac{r}{2 \pi}+o(r) & \text { for } a \neq b_{1}, b_{2}, \\
\frac{r}{2 \pi}\left(1-\delta_{g}\left(a_{j}\right)\right)+o(r) & \text { for } a=a_{j}, j=1,2\left(\text { if } b_{1} \neq b_{2}\right), \\
\frac{r}{2 \pi}\left(1-\delta_{g}\left(a_{1}\right)\right)+o(r) & \text { for } a=a_{1}\left(\text { if } b_{1}=b_{2}=a_{1}\right),\end{cases} \\
& n_{1}\left(r, a, \varphi_{\alpha \beta}\right)=\varepsilon_{g}(a) \frac{r}{2 \pi}+o(r) \text { for all } a .
\end{aligned}
$$

Now we prove the following theorem.

THEOREM 6.2. Let $\varepsilon_{1}, \varepsilon_{2}, \ldots, \varepsilon_{q}(q \geq 2), \delta_{1}, \delta_{2}$ be some numbers, such that $\delta_{j}>0, j=1,2 ; 0 \leq \varepsilon_{j} \leq 1, j=1, \ldots, q, \delta_{j}+\varepsilon_{j} \leq 1$ for $j=1,2$, and

$$
\delta_{1}+\delta_{2}+\sum_{j=1}^{q} \varepsilon_{j}=2 .
$$

Let $a_{1}, \ldots, a_{q}$ be distinct complex numbers and $\gamma$ be some $J$-curve in the $w$-plane with ends at the points $a_{1}$ and $a_{2}$, which does not pass through the points $a_{3}, \ldots, a_{q}$. Then there exists a Riemann surface $\Phi$ with almost periodic ends of the first kind, separated by a $J$-curve $\Gamma \subset \Phi$, such that $\Gamma$ is projected into $\gamma, \delta_{g}\left(a_{j}\right)=\delta_{j}, j=1,2$; $\varepsilon_{g}\left(a_{j}\right)=\varepsilon_{j}, j=1, \ldots, q ; \delta_{g}(a)=0$ for $a \neq a_{1}, a_{2} ; \varepsilon_{g}(a)=0$ for $a \neq a_{1}, \ldots, a_{q}$.

If $\delta_{1}+\delta_{2}=2$, then necessarily $\delta_{1}=\delta_{2}=1, \varepsilon_{j}=0, j=1, \ldots, q$. We can take as $\Phi$ the Riemann surface of the function $\ln \frac{w-a_{2}}{w-a_{1}}$, that is, a Riemann surface $\Phi$ with logarithmic ends. The logarithmic ends can be separated by an arbitrary $J$-curve on $\Phi$, joining the logarithmic ramification points. In particular, the $J$-curve can be selected in such a way that it is projected into $\gamma$.

Now we can assume that $\delta_{1}+\delta_{2}<2$. First we introduce an additional restriction:

A. If $\varepsilon_{j}>0$, then $\varepsilon_{j}+\delta_{j}<1, j=1,2$, and $\varepsilon_{j}<1, j=3, \ldots, q$. From here it follows that $q \geq 3, \sum_{j=3}^{q} \varepsilon_{j}>0$. We may assume without loss of generality that $\varepsilon_{3}>0$. Choose a positive integer $m$ so large that, with the notation $D_{j}=m \delta_{j}-1$, $j=1,2, E_{j}=m \varepsilon_{j}, j=1, \ldots, q$, the following conditions are satisfied: 
(a) $D_{j} \geq 0, j=1,2$, moreover, if $\delta_{j}<1$, then $D_{j} \leq m-2$;

(b) If $E_{j}>0$, then $D_{j}+E_{j} \leq m-4, j=1,2$, and $E_{j} \leq m-2, j=3, \ldots, q$;

(c) $q+3 \leq E_{3} \leq m-q-3$.

To construct the surface $\Phi$ we shall take surfaces $\Phi_{k}$ with the same number $m$ of sheets, and with algebraic ramification points which are projected into the points $a_{1}, \ldots, a_{q}$ only. There exists only a finite number ${ }^{12}$ of different surfaces $\Phi_{k}$. Since the curve $\gamma$ for the construction of the surface $\Phi$ is fixed, then $\Phi$ is composed if a finite number of bricks $\tilde{\Phi}_{k}$ of different types. Choosing arbitrarily a sufficiently small $\varepsilon$, we define $\Phi_{k}^{\prime}$ and find, that $\max _{k} p_{k}=P<\infty$. It is clear that $n_{\nu}=m \nu$, $m_{\nu}=m$, therefore the conditions $2^{\circ}$ and $4^{\circ}$ from the definition of an almost periodic end hold.

We shall take surfaces $\Phi_{k}$ which have algebraic ramification points over the points $a_{1}$ and $a_{2}$, and at the ends of the curve $\Gamma_{k}$ of orders $d_{1}^{k}$ and $d_{2}^{k}, d_{j}^{k} \geq 0$, $j=1,2$, respectively, and at most one algebraic ramification point of each of the orders $e_{1}^{k}$ and $e_{2}^{k}, e_{j}^{k} \geq 0, j=1,2$. There is at most one algebraic ramification point of order $e_{j}^{k} \geq 0,4 \leq j \leq q$, over each point $a_{j}, j=4, \ldots, q$, and there are at most two algebraic ramification points with the sum or orders $e_{3}^{k} \geq 0$ over the point $a_{3}$. Theorem 4.2 implies that the only relations between the numbers $e_{j}^{k}, 1 \leq j \leq q, d_{1}^{k}, d_{2}^{k}$ are the relations $d_{j}^{k} \leq m-1$ and $d_{j}^{k}+e_{j}^{k} \leq m-2$, if $e_{j}^{k}>0, j=1,2 ; e_{j}^{k} \leq m-1,3 \leq j \leq q$, (moreover, if there are two algebraic ramification points over $a_{3}$, we have also $e_{3}^{k} \leq m-2$ ) and the Riemann-Hurwitz formula: $d_{1}^{k}+d_{2}^{k}+\sum_{j=1}^{q} e_{j}^{k}=2(m-1)$, that is, we can construct Riemann surfaces $\Phi_{k}$ with preassigned numbers $d_{j}^{k}, e_{j}^{k}$, provided they satisfy the relations listed above.

Choosing the sequence $d_{j}^{k}, j=1,2$, we shall distinguish two variants. In the first variant $d_{j}^{k}=\left[k D_{j}\right]-\left[(k-1) D_{j}\right], k=1,2, \ldots$ Then

$$
\begin{aligned}
& \frac{n_{\nu}-n_{\nu}\left(a_{j}\right)}{n_{\nu}}-\delta_{j}=\frac{1}{\nu m} \sum_{k=1}^{k}\left(d_{j}^{k}+1\right)-\delta_{j}=\frac{1}{\nu m} \sum_{k=1}^{\nu} d_{j}^{k}-\delta_{j}+\frac{1}{m} \\
& =\frac{1}{m}\left\{\frac{1}{\nu} \sum_{k=1}^{\nu} d_{j}^{k}-D_{j}\right\}=\frac{1}{m}\left\{\frac{\left[\nu D_{j}\right]}{\nu}-D_{j}\right\}=-\frac{1}{m \nu}\left\{\nu D_{j}-\left[\nu D_{j}\right]\right\} .
\end{aligned}
$$

Hence

$$
-\frac{1}{n_{\nu}}=-\frac{1}{m \nu}<\frac{n_{\nu}-n_{\nu}\left(a_{j}\right)}{n_{\nu}}-\delta_{j} \leq 0 .
$$

In the second variant the choice of $d_{j}^{k}$ differs from the first only when we set $d_{j}^{1}=\left[D_{j}\right]+1$. The second variant is applicable only if $\delta_{j}<1$ and, by the condition (a), $D_{j} \leq m-2$. In the second variant of choice of $d_{j}^{k}$ we get, instead of $\left(6.23_{1}\right)$, the following inequality

$$
0<\frac{n_{\nu}-n_{\nu}\left(a_{j}\right)}{n_{\nu}}-\delta_{j} \leq \frac{1}{n_{\nu}}
$$

\footnotetext{
${ }^{12}$ Readers who are not acquainted with this fact, can replace this sentence by the following: "We shall use only a finite number of different surfaces $\Phi_{k}$ ".
} 
In both cases we have

$$
\left|1-\frac{n_{\nu}\left(a_{j}\right)}{n_{\nu}}-\delta_{j}\right| \leq \frac{1}{n_{\nu}}, j=1,2,
$$

that is, $\delta_{g}\left(a_{j}\right)=\delta_{j}, j=1,2$, and the surface $\Phi$ satisfies the condition $1^{\circ}$ from the definition of an almost periodic end.

Now we can choose $e_{j}^{k}, 1 \leq j \leq q, k \geq 1$, in such a way, that

$$
\lim _{\nu \rightarrow \infty} \frac{n_{\nu}\left(a_{j}\right)-\bar{n}_{\nu}\left(a_{j}\right)}{n_{\nu}}=\varepsilon_{j}, 1 \leq j \leq q .
$$

For $1 \leq j \leq q, j \neq 3$, it suffices to take

$$
e_{j}^{k}=\left[k E_{j}\right]-\left[(k-1) E_{j}\right] \leq E_{j}+1 .
$$

By the condition (b) such choice of $e_{j}^{k}$ is possible. Then $(j \neq 3)$

$$
\frac{n_{\nu}\left(a_{j}\right)-\bar{n}\left(a_{j}\right)}{n_{\nu}}=\frac{1}{\nu m} \sum_{k=1}^{\nu} e_{j}^{k}=\frac{\left[\nu E_{j}\right]}{\nu m}=\frac{\left[\nu m \varepsilon_{j}\right]}{\nu m} \rightarrow \varepsilon_{j}, \nu \rightarrow \infty .
$$

Now as $e_{3}^{k}$ we take

$$
e_{3}^{k}=2(m-1)-d_{1}^{k}-d_{2}^{k}-\sum_{\substack{j=1 \\ j \neq 3}}^{q} e_{j}^{k} .
$$

Since $m \delta_{j}-2 \leq d_{1}^{k} \leq m \delta_{j}+1, j=1,2 ; E_{j}-1 \leq e_{j}^{k} \leq E_{j}+1$ for $j \neq 3$, by the condition (c) and (6.21) we have

$$
\begin{aligned}
e_{3}^{k} & \leq 2(m-1)-\left(m \delta_{1}-2\right)-\left(m \delta_{2}-2\right)-\sum_{j \neq 3}\left(m \varepsilon_{j}-1\right) \\
& =2 m+q+1-m\left(\delta_{1}+\delta_{2}+\sum_{j \neq 3} \varepsilon_{j}\right) \\
& =2 m+q+1-m\left(2-\varepsilon_{3}\right)=E_{3}+q+1 \leq m-2, \\
e_{3}^{k} & \geq 2(m-1)-\left(m \delta_{1}+1\right)-\left(m \delta_{2}+1\right)-\sum_{j \neq 3}\left(m \varepsilon_{j}+1\right) \\
& =2 m-(q+3)-m\left(2-\varepsilon_{3}\right)=E_{3}-(q+3) \geq 0 .
\end{aligned}
$$

Hence such choice of $e_{3}^{k}$ is admissible. Now, by (6.21), (6.24), (6.26), and (6.27) we get

$$
\begin{aligned}
& \frac{n_{\nu}\left(a_{3}\right)-\bar{n}_{\nu}\left(a_{3}\right)}{n_{\nu}}=\frac{1}{\nu m} \sum_{k=1}^{\nu} e_{3}^{k}=2-\frac{1}{\nu m} \sum_{k=1}^{\nu}\left(d_{1}^{k}+1\right) \\
& -\frac{1}{\nu m} \sum_{k=1}^{\nu}\left(d_{2}^{k}+1\right)-\sum_{j \neq 3} \frac{1}{\nu m} \sum_{k=1}^{\nu} e_{j}^{k} \rightarrow 2-\delta_{1}-\delta_{2}-\sum_{j \neq 3} \varepsilon_{j}=\varepsilon_{3}(\nu \rightarrow \infty) .
\end{aligned}
$$

Thus, the relation (6.25) has been proved for all $j, 1 \leq j \leq q$. So we have proved that the condition $3^{\circ}$ from the definition of an almost periodic end is satisfied, and also, that $\varepsilon_{g}\left(a_{j}\right)=\varepsilon_{j}, 1 \leq j \leq q$. 
Note, that we not only proved (6.25), but also obtained the inequalities

$$
\left|\frac{n_{\nu}\left(a_{j}\right)-\bar{n}_{\nu}\left(a_{j}\right)}{n_{\nu}}-\varepsilon_{j}\right| \leq \frac{C_{j}}{n_{\nu}},
$$

where $C_{j}=1$ for $j \neq 3$ and $C_{3}=q+1$.

Now we shall eliminate the restriction A. We may assume that $q \geq 3$, letting $\varepsilon_{3}=0$ if necessary. We can choose numbers $\varepsilon_{1}^{(n)}, \ldots, \varepsilon_{q}^{(n)} \geq 0, n=1,2, \ldots$, such that

(a) $\delta_{1}+\delta_{2}+\sum_{j=1}^{q} \varepsilon_{j}^{(n)}=2$;

(b) If $\varepsilon_{j}^{(n)}>0$, then $\varepsilon_{j}^{(n)}+\delta_{j}<1, j=1,2$, and $\varepsilon_{j}^{(n)}<1, j=3, \ldots, q$;

(c) $\lim _{n \rightarrow \infty} \varepsilon_{j}^{(n)}=\varepsilon_{j}, 1 \leq j \leq q$.

We already know how to construct a surface $\Phi^{(n)}$ with almost periodic ends, such that $\delta_{g}\left(a_{j}\right)=\delta_{j}, j=1,2 ; \varepsilon_{g}\left(a_{j}\right)=\varepsilon_{j}^{(n)}, 1 \leq j \leq q$. We denote bricks of the surface $\Phi^{(n)}$ by $\tilde{\Phi}_{k}^{(n)}$. Now we construct a surface $\Phi$, each of whose ends consists of the following sequence of bricks:

$$
\tilde{\Phi}_{1}^{(1)}, \ldots, \tilde{\Phi}_{l_{1}}^{(1)}, \tilde{\Phi}_{1}^{(2)} \ldots, \tilde{\Phi}_{l_{2}}^{(2)}, \ldots, \tilde{\Phi}_{1}^{(n)}, \ldots, \ldots, \tilde{\Phi}_{l_{n}}^{(n)}, \ldots,
$$

the choice of the sequence $l_{1}, l_{2}, \ldots$ will be specified later. We shall use the same notation for the surface $\Phi^{(n)}$ as we used for $\Phi$, but shall add an upper index $n$. If $\delta_{j}=1(j=1,2)$, we take $d_{j}^{k,(n)}=m^{(n)}-1$ for all $n$. Then obviously $n_{\nu}\left(a_{j}\right)=0$, and the condition $1^{\circ}$ for the point $a_{j}$ is satisfied for $\Phi$. Now let $0<\delta_{j}<1$. Let us show that we can choose $d_{j}^{k,(n)}$ according to the first or the second variant, depending on $n$, in such a way that the inequality (6.24) is satisfied for $\Phi$. Let us choose $d_{j}^{k,(1)}$ according to the first variant. Then, obviously, $\left(6.23_{1}\right)$ and (6.24) are satisfied for $1 \leq \nu \leq l_{1}$. We introduce the notation $\nu_{\tau}=l_{1}+l_{2}+\cdots+l_{\tau}$. Suppose that (6.24) is satisfied for $1 \leq \nu \leq \nu_{\tau}$. Consider the expression

$$
Q_{\tau}=1-\frac{n_{\nu_{\tau}}\left(a_{j}\right)}{n_{\nu_{\tau}}}-\delta_{j} .
$$

If $Q_{\tau}>0$, then we choose $d_{j}^{k,(\tau+1)}$ according to the second variant, if $Q_{\tau} \leq 0$, we use the first variant. Then the inequality (6.24) is valid also for $\nu_{\tau}<\nu \leq \nu_{\tau+1}$. In fact, let, for example, $Q_{\tau}>0$, that is

$$
0<1-\frac{n_{\nu_{\tau}}\left(a_{j}\right)}{n_{\nu_{\tau}}}-\delta_{j} \leq \frac{1}{n_{\nu_{\tau}}} .
$$

Then we choose $d_{j}^{k,(\tau+1)}$ according to the first variant and, denoting $N=\nu-\nu_{\tau}$, by $\left(6.23_{1}\right)$, we have

$$
-\frac{1}{n_{N}^{(\tau+1)}}<\frac{n_{N}^{(\tau+1)}-n_{N}^{(\tau+1)}\left(a_{j}\right)}{n_{N}^{(\tau+1)}}-\delta_{j} \leq 0 .
$$

By (6.29) and (6.30), respectively, we have

$$
\begin{gathered}
0<n_{\nu_{\tau}}-n_{\nu_{\tau}}\left(a_{j}\right)-\delta_{j} n_{\nu_{\tau}} \leq 1, \\
-1<n_{N}^{(\tau+1)}-n_{N}^{(\tau+1)}\left(a_{j}\right)-\delta_{j} n_{N}^{(\tau+1)} \leq 0 .
\end{gathered}
$$


Adding these inequalities and observing that

$$
n_{\nu_{\tau}}+n_{N}^{(\tau+1)}=n_{\nu_{\tau}+N}=n_{\nu}, n_{\nu_{\tau}}\left(a_{j}\right)+n_{N}^{(\tau+1)}\left(a_{j}\right)=n_{\nu}\left(a_{j}\right)
$$

we find that

$$
-1<n_{\nu}-n_{\nu}\left(a_{j}\right)-\delta_{j} n_{\nu} \leq 1,
$$

the inequality (6.24) follows. The case $Q_{\tau} \leq 0$ is treated similarly. Thus, we may assume that (6.24) holds for all $\nu$ independently of the choice of the sequence $l_{1}, l_{2}, \ldots$ Hence $\delta_{g}\left(a_{j}\right)=\delta_{j}$, and the condition $1^{\circ}$ holds for $\Phi$.

Let $\nu_{\tau}<\nu \leq \nu_{\tau+1}$. By $(6.28)$ we get

$$
\left|n_{l_{s}}^{(s)}\left(a_{j}\right)-\bar{n}_{l_{s}}^{(s)}\left(a_{j}\right)-\varepsilon_{j}^{(s)} n_{l_{s}}^{(s)}\right| \leq C_{j}, 1 \leq s \leq \tau
$$

then

$$
\left|n_{l_{s}}^{(s)}\left(a_{j}\right)-\bar{n}_{l_{s}}^{(s)}\left(a_{j}\right)-\varepsilon_{j} n_{l_{s}}^{(s)}\right| \leq C_{j}+\left|\varepsilon_{j}^{(s)}-\varepsilon_{j}\right| n_{l_{s}}^{(s)}, 1 \leq s \leq \tau .
$$

Similarly for $N=\nu-\nu_{\tau}$ we have

$$
\left|n_{N}^{(\tau+1)}\left(a_{j}\right)-\bar{n}_{N}^{(\tau+1)}\left(a_{j}\right)-\varepsilon_{j} n_{N}^{(\tau+1)}\right| \leq C_{j}+\left|\varepsilon_{j}^{(\tau+1)}-\varepsilon_{j}\right| n_{N}^{(\tau+1)} .
$$

Adding the inequalities (6.31) and (6.32), and taking into account

$$
n_{\nu}=\sum_{s=1}^{\tau} n_{l_{s}}^{(s)}+n_{N}^{(\tau+1)}
$$

and similar inequalities for $n_{\nu}\left(a_{j}\right)$ and $\bar{n}_{\nu}\left(a_{j}\right)$, we get

$$
\begin{aligned}
\left|n_{\nu}\left(a_{j}\right)-\bar{n}_{\nu}\left(a_{j}\right)-\varepsilon_{j} n_{\nu}\right| \leq & (\tau+1) C_{j} \\
& +\sum_{s=1}^{\tau}\left|\varepsilon_{j}^{(s)}-\varepsilon_{j}\right| n_{l_{s}}^{(s)}+\left|\varepsilon_{j}^{(\tau+1)}-\varepsilon_{j}\right| n_{N}^{(\tau+1)} .
\end{aligned}
$$

Since $\varepsilon_{j}^{(n)} \rightarrow \varepsilon_{j}$ as $n \rightarrow \infty$, taking into account (6.33) we get $(\nu \rightarrow \infty)$

$$
\frac{1}{n_{\nu}}\left\{\sum_{s=1}^{\tau}\left|\varepsilon_{j}^{(s)}-\varepsilon_{j}\right| n_{l_{s}}^{(s)}+\left|\varepsilon_{j}^{(\tau+1)}-\varepsilon_{j}\right| n_{N}^{(\tau+1)}\right\} \rightarrow 0 .
$$

We shall assume that $l_{n} \geq n$, then $\tau=o\left(\nu_{\tau}\right)$, and for $\tau \rightarrow \infty$ we have

$$
\frac{\tau+1}{n_{\nu}} \leq \frac{\tau+1}{n_{\nu_{\tau}}} \leq \frac{\tau+1}{\nu_{\tau}} \rightarrow 0 .
$$

The relations (6.34), (6.35), and (6.36) imply that, for $\Phi$, the relation $\varepsilon_{g}\left(a_{j}\right)=\varepsilon_{j}$, $1 \leq j \leq q$, holds, and the condition $3^{\circ}$ is satisfied.

It is easy to see that ${ }^{13}\left(\nu_{0}=0\right)$

$$
\begin{aligned}
\sum_{\nu=1}^{\infty} \frac{p_{\nu} m_{\nu}}{n_{\nu}^{2}} & \leq \sum_{\tau=1}^{\infty} \sum_{\nu=\nu_{\tau-1}+1}^{\nu_{\tau}} \frac{P^{(\tau)} m^{(\tau)}}{n_{\nu}^{2}} \\
& \leq \sum_{\tau=1}^{\infty} P^{(\tau)} m^{(\tau)} \sum_{\nu=\nu_{\tau-1}+1}^{\nu_{\tau}} \frac{1}{\nu^{2}}<\sum_{\tau=1}^{\infty} P^{(\tau)} m^{(\tau)} \sum_{\nu=\nu_{\tau-1}+1}^{\infty} \frac{1}{\nu^{2}} \\
& <\sum_{\tau=2}^{\infty} \frac{P^{(\tau)} m^{(\tau)}}{\nu_{\tau-1}}+P^{(1)} m^{(1)} \sum_{\nu=1}^{\infty} \frac{1}{\nu^{2}} .
\end{aligned}
$$

\footnotetext{
${ }^{13}$ Recall that $P^{(\tau)}=\max _{k} p_{k}^{(\tau)}$
} 
It is clear that for arbitrary given sequences $\left\{P^{(\tau)}\right\},\left\{m^{(\tau)}\right\}$ we can, by means of a suitable choice of $l_{1}, l_{2}, \ldots$ make the sequence $\nu_{\tau-1}$ so rapidly increasing that

$$
\sum_{\tau=2}^{\infty} \frac{P^{(\tau)} m^{(\tau)}}{\nu_{\tau-1}}<\infty
$$

The inequality (6.37) then implies that the condition $2^{\circ}$ holds for $\Phi$. The condition (6.38) implies that $m^{(\tau)}=o\left(\nu_{\tau-1}\right)$ as $\tau \rightarrow \infty$, that is, the condition $4^{\circ}$ is satisfied.

Theorem 6.2 has been proved.

TheOREM 6.3. Let $\varepsilon_{1}, \varepsilon_{2}, \ldots, \varepsilon_{q}(q \geq 2), \delta_{1}, \delta_{2}$ be some numbers such that $\delta_{j}>0, j=1,2,1 \geq \varepsilon_{j} \geq 0, j=1, \ldots, q, \delta_{1}+\delta_{2}+\varepsilon_{1} \leq 1$, and

$$
\delta_{1}+\delta_{2}+\sum_{j=1}^{q} \varepsilon_{j}=2 .
$$

Let $a_{1}, \ldots, a_{q}$ be distinct complex numbers and let $\gamma$ be a $J^{\prime}$-curve with ends at the points $a_{1}$ and with all points $a_{2}, \ldots, a_{q}$ on the same side of the curve $\gamma$ with the possible exception of one point, which is lying on the other side of $\gamma$. Then there exists a Riemann surface $\Phi$ with almost periodic ends of the second kind, which are separated by a $J$-curve $\Gamma \subset \Phi$, such that $\Gamma$ is projected into $\gamma, \delta_{g}\left(a_{1}\right)=\delta_{1}+\delta_{2}$, $\varepsilon_{g}\left(a_{j}\right)=\varepsilon_{j}, j=1, \ldots, q, \delta_{g}(a)=0$ if $a \neq a_{1}, \varepsilon_{g}(a)=0$ if $a \neq a_{1}, \ldots, a_{q}$, and $\delta_{g}\left(b_{j}\right)=\delta_{j}, j=1,2$.

We are not going to present a proof of Theorem 6.3 because its proof is very close to the proof of Theorem 6.2. First we construct a Riemann surface $\Phi$ under the following additional restrictions:

B. All points $a_{3}, \ldots, a_{q}$ lie on the same side of the curve $\gamma$, and the point $a_{2}$ is on the other side; $1>\varepsilon\left(a_{2}\right)>0$; if $\varepsilon_{1}>0$, then $\varepsilon_{1}+\delta_{1}+\delta_{2}<1 ; \varepsilon_{j}<1$ for $3 \leq j \leq q$.

The construction is carried out in the same way as in the corresponding part of the proof of Theorem 6.2 , but the reference to Theorem 4.2 is replaced by the reference to Theorem 4.3. Then we turn to the general case, as before, but if the given points $a_{2}, \ldots, a_{q}$ lie on the same side of the curve $\gamma$, we add a point $a_{q+1}$, which is separated from $a_{2}, \ldots, a_{q}$ by the curve $\gamma$ in the construction of the auxiliary surfaces $\Phi^{(n)}$, and let $\varepsilon_{q+1}^{(n)}>0, \varepsilon_{q+1}^{(n)} \rightarrow 0$ as $n \rightarrow \infty$.

REMARK. In the condition of Theorem 6.3 we can require only that the curve $\gamma$ is a $J^{\prime}$-curve with ends at $a_{1}$, and $a_{j} \notin \gamma, 2 \leq j \leq q$. To prove this modification we replace the reference to Theorem 4.3 in the proof by a reference to the exercise at the end of Section 4.

Exercise. Suppose that we have added the condition A to the conditions of Theorem 6.2 , or the condition B to the conditions of Theorem 6.3, and, besides, the numbers $\delta_{1}, \delta_{2}, \varepsilon_{1}, \ldots, \varepsilon_{q}$ are rational. Show that in these theorems we can choose $\Phi$ to be Riemann surfaces with periodic ends.

Theorems $6.1,6.2$, and 6.3 immediately imply 
THEOREM 6.4. Let $q \geq 2$ complex numbers $a_{1}, \ldots, a_{q}$ be given, and let $\delta\left(a_{j}\right)$ and $\varepsilon\left(a_{j}\right), 1 \leq j \leq q$, be non-negative numbers, such that $0 \leq \delta\left(a_{j}\right)+\varepsilon\left(a_{j}\right) \leq 1$ and

$$
\sum_{j=1}^{q}\left\{\delta\left(a_{j}\right)+\varepsilon\left(a_{j}\right)\right\}=2
$$

moreover, only one or two of the numbers $\delta\left(a_{j}\right)$ are positive. Then there exists a meromorphic function $f(z)$ such that its deficiencies and indices at the point $a_{j}$ are equal to $\delta\left(a_{j}\right)$ and $\varepsilon\left(a_{j}\right)$, respectively, $1 \leq j \leq q$.

REMARK. We can drop the requirement that the number of index values in Theorem 6.4 is finite, extending somewhat the notion of an almost periodic end. Without giving the details, we shall provide hints, which allow the reader to reconstruct the corresponding argument. We consider the case when $\delta\left(a_{j}\right)=\delta_{j}>0$, $j=1,2$. Now we have sequences $\left\{a_{1}, a_{2}, \ldots\right\}$ and $\left\{\varepsilon\left(a_{1}\right), \varepsilon\left(a_{2}\right), \ldots\right\}$. If the points $a_{1}$ and $a_{2}$ are not accumulation points of the sequence $\left\{a_{3}, a_{4}, \ldots\right\}$, there are no any new substantial difficulties. We carry out the construction of the surface $\Phi$ in the same way as in the proof of Theorem 6.2 , but the number $q^{(n)}$ of the base points for the surface $\Phi^{(n)}$ will depend on $n$, and $q^{(n)} \rightarrow \infty$ as $n \rightarrow \infty$, while before we had $q^{(n)} \equiv q$. In general case we need to make more substantial changes in the construction of the surface $\Phi$.

As before, we choose a $J$-curve $\gamma$ with ends at the points $a_{1}, a_{2}$ in such a way that it does not pass through the points $a_{j}, 3 \leq j<\infty$. Now we cannot choose neighborhoods $U\left(\varepsilon, B_{j}^{k}\right), j=1,2$ with fixed $\varepsilon$, which does not depend on $k$, and we take $U\left(\varepsilon_{k}, B_{j}^{k}\right)$, where $\varepsilon_{k}=\varepsilon_{-k}, 0<\varepsilon_{j} \leq \varepsilon_{k}<1$ for $|k|<|j|$. Now $\Phi_{k}^{\prime}=\tilde{\Phi}_{k} \backslash\left(U\left(\varepsilon_{k}, B_{j}^{k}\right) \cup U\left(\varepsilon_{k}, B_{2}^{k}\right)\right)$, and the role of $U\left(\varepsilon, \tilde{B}_{j}\right)$ is played by

$$
U\left(\left\{\varepsilon_{k}\right\}, \tilde{B}_{j}\right)=\bigcup_{|k|=1}^{\infty}\left\{U\left(\varepsilon_{k}, B_{j}^{k}\right) \backslash \Gamma_{k}\right\} .
$$

We let $\varepsilon=\varepsilon_{1}$ in the formulas (6.5) and (6.6), then the functions (6.5) and (6.6) are conformal mappings of $U\left(\left\{\varepsilon_{k}\right\}, \tilde{B}_{1}\right)$ and $U\left(\left\{\varepsilon_{k}\right\}, \tilde{B}_{1}\right)$ onto the regions

$$
G_{2}=\bigcup_{\nu=1}^{\infty}\left\{\xi<\frac{1}{\delta_{g}\left(b_{2}\right)} \ln \frac{\varepsilon_{\nu}}{\varepsilon e}, 2 \pi \frac{n_{\nu-1}-n_{\nu-1}\left(b_{2}\right)}{\delta_{g}\left(b_{2}\right)} \leq|\eta| \leq 2 \pi \frac{n_{\nu}-n_{\nu}\left(b_{2}\right)}{\delta_{g}\left(b_{2}\right)}\right\}
$$

and

$$
G_{1}=\bigcup_{\nu=1}^{\infty}\left\{\xi>\frac{1}{\delta_{g}\left(b_{1}\right)} \ln \frac{\varepsilon e}{\varepsilon_{\nu}}, 2 \pi \frac{n_{\nu-1}-n_{\nu-1}\left(b_{1}\right)}{\delta_{g}\left(b_{1}\right)} \leq|\eta| \leq 2 \pi \frac{n_{\nu}-n_{\nu}\left(b_{1}\right)}{\delta_{g}\left(b_{1}\right)}\right\},
$$

respectively.

The definition of the number $p_{k}$ also has to be changed. Now we shall find a quasiconformal mapping of $\Phi_{k}^{\prime}$ onto the rectangle

$$
R_{k}=\left\{\frac{1}{\delta_{g}\left(b_{2}\right)} \ln \frac{\varepsilon_{k}}{\varepsilon e} \leq \xi \leq \frac{1}{\delta_{g}\left(b_{1}\right)} \ln \frac{\varepsilon e}{\varepsilon_{k}}, 0 \leq \eta \leq 2 \pi m_{k}\right\},
$$

such that: (1) The part of the boundary of $\Phi_{k}^{\prime}$ lying over the circle $\left\{\left|w-b_{2}\right|=\varepsilon_{k}\right\}$ (over $\left\{\left|w-b_{1}\right|=\varepsilon_{k}\right\}$ ) is mapped with the constant distortion onto the left (right) side of the rectangle; (2) The part of the boundary of $\Phi_{k}^{\prime}$ lying on $\Gamma_{k}^{-}$(on $\Gamma_{k}^{+}$) is mapped onto the lower (upper) side of $R_{k}$, moreover, the points on $\Gamma_{k}^{ \pm}$satisfying 
$\varepsilon_{k} \leq\left|w-b_{2}\right| \leq \varepsilon$ (satisfying $\varepsilon_{k} \leq\left|w-b_{1}\right| \leq \varepsilon$ ) are mapped onto the points with the real part equal to

$$
\xi=\frac{1}{\delta_{g}\left(b_{2}\right)} \ln \frac{\left|w-b_{2}\right|}{\varepsilon e}
$$

(with the real part equal to

$$
\left.\xi=\frac{1}{\delta_{g}\left(b_{1}\right)} \ln \frac{\varepsilon e}{\left|w-b_{1}\right|}\right)
$$

points of the remaining part of $\Gamma_{k}^{ \pm}$are mapped with the constant distortion onto intervals with $-1 / \delta_{g}\left(b_{2}\right) \leq \xi \leq 1 / \delta_{g}\left(b_{1}\right)$. After that the number $p_{k}$ is defined as before.

We replace the condition $2^{\circ}$ in the definition of an almost periodic end by the following condition.

$2^{\circ}$ bis.

$$
\sum_{\nu=1}^{\infty} \frac{p_{\nu} m_{\nu} \ln \frac{1}{\varepsilon_{\nu}}}{n_{\nu}^{2}}<\infty .
$$

Now, modifying somewhat the previous argument, we find a quasiconformal mapping of $\Phi$ onto $\{|\zeta|<\infty\}$, such that $\bigcup_{|k|=1}^{\infty} \Phi_{k}^{\prime}$ is mapped into $\{|\zeta|<\infty\} \backslash\left\{G_{1} \cup G_{2}\right\}$ by some function $\zeta=\zeta(w)$, and $U\left(\left\{\varepsilon_{k}\right\}, \tilde{B}_{2}\right)$ and $U\left(\left\{\varepsilon_{k}\right\}, \tilde{B}_{1}\right)$ are mapped by the functions (6.5) and (6.6).

Observe that now that mapping $\zeta=\psi_{k}\left(\zeta^{\prime}\right)$ is such that we still have $\xi=\xi^{\prime}$, but only the set $R_{k} \cap\left\{-1 / \delta_{g}\left(b_{2}\right) \leq \xi^{\prime} \leq 1 / \delta_{g}\left(b_{1}\right)\right\}$ is mapped into the trapezoid $T_{k}$, and the sets $R_{k} \cap\left\{\xi^{\prime} \leq-1 / \delta_{g}\left(b_{2}\right)\right\}$ and $R_{k} \cap\left\{\xi^{\prime} \geq 1 / \delta_{g}\left(b_{1}\right)\right\}$ are mapped by affine transformations into rectangles adjoining to $T_{k}$ from the left and from the right. Using the condition $2^{\circ}$ bis we can show that

$$
\iint_{|\zeta| \geq 1}\{p(\zeta, w)-1\} d \sigma(\ln \zeta) \leq \iint_{\substack{\zeta \notin G_{1} \cup G_{2} \\|\zeta| \geq 1}} p(\zeta, w) d \sigma(\ln \zeta)<\infty
$$

Let $w=\varphi(\zeta)$ be a function realizing the inverse mapping of $\{|\zeta|<\infty\}$ onto $\Phi$. If we add the condition

$$
5^{\circ} . \lim _{\nu \rightarrow \infty} \frac{\ln \varepsilon_{\nu}}{n_{\nu}}=0
$$

the arguments of $a$-points of $\varphi(\zeta)$, lying in $\left\{(-1)^{j} \eta>0\right\}, j=0,1$, tend to $(-1)^{j} \frac{\pi}{2}$, and the formulas (6.12) and (6.13) remain true. Now it is not difficult to prove analogs of Theorems $6.1,6.2$, and 6.3 for this wider class of generalized almost periodic ends. In the case of an analog of Theorem 6.3 we impose on the curve $\gamma$ the following restrictions only: $a_{j} \notin \gamma, j=2,3 \ldots ; a_{1}$ is an end of $\gamma$, and use the exercise at the end of Section 4 (cf. Remark to Theorem 6.3). The point is that the points $a_{2}, a_{3}, \ldots$ could be located in such a way (e.g. everywhere dense in the plane), that no matter how we draw a $J^{\prime}$-curve $\gamma$ with ends at $a_{1}$, there are infinitely many points of the sequence $a_{2}, a_{3}, \ldots$ on each of the sides of $\gamma$.

\section{Riemann surfaces with finitely many almost periodic ends}

After the introduction of the notion of an almost periodic end we can introduce the class of Riemann surfaces with finitely many almost periodic ends in the same 
way as, using the notion of a logarithmic end we constructed surfaces with finitely many logarithmic ends.

Let $K$ be some simply connected finite-sheeted Riemann surface with boundary, bounded by a closed Jordan curve $C$ consisting of $p J$-curves $\sigma_{k}=\widehat{B}_{k} B_{k+1}, k=$ $1, \ldots, p$. As in Section 5 , all indices $k$ will be considered $\bmod p$. Let $b_{k}$ be the projection of the point $B_{k}$. For simplicity we assume that $\infty$ is not among the points $b_{k}$. Take $p$ almost periodic ends $\Lambda_{k}, 1 \leq k \leq p$, moreover, the boundary of $\Lambda_{k}$ is a $J$-curve $\sigma_{k}^{-}=\widehat{B_{k+1} B}{ }_{k}$. Obviously, if $b_{k} \neq b_{k+1}$, then the end $\Lambda_{k}$ is of the first kind, if $b_{k}=b_{k+1}$, then the end $\Lambda_{k}$ is of the second kind. We introduce the following notation

$$
\delta_{g}\left(b_{k}, \Lambda_{k}\right)=d_{k}^{+}, \delta_{g}\left(b_{k+1}, \Lambda_{k}\right)=d_{k}^{-} .
$$

We paste to the Riemann surface $K$ with the points $B_{k}, 1 \leq k \leq p$, excluded, almost periodic ends $\Lambda_{k}$ along the curves $\sigma_{k}, 1 \leq k \leq p$. Then we get some Riemann surface $F$ which is called a Riemann surface with $p$ almost periodic ends. The part $K$ of the Riemann surface $F$ is called its pseudokernel. If we exclude from $K$ sufficiently small neighborhoods of the boundary points $B_{k}, 1 \leq k \leq p$, we get a Riemann surface with boundary $K_{0}$, which is called the kernel of the Riemann surface $F$. The Riemann surface $F$ has logarithmic ramification points $\tilde{B}_{1}, \ldots, \tilde{B}_{p}$, which are projected into $b_{1}, \ldots, b_{p}$. A sufficiently small $\varepsilon$-neighborhood $U\left(\varepsilon, \tilde{B}_{k}\right)$ of a logarithmic ramification point $\tilde{B}_{k}$ contains and $\varepsilon$-neighborhood of the point $B_{k}$ on $K$, and intersects with the almost periodic ends $\Lambda_{k}$ and $\Lambda_{k-1}$. From now on we assume that $\varepsilon>0$ does not depend on $k$ and does not exceed any of $\varepsilon$ from the definition of almost periodic ends $\Lambda_{k}$.

Obviously, the Riemann surface $F$ belongs to some class $F_{q}$. Note, that it is possible for a Riemann surface to have one almost periodic end $(p=1)$, which is, obviously, and end of the second kind. For example, the function $w=\cos \sqrt{z}$ (see Fig. 15) maps the complex $z$-plane onto a Riemann surface $F$ with one periodic end (sine-end).

Now we construct a homeomorphic mapping of the Riemann surface $F$ one the complex $\zeta$-plane, which is quasiconformal outside certain compact region on $F$.

As in Section 5, we assume that all regions on $F$ which we consider contain all of their boundary points belonging to $F$. We make a cut in $U\left(\varepsilon, \tilde{B}_{k}\right) \cap K$ along a radius of $S_{k}=\left\{0<\left|w-b_{k}\right| \leq \varepsilon\right.$, arg $\left.w=\alpha_{k}^{\prime}\right\}$ starting at $B_{k}$. This cut splits $U\left(\varepsilon, \tilde{B}_{k}\right)$ into two parts: $U^{+}\left(\varepsilon, \tilde{B}_{k}\right)$ and $U^{-}\left(\varepsilon, \tilde{B}_{k}\right)$, where $U^{+}\left(\varepsilon, \tilde{B}_{k}\right)$ has a non-empty intersection with $\Lambda_{k}$ and $U^{-}\left(\varepsilon, \tilde{B}_{k}\right)$ has a non-empty intersection with $\Lambda_{k-1}$. We denote the kernel $K \backslash \bigcup_{k=1}^{p} U\left(\varepsilon, \tilde{B}_{k}\right)$ by $K_{0}$. The regions $U_{1}^{+}\left(\varepsilon, \tilde{B}_{k}\right)=$ $U^{+}\left(\varepsilon, \tilde{B}_{k}\right) \backslash \Lambda_{k}$ and $U_{1}^{-}\left(\varepsilon, \tilde{B}_{k}\right)=U^{-}\left(\varepsilon, \tilde{B}_{k}\right) \backslash \Lambda_{k-1}$ are circular sectors with the vertex at $B_{k}$ and the angles $\tilde{\theta}_{k}^{+}$and $\tilde{\theta}_{k}^{-}$, respectively, $\tilde{\theta}_{k}^{+}+\tilde{\theta}_{k}^{-} \leq 2 \pi$. Denote by $\Lambda_{k}^{\prime}$ the following part of the Riemann surface $F$ :

$$
\Lambda_{k}^{\prime}=\Lambda_{k} \cup U^{+}\left(\varepsilon, \tilde{B}_{k}\right) \cup U^{-}\left(\varepsilon, \tilde{B}_{k+1}\right)=\Lambda_{k} \cup U_{1}^{+}\left(\varepsilon, \tilde{B}_{k}\right) \cup U_{1}^{-}\left(\varepsilon, \tilde{B}_{k+1}\right)
$$

It is clear that

$$
F=K_{0} \cup\left(\bigcup_{k=1}^{p} \Lambda_{k}^{\prime}\right) .
$$

We find a quasiconformal mapping of $\Lambda_{k}^{\prime}$ onto the region $H_{k}=\left\{\eta_{k}>0\right\} \backslash T_{k}$, $\zeta_{k}=\xi_{k}+i \eta_{k}$, where $T_{k}$ is a trapezoid with vertices at the points $-1 / d_{k}^{-}, 1 / d_{k}^{+}$, 
$1 / d_{k}^{+}+i \theta_{k}^{+},-1 / d_{k}^{-}+i \theta_{k+1}^{-}$, where $\theta_{k}^{+}=\tilde{\theta}_{k}^{+} / d_{k}^{+}, \theta_{k+1}^{-}=\tilde{\theta}_{k+1}^{-} / d_{k}^{-}$. We denote the region $\left\{\xi_{k} \leq 1 / d_{k}^{+}, 0 \leq \eta_{k} \leq \theta_{k}^{+}\right\}$by $H^{(k)}\left(\theta_{k}^{+}\right)$, the region $\left\{\xi_{k} \leq-1 / d_{k}^{-}, 0 \leq\right.$ $\left.\eta_{k} \leq \theta_{k+1}^{-}\right\}$by $H^{(k)}\left(\theta_{k+1}^{-}\right)$, and the region $H^{(k)}\left(\theta_{k+1}^{-}, \theta_{k}^{+}\right)=H_{k} \backslash\left(H^{(k)}\left(\theta_{k+1}^{-}\right) \cup\right.$ $\left.H^{(k)}\left(\theta_{k}^{+}\right)\right)$by $H^{(k)}\left(\theta_{k+1}^{-}, \theta_{k}^{+}\right)$. We map the region $H^{(k)}\left(\theta_{k+1}^{-}, \theta_{k}^{+}\right)$onto $\Lambda_{k}$ using the function $w=\tilde{\varphi}_{k}\left(\zeta_{k}\right)$, where $\tilde{\varphi}_{k}\left(\zeta_{k}\right)$ is the function $\varphi_{\alpha \beta}\left(\zeta_{k}\right)$ mentioned in Remark 2 to Theorem 6.1 (p. ???) with $\alpha=\theta_{k+1}^{-}, \beta=\theta_{k}^{+}\left(H_{\alpha \beta}=H^{(k)}\left(\theta_{k+1}^{-}, \theta_{k}^{+}\right)\right)$. This is a quasiconformal mapping. We map $H^{(k)}\left(\theta_{k+1}^{-}\right)$onto $U_{1}^{-}\left(\varepsilon, \tilde{B}_{k+1}\right)$ using the function

$$
w=b_{k+1}+\varepsilon \exp \left\{d_{k}^{-} \zeta_{k}+1-i d_{k}^{-} \alpha_{k+1}^{-}\right\}
$$

(that is, $\left.\zeta_{k}=\frac{1}{d_{k}^{-}} \ln \frac{w-b_{k+1}}{\varepsilon e}+i \alpha_{k+1}^{-}\right)$, where the real constant $\alpha_{k+1}^{-}$is chosen in such a way that $\left\{\xi \leq-1 / d_{k}^{-}, \eta_{k}=0\right\}$ is mapped into $S_{k+1}$. Similarly we map $H^{(k)}\left(\theta_{k}^{+}\right)$onto $U_{1}^{+}\left(\varepsilon, \tilde{B}_{k}\right)$ using the function

$$
w=b_{k}+\varepsilon \exp \left\{-d_{k}^{+} \zeta_{k}+1+i d_{k}^{+} \alpha_{k}^{+}\right\}
$$

(that is, $\left.\zeta_{k}=\frac{1}{d_{k}^{+}} \ln \frac{\varepsilon e}{w-b_{k}}+i \alpha_{k}^{+}\right)$, where the real constant $\alpha_{k}^{+}$is chosen in such a way that $\left\{\xi_{k} \geq 1 / d_{k}^{+}, \eta_{k}=0\right\}$ is mapped into $S_{k}$.

If we take into account the form of the function $\tilde{\varphi}_{k}\left(\zeta_{k}\right)$, which can be written explicitly in the regions $\left\{\xi_{k} \leq-1 / d_{k}^{-}, \eta_{k} \geq \theta_{k+1}^{-}\right\}$and $\left\{\xi_{k} \geq 1 / d_{k}^{+}, \eta_{k} \geq \theta_{k}^{+}\right\}$, it is easy to see that the point $\zeta_{k} \in \partial H^{(k)}\left(\theta_{k+1}^{-}, \theta_{k}^{+}\right) \cap \partial H^{(k)}\left(\theta_{k+1}^{-}\right)$, that is, $\xi_{k}=$ $\xi_{k}+i \theta_{k+1}^{-}, \xi_{k} \leq-1 / d_{k}^{-}$is mapped onto the same point both under the function $\tilde{\varphi}_{k}\left(\zeta_{k}\right)$ and the function (7.1), namely it is mapped onto the point $\sigma_{k}$ satisfying $\left|w-b_{k+1}\right|=\varepsilon \exp \left\{d_{k}^{-} \xi_{k}+1\right\}$. Similarly, the point $\zeta_{k}=\xi_{k}+i \theta_{k}^{+}, \xi_{k} \geq 1 / d_{k}^{+}$is mapped onto the point $\sigma_{k}$ such that

$$
\left|w-b_{k}\right|=\varepsilon \exp \left\{-d_{k}^{+} \xi_{k}+1\right\},
$$

both under the mapping $\tilde{\varphi}_{k}\left(\zeta_{k}\right)$ or under the function (7.2). In such a way we get a quasiconformal mapping $w=\varphi_{k}\left(\zeta_{k}\right)$ of the region $H_{k}$ onto the Riemann surface $\Lambda_{k}^{\prime}$, where $\varphi_{k}\left(\zeta_{k}\right)=\tilde{\varphi}_{k}\left(\zeta_{k}\right)$ for $\zeta_{k} \in H^{(k)}\left(\theta_{k+1}^{-}, \theta_{k}^{+}\right)$, for $\zeta_{k} \in H^{(k)}\left(\theta_{k+1}^{-}\right)$the function $\varphi_{k}\left(\zeta_{k}\right)$ is given by the formula $(7.1)$, and for $\zeta_{k} \in H^{(k)}\left(\theta_{k}^{+}\right)$, the function $\varphi_{k}\left(\zeta_{k}\right)$ is given by the formula (7.2). Taking into account (6.18) we get

$$
\begin{aligned}
\iint_{H_{k}} & \left.\left\{p\left(\zeta_{k}, w\right)-1\right)\right\} d \sigma\left(\ln \zeta_{k}\right) \\
& =\iint_{H^{(k)}\left(\theta_{k+1}^{-}, \theta_{k}^{+}\right)}\left\{p\left(\zeta_{k}, w\right)-1\right\} d \sigma\left(\ln \zeta_{k}\right)<\infty .
\end{aligned}
$$

We denote the number of $a$-points of the function $w=\varphi_{k}\left(\zeta_{k}\right)$ in $H_{k} \cap\left\{\left|\zeta_{k}\right| \leq r\right\}$ by $n\left(r, a, \varphi_{k}\right)$, similarly we define $n_{1}\left(r, a, \varphi_{k}\right)$. By (6.19) and (6.20) we have

$$
\begin{gathered}
n\left(r, a, \varphi_{k}\right)= \begin{cases}\frac{r}{2 \pi}+o(r) & \text { for } a \neq b_{k}, b_{k+1}, \\
\frac{r}{2 \pi}\left(1-\delta_{g}\left(b_{j}, \Lambda_{k}\right)\right)+o(r) & a=b_{j}, j=k, k+1\left(\text { if } b_{k} \neq b_{k+1}\right) \\
\frac{r}{2 \pi}\left(1-\delta_{g}\left(b, \Lambda_{k}\right)\right)+o(r) & \text { for } a=b\left(\text { if } b_{k}=b_{k+1}=b\right),\end{cases} \\
n_{1}\left(r, a, \varphi_{k}\right)=\varepsilon_{g}\left(a, \Lambda_{k}\right) \frac{r}{2 \pi}+o(r) \text { for all } a .
\end{gathered}
$$


Let us observe that for fixed $a$ all $a$-points of the function $\varphi_{k}\left(\zeta_{k}\right)$ lie in some strip $\left|\xi_{k}\right| \leq K=K(a)$. The quantity

$$
\chi=\chi(F)=\frac{d_{1}^{+} d_{2}^{+} \ldots d_{p}^{+}}{d_{1}^{-} d_{2}^{-} \ldots d_{p}^{-}}
$$

will be called the characteristic of the surface $F$. If we change the way in which we count the points $B_{k}$ on the curve $C$, the characteristic $\chi$ does not change. Let

$$
\beta=1+\left(\frac{\ln \chi}{\pi p}\right)^{2}
$$

Let $\zeta_{k}=\rho_{k} e^{i \theta_{k}}, 1 \leq k \leq p$. We map $H_{k}$ using the function

$$
\zeta^{\prime}=w_{k}\left(\zeta_{k}\right)=\left(\frac{d_{1}^{+} \ldots d_{k}^{+}}{d_{1}^{-} \ldots d_{k-1}^{-}} \zeta_{k}\right)^{\frac{2}{p}} e^{i \frac{2 \pi}{p}(k-1)}, 1 \leq k \leq p, d_{0}^{ \pm}=1
$$

where the branch of $\zeta_{k}^{\frac{2}{p}}$ is chosen in such a way, that $\left(\zeta^{\prime}=\rho^{\prime} e^{i \theta^{\prime}}\right)$

$$
\rho^{\prime}=\left(\frac{d_{1}^{+} \ldots d_{k}^{+}}{d_{1}^{-} \ldots d_{k}^{-}}\right)^{\frac{2}{p}} \rho_{k}^{\frac{2}{p}}, \theta^{\prime}=\frac{2}{p} \theta_{k}+(k-1) \frac{2 \pi}{p}, 1 \leq k \leq p .
$$

The function (7.6) maps $H_{k}$ onto the region

$$
\left\{(k-1) \frac{2 \pi}{p}<\arg \zeta^{\prime}<k \frac{2 \pi}{p}\right\} \backslash T_{k}^{\prime},
$$

where $T_{k}^{\prime}$ is the image of the trapezoid $T_{k}$ under the mapping (7.6). The mapping $\zeta_{k}=\varphi_{k}^{-1}(w)$ maps the point $w \in S_{k}$ onto the point

$$
\zeta_{k}=\xi_{k}=\frac{1}{d_{k}^{+}} \ln \frac{\varepsilon e}{\left|w-b_{k}\right|},
$$

the mapping $\zeta^{\prime}=\omega_{k}\left(\varphi_{k}^{-1}(w)\right)$ maps the point $w \in S_{k}$ onto the point

$$
\zeta^{\prime}=\left(\frac{d_{1}^{+} \ldots d_{k-1}^{+}}{d_{1}^{-} \ldots d_{k-1}^{-}}\right)^{\frac{2}{p}}\left(\ln \frac{\varepsilon e}{\left|w-b_{k}\right|}\right)^{\frac{2}{p}} e^{i \frac{2 \pi}{p}(k-1)}, k=1, \ldots, p
$$

The mapping $\zeta_{k-1}=\varphi_{k-1}^{-1}(w), k=2, \ldots, p$ maps the same point $w \in S_{k}$ onto the point

$$
\zeta_{k-1}=\frac{1}{d_{k-1}^{-}} \ln \frac{\left|w-b_{k}\right|}{\varepsilon e}=e^{i \pi} \frac{1}{d_{k-1}^{-}} \ln \frac{e}{\left|w-b_{k}\right|},
$$

and the mapping $\zeta^{\prime}=\omega_{k-1}\left(\varphi_{k-1}^{-1}(w)\right)$ maps it onto the point

$$
\begin{aligned}
\zeta^{\prime} & =\left(\frac{d_{1}^{+} \ldots d_{k-1}^{+}}{d_{1}^{-} \ldots d_{k-2}^{-}} e^{i \pi} \frac{1}{d_{k-1}^{-}} \ln \frac{\varepsilon e}{\left|w-b_{k}\right|}\right)^{\frac{2}{p}} e^{i \frac{2 \pi}{p}(k-2)} \\
& =\left(\frac{d_{1}^{+} \ldots d_{k-1}^{+}}{d_{k-1}^{-} \ldots d_{k-1}^{-}}\right)^{\frac{2}{p}}\left(\ln \frac{\varepsilon e}{\left|w-b_{k}\right|}\right)^{\frac{2}{p}} e^{i \frac{2 \pi}{p}(k-1)}, k=2, \ldots, p .
\end{aligned}
$$

Comparing the formulas (7.7) and (7.8) we see that the mapping $\zeta^{\prime}=\omega(w)=$ $\omega_{k}\left(\varphi_{k}^{-1}(w)\right)$ remains single-valued and continuous on the radii $S_{2}, S_{3}, \ldots, S_{p}$ for 
$w \in \Lambda_{k}^{\prime} \cup S_{k} \cup S_{k+1}, k=1, \ldots, p$, and maps $\left(\bigcup_{k=1}^{p} \Lambda_{k}^{\prime}\right) \cup\left(\bigcup_{k=2}^{p} S_{k}\right)$ onto the region $G^{\prime}$ defined as the complex $\zeta^{\prime}$-plane with $\bigcup_{k=1}^{p} T_{k}^{\prime}$ removed and with the cut along the positive real half-axis. In the general case the function $\zeta^{\prime}=\omega(w)$ is not singlevalued on $S_{1}$. In fact, under the mapping $\zeta^{\prime}=\omega_{1}\left(\varphi_{1}^{-1}(w)\right)$ the point $w \in S_{1}$ is mapped, by (7.7), onto the point

$$
\zeta^{\prime}=\left(\ln \frac{\varepsilon e}{\left|w-b_{1}\right|}\right)^{\frac{2}{p}}=\zeta^{+}
$$

and under the mapping by the function $\zeta^{\prime}=\omega_{p}\left(\varphi_{p}^{-1}(w)\right)$ it is mapped onto the point

$$
\begin{aligned}
\zeta^{\prime} & =\left(\frac{d_{1}^{+} \ldots d_{p}^{+}}{d_{1}^{-} \ldots d_{p-1}^{-}} e^{i \pi} \frac{1}{d_{p}^{-}} \ln \frac{\varepsilon e}{\left|w-b_{1}\right|}\right)^{\frac{2}{p}} e^{i \frac{2 \pi}{p}(p-1)} \\
& =\chi^{\frac{2}{p}}\left(\ln \frac{\varepsilon e}{\left|w-b_{1}\right|}\right)^{\frac{2}{p}}=\chi^{\frac{2}{p}} \zeta^{+}=\zeta^{-} .
\end{aligned}
$$

If $\chi=1$, then $\zeta^{+}=\zeta^{-}$, and the function $\zeta^{\prime}=\omega(w)$ is continuous and single-valued also on $S_{1}$. In the general case we make an additional conformal mapping:

$$
\zeta=\left(\zeta^{\prime}\right)^{1 /\left(1-i \frac{\ln \chi}{\pi p}\right)}, \zeta^{\prime}=\rho^{\prime} e^{i \theta^{\prime}}, \zeta=\rho e^{i \theta},
$$

where a single-valued branch is chosen in such a way that the region $\left\{0<\theta^{\prime}<2 \pi\right\}$ is mapped into the region

$$
\left\{\frac{\ln \rho \ln \chi}{\pi p}<\theta<\frac{\ln \rho \ln \chi}{\pi p}+2 \pi\right\}
$$

that is, into the complex $\zeta$-plane with the cut along a logarithmic spiral $\theta=$ $\frac{1}{\pi p} \ln \rho \ln \chi, 0<\rho<\infty$. Under this mapping the point $\zeta^{+}=\rho^{\prime}$ on the upper boundary of the cut in the $\zeta^{\prime}$-plane is mapped onto the point

$$
\zeta=\left(\zeta^{+}\right)^{1 /\left(1-i \frac{\ln \chi}{\pi p}\right)}=\left(\rho^{\prime}\right)^{1 / \beta} \exp \left\{i \frac{\ln \chi \ln \rho^{\prime}}{\pi p \beta}\right\}
$$

and the point $\zeta^{-}=\chi^{\frac{2}{p}} \zeta^{+} e^{2 \pi i}$ on the lower boundary of the cut is mapped onto the point

$$
\begin{aligned}
\zeta & =\left(\zeta^{+}\right)^{1 /\left(1-i \frac{\ln \chi}{\pi p}\right)} \chi^{2 /\left(p-i \frac{\ln \chi}{\pi}\right)} e^{2 \pi i /\left(1-i \frac{\ln \chi}{\pi p}\right)} \\
& =\left(\zeta^{+}\right)^{1 /\left(1-i \frac{\ln \chi}{\pi p}\right)} \exp \left\{\frac{2 \ln \chi+2 \pi i p}{p-i \frac{\ln \chi}{\pi}}\right\}=\left(\zeta^{+}\right)^{1 /\left(1-i \frac{\ln \chi}{\pi p}\right)} e^{2 \pi i},
\end{aligned}
$$

that is, under the mapping (7.9) the points $\zeta^{+}$and $\zeta^{-}$are mapped onto the points on the opposite boundaries of the cut in the $\zeta$-plane, which correspond to the same number in the complex plane. We denote the image of the region $G^{\prime}$ under the mapping (7.9) by $G$. It is easy to see that $G_{0}=\{|\zeta|<\infty\} \backslash \bar{G}$ is a simply connected region bounded by a closed Jordan curve. The mapping $\zeta=\zeta(w)$, which is a composition of the mapping $\zeta^{\prime}=\omega(w)$ and the mapping (7.9), is a homeomorphic mapping of $F \backslash K_{0}$ onto $G$. There exists a homeomorphic mapping of the kernel 
$K_{0}$ onto the region $G_{0}$ such that we get a homeomorphic mapping $\zeta=\zeta(w)$ of the whole Riemann surface $F$ onto $\{|\zeta|<\infty\}$. Denote the mapping inverse to $\zeta=\zeta(w)$ by $w=\varphi(\zeta)$. It is clear that the mapping $w=\varphi(\zeta)$ is quasiconformal in $G$. Taking into account (7.3) we get

$$
\begin{aligned}
\iint_{G}\{p(\zeta, w)-1\} d \sigma(\ln \zeta) & =\iint_{G^{\prime}}\left\{p\left(\zeta^{\prime}, w\right)-1\right\}\left|\frac{d \ln \zeta}{d \ln \zeta^{\prime}}\right|^{2} d \sigma\left(\ln \zeta^{\prime}\right) \\
& =\frac{1}{\beta} \iint_{G^{\prime}}\left\{p\left(\zeta^{\prime}, w\right)-1\right\} d \sigma\left(\ln \zeta^{\prime}\right) \\
& =\frac{1}{\beta} \sum_{k=1}^{p} \iint_{H_{k}}\left\{p\left(\zeta_{k}, w\right)-1\right\}\left|\frac{d \ln \zeta^{\prime}}{d \ln \zeta_{k}}\right|^{2} d \sigma\left(\ln \zeta_{k}\right) \\
& =\frac{4}{p^{2} \beta} \sum_{k=1}^{p} \iint_{H_{k}}\left\{p\left(\zeta_{k}, w\right)-1\right\} d \sigma\left(\ln \zeta_{k}\right)<\infty .
\end{aligned}
$$

If the function $w=f(z)$ is a conformal mapping of $\{|z|<R\}$ onto $F$, then the mapping $z=z(\zeta)=f^{-1}(\varphi(\zeta))$ satisfies, by (7.10), the conditions of Teichmüller Theorem 3.1, hence, $R=\infty$ and under the corresponding normalization of the meromorphic in $\{|z|<\infty\}$ function $w=f(z)$ we have

$$
\lim _{z \rightarrow \infty}\left|\frac{\zeta(z)}{z}\right|=\lim _{\zeta \rightarrow \infty}\left|\frac{\zeta}{z(\zeta)}\right|=1
$$

Let $d^{k}(a)=\delta_{g}\left(a, \Lambda_{k}\right), e^{k}(a)=\varepsilon_{g}\left(a, \Lambda_{k}\right)$. If $r$ is sufficiently large, then the region corresponding to $\{|\zeta| \leq r\} \backslash G_{0}$ in the $\zeta^{\prime}$-plane is the region which is the intersection of $G^{\prime}$ with the region $\left\{\rho^{\prime} \leq r^{\beta} \exp \left(\theta^{\prime} \frac{\ln \chi}{\pi p}\right)\right\}, 0<\theta^{\prime}<2 \pi$, and the corresponding regions in $\zeta_{k}$-planes are the intersections of $H_{k}$ with $\left(\zeta_{k}=\rho_{k} e^{i \theta_{k}}\right)$ :

$$
\begin{aligned}
\rho_{k} & \leq \frac{d_{1}^{-} \ldots d_{k-1}^{-}}{d_{1}^{+} \ldots d_{k}^{+}} r^{\beta p / 2} \exp \left\{\frac{\ln \chi}{2 \pi}\left(\frac{2}{p} \theta_{k}+(k-1) \frac{2 \pi}{p}\right)\right\} \\
& =\frac{d_{1}^{-} \ldots d_{k-1}^{-}}{d_{1}^{+} \ldots d_{k}^{+}} r^{\beta p / 2} \chi^{\frac{1}{\pi p}\left\{\theta_{k}+\pi(k-1)\right\}}, 0<\theta_{k}<\pi, 1 \leq k \leq p,
\end{aligned}
$$

this follows from $\left(7.6^{\prime}\right)$ and (7.9). Taking into account the relations (7.4) and (7.5), and also the fact that for each $a$ the arguments of $a$-points in the $\zeta_{k}$ plane tend to $\pi / 2$, we find that

$$
\begin{gathered}
n(r, a, \varphi)=\frac{r^{\beta p / 2}}{2 \pi} \sum_{k=1}^{p}\left\{1-d^{k}(a)\right\} \frac{d_{1}^{-} \ldots d_{k-1}^{-}}{d_{1}^{+} \ldots d_{k}^{+}} \chi^{\frac{1}{p}\left(k-\frac{1}{2}\right)}+o\left(r^{\beta p / 2}\right), \\
n_{1}(r, a, \varphi)=\frac{r^{\beta p / 2}}{2 \pi} \sum_{k=1}^{p} e^{k}(a) \frac{d_{1}^{-} \ldots d_{k-1}^{-}}{d_{1}^{+} \ldots d_{k}^{+}} \chi^{\frac{1}{p}\left(k-\frac{1}{2}\right)}+o\left(r^{\beta p / 2}\right) .
\end{gathered}
$$

By (7.11) the relations (7.12) and (7.13) remain true if we replace $n(r, a, \varphi)$ by $n(r, a, f)$ and $n_{1}(r, a, \varphi)$ by $n_{1}(r, a, f)$. From here we get

$$
N(r, a, f)=\frac{r^{\beta p / 2}}{\beta p \pi} \sum_{k=1}^{p}\left\{1-d^{k}(a)\right\} \frac{d_{1}^{-} \ldots d_{k-1}^{-}}{d_{1}^{+} \ldots d_{k}^{+}} \chi^{\frac{1}{p}\left(k-\frac{1}{2}\right)}+o\left(r^{\beta p / 2}\right),
$$




$$
N_{1}(r, a, f)=\frac{r^{\beta p / 2}}{\beta p \pi} \sum_{k=1}^{p} e^{k}(a) \frac{d_{1}^{-} \ldots d_{k-1}^{-}}{d_{1}^{+} \ldots d_{k}^{+}} \chi^{\frac{1}{p}\left(k-\frac{1}{2}\right)}+o\left(r^{\beta p / 2}\right) .
$$

Since $d^{k}(a)=0,1 \leq k \leq p$, for all $a$ except at most $p$, arguing as in the deduction of (6.16) we find that

$$
T(r, f)=\frac{r^{\beta p / 2}}{\beta p \pi} \sum_{k=1}^{p} \frac{d_{1}^{-} \ldots d_{k-1}^{-}}{d_{1}^{+} \ldots d_{k}^{+}} \chi^{\frac{1}{p}\left(k-\frac{1}{2}\right)}+o\left(r^{\beta p / 2}\right),
$$

and get the following theorem.

THEOREM 7.1. Let $w=f(z)$ be a meromorphic function mapping the complex $z$-plane onto a Riemann surface $F$ with $p$ almost periodic ends and characteristic $\chi$. Then the function $w=f(z)$ has a normal type of order

$$
\rho=\frac{\beta p}{2}=\frac{p}{2}\left\{1+\left(\frac{\ln \chi}{\pi p}\right)^{2}\right\}
$$

moreover $T(r, f) \sim K r^{\rho}, 0<K<\infty$, and its deficiencies and indices are equal to

$$
\delta(a, f)=\frac{\sum_{k=1}^{p} d^{k}(a) \frac{d_{1}^{-} \ldots d_{k-1}^{-}}{d_{1}^{+} \ldots d_{k}^{+}} \chi^{\frac{1}{p}\left(k-\frac{1}{2}\right)}}{\sum_{k=1}^{p} \frac{d_{1}^{-} \ldots d_{k-1}^{-}}{d_{1}^{+} \ldots d_{k}^{+}} \chi^{\frac{1}{p}\left(k-\frac{1}{2}\right)}}
$$

and

$$
\varepsilon(a, f)=\frac{\sum_{k=1}^{p} e^{k}(a) \frac{d_{1}^{-} \ldots d_{k-1}^{-}}{d_{1}^{+} \ldots d_{k}^{+}} \chi^{\frac{1}{p}\left(k-\frac{1}{2}\right)}}{\sum_{k=1}^{p} \frac{d_{1}^{-} \ldots d_{k-1}^{-}}{d_{1}^{+} \ldots d_{k}^{+}} \chi^{\frac{1}{p}\left(k-\frac{1}{2}\right)}}
$$

respectively.

Since $\sum_{a}\left\{d^{k}(a)+e^{k}(a)\right\}=2$ for $k=1, \ldots, p$, the equalities (7.18) and (7.19) imply that

$$
\sum_{a}\{\delta(a, f)+\varepsilon(a, f)\}=2
$$

for each function $f$ satisfying the conditions of Theorem 7.1.

For brevity we shall speak about deficiencies and indices of the surface $F$ with almost periodic ends meaning deficiencies and indices of the mapping meromorphic function $w=f(z)$, and defining $\delta(a, F)=\delta(a, f)$ and $\varepsilon(a, F)=\varepsilon(a, f)$. In what follows we shall consider surfaces with finitely many almost periodic ends for $\chi=1$ only, without mentioning this explicitly. For convenience of references we rewrite the formulas (7.17)-(7.19) for this case.

$$
\begin{gathered}
\rho=p / 2, \\
\delta(a, f)=\frac{\frac{1}{d_{1}^{+}} d^{1}(a)+\frac{d_{1}^{-}}{d_{1}^{+} d_{2}^{+}} d^{2}(a) \cdots+\frac{d_{1}^{-} \ldots d_{p-1}^{-}}{d_{1}^{+} \ldots d_{p}^{+}} d^{p}(a)}{\frac{1}{d_{1}^{+}}+\frac{d_{1}^{-}}{d_{1}^{+} d_{2}^{+}}+\cdots+\frac{d_{1}^{-} \ldots d_{p-1}^{-}}{d_{1}^{+} \ldots d_{p}^{+}}},
\end{gathered}
$$




$$
\varepsilon(a, f)=\frac{\frac{1}{d_{1}^{+}} e^{1}(a)+\frac{d_{1}^{-}}{d_{1}^{+} d_{2}^{+}} e^{2}(a)+\cdots+\frac{d_{1}^{-} \ldots d_{p-1}^{-}}{d_{1}^{+} \ldots d_{p}^{+}} e^{p}(a)}{\frac{1}{d_{1}^{+}}+\frac{d_{1}^{-}}{d_{1}^{+} d_{2}^{+}}+\cdots+\frac{d_{1}^{-} \ldots d_{p-1}^{-}}{d_{1}^{+} \ldots d_{p}^{+}}}
$$

The quantity

$$
L\left(F, B_{1}\right)=\frac{1}{d_{1}^{+}}+\frac{d_{1}^{-}}{d_{1}^{+} d_{2}^{+}}+\cdots+\frac{d_{1}^{-} \ldots d_{p-1}^{-}}{d_{1}^{+} \ldots d_{p}^{+}}
$$

( $B_{1}$ is the point on the boundary of the pseudokernel, at which we start counting), which is the denominator in both (7.21) and (7.22) is called the weight of the surface $F$ at the point $B_{1}$. Taking into account that $\chi=1$, it is easy to verify that for $1 \leq j \leq p$ we have

$$
L\left(F, B_{j}\right)=\frac{1}{d_{j}^{+}}+\frac{d_{j}^{-}}{d_{j}^{+} d_{j+1}^{+}}+\cdots+\frac{d_{j}^{-} \ldots d_{p}^{-} d_{1}^{-} \ldots d_{j-2}^{-}}{d_{j}^{+} \ldots d_{p}^{+} d_{1}^{+} \ldots d_{j-1}^{+}}=\frac{d_{1}^{+} \ldots d_{j-1}^{+}}{d_{1}^{-} \ldots d_{j-1}^{-}} L\left(F, B_{1}\right) .
$$

Thus, generally speaking, weight depends on a point at which it is taken. However, for brevity, we shall write $L(F)$ instead of $L\left(F, B_{1}\right)$.

It is clear that the expressions in the right-hand sides of (7.21) and (7.22) do not depend on the choice of the starting point $B_{1}$ such $\delta(a, f)$ and $\varepsilon(a, f)$ do not depend on this choice, but this can also be proved by a formal computation.

Let $F_{1}$ and $F_{2}$ be two surfaces with finitely many $\left(p_{1}\right.$ and $p_{2}$, respectively) almost periodic ends, moreover, each of these surfaces have at least one logarithmic ramification point $\tilde{B}^{\prime}$ and $\tilde{B}^{\prime \prime}$ which is projected onto $b$. Let $K_{1}$ and $K_{2}$ be pseudokernels of the surfaces $F_{1}$ and $F_{2}$. For sufficiently small $\varepsilon>0$ the regions $K_{1} \cap U\left(\varepsilon, \tilde{B}^{\prime}\right)$ and $K_{2} \cap U\left(\varepsilon, \tilde{B}^{\prime \prime}\right)$ are circular sectors with vertices at points $\tilde{B}^{\prime} \in \partial K_{1}$ and $\tilde{B}^{\prime \prime} \in \partial K_{2}$. Suppose that these two sectors contain two radii with the same projection. We make cuts along these radii and paste the surfaces $F_{1}$ and $F_{2}$ along them, pasting each boundary of one cut with the opposite boundary of the other cut. We get a Riemann surface $F$, which also is a surface with $p\left(=p_{1}+p_{2}\right)$ almost periodic ends.

We shall write $F=F_{1}+F_{2}$ and say that the surface $F$ is obtained by a junction of the surfaces $F_{1}$ and $F_{2}$ at the points $\tilde{B}^{\prime}$ and $\tilde{B}^{\prime \prime}$.

It is easy to see that $\chi(F)=\chi\left(F_{1}\right) \chi\left(F_{2}\right)$. Therefore, if $\chi\left(F_{1}\right)=\chi\left(F_{2}\right)=1$ (we agreed to consider such surface only), then $\chi(F)=1$. On each of the joined surfaces $F_{1}$ and $F_{2}$ we start counting the logarithmic ramification points at $\tilde{B}^{\prime}$ and $\tilde{B}^{\prime \prime}$, respectively, we start counting logarithmic ramification points on the junction $F=F_{1}+F_{2}$ at either the logarithmic ramification point corresponding to $\tilde{B}^{\prime}$, or at the logarithmic ramification point corresponding to $\tilde{B}^{\prime \prime}$, the specific choice makes no difference. It is easy to verify that

$$
L\left(F_{1}+F_{2}\right)=L\left(F_{1}\right)+L\left(F_{2}\right),
$$

and using the formulas (7.21) and (7.22) we find that

$$
\delta\left(a, F_{1}+F_{2}\right)=\frac{L\left(F_{1}\right) \delta\left(a, F_{1}\right)+L\left(F_{2}\right) \delta\left(a, F_{2}\right)}{L\left(F_{1}\right)+L\left(F_{2}\right)}
$$




$$
\varepsilon\left(a, F_{1}+F_{2}\right)=\frac{L\left(F_{1}\right) \varepsilon\left(a, F_{1}\right)+L\left(F_{2}\right) \varepsilon\left(a, F_{2}\right)}{L\left(F_{1}\right)+L\left(F_{2}\right)} .
$$

If we assume that we have already defined $F_{1}+\cdots+F_{n-1}$, we define $F_{1}+\cdots+$ $F_{n}=\sum_{j=1}^{n} F_{j}$ as $\left(F_{1}+\cdots+F_{n-1}\right)+F_{n}$.

Suppose that the surface $F_{1}+\cdots+F_{n}$ is obtained in the following way. The surfaces $F_{1}$ and $F_{2}$ are joined at the points $\tilde{B}^{\prime}$ and $\tilde{B}^{\prime \prime}$, and we start counting logarithmic ramification points of the surface of $F_{1}+F_{2}$ at one of the points corresponding to $\tilde{B}^{\prime}$ or $\tilde{B}^{\prime \prime}$ (we denote it by $\tilde{\tilde{B}}^{\prime \prime}$ ). The surfaces $F_{1}+F_{2}$ and $F_{3}$ are joined at the points $\tilde{\tilde{B}}^{\prime \prime}$ and $\tilde{B}^{\prime \prime \prime}$, and we start counting logarithmic ramification points of $F_{1}+F_{2}+F_{3}$ from one of the points corresponding to $\tilde{\tilde{B}}^{\prime \prime}$ and $\tilde{B}^{\prime \prime \prime}$ (we denote it by $\tilde{\tilde{B}}^{\prime \prime \prime}$ ), we continue in an obvious way till we get the surface $F_{1}+\cdots+F_{n}$. It is not always possible to get the surface in this way, but if it is the case, we get, by induction, that

$$
\begin{gathered}
L\left(\sum_{j=1}^{n} F_{j}\right)=\sum_{j=1}^{n} L\left(F_{j}\right), \\
\delta\left(a, \sum_{j=1}^{n} F_{j}\right)=\frac{\sum_{j=1}^{n} L\left(F_{j}\right) \delta\left(a, F_{j}\right)}{\sum_{j=1}^{n} L\left(F_{j}\right)}, \\
\varepsilon\left(a, \sum_{j=1}^{n} F_{j}\right)=\frac{\sum_{j=1}^{n} L\left(F_{j}\right) \varepsilon\left(a, F_{j}\right)}{\sum_{j=1}^{n} L\left(F_{j}\right)} .
\end{gathered}
$$

In the general case, for $n \geq 3$, these formulas are no longer true. Later on we shall need the following

TheOREM 7.2. Let $b_{1}, \ldots, b_{p}$ be some complex numbers, not necessarily distinct, $a_{1}, \ldots, a_{q}$ be distinct complex numbers, moreover, all terms of the sequence $\left\{b_{k}\right\}$ are present among terms of the sequence $\left\{a_{j}\right\}$. Then there exists a Riemann surface $F$ with $p$ almost periodic ends $\Lambda_{1}, \ldots, \Lambda_{p}$, following after each other in the indicated order, where $\delta\left(a, \Lambda_{k}\right)=0$ for $a \neq b_{k}, b_{k+1}$, and $\delta\left(b_{k}, \Lambda_{k}\right), \delta\left(b_{k+1}, \Lambda_{k}\right), \varepsilon\left(a_{j}, \Lambda_{k}\right)$, $1 \leq j \leq q, 1 \leq k \leq p$, are arbitrary preassigned numbers satisfying $\delta\left(b_{k}, \Lambda_{k}\right)>0$, $\delta\left(b_{k+1}, \Lambda_{k}\right)>0$,

$$
\sum_{j=1}^{q}\left\{\delta\left(a_{j}, \Lambda_{k}\right)+\varepsilon\left(a_{j}, \Lambda_{k}\right)+\varepsilon\left(a_{j}, \Lambda_{k}\right)\right\}=2, \quad \text { and } 0 \leq \delta\left(a_{j}, \Lambda_{k}\right)+\varepsilon\left(a_{j}, \Lambda_{k}\right) \leq 1 .
$$

First we construct a pseudokernel $K$. If $b_{k} \neq b_{k+1}$, that is, all almost periodic ends $\Lambda_{k}$ are of the first kind, the construction of the pseudokernel $K$ is the same as in the proof of Theorem 5.1. In general, the construction is somewhat more complicated.

First wee assume that not all of the numbers $b_{j}, j \geq 2$, are equal to $b_{1}$. Let $b_{1}^{\prime}, \ldots, b_{\nu}^{\prime}$ and $b_{1}^{\prime \prime}, \ldots, b_{\mu}^{\prime \prime}$ be subsequences of $b_{1}, \ldots, b_{p}$ such that each of them has the same set of values as $b_{1}, \ldots, b_{p}$, but: (a) $b_{k}^{\prime} \neq b_{k-1}^{\prime}, b_{k+1}^{\prime}\left(b_{0}^{\prime}=b_{\nu}^{\prime}, b_{\nu+1}^{\prime}=b_{1}^{\prime}\right.$ ), $k=1, \ldots, \nu$; (b) all numbers $b_{k}^{\prime \prime}$ are distinct; (c) there is no subsequence with the same properties and more terms. Let $b_{k}^{\prime}=b_{n_{k}}, 1=n_{1}<n_{2}<\cdots<n_{\nu} \leq p$; $b_{n_{k}} \neq b_{n_{k}-1}, b_{k}^{\prime \prime}=b_{m_{k}}^{\prime}, 1=m_{1}<m_{2}<\cdots<m_{\mu} \leq \nu$. We draw a closed Jordan 
curve $c$ through the points $\left\{b_{1}^{\prime \prime}, \ldots, b_{\mu}^{\prime \prime}\right\}$ which does not pass through the points $a_{1}, \ldots, a_{q}$, different from $\left\{b_{1}, \ldots, b_{p}\right\}$, has a continuous curvature, and consisting in line segments in sufficiently small neighborhoods of the points $b_{1}^{\prime \prime}, \ldots, b_{\mu}^{\prime \prime}$. After that we, in the same way as at the beginning of the proof of Theorem 5.1, construct a simply connected finite-sheeted Riemann surface $K^{\prime}$ with boundary, bounded by a closed Jordan curve $C^{\prime}$, consisting of $\nu J$-curves $\sigma_{k}^{\prime}=\widehat{B_{k}^{\prime} B^{\prime}}{ }_{k+1}$, where the point $B_{k}^{\prime}$ is projected onto $b_{k}^{\prime}$. If $\nu=p$, we let $K=K^{\prime}$ and $B_{k}=B_{k}^{\prime}$. If $\nu<p$, we carry out an additional construction. Suppose that the sequence $b_{1}, \ldots, b_{p}$ satisfies $b_{t-1} \neq b_{t}=b_{t+1}=\cdots=b_{t+s} \neq b_{t+s+1}$ (indices are taken $\bmod p$ ). Let $b_{t}=b_{\tau}^{\prime}=b_{r}^{\prime \prime}$. Take a $J^{\prime}$-curve $c_{\tau}$ with ends at $b_{r}^{\prime \prime}$ such that the region $G_{\tau}$ bounded by it is contained in the region bounded by the curve $c$, and $\bar{G}_{\tau} \backslash\left\{b_{r}^{\prime \prime}\right\}$ does not contain points $a_{1}, \ldots, a_{q}$, moreover, for different $t$ the regions $G_{\tau}$ are pairwise disjoint. We take $s$ copies of the region $G_{\tau}$ and make in each of them the same cut along a line segment with one end at $b_{r}^{\prime \prime}$ and the other end at $\pi_{\tau} \in G_{\tau}$. We make the same cut on $K^{\prime}$ starting at $B_{\tau}^{\prime}$. We paste to $K^{\prime} s$ copies of the regions $G_{\tau}$ along this cut in such a way, that the obtained Riemann surface remains simply connected, and an algebraic ramification point of order $s$ appears over $\pi_{\tau}$. Making such pasting for all $t$, we get a finite-sheeted simply connected Riemann surface $K$ with boundary,

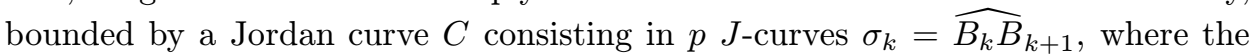
point $B_{k}$ is projected onto the point $b_{k}$, and the arc $\sigma_{k}$ is projected into the arc ${\widehat{b_{k}}}_{k+1}$ on the curve $c$ if $b_{k+1} \neq b_{k}$, and into some arc $c_{\tau}$ if $b_{k+1}=b_{k}$. The surface $K$ plays the role of pseudokernel. Now, using Theorems 6.2 and 6.3 , we construct almost periodic ends $\Lambda_{k}$ with preassigned $\delta\left(a, \Lambda_{k}\right)$ and $\varepsilon\left(a, \Lambda_{k}\right)$, and the boundary $\sigma_{k}^{-}$. Joining them to the pseudokernel $K$ we get the desired surface $F$.

If $b_{1}=\cdots=b_{p}=b$, the construction of the pseudokernel $K$ is simplified. Take a $J^{\prime}$-curve $c$ with ends at $b$, let $G$ be the interior of the curve $c$. Choose the curve $c$ in such a way that $\bar{G} \backslash\{b\}$ does not contain the points $a_{1}, \ldots, a_{q}$. Let $w_{0} \in G$. We take as $K$ the part of the surface of the function $\left(w-w_{0}\right)^{1 / p}$ lying over $G$.

\section{The inverse problem of the value distribution theory}

First we prove the following theorem.

THEOREM 8.1. Let $\delta_{j}, \varepsilon_{j}, j=1, \ldots, q$ be non-negative numbers satisfying the conditions

$\left(1^{\prime}\right) \sum_{j=1}^{q}\left\{\delta_{j}+\varepsilon_{j}\right\}=2$,

(2) $0<\delta_{j}+\varepsilon_{j} \leq 1,1 \leq j \leq q$,

(3) $0<\sum_{j=1}^{p} \delta_{j} \leq 2$, moreover, if $\sum_{j=1}^{q} \delta_{j}=2$, then all numbers $\delta_{1}, \ldots, \delta_{q}$ are rational.

Let $a_{1}, \ldots, a_{q}$ be arbitrary distinct complex numbers. Then there exists a Riemann surface $F$ with almost periodic ends such that $\delta\left(a_{j}, F\right)=\delta_{j}, \varepsilon(a, F)=\varepsilon_{j}, 1 \leq j \leq q$. 
In the case when $\sum_{j=1}^{q} \delta_{j}=2$, the desired Riemann surface $F$ was constructed in Theorem 5.1, namely, a surface with logarithmic ends. Note that for $\sum_{j=1}^{q} \delta_{j}=2$ we cannot drop the restriction that the numbers $\delta_{j}, 1 \leq j \leq q$, are rational, even if we use Riemann surfaces with almost periodic ends. In fact, if $\varepsilon\left(a_{0}, \Lambda_{k}\right)>0$ for at least one almost periodic end $\Lambda_{k}$ and at least one point $a_{0}$, then, by (7.19), we have $\varepsilon\left(a_{0}, F\right)>0$. But this contradicts the condition $\sum_{j=1}^{q} \delta_{j}=2$. Hence, for each end $\Lambda_{k}$ we have $d_{k}^{+}=d_{k}^{-}=1,1 \leq k \leq q$, and, necessarily, $\chi=1$. The equality (7.21) implies that the deficiencies $\delta(a, F)$ are rational numbers.

In what follows we assume $\sum_{j=1}^{q} \delta_{j}<2$ and $\sum_{j=1}^{q} \varepsilon_{j}>0$. The distribution of deficiencies and indices of some surface $F$ with almost periodic ends will be described using a scheme

$$
\left(\begin{array}{ccc}
a_{1} & \ldots & a_{q} \\
\delta\left(a_{1}\right) & \ldots & \delta\left(a_{q}\right) \\
\varepsilon\left(a_{1}\right) & \ldots & \varepsilon\left(a_{q}\right)
\end{array}\right)=\left(\begin{array}{ccc}
\delta_{1} & \ldots & \delta_{q} \\
\varepsilon_{1} & \ldots & \varepsilon_{q}
\end{array}\right),
$$

and the construction of a surface with this distribution of deficiencies and indices will be called a scheme solution. Junction of Riemann surfaces will be symbolically represented as addition of the corresponding schemes using the following rule:

$$
\begin{aligned}
L_{1}\left(\begin{array}{ccc}
\ldots & \delta_{j}^{(1)} & \ldots \\
\ldots & \varepsilon_{j}^{(1)} & \ldots
\end{array}\right) & +L_{2}\left(\begin{array}{ccc}
\ldots & \delta_{j}^{(2)} & \ldots \\
\ldots & \varepsilon_{j}^{(2)} & \ldots
\end{array}\right) \\
& =\left(L_{1}+L_{2}\right)\left(\begin{array}{ccc}
\ldots & \frac{L_{1} \delta_{j}^{(1)}+L_{2} \delta_{j}^{(2)}}{L_{1}+L_{2}} & \ldots \\
\ldots & \frac{L_{1} \varepsilon_{j}^{(1)}+L_{2} \varepsilon_{j}^{(2)}}{L_{1}+L_{2}} & \ldots
\end{array}\right),
\end{aligned}
$$

where the factor in front of a scheme denotes the weight of the surface taken at the point at which the junction takes place. We mean that the joined surfaces both have positive deficiencies over one of the points $a_{j_{0}}$ and the junction takes place at logarithmic ramification points over $a_{j_{0}}$. Solving schemes-summands we, obviously, can always select pseudokernels in such a way that the junction is possible. We may assume without loss of generality that $a_{j_{0}}=a_{1}$. Thus, in all schemes which we are going to join should be $\delta_{1}>0$, and the weight is always taken in a logarithmic ramification point over $a_{1}$. To solve a scheme with weight means to construct a Riemann surface with almost periodic ends, with preassigned value distribution and with the given weight up to a rational factor. If we can solve the schemes in the left-hand side of the equality (8.1) using surfaces $F_{1}$ and $F_{2}$ with weights $\frac{n_{1}}{m_{1}} L_{1}$ and $\frac{n_{2}}{m_{2}} L_{2}$, respectively, where $n_{1}, n_{2}, m_{1}, m_{2}$ are positive integers, then, joining $n_{2} m_{1}$ copies of the surface $F_{2}$ with $n_{1} m_{2}$ copies of the surface $F_{1}$, we, by (7.25), (7.26), and (7.27), get a surface $F$ solving the scheme in the right-hand side of the equality (8.1) with weight $n_{1} n_{2}\left(L_{1}+L_{2}\right)$. 
We shall reduce solutions of complicated schemes to solutions of four main schemes $(\alpha-, \beta-, \gamma-$, and $\delta$-schemes), for which we provide a solution with a preassigned weight, up to a rational factor. We use the notation $\Theta_{j}=\delta_{j}+\varepsilon_{j}, 1 \leq j \leq q$.

$\alpha$-SCHEME is a scheme with $\delta_{1}>0, \delta_{2}=\delta_{3}=\cdots=\delta_{q}=0$ :

$$
L\left(\begin{array}{cccc}
\delta_{1} & 0 & \ldots & 0 \\
\varepsilon_{1} & \varepsilon_{2} & \cdots & \varepsilon_{q}
\end{array}\right)
$$

Let $m$ be so large positive integer that $2 /(m L)<\delta_{1}$. We construct a surface $\Phi$ with almost periodic ends of the second kind solving a given $\alpha$-scheme, moreover, $d_{1}^{+}=d_{2}^{-}=2 /(m L), d_{1}^{-}=d_{2}^{+}=\delta_{1}-d_{1}^{+}$. Such surface $\Phi$ exists by Theorem 6.3. The weight of such surface $\Phi$ is equal to

$$
\frac{1}{d_{1}^{+}}+\frac{d_{1}^{-}}{d_{1}^{+} d_{2}^{+}}=\frac{2}{d_{1}^{+}}=m L .
$$

$\beta$-SCHEME is a scheme for which $\delta_{j}>0,1 \leq j \leq q, \varepsilon_{1}>0$, and all numbers $\Theta_{j}$ are rational, $1 \leq j \leq q$ :

$$
L\left(\begin{array}{llll}
\delta_{1} & \delta_{2} & \ldots & \delta_{q} \\
\varepsilon_{1} & \varepsilon_{2} & \cdots & \varepsilon_{q}
\end{array}\right)
$$

Let $\Theta_{j}=n_{j} / n, n_{j}, n$ be positive integers, $\varepsilon_{1}>1 / n$. Then $\sum_{j=1}^{q} n_{j}=2 n$. We represent $\delta_{j}$ and $\varepsilon_{j}$ as sums

$$
\delta_{j}=\sum_{k=1}^{n_{j}} \Delta_{j k}, \varepsilon_{j}=\sum_{k=1}^{n_{j}} E_{j k}
$$

where

$$
\Delta_{j k}>0, E_{j k} \geq 0, \Delta_{j k}+E_{j k}=\frac{1}{n}, 1 \leq k \leq n_{j}
$$

Let $m$ be a positive integer satisfying $2 /(m L)<1 / n$. Since $\varepsilon_{1}>0$, we can take $\Delta_{11}=2 /(m L), E_{11}=n^{-1}-\Delta_{11}>0$. We take a surface $F$ with $2 n$ almost periodic ends $\Lambda_{1}, \ldots, \Lambda_{2 n}$ and with logarithmic ramification points $\tilde{B}_{1}, \ldots, \tilde{B}_{2 n}$, where $\tilde{B}_{1}$ lies over $a_{1}$, and there are $n_{j}$ logarithmic ramification points over $a_{j}, 1 \leq j \leq q$. Since $n_{j} \leq n=\frac{1}{2} \sum_{j=1}^{q} n_{j}$, the sequence $\tilde{B}_{\nu}$ can be chosen in such a way that $b_{\nu} \neq b_{\nu \pm 1}, 1 \leq \nu \leq 2 n$, where $b_{\nu}$ is a projection of the point $\tilde{B}_{\nu}$. Here and later all indices $\nu$ are taken $\bmod 2 n$. Suppose that $\tilde{B}_{\nu}$ is the logarithmic ramification point number $s$ among those projected into $a_{j}, s=s(\nu), j=j(\nu)$. Let $\Delta_{\nu}=\Delta_{j s}$, $E_{\nu}=E_{j s}, j=j(\nu), s=s(\nu)$. Obviously, $\Delta_{1}=\Delta_{11}$. We choose an almost periodic end $\Delta_{\nu}$ in such a way that $\delta\left(a_{j(\nu)}, \Lambda_{\nu}\right)=\Delta_{\nu} n, \delta\left(a_{j(\nu+1)}, \Lambda_{\nu}\right)=\Delta_{\nu+1} n$, $\varepsilon\left(a_{j(\nu)}, \Lambda_{\nu}\right)=E_{\nu} n, \varepsilon\left(a_{j(\nu+1)}, \Lambda_{\nu}\right)=E_{\nu+1} n$. We have $d_{\nu}^{-}=d_{\nu+1}^{+}=\Delta_{\nu+1} n$. The weight of the surface $F$ is equal to

$$
\frac{1}{d_{1}^{+}} 2 n=\frac{1}{\Delta_{11} n} 2 n=\frac{2}{\Delta_{11}}=m L .
$$


The equality (7.21) implies

$$
\begin{aligned}
\delta\left(a_{j}, F\right) & =\frac{1}{2 n} \sum_{\nu=1}^{2 n} \delta\left(a_{j}, \Lambda_{\nu}\right)=\frac{1}{2 n} \sum_{j}^{\prime} \delta\left(a_{j(\nu)}, \Lambda_{\nu}\right)+\frac{1}{2 n} \sum_{j}^{\prime \prime} \delta\left(a_{j(\nu+1)}, \Lambda_{\nu}\right) \\
& =\frac{1}{2 n} \sum_{j}^{\prime}\left\{\delta\left(a_{j(\nu)}, \Lambda_{\nu}\right)+\delta\left(a_{j(\nu)}, \Lambda_{\nu-1}\right)\right\},
\end{aligned}
$$

where $\sum_{j}^{\prime}$ means that the sum is taken over those $\nu$ for which $j(\nu)=j$, and $\sum_{j}^{\prime \prime}$ means that the sum is taken over those $\nu$ for which $j(\nu+1)=j$. Therefore

$$
\delta\left(a_{j}, F\right)=\frac{1}{2 n} \sum_{j}^{\prime}\left\{\Delta_{\nu} n+\Delta_{\nu} n\right\}=\sum_{j}^{\prime} \Delta_{\nu}=\sum_{s=1}^{n_{j}} \Delta_{j s}=\delta_{j}, 1 \leq j \leq q .
$$

Similarly we get $\varepsilon\left(a_{j}, F\right)=\varepsilon_{j}, 1 \leq j \leq q$.

REMARK. If we considered the "restricted" inverse problem only and did not impose any conditions on $\varepsilon_{j}$ in the statement of Theorem 8.1, it would suffice to restrict our attention to $\alpha$ - and $\beta$-schemes. It is clear that if $\sum_{j=1}^{q} \delta_{j}<2, q \geq 2$, we can always choose non-negative numbers $\varepsilon_{j}$ such that $0<\delta_{j}+\varepsilon_{j} \leq 1,1 \leq j \leq q$; $\sum_{j=1}^{q}\left(\delta_{j}+\varepsilon_{j}\right)=2$, and $\Theta_{j}=\delta_{j}+\varepsilon_{j}$ are rational numbers. We will not have to add schemes, for this reason their weight does not matter for us, and we can disregard the requirement $\varepsilon_{1}>0$. Besides, if we refer to Theorem 6.4, we may restrict our attention by the case $q \geq 3$ and solve the "restricted" inverse problem using $\beta$-schemes only.

$\gamma$-SCHEME is a scheme for which: (1) $\delta_{j}>0$ for $1 \leq j \leq p, 2 \leq p<q$ and $\delta_{j}=0$ for $p+1 \leq j \leq q, 1<p<q$; (2) $2 \Theta_{j} \leq \sum_{j=1}^{p} \Theta_{j}=\Theta$ for $1 \leq j \leq p$; (3) $\varepsilon_{p+1}>0$ :

$$
L\left(\begin{array}{cccccc}
\delta_{1} & \ldots & \delta_{p} & 0 & \ldots & 0 \\
\varepsilon_{1} & \cdots & \varepsilon_{p} & \varepsilon_{p+1} & \ldots & \varepsilon_{q}
\end{array}\right) .
$$

Since $\varepsilon_{p+1}>0$, we have $\Theta<2$. We show that all $\Theta_{j}, 1 \leq j \leq p$, can be represented as sums of $n_{j}$ positive summands $\theta_{j k}$ in such a way that the following conditions are satisfied

$$
\begin{gathered}
\Theta_{j}=\theta_{j 1}+\cdots+\theta_{j n_{j}}, 1 \leq j \leq p, \\
\left|\frac{\theta_{j k}}{\theta_{i l}}-1\right|<2-\Theta, 1 \leq j \leq p, 1 \leq i \leq p, 1 \leq k \leq n_{j}, 1 \leq l \leq n_{j}, \\
2 n_{j} \leq \sum_{j=1}^{p} n_{j}=n .
\end{gathered}
$$

Let $\kappa$ be a positive integer, so large that

$$
\frac{2^{1-\kappa}}{1-2^{-\kappa}}<2-\Theta .
$$


Let

$$
\Theta_{j}=\sum_{k=1}^{\infty} \alpha_{k}^{j} 2^{-k}, \alpha_{k}^{j}=0 \text { or } 1,1 \leq j \leq p
$$

$\lambda$ be a positive integer so large that for each $j, 1 \leq j \leq p$, at least one of the numbers $\alpha_{1}^{j}, \alpha_{2}^{j}, \ldots, \alpha_{\lambda}^{j}$ is equal to 1 . Let

$$
n_{j}^{\prime}=2^{\lambda+\kappa} \sum_{k=1}^{\lambda+\kappa} \alpha_{k}^{j} 2^{-k}
$$

If at least two of the numbers $n_{j}^{\prime}, 1 \leq j \leq p$ attain their maximal values, then $n_{j}=n_{j}^{\prime}, 1 \leq j \leq p$. If there is $j_{0}$ such that $n_{j_{0}}^{\prime}>n_{j}^{\prime}$ for $j \neq j_{0}$, we let $n_{j_{0}}=n_{j}^{\prime}$ and $n_{j}=n_{j}^{\prime}+1$ for $j \neq j_{0}$. It is clear that (8.4) holds in the first case. In the second case the inequality (8.4) follows from the inequality

$$
\Theta_{j_{0}} \leq \sum_{\substack{1 \leq j \leq p \\ j \neq j_{0}}} \Theta_{j}
$$

which holds by the condition (2) in the definition of a $\gamma$-scheme. Now we let $\theta_{j k}=\Theta_{j} / n_{j}, 1 \leq k \leq n_{j}$. It is easy to see that

$$
\begin{aligned}
\frac{\Theta_{j}}{n_{j}} & \leq \frac{\Theta_{j}}{n_{j}^{\prime}}=2^{-\lambda-\kappa}\left(1+\frac{\sum_{k=\lambda+\kappa+1}^{\infty} \alpha_{k}^{j} 2^{-k}}{\sum_{k=1}^{\lambda+\kappa} \alpha_{k}^{j} 2^{-k}}\right) \\
& \leq 2^{-\lambda-\kappa}\left(1+\frac{\sum_{k=\lambda+\kappa+1}^{\infty} 2^{-k}}{2^{-\lambda}}\right)=2^{-\lambda-\kappa}\left(1+2^{-\kappa}\right) .
\end{aligned}
$$

On the other hand,

$$
\frac{\Theta_{j}}{n_{j}} \geq \frac{\Theta_{j}}{n_{j}^{\prime}+1}=\frac{\Theta_{j}}{n_{j}^{\prime}}\left(1-\frac{1}{n_{j}^{\prime}+1}\right) \geq 2^{-\lambda-\kappa}\left(1-\frac{1}{n_{j}^{\prime}}\right) \geq 2^{-\lambda-\kappa}\left(1-2^{-\kappa}\right) .
$$

The inequalities (8.6), (8.7) and (8.5) imply that

$$
\left|\frac{\theta_{j k}}{\theta_{i l}}-1\right| \leq \frac{1+2^{-\kappa}}{1-2^{-\kappa}}-1<2-\Theta
$$

that is, (8.3) holds.

We write $\delta_{j}$ and $\varepsilon_{j}$ in the form

$$
\delta_{j}=\sum_{k=1}^{n_{j}} \Delta_{j k}, \varepsilon_{j}=\sum_{k=1}^{n_{j}} E_{j k}, 1 \leq j \leq p
$$

where

$$
\Delta_{j k}+E_{j k}=\theta_{j k}, \Delta_{j k}>0, E_{j k} \geq 0,1 \leq k \leq n_{j} .
$$

We can always do this in such a way that $\Delta_{11}$ is equal to $r L$, where $r$ is a rational number. To achieve this in the case when $\varepsilon_{1}=0$ we possibly need to change somewhat $\theta_{11}$ and $\theta_{12}$ without changing the sum $\theta_{11}+\theta_{12}$, it is clear that this can be done without violating any of the strict inequalities in (8.3).

We take a surface $F$ with $n$ almost periodic ends $\Lambda_{1}, \ldots, \Lambda_{n}$. We use the notation $\tilde{B}_{\nu}, b_{\nu}, s=s(\nu), j=j(\nu), \Delta_{\nu}, E_{\nu}$ in the same way as in the case of a $\beta$ scheme, but now $1 \leq \nu \leq n$ and indices $\nu$ are taken $\bmod n$ rather than $\bmod 2 n$, and the set of values $\left\{b_{1}, \ldots, b_{n}\right\}$ coincides with $\left\{a_{j}\right\}, 1 \leq j \leq p$. In addition, we 
let $\theta_{\nu}=\theta_{j s}, j=j(\nu), s=s(\nu)$. The possibility to satisfy the condition $b_{\nu} \neq b_{\nu \pm 1}$ and, hence, the possibility to choose all ends to be ends of the first kind follows from (8.4).

We choose a periodic end $\Lambda_{\nu}$ in such a way that the following conditions are satisfied:

$$
\left\{\begin{array}{l}
\delta\left(a_{j(\nu)}, \Delta_{\nu}\right)=d_{\nu}^{+}=\Delta_{\nu} \frac{\Theta}{\theta_{\nu}+\theta_{\nu+1}}, \varepsilon\left(a_{j(\nu)}, \Delta_{\nu}\right)=E_{\nu} \frac{\Theta}{\theta_{\nu}+\theta_{\nu+1}}, \\
\delta\left(a_{j(\nu+1)}, \Lambda_{\nu}\right)=d_{\nu}^{-}=\Delta_{\nu+1} \frac{\Theta}{\theta_{\nu}+\theta_{\nu+1}}, \\
\varepsilon\left(a_{j(\nu+1)}, \Lambda_{\nu}\right)=E_{\nu+1} \frac{\Theta}{\theta_{\nu}+\theta_{\nu+1}}, \\
\delta\left(a, \Lambda_{\nu}\right)=0 \text { for } a \neq a_{j(\nu)}, a_{j(\nu+1)}, \\
\varepsilon\left(a_{j}, \Lambda_{\nu}\right)=\varepsilon_{j}, p+1 \leq j \leq q \\
\varepsilon\left(a, \Lambda_{\nu}\right)=0 \text { for } a \neq a_{j(\nu)}, a_{j(\nu+1)}, a_{p+1}, \ldots, a_{q} .
\end{array}\right.
$$

Such an end can be constructed since by (8.3) we have

$$
\Delta_{\nu} \frac{\Theta}{\theta_{\nu}+\theta_{\nu+1}}+E_{\nu} \frac{\Theta}{\theta_{\nu}+\theta_{\nu+1}}=\theta_{\nu} \frac{\Theta}{\theta_{\nu}+\theta_{\nu+1}}=\frac{\Theta}{1+\frac{\theta_{\nu+1}}{\theta_{\nu}}}<1
$$

$$
\Delta_{\nu+1} \frac{\Theta}{\theta_{\nu}+\theta_{\nu+1}}+E_{\nu+1} \frac{\Theta}{\theta_{\nu}+\theta_{\nu+1}}=\theta_{\nu+1} \frac{\Theta}{\theta_{\nu}+\theta_{\nu+1}}=\frac{\Theta}{1+\frac{\theta_{\nu}}{\theta_{\nu+1}}}<1
$$

and also

$$
\theta_{\nu} \frac{\Theta}{\theta_{\nu}+\theta_{\nu+1}}+\theta_{\nu+1} \frac{\Theta}{\theta_{\nu}+\theta_{\nu+1}}+\varepsilon_{p+1}+\cdots+\varepsilon_{q}=\Theta+(2-\Theta)=2
$$

The weight of the surface $F$ is equal to

$$
\begin{gathered}
\frac{1}{\Delta_{1} \frac{\Theta}{\theta_{1}+\theta_{2}}}+\frac{\Delta_{2} \frac{\Theta}{\theta_{1}+\theta_{2}}}{\Delta_{1} \frac{\Theta}{\theta_{1}+\theta_{2}} \Delta_{2} \frac{\Theta}{\theta_{2}+\theta_{3}}}+\cdots+\frac{\Delta_{2} \frac{\Theta}{\theta_{1}+\theta_{2}} \ldots \Delta_{n} \frac{\Theta}{\theta_{n-1}+\theta_{n}}}{\Delta_{1} \frac{\Theta}{\theta_{1}+\theta_{2}} \ldots \Delta_{n} \frac{\Theta}{\theta_{n}+\theta_{1}}} \\
=\frac{1}{\Delta_{1}} \frac{\sum_{j=1}^{n}\left(\theta_{j}+\theta_{j+1}\right)}{\Theta}=\frac{2}{\Delta_{1}}=\frac{2}{\Delta_{11}}=\frac{2}{r} L .
\end{gathered}
$$


Using the notation $\sum_{j}^{\prime}, \sum_{j}^{\prime \prime}$ in the same way as in the solution of a $\beta$-scheme, by (7.21) we get

$$
\begin{aligned}
\delta\left(a_{j}, F\right) & =\frac{\Delta_{1}}{2} \sum_{\nu=1}^{n} \frac{\theta_{\nu}+\theta_{\nu+1}}{\Delta_{1} \Theta} \delta\left(a_{j}, \Lambda_{\nu}\right) \\
& =\frac{1}{2 \Theta}\left\{\sum_{j}^{\prime}\left(\theta_{\nu}+\theta_{\nu+1}\right) \delta\left(a_{j(\nu)}, \Lambda_{\nu}\right)+\sum_{j}^{\prime \prime}\left(\theta_{\nu}+\theta_{\nu+1}\right) \delta\left(a_{j(\nu+1)}, \Lambda_{\nu}\right)\right\} \\
& =\frac{1}{2 \Theta} \sum_{j}^{\prime}\left\{\left(\theta_{\nu}+\theta_{\nu+1}\right) \delta\left(a_{j(\nu)}, \Lambda_{\nu}\right)+\left(\theta_{\nu-1}+\theta_{\nu}\right) \delta\left(a_{j(\nu)}, \Lambda_{\nu-1}\right)\right\} \\
& =\frac{1}{2 \Theta} \sum_{j}^{\prime}\left\{\left(\theta_{\nu}+\theta_{\nu+1}\right) \Delta_{\nu} \frac{\Theta}{\theta_{\nu}+\theta_{\nu+1}}+\left(\theta_{\nu-1}+\theta_{\nu}\right) \Delta_{\nu} \frac{\Theta}{\theta_{\nu-1}+\theta_{\nu}}\right\} \\
& =\sum_{j}^{\prime} \Delta_{\nu}=\sum_{s=1}^{n_{j}} \Delta_{j s}=\delta_{j}, 1 \leq j \leq p .
\end{aligned}
$$

Similarly we get that $\varepsilon\left(a_{j}, F\right)=\varepsilon_{j}, 1 \leq j \leq p$. For $p+1 \leq j \leq q$ the equality $\varepsilon\left(a_{j}, F\right)=\varepsilon_{j}$ is obvious.

A $\delta$-SCHEME is a scheme satisfying the conditions (1) $\delta_{j}>0$ for $1 \leq j \leq p$, $2 \leq p<q$, and $\delta_{j}=0$ for $p+1 \leq j \leq q ;\left(2^{\prime}\right) \Theta=\sum_{j=1}^{p} \Theta_{j} \leq 1 ;(3) \varepsilon_{p+1}>0$.

As we see the only difference between the conditions for a $\delta$-scheme and for a $\gamma$-scheme is that the condition (2) is replaced by the condition $\left(2^{\prime}\right)$. The scheme is solved almost in the same way as a $\gamma$-scheme, for this reason we restrict ourselves to a description of differences between solutions of $\gamma$ - and $\delta$-schemes. We used the condition (2) in the proof of the inequalities (8.4) (8.9) and (8.10). But the inequalities (8.9) and (8.10) for a $\delta$-scheme are obviously satisfied by the condition $\left(2^{\prime}\right)$.

The condition (8.4) is not satisfied in general, for this reason we cannot use ends of the first kind only. If an end $\Lambda_{\nu}$ is of the first kind, the formulas (8.8) remain true. If the end $\Lambda_{\nu}$ is of the second kind, that is, $a_{j(\nu)}=a_{j(\nu+1)}$, we choose it in such a way that

$$
\begin{gathered}
\delta\left(a_{j(\nu)}, \Lambda_{\nu}\right)=\left(\Delta_{\nu}+\Delta_{\nu+1}\right) \frac{\Theta}{\theta_{\nu}+\theta_{\nu+1}}, \\
d_{\nu}^{+}=\Delta_{\nu} \frac{\Theta}{\theta_{\nu}+\theta_{\nu+1}}, d_{\nu}^{-}=\Delta_{\nu+1} \frac{\Theta}{\theta_{\nu}+\theta_{\nu+1}}, \\
\delta\left(a, \Lambda_{\nu}\right)=0 \text { for } a \neq a_{j(\nu)}, \\
\varepsilon\left(a_{j(\nu)}, \Lambda_{\nu}\right)=\left(E_{\nu}+E_{\nu+1}\right) \frac{\Theta}{\theta_{\nu}+\theta_{\nu+1}} \\
\varepsilon\left(a_{j}, \Lambda_{\nu}\right)=\varepsilon_{j}, p+1 \leq j \leq q, \\
\varepsilon\left(a, \Lambda_{\nu}\right)=0 \text { for } a \neq a_{j(\nu)}, a_{p+1}, \ldots, a_{q} .
\end{gathered}
$$


Such an end can be chosen because

$$
\delta\left(a_{j(\nu)}, \Lambda_{\nu}\right)+\varepsilon\left(a_{j(\nu)}, \Lambda_{\nu}\right)=\left(\Delta_{\nu}+\Delta_{\nu+1}+E_{\nu}+E_{\nu+1}\right) \frac{\Theta}{\theta_{\nu}+\theta_{\nu+1}}=\Theta \leq 1
$$

by the condition $\left(2^{\prime}\right)$. The remaining computations are carried out in the same way as for a $\gamma$-scheme.

Observe that in the solution of a $\delta$-scheme we do not need to obtain (8.3), therefore we can take $\Theta_{1}=\theta_{11}+\theta_{12}, \Theta_{j}=\theta_{j 1}, 2 \leq j \leq p$, that is, $n_{1}=2$, $n_{2}=\cdots=n_{p}=1$. With this choice of $n_{j}$ the condition (8.4) is not satisfied for $p=2$ only, and only in this case it is necessary to use an end of the second kind. Although for $p=2$ we can solve a $\delta$-scheme with the help of a surface $\Phi$ with two almost periodic ends of the first kind (see Theorem 6.2), but, generally speaking, we will not be able to ensure the preassigned, up to a rational factor, weight of our scheme.

It is easy to see that a scheme can be a $\gamma$-scheme and a $\delta$-scheme simultaneously.

Now we consider schemes of the general form. Let $\delta\left(a_{j}\right)>0$ for $1 \leq j \leq p$, $p \leq q$, and $\delta\left(a_{j}\right)=0$ for $p+1 \leq j \leq q$ (if $p<q$ ). We may assume that $p \geq 2$, since for $p=1$ we get an $\alpha$-scheme. Set $\Theta=\sum_{j=1}^{p} \Theta_{j}$. We may assume without loss of generality that $\Theta_{1}=\max _{1 \leq j \leq p} \Theta_{j}$. We consider two cases: Case A: $\Theta<2$; Case $\mathrm{B}: \Theta=2$. We divide the case $\mathrm{A}$ into three subcases: (1) $\mathrm{A}^{\prime}$, if $\delta_{1}=1,(2) \mathrm{A}^{\prime \prime}$, if $\Theta_{1}=1$ and $\varepsilon_{1}>0,(3) \mathrm{A}^{\prime \prime \prime}$, if $\Theta_{1}<1$.

Subcase A'. Set

$$
\Theta^{\prime}=\sum_{j=2}^{p} \Theta_{j}, \Theta^{\prime \prime}=\sum_{j=p+1}^{q} \varepsilon_{j} .
$$

It is clear that $\Theta^{\prime}<1$ and $\Theta^{\prime \prime}>0$. We write $\Theta_{j}, 2 \leq j \leq p$, as a sum of $n_{j}$ positive summands $\theta_{j k}$ (see (8.2)) in such a way that

$$
\left|\frac{\theta_{j k}}{\theta_{i l}}-1\right|<2-2 \Theta^{\prime}, 2 \leq j \leq p, 2 \leq i \leq p, 1 \leq k \leq n_{j}, 1 \leq l \leq n_{i} .
$$

The possibility of such representation can be proved similarly to the proof of representation (8.2) under the condition (8.3). Now we write $\delta_{j}$ and $\varepsilon_{j}$ in the form

$$
\delta_{j}=\sum_{k=1}^{n_{j}} \Delta_{j k}, \varepsilon_{j}=\sum_{k=1}^{n_{j}} E_{j k}, 2 \leq j \leq p,
$$

where

$$
\Delta_{j k}+E_{j k}=\theta_{j k}, \Delta_{j k}>0, E_{j k} \geq 0,1 \leq k \leq n_{j}, 2 \leq j \leq p .
$$

We take a surface $F$ with $2 n=2 \sum_{j=2}^{p} n_{j}$ almost periodic ends $\Lambda_{1}, \ldots, \Lambda_{2 n}$ and with logarithmic ramification points $\tilde{B}_{1}, \ldots, \tilde{B}_{2 n}$. The points $\tilde{B}_{2 \nu}, 1 \leq \nu \leq n$ are projected onto $a_{1}$, and there are $n_{j}$ logarithmic ramification points lying over $a_{j}$, $2 \leq j \leq p$. Suppose that $\tilde{B}_{2 \nu-1}, 1 \leq \nu \leq n$, is the logarithmic ramification point number $s$ among points projected into $a_{j}, s=s(\nu), j=j(\nu)$. Let $\Delta_{\nu}=\Delta_{j s}$, 
$E_{\nu}=E_{j s}, \theta_{\nu}=\theta_{j s}, j=j(\nu), s=s(\nu)$. We take all numbers $\nu \bmod n$. We take ends $\Lambda_{k}$ of the first kind in such a way, that for $\Lambda_{2 \nu-1}$ the following is true:

$$
\begin{gathered}
\delta\left(a_{1}, \Lambda_{2 \nu-1}\right)=d_{2 \nu-1}^{-}=1, \\
\delta\left(a_{j(\nu)}, \Lambda_{2 \nu-1}\right)=d_{2 \nu-1}^{+}=\Delta_{\nu} \frac{2 \Theta^{\prime}}{\theta_{\nu}+\theta_{\nu+1}}, \\
\delta\left(a, \Lambda_{2 \nu-1}\right)=0 \text { for } a \neq a_{1}, a_{j(\nu)}, \\
\varepsilon\left(a_{j(\nu)}, \Lambda_{2 \nu-1}\right)=E_{\nu} \frac{2 \Theta^{\prime}}{\theta_{\nu}+\theta_{\nu+1}}, \\
\varepsilon\left(a_{j}, \Lambda_{2 \nu-1}\right)=k_{\nu} \varepsilon_{j}, p+1 \leq j \leq q,
\end{gathered}
$$

where

$$
\begin{aligned}
k_{\nu} & =\frac{1}{\Theta^{\prime \prime}}\left(1-\frac{2 \theta_{\nu} \Theta^{\prime}}{\theta_{\nu}+\theta_{\nu+1}}\right), \\
\varepsilon\left(a, \Lambda_{2 \nu-1}\right) & =0 \text { for } a \neq a_{j(\nu)}, a_{p+1}, \ldots, a_{q},
\end{aligned}
$$

and for $\Lambda_{2 \nu}$ the following is true:

$$
\begin{gathered}
\delta\left(a_{1}, \Lambda_{2 \nu}\right)=d_{2 \nu}^{+}=1, \\
\delta\left(a_{j(\nu+1)}, \Lambda_{2 \nu}\right)=d_{2 \nu}^{-}=\Delta_{\nu+1} \frac{2 \Theta^{\prime}}{\theta_{\nu}+\theta_{\nu+1}}, \\
\delta\left(a, \Lambda_{2 \nu}\right)=0 \text { for } a \neq a_{1}, a_{j(\nu+1)}, \\
\varepsilon\left(a_{j(\nu+1)}, \Lambda_{2 \nu}\right)=E_{\nu+1} \frac{2 \Theta^{\prime}}{\theta_{\nu}+\theta_{\nu+1}}, \\
\varepsilon\left(a_{j}, \Lambda_{2 \nu}\right)=l_{\nu} \varepsilon_{j}, p+1 \leq j \leq q,
\end{gathered}
$$

where

$$
\begin{gathered}
l_{\nu}=\frac{1}{\Theta^{\prime \prime}}\left(1-\frac{2 \theta_{\nu+1} \Theta^{\prime}}{\theta_{\nu}+\theta_{\nu+1}}\right), \\
\varepsilon\left(a, \Lambda_{2 \nu}\right)=0 \text { for } a \neq a_{j(\nu+1)}, a_{p+1}, \ldots, a_{q} .
\end{gathered}
$$

Let us show that such almost periodic ends can be constructed. In fact, the inequalities (8.11) imply that

$$
\frac{2 \theta_{\nu} \Theta^{\prime}}{\theta_{\nu}+\theta_{\nu+1}}=\frac{2 \Theta^{\prime}}{1+\frac{\theta_{\nu+1}}{\theta_{\nu}}}<\frac{2 \Theta^{\prime}}{1+\left(-1+2 \Theta^{\prime}\right)}=1
$$

and similarly

$$
\frac{2 \theta_{\nu+1} \Theta^{\prime}}{\theta_{\nu}+\theta_{\nu+1}}<1
$$

hence the numbers $k_{\nu}$ and $l_{\nu}$ are positive, and $\delta\left(a_{j(\nu)}, \Lambda_{2 \nu-1}\right)+\varepsilon\left(a_{j(\nu)}, \Lambda_{2 \nu-1}\right)<1$, $\delta\left(a_{j(\nu+1)}, \Lambda_{2 \nu}\right)+\varepsilon\left(a_{j(\nu+1)}, \Lambda_{2 \nu}\right)<1$. Besides, for $p+1 \leq j \leq q$ we have

$$
k_{\nu} \varepsilon_{j} \leq k_{\nu} \Theta^{\prime \prime}<1 \text { and } l_{\nu} \varepsilon_{j} \leq l_{\nu} \Theta^{\prime \prime}<1 .
$$

Thus, by Theorem 7.2 we can construct a Riemann surface $F$ with the indicated distribution of values of almost periodic ends. 
Since $\delta\left(a_{1}, \Lambda_{k}\right)=1$ for all $\Lambda_{k}$, we have $\delta\left(a_{1}, F\right)=1^{14}$.

Now let $2 \leq j \leq p$. Using the notation $\sum_{j}^{\prime}$ and $\sum_{j}^{\prime \prime}$ in the same way as before, we get

$$
\begin{gathered}
\delta\left(a_{j}, F\right)=\frac{\sum_{j}^{\prime} \frac{d_{1}^{-} \ldots d_{2 \nu-2}^{-}}{d_{1}^{+} \ldots d_{2 \nu-1}^{+}} \delta\left(a_{j(\nu)}, \Lambda_{2 \nu-1}\right)+\sum_{j}^{\prime \prime} \frac{d_{1}^{-} \ldots d_{2 \nu-1}^{-}}{d_{1}^{+} \ldots d_{2 \nu}^{+}} \delta\left(a_{j(\nu+1)}, \Lambda_{2 \nu}\right)}{\sum_{k=1}^{2 n} \frac{d_{1}^{-} \ldots d_{k-1}^{-}}{d_{1}^{+} \ldots d_{k}^{+}}} \\
=\frac{2 \sum_{j}^{\prime} \frac{d_{1}^{-} \ldots d_{2 \nu-2}^{-}}{d_{1}^{+} \ldots d_{2 \nu-1}^{+}} \delta\left(a_{j(\nu)}, \Lambda_{2 \nu-1}\right)}{\sum_{k=1}^{2 n} \frac{d_{1}^{-} \ldots d_{k-1}^{-}}{d_{1}^{+} \ldots d_{k}^{+}}}
\end{gathered}
$$

It is easy to see that

$$
\frac{d_{1}^{-} \ldots d_{k-1}^{-}}{d_{1}^{+} \ldots d_{k}^{+}}=\frac{\theta_{l}+\theta_{l+1}}{2 \Theta^{\prime} \Delta_{1}}, k=2 l-1,2 l(1 \leq l \leq n)
$$

Therefore

$$
\begin{aligned}
& L(F)=\sum_{k=1}^{2 n} \frac{d_{1}^{-} \ldots d_{k-1}^{-}}{d_{1}^{+} \ldots d_{k}^{+}}=2 \sum_{l=1}^{n} \frac{\theta_{l}+\theta_{l+1}}{2 \Theta^{\prime} \Delta_{1}}=\frac{1}{\Theta^{\prime} \Delta_{1}} \sum_{l=1}^{n}\left(\theta_{l}+\theta_{l+1}\right)=\frac{2}{\Delta_{1}} \\
& \sum_{j}^{\prime} \frac{d_{1}^{-} \ldots d_{2 \nu-2}^{-}}{d_{1}^{+} \ldots d_{2 \nu-1}^{+}} \delta\left(a_{j(\nu)}, \Lambda_{2 \nu-1}\right) \\
& =\sum_{j}^{\prime} \frac{\theta_{\nu}+\theta_{\nu+1}}{2 \Theta^{\prime} \Delta_{1}} \Delta_{\nu} \frac{2 \Theta^{\prime}}{\theta_{\nu}+\theta_{\nu+1}}=\frac{1}{\Delta_{1}} \sum_{j}^{\prime} \Delta_{\nu}=\frac{1}{\Delta_{1}} \sum_{s=1}^{n_{j}} \Delta_{j s}=\frac{\delta_{j}}{\Delta_{1}} .
\end{aligned}
$$

¿From (8.12), (8.13), and (8.14) we get that $\delta\left(a_{j}, F\right)=\delta_{j}$. Similarly we find that $\varepsilon\left(a_{j}, F\right)=\varepsilon_{j}, 2 \leq j \leq p$.

For $p+1 \leq j \leq q$, taking into account (7.22) and the equality $k_{\nu}+l_{\nu}=$ $\frac{2}{\Theta^{\prime \prime}}\left(1-\Theta^{\prime}\right)=2$, we find that

$$
\begin{aligned}
\varepsilon\left(a_{j}, F\right) & =\frac{\Delta_{1}}{2}\left\{\sum_{\nu=1}^{n} \frac{d_{1}^{-} \ldots d_{2 \nu-2}^{-}}{d_{1}^{+} \ldots d_{2 \nu-1}^{+}} k_{\nu} \varepsilon_{j}+\sum_{\nu=1}^{n} \frac{d_{1}^{-} \ldots d_{2 \nu-1}^{-}}{d_{1}^{+} \ldots d_{2 \nu}^{+}} l_{\nu} \varepsilon_{j}\right\} \\
& =\frac{\Delta_{1}}{2} \varepsilon_{j}\left\{\sum_{\nu=1}^{n} \frac{\theta_{\nu}+\theta_{\nu+1}}{2 \Theta^{\prime} \Delta_{1}} k_{\nu}+\sum_{\nu=1}^{n} \frac{\theta_{\nu}+\theta_{\nu+1}}{2 \Theta^{\prime} \Delta_{1}} l_{\nu}\right\} \\
& =\frac{\varepsilon_{j}}{4 \Theta^{\prime}} \sum_{\nu=1}^{n}\left(\theta_{\nu}+\theta_{\nu+1}\right)\left(k_{\nu}+l_{\nu}\right)=\frac{\varepsilon_{j}}{2 \Theta^{\prime}} \sum_{\nu=1}^{n}\left(\theta_{\nu}+\theta_{\nu+1}\right)=\varepsilon_{j} .
\end{aligned}
$$

Thus, the constructed surface $F$ has all desired properties.

${ }^{14}$ Obviously, the surface $F$ can be constructed in such a way that it does not cover the point $a_{1}$, in such a case the value $a_{1}$ is a Picard exceptional value for the mapping function $f(z)$. 
Subcases $A^{\prime \prime}$ AND $A^{\prime \prime \prime}$. The corresponding schemes are solved in the following way. After finitely many operations of the form

$$
\begin{aligned}
& \left(\delta_{\nu}+\varepsilon_{\nu}\right)\left(\begin{array}{cccc}
\delta_{1} & \ldots & \delta_{\nu} & \ldots \\
\ldots & \ldots & \varepsilon_{\nu} & \ldots
\end{array}\right) \\
& =\delta_{\nu}\left(\begin{array}{cccc}
\delta_{1} & \ldots & \delta_{\nu}+\varepsilon_{\nu} & \ldots \\
\ldots & \ldots & 0 & \ldots
\end{array}\right)+\varepsilon_{\nu}\left(\begin{array}{cccc}
\delta_{1} & \ldots & 0 & \ldots \\
\ldots & \ldots & \delta_{\nu}+\varepsilon_{\nu} & \ldots
\end{array}\right),
\end{aligned}
$$

where $\delta_{1}>0$, the problem is reduced to a solution with weight of schemes of the form

$$
\left(\begin{array}{cccc}
\delta_{1} & 0 & 0 & \ldots \\
\varepsilon_{1} & \varepsilon_{2} & \varepsilon_{3} & \ldots
\end{array}\right),\left(\begin{array}{ccccccc}
\delta_{1} & \delta_{2} & \ldots & \delta_{n} & 0 & \ldots & 0 \\
\varepsilon_{1} & 0 & \ldots & 0 & \varepsilon_{n+1} & \ldots & \varepsilon_{q}
\end{array}\right),
$$

where $\delta_{1}, \ldots, \delta_{n}>0$, moreover, the numbers $\delta_{j}, \varepsilon_{j}, 2 \leq j \leq q$ in these schemes are, generally speaking, different from the original, also the numbers $a_{j}, 2 \leq j \leq q$ could be subjected to a permutation. The first of these schemes is an $\alpha$-scheme. The second scheme is either a $\gamma$-scheme, or it satisfies the inequality $2 \Theta_{1}>\sum_{j=1}^{n} \Theta_{j}$ (remind that for the original scheme we assumed that $\Theta_{1} \geq \Theta_{j}, 2 \leq j \leq p$, and in decompositions of the form (8.15) the quantities $\Theta_{j}$ do not change). In the latter case, taking into account $\sum_{j=2}^{n} \Theta_{j}<\Theta_{1} \leq 1$, by a sequence of operations of the following type

$$
\begin{array}{r}
\left(\delta_{n-1}+\delta_{n}\right)\left(\begin{array}{cccccc}
\delta_{1} & \ldots & \delta_{n-1} & \delta_{n} & \ldots & 0 \\
\varepsilon_{1} & \ldots & 0 & 0 & \ldots & \varepsilon_{q}
\end{array}\right) \\
=\delta_{n-1}\left(\begin{array}{cccccc}
\delta_{1} & \ldots & \delta_{n-1}+\delta_{n} & 0 & \ldots & 0 \\
\varepsilon_{1} & \ldots & 0 & 0 & \ldots & \varepsilon_{q}
\end{array}\right) \\
+\delta_{n}\left(\begin{array}{cccccc}
\delta_{1} & \ldots & 0 & \delta_{n-1}+\delta_{n} & \ldots & 0 \\
\varepsilon_{1} & \ldots & 0 & 0 & \ldots & \varepsilon_{q}
\end{array}\right)
\end{array}
$$

we reduce the problem to a solution with weight of a scheme of the form

$$
\left(\begin{array}{ccccc}
\delta_{1} & \delta_{2} & 0 & \ldots & 0 \\
\varepsilon_{1} & 0 & \varepsilon_{3} & \ldots & \varepsilon_{q}
\end{array}\right)
$$

where $\delta_{2}=\Theta_{2}<\delta_{1}+\varepsilon_{1}+\Theta_{1}$. If we needed just to solve this scheme, we would be able to refer to Theorem 6.4, however, we need to solve this scheme with weight. Set $\Theta^{\prime \prime}=\sum_{j=3}^{q} \varepsilon_{j}$. If $\Theta^{\prime \prime} \geq 1$, then $\Theta_{1}+\Theta_{2} \leq 1$, and our scheme is a $\delta$-scheme. Further consideration is needed in the case $\Theta^{\prime \prime}<1$.

In the subcase $\mathrm{A}^{\prime \prime}$ the equalities $\delta_{1}+\varepsilon_{1}=1, \delta_{2}+\Theta^{\prime \prime}=1$ hold, and we decompose

$$
\begin{aligned}
& 1\left(\begin{array}{ccccc}
\delta_{1} & \delta_{2} & 0 & \ldots & 0 \\
\varepsilon_{1} & 0 & \varepsilon_{3} & \ldots & \varepsilon_{q}
\end{array}\right) \\
& \quad=\delta_{2}\left(\begin{array}{ccccc}
\delta_{1} & 1 & 0 & \ldots & 0 \\
\varepsilon_{1} & 0 & 0 & \ldots & 0
\end{array}\right)+\Theta^{\prime \prime}\left(\begin{array}{ccccc}
\delta_{1} & 0 & 0 & \ldots & 0 \\
\varepsilon_{1} & 0 & \frac{\varepsilon_{3}}{\Theta^{\prime \prime}} & \ldots & \frac{\varepsilon_{q}}{\Theta^{\prime \prime}}
\end{array}\right) .
\end{aligned}
$$

The first scheme in the right-hand side of (8.16) is a $\beta$-scheme, the second is an $\alpha$-scheme. 
In the subcase $\mathrm{A}^{\prime \prime \prime}$ we observe that $0<1-\Theta^{\prime \prime}=\Theta_{1}+\delta_{2}-1<2 \Theta_{1}-1$. Then

$$
\begin{aligned}
\left(2 \Theta_{1}-1\right) & \left(\begin{array}{ccccc}
\delta_{1} & \delta_{2} & 0 & \ldots & 0 \\
\varepsilon_{1} & 0 & \varepsilon_{3} & \ldots & \varepsilon_{q}
\end{array}\right) \\
= & \left(\Theta_{1}+\delta_{2}-1\right)\left(\begin{array}{ccccc}
\delta_{1} & \Theta_{1} & 0 & \ldots & 0 \\
\varepsilon_{1} & 0 & \varepsilon_{3} \frac{2\left(1-\Theta_{1}\right)}{\Theta^{\prime \prime}} & \ldots & \varepsilon_{q} \frac{2\left(1-\Theta_{1}\right)}{\Theta^{\prime \prime}}
\end{array}\right) \\
& +\left(\Theta_{1}-\delta_{2}\right)\left(\begin{array}{ccccc}
\delta_{1} & 1-\Theta_{1} & 0 & \ldots & 0 \\
\varepsilon_{1} & 0 & \varepsilon_{3} / \Theta^{\prime \prime} & \ldots & \varepsilon_{q} / \Theta^{\prime \prime}
\end{array}\right) .
\end{aligned}
$$

Here we take into account that

$$
\left(2 \Theta_{1}-1\right) \varepsilon_{j}=\left(\Theta_{1}+\delta_{2}-1\right) \varepsilon_{j} \frac{2\left(1-\Theta_{1}\right)}{\Theta^{\prime \prime}}+\left(\Theta_{1}-\delta_{2}\right) \frac{\varepsilon_{j}}{\Theta^{\prime \prime}}, 3 \leq j \leq q,
$$

by the equality $\left(\Theta_{1}+\delta_{2}-1\right) 2\left(1-\Theta_{1}\right)+\left(\Theta_{1}-\delta_{2}\right)=\left(1-\Theta^{\prime \prime}\right) 2\left(1-\Theta_{1}\right)+\left(\Theta^{\prime \prime}+2 \Theta_{1}-2\right)=$ $\Theta^{\prime \prime}\left(2 \Theta_{1}-1\right)$. The first scheme in the right-hand side of the equality (8.17) is a $\gamma$-scheme, the second is a $\delta$-scheme.

CASE B. We may assume that $\varepsilon_{1}>0$, but $\Theta_{1}$ is not necessarily the largest number among $\Theta_{j}, j=1, \ldots, q$. The scheme is of the form

$$
\left(\begin{array}{lll}
\delta_{1} & \ldots & \delta_{q} \\
\varepsilon_{1} & \cdots & \varepsilon_{q}
\end{array}\right), \delta_{j}>0,1 \leq j \leq q, \varepsilon_{1}>0 .
$$

Let $\Theta_{1}<1$. We choose rational numbers $r_{j}, 1 \leq j \leq q$, such that (a) $0<r_{1}<$ $\Theta_{1}<1$; (b) $1 \geq r_{j} \geq \Theta_{j}$ for $2 \leq j \leq q$; (c) $\sum_{j=1}^{q} r_{j}=2 ;$ (d) $r_{j}-\Theta_{j}<\Theta_{j} \frac{\Theta_{1}-r_{1}}{2\left(1-\Theta_{1}\right)}$ for $2 \leq j \leq q$. The fact that $r_{j}$ can be chosen in such a way that (a), (b), and (c) are satisfied, is obvious. The fact that the condition (d) also can be satisfied follows from the inequality

$$
\sum_{j=2}^{q} \Theta_{j} \frac{\Theta_{1}-r_{1}}{2\left(1-\Theta_{1}\right)}=\frac{2-\Theta_{1}}{2-2 \Theta_{1}}\left(\Theta_{1}-r_{1}\right)>\Theta_{1}-r_{1}=\sum_{j=2}^{q}\left(r_{j}-\Theta_{j}\right) .
$$

We decompose the scheme (8.18) in the following way:

$$
\begin{aligned}
\left(1-r_{1}\right)\left(\begin{array}{ccc}
\delta_{1} & \ldots & \delta_{q} \\
\varepsilon_{1} & \ldots & \varepsilon_{q}
\end{array}\right)= & \left(1-\Theta_{1}\right)\left(\begin{array}{ccc}
\frac{\delta_{1}}{\Theta_{1}} r_{1} & \ldots & \frac{\delta_{q}}{\Theta_{q}} r_{q} \\
\frac{\varepsilon_{1}}{\Theta_{1}} r_{1} & \ldots & \frac{\varepsilon_{q}}{\Theta_{q}} r_{q}
\end{array}\right) \\
& +\left(\Theta_{1}-r_{1}\right)\left(\begin{array}{cccc}
\delta_{1}^{\prime} & \delta_{2}^{\prime} & \ldots & \delta_{q}^{\prime} \\
\varepsilon_{1}^{\prime} & \varepsilon_{2}^{\prime} & \ldots & \varepsilon_{q}^{\prime}
\end{array}\right),
\end{aligned}
$$

where $\delta_{1}^{\prime}=\delta_{1} / \Theta_{1}, \varepsilon_{1}^{\prime}=\varepsilon_{1} / \Theta_{1}$, and for $2 \leq j \leq q$ we have

$$
\begin{aligned}
& \delta_{j}^{\prime}=\frac{\delta_{j}}{\Theta_{j}}\left\{\Theta_{j}-\frac{1-\Theta_{1}}{\Theta_{1}-r_{1}}\left(r_{j}-\Theta_{j}\right)\right\}>\frac{\delta_{j}}{2}>0, \\
& \varepsilon_{j}^{\prime}=\frac{\varepsilon_{j}}{\Theta_{j}}\left\{\Theta_{j}-\frac{1-\Theta_{1}}{\Theta_{1}-r_{1}}\left(r_{j}-\Theta_{j}\right)\right\}>\frac{\varepsilon_{j}}{2} \geq 0,
\end{aligned}
$$

by the condition (d). The first scheme in the right-hand side of (8.19) is a $\beta$-scheme, and $\Theta_{1}^{\prime}=\delta_{1}^{\prime}+\varepsilon_{1}^{\prime}=1$ in the second scheme. Therefore we may assume that $\Theta_{1}=1$ in (8.18). 
Let $\varepsilon_{\nu}>0,2 \leq \nu \leq q$. We represent the scheme (8.18) as a sum of two schemes:

$$
\begin{aligned}
& \left(\delta_{\nu}+\varepsilon_{\nu}\right)\left(\begin{array}{ccccc}
\delta_{1} & \ldots & \delta_{\nu} & \ldots & \delta_{q} \\
\varepsilon_{1} & \ldots & \varepsilon_{\nu} & \ldots & \ldots
\end{array}\right) \\
& =\delta_{\nu}\left(\begin{array}{ccccc}
\delta_{1} & \ldots & \delta_{\nu}+\varepsilon_{\nu} & \ldots & \delta_{q} \\
\varepsilon_{1} & \ldots & 0 & \ldots & \ldots
\end{array}\right)+\varepsilon_{\nu}\left(\begin{array}{cccc}
\delta_{1} & \ldots & 0 & \ldots \\
\varepsilon_{1} & \ldots & \delta_{\nu}+\varepsilon_{\nu} & \ldots
\end{array}\right) .
\end{aligned}
$$

The second scheme $(S)$ in the right-hand side of the equality (8.19) corresponds to the case $\mathrm{A}^{\prime \prime}$. As we saw, it is possible to solve the scheme $(S)$ constructing a surface $F$ with weight $L$. The scheme $(S)$ can be represented as a finite sum of basic schemes $\left(S_{1}\right), \ldots,\left(S_{t}\right)$ with weights $L_{1}, \ldots, L_{t}$, respectively, where, by $(7.25)$, $L=L_{1}+\cdots+L_{t}$. We shall write this junction in the form of the following equality: $L(S)=L_{1}\left(S_{1}\right)+\cdots+L_{t}\left(S_{t}\right)$. However, we need to get a scheme $(S)$ with weight equal to $\varepsilon_{\nu}$, up to a rational factor. We do the following. We solve a basic scheme $\left(S_{j}\right)$ with weight equal to $r_{j} \varepsilon_{\nu} L_{j} / L, 1 \leq j \leq t$, where $r_{j}$ is a rational number, and denote the corresponding surface by $F_{j}$. Let $r_{j}=m_{j} / n$, where $n$ is the least common denominator of the fractions $r_{1}, \ldots, r_{t}$. Now we join $m_{2} m_{3} \ldots m_{t}$ copies of the surface $F_{1}, m_{1} m_{3} \ldots m_{t}$ copies of the surface $F_{2}, \ldots, m_{1} m_{2} \ldots m_{t-1}$ copies of the surface $F_{t}$. By $(7.25)$, the weight of the obtained surface $F^{\prime}$ is equal to

$$
\begin{aligned}
m_{2} m_{3} \ldots m_{t} \frac{r_{1} \varepsilon_{\nu} L_{1}}{L} & +m_{1} m_{3} \ldots m_{t} \frac{r_{2} \varepsilon_{\nu} L_{2}}{L}+\ldots \\
+m_{1} m_{2} \ldots m_{t-1} \frac{r_{t} \varepsilon_{\nu} L_{t}}{L} & =m_{1} m_{2} \ldots m_{t} \frac{\varepsilon_{\nu}}{n L}\left(L_{1}+\ldots+L_{t}\right) \\
& =\frac{m_{1} \ldots m_{t}}{n} \varepsilon_{\nu}
\end{aligned}
$$

that is, equal to a rational multiple of $\varepsilon_{\nu}$.

Using the formulas (7.26) and (7.27) it is easy to see that the surface $F^{\prime}$ solves the scheme $(S)$, that is, the second scheme in the right-hand side of $(8.20)$, with the desired weight.

As for the first scheme in the right-hand side of (8.20), we decompose it again according to the formula (8.20), but now with another $\nu$. Repeating this operation finitely many times we get, besides schemes corresponding to the case $\mathrm{A}^{\prime \prime}$, a scheme of the form (8.18) with $\varepsilon_{2}=\cdots=\varepsilon_{q}=0$. Next, using successive operations

$$
\begin{aligned}
& \left(\delta_{n-1}+\delta_{n}\right)\left(\begin{array}{ccccc}
\delta_{1} & \ldots & \delta_{n-1} & \delta_{n} & \ldots \\
\varepsilon_{1} & \ldots & 0 & 0 & \ldots
\end{array}\right) \\
& =\delta_{n-1}\left(\begin{array}{ccccc}
\delta_{1} & \ldots & \delta_{n-1}+\delta_{n} & 0 & \ldots \\
\varepsilon_{1} & \ldots & 0 & 0 & \ldots
\end{array}\right)+\delta_{n}\left(\begin{array}{ccccc}
\delta_{1} & \ldots & 0 & \delta_{n-1}+\delta_{n} & \ldots \\
\varepsilon_{1} & \ldots & 0 & 0 & \ldots
\end{array}\right)
\end{aligned}
$$

we reduce the scheme to schemes of the form

$$
\left(\begin{array}{ccccccc}
\delta_{1} & 0 & \ldots & 0 & 1 & 0 & \ldots \\
\varepsilon_{1} & 0 & \ldots & 0 & 0 & 0 & \ldots
\end{array}\right)
$$

which are $\beta$-schemes.

This completes the proof of Theorem 8.1.

REMARK. We can drop the restriction that there are finitely many numbers $\varepsilon_{j}$ and consider the case of infinite sequences $\varepsilon_{1}, \varepsilon_{2}, \ldots, 0 \leq \varepsilon_{j} \leq 1$ and $a_{1}, a_{2}, \ldots$ In 
such a case the condition $\left(1^{\prime}\right)$ should be replaced by the condition

$$
\sum_{j=1}^{q} \delta_{j}+\sum_{j=1}^{\infty} \varepsilon_{j}=2
$$

and instead of Riemann surfaces with almost periodic ends we consider surfaces with generalized almost periodic ends (see the remark at the end of Section 6), which are constructed in the same way as surfaces with almost periodic ends. The main results of Section 7 can be transferred onto this wider class of surfaces without substantial changes. In order to get the indicated generalization of Theorem 8.1 it will be necessary to solve schemes with infinitely many columns:

$$
\left(\begin{array}{cccccc}
\delta_{1} & \cdots & \delta_{q} & 0 & 0 & \cdots \\
\varepsilon_{1} & \cdots & \varepsilon_{q} & \varepsilon_{q+1} & \varepsilon_{q+2} & \cdots
\end{array}\right)
$$

where $\varepsilon_{j}>0$ for $j \geq q+1$. These schemes correspond to the case A, and we solve them, as before, joining to each of the schemes the same set of columns of the form $\left(\begin{array}{c}0 \\ \varepsilon_{j}\end{array}\right)$ with numbers $j \geq q+1$, including the case of $\alpha-, \gamma-$, and $\delta$-schemes. We cannot join any columns to $\beta$-schemes, but $\beta$-schemes occur in the case B only.

Now we prove the following theorem.

THEOREM 8.2. Let $\delta_{j}, \varepsilon_{j}, j=1, \ldots, q$ be non-negative numbers satisfying

(1) $\sum_{j=1}^{q}\left\{\delta_{j}+\varepsilon_{j}\right\} \leq 2$,

(2) $0<\delta_{j}+\varepsilon_{j} \leq 1,1 \leq j \leq q$,

(3) $0<\sum_{j=1}^{q} \delta_{j} \leq 2$,

moreover, if $\sum_{j=1}^{q} \delta_{j}=2$, then all numbers $\delta_{1}, \ldots, \delta_{q}$ are rational. Let $a_{1}, \ldots, a_{q}$ be arbitrary distinct complex numbers. Then there exists a meromorphic function $f(z)$ of finite order, such that $\delta\left(a_{j}, f\right)=\delta_{j}, \varepsilon\left(a_{j}, f\right)=\varepsilon_{j}, 1 \leq j \leq q ; \delta(a, f)=0$, $\varepsilon(a, f)=0$ for $a \neq a_{j}, 1 \leq j \leq q$.

The theorem has been already proved in the case when the inequality (1) holds with equality: in such a case we can take $f(z)$ to be a mapping function of the Riemann surface $F$ which was mentioned in Theorem 8.1. So now we can assume that

$$
E=2-\sum_{j=1}^{q}\left\{\delta_{j}+\varepsilon_{j}\right\}>0 .
$$

We take unequal numbers $a_{q+1}$ and $a_{q+2}$, different from $a_{1}, \ldots, a_{q}$. Let $\varepsilon_{q+1}=$ $\varepsilon_{q+2}=E / 2$. Then there exists a meromorphic function $w=f_{1}(z)$, such that

$$
\left\{\begin{array}{l}
n\left(r, a, f_{1}\right)=K r^{\rho}+o\left(r^{\rho}\right) \text { for } a \neq a_{1}, \ldots, a_{q} \\
n\left(r, a_{j}, f_{1}\right)=K\left(1-\delta_{j}\right) r^{\rho}+o\left(r^{\rho}\right) \text { for } 1 \leq j \leq q, \\
n_{1}\left(r, a_{j}, f_{1}\right)=K \varepsilon_{j} r^{\rho}+o\left(r^{\rho}\right) \text { for } 1 \leq j \leq q+2, \\
n_{1}\left(r, a, f_{1}\right)=o\left(r^{\rho}\right) \text { for } a \neq a_{j}, 1 \leq j \leq q+2,
\end{array}\right.
$$


where $K$ and $\rho$ are some positive constants. This statement follows from Theorem 8.1 and formulas $(7.12),(7.13),(7.18),(7.19)$ (using these formulas we assume, as we agreed on p. ???, that $\chi=1)$.

The conditions (8.21) imply that

$$
n\left(r+1, a, f_{1}\right)-n\left(r-1, a, f_{1}\right)=o\left(r^{\rho}\right)
$$

for each $a$. Let $\left\{z_{k \nu}\right\}_{k=1}^{\infty}$ be the sequence of all multiple $a_{q+\nu}$-points of the function $f_{1}(z), \nu=1,2$. Take pairwise disjoint discs $C_{k \nu}=\left\{\left|z-z_{k \nu}\right|<r_{k \nu}\right\}, k=1,2, \ldots$; $\nu=1,2$, such that $r_{k \nu}<1$ and

$$
\sum_{\nu=1}^{2} \sum_{k=1}^{\infty} \iint_{C_{k \nu} \cap\{|z|>1\}} d \sigma(\ln z)<\infty .
$$

Suppose that the function $w_{1}=f_{1}(z)$ maps the complex $z$-plane onto some Riemann surface $F_{1}$. We denote the image of the disc $C_{k \nu}$ under this mapping by $D_{k \nu}, D_{k \nu} \subset F_{1}$. The region $D_{k \nu}$ contains one algebraic ramification point $W_{k \nu}^{1}$ which is projected into $a_{q+\nu}$, that is, into the image of the point $z_{k \nu}$. We take so small circular neighborhood $U_{k \nu}^{1}$ of this ramification point, that $U_{k \nu}^{1}$ does not contain other ramification points, and $U_{k \nu}^{1} \subset D_{k \nu}$. The region $U_{k \nu}^{1}$ is the Riemann surface of the function $\left(w-a_{q+\nu}\right)^{1 / m_{k \nu}}$, lying over the disc $\left\{\left|w-a_{q+\nu}\right|<\varepsilon_{k \nu}\right\}$, where $m_{k \nu}-1$ is the order of the ramification point $W_{k \nu}^{1}$, and $\varepsilon_{k \nu}$ is a sufficiently small positive number. Let $U_{k \nu}$ be the Riemann surface of the function $\left(w-a_{k \nu}\right)^{1 / m_{k \nu}}$, $\left|a_{k \nu}-a_{q+\nu}\right|<\frac{1}{2} \varepsilon_{k \nu}$, lying over the disc $\left\{\left|w-a_{q+\nu}\right|<\varepsilon_{k \nu}\right\}$. We choose the points $a_{k \nu}$ in such a way that all of them are distinct when $k=1,2, \ldots, \nu=1,2$, and all of them are different from projections of algebraic ramification points of the Riemann surface $F_{1}$.

Let $w=\varphi_{k \nu}\left(w_{1}\right)$ be a quasiconformal mapping of $U_{k \nu}^{1}$ onto $U_{k \nu}$, such that points on the boundary of $U_{k \nu}^{1}$ are fixed and the ramification point $W_{k \nu}^{1}$ is mapped onto the ramification point $W_{k \nu}$ of the same order $m_{k \nu}-1$, which is projected onto $a_{k \nu}$. The mapping $w=\varphi_{k \nu}\left(w_{1}\right)$ can be chosen in such a way that $p\left(w_{1}, w\right)<3$ (see Example 8 from Section 2).

Let $F_{0}=F_{1} \backslash \bigcup_{k, \nu} U_{k \nu}^{1}$. Denote by $F$ the Riemann surface which we get if we replace regions $U_{k \nu}^{1}$ by regions $U_{k \nu}$ on the surface $F_{1}$. It is clear that $F_{0}=F \backslash \bigcup U_{k \nu}$. Denote the preimages of $F_{0}$ and $U_{k \nu}^{1}$ under the mapping $w=f_{1}(z)$ by $G_{0}$ and $G_{k \nu}$, respectively. Obviously, $G_{k \nu} \subset C_{k \nu}$. Let $w=\varphi(\zeta)$ be a quasiconformal mapping of the complex $\zeta$-plane onto the Riemann surface $F$, given by the following formulas

$$
\varphi(\zeta)= \begin{cases}f_{1}(\zeta) & \text { for } \zeta \in G_{0}, \\ \varphi_{k \nu}\left(f_{1}(\zeta)\right) & \text { for } \zeta \in G_{k \nu}, k=1,2, \ldots, \nu=1,2 .\end{cases}
$$

It is clear that for each $a$ the functions $f_{1}(\zeta)$ and $\varphi(\zeta)$ have the same amount of $a$-points in $G_{k \nu}$. Since the diameter of each of the regions $G_{k \nu}$ is less than 2 , the relations (8.22) and (8.21) imply that

$$
\left\{\begin{array}{l}
n(r, a, \varphi)=K r^{\rho}+o\left(r^{\rho}\right) \text { for } a \neq a_{1}, \ldots, a_{q} \\
n\left(r, a_{j}, \varphi\right)=K\left(1-\delta_{j}\right) r^{\rho}+o\left(r^{\rho}\right) \text { for } 1 \leq j \leq q \\
n_{1}\left(r, a_{j}, \varphi\right)=K \varepsilon_{j} r^{\rho}+o\left(r^{\rho}\right) \text { for } 1 \leq j \leq q \\
n_{1}(r, a, \varphi)=o\left(r^{\rho}\right) \text { for } a \neq a_{j}, 1 \leq j \leq q
\end{array}\right.
$$


We can even claim that $n_{1}(r, a, \varphi)=n_{1}\left(r, a, f_{1}\right)$ for $a \neq a_{1}, \ldots, a_{q+2}, k=1,2, \ldots$, $\nu=1,2, n_{1}(r, a, \varphi)=0$ for $a=a_{q+1}, a_{q+2}$, and $n_{1}(r, a, \varphi)=O(1)$ for $a=a_{k \nu}$.

Taking into account (8.23) we get that the mapping $w=\varphi(\zeta)$ satisfies

$$
\begin{aligned}
\iint_{|\zeta|>1}\{p(\zeta, w)-1\} d \sigma(\ln \zeta)=\sum_{\nu=1}^{2} \sum_{k=1}^{\infty} \iint_{G_{k \nu} \cap\{|\zeta|>1\}}\{p(\zeta, w)-1\} d \sigma(\ln \zeta) \\
\leq 3 \sum_{\nu=1}^{2} \sum_{k=1}^{\infty} \iint_{G_{k \nu} \cap\{|\zeta|>1\}} d \sigma(\ln \zeta) \leq 3 \sum_{\nu=1}^{2} \sum_{k=1}^{\infty} \iint_{C_{k \nu} \cap\{|\zeta|>1\}} d \sigma(\ln \zeta)<\infty .
\end{aligned}
$$

Using Teichmüller Theorem 3.1 in the same way as we have repeatedly done, we find that the surface $F$ is of parabolic type, and the relations (8.24) remain true for the meromorphic function $w=f(z)$ which maps $\{|z|<\infty\}$ onto $F$, after an appropriate normalization of $f(z)$. From here we get that

$$
\begin{gathered}
T(r, f)=\frac{K}{\rho} r^{\rho}+o\left(r^{\rho}\right), \\
N(r, a, f)=\frac{K}{\rho} r^{\rho}+o\left(r^{\rho}\right) \text { for } a \neq a_{1}, \ldots, a_{q}, \\
N\left(r, a_{j}, f\right)=\frac{K}{\rho}\left(1-\delta_{j}\right) r^{\rho}+o\left(r^{\rho}\right) \text { for } 1 \leq j \leq q, \\
N_{1}\left(r, a_{j}, f\right)=\frac{K}{\rho} \varepsilon_{j} r^{\rho}+o\left(r^{\rho}\right) \text { for } 1 \leq j \leq q, \\
N_{1}(r, a, f)=o\left(r^{\rho}\right) \text { for } a \neq a_{j}, 1 \leq j \leq q .
\end{gathered}
$$

These relations imply that the function $w=f(z)$ is a desired function, whose existence was claimed in Theorem 8.2. It is clear that our remark to Theorem 8.1 is applicable to Theorem 8.2 also. It is clear from our argument that if $\delta_{j}=1$, then the function $f(z)$ can be chosen in such a way that $f(z) \neq a_{j}$.

We can drop the restriction $\sum_{j=1}^{q} \delta_{j}>0$ in Theorem 8.2 , but in such a case we have to avoid usage of Riemann surfaces with almost periodic ends, because their mapping functions necessarily have deficient values.

We prove the following theorem.

THEOREM 8.3. Let $\varepsilon_{1}, \varepsilon_{2}, \ldots$ be a sequence of numbers satisfying $0 \leq \varepsilon_{j} \leq 1$, $\sum_{j=1}^{\infty} \varepsilon_{j} \leq 2$, and $a_{1}, a_{2}, \ldots$ be a sequence of pairwise distinct complex numbers. There exists a meromorphic function $w=f(z)$ such that $\varepsilon\left(a_{j}, f\right)=\varepsilon_{j}$ for $j=1,2, \ldots$, $\varepsilon(a, f)=0$ for $a \neq a_{j}, j \geq 1 ; \delta(a, f)=0$ for all $a$.

Let $b_{1}, b_{2}, \ldots$ be a sequence of distinct complex numbers, $b_{j} \neq a_{k}, j, k=$ $1,2, \ldots$ Let $e_{j k}, 1 \leq j \leq k$, be integers satisfying

(1) $0 \leq e_{j k} \leq k-1,1 \leq j \leq k-1$,

(2) $0 \leq \sum_{j=1}^{k} e_{j k} \leq 2(k-1)$ 
(3) $\lim _{k \rightarrow \infty} \frac{e_{j k}}{k}=\varepsilon_{j}$ for each $j$.

It is clear that such numbers $e_{j k}$ can be selected. Let $\beta_{k}^{\prime}$ and $\beta_{k}^{\prime \prime}$ be some integers, such that $0 \leq \beta_{j k}^{\prime}, \beta_{k}^{\prime \prime} \leq k-1$ and

$$
\sum_{j=1}^{k} e_{j k}+\beta_{k}^{\prime}+\beta_{k}^{\prime \prime}=2(k-1) .
$$

Let $\Phi_{k}$ be a Riemann surface from the class

$$
\mathfrak{F}\left(k ; a_{1}: e_{1 k} ; a_{2}: e_{2 k} ; \ldots ; a_{k}: e_{k k} ; b_{2 k-1}: \beta_{k}^{\prime} ; b_{2 k}: \beta_{k}^{\prime \prime}\right), \quad k=1,2, \ldots
$$

By Theorem 4.1 such surface exists.

It is always possible to find a positive number $u_{k}$ and a number $r_{k}, 0<r_{k}<1 / 4$, such that $\left|u_{k}-k\right|<1 / 4$ and there are no ramification points of the surface $\Phi_{k}$ over the $\operatorname{disc}\left\{\left|w-u_{k}\right| \leq r_{k}\right\}$. Denote by $G_{k}$ a fixed one-sheeted region on $\Phi_{k}$ lying over $\left\{\left|w-u_{k}\right|<r_{k}\right\}$. Let $\Phi_{k}^{0}=\Phi_{k} \backslash G_{k}$. Let $w_{1}=\psi_{k}(w)$ be a quasiconformal mapping of the surface $\Phi_{k}^{0}$ onto the region $\left\{\left|w_{1}-u_{k}\right| \geq r_{k}\right\}$, under which points on the boundary of $\Phi_{k}^{0}$ remain fixed. The existence of such a quasiconformal mapping follows from Example 9 in Section 2. Let the characteristic of this quasiconformal mapping be bounded by a constant $p_{k}$. Let $r^{\prime}=\min \left(r_{k}, p_{k}^{-1 / 2}\right)$. We introduce the notation $G_{k}^{\prime}=G_{k} \cap\left\{\left|w-u_{k}\right|<r_{k}^{\prime}\right\}, \Phi_{k}^{\prime}=\Phi_{k} \backslash G_{k}^{1}$.

It is easy to see that there exists a quasiconformal mapping of $\Phi_{k}^{\prime}$ onto the disc $\left\{\left|\zeta-u_{k}\right| \leq r_{k}^{\prime} / 4\right\}$ with constant distortion on the boundary and with characteristic at most $p_{k}$. In fact, first we map $\Phi_{k}^{\prime}$ onto $\left\{\left|w-u_{k}\right| \geq r_{k}^{\prime}\right\}$; to this end we map $\Phi_{k}^{0} \subset \Phi_{k}^{\prime}$ using the function $\psi_{k}(w)$, and leave the region $G_{k} \backslash G_{k}^{\prime}$ fixed. Then we map the region $\left\{\left|w-u_{k}\right| \geq r_{k}^{\prime}\right\}$ onto $\left\{\left|\zeta-u_{k}\right| \leq r_{k}^{\prime} / 4\right\}$ using the linear-fractional transformation $\zeta=\left(r_{k}^{\prime 2} / 4\right)\left(w-u_{k}\right)^{-1}+u_{k}$. In this case the point on $\partial \Phi_{k}^{\prime}$ which is projected onto $w=u_{k}+r_{k}^{\prime}$ is mapped onto the point $w_{1}=u_{k}+r_{k}^{\prime} / 4$. Denote the constructed mapping of $\Phi_{k}^{\prime}$ onto $\left\{\left|\zeta-u_{k}\right| \leq r_{k}^{\prime} / 4\right\}$ by $\zeta=\omega_{k}(w)$, denote by $w=\Omega_{k}(\zeta)$ the inverse mapping.

We make a cut on $\Phi_{k}$ along the line segment $\Gamma_{k} \subset G_{k}^{\prime}$, which is projected into the line segment $\gamma_{k}=\left\{u_{k}-\frac{r_{k}^{\prime}}{2} \leq u \leq u_{k}+\frac{r_{k}^{\prime}}{2}, v=0\right\}, w=u+i v$. Take the complex $w$-plane with cuts along $\gamma_{k}, k=1,2, \ldots$, and denote it by $P$. We paste to $P$ the surfaces $\Phi_{k}$ with cuts along $\Gamma_{k}$ pasting the upper (lower) side of the cut $\Gamma_{k}$ to the lower (upper) side of the cut $\gamma_{k}$. We get a simply connected Riemann surface $F$.

Let $\tilde{G}_{k}^{\prime}=G_{k}^{\prime} \backslash \Gamma_{k}, G_{k}^{\prime \prime}=\left\{\left|w-u_{k}\right|<r_{k}^{\prime}\right\} \cap P$. Denote the part of the Riemann surface $F$ consisting of $\tilde{G}_{k}^{\prime}$ and $G_{k}^{\prime \prime}$ by $W_{k}$. It is a doubly connected Riemann surface with two algebraic ramification points of the first order over the points $u_{k} \pm r_{k}^{\prime} / 2$. We denote the boundary curves of $W_{k}$ by $C_{1 k}$ and $C_{2 k}$, both of them are projected into $\left\{\left|w-u_{k}\right|=r_{k}^{\prime}\right\}$, moreover, $C_{1 k} \subset \partial G_{k}^{\prime \prime}, C_{2 k} \subset \partial \tilde{G}_{k}^{\prime}$. By Example 10 from Section 2 there exists a quasiconformal mapping of the surface $W_{k}$ onto the annulus $\left\{r_{k}^{\prime} / 4<\left|\zeta-u_{k}\right|<r_{k}^{\prime}\right\}$, such that the point $w=u_{k}+r_{k}^{\prime} e^{i \theta} \in C_{1 k}$ is mapped onto the point $\zeta=u_{k}+r_{k}^{\prime} e^{i \theta}$, and the point $w=u_{k}+r_{k}^{\prime} e^{-i \theta} \in C_{2 k}$ is mapped onto the point $\zeta=u_{k}+\frac{r_{k}^{\prime}}{4} e^{i \theta}$. Denote this quasiconformal mapping by $\zeta=s_{k}(w)$, and its inverse by $w=S_{k}(\zeta)$. As it was shown in Example 10, the 
characteristic of this mapping is bounded by a constant $Q$, which obviously does not depend on $k$.

Let $P_{0}=P \backslash\left(\bigcup_{k=1}^{\infty} G_{k}^{\prime \prime}\right)$. The Riemann surface $F$ can be partitioned into pairwise disjoint parts $P_{0}, W_{1}, W_{2}, \ldots, \Phi_{1}^{\prime}, \Phi_{2}^{\prime}, \ldots$ There exist a quasiconformal mapping of the surface $F$ onto the complex $\zeta$-plane which leaves $P_{0}$ fixed, maps the surfaces $W_{k}$ by $\zeta=s_{k}(w)$, and the surfaces $\Phi_{k}^{\prime}$ by $\zeta=\omega_{k}(w)$. It is easy to verify that such mapping is one-to-one and continuous everywhere on the surface $F$. We denote its inverse quasiconformal mapping of $\{|\zeta|<\infty\}$ onto $F$ by $w=\varphi(\zeta)$ :

$$
w=\varphi(\zeta)= \begin{cases}\zeta & \text { for } \zeta \in P_{0}, \\ S_{k}(\zeta) & \text { for } r_{k}^{\prime} / 4<\left|\zeta-u_{k}\right|<r_{k}^{\prime}, k=1,2, \ldots \\ \Omega_{k}(\zeta) & \text { for }\left|\zeta-u_{k}\right| \leq r_{k}^{\prime} / 4, k=1,2 \ldots\end{cases}
$$

It is clear that $p(\zeta, w) \equiv 1$ for $\zeta \in P_{0}, p(\zeta, w) \leq Q$ for $r_{k}^{\prime} / 4<\left|\zeta-u_{k}\right|<r_{k}^{\prime}$, $k=1,2, \ldots ; p(\zeta, w) \leq p_{k}$ for $\left|\zeta-u_{k}\right| \leq r_{k}^{\prime} / 4$. Hence

$$
\begin{gathered}
\iint_{|\zeta|>1 / 2}\{p(\zeta, w)-1\} d \sigma(\ln \zeta)=\sum_{k=1}^{\infty} \iint_{\left|\zeta-u_{k}\right| \leq r_{k}^{\prime}}\{p(\zeta, w)-1\} d \sigma(\ln \zeta) \\
\leq \sum_{k=1}^{\infty} \max \left(Q, p_{k}\right) \iint_{\left|\zeta-u_{k}\right| \leq r_{k}^{\prime}} d \sigma(\ln \zeta) \\
\leq \sum_{k=1}^{\infty} \max \left(Q, p_{k}\right) O\left(\frac{r_{k}^{\prime 2}}{k^{2}}\right)=\sum_{k=1}^{\infty} O\left(\frac{1}{k^{2}}\right)<\infty .
\end{gathered}
$$

Here we used the condition $r_{k}^{\prime 2} \leq 1 / p_{k}$.

Now we consider the distribution of $a$-points of the function $w=\varphi(z)$. It is clear that all $a$-points except at most one are lying in the discs $\left\{\left|\zeta-u_{k}\right|<r_{k}^{\prime}<1 / 4\right\}$, moreover, the number of $a$-points in each of these discs is equal to $k$ if $\left|a-u_{k}\right| \geq r_{k}^{\prime}$, and is equal to $k+1$ if $\left|a-u_{k}\right|<r_{k}^{\prime}$. Therefore

$$
n(r, a, \varphi)=\sum_{k=1}^{[r]} k+O(r)=\frac{r^{2}}{2}+O(r)
$$

for each $a$. Similarly we get that

$$
n_{1}(r, a, \varphi)=O(1)
$$

for $a \neq a_{j}, j=1,2, \ldots$, and

$$
\begin{aligned}
n_{1}\left(r, a_{j}, \varphi\right) & =\sum_{k=1}^{[r]} e_{j k}+O(r) \\
& =\varepsilon_{j} \sum_{k=1}^{[r]} k+\sum_{k=1}^{[r]} o(k)+O(r)=\varepsilon_{j} \frac{r^{2}}{2}+o\left(r^{2}\right) .
\end{aligned}
$$

for $a=a_{j}$.

Using Teichmüller Theorem 3.1, which is applicable by (8.25), in a standard for this chapter way we find that there exists a meromorphic function $w=f(z)$ for 
which the relations $(8.26),(8.27),(8.28)$ remain true if we replace $\varphi$ by $f$ and $O(r)$ by $o\left(r^{2}\right)$ in them. Now we get that

$$
\begin{gathered}
N(r, a, f)=\frac{r^{2}}{4}+o\left(r^{2}\right) \text { for all } a, \\
T(r, f)=\frac{r^{2}}{4}+o\left(r^{2}\right), \\
N_{1}\left(r, a_{j}, f\right)=\varepsilon_{j} \frac{r^{2}}{4}+o\left(r^{2}\right), j=1,2, \ldots, \\
N_{1}(r, a, f)=O(\ln r) \text { for } a \neq a_{j}, j=1,2, \ldots
\end{gathered}
$$

It is clear that the function $w=f(z)$ satisfies the conditions of Theorem 8.3. 


\section{On the magnitude of type of an entire function}

It was proved in Theorem 1.3 from Chapter 2 that for an entire function $f(z)$ the characteristics $T(r, f)$ and $\ln M(r, f)$ have the same growth category. Let $\rho(r)$ be some proximate order, such that $\rho(r) \rightarrow \rho$ as $r \rightarrow \infty$, where $\rho<\infty$ is the order of an entire function.

Set

$$
\Delta_{T}=\limsup _{r \rightarrow \infty} \frac{T(r, f)}{r^{\rho(r)}}, \Delta_{M}=\limsup _{r \rightarrow \infty} \frac{\ln M(r, f)}{r^{\rho(r)}} .
$$

Theorem 1.3 from Chapter 2 implies that $\Delta_{T}$ and $\Delta_{M}$ are equal to 0 or $\infty$ simultaneously, since $0<\Delta_{T}<\infty$ implies $0<\Delta_{M}<\infty$, and vice versa. Now we shall establish more precise relations between $\Delta_{T}$ and $\Delta_{M}$. The inequality $\Delta_{T} \leq \Delta_{M}$ is obvious, because $T(r, f) \leq \ln ^{+} M(r, f)$. We shall now prove the following theorem.

THEOREM 0.4. The inequalities

$$
\begin{gathered}
\Delta_{M} \leq \pi \rho \csc (\pi \rho) \Delta_{T} \text { for } 0 \leq \rho \leq 1 / 2, \\
\Delta_{M} \leq \pi \rho \Delta_{T} \text { for } 1 / 2<\rho<\infty .
\end{gathered}
$$

hold.

Observe that the inequalities (0.29) and (0.30) hold with equalities for the Mittag-Leffler function (5.31) from Chapter 2 for $\rho(r) \equiv \rho, 0<\rho<\infty^{15}$. If $\rho=0$ the equality $\Delta_{M}=\Delta_{T}$ holds for each entire function (see Remark 1 to Theorem 4.5 from Chapter 2). Therefore we can restrict our attention to the case $\rho>0$.

It is relatively easy to prove the inequality $(0.29)$. We may assume without loss of generality that $f(0)=1$. Then $N(r, 0, f) \leq T(r, f)$. Let $\left\{a_{k}\right\}$ denote the sequence of zeros. Then (cf. (4.3) from Chapter 2)

$$
\begin{aligned}
\ln M(r, f) & =\ln M\left(r, \prod_{k=1}^{\infty} E\left(\frac{z}{a_{k}}, 0\right)\right) \leq \sum_{k=1}^{\infty} \ln \left(1+\frac{r}{\left|a_{k}\right|}\right) \\
& =\int_{0}^{\infty} \ln \left(1+\frac{r}{t}\right) d n(t, 0, f)=r \int_{0}^{\infty} \frac{n(t, 0, f)}{t} \frac{d t}{r+t} \\
& =r \int_{0}^{\infty} N(t, 0, f) \frac{d t}{(r+t)^{2}} \\
& \leq r \int_{0}^{\infty} T(t, f) \frac{d t}{(r+t)^{2}}=\int_{0}^{\infty} T(\tau r, f) \frac{d \tau}{(1+\tau)^{2}} .
\end{aligned}
$$

\footnotetext{
${ }^{15}$ Similar examples can be constructed in the case when $\rho(r)$ is not identically equal to $\rho$. For example, if $0<\rho<1$ we can take the function (5.1) from Chapter 2 with $l(r) \equiv \rho(r)$ and use the formulas $\left(5.4^{\prime}\right)$ and $(5.11)$.
} 
We may assume that $\Delta_{T}<\infty$, since for $\Delta_{T}=\infty$ the inequality (0.29) is obvious. Let $T(r, f) \leq\left(\Delta_{T}+\varepsilon\right) r^{\rho(r)}$ hold for $r \geq r_{0}(\varepsilon)$. Then (0.31) implies

$$
\ln M(r, f) \leq T\left(r_{0}, f\right) \int_{0}^{r_{0} / r} \frac{d \tau}{(1+\tau)^{2}}+\left(\Delta_{T}+\varepsilon\right) \int_{r_{0} / r}^{\infty}(r \tau)^{\rho(r \tau)} \frac{d \tau}{(1+\tau)^{2}},
$$

$$
\begin{aligned}
\Delta_{M} & =\limsup _{r \rightarrow \infty} \frac{\ln M(r, f)}{r^{\rho(r)}} \leq\left(\Delta_{T}+\varepsilon\right) \lim _{r \rightarrow \infty} \int_{r_{0} / r}^{\infty} \frac{(r \tau)^{\rho(r \tau)}}{r^{\rho(r)}} \frac{d \tau}{(1+\tau)^{2}} \\
& =\left(\Delta_{T}+\varepsilon\right) \int_{0}^{\infty} \frac{\tau^{\rho} d \tau}{(1+\tau)^{2}}=\left(\Delta_{T}+\varepsilon\right) \frac{\pi \rho}{\sin \pi \rho} .
\end{aligned}
$$

The possibility of passing to the limit under the integral sign is proved in the same way as in Exercise 1 in Section 2 of Chapter 2; the last integral can be easily computed by using the residue calculus. Since $\varepsilon$ can be chosen arbitrarily small, the inequality (0.32) implies (0.29).

The inequality (0.30) is proved in a much more complicated way; it was obtained only recently by N.V. Govorov [Gov69], although it was conjectured long time ago (see, for example, [Hay64, Ch. 1, 1.8]).

Let $f(z)$ be an entire function of order $\rho, 1 / 2<\rho<\infty, \rho(r)$ be a proximate order, $\rho(r) \rightarrow \rho$ as $r \rightarrow \infty$. We may restrict ourselves to the case when $0<\Delta_{M}<$ $\infty$. Besides, without loss of generality, we may assume $f(0)=1$.

Let $\varepsilon_{0}$ be an arbitrary number satisfying the condition $0<\varepsilon_{0}<\min \left(2 \rho-1, \frac{2}{3} \rho\right)$. Choose $\varepsilon, 0<\varepsilon<\varepsilon_{0}$, in such a way that the function $f(z)$ does not have zeros on the rays $\arg z= \pm \pi /(2 \rho-\varepsilon)$. Set $\sigma=1 /(2 \rho-\varepsilon), 0<\sigma<1$,

$$
T_{1}(r)=\frac{1}{2 \pi} \int_{-\pi \sigma}^{\pi \sigma} \ln \left|f\left(r e^{i \theta}\right)\right| d \theta .
$$

It is clear that

$$
T_{1}(r) \leq m(r, f)=T(r, f) .
$$

The function $\zeta=-z^{2 \rho-\varepsilon}$ is a conformal mapping of the angle $\{|\arg z|<\pi \sigma\}$ onto the region $D=\{0<\arg \zeta<2 \pi\}$, that is, $\zeta$-plane with a cut along the positive real half-axis. We consider the function

$$
\Phi(\zeta)=f\left\{(-\zeta)^{\sigma}\right\}
$$

in $D$, where the single-valued branch of the function $(-\zeta)^{\sigma}$ is chosen in such a way that $(-\zeta)^{\sigma}>0$ for $\zeta<0$. Let $\zeta=\xi+i \eta$. We denote the points $\zeta=\xi>0$ lying on the upper and lower sides of the cut $S$ by $\xi^{+}$and $\xi^{-}$, respectively. Obviously,

$$
\Phi\left(\xi^{ \pm}\right)=f\left(\xi^{\sigma} e^{ \pm i \pi \sigma}\right) .
$$

Let

$$
G(\xi)=\Phi\left(\xi^{+}\right) / \Phi\left(\xi^{-}\right)=f\left(\xi^{\sigma} e^{-i \pi \sigma}\right) / f\left(\xi^{\sigma} e^{i \pi \sigma}\right), \xi>0, G(0)=1 .
$$

It is clear that the function $G(\xi)$ is analytic on the ray $\{\xi>0\}$, and the function $G\left(\xi^{1 / \sigma}\right)$ is analytic, also, at the point $\xi=0$, moreover, $G(\xi) \neq 0$ for $\xi \geq 0$.

We choose a branch of $\ln G(\xi)$ which is continuous for $\xi \geq 0$ and satisfies the condition $\ln G(0)=0$. Let

$$
s(r)=\int_{0}^{r} \ln G(\xi) \frac{d \xi}{\xi} .
$$


Let us show that

$$
s(r)=o\left(r^{3 / 4}\right) \text { for } r \rightarrow \infty .
$$

In fact, let $f(z)$ be without zeros on the ray $\left\{\arg z=\theta_{0}\right\}$. We choose a continuous branch of $\ln f(z)$ on this ray, such that $\ln f(0)=0$. Applying Theorem 2.4 from Chapter 1 to $\ln f(z)$ we get $(R=2 r>r>t>0)$

$$
\begin{aligned}
\ln f\left(t e^{i \theta_{0}}\right)= & \frac{1}{2 \pi} \int_{0}^{2 \pi} \ln \left|f\left(R e^{i \theta}\right)\right| \frac{R e^{i \theta}+t e^{i \theta_{0}}}{R e^{i \theta}-t e^{i \theta_{0}}} d \theta \\
& -\sum_{\left|a_{m}\right|<R} \ln \left\{\frac{\left|a_{m}\right|}{\bar{a}_{m}} \frac{R^{2}-\bar{a}_{m} t e^{i \theta_{0}}}{R\left(a_{m}-t e^{i \theta_{0}}\right)}\right\},
\end{aligned}
$$

where $a_{m}$ are the zeros of the function $f(z)$, and the branches of the logarithms in the sum are chosen in such a way that $\operatorname{Im} \ln \frac{R}{\left|a_{m}\right|}=0$. Then

$$
\left|\operatorname{Im} \ln \left\{\frac{\left|a_{m}\right|}{\bar{a}_{m}} \frac{R^{2}-\bar{a}_{m} t e^{i \theta_{0}}}{R\left(a_{m}-t e^{i \theta_{0}}\right)}\right\}\right| \leq 2 \pi
$$

for each $t, 0 \leq t<\infty$. Since

$$
0<\operatorname{Re} \ln \left\{\frac{\left|a_{m}\right|}{\bar{a}_{m}} \frac{R^{2}-\bar{a}_{m} t e^{i \theta_{0}}}{R\left(a_{m}-t e^{i \theta_{0}}\right)}\right\}=\ln \left|\frac{R^{2}-\bar{a}_{m} t e^{i \theta_{0}}}{R\left(a_{m}-t e^{i \theta_{0}}\right)}\right| \leq \ln \frac{2 R}{|| a_{m}|-t|},
$$

from $(0.36)$ we get

$$
\begin{aligned}
\left|\ln f\left(t e^{i \theta_{0}}\right)\right| & \leq \frac{R+t}{R-t} \cdot \frac{1}{2 \pi} \int_{0}^{2 \pi}|\ln | f\left(R e^{i \theta}\right)|| d \theta+\sum_{\left|a_{m}\right|<R} \ln \frac{2 R}{|| a_{m}|-t|}+n(R, 0, f) 2 \pi \\
& \leq \frac{R+r}{R-r}\left\{m(R, f)+m\left(R, \frac{1}{f}\right)\right\}+\sum_{\left|a_{m}\right|<R} \ln \frac{2 R}{|| a_{m}|-t|}+2 \pi n(R, 0, f) \\
& \leq 6 T(R, f)+2 \pi n(R, 0, f)+\sum_{\left|a_{m}\right|<R} \ln \frac{2 R}{|| a_{m}|-t|} .
\end{aligned}
$$

We integrate this inequality with respect to $d \ln t$ from $t=1$ to $t=r$. We get

$$
\int_{1}^{r}\left|\ln f\left(t e^{i \theta_{0}}\right)\right| \frac{d t}{t} \leq 6 T(R, f) \ln R+2 \pi n(R, 0, f) \ln R+\sum_{\left|a_{m}\right|<R} \int_{1}^{r} \ln \frac{2 R}{|| a_{m}|-t|} \frac{d t}{t} .
$$

But

$$
\begin{aligned}
\int_{1}^{r} \ln \frac{2 R}{|| a_{m}|-t|} \frac{d t}{t} & \leq \int_{\left|a_{m}\right|-1}^{\left|a_{m}\right|+1} \ln \frac{2 R}{|| a_{m}|-t|} d t+\ln 2 R \int_{1}^{r} \frac{d t}{t} \\
& =2 \int_{0}^{1} \ln \frac{2 R}{\tau} d \tau+\ln 2 R \cdot \ln r \\
& =2\{\ln (2 R)+1\}+\ln 2 R \cdot \ln r<(\ln r)^{2}+4 \ln r+6 .
\end{aligned}
$$

Therefore

$$
\int_{1}^{r} \frac{\left|\ln f\left(t e^{i \theta_{0}}\right)\right|}{t} d t \leq 6 T(2 r, f) \ln 2 r+n(2 r, 0, f)\left\{2 \pi \ln (2 r)+(\ln r)^{2}+4 \ln r+6\right\} .
$$


Since the order of the functions $T(r, f)$ and $n(r, 0, f)$ does not exceed $\rho$, then the relations

$$
\int_{0}^{r} \frac{\left|\ln f\left(t e^{i \theta_{0}}\right)\right|}{t} d t=O\left(r^{\rho^{\prime}}\right)
$$

and

$$
\int_{0}^{r} \frac{\left|\ln f\left(t^{\sigma} e^{i \theta_{0}}\right)\right|}{t} d t=O\left(r^{\rho^{\prime} \sigma}\right)=o\left(r^{3 / 4}\right)
$$

hold for each $\rho^{\prime}, 3 /(4 \sigma)>\rho^{\prime}>\rho$. The relation $(0.35)$ follows.

Denote the number of zeros of the function $\Phi(\zeta)$ in the region $\{0<\arg \zeta<$ $2 \pi,|\zeta| \leq r\}$ or, which is the same, the number of zeros of the function $f(z)$ in the sector $\left\{|\arg z|<\pi \sigma,|z| \leq r^{\sigma}\right\}$, by $n_{2}(r)$. Then $n_{2}(r)=O\left(r^{\rho^{\prime} \sigma}\right)$ and

$$
n_{2}(r)=o\left(r^{3 / 4}\right) \text {. }
$$

Suppose that the $\operatorname{arc}\{|\zeta|=t, 0<\arg <2 \pi\}$ does not contain zeros of the function $\Phi(\zeta)$. Let

$$
T_{2}(r)=\frac{1}{2 \pi} \int_{0}^{2 \pi} \ln \left|\Phi\left(r e^{i \alpha}\right)\right| d \alpha .
$$

Then, applying the argument principle and the Cauchy-Riemann equations we find that

$$
\begin{aligned}
n_{2}(t) & =\frac{1}{2 \pi} \int_{0}^{t} d \arg G(\xi)+\frac{1}{2 \pi} \int_{\alpha=0}^{2 \pi} d \arg \Phi\left(t e^{i \alpha}\right) \\
& =\frac{1}{2 \pi} \arg G(t)+\frac{1}{2 \pi} \int_{0}^{2 \pi} \frac{\partial \arg \Phi\left(t e^{i \alpha}\right)}{\partial \alpha} d \alpha \\
& =\frac{1}{2 \pi} \arg G(t)+t \frac{d}{d t}\left\{\frac{1}{2 \pi} \int_{0}^{2 \pi} \ln \left|\Phi\left(t e^{i \alpha}\right)\right| d \alpha\right\} \\
& =\frac{1}{2 \pi} \arg G(t)+t \frac{d T_{2}(t)}{d t} .
\end{aligned}
$$

We divide this equality by $t$ and integrate from 0 to $r$. Taking into account $n_{2}(0)=$ $T_{2}(0)=0$ we get the equality

$$
T_{2}(r)=\int_{0}^{r}\left\{n_{2}(t)-\frac{1}{2 \pi} \arg G(t)\right\} \frac{d t}{t} .
$$

Now we consider the analytic function

$$
F(\zeta)=\exp \left\{\frac{\zeta}{2 \pi i} \int_{0}^{\infty} \frac{s(t)}{(t-\zeta)^{2}} d t\right\}=\exp \{\zeta \psi(\zeta)\}
$$

in the region $D$. The relation (0.35) implies the absolute convergence of the integral in (0.39). Integrating it by parts we get

$$
\psi(\zeta)=\frac{1}{2 \pi i} \int_{0}^{\infty} \frac{s(t)}{(t-\zeta)^{2}} d t=\frac{1}{2 \pi i} \int_{0}^{\infty} \frac{\ln G(t)}{t(t-\zeta)} d t .
$$

Let $0<\xi_{0}<\infty$. We show that

$$
\psi\left(\xi_{0}^{+}\right)-\psi\left(\xi_{0}^{-}\right)=\frac{\ln G\left(\xi_{0}\right)}{\xi_{0}} .
$$

The equality (0.40) is a special case of the well-known formulas due to Yu.V. Sokhotskii; we prove only the special case that we need. 
Take a circle $\gamma=\left\{\left|\zeta-\xi_{0}\right|=r\right\}$, where $r<\xi_{0}$ is chosen so small, that the function $\ln G(\zeta)$ is analytic in the disc $\left\{\left|\zeta-\xi_{0}\right| \leq r\right\}$. If $\operatorname{Im} \zeta>0$, then by the Cauchy theorem, we can write the function $\psi(\zeta)$ as

$$
\psi(\zeta)=\frac{1}{2 \pi i} \int_{\Gamma^{-}} \frac{\ln G(t)}{t(t-\zeta)} d t
$$

where the curve $\Gamma^{-}$consists of the positive real half-axis without the interval $\{\mid \xi-$ $\left.\xi_{0} \mid<r\right\}$ and the lower semicircle $\left\{\left|\zeta-\xi_{0}\right|=r, \operatorname{Im} \zeta<0\right\}$. It is clear that with the help of $(0.41)$ the function $\psi(\zeta)$ can be continued analytically from $\{\operatorname{Im} \zeta>0\}$ into the half-disc $\left\{\left|\zeta-\xi_{0}\right|<r, \operatorname{Im} \zeta<0\right\}$ through the interval $\left\{\eta=0,\left|\xi-\xi_{0}\right|<r\right\}$. Therefore $\psi\left(\xi_{0}^{+}\right)$exists and

$$
\psi\left(\xi_{0}^{+}\right)=\frac{1}{2 \pi i} \int_{\Gamma^{-}} \frac{\ln G(t)}{t\left(t-\xi_{0}\right)} d t .
$$

Similarly we get that

$$
\psi\left(\xi_{0}^{-}\right)=\frac{1}{2 \pi i} \int_{\Gamma^{+}} \frac{\ln G(t)}{t\left(t-\xi_{0}\right)} d t
$$

where the curve $\Gamma^{+}$consists of positive real half-axis without the interval $\left\{\left|\xi-\xi_{0}\right|<\right.$ $r\}$ and the upper semicircle $\left\{\left|\zeta-\xi_{0}\right|=r, \operatorname{Im} \zeta>0\right\}$. Since $\Gamma^{-}-\Gamma^{+}=\gamma$, we have

$$
\psi\left(\xi_{0}^{+}\right)-\psi\left(\xi_{0}^{-}\right)=\frac{1}{2 \pi i} \int_{\gamma} \frac{\ln G(t)}{t\left(t-\xi_{0}\right)} d t=\frac{\ln G\left(\xi_{0}\right)}{\xi_{0}} .
$$

It is also clear from the preceding argument that the functions $\psi\left(\xi^{+}\right)$and $\psi\left(\xi^{-}\right)$ are continuous for $0<\xi<\infty$.

Let us show that

$$
\zeta \psi(\zeta) \rightarrow 0 \text { as } \zeta \rightarrow 0, \zeta \in D .
$$

The function $\ln G\left(\zeta^{1 / \sigma}\right)$ is analytic in a sufficiently small neighborhood of $\zeta=0$ and

$$
\ln G\left(\zeta^{1 / \sigma}\right)=\sum_{k=1}^{\infty} c_{k} \zeta^{k}
$$

hence, the function $\ln G(\zeta) / \zeta$ is analytic for $\left\{|\arg \zeta|<\pi,|\zeta| \leq r_{0}\right\}$, and

$$
|\ln G(\zeta) / \zeta|=O\left(|\zeta|^{\sigma-1}\right) \text { as } \zeta \rightarrow 0 .
$$

Let $\operatorname{Im} \zeta \leq 0$. We write

$$
\begin{aligned}
\psi(\zeta) & =\frac{1}{2 \pi i} \int_{S_{1}} \frac{\ln G(t)}{t(t-\zeta)} d t+\frac{1}{2 \pi i} \int \frac{\ln G(t)}{t(t-\zeta)} \\
& =\frac{1}{2 \pi i} \int_{S_{1}} \frac{\ln G(t)}{t(t-\zeta)} d t+O(1), \quad \zeta \rightarrow 0,
\end{aligned}
$$

where $S_{1}$ is the line segment joining 0 and $i r_{0}$, and $S_{2}$ is a curve consisting of the line segment joining the points $i r_{0}$ and $r_{0}$, and the ray $\left\{\xi \geq r_{0}, \eta=0\right\}$. If $t \in S_{1}$ and $\operatorname{Im} \zeta \leq 0$, then $|t-\zeta|>|\zeta|$. Taking into account (0.43) and (0.44) we obtain that

$$
|\psi(\zeta)| \leq \frac{1}{|\zeta|} \frac{1}{2 \pi} \int_{0}^{r_{0}} O\left(t^{\sigma-1}\right) d t+O(1)=\frac{1}{|\zeta|} O\left(r_{0}^{\sigma}\right)+O(1) .
$$


Hence

$$
\limsup _{\zeta \rightarrow 0, \operatorname{Im} \leq 0}|\zeta \psi(\zeta)| \leq O\left(r_{0}^{\sigma}\right)
$$

Since $r_{0}$ can be chosen to be arbitrarily small, the relation (0.42) follows. Similar argument works in the case when $\operatorname{Im} \zeta \geq 0$. Thus, the relation $(0.42)$ has been proved.

The formulas (0.39), (0.40), and (0.42) imply that

$$
\frac{F\left(\xi^{+}\right)}{F\left(\xi^{-}\right)}=G(\xi)=\frac{\Phi\left(\xi^{+}\right)}{\Phi\left(\xi^{-}\right)}, \frac{\Phi\left(\xi^{+}\right)}{F\left(\xi^{+}\right)}=\frac{\Phi\left(\xi^{-}\right)}{F\left(\xi^{-}\right)} .
$$

and

$$
\frac{1}{K}<|F(\zeta)|<K<\infty
$$

in a neighborhood of $\zeta=0$, where $K$ is some constant. Therefore the analytic in $D$ function

$$
\Omega(\zeta)=\Phi(\zeta) / F(\zeta)
$$

admits an analytic continuation into the whole complex $\zeta$-plane and is an entire function. Taking into account (0.34) and $(0.42)$ we see that $\Omega(0)=1$.

The equality $(0.39)$ implies that $(0<\varphi<2 \pi)$

$$
|\ln | F\left(r e^{i \varphi}\right)|| \leq \frac{r}{2 \pi} \int_{0}^{\infty} \frac{|s(t)| d t}{\left|t-r e^{i \varphi}\right|^{2}}=\frac{1}{2 \pi} \int_{0}^{\infty} \frac{|s(r \tau)| d \tau}{\left|\tau-e^{i \varphi}\right|^{2}} .
$$

Since $\left|\tau-e^{i \varphi}\right|>(\tau+1) \sin \frac{\varphi}{2}$ (see Section 5 of Chapter 2), taking into account $(0.35)$, we get that

$$
|\ln | F\left(r e^{i \varphi}\right)|| \leq \frac{1}{\sin ^{2} \frac{\varphi}{2}} \int_{0}^{\infty} \frac{o\left\{(r t)^{3 / 4}\right\}}{(t+1)^{2}} d t=o\left(r^{3 / 4}\right) \csc ^{2} \frac{\varphi}{2} .
$$

Besides, it is obvious that $\ln \left|\Phi\left(r e^{i \varphi}\right)\right| \leq o\left(r^{3 / 4}\right)$. Therefore

$$
\ln \left|\Omega\left(r e^{i \varphi}\right)\right| \leq C_{1} r^{3 / 4} \csc ^{2} \frac{\varphi}{2}+C_{1},
$$

where $C_{1}$ is some positive constant. Positive constants which do not depend on $r$ and $\varphi$ will be denoted $C_{j}$.

Using the method due to V.I. Matsaev [Mat60] we show that the estimate

$$
\ln \left|\Omega\left(r e^{i \varphi}\right)\right| \leq C_{2} r^{3 / 4}+C_{2}
$$

holds.

Denote the region $\left\{|\arg (\zeta-1)-\pi|<\frac{\pi}{16}\right\} \cap\{|\zeta|<1\}$ by $G$, the boundary of this region by $L$, and the part $L \cap\{|\zeta|<1\}$ of the boundary $L$ by $L_{1}$. Let $d=\sin \frac{\pi}{16}$. It is clear that $\{|\zeta|<d\} \subset G$. The function $\mu(\zeta)=\exp \left\{-(\zeta-1)^{-4}\right\}$ is, as it is easy to show, analytic in $G$ and continuous in $\bar{G}$. For $\zeta \in L_{1}$ we have

$$
|\mu(\zeta)|=\left|\exp \left\{-\frac{e^{-4 i \arg (\zeta-1)}}{|\zeta-1|^{4}}\right\}\right|=\exp \left\{-\frac{\cos \frac{\pi}{4}}{|\zeta-1|^{4}}\right\} .
$$

If $\zeta \in L_{1}$, then, by the sine theorem,

$$
\frac{1}{d} \geq \frac{|\zeta|}{d}=\frac{|\zeta-1|}{|\sin \varphi|}, \varphi=\arg \zeta
$$


Therefore $\left(\zeta \in L_{1}\right)$

$$
|\mu(\zeta)| \leq \exp \left\{-\frac{d^{4}}{\sqrt{2} \sin ^{4} \varphi}\right\} \leq \exp \left\{-\frac{d^{4} 2^{-\frac{9}{2}}}{\sin ^{4} \frac{\varphi}{2}}\right\} .
$$

For $\zeta \in L \backslash L_{1}$ we have

$$
|\mu(\zeta)| \leq \exp \left\{-\frac{\cos \frac{\pi}{4}}{|\zeta-1|^{4}}\right\} \leq \exp \left\{-2^{-\frac{9}{2}}\right\} .
$$

Combining the estimates (0.48) and (0.49) we obtain that the inequality

$$
|\mu(\zeta)| \leq \exp \left\{-\frac{C_{3}}{\sin ^{4} \frac{\varphi}{2}}\right\}
$$

holds on $L$. Let $|\zeta|<\frac{d}{2} r, r \geq 1$. Then $\zeta / r \in G$, and, by the Cauchy formula, we get

$$
\Omega(\zeta)=\frac{1}{2 \pi i \mu(\zeta / r)} \int_{L} \frac{\Omega(r t) \mu(t)}{t-\frac{\zeta}{r}} d t .
$$

It is easy to see that

$$
\begin{gathered}
\max _{|\zeta|=\frac{d}{2}} \frac{1}{|\mu(\zeta)|}=e^{\left(1-\frac{d}{2}\right)^{-4}}=C_{4}, \\
\int_{L} \frac{|d t|}{\left|t-\frac{\zeta}{r}\right|} \leq \frac{1}{\left(\frac{d}{2}\right)} \int_{L}|d t|=C_{5},|\zeta|=\frac{d}{2} r .
\end{gathered}
$$

Taking into account (0.46) and (0.50) we conclude that (0.51) implies

$$
M\left(\frac{d r}{2}, \Omega\right) \leq \frac{1}{2 \pi} C_{4} C_{5} e^{C_{1}} \max _{0<\varphi<2 \pi} \exp \left\{\frac{C_{1} r^{3 / 4}}{\sin ^{2} \frac{\varphi}{2}}-\frac{C_{3}}{\sin ^{4} \frac{\varphi}{2}}\right\} .
$$

We assume that $r$ is so large that $2 C_{3} \leq C_{1} r^{3 / 4}$. Than, finding the maximum in (0.52) using the standard methods of calculus, we get

$$
M\left(\frac{d r}{2}, \Omega\right) \leq \frac{1}{2 \pi} C_{4} C_{5} e^{C_{1}} \exp \left\{\frac{C_{1}^{2}}{4 C_{3}} r^{3 / 2}\right\}
$$

and

$$
M(r, \Omega) \leq C_{6} e^{C_{6} r^{3 / 2}}, r>0 .
$$

Now we consider the auxiliary function

$$
\Omega_{\eta}(\zeta)=\Omega(\zeta) \exp \left\{-7 C_{1} \zeta^{3 / 4} \sec \frac{3 \pi}{16}-\eta \zeta^{5 / 3}\right\}
$$

in the angle $W=\left\{|\arg \zeta|<\frac{\pi}{4}\right\}$, where $\eta$ is an arbitrary positive number. If $|\theta| \leq \frac{\pi}{4}$, taking into account (0.53), we obtain the estimate

$$
\begin{aligned}
\left|\Omega_{\eta}\left(r e^{i \theta}\right)\right| & \leq C_{6} \exp \left\{C_{6} r^{3 / 2}-7 C_{1} \sec \frac{3 \pi}{16} r^{3 / 4} \cos \frac{3 \theta}{4}-\eta r^{5 / 3} \cos \frac{5 \theta}{3}\right\} \\
& \leq C_{6} \exp \left\{C_{6} r^{3 / 2}-7 C_{1} r^{3 / 4}-\eta r^{5 / 3} \cos \frac{5 \pi}{12}\right\} .
\end{aligned}
$$


Hence $\Omega_{\eta}\left(r e^{i \theta}\right) \rightarrow 0$ for $r \rightarrow \infty,|\theta| \leq \frac{\pi}{4}$. The function $\Omega_{\eta}(\zeta)$ is analytic in the region $W$ and is continuous in $\bar{W}$. Now we estimate $\left|\Omega_{\eta}(\zeta)\right|$ on the boundary of $W$ using (0.46). We get

$$
\left|\Omega_{\eta}\left(r e^{ \pm i \frac{\pi}{4}}\right)\right| \leq e^{C_{1}} \exp \left\{7 C_{1} r^{3 / 4}-7 C_{1} r^{3 / 4}-\eta r^{5 / 3} \cos \frac{5 \pi}{12}\right\} \leq e^{C_{1}}
$$

Hence, by the principle of the maximum modulus, the inequality

$$
\left|\Omega_{\eta}(\zeta)\right| \leq e^{C_{1}}
$$

holds in $W$. Letting $\eta$ tend to 0 in this inequality, we get that the inequalities

$$
\left|\Omega(\zeta) e^{-7 C_{1} \zeta^{3 / 4} \sec \frac{3 \pi}{16}}\right| \leq e^{C_{1}}
$$

and

$$
|\Omega(\zeta)| \leq \exp \left\{C_{1}+7 C_{1}|\zeta|^{3 / 4} \sec \frac{3 \pi}{16}\right\}
$$

hold in $W$. The inequality (0.46) implies that the inequality

$$
\ln |\Omega(\zeta)| \leq 7 C_{1}|\zeta|^{3 / 4}+C_{1}
$$

holds for $\frac{\pi}{4} \leq \arg \zeta \leq \frac{7 \pi}{4}$. Thus, the estimate (0.54) holds for $|\zeta|<\infty$. Since the order of the entire function $\Omega(\zeta)$ does not exceed $3 / 4, \Omega(\zeta) \neq 0$ for $\zeta \geq 0$, and $\Omega(0)=1$, we have

$$
\Omega(\zeta)=\prod_{n=1}^{\infty}\left(1-\frac{\zeta}{\zeta_{n}}\right), \zeta_{n} \rightarrow \infty, 0<\arg \zeta_{n}<2 \pi
$$

Since the equality $\Phi(\zeta)=F(\zeta) \Omega(\zeta)$ holds in $D$, zeros of the function $\Phi(\zeta)$ in $D$ coincide with zeros of $\Omega(\zeta)$, and $n(r, 0, \Omega)=n_{2}(r)$. Therefore (cf. (0.31))

$$
\ln M(r, \Omega) \leq r \int_{0}^{\infty} \frac{n_{2}(t) d t}{t(t+r)}
$$

By $(0.39),\left(\mathrm{A} .11^{\prime}\right),\left(\mathrm{A} .26^{\prime}\right),(0.38)$, we have that

$$
\begin{aligned}
\ln |\Phi(-r)| & \leq \ln |F(-r)|+\ln M(r, \Omega) \\
& \leq \operatorname{Re}\left\{-\frac{r}{2 \pi i} \int_{0}^{\infty} \frac{\ln G(t)}{t(t+r)} d t\right\}+r \int_{0}^{\infty} \frac{n_{2}(t) d t}{t(t+r)} \\
& =-r \int_{0}^{\infty} \frac{\frac{1}{2 \pi} \arg G(t)-n_{2}(t)}{t} \frac{d t}{t+r}=r \int_{0}^{\infty} \frac{d T_{2}(t)}{t+r} \\
& =r \int_{0}^{\infty} T_{2}(t) \frac{d t}{(t+r)^{2}}=\int_{0}^{\infty} T_{2}(\tau r) \frac{d \tau}{(\tau+1)^{2}} .
\end{aligned}
$$

Taking into account (0.33) and (0.34) it is easy to get that

$$
T_{2}(r)=(2 \rho-\varepsilon) T_{1}\left(r^{\sigma}\right)<2 \rho T\left(r^{\sigma}, f\right) .
$$

Then (0.55) implies that

whence

$$
\ln \left|f\left(r^{\sigma}\right)\right| \leq 2 \rho \int_{0}^{\infty} T\left(\tau^{\sigma} r^{\sigma}, f\right) \frac{d \tau}{(\tau+1)^{2}}
$$

$$
\ln |f(r)| \leq 2 \rho \int_{0}^{\infty} T\left(\tau^{\sigma} r, f\right) \frac{d \tau}{(\tau+1)^{2}}=\frac{2 \rho}{\sigma} \int_{0}^{\infty} T(t r, f) \frac{t^{1 / \sigma}}{\left(t^{1 / \sigma}+1\right)^{2}} \frac{d t}{t}
$$


Since the function $x(x+1)^{-2}$ increases on $[0,1]$ and decreases on $[1, \infty)$, we have

$$
\frac{t^{1 / \sigma}}{\left(t^{1 / \sigma}+1\right)^{2}} \leq \frac{t^{1 / \sigma_{0}}}{\left(t^{1 / \sigma_{0}}+1\right)^{2}}, 0<\sigma<\sigma_{0}=\frac{1}{2 \rho-\varepsilon_{0}}
$$

Therefore (0.56) implies that

$$
\ln |f(r)| \leq(2 \rho)^{2} \int_{0}^{\infty} T(t r, f) \frac{t^{1 / \sigma_{0}}}{\left(t^{1 / \sigma_{0}}+1\right)^{2}} \frac{d t}{t} .
$$

Applying this inequality to the function $f\left(z e^{i \theta}\right), 0 \leq \theta<2 \pi$ we get that $\ln |f(r)|$ in the left-hand side of the inequality (0.57) can be replaced by $\ln \left|f\left(r e^{i \theta}\right)\right|$ (we cannot do the same for the inequality (0.56) since $\sigma$ unlike $\sigma_{0}$ depends on $\theta$ ). Therefore

$$
\ln M(r, f) \leq(2 \rho)^{2} \int_{0}^{\infty} T(t r, f) \frac{t^{1 / \sigma_{0}}}{\left(t^{1 / \sigma_{0}}+1\right)^{2}} \frac{d t}{t} .
$$

In the same way as we obtained (0.32) from (0.31), we find that for an arbitrary small $\varepsilon_{1}$ the inequality

$$
\begin{aligned}
\Delta_{M} & \leq\left(\Delta_{T}+\varepsilon_{1}\right)(2 \rho)^{2} \int_{0}^{\infty} t^{\rho-1} \frac{t^{1 / \sigma_{0}}}{\left(t^{1 / \sigma_{0}}+1\right)^{2}} d t \\
& =\left(\Delta_{T}+\varepsilon_{1}\right)(2 \rho)^{2} \sigma_{0} \int_{0}^{\infty} \frac{\tau^{\sigma_{0} \rho}}{(\tau+1)^{2}} d \tau=\left(\Delta_{T}+\varepsilon_{1}\right)(2 \rho)^{2} \sigma_{0} \frac{\pi \sigma_{0} \rho}{\sin \pi \sigma_{0} \rho}
\end{aligned}
$$

holds. Letting $\varepsilon_{1}$ and $\varepsilon_{0}$ tend to 0 in the right-hand side of this inequality, we get (0.30). This completes our proof of Theorem 0.4 .

Theorem 0.4 immediately implies

Corollary. Let $f(z)$ be an entire function of order $\rho, 0 \leq \rho \leq \infty$. Then

$$
\begin{gathered}
\limsup _{r \rightarrow \infty} \frac{T(r, f)}{\ln M(r, f)} \geq \frac{\sin \pi \rho}{\pi \rho} \quad \text { if } \quad 0 \leq \rho \leq \frac{1}{2}, \\
\limsup _{r \rightarrow \infty} \frac{T(r, f)}{\ln M(r, f)} \geq \frac{1}{\pi \rho} \quad \text { if } \rho>\frac{1}{2} .
\end{gathered}
$$

In fact, if $\rho=\infty$, the statement of the corollary is trivial. If $\rho<\infty$, then let $\rho(r)$ be a proximate order, such that

$$
\limsup _{r \rightarrow \infty} \frac{\ln M(r, f)}{r^{\rho(r)}}=1 .
$$

Then

$$
\limsup _{r \rightarrow \infty} \frac{T(r, f)}{\ln M(r, f)} \geq \limsup _{r \rightarrow \infty} \frac{T(r, f)}{r^{\rho(r)}},
$$

and (0.58) and (0.59) follow from (0.29) and (0.30), respectively.

The fact that the estimates $(0.58)$ and $(0.59)$ are sharp follows from the same examples which were used to show that the estimates (0.29) and (0.30) are sharp. If $\rho=\infty$, we can use the function $f(z)=\exp \left(e^{z}\right)$, for which $T(r, f)=o(\ln M(r, f))=$ $o\left(e^{r}\right)$ as $r \rightarrow \infty$ (see (6.4) from Chapter 4$)$.

The inequality (0.58) was first proved by G. Valiron [Val30] and A. Wahlund [Wah29]. The inequality (0.59) was conjectured by R.E.A.C. Paley [Pal32] and proved only 35 years later by N.V. Govorov [Gov69]. V.P. Petrenko [Pet69] proved that the inequalities $(0.58)$ and $(0.59)$ remain true for meromorphic functions even 
if we replace the order $\rho$ by the lower order $\lambda$. The fact that it is possible to replace $\rho$ by $\lambda$ in (0.58) for entire functions and for $\lambda \leq 1 / 2$ was proved in Section 3 of Chapter 5 (see the inequality (3.30)). 


\section{Notes}

\section{Chapter 1}

Theorem 2.1 was apparently first proved by F. Nevanlinna and R. Nevanlinna [NN22]. Formula (2.2) was first obtained by R. Nevanlinna [NevR24] who named it the Poisson-Jensen formula, since it is an immediate generalization of the classical formulas due to Poisson and Jensen [Jen99] (Theorem 2.5). Formula (2.4) was obtained in the same paper of R. Nevanlinna, it is often called the SchwarzJensen formula. Formula (2.3) was also discovered by R. Nevanlinna [NevR25b]. Important generalization of the formulas (2.2), (2.4), and (2.8) were found by M.M. Dzhrbashyan [Dzh66, Chapter IX]. Theorems equivalent to Theorem 2.6 were independently proved by T. Shimizu [Shi29] and L. Ahlfors [Ahl29].

Theorem 3.1 was first proved by F. Nevanlinna [NevF22a] (see, also, [NevF22b]), and somewhat later by T. Carleman [Carl23]. Following the existing tradition we use the name "Carleman formula" for (3.1). Theorem 3.4 was proved by B.Ya. Levin [Lev41], and later independently by M. Tsuji [Tsu50].

Theorem 4.1 is due to R. Nevanlinna [NevR25a]. Theorems 4.2 and 4.4 were obtained by T. Shimizu [Shi29] and L. Ahlfors [Ahl29]. Formulas (4.8) and (4.13) were obtained by H. Cartan [Cart29b], [Cart29a], [Cart33].

Theorems 5.1 and 5.2 were proved by R. Nevanlinna [NevR25b]. Theorem 5.3 was proved by M. Tsuji [Tsu50].

Relations (6.1)-(6.15) are well known. Inequalities (6.16)-(6.18) were proved by S. Hellerstein and L. Rubel [HR64]. G. Valiron [Val31] proved the following theorem containing Theorem 6.2 and the equality (6.29).

Let $R(u, v)$ be a rational function of $u$ and $v$, of degree $d$ in $v$. If $f(z)$ is a meromorphic function, then $T(r, R(z, f(z)))=d T(r, f)+O(\ln r)$. Theorem 6.4 is due to R. Nevanlinna [NevR29], [NevR74, Ch. X, sect. 214].

Theorems 7.1 and 7.2 were proved by R. Nevanlinna [NevR29, pp. 24-27]. Theorem 7.3 was proved by A. Edrei and W.H.J. Fuchs [EF62a].

\section{Chapter 2}

Section 1 contains well-known facts presented in many texts. In using the term "growth category" we follow B.Ya. Levin [Lev80]. The term "magnitude of type" is not standard and apparently is introduced for the first time here. Usually they call the number $\sigma[\alpha]$ simply the "type". We find this inconvenient. We note also that the well-known Cauchy-Hadamard formulas allow to determine the order and the type of the function $\ln M(r, f)$ in terms of Taylor coefficients of an entire function $f(z)$ (see, for example, B.Ya. Levin [Lev80, Chapter I, §2]). Sometimes Picard exceptional values are defined as values which are never taken by a function $f(z)$ 
and Borel exceptional values are defined as values for which the order of $n(r, a)$ is less than the order of the function.

Various comparison functions for building the scale of growth, different from the function $\exp \left(r^{\rho}\right)$ were introduced at the beginning of the 20th century by E. Lindelöf, P. Boutroux, O. Blumenthal, G. Valiron, and others. G. Valiron in [Val23] calls the proximate order, defined as in our Section 2 a proximate order of Lindelöf (although Lindelöf considered much more narrow class of comparison functions), and he calls the function $l(r)$ which we introduce in Section 5 a proximate order of Boutroux. Today the definition of a proximate order, as we introduce it in Section 2 , is standard, and the results proved in Section 2 are well known (see, for example, [Lev80]). Theorem 2.1 is due to G. Valiron [Val23] (in a bit weaker form it was proved already in [Val14, p. 213]), who, however, did not consider a lower proximate order. The presented proof of Theorem 2.1 is mostly due to S.M. Shah [Sha46b], he also introduced [Sha48] a lower proximate order. In the definition of a slowly varying function one can omit the condition of the uniform convergence of $L(k r) / L(r)$ to 1 . In fact, J. Karamata [Kar30] proved that if $\lim _{r \rightarrow \infty} L(k r) / L(r)=1$ for all $k, 0<k<\infty$, then the convergence of $L(k r) / L(r)$ to 1 is uniform in $k$ for $0<a \leq k \leq b<\infty$. See, also, J. Korevaar, T. Aardenne-Ehrenfest and N.G. de Bruijn [KAB49].

Theorems 3.2 and 3.3 in this generality are apparently published here for the first time, although some of their corollaries, for example, the inequality (3.6) are well known (see, for example, B.Ya. Levin [Lev80]). Theorem 3.4 is due to E. Borel [Bor97] and is contained in all standard courses of the theory of entire functions.

Theorem 4.1 was proved by J. Hadamard [Had93] for entire functions. Theorem 4.2, essentially, goes back to E. Borel [Bor00], who however did not use proximate orders. The corollary of Theorem 4.2 was proved by R. Nevanlinna [NevR29] in a different way.

Theorem 4.4 was proved by E. Lindelöf [Lin05] for entire functions without using proximate orders. The generalization of the Lindelöf theorem consisting in the usage of proximate orders apparently was first done by B.Ya. Levin [Lev80, Chapter I, §13]. The Lindelöf theorem was carried over to meromorphic functions by G. Valiron [Val33]. Our proofs of Theorems 4.3, 4.4, and 4.5 are close to the presentation of S.M. Shah [Sha60]. Theorem 4.6 was proved by G. Valiron [Val50], our proof is different from the original one, although it uses the same idea.

The asymptotics of functions (5.1) was found by G. Valiron [Val14], however in some particular cases, for example, when $n(r, 0) \sim \Delta r^{\rho}(\ln r)^{\alpha}$, it was known already to E. Lindelöf [Lin02]. Our presentation is different from Valiron's method. Stronger results than those presented here are known in the case when $l(r)=\rho(r)$ is a proximate order (B.Ya. Levin [Lev80]). Examples 1, 2 and $1^{\prime}$ are due to A.A. Goldberg [Gol63]. The asymptotics of the functions considered in $2^{\circ}$ in the case $n(r, 0) \sim \Delta r^{\rho}(\ln r)^{\alpha}, \rho>0$ was studied already by E. Lindelöf [Lin02]. For an arbitrary proximate order $\rho(r)$ the results from Section $5,2^{\circ}$ follow from more general results of A.A. Goldberg [Gol64a]. The function $E_{\rho}(z)$ (5.31) was introduced (including the case of complex $\rho$ ) by G. Mittag-Leffler [Mit05], estimates (5.40), (5.43) are due to him. Asymptotic properties of functions $E_{\rho}(z)$ have been studied in detail, including the case of imaginary values of the parameter $\rho$. A series of papers by different authors devoted to this function was published in Acta 
Mathematica, 29 (1905); from recent works we would like to mention the work of M.M. Dzhrbashyan (see [Dzh66]) and a paper by M.N. Sheremeta [She69].

\section{Chapter 3}

The main in Section 1 Theorem 1.3 (the lemma on the logarithmic derivative) was first proved by R. Nevanlinna [NevR25a], [NevR29]. Theorem 1.1, giving the sharpest known estimate of $m\left(r, f^{\prime} / f\right)$, and the inequality (1.17) following from it, are due to $\mathrm{Vu}$ Ngoan and I.V. Ostrovskii [NO65]. Theorem 1.2 was proved by R. Nevanlinna [NevR31], who generalized an earlier result (Exercise 1) of E. Borel [Bor97].

Theorem 2.1 - the second fundamental theorem - was proved by R. Nevanlinna [NevR25a], [NevR29]. We would like to mention that, as it was discovered later, there are proofs of the second fundamental theorem which do not use the lemma on the logarithmic derivative. We refer only to proofs due to L. Ahlfors, which can be found in M.A. Evgrafov [Evg68, Chapter X, §6], R. Nevanlinna [NevR74, Chapter XIII], L. Ahlfors [Ahl35], S. Stoilow [Sto62, Vol. II, Chapter X]. Remark 1 to Theorem 2.1 is due to K. Yosida [Yos34].

Theorem 2.4 is due to H. Milloux [Mil40], [Mil47]. We present the proof given by K.-L. Hiong [Hio55]. He also proved Theorem 2.5 (K.-L. Hiong [Hio56]). Theorems 2.6 and 2.7 are due to W.K. Hayman [Hay59], [Hay64, §3.3].

Theorem 3.1 for meromorphic functions of finite order was proved by R. Nevanlinna [NevR25b], in the stated form it was proved by I.V. Ostrovskii [Ost60c], [Ost61]. Note that J. Dufresnoy [Duf39] gave an estimate of the sum $A_{\alpha \beta}\left(r, f^{\prime} / f\right)+$ $B_{\alpha \beta}\left(r, f^{\prime} / f\right)$ in terms of $S_{\alpha_{1} \beta_{1}}(r, f), \alpha_{1}<\alpha<\beta<\beta_{1}$; some estimates of different character were given by K. Habetha [Hab61a], [Hab61b].

Theorem 3.2 is due to M. Tsuji [Tsu50]. Our presentation follows the paper B.Ya. Levin and I.V. Ostrovskii [LO60].

\section{Chapter 4}

The notions of deficient values were introduced by R. Nevanlinna [NevR25a] and G. Valiron [Val26] in 1925-1926. A stronger result than Theorem 2.1 was obtained by G. Valiron [Val26]. In our proof of Theorem 2.1 we follow [Val60]. The strongest result showing 'sparseness' of the set $E_{V}(f)$ can be found in R. Navanlinna's book [NevR74, Chapter X, §3]. We state a weaker result: for each $\varepsilon>0$ and each continuous strictly monotone increasing function $h(r), 0 \leq r \leq 1$, $h(0)=0$, such that $\int_{0}^{1} h(r) d \ln r<\infty$, the set $E_{V}(f)$ can be covered by a sequence of discs with radii $r_{\nu}$, such that $\sum_{\nu} h\left(r_{\nu}\right)<\varepsilon$. Theorem 2.1 corresponds to the case $h(r)=\pi r^{2}$. Example 1 from Section 2 is due to G. Valiron [Val19] (in our presentation we have changed some details). D. Drasin and D.F. Shea [DS69] constructed a similar example of an entire function $f(z)$ with $T(r, f)=O\left(\psi(r) \ln ^{2} r\right)$, where $\psi(r) \rightarrow \infty$ is an arbitrary preassigned function. On the other hand, if an entire function $f(z)$ satisfies $T(r, f)=O\left(\ln ^{2} r\right)$, then $E_{V}(f) \backslash\{\infty\}=\emptyset$ (G. Valiron [Val14]).

For $0<\rho<\infty$ examples of meromorphic functions without deficient values were found by S.K. Singh [Sin61] (the idea of his construction can be easily extended to the cases $\rho=0$ and $\rho=\infty$ ). Our construction uses a different idea. 
Examples of meromorphic functions of order $\rho>0$ with preassigned at most countable set of deficient values were first constructed by A.A. Goldberg [Gol54a], [Gol59a]. W.K. Hayman [Hay64, §4.3] using A.A. Goldberg's idea, significantly simplified the construction. Our presentation in $\S 4$ is close to the presentation of W.K. Hayman, here we used also some of his observations made during our meeting in 1965. Lemma 4.1 (with a slightly different statement) was proved by W.H.J. Fuchs and W.K. Hayman [FH62] (see, also, [Hay64, §4.1.3]).

In our presentation of N.U. Arakelyan's example we, in addition to his paper [Ara66] used notes of his lectures at the Kharkov university in April 1967. Theorems 5.2 and 5.3 are special cases of certain results of M.V. Keldysh presented in S.N. Mergelyan's papers [Mer52], [Mer53].

Example 1 from Section 6 is due to D. Dugué [Dug47], Example 6 from Section 6 is due to W.K. Hayman [Hay53]. Result which we obtained as a corollary of Theorem 6.5 was first obtained (but under somewhat stronger restriction $\rho-$ $\lambda<1$ ) by G. Valiron [Val47]. The remaining results of Section 6 are due to A.A. Goldberg (see $\S 2$ of the paper P.P. Belinskiu and A.A. Goldberg [BG54]). Originally one of the essential steps of the construction of Example 2 was based on P.P. Belinskiu's theorem on univalent conformal mappings (see $\S 1$ of [BG54]), but later B.Ya. Levin pointed out that it is possible to construct such an example using the same idea, but not the theorem of P.P. Belinskil. This construction was carried out by A.A. Goldberg [Gol60b]. In Section 6 these results are presented in more detail than in the paper, some of them are published for the first time.

W.K. Hayman [Hay52] considered more general functions than in Example 6. His purpose was to establish the possibility of realization of (6.61) and to get more precise statements about the speed of convergence to $-\infty$ in (6.61).

\section{Chapter 5}

To Section 1. The notion of an asymptotic spot is essentially present already in the work of P. Boutroux [Bou08]. The term "asymptotic spot" was introduced by M. Heins [Hei57]. Another term used for the same notion is "asymptotic tract" (see, for example, G.R. MacLane [Mac63]). Theorem 1.1 is due to F. Iversen [Ive14] (see, also [NevR74, $\left.\mathrm{n}^{\circ} .239\right]$ ). Lemma 6.1 is due to E. Phragmén and E. Lindelöf (see, for example, B.Ya. Levin [Lev80, Chapter 1, §14]).

Theorem 1.2 is a weakened version of L. Ahlfors's theorem [Ahl32a] (see, also, R. Nevanlinna [NevR74, $\left.\mathrm{n}^{\circ} .258\right]$ ). In our proof we use the method due to T. Carleman [Car133] (see, also, A. Dinghas [Din36]).

Theorem 1.3 was proved by A. Wiman [Wim05] under the assumption that $\rho<\frac{1}{2}$, we state this theorem in the form in which it was first stated in M. Heins [Hei48]. Corollary 1 of Theorem 1.3 was first found by A. Edrei and W.H.J. Fuchs [EF56], Corollary 3 (in which the order $\rho$ appears instead of the lower order $\lambda$ ) was proved by J.E. Littlewood [Lit08]. W.K. Hayman [Hay52] proved that the quantity

$$
C(\rho)=\inf \limsup _{r \rightarrow \infty} \frac{\ln \mu(r, f)}{\ln M(r, f)},
$$

where the infimum is taken over all entire functions of order $\rho$, satisfies the inequality

$$
-K_{1}(\ln \rho+1) \leq C(\rho) \leq-K_{2}(\ln \rho+1), \rho \geq 1,
$$

where $0<K_{2}<K_{1}<\infty$ are absolute constants. 
Theorem $1.3^{\prime}$ was proved by K. Arima [Ari52]. V.P. Petrenko [Pet67] proved that if $f(z)$ is a meromorphic function of finite lower order $\lambda$, and $l$ is a number satisfying the conditions

$$
0 \leq l \leq 2 \pi, l<K \lambda^{-1} \delta^{\frac{1}{2}}(a, f) / \ln \frac{e}{\delta(a, f)},
$$

where $K$ is an absolute constant, then there exists a sequence of $\operatorname{arcs}\left\{|z|=r_{k}, \theta_{k} \leq\right.$ $\left.\arg z \leq \theta_{k}+l\right\}, r_{k} \rightarrow \infty$, on which $f(z)$ tends to $a$ uniformly in $\arg z$.

Theorem 1.4 was conjectured by A. Denjoy [Den07], who proved it under the additional assumption that $C_{j}$ are rays going out of $z=0$. T. Carleman [Carl21] proved Theorem 1.4 with a weaker estimate than (1.1). Theorem 1.4 in its full extent was first proved by L. Ahlfors [Ahl30]. Proving E. Lindelöf's Lemma 1.2 we used the presentations in G. Valiron [Val23, Chapter V, $\S 4$ ] and in N.G. Chebotarev and N.N. Mel̆man [CM49, Chapter II, §7].

To Section 2. The first example of an entire function (of order $\rho=\infty$ ) with deficient nonasymptotic value was constructed by W.K. Hayman [Hay53]; later A.A. Goldberg [Gol57b] constructed an analogous example in the case when $1<$ $\rho<\infty$, and, finally, N.U. Arakelyan [Ara66] in the case when $1 / 2<\rho<\infty$.

J.M. Anderson and J. Clunie [AC66] proved that if a meromorphic function $f(z)$ satisfies $T(r, f)=O\left(\ln ^{2} r\right)$, then each deficient value of $f(z)$ should be asymptotic (by Theorem 4.6 the function $f(z)$ has at most one deficient value).

Example 1 is due to O. Teichmüller [Tei39]. He considered the case $\rho=1 / 2$ only, but his construction can be easily carried over to the case $0<\rho<\infty$. Example 2 was constructed by A.A. Goldberg [Gol66]. Using the idea of Example 2 it is possible to construct an analogous example of a meromorphic function of the first order and of the maximal type (F.A. Baraket and A.A. Goldberg [BG69]). Such examples are not known for lower growth categories. The statement of Lemma 2.1 was first published in A. Wiman [Wim15]. Today much more general results are known (B.Ya. Levin [Lev80, Chapter II]).

Theorem 2.1 is well known, however, we did not succeed to find out the corresponding reference. In any case, all estimates needed for its prove were known to E. Borel [Bor00].

Examples 3 and 4 are published here for the first time. The observation that there are Borel exceptional values which are not deficient is due to G. Valiron [Val33]. Example 5 is due to A.A. Goldberg [Gol67].

In examples of meromorphic functions of order $\rho>0$ constructed in Section 3 of Chapter 4, each deficient value was asymptotic, in particular, these functions can have a countable set of asymptotic values. G. Valiron [Val25] proved that for each function $\psi(r) \rightarrow \infty$ as $r \rightarrow \infty$ there exists a meromorphic function $f(z)$ such that $T(r, f)<\psi(r) \ln ^{2} r, r>r_{0}$, and the set of asymptotic values of $f(z)$ has the cardinality of continuum. As was shown by G. Valiron [Val35b], if $T(r, f)=O\left(\ln ^{2} r\right)$, then $f(z)$ cannot have more than one asymptotic value. Y. Tumura [Tum43a] showed that the last condition can be replaced by the condition

$$
\liminf _{r \rightarrow \infty} T(r, f) / \ln ^{2} r<\infty .
$$

If we do not impose any restrictions onto $T(r, f)$, the structure of the set of asymptotic values is known. Namely, M. Heins [Hei53], [Hei55] proved that if a set $M, \infty \in M$ is analytic (Suslin), then it is a set of asymptotic values of an entire function; the necessity of this condition was proved earlier by S. Mazurkiewicz 
[Maz31]. For meromorphic functions a result, similar to Heins's was proved earlier by D.B. Potyagailo [Pot51], but his result was not published. We do not have an opportunity to present here these interesting results.

To Section 3. Theorem 3.1 sums up works of several authors. First O. Teichmüller [Tei39] proved that under the condition $\delta(\infty, f)>1-\cos \pi \rho$, where $\rho<1 / 2$ is the order of the function $f(z)$, the equality $\lim _{\sup } \rightarrow \infty\left|f\left(r e^{i \theta}\right)\right|=\infty$ holds for all $\theta, 0 \leq \theta<2 \pi$, and proved the statement of Theorem 3.1 under a stronger assumption $\delta(\infty, f)>\frac{1-\cos \pi \rho}{1-\varepsilon \cos \pi \rho}$, where $\varepsilon \geq 0$ is an absolute constant. Under the condition $\delta(\infty, f)>1-\cos \pi \rho$ the statement of Theorem 3.1 was proved by A.A. Goldberg [Gol54a], [Gol56].

The mentioned works of O. Teichmüller and A.A. Goldberg relied on the Hadamard theorem on canonical representation of meromorphic functions of finite order. The Hadamard theorem is inapplicable in the study of functions of finite lower order. A generalization of the results to the case of functions of finite lower order became possible due to the idea of B. Kjellberg [Kje60], which can be described in the following way: a function of finite lower order for some sequence $R=R_{k} \rightarrow \infty$ can be represented as a product of two factors, one of which is a quotient of two canonical products, and the second is very close to one in the disc $\left\{|z| \leq R_{k}\right\}$. A precise statement, somewhat more general than the one due to B. Kjellberg, is contained in Lemma 3.1, which was essentially proved already in the paper by F. Nevanlinna [NevF23] (see, also, R. Nevanlinna [NevR74, Ch. VIII, sect. 177]).

Theorem 3.1, its corollary, Theorems 3.2, 3.3, and the inequality (3.29) were obtained by A.A. Goldberg and I.V. Ostrovskii (published in I.V. Ostrovskii [Ost63a]). See, also, the preceding paper A.A. Goldberg and I.V. Ostrovskii [GO61a], containing a somewhat weaker result. Theorem 3.1 and its corollary were independently obtained by A. Edrei [Edr64]. The statement of the corollary for $\lambda=0$ was obtained significantly earlier by A. Edrei, W.H.J. Fuchs [EF59b].

The inequality $\limsup _{r \rightarrow \infty} \frac{N(r, 0, f)}{\ln M(r, f)} \geq \frac{\sin \pi \rho}{\pi \rho}$ for entire functions of order $\rho<1$ is due to A. Wahlund [Wah29] and G. Valiron [Val30] (a simple proof was shown by G. Valiron [Val35a]).

Theorem 3.4 is due to B. Kjellberg [Kje60] (see, also, B. Kjellberg [Kje63], where its refinement is presented). It is a generalization of the classical theorem proved independently by A. Wiman [Wim15] and G. Valiron [Val14]: each entire function $f(z)$ of order $\rho<1$ satisfies

$$
\limsup _{r \rightarrow \infty} \frac{\ln \mu(r, f)}{\ln M(r, f)} \geq \cos \pi \rho .
$$

Different problems related to the Wiman-Valiron theorem are treated in many papers. A survey of these papers is given in A.A. Goldberg and I.V. Ostrovskii [GO61b].

The main idea of the method used in the proofs of Theorems 3.2-3.4 is due to A. Denjoy [Den30]. This method was later developed in G. Valiron [Val35a], B. Kjellberg [Kje54], [Kje60], A.A. Goldberg and I.V. Ostrovskii [GO61a]. The last paper contains a general formulation of the method, using it one can immediately get Theorems 3.2, 3.3, and several other relations. We decided not to give here this general formulation because of its complexity. 
The construction of the examples showing how sharp are Theorems 3.2-3.4 in the case $\lambda=\rho$ is due to A. Edrei and W.H.J. Fuchs [EF60], and of the example showing how sharp is Theorem 3.1 is due to O. Teichmüller [Tei39]. These authors considered functions with positive zeros and $n(r, 0) \sim \alpha r^{\rho}$ instead of the functions $f(z, \alpha)$.

To Section 4. The estimate (4.1) with $d(\rho)=|\sin \pi \rho| / C_{1}([\rho])$ was obtained by R. Nevanlinna [NevR29] and later sharpened by S.M. Shah [Sha44] and A.A. Goldberg [Gol57a]. The parameter $\kappa(f)$ was introduced by R. Nevanlinna [NevR29], who conjectured that the best estimate of this parameter from below in terms of $\rho$ is $\kappa(f) \geq \nu_{1}(\rho)$.

The estimate (4.4) confirming R. Nevanlinna's conjecture for $\rho<1$ and close to the best possible for $\rho>1$ is due to A. Edrei and W.H.J. Fuchs [EF59a], [EF60]. The estimate (4.3) is due to I.V. Ostrovskii [Ost63b]; close but somewhat weaker estimate was obtained independently by A. Edrei [Edr64]. The method consists in extension of the method of works A. Edrei and W.H.J. Fuchs [EF59a], [EF60] with the help of the idea of B. Kjellberg mentioned in our notes to Section 3.

Lemmas 4.2 and 4.5 are due to A. Edrei and W.H.J. Fuchs [EF59a], [EF60].

Lemma 4.4 is due to A.A. Goldberg [Gol54a], [Gol56], who also found its generalizations [Gol57c]. The presented proof of Lemma 4.4 is taken from A. Edrei [Edr64]. Lemma 4.6, part B is an analogue of G. Pólya's theorem on sequences (G. Pólya, G. Szegö [PS98, Vol. 1, Ch. 3, probl. 107-110], it was obtained in a more general form by S.M. Shah [Sha40], [Sha41]. Lemma 4.6, part A is due to I.V. Ostrovskii [Ost63b].

A version of Theorem 4.4 with the lower order $\lambda$ replaced by the order $\rho$ is due to A. Edrei and W.H.J. Fuchs [EF60]. Theorem 4.4 was proved independently by A. Edrei [Edr62] and I.V. Ostrovskii [Ost63a]. The method is an extension of the method from A. Edrei and W.H.J. Fuchs [EF60].

To Section 5. Examples similar to Examples 1-5 can be constructed in a uniform manner using a result from S.M. Shah [Sha46a]. In this paper entire functions $f(z)=\sum_{k=0}^{\infty} a_{k} z^{k}$, such that the sequence $\left|a_{k} / a_{k+1}\right|$ is non-decreasing, are considered, and the formulas

$$
\rho[f]=\limsup _{k \rightarrow \infty} \frac{\ln k}{\ln \left|a_{k} / a_{k+1}\right|}, \lambda[f]=\liminf _{k \rightarrow \infty} \frac{\ln k}{\ln \left|a_{k} / a_{k+1}\right|}
$$

are obtained.

Theorem 5.2 with less sharp estimate than (5.5) was in a different way obtained by A. Edrei and W.H.J. Fuchs [EF59b]. Our presentation follows I.V. Ostrovskii [Ost63b]. The corollary of Theorem 5.2 and Theorem 5.3 with somewhat less sharp estimates were obtained by A. Edrei and W.H.J. Fuchs [EF59b], they also proved Lemma 5.1. Much earlier A. Pfluger [Pfl46] established that for an entire function $f(z)$ of finite order $\rho$ with $\sum_{a \neq \infty} \delta(a, f)=1$ the number $\rho$ is a positive integer, and all deficiencies are of the form $k / \rho$, where $k$ is an integer; thus the number of finite deficient values in this case does not exceed $\rho$. A. Edrei and W.H.J. Fuchs [EF59a] complemented this result of A. Pfluger by showing that all deficient values are asymptotic. They established an analogous result for "almost all" deficient values of meromorphic functions satisfying the condition (5.7) with sufficiently small value of $\gamma$. 
Example 8 is a representative of a wide class of similar examples considered by F. Nevanlinna [NevF29]. ${ }^{16}$ F. Nevanlinna [NevF29] conjectured that if a meromorphic function $f(z)$ satisfies $\sum_{a} \delta(a, f)=2$, then $\rho[f]=q / 2$, where $q$ is a positive integer. This conjecture is still unproved. ${ }^{17}$

Example 9 was constructed by A.A. Goldberg [Gol64b], however his construction of the auxiliary function $g(z)$ is different. Presented construction of the function $g(z)$ is due to B.Ya. Levin and I.V. Ostrovskii.

To Section 6. The relation $m(r, f)=O(1)$ for functions of the form (6.1) was proved by M.V. Keldysh [Kel54]. Theorem 6.1 representing a strengthening of this result was obtained by I.V. Ostrovskii (published in A.A. Goldberg [Gol60b]). Lemma 6.1, making possible the sharpening of the method of M.V. Keldysh, is due to V.I. Smirnov [Smi28].

Theorem 6.2 is due to M.V. Keldysh [Kel54], the relation (6.4) makes it possible to simplify the original proof.

Theorem 6.3 is due to I.V. Ostrovskii [Ost61].

\section{Chapter 6}

To Section 1. Functions with $(\rho, \eta)$-separated zeros and poles were introduced by A.A. Goldberg [Gol60a], he also proved ([Gol60a], [Gol61]) Theorems 1.1, 1.2, 1.4, the corollaries of Theorem 1.1, constructed Examples 1 and 3. All other results of this section are due to A. Edrei and W.H.J. Fuchs, S. Hellerstein [EFH61]. In the case when $f(z)$ is an entire function, Corollary 3 of Theorem 1.5 was proved by A. Edrei and W.H.J. Fuchs [EF59b]. The same paper contains the first proof of Corollary 1 under the a priori assumption $\rho[f]<\infty$.

The papers A. Edrei, W.H.J. Fuchs and S. Hellerstein [EFH61] and [Hel63] contain results showing that under certain condition of arithmetic character imposed onto arguments of zeros and poles of a meromorphic function $f(z)$ for which $\rho[N(r, 0, \infty)]$ is finite but sufficiently large, the inequalities $\delta(0, f)>0, \delta(\infty, f)>0$ hold.

To Section 2. The first results on connections between the growth of a meromorphic function and the distribution of its values with respect to the arguments are due to L. Bieberbach [Bie19] and R. Nevanlinna [NevR25b].

The paper of L. Bieberbach [Bie19] contains in implicit form the following statement. Let $f(z)$ be an entire function of finite order such that all of its $a$ - and $b$-points $(a \neq b ; a, b \neq \infty)$ belong to the system of rays $D=D\left(\alpha_{1}, \ldots, \alpha_{n}\right)$. Then the growth of the function $f(z)$ is does not exceed the normal type of order $\omega(D)$. R. Nevanlinna [NevR25b] showed that this estimate is valid also in the case when $a$ - and $b$-points satisfy the condition

$$
\sum_{j=1}^{n}\left\{C_{\alpha_{j} \alpha_{j+1}}(r, a)+C_{\alpha_{j} \alpha_{j+1}}(r, b)\right\}=O(1) .
$$

\footnotetext{
${ }^{16}$ In fact, the function from example 8 belongs to the class of functions constructed in Theorem 5.1, Chapter VII. (Editor).

${ }^{17}$ It is proved now in [A44], see the Editor's comments.
} 
Besides, R. Nevanlinna [NevR25b] proved that this growth estimate holds for a meromorphic function $f(z)$ of finite order, if

$$
\sum_{j=1}^{n}\left\{C_{\alpha_{j} \alpha_{j+1}}(r, a)+C_{\alpha_{j} \alpha_{j+1}}(r, b)+C_{\alpha_{j} \alpha_{j+1}}(r, c)\right\}=O(1)
$$

( $a, b$, and $c$ are three different points from the extended complex plane) and the growth categories of at least two of the functions $N(r, a), N(r, b), N(r, c)$ do not exceed the convergence class of order $\omega(D)$.

Note that these results are contained in Corollary 3 from Theorem 2.1 and in Theorem 2.4.

In the cited above results of L. Bieberbach and R. Nevanlinna finiteness of the order of $f(z)$ is an a priori assumption. M.G. Krein [Kre47] considered entire functions $f(z)$ with zeros close to the real line, such that $1 / f(z)$ can be represented in the form (2.29), and found growth estimates without an a priori assumption of finiteness of the order. We stated the result of M.G. Krein as Corollary 2 of Theorem 2.10. Theorems 2.9 and 2.10 can be considered as generalizations of this result.

A. Edrei $[\mathbf{E d r 5 5}]$ proved the following theorem. Let $D=D\left(\alpha_{1}, \ldots, \alpha_{n}\right)$ be a system of rays, $f(z)$ be a meromorphic function, such that for some $a \neq 0, \infty$ and some integer $l \geq 0$ all solutions of the equations

$$
f(z)=0, f(z)=\infty, f^{(l)}(z)=a
$$

except, possibly, finitely many of them lie on the rays of the system $D$. If $\delta(0, f)+$ $\delta(\infty, f)+\delta\left(a, f^{(l)}\right)>0$, then the order of the function $f(z)$ does not exceed $\omega(D)$. In the case when $\delta\left(a, f^{(l)}\right)>0$, this theorem of A. Edrei is contained in the corollary of Theorem 2.5; the growth estimate in the Edrei theorem is less precise, and the argument constraints are more restrictive. It is essential that in the corollary of Theorem 2.5 the argument restrictions are imposed onto solutions of only two of the equations (K.1). In the case that $\delta\left(a, f^{(l)}\right)>0$, Edrei's theorem cannot be derived from Theorem 2.5. However, using the method of the proof of Theorem 2.5, it is not difficult to prove (see I.V. Ostrovskii [Ost61]) a generalization of Theorem 2.5, also containing these cases of the Edrei theorem.

All results of the present section, except Theorems 2.7, 2.8, Corollary 2 of Theorem 2.10, and Examples 2 and 4 are due to I.V. Ostrovskii [Ost57], [Ost58], [Ost60a], [Ost61].

Using a method, similar to the method of I.V. Ostrovskii [Ost58], [Ost61], A. Edrei and W.H.J. Fuchs [EF62b] proved the following theorem:

Let

$$
L_{j}=\left\{z=r e^{i \alpha_{j}(r)}, r_{0} \leq r<\infty\right\}, j=1,2, \ldots, n,
$$

be a system of $n$ rectifiable curves in the plane, where $\alpha_{j}(r), r \geq r_{0}, j=1, \ldots, n$, are continuous functions, and

$$
\alpha_{1}(r)<\alpha_{2}(r)<\cdots<\alpha_{n}(r)<\alpha_{1}(r)+2 \pi=\alpha_{n+1}(r), r \geq r_{0} .
$$

Suppose that the length $s_{j}\left(r_{1}, r_{2}\right)$ of the curve $L_{j} \cap\left\{r_{1} \leq|z| \leq r_{2}\right\}$ admits the estimate

$$
s_{j}\left(r_{1}, r_{2}\right) \leq B\left(r_{2}-r_{1}\right)
$$

where $B$ is a constant. If all zeros and poles of the meromorphic function $f(z)$ except, possibly, finitely many lie on the indicated system of curves, and for some 
$a \neq 0, \infty$ and integer $l \geq 0$ the inequality $\delta\left(a, f^{(l)}\right)>0$ holds, then the order of the function $f(z)$ does not exceed

$$
\max _{1 \leq j \leq n} \limsup _{r \rightarrow \infty} \frac{9 \pi B^{2}}{\alpha_{j+1}(r)-\alpha_{j}(r)} .
$$

Theorem 2.7 is a simple corollary of results of R. Nevanlinna [NevR25b] (Theorem 3.1 in Section 3) and W.K. Hayman [Hay56] (the corollary of Theorem 3.2 in Section 3).

Theorem 2.8 somewhat complements the following result due to A. Edrei and W.H.J. Fuchs [EF62a] (some other results of the same character can be found in A. Edrei and W.H.J. Fuchs $[\mathbf{E F 6 2 b}]$, A. Edrei [Edr62]).

Let $f(z)$ be a meromorphic function. Suppose that there exists a number $\delta$, $0 \leq \delta<1$, and a sequence $\left\{r_{k}\right\}, r_{k} \uparrow \infty$, such that the annuli

$$
S_{k}=\left\{\frac{r_{k}}{\sigma_{k}} \leq|z| \leq \sigma_{k} r_{k}\right\}, k=1,2, \ldots,
$$

where $\sigma_{k}=1+\left\{\ln T\left(r_{k}, f\right)\right\}^{-\delta}$ have the following property: to each $S_{k}$ there correspond $n \geq 1$ numbers

$$
\alpha_{k 1}, \alpha_{k 2}, \ldots, \alpha_{k n} ; \quad \alpha_{k 1}<\alpha_{k 2}<\ldots \alpha_{k n}<\alpha_{k 1}+2 \pi
$$

in such a way that the number of zeros and poles of the function $f(z)$ lying in

$$
S_{k} \backslash \bigcup_{j=1}^{n}\left\{\left|\arg z-\alpha_{k j}\right| \leq\left\{\ln T\left(\sigma_{k} r_{k}, f\right)\right\}^{-1-\eta}\right\}
$$

does not exceed

$$
O\left(T^{c}\left(r_{k}\right)\right)
$$

$(\eta>\delta, c<1$ are constants).

Then the function $f(z)$ has at most $n+1$ deficient values. If the function $f(z)$ has $n+1$ deficient values, then 0 and $\infty$ are among deficient values.

Example 2 is taken from A. Edrei [Edr55] and Example 4 is taken from A. Edrei and W.H.J. Fuchs [EF62a].

To Section 3. Theorem 3.1 is due to R. Nevanlinna [NevR25b]. We prove Theorem 3.2 using the Cartan-Ahlfors method (see R. Nevanlinna [NevR74, Chapter V, §5]), which W.K. Hayman [Hay56] used to prove the result which we state as a corollary of Theorem 3.2. Our presentation follows this paper of W.K. Hayman. Theorem 3.3 is published here for the first time. Theorem 2.3 which is an immediate corollary of Theorem 3.3 originally was obtained (I.V. Ostrovskii [Ost61]) in a different way.

To Section 4. Theorem 4.1 (I.V. Ostrovskii [Ost60b]) is a generalization of L. Bieberbach's [Bie19] theorem, which we state as Corollary 2 of Theorem 4.1. Example 1 is due to L. Bieberbach [Bie19], Lemma 4.1 is due to O. Teichmüller [Tei39], Example 2 is due to I.V. Ostrovskii [Ost60b].

To Section 5. Theorem 5.2 was obtained by I.V. Ostrovskii [Ost60c]. Theorems 5.3, 5.4, and 5.5 are due to B.Ya. Levin and I.V. Ostrovskii [LO60]. Lemma 5.3 is a classical result due to C. Carathéodory. Theorem 5.3 is a strengthening of the following result of A. Edrei [Edr55]: If an entire function $f(z)$ is representable in the form $f(z)=e^{g(z)} h(z)$, where $g(z)$ is an entire function, $h(z)$ is an entire function of finite order, and all zeros of the function $f(z) f^{\prime}(z) f^{\prime \prime}(z)$ except, possibly, 
finitely many are real, then $\rho[g] \leq 1$. S. Hellerstein $[$ Hel66] found a generalization of Theorem 5.3. An extensive bibliography of works devoted to the question of G. Pólya and A. Wiman can be found in B.Ya. Levin and I.V. Ostrovskii [LO60]. ${ }^{18}$

\section{Chapter 7}

We present Theorem 1.2 following S. Kakutani [Kak35], who stated it in a somewhat more general form and made a small error. Theorem 1.2 is contained in a stronger result of Y. Tumura [Tum43b] and H.L. Selberg [Sel44], [Sel46], who, in particular, proved that under the conditions of Theorem 1.2 the relation $m(r, a)=O(\ln r)$ holds. Under the additional restriction that all algebraic branch points of $P$ are projected to $a$ (each region $F_{\nu}$ can have at most one branch point), Theorem 1.2 was proved by E.F. Collingwood [Col24] and H. Cartan [Cart30].

In Section 2 we present the necessary preliminaries from the theory of quasiconformal mappings. The theory of quasiconformal mappings is presented in L.I. Volkovyskiū [Vol54] and L. Ahlfors [Ahl66].

Most of the examples is contained (sometimes implicitly) in the monograph L.I. Volkovyskiı̌ [Vol50]. O. Teichmüller [Tei44] considered a class of quasiconformal mappings solving the problem from Example 7, and found a mapping for which $\max _{|z| \leq R} p(z, w)$ attains its minimum. Comparison with Teichmüller's result shows that the estimate (2.17) (see L.I. Volkovyskiı [Vol50, p. 110]) approaches the best possible as $x_{0} \rightarrow 0$.

Theorem 3.1 was obtained by O. Teichmüller ([Tei38], see, also, H. Wittich [Wit68, Chapter VI]) as a corollary of his theorem on moduli of ring domains. H. Wittich [Wit48a] gave another proof of the Teichmüller theorem, using a method of L. Ahlfors [Ahl32b] (Step III of our proof). We present H. Wittich's proof with significant simplifications. P.P. Belinskiı [Bel53], [Bel54] proved that under the conditions of Theorem 3.1 the $\operatorname{limit}_{z \rightarrow \infty} w(z) / z$ exists.

The class $F_{q}$ of Riemann surfaces, as well as the notion of a line complex, were introduced by R. Nevanlinna [NevR32a], [NevR32b]. A detailed theory of line complexes was developed by G. Elfving [Elf34]. Our presentation differs of that of G. Elfving. The dependence of the line complex on the choice of the base curve was studied by E. Drape [Dra36], H. Habsch [Hab52], V.G. Tairova [Tai62], [Tai64]. F. Huckemann [Huc56a] extended the class $F_{q}$ and the notion of a line complex.

Theorem 4.1 should follow from a more general result of H. Weyl [Wey31], but this result, in author's own words, is stated in a so inefficient form, that it is not clear how to get any specific corollaries of it.

Our proof, which is close to certain argument of V.G. Tairova [Tai64] allows us to make an important for our purposes remark at the end of the proof. The corollary of Theorem 4.1 was proved, using line complexes, by Le-Van-Thiem [LeV50]. In familiar sources (A. Hurwitz [Hur91], [Hur02], H. Weyl [Wey31], H. Röhrl [Röh63]) we have not found anything indicating that the problem posed in Section 4 before Example 1 is not always solvable. Theorems 4.2 and 4.3 are published here for the first time.

The statement of the inverse value distribution problem is due to R. Nevanlinna (see, for example, [NevR74, Sect. 220]).

\footnotetext{
${ }^{18}$ This question is now completely solved. See the Editor's comments at the end of the book.
} 
The hypothesis that the deficiencies of a meromorphic function of a finite order should satisfy some other relations besides $\sum_{a} \delta(a, f) \leq 2$ was stated by $\mathrm{O}$. Teichmüller [Tei39]. W.H.J. Fuchs [Fuc58] proved that for functions of finite order the relation $\sum_{a} \sqrt{\delta(a, f)}<\infty$ holds. This result was strengthened by W.K. Hayman [Hay64], who established that for functions of finite lower order and for $\alpha>1 / 3$ the series $\sum_{a} \delta^{\alpha}(a, f)$ is convergent, and observed that for $\alpha<1 / 3$ such series can diverge. The behavior of this series for $\alpha=1 / 3$ has not been studied, ${ }^{19}$ but V.P. Petrenko [Pet67] proved that for $\beta>1 / 3$ the series $\sum_{a} \delta^{1 / 3}(a, f) \ln ^{-\beta} \frac{e}{\delta(a, f)}$ converges.

W.H.J. Fuchs [Fuc58] and W.K. Hayman [Hay64] obtained also estimates for the sum of the series $\sum_{a} \delta^{\alpha}(a, f)$ in terms of $\lambda[f]$; the sharpest estimates are due to V.P. Petrenko [Pet66], [Pet67].

R. Nevanlinna [NevR32b] proved Theorem 5.1 using Riemann surfaces with finitely many logarithmic ends and without any algebraic branch points. To study the value distribution of the uniformizing functions he used some results of the analytic theory of differential equations. Using the same method G. Elfving [Elf34] studied uniformizing functions of surfaces with finitely many logarithmic ends, defined as in our Section 5. L. Ahlfors $[\mathbf{A h l 3 2 b}]$ proved the results of R. Nevanlinna [NevR32b] without using differential equations.

Results of Sections 6, 7, 8 are due to A.A. Goldberg [Gol54b] (this paper contains some errors). However various particular cases of these results were known before. A detailed survey of previous results can be found in Chapter VIII of the book H. Wittich [Wit68]. Here we mention the most important papers. The class of Riemann surfaces with periodic ends was introduced by E. Ullrich [Ull36]. H. Wittich [Wit47], [Wit48b] used quasiconformal mappings for the study of the value distribution of the uniformizing functions of such surfaces, and obtained the formulas (7.17), (7.18), (7.19) for surfaces with periodic ends. Note that for surfaces $\Phi$ with periodic ends it is not necessary to construct a quasiconformal mapping onto the complex plane, the problem we were concerned with in Section 6 , since the uniformizing meromorphic function can be easily written in an explicit form (see the exercise after Theorem 6.1). Le-Van-Thiem [LeV49], [LeV50] solved the inverse problem under the following restrictions: 1) The number of deficient and index values is finite; 2) The deficiencies and indexes are rational numbers; 3) If $\varepsilon(a)>0$, then $\delta(a)+\varepsilon(a)<1$. In the case when $\sum_{a} \delta(a)=0$ Le-Van-Thiem solved the problem using elliptic functions; in the case $\sum_{a} \delta(a)>0$ he used Riemann surfaces with finitely many periodic ends and characteristic $\chi=1$. K. Pöschl [Pös51] noticed that if drop the restriction $\chi=1$, then, with the help of Riemann surfaces with finitely many periodic ends, the inverse problem can be solved, at best (for $0<\sum_{a} \delta(a)<2$ ), if the deficiencies and indexes belong to a very narrow subclass of the set of algebraic numbers.

\footnotetext{
${ }^{19}$ Weitsman [A157] proved the convergence for $\alpha=1 / 3$.
} 
Le-Van-Thiem [LeV49], [LeV50] introduced the notion of a conjunction of surfaces with periodic ends and obtained the formulas (7.23)-(7.27) for them. However he did not take into consideration the conditions under which conjunction is possible and the conditions under which the formulas (7.25)-(7.27) are valid, for this reason some parts of his argument are not convincing. The idea to use the calculus of diagrams (see Section 8) is also due to Le-Van-Thiem.

It was conjectured that, if in Theorem 5.1 we restrict our attention to functions of finite order, then the requirements of rationality of deficiencies and of the finiteness of the number of deficient values become necessary. ${ }^{20}$ For entire functions this conjecture has been proved (A. Pfluger [Pfl46]).

In connection with Theorem 8.3 we note that the first example of a meromorphic function with infinitely many index values was constructed by G. af Hällström [Häl52], but in his construction severe restrictions were imposed both on the location of index values and on the values of indexes. G. af Hällström [Häl41] also found the first example of a function with $\varepsilon(a)=1$ (see, also, H. Wittich [Wit68, Chapter VIII, §5]).

The class of Riemann surfaces with almost periodic ends is, as it was mentioned, a generalization of a class of Riemann surfaces with periodic ends. The class of Riemann surfaces with periodic ends was generalized also in other directions. We mention in this connection the papers of F. Huckemann [Huc56b], A.A. Goldberg [Gol59b], H.P. Künzi and H. Wittich [KW59], where the reader can find further references.

\footnotetext{
${ }^{20}$ This is proved in [A44].
} 



\section{Bibliography}

[Ahl29] Ahlfors, L.: Beiträge zur Theorie der meromorphen Funktionen. In: VII Congrés des mathematiciens scand., Oslo, 84-88 (1929).

[Ahl30] Ahlfors, L.: Untersuchungen zur Theorie der konformen Abbildung und der ganzen Funktionen. Acta Soc. Sci. Fenn., 1, no. 9, 1-40 (1930)

[Ahl32a] Ahlfors, L.: Über die asymptotischen Werte der meromorphen Funktionen endlicher Ordnung. Acta Acad. Aboensis. Math. et Phys., 6, no. 9, 1-8 (1932)

[Ahl32b] Ahlfors, L.: Über eine in der neueren Wertverteilungstheorie betrachtete Klasse transzendenter Funktionen. Acta math., 58, 375-406 (1932)

[Ahl35] Ahlfors, L.: Zur Theorie der Überlagerungsflächen. Acta Math., 65, 157-194 (1935)

[Ahl66] Ahlfors, L.: Lectures on quasiconformal mappings. Van Nostrand Mathematical Studies, Toronto (1966)

[Akh27] Akhiezer, N.I.: A new approach to necessary conditions under which an entire function of integer order has a given type (Ukrainian). Zap. fiz.-mat. otd. AN USSR, Kiev, 2 (1927), no. 3, 29-33.

[AC66] Anderson, J. M., Clunie, J.: Slowly growing meromorphic functions. Comment. Math. Helv., 40, 267-280 (1966)

[Ara66] Arakelyan, N. U.: Entire functions of finite order with an infinite set of deficient values. (Russian) Dokl. Akad. Nauk SSSR, 170, 999-1002 (1966). English transl.: Sov. Math., Dokl. 7, 1303-1306 (1966).

[Ari52] Arima, K.: On maximum modulus of integral functions. Journ. Math. Soc. Japan, 4, 62-66 (1952).

[BG69] Baraket, F. A., Goldberg, A. A.: Deficient nonasymptotic values of first order meromorphic functions. (Russian) Sibirsk. Mat. Zh., 10, 483-487 (1969). English transl.: Sib. Math. J. 10, 345-348 (1969).

[Bel53] Belinskiū, P. P.: Behavior of a quasi-conformal mapping at an isolated point. (Russian) Doklady Akad. Nauk SSSR (N.S.), 91, 709-710 (1953)

[Bel54] Belinskiǔ, P. P.: Behavior of a quasi-conformal mapping at an isolated singular point. (Russian) Lvov. Gos. Univ. Uch. Zap. Ser. Meh.-Mat., 29, no. 6, 58-70 (1954)

[BG54] Belinskiü, P. P., Goldberg, A. A.: Application of a theorem on conformal mappings to questions of invariance of deficiencies of meromorphic functions. (Russian) Ukraïn. Mat. Zh., 6, 263-269 (1954)

[Bie19] Bieberbach, L.: Über eine Vertiefung des Picardschen Satzes bei ganzen Funktionen endlicher Ordnung. Math. Z., 3, 175-190 (1919)

[Bor97] Borel, É.: Sur les zeros des fonctions entieres. Acta math., 20, 357-396 (1897)

[Bor00] Borel, É.: Leçons sur les fonctions entieres. Paris (1900); Second edition (1921)

[Bou08] Boutroux, P.: Sur l'indetermination d'une fonction uniforme au voisinage d'une singularite transcendente. Ann. Sci. Ecole Norm. Sup., 25, 318-370 (1908)

[Carl21] Carleman, T.: Sur les fonctions inverses des fonctions entieres d'ordre fini. Ark. for Mat. Astr. och Fys., 15, no. 10 (1921)

[Carl23] Carleman, T.: Über die Approximation analytischer Funktionen durch lineare Aggregate von vorgegebenen Potenzen. Ark. for Mat., Astr. och Fysik, 17, no. 9, 1-30 (1923)

[Carl33] Carleman, T.: Sur une inegalite differentielle dans la theorie des fonctions analytiques. C. r. Acad. sci., 196, 995-997 (1933)

[Cart29a] Cartan, H.: Sur la fonction de croissance attachee a une fonction meromorphe de deux variables et ses applications aux fonctions meromorphes d'une variable. C. r. Acad. sci., 189, 521-523 (1929) 
[Cart29b] Cartan, H.: Sur la croissance des fonctions meromorphes d'une ou de plusieurs variables complexes. C. r. Acad. sci., 188, 1374-1376 (1929)

[Cart30] Cartan, H.: Sur les valeurs exceptionnelles d'une fonction meromorphe dans tout le plan. C. r. Acad. sci., 190, 1003-1005 (1930)

[Cart33] Cartan, H.: Sur les zeros des combinaisons lineaires de p fonctions holomorphes donnees. Mathematica, 7, 5-31 (1933)

[CM49] Chebotarev, N. G., Meimman, N. N.: The Routh-Hurwitz problem for polynomials and entire functions. Real quasipolynomials with $r=3, s=1$. (Russian) Appendix by G. S. Barhin and A. N. Hovanskiı̌. Trudy Mat. Inst. Steklov., 26, 1-331 (1949)

[Col24] Collingwood, E. F.: Sur les valeurs exceptionnelles des fonctions entieres d'ordre fini. C. r. Acad. sci., 179, 1125-1127 (1924)

[Den07] Denjoy, A.: Sur les fonctions entieres de genre fini. C. r. Acad. sci., 145 (1907)

[Den30] Denjoy, A.: Sur une theoreme de Wiman. C. r. Acad. sci., 193, 828-830 (1930)

[Din36] Dinghas, A.: Bemerkung zu einer Differentialungleichung von Carleman. Math. Z., 41, 713-716 (1936)

[Dra36] Drape, E.: Über die Darstellung Riemannscher Flächen durch Streckenkomplexe. Deutsche Math., 1, 805-824 (1936)

[DS69] Drasin, D., Shea, D. F.: On the Valiron deficiencies of integral functions. Bull. London Math. Soc., 1, 174-178 (1969)

[Duf39] Dufresnoy J.: Sur les fonctions meromorphes dans une angle. C. r. Acad. sci., 208, 718-720 (1939)

[Dug47] Dugué, D.: Le défaut au sens de M. Nevanlinna depend de l'origine choisie. C. r. Acad. sci., 225, 555-557 (1947)

[Dzh66] Dzhrbashyan, M. M.: Integral transforms and representations of functions in the complex domain. (Russian) Nauka, Moscow (1966)

[Edr55] Edrei, A.: Meromorphic functions with three radially distributed values. Trans. Amer. Math. Soc., 78, no. 2, 276-293 (1955)

[Edr62] Edrei, A.: Meromorphic functions with values that are both deficient and asymptotic. Studies in mathematical analysis and related topics. Essays in honor of George Pólya. Stanford University Press, 93-103 (1962)

[Edr64] Edrei, A.: The deficiencies of meromorphic functions of finite lower order. Duke Math. J., 31, 1-21 (1964)

[EF56] Edrei, A., Fuchs, W. H. J.: On the regularity of the growth of meromorphic functions with highly deficient zeros and poles. Report no. 59, Cornell University, Ithaca, N. Y. (1956)

[EF59a] Edrei, A., Fuchs, W. H. J.: Valeurs deficientes et valeurs asymptotiques des fonctions meromorphes. Comment. Math. Helv., 33, 258-295 (1959)

[EF59b] Edrei, A., Fuchs, W. H. J.: On the growth of meromorphic functions with several deficient values. Trans. Amer. Math. Soc., 93, no. 2, 292-328 (1959)

[EF60] Edrei, A., Fuchs, W. H. J.: On the deficiencies of meromorphic functions of order less than one. Duke Math. J., 27, no. 2, 233-249 (1960)

[EF62a] Edrei, A., Fuchs, W. H. J.: Bounds for the number of deficient values of certain classes of meromorphic functions. Proc. London Math. Soc., 12, 315-344 (1962)

[EF62b] Edrei, A., Fuchs, W. H. J.: On meromorphic functions with regions free of poles and zeros. Acta math., 108, 113-145 (1962)

[EFH61] Edrei, A., Fuchs, W. H. J., Hellerstein, S.: Radial distribution and deficiencies of the values of a meromorphic function. Pacif. J. Math., 2, 135-151 (1961)

[Elf34] Elfving, G.: Über eine Klasse von Riemannschen Flachen and ihre Uniformisierung. Acta Soc. Sci. Fenn., N. S., 2, no. 3, 1-60 (1934)

[Evg68] Evgrafov, M. A.: Analytic functions. Second revised and enlarged edition. Nauka, Moscow (1968). English transl.: Dover, New York, 1978.

[Fuc58] Fuchs, W. H. J.: A theorem on the Nevanlinnas deficiencies of meromorphic functions of finite order. Ann. of Math., 68, 203-209 (1958)

[FH62] Fuchs, W. H. J., Hayman, W. K.: An entire function with assigned deficiencies. Studies in mathematical analysis and related topics. Essays in honor of George Pólya. Stanford University Press, 117-125 (1962)

[Gol54a] Goldberg, A. A.: On deficiencies of meromorphic functions. (Russian) Dokl. Akad. Nauk SSSR (N.S.), 98, 893-895 (1954) 
[Gol54b] Goldberg, A. A.: On the inverse problem of the theory of the distribution of the values of meromorphic functions. (Russian) Ukraïn. Mat. Zh., 6, 385-397 (1954)

[Gol56] Goldberg, A. A.: Some asymptotic properties of meromorphic functions. (Russian) Uchen. Zap. Lvov. Univ., Ser. Mekh.-Mat., 38, no. 7 (1956)

[Gol57a] Goldberg, A. A.: An estimate of the sum of the deficiencies of a meromorphic function of order less than unity. (Russian) Dokl. Akad. Nauk SSSR (N.S.), 114, 245-248 (1957)

[Gol57b] Goldberg, A. A.: An example of an entire function of finite order with a non-asymptotic deficient value. (Russian) Uzhgorod. Gos. Univ. Nauchn. Zap., 18, 191-194 (1957); English translation: Amer. Math. Soc. Transl. (2), 18, 45-47 (1961)

[Gol57c] Goldberg, A. A.: On an inequality connected with logarithmically convex functions. (Ukrainian) Dopovidi Akad. Nauk Ukraïn. RSR, no. 3, 227-230 (1957)

[Gol59a] Goldberg, A. A.: On the set of deficient values of meromorphic functions of finite order. (Russian) Ukraïn. Mat. Zh., 11, 438-443 (1959)

[Gol59b] Goldberg, A. A.: On a class of Riemann surfaces. (Russian) Mat. Sb. (N.S.), 49 (91), 447-458 (1959)

[Gol60a] Goldberg, A. A.: Meromorphic functions with separated zeros and poles. (Russian) Izv. Vyssh. Uchebn. Zaved. Matematika, no. 4 (17), 67-72 (1960)

[Gol60b] Goldberg, A. A.: Some problems of the value distribution theory (Russian). An appendix in the Russian translation of [Wit68], pp. 263-300.

[Gol61] Goldberg, A. A.: Distribution of values of meromorphic functions with separated zeroes and poles.(Russian) Dokl. Akad. Nauk SSSR, 137, 1030-1033 (1961); English translation: Soviet Math. Dokl., 2, 389-392 (1961)

[Gol63] Goldberg, A. A.: Three examples of entire functions. (Ukrainian) Dopovidi Akad. Nauk Ukraïn. RSR, no. 4, 443-446 (1963)

[Gol64a] Goldberg, A. A.: The integral with respect to a semi-additive measure and its application to the theory of entire functions. III. (Russian) Mat. Sb. (N.S.), 65 (107), 414-453 (1964)

[Gol64b] Goldberg, A. A.: On the possible value of the lower order of an entire function with a finite deficient value. (Russian) Dokl. Akad. Nauk SSSR, 159, 968-970 (1964)

[Gol66] Goldberg, A. A.: Non-asymptotic deficient values of meromorphic functions. (Russian) Dokl. Akad. Nauk SSSR, 171, 254-257 (1966)

[Gol67] Goldberg, A. A.: Non-asymptotic deficient values of entire functions of infinite order. (Russian) Mat. Zametki, 1, no. 1, 81-86 (1967)

[GO61a] Goldberg, A. A., Ostrovskiı̌, I. V.: Some theorems on the growth of meromorphic functions. (Russian) Zap. Matem. Otd. Kharkov. Univ. and Kharkov. Mat. Ob., 27, 3-37 (1961)

[GO61b] Goldberg, A. A., Ostrovskiı̌, I. V.: New investigations on the growth and distribution of values of entire and meromorphic functions of genus zero. (Russian) Uspekhi Mat. Nauk, 16, no. 4, 51-62 (1961)

[Gol69] Goluzin, G. M.: Geometric theory of functions of a complex variable. American Mathematical Society, Providence, R.I. (1969)

[Gov69] Govorov, N. V.: The Paley conjecture. (Russian) Funktsional. Anal. i Prilozhen., 3, no. 2, 41-45 (1969)

[Hab61a] Habetha, K.: Eine Bemerkung zur Werteverteilung meromorpher Funktionen in der Halbebene. Arch. Math., 12, 43-50 (1961)

[Hab61b] Habetha, K.: Über die Werteverteilung in Winkelraumen. Math. Z., 77, 453-467 (1961)

[Hab52] Habsch, H.: Die Theorie der Grundkurven und das Aquivalenzproblem bei der Darstellung Riemannscher Flächen. Mitt. Math. Seminar Giefien, no. 42, 1-51 (1952)

[Had93] Hadamard J.: Sur les proprietes des fonctions entieres et en particulier une fonction etudiee par Riemann. Journ. math, pure appl., 9, 171-215 (1893)

[Häl41] Hällström, G. af: Zwei Beispiele ganzer Funktionen mil algebraischem Hochstindex einer Stellensorte. Math. Z., 47, 161-174 (1941)

[Häl52] Hällström, G. af: Eine quasikonforme Abbildung mit Anwendungen aui die Wertverteilungsiehre. Acta Acad. Aboensis. Math, et Phys., 18, no. 8, 1-16 (1952)

[Hay52] Hayman, W. K.: The minimum modulus of large integral functions. Proc. London Math. Soc., 2, 469-512 (1952)

[Hay53] Hayman, W. K.: An integral function with a deficient value that is neither asymptotic nor invariant under change of origin. J. London Math. Soc., 28, 369-376 (1953) 
[Hay56] Hayman, W. K.: Questions of regularity connected with the Phragmen-Lindelof principle. J. Math, pures et appl., 35, 115-126 (1956)

[Hay59] Hayman, W. K.: Picard values of meromorphic functions and their derivatives. Ann. of Math., 70, 9-42 (1959)

[Hay64] Hayman, W. K.: Meromorphic functions. Clarendon Press, Oxford (1964)

[Hay65] Hayman, W. K.: On the characteristic of functions meromorphic in the plane and of their integrals. Proc. London Math. Soc., 14A, 93-128 (1965)

[Hei48] Heins, M.: Entire functions with bounded minimum modulus; subharmonic functions analogues. Ann. of Math., 49, 200-213 (1948)

[Hei53] Heins, M.: The set of asymptotic values of an entire function. 12-te Skand. matematikerkongr., Lund, 56-60 (1953)

[Hei55] Heins, M.: Meromorphic functions with assigned asymptotic values. Duke Math. J., 22, 353-356 (1955)

[Hei57] Heins, M.: Asymptotic spots of entire and meromorphic functions. Ann. of Math., 66, 430-439 (1957)

[Hel63] Hellerstein, S.: On a class of meromorphic functions with deficient zeros and poles. Pacif. J. Math., 13, 115-124 (1963)

[Hel66] Hellerstein, S.: On the zeros of an entire function and its second derivative. Ill. J. Math., 10, 488-496 (1966)

[HR64] Hellerstein, S., Rubel, L. A.: Subfields that are algebraically closed in the field of all meromorphic functions. Journ. analyse math., 12, 105-111 (1964)

[Hio55] Hiong, King-Lai: Nouvelle demonstration et amelioration d'une inegalite de M. Milloux. Bull. sci. math., 79, 135-160 (1955)

[Hio56] Hiong, King-Lai: Sur la limitation de $T(r, f)$ sans intervention des poles. Bull. sci. math., 80, 175-190 (1956)

[Huc56a] Huckemann, F.: Zur Darstellung von Riemannschen Flächen durch Streckenkomplexe. Math. Z., 65, 215-239 (1956)

[Huc56b] Huckemann, F.: Über den Einfluß von Randstellen Riemannschen Flächen auf die Wertverteilung. Math. Z., 65, 240-282 (1956)

[Hur91] Hurwitz, A.: Über Riemann'sche Flachen mit gegebenen Verzweigungspunkten. Math. Ann., 39, 1-61 (1891)

[Hur02] Hurwitz, A.: Über die Anzahl der Riemann'schen Flachen mit gegebenen Verzweigungspunkten. Math. Ann., 55, 53-66 (1902)

[Ive14] Iversen, F.: Recherches sur les fonctions inverses des fonctions meromorphes. These, Helsingfors, 1-67 (1914)

[Jen99] Jensen, J. L. W. V.: Sur un nouvel et important théorème de la théorie des fonctions. Acta math., 22, 359-364 (1899)

[Kak35] Kakutani, S.: On the exceptional value of meromorphic functions. Proc. Phys.-Math. Soc. Japan, 17, 174-176 (1935)

[Kar30] Karamata, J.: Sur un mode de croissance reguliere des fonctions. Mathematica, 4, 38-53 (1930)

[Kel54] Keldysh, M. V.: On series of rational fractions. (Russian) Doklady Akad. Nauk SSSR (N.S.), 94, 377-380 (1954)

[Kje54] Kjellberg, B.: A relation between the maximum and minimum modulus of a class of entire functions. 12-te Scand. matematikerkongr., Lund, 135-138 (1954)

[Kje60] Kjellberg, B.: On the minimum modulus of entire functions of lower order less than one. Math. Scand., 8, 189-197 (1960)

[Kje63] Kjellberg, B.: A theorem on the minimum modulus of entire functions. Math. Scand., 12, 5-11 (1963)

[KAB49] Korevaar, J., Aardenne-Ehrenfest, T., van, Bruijn, N. Q., de: A note on slowly oscillating functions. Nieuw Archief voor Wiskunde, 23, 77-86 (1949)

[Kre47] Krein, M.: A contribution to the theory of entire functions of exponential type. (Russian) Izvestiya Akad. Nauk SSSR. Ser. Math., 11, 309-326 (1947)

[KW59] Künzi, H. P., Wittich, H.: The distribution of $a$-points of certain meromorphic functions. Michigan Math. J., 6, 105-121 (1959)

[LeV49] Le-Van-Thiem: Über das Umkehrproblem der Wertverteilungslehre, Comment. Math. Helv., 23, 26-49 (1949) 
[LeV50] Le-Van-Thiem: Sur un problème d'inversion dans la théorie des fonctions méromorphes. Ann. Sci. Ecole Norm. Sup., 67, 51-98 (1950)

[Lev41] Levin, B. Ja.: On functions holomorphic in a half-plane, Trudy Odess. Derzh. Univ., 3, 5-14 (1941)

[Lev80] Levin, B. Ja.: Distribution of zeros of entire functions. American Mathematical Society, Providence, R.I. (1980)

[LO60] Levin, B. Ja., Ostrovskiı̌, I. V.: The dependence of the growth of an entire function on the distribution of zeros of its derivatives. (Russian) Sibirsk. Mat. Zh., 1, 427-455 (1960) English transl.: AMS Translations (2) 32 (1963), 323-357.

[Lin02] Lindelöf, E.: Mémoire sur la théorie des fonctions entières de genre fini. Acta Soc. Sci Fenn., 31, no. 1 (1902)

[Lin05] Lindelöf, E.: Sur les fonctions entières d'ordre entier. Ann. Sci. École Norm. Sup., 22, 365-395 (1905)

[Lit08] Littlewood, J. E.: A general theorem on integral functions of finite order. Proc. London Math. Soc., 6, 189-204 (1908)

[Mac63] MacLane, G. R.: Asymptotic values of holomorphic functions. Rice Univ. Studies, 49, no. 1,83 pp. (1963)

[Mar77] Markushevich, A. I.: Theory of functions of a complex variable. Vol. I, II, III. Chelsea Publishing Co., New York (1977)

[Mat60] Matsaev, V. I.: On the growth of entire functions that admit a certain estimate from below. (Russian) Dokl. Akad. Nauk SSSR, 132, 283-286 (1960); English translation: Soviet Math. Dokl., 1, 548-552 (1960)

[Maz31] Mazurkiewicz, S.: Sur les points singuliers d'une fonction analytique. Fund. math., 17, 26-29 (1931)

[Mer52] Mergelyan, S. N.: Uniform approximations of functions of a complex variable. (Russian) Uspehi Matem. Nauk (N.S.), 7, no. 2(48), 31-122 (1952)

[Mer53] Mergelyan, S. N.: On completeness of systems of analytic functions. (Russian) Uspehi Matem. Nauk (N.S.), 8, no. 4(56), 3-63 (1953)

[Mil40] Milloux, H.: Les fonctions méromorphes et leurs dérivées. Extensions d'un théorème de M. R. Nevanlinna. Applications, Paris (1940)

[Mil47] Milloux, H.: Les derivées des fonctions meromorphes et la theorie des defauts. Ann Sci. Ecole Norm. Sup., 3, 289-316 (1947)

[Mit05] Mittag-Leffler, G.: Sur la représentation analytique d'une branche uniforme d'une fonction monogène. Acta math., 29, 101-181 (1905)

[NevF22a] Nevanlinna, F.: Über die Beziehungen zwischen dem Anwachsen einer analytischen Funktion und der Verteilung ihrer Nullstellen und Pole. V Congrès des mathématiciens scand., Helsingfors (1922)

[NevF22b] Nevanlinna, F.: Sur les relations qui existent entre la distribution des zéros et des pôles d'une fonction monogène et la croissance de son module. C. r. Acad. sci., 175, 676-679 (1922)

[NevF23] Nevanlinna, F.: Bemerkungen zur Theorie der ganzen Funktionen endlicher Ordnung. Soc. Sci. Fenn. Comment. Phys.-math., 2, no. 4, 1-7 (1923)

[NevF29] Nevanlinna, F.: Über eine Klasse meromorpher Funktionen. 7-te Congrés Math. Scand., Oslo, 81-83 (1929)

[NN22] Nevanlinna, F., Nevanlinna, R.: Über die Eigenschaften analytischer Funktionen in der Umgebung einer singulären Stelle oder Linie. Acta Soc. sci. Fenn., 50, no. 5, 1-46 (1922)

[NevR24] Nevanlinna, R.: Untersuchungen über den Picard'schen Satz. Acta Soc. sci. Fenn., 50, no. $6,1-42(1924)$

[NevR25a] Nevanlinna, R.: Zur Theorie der meromorphen Funktionen. Acta math., 46, 1-99 (1925)

[NevR25b] Nevanlinna, R.: Über die Eigenschaften meromorpher Funktionen in einem Winkelraum. Acta Soc. Sci. Fenn., 50, no. 12, 1-45 (1925)

[NevR29] Nevanlinna, R.: Le théorème de Picard-Borel et la théorie des fonctions méromorphes. Paris (1929)

[NevR31] Nevanlinna, R.: Remarques sur les fonctions monotones. Bull. sci. math., 55, 140-144 (1931) 
[NevR32a] Nevanlinna, R.: Über die Riemannsche Fläche einer analytischen Funktion. Verhandl. Iinternat. Mathematiker-Kongr., Zurich, B. I. (1932)

[NevR32b] Nevanlinna, R.: Über Riemannsche Flächen mit endlich vielen Windungspunkten. Acta math., 58, 295-373 (1932)

[NevR74] Nevanlinna, R.: Eindeutige analytische Funktionen. Springer-Verlag, Berlin (1974)

[NO65] Ngoan, Vu., Ostrovskiı̌, I. V.: The logarithmic derivative of a meromorphic function. (Russian) Akad. Nauk Armjan. SSR Dokl., 41, 272-277 (1965)

[Ost57] Ostrovskǐ̌, I. V.: A generalization of a theorem of M. G. Kreı̌n. (Russian) Dokl. Akad. Nauk SSSR (N.S.), 116, 742-745 (1957)

[Ost58] Ostrovskiı̌, I. V.: On meromorphic functions taking certain values at points lying near a finite system of rays. (Russian) Dokl. Akad. Nauk SSSR, 120, 970-972 (1958)

[Ost60a] Ostrovskiŭ, I. V.: On the relation of the growth of a meromorphic function to the distribution of its values according to their arguments. (Russian) Dokl. Akad. Nauk SSSR, 132, 48-51 (1960); English translation: Soviet Math. Dokl., 1, 485-488 (1960)

[Ost60b] Ostrovskiı̌, I. V.: An estimate for the deficiency of a meromorphic function for which two values are distributed inside a certain angle. (Russian) Izv. Vyssh. Uchebn. Zaved. Matematika, no. 2 (15), 138-148 (1960)

[Ost60c] Ostrovskiur, I. V.: On the location of the zeros of the derivative of an entire function whose zeros lie close to the real axis. (Russian) Dokl. Akad. Nauk SSSR, 130, 973-976 (1960); English translation: Soviet Math. Dokl., 1, 114-117 (1960)

[Ost61] Ostrovskǐ̌, I. V.: The connection between the growth of a meromorphic function and the distribution of the arguments of its values. (Russian) Izv. Akad. Nauk SSSR. Ser. Mat., 25, 277-328 (1961)

[Ost63a] Ostrovskǐ̌, I. V.: Deficiencies of meromorphic functions of order less than one. (Russian) Dokl. Akad. Nauk SSSR, 150, 32-35 (1963)

[Ost63b] Ostrovskǐr, I. V.: A problem from the theory of distribution of values. (Russian) Dokl. Akad. Nauk SSSR, 151, 34-37 (1963)

[Pal32] Paley, R. E. A. C.: A note on integral functions. Proc. Cambridge Philos. Soc., 28, 262-265 (1932)

[Pet66] Petrenko, V. P.: Certain estimates for the values of the deficiencies of a meromorphic function. (Russian) Sibirsk. Mat. Zh., 7, 1319-1336 (1966), English transl.: Sib. Math. J. 7, 1035-1047 (1966).

[Pet67] Petrenko, V. P.: On the growth of meromorphic functions of finite lower order and on the magnitudes of their deficiencies. (Russian) Sibirsk. Mat. Zh., 8, 1156-1189 (1967). English transl.: Sib. Math. J. 8, 883-909 (1967).

[Pet69] Petrenko, V. P.: Growth of meromorphic functions of finite lower order. (Russian) Izv. Akad. Nauk SSSR Ser. Mat., 33, 414-454 (1969). English transl.: Math. USSR, Izv. 3, 391-432 (1969).

[Pfl46] Pfluger, A.: Zur Defektrelation ganzer Funktionen endlicher Ordnung. Comment. Math. Helv., 19, 91-104 (1946)

[PS98] Pólya, G., Szegö, G.: Problems and theorems in analysis. I. Series, integral calculus, theory of functions. Springer-Verlag, Berlin (1998)

[Pös51] Pöschl, K.: Über die Wertverteilung der erzeugenden Funktionen Riemannscher Flächen mit endlich vielen periodischen Enden. Math. Ann., 123, 79-95 (1951)

[Pot51] Potyagailo, D. B.: Asymptotic and boundary properties of meromorphic functions. Master's Thesis, Lvov University (1951)

[Pri56] Priwalow, I. I.: Randeigenschaften analytischer Funktionen. (German) VEB Deutscher Verlag der Wissenschaften, Berlin (1956)

[Röh63] Röhrl, H.: Unbounded coverings of Riemann surfaces and extensions of ring of meromorphic functions. Trans. Amer. Math. Soc., 107, 320-346 (1963)

[Rub63] Rubel, L. A.: A Fourier series method for entire functions. Duke Math. J., 30, 437-442 (1963)

[Sel44] Selberg, H. L.: Über einen Satz von Collingwood. Arch. Math. Naturv., B 48, no. 9, 119-126 (1944)

[Sel46] Selberg, H. L.: Eine Ungleichung der Potentialtheorie und ihre Anwendung in der Theorie der meromorphen Funktionen. Comment. Math. Helv., 18, 309-326 (1946)

[Sha40] Shah, S. M.: A theorem on integral functions of integral order. J. London Math. Soc., 15, 23-31 (1940) 
[Sha41] Shah, S. M.: A theorem on integral functions of integral order, II. J. Indian Math. Soc., N. S., 5, 179-188 (1941)

[Sha44] Shah, S. M.: A note on meromorphic functions. Math. Student, 12, 67-70 (1944)

[Sha46a] Shah, S. M.: On the lower order of integral functions. Bull. Amer. Math. Soc., 52, 1046-1052 (1946)

[Sha46b] Shah, S. M.: On proximate orders of integral functions. Bull. Amer. Math. Soc., 52, 326-328 (1946)

[Sha48] Shah, S. M.: A note on lower proximate orders. J. Indian Math. Soc., 12, no. 1-2, 31-32 (1948)

[Sha60] Shah, S. M.: An extension of Lindelöf's theorem to meromorphic functions. Tôhoku Math. J., 12, 333-338 (1960)

[She69] Sheremeta, M. N.: Asymptotic behavior of functions of Mittag-Leffler type and their application. (Russian) Izv. AN Arm. SSR. Matematika, 4, no. 2, 144-148 (1969)

[Shi29] Shimizu, T.: On the theory of meromorphic functions. Japanese J. Math., 6, 119-171, (1929)

[Sin61] Singh, S. K.: On exceptional values of entire and meromorphic functions. Tôhoku Math. J., 13, 373-380 (1961)

[Smi28] Smirnov, V. I.: Sur les valeurs limites des fonctions régulières à l'interieur d'un cercle. Zhurnal Leningr. Fiz.-Mat. Ob-va, 2, no. 2, 22-37 (1928)

[Sto62] Stoïlow, S.: Teoria funcţiilor de o variabilă complexă, vol. 1-2, Editura Academiei Republicii Populare Române, 1954. Russian transl.: Theory of functions of a complex variable. Inostrannaya Literatura, Moscow (1962).

[Tai62] Tairova, V. G.: On "Streckenkomplexe" of closed Riemann surfaces of genus zero. (Russian) Izv. Vyssh. Uchebn. Zaved. Matematika, no. 4 (29), 155-160 (1962)

[Tai64] Tairova, V. G.: "Streckenkomplexe" of a certain class of closed Riemann surfaces. (Russian) Sibirsk. Mat. Zh., 5, 929-951 (1964)

[Tei38] Teichmüller, O.: Untersuchungen über konforme und quasikonforme Abbildung. Deutsche Math., 3, 621-678 (1938)

[Tei39] Teichmüller, O.: Vermutungen und Sätze über die Wertverteilung gebrochener Funktionen endlicher Ordnung. Deutsche Math., 4, 163-190 (1939)

[Tei44] Teichmüller, O.: Ein Verschiebungssatz der quasikonformen Abbildung. Deutsche Math., 7, 336-343 (1944)

[Tsu50] Tsuji, M.: On Borel's directions of meromorphic functions of finite order. Tôhoku Math. J., 2, no. 2, 97-112 (1950)

[Tum43a] Tumura, Y.: Recherches sur la distribution des valeurs des fonctions analytiques. Japanese J. Math., 18, 797-876 (1943)

[Tum43b] Tumura, Y.: Sur une extension d'un théorème de M. Teichmüller. Proc. Imp. Acad. Tokyo, 19, 55-59 (1943)

[Ull36] Ullrich, E.: Zum Umkehrproblem der Wertverteilungslehre. Nachr. Qes. Wiss. Göttingen, Math.-Phys. Kl., 1, no. 9, 135-150 (1936)

[Val14] Valiron, G.: Sur les fonctions entières d'ordre nul et d'ordre fini et en particulier les fonctions à correspondance régulière. Ann. fac. sci. Univ. Toulouse, 5, 117-257 (1914)

[Val19] Valiron, G.: Sur les zéros des fonctions entières d'ordre fini. Rend. Circ. mat. Palermo, 43, 255-268 (1919)

[Val23] Valiron, G.: Lectures on the general theory of integral functions. Toulouse, (1923)

[Val25] Valiron, G.: Sur les valeurs asymptotiques de quelques fonctions méromorphes. Rend. Circ. mat. Palermo, 49, 415-421 (1925)

[Val26] Valiron, G.: Sur la distribution des valeurs des fonctions méromorphes. Acta math., 47, 117-142 (1926)

[Val30] Valiron, G.: Sur un théorème de M. Wiman. Opuscula math. A. Wiman dedicata, 1-12 (1930)

[Val31] Valiron, G.: Sur la dérivée des fonctions algébrö̈des. Bull. Soc. math. France, 59, no. 1-2, 17-39 (1931)

[Val33] Valiron, G.: Remarques sur les valuers exceptionnelles des fonctions méromorphes. Rend. Circ. mat. Palermo, 57, 71-86 (1933)

[Val35a] Valiron, G.: Sur le minimum du module des fonctions entières d'ordre inferieur à un. Mathematica, 11, 264-269 (1935) 
[Val35b] Valiron, G.: Sur le nombre des singularités transcendantes des fonctions inverses d'une classe d'algébroïdes. C. r. Acad. sci., 200, 713-715 (1935)

[Val47] Valiron, G.: Valeurs exceptionnelles et valeurs déficientes des fonctions méromorphes. C. r. Acad. sci., 225, 556-558 (1947)

[Val50] Valiron, G.: Sur les valeurs déficientes des fonctions méromorphes d'ordre nul. C. r. Acad. sci., 230, 40-42 (1950)

[Val60] Valiron, G.: Fonctions entières d'ordre fini et fonctions méromorphes. Genève (1960)

[Vol50] Volkovyskiǔ, L. I.: Investigation of the type problem for a simply connected Riemann surface. (Russian) Trudy Mat. Inst. Steklov., 34, 1-171 (1950)

[Vol54] Volkovyskiı̌, L. I.: Quasiconformal mappings (Russian), Lvov, Lvov State University Publishers, 1954.

[VLA91] Volkovyskiı̌, L. I., Lunts, G. L., Aramanovich, I. G.: A collection of problems on complex analysis. Dover Publications, Inc., New York (1991)

[Wah29] Wahlund, A.: Über einen Zusammenhang zwischen dem Maxirnalbetrage der ganzen Funktion und seiner unteren Grenze nach dem Jensensche Theoreme. Arkiv Math., 21 A, no. 23, 1-34 (1929)

[Wey31] Weyl, H.: Über das Hurwitzsche Problem der Bestimmung der Anzahl Riemannscher Flächen von gegebener Verzweigungsart. Comment. Math. Helv., 3, 103-113 (1931)

[WW96] Whittaker, E. T., Watson, G. N.: A course of modern analysis. An introduction to the general theory of infinite processes and of analytic functions; with an account of the principal transcendental functions. Cambridge University Press, Cambridge (1996)

[Wim05] Wiman, A.: Sur une extension d'une théorème de M. Hadamard. Ark. för Math., Astr., Fys., 2, no. 14, 1-5 (1905)

[Wim15] Wiman, A.: Über eine Eigenschaft der ganzen Funktionen von der Höhe Null. Math. Ann., 76, 197-211 (1915)

[Wit47] Wittich, H.: Über die Wachstumsordnung einer ganzen transzendenten Funktion. Math. Z., 51, 1-16 (1947)

[Wit48a] Wittich, H.: Zum Beweis eines Satzes über quasikonforme Abbildungen. Math. Z., 51, 278-288 (1948)

[Wit48b] Wittich, H.: Über eine Klasse meromorpher Funktionen. Arch. Math., 1, 160-166 (1948)

[Wit68] Wittich, H.: Neuere Untersuchungen über eindeutige analytische Funktionen. SpringerVerlag, Berlin (1968)

[Yos34] Yosida, K.: On a class of meromorphic functions. Proc. Phys.-Math. Soc. Japan, 16, 227-235 (1934) 


\section{Index}

Note: the pages are still according to the Russian version

Aardenne-Ehrenfest T. van, 567. 584

Ahlfors L. V., 15, 18, 21, 23, 29, 30, 43, 151, 226. $234,430,433,566,568569,574-$ 576,581

Akhiezer N. I., 85. 578

Anderson J. M., 570, 581

Arakeljan N. U., 172, 563, 569, 578

Aramanovich I. G., 446. 578

Arima K., 569, 531

Baraket F. A., 570. 578

Belinskiǔ P. P., 569, 575, 578

Bessel F. W., 317

Bieberbach L., 573, 574. 581

Blumenthal O. 567

Bolzano B., 448, 455

Boutroux P., 567. 569, 581

Borel É., 5, 79, Il5, 116, 119, 120, 130, 147. 150, 158. 171. 177. 226. 334. 369, 380. 392. $438,567.570,581$

Bruijn N. G. de, 567, 584

Buniakowsky V. Ya. 88, 229, 230. 433, 449, 450

Carathéodory C., 575

Carleman T., 19. 38, 39, 234 566, 569, 581

Cartan H., 33, 153, 432, 566, 674, 575, 581

Casorati F., 5

Cauchy A.-L., 12, 17, 88, 112, 126, 178, 181, 189, 192. 193, 229, 230. 242, 256. 257, 401, 413, 433, 438. 448-450, 455, 558, 559. 562,566

Chebotarev N. G., 569, 580

Clunie J., 137, 570, 581

Collingwood E. F., 575, 581

Denjoy A., 234, 569, 571, 581

Dinghas A., 569, 581

Drape E., 575, 581

Drasin D., 568,582

Dufresnoy J., 568, 582

Dugué D., 195, 196, 193, 202, 205. 568, 582
Dzhrbashyan M. M., 566, 567, 579

Edrei A., 256, 308. 566, 569. 571-575 582

Elfving G., 575, 576, 582

Euler L., 108, 291. 467

Evgrafov M. A., 568, 579

Fatou P., 328

Fourier J.-B., 86, 87

Fubini G., 35, 37

Fuchs W. H. J., 167, 256, 308. 488, 566, 568, 569, 571-574. 576.582

Goldberg A. A., 567-572, 576-579

Goluzin G. M., 429, 443, 578

Govorov N. V. 555, 565, 578

Green G., 9-11. 13, 20, 22, 54, 393

Habetha K., 568, 582

Habsch H., 575, 582

Hadamard J., 5, 80, 148. 566, 567, 570, 582

Hällström G. af, 577, 583

Hankel H., Ill, 1)3

Hayman W. K., 6, 78, 123, 134, 136, 137, 167, 216, 488, 555, 568, 569, 574, 576 . 583

Heine E., 115. 177. 438

Heins M., 232, 262. 569, 570, 583

Hellerstein S., 566, 572, 573, 575, 582, 583

Hiong King-Lai, 133, 568, 583

Huckemann F., 575. 577, 583

Hurwitz A., 468, 500, 512, 575. 583

Iversen F., 224, 240, 569, 683

Jensen J. L. W. V., 15-18, 24. 27. 28, 33, $54,56,87,133.433,566,583$

Kakutani S., 575, 584

Karamata J., 567, 584

Keldysh M. V., 173, 177, 558, 572. 579

Kellogg O. D., 443

Kjellberg B., 570. 571. 584

Korevaar J., 567, 583

Krein M. G., 573, 579

Künzi H. P., 577, 584 
Lagrange J. L., 73. 220

Laguerre E., 413, 415

Laurent P., 17, 27, 30. 32, 173. 289, 272

Lebesgue H., 386

Leibniz G. W., 397, 425

Le-Van-Thiem, 575-577, 584

Levin B. Ja., 5, 19, 21, 41, 42, 171. 423, 566-570. 572, 575, 58x

L'Hospital G. F. A. de, 67, 74.' 108, 266

Lindelöf E., 80, 85, 234, 388, 566, 667, 569, 584

Littlewood J. E., 569, 584

Lunts G. L., 446. 578

MacLane G. R., 569, 579

Markushevich A. I., 459, 579

Mascheroni L., 108, 291

Matsaev V. I., 561, 579

Mazurkiewicz S., 570, 584

Meĭman N. N., 569, 580

Mergelyan S. N., 568. 580

Milloux H., 132, 568, 584

Mittag-Leffler G., Ill, 150. 367, 399, 554, 567.

Morera G., ) 12

Nevanlinna R., 5. 6, 9, 15, 17, 23, 27, 31, $36,37,39,40,43,55,58,116,119-121$, $123,126,127,137,145-147,150,158$, 172. 195. 196, 226, 236, 337, 358, 362, 373, 392, 403. 418, 421, 489, 566-569. 571. 573-576. 585

Nevanlinna F., 566, 571, 572. 584, 585

Ngoan $\mathrm{Vu}, 567,578$

Ostrovskiŭ I. V., 423, 567, 568, 571. 572. 574. $575,578-580$

Paley R. E. A. C., 565, 585

Parseval M. A., 88

Petrenko V. P., 565, 569, 576, 580

Pfluger A., 572, 577, 585

Phragmén E., 569

Picard E., 5, 116, 131, 147, 158

Poisson S. D., 12, 15, 16, 18, 54, 56. 566

Pólya G., 417, 423. 428, 571, 575, 58C

Pöschl K., 576, 585

Potyagailo D. B., 570, 580

Priwalow (or Privalov? In the text we used Privalov, reference is German) I. I., $11,75,580$

Riemann B., 11, 12, 17, 29, 32, 401. 438, $467,468,500,512.558$

Röhrl H., 575, 585

Rolle M., 426

Rubel L. A., 85, 566, 583. 585

Schwarz H. A., 11, 17, 424. 566

Selberg H. L., 575, 585
Shah S. M., 567, 571, 572, 585

Shea D. F., 568,582

Sheremeta M. N., 280, 567, 580

Shimizu T., 15, 18, 21. 23, 29, 30, 43, 433, 556,585

Singh S. K., 568. 586

Smirnov V. I., 572, 580

Sokhotskii Yu. V., 5, 569

Stieltjes T. J., 24, 386

Stirling J., 111

Stoïlow, S., 429, 430, 434, 469. 489 568. 580

Szegö G., 571, 580

Tairova V. G., 462, 575, 580

Teichmüller O., 447, 496, 526, 549, 553, 570, $571,574-576,586$

Tsuji M., 37, 41, 43, 137. 141, 146, 337, 418, $420,421,566,568,586$

Tumura Y., 137, 570, 575, 586

Ullrich E., 576. 586

Valiron G., 5, 90，147，150. 151，158. 195, $224,226,262$. 565-571, 586

Volkovyskiı̌ L. I., 5, 446, 499, 575, 578

Wahlund A., 565, 571, 586

Watson G. N., 290, 317, 378, 580

Weierstrass K., 5, 76, 78-80. 165, 171

Weyl H., 575, 586

Whittaker E. T., 290. 317. 378, 580

Wiman A., 232, 262, 417. 423, 428. 569-571, 575,587

Wirtinger W., 227, 229

Wittich H., 575-577, 584, 587,

Yosida K., 568, 587 


\section{Index}

Almost periodic end

Angular Nevanlinna characteristic

Asymptotic curve

Asymptotic spot

Asymptotic value

Base curve

Base point

Borel exceptional value

Canonical product

Carleman formula

Characteristic of a quasiconformal mapping

Characteristic of a surface with almost periodic ends

Class $A$

Class $F_{q}$

Closed simply connected Riemann surface

Constant dilation on a curve

Convergence class

$J$-curve

$J^{\prime}$-curve

Deficiency

Deficiency relation

Degree of a rational function

Divergence class

Dugué's example

Edge (otrezok on p. 460 originala)

Edge bundle

Elementary algebraic domain

Elementary logarithmic domain

Elliptic type

End of the first kind

End of the second kind

Equivalence of proximate orders

Finite order

First fundamental theorem

First fundamental theorem for angular Nevanlinna characteristics

First fundamental theorem for Nevanlinna characteristics for half-plane
First fundamental theorem for Tsuji characteristics

Fuchs-Hayman lemma

Function with separated zeros and poles

Genus of a canonical product

Genus of a meromorphic function

Genus of a sequence

Geometric deficiency

Geometric index

Green's formula

Green's function

Growth category

Hankel's formula

Hayman's example

Hyperbolic type

Index

Index value

Infinite order

Integral representation of the Gamma-function

Integral representation of the Mittag-Leffler function

Inverse problem of the value distribution theory

Jacobian

Jensen's formula

Kernel of a Riemann surface

Lemma on the logarithmic derivative

Levin's formula

Lindelöf's lemma

Line complex

Line complex of a Riemann surface

Logarithmic end

Lower order of a function

Lower proximate order of a function

Magnitude of type

Magnitude of type with respect to a proximate order

Mapping function of a Riemann surface

Maximal type 
Mean type

Minimal type

Mittag-Leffler function

Narrow inverse problem of the value distribution theory

Nevanlinna characteristic

Nevanlinna characteristic for half-plane

Nevanlinna deficiency

Nevanlinna exceptional value

Nevanlinna formula

Normal type

Order of a function

Order of an $a$-point

Parabolic type

Periodic end

Picard exceptional value

$a$-point

$a$-point of a quasiconformal mapping

Poisson kernel

Poisson-Jensen formula

Proximate order

Proximate order of a function

Pseudokernel

Quasiconformal mapping

Ramification index

Rational function

$j$-region

Relative upper measure

Riemann-Hurwitz formula

Riemann surface corresponding to a function

Riemann surface of elliptic type

Riemann surface of hyperbolic type

Riemann surface of parabolic type

Riemann surface with finitely many logarithmic ends

Riemann surface with finitely many almost periodic ends

Scheme

Scheme solution (reshenie skhemy)

Second fundamental theorem

Second fundamental theorem for angular Nevanlinna characteristics

Second fundamental theorem for Tsuji characteristics

Second Green's formula

Set of finite logarithmic measure

Shimizu-Ahlfors formula

Simple point (prostaya tochka)

Simply connected Riemann surface

Slowly varying function

Spherical characteristic

Spherical derivative

Theorem of

\author{
Ahlfors \\ Arakelyan \\ Borel \\ Borel-Nevanlinna \\ Cartan \\ Denjoy-Carleman-Ahlfors \\ Edrei-Fuchs \\ Hadamard \\ Hayman \\ Hiong King-Lai \\ Hadamard \\ Keldysh \\ Laguerre \\ Lindelöf \\ Milluox \\ Nevanlinna \\ Picard \\ Teichmüller \\ Valiron \\ Wiman-Heins \\ Transcendental function \\ Tsuji characteristic
}

Valiron deficiency theorem Vertex (uzel)

Weierstrass primary factor

Weight of a surface

Wirtinger inequality 


\section{Index}

\begin{tabular}{|c|c|}
\hline Note: the pages are still according to & $\stackrel{\circ}{m}(r, a)$ \\
\hline the Russian version & $m(r, f)$ \\
\hline$A(r, a) 39$ & $\mathfrak{m}(r, a)$ \\
\hline$A_{\alpha \beta}(r, a) 40$ & $\mathfrak{m}(r, f)$ \\
\hline$A(r, f) 39$ & \\
\hline$\stackrel{\circ}{A}(r, f) 30$ & $N_{1}(r)$ \\
\hline$A_{\alpha \beta}(r, f)$ & $N(r, a)$ \\
\hline $\begin{array}{l}A_{\alpha \beta}(r, J) \\
\{a C\} 223\end{array}$ & $N_{1}(r, a)$ \\
\hline$\{a, c\} 223$ & $N(r, a, f)$ \\
\hline$B(r, a) 39$ & $\bar{N}(r, a)$ \\
\hline$B_{\alpha, \beta}(r, a) 40$ & $N(r ; a, b)$ \\
\hline$B(r, f) 39$ & $\widehat{N}(r ; a, b)$ \\
\hline$B_{\alpha \beta}(r, f) 40$ & $N(r, f)$ \\
\hline & $N_{1}(r, f)$ \\
\hline$C(r, a) 39$ & $\mathfrak{N}(r, a)$ \\
\hline$C_{\alpha \beta}(r, a) 40$ & $\overline{\mathfrak{N}}(r, a)$ \\
\hline $\bar{C}_{\alpha \beta}(r, a) 145$ & $\mathfrak{N}_{1}(r, a)$ \\
\hline$C_{1 \alpha \beta}(r, a) 145$ & $\mathfrak{N}(r ; a, b)$ \\
\hline$C(r, f) 38$ & $\mathfrak{N}(r, f)$ \\
\hline$C_{\alpha \beta}(r, f)$ & $n_{1}(r)$ \\
\hline$c(r, f) 38$ & $n(r, a)$ \\
\hline$c_{\alpha \beta}(r, f) 40$ & $n_{1}(r, a)$ \\
\hline & $\bar{n}(r, a)$ \\
\hline$D\left(\alpha_{1}, \ldots, \alpha_{n}\right) 358$ & $n(r ; a, b)$ \\
\hline$E_{B}(f) 147$ & $n(r, a, f)$ \\
\hline$E_{N}(f) 147$ & $n_{1}(r, a, f)$ \\
\hline$E_{P}(f) 147$ & $n(r, f)$ \\
\hline$E_{V}(f) 147$ & $\mathfrak{n}(r, a)$ \\
\hline$E(z, p) 76$ & $\mathfrak{n}(r ; a, b)$ \\
\hline$E_{\rho}(z)$ & $\mathfrak{n}(r, f)$ \\
\hline$F_{q} 456$ & $p(w)$ \\
\hline$F_{q}\left(a_{1}, \ldots, a_{q}\right) 456$ & $p(z, w)$ \\
\hline$(F)\left(n ; a_{1}: \lambda_{1}\left(a_{1}\right), \ldots\right) 469$ & \\
\hline$f_{h}(z) 195$ & $Q(r, f)$ \\
\hline$f(z)$ & $\begin{array}{l}Q_{\alpha \beta}(r, f) \\
\mathfrak{Q}(r, f)\end{array}$ \\
\hline$L(F)$ & \\
\hline $\ln ^{+} x$ & $S_{0}$ \\
\hline $\ln _{j} x$ & $S(r, f)$ \\
\hline & $S_{a l p h a \beta}(r, f)$ \\
\hline$M(r, f)$ & $\stackrel{\circ}{S}_{\alpha \beta}(r, f)$ \\
\hline$m(r, a)$ & \\
\hline$m(r, a, f)$ & $T(r, f)$ \\
\hline
\end{tabular}




$\stackrel{\circ}{T}(r, f)$
$\mathfrak{T}(r, f)$
$\mathfrak{T}(r, f)$
$U(a, \eta)$
$U_{D}(r, a)$
$U_{D}(r, a, b)$
$U_{D}(r, a, f)$
$U_{D}(r, a, b ; f)$
$[w, a]$
$x^{\wedge}$
$\alpha^{+}$
$[\alpha]$
$\Delta(a)$
$\Delta^{*}(a)$
$\Delta(a, f)$
$\delta(a)$
$\delta^{*}(a)$
$\delta_{g}(a)$
$\delta(a, f)$
$\delta^{\prime}(a, f)$
$\varepsilon(a)$
$\varepsilon^{*}(a)$
$\varepsilon_{g}(a)$
$\kappa(f)$
$\Lambda$
$\lambda=\lambda[\alpha]$
$\mu(r, f)$
$\rho=\rho[\alpha]$
$\sigma=\sigma[\alpha]$
$\chi=\chi(F)$
$\omega(D)$




\section{APPENDIX A}

\section{A survey of some results after 1970 (by A. Eremenko and J. K. Langley)}

The literature on meromorphic functions ${ }^{1}$ is very large. There is a comprehensive survey [62] that contains everything that was reviewed on the topic in the Soviet "Referativnyi Zhurnal" in 1953-1970, and a later large survey [67]. More recent surveys $[\mathbf{3 0}],[\mathbf{8 0}]$ and $[\mathbf{4 8}]$ are shorter and have narrower scope.

Some books on specific topics in the theory of meromorphic functions published after 1970 are $[\mathbf{1 8}],[\mathbf{2 0}],[\mathbf{7 9}],[\mathbf{1 0 0}],[\mathbf{1 3 6}],[\mathbf{1 3 3}],[\mathbf{1 3 7}],[\mathbf{1 6 2}]$. A survey of the fast developing subject of iteration of meromorphic functions is $[\mathbf{7}]$.

Here we give a short survey of some results which are closely related to the problems considered in this book. Thus we do not include many important topics, like geometric theory of meromorphic functions, iteration, composition, differential and functional equations, normal families, Borel and Julia directions, uniqueness theorems, and most regrettably, holomorphic curves and quasiregular mappings.

\section{Chapter II.}

Mokhon'ko [121] proved the following generalization of (6.29), Ch. I. Let $R(w, z)$ be a rational function of $w$ whose coefficients are meromorphic functions $h(z)$ satisfying $T(r, h)=O(\phi(r))$, where $\phi$ is a fixed positive increasing function on $[0, \infty)$. Then for every meromorphic function $f$ we have

$$
T(r, R(f(z), z))=\operatorname{deg}_{w} R T(r, f)+O(\phi(r)) .
$$

This is a purely algebraic result; its proof uses only properties $(6.5),(6.6),\left(6.8^{\prime}\right)$ and the property $T\left(r, f^{2}\right)=2 T(r, f)$.

Another result in the same direction is due to Eremenko [39]. Let $F(u, v, z)$ be a polynomial in $u$ and $v$ whose coefficients are meromorphic functions $h$ of $z$ satisfying $T(r, h)=O(\phi(r))$, where $\phi$ is a function as above, and assume that $F$ is irreducible over the algebraic closure of the field of meromorphic functions. If $f$ and $g$ are meromorphic functions satisfying

$$
F(f(z), g(z), z) \equiv 0,
$$

then

$$
\operatorname{deg}_{u} F T(r, f)=\left(\operatorname{deg}_{v} F+o(1)\right) T(r, g)+O(\phi(r)) .
$$

Both theorems have applications in the analytic theory of differential equations.

Vojta [153] noticed a formal analogy between the definition of the Nevanlinna characteristic and the definition of height in number theory. This analogy extends quite far, and it has been a source of many interesting results and conjectures in the recent years. For example, under Vojta's analogy, Jensen's formula corresponds

${ }^{1}$ If the domain is not specified explicitly, we mean meromorphic functions in $\mathbf{C}$. 
to the fundamental theorem of arithmetic, while the second main theorem without the ramification term corresponds to the Thue-Siegel-Roth theorem on Diophantine approximation. The full second main Theorem corresponds to the famous unproved conjecture in number theory which is known as the "abc-conjecture". The analogy extends to the multi-dimensional generalizations of Nevanlinna theory, where it has been especially fruitful $[\mathbf{1 3 6}, \mathbf{1 0 1}]$. As an example of the influence of Vojta's analogy on the one-dimensional value distribution theory, we mention the recent precise results on the error term in the second main theorem (see comments to Chapter III).

Analogies between function theory and number theory were noticed before Vojta. Osgood was the first to view the second main theorem as an analogue of the Thue-Siegel Roth theorem $[\mathbf{1 2 8}, \mathbf{1 2 9}, \mathbf{1 3 0}]$. See also $[\mathbf{6 9}, \mathbf{7 7}]$.

Formula (2.6), Ch. I is the basis of the so-called "Fourier method" in the theory of entire and meromorphic functions, which was developed in the work of Rubel [137], Taylor, Miles and others. The Fourier method is considered as a substitute for the Weierstrass representation, which is more effective in certain cases. One of the main results achieved with this technique is the theorem of Miles [114] on the quotient representation of meromorphic functions: Every meromorphic function $f$ can be written as $f=g_{1} / g_{0}$, where $g_{j}$ are entire functions, possibly with common zeros, satisfying

$$
T\left(r, g_{j}\right) \leq A T(B r, f), \quad j=0,1,
$$

where $A$ and $B$ are absolute constants (independent of $f$ ). Moreover, for every $B>1$ there exists $A$ such that the above statement holds for every $f$. Simple examples show that one cannot in general take $B=1$. This result was improved by Khabibullin [97]: Let $f$ be a meromorphic function and $\epsilon>0$ a non-increasing convex function on $[0, \infty)$. Then there esists a representation $f=g_{1} / g_{0}$ such that $g_{j}$ are entire functions, and

$$
\ln \left(\left|g_{1}(z)\right|+\left|g_{0}(z)\right|\right) \leq \frac{A_{\epsilon}}{\epsilon(|z|)} T((1+\epsilon(|z|))|z|, f)+B_{\epsilon} .
$$

Khabibullin also extended Miles's theorem to meromorphic functions in $\mathbf{C}^{n}$. A survey of his results is $[\mathbf{9 8}]$.

The Fourier method is also one of the main tools for the study of functions which satisfy various restrictions on the arguments of $a$-points (see comments to Chapter VI). A recent book on the Fourier method is [99].

Chapter II.

Various properties of increasing functions on $[0, \infty)$ play an important role in the theory of meromorphic functions. By 1970 it became clear that the lower order $\lambda$ is at least as important as the order $\rho$. The main development since 1970 was "localizing" the notion of order.

Let $\Phi$ be an unbounded increasing function. A sequence $r_{k} \rightarrow \infty$ is called a sequence of Pólya peaks (of the first kind) of order $\mu$ if the inequalities

$$
\Phi\left(r r_{k}\right) \leq\left(1+\epsilon_{k}\right) r^{\mu} \Phi\left(r_{k}\right), \quad \epsilon_{k}<r<\epsilon_{k}^{-1}
$$

hold with some $\epsilon_{k} \rightarrow 0$. Pólya peaks were formally introduced by Edrei [33], though they were used by Edrei and Fuchs already in 1963. Pólya peaks of order $\mu$ exist for all $\mu$ in certain interval $\left[\lambda^{*}, \rho^{*}\right]$, which contains the interval $[\lambda, \rho]$. The endpoints 
$\lambda^{*}$ and $\rho^{*}$ of this interval are called the order and lower order in the sense of Pólya, respectively. The following formulas were given in [31]:

$$
\rho^{*}=\sup \left\{p: \limsup _{x, A \rightarrow \infty} g(A x) / A^{p} g(x)=\infty\right\}
$$

and

$$
\lambda^{*}=\inf \left\{p: \liminf _{x, A \rightarrow \infty} g(A x) / A^{p} g(x)=0\right\} .
$$

Most contemporary results on functions of finite lower order use Pólya peaks.

\section{Chapter III.}

Milloux' inequality (Ch. III, Theorem 2.4) has led to a rich vein of results developing the value distribution properties of meromorphic functions and their derivatives, in which a decisive role has been played by the paper [71] of Hayman.

Perhaps the most striking of the many results from [71] is Hayman's alternative (Ch. III, Theorem 2.6): if a function $f$ meromorphic in the plane omits a finite value $a$, and its $k$ th derivative $f^{(k)}$, for some $k \geq 1$, omits a finite non-zero value $b$, then $f$ is constant. Two principal questions arising in connection with Hayman's alternative are: (i) whether a version of Hayman's main inequality (Ch. III, (2.23)) holds with $N(r, 1 /(f-a))$ replaced by $\bar{N}(r, 1 /(f-a))$; (ii) whether $f^{(k)}$ can be replaced by a more general term, such as a linear differential polynomial

$$
F=L[f]=f^{(k)}+a_{k-1} f^{(k-1)}+\ldots+a_{0} f,
$$

with suitable coefficients $a_{j}$ of small growth compared to $f$. A positive answer to (i) was given by Chen [17]. Question (ii) was answered affirmatively in [102], although there do exist exceptional functions $f$, which may be determined from the $a_{j}$, for which $f$ and $F-1$ have no zeros. A unified approach to the questions from $[\mathbf{1 7}, \mathbf{1 0 2}]$ may be found in $[\mathbf{1 4}]$. It was shown further in $[\mathbf{1 9}, \mathbf{8 2}]$ that if $P$ is a non-constant differential polynomial in $f$, all of whose terms have degree at least 2 in $f$ and its derivatives, then a version of Hayman's inequality holds with $f^{(k)}$ replaced by $P$, and with $\bar{N}$ counting functions.

Question (i) is related to the issue of whether $f-a$ and $f^{(k)}-b(k \geq 1, b \neq$ $0)$ can both fail to have simple zeros, in analogy with the sharp result that a nonconstant meromorphic function cannot be completely branched over five distinct values. It has recently been shown $[\mathbf{1 2 7}]$ using normal family methods that if $f$ is transcendental and meromorphic in the plane with only multiple zeros then $f^{\prime}$ takes every finite non-zero value infinitely often (see also $[\mathbf{1 4}, \mathbf{1 5 5}]$ ).

The obvious example $f(z)=e^{z}$ shows that a transcendental entire function $f$ may have the property that $f$ and all its derivatives omit 0 : thus the condition $b \neq 0$ is necessary in Hayman's alternative. However, Hayman showed in [71] (see Ch. III, Theorem 2.7) that if $f$ is an entire function such that $f f^{\prime \prime}$ has no zeros then $f(z)=\exp (A z+B)$ with $A, B$ constants: this follows from applying (Ch. III, Theorem 2.6) to $f / f^{\prime}$. Clunie [21] established the corresponding result with $f^{\prime \prime}$ replaced by a higher derivative $f^{(k)}$, which on combination with Hayman's theorem on $f f^{\prime \prime}$ significantly improved earlier results of Pólya and others on the zeros of entire functions and their derivatives $[\mathbf{2 4}, \mathbf{1 3 4}, \mathbf{1 3 9}, \mathbf{1 4 0}]$. The Tumura-Clunie method, as developed in $[\mathbf{2 1}]$, shows that if $\Psi_{k}[g]$ is a differential polynomial of form $\Psi_{k}[g]=g^{k}+P_{k-1}[g]$, where $P_{k-1}[g]$ is a polynomial in $g$ and its derivatives of total degree at most $k-1$, and with coefficients of small growth compared to 
$g$, and if $g$ has few poles and $\Psi_{k}[g]$ has few zeros, then $\Psi_{k}[g]$ admits a simple factorisation. The application to zeros of $f f^{(k)}$ then follows by writing $f^{(k)} / f$ in the form $\Psi_{k}\left[f^{\prime} / f\right]$. Variants and generalisations of the Tumura-Clunie method, allowing functions $g$ with unrestricted poles, appear in a large number of papers including notably $[\mathbf{1 2 5}, \mathbf{1 5 1}, \mathbf{1 6 4}]$.

It was conjectured by Hayman in [71] that if $f$ is meromorphic in the plane and $f f^{(k)}$ has no zeros, for some $k \geq 2$, then $f(z)=\exp (A z+B)$ or $f(z)=(A z+B)^{-m}$, with $A, B$ constants and $m \in \mathbf{N}$. This was proved by Frank [52] for $k \geq 3$ and by Langley $[\mathbf{1 0 3}]$ for $k=2$ (see also $[\mathbf{9}, \mathbf{1 0 5}])$, while simple examples show that no such result holds for $k=1$. Generalisations include replacing $f f^{(k)}$ by $f F$, where $F$ is given by $(0.61)$ with rational coefficients $[\mathbf{1 5}, \mathbf{5 4}, \mathbf{1 0 4}, \mathbf{1 4 7}]$, and by $f f^{\prime \prime}-\alpha f^{\prime 2}, \alpha \in$ $\mathbf{C}[\mathbf{8}, \mathbf{1 0 5}, \mathbf{1 2 3}]$. Closely linked to Milloux' inequality and Hayman's alternative is the question of whether $G=f^{n} f^{\prime}$ must take every finite non-zero value, when $f$ is non-constant meromorphic and $n \in \mathbf{N}$, the connection being that $(n+1) G$ is the derivative of $f^{n+1}$, which has only multiple zeros and poles. Hayman proved in [71] that this is the case for $n \geq 2$ and $f$ entire, and for $n \geq 3$ when $f$ has poles. Following incremental results by a number of authors $[\mathbf{2 2}, \mathbf{8 1}, \mathbf{1 2 4}]$, the definitive theorem in this direction was proved in [11]: if $f$ is a transcendental meromorphic function in the plane and $m>k \geq 1$ then $\left(f^{m}\right)^{(k)}$ has infinitely many zeros. Here the result is proved first for finite order, and the infinite order case is then deduced by applying a renormalisation method from normal families $[131,132,163]$. The closely related question of whether $f^{\prime}+f^{n}$ may omit a finite value, which in turn is related to the Tumura-Clunie method, is resolved in $[\mathbf{1 1}, \mathbf{7 1}, \mathbf{1 2 4}, \mathbf{1 4 5}]$. A more recent conjecture [156] asserts that if $f$ is a transcendental meromorphic function then $f f^{(k)}$ takes every finite, non-zero value infinitely often: this is known to be true for $k=1$ [11], and for $k=2$ and $f$ entire [107].

A further development from [71] leads to two conjectures which remain open. Hayman observed in $[\mathbf{7 1}]$ that since the derivative $f^{\prime}$ of a transcendental meromorphic function $f$ has only multiple poles, it follows that $f^{\prime}$ has at most one finite Picard value. It was subsequently conjectured by Mues [122] that the Nevanlinna deficiencies of $f^{(m)}$ satisfy, for $m \geq 1$,

$$
\sum_{a \in \mathbf{C}} \delta\left(a, f^{(m)}\right) \leq 1 .
$$

This was proved by Mues [122] for $m \geq 2$, provided all poles of $f$ are simple. In the general case the best known upper bound for the sum in (0.62) appears to be $4 / 3[\mathbf{8 4}, \mathbf{1 6 1}]$.

The Mues conjecture (0.62) would follow from a positive resolution of the Gol'dberg conjecture that, for a transcendental meromorphic function $f$ and $k \geq 2$,

$$
\bar{N}(r, f) \leq N\left(r, 1 / f^{(k)}\right)+o(T(r, f))
$$

as $r \rightarrow \infty$, possibly outside an exceptional set. This is in turn linked to a classical result of Pólya [134] that if $f$ has at least two distinct poles then $f^{(k)}$ has at least one zero, for all sufficiently large $k$. When $f$ has poles of multiplicity at most $k-1$ the inequality (0.63) follows from a lemma of Frank and Weissenborn [55] (see also [154]), so that in particular if $f$ has only simple poles then $(0.62)$ is true for every positive $m$. A related inequality was proved in $[\mathbf{5 3}, \mathbf{1 4 8}]:$ if $F=L[f]$ as in (0.61), 
where the $a_{j}$ are small functions compared to $f$, then either

$$
N(r, F) \leq N(r, 1 / F)+2 N(r, f)+o(T(r, f))
$$

outside a set of finite measure or $f$ is a rational function in solutions of the homogeneous equation $L[w]=0$. This method is connected to Steinmetz' proof [146] of the second main theorem for small functions discussed below. No further results in the direction of (0.63) appear to be known, although it was proved by Langley in $[\mathbf{1 0 6}]$ that if $f$ is meromorphic of finite order in the plane and $f^{(k)}$ has finitely many zeros, for some $k \geq 2$, then $f$ has finitely many poles.

Examples abound of meromorphic functions with infinitely many poles such that the first derivative has no zeros, but it was proved in [50] (see also [11]) that if $f$ is transcendental and meromorphic with $\limsup _{r \rightarrow \infty} T(r, f) / r=0$ then $f^{\prime}$ has infinitely many zeros: the corresponding result with lim sup replaced by lim inf may be found in $[\mathbf{8 3}]$.

We remark that the above results have all been stated for functions meromorphic in the plane. Those which are proved only using properties of the Nevanlinna characteristic admit in some cases generalisation to functions of sufficiently rapid growth in a disk [71] or a half-plane [110]. Some related results for functions of slower growth in the disc appear in $[\mathbf{1 4 2}, \mathbf{1 4 4}]$.

An old conjecture of Nevanlinna was that one can replace constants $a_{k}$ in the second main theorem by meromorphic functions $a_{k}(z)$ with the property $T\left(r, a_{k}\right)=$ $o(T(r, f))$. Such functions $a_{k}$ are usually called "small targets". For the case of an entire function $f$ such a generalization was obtained by Chuang Chi Tai in 1964. Much later, the second main theorem without the ramification term, was proved for meromorphic functions by Osgood, who used methods from number theory $[\mathbf{1 2 8}, \mathbf{1 2 9}, \mathbf{1 3 0}]$. A substantial simplification of Osgood's proof was made by Steinmetz [146], who also used a beautiful idea of Frank and Weissenborn [55]. Osgood and Steinmetz proved that

$$
\sum_{k=1}^{q} N\left(r,\left(f-a_{k}\right)^{-1}\right) \geq(q-2+o(1)) T(r, f),
$$

outside of the usual exceptional set. The proof in [146] is simple and elegant; and uses only manipulations with Wronski determinants and the classical lemma on the logarithmic derivative. This makes it suitable for generalizations to holomorphic curves $[\mathbf{1 2 9}, \mathbf{1 3 0}, \mathbf{1 3 6}]$. However, this version of the second main theorem does not take ramification into account. Simple examples like $f(z)=e^{z}+z$ where $\delta(\infty, f)=\delta(z, f)=1$ and $N_{1}(r, f) \sim T(r, f)$ show that one cannot include the term $N_{1}$. However, the following form of the second main theorem holds with small targets:

$$
\sum_{k=1}^{q} \bar{N}\left(r,\left(f-a_{k}\right)^{-1}\right) \geq(q-2+o(1)) T(r, f),
$$

where $\bar{N}\left(r,\left(f-a_{k}\right)^{-1}\right)$ is the usual function counting zeros of $f-a$ disregarding multiplicity. This result was recently obtained by Yamanoi $[\mathbf{1 6 0}]$. In [159] he separately treats the case of rational functions $a_{k}$ when the proof is technically simpler. Yamanoi's proof is very complicated, and it will be hard to generalize to holomorphic curves. Surprisingly, it uses Ahlfors's theory of covering surfaces (and also algebraic geometry, moduli spaces of curves, and combinatorics). The idea to bring Ahlfors's theory to this context has its origin in the work of Sauer $[\mathbf{1 3 8}]$ who 
obtained a partial result for rational small targets. One application of Yamanoi's generalization of the second main theorem is the following. Suppose that $f$ and $g$ are meromorphic functions in $\mathbf{C}$ satisfying a relation of the form (0.60). If the genus of the curve $F(u, v)=0$ is greater than 1 , then $T(r, f)+T(r, g)=O(\phi(r))$. This was conjectured by Eremenko in 1982, and the important special case that $\phi(r)=\ln r$, that is $F$ is a polynomial in all three variables, was proved by Zaidenberg in 1990 .

Now we turn to the classic setting. The estimate in the lemma on the logarithmic derivative was improved by Gol'dberg and Grinstein $[\mathbf{6 6}]$ :

$$
m\left(r, f^{\prime} / f\right) \leq \ln ^{+}\left\{T(\rho, f)(1-(r / \rho))^{-1}\right\}+\text { const },
$$

where the constant depends on $f$. Vojta's analogy (see comments to Chapter I) stimulated new interest in refined estimates for the logarithmic derivative, as well as for the error term

$$
S(r, f)=\sum_{j=1}^{q} m\left(r, a_{j}, f\right)+N_{1}(r, f)-2 T(r, f)
$$

in the second main theorem. Miles [118] derived from Gol'dberg's and Grinstein's estimate the following. Let $\psi$ be a continuous non-decreasing function such that

$$
\int_{1}^{\infty} \frac{d t}{t \psi(t)}<\infty
$$

Then for every meromorphic function $f$ we have

$$
m\left(r, f^{\prime} / f\right) \leq \ln ^{+} \psi(T(r, f))+O(1),
$$

outside an exceptional set of finite logarithmic measure. The strongest results on the error term $S(r, f)$ belong to Hinkkanen [85], for example:

$$
S(r, f) \leq \ln ^{+} \psi(T(r, f))+O(1),
$$

outside an exceptional set of finite logarithmic measure, where $\phi$ is as before. If one replaces $\psi$ by $t \psi(t)$, then both results will hold outside an exceptional set of finite measure. All presently known results on the error terms in one-dimensional Nevanlinna theory are collected in the book [18].

No analog of the second main theorem holds without an exceptional set of $r$ 's. This can be seen from the result of Hayman [72]: Let $\left\{E_{k}\right\}$ be closed sets of zero logarithmic capacity, and $\phi$ and $\psi$ arbitrary unbounded increasing functions. Then there exist an entire function $f$ and a sequence $r_{k} \rightarrow \infty$ such that $N\left(r_{k}, a, f\right) \leq$ $\phi\left(r_{k}\right) \ln r_{k}$ for all $a \in E_{k}$, while $T\left(r_{k}, f\right)>\psi\left(r_{k}\right)$.

\section{Chapter IV.}

The result of Hayman we just cited shows that the set $E_{V}(f)$ of Valiron exceptional values, which always has zero capacity, can contain any $F_{\sigma}$ set of zero capacity, but a complete description of possible sets $E_{V}(f)$ is not known.

For meromorphic functions $f$ of finite order, Hyllengren [96] obtained a very precise description of the sets $E_{V}(f)$. Let us say that a set $E$ satisfies the $H$ condition if there exist a sequence $\left(a_{n}\right)$ of complex numbers and $\eta>0$ such that every point of $E$ belongs to infinitely many discs $\left\{w:\left|w-a_{n}\right|<\exp (-\exp (\eta n))\right\}$. For every meromorphic function $f$ of finite order, and every $x \in(0,1)$, the set $E_{V}(x, f)$ of those $a \in \mathbf{C}$ for which $\Delta(a, f)>x$ satisfies the $H$-condition, and vise versa, for every set $E$ satisfying the $H$-condition, there exist an entire function $f$ 
and a number $x \in(0,1)$ such that $\Delta(a, f)>x$, for all $a \in E$. Notice that the $H$-condition is much stronger than the condition of zero capacity.

The first example of an entire function of finite order whose deficiency depends on the choice of the origin was constructed by Miles [116]. The order of this function was very large. Then Gol'dberg, Eremenko and Sodin [65] constructed such examples with any given order greater than 5. (For entire functions of order less than $3 / 2$, deficiencies are independent of the choice of the origin.)

\section{Chapter V.}

Put $L(r, f)=\ln \mu(r, f) / \ln M(r, f)$, where $\mu(r, f)=\min \{|f(z):| z \mid=r\}$. Corollary 3 on p. 232 says that $\lim _{\sup _{r \rightarrow \infty}} L(r, f) \geq-[2 \lambda]$. Hayman [70] showed that the same holds with $-2[\lambda]$ replaced by $-2.19 \ln \rho$ when $\rho$ is large enough. For functions of infinite order, he proved

$$
\limsup _{r \rightarrow \infty} \frac{L(r, f)}{\ln \ln \ln M(r, f)} \geq-2.19 .
$$

He also constructed examples of entire functions of large finite order for which $\lim \sup _{r \rightarrow \infty} L(r, f)<-1$. Then Fryntov [56], answering a question of Hayman, constructed entire functions of any given order $\rho>1$ with the same property. Drasin [29] constructed entire functions of order one, maximal type, with the property $M(r, f) \mu(r, f) \rightarrow 0$. This may be contrasted with a remarkable theorem of Hayman [73] which says that if $f$ is an entire function of order one and normal type, and $M(r, f) \mu(r, f)$ is bounded, then $f(z)=c \exp (a z)$ for some constants $c$ and $a$.

Thus for an entire function of order at least one, $\mu(r, f)$ can decrease at a higher rate than that of increase of $M(r, f)$. The situation changes dramatically if we consider the rate of decrease of $|f(z)|$ on an unbounded connected set. Hayman and Kjellberg [78] proved that for every entire function $f$ and every $K>1$ all components of the set $\{z: \ln |f(z)|+K \ln M(|z|, f)<0\}$ are bounded.

Theorem $1.3^{\prime}$ has been the subject of many deep generalizations. First we mention the famous "spread relation" of Baernstein $[\mathbf{2}][\mathbf{4}]$ conjectured by Edrei in [34]: If $f$ is a meromorphic function of lower order $\lambda$, then for every $\epsilon>0$ there are arbitrary large values of $r$ such that the set of arguments $\theta$ where $\left|f\left(r e^{i \theta}\right)\right|>1$ has measure at least

$$
\min \left\{\frac{4}{\lambda} \arcsin \sqrt{\frac{\delta(\infty, f)}{2}}, 2 \pi\right\}-\epsilon .
$$

Similar sharp estimates of the measure of the set where

$$
\ln \left|f\left(r e^{i \theta}\right)\right|>\alpha T(r, f)
$$

were given in $[\mathbf{1}]$.

Fryntov, Rossi and Weitsman $[\mathbf{5 7}, \mathbf{5 8}]$ proved that under the assumptions of the spread conjecture, the set $\left|f\left(r e^{i \theta}\right)\right|>1$ must contain an arc of length (0.64). See also $[\mathbf{3}]$ for the sharp lower estimate of the length of the arcs in the set $\{\theta$ : $\ln \mid f\left(r e^{i \theta} \mid>\alpha \ln M(r, f)\right\}$.

Extremal functions for the spread relation and its generalizations were studied extensively, $[\mathbf{5}, \mathbf{3 6}, \mathbf{3 7}, \mathbf{1 4 1}]$. 
The new methods introduced by Baernstein [2], [4] are based on the use of subharmonic functions, and especially, on a new type of maximal function, the socalled "star-function", which turned out to be very useful in solving a wide variety of extremal problems of function theory. An account of Baernstein's star function and its main applications is contained in the monograph [79].

One important application of the spread relation is the sharp estimate of the sum of deficiencies of a meromorphic function of lower order $\lambda \leq 1$ [35]. If $a$ meromorphic function $f$ of lower order $\lambda$ has at least two deficient values, then

$$
\sum_{a \in \overline{\mathbf{C}}} \delta(a, f) \leq\left\{\begin{array}{ll}
1-\cos \pi \lambda, & 0<\lambda \leq 1 / 2 \\
2-\sin \pi \lambda, & 1 / 2<\lambda \leq 1
\end{array},\right.
$$

The sharp estimate of the sum of deficiencies of a meromorphic function in terms of its order or lower order $\lambda$ is still not established for $\lambda>1$. The conjectured extremal functions are described in $[\mathbf{3 2}]$.

The results of $\S 2$ show that neither Nevanlinna nor Borel exceptional values need be asymptotic values. On the other hand, Picard exceptional values are asymptotic. A natural question arises, whether any condition of smallness of $N(r, a, f)$ in comparison with $T(r, f)$ will imply that $a$ is an asymptotic value. The basic result belongs to the intersection of the papers [16], [74], and [38]. Let $f$ be $a$ meromorphic function of lower order $\lambda \leq \infty$. If the order of $N(r, a, f)$ is strictly less than $\min \{1 / 2, \lambda\}$ then $a$ is an asymptotic value. Example 3 on p. 249 shows that this condition is sharp, if only the lower order of $f$ is taken into account. In [38], a weaker sufficient condition for $a$ to be an asymptotic value is given, that uses both the order and lower order of $f$. Hayman [74] gives the following refined condition: if

$$
T(r, f)-\frac{1}{2} r^{1 / 2} \int_{r}^{\infty} t^{-3 / 2} N(t, a, f) d t \rightarrow \infty,
$$

then $a$ is an asymptotic value.

The problem on p. 285 of optimal estimation of $\kappa(f)$ for functions of lower order greater than 1 is still open, even for entire functions. It has been solved only for entire functions with zeros on a ray $[\mathbf{9 4}]$.

The best estimates known at this time for entire and meromorphic functions with fixed $\lambda>1$ are contained in $[\mathbf{1 1 9}, \mathbf{1 2 0}]$. They are derived from the following sharp inequality which is obtained by the Fourier method:

$$
\limsup \frac{N(r, 0)+N(r, \infty)}{m_{2}(r, f)} \geq \sup _{\lambda^{*} \leq \lambda \leq \rho^{*}} \sqrt{2} \frac{|\sin \pi \lambda|}{\pi \lambda}\left\{1+\frac{\sin 2 \pi \lambda}{2 \pi \lambda}\right\}^{-1 / 2},
$$

where $m_{2}$ is the $L^{2}$-norm,

$$
m_{2}^{2}(r, f)=\frac{1}{2 \pi} \int_{0}^{2 \pi}\left(\ln \left|f\left(r e^{i \theta}\right)\right|\right)^{2} d \theta
$$

and $\lambda^{*}$ and $\rho^{*}$ are the order and lower order in the sense of Pólya, [31] (see also comments to Chapter II).

In 1929, F. Nevanlinna [126] found that meromorphic functions of finite order satisfying $N_{1}(r, f) \equiv 0$ have the following properties:

a) $2 \rho$ is an integer, $2 \rho \geq 2$,

b) all deficient values are asymptotic, and 
c) all deficiencies are rational numbers with denominators at most $2 \rho$, and their sum equals 2 .

It was natural to conjecture that one of the conditions

$$
N_{1}(r, f)=o(T(r, f)),
$$

or

$$
\sum_{a \in \overline{\mathbf{C}}} \delta(a, f)=2
$$

implies the properties a), b) and c). Notice, that by the second main theorem, (0.66) implies (0.65) for functions of finite order.

It turns out that a strong form of this conjecture holds:

Small Ramification Theorem. If $f$ is a meromorphic function of finite lower order with the property (0.65) then a), b) and c) hold, and:

$$
T(r, f)=r^{\rho} \ell(r) \text {, }
$$

where $\ell$ is a slowly varying function in the sense of Karamata. ${ }^{2}$

As a corollary we obtain that conditions $(0.65)$ and (0.66) for functions of finite lower order are equivalent.

This result has a long history which begins with theorems of Pfluger and Edrei and Fuchs establishing the case of entire functions (see Corollary 2 on p. 315). Weitsman [158] proved that (0.66) implies that the number of deficient values is at most $2 \rho$. Then Drasin $[\mathbf{2 7}],[\mathbf{2 8}]$ proved that for functions of finite order $(0.66)$ implies a), b) and c) and the regularity condition (0.67). Eremenko proposed a new potential-theoretic method (see, for example, $[\mathbf{4 8}]$ ) which finally led to a proof of a simpler proof of Drasin's theorem. The small ramification theorem in its present form stated above is proved in [44].

These results show that besides the defect relation, there is an additional restriction on defects of functions of finite order: (0.66) implies that the number of deficient values is finite and all defects are rational.

There are other restrictions as well. Weitsman's theorem [157] says that for functions $f$ of finite lower order

$$
\sum_{a \in \overline{\mathbf{C}}} \delta(a, f)^{1 / 3}<\infty
$$

The story of this theorem is described in Comments to Chapter VII (see p. 576). Weitsman's proof can actually be modified to produce an upper bound depending only on the lower order of $f$.

The second restriction concerns functions of finite order having a defect equal to 1 . Lewis and $\mathrm{Wu}$ proved that such functions satisfy

$$
\sum_{a \in \overline{\mathbf{C}}} \delta(a, f)^{1 / 3-\alpha}<\infty
$$

with some absolute constant $\alpha>0$. Their proof gives $\alpha=2^{-264}$, which is far from what is expected. (Lewis and $\mathrm{Wu}$ state their result for entire functions but their proof applies to all functions with $\delta(a, f)=1$ for some $a$.)

\footnotetext{
${ }^{2}$ That is $\ell(c r) / \ell(r) \rightarrow 1$, as $r \rightarrow=\infty$, uniformly with respect to $c \in[1,2]$.
} 
Examples of entire functions of finite order with the property $\delta\left(a_{n}, f\right) \geq c^{n}$ for some $c \in(0,1)$ are constructed in $[\mathbf{4 5}]$, but a large gap remains between these examples and the result of Lewis and $\mathrm{Wu}$.

Recent research on value distribution meromorphic functions of the form

$$
\sum \frac{c_{k}}{z-z_{k}}, \quad \sum \frac{\left|c_{k}\right|}{\left|z_{k}\right|}<\infty
$$

was mainly concentrated on the functions with $c_{k}>0$. Such functions are (complex conjugate to) gradients of subharmonic functions of genus zero with discrete mass. The main conjecture is that every function of the form (0.69) has zeros. This was proved in [50] under the additional assumption that inf $c_{k}>0$.

\section{Chapter VI}

Entire functions whose zeros lie on (or are close to) finitely many rays were intensively studied. Under certain conditions, one can estimate $\delta(0, f)$ from below, as in Corollary 4 on p. 350. The strongest results in this direction belong to Hellerstein and Shea [91] and Miles [115]. One of the results of [91] says that $\delta(0, f)>B_{q}\left(\theta_{1}, \ldots, \theta_{n}\right)$ for all entire functions of genus $q$ with zeros on the rays $\arg z \in\left\{\theta_{1}, \ldots, \theta_{q}\right)$, and $B_{q} \rightarrow 1$ when $q \rightarrow \infty$ while the rays remain fixed. In the case of one ray, they obtained $B_{q}(\theta)=1-\left(\pi^{2} e^{-1}+o(1)\right) / \ln q, q \rightarrow \infty$. For entire functions of infinite order with zeros on a ray, Miles [115] proved that $N(r, 0, f) / T(r, f) \rightarrow 0$ as $r \rightarrow \infty$ avoiding an exceptional set of zero logarithmic density. However, it may happen that $\delta(0, f)=0$ for such functions, as Miles shows by an example constructed in the same paper.

Hellerstein and Shea $[\mathbf{9 1}]$ also considered meromorphic functions of finite order whose zeros $\left\{z_{n}\right\}$ and poles $\left\{w_{n}\right\}$ lie in opposite sectors $\left|\arg z_{n}\right| \leq \eta$ and $\mid \arg w_{n}-$ $\pi \mid \leq \eta$, where $0 \leq \eta<\pi / 6$. For such functions, they obtained a sharp estimate of $\kappa(f)$ (definition on p. 285) from above.

For entire functions with zeros on finitely many rays, there are relations between the order and lower order (see p. 344). There relations were further investigated in $[\mathbf{1 1 7}, \mathbf{6 0}]$ and $[\mathbf{1 3 5}]$.

Miles [117] considers the class of meromorphic functions $f$ whose zeros belong to a finite union of rays $X$ and poles belong to a finite union of rays $Y$, where $X \cap Y=\emptyset$, and such that the exponent of convergence of the union of zeros and poles is a given number $q$. He then produces a non-negative integer $p=p(q, X, Y)$ such that

$$
\lim _{r \rightarrow \infty} \frac{T(r, f)}{r^{p}}=\infty \quad \text { if } \quad p>0 \text { and } \quad \lim _{r \rightarrow \infty} \frac{T(r, f)}{\ln r}=\infty \quad \text { if } \quad p=0,
$$

and these growth estimates are sharp in the considered class. The integer $p$ depends in a subtle way on the arithmetical properties of the arguments of the rays $X$ and $Y$, and this integer is in general hard to compute or estimate.

Gleizer [60] considers entire functions with zeros on $n$ rays. If $n=1$ or $n=2$, we have $\rho \leq[\lambda]+n$, where [ ] is the integer part. This follows from Theorem 1.1, Chapter VI. However, if $n=3$, then the difference $\rho-\lambda$ can be arbitrarily large. In this case, Gleizer proved that $[\rho] \leq 3([\lambda]+1)$. For arbitrary $n$, Qiao $[\mathbf{1 3 5}]$ proved that $\rho \leq 4^{q-1}([\lambda]+1)$. 
In $[59,61]$, Gleizer extended Theorem 4.1 by taking into account not only the order but the lower order in the sense of Pólya. He used Baernstein's star-function.

There has been remarkable progress in the problems considered in $\S 5$. The conjecture of Pólya and Wiman stated on p. 417 was proved by Hellerstein and Williamson $[\mathbf{9 2}, \mathbf{9 3}]$. If $f$ is a real entire function such that all zeros of $f f^{\prime} f^{\prime \prime}$ are real then $f$ belongs to the Laguerre-Pólya class. In [95] the same authors with Shen classified all entire functions (not necessarily real) with the property that $f f^{\prime} f^{\prime \prime}$ has only real zeros. The classification of meromorphic functions with the property that all their derivatives have only real zeros was achieved by Hinkkanen $[86,87,88,89,90]$.

Sheil-Small [143] proved a conjecture of Wiman (1911), that every real entire function of finite order with the property that $f f^{\prime \prime}$ has only real zeros belongs to the Laguerre-Pólya class. Bergweiler, Eremenko and Langley [13] extended SheilSmall's result to functions of infinite order. Then Langley [109] extended this result to the derivatives of higher orders: If $f$ is a real entire function of infinite order, with finitely many non-real zeros, then $f^{(k)}$ has infinitely many non-real zeros for every $k \geq 2$.

For real entire functions of finite order with finitely many non-real zeros, that do not belong to the Laguerre-Pólya class, Bergweiler and Eremenko [12] proved that the number of non-real zeros of $f^{(k)}$ tends to infinity as $k \rightarrow \infty$. Together with Langley's result, this confirms another conjecture of Pólya (1943).

\section{Chapter VII}

The inverse problem (as stated on p. 487) was completely solved by Drasin [25]. A simplified proof is given in [26]. The general idea is the same as in Chapter VII: quasiconformal surgery and a version of the theorem of Belinskii and Teichmüller are involved. However, unlike in Chapter VII, Drasin does not construct the Riemann surface spread over the sphere explicitly but uses a more flexible technique.

Theorem 8.1 in Chapter VII actually gives a complete solution of the inverse problem for finitely many deficient values in the class of meromorphic functions of finite order. (This was not known in 1970 when the book was written. That condition 3 of this Theorem 8.1 is necessary follows from the small ramification theorem above).

On the narrow inverse problem in the class of meromorphic functions of finite order with infinitely many deficiencies, there is the following result $[\mathbf{4 2}]$ :

Let $\{a\}$ be an arbitrary infinite countable subset of $\overline{\mathbf{C}}$, and $\left\{\delta_{n}\right\}$ positive numbers satisfying the following conditions:

(i) $\delta_{n}<1$

(ii) $\sum_{n} \delta_{n}<2$, and

(iii) $\sum_{n} \delta_{n}^{1 / 3}<\infty$.

Then there exists a meromorphic function $f$ of (large) finite order such that $\delta\left(a_{n}, f\right)=\delta_{n}$, and $f$ has no other deficient values.

The order of this function depends on the quantities in the right hand sides of (i), (ii) and (iii). 
Conditions (ii) and (iii) are necessary because of the small ramification theorem, and Weitsman's theorem (see comments to Chapter V above). Condition (i) cannot be removed because of the Lewis and $\mathrm{Wu}$ theorem stated above, but it is not known what the precise condition on $\delta_{n}$ is, if $\delta_{1}=1$.

The class of meromorphic functions with finitely many critical and asymptotic values which was used in Chapter VII to investigate the inverse problem is interesting independently of this application. Let us call this class $S$. The first general result on functions of this class belongs to Teichmüller [149], who proved that the second main theorem becomes an asymptotic equality for functions of this class:

$$
\sum_{j=1}^{q} m\left(r, a_{j}, f\right)+N_{1}(r, f)=2 T(r, f)+Q(r, f),
$$

where $a_{j}$ are all critical and asymptotic values.

Langley [108] found that the growth of a function $f \in S$ cannot be arbitrary:

$$
c(f):=\liminf _{r \rightarrow \infty} \frac{T(r, f)}{\ln ^{2} r}>0 .
$$

This constant $c(f)$ can be arbitrarily small, but in the case that $f$ has only three critical and asymptotic values, we have $[49] c(f) \geq \sqrt{3} /(2 \pi)$ and this is best possible. On the other hand, there are no restrictions from above on the growth of functions of class $S$ [113].

Class $S$ plays an important role in holomorphic dynamics (iteration of entire and meromorphic functions), see, for example, the survey [7]. In [23] an application of almost periodic ends is given. In [32] the method of Chapter VII is extended to a new class of Riemann surfaces which the authors call "Lindelöfian ends". The corresponding functions have infinitely many critical values and thus do not belong to the class $S$.

Appendix.

Govorov's original proof of the Paley conjecture was a byproduct of his research on the Riemann Boundary problem with infinite index [68]. His theorem was generalized to meromorphic functions by Petrenko, to subharmonic functions in $\mathbf{R}^{n}$ by Dahlberg, and to entire functions of several complex variables by Khabubullin, see his survey $[\mathbf{9 8}]$.

Petrenko introduced the following quantity which he called the "deviation of $f$ from the point $a$ ".

$$
\beta(a, f)=\liminf _{r \rightarrow \infty} \frac{\ln ^{+} M\left(r,(f-a)^{-1}\right)}{T(r, f)} .
$$

This differs from the defect in one respect: the uniform norm of $\ln ^{+} \mid f\left(r e^{i \theta}\right)-$ $\left.a\right|^{-1}$ stands in the numerator instead of the $L^{1}$ norm. Petrenko's generalization of Govorov's theorem proved in the Appendix can be restated as:

$$
\beta(a, f) \leq \pi \lambda
$$

for all meromorphic functions of lower order $\lambda$ and all $a \in \overline{\mathbf{C}}$. This was the starting point of a study of deviations $\beta(a, f)$ by Petrenko and others. The results obtained before 1978 are summarized in his book [133]. The main difference between the theory of deviations and the theory of defects is the absence of a first main theorem: there is no simple relation between $\beta(a, f)$ and solutions of the equation $f(z)=a$. 
We only present a sample of the results. By analogy with defects, one can expect that the set of exceptional values in the sense of Petrenko

$$
P(f):=\{a \in \overline{\mathbf{C}}: \beta(a, f)>0\}
$$

is small. This is indeed the case: for every meromorphic function $f$, the set $P(f)$ has zero logarithmic capacity; for functions of finite lower order it is at most countable (but may have the power of continuum for functions of infinite lower order). The following analog of the Defect relation for functions of finite lower order was established by Marchenko and Shcherba [112]:

$$
\sum_{a \in \overline{\mathbf{C}}} \beta(a, f) \leq \begin{cases}2 \pi \lambda, & \lambda \geq 1 / 2 \\ \pi \lambda \csc \pi \lambda, & \lambda<1 / 2\end{cases}
$$

Moreover, an analog of Weitsman's theorem (see comments to Chapter V) holds: for functions $f$ of finite lower order we have

$$
\sum_{a \in \overline{\mathbf{C}}} \beta(a, f)^{1 / 2}<\infty
$$

and the exponent $1 / 2$ is best possible. A version Baernstein's spread relation also holds with deviations instead of deficiencies [133]. It is worth mentioning here that, according to Baernstein [4], the idea of introducing the star function that led to the proof of the spread relation occurred under the influence of Petrenko's proof of (0.71).

The inverse problem for deviations turned out to be simpler than the inverse problem for deficiencies. A complete solution for functions of finite order is given in [42]: For every at most countable set $\left\{a_{n}\right\}$ of points and every sequence of positive numbers $\beta_{n}$ satisfying the condition $\sum \beta_{n}^{1 / 2}<\infty$, there exists a meromorphic function $f$ of finite order such that $\beta\left(a_{n}, f\right)=\beta_{n}$ and $\beta(a, f)=0$ for $a \notin\left\{a_{n}\right\}$.

In general, there is no relation between the sets $E_{N}(f)$ and $P(f)$ : for every pair $(A, B)$ of at most countable subsets of $\overline{\mathbf{C}}$, there exists a meromorphic function $f$ of any given non-zero order such that $E_{N}(f)=A$ and $P(f)=B[\mathbf{6 4}, \mathbf{6 5}]$. On the other hand, if $T(2 r, f)=O(T(r, f))$ then $P(f)=E_{V}(f)[\mathbf{4 1}]$.

Bergweiler and Bock [10] found an analog of $(0.71)$ for functions of infinite lower order. The idea was to replace $T(r, f)$ in the denominator of $(0.70)$ by $A(r, f)$. Notice that if one uses the Ahlfors definition of $T(r, f)$ then $A(r, f)=d T(r, f) / d \ln r$, for example, if $T(r, f)=r^{\lambda}$ then $A(r, f)=\lambda T(r, f)$. Bergweiler and Bock proved that for every meromorphic function $f$ of order at least $1 / 2$ and every $a \in \overline{\mathbf{C}}$ we have

$$
b(a, f):=\liminf _{r \rightarrow \infty} \frac{\ln ^{+} M\left(r,(f-a)^{-1}\right)}{A(r, f)} \leq \pi,
$$

and then Eremenko [46] established the following analog of the Defect Relation:

$$
\sum_{a \in \overline{\mathbf{C}}} b(a, f) \leq\left\{\begin{array}{ll}
2 \pi, & \lambda>1 / 2 \\
2 \pi \sin \pi \lambda, & \lambda \leq 1 / 2
\end{array},\right.
$$

assuming that there are at least two values $a$ with $b(a, f)>0$. It follows that for every meromorphic function the set $\{a \in \overline{\mathbf{C}}: b(a, f)>0\}$ is at most countable.

Even Drasin's theorem on the extremal functions for the defect relation (see Comments to Chapter V) has its analog for $b(a, f)[\mathbf{4 7}]$ : 
If $f$ is of finite lower order and

$$
\sum_{a \in \overline{\mathbf{C}}} b(a, f)=2 \pi
$$

then the following limit exists

$$
\lim _{r \rightarrow \infty} \frac{\ln T(r, f)}{\ln r}=\frac{n}{2},
$$

where $n$ is an integer, and $b(a, f)=\pi / n$ or 0 for every $a \in \overline{\mathbf{C}}$. 


\section{Bibliography}

[1] Anderson, J. M.; Baernstein, A. The size of the set on which a meromorphic function is large. Proc. London Math. Soc. (3) 36 (1978), no. 3, 518-539.

[2] Baernstein, A. Proof of Edrei's spread conjecture. Proc. London Math. Soc. (3) 26 (1973), 418-434.

[3] Baernstein, A. A generalization of the $\cos \pi \rho$ theorem. Trans. Amer. Math. Soc. 193 (1974), 181-197.

[4] Baernstein, A. How the $*$-function solves extremal problems. Proceedings of the International Congress of Mathematicians (Helsinki, 1978), pp. 639-644, Acad. Sci. Fennica, Helsinki, 1980.

[5] Baernstein, A. Regularity theorems associated with the spread relation. J. Analyse Math. 31 (1977), 76-111.

[6] Barth, K. F.; Brannan, D. A.; Hayman, W. K. The growth of plane harmonic functions along an asymptotic path. Proc. London Math. Soc. (3) 37 (1978), no. 2, 363-384.

[7] Bergweiler, W. Iteration of meromorphic functions. Bull. Amer. Math. Soc. (N.S.) 29 (1993), no. 2, 151-188

[8] Bergweiler, W. On the zeros of certain homogeneous differential polynomials, Archiv der Math. 64 (1995), 199-202.

[9] Bergweiler, W. Normality and exceptional values of derivatives, Proc. Amer. Math. Soc. 129 (2001), 121-129.

[10] Bergweiler, W. and Bock, H. On the growth of meromorphic functions of infinite order. J. Anal. Math. 64 (1994), 327-336.

[11] Bergweiler, W. and Eremenko, A. On the singularities of the inverse to a meromorphic function of finite order, Rev. Mat. Iberoamericana 11 (1995), 355-373.

[12] Bergweiler, W; Eremenko, A. Proof of a conjecture of Pólya on the zeros of successive derivatives of real entire functions, To appear in Acta Math.

[13] Bergweiler, W.; Eremenko, A.; Langley, J. K. Real entire functions of infinite order and a conjecture of Wiman. Geom. Funct. Anal. 13 (2003), no. 5, 975-991.

[14] Bergweiler, W. and Langley, J. K. Multiplicities in Hayman's alternative. J. Aust. Math. Soc. 78 (2005), no. 1, 37-57.

[15] Brüggemann, F. Proof of a conjecture of Frank and Langley concerning zeros of meromorphic functions and linear differential polynomials, Analysis 12 no. 1/2 (1992), 5-30.

[16] Chang, Kuan Heo. Asymptotic values of entire and meromorphic functions. Sci. Sinica 20 (1977), no. 6, 720-739.

[17] Chen Zhen Hua, Normality of families of meromorphic functions with multiple valued derivatives, Acta Math. Sinica 30 (1987), 97-105.

[18] Cherry, W. and Ye, Zhuan. Nevanlinna's theory of value distribution. The second main theorem and its error terms. Springer-Verlag, Berlin, 2001.

[19] Chuang, C. T., On differential polynomials, in: Analysis of One Complex Variable, World Sci. Publishing, Singapore 1987, 12-32.

[20] Chuang, Chi-Tai; Yang, Chung-Chun. Fix-points and factorization of meromorphic functions. Translated from the Chinese. World Scientific Publishing Co., Inc., Teaneck, NJ, 1990.

[21] Clunie, J.G. On integral and meromorphic functions, J. London Math. Soc. 37 (1962), 17-27.

[22] Clunie, J.G. On a result of Hayman, J. London Math. Soc. 42 (1967), 389-392.

[23] Clunie, J.; Eremenko, A.; Rossi, J. on equilibrium points of logarithmic and Newtonian potentials. J. London Math. Soc. (2) 47 (1993), no. 2, 309-320.

[24] Csillag, P. Über ganze Funktionen, welche drei nicht verschwindende Ableitungen besitzen, Math. Ann. 110 (1935), 745-752. 
[25] Drasin, D. The inverse problem of the Nevanlinna theory. Acta Math. 138 (1976), no. 1-2, 83-151.

[26] Drasin, D. On Nevanlinna's inverse problem. Complex Variables Theory Appl. 37 (1998), no. $1-4,123-143$.

[27] Drasin, D. Quasiconformal modifications of functions having deficiency sum two. Ann. of Math. (2) 114 (1981), no. 3, 493-518.

[28] Drasin, D. Proof of a conjecture of F. Nevanlinna concerning functions which have deficiency sum two. Acta Math. 158 (1987), no. 1-2, 1-94.

[29] Drasin, D. The minimum modulus of subharmonic functions of order one and a method of Fryntov. J. London Math. Soc. (2) 54 (1996), no. 2, 239-250.

[30] Drasin, D. Meromorphic functions: progress and problems, Proc. Intl. Congr. Math., (Zürich, 1994) vol. 2, 828-835. Birkhäuser, Basel, 1995.

[31] Drasin, D. and Shea, D. Pólya peaks and the oscillation of positive functions. Proc. Amer. Math. Soc. 34 (1972), 403-411

[32] Drasin, D. and Weitsman, A. Meromorphic functions with large sums of deficiencies. Advances in Math. 15 (1975), 93-126.

[33] Edrei, A. Sums of deficiencies of meromorphic functions. J. Analyse Math. $14196579-107$

[34] Edrei, A. Sum of deficiencies of meromorphic functions. J. d'Analyse Math. 19 (1967), 53-74.

[35] Edrei, A. Solution of the deficiency problem for functions of small lower order. Proc. London Math. Soc. (3) 26 (1973), 435-445.

[36] Edrei, A. and Fuchs, W. H. J. Asymptotic behavior of meromorphic functions with extremal spread. I. Ann. Acad. Sci. Fenn. Ser A I Math. 2 (1976), 67-111.

[37] Edrei, A. and Fuchs, W. H. J. Asymptotic behavior of meromorphic functions with extremal spread. II. Ann. Acad. Sci. Fenn. Ser A I Math. 3 (1977), 141-168.

[38] Eremenko, A. The growth of entire and subharmonic functions on asymptotic curves. Siberian Math. J., 21, 5 (1981), 573-683.

[39] Eremenko, A. Meromorphic solutions of algebraic differential equations. Russian Math. Surveys, 37, 4 (1982), 61-95. Correction: 38, 6 (1983).

[40] Eremenko A. Deviations of meromorphic functions of finite lower order. (Russian) Teor.

Funktsiı̌ Funktsional. Anal. i Prilozhen. No. 40 (1983), 56-64.

[41] Eremenko, A. Defects and deviations of finite-order meromorphic functions. (Russian) Dokl.

Akad. Nauk Ukrain. SSR Ser. A 1985, no. 1, 18-21.

[42] Eremenko, A. Inverse problem of the theory of distribution of values for finite-order meromorphic functions. Siberian Math. J., 27, 3 (1987), 377-390.

[43] Eremenko, A. On the set of defect values of a finite-order entire function. Ukrainian Math. J., 39, 3 (1987), 225-228.

[44] Eremenko, A. Meromorphic functions with small ramification. Indiana Univ. Math. J. 42 (1993), no. 4, 1193-1218.

[45] Eremenko, A. A counterexample to the Arakelyan conjecture. Bull. Amer. Math. Soc. (N.S.) 27 (1992), no. 1, 159-164.

[46] Eremenko, A. An analogue of the defect relation for the uniform metric. Complex Variables Theory Appl. 34 (1997), no. 1-2, 83-97.

[47] Eremenko, A. An analogue of the defect relation for the uniform metric. II. Complex Variables Theory Appl. 37 (1998), no. 1-4, 145-159.

[48] Eremenko, A. Value distribution and potential theory. Proceedings of the International Congress of Mathematicians, Vol. II (Beijing, 2002), 681-690, Higher Ed. Press, Beijing, 2002.

[49] Eremenko, A. Transcendental meromorphic functions with three singular values. Illinois J. Math. 48 (2004), no. 2, 701-709.

[50] Eremenko, A., Langley, J., and Rossi, J. On the zeros of meromorphic functions of the form $f(z)=\sum_{k=1}^{\infty} a_{k} /\left(z-z_{k}\right)$. J. Anal. Math. 62 (1994), 271-286.

[51] Fedorov, M. A.; Grishin, A. F. Some questions of the Nevanlinna theory for the complex half-plane. Math. Physics, Analysis and Geometry, 1 (1998), 223-271.

[52] Frank, G. Eine Vermutung von Hayman über Nullstellen meromorpher Funktionen. Math. Z. 149 (1976), no. 1, 29-36.

[53] Frank, G. Über die Nullstellen von linearen Differentialpolynomen mit meromorphen Koeffizienten, Complex Methods on Partial Differential Equations, 39-48, Math. Res. 53, AkademieVerlag, Berlin 1989. 
[54] Frank, G. and Hellerstein, S. On the meromorphic solutions of nonhomogeneous linear differential equations with polynomial coefficients, Proc. London Math. Soc. (3) 53 (1986), 407-428.

[55] Frank, G. and Weissenborn, G. Rational deficient functions of meromorphic functions. Bull. London Math. Soc. 18 (1986), no. 1, 29-33.

[56] Fryntov, A. A counterexample concerning the maximum and minimum of a subharmonic function. Proc. Amer. Math. Soc. 122 (1994), no. 1, 97-103.

[57] Fryntov, A., Rossi, J. and Weitsman, A. On the longest arc relation for $\delta$-subharmonic functions. Complex Variables Theory Appl. 34 (1997), no. 1-2, 99-108.

[58] Fryntov, A., Rossi, J. and Weitsman, A. Circular means of fine Green's functions and the longest arc relation. Complex Variables Theory Appl. 37 (1998), no. 1-4, 211-224.

[59] Gleı̆zer, E. V. Meromorphic functions with zeros and poles in small angles. (Russian) Sibirsk. Mat. Zh. 26 (1985), no. 4, 22-37, 203.

[60] Gleǐzer, E. V. The growth of entire functions with zeros on a system of rays. (Russian) Ukrain. Mat. Zh. 38 (1986), no. 3, 297-302, 404.

[61] Gleı̌zer, E. V. Meromorphic functions with zeros and poles in small angles. II. (Russian) Sibirsk. Mat. Zh. 31 (1990), no. 2, 9-20, 223; translation in Siberian Math. J. 31 (1990), no. 2, 192-202

[62] Goldberg, A. A. Meromorphic functions. Mathematical analysis, Vol. 10 (Russian), pp. 5-97. Akad. Nauk SSSR Vsesojuz. Inst. Naučn. i Tehn. Informacii, Moscow, 1973.

[63] Goldberg, A. A. Nevanlinna's lemma on the logarithmic derivative of a meromorphic function. (Russian) Mat. Zametki 17 (1975), no. 4, 525-529.

[64] Goldberg, A. A.; Eremenko, A.; Sodin, M. L. Exceptional values in the sense of R. Nevanlinna and in the sense of V. P. Petrenko. I. (Russian) Teor. Funktsiu Funktsional. Anal. i Prilozhen. No. 47 (1987), 41-51; translation in J. Soviet Math. 48 (1990), no. 6, 648-655

[65] Goldberg, A. A.; Eremenko, A.; Sodin, M. L. Exceptional values in the sense of R. Nevanlinna and in the sense of V. P. Petrenko. II. (Russian) Teor. Funktsir Funktsional. Anal. i Prilozhen. No. 4 (1987), 58-70; translation in J. Soviet Math. 49 (1990), no. 2, 891-899

[66] Goldberg, A. A.; Grinštein, V. A. The logarithmic derivative of a meromorphic function. (Russian) Mat. Zametki 19 (1976), no. 4, 525-530.

[67] Goldberg, A. A.; Levin, B. Ya.; Ostrovskii, I. V. Entire and meromorphic functions. Complex analysis, I, 1-193, 254-261, Encyclopaedia Math. Sci., 85, Springer, Berlin, 1997.

[68] Govorov, N. V. Riemann's boundary problem with infinite index. Edited and with an introduction and an appendix by I. V. Ostrovskiu. Translated from the 1986 Russian original by Yu. I. Lyubarskiĭ. Birkhäuser Verlag, Basel, 1994. xii+252 pp.

[69] Gundersen, G. and Hayman, W. K. The strength of Cartan's version of Nevanlinna theory. Bull. London Math. Soc. 36 (2004), no. 4, 433-454.

[70] Hayman, W. The minimum modulus of large integral functions, Proc. London Math. Soc., 2 (1952) 469-512.

[71] Hayman, W. K. Picard values of meromorphic functions and their derivatives, Ann. of Math. 70 (1959), 9-42.

[72] Hayman, W. K. On the Valiron deficiencies of integral functions of infinite order. Ark. Mat. 10, 163-172. (1972).

[73] Hayman, W. K. The minimum modulus of integral functions of order one, J. Analyse Math., 28 (1975), 171-212.

[74] Hayman, W. K. On Iversen's theorem for meromorphic functions with few poles. Acta Math. 141 (1978), no. 1-2, 115-145.

[75] Hayman, W. K. An approximation theorem with applications to Iversen's theorem. Aspects of contemporary complex analysis (Proc. NATO Adv. Study Inst., Univ. Durham, Durham, 1979), pp. 209-223, Academic Press, London-New York, 1980.

[76] Hayman, W. K. On a meromorphic function having few poles but not tending to infinity along a path. Ann. Polon. Math. 39 (1981), 83-91.

[77] Hayman, W. K. Warings Problem für analytische Funktionen. Bayer. Akad. Wiss. Math.Natur. Kl. Sitzungsber. 1984, 1-13 (1985).

[78] Hayman, W. K.; Kjellberg, B. On the minimum of a subharmonic function on a connected set. Studies in pure mathematics, 291-322, Birkhäuser, Basel, 1983.

[79] Hayman, W. K. Subharmonic functions. Vol. 2. London Mathematical Society Monographs, 20. Academic Press, Inc. [Harcourt Brace Jovanovich, Publishers], London, 1989. 
[80] Hayman, W. K. Nevanlinna theory 1945-1995. XVIth Rolf Nevanlinna Colloquium, Eds.: Laine/Martio, Walter de Gryuyter and Co., Berlin, New York, 1996.

[81] Hennekemper, W. Über die Werteverteilung von $\left(f^{k+1}\right)^{(k)}$, Math. Z. 177 (1981), 375-380.

[82] Hinchliffe, J. D. On a result of Chuang related to Hayman's alternative, Comput. Methods Funct. Thy. 1 (2002), 293-297.

[83] Hinchliffe, J. D. The Bergweiler-Eremenko theorem for finite lower order, Result. Math. 43 (2003), 121-128.

[84] Ishizaki, K. Some remarks on results of Mues about deficiency sums of derivatives, Arch. Math. (Basel) 55 (1990), 374-379.

[85] Hinkkanen, A. A sharp form of Nevanlinna's second fundamental theorem. Invent. Math. 108 (1992), no. 3, 549-574.

[86] Hinkkanen, A. Iteration and the zeros of the second derivative of a meromorphic function. Proc. London Math. Soc. (3) 65 (1992), no. 3, 629-650

[87] Hinkkanen, A. Reality of zeros of derivatives of meromorphic functions. Ann. Acad. Sci. Fenn. Math. 22 (1997), no. 1, 21-38

[88] Hinkkanen, A. Zeros of derivatives of strictly non-real meromorphic functions. Ann. Acad. Sci. Fenn. Math. 22 (1997), no. 1, 39-74.

[89] Hinkkanen, A. Iteration, level sets, and zeros of derivatives of meromorphic functions. Ann. Acad. Sci. Fenn. Math. 23 (1998), no. 2, 317-388

[90] Hinkkanen, A. Zeros of derivatives of meromorphic functions with one pole. Complex Variables Theory Appl. 37 (1998), no. 1-4, 279-369.

[91] Hellerstein, S. and Shea, D. Minimal deficiencies for entire functions with radially distributed zeros. Proc. London Math. Soc. (3) 37 (1978), no. 1, 35-55.

[92] Hellerstein, S. and Williamson, J. Derivatives of entire functions and a question of Pólya. Trans. Amer. Math. Soc. 227 (1977), 227-249

[93] Hellerstein, S. and Williamson, J. Derivatives of entire functions and a question of Pólya. II. Trans. Amer. Math. Soc. 234 (1977), no. 2, 497-503.

[94] Hellerstein, S. and Williamson, J. Entire functions with negative zeros and a problem of R. Nevanlinna. J. Analyse Math. 221969 233-267.

[95] Hellerstein, S., Shen, Li Chien and Williamson, J. Reality of the zeros of an entire function and its derivatives. Trans. Amer. Math. Soc. 275 (1983), no. 1, 319-331.

[96] Hyllengren, A. Valiron deficient values for meromorphic functions in the plane. Acta Math. $12419701-8$.

[97] Khabibullin, B. N. Growth of entire functions with prescribed zeros and representation of meromorphic functions. (Russian. ) Mat. Zametki 73 (2003), no. 1, 120-134; translation in Math. Notes 73 (2003), no. 1-2, 110-124

[98] Khabibullin, B. N. The representation of a meromorphic function as the quotient of entire functions and Paley problem in $\overline{\mathbf{C}}^{n}$ : survey of some results. Mat. Fiz. Anal. Geom. 9 (2002), no. $2,146-167$.

[99] Kondratyuk, A. A. Fourier series and meromorphic functions, L'viv, Vyshcha shkola, 1988.

[100] Lang, S. Introduction to complex hyperbolic spaces. Springer-Verlag, New York, 1987.

[101] Lang, S. Number theory. III. Diophantine geometry. Encyclopaedia of Mathematical Sciences, 60. Springer-Verlag, Berlin, 1991. xiv+296 pp.

[102] Langley, J. K. On Hayman's alternative, Mathematika 32 (1985), 139-146.

[103] Langley, J. K. Proof of a conjecture of Hayman concerning $f$ and $f^{\prime \prime}$. J. London Math. Soc.

(2) 48 (1993), no. 3, 500-514.

[104] Langley, J. K. On second order linear differential polynomials, Result. Math. 26 (1994), $51-82$.

[105] Langley, J. K. A lower bound for the number of zeros of a meromorphic function and its second derivative, Proc. Edinburgh Math. Soc. 39 (1996), 171-185.

[106] Langley, J. K. The second derivative of a meromorphic function of finite order, Bulletin London Math. Soc. 35 (2003), 97-108.

[107] Langley, J. K. The zeros of $f f^{\prime \prime}-b$. Results Math. 44 (2003), no. 1-2, 130-140.

[108] Langley, J. K. Critical values of slowly growing meromorphic functions, Comput. Methods Funct. Theory. 2 (2002), 539-547.

[109] Langley, J. K. Nonreal zeros of higher derivatives of real entire functions of infinite order, J. d'Analyse 97 (2005), 357-396. 
[110] Levin B. Ja. and Ostrovskii, I. V. The dependence of the growth of an entire function on the distribution of zeros of its derivatives. Sibirsk. Mat. Zh. 1 (1960) 427-455. English transl., AMS Transl. (2) 32 (1963) 323-357.

[111] Lewis, J. and Wu, Jang-Mei. On conjectures of Arakelyan and Littlewood. J. Analyse Math. 50 (1988), 259-283.

[112] Marchenko, I. I.; Shcherba, A. I. On the magnitudes of deviations of meromorphic functions. (Russian) Mat. Sb. 181 (1990), no. 1, 3-24; translation in Math. USSR-Sb. 69 (1991), no. 1, 1-24

[113] Merenkov, S. Rapidly growing entire functions with three singular values, preprint, http://www.math.lsa.umich.edu/ merenkov/entire1.pdf

[114] Miles, J. Quotient representations of meromorphic functions. J. Analyse Math. 25 (1972), 371-388.

[115] Miles, J. On entire functions of infinite order with radially distributed zeros. Pacific J. Math. 81 (1979), no. 1, 131-157.

[116] Miles, J. Some examples of the dependence of the Nevanlinna deficiency upon the choice of origin. Proc. London Math. Soc. (3) 47 (1983), no. 1, 145-176.

[117] Miles, J. On the growth of meromorphic functions with radially distributed zeros and poles. Pacific J. Math. 122 (1986), no. 1, 147-167.

[118] Miles, J. A sharp form of the lemma on the logarithmic derivative. J. London Math. Soc.

(2) 45 (1992), no. 2, 243-254.

[119] Miles, J. and Shea, D. An extremal problem in value-distribution theory. Quart. J. Math. Oxford Ser. (2) 24 (1973), 377-383.

[120] Miles, J. and Shea, D. On the growth of meromorphic functions having at least one deficient value. Duke Math. J. 43 (1976), no. 1, 171-186.

[121] Mohonko A. Z. Characteristics of certain meromorphic functions. (Russian) Teor. Funkciu Funkcional. Anal. i Priložen. No. 14 (1971), 83-87.

[122] Mues, E. Über eine Defekt- und Verzweigungsrelation für die Ableitung meromorpher Funktionen, Manuscripta Math. 5 (1971), 275-297.

[123] Mues, E. Über die Nullstellen homogener Differentialpolynome, Manuscripta Math. 23 (1978), 325-341.

[124] Mues, E. Über ein Problem von Hayman, Math. Z. 164 (1979), 239-259.

[125] Mues E. and Steinmetz, N. The theorem of Tumura-Clunie for meromorphic functions, J. London Math. Soc. (2) 23 (1981), 113-122.

[126] F. Nevanlinna (see the old ref list).

[127] Nevo, S., Pang, X. and Zalcman, L. Picard-Hayman behavior of derivatives of meromorphic functions with multiple zeros, Electron. Res. Announc. Amer. Math. Soc. 12 (2006), 37-43.

[128] Osgood, C. F. A number theoretic-differential equations approach to generalizing Nevanlinna theory. Indian J. Math. 23 (1981), no. 1-3, 1-15.

[129] Osgood, C. F. Sometimes effective Thue-Siegel-Roth-Schmidt-Nevanlinna bounds, or better. J. Number Theory 21 (1985), no. 3, 347-389.

[130] Osgood, C. F. Simplified proofs of generalizations of the $n$-small function theorem. Analysis of one complex variable (Laramie, Wyo., 1985), 158-174, World Sci. Publishing, Singapore, 1987.

[131] Pang Xuecheng, Bloch's principle and normal criterion, Sci. China Ser. A 32 (1989), 782-791.

[132] Pang Xuecheng, On normal criterion of meromorphic functions, Sci. China Ser. A (33) (1990), 521-527.

[133] Petrenko, V. P. Growth of meromorphic functions (Russian) Izdat. pri Harkov. Gos. Univ. Izdat. Ob'ed. "Vyshcha shkola", Kharkov, 1978.

[134] Pólya, G. Über die Nullstellen sukzessiver Derivierten, Math. Z. 12 (1922), 36-60.

[135] Qiao, Jianyong. Two problems in the value distribution theory. Acta Math. Sinica 11 (1995), 4, 365-371.

[136] Ru, Min. Nevanlinna theory and its relation to Diophantine approximation. World Scientific Publishing Co., Inc., River Edge, NJ, 2001.

[137] Rubel, L. Entire and meromorphic functions. Springer-Verlag, New York, 1996.

[138] Sauer, A. Deficient rational functions and Ahlfors's theory of covering surfaces. Ark. Mat. 39 (2001), no. 1, 151-155.

[139] Saxer, W. Sur les valeurs exceptionelles des dérivées successives des fonctions méromorphes, C.R. Acad. Sci. Paris 182 (1926), 831-3.

[140] Saxer, W. Über die Picardschen Ausnahmewerte successiver Derivierten, Math. Z. 17 (1928), 206-227. 
[141] Shea, D. On the set where a meromorphic function is large. Complex analysis and its applications (Hong Kong, 1993), 95-106, Pitman Res. Notes Math. Ser., 305, Longman Sci. Tech., Harlow, 1994.

[142] Shea, D. F. and Sons, L.R. Value distribution theory for meromorphic functions of slow growth in the disk, Houston Math. J. 12 (1986), 249-266.

[143] Sheil-Small, T. On the zeros of the derivatives of real entire functions and Wiman's conjecture. Ann. of Math. (2) 129 (1989), no. 1, 179-193.

[144] Sons, L.R. Value distribution for unbounded functions in the unit disc, Complex Var. Theory Appl. 7 (1987), 337-431.

[145] Steinmetz, N. Über die Nullstellen von Differentialpolynomen, Math. Z. 176 (1981), 255-264.

[146] Steinmetz, N. Eine Verallgemeinerung des zweiten Nevanlinnaschen Hauptsatzes, J. für die reine u. angewandte Mathematik 368 (1986), 134-141.

[147] Steinmetz, N. On the zeros of $\left(f^{(p)}+a_{p-1} f^{(p-1)}+\ldots+a_{0} f\right) f$, Analysis 7 (1987), 375-389.

[148] Steinmetz, N. On the zeros of a certain Wronskian, Bull. London Math. Soc. 20 (1988), 525-531.

[149] O. Teichmüller, Eine Umkehrung des zweiten Hauptsatzes der Wertwerteilungslehre, Deutsche Math., 2 (1937) 96-107.

[150] Ter-Israeljan, L. A. A meromorphic function of zero order with a non-asymptotic defect value. (Russian) Mat. Zametki 13 (1973), 195-206. English translation: Math. Notes 13 (1973), $116-123$.

[151] Tohge, K. Logarithmic derivatives of meromorphic or algebroid solutions of some homogeneous linear differential equation, Analysis (Munich) 19 (1999), 273-297.

[152] Tumura, Y. On the extensions of Borel's theorem and Saxer-Csillag's theorem, Proc. Phys. Math. Soc. Japan (3) 19 (1937), 29-35.

[153] Vojta, P. Diophantine approximations and value distribution theory. Lecture Notes in Mathematics, 1239. Springer-Verlag, Berlin, 1987.

[154] Wang, Y. F. On Mues' conjecture and Picard values, Sci. China Ser. A 36 (1993), 28-35.

[155] Wang Y. F. and Fang, M. Picard values and normal families of meromorphic functions with multiple zeros, Acta Math. Sinica New Ser. 14 (1998), 17-26.

[156] Wang, Y. F., Yang, C. C. Yang, Lo. On the zeros of $\left(f^{(k)}\right)^{n} f-a$, Kexue Tongbao 38 (1993), $2215-2218$

[157] Weitsman, A. A theorem on Nevanlinna deficiencies. Acta Math. 128 (1972), no. 1-2, 41-52.

[158] Weitsman, A. Meromorphic functions with maximal deficiency sum and a conjecture of F. Nevanlinna. Acta Math. 1231969 115-139.

[159] Yamanoi, K. Defect relation for rational functions as targets. With errata by the author. Forum Math. 17 (2005), no. 2, 169-189.

[160] Yamanoi, K. The second main theorem for small functions and related problems. Acta Math. 192 (2004), no. 2, 225-294.

[161] Yang, Lo. Precise estimate of total deficiency of meromorphic derivatives, J. d'Analyse Math. 55 (1990), 287-296.

[162] Yang, Lo. Value distribution theory. Translated and revised from the 1982 Chinese original. Springer-Verlag, Berlin; Science Press, Beijing, 1993.

[163] Zalcman, L. Normal families: new perspectives, Bull. Amer. Math. Soc. (N.S.) 35 (1998), 215-230.

[164] Zhang Z.-L. and Li, W. Tumura-Clunie's theorem for differential polynomials, Complex Variables 25 (1994), 97-105. 